\title{
Basis to Demonstrate Compliance with the National Emission Standards for Hazardous Air Pollutants for the Stand-off Experiments Range
}

Michael Sandvig James Sterbentz Arthur Rood James Jones

January 2011

The INL is a U.S. Department of Energy National Laboratory operated by Battelle Energy Alliance 

INL/EXT-10-20220

Revision 1

\title{
Basis to Demonstrate Compliance with the National Emission Standards for Hazardous Air Pollutants for the Stand-off Experiments Range
}

\author{
Michael Sandvig \\ James Sterbentz \\ Arthur Rood \\ James Jones
}

January 2011

\author{
Idaho National Laboratory \\ National and Homeland Security \\ Idaho Falls, Idaho 83415
}

http://www.inl.gov

Prepared for the

U.S. Department of Energy

Office of Nuclear Energy

Under DOE Idaho Operations Office

Contract DE-AC07-05ID14517 



\section{SUMMARY}

This report provides the proposed methodology for evaluating diffuse radionuclide emissions for the outdoor linear accelerator (linac) operations at the Idaho National Laboratory (INL) Stand-off Experiments Range (SOX). In accordance with the National Emission Standards for Hazardous Air Pollutants (NESHAP), 40 CFR 61 Subpart H, "National Emission Standards for Emissions of Radionuclides Other Than Radon from Department of Energy Facilities," section 61.96(b) and the 1995 Memorandum of Understanding (MOU) (EPA 1995), U.S. Department of Energy (DOE) is required to obtain U.S. Environmental Protection Agency (EPA) approval of alternate procedures for estimating source terms, and specifically per the MOU, for diffuse emission sources.

Photonuclear active interrogation research at INL uses high-energy x-ray (photon) radiation (bremsstrahlung), up to a nominal $60 \mathrm{MeV}$, to interrogate inspection objects up to several thousand meters away. This research supports the Department of Defense, Department of Homeland Security, and Department of Energy's research and evaluation of deployment issues associated with outdoor inspections of configurations of shielded nuclear material. The photons are generated by open installation electron linacs. Some of the photons create airborne radioactive gaseous isotopes as the photon beam transits the air to the inspection object. High-energy linacs in this INL program are not enclosed in a shielded building or other enclosure that confines the radioactive isotopes.

Calculation of the source terms is performed using methods principally from the National Council on Radiation Protection and Measurements (NCRP) report 144, "Radiation Protection for Particle Accelerator Facilities" and Monte Carlo N-Particle eXtended (MCNPX) codes. The bounding limit at the SOX Range is a nominal $60 \mathrm{MeV}$ maximum electron energy and 100 microamps average beam current. A range of linac energies and power up to that limit is expected, and source terms have been calculated based on anticipated operating configurations. In accordance with Federal regulations 40 CFR 61 subpart $\mathrm{H}$, the atmospheric transport and radiological dose code, CAP88-PC-Version 3 (EPA 2007), is used to calculate effective dose equivalent (EDE) to a maximally exposed individual (MEI) located off the INL Site boundary using the methodology described in the 2009 Radiological NESHAP report (DOE-ID 2010). The EDE is calculated prior to any linac operation at the SOX Range to maintain the dose to the MEI less than 0.1 mrem per year.

Prior to any linac operation at the SOX Range, a beam authorization sheet must be prepared and approved by the Accelerator Safety Officer. The beam authorization sheet identifies the linac to be operated, the maximum beam energy and average beam current (maximum SOX Range values for these parameters are $60 \mathrm{MeV}$ and 100 microamperes, respectively), the type of converter and collimator used, and the maximum hours of operation for the specified parameters. The Accelerator Safety Officer uses this information to calculate the maximum radioactive source term that could be generated for the particular linac operation. This source term is then analyzed using CAP88 to determine the EDE that would result to the MEI. The Accelerator Safety Officer tallies the cumulative EDE for all linac operations during a calendar year. If the cumulative dose to the MEI for proposed linac operations when summed with all other approved linac operations within that calendar year is less than $0.1 \mathrm{mrem}$, then the beam authorization sheet is approved, and the proposed linac operation may commence. Any proposed operation of a linac that would result in a cumulative EDE that equals or exceeds 0.1 mrem in a calendar year would not be approved. This process controls the dose to the MEI resulting from SOX Range operations to less than $1 \%$ of the NESHAP standard, the threshold that would require an EPA approval to construct. As a comparison, in 2009, radionuclide air emissions and the resulting EDE to the MEI from all operations at the INL Site was $0.0687 \mathrm{mrem} / \mathrm{yr}, 0.69 \%$ of the 10 -mrem standard (DOE/ID-10890 2010). The cumulative EDE from SOX Range and other INL operations would remain far below the 10 mrem regulatory limit. The source terms are calculated without any credit for pollution control equipment, as it is not practical for the types of linac operations at the SOX Range. 
The average electron beam power and operating time are measured, recorded, and used to calculate radionuclide emissions for inclusion in INL's annual NESHAP report. 


\section{CONTENTS}

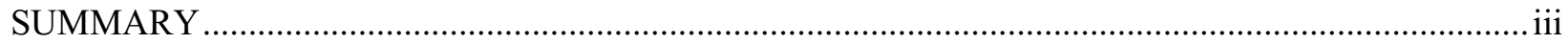

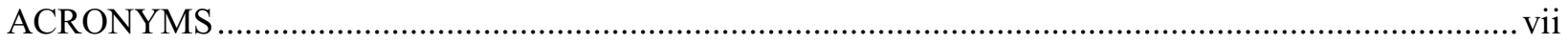

1. OVERVIEW OF THE ACTIVE INTERROGATION

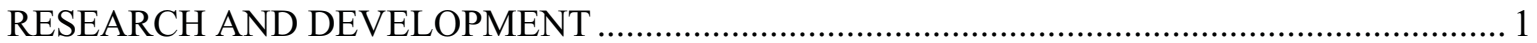

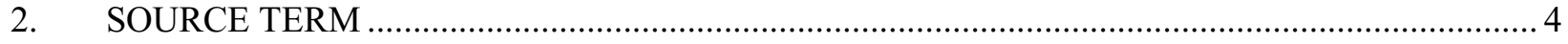

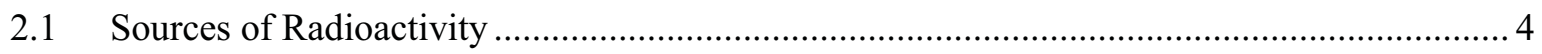

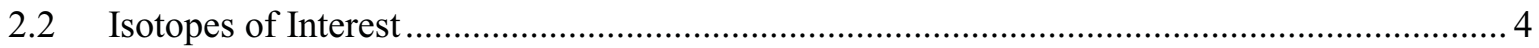

3. CALCULATION OF RADIOACTIVITY USING NCRP-144 METHODS ................................ 5

3.1 Beam Length in Open Installations.................................................................................. 5

3.2 Determining Activity Production Rate from the Maximum Saturated Activity ..................... 6 3.2.1 Determining the Production of Argon-41 ............................................................. 7

3.3 Assumptions and Adjustment Factors (Ground, Convertor, Energy Yield) .......................... 8

3.4 Calculation of Radionuclide Production Rates ................................................................ 11

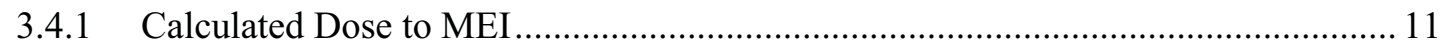

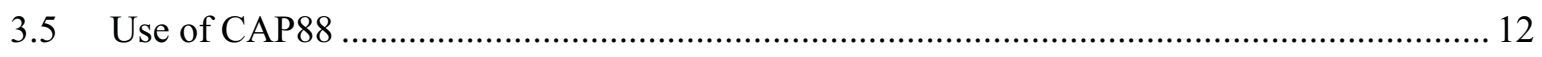

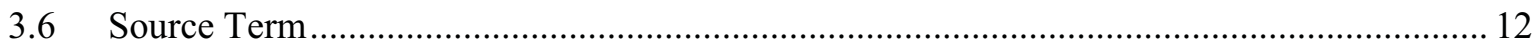

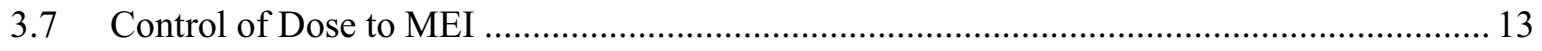

3.8 Annual NESHAP Report of Dose to MEI..................................................................... 15

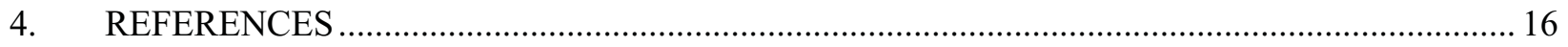

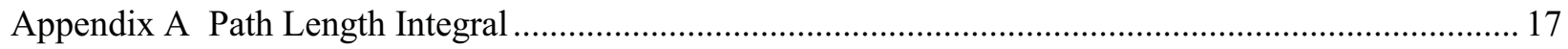

Appendix B Bremsstrahlung Photon Flux versus Electron Energy (MeV) ......................................... 21

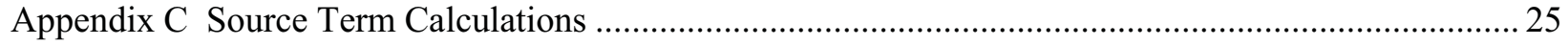

Appendix D Relative Capture Fractions for Thermal Neutrons in Isotopes of Air ................................ 35

Appendix E Neutrons Production from Thick Targets (Convertor) …................................................... 39

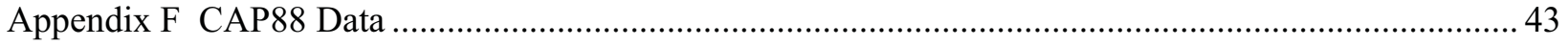




\section{FIGURES}

Figure 1. Linac example with inspection object and monostatic and bistatic detector configurations.

Figure 2. Photon flux field at $60 \mathrm{MeV}$ maximum electron beam energy and $100 \mu \mathrm{A}$ with high $\mathrm{Z}$ convertor in the linac, no collimator or ground plane.

Figure 3. Photon flux field at $60 \mathrm{MeV}$ electron beam energy and $100 \mu \mathrm{A}$ using air as the convertor, without a collimator or ground plane.

Figure 4. Bremsstrahlung distribution curves at various maximum electron beam energies. 10

\section{TABLES}

Table 1. Radiation yields and convertor factors for various materials extracted from Figure 3.3 of NCRP-144.

Table 2. Threshold values for selected photonuclear reactions and radiation fraction values for 30 and $60 \mathrm{MeV}$ linacs. 10

Table 3. operational configurations and calculated MEI dose and operating hour results. 13

Table 4. Calculated annual MEI dose. 14 


\section{ACRONYMS}

\begin{tabular}{|c|c|}
\hline$\mu$ & linear attenuation coefficient \\
\hline$\mu \mathrm{A}$ & microamperes \\
\hline$\mu \mathrm{m}$ & micrometer \\
\hline $\mathrm{Ar}$ & Argon \\
\hline $\mathrm{Be}$ & beryllium \\
\hline $\mathrm{Bq}$ & Becquerel \\
\hline $\mathrm{C}$ & carbon \\
\hline $\mathrm{Cl}$ & Chlorine \\
\hline $\mathrm{D}$ & dose \\
\hline $\mathrm{H}$ & hydrogen \\
\hline INL & Idaho National Laboratory \\
\hline $\operatorname{Ir}$ & iridium \\
\hline $\mathrm{keV}$ & kilo electron volts \\
\hline linac & linear accelerator \\
\hline MCNPX & Monte Carlo N-Particle eXtended \\
\hline MEI & maximally exposed individual \\
\hline $\mathrm{MeV}$ & mega electron volts \\
\hline mrem & millirem \\
\hline $\mathrm{N}$ & nitrogen \\
\hline NCRP & National Council on Radiation Protection and Measurements \\
\hline NESHAP & National Emission Standards for Hazardous Air Pollutants \\
\hline $\mathrm{O}$ & oxygen \\
\hline $\mathrm{Pb}$ & lead \\
\hline PR & production rate \\
\hline $\mathrm{S}$ & saturated activity \\
\hline SOX & Stand-off Experiments \\
\hline $\mathrm{W}$ & tungsten \\
\hline YF & yield factor \\
\hline Z & atomic number \\
\hline
\end{tabular}




\section{Basis to Demonstrate Compliance with the National Emission Standards for Hazardous Air Pollutants for the Stand-off Experiments Range}

\section{OVERVIEW OF THE ACTIVE INTERROGATION RESEARCH AND DEVELOPMENT}

Photonuclear active interrogation research at INL uses high-energy x-ray (photon) radiation (bremsstrahlung) to interrogate threat objects to detect, identify, and characterize shielded fissionable materials, including uranium and plutonium that may be contained in the threat objects. In this work, the threat objects vary in size from small shipping drums to large, full-size cargo containers that may be empty or filled with different shield materials. Shield materials used in this work are chosen based upon their potential ability to challenge and/or defeat the photonuclear active interrogation process and include low-atomic number $(Z)$ materials, such as plastic or borated plastic (which attenuate neutron radiation) and high- $Z$ materials such as iron, lead, or tungsten (which attenuate photon radiation).

The interrogating photon radiation used in this work is produced from high-energy linacs. In these devices, electrons with nominal energies of up to $60 \mathrm{MeV}$, depending upon the configuration, are directed against a converter such as carbon, aluminum, copper, tantalum, tungsten, or air, which causes the electrons to decelerate and lose energy. This process results in the creation of high-energy photons. The photons are produced in a continuous energy spectrum, with small numbers produced at the very highest energy, corresponding to the maximum electron beam energy, and progressively more photons produced at lower energies. Typically a thin "window" is used to allow the electron beam to exit the linac while maintaining the vacuum in the system before it strikes the converter. The linacs almost exclusively operate as pulsed machines and generate photon pulses on the order of 5 microseconds in duration with repetition rates typically in the range of 60 to $120 \mathrm{~Hz}$.

Linacs are commonly used in medical, commercial/industrial, university, and government laboratories throughout the United States and the world. In the medical field, linacs are used for radiation therapy and for surgical equipment and materials sterilization. In the commercial/industrial world, linacs are used for food safety inspection and treatment, water treatment, material manufacturing, large and/or dense object $\mathrm{x}$-ray inspections, etc. Universities use linacs for anything from the nature-of-matter basic research to assessing the age of ancient artifacts. Government laboratories use linacs in ways similar to universities, but have also used them to conduct research and development for waste characterization, material identification, and national defense needs. The uses here are not all inclusive, but are provided to highlight the fact that linac machines are relatively common. Most major cities in the U.S. have at least one, though linac use is typically conducted indoors.

To better understand electron linacs and the effects of their accelerated particles, there are two characteristics that are important: the energy level and the current of the electrons. The energy level determines the types of possible interactions with matter. The current determines the interaction rate or number of interactions per unit time. While the primary interaction of electrons with matter is ionization and heating, there is a process that is referred to as bremsstrahlung where the electron releases its excess energy as electromagnetic waves (or photons) commonly called x-rays. The difference between the photons that are generated by an x-ray machine in a dental office and the photons used for INL's research and development is that the dental office photons are of the order of tens of $\mathrm{keV}$ and those at INL are about 1,000 times more energetic or tens of $\mathrm{MeV}$. The difference between the $\mathrm{keV}$ and $\mathrm{MeV}$ photons is that, as the energy increases, the electromagnetic wave length shortens, causing different interaction mechanisms in various materials. 
The linacs used at INL's Stand-off Experiments (SOX) Range are modest in size and capable of being transported in small cargo trucks or containers. The linacs are comparable to those used for photon and electron beam therapy in a hospital or cancer center. They contrast with the very large, complex, and very high-energy linacs found at major accelerator facilities. Historic use of linacs at INL has been at low energies and current settings that do not result in photonuclear reactions in the air.

Unshielded photon radiation from high energy linacs tend to be forward focused and can travel very long distances. This effect proves beneficial in measurement situations focused at interrogating objects long distances from the linac (often called stand-off interrogation).

Part of the proposed research may include methods to make the system more radiation safe. A collimator may be used during the research to modify the beam generated by the linac. The collimator is essentially a radiation shield that attenuates the incident radiation emitted from the linac. The collimator aperture is in line with the primary electron beam. This allows a desirable component of the generated radiation to continue downrange without interacting with the collimation materials. The collimator is also a key topical area in research and development efforts to optimize the high-energy linac parameters. The collimator can substantially reduce off-axis dose rates when a converter is integrated with the linac at the end of the electron beam. The collimator effectively reduces the broad beam in the forward direction, leaving a narrower unaffected beam and minimizes the off-axis radiation from the converter. This reduces the overall width of the radiation area and the resulting photonuclear reactions in the air. The residual linac photon beam is best characterized as a narrow cone gradually expanding from the point of origin (collimator).

When energetic photons interact with materials, they have the potential to cause the ejection of neutrons, protons, and other charged particles from atomic nuclei via photonuclear reactions. These include photo-neutron $(\gamma, n)$, photon-dual neutron $(\gamma, 2 n)$, and photon-proton $(\gamma, p)$ reactions. Giant dipole resonance (giant resonance) is the photonuclear mechanism that will dominate reactions from the INL linac photons.

In fissionable materials, including uranium and plutonium, incident energetic photons can induce fission via the photofission ( $\gamma$, fission) reaction. The photon energy thresholds and reaction probabilities for these reactions vary from isotope to isotope. Photoneutrons and neutrons produced during fission occur nearly simultaneously with the reactions and are referred to as prompt neutrons.

In a similar fashion, prompt gamma rays can also be generated within an inspection object subjected to irradiation from energetic photons. In addition to these prompt radiation emissions, a second source of neutrons and photons exists in inspection objects when fission takes place. This secondary radiation source is a result of the decay of the fission products created during fission. Nearly all radioactive fission products will emit gamma rays as they decay; these emissions are referred to as delayed gamma rays and they are generated in relation to the decay rates of the fission products. A small subset of fission products also yields neutrons during the decay processes; these are referred to as delayed neutrons.

Unique differences exist in the energy, emission rates, and emission properties between prompt and delayed radiations among different materials. Photonuclear active interrogation exploits these unique signatures to detect, identify, and characterize different fissionable materials. Prompt and delayed photofission neutrons can remain in a test object for short periods of time (milliseconds) after each linac photon pulse. To measure these signatures, special detector systems must be employed that are simultaneously capable of withstanding the radiation fields generated when the linac pulses and, also, of achieving very sensitive detection efficiencies for the delayed radiation products. See Figure 1 for a simple representation of the linac detector systems and inspected object. 


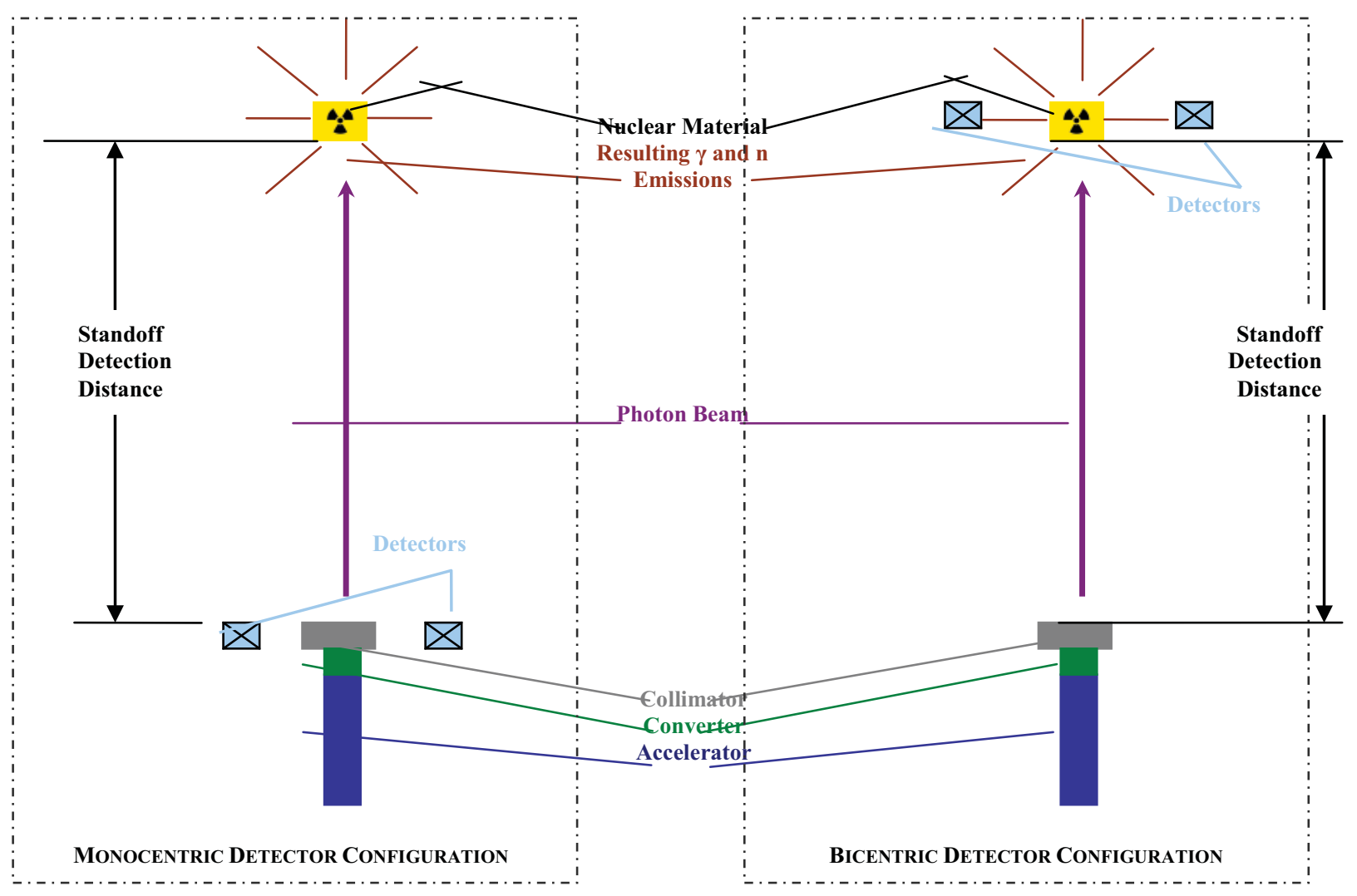

Figure 1. Linac example with inspection object and monostatic and bistatic detector configurations. 


\section{SOURCE TERM \\ 2.1 Sources of Radioactivity}

The principal source of radioactivity from INL linac operations is from photonuclear interactions with atoms in the air, primarily ${ }^{16} \mathrm{O},{ }^{14} \mathrm{~N},{ }^{12} \mathrm{C}$, and ${ }^{40} \mathrm{Ar}$. As previously noted, linac photons have the potential to cause the ejection of neutrons, as well as protons and other charged particles, from atomic nuclei.

The next most important source of radioactivity in air is from thermal neutron capture (such as creation of ${ }^{41} \mathrm{Ar}$ from ${ }^{40} \mathrm{Ar}$ ). The source of these thermal neutrons is from the neutrons ejected by the giant resonance mechanism in the air and converter. They have an energy spectra compared to the fission spectra that averages about $1-3 \mathrm{MeV}$. Activation of air by interaction with high-energy neutrons $(>20$ $\mathrm{MeV}$ neutron energy) is not an important source of radioactivity unless a linac is operated at electron beam energies greater than $100 \mathrm{MeV}$. INL linacs will not be operated at energies greater than $60 \mathrm{MeV}$, so high-energy neutron activation is not significant.

\subsection{Isotopes of Interest}

The airborne radionuclides of significance for environmental release are ${ }^{13} \mathrm{~N},{ }^{15} \mathrm{O},{ }^{11} \mathrm{C}$, and ${ }^{41} \mathrm{Ar}$. These radionuclides contribute approximately $99 \%$ of the effective dose. Of lesser importance are ${ }^{39} \mathrm{Cl}$, ${ }^{38} \mathrm{Cl},{ }^{39} \mathrm{Ar},{ }^{3} \mathrm{H}$, and ${ }^{7} \mathrm{Be}$. With the exception of ${ }^{39} \mathrm{Ar},{ }^{3} \mathrm{H}$, and ${ }^{7} \mathrm{Be}$, the half-lives of these radionuclides are relatively short. All isotopes of interest and their half-lives are appropriately modeled by CAP88. 


\section{CALCULATION OF RADIOACTIVITY USING NCRP-144 METHODS}

The production of airborne radioactivity may be analyzed using calculated yields listed in Table 6.4 of NCRP report 144, "Radiation Protection for Particle Accelerator Facilities." The table provides the yield factor that may be used to obtain the saturated activity $(\mathrm{S})$ in Becquerel (Bq) per unit electron beam power $(\mathrm{kW})$ and per unit bremsstrahlung path length ( $\mathrm{m}$ - meters of air).

For example, the yield factor (YF) for ${ }^{13} \mathrm{~N}=1.1 \times 10^{8} \mathrm{~Bq} \mathrm{~kW}^{-1} \mathrm{~m}^{-1}$.

Beam power in kilowatts $(\mathrm{kW})$ is determined by multiplying the maximum electron beam energy in $\mathrm{MeV}$ by the average electron beam current in $\mu \mathrm{A}$ and dividing by 1000 to convert watts into $\mathrm{kW}$. The following example is for a $60 \mathrm{MeV}$ linac operating at $100 \mu \mathrm{A}$.

Power $=60 \mathrm{MeV} \times 100 \mu \mathrm{A} / 1000=6 \mathrm{~kW}$

The photon path length in air at a typical accelerator facility is generally very short, in the range of a few meters to 10 meters. The generation of airborne radioactivity by the photon radiation ceases once it begins striking the shield wall. The path length is taken to be the distance from where the electron beam strikes the bremsstrahlung converter (either an electron convertor or linac component) and the shield wall. The saturated activity for ${ }^{13} \mathrm{~N}$ within the shielded facility assuming $6 \mathrm{~kW}$ power and 5 meter path length is calculated as follows:

$\mathrm{S}=1.1 \times 10^{8} \mathrm{~Bq} \mathrm{~kW}^{-1} \mathrm{~m}^{-1} \times 6 \mathrm{~kW} \times 5 \mathrm{~m}=3.3 \times 10^{9} \mathrm{~Bq}$

The concept of saturation is useful for an enclosed installation or room where equilibrium can be reached between the production and decay rates of individual radionuclides. However, when applied to an open installation with short run times, such as the SOX Range, it becomes less instructive due to the variable atmospheric conditions to which this type of configuration is exposed. For the SOX Range saturation rate is only used in the determination of production rates.

\subsection{Beam Length in Open Installations}

The photon path length is significantly longer for the open installation used at INL. The path length is potentially thousands of meters unless a backstop such as a hill or shield wall is encountered. In this case, the photon flux is greatly affected by the air acting as shielding. The radioactivity yield in the first meter of the photon path would be significantly higher than at the $1000^{\text {th }}$ meter. Therefore, the yield for each increment along the path length must take into account the reduction of the photon flux based on the shielding effect of air. The reduction of the photon flux from the original strength to any distance (s) can be determined by multiplying the original flux by $\mathrm{e}^{-\mu \mathrm{s}}$, where $\mu$ is the linear attenuation coefficient. The following equation may be substituted for the beam path length and solved for a particular path length to integrate the effect of air as shielding: $\int \mathrm{e}^{-\mu \mathrm{s}} \mathrm{ds}$.

The linear attenuation coefficient $(\mu)$ may be determined by taking the mass attenuation coefficient and multiplying by the density of air. A selected single value of the mass attenuation coefficient $(\mu \mathrm{m})$ for photons in the range of $10 \mathrm{MeV}$ through $60 \mathrm{MeV}$ is $0.016 \mathrm{~cm}^{2} / \mathrm{g}$. This value was taken from the NIST "Tables of X-Ray Mass Attenuation Coefficients and Mass Energy-Absorption Coefficients" (http://physics.nist.gov/PhysRefData/XrayMassCoef/cover.html). This is the value for a $40 \mathrm{MeV}$ photon, which is the minimum cross section through air. This value significantly underestimates the attenuation from air as the photon energy decreases to $1 \mathrm{MeV}$ and less. The density of air at 1,500 meters elevation, the altitude at the INL, is $0.001056 \mathrm{~g} / \mathrm{cm}^{3}$ compared to $0.0012 \mathrm{~g} / \mathrm{cm}^{3}$ at sea level. The linear attenuation coefficient $(\mu)$ is the product of the mass attenuation coefficient and the density of air. The value of the linear attenuation coefficient at 1500 meters is $1.69 \times 10^{-5} / \mathrm{cm}$.

The following example is used for clarity. Assume a distance from the linac to the shield wall of 500 meters in a large (unrealistic) shielded facility. The saturated activity for ${ }^{13} \mathrm{~N}$ within the shielded 
facility assuming $6 \mathrm{~kW}$ power and integrating $\int \mathrm{e}^{-\mu \mathrm{s}} \mathrm{ds}$ from zero to 500 meters as the path length is calculated as follows:

$\mathrm{S}=1.1 \times 10^{8} \mathrm{~Bq} \mathrm{~kW}^{-1} \mathrm{~m}^{-1} \times 6 \mathrm{~kW} \times \int \mathrm{e}^{-\mu s} \mathrm{ds}($ see Appendix A)

$\mathrm{S}=1.1 \times 10^{8} \mathrm{~Bq} \mathrm{~kW}{ }^{-1} \mathrm{~m}^{-1} \times 6 \mathrm{~kW} \times 338 \mathrm{~m}$

$\mathrm{S}=2.2 \times 10^{11} \mathrm{~Bq}$

Assume the same example with an infinite distance for beam length. The saturated activity for ${ }^{13} \mathrm{~N}$ within the shielded facility assuming $6 \mathrm{~kW}$ power and integrating $\int \mathrm{e}^{-\mu \mathrm{s}} \mathrm{ds}$ from zero to $\infty$ meters as the path length is calculated as follows:

$\mathrm{S}=1.1 \times 10^{8} \mathrm{~Bq} \mathrm{~kW}^{-1} \mathrm{~m}^{-1} \times 6 \mathrm{~kW} \times \int \mathrm{e}^{-\mu \mathrm{s}} \mathrm{ds} \mathrm{m}$

$\mathrm{S}=1.1 \times 10^{8} \mathrm{~Bq} \mathrm{~kW}^{-1} \mathrm{~m}^{-1} \times 6 \mathrm{~kW} \times 592 \mathrm{~m}$

$\mathrm{S}=3.9 \times 10^{11} \mathrm{~Bq}$

This saturated activity result converges on this maximum at about 2500 meters of the photon path length. This concept is useful for open installations for determining maximum production rates given the power of the linac. It may also be used for determining the average saturated activity concentration in a virtual unshielded box that has mixing and no dispersion.

\subsection{Determining Activity Production Rate from the Maximum Saturated Activity}

For radioactivity, saturation is achieved when the rate of decay is equal to the build-up. That is, one atom is lost to decay and one atom replaces it. The unit $\mathrm{Bq}$ is equal to a single disintegration per second. It follows that if a linac has a saturated activity of $3.9 \times 10^{11} \mathrm{~Bq}$, it has a production rate $(\mathrm{PR})$ of $3.9 \times 10^{11}$ atoms $\left({ }^{13} \mathrm{~N}\right)$ per second. The operating time at a particular power can be used to determine the number of radioactive atoms produced. For example, the number of ${ }^{13} \mathrm{~N}$ atoms produced for a 3 minute run of the linac at $6 \mathrm{~kW}$ would be as follows:

$\mathrm{S}=1.1 \times 10^{8} \mathrm{~Bq} \mathrm{~kW}^{-1} \mathrm{~m}^{-1} \times 6 \mathrm{~kW} \times \int \mathrm{e}^{-\mu \mathrm{s}} \mathrm{ds} \mathrm{m}$

$\mathrm{S}=1.1 \times 10^{8} \mathrm{~Bq} \mathrm{~kW} \mathrm{~kW}^{-1} \times 6 \mathrm{~kW} \times 592 \mathrm{~m}$

from Section 3.1

$\mathrm{S}=3.9 \times 10^{11} \mathrm{~Bq}$

$\mathrm{PR}=3.9 \times 10^{11}$ atoms $/ \mathrm{sec}$

Production $(\mathrm{N})=\mathrm{PR} \times$ time

$\mathrm{N}=3.9 \times 10^{11}$ atoms $/ \mathrm{sec} \times 180 \mathrm{sec}$

$\mathrm{N}=7.02 \times 10^{13}$ atoms

The activity may be determined by multiplying the number of atoms by $\lambda$, which is 0.693 divided by the half-life $(\mathrm{A}=\mathrm{N} \lambda)$. The production may then be expressed in units of activity. The activity production for the ${ }^{13} \mathrm{~N}$ example assuming a 180 second run would be: 
$\mathrm{S}=1.1 \times 10^{8} \mathrm{~Bq} \mathrm{~kW}^{-1} \mathrm{~m}^{-1} \times 6 \mathrm{~kW} \times \int \mathrm{e}^{-\mu \mathrm{s}} \mathrm{ds} \mathrm{m}$

$\mathrm{S}=1.1 \times 10^{8} \mathrm{~Bq} \mathrm{~kW}^{-1} \mathrm{~m}^{-1} \times 6 \mathrm{~kW} \times 592 \mathrm{~m}$

$\mathrm{S}=3.9 \times 10^{11} \mathrm{~Bq}$

$\mathrm{PR}=3.9 \times 10^{11}$ atoms $/ \mathrm{sec}$

Production $=\mathrm{PR} \times$ time

Production $=3.9 \times 10^{11}$ atoms $/ \mathrm{sec} \times 180 \mathrm{sec}$

Production $=7.02 \times 10^{13}$ atoms

$\mathrm{A}=\mathrm{N} \lambda$

$\mathrm{A}=7.02 \times 10^{13}$ atoms $\times 0.693 / 10 \mathrm{~min}$

$\mathrm{A}=7.02 \times 10^{13}$ atoms $\times 0.693 / 600 \mathrm{sec}$

$\mathrm{A}=8.1 \times 10^{10} \mathrm{~Bq}$

\subsubsection{Determining the Production of Argon-41}

Neutrons are created by photonuclear reactions in the convertor and during the production of ${ }^{13} \mathrm{~N},{ }^{15} \mathrm{O}$, ${ }^{11} \mathrm{C},{ }^{38} \mathrm{Cl}$, and ${ }^{39} \mathrm{Ar}$ in the air. While other minor sources of neutrons exist, the addition of these sources is not a significant contribution to the total neutron production. A conservative assumption has been made in this analysis that all of the neutrons created slow down and become thermal energy neutrons. The thermal neutrons are captured by various isotopes in air, predominately nitrogen, in proportion to the number of atoms in air and the cross section for absorption of thermal neutrons. An ${ }^{41} \mathrm{Ar}$ atom is created when a neutron is captured in an ${ }^{40} \mathrm{Ar}$ atom. ${ }^{40} \mathrm{Ar}$ captures a small fraction $(0.035)$ of all neutrons generated by the photonuclear reactions (see Appendix D). This factor is applied to the neutron production in the air and from the convertor. The neutron generation at the convertor is isotropic, distributing neutrons in all directions. About half of these neutrons are absorbed into the ground below the convertor and along the photon beam path.

Example: The neutron production rate for a high $\mathrm{Z}$ convertor (such as tungsten) is about $2.3 \times 10^{12}$ neutrons per second per $\mathrm{kW}$ power at $60 \mathrm{MeV}$ electron beam energy. This value is taken from Figure 3.12 of NCRP-144. This corresponds to a neutron production rate of $1.3 \times 10^{13}$ neutrons per second at $6 \mathrm{~kW}$ power. See Appendix E. The ${ }^{41} \mathrm{Ar}$ atom production rate for this convertor is equal to:

$\mathrm{PR}($ atoms $/ \mathrm{sec}){ }^{41} \mathrm{Ar}=1.38 \times 10^{13}$ neutrons $/ \mathrm{sec} \times 0.035^{41} \mathrm{Ar}$ atoms/neutron $\mathrm{x} 0.5$

$\mathrm{PR}($ atoms $/ \mathrm{sec}){ }^{41} \mathrm{Ar}=2.42 \times 10^{11}{ }^{41} \mathrm{Ar}$ atoms $/$ second.

Production $(\mathrm{N})=\mathrm{PR} \times$ time

Activity $=\mathrm{N} \times \lambda$

Note: $\lambda=.693 / \mathrm{T}^{1} / 2=.693 /$ half-life $=.693 / 1.827$ hours $=0.693 / 6577$ seconds

The neutron production rate of ${ }^{41} \mathrm{Ar}$ from photonuclear reactions in the air may be calculated by adding together the production rates of ${ }^{13} \mathrm{~N},{ }^{15} \mathrm{O},{ }^{11} \mathrm{C},{ }^{38} \mathrm{Cl}$, and ${ }^{39} \mathrm{Ar}$ and assuming one neutron is created from the creation of these radionuclides. The production values for these neutron sources are located in row 13 of the source term spreadsheets in Appendix C. The activity production rate of ${ }^{41} \mathrm{Ar}$ is calculated in the same manner as the example above.

The total production rate for ${ }^{41} \mathrm{Ar}$ may be calculated by adding together the neutron production rates from the convertor and the production of ${ }^{13} \mathrm{~N},{ }^{15} \mathrm{O},{ }^{11} \mathrm{C},{ }^{38} \mathrm{Cl}$, and ${ }^{39} \mathrm{Ar}$ then multiply by the ${ }^{40} \mathrm{Ar}$ neutron capture fraction. 


\subsection{Assumptions and Adjustment Factors (Ground, Convertor, Energy Yield)}

The radiation flux pattern of these open installation high energy linacs is broad and heavily weighted in the forward directions. This contrasts with lower energy sources, such as an ${ }^{192}$ Ir radiography source or a 1 or $2 \mathrm{MeV}$ linac, which emits radiation more evenly in all directions. Figure 2 depicts the radiation flux pattern of a high-energy linac. The image was created using MCNPX. The photon flux field was generated by $60 \mathrm{MeV}$ maximum electron beam energy, $100 \mu \mathrm{A}$ average beam current, no collimator at the convertor, and a $0.84 \mathrm{~cm}$ thick tungsten converter on the linac. The image scale is 520 meters long down the beam axis and 100 meters wide in each direction at 90 degrees from the beam axis. The flux pattern is symmetrical in full rotation around the beam axis assuming the linac was suspended at least 100 meters above the ground.

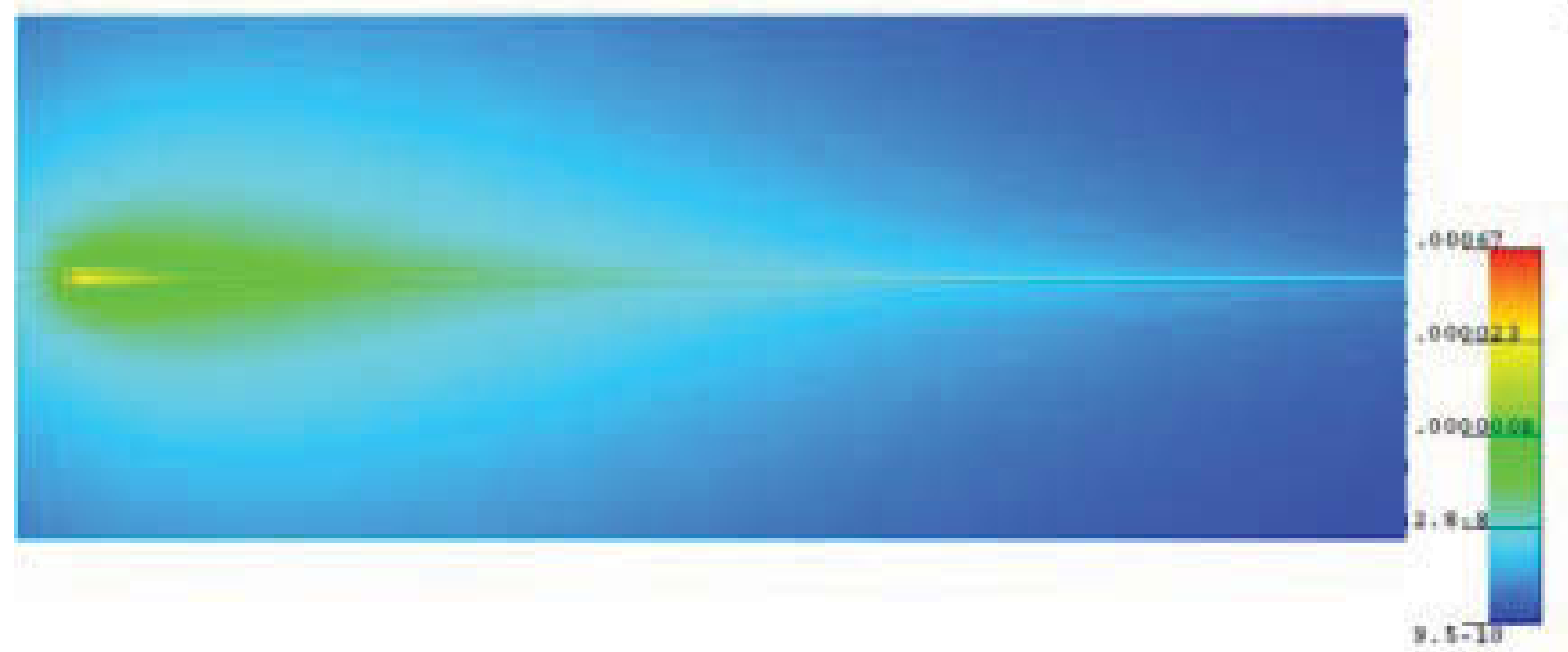

Figure 2. Photon flux field at $60 \mathrm{MeV}$ maximum electron beam energy and $100 \mu \mathrm{A}$ with high $\mathrm{Z}$ convertor in the linac, no collimator or ground plane.

The INL open installation linac is at ground level. The beam line is typically $1-2$ meters above the ground at the point of creation. It may be seen by inspection that about $50 \%$ of the broad beam from a linac without a collimator is absorbed or attenuated in the ground. MCNPX modeling has shown high energy photons are absorbed in the ground, not reflected. It is realistic to reduce the airborne radioactivity production rate by a fraction to account for ground attenuation. A conservative factor of 0.6 (a beam reduction of $40 \%$ ), rather than 0.5 , has been chosen to take credit for the ground absorption effect and to make allowance for the linac placement slightly above the ground.

Electrons cause ionization and excitation or create bremsstrahlung photons when they interact with materials. The number of photons created by bremsstrahlung is dependent on the energy of the electron beam and the atomic number of the convertor being struck by the electrons. The fraction of bremsstrahlung photons created increases as the electron energy increases. Conversely, the fraction of bremsstrahlung photons decrease with a smaller atomic number convertor, such as carbon (C), compared to a high- $\mathrm{Z}$ convertor such $\mathrm{Pb}$. The yield factors assume the electrons interact with an optimally thick high- $\mathrm{Z}$ convertor, such as $\mathrm{Pb}$ or $\mathrm{W}$. A radiation yield fraction (convertor factor) for the convertor in use can be multiplied with the yield factor when determining production of airborne radionuclides. The convertor factor would be one (1) when using $\mathrm{Pb}$ or $\mathrm{W}$. For example, at $60 \mathrm{MeV}$ electron beam energy, the radiation yield for $\mathrm{Pb}$ is about 0.67 ; the radiation yield for $\mathrm{C}$ is about 0.22 . A convertor factor is calculated by dividing the carbon radiation yield by the $\mathrm{Pb}$ radiation yield $(0.22 / 0.67=0.33)$. This carbon convertor factor $(0.33)$ can be multiplied with the airborne radionuclide yield values to determine a 
realistic source term production rate. Table 1 is a summary of these calculations. Figure 3 is similar to Figure 2 and uses the same parameters, except the $\mathrm{W}$ converter is removed, and the electron beam interacts directly with the air. Note that the scale changes. The flux is reduced by at least a factor of 10 down the beam line axis. This is due to the reduced radiation yield of the air atoms (low Z) as a convertor compared to $\mathrm{W}$, and scattering attributed to the delayed conversion to bremsstrahlung due to the density of the convertor air atoms. This scattering attribute does not reduce the overall production of radionuclides. Therefore, no reduction factor is applied for the scattering when determining total radionuclide production.

Table 1. Radiation yields and convertor factors for various materials extracted from Figure 3.3 of NCRP-144.

\begin{tabular}{|l|c|c|c|c|}
\hline Material & $\begin{array}{c}\text { Radiation Yield } \\
\text { at } 30 \mathrm{MeV}\end{array}$ & $\begin{array}{c}\text { Radiation Yield } \\
\text { at } 60 \mathrm{MeV}\end{array}$ & $\begin{array}{c}\text { Convertor Factor } \\
\text { at } 30 \mathrm{MeV}\end{array}$ & $\begin{array}{c}\text { Convertor Factor } \\
\text { at } 60 \mathrm{MeV}\end{array}$ \\
\hline $\mathrm{Pb}$ or $\mathrm{W}$ & .55 & .67 & 1 & 1 \\
\hline $\mathrm{Al}$ or air & .2 & .33 & .37 & .5 \\
\hline $\mathrm{C}$ & .13 & .22 & .24 & .33 \\
\hline
\end{tabular}

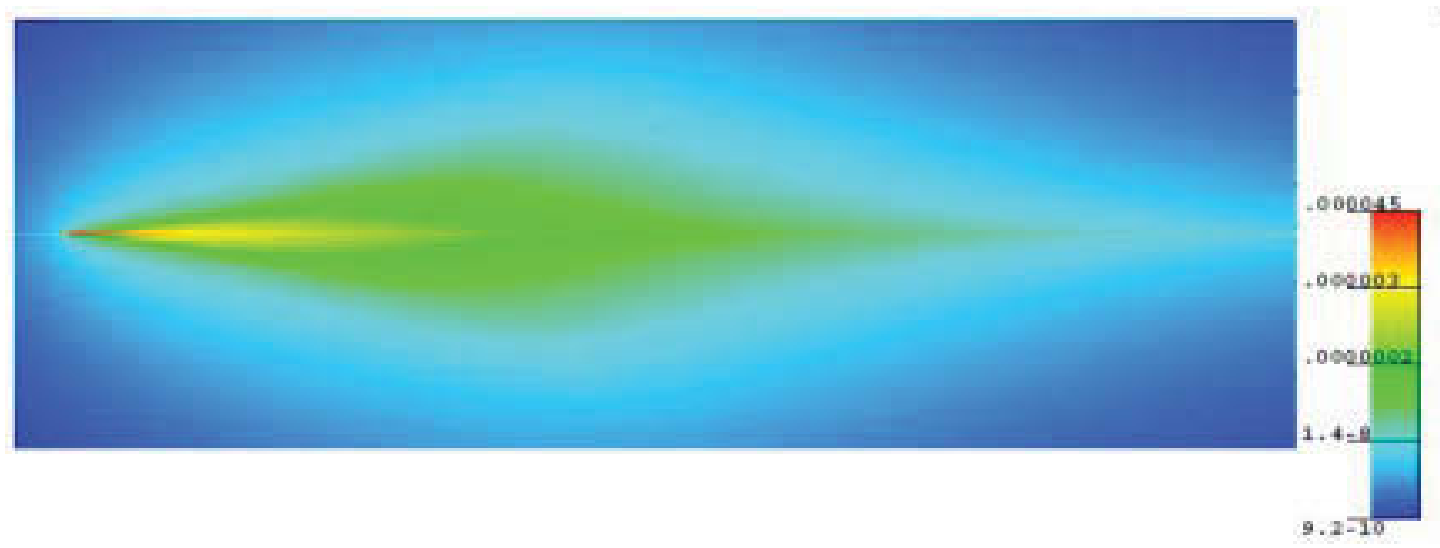

Figure 3. Photon flux field at $60 \mathrm{MeV}$ electron beam energy and $100 \mu \mathrm{A}$ using air as the convertor, without a collimator or ground plane.

The shape of the broad field photon flux is similar at lower power levels (lower energies and/or average beam currents) and convertor configurations shown in the two images above. However, the photon fluxes are reduced.

The yield factors for the creation of airborne radionuclides by photonuclear reactions assume all photons are above the threshold for those reactions. Thresholds for radionuclides of interest are shown in Table 2. This is a realistic assumption if the maximum electron beam energy is hundreds of $\mathrm{MeV}$. This is not the case for the $60 \mathrm{MeV}$ maximum electron beam energy linacs proposed for use at INL. The photons created by bremsstrahlung are produced in a continuous energy spectrum, with small numbers produced at the very highest energy, corresponding to the maximum electron beam energy, and progressively more photons produced at lower and lower energies. Figure 4 shows the relative number of photons at the various energy levels for a range of electron beam energies. The data for the figure was created using MCNPX and placed in a spreadsheet, shown in Appendix B. Using this spreadsheet, the fraction of the photon flux above specific threshold energies may be determined from the tally equal to or greater than the threshold divided by the total number of photons. Table 2 shows the resulting fraction of photons above the minimum threshold for photonuclear reactions that result in the selected radioactive isotopes at two different maximum electron beam energy levels. 


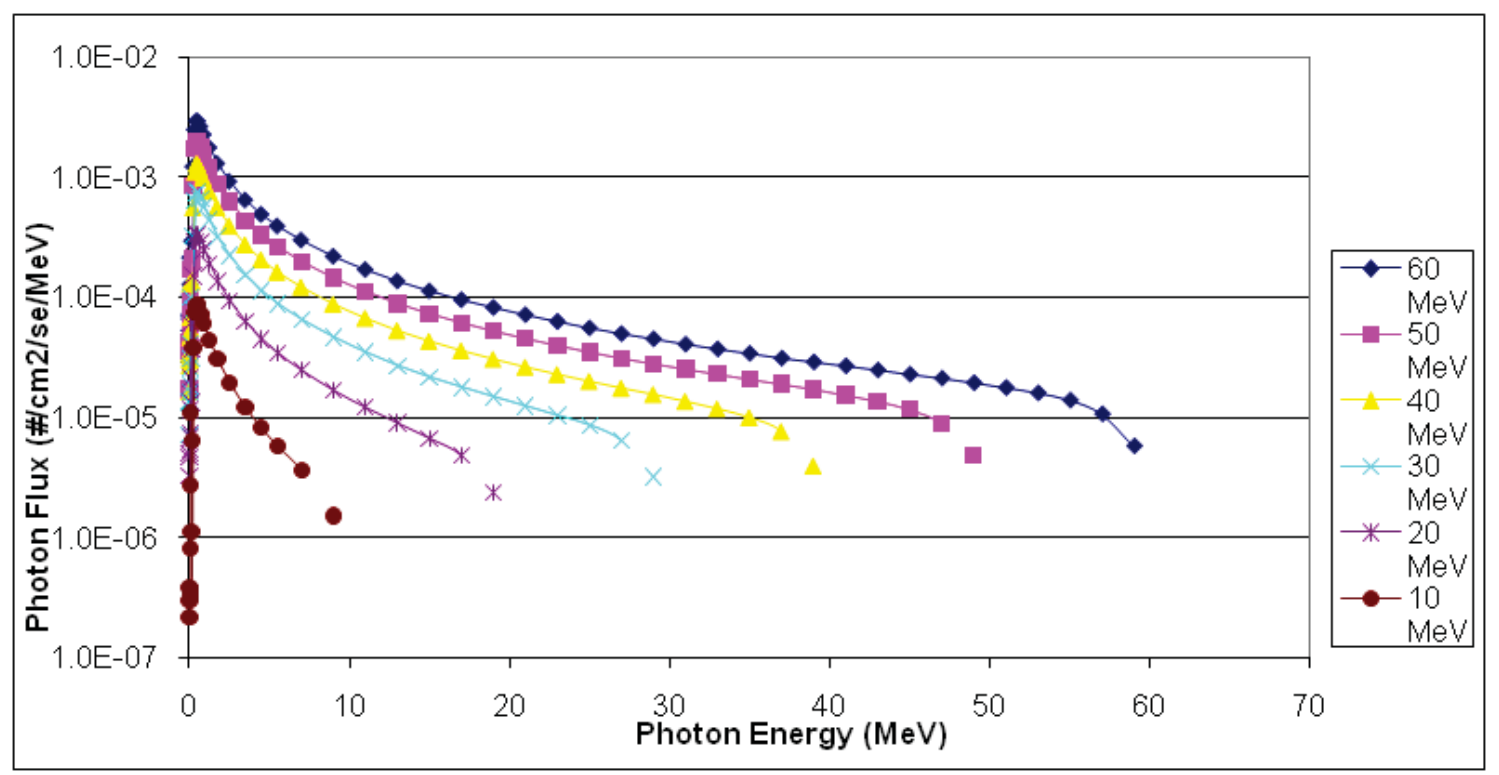

Figure 4. Bremsstrahlung distribution curves at various maximum electron beam energies.

Table 2. Threshold values for selected photonuclear reactions and radiation fraction values for 30 and $60 \mathrm{MeV}$ linacs.

\begin{tabular}{|c|c|c|c|c|}
\hline Radionuclide & Half-Life & Threshold & $\begin{array}{c}\text { Fraction of } 30 \mathrm{MeV} \\
\text { Radiation above } \\
\text { Minimum } \\
\text { Threshold }\end{array}$ & $\begin{array}{c}\text { Fraction of } 60 \mathrm{MeV} \\
\text { Radiation above } \\
\text { Minimum } \\
\text { Threshold }\end{array}$ \\
\hline 39Ar & 269 years & $9.37 \mathrm{MeV}$ & .21 & .31 \\
\hline $13 \mathrm{~N}$ & 10 minutes & $10.6 \mathrm{MeV}$ & .16 & .26 \\
\hline $39 \mathrm{Cl}$ & 55 minutes & $12.5 \mathrm{MeV}$ & .12 & .23 \\
\hline $15 \mathrm{O}$ & 2.1 minutes & $15.7 \mathrm{MeV}$ & .07 & .17 \\
\hline $11 \mathrm{C}$ & 20.5 minutes & $18.7,22.7$ and $25.9 \mathrm{MeV}$ & .06 & .15 \\
\hline $38 \mathrm{Cl}$ & 37 minutes & $20.6 \mathrm{MeV}$ & .04 & .13 \\
\hline $3 \mathrm{H}$ & 12.3 years & 22.7 and $25 \mathrm{MeV}$ & .03 & .12 \\
\hline $7 \mathrm{Be}$ & 54 days & 31.9 and $37.8 \mathrm{MeV}$ & 0 & .07 \\
\hline
\end{tabular}

Application of the factors is a simple multiplication with the production elements. The following is an example using the factors discussed in this section. The ${ }^{13} \mathrm{~N}$ activity produced for a 3 minute run of the linac at $6 \mathrm{~kW}$ would be:

$$
\left.\begin{array}{l}
\mathrm{S}=1.1 \times 10^{8} \mathrm{~Bq} \mathrm{~kW}^{-1} \mathrm{~m}^{-1} \times 6 \mathrm{~kW} \times \int \mathrm{e}^{-\mu \mathrm{s}} \mathrm{ds} \mathrm{m} \\
\mathrm{S}=1.1 \times 10^{8} \mathrm{~Bq} \mathrm{~kW}^{-1} \mathrm{~m}^{-1} \times 6 \mathrm{~kW} \times 592 \mathrm{~m} \\
\mathrm{~S}=3.9 \times 10^{11} \mathrm{~Bq} \\
\mathrm{PR}=3.9 \times 10^{11} \text { atoms } / \mathrm{sec}
\end{array}\right\} \text { from Section } 3.2
$$

Production $(\mathrm{N})=3.9 \times 10^{11}$ atoms $/ \mathrm{sec} \times$ time $\times$ ground factor $\times$ convertor factor $\times$ threshold factor Maximum electron beam energy $=60 \mathrm{MeV}$

Ground factor $=0.6$ 
Carbon convertor factor $=0.33$

Fraction of radiation above threshold $=0.26$

$\mathrm{N}=3.9 \times 10^{11}$ atoms $/ \mathrm{sec} \times 180 \mathrm{sec} \times 0.6 \times 0.33 \times 0.26$

$\mathrm{N}=3.6 \times 10^{12}$ atoms

$\mathrm{A}=\mathrm{N} \lambda$

$\mathrm{A}=3.6 \times 10^{12}$ atoms $\times 0.693 / 10 \mathrm{~min}$

$\mathrm{A}=3.6 \times 10^{12}$ atoms $\times 0.693 / 600 \mathrm{sec}$

$\mathrm{A}=4.2 \times 10^{9} \mathrm{~Bq}$

\subsection{Calculation of Radionuclide Production Rates}

The radiation generated by the linac is used to stimulate photonuclear reaction in the inspection object. Any radiation not striking the inspection object has little or no value in the process. Additionally, the low-energy photons are also of no value since they do not cause photonuclear reactions. The linac radiation may be considered noise to the detector instrumentation in some cases. Therefore, project personnel will continue to develop techniques to optimize the characteristics of the radiation beam directed at the inspection object. Various methods may be used to narrow the forward beam of radiation and remove the lower-energy photons. For example, a collimator may be used during the research to modify the beam generated by the linac. The collimator is essentially a radiation shield that attenuates the incident radiation emitted from the linac. The collimator aperture is in line with the primary electron beam that allows a desirable component of the generated radiation to continue down-range without interacting with the collimation materials. The collimator is also a key topical area in the research and development efforts to optimize the high-energy accelerator linac parameters. The collimator can substantially reduce off-axis dose rates when a converter is integrated with the linac at the end of the electron beam. The collimator can effectively reduce the broad beam in the forward direction, leaving a narrower beam and minimizing the off-axis radiation field. This reduces the volume of air that is susceptible to photonuclear reactions. The resulting linac beam in this case is best characterized as a narrow cone gradually expanding from the point of origin (collimator).

These techniques and methods have the potential to significantly reduce the overall source term of radionuclides from photonuclear reactions, particularly in the collimated down-range radiation beam compared to the broad field radiation previously described. It may in some cases concentrate a portion of the neutron generation to the vicinity of the convertor. The quantitative impact on the generation of radionuclides may be very complex to calculate. Monte Carlo codes could be used in these cases to determine the photonuclear production rates based on equipment-specific configurations. The most common code used for this type of operation is MCNPX. Monte Carlo modeling parameters take into account the converter material Z, thickness and shape, collimator materials and shape, beam hardening filters, electron beam energy, and current. The modeling of equipment-specific parameters requires significant computer resources and labor to calculate and verify the radionuclide source term. This level of modeling may be employed in those cases where there has been significant operating time with a specific configuration and a more realistic radionuclide source term is desired for annual reporting.

\subsubsection{Calculated Dose to MEI}

High-energy linacs in this INL program are diffuse sources that will not be enclosed in a shielded building or other enclosure that confines the radioactive isotopes in air. There is no pollution control equipment, nor is it practical to install pollution-control equipment since the primary isotopes are gases in air. The airborne radionuclides produced during linac operation are released continuously from the photon field near the vicinity of the linac and along the beam path. This condition complicates the collection and comparison of sampling and monitoring data. Factors that make it difficult to monitor or sample include 
creation of radionuclides throughout a large volume resulting in a low activity concentration; creation of isotopes with short half-lives; continuous mixing by molecular movement and atmospheric conditions; natural airborne radioactivity; and extremely high ambient radiation levels during linac operations.

In addition, the radionuclides do not reach equilibrium since typical linac operations consist of multiple short operating periods each day. The source term for each individual run could be determined by the production rate multiplied by the operating time. Each source term could then be entered into CAP88 to determine the dose to the MEI for the specific run. Alternatively, total radionuclide production for each run, or proposed run, may be summed with other runs and a cumulative source term used in CAP88. The resulting dose to the MEI is the same as calculating and adding the individual run doses. Determining the annual source term for reporting is accomplished by tracking the total operating run time for a specific linac configuration. This annual run time is then multiplied by the production rate to determine the source term.

Physical and operational constraints associated with the linacs utilized at the INL SOX Range maintain the calculated dose to less than $0.1 \mathrm{mrem} / \mathrm{yr}$. Emissions will be evaluated and reported as proposed herein and approved in accordance with 40 CFR 61.96(b) and the 1995 Memorandum of Understanding (EPA 1995) that requires EPA and DOE to review and agree on procedures for evaluating diffuse emissions.

\subsection{Use of CAP88}

Operational controls associated with the linacs utilized at the SOX Range maintain the dose to the MEI to less than $0.1 \mathrm{mrem} / \mathrm{year}$. There are 62 potential MEI locations surrounding the operating location at INL. The distance to these MEI locations ranges between 10,344 meters and 58,610 meters from the Test Area North SOX Range location. A broad field calculation in CAP88 was performed (See Appendix F). The dose to the MEI was the same as the point release because of the significant distance from the release location. Therefore, the calculated source term from the broad field generation is assumed to be discharged from a single point for this evaluation.

The dose to the MEIs was modeled from the SOX Range at Test Area North. The radionuclide release rates for each nuclide were applied to this location. Doses are calculated using a unit dose factor for each nuclide at each of the 62 potential MEI locations surrounding INL. Unit dose factors are by receptor location where the release occurs, and by nuclide. The dose (D) is given by

$\mathrm{D}=\mathrm{UDF} \cdot \mathrm{Q}$

where

$\mathrm{UDF}=$ the unit dose factor $(\mathrm{mrem} /$ year per $\mathrm{Ci} /$ year released)

$\mathrm{Q}=$ the actual release rate $(\mathrm{Ci} /$ year $)$.

Unit dose factor values are calculated using CAP88-PC-Version 3 code, INL-specific meteorology, and parameter values used in the annual INL NESHAP reporting.

\subsection{Source Term}

Source terms were calculated with various combinations of energies, currents and convertors based on currently available linac configurations. The following parameters and assumptions were incorporated:

- $\quad 12,30$ and $60 \mathrm{MeV}$ maximum electron energy

- 11,40 and $100 \mu \mathrm{A}$ average beam current

- No collimator

- Tungsten, aluminum, carbon and air convertors 
- Ground absorption effect (ground plane factor - 0.6)

- Ground absorption effect for neutrons (ground plane factor - 0.5)

- 800 hours operation (considered normal operations based on historical linac operating experience)

- Hours corresponding to a dose of $0.1 \mathrm{mrem} / \mathrm{year}$ to the MEI.

See Appendix $\mathrm{C}$ for the source term calculations. Table 3 lists the physical and operations parameters, MEI dose for 800 hours of operation and hours of operation corresponding to $0.1 \mathrm{mrem} / \mathrm{year}$ to the MEI. The source term data is contained in Appendix C.

Table 3. Operational configurations, calculated MEI dose, and operating hour results.

\begin{tabular}{|c|c|l|c|c|}
\hline $\begin{array}{c}\text { Electron } \\
\text { Beam Energy } \\
(\mathrm{MeV})\end{array}$ & $\begin{array}{c}\text { Average Electron } \\
\text { Beam Current } \\
\text { (microamperes) }\end{array}$ & $\begin{array}{c}\text { Convertor } \\
\text { Material }\end{array}$ & $\begin{array}{c}\text { MEI Dose for 800 } \\
\text { Hours of Operation } \\
\text { (mrem/year) }\end{array}$ & $\begin{array}{c}\text { Hours of Operation } \\
\text { for an MEI Dose of } \\
0.1 \text { mrem/year }\end{array}$ \\
\hline 60 & 100 & Tungsten & 0.07 & 1,146 \\
\hline 60 & 100 & Air & 0.021 & 3,809 \\
\hline 60 & 100 & Carbon & 0.016 & 4,908 \\
\hline 30 & 40 & Tungsten & 0.009 & 8,676 \\
\hline 30 & 40 & Aluminum & 0.002 & 39,024 \\
\hline 30 & 40 & Air & 0.0018 & 44,198 \\
\hline 12 & 11 & Tungsten & 0.000005 & $15,037,593$ \\
\hline
\end{tabular}

\subsection{Control of Dose to MEI}

Linacs at the INL are used in research and development, particularly relating to detector and signal processing systems. The Laboratory typically works 10 hour days, four days a week, and fifty weeks a year at TAN. Daily set up and take down of the systems are expected to take an hour each. This leaves an average of 8 hours per day to conduct the R\&D activities. Much of the time is used to prepare the detection and acquisition systems, clear the area, position materials and detectors, prepare the linac for operations, review data, change material and detector configurations, put materials and detectors away, and shut down the linac. Our experience during focused campaigns at a non-DOE offsite facility revealed only half the potential work hours are typically used operating the linacs. Using these factors, the typical linac operations would be:

Typical operations $=(10 \mathrm{hrs}-2 \mathrm{hrs}) \times 0.5 \times 4$ days $/$ week $\times 50$ weeks $/$ year $\approx 800$ hours per year.

The average number of hours per day could be greater in the summer months when the weather is mild and the days are longer. The average number of hours would be significantly fewer in the winter during the extreme cold and shorter daylight hours. On rare occasions the operations could be expanded to seven days a week in case of high customer demand.

The most frequent operations are expected to use beam energies of $2 \mathrm{MeV}$ to $30 \mathrm{MeV}$ at beam currents between 1 to $30 \mu \mathrm{A}$. The SOX Range will support the use of linacs operating at beam energies up to $60 \mathrm{MeV}$ and beam currents up to $100 \mu \mathrm{A}$. These are the maximum allowed parameters on the SOX Range. Converters will typically be composed of aluminum, carbon, or air, but tungsten converters may also be used on occasion.

Before any linac operation at the SOX Range may commence, a beam authorization sheet must be prepared and approved by the Accelerator Safety Officer. The beam authorization sheet identifies the linac to be operated, the maximum beam energy and average beam current (maximum SOX Range values for these parameters are $60 \mathrm{MeV}$ and 100 microamperes, respectively), the type of converter used, and the 
maximum hours of operation for the specified parameters. The Accelerator Safety Officer uses this information to calculate the maximum radioactive source term that could be generated for the particular linac operation. This source term is then analyzed using CAP88 to determine the EDE that would result to the MEI. The Accelerator Safety Officer tallies the cumulative EDE for all linac operations at the SOX Range during a calendar year. If the cumulative dose to the MEI for proposed linac operations, when summed with all other approved linac operations within that calendar year is less than $0.1 \mathrm{mrem}$, then the beam authorization sheet is approved and the proposed linac operation may proceed. Any proposed operation of a linac that would result in a cumulative EDE that equals or exceeds 0.1 mrem in a calendar year would not be approved. This process controls the dose to the MEI resulting from SOX Range operations to less than $1 \%$ of the NESHAP standard: the threshold that would require an EPA approval to construct. As a comparison, in 2009, radionuclide air emissions and the resulting EDE to the MEI from all operations at the INL Site was $0.0687 \mathrm{mrem} /$ year, $0.69 \%$ of the 10 -mrem standard (DOE/ID-10890 2010). The cumulative EDE from Site operations and linac operations at the SOX Range would not challenge the annual limit.

The source terms are calculated without any credit for pollution control equipment, as they are not practical to this operation, and the linacs at INL do not have them. The configurations and associated source terms in Table 3 have been analyzed using CAP88. These configurations represent likely configurations known at this time. If a different linac configuration is identified for use at the SOX Range, the source term will be calculated and recorded as given in Table 3. A beam authorization sheet must be completed and approved by the Accelerator Safety Officer prior to operation of any linac at the SOX Range. The proposed and/or actual hours of operation divided by the hours associated with the 0.1 $\mathrm{mrem} /$ year dose may be used to calculate the dose for a certain configuration. The sum of the fractions can be used to evaluate a combination of linac configurations. For example, suppose linac A has operated 420 hours this calendar year at $60 \mathrm{MeV}$ and $100 \mu \mathrm{A}$ with a carbon convertor. Linac B is scheduled to operate 300 hours at $30 \mathrm{MeV}$ and $40 \mu \mathrm{A}$ with an aluminum convertor, and linac $\mathrm{C}$ has operated 200 hours and is scheduled to operate another 100 hours at $12 \mathrm{MeV}$ and $11 \mu \mathrm{A}$ using a tungsten convertor.

The calculated annual MEI dose is shown in Table 4:

Table 4. Calculated annual MEI dose.

\begin{tabular}{|l|l|l|l|l|l|l|l|l|}
\hline \multicolumn{1}{|c|}{$\operatorname{linac} \mathrm{A}$} & & \multicolumn{1}{c|}{$\operatorname{linac} \mathrm{B}$} & & \multicolumn{1}{|c|}{$\operatorname{linac} \mathrm{C}$} & & & \multicolumn{1}{c|}{ Annual Dose } \\
\hline$(420 / 4908$ & + & $300 / 39024$ & + & $300 / 15037593)$ & $\times$ & $0.1 \mathrm{mrem}$ & $=$ & EDE to the MEI \\
\hline$(0.09$ & + & 0.008 & + & $0.00002)$ & $\times$ & $0.1 \mathrm{mrem}$ & $=0.0098 \mathrm{mrem}$ \\
\hline
\end{tabular}

Several layers of conservatism are applied to the calculation of the radioactive source term and determination of the effective dose to the MEI. These include:

1. Linear attenuation coefficient $(\mu)$ - the coefficient used applies to the minimum cross section in air for $\mathrm{x}$-rays less than $60 \mathrm{MeV}$, which significantly underestimates the attenuation of photon energy from air, thus resulting in a high estimate of the saturated activity and production rate.

2. Neutron absorption - all neutrons created are assumed to slow down and undergo radiative capture in the air or entrained dust particles, which overestimates the production rate, thus resulting in a high estimate of the activity generated by the linac.

3. Collimator - no credit is being taken for collimators that would greatly reduce the photon flux off the beam axis, thus resulting in an overestimate of the activity generated by the linac.

4. Exposure scenario - although it is physically impossible for an individual to be exposed to all activated atoms generated in the linac beam path, CAP88 modeling for the SOX Range assumes that every atom of ${ }^{39} \mathrm{Ar},{ }^{41} \mathrm{Ar},{ }^{7} \mathrm{Be},{ }^{11} \mathrm{C},{ }^{38} \mathrm{Cl},{ }^{39} \mathrm{Cl},{ }^{3} \mathrm{H},{ }^{13} \mathrm{~N}$ and ${ }^{15} \mathrm{O}$ is released from a single point source and contributes to the effective dose to the MEI. 
5. The linacs operate over a range of power levels for a given maximum electron beam energy. The maximum power level for each maximum energy level will be used for the annual dose report to avoid detailed calculations at the lower power levels. The use of the higher power level overestimates the radionuclide emissions.

These practices produce an estimated dose that is bounding, whereas the actual impact to any member of the public is likely much lower.

The photon and neutron fluxes do cause activation of dust particles in the beam path. However, the activation potential of the dust is many orders of magnitude smaller than that of air due to the very small mass of the dust compared to air. A macroscopic evaluation of the potential to activate, assuming 10 micrograms of dust in a cubic meter of air, showed 8 orders of magnitude greater activation potential for air compared to dust. Therefore, the addition of the dust particles in the dose calculation will not change the resulting dose to the MEI. In addition, the linac would not likely operate in high dust conditions due to the impaired ability to view the downrange area. Furthermore, heavy dust particles would fall out of the air in close proximity to the SOX Range and would therefore not contribute to exposure to the nearest MEI, who is located more than 10,000 meters away from the SOX Range. The level of conservatism applied to the calculation of source term and modeling to the MEI would envelope any particulate activation that could occur.

\subsection{Annual NESHAP Report of Dose to MEI}

Radionuclide emissions evaluations, based on operating data, will be submitted to the Accelerator Safety Officer for evaluation and inclusion in INL's annual NESHAP report. The accelerator operations data and evaluation of the dose for inclusion in the INL NESHAP report will be documented. 


\section{REFERENCES}

CAP88-PC-Version 3, "Clean Air Act Assessment Package," 1988

Guidebook for the ENDF/B-V Nuclear Data Files, ENDF-238, Brookhaven National Laboratory, July 1982

Monte Carlo N-Particle eXtended (MCNPX)

National Council on Radiation Protection and Measurements (NCRP) report 144, "Radiation Protection for Particle Accelerator Facilities"

National Emission Standard for Hazardous Air Pollutants (NESHAPS) 40 CFR 61 Subpart H, "National Emission Standards for Emissions of Radionuclides Other Than Radon from Department of Energy Facilities"

National Institute of Science and Technology - NIST XCOM: Photon Cross Section Database

U.S. Environmental Protection Agency, U.S. Department of Energy, 1995, Memorandum of Understanding Between the U.S. EPA and the U.S. DOE concerning the Clean Air Act Emission Standards for Radionuclides, 40 CFR Part 61 Including Subparts H, I, Q, and T. 
Appendix A

\section{Path Length Integral}




\title{
Appendix A
}

\section{Path Length Integral}

\author{
Essential Integral
}

Substitute terms

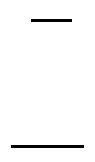

Substitute terms

Solve for
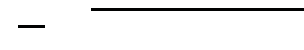

$-$ 
Solve for

Round up

Solve for

Solve for $\mathrm{s}=2500$ meters 


\section{Appendix B}

\section{Bremsstrahlung Photon Flux versus Electron Energy (MeV)}




\section{Appendix B}

\section{Bremsstrahlung Photon Flux versus Electron Energy (MeV)}

Bremsstrahlung Photon Flux versus Electron Energy (MeV)

0.30-cm thick, 1.0-cm diameter Tungsten $(\mathrm{W})$ converter

Photon flux at 1.0-meter away from converter on beamline

0.5- $\mathrm{cm}$ radius electron beam on converter disk (3- $\mathrm{mm})$

Electron source energies at $10,20,30,40,50,60 \mathrm{MeV}$

\begin{tabular}{|c|c|c|c|c|c|c|c|c|c|}
\hline Photon & Photon & Photon & Photon & $10 \mathrm{MeV}$ & $20 \mathrm{MeV}$ & $30 \mathrm{MeV}$ & $40 \mathrm{MeV}$ & $50 \mathrm{MeV}$ & $60 \mathrm{MeV}$ \\
\hline Energy & Energy & Energy & Energy & Photon & Photon & Photon & Photon & Photon & Photon \\
\hline $\begin{array}{l}\text { Lower Bin } \\
(\mathrm{MeV})\end{array}$ & $\begin{array}{l}\text { Upper Bin } \\
(\mathrm{MeV})\end{array}$ & $\begin{array}{c}\text { AVERAGE } \\
\text { (MeV) }\end{array}$ & $\begin{array}{c}\text { Interval } \\
(\mathrm{MeV})\end{array}$ & $\begin{array}{l}\text { Tally Flux } \\
\text { (p/cm2/se) }\end{array}$ & $\begin{array}{l}\text { Tally Flux } \\
\text { (p/cm/se) }\end{array}$ & $\begin{array}{l}\text { Tally Flux } \\
(\mathrm{p} / \mathrm{cm} / \mathrm{se})\end{array}$ & $\begin{array}{l}\text { Tally Flux } \\
\text { (p/cm2/se) }\end{array}$ & $\begin{array}{l}\text { Tally Flux } \\
(\mathrm{p} / \mathrm{cm} 2 / \mathrm{se})\end{array}$ & $\begin{array}{l}\text { Tally Flux } \\
\text { (p/cm2/se) }\end{array}$ \\
\hline 0.0 & $1.00 \mathrm{E}-02$ & 0.0050 & 0.0100 & 2.20934E-09 & 3.32179E-08 & 7.15963E-08 & $1.66179 \mathrm{E}-07$ & $1.72047 \mathrm{E}-07$ & 2.90244E-07 \\
\hline $1.00 \mathrm{E}-02$ & $1.50 \mathrm{E}-02$ & 0.0125 & 0.0050 & $1.96523 \mathrm{E}-09$ & 2.33999E-08 & $6.66820 \mathrm{E}-08$ & 1.69703E-07 & $2.12088 \mathrm{E}-07$ & 3.52783E-07 \\
\hline $1.50 \mathrm{E}-02$ & $2.00 \mathrm{E}-02$ & 0.0175 & 0.0050 & $1.52089 \mathrm{E}-09$ & 2.54657E-08 & $6.85782 \mathrm{E}-08$ & $1.53587 \mathrm{E}-07$ & $1.78159 \mathrm{E}-07$ & 2.66987E-07 \\
\hline $2.00 \mathrm{E}-02$ & $3.00 \mathrm{E}-02$ & 0.0250 & 0.0100 & 3.56753E-09 & 5.46877E-08 & $1.33296 \mathrm{E}-07$ & 2.72497E-07 & 4.35448E-07 & $6.69047 \mathrm{E}-07$ \\
\hline $3.00 \mathrm{E}-02$ & $4.00 \mathrm{E}-02$ & 0.0350 & 0.0100 & 3.77761E-09 & 7.31764E-08 & $1.84268 \mathrm{E}-07$ & $3.48658 \mathrm{E}-07$ & $5.35090 \mathrm{E}-07$ & $7.36268 \mathrm{E}-07$ \\
\hline $4.00 \mathrm{E}-02$ & $5.00 \mathrm{E}-02$ & 0.0450 & 0.0100 & $8.30240 \mathrm{E}-09$ & $1.15686 \mathrm{E}-07$ & 2.63394E-07 & 4.69410E-07 & 6.91197E-07 & $1.05961 \mathrm{E}-06$ \\
\hline $5.00 \mathrm{E}-02$ & $6.00 \mathrm{E}-02$ & 0.0550 & 0.0100 & 1.10976E-07 & 6.15714E-07 & $1.00863 \mathrm{E}-06$ & $1.31468 \mathrm{E}-06$ & $1.69692 \mathrm{E}-06$ & $2.15729 \mathrm{E}-06$ \\
\hline $6.00 \mathrm{E}-02$ & $8.00 \mathrm{E}-02$ & 0.0700 & 0.0200 & 5.52560E-08 & 4.25628E-07 & $8.26935 \mathrm{E}-07$ & $1.35728 \mathrm{E}-06$ & 1.89980E-06 & 2.58227E-06 \\
\hline $8.00 \mathrm{E}-02$ & $1.00 \mathrm{E}-01$ & 0.0900 & 0.0200 & $6.55782 \mathrm{E}-09$ & 1.35634E-07 & 3.29849E-07 & $6.06839 \mathrm{E}-07$ & $9.84637 \mathrm{E}-07$ & $1.33152 \mathrm{E}-06$ \\
\hline $1.00 \mathrm{E}-01$ & $1.50 \mathrm{E}-01$ & 0.1250 & 0.0500 & $5.65552 \mathrm{E}-08$ & $6.72109 \mathrm{E}-07$ & $1.55312 \mathrm{E}-06$ & $2.74271 \mathrm{E}-06$ & 4.26121E-06 & $5.76728 \mathrm{E}-06$ \\
\hline $1.50 \mathrm{E}-01$ & $2.00 \mathrm{E}-01$ & 0.1750 & 0.0500 & $3.25065 \mathrm{E}-07$ & $1.87835 \mathrm{E}-06$ & 3.95333E-06 & $6.89821 E-06$ & $1.03468 \mathrm{E}-05$ & $1.48317 \mathrm{E}-05$ \\
\hline $2.00 \mathrm{E}-01$ & $3.00 \mathrm{E}-01$ & 0.2500 & 0.1000 & 3.78913E-06 & 1.51115E-05 & 3.25198E-05 & 5.59278E-05 & $8.50023 E-05$ & $1.23720 \mathrm{E}-04$ \\
\hline $3.00 \mathrm{E}-01$ & $4.00 \mathrm{E}-01$ & 0.3500 & 0.1000 & $7.59826 \mathrm{E}-06$ & $2.92028 \mathrm{E}-05$ & $6.44405 \mathrm{E}-05$ & $1.10978 \mathrm{E}-04$ & $1.70983 \mathrm{E}-04$ & $2.51379 \mathrm{E}-04$ \\
\hline $4.00 \mathrm{E}-01$ & $5.00 E-01$ & 0.4500 & 0.1000 & $8.62609 \mathrm{E}-06$ & 3.35399E-05 & $7.47706 \mathrm{E}-05$ & $1.29864 \mathrm{E}-04$ & $2.00340 \mathrm{E}-04$ & 2.95293E-04 \\
\hline $5.00 \mathrm{E}-01$ & $6.00 \mathrm{E}-01$ & 0.5500 & 0.1000 & $8.67305 E-06$ & 3.37814E-05 & $7.52447 \mathrm{E}-05$ & $1.29557 \mathrm{E}-04$ & $2.00142 \mathrm{E}-04$ & $2.95579 \mathrm{E}-04$ \\
\hline $6.00 \mathrm{E}-01$ & $8.00 \mathrm{E}-01$ & 0.7000 & 0.2000 & $1.46307 \mathrm{E}-05$ & $5.87279 \mathrm{E}-05$ & $1.33201 \mathrm{E}-04$ & $2.32478 \mathrm{E}-04$ & $3.61322 E-04$ & $5.34496 \mathrm{E}-04$ \\
\hline $8.00 \mathrm{E}-01$ & $1.00 E+00$ & 0.9000 & 0.2000 & $1.20351 \mathrm{E}-05$ & 4.94833E-05 & $1.12970 \mathrm{E}-04$ & 1.97991E-04 & $3.08700 \mathrm{E}-04$ & 4.56719E-04 \\
\hline $1.00 \mathrm{E}+00$ & $1.50 E+00$ & 1.2500 & 0.5000 & $2.23379 \mathrm{E}-05$ & $9.45646 \mathrm{E}-05$ & $2.18401 \mathrm{E}-04$ & 3.84713E-04 & $6.00608 \mathrm{E}-04$ & $8.91938 \mathrm{E}-04$ \\
\hline $1.50 E+00$ & $2.00 E+00$ & 1.7500 & 0.5000 & $1.53222 \mathrm{E}-05$ & 6.79926E-05 & $1.58960 \mathrm{E}-04$ & $2.80472 \mathrm{E}-04$ & 4.42831E-04 & $6.57306 \mathrm{E}-04$ \\
\hline $2.00 E+00$ & $3.00 E+00$ & 2.5000 & 1.0000 & $1.97076 \mathrm{E}-05$ & $9.32820 \mathrm{E}-05$ & 2.22145E-04 & $3.95871 E-04$ & $6.24048 \mathrm{E}-04$ & $9.31064 E-04$ \\
\hline $3.00 E+00$ & $4.00 E+00$ & 3.5000 & 1.0000 & $1.21824 \mathrm{E}-05$ & $6.24140 \mathrm{E}-05$ & 1.53447E-04 & $2.74876 \mathrm{E}-04$ & 4.36199E-04 & $6.50887 \mathrm{E}-04$ \\
\hline $4.00 E+00$ & $5.00 E+00$ & 4.5000 & 1.0000 & $8.20985 E-06$ & 4.51584E- 05 & $1.13334 \mathrm{E}-04$ & 2.06765E-04 & $3.30257 \mathrm{E}-04$ & 4.95099E-04 \\
\hline $5.00 \mathrm{E}+00$ & $6.00 E+00$ & 5.5000 & 1.0000 & $5.81526 \mathrm{E}-06$ & $3.45036 \mathrm{E}-05$ & $8.86555 \mathrm{E}-05$ & $1.63130 \mathrm{E}-04$ & 2.63241E-04 & $3.95767 \mathrm{E}-04$ \\
\hline $6.00 \mathrm{E}+00$ & $8.00 E+00$ & 7.0000 & 2.0000 & $7.30908 \mathrm{E}-06$ & 4.94316E-05 & $1.31153 \mathrm{E}-04$ & $2.44774 E-04$ & 3.97362E-04 & $6.03685 \mathrm{E}-04$ \\
\hline $8.00 E+00$ & $1.00 E+01$ & 9.0000 & 2.0000 & $3.07251 \mathrm{E}-06$ & 3.37476E-05 & $9.33400 \mathrm{E}-05$ & $1.77037 \mathrm{E}-04$ & 2.90217E-04 & 4.42201E-04 \\
\hline $1.00 \mathrm{E}+01$ & $1.20 \mathrm{E}+01$ & 11.0000 & 2.0000 & $0.00000 E+00$ & 2.43444E-05 & $6.98237 \mathrm{E}-05$ & $1.34869 \mathrm{E}-04$ & $2.24038 \mathrm{E}-04$ & $3.43279 \mathrm{E}-04$ \\
\hline $1.20 E+01$ & $1.40 E+01$ & 13.0000 & 2.0000 & $0.00000 E+00$ & $1.80923 \mathrm{E}-05$ & $5.40808 \mathrm{E}-05$ & $1.06181 \mathrm{E}-04$ & $1.77909 \mathrm{E}-04$ & $2.75375 \mathrm{E}-04$ \\
\hline $1.40 \mathrm{E}+01$ & $1.60 \mathrm{E}+01$ & 15.0000 & 2.0000 & $0.00000 E+00$ & 1.35553E-05 & 4.33805E-05 & $8.67475 E-05$ & $1.46406 \mathrm{E}-04$ & $2.27998 \mathrm{E}-04$ \\
\hline $1.60 E+01$ & $1.80 E+01$ & 17.0000 & 2.0000 & $0.00000 E+00$ & $9.73829 \mathrm{E}-06$ & 3.58337E-05 & $7.24855 E-05$ & $1.23183 E-04$ & $1.92256 \mathrm{E}-04$ \\
\hline $1.80 \mathrm{E}+01$ & $2.00 E+01$ & 19.0000 & 2.0000 & $0.00000 E+00$ & 4.71793E-06 & 2.98188E-05 & $6.15232 \mathrm{E}-05$ & $1.05314 \mathrm{E}-04$ & $1.65917 \mathrm{E}-04$ \\
\hline $2.00 \mathrm{E}+01$ & $2.20 \mathrm{E}+01$ & 21.0000 & 2.0000 & $0.00000 E+00$ & $0.00000 E+00$ & 2.49331E-05 & $5.29158 \mathrm{E}-05$ & $9.10758 \mathrm{E}-05$ & $1.44227 \mathrm{E}-04$ \\
\hline $2.20 \mathrm{E}+01$ & $2.40 \mathrm{E}+01$ & 23.0000 & 2.0 & $0.00000 E+00$ & $0.00000 E+00$ & $2.08168 \mathrm{E}-05$ & 4.59912E-05 & 7.98312E-05 & $1.26677 \mathrm{E}-04$ \\
\hline $2.40 \mathrm{E}+01$ & $2.60 E+01$ & 25.0000 & 2.0000 & $0.00000 E+00$ & $0.00000 E+00$ & $1.72844 \mathrm{E}-05$ & $4.01681 \mathrm{E}-05$ & 7.03849E-05 & $1.12041 \mathrm{E}-04$ \\
\hline $2.60 \mathrm{E}+01$ & $2.80 E+01$ & 27.0000 & 2.0000 & $0.00000 E+00$ & $0.00000 E+00$ & $1.27941 \mathrm{E}-05$ & 3.53269E-05 & $6.23726 \mathrm{E}-05$ & $1.00380 \mathrm{E}-04$ \\
\hline $2.80 \mathrm{E}+01$ & $3.00 E+01$ & 29.0000 & 2.0000 & $0.00000 E+00$ & $0.00000 E+00$ & $6.45402 \mathrm{E}-06$ & 3.13151E-05 & $5.62604 \mathrm{E}-05$ & $9.08978 \mathrm{E}-05$ \\
\hline $3.00 \mathrm{E}+01$ & $3.20 \mathrm{E}+01$ & 31.0000 & 2.0000 & $0.00000 E+00$ & $0.00000 E+00$ & $0.00000 E+00$ & $2.74204 E-05$ & 5.07997E-05 & $8.19737 \mathrm{E}-05$ \\
\hline $3.20 \mathrm{E}+01$ & $3.40 \mathrm{E}+01$ & 33.0000 & 2.0000 & $0.00000 E+00$ & $0.00000 E+00$ & $0.00000 E+00$ & 2.37713E-05 & 4.60648E-05 & $7.47726 \mathrm{E}-05$ \\
\hline $3.40 \mathrm{E}+01$ & $3.60 E+01$ & 35.0000 & 2.0000 & $0.00000 E+00$ & $0.00000 E+00$ & $0.00000 E+00$ & $2.01056 \mathrm{E}-05$ & 4.18033E-05 & $6.86906 \mathrm{E}-05$ \\
\hline $3.60 E+01$ & $3.80 E+01$ & 37.0000 & 2.0000 & $0.00000 E+00$ & $0.00000 E+00$ & $0.00000 E+00$ & $1.54232 \mathrm{E}-05$ & 3.81133E-05 & $6.27539 \mathrm{E}-05$ \\
\hline $3.80 \mathrm{E}+01$ & $4.00 E+01$ & 39.0000 & 2.0000 & $0.00000 E+00$ & $0.00000 E+00$ & $0.00000 E+00$ & 7.93897E-06 & 3.45958E-05 & $5.83320 \mathrm{E}-05$ \\
\hline $4.00 \mathrm{E}+01$ & $4.20 E+01$ & 41.0000 & 2.0000 & $0.00000 E+00$ & $0.00000 E+00$ & $0.00000 E+00$ & $0.00000 E+00$ & 3.08995E-05 & $5.41066 \mathrm{E}-05$ \\
\hline $4.20 \mathrm{E}+01$ & $4.40 E+01$ & 43.0000 & 2.0000 & $0.00000 E+00$ & $0.00000 E+00$ & $0.00000 E+00$ & $0.00000 E+00$ & $2.74655 E-05$ & 4.99656E-05 \\
\hline $4.40 \mathrm{E}+01$ & $4.60 E+01$ & 45.0000 & 2.0000 & $0.00000 E+00$ & $0.00000 E+00$ & $0.00000 E+00$ & $0.00000 E+00$ & 2.34454E-05 & 4.60677E-05 \\
\hline $4.60 E+01$ & $4.80 \mathrm{E}+01$ & 47.0000 & 2.0000 & $0.00000 E+00$ & $0.00000 E+00$ & $0.00000 E+00$ & $0.00000 E+00$ & $1.80060 \mathrm{E}-05$ & 4.27213E-05 \\
\hline $4.80 E+01$ & $5.00 E+01$ & 49.0000 & 2.0000 & $0.00000 E+00$ & $0.00000 E+00$ & $0.00000 E+00$ & $0.00000 E+00$ & $9.62111 \mathrm{E}-06$ & $3.90946 \mathrm{E}-05$ \\
\hline $5.00 E+01$ & $5.20 E+01$ & 51.0000 & 2.0000 & $0.00000 E+00$ & $0.00000 E+00$ & $0.00000 E+00$ & $0.00000 E+00$ & $0.00000 E+00$ & 3.56198E-05 \\
\hline $5.20 \mathrm{E}+01$ & $5.40 E+01$ & 53.0000 & 2.0000 & $0.00000 E+00$ & $0.00000 E+00$ & $0.00000 E+00$ & $0.00000 E+00$ & $0.00000 E+00$ & 3.22393E-05 \\
\hline $5.40 E+01$ & $5.60 E+01$ & 55.0000 & 2.0000 & $0.00000 E+00$ & $0.00000 E+00$ & $0.00000 E+00$ & $0.00000 E+00$ & $0.00000 E+00$ & $2.80411 \mathrm{E}-05$ \\
\hline $5.60 E+01$ & $5.80 E+01$ & 57.0000 & 2.0000 & $0.00000 E+00$ & $0.00000 E+00$ & $0.00000 E+00$ & $0.00000 E+00$ & $0.00000 E+00$ & $2.15413 E-05$ \\
\hline $5.80 E+01$ & $6.00 E+01$ & 59.0000 & 2.0000 & $0.00000 E+00$ & $0.00000 E+00$ & $0.00000 E+00$ & $0.00000 E+00$ & $0.00000 E+00$ & 1.16454E-05 \\
\hline
\end{tabular}

$\begin{array}{llllll}1.49885 E-04 & 7.75442 E-04 & 1.99626 E-03 & 3.76112 E-03 & 6.19026 E-03 & 9.54179 E-03\end{array}$

Energy (MeV) Fraction $>6 \mathrm{MeV}$ fraction $>9.37 \mathrm{MeV}$ Fraction $>10.6 \mathrm{MeV}$ Fraction $>12.5 \mathrm{MeV}$ Fraction $>15.7 \mathrm{MeV}$ Fraction $>18.7 \mathrm{MeV}$ Fraction $>20.6 \mathrm{MeV}$ Fraction $>22.7 \mathrm{MeV}$ Fraction $>31.9 \mathrm{MeV}$

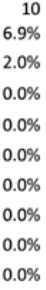

$$
\begin{array}{r}
20 \\
19.8 \%
\end{array}
$$$$
\begin{aligned}
& 19.8 \% \\
& 13.4 \%
\end{aligned}
$$$$
\begin{aligned}
& 9.1 \% \\
& 5.9 \%
\end{aligned}
$$$$
1.9 \%
$$$$
0.6 \%
$$$$
0.0 \%
$$$$
0.0 \%
$$$$
0.0 \%
$$

30
$27.0 \%$
$20.5 \%$
$15.8 \%$
$12.3 \%$
$7.4 \%$
$5.6 \%$
$4.1 \%$
$2.9 \%$
$0.0 \%$

$\begin{array}{rr}50 & 60 \\ 34.7 \% & 37.0 \% \\ 28.2 \% & 30.7 \% \\ 23.5 \% & 26.1 \% \\ 19.9 \% & 22.5 \% \\ 14.7 \% & 17.2 \% \\ 12.7 \% & 15.2 \% \\ 11.0 \% & 13.4 \% \\ 9.5 \% & 11.9 \% \\ 4.4 \% & 6.6 \%\end{array}$


Appendix C

\section{Source Term Calculations}




\section{Appendix C}

\section{Source Term Calculations}

Glossary

- $\quad$ S sat $=$ Saturated airborne radioactivity of a specific nuclide

- $\quad \mathrm{YF}=$ Yield Factor - Specific yields $\left[\mathrm{Bq} /\left(\mathrm{kW}^{*} \mathrm{~m}\right)\right]$ of radionuclides from photoactivated air. (NCRP144, pg 342 Table 6.4) (For neutrons, the yield is 10E+12 taken from Figure 3.12 of NCRP-144.)

- $\quad \mathrm{f}($ ground plane $)=$ The fraction of the radiation not absorbed in the ground.

- $\mathrm{f}($ convertor $)=$ For bremsstrahlung, the effectiveness for a convertor to produce photons compared to tungsten. Derived from Figure 3.3 of NCRP-144. See Table 1. For neutrons, it is the factor that is multiplied by the YF (1E-12) to determine the photo neutron yield of a thick target (convertor). This is taken from Figure 3.12 of NCRP-144. See Appendix E.

- $\mathrm{f}$ (energy factor) $=$ The fraction of bremsstrahlung photons that are equal to or greater than the threshold for photonuclear reactions. See Table 2.

- $\mathrm{f}($ neutron capture $)=$ The fraction of thermal neutrons that will be captured by ${ }^{40} \mathrm{Ar}$ to create ${ }^{41} \mathrm{Ar}$. The value is 0.035 . See Appendix D.

- $\quad$ YF adjusted $=$ YF $\times f$ (ground plane) $\mathrm{x} f$ (convertor) $\mathrm{x} f$ (energy factor) $\mathrm{x} f$ (neutron capture) as applicable.

- $\quad \mathrm{P}=$ power $(\mathrm{kW})=$ Eo $\times \mathrm{I} / 1000(\mathrm{MeV} \times \mu \mathrm{A})$

- $\quad$ Eo $=$ maximum electron beam energy $(\mathrm{MeV})$

- $\quad \mathrm{I}=$ electron beam current $(\mu \mathrm{A})$

- $\mathrm{s}=$ Integrated beam distance

- $\quad \mathrm{PR}=$ Production rate of a radionuclide $($ atoms per $\mathrm{sec})=\mathrm{YF}$ adjusted $\times \mathrm{P} \times \mathrm{s}$

- $\mathrm{N}=\mathrm{PR} \times$ operating time

- $\mathrm{A}=\mathrm{N} \times \lambda=\mathrm{N} \times .693 / \mathrm{T}^{1} / 2$

- $\mathrm{A}(\mathrm{xxxx})=$ Activity for an operating time of $\mathrm{xxxx}$ hours. 


\begin{tabular}{|c|c|c|c|c|c|c|c|c|c|c|c|c|}
\hline \multirow{2}{*}{\multicolumn{2}{|c|}{1 Convertor Type = }} & B & C & D & $\mathrm{E}$ & $\mathrm{F}$ & G & $\mathrm{H}$ & 1 & J & K & L \\
\hline & & & \multicolumn{2}{|c|}{ Tungsten (W) } & \multicolumn{2}{|c|}{$\begin{array}{l}\text { Bremsstralung } \\
\text { Production } \\
\text { Convertor Factor } \\
\text { (Cells B8, C8, D8, E8, } \\
\text { F8, G8, H8, \& J8 only) }\end{array}$} & & \multicolumn{2}{|c|}{$\begin{array}{l}\text { Neutron Production } \\
\text { Convertor Factor } \\
1 \text { (Cell K8 only) }=\end{array}$} & \multirow[t]{2}{*}{2.3} & & \\
\hline & Energy Eo $=$ & 60 & Current I = & 100 & Power $\mathrm{P}=$ & & Beam Dist & ance $s=$ & 592 & & & \\
\hline \multicolumn{13}{|c|}{3} \\
\hline 4 & Radionuclide & $\mathrm{H}-3$ & Be-7 & C-11 & $\mathrm{N}-13$ & $0-15$ & $\mathrm{Cl}-38$ & $\mathrm{Cl}-39$ & $\begin{array}{l}\text { Ar-41 } \\
\text { Beam } \\
\text { Path } \\
\end{array}$ & Ar-39 & $\begin{array}{l}\text { Ar-41 } \\
\text { Convertor }\end{array}$ & \begin{tabular}{|l|} 
Total Ar- \\
41 \\
Activity \\
(columns \\
I + K) \\
\end{tabular} \\
\hline 5 & Half-life & $12.3 y$ & $54 \mathrm{~d}$ & $20.5 \mathrm{~min}$ & $10 \mathrm{~min}$ & $2.1 \mathrm{~min}$ & $37 \mathrm{~min}$ & $55 \mathrm{~min}$ & $1.827 \mathrm{hr}$ & $269 y$ & $1.827 \mathrm{hr}$ & \\
\hline & Half-life (sec) & 387892800 & 4665600 & 1230 & 600 & 126 & 2220 & 3300 & 6577.2 & $8.483 \mathrm{E}+09$ & 6577.2 & \\
\hline & f (ground plane) & $6.00 \mathrm{E}-01$ & $6.00 \mathrm{E}-01$ & $6.00 \mathrm{E}-01$ & $6.00 \mathrm{E}-01$ & $6.00 \mathrm{E}-01$ & $6.00 \mathrm{E}-01$ & $6.00 \mathrm{E}-01$ & 5.00E-01 & 6.00E-01 & 5.00E-01 & \\
\hline 8 & f (convertor) & $1.00 \mathrm{E}+00$ & $1.00 \mathrm{E}+00$ & $1.00 \mathrm{E}+00$ & $1.00 \mathrm{E}+00$ & $1.00 \mathrm{E}+00$ & $1.00 \mathrm{E}+00$ & $1.00 \mathrm{E}+00$ & $1.00 \mathrm{E}+00$ & $1.00 \mathrm{E}+00$ & $2.30 \mathrm{E}+00$ & \\
\hline & $f$ (energy fraction) & 1.20E-01 & $7.00 \mathrm{E}-02$ & $1.50 \mathrm{E}-01$ & $2.60 \mathrm{E}-01$ & $1.70 \mathrm{E}-01$ & $1.30 \mathrm{E}-01$ & $2.30 \mathrm{E}-01$ & $1.00 \mathrm{E}+00$ & $3.10 \mathrm{E}-01$ & $1.00 \mathrm{E}+00$ & \\
\hline 10 & f(neutron capture) & $1.00 \mathrm{E}+00$ & $1.00 \mathrm{E}+00$ & $1.00 \mathrm{E}+00$ & $1.00 \mathrm{E}+00$ & $1.00 \mathrm{E}+00$ & $1.00 \mathrm{E}+00$ & $1.00 \mathrm{E}+00$ & $3.50 \mathrm{E}-02$ & $1.00 \mathrm{E}+00$ & $3.50 \mathrm{E}-02$ & \\
\hline 11 & $\mathrm{YF}$ & $7.10 \mathrm{E}+06$ & $1.10 \mathrm{E}+06$ & $1.10 \mathrm{E}+07$ & $1.10 \mathrm{E}+08$ & $5.60 \mathrm{E}+07$ & $6.80 \mathrm{E}+05$ & $8.50 \mathrm{E}+06$ & $9.06 \mathrm{E}+10$ & $8.50 \mathrm{E}+06$ & $1.00 \mathrm{E}+12$ & \\
\hline 12 & YF adjusted & $5.11 \mathrm{E}+05$ & \begin{tabular}{|l|}
$4.62 E+04$ \\
\end{tabular} & $9.90 \mathrm{E}+05$ & $1.72 \mathrm{E}+07$ & $5.71 E+06$ & $5.30 \mathrm{E}+04$ & $1.17 \mathrm{E}+06$ & $1.58 \mathrm{E}+09$ & $1.58 \mathrm{E}+06$ & $4.03 E+10$ & \\
\hline 13 & Ssat (Bq) & $1.82 \mathrm{E}+09$ & $1.64 \mathrm{E}+08$ & $3.52 E+09$ & $6.10 \mathrm{E}+10$ & $2.03 \mathrm{E}+10$ & $1.88 \mathrm{E}+08$ & $4.17 \mathrm{E}+09$ & $1.58 \mathrm{E}+09$ & $5.62 \mathrm{E}+09$ & $2.42 \mathrm{E}+11$ & \\
\hline 14 & $\mathrm{PR}$ (atoms/s) & $1.82 \mathrm{E}+09$ & $1.64 \mathrm{E}+08$ & $3.52 E+09$ & $6.10 \mathrm{E}+10$ & $2.03 \mathrm{E}+10$ & $1.88 \mathrm{E}+08$ & $4.17 \mathrm{E}+09$ & $1.58 \mathrm{E}+09$ & $5.62 \mathrm{E}+09$ & $2.42 \mathrm{E}+11$ & \\
\hline 15 & $\mathrm{~N}(\mathrm{PR} * 1146 \mathrm{~h})$ & $7.49 \mathrm{E}+15$ & $6.77 \mathrm{E}+14$ & $1.45 \mathrm{E}+16$ & $2.51 \mathrm{E}+17$ & $8.37 \mathrm{E}+16$ & $7.77 \mathrm{E}+14$ & $1.72 \mathrm{E}+16$ & $6.54 \mathrm{E}+15$ & $2.32 \mathrm{E}+16$ & $9.96 \mathrm{E}+17$ & \\
\hline 16 & $\mathrm{~A}(1146 \mathrm{~h})(\mathrm{Bq})$ & $1.34 \mathrm{E}+07$ & \begin{tabular}{|c|}
$1.01 \mathrm{E}+08$ \\
\end{tabular} & $8.17 E+12$ & $2.90 \mathrm{E}+14$ & $4.60 \mathrm{E}+14$ & $2.43 \mathrm{E}+11$ & \begin{tabular}{|c|}
$3.61 \mathrm{E}+12$ \\
\end{tabular} & $6.89 \mathrm{E}+11$ & $1.89 \mathrm{E}+06$ & $1.05 \mathrm{E}+14$ & $1.06 \mathrm{E}+14$ \\
\hline 17 & $\mathrm{~A}(1146 \mathrm{~h})(\mathrm{Ci})$ & $3.62 \mathrm{E}-04$ & \begin{tabular}{|l|}
$2.72 \mathrm{E}-03$ \\
\end{tabular} & $2.21 \mathrm{E}+02$ & $7.85 \mathrm{E}+03$ & $1.24 \mathrm{E}+04$ & $6.56 \mathrm{E}+00$ & $9.76 \mathrm{E}+01$ & $1.86 \mathrm{E}+01$ & $5.12 \mathrm{E}-05$ & $2.84 \mathrm{E}+03$ & $2.86 \mathrm{E}+03$ \\
\hline 18 & $\mathrm{~N}(\mathrm{PR} * 800 \mathrm{~h})$ & $5.23 \mathrm{E}+15$ & $4.73 \mathrm{E}+14$ & $1.01 \mathrm{E}+16$ & $1.76 \mathrm{E}+17$ & $5.84 \mathrm{E}+16$ & $5.43 \mathrm{E}+14$ & $1.20 \mathrm{E}+16$ & $4.56 \mathrm{E}+15$ & $1.62 \mathrm{E}+16$ & $6.96 \mathrm{E}+17$ & \\
\hline 19 & $\mathrm{~A}(800 \mathrm{~h})(\mathrm{Bq})$ & $9.34 \mathrm{E}+06$ & $7.02 \mathrm{E}+07$ & $5.71 \mathrm{E}+12$ & $2.03 E+14$ & $3.21 \mathrm{E}+14$ & $1.69 \mathrm{E}+11$ & $2.52 \mathrm{E}+12$ & $4.81 \mathrm{E}+11$ & $1.32 \mathrm{E}+06$ & $7.33 \mathrm{E}+13$ & $7.38 \mathrm{E}+13$ \\
\hline 20 & $\mathrm{~A}(800 \mathrm{~h})(\mathrm{Ci})$ & $2.53 \mathrm{E}-04$ & \begin{tabular}{|l|l|}
$4.90 \mathrm{E}-03$ \\
\end{tabular} & $1.54 E+02$ & $5.48 \mathrm{E}+03$ & $8.69 \mathrm{E}+03$ & $4.58 \mathrm{E}+00$ & $6.81 \mathrm{E}+01$ & $1.30 \mathrm{E}+01$ & $3.57 E-05$ & $1.98 \mathrm{E}+03$ & $1.99 \mathrm{E}+03$ \\
\hline 21 & Threshold (MeV) & 22.7 & 31.9 & 18.7 & 10.6 & 15.7 & 20.6 & 12.5 & & 9.37 & & \\
\hline 22 & & $\mathrm{H}-3$ & Be-7 & C-11 & $\mathrm{N}-13$ & $0-15$ & $\mathrm{Cl}-38$ & $\mathrm{Cl}-39$ & $\begin{array}{l}\text { Ar-41 } \\
\text { Beam } \\
\text { Path } \\
\end{array}$ & Ar-39 & \begin{tabular}{|l} 
Ar-41 \\
Convertor
\end{tabular} & \begin{tabular}{|l|} 
Total Ar- \\
41 \\
Activity \\
(columns \\
I + K) \\
\end{tabular} \\
\hline \multicolumn{4}{|c|}{23 Maximum MEl Dose at 800 Hours = } & \multicolumn{2}{|c|}{$0.0698 \mathrm{mrem} / \mathrm{yr}$} & & & & & & & \\
\hline 24 & Maximum MEI Dose & at $1146 \mathrm{Hou}$ & urs $=$ & & mrem/yr & & & & & & & \\
\hline
\end{tabular}




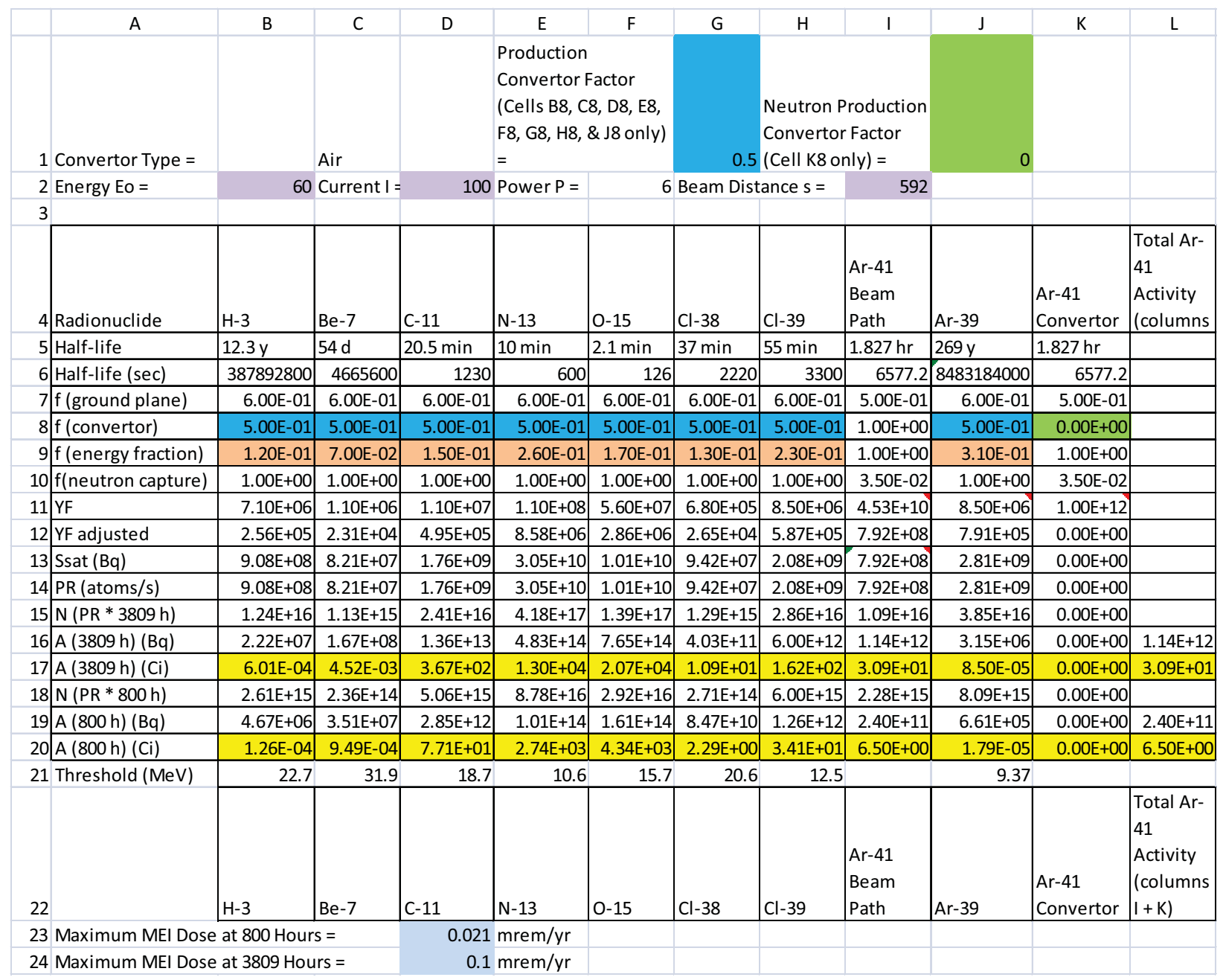




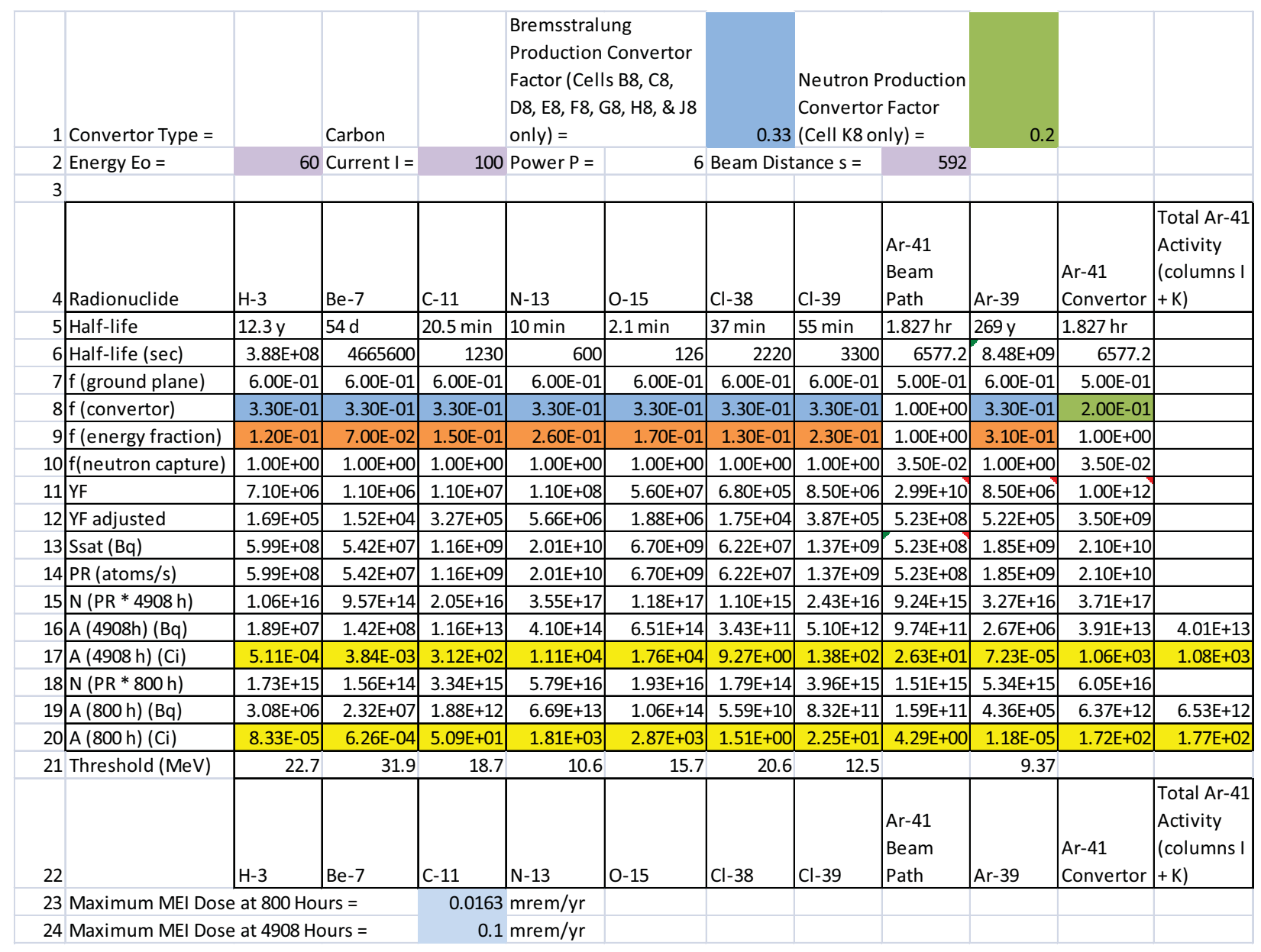




\begin{tabular}{|c|c|c|c|c|c|c|c|c|c|c|c|c|}
\hline & A & B & C & D & $E$ & $\mathrm{~F}$ & G & $\mathrm{H}$ & I & J & K & L \\
\hline & Convertor Type $=$ & & Tungsten & & \multicolumn{2}{|c|}{$\begin{array}{l}\text { Production } \\
\text { Convertor Factor } \\
\text { (Cells B8, C8, D8, E8, } \\
\text { F8, G8, H8, \& J8 only) } \\
=\end{array}$} & \multicolumn{3}{|c|}{$\begin{array}{l}\text { Neutron Production } \\
\text { Convertor Factor } \\
1 \text { (Cell K8 only) }=\end{array}$} & 1.8 & & \\
\hline \multicolumn{2}{|r|}{2 Energy Eo = } & 30 & Current I = & 40 & Power $\mathrm{P}=$ & 1.2 & Beam Dist & tance $s=$ & 592 & & & \\
\hline \multicolumn{13}{|l|}{3} \\
\hline 4 & Radionuclide & $\mathrm{H}-3$ & Be-7 & C-11 & $\mathrm{N}-13$ & $0-15$ & $\mathrm{Cl}-38$ & $\mathrm{Cl}-39$ & \begin{tabular}{|l} 
Ar-41 \\
Beam \\
Path \\
\end{tabular} & Ar-39 & \begin{tabular}{|l|} 
Ar-41 \\
Convertor
\end{tabular} & \begin{tabular}{|l|} 
Total Ar- \\
41 \\
Activity \\
(columns \\
\end{tabular} \\
\hline 5 & Half-life & $12.3 y$ & $54 \mathrm{~d}$ & $20.5 \mathrm{~min}$ & $10 \mathrm{~min}$ & $2.1 \mathrm{~min}$ & $37 \mathrm{~min}$ & $55 \mathrm{~min}$ & $1.827 \mathrm{hr}$ & $269 y$ & $1.827 \mathrm{hr}$ & \\
\hline 6 & Half-life (sec) & 387892800 & 4665600 & 1230 & 600 & 126 & 2220 & 3300 & 6577.2 & $8.483 E+09$ & 6577.2 & \\
\hline 7 & $f$ (ground plane) & $6.00 \mathrm{E}-01$ & $6.00 \mathrm{E}-01$ & $6.00 \mathrm{E}-01$ & $6.00 \mathrm{E}-01$ & $6.00 \mathrm{E}-01$ & $6.00 \mathrm{E}-01$ & $6.00 \mathrm{E}-01$ & $5.00 \mathrm{E}-01$ & $6.00 \mathrm{E}-01$ & $5.00 \mathrm{E}-01$ & \\
\hline 8 & f (convertor) & $1.00 \mathrm{E}+00$ & $1.00 \mathrm{E}+00$ & $1.00 \mathrm{E}+00$ & $1.00 \mathrm{E}+00$ & $1.00 \mathrm{E}+00$ & $1.00 \mathrm{E}+00$ & $1.00 \mathrm{E}+00$ & $1.00 \mathrm{E}+00$ & $1.00 \mathrm{E}+00$ & $1.80 \mathrm{E}+00$ & \\
\hline 9 & $\mathrm{f}$ (energy fraction) & $3.00 \mathrm{E}-02$ & $0.00 \mathrm{E}+00$ & 6.00E-02 & 1.60E-01 & 7.00E-02 & 4.00E-02 & $1.20 \mathrm{E}-01$ & $1.00 \mathrm{E}+00$ & $2.10 \mathrm{E}-01$ & $1.00 \mathrm{E}+00$ & \\
\hline 10 & f(neutron capture) & $1.00 \mathrm{E}+00$ & $1.00 \mathrm{E}+00$ & $1.00 \mathrm{E}+00$ & $1.00 \mathrm{E}+00$ & $1.00 \mathrm{E}+00$ & $1.00 \mathrm{E}+00$ & $1.00 \mathrm{E}+00$ & $3.50 \mathrm{E}-02$ & $1.00 \mathrm{E}+00$ & $3.50 \mathrm{E}-02$ & \\
\hline 11 & $\mathrm{YF}$ & $7.10 E+06$ & $1.10 \mathrm{E}+06$ & $1.10 \mathrm{E}+07$ & $1.10 \mathrm{E}+08$ & $5.60 \mathrm{E}+07$ & $6.80 \mathrm{E}+05$ & $8.50 \mathrm{E}+06$ & $1.02 \mathrm{E}+10$ & $8.50 E+06$ & $1.00 \mathrm{E}+12$ & \\
\hline 12 & YF adjusted & $1.28 \mathrm{E}+05$ & $0.00 \mathrm{E}+00$ & $3.96 \mathrm{E}+05$ & $1.06 \mathrm{E}+07$ & $2.35 \mathrm{E}+06$ & $1.63 \mathrm{E}+04$ & $6.12 \mathrm{E}+05$ & $1.79 \mathrm{E}+08$ & $1.07 \mathrm{E}+06$ & $3.15 \mathrm{E}+10$ & \\
\hline 13 & Ssat $(\mathrm{Bq})$ & $9.08 \mathrm{E}+07$ & $0.00 \mathrm{E}+00$ & $2.81 \mathrm{E}+08$ & $7.50 \mathrm{E}+09$ & $1.67 \mathrm{E}+09$ & $1.16 \mathrm{E}+07$ & $4.35 \mathrm{E}+08$ & $1.79 \mathrm{E}+08$ & $7.61 \mathrm{E}+08$ & $3.78 \mathrm{E}+10$ & \\
\hline 14 & $\mathrm{PR}$ (atoms/s) & $9.08 \mathrm{E}+07$ & $0.00 \mathrm{E}+00$ & $2.81 \mathrm{E}+08$ & $7.50 \mathrm{E}+09$ & $1.67 \mathrm{E}+09$ & $1.16 \mathrm{E}+07$ & $4.35 \mathrm{E}+08$ & $1.79 \mathrm{E}+08$ & $7.61 \mathrm{E}+08$ & $3.78 \mathrm{E}+10$ & \\
\hline 15 & $\mathrm{~N}(\mathrm{PR} * 8676 \mathrm{~h})$ & $2.84 \mathrm{E}+15$ & $0.00 \mathrm{E}+00$ & $8.79 \mathrm{E}+15$ & $2.34 \mathrm{E}+17$ & $5.22 \mathrm{E}+16$ & $3.62 \mathrm{E}+14$ & $1.36 \mathrm{E}+16$ & $5.59 \mathrm{E}+15$ & $2.38 \mathrm{E}+16$ & $1.18 \mathrm{E}+18$ & \\
\hline 16 & $A(8676 h)(B q)$ & $5.07 \mathrm{E}+06$ & $0.00 \mathrm{E}+00$ & $4.95 \mathrm{E}+12$ & $2.71 \mathrm{E}+14$ & $2.87 \mathrm{E}+14$ & $1.13 \mathrm{E}+11$ & $2.85 \mathrm{E}+12$ & $5.89 \mathrm{E}+11$ & $1.94 \mathrm{E}+06$ & $1.24 \mathrm{E}+14$ & $1.25 \mathrm{E}+14$ \\
\hline 17 & A (8676 h) (Ci) & $1.37 \mathrm{E}-04$ & $0.00 \mathrm{E}+00$ & $1.34 \mathrm{E}+02$ & $7.31 E+03$ & $7.76 E+03$ & $3.06 \mathrm{E}+00$ & $7.71 \mathrm{E}+01$ & $1.59 \mathrm{E}+01$ & 5.25E-05 & $3.36 \mathrm{E}+03$ & \begin{tabular}{l|l}
$3.38 \mathrm{E}+03$ \\
\end{tabular} \\
\hline 18 & $N(P R * 800 h)$ & $2.61 \mathrm{E}+14$ & $0.00 E+00$ & $8.10 \mathrm{E}+14$ & $2.16 \mathrm{E}+16$ & $4.81 \mathrm{E}+15$ & $3.34 \mathrm{E}+13$ & $1.25 \mathrm{E}+15$ & $5.15 \mathrm{E}+14$ & $2.19 \mathrm{E}+15$ & $1.09 \mathrm{E}+17$ & \\
\hline 19 & $\mathrm{~A}(800 \mathrm{~h})(\mathrm{Bq})$ & $4.67 E+05$ & $0.00 \mathrm{E}+00$ & $4.56 \mathrm{E}+11$ & $2.50 \mathrm{E}+13$ & $2.65 \mathrm{E}+13$ & $1.04 \mathrm{E}+10$ & $2.63 \mathrm{E}+11$ & $5.43 \mathrm{E}+10$ & $1.79 \mathrm{E}+05$ & $1.15 \mathrm{E}+13$ & $1.15 \mathrm{E}+13$ \\
\hline 20 & $\mathrm{~A}(800 \mathrm{~h})(\mathrm{Ci})$ & $1.26 \mathrm{E}-05$ & $0.00 \mathrm{E}+00$ & $1.23 \mathrm{E}+01$ & $6.74 \mathrm{E}+02$ & $7.15 E+02$ & $2.82 \mathrm{E}-01$ & $7.11 \mathrm{E}+00$ & $1.47 \mathrm{E}+00$ & 4.84E-06 & $3.10 \mathrm{E}+02$ & $3.11 \mathrm{E}+02$ \\
\hline 21 & Threshold (MeV) & 22.7 & 31.9 & 18.7 & 10.6 & 15.7 & 20.6 & 12.5 & & 9.37 & & \\
\hline 22 & & $\mathrm{H}-3$ & Be-7 & C-11 & $\mathrm{N}-13$ & $0-15$ & $\mathrm{Cl}-38$ & $\mathrm{Cl}-39$ & \begin{tabular}{|l} 
Ar-41 \\
Beam \\
Path \\
\end{tabular} & Ar-39 & $\begin{array}{l}\text { Ar-41 } \\
\text { Convertor }\end{array}$ & \begin{tabular}{|l|} 
Total Ar- \\
41 \\
Activity \\
(columns \\
I +K) \\
\end{tabular} \\
\hline \multicolumn{4}{|c|}{23 Maximum MEl Dose at 800 Hours = } & \multicolumn{2}{|c|}{$0.00922 \mathrm{mrem} / \mathrm{yr}$} & & & & & & & \\
\hline 24 & Maximum MEI Dose & at $8676 \mathrm{Hou}$ & urs $=$ & 0.1 & $\mathrm{mrem} / \mathrm{yr}$ & & & & & & & \\
\hline
\end{tabular}




\begin{tabular}{|c|c|c|c|c|c|c|c|c|c|c|c|c|}
\hline & A & B & C & $\mathrm{D}$ & $E$ & $\mathrm{~F}$ & G & $\mathrm{H}$ & 1 & $\mathrm{~J}$ & K & L \\
\hline & Convertor Type $=$ & & \multirow{2}{*}{\multicolumn{2}{|c|}{ Aluminum (Al) }} & \multicolumn{2}{|c|}{$\begin{array}{l}\text { Bremsstralung } \\
\text { Production Convertor } \\
\text { Factor (Cells B8, C8, } \\
\text { D8, E8, F8, G8, H8, \& } \\
\text { J8 only) = }\end{array}$} & \multicolumn{3}{|c|}{$\begin{array}{l}\text { Neutron Production } \\
\text { Convertor Factor } \\
7 \text { (Cell K8 only) = }\end{array}$} & \multirow[t]{2}{*}{0.1} & & \\
\hline & Energy Eo = & 30 & & 40 & Power $\mathrm{P}=$ & 1.2 & Beam Dist & tance $s=$ & 592 & & & \\
\hline \multicolumn{13}{|l|}{3} \\
\hline 4 & Radionuclide & $\mathrm{H}-3$ & Be-7 & C-11 & $\mathrm{N}-13$ & $0-15$ & $\mathrm{Cl}-38$ & $\mathrm{Cl}-39$ & $\begin{array}{l}\text { Ar-41 } \\
\text { Beam } \\
\text { Path }\end{array}$ & Ar-39 & $\begin{array}{l}\text { Ar-41 } \\
\text { Convertor }\end{array}$ & \begin{tabular}{|l|} 
Total Ar- \\
41 \\
Activity \\
(columns \\
I +K)
\end{tabular} \\
\hline 5 & Half-life & $12.3 y$ & $54 d$ & $20.5 \mathrm{~min}$ & $10 \mathrm{~min}$ & $2.1 \mathrm{~min}$ & $37 \mathrm{~min}$ & $55 \mathrm{~min}$ & $1.827 \mathrm{hr}$ & $269 y$ & $1.827 \mathrm{hr}$ & \\
\hline 6 & Half-life (sec) & 387892800 & 4665600 & 1230 & 600 & 126 & 2220 & 3300 & 6577.2 & $8.483 E+09$ & 6577.2 & \\
\hline 7 & $f$ (ground plane) & $6.00 \mathrm{E}-01$ & $6.00 \mathrm{E}-01$ & $6.00 \mathrm{E}-01$ & $6.00 \mathrm{E}-01$ & $6.00 \mathrm{E}-01$ & 6.00E-01 & $6.00 \mathrm{E}-01$ & 5.00E-01 & $6.00 \mathrm{E}-01$ & 5.00E-01 & \\
\hline 8 & $f$ (convertor) & $3.70 \mathrm{E}-01$ & $3.70 \mathrm{E}-01$ & $3.70 \mathrm{E}-01$ & 3.70E-01 & $3.70 \mathrm{E}-01$ & $3.70 \mathrm{E}-01$ & 3.70E-01 & $1.00 \mathrm{E}+00$ & $3.70 \mathrm{E}-01$ & 1.00E-01 & \\
\hline 9 & $f$ (energy fraction) & $3.00 \mathrm{E}-02$ & $0.00 \mathrm{E}+00$ & $6.00 \mathrm{E}-02$ & $1.60 \mathrm{E}-01$ & $7.00 \mathrm{E}-02$ & $4.00 \mathrm{E}-02$ & $1.20 \mathrm{E}-01$ & $1.00 \mathrm{E}+00$ & $2.10 \mathrm{E}-01$ & $1.00 \mathrm{E}+00$ & \\
\hline 10 & $f($ neutron capture) & $1.00 \mathrm{E}+00$ & $1.00 \mathrm{E}+00$ & $1.00 \mathrm{E}+00$ & $1.00 \mathrm{E}+00$ & $1.00 \mathrm{E}+00$ & $1.00 \mathrm{E}+00$ & $1.00 \mathrm{E}+00$ & $3.50 \mathrm{E}-02$ & $1.00 \mathrm{E}+00$ & $3.50 \mathrm{E}-02$ & \\
\hline 11 & $\mathrm{YF}$ & $7.10 E+06$ & $1.10 \mathrm{E}+06$ & $1.10 \mathrm{E}+07$ & $1.10 \mathrm{E}+08$ & $5.60 \mathrm{E}+07$ & $6.80 \mathrm{E}+05$ & $8.50 E+06$ & $3.78 \mathrm{E}+09$ & $8.50 E+06$ & $1.00 \mathrm{E}+12$ & \\
\hline 12 & YF adjusted & $4.73 \mathrm{E}+04$ & $0.00 \mathrm{E}+00$ & $1.47 \mathrm{E}+05$ & $3.91 \mathrm{E}+06$ & $8.70 \mathrm{E}+05$ & $6.04 E+03$ & $2.26 \mathrm{E}+05$ & $6.62 \mathrm{E}+07$ & $3.96 \mathrm{E}+05$ & $1.75 \mathrm{E}+09$ & \\
\hline 13 & Ssat (Bq) & $3.36 \mathrm{E}+07$ & $0.00 E+00$ & $1.04 \mathrm{E}+08$ & $2.78 \mathrm{E}+09$ & $6.18 \mathrm{E}+08$ & $4.29 \mathrm{E}+06$ & $1.61 \mathrm{E}+08$ & $6.62 \mathrm{E}+07$ & $2.82 \mathrm{E}+08$ & $2.10 \mathrm{E}+09$ & \\
\hline 14 & PR (atoms/s) & $3.36 \mathrm{E}+07$ & $0.00 \mathrm{E}+00$ & $1.04 \mathrm{E}+08$ & $2.78 \mathrm{E}+09$ & $6.18 \mathrm{E}+08$ & $4.29 E+06$ & $1.61 \mathrm{E}+08$ & $6.62 \mathrm{E}+07$ & $2.82 \mathrm{E}+08$ & $2.10 \mathrm{E}+09$ & \\
\hline 15 & $\mathrm{~N}(\mathrm{PR} * 39024 \mathrm{~h})$ & $4.72 \mathrm{E}+15$ & $0.00 \mathrm{E}+00$ & $1.46 \mathrm{E}+16$ & $3.90 \mathrm{E}+17$ & $8.69 \mathrm{E}+16$ & $6.03 E+14$ & $2.26 \mathrm{E}+16$ & $9.30 \mathrm{E}+15$ & $3.95 \mathrm{E}+16$ & $2.95 \mathrm{E}+17$ & \\
\hline 16 & $\mathrm{~A}(39024 \mathrm{~h})(\mathrm{Bq})$ & $8.43 E+06$ & $0.00 \mathrm{E}+00$ & $8.24 \mathrm{E}+12$ & $4.50 E+14$ & $4.78 \mathrm{E}+14$ & $1.88 \mathrm{E}+11$ & $4.75 \mathrm{E}+12$ & $9.80 \mathrm{E}+11$ & $3.23 E+06$ & $3.11 \mathrm{E}+13$ & $3.21 \mathrm{E}+13$ \\
\hline 17 & $\mathrm{~A}(39024 \mathrm{~h})(\mathrm{Ci})$ & $2.28 \mathrm{E}-04$ & $0.00 \mathrm{E}+00$ & $2.23 \mathrm{E}+02$ & $1.22 \mathrm{E}+04$ & $1.29 \mathrm{E}+04$ & $5.08 \mathrm{E}+00$ & $1.28 \mathrm{E}+02$ & $2.65 \mathrm{E}+01$ & $8.73 \mathrm{E}-05$ & $8.40 \mathrm{E}+02$ & $8.67 \mathrm{E}+02$ \\
\hline 18 & $\mathrm{~N}(\mathrm{PR} * 800 \mathrm{~h})$ & $9.67 E+13$ & $0.00 \mathrm{E}+00$ & $3.00 \mathrm{E}+14$ & $7.99 \mathrm{E}+15$ & $1.78 \mathrm{E}+15$ & $1.24 \mathrm{E}+13$ & $4.63 \mathrm{E}+14$ & $1.91 \mathrm{E}+14$ & $8.11 \mathrm{E}+14$ & $6.05 E+15$ & \\
\hline 19 & $A(800 h)(B q)$ & $1.73 \mathrm{E}+05$ & $0.00 \mathrm{E}+00$ & $1.69 \mathrm{E}+11$ & $9.23 \mathrm{E}+12$ & $9.79 \mathrm{E}+12$ & $3.86 \mathrm{E}+09$ & $9.73 \mathrm{E}+10$ & $2.01 \mathrm{E}+10$ & $6.62 \mathrm{E}+04$ & $6.37 \mathrm{E}+11$ & $6.57 \mathrm{E}+11$ \\
\hline 20 & $A(800 h)(C i)$ & 4.67E-06 & $0.00 E+00$ & $4.56 \mathrm{E}+00$ & $2.50 \mathrm{E}+02$ & $2.65 \mathrm{E}+02$ & 1.04E-01 & $2.63 \mathrm{E}+00$ & 5.43E-01 & $1.79 \mathrm{E}-06$ & $1.72 \mathrm{E}+01$ & $1.78 \mathrm{E}+01$ \\
\hline 21 & Threshold (MeV) & 22.7 & 31.9 & 18.7 & 10.6 & 15.7 & 20.6 & 12.5 & & 9.37 & & \\
\hline 22 & & $\mathrm{H}-3$ & $\mathrm{Be}-7$ & C-11 & $\mathrm{N}-13$ & $0-15$ & $\mathrm{Cl}-38$ & $\mathrm{Cl}-39$ & \begin{tabular}{|l} 
Ar-41 \\
Beam \\
Path \\
\end{tabular} & Ar-39 & $\begin{array}{l}\text { Ar-41 } \\
\text { Convertor }\end{array}$ & \begin{tabular}{|l|} 
Total Ar- \\
41 \\
Activity \\
(columns \\
$+K$ ) \\
\end{tabular} \\
\hline \multicolumn{4}{|c|}{23 Maximum MEI Dose at 800 Hours $=$} & \multicolumn{2}{|c|}{$0.00205 \mathrm{mrem} / \mathrm{yr}$} & & & & & & & \\
\hline & Maximum MEI Dos & at $39024 \mathrm{Hc}$ & ours $=$ & & $\mathrm{mrem} / \mathrm{yr}$ & & & & & & & \\
\hline
\end{tabular}




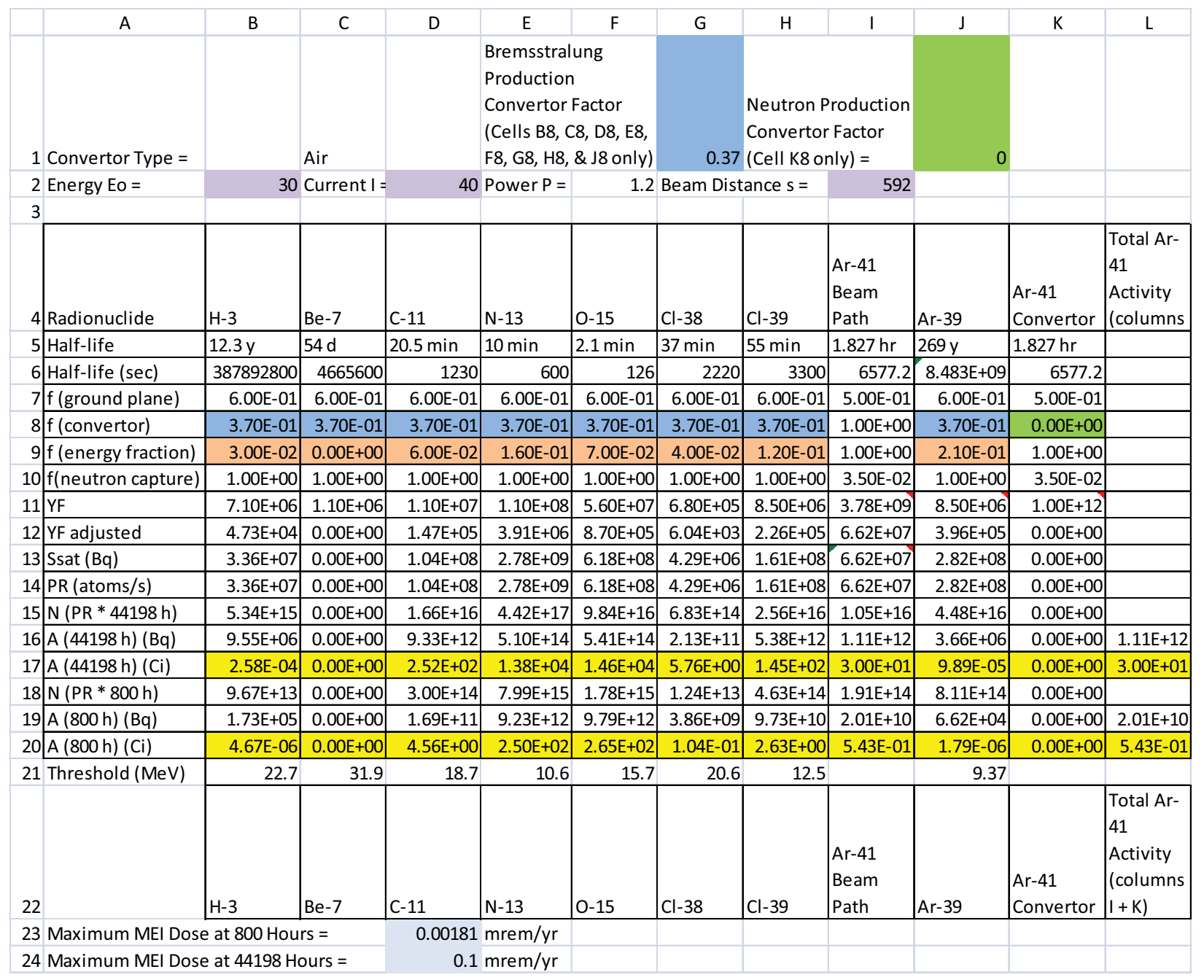




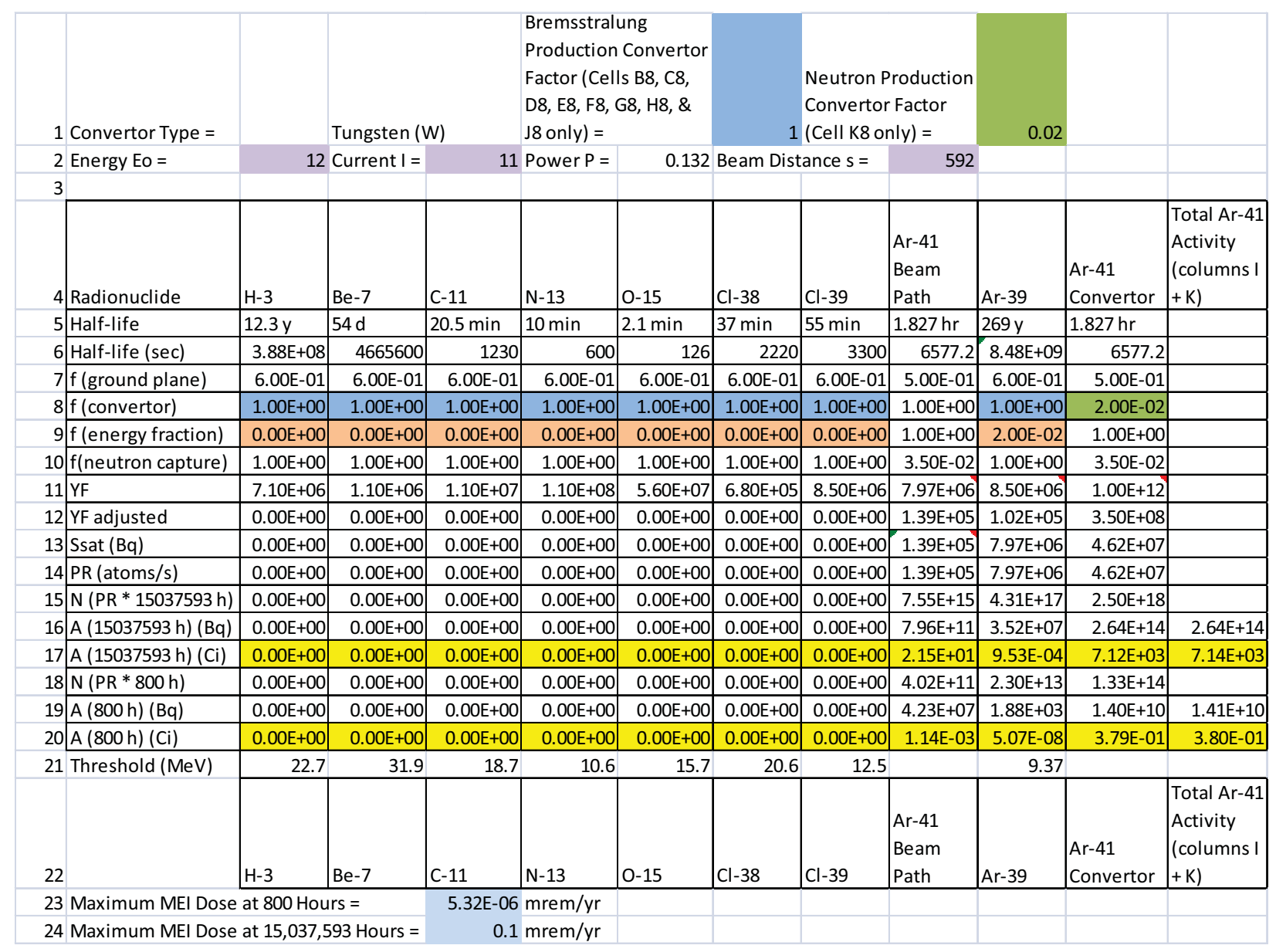




\section{Appendix D}

Relative Capture Fractions for Thermal Neutrons in Isotopes of Air 


\section{Appendix D}

\section{Relative Capture Fractions for Thermal Neutrons in Isotopes of Air}

\begin{tabular}{|c|c|c|c|c|}
\hline $\begin{array}{c}\text { STP } \\
\text { Air } \\
\text { Isotope }\end{array}$ & $\begin{array}{c}\text { No. density } \\
\text { (atoms/barn/cm) }\end{array}$ & $\begin{array}{c}\text { Microscopic } \\
\text { Radiative Capture } \\
\text { Cross Section } \\
\text { at } 0.02 \mathrm{eV} \\
\text { (barn) }\end{array}$ & $\begin{array}{c}\text { Macroscopic } \\
\text { Cross Section } \\
\text { Capital Sigma } \\
\text { Product } \\
(1 / \mathrm{cm})\end{array}$ & $\begin{array}{l}\text { Capture } \\
\text { Fraction }\end{array}$ \\
\hline $\mathrm{N}-14$ & 3.89414E-05 & 0.1 & 3.89414E-06 & 0.96298 \\
\hline $\mathrm{N}-15$ & $1.44648 \mathrm{E}-07$ & $3.00 \mathrm{E}-05$ & 4.33944E-12 & 0.00000 \\
\hline $0-16$ & $1.04878 \mathrm{E}-05$ & 0.0003 & 3.14634E-09 & 0.00078 \\
\hline 0-17 & 4.20520E-09 & 0.005 & $2.10260 \mathrm{E}-11$ & 0.00001 \\
\hline $0-18$ & $2.10260 \mathrm{E}-08$ & 0.00016 & $3.36416 \mathrm{E}-12$ & 0.00000 \\
\hline C-12 & 8.14756E-09 & 0.004 & $3.25902 \mathrm{E}-11$ & 0.00001 \\
\hline$C-13$ & $9.06199 \mathrm{E}-11$ & 0.00137 & 1.24149E-13 & 0.00000 \\
\hline Ar-36 & $7.89106 \mathrm{E}-10$ & 5.2 & 4.10335E-09 & 0.00101 \\
\hline Ar-38 & $1.47518 \mathrm{E}-10$ & 0.8 & $1.18014 \mathrm{E}-10$ & 0.00003 \\
\hline Ar-40 & $2.33219 \mathrm{E}-07$ & 0.61 & $1.42264 \mathrm{E}-07$ & 0.03518 \\
\hline
\end{tabular}

The capture factor is the macroscopic cross section $(\Sigma)$ product for a specific air isotope divided by the sum of all $\Sigma$ values. This represents the fraction of thermal neutrons that will be captured by a specific air isotope.

Cross section data from "Guidebook for the ENDF/B-V Nuclear Data Files, NP-2510, ENDF328, July 1982 


\section{Appendix E}

Neutrons Production from Thick Targets (Convertor) 


\section{Appendix E}

\section{Neutrons Production from Thick Targets (Convertor)}

\begin{tabular}{|l|l|l|l|l|}
\hline $\begin{array}{l}\text { Maximum } \\
\text { Electron Beam } \\
\text { Energy } \\
(\mathrm{MeV})\end{array}$ & Tungsten* & Aluminum* & Carbon* & Air* \\
\hline 60 & 2.3 & 0.4 & 0.2 & 0 \\
\hline 30 & 1.8 & 0.1 & 0.04 & 0 \\
\hline 12 & 0.02 & 0 & 0 & 0 \\
\hline & & & & \\
\hline
\end{tabular}

*Neutrons $\times 10^{12}$ per $\mathrm{kW}$

Reference - Figure 3.12, NCRP-144 
Appendix $F$

CAP88 Data 


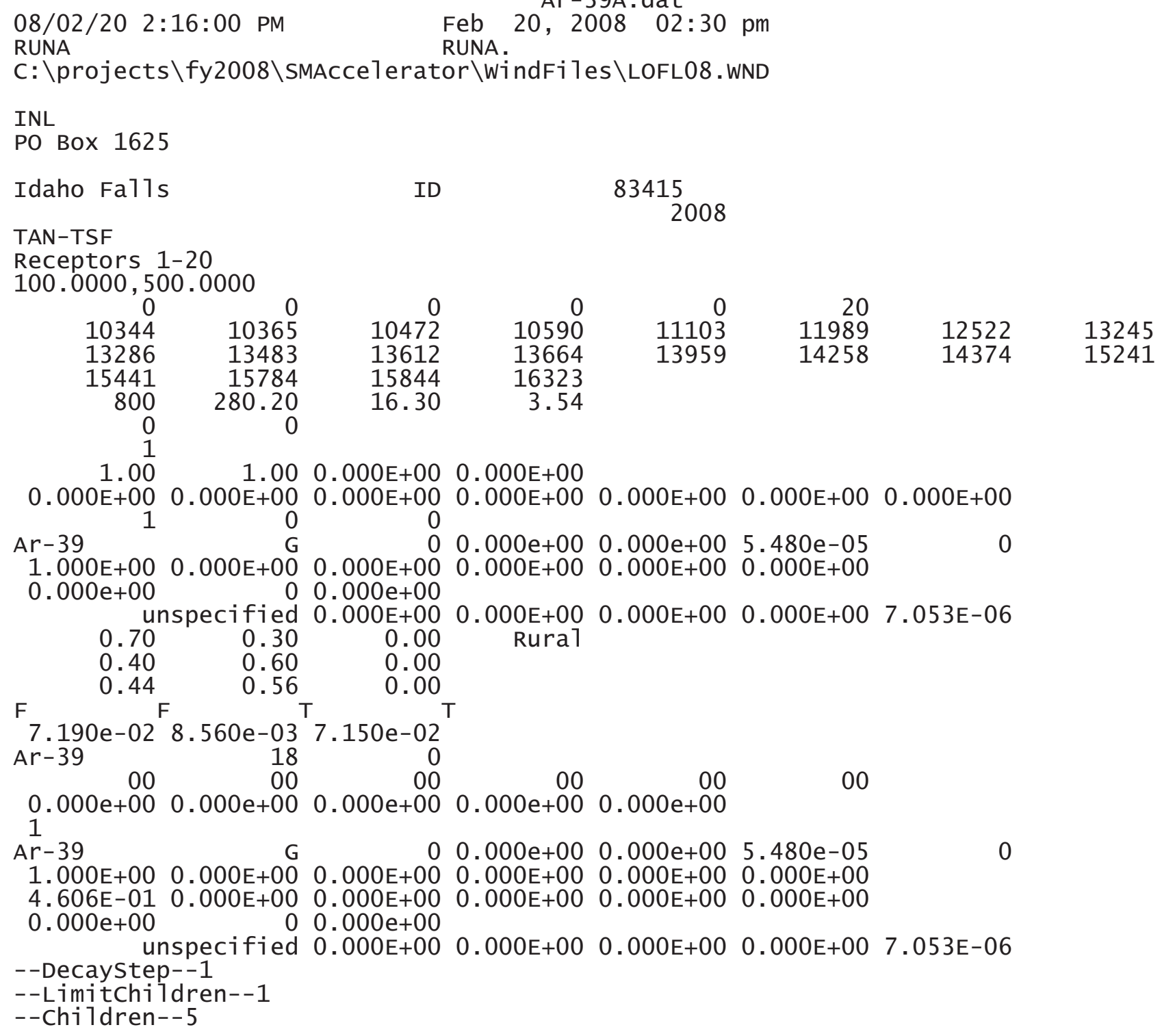




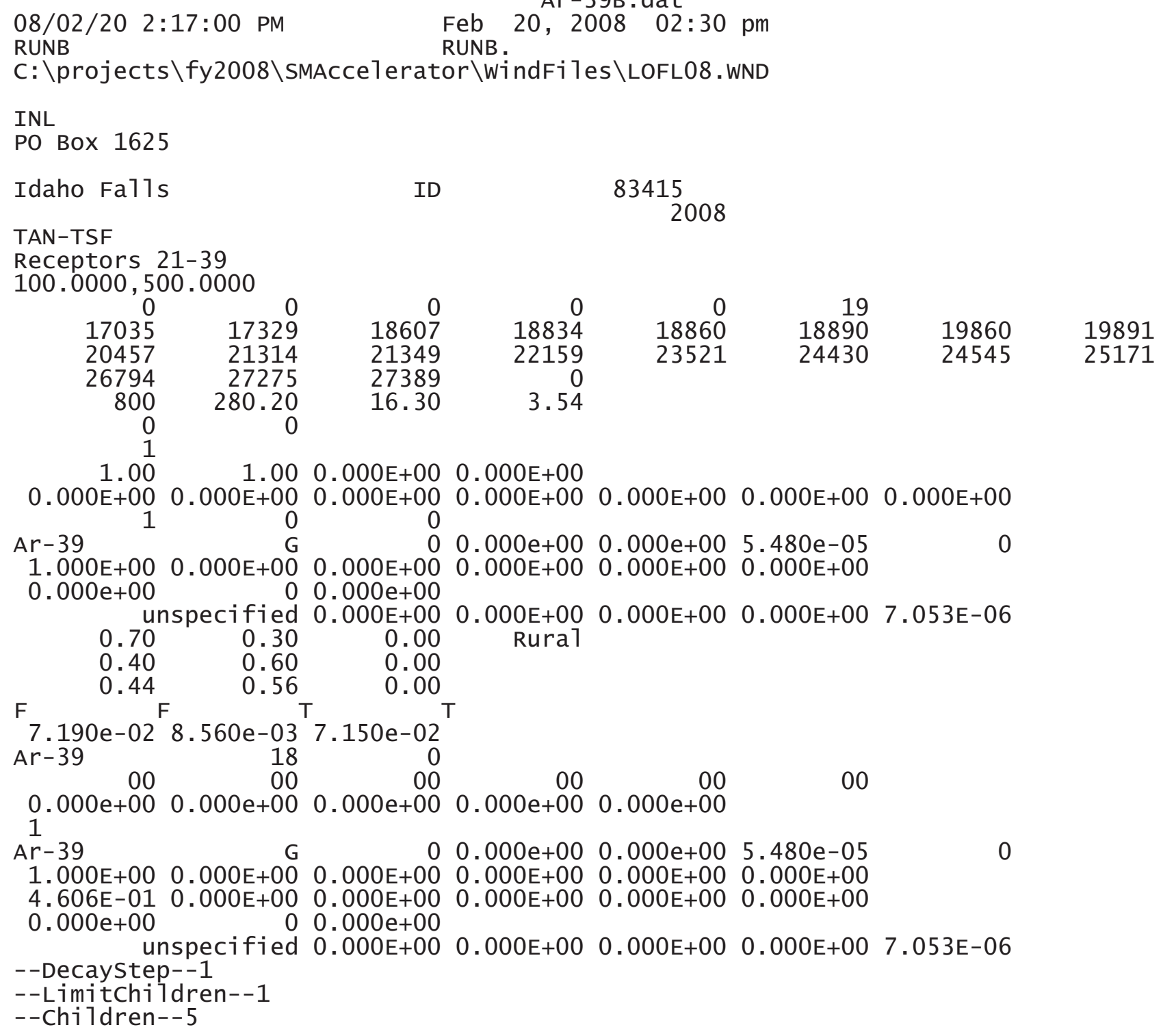




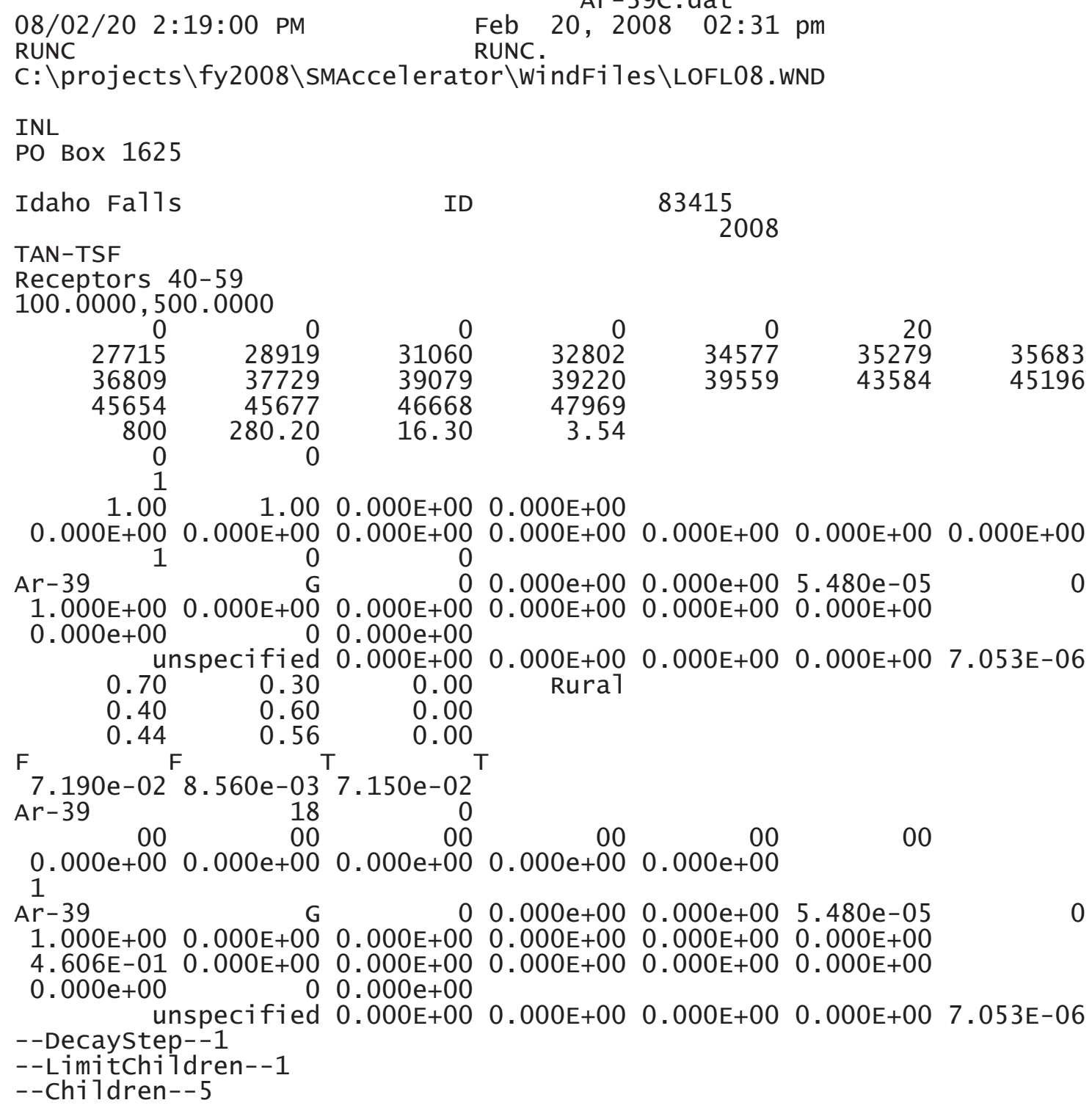

ID

83415

TAN-TSF

Receptors 40-59

$100.0000,500.0000$

$\begin{array}{rrrr}0 & 0 & 0 & \\ 27715 & 28919 & 31060 & 32802 \\ 36809 & 37729 & 39079 & 39220 \\ 45654 & 45677 & 46668 & 47969 \\ 800 & 280.20 & 16.30 & 3.54 \\ 0 & 0 & & \end{array}$




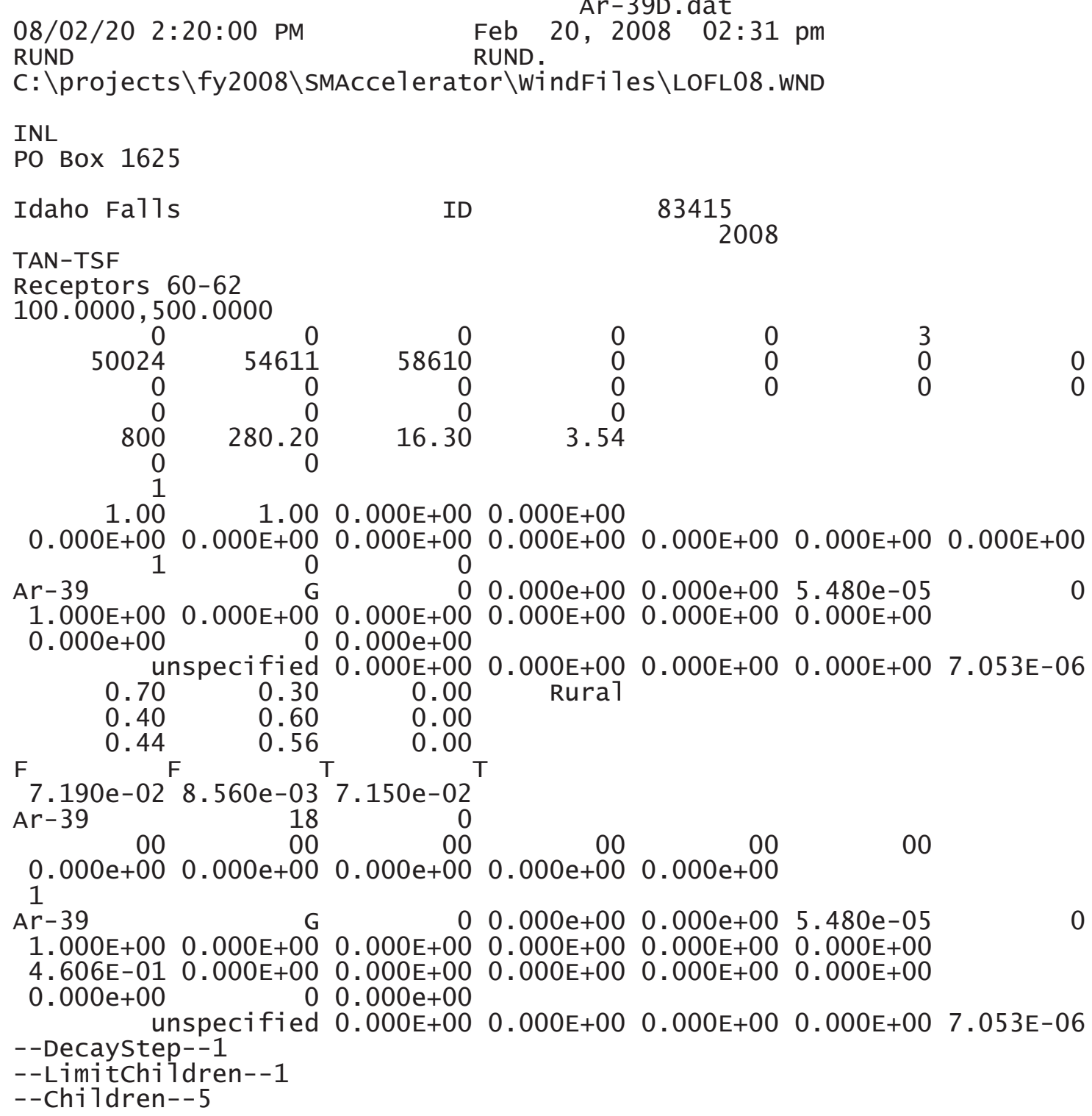




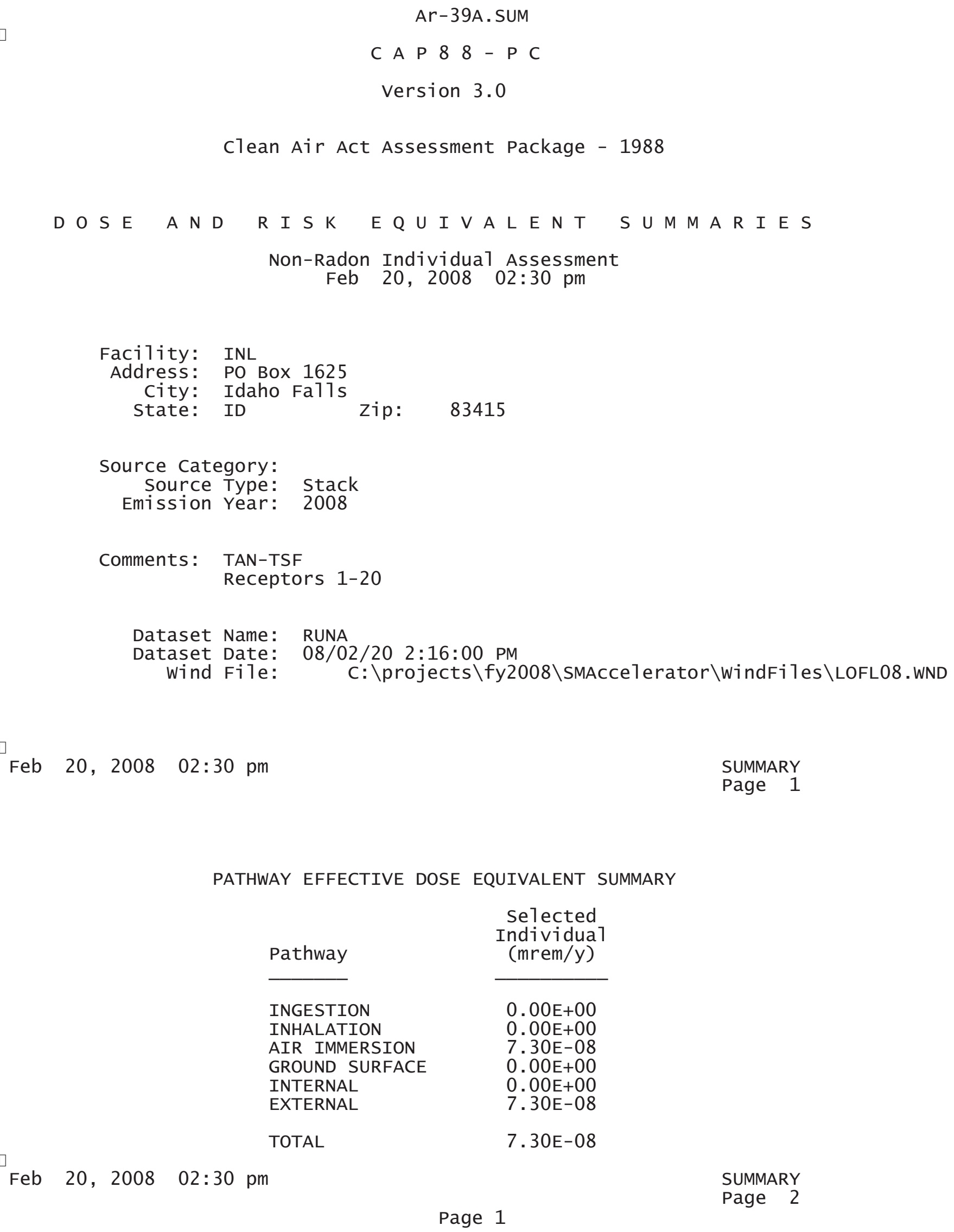

$\begin{array}{lc}\text { Pathway } & \begin{array}{c}\text { } \begin{array}{c}\text { Individual } \\ \text { (mrem/y) }\end{array} \\$\cline { 2 - 2 } \text {$INGESTION }\end{array} \\ \text { INHALATION } & 0.00 \mathrm{E}+00 \\ \text { AIR IMMERSION } & 0.00 \mathrm{E}+00 \\ \text { GROUND SURFACE } & 7.30 \mathrm{E}-08 \\ \text { INTERNAL } & 0.00 \mathrm{E}+00 \\ \text { EXTERNAL } & 0.00 \mathrm{E}+00 \\ \text { TOTAL } & 7.30 \mathrm{E}-08 \\ & 7.30 \mathrm{E}-08\end{array}$

Feb 20, $2008 \quad 02: 30$ pm 
Ar-39A. SUM

NUCLIDE EFFECTIVE DOSE EQUIVALENT SUMMARY

Nuclide

Ar-39

TOTAL

Feb 20, $2008 \quad 02: 30 \mathrm{pm}$

$$
\begin{gathered}
\begin{array}{c}
\text { Selected } \\
\text { Individual } \\
(\mathrm{mrem} / \mathrm{y})
\end{array} \\
\hline 7.30 \mathrm{E}-08 \\
7.30 \mathrm{E}-08
\end{gathered}
$$

SUMMARY

Page 3

CANCER RISK SUMMARY

\section{Cancer \\ Esophagu \\ Stomach \\ colon \\ Liver \\ LUNG \\ Bone \\ skin \\ Breast \\ ovary \\ Bladder \\ Kidneys \\ Thyroid \\ Leukemia \\ Residua 1 \\ Total}

TOTAL selected Individual Total Lifetime Fatal Cancer Risk

\section{3. $71 \mathrm{E}-17$}

1. $70 \mathrm{E}-16$

$3.63 \mathrm{E}-16$

$6.48 \mathrm{E}-17$

$4.86 \mathrm{E}-16$

$1.65 \mathrm{E}-17$

$6.79 \mathrm{E}-15$

$3.50 \mathrm{E}-16$

$4.45 \mathrm{E}-17$

9. $59 \mathrm{E}-17$

2. $32 \mathrm{E}-17$

1. $79 \mathrm{E}-17$

2. $36 \mathrm{E}-16$

$5.99 \mathrm{E}-16$

$9.27 \mathrm{E}-15$

1. $86 \mathrm{E}-14$

PATHWAY RISK SUMMARY

Pathway

INGESTION

INHALATION

AIR IMMERSION

GROUND SURFACE

INTERNAL

EXTERNAL

TOTAL
Selected Individual

Total Lifetime Fatal Cancer Risk

\section{$0.00 \mathrm{E}+00$}

$0.00 \mathrm{E}+00$

9.27E-15

$0.00 \mathrm{E}+00$

$0.00 \mathrm{E}+00$

9. 27E-15

$9.27 E-15$

Page 2 
Ar-39A. SUM

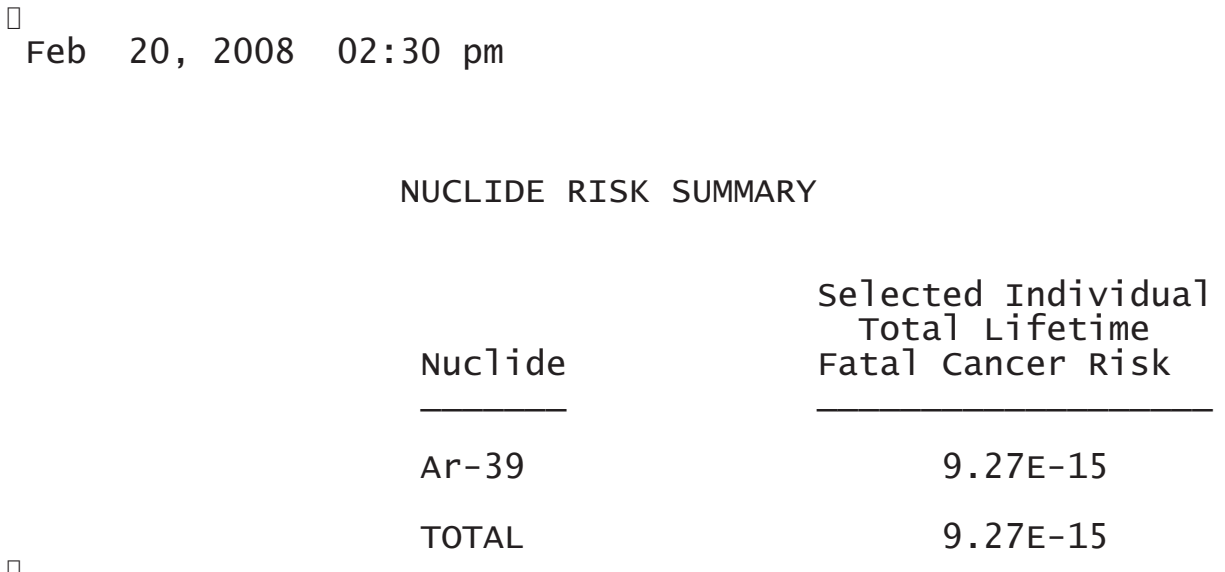

Feb 20, $2008 \quad 02: 30$ pm

SUMMARY

Page 5

INDIVIDUAL EFFECTIVE DOSE EQUIVALENT RATE (mrem/y)

(A11 Radionuclides and Pathways)

\begin{tabular}{|c|c|c|c|c|c|c|c|}
\hline \multicolumn{8}{|c|}{ Distance (m) } \\
\hline Direction & 10344 & 10365 & 10472 & 10590 & 11103 & 11989 & 12522 \\
\hline $\begin{array}{r}N \\
N N W \\
N W \\
W N W \\
W \\
W S W \\
\text { SW } \\
\text { SSW } \\
S \\
\text { SSE } \\
\text { SE } \\
\text { ESE } \\
E \\
\text { ENE } \\
N E \\
N N E\end{array}$ & $\begin{array}{l}1.8 \mathrm{E}-08 \\
6.7 \mathrm{E}-09 \\
9.2 \mathrm{E}-09 \\
1.2 \mathrm{E}-08 \\
1.4 \mathrm{E}-08 \\
1.9 \mathrm{E}-08 \\
3.1 \mathrm{E}-08 \\
5.2 \mathrm{E}-08 \\
7.3 \mathrm{E}-08 \\
6.1 \mathrm{E}-08 \\
3.2 \mathrm{E}-08 \\
2.1 \mathrm{E}-08 \\
2.7 \mathrm{E}-08 \\
2.3 \mathrm{E}-08 \\
3.6 \mathrm{E}-08 \\
2.3 \mathrm{E}-08\end{array}$ & $\begin{array}{l}1.8 \mathrm{E}-08 \\
6.7 \mathrm{E}-09 \\
9.2 \mathrm{E}-09 \\
1.2 \mathrm{E}-08 \\
1.4 \mathrm{E}-08 \\
1.9 \mathrm{E}-08 \\
3.1 \mathrm{E}-08 \\
5.2 \mathrm{E}-08 \\
7.3 \mathrm{E}-08 \\
6.1 \mathrm{E}-08 \\
3.2 \mathrm{E}-08 \\
2.1 \mathrm{E}-08 \\
2.7 \mathrm{E}-08 \\
2.3 \mathrm{E}-08 \\
3.6 \mathrm{E}-08 \\
2.2 \mathrm{E}-08\end{array}$ & $\begin{array}{l}1.7 \mathrm{E}-08 \\
6.6 \mathrm{E}-09 \\
9.1 \mathrm{E}-09 \\
1.2 \mathrm{E}-08 \\
1.4 \mathrm{E}-08 \\
1.9 \mathrm{E}-08 \\
3.1 \mathrm{E}-08 \\
5.1 \mathrm{E}-08 \\
7.2 \mathrm{E}-08 \\
6.0 \mathrm{E}-08 \\
3.1 \mathrm{E}-08 \\
2.1 \mathrm{E}-08 \\
2.7 \mathrm{E}-08 \\
2.3 \mathrm{E}-08 \\
3.5 \mathrm{E}-08 \\
2.2 \mathrm{E}-08\end{array}$ & $\begin{array}{l}1.7 \mathrm{E}-08 \\
6.5 \mathrm{E}-09 \\
9.0 \mathrm{E}-09 \\
1.2 \mathrm{E}-08 \\
1.4 \mathrm{E}-08 \\
1.9 \mathrm{E}-08 \\
3.0 \mathrm{E}-08 \\
5.0 \mathrm{E}-08 \\
7.1 \mathrm{E}-08 \\
5.9 \mathrm{E}-08 \\
3.1 \mathrm{E}-08 \\
2.1 \mathrm{E}-08 \\
2.6 \mathrm{E}-08 \\
2.3 \mathrm{E}-08 \\
3.5 \mathrm{E}-08 \\
2.2 \mathrm{E}-08\end{array}$ & $\begin{array}{l}1.6 \mathrm{E}-08 \\
6.2 \mathrm{E}-09 \\
8.5 \mathrm{E}-09 \\
1.1 \mathrm{E}-08 \\
1.3 \mathrm{E}-08 \\
1.8 \mathrm{E}-08 \\
2.9 \mathrm{E}-08 \\
4.8 \mathrm{E}-08 \\
6.7 \mathrm{E}-08 \\
5.6 \mathrm{E}-08 \\
2.9 \mathrm{E}-08 \\
1.9 \mathrm{E}-08 \\
2.5 \mathrm{E}-08 \\
2.2 \mathrm{E}-08 \\
3.3 \mathrm{E}-08 \\
2.1 \mathrm{E}-08\end{array}$ & $\begin{array}{l}1.5 \mathrm{E}-08 \\
5.6 \mathrm{E}-09 \\
7.8 \mathrm{E}-09 \\
1.0 \mathrm{E}-08 \\
1.2 \mathrm{E}-08 \\
1.6 \mathrm{E}-08 \\
2.6 \mathrm{E}-08 \\
4.3 \mathrm{E}-08 \\
6.1 \mathrm{E}-08 \\
5.1 \mathrm{E}-08 \\
2.7 \mathrm{E}-08 \\
1.8 \mathrm{E}-08 \\
2.3 \mathrm{E}-08 \\
2.0 \mathrm{E}-08 \\
3.0 \mathrm{E}-08 \\
1.9 \mathrm{E}-08\end{array}$ & $\begin{array}{l}1.4 \mathrm{E}-08 \\
5.4 \mathrm{E}-09 \\
7.5 \mathrm{E}-09 \\
1.0 \mathrm{E}-08 \\
1.1 \mathrm{E}-08 \\
1.5 \mathrm{E}-08 \\
2.5 \mathrm{E}-08 \\
4.1 \mathrm{E}-08 \\
5.8 \mathrm{E}-08 \\
4.8 \mathrm{E}-08 \\
2.6 \mathrm{E}-08 \\
1.7 \mathrm{E}-08 \\
2.2 \mathrm{E}-08 \\
1.9 \mathrm{E}-08 \\
2.8 \mathrm{E}-08 \\
1.8 \mathrm{E}-08\end{array}$ \\
\hline \multicolumn{8}{|c|}{ Distance (m) } \\
\hline Direction & 13245 & 13286 & 13483 & 13612 & 13664 & 13959 & 14258 \\
\hline $\begin{array}{r}N \\
N N W \\
N W \\
\text { WNW } \\
W \\
\text { WSW } \\
\text { SW } \\
\text { SSW }\end{array}$ & $\begin{array}{l}1.3 \mathrm{E}-08 \\
5.0 \mathrm{E}-09 \\
7.0 \mathrm{E}-09 \\
9.4 \mathrm{E}-09 \\
1.1 \mathrm{E}-08 \\
1.4 \mathrm{E}-08 \\
2.3 \mathrm{E}-08 \\
3.8 \mathrm{E}-08\end{array}$ & $\begin{array}{l}1.3 \mathrm{E}-08 \\
5.0 \mathrm{E}-09 \\
7.0 \mathrm{E}-09 \\
9.4 \mathrm{E}-09 \\
1.1 \mathrm{E}-08 \\
1.4 \mathrm{E}-08 \\
2.3 \mathrm{E}-08 \\
3.8 \mathrm{E}-08\end{array}$ & $\begin{array}{l}1.3 \mathrm{E}-08 \\
4.9 \mathrm{E}-09 \\
6.9 \mathrm{E}-09 \\
9.2 \mathrm{E}-09 \\
1.0 \mathrm{E}-08 \\
1.4 \mathrm{E}-08 \\
2.3 \mathrm{E}-08 \\
3.8 \mathrm{E}-08\end{array}$ & $\begin{array}{r}1.3 \mathrm{E}-08 \\
4.9 \mathrm{E}-09 \\
6.8 \mathrm{E}-09 \\
9.1 \mathrm{E}-09 \\
1.0 \mathrm{E}-08 \\
1.4 \mathrm{E}-08 \\
2.2 \mathrm{E}-08 \\
3.7 \mathrm{E}-08 \\
\text { Page }\end{array}$ & $\begin{array}{l}1.3 \mathrm{E}-08 \\
4.9 \mathrm{E}-09 \\
6.8 \mathrm{E}-09 \\
9.1 \mathrm{E}-09 \\
1.0 \mathrm{E}-08 \\
1.4 \mathrm{E}-08 \\
2.2 \mathrm{E}-08 \\
3.7 \mathrm{E}-08\end{array}$ & $\begin{array}{l}1.3 \mathrm{E}-08 \\
4.7 \mathrm{E}-09 \\
6.6 \mathrm{E}-09 \\
8.9 \mathrm{E}-09 \\
1.0 \mathrm{E}-08 \\
1.3 \mathrm{E}-08 \\
2.2 \mathrm{E}-08 \\
3.6 \mathrm{E}-08\end{array}$ & $\begin{array}{l}1.2 \mathrm{E}-08 \\
4.6 \mathrm{E}-09 \\
6.5 \mathrm{E}-09 \\
8.6 \mathrm{E}-09 \\
9.9 \mathrm{E}-09 \\
1.3 \mathrm{E}-08 \\
2.1 \mathrm{E}-08 \\
3.5 \mathrm{E}-08\end{array}$ \\
\hline
\end{tabular}




\begin{tabular}{rlllllll}
\multicolumn{7}{c}{ Ar-39A.SUM } \\
S & $5.4 \mathrm{E}-08$ & $5.4 \mathrm{E}-08$ & $5.3 \mathrm{E}-08$ & $5.2 \mathrm{E}-08$ & $5.2 \mathrm{E}-08$ & $5.1 \mathrm{E}-08$ & $4.9 \mathrm{E}-08$ \\
SSE & $4.5 \mathrm{E}-08$ & $4.5 \mathrm{E}-08$ & $4.4 \mathrm{E}-08$ & $4.4 \mathrm{E}-08$ & $4.3 \mathrm{E}-08$ & $4.2 \mathrm{E}-08$ & $4.1 \mathrm{E}-08$ \\
$\mathrm{SE}$ & $2.4 \mathrm{E}-08$ & $2.4 \mathrm{E}-08$ & $2.3 \mathrm{E}-08$ & $2.3 \mathrm{E}-08$ & $2.3 \mathrm{E}-08$ & $2.3 \mathrm{E}-08$ & $2.2 \mathrm{E}-08$ \\
$\mathrm{ESE}$ & $1.6 \mathrm{E}-08$ & $1.6 \mathrm{E}-08$ & $1.6 \mathrm{E}-08$ & $1.5 \mathrm{E}-08$ & $1.5 \mathrm{E}-08$ & $1.5 \mathrm{E}-08$ & $1.5 \mathrm{E}-08$ \\
$\mathrm{E}$ & $2.1 \mathrm{E}-08$ & $2.0 \mathrm{E}-08$ & $2.0 \mathrm{E}-08$ & $2.0 \mathrm{E}-08$ & $2.0 \mathrm{E}-08$ & $1.9 \mathrm{E}-08$ & $1.9 \mathrm{E}-08$ \\
$\mathrm{ENE}$ & $1.8 \mathrm{E}-08$ & $1.8 \mathrm{E}-08$ & $1.7 \mathrm{E}-08$ & $1.7 \mathrm{E}-08$ & $1.7 \mathrm{E}-08$ & $1.7 \mathrm{E}-08$ & $1.6 \mathrm{E}-08$ \\
$\mathrm{NE}$ & $2.6 \mathrm{E}-08$ & $2.6 \mathrm{E}-08$ & $2.6 \mathrm{E}-08$ & $2.6 \mathrm{E}-08$ & $2.5 \mathrm{E}-08$ & $2.5 \mathrm{E}-08$ & $2.4 \mathrm{E}-08$ \\
$\mathrm{NNE}$ & $1.7 \mathrm{E}-08$ & $1.7 \mathrm{E}-08$ & $1.6 \mathrm{E}-08$ & $1.6 \mathrm{E}-08$ & $1.6 \mathrm{E}-08$ & $1.6 \mathrm{E}-08$ & $1.5 \mathrm{E}-08$
\end{tabular}

SUMMARY

Feb 20, $2008 \quad 02: 30 \mathrm{pm}$

Page 6

INDIVIDUAL EFFECTIVE DOSE EQUIVALENT RATE (mrem/y)

(A11 Radionuclides and Pathways)

\begin{tabular}{|c|c|c|c|c|c|c|c|}
\hline \multicolumn{8}{|c|}{ Distance $(\mathrm{m})$} \\
\hline Direction & 14374 & 15241 & 15441 & 15784 & 15844 & 16323 & \\
\hline $\begin{array}{r}\text { N } \\
\text { NNW } \\
N W \\
\text { WNW } \\
W \\
\text { WSW } \\
\text { SW } \\
\text { SSW } \\
\text { S } \\
\text { SSE } \\
\text { SE } \\
\text { ESE } \\
\text { E } \\
\text { ENE } \\
\text { NE } \\
\text { NNE }\end{array}$ & $\begin{array}{l}1.2 \mathrm{E}-08 \\
4.6 \mathrm{E}-09 \\
6.4 \mathrm{E}-09 \\
8.6 \mathrm{E}-09 \\
9.8 \mathrm{E}-09 \\
1.3 \mathrm{E}-08 \\
2.1 \mathrm{E}-08 \\
3.5 \mathrm{E}-08 \\
4.9 \mathrm{E}-08 \\
4.1 \mathrm{E}-08 \\
2.2 \mathrm{E}-08 \\
1.5 \mathrm{E}-08 \\
1.9 \mathrm{E}-08 \\
1.6 \mathrm{E}-08 \\
2.4 \mathrm{E}-08 \\
1.5 \mathrm{E}-08\end{array}$ & $\begin{array}{l}1.1 \mathrm{E}-08 \\
4.3 \mathrm{E}-09 \\
6.0 \mathrm{E}-09 \\
8.0 \mathrm{E}-09 \\
9.1 \mathrm{E}-09 \\
1.2 \mathrm{E}-08 \\
2.0 \mathrm{E}-08 \\
3.3 \mathrm{E}-08 \\
4.6 \mathrm{E}-08 \\
3.8 \mathrm{E}-08 \\
2.0 \mathrm{E}-08 \\
1.4 \mathrm{E}-08 \\
1.8 \mathrm{E}-08 \\
1.5 \mathrm{E}-08 \\
2.2 \mathrm{E}-08 \\
1.4 \mathrm{E}-08\end{array}$ & $\begin{array}{l}1.1 \mathrm{E}-08 \\
4.2 \mathrm{E}-09 \\
5.9 \mathrm{E}-09 \\
7.9 \mathrm{E}-09 \\
9.0 \mathrm{E}-09 \\
1.2 \mathrm{E}-08 \\
1.9 \mathrm{E}-08 \\
3.2 \mathrm{E}-08 \\
4.5 \mathrm{E}-08 \\
3.8 \mathrm{E}-08 \\
2.0 \mathrm{E}-08 \\
1.3 \mathrm{E}-08 \\
1.7 \mathrm{E}-08 \\
1.5 \mathrm{E}-08 \\
2.2 \mathrm{E}-08 \\
1.4 \mathrm{E}-08\end{array}$ & $\begin{array}{l}1.1 \mathrm{E}-08 \\
4.1 \mathrm{E}-09 \\
5.8 \mathrm{E}-09 \\
7.7 \mathrm{E}-09 \\
8.8 \mathrm{E}-09 \\
1.2 \mathrm{E}-08 \\
1.9 \mathrm{E}-08 \\
3.1 \mathrm{E}-08 \\
4.4 \mathrm{E}-08 \\
3.7 \mathrm{E}-08 \\
2.0 \mathrm{E}-08 \\
1.3 \mathrm{E}-08 \\
1.7 \mathrm{E}-08 \\
1.4 \mathrm{E}-08 \\
2.1 \mathrm{E}-08 \\
1.4 \mathrm{E}-08\end{array}$ & $\begin{array}{l}1.1 \mathrm{E}-08 \\
4.1 \mathrm{E}-09 \\
5.7 \mathrm{E}-09 \\
7.7 \mathrm{E}-09 \\
8.8 \mathrm{E}-09 \\
1.2 \mathrm{E}-08 \\
1.9 \mathrm{E}-08 \\
3.1 \mathrm{E}-08 \\
4.4 \mathrm{E}-08 \\
3.6 \mathrm{E}-08 \\
2.0 \mathrm{E}-08 \\
1.3 \mathrm{E}-08 \\
1.7 \mathrm{E}-08 \\
1.4 \mathrm{E}-08 \\
2.1 \mathrm{E}-08 \\
1.4 \mathrm{E}-08\end{array}$ & $\begin{array}{l}1.1 \mathrm{E}-08 \\
4.0 \mathrm{E}-09 \\
5.6 \mathrm{E}-09 \\
7.4 \mathrm{E}-09 \\
8.5 \mathrm{E}-09 \\
1.1 \mathrm{E}-08 \\
1.8 \mathrm{E}-08 \\
3.0 \mathrm{E}-08 \\
4.2 \mathrm{E}-08 \\
3.5 \mathrm{E}-08 \\
1.9 \mathrm{E}-08 \\
1.3 \mathrm{E}-08 \\
1.6 \mathrm{E}-08 \\
1.4 \mathrm{E}-08 \\
2.1 \mathrm{E}-08 \\
1.3 \mathrm{E}-08\end{array}$ & \\
\hline \multirow[t]{2}{*}{ Feb 20, } & 2008 & $2: 30$ pm & & & & & $\begin{array}{l}\text { SUMMARY } \\
\text { Page } 7\end{array}$ \\
\hline & \multicolumn{7}{|c|}{$\begin{array}{l}\text { INDIVIDUAL LIFETIME RISK (deaths) } \\
\text { (A11 Radionuclides and Pathways) }\end{array}$} \\
\hline \multicolumn{8}{|c|}{ Distance (m) } \\
\hline Direction & 10344 & 10365 & 10472 & 10590 & 11103 & 11989 & 12522 \\
\hline $\begin{array}{r}N \\
N N W \\
N W \\
W N W\end{array}$ & $\begin{array}{l}\text { 2. } 3 \mathrm{E}-15 \\
\text { 8. } 5 \mathrm{E}-16 \\
1.2 \mathrm{E}-15 \\
1.6 \mathrm{E}-15\end{array}$ & $\begin{array}{l}\text { 2. } 2 \mathrm{E}-15 \\
\text { 8. } 5 \mathrm{E}-16 \\
1.2 \mathrm{E}-15 \\
1.6 \mathrm{E}-15\end{array}$ & $\begin{array}{l}\text { 2. } 2 \mathrm{E}-15 \\
\text { 8. } 4 \mathrm{E}-16 \\
1.2 \mathrm{E}-15 \\
1.5 \mathrm{E}-15\end{array}$ & $\begin{array}{r}2.2 \mathrm{E}-15 \\
8.3 \mathrm{E}-16 \\
1.1 \mathrm{E}-15 \\
1.5 \mathrm{E}-15 \\
\text { Page }\end{array}$ & $\begin{array}{l}2.1 \mathrm{E}-15 \\
7.8 \mathrm{E}-16 \\
1.1 \mathrm{E}-15 \\
1.5 \mathrm{E}-15\end{array}$ & $\begin{array}{l}1.9 \mathrm{E}-15 \\
7.2 \mathrm{E}-16 \\
1.0 \mathrm{E}-15 \\
1.3 \mathrm{E}-15\end{array}$ & $\begin{array}{l}1.8 \mathrm{E}-15 \\
6.8 \mathrm{E}-16 \\
9.5 \mathrm{E}-16 \\
1.3 \mathrm{E}-15\end{array}$ \\
\hline
\end{tabular}




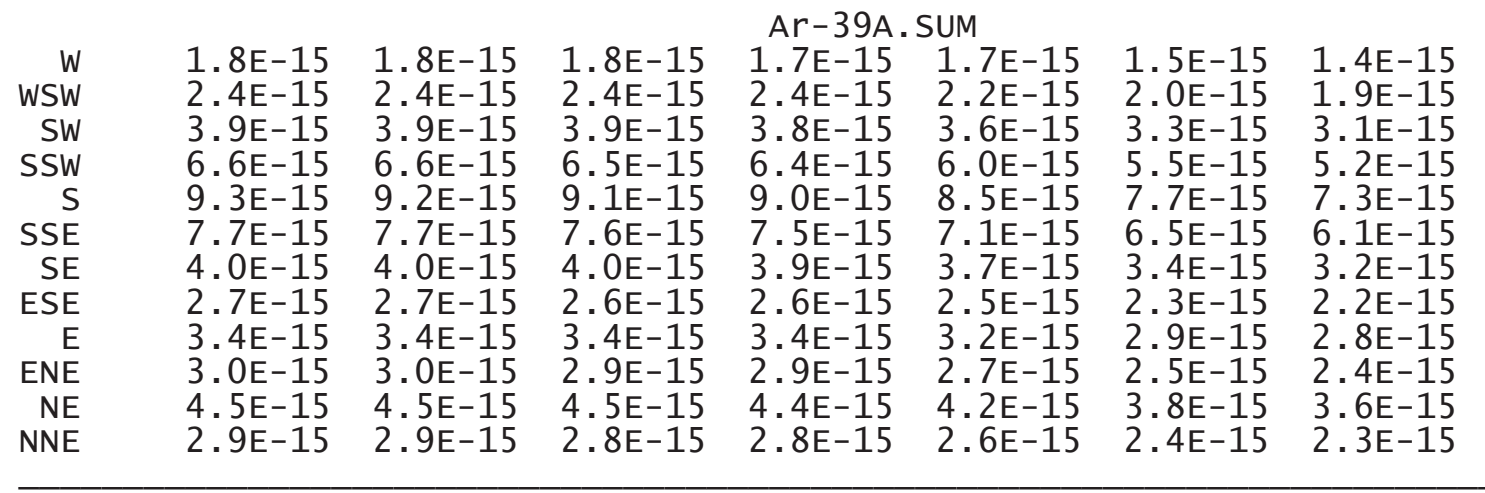

Distance (m)

\begin{tabular}{|c|c|c|c|c|c|c|c|c|}
\hline \multicolumn{2}{|c|}{ Direction } & 13245 & 13286 & 13483 & 13612 & 13664 & 13959 & 14258 \\
\hline $\begin{array}{r}\mathrm{N} \\
\mathrm{NNW} \\
\mathrm{NW} \\
\text { WNW } \\
W \\
W S W \\
\mathrm{SW} \\
\mathrm{SSW} \\
\mathrm{S} \\
\mathrm{SSE} \\
\mathrm{SE} \\
\mathrm{ESE} \\
\mathrm{E} \\
\mathrm{ENE} \\
\mathrm{NE} \\
\mathrm{NNE}\end{array}$ & & $\begin{array}{l}1.7 \mathrm{E}-15 \\
6.4 \mathrm{E}-16 \\
8.9 \mathrm{E}-16 \\
1.2 \mathrm{E}-15 \\
1.4 \mathrm{E}-15 \\
1.8 \mathrm{E}-15 \\
2.9 \mathrm{E}-15 \\
4.9 \mathrm{E}-15 \\
6.9 \mathrm{E}-15 \\
5.7 \mathrm{E}-15 \\
3.0 \mathrm{E}-15 \\
2.0 \mathrm{E}-15 \\
2.6 \mathrm{E}-15 \\
2.2 \mathrm{E}-15 \\
3.3 \mathrm{E}-15 \\
2.1 \mathrm{E}-15\end{array}$ & $\begin{array}{l}1.7 \mathrm{E}-15 \\
6.4 \mathrm{E}-16 \\
8.9 \mathrm{E}-16 \\
1.2 \mathrm{E}-15 \\
1.4 \mathrm{E}-15 \\
1.8 \mathrm{E}-15 \\
2.9 \mathrm{E}-15 \\
4.9 \mathrm{E}-15 \\
6.8 \mathrm{E}-15 \\
5.7 \mathrm{E}-15 \\
3.0 \mathrm{E}-15 \\
2.0 \mathrm{E}-15 \\
2.6 \mathrm{E}-15 \\
2.2 \mathrm{E}-15 \\
3.3 \mathrm{E}-15 \\
2.1 \mathrm{E}-15\end{array}$ & $\begin{array}{l}1.7 \mathrm{E}-15 \\
6.3 \mathrm{E}-16 \\
8.7 \mathrm{E}-16 \\
1.2 \mathrm{E}-15 \\
1.3 \mathrm{E}-15 \\
1.8 \mathrm{E}-15 \\
2.9 \mathrm{E}-15 \\
4.8 \mathrm{E}-15 \\
6.7 \mathrm{E}-15 \\
5.6 \mathrm{E}-15 \\
3.0 \mathrm{E}-15 \\
2.0 \mathrm{E}-15 \\
2.6 \mathrm{E}-15 \\
2.2 \mathrm{E}-15 \\
3.3 \mathrm{E}-15 \\
2.1 \mathrm{E}-15\end{array}$ & $\begin{array}{l}1.7 \mathrm{E}-15 \\
6.2 \mathrm{E}-16 \\
8.6 \mathrm{E}-16 \\
1.2 \mathrm{E}-15 \\
1.3 \mathrm{E}-15 \\
1.8 \mathrm{E}-15 \\
2.8 \mathrm{E}-15 \\
4.7 \mathrm{E}-15 \\
6.6 \mathrm{E}-15 \\
5.5 \mathrm{E}-15 \\
3.0 \mathrm{E}-15 \\
2.0 \mathrm{E}-15 \\
2.5 \mathrm{E}-15 \\
2.2 \mathrm{E}-15 \\
3.2 \mathrm{E}-15 \\
2.1 \mathrm{E}-15\end{array}$ & $\begin{array}{l}1.6 \mathrm{E}-15 \\
6.2 \mathrm{E}-16 \\
8.6 \mathrm{E}-16 \\
1.2 \mathrm{E}-15 \\
1.3 \mathrm{E}-15 \\
1.7 \mathrm{E}-15 \\
2.8 \mathrm{E}-15 \\
4.7 \mathrm{E}-15 \\
6.6 \mathrm{E}-15 \\
5.5 \mathrm{E}-15 \\
2.9 \mathrm{E}-15 \\
2.0 \mathrm{E}-15 \\
2.5 \mathrm{E}-15 \\
2.2 \mathrm{E}-15 \\
3.2 \mathrm{E}-15 \\
2.1 \mathrm{E}-15\end{array}$ & $\begin{array}{l}1.6 \mathrm{E}-15 \\
6.0 \mathrm{E}-16 \\
8.4 \mathrm{E}-16 \\
1.1 \mathrm{E}-15 \\
1.3 \mathrm{E}-15 \\
1.7 \mathrm{E}-15 \\
2.7 \mathrm{E}-15 \\
4.6 \mathrm{E}-15 \\
6.4 \mathrm{E}-15 \\
5.4 \mathrm{E}-15 \\
2.9 \mathrm{E}-15 \\
1.9 \mathrm{E}-15 \\
2.5 \mathrm{E}-15 \\
2.1 \mathrm{E}-15 \\
3.1 \mathrm{E}-15 \\
2.0 \mathrm{E}-15\end{array}$ & $\begin{array}{l}1.6 \mathrm{E}-15 \\
5.9 \mathrm{E}-16 \\
8.2 \mathrm{E}-16 \\
1.1 \mathrm{E}-15 \\
1.3 \mathrm{E}-15 \\
1.7 \mathrm{E}-15 \\
2.7 \mathrm{E}-15 \\
4.5 \mathrm{E}-15 \\
6.3 \mathrm{E}-15 \\
5.2 \mathrm{E}-15 \\
2.8 \mathrm{E}-15 \\
1.9 \mathrm{E}-15 \\
2.4 \mathrm{E}-15 \\
2.1 \mathrm{E}-15 \\
3.1 \mathrm{E}-15 \\
2.0 \mathrm{E}-15\end{array}$ \\
\hline \multirow[t]{2}{*}{ Feb } & 20 & 2008 & $02: 30 \mathrm{pm}$ & & & & & $\begin{array}{l}\text { SUMMARY } \\
\text { Page } 8\end{array}$ \\
\hline & \multicolumn{7}{|c|}{$\begin{array}{l}\text { INDIVIDUAL LIFETIME RISK (deaths) } \\
\text { (A } 11 \text { Radionuclides and Pathways) }\end{array}$} & \\
\hline
\end{tabular}

Distance (m)

\begin{tabular}{lllllll}
\cline { 2 - 5 } Direction & 14374 & 15241 & 15441 & 15784 & 15844 & 16323 \\
\hline
\end{tabular}

\begin{tabular}{rrrrrrr}
\hline & & & & \\
$N$ & $1.6 \mathrm{E}-15$ & $1.5 \mathrm{E}-15$ & $1.4 \mathrm{E}-15$ & $1.4 \mathrm{E}-15$ & $1.4 \mathrm{E}-15$ & $1.3 \mathrm{E}-15$ \\
$\mathrm{NNW}$ & $5.8 \mathrm{E}-16$ & $5.5 \mathrm{E}-16$ & $5.4 \mathrm{E}-16$ & $5.3 \mathrm{E}-16$ & $5.2 \mathrm{E}-16$ & $5.1 \mathrm{E}-16$ \\
$\mathrm{NW}$ & $8.1 \mathrm{E}-16$ & $7.6 \mathrm{E}-16$ & $7.5 \mathrm{E}-16$ & $7.3 \mathrm{E}-16$ & $7.3 \mathrm{E}-16$ & $7.1 \mathrm{E}-16$ \\
$\mathrm{WNW}$ & $1.1 \mathrm{E}-15$ & $1.0 \mathrm{E}-15$ & $1.0 \mathrm{E}-15$ & $9.8 \mathrm{E}-16$ & $9.8 \mathrm{E}-16$ & $9.5 \mathrm{E}-16$ \\
$\mathrm{~W}$ & $1.2 \mathrm{E}-15$ & $1.2 \mathrm{E}-15$ & $1.1 \mathrm{E}-15$ & $1.1 \mathrm{E}-15$ & $1.1 \mathrm{E}-15$ & $1.1 \mathrm{E}-15$ \\
$\mathrm{WSW}$ & $1.6 \mathrm{E}-15$ & $1.5 \mathrm{E}-15$ & $1.5 \mathrm{E}-15$ & $1.5 \mathrm{E}-15$ & $1.5 \mathrm{E}-15$ & $1.4 \mathrm{E}-15$ \\
$\mathrm{SW}$ & $2.7 \mathrm{E}-15$ & $2.5 \mathrm{E}-15$ & $2.4 \mathrm{E}-15$ & $2.4 \mathrm{E}-15$ & $2.4 \mathrm{E}-15$ & $2.3 \mathrm{E}-15$ \\
$\mathrm{SSW}$ & $4.4 \mathrm{E}-15$ & $4.1 \mathrm{E}-15$ & $4.1 \mathrm{E}-15$ & $4.0 \mathrm{E}-15$ & $3.9 \mathrm{E}-15$ & $3.8 \mathrm{E}-15$ \\
$\mathrm{~S}$ & $6.2 \mathrm{E}-15$ & $5.8 \mathrm{E}-15$ & $5.7 \mathrm{E}-15$ & $\begin{array}{c}5.5 \mathrm{E}-15 \\
\text { Page }\end{array}$ & $5.5 \mathrm{E}-15$ & $5.3 \mathrm{E}-15$
\end{tabular}




\begin{tabular}{rllllll} 
& \multicolumn{5}{c}{ Ar-39A. SUM } \\
SSE & $5.2 \mathrm{E}-15$ & $4.8 \mathrm{E}-15$ & $4.8 \mathrm{E}-15$ & $4.6 \mathrm{E}-15$ & $4.6 \mathrm{E}-15$ & $4.5 \mathrm{E}-15$ \\
SE & $2.8 \mathrm{E}-15$ & $2.6 \mathrm{E}-15$ & $2.6 \mathrm{E}-15$ & $2.5 \mathrm{E}-15$ & $2.5 \mathrm{E}-15$ & $2.4 \mathrm{E}-15$ \\
ESE & $1.8 \mathrm{E}-15$ & $1.7 \mathrm{E}-15$ & $1.7 \mathrm{E}-15$ & $1.7 \mathrm{E}-15$ & $1.7 \mathrm{E}-15$ & $1.6 \mathrm{E}-15$ \\
$\mathrm{E}$ & $2.4 \mathrm{E}-15$ & $2.2 \mathrm{E}-15$ & $2.2 \mathrm{E}-15$ & $2.1 \mathrm{E}-15$ & $2.1 \mathrm{E}-15$ & $2.1 \mathrm{E}-15$ \\
ENE & $2.0 \mathrm{E}-15$ & $1.9 \mathrm{E}-15$ & $1.9 \mathrm{E}-15$ & $1.8 \mathrm{E}-15$ & $1.8 \mathrm{E}-15$ & $1.8 \mathrm{E}-15$ \\
NE & $3.0 \mathrm{E}-15$ & $2.8 \mathrm{E}-15$ & $2.8 \mathrm{E}-15$ & $2.7 \mathrm{E}-15$ & $2.7 \mathrm{E}-15$ & $2.6 \mathrm{E}-15$ \\
NNE & $1.9 \mathrm{E}-15$ & $1.8 \mathrm{E}-15$ & $1.8 \mathrm{E}-15$ & $1.7 \mathrm{E}-15$ & $1.7 \mathrm{E}-15$ & $1.7 \mathrm{E}-15$ \\
\hline
\end{tabular}




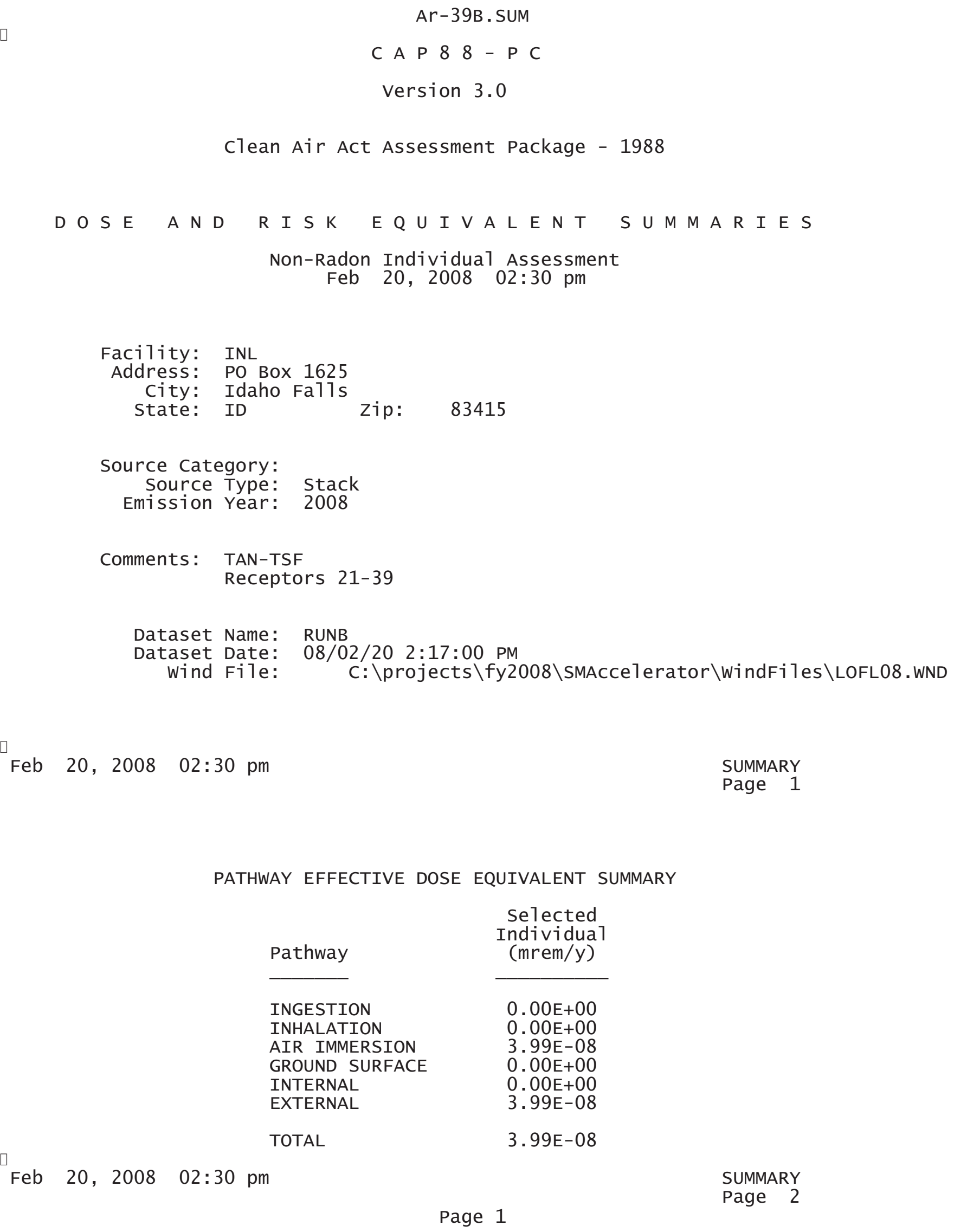

Pathway

INGESTION

INHALATION

AIR IMMERSION GROUND SURFACE INTERNAL

EXTERNAL

TOTAL

$$
\begin{gathered}
\begin{array}{c}
\text { selected } \\
\text { Individual } \\
\text { (mrem/y) }
\end{array} \\
\hline 0.00 \mathrm{E}+00 \\
0.00 \mathrm{E}+00 \\
3.99 \mathrm{E}-08 \\
0.00 \mathrm{E}+00 \\
0.00 \mathrm{E}+00 \\
3.99 \mathrm{E}-08 \\
3.99 \mathrm{E}-08
\end{gathered}
$$

Feb 20, $2008 \quad 02: 30$ pm 
NUCLIDE EFFECTIVE DOSE EQUIVALENT SUMMARY

Nuclide

Ar-39

TOTAL

Feb 20, $2008 \quad 02: 30 \mathrm{pm}$

$$
\begin{gathered}
\begin{array}{c}
\text { Selected } \\
\text { Individual } \\
(\mathrm{mrem} / \mathrm{y})
\end{array} \\
\hline 3.99 \mathrm{E}-08 \\
3.99 \mathrm{E}-08
\end{gathered}
$$

SUMMARY

Page 3

CANCER RISK SUMMARY

\section{Cancer}

Esophagu

Stomach

colon

Liver

LUNG

Bone

Skin

Breast

ovary

Bladder

Kidneys

Thyroid

Leukemia

Residual

Total

TOTAL
Selected Individual Total Lifetime Fatal Cancer Risk

2. $02 \mathrm{E}-17$

9. $26 \mathrm{E}-17$

$1.98 \mathrm{E}-16$

3. $54 \mathrm{E}-17$

2. $65 \mathrm{E}-16$

9. $01 \mathrm{E}-18$

$3.71 \mathrm{E}-15$

$1.91 \mathrm{E}-16$

$2.43 \mathrm{E}-17$

$5.24 \mathrm{E}-17$

1.27E-17

9. $78 \mathrm{E}-18$

1. $29 \mathrm{E}-16$

$3.27 \mathrm{E}-16$

$5.06 \mathrm{E}-15$

1. 01E-14

PATHWAY RISK SUMMARY

Pathway

INGESTION

INHALATION

AIR IMMERSION

GROUND SURFACE

INTERNAL

EXTERNAL

TOTAL
Selected Individual

Total Lifetime

Fatal Cancer Risk

$0.00 \mathrm{E}+00$

$0.00 \mathrm{E}+00$

$5.06 \mathrm{E}-15$

$0.00 \mathrm{E}+00$

$0.00 \mathrm{E}+00$

5. 06E-15

5. $06 \mathrm{E}-15$

Page 2 


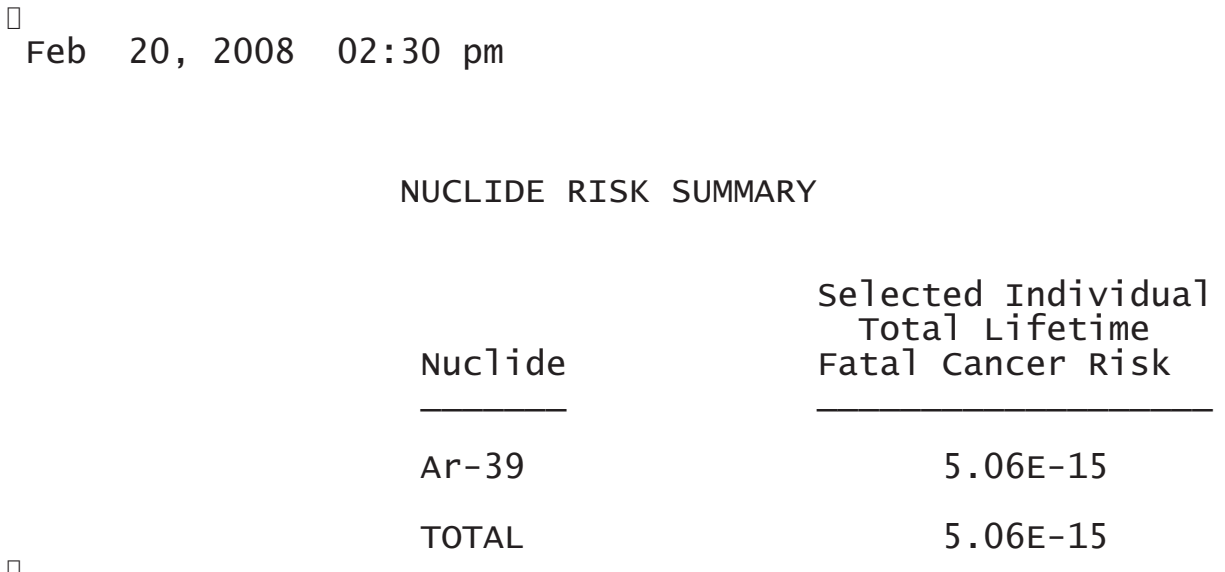

Feb 20, $2008 \quad 02: 30$ pm

SUMMARY

Page 5

INDIVIDUAL EFFECTIVE DOSE EQUIVALENT RATE (mrem/y) (A11 Radionuclides and Pathways)

\begin{tabular}{|c|c|c|c|c|c|c|c|}
\hline \multicolumn{8}{|c|}{ Distance (m) } \\
\hline Direction & 17035 & 17329 & 18607 & 18834 & 18860 & 18890 & 19860 \\
\hline $\begin{array}{r}N \\
N N W \\
N W \\
\text { WNW } \\
W \\
\text { WSW } \\
\text { SW } \\
\text { SSW } \\
\text { S } \\
\text { SSE } \\
\text { SE } \\
\text { ESE } \\
\text { E } \\
\text { ENE } \\
N E \\
\text { NNE }\end{array}$ & $\begin{array}{l}1.0 \mathrm{E}-08 \\
3.8 \mathrm{E}-09 \\
5.3 \mathrm{E}-09 \\
7 \cdot 1 \mathrm{E}-09 \\
8.1 \mathrm{E}-09 \\
1.1 \mathrm{E}-08 \\
1.7 \mathrm{E}-08 \\
2 \cdot 9 \mathrm{E}-08 \\
4 \cdot 0 \mathrm{E}-08 \\
3 \cdot 3 \mathrm{E}-08 \\
1.8 \mathrm{E}-08 \\
1 \cdot 2 \mathrm{E}-08 \\
1 \cdot 5 \mathrm{E}-08 \\
1.3 \mathrm{E}-08 \\
2.0 \mathrm{E}-08 \\
1.3 \mathrm{E}-08\end{array}$ & $\begin{array}{l}9.9 \mathrm{E}-09 \\
3.7 \mathrm{E}-09 \\
5.2 \mathrm{E}-09 \\
7.0 \mathrm{E}-09 \\
7.9 \mathrm{E}-09 \\
1.0 \mathrm{E}-08 \\
1.7 \mathrm{E}-08 \\
2.8 \mathrm{E}-08 \\
3.9 \mathrm{E}-08 \\
3.3 \mathrm{E}-08 \\
1.8 \mathrm{E}-08 \\
1.2 \mathrm{E}-08 \\
1.5 \mathrm{E}-08 \\
1.3 \mathrm{E}-08 \\
1.9 \mathrm{E}-08 \\
1.2 \mathrm{E}-08\end{array}$ & $\begin{array}{l}9.2 \mathrm{E}-09 \\
3.4 \mathrm{E}-09 \\
4.8 \mathrm{E}-09 \\
6.4 \mathrm{E}-09 \\
7.3 \mathrm{E}-09 \\
9.7 \mathrm{E}-09 \\
1.5 \mathrm{E}-08 \\
2.6 \mathrm{E}-08 \\
3.6 \mathrm{E}-08 \\
3.0 \mathrm{E}-08 \\
1.6 \mathrm{E}-08 \\
1.1 \mathrm{E}-08 \\
1.4 \mathrm{E}-08 \\
1.2 \mathrm{E}-08 \\
1.8 \mathrm{E}-08 \\
1.1 \mathrm{E}-08\end{array}$ & $\begin{array}{l}9.0 \mathrm{E}-09 \\
3.4 \mathrm{E}-09 \\
4.7 \mathrm{E}-09 \\
6.4 \mathrm{E}-09 \\
7.2 \mathrm{E}-09 \\
9.6 \mathrm{E}-09 \\
1.5 \mathrm{E}-08 \\
2.5 \mathrm{E}-08 \\
3.5 \mathrm{E}-08 \\
3.0 \mathrm{E}-08 \\
1.6 \mathrm{E}-08 \\
1.1 \mathrm{E}-08 \\
1.4 \mathrm{E}-08 \\
1.2 \mathrm{E}-08 \\
1.7 \mathrm{E}-08 \\
1.1 \mathrm{E}-08\end{array}$ & $\begin{array}{l}9.0 \mathrm{E}-09 \\
3.4 \mathrm{E}-09 \\
4.7 \mathrm{E}-09 \\
6.3 \mathrm{E}-09 \\
7.2 \mathrm{E}-09 \\
9.5 \mathrm{E}-09 \\
1.5 \mathrm{E}-08 \\
2.5 \mathrm{E}-08 \\
3.5 \mathrm{E}-08 \\
3.0 \mathrm{E}-08 \\
1.6 \mathrm{E}-08 \\
1.1 \mathrm{E}-08 \\
1.4 \mathrm{E}-08 \\
1.2 \mathrm{E}-08 \\
1.7 \mathrm{E}-08 \\
1.1 \mathrm{E}-08\end{array}$ & $\begin{array}{l}9.0 \mathrm{E}-09 \\
3.4 \mathrm{E}-09 \\
4.7 \mathrm{E}-09 \\
6.3 \mathrm{E}-09 \\
7.2 \mathrm{E}-09 \\
9.5 \mathrm{E}-09 \\
1.5 \mathrm{E}-08 \\
2.5 \mathrm{E}-08 \\
3.5 \mathrm{E}-08 \\
3.0 \mathrm{E}-08 \\
1.6 \mathrm{E}-08 \\
1.1 \mathrm{E}-08 \\
1.4 \mathrm{E}-08 \\
1.2 \mathrm{E}-08 \\
1.7 \mathrm{E}-08 \\
1.1 \mathrm{E}-08\end{array}$ & $\begin{array}{l}8.5 \mathrm{E}-09 \\
3.2 \mathrm{E}-09 \\
4.5 \mathrm{E}-09 \\
6.0 \mathrm{E}-09 \\
6.8 \mathrm{E}-09 \\
9.0 \mathrm{E}-09 \\
1.4 \mathrm{E}-08 \\
2.4 \mathrm{E}-08 \\
3.3 \mathrm{E}-08 \\
2.8 \mathrm{E}-08 \\
1.5 \mathrm{E}-08 \\
1.0 \mathrm{E}-08 \\
1.3 \mathrm{E}-08 \\
1.1 \mathrm{E}-08 \\
1.6 \mathrm{E}-08 \\
1.1 \mathrm{E}-08\end{array}$ \\
\hline \multicolumn{8}{|c|}{ Distance (m) } \\
\hline Direction & 19891 & 20457 & 21314 & 21349 & 22159 & 23521 & 24430 \\
\hline $\begin{array}{r}N \\
N N W \\
N W \\
W N W \\
W \\
\text { WSW } \\
\text { SW } \\
\text { SSW }\end{array}$ & $\begin{array}{l}8.5 \mathrm{E}-09 \\
3.2 \mathrm{E}-09 \\
4.5 \mathrm{E}-09 \\
6.0 \mathrm{E}-09 \\
6.8 \mathrm{E}-09 \\
9.0 \mathrm{E}-09 \\
1.4 \mathrm{E}-08 \\
2.4 \mathrm{E}-08\end{array}$ & $\begin{array}{l}8.2 \mathrm{E}-09 \\
3.1 \mathrm{E}-09 \\
4.3 \mathrm{E}-09 \\
5.8 \mathrm{E}-09 \\
6.6 \mathrm{E}-09 \\
8.7 \mathrm{E}-09 \\
1.4 \mathrm{E}-08 \\
2.3 \mathrm{E}-08\end{array}$ & $\begin{array}{l}7.9 \mathrm{E}-09 \\
3.0 \mathrm{E}-09 \\
4.1 \mathrm{E}-09 \\
5.5 \mathrm{E}-09 \\
6.3 \mathrm{E}-09 \\
8.3 \mathrm{E}-09 \\
1.3 \mathrm{E}-08 \\
2.2 \mathrm{E}-08\end{array}$ & $\begin{array}{r}7.9 \mathrm{E}-09 \\
3.0 \mathrm{E}-09 \\
4.1 \mathrm{E}-09 \\
5.5 \mathrm{E}-09 \\
6.3 \mathrm{E}-09 \\
8.3 \mathrm{E}-09 \\
1.3 \mathrm{E}-08 \\
2.2 \mathrm{E}-08 \\
\text { Page }\end{array}$ & $\begin{array}{l}7.5 E-09 \\
2.8 E-09 \\
4.0 E-09 \\
5.3 E-09 \\
6.0 E-09 \\
8.0 E-09 \\
1.3 E-08 \\
2.1 E-08\end{array}$ & $\begin{array}{l}\text { 7. } 1 \mathrm{E}-09 \\
2.6 \mathrm{E}-09 \\
3.7 \mathrm{E}-09 \\
5.0 \mathrm{E}-09 \\
5.6 \mathrm{E}-09 \\
7.4 \mathrm{E}-09 \\
1.2 \mathrm{E}-08 \\
2.0 \mathrm{E}-08\end{array}$ & $\begin{array}{l}6.8 \mathrm{E}-09 \\
2.5 \mathrm{E}-09 \\
3.6 \mathrm{E}-09 \\
4.8 \mathrm{E}-09 \\
5.4 \mathrm{E}-09 \\
7.1 \mathrm{E}-09 \\
1.1 \mathrm{E}-08 \\
1.9 \mathrm{E}-08\end{array}$ \\
\hline
\end{tabular}




\begin{tabular}{rccccccc}
\multicolumn{7}{c}{$\mathrm{Ar}-39 \mathrm{~B} . \mathrm{SUM}$} \\
$\mathrm{S}$ & $3.3 \mathrm{E}-08$ & $3.2 \mathrm{E}-08$ & $3.0 \mathrm{E}-08$ & $3.0 \mathrm{E}-08$ & $2.9 \mathrm{E}-08$ & $2.7 \mathrm{E}-08$ & $2.6 \mathrm{E}-08$ \\
SSE & $2.8 \mathrm{E}-08$ & $2.7 \mathrm{E}-08$ & $2.6 \mathrm{E}-08$ & $2.6 \mathrm{E}-08$ & $2.4 \mathrm{E}-08$ & $2.3 \mathrm{E}-08$ & $2.2 \mathrm{E}-08$ \\
SE & $1.5 \mathrm{E}-08$ & $1.5 \mathrm{E}-08$ & $1.4 \mathrm{E}-08$ & $1.4 \mathrm{E}-08$ & $1.3 \mathrm{E}-08$ & $1.2 \mathrm{E}-08$ & $1.2 \mathrm{E}-08$ \\
ESE & $1.0 \mathrm{E}-08$ & $9.8 \mathrm{E}-09$ & $9.3 \mathrm{E}-09$ & $9.3 \mathrm{E}-09$ & $8.9 \mathrm{E}-09$ & $8.3 \mathrm{E}-09$ & $8.0 \mathrm{E}-09$ \\
$\mathrm{E}$ & $1.3 \mathrm{E}-08$ & $1.3 \mathrm{E}-08$ & $1.2 \mathrm{E}-08$ & $1.2 \mathrm{E}-08$ & $1.1 \mathrm{E}-08$ & $1.1 \mathrm{E}-08$ & $1.0 \mathrm{E}-08$ \\
$\mathrm{ENE}$ & $1.1 \mathrm{E}-08$ & $1.1 \mathrm{E}-08$ & $1.0 \mathrm{E}-08$ & $1.0 \mathrm{E}-08$ & $9.8 \mathrm{E}-09$ & $9.1 \mathrm{E}-09$ & $8.7 \mathrm{E}-09$ \\
$\mathrm{NE}$ & $1.6 \mathrm{E}-08$ & $1.6 \mathrm{E}-08$ & $1.5 \mathrm{E}-08$ & $1.5 \mathrm{E}-08$ & $1.4 \mathrm{E}-08$ & $1.3 \mathrm{E}-08$ & $1.3 \mathrm{E}-08$ \\
NNE & $1.1 \mathrm{E}-08$ & $1.0 \mathrm{E}-08$ & $9.8 \mathrm{E}-09$ & $9.7 \mathrm{E}-09$ & $9.3 \mathrm{E}-09$ & $8.7 \mathrm{E}-09$ & $8.3 \mathrm{E}-09$
\end{tabular}

Feb 20, $2008 \quad 02: 30$ pm

SUMMARY

Page 6

INDIVIDUAL EFFECTIVE DOSE EQUIVALENT RATE (mrem/y)

(A11 Radionuclides and Pathways)

\begin{tabular}{|c|c|c|c|c|c|c|c|}
\hline \multicolumn{8}{|c|}{ Distance $(\mathrm{m})$} \\
\hline Direction & 24545 & 25171 & 26794 & 27275 & 27389 & & \\
\hline $\begin{array}{r}\text { N } \\
\text { NNW } \\
N W \\
\text { WNW } \\
W \\
\text { WSW } \\
\text { SW } \\
\text { SSW } \\
\text { S } \\
\text { SSE } \\
\text { SE } \\
\text { ESE } \\
\text { E } \\
\text { ENE } \\
\text { NE } \\
\text { NNE }\end{array}$ & $\begin{array}{l}6.7 \mathrm{E}-09 \\
2.5 \mathrm{E}-09 \\
3.5 \mathrm{E}-09 \\
4.7 \mathrm{E}-09 \\
5.4 \mathrm{E}-09 \\
7.1 \mathrm{E}-09 \\
1.1 \mathrm{E}-08 \\
1.9 \mathrm{E}-08 \\
2.6 \mathrm{E}-08 \\
2.2 \mathrm{E}-08 \\
1.2 \mathrm{E}-08 \\
8.0 \mathrm{E}-09 \\
1.0 \mathrm{E}-08 \\
8.7 \mathrm{E}-09 \\
1.3 \mathrm{E}-08 \\
8.3 \mathrm{E}-09\end{array}$ & $\begin{array}{l}6.5 \mathrm{E}-09 \\
2.5 \mathrm{E}-09 \\
3.4 \mathrm{E}-09 \\
4.6 \mathrm{E}-09 \\
5.2 \mathrm{E}-09 \\
6.9 \mathrm{E}-09 \\
1.1 \mathrm{E}-08 \\
1.8 \mathrm{E}-08 \\
2.5 \mathrm{E}-08 \\
2.1 \mathrm{E}-08 \\
1.2 \mathrm{E}-08 \\
7.7 \mathrm{E}-09 \\
1.0 \mathrm{E}-08 \\
8.4 \mathrm{E}-09 \\
1.2 \mathrm{E}-08 \\
8.1 \mathrm{E}-09\end{array}$ & $\begin{array}{l}6.1 \mathrm{E}-09 \\
2.3 \mathrm{E}-09 \\
3.2 \mathrm{E}-09 \\
4.3 \mathrm{E}-09 \\
4.9 \mathrm{E}-09 \\
6.4 \mathrm{E}-09 \\
1.0 \mathrm{E}-08 \\
1.7 \mathrm{E}-08 \\
2.3 \mathrm{E}-08 \\
2.0 \mathrm{E}-08 \\
1.1 \mathrm{E}-08 \\
7.2 \mathrm{E}-09 \\
9.3 \mathrm{E}-09 \\
7.9 \mathrm{E}-09 \\
1.1 \mathrm{E}-08 \\
7.5 \mathrm{E}-09\end{array}$ & $\begin{array}{l}6.0 \mathrm{E}-09 \\
2.2 \mathrm{E}-09 \\
3.2 \mathrm{E}-09 \\
4.2 \mathrm{E}-09 \\
4.8 \mathrm{E}-09 \\
6.3 \mathrm{E}-09 \\
9.9 \mathrm{E}-09 \\
1.6 \mathrm{E}-08 \\
2.3 \mathrm{E}-08 \\
1.9 \mathrm{E}-08 \\
1.1 \mathrm{E}-08 \\
7.1 \mathrm{E}-09 \\
9.1 \mathrm{E}-09 \\
7.7 \mathrm{E}-09 \\
1.1 \mathrm{E}-08 \\
7.4 \mathrm{E}-09\end{array}$ & $\begin{array}{l}6.0 \mathrm{E}-09 \\
2.2 \mathrm{E}-09 \\
3.1 \mathrm{E}-09 \\
4.2 \mathrm{E}-09 \\
4.8 \mathrm{E}-09 \\
6.3 \mathrm{E}-09 \\
9.9 \mathrm{E}-09 \\
1.6 \mathrm{E}-08 \\
2.3 \mathrm{E}-08 \\
1.9 \mathrm{E}-08 \\
1.0 \mathrm{E}-08 \\
7.0 \mathrm{E}-09 \\
9.1 \mathrm{E}-09 \\
7.7 \mathrm{E}-09 \\
1.1 \mathrm{E}-08 \\
7.3 \mathrm{E}-09\end{array}$ & & \\
\hline \multirow[t]{2}{*}{ Feb 20 , } & 2008 & $2: 30$ pm & & & & & $\begin{array}{l}\text { SUMMARY } \\
\text { Page } 7\end{array}$ \\
\hline & \multicolumn{7}{|c|}{$\begin{array}{l}\text { INDIVIDUAL LIFETIME RISK (deaths) } \\
\text { (A11 Radionuclides and Pathways) }\end{array}$} \\
\hline \multicolumn{8}{|c|}{ Distance (m) } \\
\hline Direction & 17035 & 17329 & 18607 & 18834 & 18860 & 18890 & 19860 \\
\hline $\begin{array}{r}N \\
N N W \\
N W \\
W N W\end{array}$ & $\begin{array}{l}\text { 1. 3E-15 } \\
\text { 4.8E-16 } \\
6.7 \mathrm{E}-16 \\
9.0 \mathrm{E}-16\end{array}$ & $\begin{array}{l}\text { 1. } 3 \mathrm{E}-15 \\
4.7 \mathrm{E}-16 \\
6.6 \mathrm{E}-16 \\
8.8 \mathrm{E}-16\end{array}$ & $\begin{array}{l}1.2 \mathrm{E}-15 \\
4.4 \mathrm{E}-16 \\
6.1 \mathrm{E}-16 \\
8.2 \mathrm{E}-16\end{array}$ & $\begin{array}{r}1.1 \mathrm{E}-15 \\
4.3 \mathrm{E}-16 \\
6.0 \mathrm{E}-16 \\
8.1 \mathrm{E}-16 \\
\text { Page }\end{array}$ & $\begin{array}{r}1.1 \mathrm{E}-15 \\
4.3 \mathrm{E}-16 \\
6.0 \mathrm{E}-16 \\
8.1 \mathrm{E}-16\end{array}$ & $\begin{array}{l}\text { 1. } 1 \mathrm{E}-15 \\
\text { 4.3E-16 } \\
6.0 \mathrm{E}-16 \\
8.0 \mathrm{E}-16\end{array}$ & $\begin{array}{l}1.1 \mathrm{E}-15 \\
4.1 \mathrm{E}-16 \\
5.7 \mathrm{E}-16 \\
7.6 \mathrm{E}-16\end{array}$ \\
\hline
\end{tabular}




\begin{tabular}{rlllllll} 
W & $1.0 \mathrm{E}-15$ & $1.0 \mathrm{E}-15$ & $9.3 \mathrm{E}-16$ & $9.2 \mathrm{E}-16$ & $9.2 \mathrm{E}-16$ & $9.1 \mathrm{E}-16$ & $8.6 \mathrm{E}-16$ \\
WSW & $1.4 \mathrm{E}-15$ & $1.3 \mathrm{E}-15$ & $1.2 \mathrm{E}-15$ & $1.2 \mathrm{E}-15$ & $1.2 \mathrm{E}-15$ & $1.2 \mathrm{E}-15$ & $1.1 \mathrm{E}-15$ \\
SW & $2.2 \mathrm{E}-15$ & $2.1 \mathrm{E}-15$ & $2.0 \mathrm{E}-15$ & $1.9 \mathrm{E}-15$ & $1.9 \mathrm{E}-15$ & $1.9 \mathrm{E}-15$ & $1.8 \mathrm{E}-15$ \\
SSW & $3.6 \mathrm{E}-15$ & $3.6 \mathrm{E}-15$ & $3.3 \mathrm{E}-15$ & $3.2 \mathrm{E}-15$ & $3.2 \mathrm{E}-15$ & $3.2 \mathrm{E}-15$ & $3.0 \mathrm{E}-15$ \\
S & $5.1 \mathrm{E}-15$ & $5.0 \mathrm{E}-15$ & $4.6 \mathrm{E}-15$ & $4.5 \mathrm{E}-15$ & $4.5 \mathrm{E}-15$ & $4.5 \mathrm{E}-15$ & $4.2 \mathrm{E}-15$ \\
SSE & $4.2 \mathrm{E}-15$ & $4.2 \mathrm{E}-15$ & $3.8 \mathrm{E}-15$ & $3.8 \mathrm{E}-15$ & $3.8 \mathrm{E}-15$ & $3.8 \mathrm{E}-15$ & $3.5 \mathrm{E}-15$ \\
SE & $2.3 \mathrm{E}-15$ & $2.2 \mathrm{E}-15$ & $2.1 \mathrm{E}-15$ & $2.0 \mathrm{E}-15$ & $2.0 \mathrm{E}-15$ & $2.0 \mathrm{E}-15$ & $1.9 \mathrm{E}-15$ \\
ESE & $1.5 \mathrm{E}-15$ & $1.5 \mathrm{E}-15$ & $1.4 \mathrm{E}-15$ & $1.4 \mathrm{E}-15$ & $1.4 \mathrm{E}-15$ & $1.4 \mathrm{E}-15$ & $1.3 \mathrm{E}-15$ \\
E & $2.0 \mathrm{E}-15$ & $1.9 \mathrm{E}-15$ & $1.8 \mathrm{E}-15$ & $1.8 \mathrm{E}-15$ & $1.7 \mathrm{E}-15$ & $1.7 \mathrm{E}-15$ & $1.7 \mathrm{E}-15$ \\
ENE & $1.7 \mathrm{E}-15$ & $1.6 \mathrm{E}-15$ & $1.5 \mathrm{E}-15$ & $1.5 \mathrm{E}-15$ & $1.5 \mathrm{E}-15$ & $1.5 \mathrm{E}-15$ & $1.4 \mathrm{E}-15$ \\
NE & $2.5 \mathrm{E}-15$ & $2.4 \mathrm{E}-15$ & $2.2 \mathrm{E}-15$ & $2.2 \mathrm{E}-15$ & $2.2 \mathrm{E}-15$ & $2.2 \mathrm{E}-15$ & $2.1 \mathrm{E}-15$ \\
NNE & $1.6 \mathrm{E}-15$ & $1.6 \mathrm{E}-15$ & $1.4 \mathrm{E}-15$ & $1.4 \mathrm{E}-15$ & $1.4 \mathrm{E}-15$ & $1.4 \mathrm{E}-15$ & $1.3 \mathrm{E}-15$ \\
\hline
\end{tabular}

Distance (m)

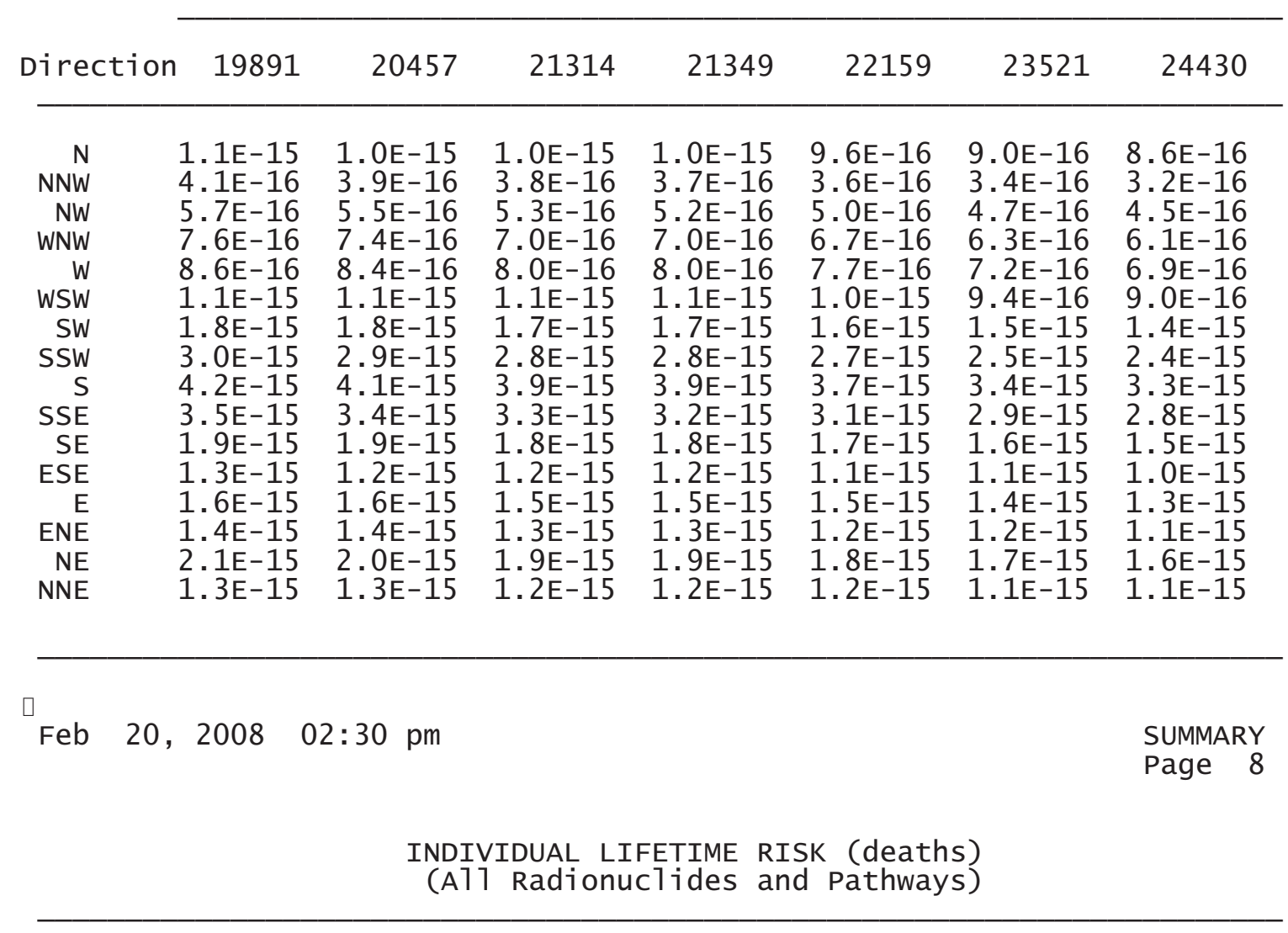

Distance (m)

\begin{tabular}{|c|c|c|c|c|c|}
\hline Direction & 24545 & 25171 & 26794 & 27275 & 27389 \\
\hline
\end{tabular}

\begin{tabular}{rlllll}
\hline$N$ & $8.5 \mathrm{E}-16$ & $8.3 \mathrm{E}-16$ & $7.7 \mathrm{E}-16$ & $7.6 \mathrm{E}-16$ & $7.6 \mathrm{E}-16$ \\
$\mathrm{~N} W$ & $3.2 \mathrm{E}-16$ & $3.1 \mathrm{E}-16$ & $2.9 \mathrm{E}-16$ & $2.9 \mathrm{E}-16$ & $2.8 \mathrm{E}-16$ \\
$\mathrm{NW}$ & $4.5 \mathrm{E}-16$ & $4.4 \mathrm{E}-16$ & $4.1 \mathrm{E}-16$ & $4.0 \mathrm{E}-16$ & $4.0 \mathrm{E}-16$ \\
$\mathrm{WNW}$ & $6.0 \mathrm{E}-16$ & $5.9 \mathrm{E}-16$ & $5.5 \mathrm{E}-16$ & $5.4 \mathrm{E}-16$ & $5.3 \mathrm{E}-16$ \\
$\mathrm{~W}$ & $6.8 \mathrm{E}-16$ & $6.6 \mathrm{E}-16$ & $6.2 \mathrm{E}-16$ & $6.1 \mathrm{E}-16$ & $6.1 \mathrm{E}-16$ \\
$\mathrm{WSW}$ & $9.0 \mathrm{E}-16$ & $8.7 \mathrm{E}-16$ & $8.1 \mathrm{E}-16$ & $8.0 \mathrm{E}-16$ & $8.0 \mathrm{E}-16$ \\
$\mathrm{SW}$ & $1.4 \mathrm{E}-15$ & $1.4 \mathrm{E}-15$ & $1.3 \mathrm{E}-15$ & $1.3 \mathrm{E}-15$ & $1.3 \mathrm{E}-15$ \\
$\mathrm{SSW}$ & $2.4 \mathrm{E}-15$ & $2.3 \mathrm{E}-15$ & $2.1 \mathrm{E}-15$ & $2.1 \mathrm{E}-15$ & $2.1 \mathrm{E}-15$ \\
$\mathrm{~S}$ & $3.3 \mathrm{E}-15$ & $3.2 \mathrm{E}-15$ & $2.9 \mathrm{E}-15$ & $2.9 \mathrm{E}-15$ & $2.9 \mathrm{E}-15$ \\
& & & \multicolumn{4}{c}{ Page 5} \\
& & & &
\end{tabular}




\begin{tabular}{rlllll} 
& & & \multicolumn{3}{c}{ Ar-39B.SUM } \\
SSE & $2.8 \mathrm{E}-15$ & $2.7 \mathrm{E}-15$ & $2.5 \mathrm{E}-15$ & $2.4 \mathrm{E}-15$ & $2.4 \mathrm{E}-15$ \\
SE & $1.5 \mathrm{E}-15$ & $1.5 \mathrm{E}-15$ & $1.4 \mathrm{E}-15$ & $1.3 \mathrm{E}-15$ & $1.3 \mathrm{E}-15$ \\
ESE & $1.0 \mathrm{E}-15$ & $9.8 \mathrm{E}-16$ & $9.2 \mathrm{E}-16$ & $9.0 \mathrm{E}-16$ & $8.9 \mathrm{E}-16$ \\
$\mathrm{E}$ & $1.3 \mathrm{E}-15$ & $1.3 \mathrm{E}-15$ & $1.2 \mathrm{E}-15$ & $1.2 \mathrm{E}-15$ & $1.1 \mathrm{E}-15$ \\
ENE & $1.1 \mathrm{E}-15$ & $1.1 \mathrm{E}-15$ & $1.0 \mathrm{E}-15$ & $9.8 \mathrm{E}-16$ & $9.7 \mathrm{E}-16$ \\
$\mathrm{NE}$ & $1.6 \mathrm{E}-15$ & $1.6 \mathrm{E}-15$ & $1.4 \mathrm{E}-15$ & $1.4 \mathrm{E}-15$ & $1.4 \mathrm{E}-15$ \\
NNE & $1.1 \mathrm{E}-15$ & $1.0 \mathrm{E}-15$ & $9.5 \mathrm{E}-16$ & $9.3 \mathrm{E}-16$ & $9.3 \mathrm{E}-16$
\end{tabular}




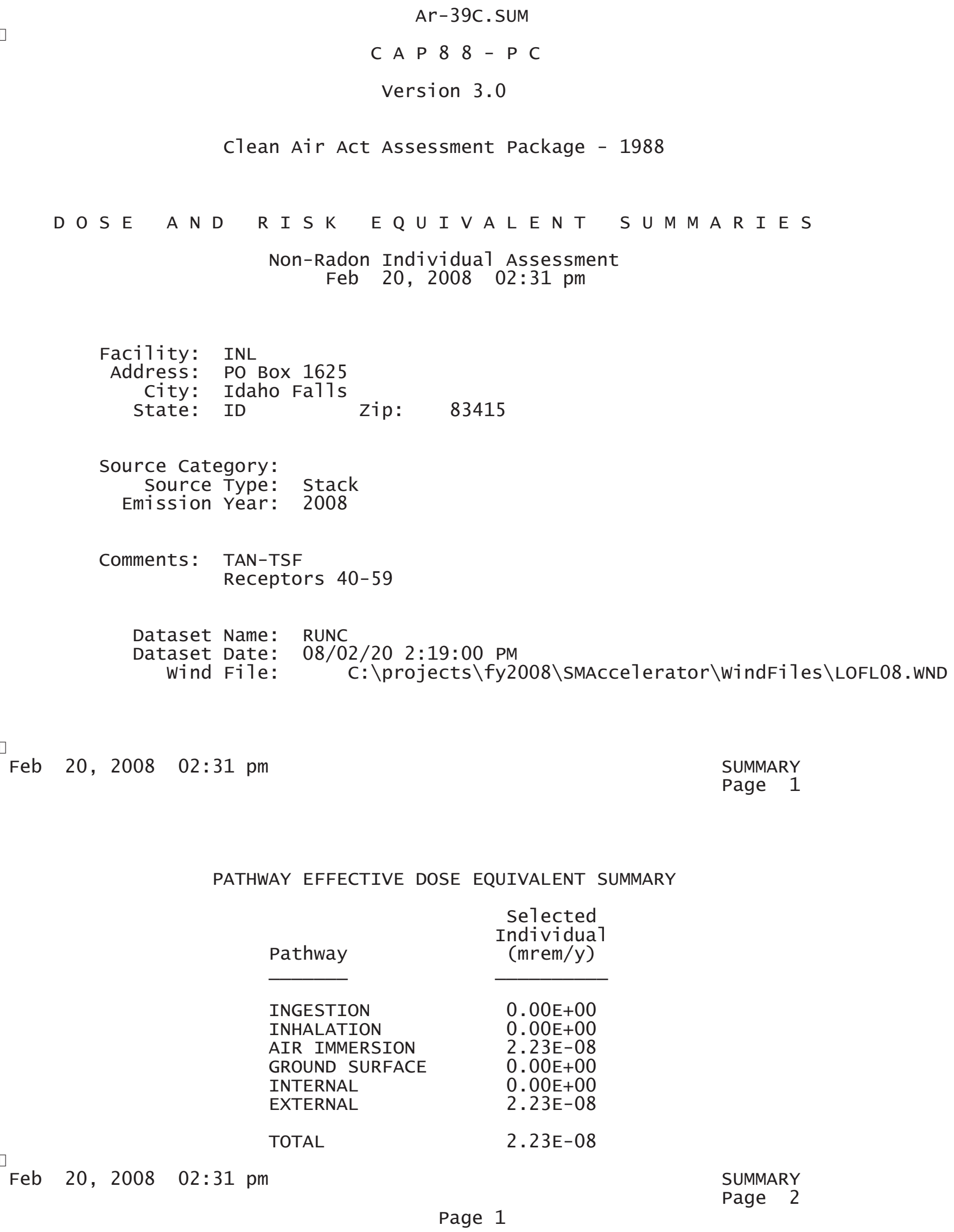

$\begin{array}{lc}\text { Pathway } & \begin{array}{c}\text { } \begin{array}{c}\text { Individual } \\ \text { (mrem/y) }\end{array} \\$\cline { 2 - 2 } \text {$INGESTION }\end{array} \\ \text { INHALATION } & 0.00 \mathrm{E}+00 \\ \text { AIR IMMERSION } & 0.00 \mathrm{E}+00 \\ \text { GROUND SURFACE } & 2.23 \mathrm{E}-08 \\ \text { INTERNAL } & 0.00 \mathrm{E}+00 \\ \text { EXTERNAL } & 0.00 \mathrm{E}+00 \\ \text { TOTAL } & 2.23 \mathrm{E}-08 \\ & 2.23 \mathrm{E}-08\end{array}$

Feb 20, $2008 \quad 02: 31$ pm 
NUCLIDE EFFECTIVE DOSE EQUIVALENT SUMMARY

Nuclide

Ar-39

TOTAL

$$
\begin{gathered}
\begin{array}{c}
\text { Selected } \\
\text { Individual } \\
(\mathrm{mrem} / \mathrm{y})
\end{array} \\
\hline 2.23 \mathrm{E}-08 \\
2.23 \mathrm{E}-08
\end{gathered}
$$

Feb 20, $2008 \quad 02: 31 \mathrm{pm}$

SUMMARY

Page 3

CANCER RISK SUMMARY

\section{Cancer \\ Esophagu \\ stomach \\ colon \\ Liver \\ LUNG \\ Bone \\ Skin \\ Breast \\ Ovary \\ Bladder \\ Kidneys \\ Thyroid \\ Leukemia \\ Residua 1 \\ Tota 1}

TOTAL

$$
\text { Selected Individual }
$$$$
\text { Tota1 Lifetime }
$$$$
\text { Fatal Cancer Risk }
$$

1. $13 \mathrm{E}-17$

5. $18 \mathrm{E}-17$

1. $11 \mathrm{E}-16$

1. $98 \mathrm{E}-17$

1. $48 \mathrm{E}-16$

5. 04E-18

2.07E-15

1.07E-16

1. $36 \mathrm{E}-17$

2. $93 \mathrm{E}-17$

7. $08 \mathrm{E}-18$

5. $47 \mathrm{E}-18$

7. $21 \mathrm{E}-17$

$1.83 \mathrm{E}-16$

2. 83E-15

$5.67 \mathrm{E}-15$

PATHWAY RISK SUMMARY

Pathway

INGESTION

INHALATION

AIR IMMERSION

GROUND SURFACE

INTERNAL

EXTERNAL

TOTAL
Selected Individual

Total Lifetime Fatal Cancer Risk

$0.00 \mathrm{E}+00$

$0.00 \mathrm{E}+00$

$2.83 \mathrm{E}-15$

$0.00 \mathrm{E}+00$

$0.00 \mathrm{E}+00$

$2.83 \mathrm{E}-15$

$2.83 \mathrm{E}-15$

Page 2 


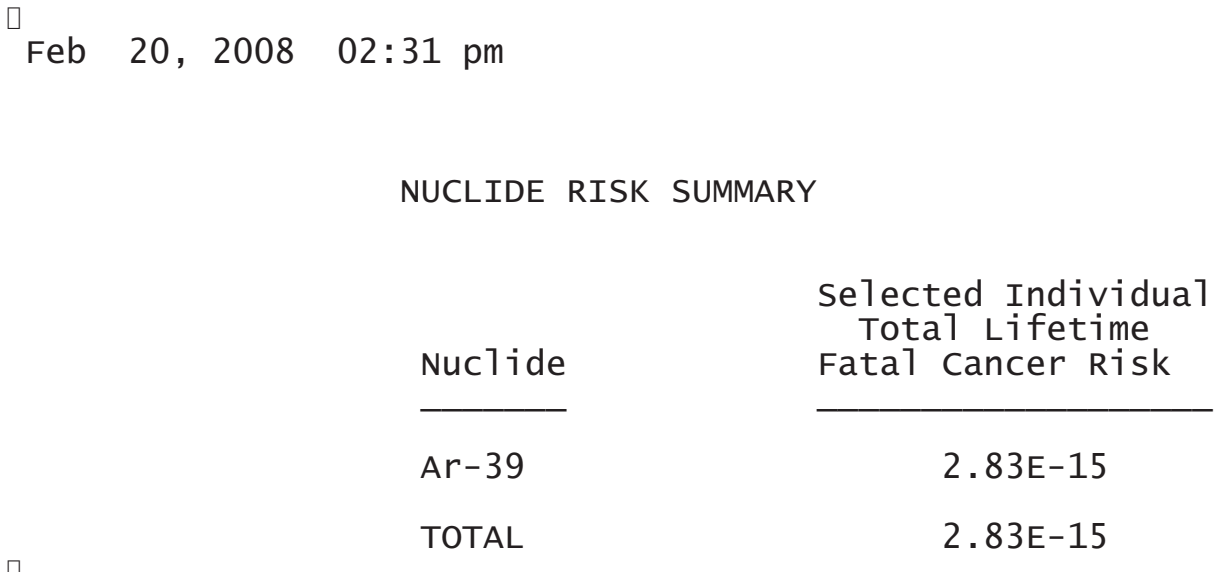

Feb 20, $2008 \quad 02: 31$ pm

SUMMARY

Page 5

INDIVIDUAL EFFECTIVE DOSE EQUIVALENT RATE (mrem/y) (A11 Radionuclides and Pathways)

\begin{tabular}{|c|c|c|c|c|c|c|c|}
\hline \multicolumn{8}{|c|}{ Distance (m) } \\
\hline Direction & 27715 & 28919 & 31060 & 32802 & 34577 & 35279 & 35683 \\
\hline $\begin{array}{r}N \\
N N W \\
N W \\
W N W \\
W \\
W S W \\
\text { SW } \\
\text { SSW } \\
S \\
\text { SSE } \\
\text { SE } \\
\text { ESE } \\
\text { E } \\
\text { ENE } \\
N E \\
\text { NNE }\end{array}$ & $\begin{array}{l}5.9 \mathrm{E}-09 \\
2.2 \mathrm{E}-09 \\
3.1 \mathrm{E}-09 \\
4.1 \mathrm{E}-09 \\
4.7 \mathrm{E}-09 \\
6.2 \mathrm{E}-09 \\
9.7 \mathrm{E}-09 \\
1.6 \mathrm{E}-08 \\
2.2 \mathrm{E}-08 \\
1.9 \mathrm{E}-08 \\
1.0 \mathrm{E}-08 \\
6.9 \mathrm{E}-09 \\
8.9 \mathrm{E}-09 \\
7.6 \mathrm{E}-09 \\
1.1 \mathrm{E}-08 \\
7.2 \mathrm{E}-09\end{array}$ & $\begin{array}{l}5.6 \mathrm{E}-09 \\
2.1 \mathrm{E}-09 \\
3.0 \mathrm{E}-09 \\
4.0 \mathrm{E}-09 \\
4.5 \mathrm{E}-09 \\
5.9 \mathrm{E}-09 \\
9.3 \mathrm{E}-09 \\
1.5 \mathrm{E}-08 \\
2.1 \mathrm{E}-08 \\
1.8 \mathrm{E}-08 \\
9.8 \mathrm{E}-09 \\
6.6 \mathrm{E}-09 \\
8.5 \mathrm{E}-09 \\
7.2 \mathrm{E}-09 \\
1.0 \mathrm{E}-08 \\
6.9 \mathrm{E}-09\end{array}$ & $\begin{array}{l}5.2 \mathrm{E}-09 \\
1.9 \mathrm{E}-09 \\
2.7 \mathrm{E}-09 \\
3.7 \mathrm{E}-09 \\
4.1 \mathrm{E}-09 \\
5.4 \mathrm{E}-09 \\
8.5 \mathrm{E}-09 \\
1.4 \mathrm{E}-08 \\
1.9 \mathrm{E}-08 \\
1.6 \mathrm{E}-08 \\
9.1 \mathrm{E}-09 \\
6.1 \mathrm{E}-09 \\
7.9 \mathrm{E}-09 \\
6.6 \mathrm{E}-09 \\
9.6 \mathrm{E}-09 \\
6.3 \mathrm{E}-09\end{array}$ & $\begin{array}{l}4.9 \mathrm{E}-09 \\
1.8 \mathrm{E}-09 \\
2.6 \mathrm{E}-09 \\
3.4 \mathrm{E}-09 \\
3.9 \mathrm{E}-09 \\
5.1 \mathrm{E}-09 \\
8.0 \mathrm{E}-09 \\
1.3 \mathrm{E}-08 \\
1.8 \mathrm{E}-08 \\
1.5 \mathrm{E}-08 \\
8.5 \mathrm{E}-09 \\
5.7 \mathrm{E}-09 \\
7.4 \mathrm{E}-09 \\
6.2 \mathrm{E}-09 \\
9.0 \mathrm{E}-09 \\
6.0 \mathrm{E}-09\end{array}$ & $\begin{array}{l}4.6 \mathrm{E}-09 \\
1.7 \mathrm{E}-09 \\
2.4 \mathrm{E}-09 \\
3.3 \mathrm{E}-09 \\
3.7 \mathrm{E}-09 \\
4.8 \mathrm{E}-09 \\
7.5 \mathrm{E}-09 \\
1.2 \mathrm{E}-08 \\
1.7 \mathrm{E}-08 \\
1.5 \mathrm{E}-08 \\
8.0 \mathrm{E}-09 \\
5.4 \mathrm{E}-09 \\
7.0 \mathrm{E}-09 \\
5.9 \mathrm{E}-09 \\
8.5 \mathrm{E}-09 \\
5.6 \mathrm{E}-09\end{array}$ & $\begin{array}{l}4.5 \mathrm{E}-09 \\
1.7 \mathrm{E}-09 \\
2.4 \mathrm{E}-09 \\
3.2 \mathrm{E}-09 \\
3.6 \mathrm{E}-09 \\
4.7 \mathrm{E}-09 \\
7.4 \mathrm{E}-09 \\
1.2 \mathrm{E}-08 \\
1.7 \mathrm{E}-08 \\
1.4 \mathrm{E}-08 \\
7.9 \mathrm{E}-09 \\
5.3 \mathrm{E}-09 \\
6.8 \mathrm{E}-09 \\
5.7 \mathrm{E}-09 \\
8.3 \mathrm{E}-09 \\
5.5 \mathrm{E}-09\end{array}$ & $\begin{array}{l}4.4 \mathrm{E}-09 \\
1.7 \mathrm{E}-09 \\
2.3 \mathrm{E}-09 \\
3.1 \mathrm{E}-09 \\
3.6 \mathrm{E}-09 \\
4.7 \mathrm{E}-09 \\
7.3 \mathrm{E}-09 \\
1.2 \mathrm{E}-08 \\
1.7 \mathrm{E}-08 \\
1.4 \mathrm{E}-08 \\
7.8 \mathrm{E}-09 \\
5.2 \mathrm{E}-09 \\
6.7 \mathrm{E}-09 \\
5.7 \mathrm{E}-09 \\
8.1 \mathrm{E}-09 \\
5.4 \mathrm{E}-09\end{array}$ \\
\hline \multicolumn{8}{|c|}{ Distance (m) } \\
\hline Direction & 36721 & 36809 & 37729 & 39079 & 39220 & 39559 & 43584 \\
\hline $\begin{array}{r}N \\
N N W \\
N W \\
W N W \\
W \\
\text { WSW } \\
\text { SW } \\
\text { SSW }\end{array}$ & $\begin{array}{l}4.3 \mathrm{E}-09 \\
1.6 \mathrm{E}-09 \\
2.3 \mathrm{E}-09 \\
3.0 \mathrm{E}-09 \\
3.4 \mathrm{E}-09 \\
4.5 \mathrm{E}-09 \\
7.0 \mathrm{E}-09 \\
1.2 \mathrm{E}-08\end{array}$ & $\begin{array}{l}4.3 E-09 \\
1.6 E-09 \\
2.3 E-09 \\
3.0 E-09 \\
3.4 E-09 \\
4.5 E-09 \\
7.0 E-09 \\
1.2 E-08\end{array}$ & $\begin{array}{l}4.2 \mathrm{E}-09 \\
1.6 \mathrm{E}-09 \\
2.2 \mathrm{E}-09 \\
3.0 \mathrm{E}-09 \\
3.3 \mathrm{E}-09 \\
4.4 \mathrm{E}-09 \\
6.8 \mathrm{E}-09 \\
1.1 \mathrm{E}-08\end{array}$ & $\begin{array}{r}4.0 \mathrm{E}-09 \\
1.5 \mathrm{E}-09 \\
2.1 \mathrm{E}-09 \\
2.8 \mathrm{E}-09 \\
3.2 \mathrm{E}-09 \\
4.2 \mathrm{E}-09 \\
6.6 \mathrm{E}-09 \\
1.1 \mathrm{E}-08 \\
\text { Page }\end{array}$ & $\begin{array}{l}4.0 E-09 \\
1.5 E-09 \\
2.1 E-09 \\
2.8 E-09 \\
3.2 E-09 \\
4.2 E-09 \\
6.5 E-09 \\
1.1 E-08\end{array}$ & $\begin{array}{l}4.0 \mathrm{E}-09 \\
1.5 \mathrm{E}-09 \\
2.1 \mathrm{E}-09 \\
2.8 \mathrm{E}-09 \\
3.2 \mathrm{E}-09 \\
4.1 \mathrm{E}-09 \\
6.5 \mathrm{E}-09 \\
1.1 \mathrm{E}-08\end{array}$ & $\begin{array}{l}3.5 \mathrm{E}-09 \\
1.3 \mathrm{E}-09 \\
1.9 \mathrm{E}-09 \\
2.5 \mathrm{E}-09 \\
2.8 \mathrm{E}-09 \\
3.7 \mathrm{E}-09 \\
5.8 \mathrm{E}-09 \\
9.5 \mathrm{E}-09\end{array}$ \\
\hline
\end{tabular}




\begin{tabular}{rllllllll}
\multicolumn{10}{c}{ Ar-39C.SUM } \\
S & $1.6 \mathrm{E}-08$ & $1.6 \mathrm{E}-08$ & $1.5 \mathrm{E}-08$ & $1.5 \mathrm{E}-08$ & $1.5 \mathrm{E}-08$ & $1.5 \mathrm{E}-08$ & $1.3 \mathrm{E}-08$ \\
SSE & $1.4 \mathrm{E}-08$ & $1.3 \mathrm{E}-08$ & $1.3 \mathrm{E}-08$ & $1.3 \mathrm{E}-08$ & $1.3 \mathrm{E}-08$ & $1.2 \mathrm{E}-08$ & $1.1 \mathrm{E}-08$ \\
SE & $7.5 \mathrm{E}-09$ & $7.5 \mathrm{E}-09$ & $7.3 \mathrm{E}-09$ & $7.0 \mathrm{E}-09$ & $7.0 \mathrm{E}-09$ & $6.9 \mathrm{E}-09$ & $6.2 \mathrm{E}-09$ \\
ESE & $5.1 \mathrm{E}-09$ & $5.0 \mathrm{E}-09$ & $4.9 \mathrm{E}-09$ & $4.7 \mathrm{E}-09$ & $4.7 \mathrm{E}-09$ & $4.7 \mathrm{E}-09$ & $4.2 \mathrm{E}-09$ \\
$\mathrm{E}$ & $6.5 \mathrm{E}-09$ & $6.5 \mathrm{E}-09$ & $6.3 \mathrm{E}-09$ & $6.1 \mathrm{E}-09$ & $6.1 \mathrm{E}-09$ & $6.0 \mathrm{E}-09$ & $5.4 \mathrm{E}-09$ \\
ENE & $5.5 \mathrm{E}-09$ & $5.5 \mathrm{E}-09$ & $5.3 \mathrm{E}-09$ & $5.1 \mathrm{E}-09$ & $5.1 \mathrm{E}-09$ & $5.0 \mathrm{E}-09$ & $4.5 \mathrm{E}-09$ \\
NE & $7.9 \mathrm{E}-09$ & $7.9 \mathrm{E}-09$ & $7.6 \mathrm{E}-09$ & $7.3 \mathrm{E}-09$ & $7.3 \mathrm{E}-09$ & $7.2 \mathrm{E}-09$ & $6.4 \mathrm{E}-09$ \\
NNE & $5.2 \mathrm{E}-09$ & $5.2 \mathrm{E}-09$ & $5.1 \mathrm{E}-09$ & $4.9 \mathrm{E}-09$ & $4.9 \mathrm{E}-09$ & $4.8 \mathrm{E}-09$ & $4.3 \mathrm{E}-09$
\end{tabular}

Feb 20, 2008 02:31 pm

SUMMARY

Page 6

INDIVIDUAL EFFECTIVE DOSE EQUIVALENT RATE (mrem/y)

(A11 Radionuclides and Pathways)

\begin{tabular}{|c|c|c|c|c|c|c|c|}
\hline \multicolumn{8}{|c|}{ Distance (m) } \\
\hline Direction & 45196 & 45275 & 45654 & 45677 & 46668 & 47969 & \\
\hline $\begin{array}{r}N \\
N N W \\
N W \\
W N W \\
W \\
W S W \\
\text { SW } \\
\text { SSW } \\
S \\
\text { SSE } \\
\text { SE } \\
\text { ESE } \\
E \\
\text { ENE } \\
N E \\
N N E\end{array}$ & $\begin{array}{l}3.4 \mathrm{E}-09 \\
1.3 \mathrm{E}-09 \\
1.8 \mathrm{E}-09 \\
2.4 \mathrm{E}-09 \\
2.7 \mathrm{E}-09 \\
3.6 \mathrm{E}-09 \\
5.5 \mathrm{E}-09 \\
9.1 \mathrm{E}-09 \\
1.3 \mathrm{E}-08 \\
1.1 \mathrm{E}-08 \\
5.9 \mathrm{E}-09 \\
4.0 \mathrm{E}-09 \\
5.2 \mathrm{E}-09 \\
4.3 \mathrm{E}-09 \\
6.2 \mathrm{E}-09 \\
4.1 \mathrm{E}-09\end{array}$ & $\begin{array}{l}3.4 \mathrm{E}-09 \\
1.3 \mathrm{E}-09 \\
1.8 \mathrm{E}-09 \\
2.4 \mathrm{E}-09 \\
2.7 \mathrm{E}-09 \\
3.6 \mathrm{E}-09 \\
5.5 \mathrm{E}-09 \\
9.1 \mathrm{E}-09 \\
1.2 \mathrm{E}-08 \\
1.1 \mathrm{E}-08 \\
5.9 \mathrm{E}-09 \\
4.0 \mathrm{E}-09 \\
5.2 \mathrm{E}-09 \\
4.3 \mathrm{E}-09 \\
6.2 \mathrm{E}-09 \\
4.1 \mathrm{E}-09\end{array}$ & $\begin{array}{l}3.4 \mathrm{E}-09 \\
1.3 \mathrm{E}-09 \\
1.8 \mathrm{E}-09 \\
2.4 \mathrm{E}-09 \\
2.7 \mathrm{E}-09 \\
3.5 \mathrm{E}-09 \\
5.5 \mathrm{E}-09 \\
9.0 \mathrm{E}-09 \\
1.2 \mathrm{E}-08 \\
1.0 \mathrm{E}-08 \\
5.9 \mathrm{E}-09 \\
4.0 \mathrm{E}-09 \\
5.1 \mathrm{E}-09 \\
4.3 \mathrm{E}-09 \\
6.1 \mathrm{E}-09 \\
4.1 \mathrm{E}-09\end{array}$ & $\begin{array}{l}3.4 \mathrm{E}-09 \\
1.3 \mathrm{E}-09 \\
1.8 \mathrm{E}-09 \\
2.4 \mathrm{E}-09 \\
2.7 \mathrm{E}-09 \\
3.5 \mathrm{E}-09 \\
5.5 \mathrm{E}-09 \\
9.0 \mathrm{E}-09 \\
1.2 \mathrm{E}-08 \\
1.0 \mathrm{E}-08 \\
5.9 \mathrm{E}-09 \\
4.0 \mathrm{E}-09 \\
5.1 \mathrm{E}-09 \\
4.3 \mathrm{E}-09 \\
6.1 \mathrm{E}-09 \\
4.1 \mathrm{E}-09\end{array}$ & $\begin{array}{l}3.3 \mathrm{E}-09 \\
1.2 \mathrm{E}-09 \\
1.7 \mathrm{E}-09 \\
2.3 \mathrm{E}-09 \\
2.6 \mathrm{E}-09 \\
3.4 \mathrm{E}-09 \\
5.3 \mathrm{E}-09 \\
8.8 \mathrm{E}-09 \\
1.2 \mathrm{E}-08 \\
1.0 \mathrm{E}-08 \\
5.7 \mathrm{E}-09 \\
3.9 \mathrm{E}-09 \\
5.0 \mathrm{E}-09 \\
4.2 \mathrm{E}-09 \\
6.0 \mathrm{E}-09 \\
4.0 \mathrm{E}-09\end{array}$ & $\begin{array}{l}3.2 \mathrm{E}-09 \\
1.2 \mathrm{E}-09 \\
1.7 \mathrm{E}-09 \\
2.3 \mathrm{E}-09 \\
2.6 \mathrm{E}-09 \\
3.3 \mathrm{E}-09 \\
5.2 \mathrm{E}-09 \\
8.5 \mathrm{E}-09 \\
1.2 \mathrm{E}-08 \\
9.9 \mathrm{E}-09 \\
5.5 \mathrm{E}-09 \\
3.8 \mathrm{E}-09 \\
4.8 \mathrm{E}-09 \\
4.0 \mathrm{E}-09 \\
5.8 \mathrm{E}-09 \\
3.9 \mathrm{E}-09\end{array}$ & \\
\hline \multirow[t]{2}{*}{ Feb } & 2008 & $2: 31 \mathrm{pm}$ & & & & & $\begin{array}{l}\text { SUMMARY } \\
\text { Page } 7\end{array}$ \\
\hline & \multicolumn{7}{|c|}{$\begin{array}{l}\text { INDIVIDUAL LIFETIME RISK (deaths) } \\
\text { (A11 Radionuclides and pathways) }\end{array}$} \\
\hline \multicolumn{8}{|c|}{ Distance (m) } \\
\hline Direction & 27715 & 28919 & 31060 & 32802 & 34577 & 35279 & 35683 \\
\hline $\begin{array}{r}N \\
N N W \\
N W \\
W N W\end{array}$ & $\begin{array}{l}7.5 \mathrm{E}-16 \\
2.8 \mathrm{E}-16 \\
3.9 \mathrm{E}-16 \\
5.3 \mathrm{E}-16\end{array}$ & $\begin{array}{l}7.1 E-16 \\
2.7 E-16 \\
3.8 E-16 \\
5.0 E-16\end{array}$ & $\begin{array}{l}6 \cdot 6 \mathrm{E}-16 \\
2 \cdot 5 \mathrm{E}-16 \\
3 \cdot 5 \mathrm{E}-16 \\
4 \cdot 6 \mathrm{E}-16\end{array}$ & $\begin{array}{r}6.2 E-16 \\
2.3 E-16 \\
3.3 E-16 \\
4.4 E-16 \\
\text { Page }\end{array}$ & $\begin{array}{l}5.8 E-16 \\
2.2 E-16 \\
3.1 E-16 \\
4.1 E-16\end{array}$ & $\begin{array}{l}5.7 \mathrm{E}-16 \\
2.1 \mathrm{E}-16 \\
3.0 \mathrm{E}-16 \\
4.0 \mathrm{E}-16\end{array}$ & $\begin{array}{l}5.6 E-16 \\
2.1 E-16 \\
3.0 E-16 \\
4.0 E-16\end{array}$ \\
\hline
\end{tabular}




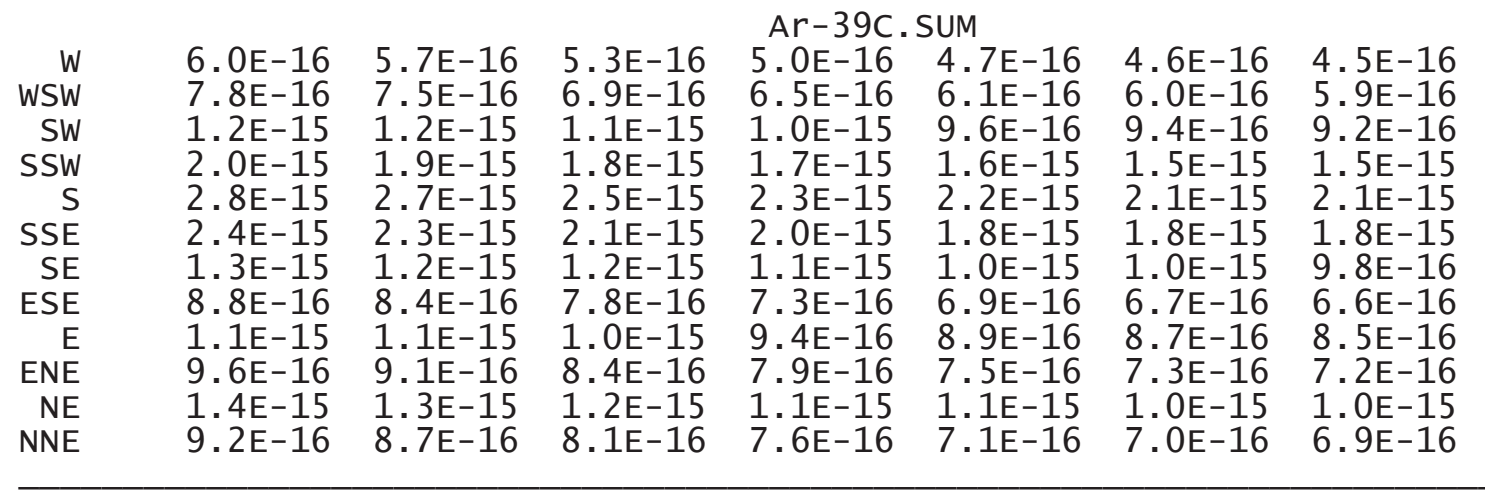

Distance (m)

\begin{tabular}{|c|c|c|c|c|c|c|c|}
\hline Direction & 36721 & 36809 & 37729 & 39079 & 39220 & 39559 & 43584 \\
\hline $\mathrm{N}$ & $5.4 \mathrm{E}-16$ & $5.4 \mathrm{E}-16$ & $5.3 \mathrm{E}-16$ & $5.1 \mathrm{E}-16$ & $5.1 \mathrm{E}-16$ & $5.0 \mathrm{E}-16$ & $4.5 \mathrm{E}-16$ \\
\hline NNW & $2.0 \mathrm{E}-16$ & $2.0 \mathrm{E}-16$ & $2.0 \mathrm{E}-16$ & $1.9 \mathrm{E}-16$ & $1.9 \mathrm{E}-16$ & $1.9 \mathrm{E}-16$ & 1.7E-16 \\
\hline NW & $2.9 E-16$ & $2.9 \mathrm{E}-16$ & $2.8 \mathrm{E}-16$ & $2.7 \mathrm{E}-16$ & $2.7 \mathrm{E}-16$ & $2.7 \mathrm{E}-16$ & $2.4 \mathrm{E}-16$ \\
\hline WNW & $3.9 \mathrm{E}-16$ & $3.9 \mathrm{E}-16$ & $3.7 \mathrm{E}-16$ & $3.6 \mathrm{E}-16$ & $3.6 \mathrm{E}-16$ & $3.6 \mathrm{E}-16$ & $3.2 \mathrm{E}-16$ \\
\hline W & $4.4 \mathrm{E}-16$ & 4. $4 \mathrm{E}-16$ & $4.2 \mathrm{E}-16$ & $4.1 \mathrm{E}-16$ & 4.1E-16 & $4.0 \mathrm{E}-16$ & $3.6 \mathrm{E}-16$ \\
\hline WSW & $5.7 \mathrm{E}-16$ & $5.7 \mathrm{E}-16$ & $5.5 \mathrm{E}-16$ & $5.3 \mathrm{E}-16$ & $5.3 \mathrm{E}-16$ & $5.3 \mathrm{E}-16$ & $4.7 \mathrm{E}-16$ \\
\hline SW & $8.9 \mathrm{E}-16$ & 8.9E-16 & 8.7E-16 & 8. $3 \mathrm{E}-16$ & $8.3 \mathrm{E}-16$ & $8.2 \mathrm{E}-16$ & 7. $3 \mathrm{E}-16$ \\
\hline SSW & 1. $5 \mathrm{E}-15$ & 1. $5 \mathrm{E}-15$ & 1. $4 \mathrm{E}-15$ & 1. $4 \mathrm{E}-15$ & 1. $4 \mathrm{E}-15$ & $1.4 \mathrm{E}-15$ & 1. $2 \mathrm{E}-15$ \\
\hline$S$ & 2.0E-15 & 2.0E-15 & 2.0E-15 & 1.9E-15 & 1.9E-15 & $1.9 \mathrm{E}-15$ & $1.7 \mathrm{E}-15$ \\
\hline SSE & $1.7 \mathrm{E}-15$ & 1.7E-15 & 1.7E-15 & $1.6 \mathrm{E}-15$ & $1.6 \mathrm{E}-15$ & $1.6 \mathrm{E}-15$ & $1.4 \mathrm{E}-15$ \\
\hline SE & $9.5 \mathrm{E}-16$ & $9.5 \mathrm{E}-16$ & $9.2 \mathrm{E}-16$ & 8. $9 \mathrm{E}-16$ & $8.8 \mathrm{E}-16$ & $8.8 \mathrm{E}-16$ & $7.9 \mathrm{E}-16$ \\
\hline ESE & $6.4 \mathrm{E}-16$ & $6.4 \mathrm{E}-16$ & $6.2 \mathrm{E}-16$ & 6. $0 \mathrm{E}-16$ & 6. $0 \mathrm{E}-16$ & $5.9 \mathrm{E}-16$ & $5.3 E-16$ \\
\hline E & $8.3 \mathrm{E}-16$ & 8. $3 \mathrm{E}-16$ & 8. $0 \mathrm{E}-16$ & $7.7 \mathrm{E}-16$ & $7.7 \mathrm{E}-16$ & $7.6 \mathrm{E}-16$ & $6.8 \mathrm{E}-16$ \\
\hline ENE & $7.0 \mathrm{E}-16$ & $6.9 \mathrm{E}-16$ & 6.7E-16 & $6.5 \mathrm{E}-16$ & $6.5 \mathrm{E}-16$ & $6.4 \mathrm{E}-16$ & $5.7 \mathrm{E}-16$ \\
\hline $\mathrm{NE}$ & $1.0 \mathrm{E}-15$ & 1. $0 \mathrm{E}-15$ & $9.7 \mathrm{E}-16$ & $9.3 \mathrm{E}-16$ & $9.3 \mathrm{E}-16$ & $9.2 \mathrm{E}-16$ & $8.2 \mathrm{E}-16$ \\
\hline NNE & $6.7 \mathrm{E}-16$ & $6.6 \mathrm{E}-16$ & $6.5 \mathrm{E}-16$ & $6.2 \mathrm{E}-16$ & $6.2 \mathrm{E}-16$ & $6.1 \mathrm{E}-16$ & $5.5 \mathrm{E}-16$ \\
\hline
\end{tabular}

$\square$

Feb 20, $2008 \quad 02: 31$ pm

SUMMARY

Page 8

INDIVIDUAL LIFETIME RISK (deaths)

(A11 Radionuclides and Pathways)

\begin{tabular}{|c|c|c|c|c|c|c|}
\hline \multirow[b]{2}{*}{ Direction } & \multicolumn{6}{|c|}{ Distance (m) } \\
\hline & n 45196 & 45275 & 45654 & 45677 & 46668 & 47969 \\
\hline $\begin{array}{r}\mathrm{N} \\
\mathrm{NNW} \\
\mathrm{NW} \\
\text { WNW } \\
W \\
\text { WSW } \\
\text { SW } \\
\text { SSW } \\
\mathrm{S}\end{array}$ & $\begin{array}{l}4 \cdot 3 \mathrm{E}-16 \\
1 \cdot 6 \mathrm{E}-16 \\
2 \cdot 3 \mathrm{E}-16 \\
3 \cdot 1 \mathrm{E}-16 \\
3 \cdot 5 \mathrm{E}-16 \\
4 \cdot 5 \mathrm{E}-16 \\
7 \cdot 0 \mathrm{E}-16 \\
1 \cdot 2 \mathrm{E}-15 \\
1 \cdot 6 \mathrm{E}-15\end{array}$ & $\begin{array}{l}4 \cdot 3 \mathrm{E}-16 \\
1 \cdot 6 \mathrm{E}-16 \\
2 \cdot 3 \mathrm{E}-16 \\
3 \cdot 1 \mathrm{E}-16 \\
3 \cdot 5 \mathrm{E}-16 \\
4 \cdot 5 \mathrm{E}-16 \\
7 \cdot 0 \mathrm{E}-16 \\
1 \cdot 2 \mathrm{E}-15 \\
1 \cdot 6 \mathrm{E}-15\end{array}$ & $\begin{array}{l}\text { 4. 3E-16 } \\
1.6 \mathrm{E}-16 \\
2.3 \mathrm{E}-16 \\
3.0 \mathrm{E}-16 \\
3.4 \mathrm{E}-16 \\
4.5 \mathrm{E}-16 \\
7.0 \mathrm{E}-16 \\
1.1 \mathrm{E}-15 \\
1.6 \mathrm{E}-15\end{array}$ & $\begin{array}{r}4.3 \mathrm{E}-16 \\
1.6 \mathrm{E}-16 \\
2.3 \mathrm{E}-16 \\
3.0 \mathrm{E}-16 \\
3.4 \mathrm{E}-16 \\
4.5 \mathrm{E}-16 \\
7.0 \mathrm{E}-16 \\
1.1 \mathrm{E}-15 \\
1.6 \mathrm{E}-15 \\
\text { Page }\end{array}$ & $\begin{array}{l}\text { 4. } 2 \mathrm{E}-16 \\
1.6 \mathrm{E}-16 \\
2.2 \mathrm{E}-16 \\
3.0 \mathrm{E}-16 \\
3.4 \mathrm{E}-16 \\
4.4 \mathrm{E}-16 \\
6.8 \mathrm{E}-16 \\
1.1 \mathrm{E}-15 \\
1.5 \mathrm{E}-15\end{array}$ & $\begin{array}{l}4.0 \mathrm{E}-16 \\
1.5 \mathrm{E}-16 \\
2.1 \mathrm{E}-16 \\
2.9 \mathrm{E}-16 \\
3.3 \mathrm{E}-16 \\
4.2 \mathrm{E}-16 \\
6.6 \mathrm{E}-16 \\
1.1 \mathrm{E}-15 \\
1.5 \mathrm{E}-15\end{array}$ \\
\hline
\end{tabular}




\begin{tabular}{rllllll} 
& \multicolumn{5}{c}{ Ar-39C. SUM } \\
SSE & $1.3 \mathrm{E}-15$ & $1.3 \mathrm{E}-15$ & $1.3 \mathrm{E}-15$ & $1.3 \mathrm{E}-15$ & $1.3 \mathrm{E}-15$ & $1.3 \mathrm{E}-15$ \\
SE & $7.5 \mathrm{E}-16$ & $7.5 \mathrm{E}-16$ & $7.4 \mathrm{E}-16$ & $7.4 \mathrm{E}-16$ & $7.3 \mathrm{E}-16$ & $7.0 \mathrm{E}-16$ \\
ESE & $5.1 \mathrm{E}-16$ & $5.1 \mathrm{E}-16$ & $5.0 \mathrm{E}-16$ & $5.0 \mathrm{E}-16$ & $4.9 \mathrm{E}-16$ & $4.8 \mathrm{E}-16$ \\
E & $6.6 \mathrm{E}-16$ & $6.5 \mathrm{E}-16$ & $6.5 \mathrm{E}-16$ & $6.5 \mathrm{E}-16$ & $6.3 \mathrm{E}-16$ & $6.1 \mathrm{E}-16$ \\
ENE & $5.5 \mathrm{E}-16$ & $5.5 \mathrm{E}-16$ & $5.4 \mathrm{E}-16$ & $5.4 \mathrm{E}-16$ & $5.3 \mathrm{E}-16$ & $5.1 \mathrm{E}-16$ \\
NE & $7.8 \mathrm{E}-16$ & $7.8 \mathrm{E}-16$ & $7.8 \mathrm{E}-16$ & $7.7 \mathrm{E}-16$ & $7.6 \mathrm{E}-16$ & $7.3 \mathrm{E}-16$ \\
NNE & $5.3 \mathrm{E}-16$ & $5.3 \mathrm{E}-16$ & $5.2 \mathrm{E}-16$ & $5.2 \mathrm{E}-16$ & $5.1 \mathrm{E}-16$ & $4.9 \mathrm{E}-16$ \\
\hline
\end{tabular}




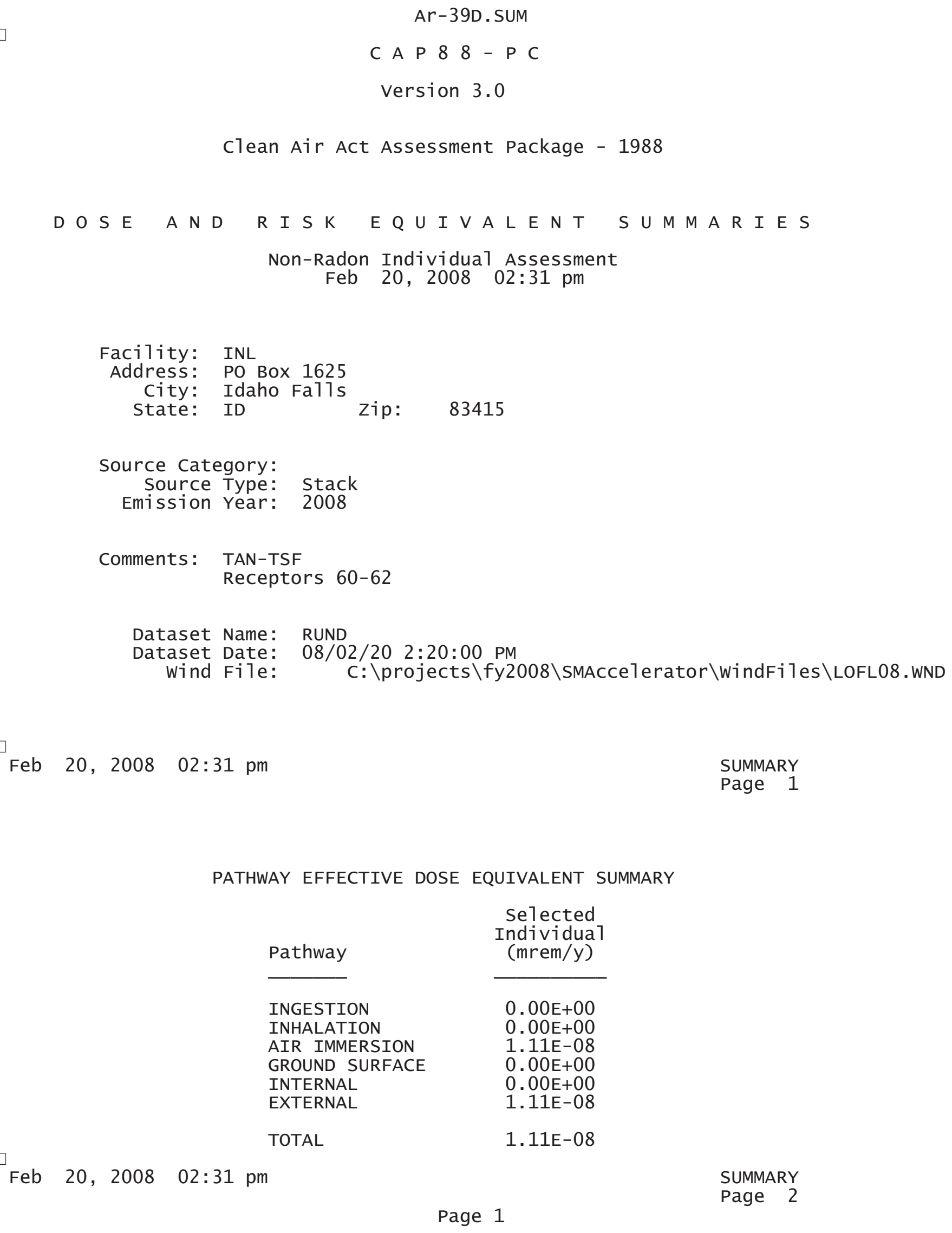

Pathway

INGESTION

INHALATION

AIR IMMERSION GROUND SURFACE INTERNAL

EXTERNAL

TOTAL

$$
\begin{gathered}
\begin{array}{c}
\text { selected } \\
\text { Individual } \\
\text { (mrem/y) }
\end{array} \\
\hline 0.00 \mathrm{E}+00 \\
0.00 \mathrm{E}+00 \\
1.11 \mathrm{E}-08 \\
0.00 \mathrm{E}+00 \\
0.00 \mathrm{E}+00 \\
1.11 \mathrm{E}-08 \\
1.11 \mathrm{E}-08
\end{gathered}
$$

Feb $20,2008 \quad 02: 31 \mathrm{pm}$

SUMMARY

Page 2 
Ar-39D. SUM

NUCLIDE EFFECTIVE DOSE EQUIVALENT SUMMARY

Nuclide

Ar-39

TOTAL

Feb 20, $2008 \quad 02: 31 \mathrm{pm}$ selected Individual

(mrem/y)

1. $11 \mathrm{E}-08$

1. $11 \mathrm{E}-08$
SUMMARY

Page 3

CANCER RISK SUMMARY

Cancer

Esophagu

Stomach

colon

Liver

LUNG

Bone

Skin

Breast

Ovary

Bladder

Kidneys

Thyroid

Leukemia

Residua 1

Total

TOTAL
Selected Individual

Total Lifetime

Fatal Cancer Risk

5. $64 \mathrm{E}-18$

2. $58 \mathrm{E}-17$

5. 51E-17

9. $85 \mathrm{E}-18$

7. 39E-17

2. 51E-18

1. $03 \mathrm{E}-15$

5. 32E-17

$6.77 \mathrm{E}-18$

1. $46 \mathrm{E}-17$

3. $52 \mathrm{E}-18$

2. $72 \mathrm{E}-18$

3. $59 \mathrm{E}-17$

9. $12 \mathrm{E}-17$

1. $41 \mathrm{E}-15$

$2.82 \mathrm{E}-15$

PATHWAY RISK SUMMARY

Pathway

INGESTION

INHALATION

AIR IMMERSION

GROUND SURFACE

INTERNAL

EXTERNAL

TOTAL selected Individual

Total Lifetime

Fatal Cancer Risk

$0.00 \mathrm{E}+00$

$0.00 \mathrm{E}+00$

1. 41E-15

$0.00 \mathrm{E}+00$

$0.00 \mathrm{E}+00$

1. 41E-15

1. $41 \mathrm{E}-15$

Page 2 


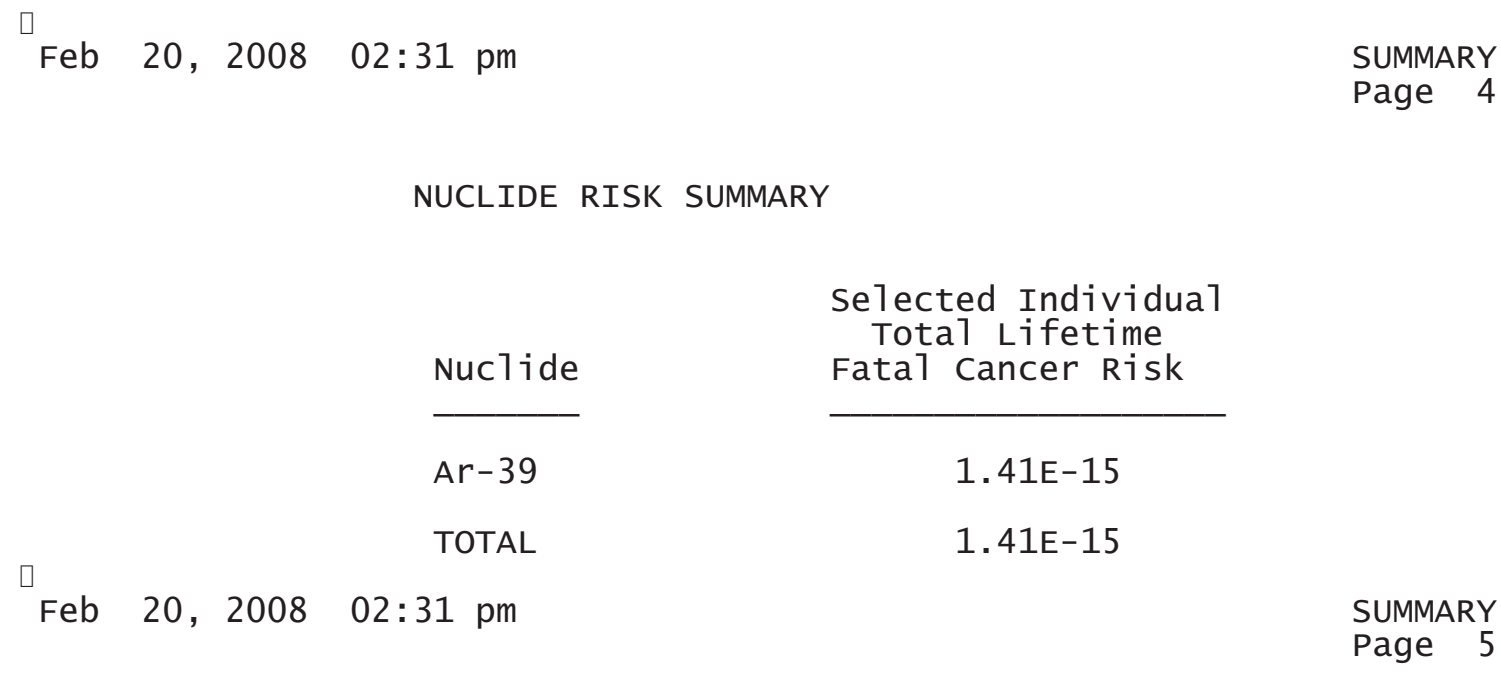

INDIVIDUAL EFFECTIVE DOSE EQUIVALENT RATE (mrem/y) (A11 Radionuclides and Pathways)

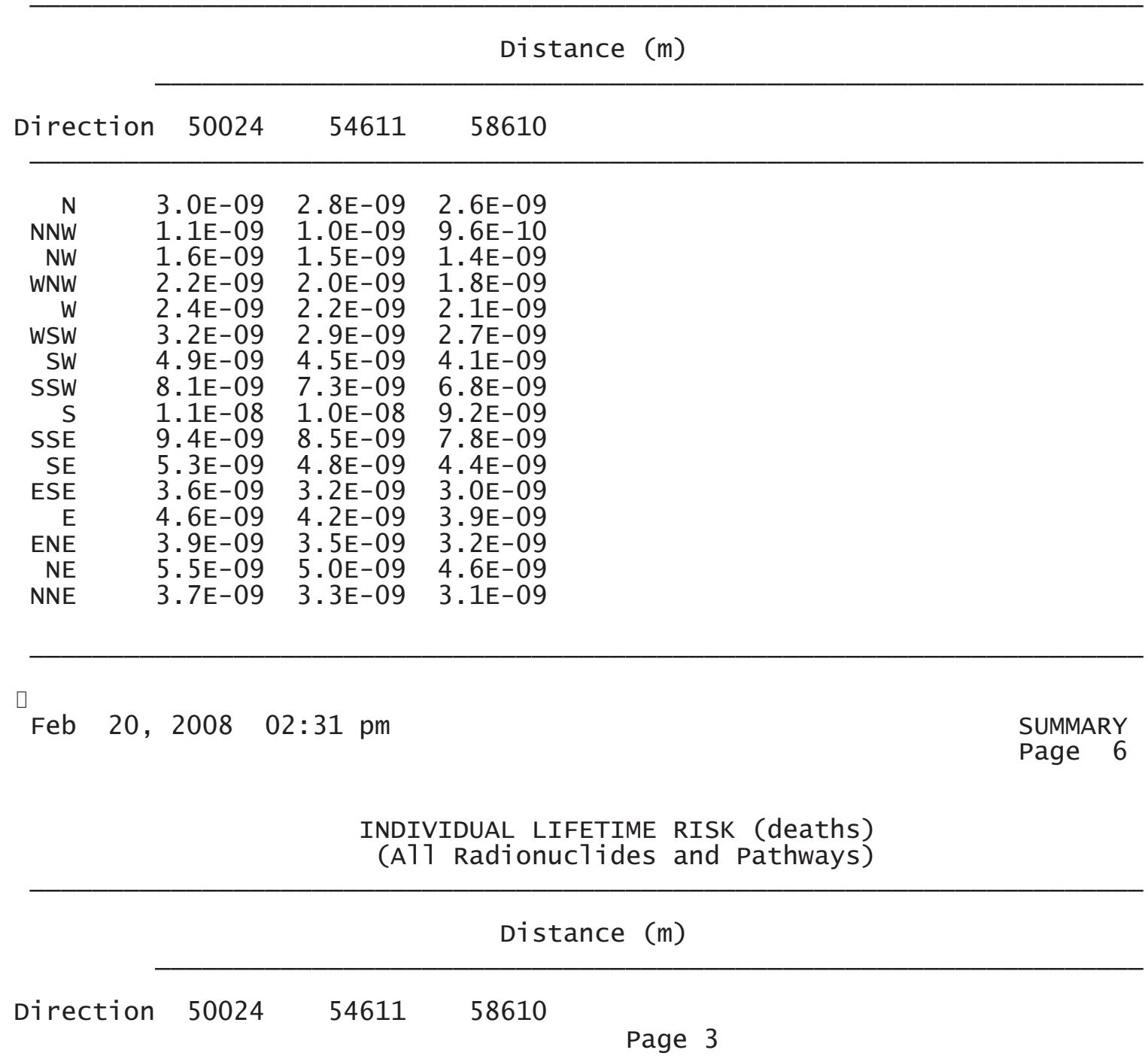


Ar-39D. SUM

\begin{tabular}{|c|c|c|c|}
\hline $\begin{array}{r}\text { N } \\
N N W \\
N W \\
\text { WNW } \\
W \\
W S W \\
\text { SW } \\
\text { SSW } \\
S \\
\text { SSE } \\
\text { SE } \\
\text { ESE } \\
\text { E } \\
\text { ENE } \\
N E \\
\text { NNE }\end{array}$ & $\begin{array}{l}3.9 \mathrm{E}-16 \\
1.5 \mathrm{E}-16 \\
2.1 \mathrm{E}-16 \\
2.7 \mathrm{E}-16 \\
3.1 \mathrm{E}-16 \\
4.0 \mathrm{E}-16 \\
6.3 \mathrm{E}-16 \\
1.0 \mathrm{E}-15 \\
1.4 \mathrm{E}-15 \\
1.2 \mathrm{E}-15 \\
6.7 \mathrm{E}-16 \\
4.5 \mathrm{E}-16 \\
5.9 \mathrm{E}-16 \\
4.9 \mathrm{E}-16 \\
7.0 \mathrm{E}-16 \\
4.7 \mathrm{E}-16\end{array}$ & $\begin{array}{l}3 \cdot 5 \mathrm{E}-16 \\
1 \cdot 3 \mathrm{E}-16 \\
1 \cdot 9 \mathrm{E}-16 \\
2.5 \mathrm{E}-16 \\
2.8 \mathrm{E}-16 \\
3.7 \mathrm{E}-16 \\
5 \cdot 7 \mathrm{E}-16 \\
9 \cdot 3 \mathrm{E}-16 \\
1.3 \mathrm{E}-15 \\
1.1 \mathrm{E}-15 \\
6 \cdot 1 \mathrm{E}-16 \\
4 \cdot 1 \mathrm{E}-16 \\
5 \cdot 3 \mathrm{E}-16 \\
4 \cdot 4 \mathrm{E}-16 \\
6.3 \mathrm{E}-16 \\
4 \cdot 2 \mathrm{E}-16\end{array}$ & $\begin{array}{l}3.2 \mathrm{E}-16 \\
1.2 \mathrm{E}-16 \\
1.7 \mathrm{E}-16 \\
2.3 \mathrm{E}-16 \\
2.6 \mathrm{E}-16 \\
3.4 \mathrm{E}-16 \\
5.2 \mathrm{E}-16 \\
8.6 \mathrm{E}-16 \\
1.2 \mathrm{E}-15 \\
1.0 \mathrm{E}-15 \\
5.6 \mathrm{E}-16 \\
3.8 \mathrm{E}-16 \\
4.9 \mathrm{E}-16 \\
4.1 \mathrm{E}-16 \\
5.8 \mathrm{E}-16 \\
3.9 \mathrm{E}-16\end{array}$ \\
\hline
\end{tabular}




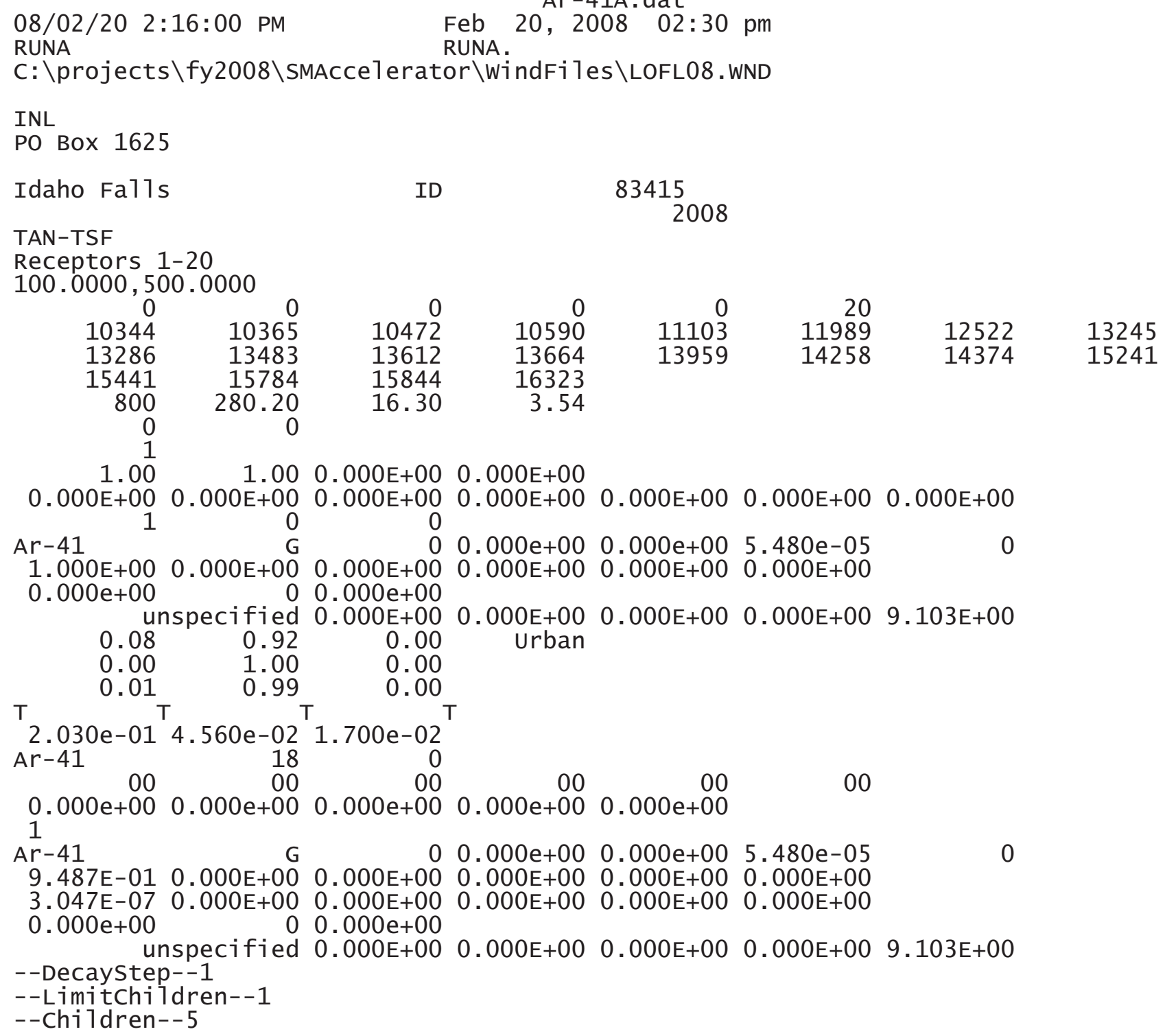




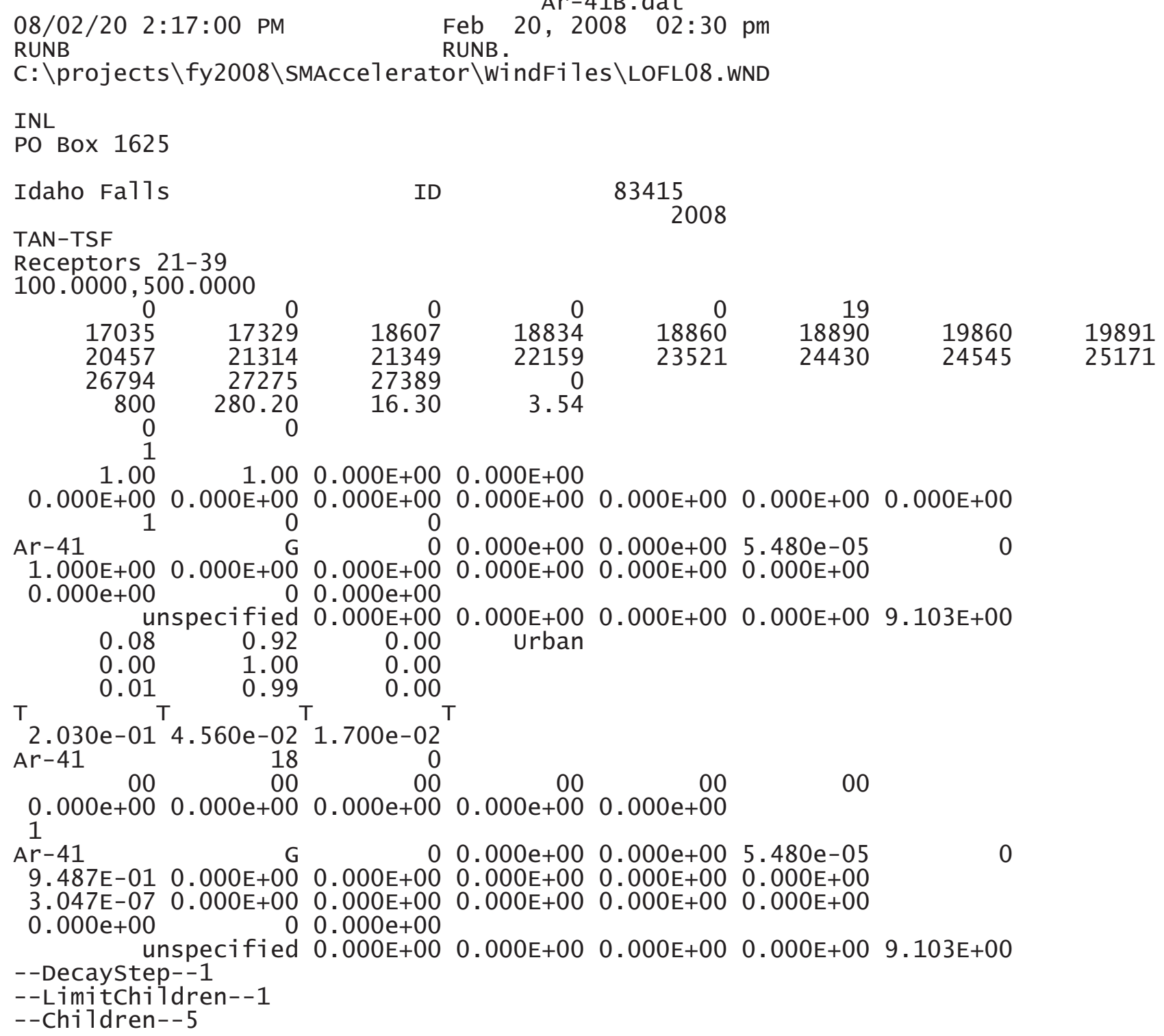




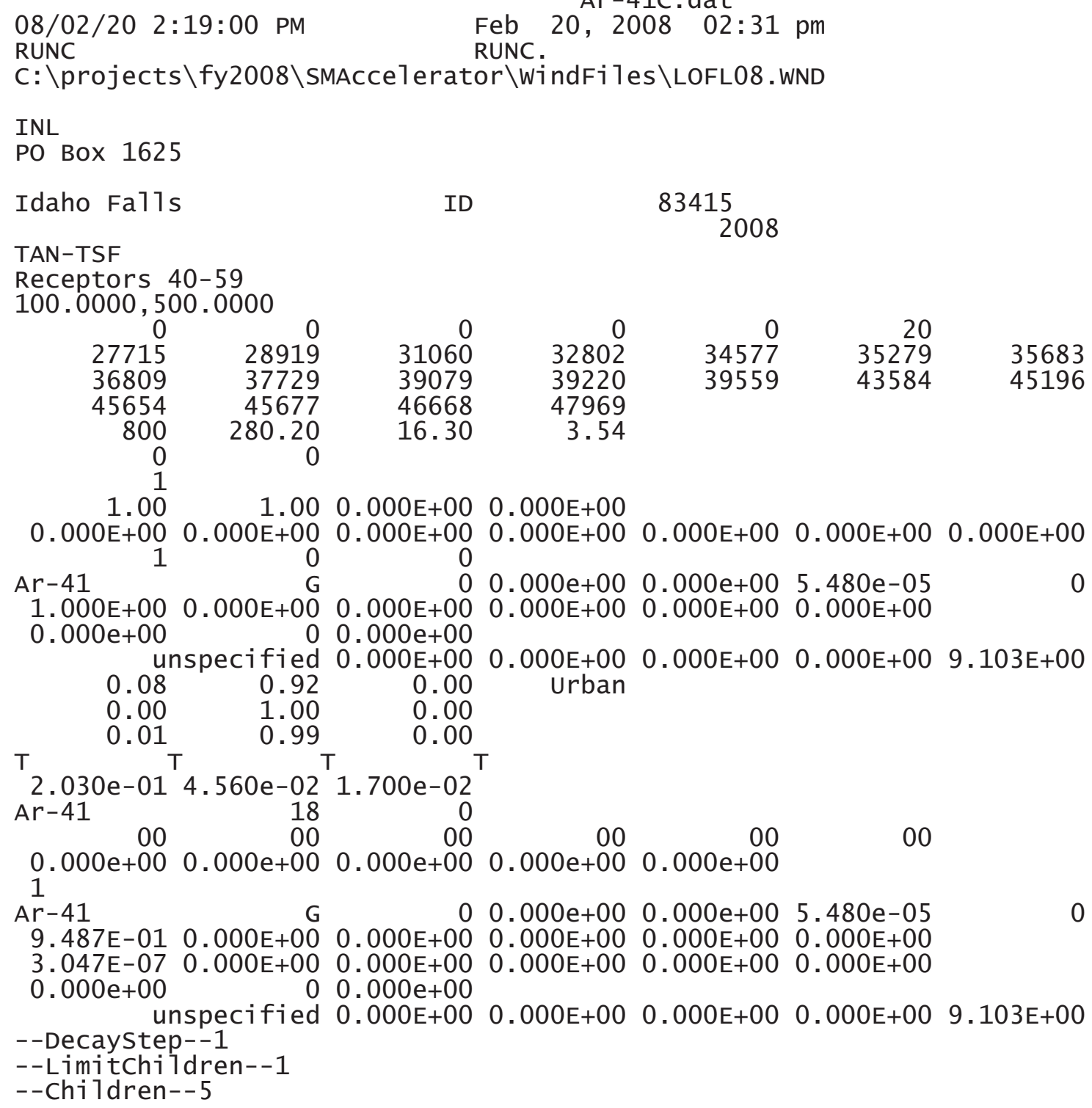

ID

83415

TAN-TSF

Receptors 40-59

$100.0000,500.0000$

$\begin{array}{rrrr}0 & 0 & 0 & \\ 27715 & 28919 & 31060 & 3280 \\ 36809 & 37729 & 39079 & 3922 \\ 45654 & 45677 & 46668 & 4796 \\ 800 & 280.20 & 16.30 & 3.5 \\ 0 & 0 & & \end{array}$




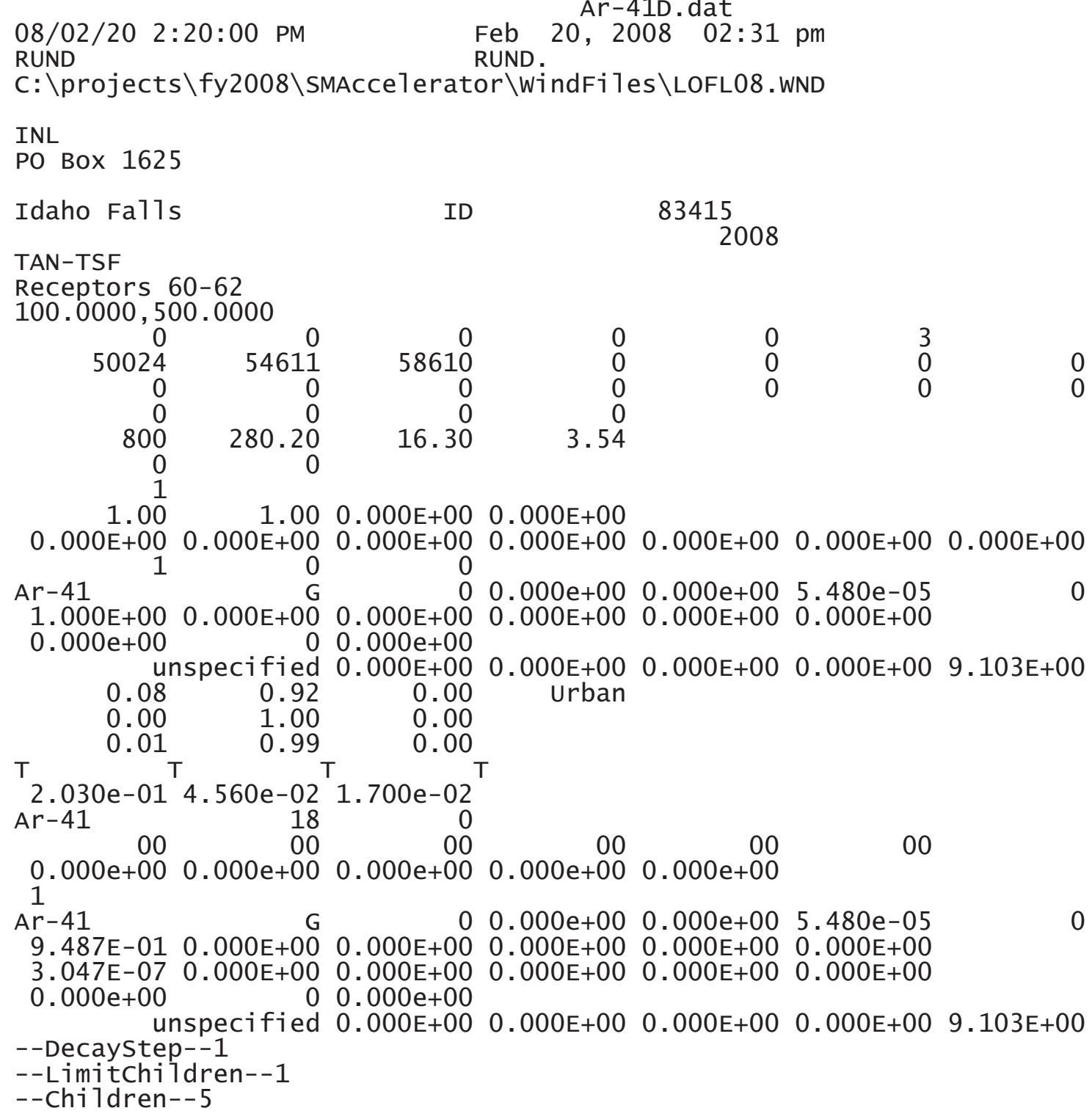




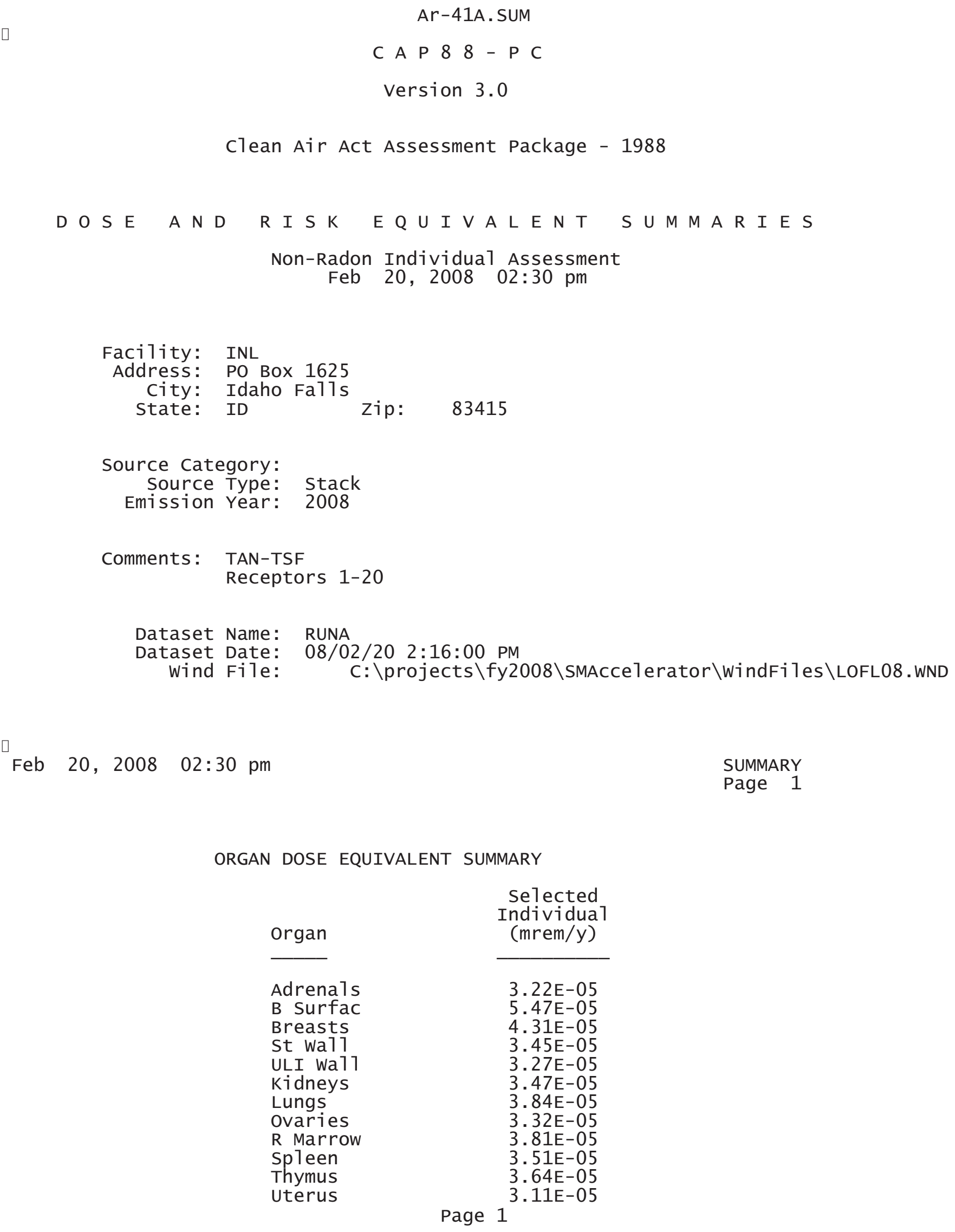

\begin{tabular}{lr} 
ORGAN DOSE EQUIVALENT SUMMARY \\
Organ & $\begin{array}{r}\text { Selected } \\
\text { Individual } \\
\text { (mrem/y) }\end{array}$ \\
\cline { 2 - 2 } Adrenals & $3.22 \mathrm{E}-05$ \\
B Surfac & $5.47 \mathrm{E}-05$ \\
Breasts & $4.31 \mathrm{E}-05$ \\
St wa71 & $3.45 \mathrm{E}-05$ \\
ULI wa11 & $3.27 \mathrm{E}-05$ \\
Kidneys & $3.47 \mathrm{E}-05$ \\
Lungs & $3.84 \mathrm{E}-05$ \\
Ovaries & $3.32 \mathrm{E}-05$ \\
R Marrow & $3.81 \mathrm{E}-05$ \\
Spleen & $3.51 \mathrm{E}-05$ \\
Thymus & $3.64 \mathrm{E}-05$ \\
Uterus & $3.11 \mathrm{E}-05$ \\
& 1
\end{tabular}




$\begin{array}{lr} & \text { Ar-41A. SUM } \\ \text { B1d wa11 } & 3.22 \mathrm{E}-05 \\ \text { Brain } & 4.14 \mathrm{E}-05 \\ \text { Esophagu } & 3.33 \mathrm{E}-05 \\ \text { SI wa11 } & 3.19 \mathrm{E}-05 \\ \text { LLI wa11 } & 3.24 \mathrm{E}-05 \\ \text { Liver } & 3.51 \mathrm{E}-05 \\ \text { Muscle } & 3.75 \mathrm{E}-05 \\ \text { Pancreas } & 3.14 \mathrm{E}-05 \\ \text { Skin } & 6.08 \mathrm{E}-05 \\ \text { Testes } & 3.81 \mathrm{E}-05 \\ \text { Thyroid } & 3.93 \mathrm{E}-05 \\ & \\ \text { EFFEC } & 3.70 \mathrm{E}-05\end{array}$

PATHWAY EFFECTIVE DOSE EQUIVALENT SUMMARY

\begin{tabular}{lcc} 
Pathway & $\begin{array}{c}\text { selected } \\
\text { Individua1 } \\
\text { (mrem/y) }\end{array}$ \\
\cline { 2 - 3 } INGESTION & $0.00 \mathrm{E}+00$ \\
INHALATION & $0.00 \mathrm{E}+00$ \\
AIR IMMERSION & $3.70 \mathrm{E}-05$ & \\
GROUND SURFACE & $0.00 \mathrm{E}+00$ & \\
INTERNAL & $0.00 \mathrm{E}+00$ & \\
EXTERNAL & $3.70 \mathrm{E}-05$ & \\
TOTAL & $3.70 \mathrm{E}-05$ & SUMMARY \\
Page 2
\end{tabular}

NUCLIDE EFFECTIVE DOSE EQUIVALENT SUMMARY

Nuclide

Ar-41

TOTAL

Feb 20, $2008 \quad 02: 30$ pm

$$
\begin{gathered}
\begin{array}{c}
\text { Selected } \\
\text { Individua } \\
\text { (mrem/y) }
\end{array} \\
\hline 3.70 \mathrm{E}-05 \\
3.70 \mathrm{E}-05
\end{gathered}
$$$$
3.70 \mathrm{E}-05
$$$$
3.70 \mathrm{E}-05
$$ \\ 3. $70 \mathrm{E}-05$ \\ $3.70 \mathrm{E}-05$}

CANCER RISK SUMMARY

Cancer

Esophagu
SUMMARY

Page 3 


$\begin{array}{lr} & \text { Ar-41A. SUM } \\ \text { Stomach } & 1.39 \mathrm{E}-12 \\ \text { Colon } & 3.36 \mathrm{E}-12 \\ \text { Liver } & 5.31 \mathrm{E}-13 \\ \text { LUNG } & 3.76 \mathrm{E}-12 \\ \text { Bone } & 5.19 \mathrm{E}-14 \\ \text { Skin } & 6.08 \mathrm{E}-14 \\ \text { Breast } & 2.08 \mathrm{E}-12 \\ \text { Ovary } & 4.72 \mathrm{E}-13 \\ \text { B Tadder } & 7.77 \mathrm{E}-13 \\ \text { Kidneys } & 1.81 \mathrm{E}-13 \\ \text { Thyroid } & 1.25 \mathrm{E}-13 \\ \text { Leukemia } & 2.14 \mathrm{E}-12 \\ \text { Residua1 } & 5.02 \mathrm{E}-12 \\ \text { Tota1 } & 2.04 \mathrm{E}-11 \\ & \\ \text { TOTAL } & 4.07 \mathrm{E}-11\end{array}$

PATHWAY RISK SUMMARY

\begin{tabular}{lc} 
Pathway & $\begin{array}{c}\text { Selected Individual } \\
\text { Total Lifetime } \\
\text { Fatal Cancer Risk }\end{array}$ \\
\cline { 2 - 2 } INGESTION & $0.00 \mathrm{E}+00$ \\
INHALATION & $0.00 \mathrm{E}+00$ \\
AIR IMMERSION & $2.04 \mathrm{E}-11$ \\
GROUND SURFACE & $0.00 \mathrm{E}+00$ \\
INTERNAL & $0.00 \mathrm{E}+00$ \\
EXTERNAL & $2.04 \mathrm{E}-11$ \\
TOTAL & $2.04 \mathrm{E}-11$
\end{tabular}

SUMMARY Page 4

NUCLIDE RISK SUMMARY

Nuc7ide

Ar -41

TOTAL

口

Feb 20, 2008 02:30 pm
Selected Individual

Total Lifetime Fatal Cancer Risk

2. 04E-11

2. 04E-11

SUMMARY

Page 5

INDIVIDUAL EFFECTIVE DOSE EQUIVALENT RATE (mrem/y) (A11 Radionuclides and Pathways)

Distance $(m)$ 


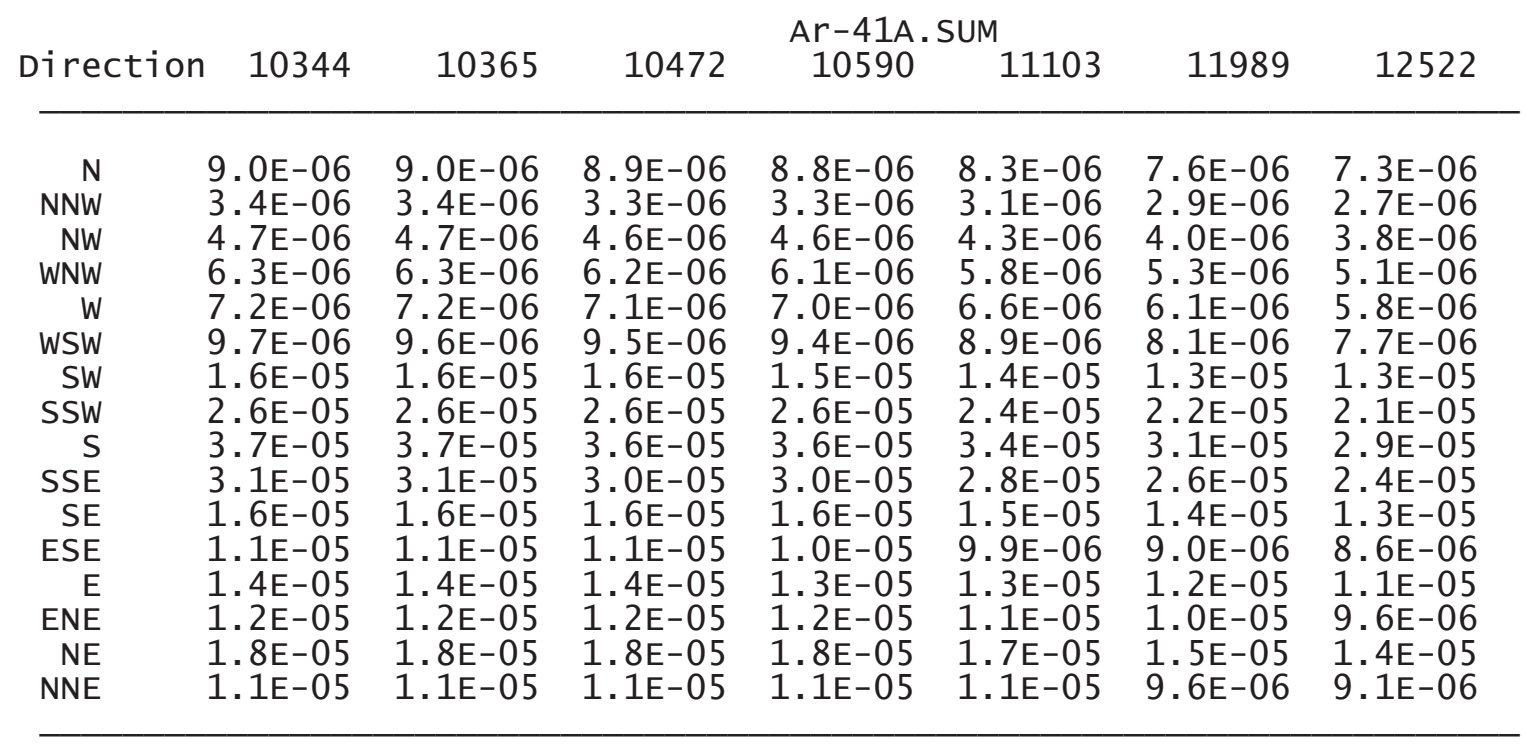

Distance (m)

\begin{tabular}{|c|c|c|c|c|c|c|c|}
\hline Direction & 13245 & 13286 & 13483 & 13612 & 13664 & 13959 & 14258 \\
\hline $\begin{array}{r}\text { N } \\
N N W \\
N W \\
W N W \\
W \\
W S W \\
S W \\
S S W \\
S \\
\text { SSE } \\
\text { SE } \\
\text { ESE } \\
\text { E } \\
\text { ENE } \\
N E \\
\text { NNE }\end{array}$ & $\begin{array}{l}6.8 \mathrm{E}-06 \\
2.6 \mathrm{E}-06 \\
3.6 \mathrm{E}-06 \\
4.8 \mathrm{E}-06 \\
5.4 \mathrm{E}-06 \\
7.2 \mathrm{E}-06 \\
1.2 \mathrm{E}-05 \\
2.0 \mathrm{E}-05 \\
2.7 \mathrm{E}-05 \\
2.3 \mathrm{E}-05 \\
1.2 \mathrm{E}-05 \\
8.1 \mathrm{E}-06 \\
1.0 \mathrm{E}-05 \\
8.9 \mathrm{E}-06 \\
1.3 \mathrm{E}-05 \\
8.5 \mathrm{E}-06\end{array}$ & $\begin{array}{l}6.8 \mathrm{E}-06 \\
2.5 \mathrm{E}-06 \\
3.5 \mathrm{E}-06 \\
4.7 \mathrm{E}-06 \\
5.4 \mathrm{E}-06 \\
7.2 \mathrm{E}-06 \\
1.2 \mathrm{E}-05 \\
1.9 \mathrm{E}-05 \\
2.7 \mathrm{E}-05 \\
2.3 \mathrm{E}-05 \\
1.2 \mathrm{E}-05 \\
8.1 \mathrm{E}-06 \\
1.0 \mathrm{E}-05 \\
8.9 \mathrm{E}-06 \\
1.3 \mathrm{E}-05 \\
8.5 \mathrm{E}-06\end{array}$ & $\begin{array}{l}6.7 \mathrm{E}-06 \\
2.5 \mathrm{E}-06 \\
3.5 \mathrm{E}-06 \\
4.7 \mathrm{E}-06 \\
5.3 \mathrm{E}-06 \\
7.1 \mathrm{E}-06 \\
1.1 \mathrm{E}-05 \\
1.9 \mathrm{E}-05 \\
2.7 \mathrm{E}-05 \\
2.2 \mathrm{E}-05 \\
1.2 \mathrm{E}-05 \\
7.9 \mathrm{E}-06 \\
1.0 \mathrm{E}-05 \\
8.8 \mathrm{E}-06 \\
1.3 \mathrm{E}-05 \\
8.4 \mathrm{E}-06\end{array}$ & $\begin{array}{l}6.6 \mathrm{E}-06 \\
2.5 \mathrm{E}-06 \\
3.5 \mathrm{E}-06 \\
4.6 \mathrm{E}-06 \\
5.3 \mathrm{E}-06 \\
7.0 \mathrm{E}-06 \\
1.1 \mathrm{E}-05 \\
1.9 \mathrm{E}-05 \\
2.7 \mathrm{E}-05 \\
2.2 \mathrm{E}-05 \\
1.2 \mathrm{E}-05 \\
7.8 \mathrm{E}-06 \\
1.0 \mathrm{E}-05 \\
8.7 \mathrm{E}-06 \\
1.3 \mathrm{E}-05 \\
8.3 \mathrm{E}-06\end{array}$ & $\begin{array}{l}6.6 \mathrm{E}-06 \\
2.5 \mathrm{E}-06 \\
3.4 \mathrm{E}-06 \\
4.6 \mathrm{E}-06 \\
5.2 \mathrm{E}-06 \\
7.0 \mathrm{E}-06 \\
1.1 \mathrm{E}-05 \\
1.9 \mathrm{E}-05 \\
2.6 \mathrm{E}-05 \\
2.2 \mathrm{E}-05 \\
1.2 \mathrm{E}-05 \\
7.8 \mathrm{E}-06 \\
1.0 \mathrm{E}-05 \\
8.6 \mathrm{E}-06 \\
1.3 \mathrm{E}-05 \\
8.2 \mathrm{E}-06\end{array}$ & $\begin{array}{l}6.4 \mathrm{E}-06 \\
2.4 \mathrm{E}-06 \\
3.4 \mathrm{E}-06 \\
4.5 \mathrm{E}-06 \\
5.1 \mathrm{E}-06 \\
6.8 \mathrm{E}-06 \\
1.1 \mathrm{E}-05 \\
1.8 \mathrm{E}-05 \\
2.6 \mathrm{E}-05 \\
2.1 \mathrm{E}-05 \\
1.1 \mathrm{E}-05 \\
7.6 \mathrm{E}-06 \\
9.8 \mathrm{E}-06 \\
8.4 \mathrm{E}-06 \\
1.3 \mathrm{E}-05 \\
8.0 \mathrm{E}-06\end{array}$ & $\begin{array}{l}6.3 \mathrm{E}-06 \\
2.4 \mathrm{E}-06 \\
3.3 \mathrm{E}-06 \\
4.4 \mathrm{E}-06 \\
5.0 \mathrm{E}-06 \\
6.6 \mathrm{E}-06 \\
1.1 \mathrm{E}-05 \\
1.8 \mathrm{E}-05 \\
2.5 \mathrm{E}-05 \\
2.1 \mathrm{E}-05 \\
1.1 \mathrm{E}-05 \\
7.4 \mathrm{E}-06 \\
9.6 \mathrm{E}-06 \\
8.2 \mathrm{E}-06 \\
1.2 \mathrm{E}-05 \\
7.8 \mathrm{E}-06\end{array}$ \\
\hline
\end{tabular}

$\square$

Feb 20, 2008 02:30 pm

SUMMARY

Page 6

INDIVIDUAL EFFECTIVE DOSE EQUIVALENT RATE (mrem/y) (A11 Radionuclides and Pathways)

\begin{tabular}{|c|c|c|c|c|c|c|}
\hline \multicolumn{7}{|c|}{ Distance (m) } \\
\hline Direction & 14374 & 15241 & 15441 & 15784 & 15844 & 16323 \\
\hline $\begin{array}{r}N \\
N N W\end{array}$ & $\begin{array}{l}6.2 \mathrm{E}-06 \\
2.3 \mathrm{E}-06\end{array}$ & $\begin{array}{l}5.8 \mathrm{E}-06 \\
2.2 \mathrm{E}-06\end{array}$ & $\begin{array}{l}5.7 \mathrm{E}-06 \\
2.2 \mathrm{E}-06\end{array}$ & $\begin{array}{r}5.6 \mathrm{E}-06 \\
2.1 \mathrm{E}-06 \\
\text { Page }\end{array}$ & $\begin{array}{r}5.6 \mathrm{E}-06 \\
2.1 \mathrm{E}-06 \\
4\end{array}$ & $\begin{array}{l}5.4 \mathrm{E}-06 \\
2.0 \mathrm{E}-06\end{array}$ \\
\hline
\end{tabular}




\begin{tabular}{rllllll} 
& \multicolumn{1}{c}{ Ar-41A. SUM } \\
NW & $3.2 \mathrm{E}-06$ & $3.0 \mathrm{E}-06$ & $3.0 \mathrm{E}-06$ & $2.9 \mathrm{E}-06$ & $2.9 \mathrm{E}-06$ & $2.8 \mathrm{E}-06$ \\
WNW & $4.3 \mathrm{E}-06$ & $4.1 \mathrm{E}-06$ & $4.0 \mathrm{E}-06$ & $3.9 \mathrm{E}-06$ & $3.9 \mathrm{E}-06$ & $3.8 \mathrm{E}-06$ \\
$\mathrm{~W}$ & $5.0 \mathrm{E}-06$ & $4.6 \mathrm{E}-06$ & $4.6 \mathrm{E}-06$ & $4.5 \mathrm{E}-06$ & $4.4 \mathrm{E}-06$ & $4.3 \mathrm{E}-06$ \\
WSW & $6.6 \mathrm{E}-06$ & $6.2 \mathrm{E}-06$ & $6.1 \mathrm{E}-06$ & $5.9 \mathrm{E}-06$ & $5.9 \mathrm{E}-06$ & $5.7 \mathrm{E}-06$ \\
$\mathrm{SW}$ & $1.1 \mathrm{E}-05$ & $9.9 \mathrm{E}-06$ & $9.8 \mathrm{E}-06$ & $9.5 \mathrm{E}-06$ & $9.5 \mathrm{E}-06$ & $9.1 \mathrm{E}-06$ \\
SSW & $1.8 \mathrm{E}-05$ & $1.7 \mathrm{E}-05$ & $1.6 \mathrm{E}-05$ & $1.6 \mathrm{E}-05$ & $1.6 \mathrm{E}-05$ & $1.5 \mathrm{E}-05$ \\
$\mathrm{~S}$ & $2.5 \mathrm{E}-05$ & $2.3 \mathrm{E}-05$ & $2.3 \mathrm{E}-05$ & $2.2 \mathrm{E}-05$ & $2.2 \mathrm{E}-05$ & $2.1 \mathrm{E}-05$ \\
SSE & $2.1 \mathrm{E}-05$ & $1.9 \mathrm{E}-05$ & $1.9 \mathrm{E}-05$ & $1.9 \mathrm{E}-05$ & $1.8 \mathrm{E}-05$ & $1.8 \mathrm{E}-05$ \\
SE & $1.1 \mathrm{E}-05$ & $1.0 \mathrm{E}-05$ & $1.0 \mathrm{E}-05$ & $1.0 \mathrm{E}-05$ & $9.9 \mathrm{E}-06$ & $9.6 \mathrm{E}-06$ \\
ESE & $7.4 \mathrm{E}-06$ & $6.9 \mathrm{E}-06$ & $6.8 \mathrm{E}-06$ & $6.6 \mathrm{E}-06$ & $6.6 \mathrm{E}-06$ & $6.4 \mathrm{E}-06$ \\
$\mathrm{E}$ & $9.5 \mathrm{E}-06$ & $8.9 \mathrm{E}-06$ & $8.8 \mathrm{E}-06$ & $8.5 \mathrm{E}-06$ & $8.5 \mathrm{E}-06$ & $8.2 \mathrm{E}-06$ \\
ENE & $8.1 \mathrm{E}-06$ & $7.6 \mathrm{E}-06$ & $7.5 \mathrm{E}-06$ & $7.3 \mathrm{E}-06$ & $7.3 \mathrm{E}-06$ & $7.0 \mathrm{E}-06$ \\
NE & $1.2 \mathrm{E}-05$ & $1.1 \mathrm{E}-05$ & $1.1 \mathrm{E}-05$ & $1.1 \mathrm{E}-05$ & $1.1 \mathrm{E}-05$ & $1.0 \mathrm{E}-05$ \\
NNE & $7.8 \mathrm{E}-06$ & $7.3 \mathrm{E}-06$ & $7.2 \mathrm{E}-06$ & $7.0 \mathrm{E}-06$ & $7.0 \mathrm{E}-06$ & $6.7 \mathrm{E}-06$
\end{tabular}

Feb 20, 2008 02:30 pm

SUMMARY

Page 7

INDIVIDUAL LIFETIME RISK (deaths)

(A11 Radionuclides and Pathways)

\begin{tabular}{|c|c|c|c|c|c|c|c|}
\hline \multicolumn{8}{|c|}{ Distance (m) } \\
\hline Direction & 10344 & 10365 & 10472 & 10590 & 11103 & 11989 & 12522 \\
\hline $\begin{array}{r}\text { N } \\
\text { NNW } \\
N W \\
\text { WNW } \\
W \\
W S W \\
\text { SW } \\
\text { SSW } \\
\text { S } \\
\text { SSE } \\
\text { SE } \\
\text { ESE } \\
\text { E } \\
\text { ENE } \\
N E \\
\text { NNE }\end{array}$ & $\begin{array}{l}4.9 \mathrm{E}-12 \\
1.9 \mathrm{E}-12 \\
2.6 \mathrm{E}-12 \\
3.5 \mathrm{E}-12 \\
3.9 \mathrm{E}-12 \\
5.3 \mathrm{E}-12 \\
8.7 \mathrm{E}-12 \\
1.4 \mathrm{E}-11 \\
2.0 \mathrm{E}-11 \\
1.7 \mathrm{E}-11 \\
8.9 \mathrm{E}-12 \\
5.9 \mathrm{E}-12 \\
7.6 \mathrm{E}-12 \\
6.6 \mathrm{E}-12 \\
1.0 \mathrm{E}-11 \\
6.3 \mathrm{E}-12\end{array}$ & $\begin{array}{l}4.9 \mathrm{E}-12 \\
1.9 \mathrm{E}-12 \\
2.6 \mathrm{E}-12 \\
3.4 \mathrm{E}-12 \\
3.9 \mathrm{E}-12 \\
5.3 \mathrm{E}-12 \\
8.6 \mathrm{E}-12 \\
1.4 \mathrm{E}-11 \\
2.0 \mathrm{E}-11 \\
1.7 \mathrm{E}-11 \\
8.9 \mathrm{E}-12 \\
5.9 \mathrm{E}-12 \\
7.5 \mathrm{E}-12 \\
6.5 \mathrm{E}-12 \\
9.9 \mathrm{E}-12 \\
6.3 \mathrm{E}-12\end{array}$ & $\begin{array}{l}4.9 \mathrm{E}-12 \\
1.8 \mathrm{E}-12 \\
2.5 \mathrm{E}-12 \\
3.4 \mathrm{E}-12 \\
3.9 \mathrm{E}-12 \\
5.2 \mathrm{E}-12 \\
8.5 \mathrm{E}-12 \\
1.4 \mathrm{E}-11 \\
2.0 \mathrm{E}-11 \\
1.7 \mathrm{E}-11 \\
8.8 \mathrm{E}-12 \\
5.8 \mathrm{E}-12 \\
7.5 \mathrm{E}-12 \\
6.5 \mathrm{E}-12 \\
9.8 \mathrm{E}-12 \\
6.2 \mathrm{E}-12\end{array}$ & $\begin{array}{l}4.8 \mathrm{E}-12 \\
1.8 \mathrm{E}-12 \\
2.5 \mathrm{E}-12 \\
3.4 \mathrm{E}-12 \\
3.8 \mathrm{E}-12 \\
5.2 \mathrm{E}-12 \\
8.4 \mathrm{E}-12 \\
1.4 \mathrm{E}-11 \\
2.0 \mathrm{E}-11 \\
1.6 \mathrm{E}-11 \\
8.6 \mathrm{E}-12 \\
5.7 \mathrm{E}-12 \\
7.4 \mathrm{E}-12 \\
6.4 \mathrm{E}-12 \\
9.7 \mathrm{E}-12 \\
6.1 \mathrm{E}-12\end{array}$ & $\begin{array}{l}4.6 \mathrm{E}-12 \\
1.7 \mathrm{E}-12 \\
2.4 \mathrm{E}-12 \\
3.2 \mathrm{E}-12 \\
3.6 \mathrm{E}-12 \\
4.9 \mathrm{E}-12 \\
8.0 \mathrm{E}-12 \\
1.3 \mathrm{E}-11 \\
1.9 \mathrm{E}-11 \\
1.6 \mathrm{E}-11 \\
8.2 \mathrm{E}-12 \\
5.4 \mathrm{E}-12 \\
7.0 \mathrm{E}-12 \\
6.0 \mathrm{E}-12 \\
9.1 \mathrm{E}-12 \\
5.8 \mathrm{E}-12\end{array}$ & $\begin{array}{l}4.2 \mathrm{E}-12 \\
1.6 \mathrm{E}-12 \\
2.2 \mathrm{E}-12 \\
2.9 \mathrm{E}-12 \\
3.3 \mathrm{E}-12 \\
4.5 \mathrm{E}-12 \\
7.3 \mathrm{E}-12 \\
1.2 \mathrm{E}-11 \\
1.7 \mathrm{E}-11 \\
1.4 \mathrm{E}-11 \\
7.5 \mathrm{E}-12 \\
5.0 \mathrm{E}-12 \\
6.4 \mathrm{E}-12 \\
5.5 \mathrm{E}-12 \\
8.3 \mathrm{E}-12 \\
5.3 \mathrm{E}-12\end{array}$ & $\begin{array}{l}4.0 \mathrm{E}-12 \\
1.5 \mathrm{E}-12 \\
2.1 \mathrm{E}-12 \\
2.8 \mathrm{E}-12 \\
3.2 \mathrm{E}-12 \\
4.3 \mathrm{E}-12 \\
6.9 \mathrm{E}-12 \\
1.1 \mathrm{E}-11 \\
1.6 \mathrm{E}-11 \\
1.3 \mathrm{E}-11 \\
7.1 \mathrm{E}-12 \\
4.7 \mathrm{E}-12 \\
6.1 \mathrm{E}-12 \\
5.2 \mathrm{E}-12 \\
7.9 \mathrm{E}-12 \\
5.0 \mathrm{E}-12\end{array}$ \\
\hline \multicolumn{8}{|c|}{ Distance (m) } \\
\hline Direction & 13245 & 13286 & 13483 & 13612 & 13664 & 13959 & 14258 \\
\hline $\begin{array}{r}\text { N } \\
\text { NNW } \\
\text { NW } \\
\text { WNW } \\
\text { W } \\
\text { WSW } \\
\text { SW } \\
\text { SSW }\end{array}$ & $\begin{array}{l}3.7 \mathrm{E}-12 \\
1.4 \mathrm{E}-12 \\
2.0 \mathrm{E}-12 \\
2.6 \mathrm{E}-12 \\
3.0 \mathrm{E}-12 \\
4.0 \mathrm{E}-12 \\
6.4 \mathrm{E}-12 \\
1.1 \mathrm{E}-11\end{array}$ & $\begin{array}{l}3.7 \mathrm{E}-12 \\
1.4 \mathrm{E}-12 \\
1.9 \mathrm{E}-12 \\
2.6 \mathrm{E}-12 \\
3.0 \mathrm{E}-12 \\
4.0 \mathrm{E}-12 \\
6.4 \mathrm{E}-12 \\
1.1 \mathrm{E}-11\end{array}$ & $\begin{array}{l}3.7 \mathrm{E}-12 \\
1.4 \mathrm{E}-12 \\
1.9 \mathrm{E}-12 \\
2.6 \mathrm{E}-12 \\
2.9 \mathrm{E}-12 \\
3.9 \mathrm{E}-12 \\
6.3 \mathrm{E}-12 \\
1.0 \mathrm{E}-11\end{array}$ & $\begin{array}{r}3.6 \mathrm{E}-12 \\
1.4 \mathrm{E}-12 \\
1.9 \mathrm{E}-12 \\
2.5 \mathrm{E}-12 \\
2.9 \mathrm{E}-12 \\
3.9 \mathrm{E}-12 \\
6.2 \mathrm{E}-12 \\
1.0 \mathrm{E}-11 \\
\text { Page }\end{array}$ & $\begin{array}{l}3.6 \mathrm{E}-12 \\
1.4 \mathrm{E}-12 \\
1.9 \mathrm{E}-12 \\
2.5 \mathrm{E}-12 \\
2.9 \mathrm{E}-12 \\
3.8 \mathrm{E}-12 \\
6.2 \mathrm{E}-12 \\
1.0 \mathrm{E}-11\end{array}$ & $\begin{array}{l}3.5 \mathrm{E}-12 \\
1.3 \mathrm{E}-12 \\
1.8 \mathrm{E}-12 \\
2.5 \mathrm{E}-12 \\
2.8 \mathrm{E}-12 \\
3.7 \mathrm{E}-12 \\
6.0 \mathrm{E}-12 \\
1.0 \mathrm{E}-11\end{array}$ & $\begin{array}{l}3.4 \mathrm{E}-12 \\
1.3 \mathrm{E}-12 \\
1.8 \mathrm{E}-12 \\
2.4 \mathrm{E}-12 \\
2.7 \mathrm{E}-12 \\
3.7 \mathrm{E}-12 \\
5.9 \mathrm{E}-12 \\
9.8 \mathrm{E}-12\end{array}$ \\
\hline
\end{tabular}




\begin{tabular}{rlllllll}
\multicolumn{7}{c}{ Ar-41A.SUM } \\
S & $1.5 \mathrm{E}-11$ & $1.5 \mathrm{E}-11$ & $1.5 \mathrm{E}-11$ & $1.5 \mathrm{E}-11$ & $1.5 \mathrm{E}-11$ & $1.4 \mathrm{E}-11$ & $1.4 \mathrm{E}-11$ \\
SSE & $1.3 \mathrm{E}-11$ & $1.3 \mathrm{E}-11$ & $1.2 \mathrm{E}-11$ & $1.2 \mathrm{E}-11$ & $1.2 \mathrm{E}-11$ & $1.2 \mathrm{E}-11$ & $1.2 \mathrm{E}-11$ \\
SE & $6.7 \mathrm{E}-12$ & $6.7 \mathrm{E}-12$ & $6.6 \mathrm{E}-12$ & $6.5 \mathrm{E}-12$ & $6.5 \mathrm{E}-12$ & $6.3 \mathrm{E}-12$ & $6.1 \mathrm{E}-12$ \\
$\mathrm{ESE}$ & $4.4 \mathrm{E}-12$ & $4.4 \mathrm{E}-12$ & $4.4 \mathrm{E}-12$ & $4.3 \mathrm{E}-12$ & $4.3 \mathrm{E}-12$ & $4.2 \mathrm{E}-12$ & $4.1 \mathrm{E}-12$ \\
$\mathrm{E}$ & $5.7 \mathrm{E}-12$ & $5.7 \mathrm{E}-12$ & $5.6 \mathrm{E}-12$ & $5.5 \mathrm{E}-12$ & $5.5 \mathrm{E}-12$ & $5.4 \mathrm{E}-12$ & $5.3 \mathrm{E}-12$ \\
$\mathrm{ENE}$ & $4.9 \mathrm{E}-12$ & $4.9 \mathrm{E}-12$ & $4.8 \mathrm{E}-12$ & $4.8 \mathrm{E}-12$ & $4.7 \mathrm{E}-12$ & $4.6 \mathrm{E}-12$ & $4.5 \mathrm{E}-12$ \\
$\mathrm{NE}$ & $7.4 \mathrm{E}-12$ & $7.3 \mathrm{E}-12$ & $7.2 \mathrm{E}-12$ & $7.1 \mathrm{E}-12$ & $7.1 \mathrm{E}-12$ & $6.9 \mathrm{E}-12$ & $6.7 \mathrm{E}-12$ \\
$\mathrm{NNE}$ & $4.7 \mathrm{E}-12$ & $4.7 \mathrm{E}-12$ & $4.6 \mathrm{E}-12$ & $4.5 \mathrm{E}-12$ & $4.5 \mathrm{E}-12$ & $4.4 \mathrm{E}-12$ & $4.3 \mathrm{E}-12$
\end{tabular}

Feb 20, $2008 \quad 02: 30 \mathrm{pm}$

SUMMARY

Page 8

INDIVIDUAL LIFETIME RISK (deaths)

(A11 Radionuclides and Pathways)

\begin{tabular}{|c|c|c|c|c|c|c|}
\hline \multirow[b]{2}{*}{ Direction } & \multicolumn{6}{|c|}{ Distance $(\mathrm{m})$} \\
\hline & 14374 & 15241 & 15441 & 15784 & 15844 & 16323 \\
\hline $\begin{array}{r}N \\
N N W \\
N W \\
W N W \\
W \\
W S W \\
S W \\
S S W \\
S \\
S S E \\
S E \\
\text { ESE } \\
E \\
\text { ENE } \\
N E \\
N N E\end{array}$ & $\begin{array}{l}3.4 \mathrm{E}-12 \\
1.3 \mathrm{E}-12 \\
1.8 \mathrm{E}-12 \\
2.4 \mathrm{E}-12 \\
2.7 \mathrm{E}-12 \\
3.6 \mathrm{E}-12 \\
5.8 \mathrm{E}-12 \\
9.7 \mathrm{E}-12 \\
1.4 \mathrm{E}-11 \\
1.1 \mathrm{E}-11 \\
6.1 \mathrm{E}-12 \\
4.1 \mathrm{E}-12 \\
5.2 \mathrm{E}-12 \\
4.5 \mathrm{E}-12 \\
6.7 \mathrm{E}-12 \\
4.3 \mathrm{E}-12\end{array}$ & $\begin{array}{l}3.2 \mathrm{E}-12 \\
1.2 \mathrm{E}-12 \\
1.7 \mathrm{E}-12 \\
2.2 \mathrm{E}-12 \\
2.6 \mathrm{E}-12 \\
3.4 \mathrm{E}-12 \\
5.4 \mathrm{E}-12 \\
9.1 \mathrm{E}-12 \\
1.3 \mathrm{E}-11 \\
1.1 \mathrm{E}-11 \\
5.7 \mathrm{E}-12 \\
3.8 \mathrm{E}-12 \\
4.9 \mathrm{E}-12 \\
4.2 \mathrm{E}-12 \\
6.2 \mathrm{E}-12 \\
4.0 \mathrm{E}-12\end{array}$ & $\begin{array}{l}3.2 \mathrm{E}-12 \\
1.2 \mathrm{E}-12 \\
1.6 \mathrm{E}-12 \\
2.2 \mathrm{E}-12 \\
2.5 \mathrm{E}-12 \\
3.3 \mathrm{E}-12 \\
5.4 \mathrm{E}-12 \\
8.9 \mathrm{E}-12 \\
1.3 \mathrm{E}-11 \\
1.0 \mathrm{E}-11 \\
5.6 \mathrm{E}-12 \\
3.7 \mathrm{E}-12 \\
4.8 \mathrm{E}-12 \\
4.1 \mathrm{E}-12 \\
6.1 \mathrm{E}-12 \\
3.9 \mathrm{E}-12\end{array}$ & $\begin{array}{l}3.1 \mathrm{E}-12 \\
1.2 \mathrm{E}-12 \\
1.6 \mathrm{E}-12 \\
2.2 \mathrm{E}-12 \\
2.5 \mathrm{E}-12 \\
3.3 \mathrm{E}-12 \\
5.2 \mathrm{E}-12 \\
8.7 \mathrm{E}-12 \\
1.2 \mathrm{E}-11 \\
1.0 \mathrm{E}-11 \\
5.5 \mathrm{E}-12 \\
3.6 \mathrm{E}-12 \\
4.7 \mathrm{E}-12 \\
4.0 \mathrm{E}-12 \\
6.0 \mathrm{E}-12 \\
3.8 \mathrm{E}-12\end{array}$ & $\begin{array}{l}3.1 \mathrm{E}-12 \\
1.1 \mathrm{E}-12 \\
1.6 \mathrm{E}-12 \\
2.1 \mathrm{E}-12 \\
2.4 \mathrm{E}-12 \\
3.2 \mathrm{E}-12 \\
5.2 \mathrm{E}-12 \\
8.7 \mathrm{E}-12 \\
1.2 \mathrm{E}-11 \\
1.0 \mathrm{E}-11 \\
5.4 \mathrm{E}-12 \\
3.6 \mathrm{E}-12 \\
4.7 \mathrm{E}-12 \\
4.0 \mathrm{E}-12 \\
5.9 \mathrm{E}-12 \\
3.8 \mathrm{E}-12\end{array}$ & $\begin{array}{l}3.0 \mathrm{E}-12 \\
1.1 \mathrm{E}-12 \\
1.6 \mathrm{E}-12 \\
2.1 \mathrm{E}-12 \\
2.4 \mathrm{E}-12 \\
3.1 \mathrm{E}-12 \\
5.0 \mathrm{E}-12 \\
8.4 \mathrm{E}-12 \\
1.2 \mathrm{E}-11 \\
9.8 \mathrm{E}-12 \\
5 \cdot 3 \mathrm{E}-12 \\
3 \cdot 5 \mathrm{E}-12 \\
4 \cdot 5 \mathrm{E}-12 \\
3.9 \mathrm{E}-12 \\
5.7 \mathrm{E}-12 \\
3.7 \mathrm{E}-12\end{array}$ \\
\hline
\end{tabular}




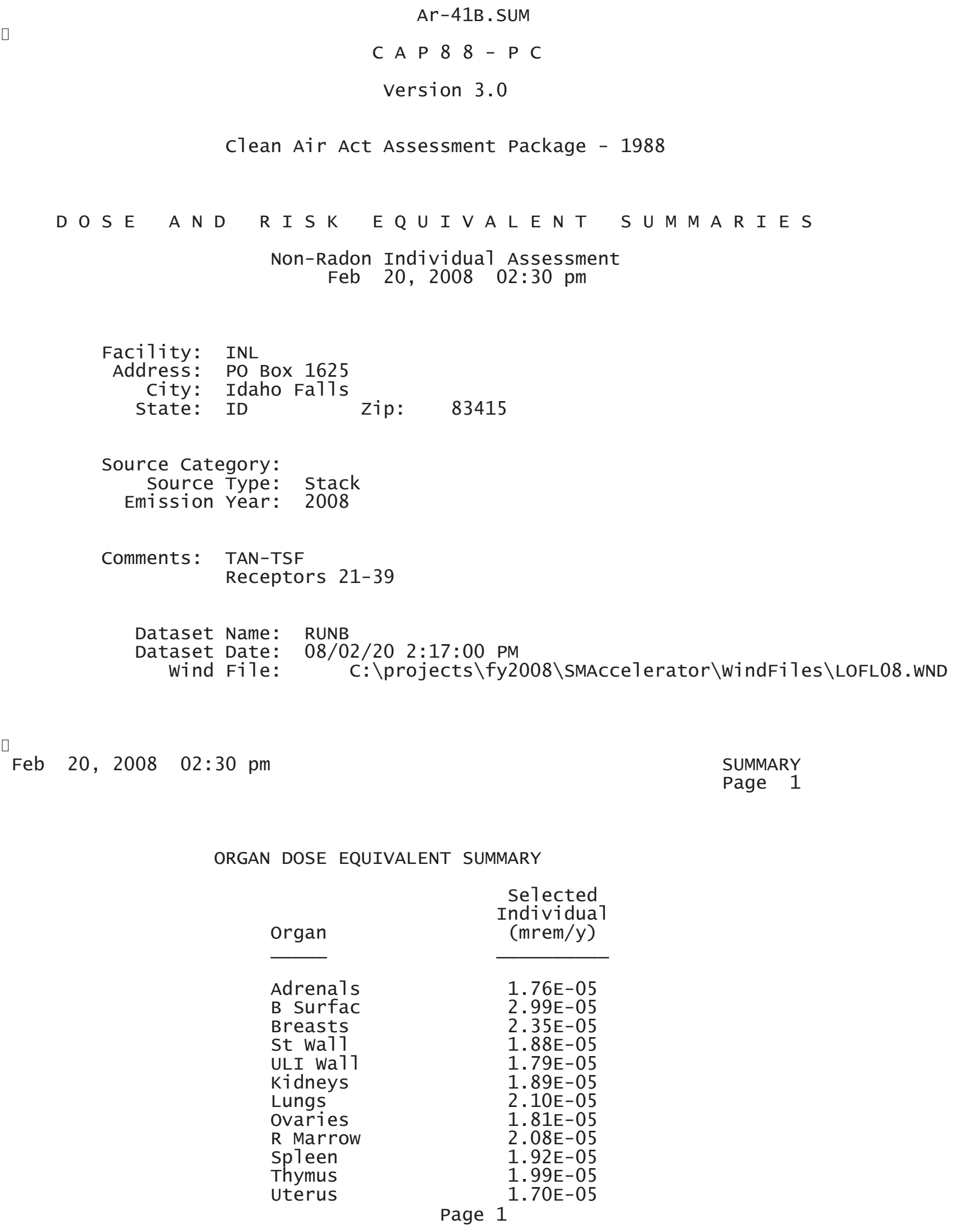

\begin{tabular}{lr} 
ORGAN DOSE EQUIVALENT SUMMARY \\
Organ & $\begin{array}{r}\text { Selected } \\
\text { Individual } \\
\text { (mrem/y) }\end{array}$ \\
\cline { 2 - 2 } Adrenals & $1.76 \mathrm{E}-05$ \\
B Surfac & $2.99 \mathrm{E}-05$ \\
Breasts & $2.35 \mathrm{E}-05$ \\
St wa11 & $1.88 \mathrm{E}-05$ \\
ULI wa11 & $1.79 \mathrm{E}-05$ \\
Kidneys & $1.89 \mathrm{E}-05$ \\
Lungs & $2.10 \mathrm{E}-05$ \\
Ovaries & $1.81 \mathrm{E}-05$ \\
R Marrow & $2.08 \mathrm{E}-05$ \\
Spleen & $1.92 \mathrm{E}-05$ \\
Thymus & $1.99 \mathrm{E}-05$ \\
Uterus & $1.70 \mathrm{E}-05$ \\
& 1
\end{tabular}




$\begin{array}{lr} & \text { Ar-41B. SUM } \\ \text { B1d wa11 } & 1.76 \mathrm{E}-05 \\ \text { Brain } & 2.26 \mathrm{E}-05 \\ \text { Esophagu } & 1.82 \mathrm{E}-05 \\ \text { SI wa11 } & 1.74 \mathrm{E}-05 \\ \text { LLI wa11 } & 1.77 \mathrm{E}-05 \\ \text { Liver } & 1.91 \mathrm{E}-05 \\ \text { Muscle } & 2.05 \mathrm{E}-05 \\ \text { Pancreas } & 1.72 \mathrm{E}-05 \\ \text { Skin } & 3.32 \mathrm{E}-05 \\ \text { Testes } & 2.08 \mathrm{E}-05 \\ \text { Thyroid } & 2.14 \mathrm{E}-05 \\ & \\ \text { EFFEC } & 2.02 \mathrm{E}-05\end{array}$

PATHWAY EFFECTIVE DOSE EQUIVALENT SUMMARY

\begin{tabular}{lc} 
Pathway & $\begin{array}{c}\text { selected } \\
\text { Individual } \\
\text { (mrem/y) }\end{array}$ \\
\cline { 2 - 2 } INGESTION & $0.00 \mathrm{E}+00$ \\
INHALATION & $0.00 \mathrm{E}+00$ \\
AIR IMMERSION & $2.02 \mathrm{E}-05$ \\
GROUND SURFACE & $0.00 \mathrm{E}+00$ \\
INTERNAL & $0.00 \mathrm{E}+00$ \\
EXTERNAL & $2.02 \mathrm{E}-05$ \\
TOTAL & $2.02 \mathrm{E}-05$
\end{tabular}

Feb 20, 2008 02:30 pm

SUMMARY

Page 2

NUCLIDE EFFECTIVE DOSE EQUIVALENT SUMMARY

Nuclide

Ar -41

TOTAL

Feb 20, $2008 \quad 02: 30$ pm

$$
\begin{gathered}
\begin{array}{c}
\text { Selected } \\
\text { Individua } \\
\text { (mrem/y) }
\end{array} \\
\hline 2.02 \mathrm{E}-05 \\
2.02 \mathrm{E}-05
\end{gathered}
$$$$
2.02 \mathrm{E}-05
$$$$
2.02 \mathrm{E}-05
$$
2.02E-05
$2.02 \mathrm{E}-05$

SUMMARY

Page 3

\section{CANCER RISK SUMMARY}

Cancer

Esophagu
Selected Individual

Total Lifetime Fatal Cancer Risk

$$
\text { Page } 2^{2.09 \mathrm{E}-13}
$$




$\begin{array}{lr} & \text { Ar-41B. SUM } \\ \text { Stomach } & 7.60 \mathrm{E}-13 \\ \text { Colon } & 1.84 \mathrm{E}-12 \\ \text { Liver } & 2.90 \mathrm{E}-13 \\ \text { LUNG } & 2.05 \mathrm{E}-12 \\ \text { Bone } & 2.84 \mathrm{E}-14 \\ \text { Skin } & 3.32 \mathrm{E}-14 \\ \text { Breast } & 1.14 \mathrm{E}-12 \\ \text { Ovary } & 2.58 \mathrm{E}-13 \\ \text { B Tadder } & 4.24 \mathrm{E}-13 \\ \text { Kidneys } & 9.87 \mathrm{E}-14 \\ \text { Thyroid } & 6.84 \mathrm{E}-14 \\ \text { Leukemia } & 1.17 \mathrm{E}-12 \\ \text { Residua1 } & 2.74 \mathrm{E}-12 \\ \text { Tota1 } & 1.11 \mathrm{E}-11 \\ & \\ \text { TOTAL } & 2.22 \mathrm{E}-11\end{array}$

PATHWAY RISK SUMMARY

\begin{tabular}{lc} 
Pathway & $\begin{array}{c}\text { Selected Individua1 } \\
\text { Total Lifetime } \\
\text { Fatal Cancer Risk }\end{array}$ \\
\cline { 2 - 2 } INGESTION & $0.00 \mathrm{E}+00$ \\
INHALATION & $0.00 \mathrm{E}+00$ \\
AIR IMMERSION & $1.11 \mathrm{E}-11$ \\
GROUND SURFACE & $0.00 \mathrm{E}+00$ \\
INTERNAL & $0.00 \mathrm{E}+00$ \\
EXTERNAL & $1.11 \mathrm{E}-11$ \\
TOTAL & $1.11 \mathrm{E}-11$
\end{tabular}

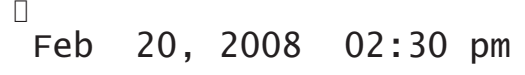

NUCLIDE RISK SUMMARY

Nuclide

Ar -41

TOTAL

口

Feb 20, 2008 02:30 pm
Selected Individual

Total Lifetime Fatal Cancer Risk

1. $11 \mathrm{E}-11$

1. $11 \mathrm{E}-11$

SUMMARY

Page 5

INDIVIDUAL EFFECTIVE DOSE EQUIVALENT RATE (mrem/y) (A11 Radionuclides and Pathways)

Distance $(m)$ 


\begin{tabular}{|c|c|c|c|c|c|c|c|}
\hline Direction & 17035 & 17329 & 18607 & $\begin{array}{c}\mathrm{Ar}-41 \mathrm{~B} \\
18834\end{array}$ & $\begin{array}{l}{ }_{18 M} \\
18860\end{array}$ & 18890 & 19860 \\
\hline$N$ & $5.1 E-06$ & $5.0 E-06$ & $4.7 E-06$ & $4.6 \mathrm{E}-06$ & $4.6 \mathrm{E}-06$ & $4.6 \mathrm{E}-06$ & $4.3 E-06$ \\
\hline NNW & $1.9 \mathrm{E}-06$ & $1.9 \mathrm{E}-06$ & $1.7 \mathrm{E}-06$ & $1.7 \mathrm{E}-06$ & $1.7 \mathrm{E}-06$ & $1.7 \mathrm{E}-06$ & 1. $6 \mathrm{E}-06$ \\
\hline NW & $2.7 \mathrm{E}-06$ & $2.6 \mathrm{E}-06$ & $2.4 \mathrm{E}-06$ & $2.4 \mathrm{E}-06$ & $2.4 \mathrm{E}-06$ & $2.4 \mathrm{E}-06$ & 2. $3 \mathrm{E}-06$ \\
\hline WNW & $3.6 \mathrm{E}-06$ & $3.5 \mathrm{E}-06$ & $3.3 E-06$ & $3.2 \mathrm{E}-06$ & $3.2 \mathrm{E}-06$ & $3.2 \mathrm{E}-06$ & $3.0 \mathrm{E}-06$ \\
\hline W & $4.1 \mathrm{E}-06$ & 4.0E-06 & $3.7 \mathrm{E}-06$ & $3.7 \mathrm{E}-06$ & $3.7 \mathrm{E}-06$ & $3.7 \mathrm{E}-06$ & $3.5 \mathrm{E}-06$ \\
\hline WSW & $5.4 \mathrm{E}-06$ & $5.3 \mathrm{E}-06$ & $4.9 \mathrm{E}-06$ & $4.8 \mathrm{E}-06$ & $4.8 \mathrm{E}-06$ & $4.8 \mathrm{E}-06$ & 4. $6 \mathrm{E}-06$ \\
\hline SW & $8.7 \mathrm{E}-06$ & $8.5 \mathrm{E}-06$ & $7.9 \mathrm{E}-06$ & $7.7 \mathrm{E}-06$ & $7.7 \mathrm{E}-06$ & $7.7 \mathrm{E}-06$ & 7. $3 \mathrm{E}-06$ \\
\hline SSW & 1. $4 \mathrm{E}-05$ & 1. $4 \mathrm{E}-05$ & 1. $3 \mathrm{E}-05$ & 1. $3 \mathrm{E}-05$ & 1. $3 \mathrm{E}-05$ & 1.3E-05 & 1. $2 \mathrm{E}-05$ \\
\hline $\mathrm{S}$ & 2.0E-05 & $2.0 \mathrm{E}-05$ & $1.8 \mathrm{E}-05$ & $1.8 \mathrm{E}-05$ & $1.8 \mathrm{E}-05$ & $1.8 \mathrm{E}-05$ & $1.7 \mathrm{E}-05$ \\
\hline SSE & 1.7E-05 & $1.7 \mathrm{E}-05$ & 1. $5 \mathrm{E}-05$ & $1.5 \mathrm{E}-05$ & 1. $5 \mathrm{E}-05$ & 1.5E-05 & 1. $4 \mathrm{E}-05$ \\
\hline SE & $9.1 \mathrm{E}-06$ & $8.9 \mathrm{E}-06$ & $8.2 \mathrm{E}-06$ & $8.1 \mathrm{E}-06$ & $8.1 \mathrm{E}-06$ & $8.1 \mathrm{E}-06$ & $7.7 \mathrm{E}-06$ \\
\hline ESE & $6.1 \mathrm{E}-06$ & $6.0 \mathrm{E}-06$ & $5.5 \mathrm{E}-06$ & $5.4 \mathrm{E}-06$ & $5.4 \mathrm{E}-06$ & $5.4 \mathrm{E}-06$ & $5.1 \mathrm{E}-06$ \\
\hline $\mathrm{E}$ & $7.8 \mathrm{E}-06$ & $7.7 \mathrm{E}-06$ & $7.1 \mathrm{E}-06$ & $7.0 \mathrm{E}-06$ & $7.0 \mathrm{E}-06$ & $7.0 \mathrm{E}-06$ & $6.6 \mathrm{E}-06$ \\
\hline ENE & $6.7 \mathrm{E}-06$ & $6.6 \mathrm{E}-06$ & $6.0 \mathrm{E}-06$ & $6.0 \mathrm{E}-06$ & $6.0 \mathrm{E}-06$ & $5.9 \mathrm{E}-06$ & $5.6 \mathrm{E}-06$ \\
\hline $\mathrm{NE}$ & $9.9 \mathrm{E}-06$ & $9.7 \mathrm{E}-06$ & 8.9E-06 & $8.8 \mathrm{E}-06$ & $8.8 \mathrm{E}-06$ & $8.8 \mathrm{E}-06$ & $8.2 \mathrm{E}-06$ \\
\hline NNE & $6.4 \mathrm{E}-06$ & $6.3 \mathrm{E}-06$ & $5.8 \mathrm{E}-06$ & $5.7 \mathrm{E}-06$ & $5.7 \mathrm{E}-06$ & $5.7 \mathrm{E}-06$ & $5.4 \mathrm{E}-06$ \\
\hline
\end{tabular}

Distance (m)

\begin{tabular}{|c|c|c|c|c|c|c|c|}
\hline Direction & 19891 & 20457 & 21314 & 21349 & 22159 & 23521 & 24430 \\
\hline$N$ & $4.3 E-06$ & $4.2 E-06$ & $4.0 \mathrm{E}-06$ & $4.0 E-06$ & $3.8 \mathrm{E}-06$ & $3.6 E-06$ & $3.4 E-06$ \\
\hline NNW & $1.6 \mathrm{E}-06$ & $1.6 \mathrm{E}-06$ & $1.5 \mathrm{E}-06$ & $1.5 \mathrm{E}-06$ & $1.4 \mathrm{E}-06$ & $1.3 \mathrm{E}-06$ & 1. $3 \mathrm{E}-06$ \\
\hline NW & $2.3 \mathrm{E}-06$ & $2.2 \mathrm{E}-06$ & $2.1 \mathrm{E}-06$ & $2.1 \mathrm{E}-06$ & $2.0 \mathrm{E}-06$ & $1.9 \mathrm{E}-06$ & $1.8 \mathrm{E}-06$ \\
\hline WNW & $3.0 \mathrm{E}-06$ & $2.9 \mathrm{E}-06$ & $2.8 \mathrm{E}-06$ & $2.8 \mathrm{E}-06$ & $2.7 \mathrm{E}-06$ & $2.5 \mathrm{E}-06$ & $2.4 \mathrm{E}-06$ \\
\hline W & $3.5 \mathrm{E}-06$ & 3. $3 \mathrm{E}-06$ & $3.2 \mathrm{E}-06$ & $3.2 \mathrm{E}-06$ & $3.1 \mathrm{E}-06$ & $2.9 \mathrm{E}-06$ & $2.7 \mathrm{E}-06$ \\
\hline WSW & $4.6 \mathrm{E}-06$ & $4.4 \mathrm{E}-06$ & $4.2 \mathrm{E}-06$ & $4.2 \mathrm{E}-06$ & $4.0 \mathrm{E}-06$ & $3.8 \mathrm{E}-06$ & $3.6 \mathrm{E}-06$ \\
\hline SW & $7.3 \mathrm{E}-06$ & 7.0E-06 & $6.7 \mathrm{E}-06$ & $6.7 \mathrm{E}-06$ & $6.4 \mathrm{E}-06$ & $6.0 \mathrm{E}-06$ & $5.7 \mathrm{E}-06$ \\
\hline SSW & $1.2 \mathrm{E}-05$ & 1. $2 \mathrm{E}-05$ & $1.1 \mathrm{E}-05$ & $1.1 \mathrm{E}-05$ & $1.1 \mathrm{E}-05$ & $9.9 \mathrm{E}-06$ & $9.5 \mathrm{E}-06$ \\
\hline & $1.7 \mathrm{E}-05$ & $1.6 \mathrm{E}-05$ & $1.5 \mathrm{E}-05$ & $1.5 \mathrm{E}-05$ & $1.5 \mathrm{E}-05$ & $1.4 \mathrm{E}-05$ & 1. $3 \mathrm{E}-05$ \\
\hline SSE & $1.4 \mathrm{E}-05$ & $1.4 \mathrm{E}-05$ & 1. $3 \mathrm{E}-05$ & $1.3 \mathrm{E}-05$ & $1.2 \mathrm{E}-05$ & $1.2 \mathrm{E}-05$ & $1.1 \mathrm{E}-05$ \\
\hline $\mathrm{SE}$ & $7.6 \mathrm{E}-06$ & $7.4 \mathrm{E}-06$ & $7.1 \mathrm{E}-06$ & $7.1 \mathrm{E}-06$ & $6.8 \mathrm{E}-06$ & $6.3 \mathrm{E}-06$ & $6.0 \mathrm{E}-06$ \\
\hline ESE & $5.1 \mathrm{E}-06$ & $5.0 \mathrm{E}-06$ & $4.7 \mathrm{E}-06$ & $4.7 \mathrm{E}-06$ & $4.5 \mathrm{E}-06$ & $4.2 \mathrm{E}-06$ & $4.1 \mathrm{E}-06$ \\
\hline $\mathrm{E}$ & $6.6 \mathrm{E}-06$ & $6.4 \mathrm{E}-06$ & $6.1 \mathrm{E}-06$ & $6.1 \mathrm{E}-06$ & $5.8 \mathrm{E}-06$ & $5.5 \mathrm{E}-06$ & $5.2 \mathrm{E}-06$ \\
\hline ENE & $5.6 \mathrm{E}-06$ & $5.4 \mathrm{E}-06$ & $5.2 \mathrm{E}-06$ & $5.2 \mathrm{E}-06$ & $5.0 \mathrm{E}-06$ & $4.6 \mathrm{E}-06$ & $4.4 \mathrm{E}-06$ \\
\hline $\mathrm{NE}$ & $8.2 \mathrm{E}-06$ & $8.0 \mathrm{E}-06$ & $7.6 \mathrm{E}-06$ & $7.6 \mathrm{E}-06$ & $7.2 \mathrm{E}-06$ & $6.8 \mathrm{E}-06$ & $6.5 \mathrm{E}-06$ \\
\hline NNE & $5.4 \mathrm{E}-06$ & $5.2 \mathrm{E}-06$ & $4.9 \mathrm{E}-06$ & $4.9 \mathrm{E}-06$ & $4.7 \mathrm{E}-06$ & $4.4 \mathrm{E}-06$ & $4.2 \mathrm{E}-06$ \\
\hline
\end{tabular}

口

Feb 20, 2008 02:30 pm

SUMMARY

Page 6

INDIVIDUAL EFFECTIVE DOSE EQUIVALENT RATE (mrem/y) (A11 Radionuclides and Pathways)

\begin{tabular}{|c|c|c|c|c|c|}
\hline \multirow[b]{2}{*}{ Direction } & \multicolumn{5}{|c|}{ Distance (m) } \\
\hline & 24545 & 25171 & 26794 & 27275 & 27389 \\
\hline $\begin{array}{r}N \\
\text { NNW }\end{array}$ & $\begin{array}{l}3.4 \mathrm{E}-06 \\
1.3 \mathrm{E}-06\end{array}$ & $\begin{array}{l}3.3 \mathrm{E}-06 \\
1.2 \mathrm{E}-06\end{array}$ & $\begin{array}{l}3.1 \mathrm{E}-06 \\
1.2 \mathrm{E}-06\end{array}$ & $\begin{array}{r}3.0 \mathrm{E}-06 \\
1.1 \mathrm{E}-06 \\
\text { Page }\end{array}$ & $\begin{array}{l}3.0 \mathrm{E}-06 \\
1.1 \mathrm{E}-06\end{array}$ \\
\hline
\end{tabular}




\begin{tabular}{rlllll} 
& & & \multicolumn{3}{c}{ Ar-41B.SUM } \\
NW & $1.8 \mathrm{E}-06$ & $1.7 \mathrm{E}-06$ & $1.6 \mathrm{E}-06$ & $1.6 \mathrm{E}-06$ & $1.6 \mathrm{E}-06$ \\
WNW & $2.4 \mathrm{E}-06$ & $2.3 \mathrm{E}-06$ & $2.2 \mathrm{E}-06$ & $2.1 \mathrm{E}-06$ & $2.1 \mathrm{E}-06$ \\
$\mathrm{~W}$ & $2.7 \mathrm{E}-06$ & $2.7 \mathrm{E}-06$ & $2.5 \mathrm{E}-06$ & $2.4 \mathrm{E}-06$ & $2.4 \mathrm{E}-06$ \\
WSW & $3.6 \mathrm{E}-06$ & $3.5 \mathrm{E}-06$ & $3.3 \mathrm{E}-06$ & $3.2 \mathrm{E}-06$ & $3.2 \mathrm{E}-06$ \\
$\mathrm{SW}$ & $5.7 \mathrm{E}-06$ & $5.5 \mathrm{E}-06$ & $5.1 \mathrm{E}-06$ & $5.0 \mathrm{E}-06$ & $5.0 \mathrm{E}-06$ \\
$\mathrm{SSW}$ & $9.4 \mathrm{E}-06$ & $9.2 \mathrm{E}-06$ & $8.5 \mathrm{E}-06$ & $8.3 \mathrm{E}-06$ & $8.3 \mathrm{E}-06$ \\
$\mathrm{~S}$ & $1.3 \mathrm{E}-05$ & $1.3 \mathrm{E}-05$ & $1.2 \mathrm{E}-05$ & $1.2 \mathrm{E}-05$ & $1.1 \mathrm{E}-05$ \\
$\mathrm{SSE}$ & $1.1 \mathrm{E}-05$ & $1.1 \mathrm{E}-05$ & $9.9 \mathrm{E}-06$ & $9.7 \mathrm{E}-06$ & $9.7 \mathrm{E}-06$ \\
$\mathrm{SE}$ & $6.0 \mathrm{E}-06$ & $5.8 \mathrm{E}-06$ & $5.4 \mathrm{E}-06$ & $5.3 \mathrm{E}-06$ & $5.3 \mathrm{E}-06$ \\
$\mathrm{ESE}$ & $4.0 \mathrm{E}-06$ & $3.9 \mathrm{E}-06$ & $3.7 \mathrm{E}-06$ & $3.6 \mathrm{E}-06$ & $3.6 \mathrm{E}-06$ \\
$\mathrm{E}$ & $5.2 \mathrm{E}-06$ & $5.1 \mathrm{E}-06$ & $4.7 \mathrm{E}-06$ & $4.6 \mathrm{E}-06$ & $4.6 \mathrm{E}-06$ \\
$\mathrm{ENE}$ & $4.4 \mathrm{E}-06$ & $4.3 \mathrm{E}-06$ & $4.0 \mathrm{E}-06$ & $3.9 \mathrm{E}-06$ & $3.9 \mathrm{E}-06$ \\
$\mathrm{NE}$ & $6.4 \mathrm{E}-06$ & $6.2 \mathrm{E}-06$ & $5.8 \mathrm{E}-06$ & $5.7 \mathrm{E}-06$ & $5.6 \mathrm{E}-06$ \\
NNE & $4.2 \mathrm{E}-06$ & $4.1 \mathrm{E}-06$ & $3.8 \mathrm{E}-06$ & $3.7 \mathrm{E}-06$ & $3.7 \mathrm{E}-06$ \\
& & & & &
\end{tabular}

Feb 20, 2008 02:30 pm
SUMMARY

Page 7

INDIVIDUAL LIFETIME RISK (deaths)

(A11 Radionuclides and Pathways)

\begin{tabular}{|c|c|c|c|c|c|c|c|}
\hline \multicolumn{8}{|c|}{ Distance $(\mathrm{m})$} \\
\hline Direction & 17035 & 17329 & 18607 & 18834 & 18860 & 18890 & 19860 \\
\hline $\begin{array}{r}\text { N } \\
N N W \\
N W \\
W N W \\
W \\
W S W \\
\text { SW } \\
\text { SSW } \\
S \\
\text { SSE } \\
\text { SE } \\
\text { ESE } \\
\text { E } \\
\text { ENE } \\
N E \\
\text { NNE }\end{array}$ & $\begin{array}{l}2.8 \mathrm{E}-12 \\
1.1 \mathrm{E}-12 \\
1.5 \mathrm{E}-12 \\
2.0 \mathrm{E}-12 \\
2.3 \mathrm{E}-12 \\
3.0 \mathrm{E}-12 \\
4.8 \mathrm{E}-12 \\
8.0 \mathrm{E}-12 \\
1.1 \mathrm{E}-11 \\
9.3 \mathrm{E}-12 \\
5.0 \mathrm{E}-12 \\
3.3 \mathrm{E}-12 \\
4.3 \mathrm{E}-12 \\
3.7 \mathrm{E}-12 \\
5.4 \mathrm{E}-12 \\
3.5 \mathrm{E}-12\end{array}$ & $\begin{array}{l}2.8 \mathrm{E}-12 \\
1.0 \mathrm{E}-12 \\
1.5 \mathrm{E}-12 \\
1.9 \mathrm{E}-12 \\
2.2 \mathrm{E}-12 \\
2.9 \mathrm{E}-12 \\
4.7 \mathrm{E}-12 \\
7.8 \mathrm{E}-12 \\
1.1 \mathrm{E}-11 \\
9.1 \mathrm{E}-12 \\
4.9 \mathrm{E}-12 \\
3.3 \mathrm{E}-12 \\
4.2 \mathrm{E}-12 \\
3.6 \mathrm{E}-12 \\
5.3 \mathrm{E}-12 \\
3.4 \mathrm{E}-12\end{array}$ & $\begin{array}{l}2.6 \mathrm{E}-12 \\
9.6 \mathrm{E}-13 \\
1.3 \mathrm{E}-12 \\
1.8 \mathrm{E}-12 \\
2.0 \mathrm{E}-12 \\
2.7 \mathrm{E}-12 \\
4.3 \mathrm{E}-12 \\
7.2 \mathrm{E}-12 \\
1.0 \mathrm{E}-11 \\
8.4 \mathrm{E}-12 \\
4.5 \mathrm{E}-12 \\
3.0 \mathrm{E}-12 \\
3.9 \mathrm{E}-12 \\
3.3 \mathrm{E}-12 \\
4.9 \mathrm{E}-12 \\
3.2 \mathrm{E}-12\end{array}$ & $\begin{array}{l}2.5 \mathrm{E}-12 \\
9.5 \mathrm{E}-13 \\
1.3 \mathrm{E}-12 \\
1.8 \mathrm{E}-12 \\
2.0 \mathrm{E}-12 \\
2.7 \mathrm{E}-12 \\
4.3 \mathrm{E}-12 \\
7.1 \mathrm{E}-12 \\
9.9 \mathrm{E}-12 \\
8.3 \mathrm{E}-12 \\
4.5 \mathrm{E}-12 \\
3.0 \mathrm{E}-12 \\
3.8 \mathrm{E}-12 \\
3.3 \mathrm{E}-12 \\
4.8 \mathrm{E}-12 \\
3.1 \mathrm{E}-12\end{array}$ & $\begin{array}{l}2.5 \mathrm{E}-12 \\
9.5 \mathrm{E}-13 \\
1.3 \mathrm{E}-12 \\
1.8 \mathrm{E}-12 \\
2.0 \mathrm{E}-12 \\
2.7 \mathrm{E}-12 \\
4.2 \mathrm{E}-12 \\
7.1 \mathrm{E}-12 \\
9.8 \mathrm{E}-12 \\
8.3 \mathrm{E}-12 \\
4.5 \mathrm{E}-12 \\
3.0 \mathrm{E}-12 \\
3.8 \mathrm{E}-12 \\
3.3 \mathrm{E}-12 \\
4.8 \mathrm{E}-12 \\
3.1 \mathrm{E}-12\end{array}$ & $\begin{array}{l}2.5 \mathrm{E}-12 \\
9.4 \mathrm{E}-13 \\
1.3 \mathrm{E}-12 \\
1.8 \mathrm{E}-12 \\
2.0 \mathrm{E}-12 \\
2.7 \mathrm{E}-12 \\
4.2 \mathrm{E}-12 \\
7.0 \mathrm{E}-12 \\
9.8 \mathrm{E}-12 \\
8.2 \mathrm{E}-12 \\
4.5 \mathrm{E}-12 \\
3.0 \mathrm{E}-12 \\
3.8 \mathrm{E}-12 \\
3.3 \mathrm{E}-12 \\
4.8 \mathrm{E}-12 \\
3.1 \mathrm{E}-12\end{array}$ & $\begin{array}{l}2.4 \mathrm{E}-12 \\
8.9 \mathrm{E}-13 \\
1.2 \mathrm{E}-12 \\
1.7 \mathrm{E}-12 \\
1.9 \mathrm{E}-12 \\
2.5 \mathrm{E}-12 \\
4.0 \mathrm{E}-12 \\
6.6 \mathrm{E}-12 \\
9.2 \mathrm{E}-12 \\
7.8 \mathrm{E}-12 \\
4.2 \mathrm{E}-12 \\
2.8 \mathrm{E}-12 \\
3.6 \mathrm{E}-12 \\
3.1 \mathrm{E}-12 \\
4.5 \mathrm{E}-12 \\
2.9 \mathrm{E}-12\end{array}$ \\
\hline \multicolumn{8}{|c|}{ Distance (m) } \\
\hline Direction & 19891 & 20457 & 21314 & 21349 & 22159 & 23521 & 24430 \\
\hline $\begin{array}{r}\text { N } \\
\text { NNW } \\
\text { NW } \\
\text { WNW } \\
W \\
\text { WSW } \\
\text { SW } \\
\text { SSW }\end{array}$ & $\begin{array}{l}2.4 \mathrm{E}-12 \\
8.9 \mathrm{E}-13 \\
1.2 \mathrm{E}-12 \\
1.7 \mathrm{E}-12 \\
1.9 \mathrm{E}-12 \\
2.5 \mathrm{E}-12 \\
4.0 \mathrm{E}-12 \\
6.6 \mathrm{E}-12\end{array}$ & $\begin{array}{l}2.3 \mathrm{E}-12 \\
8.6 \mathrm{E}-13 \\
1.2 \mathrm{E}-12 \\
1.6 \mathrm{E}-12 \\
1.8 \mathrm{E}-12 \\
2.4 \mathrm{E}-12 \\
3.9 \mathrm{E}-12 \\
6.4 \mathrm{E}-12\end{array}$ & $\begin{array}{l}2.2 \mathrm{E}-12 \\
8.2 \mathrm{E}-13 \\
1.2 \mathrm{E}-12 \\
1.5 \mathrm{E}-12 \\
1.8 \mathrm{E}-12 \\
2.3 \mathrm{E}-12 \\
3.7 \mathrm{E}-12 \\
6.1 \mathrm{E}-12\end{array}$ & $\begin{array}{r}2.2 \mathrm{E}-12 \\
8.2 \mathrm{E}-13 \\
1.2 \mathrm{E}-12 \\
1.5 \mathrm{E}-12 \\
1.8 \mathrm{E}-12 \\
2.3 \mathrm{E}-12 \\
3.7 \mathrm{E}-12 \\
6.1 \mathrm{E}-12 \\
\text { Page }\end{array}$ & $\begin{array}{l}2.1 \mathrm{E}-12 \\
7.9 \mathrm{E}-13 \\
1.1 \mathrm{E}-12 \\
1.5 \mathrm{E}-12 \\
1.7 \mathrm{E}-12 \\
2.2 \mathrm{E}-12 \\
3.5 \mathrm{E}-12 \\
5.8 \mathrm{E}-12\end{array}$ & $\begin{array}{l}2.0 \mathrm{E}-12 \\
7.4 \mathrm{E}-13 \\
1.0 \mathrm{E}-12 \\
1.4 \mathrm{E}-12 \\
1.6 \mathrm{E}-12 \\
2.1 \mathrm{E}-12 \\
3.3 \mathrm{E}-12 \\
5.4 \mathrm{E}-12\end{array}$ & $\begin{array}{l}1.9 \mathrm{E}-12 \\
7.1 \mathrm{E}-13 \\
9.9 \mathrm{E}-13 \\
1.3 \mathrm{E}-12 \\
1.5 \mathrm{E}-12 \\
2.0 \mathrm{E}-12 \\
3.1 \mathrm{E}-12 \\
5.2 \mathrm{E}-12\end{array}$ \\
\hline
\end{tabular}




\begin{tabular}{rlllllll} 
& \multicolumn{7}{c}{ Ar-41B. SUM } \\
S & $9.2 \mathrm{E}-12$ & $8.9 \mathrm{E}-12$ & $8.5 \mathrm{E}-12$ & $8.5 \mathrm{E}-12$ & $8.1 \mathrm{E}-12$ & $7.6 \mathrm{E}-12$ & $7.2 \mathrm{E}-12$ \\
$\mathrm{SSE}$ & $7.8 \mathrm{E}-12$ & $7.5 \mathrm{E}-12$ & $7.1 \mathrm{E}-12$ & $7.1 \mathrm{E}-12$ & $6.8 \mathrm{E}-12$ & $6.4 \mathrm{E}-12$ & $6.1 \mathrm{E}-12$ \\
$\mathrm{SE}$ & $4.2 \mathrm{E}-12$ & $4.1 \mathrm{E}-12$ & $3.9 \mathrm{E}-12$ & $3.9 \mathrm{E}-12$ & $3.7 \mathrm{E}-12$ & $3.5 \mathrm{E}-12$ & $3.3 \mathrm{E}-12$ \\
$\mathrm{ESE}$ & $2.8 \mathrm{E}-12$ & $2.7 \mathrm{E}-12$ & $2.6 \mathrm{E}-12$ & $2.6 \mathrm{E}-12$ & $2.5 \mathrm{E}-12$ & $2.3 \mathrm{E}-12$ & $2.2 \mathrm{E}-12$ \\
$\mathrm{E}$ & $3.6 \mathrm{E}-12$ & $3.5 \mathrm{E}-12$ & $3.3 \mathrm{E}-12$ & $3.3 \mathrm{E}-12$ & $3.2 \mathrm{E}-12$ & $3.0 \mathrm{E}-12$ & $2.9 \mathrm{E}-12$ \\
$\mathrm{ENE}$ & $3.1 \mathrm{E}-12$ & $3.0 \mathrm{E}-12$ & $2.8 \mathrm{E}-12$ & $2.8 \mathrm{E}-12$ & $2.7 \mathrm{E}-12$ & $2.5 \mathrm{E}-12$ & $2.4 \mathrm{E}-12$ \\
$\mathrm{NE}$ & $4.5 \mathrm{E}-12$ & $4.4 \mathrm{E}-12$ & $4.2 \mathrm{E}-12$ & $4.2 \mathrm{E}-12$ & $4.0 \mathrm{E}-12$ & $3.7 \mathrm{E}-12$ & $3.5 \mathrm{E}-12$ \\
$\mathrm{NNE}$ & $2.9 \mathrm{E}-12$ & $2.8 \mathrm{E}-12$ & $2.7 \mathrm{E}-12$ & $2.7 \mathrm{E}-12$ & $2.6 \mathrm{E}-12$ & $2.4 \mathrm{E}-12$ & $2.3 \mathrm{E}-12$
\end{tabular}

Feb 20, $2008 \quad 02: 30 \mathrm{pm}$

SUMMARY

Page 8

INDIVIDUAL LIFETIME RISK (deaths)

(A11 Radionuclides and Pathways)

\begin{tabular}{|c|c|c|c|c|c|}
\hline \multirow[b]{2}{*}{ Direction } & \multicolumn{5}{|c|}{ Distance (m) } \\
\hline & 24545 & 25171 & 26794 & 27275 & 27389 \\
\hline $\begin{array}{r}\text { N } \\
N N W \\
N W \\
\text { WNW } \\
W \\
W S W \\
\text { SW } \\
\text { SSW } \\
S \\
\text { SSE } \\
\text { SE } \\
\text { ESE } \\
\text { E } \\
\text { ENE } \\
N E \\
\text { NNE }\end{array}$ & $\begin{array}{l}1.9 \mathrm{E}-12 \\
7.0 \mathrm{E}-13 \\
9.9 \mathrm{E}-13 \\
1.3 \mathrm{E}-12 \\
1.5 \mathrm{E}-12 \\
2.0 \mathrm{E}-12 \\
3.1 \mathrm{E}-12 \\
5.2 \mathrm{E}-12 \\
7.2 \mathrm{E}-12 \\
6.0 \mathrm{E}-12 \\
3.3 \mathrm{E}-12 \\
2.2 \mathrm{E}-12 \\
2.9 \mathrm{E}-12 \\
2.4 \mathrm{E}-12 \\
3.5 \mathrm{E}-12 \\
2.3 \mathrm{E}-12\end{array}$ & $\begin{array}{l}1.8 \mathrm{E}-12 \\
6.8 \mathrm{E}-13 \\
9.6 \mathrm{E}-13 \\
1.3 \mathrm{E}-12 \\
1.5 \mathrm{E}-12 \\
1.9 \mathrm{E}-12 \\
3.0 \mathrm{E}-12 \\
5.0 \mathrm{E}-12 \\
7.0 \mathrm{E}-12 \\
5.9 \mathrm{E}-12 \\
3.2 \mathrm{E}-12 \\
2.2 \mathrm{E}-12 \\
2.8 \mathrm{E}-12 \\
2.4 \mathrm{E}-12 \\
3.4 \mathrm{E}-12 \\
2.2 \mathrm{E}-12\end{array}$ & $\begin{array}{l}1.7 \mathrm{E}-12 \\
6.4 \mathrm{E}-13 \\
9.0 \mathrm{E}-13 \\
1.2 \mathrm{E}-12 \\
1.4 \mathrm{E}-12 \\
1.8 \mathrm{E}-12 \\
2.8 \mathrm{E}-12 \\
4.7 \mathrm{E}-12 \\
6.5 \mathrm{E}-12 \\
5.5 \mathrm{E}-12 \\
3.0 \mathrm{E}-12 \\
2.0 \mathrm{E}-12 \\
2.6 \mathrm{E}-12 \\
2.2 \mathrm{E}-12 \\
3.2 \mathrm{E}-12 \\
2.1 \mathrm{E}-12\end{array}$ & $\begin{array}{l}1.7 \mathrm{E}-12 \\
6.3 \mathrm{E}-13 \\
8.8 \mathrm{E}-13 \\
1.2 \mathrm{E}-12 \\
1.3 \mathrm{E}-12 \\
1.8 \mathrm{E}-12 \\
2.8 \mathrm{E}-12 \\
4.6 \mathrm{E}-12 \\
6.3 \mathrm{E}-12 \\
5.3 \mathrm{E}-12 \\
2.9 \mathrm{E}-12 \\
2.0 \mathrm{E}-12 \\
2.5 \mathrm{E}-12 \\
2.1 \mathrm{E}-12 \\
3.1 \mathrm{E}-12 \\
2.1 \mathrm{E}-12\end{array}$ & $\begin{array}{l}1.7 \mathrm{E}-12 \\
6.2 \mathrm{E}-13 \\
8.8 \mathrm{E}-13 \\
1.2 \mathrm{E}-12 \\
1.3 \mathrm{E}-12 \\
1.7 \mathrm{E}-12 \\
2.8 \mathrm{E}-12 \\
4.6 \mathrm{E}-12 \\
6.3 \mathrm{E}-12 \\
5.3 \mathrm{E}-12 \\
2.9 \mathrm{E}-12 \\
2.0 \mathrm{E}-12 \\
2.5 \mathrm{E}-12 \\
2.1 \mathrm{E}-12 \\
3.1 \mathrm{E}-12 \\
2.0 \mathrm{E}-12\end{array}$ \\
\hline
\end{tabular}




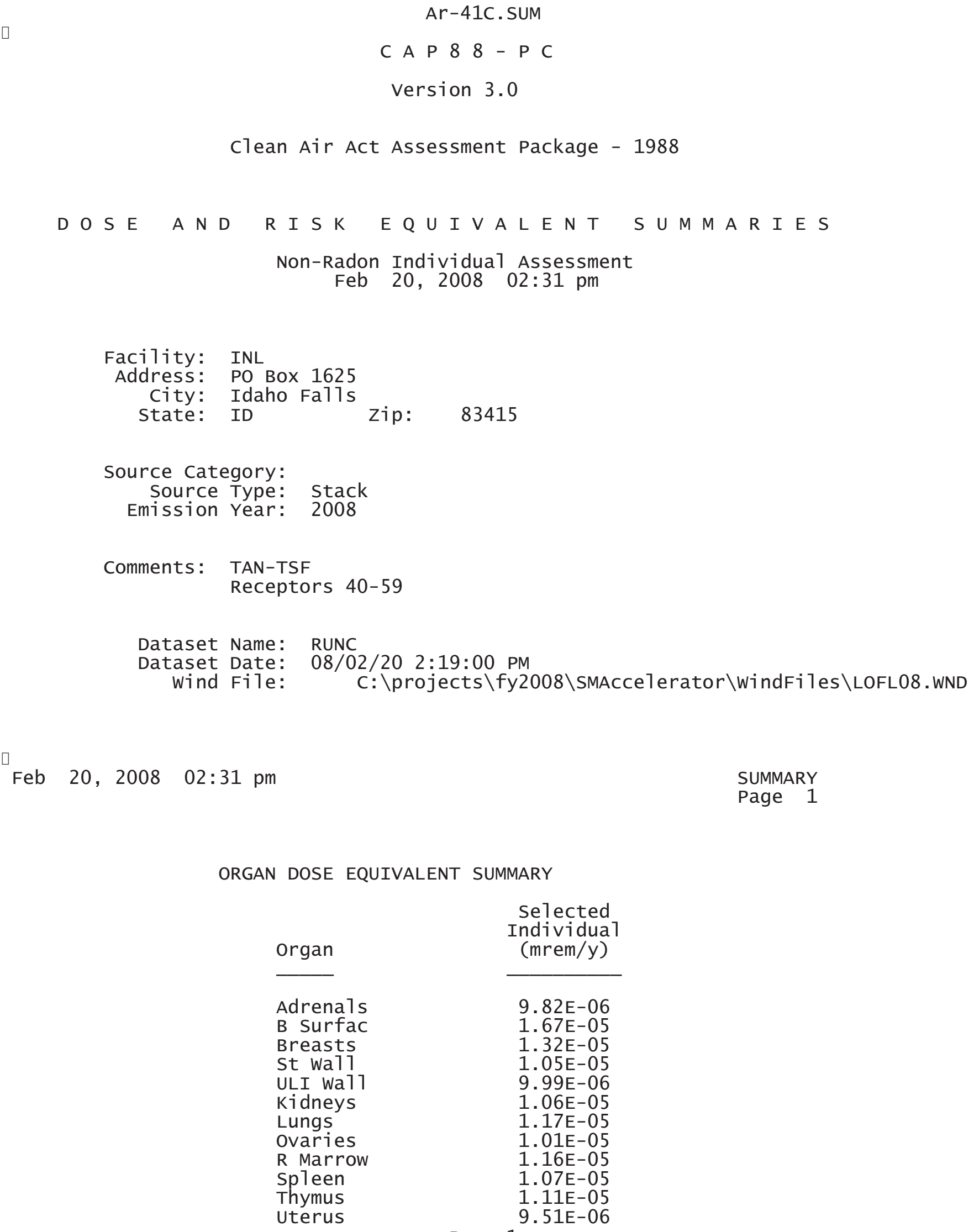

9. $82 \mathrm{E}-06$

1. $67 \mathrm{E}-05$

1. $32 \mathrm{E}-05$

1. $05 \mathrm{E}-05$

9. $99 \mathrm{E}-06$

1.06E-05

1. 17E-05

1. $01 \mathrm{E}-05$

1. 16E-05

$1.07 \mathrm{E}-05$

1. $11 \mathrm{E}-05$

9. 51E-06 


$\begin{array}{lr} & \text { Ar-41C. SUM } \\ \text { B1d wa11 } & 9.84 \mathrm{E}-06 \\ \text { Brain } & 1.26 \mathrm{E}-05 \\ \text { Esophagu } & 1.02 \mathrm{E}-05 \\ \text { SI wa11 } & 9.75 \mathrm{E}-06 \\ \text { LLI wa11 } & 9.89 \mathrm{E}-06 \\ \text { Liver } & 1.07 \mathrm{E}-05 \\ \text { Muscle } & 1.14 \mathrm{E}-05 \\ \text { Pancreas } & 9.60 \mathrm{E}-06 \\ \text { Skin } & 1.86 \mathrm{E}-05 \\ \text { Testes } & 1.16 \mathrm{E}-05 \\ \text { Thyroid } & 1.20 \mathrm{E}-05 \\ & \\ \text { EFFEC } & 1.13 \mathrm{E}-05\end{array}$

PATHWAY EFFECTIVE DOSE EQUIVALENT SUMMARY

$\begin{array}{lc}\text { Pathway } & \begin{array}{c}\text { selected } \\ \text { Individual } \\ \text { (mrem/y) }\end{array} \\ & \\ \text { INGESTION } & 0.00 \mathrm{E}+00 \\ \text { INHALATION } & 0.00 \mathrm{E}+00 \\ \text { AIR IMMERSION } & 1.13 \mathrm{E}-05 \\ \text { GROUND SURFACE } & 0.00 \mathrm{E}+00 \\ \text { INTERNAL } & 0.00 \mathrm{E}+00 \\ \text { EXTERNAL } & 1.13 \mathrm{E}-05 \\ \text { TOTAL } & 1.13 \mathrm{E}-05\end{array}$

Feb 20, 2008 02:31 pm

SUMMARY

Page 2

NUCLIDE EFFECTIVE DOSE EQUIVALENT SUMMARY

Nuclide

$\operatorname{Ar}-41$

TOTAL

Feb 20, 2008 02:31 pm

$$
\begin{gathered}
\begin{array}{c}
\text { Selected } \\
\text { Individual } \\
\text { (mrem/y) }
\end{array} \\
\hline 1.13 \mathrm{E}-05 \\
1.13 \mathrm{E}-05
\end{gathered}
$$

SUMMARY

Page 3

CANCER RISK SUMMARY

Cancer

Esophagu
Selected Individual

Total Lifetime Fatal Cancer Risk

$$
\text { Page } 2^{1 \cdot 17 \mathrm{E}-13}
$$




$\begin{array}{lr} & \text { Ar-41C. SUM } \\ \text { Stomach } & 4.25 \mathrm{E}-13 \\ \text { Colon } & 1.03 \mathrm{E}-12 \\ \text { Liver } & 1.62 \mathrm{E}-13 \\ \text { LUNG } & 1.15 \mathrm{E}-12 \\ \text { Bone } & 1.59 \mathrm{E}-14 \\ \text { Skin } & 1.86 \mathrm{E}-14 \\ \text { Breast } & 6.36 \mathrm{E}-13 \\ \text { Ovary } & 1.44 \mathrm{E}-13 \\ \text { Bladder } & 2.37 \mathrm{E}-13 \\ \text { Kidneys } & 5.52 \mathrm{E}-14 \\ \text { Thyroid } & 3.82 \mathrm{E}-14 \\ \text { Leukemia } & 6.53 \mathrm{E}-13 \\ \text { Residua1 } & 1.53 \mathrm{E}-12 \\ \text { Total } & 6.22 \mathrm{E}-12 \\ & 1.24 \mathrm{E}-11 \\ \text { TOTAL } & \end{array}$

PATHWAY RISK SUMMARY

\begin{tabular}{|c|c|}
\hline Pathway & $\begin{array}{c}\text { Total Lifetime } \\
\text { Fatal Cancer Risk }\end{array}$ \\
\hline $\begin{array}{l}\text { INGESTION } \\
\text { INHALATION } \\
\text { AIR IMMERSION } \\
\text { GROUND SURFACE } \\
\text { INTERNAL } \\
\text { EXTERNAL }\end{array}$ & $\begin{array}{l}0.00 \mathrm{E}+00 \\
0.00 \mathrm{E}+00 \\
6.22 \mathrm{E}-12 \\
0.00 \mathrm{E}+00 \\
0.00 \mathrm{E}+00 \\
6.22 \mathrm{E}-12\end{array}$ \\
\hline TOTAL & $6.22 \mathrm{E}-12$ \\
\hline
\end{tabular}

Feb 20, 2008 02:31 pm

SUMMARY Page 4

NUCLIDE RISK SUMMARY

Nuclide

Ar -41

TOTAL

口

Feb 20, 2008 02:31 pm
Selected Individual

Total Lifetime Fatal Cancer Risk

$6.22 \mathrm{E}-12$

$6.22 \mathrm{E}-12$

INDIVIDUAL EFFECTIVE DOSE EQUIVALENT RATE (mrem/y) (A11 Radionuclides and Pathways)

Distance $(m)$ 


\begin{tabular}{|c|c|c|c|c|c|c|c|}
\hline Direction & 27715 & 28919 & 31060 & $\begin{array}{c}\mathrm{Ar}-41 \mathrm{C} \\
32802\end{array}$ & ${ }^{U M}{ }_{34577}$ & 35279 & 35683 \\
\hline$N$ & $3.0 \mathrm{E}-06$ & $2.8 \mathrm{E}-06$ & $2.6 \mathrm{E}-06$ & $2.5 \mathrm{E}-06$ & $2.3 \mathrm{E}-06$ & $2.3 \mathrm{E}-06$ & 2. $2 \mathrm{E}-06$ \\
\hline NNW & $1.1 \mathrm{E}-06$ & $1.1 \mathrm{E}-06$ & $9.9 \mathrm{E}-07$ & $9.3 \mathrm{E}-07$ & $8.8 \mathrm{E}-07$ & $8.6 \mathrm{E}-07$ & $8.5 \mathrm{E}-07$ \\
\hline NW & $1.6 \mathrm{E}-06$ & 1. $5 \mathrm{E}-06$ & $1.4 \mathrm{E}-06$ & 1. $3 \mathrm{E}-06$ & $1.2 \mathrm{E}-06$ & $1.2 \mathrm{E}-06$ & $1.2 \mathrm{E}-06$ \\
\hline WNW & $2.1 \mathrm{E}-06$ & $2.0 \mathrm{E}-06$ & 1.9E-06 & $1.7 \mathrm{E}-06$ & $1.6 \mathrm{E}-06$ & $1.6 \mathrm{E}-06$ & $1.6 \mathrm{E}-06$ \\
\hline W & 2. $4 \mathrm{E}-06$ & 2. $3 \mathrm{E}-06$ & $2.1 \mathrm{E}-06$ & 2.0E-06 & $1.9 \mathrm{E}-06$ & $1.8 \mathrm{E}-06$ & $1.8 \mathrm{E}-06$ \\
\hline WSW & $3.1 \mathrm{E}-06$ & $3.0 \mathrm{E}-06$ & $2.8 \mathrm{E}-06$ & $2.6 \mathrm{E}-06$ & $2.4 \mathrm{E}-06$ & $2.4 \mathrm{E}-06$ & $2.4 \mathrm{E}-06$ \\
\hline SW & $4.9 \mathrm{E}-06$ & $4.7 \mathrm{E}-06$ & 4. 3E-06 & $4.1 \mathrm{E}-06$ & $3.8 \mathrm{E}-06$ & $3.7 \mathrm{E}-06$ & $3.7 \mathrm{E}-06$ \\
\hline SSW & $8.2 \mathrm{E}-06$ & $7.8 \mathrm{E}-06$ & $7.2 \mathrm{E}-06$ & $6.7 \mathrm{E}-06$ & $6.3 \mathrm{E}-06$ & $6.2 \mathrm{E}-06$ & $6.1 \mathrm{E}-06$ \\
\hline $\mathrm{S}$ & $1.1 \mathrm{E}-05$ & $1.1 \mathrm{E}-05$ & $9.9 \mathrm{E}-06$ & $9.3 \mathrm{E}-06$ & $8.7 \mathrm{E}-06$ & 8. 5E-06 & $8.4 \mathrm{E}-06$ \\
\hline SSE & $9.5 \mathrm{E}-06$ & $9.1 \mathrm{E}-06$ & $8.3 \mathrm{E}-06$ & $7.8 \mathrm{E}-06$ & $7.4 \mathrm{E}-06$ & $7.2 \mathrm{E}-06$ & $7.1 \mathrm{E}-06$ \\
\hline SE & $5.2 \mathrm{E}-06$ & $5.0 \mathrm{E}-06$ & $4.6 \mathrm{E}-06$ & $4.3 \mathrm{E}-06$ & $4.1 \mathrm{E}-06$ & $4.0 \mathrm{E}-06$ & $3.9 \mathrm{E}-06$ \\
\hline ESE & $3.5 \mathrm{E}-06$ & $3.4 \mathrm{E}-06$ & $3.1 \mathrm{E}-06$ & $2.9 \mathrm{E}-06$ & $2.7 \mathrm{E}-06$ & $2.7 \mathrm{E}-06$ & $2.7 \mathrm{E}-06$ \\
\hline $\mathrm{E}$ & $4.5 \mathrm{E}-06$ & $4.3 \mathrm{E}-06$ & $4.0 \mathrm{E}-06$ & $3.8 \mathrm{E}-06$ & $3.5 \mathrm{E}-06$ & $3.5 \mathrm{E}-06$ & $3.4 \mathrm{E}-06$ \\
\hline ENE & $3.8 \mathrm{E}-06$ & $3.7 \mathrm{E}-06$ & $3.4 \mathrm{E}-06$ & $3.2 \mathrm{E}-06$ & $3.0 \mathrm{E}-06$ & $2.9 \mathrm{E}-06$ & $2.9 \mathrm{E}-06$ \\
\hline $\mathrm{NE}$ & $5.6 \mathrm{E}-06$ & $5.3 \mathrm{E}-06$ & $4.9 \mathrm{E}-06$ & $4.6 \mathrm{E}-06$ & $4.3 \mathrm{E}-06$ & $4.2 \mathrm{E}-06$ & $4.1 \mathrm{E}-06$ \\
\hline NNE & $3.7 \mathrm{E}-06$ & $3.5 E-06$ & $3.2 \mathrm{E}-06$ & $3.0 \mathrm{E}-06$ & $2.9 \mathrm{E}-06$ & $2.8 \mathrm{E}-06$ & $2.7 \mathrm{E}-06$ \\
\hline
\end{tabular}

Distance (m)

\begin{tabular}{|c|c|c|c|c|c|c|c|}
\hline Direction & 36721 & 36809 & 37729 & 39079 & 39220 & 39559 & 43584 \\
\hline $\mathrm{N}$ & $2.2 \mathrm{E}-06$ & $2.2 \mathrm{E}-06$ & $2.1 \mathrm{E}-06$ & $2.0 \mathrm{E}-06$ & $2.0 \mathrm{E}-06$ & $2.0 \mathrm{E}-06$ & $1.8 \mathrm{E}-06$ \\
\hline NNW & $8.2 \mathrm{E}-07$ & $8.2 \mathrm{E}-07$ & $7.9 \mathrm{E}-07$ & $7.6 \mathrm{E}-07$ & $7.6 \mathrm{E}-07$ & $7.5 \mathrm{E}-07$ & $6.8 \mathrm{E}-07$ \\
\hline NW & $1.2 \mathrm{E}-06$ & $1.1 \mathrm{E}-06$ & $1.1 \mathrm{E}-06$ & $1.1 \mathrm{E}-06$ & $1.1 \mathrm{E}-06$ & 1.1E-06 & $9.5 \mathrm{E}-07$ \\
\hline WNW & $1.5 \mathrm{E}-06$ & $1.5 \mathrm{E}-06$ & $1.5 \mathrm{E}-06$ & $1.4 \mathrm{E}-06$ & $1.4 \mathrm{E}-06$ & $1.4 \mathrm{E}-06$ & 1. 3E-06 \\
\hline W & $1.7 \mathrm{E}-06$ & $1.7 \mathrm{E}-06$ & $1.7 \mathrm{E}-06$ & $1.6 \mathrm{E}-06$ & $1.6 \mathrm{E}-06$ & $1.6 \mathrm{E}-06$ & $1.4 \mathrm{E}-06$ \\
\hline WSW & $2.3 \mathrm{E}-06$ & $2.3 \mathrm{E}-06$ & $2.2 \mathrm{E}-06$ & $2.1 \mathrm{E}-06$ & $2.1 \mathrm{E}-06$ & $2.1 \mathrm{E}-06$ & 1. $9 \mathrm{E}-06$ \\
\hline SW & $3.6 \mathrm{E}-06$ & $3.6 \mathrm{E}-06$ & $3.5 \mathrm{E}-06$ & $3.3 \mathrm{E}-06$ & $3.3 \mathrm{E}-06$ & $3.3 \mathrm{E}-06$ & $2.9 \mathrm{E}-06$ \\
\hline SSW & $5.9 \mathrm{E}-06$ & $5.9 \mathrm{E}-06$ & $5.7 \mathrm{E}-06$ & $5.5 \mathrm{E}-06$ & $5.5 \mathrm{E}-06$ & $5.4 \mathrm{E}-06$ & $4.8 \mathrm{E}-06$ \\
\hline $\mathrm{s}$ & $8.1 \mathrm{E}-06$ & $8.1 \mathrm{E}-06$ & 7. $9 \mathrm{E}-06$ & $7.5 \mathrm{E}-06$ & $7.5 \mathrm{E}-06$ & $7.4 \mathrm{E}-06$ & $6.6 \mathrm{E}-06$ \\
\hline SSE & $6.9 \mathrm{E}-06$ & $6.8 \mathrm{E}-06$ & $6.6 \mathrm{E}-06$ & $6.4 \mathrm{E}-06$ & $6.4 \mathrm{E}-06$ & $6.3 \mathrm{E}-06$ & $5.6 \mathrm{E}-06$ \\
\hline SE & $3.8 \mathrm{E}-06$ & $3.8 \mathrm{E}-06$ & $3.7 \mathrm{E}-06$ & $3.5 \mathrm{E}-06$ & $3.5 \mathrm{E}-06$ & $3.5 \mathrm{E}-06$ & $3.1 \mathrm{E}-06$ \\
\hline ESE & $2.6 \mathrm{E}-06$ & $2.6 \mathrm{E}-06$ & $2.5 \mathrm{E}-06$ & $2.4 \mathrm{E}-06$ & $2.4 \mathrm{E}-06$ & $2.4 \mathrm{E}-06$ & $2.1 \mathrm{E}-06$ \\
\hline & $3.3 E-06$ & $3.3 \mathrm{E}-06$ & $3.2 \mathrm{E}-06$ & $3.1 \mathrm{E}-06$ & $3.1 \mathrm{E}-06$ & $3.0 \mathrm{E}-06$ & $2.7 \mathrm{E}-06$ \\
\hline ENE & $2.8 \mathrm{E}-06$ & $2.8 \mathrm{E}-06$ & $2.7 \mathrm{E}-06$ & $2.6 \mathrm{E}-06$ & $2.6 \mathrm{E}-06$ & $2.6 \mathrm{E}-06$ & $2.3 \mathrm{E}-06$ \\
\hline $\mathrm{NE}$ & $4.0 \mathrm{E}-06$ & $4.0 \mathrm{E}-06$ & $3.9 \mathrm{E}-06$ & $3.7 \mathrm{E}-06$ & $3.7 \mathrm{E}-06$ & $3.7 \mathrm{E}-06$ & $3.3 \mathrm{E}-06$ \\
\hline NNE & $2.7 \mathrm{E}-06$ & $2.7 \mathrm{E}-06$ & $2.6 \mathrm{E}-06$ & $2.5 \mathrm{E}-06$ & $2.5 \mathrm{E}-06$ & $2.4 \mathrm{E}-06$ & $2.2 \mathrm{E}-06$ \\
\hline
\end{tabular}

口

Feb 20, 2008 02:31 pm

SUMMARY

Page 6

INDIVIDUAL EFFECTIVE DOSE EQUIVALENT RATE (mrem/y) (A11 Radionuclides and Pathways)

\begin{tabular}{|c|c|c|c|c|c|c|}
\hline \multirow[b]{2}{*}{ Direction } & \multicolumn{6}{|c|}{ Distance (m) } \\
\hline & 45196 & 45275 & 45654 & 45677 & 46668 & 47969 \\
\hline
\end{tabular}

\begin{tabular}{|c|c|c|c|c|c|c|}
\hline $\begin{array}{r}\text { N } \\
\text { NNW }\end{array}$ & $\begin{array}{l}1.7 \mathrm{E}-06 \\
6.5 \mathrm{E}-07\end{array}$ & $\begin{array}{l}1.7 \mathrm{E}-06 \\
6.5 \mathrm{E}-07\end{array}$ & $\begin{array}{l}1.7 \mathrm{E}-06 \\
6.4 \mathrm{E}-07\end{array}$ & $\begin{array}{r}1.7 \mathrm{E}-06 \\
6.4 \mathrm{E}-07 \\
\text { Page }\end{array}$ & $\begin{array}{r}1.7 \mathrm{E}-06 \\
6.3 \mathrm{E}-07 \\
4\end{array}$ & $\begin{array}{l}1.6 \mathrm{E}-06 \\
6.1 \mathrm{E}-07\end{array}$ \\
\hline
\end{tabular}




\begin{tabular}{rllllll} 
& & \multicolumn{5}{c}{ Ar-41C.SUM } \\
NW & $9.2 \mathrm{E}-07$ & $9.2 \mathrm{E}-07$ & $9.1 \mathrm{E}-07$ & $9.1 \mathrm{E}-07$ & $8.9 \mathrm{E}-07$ & $8.6 \mathrm{E}-07$ \\
WNW & $1.2 \mathrm{E}-06$ & $1.2 \mathrm{E}-06$ & $1.2 \mathrm{E}-06$ & $1.2 \mathrm{E}-06$ & $1.2 \mathrm{E}-06$ & $1.2 \mathrm{E}-06$ \\
$\mathrm{~W}$ & $1.4 \mathrm{E}-06$ & $1.4 \mathrm{E}-06$ & $1.4 \mathrm{E}-06$ & $1.4 \mathrm{E}-06$ & $1.3 \mathrm{E}-06$ & $1.3 \mathrm{E}-06$ \\
WSW & $1.8 \mathrm{E}-06$ & $1.8 \mathrm{E}-06$ & $1.8 \mathrm{E}-06$ & $1.8 \mathrm{E}-06$ & $1.7 \mathrm{E}-06$ & $1.7 \mathrm{E}-06$ \\
$\mathrm{SW}$ & $2.8 \mathrm{E}-06$ & $2.8 \mathrm{E}-06$ & $2.8 \mathrm{E}-06$ & $2.8 \mathrm{E}-06$ & $2.7 \mathrm{E}-06$ & $2.6 \mathrm{E}-06$ \\
$\mathrm{SSW}$ & $4.6 \mathrm{E}-06$ & $4.6 \mathrm{E}-06$ & $4.6 \mathrm{E}-06$ & $4.6 \mathrm{E}-06$ & $4.5 \mathrm{E}-06$ & $4.3 \mathrm{E}-06$ \\
$\mathrm{~S}$ & $6.3 \mathrm{E}-06$ & $6.3 \mathrm{E}-06$ & $6.3 \mathrm{E}-06$ & $6.3 \mathrm{E}-06$ & $6.1 \mathrm{E}-06$ & $5.9 \mathrm{E}-06$ \\
$\mathrm{SSE}$ & $5.4 \mathrm{E}-06$ & $5.4 \mathrm{E}-06$ & $5.3 \mathrm{E}-06$ & $5.3 \mathrm{E}-06$ & $5.2 \mathrm{E}-06$ & $5.0 \mathrm{E}-06$ \\
$\mathrm{SE}$ & $3.0 \mathrm{E}-06$ & $3.0 \mathrm{E}-06$ & $3.0 \mathrm{E}-06$ & $3.0 \mathrm{E}-06$ & $2.9 \mathrm{E}-06$ & $2.8 \mathrm{E}-06$ \\
$\mathrm{ESE}$ & $2.0 \mathrm{E}-06$ & $2.0 \mathrm{E}-06$ & $2.0 \mathrm{E}-06$ & $2.0 \mathrm{E}-06$ & $2.0 \mathrm{E}-06$ & $1.9 \mathrm{E}-06$ \\
$\mathrm{E}$ & $2.6 \mathrm{E}-06$ & $2.6 \mathrm{E}-06$ & $2.6 \mathrm{E}-06$ & $2.6 \mathrm{E}-06$ & $2.5 \mathrm{E}-06$ & $2.5 \mathrm{E}-06$ \\
$\mathrm{ENE}$ & $2.2 \mathrm{E}-06$ & $2.2 \mathrm{E}-06$ & $2.2 \mathrm{E}-06$ & $2.2 \mathrm{E}-06$ & $2.1 \mathrm{E}-06$ & $2.1 \mathrm{E}-06$ \\
$\mathrm{NE}$ & $3.1 \mathrm{E}-06$ & $3.1 \mathrm{E}-06$ & $3.1 \mathrm{E}-06$ & $3.1 \mathrm{E}-06$ & $3.0 \mathrm{E}-06$ & $2.9 \mathrm{E}-06$ \\
$\mathrm{NNE}$ & $2.1 \mathrm{E}-06$ & $2.1 \mathrm{E}-06$ & $2.1 \mathrm{E}-06$ & $2.1 \mathrm{E}-06$ & $2.0 \mathrm{E}-06$ & $2.0 \mathrm{E}-06$ \\
& & & & & &
\end{tabular}

Feb 20, 2008 02:31 pm

SUMMARY

Page 7

INDIVIDUAL LIFETIME RISK (deaths)

(A11 Radionuclides and Pathways)

\begin{tabular}{|c|c|c|c|c|c|c|c|}
\hline \multicolumn{8}{|c|}{ Distance $(\mathrm{m})$} \\
\hline Direction & 27715 & 28919 & 31060 & 32802 & 34577 & 35279 & 35683 \\
\hline $\begin{array}{r}N \\
N N W \\
N W \\
W N W \\
W \\
W S W \\
S W \\
\text { SSW } \\
S \\
\text { SSE } \\
\text { SE } \\
\text { ESE } \\
\text { E } \\
\text { ENE } \\
N E \\
\text { NNE }\end{array}$ & $\begin{array}{l}1.6 \mathrm{E}-12 \\
6.2 \mathrm{E}-13 \\
8.6 \mathrm{E}-13 \\
1.2 \mathrm{E}-12 \\
1.3 \mathrm{E}-12 \\
1.7 \mathrm{E}-12 \\
2.7 \mathrm{E}-12 \\
4.5 \mathrm{E}-12 \\
6.2 \mathrm{E}-12 \\
5.2 \mathrm{E}-12 \\
2.9 \mathrm{E}-12 \\
1.9 \mathrm{E}-12 \\
2.5 \mathrm{E}-12 \\
2.1 \mathrm{E}-12 \\
3.1 \mathrm{E}-12 \\
2.0 \mathrm{E}-12\end{array}$ & $\begin{array}{l}1.6 \mathrm{E}-12 \\
5.9 \mathrm{E}-13 \\
8.2 \mathrm{E}-13 \\
1.1 \mathrm{E}-12 \\
1.3 \mathrm{E}-12 \\
1.6 \mathrm{E}-12 \\
2.6 \mathrm{E}-12 \\
4.3 \mathrm{E}-12 \\
5.9 \mathrm{E}-12 \\
5.0 \mathrm{E}-12 \\
2.7 \mathrm{E}-12 \\
1.8 \mathrm{E}-12 \\
2.4 \mathrm{E}-12 \\
2.0 \mathrm{E}-12 \\
2.9 \mathrm{E}-12 \\
1.9 \mathrm{E}-12\end{array}$ & $\begin{array}{l}1.4 \mathrm{E}-12 \\
5.4 \mathrm{E}-13 \\
7.6 \mathrm{E}-13 \\
1.0 \mathrm{E}-12 \\
1.2 \mathrm{E}-12 \\
1.5 \mathrm{E}-12 \\
2.4 \mathrm{E}-12 \\
3.9 \mathrm{E}-12 \\
5.4 \mathrm{E}-12 \\
4.6 \mathrm{E}-12 \\
2.5 \mathrm{E}-12 \\
1.7 \mathrm{E}-12 \\
2.2 \mathrm{E}-12 \\
1.8 \mathrm{E}-12 \\
2.7 \mathrm{E}-12 \\
1.8 \mathrm{E}-12\end{array}$ & $\begin{array}{l}1.4 \mathrm{E}-12 \\
5.1 \mathrm{E}-13 \\
7.2 \mathrm{E}-13 \\
9.6 \mathrm{E}-13 \\
1.1 \mathrm{E}-12 \\
1.4 \mathrm{E}-12 \\
2.2 \mathrm{E}-12 \\
3.7 \mathrm{E}-12 \\
5.1 \mathrm{E}-12 \\
4.3 \mathrm{E}-12 \\
2.4 \mathrm{E}-12 \\
1.6 \mathrm{E}-12 \\
2.1 \mathrm{E}-12 \\
1.7 \mathrm{E}-12 \\
2.5 \mathrm{E}-12 \\
1.7 \mathrm{E}-12\end{array}$ & $\begin{array}{l}1.3 \mathrm{E}-12 \\
4.8 \mathrm{E}-13 \\
6.8 \mathrm{E}-13 \\
9.1 \mathrm{E}-13 \\
1.0 \mathrm{E}-12 \\
1.3 \mathrm{E}-12 \\
2.1 \mathrm{E}-12 \\
3.5 \mathrm{E}-12 \\
4.8 \mathrm{E}-12 \\
4.0 \mathrm{E}-12 \\
2.2 \mathrm{E}-12 \\
1.5 \mathrm{E}-12 \\
1.9 \mathrm{E}-12 \\
1.6 \mathrm{E}-12 \\
2.4 \mathrm{E}-12 \\
1.6 \mathrm{E}-12\end{array}$ & $\begin{array}{l}1.3 \mathrm{E}-12 \\
4.7 \mathrm{E}-13 \\
6.6 \mathrm{E}-13 \\
8.9 \mathrm{E}-13 \\
1.0 \mathrm{E}-12 \\
1.3 \mathrm{E}-12 \\
2.1 \mathrm{E}-12 \\
3.4 \mathrm{E}-12 \\
4.7 \mathrm{E}-12 \\
4.0 \mathrm{E}-12 \\
2.2 \mathrm{E}-12 \\
1.5 \mathrm{E}-12 \\
1.9 \mathrm{E}-12 \\
1.6 \mathrm{E}-12 \\
2.3 \mathrm{E}-12 \\
1.5 \mathrm{E}-12\end{array}$ & $\begin{array}{l}1.2 \mathrm{E}-12 \\
4.6 \mathrm{E}-13 \\
6.5 \mathrm{E}-13 \\
8.8 \mathrm{E}-13 \\
9.9 \mathrm{E}-13 \\
1.3 \mathrm{E}-12 \\
2.0 \mathrm{E}-12 \\
3.3 \mathrm{E}-12 \\
4.6 \mathrm{E}-12 \\
3.9 \mathrm{E}-12 \\
2.2 \mathrm{E}-12 \\
1.5 \mathrm{E}-12 \\
1.9 \mathrm{E}-12 \\
1.6 \mathrm{E}-12 \\
2.3 \mathrm{E}-12 \\
1.5 \mathrm{E}-12\end{array}$ \\
\hline \multicolumn{8}{|c|}{ Distance (m) } \\
\hline Direction & 36721 & 36809 & 37729 & 39079 & 39220 & 39559 & 43584 \\
\hline $\begin{array}{r}\text { N } \\
\text { NNW } \\
N W \\
\text { WNW } \\
W \\
\text { WSW } \\
\text { SW } \\
\text { SSW }\end{array}$ & $\begin{array}{l}1.2 \mathrm{E}-12 \\
4.5 \mathrm{E}-13 \\
6.3 \mathrm{E}-13 \\
8.5 \mathrm{E}-13 \\
9.6 \mathrm{E}-13 \\
1.3 \mathrm{E}-12 \\
2.0 \mathrm{E}-12 \\
3.2 \mathrm{E}-12\end{array}$ & $\begin{array}{l}1.2 \mathrm{E}-12 \\
4.5 \mathrm{E}-13 \\
6.3 \mathrm{E}-13 \\
8.5 \mathrm{E}-13 \\
9.6 \mathrm{E}-13 \\
1.3 \mathrm{E}-12 \\
2.0 \mathrm{E}-12 \\
3.2 \mathrm{E}-12\end{array}$ & $\begin{array}{l}1.2 \mathrm{E}-12 \\
4.4 \mathrm{E}-13 \\
6.1 \mathrm{E}-13 \\
8.2 \mathrm{E}-13 \\
9.3 \mathrm{E}-13 \\
1.2 \mathrm{E}-12 \\
1.9 \mathrm{E}-12 \\
3.1 \mathrm{E}-12\end{array}$ & $\begin{array}{r}1.1 \mathrm{E}-12 \\
4.2 \mathrm{E}-13 \\
5.9 \mathrm{E}-13 \\
7.9 \mathrm{E}-13 \\
9.0 \mathrm{E}-13 \\
1.2 \mathrm{E}-12 \\
1.8 \mathrm{E}-12 \\
3.0 \mathrm{E}-12 \\
\text { Page }\end{array}$ & $\begin{array}{l}1.1 \mathrm{E}-12 \\
4.2 \mathrm{E}-13 \\
5.9 \mathrm{E}-13 \\
7.9 \mathrm{E}-13 \\
8.9 \mathrm{E}-13 \\
1.2 \mathrm{E}-12 \\
1.8 \mathrm{E}-12 \\
3.0 \mathrm{E}-12 \\
5\end{array}$ & $\begin{array}{l}1.1 \mathrm{E}-12 \\
4.1 \mathrm{E}-13 \\
5.8 \mathrm{E}-13 \\
7.8 \mathrm{E}-13 \\
8.8 \mathrm{E}-13 \\
1.2 \mathrm{E}-12 \\
1.8 \mathrm{E}-12 \\
3.0 \mathrm{E}-12\end{array}$ & $\begin{array}{l}9.9 \mathrm{E}-13 \\
3.7 \mathrm{E}-13 \\
5.2 \mathrm{E}-13 \\
7.0 \mathrm{E}-13 \\
7.9 \mathrm{E}-13 \\
1.0 \mathrm{E}-12 \\
1.6 \mathrm{E}-12 \\
2.7 \mathrm{E}-12\end{array}$ \\
\hline
\end{tabular}




\begin{tabular}{rlllllll} 
& \multicolumn{7}{c}{$\mathrm{Ar}-41 \mathrm{C} \cdot \mathrm{SUM}$} \\
$\mathrm{S}$ & $4.5 \mathrm{E}-12$ & $4.4 \mathrm{E}-12$ & $4.3 \mathrm{E}-12$ & $4.1 \mathrm{E}-12$ & $4.1 \mathrm{E}-12$ & $4.1 \mathrm{E}-12$ & $3.6 \mathrm{E}-12$ \\
SSE & $3.8 \mathrm{E}-12$ & $3.8 \mathrm{E}-12$ & $3.7 \mathrm{E}-12$ & $3.5 \mathrm{E}-12$ & $3.5 \mathrm{E}-12$ & $3.5 \mathrm{E}-12$ & $3.1 \mathrm{E}-12$ \\
$\mathrm{SE}$ & $2.1 \mathrm{E}-12$ & $2.1 \mathrm{E}-12$ & $2.0 \mathrm{E}-12$ & $2.0 \mathrm{E}-12$ & $1.9 \mathrm{E}-12$ & $1.9 \mathrm{E}-12$ & $1.7 \mathrm{E}-12$ \\
$\mathrm{ESE}$ & $1.4 \mathrm{E}-12$ & $1.4 \mathrm{E}-12$ & $1.4 \mathrm{E}-12$ & $1.3 \mathrm{E}-12$ & $1.3 \mathrm{E}-12$ & $1.3 \mathrm{E}-12$ & $1.2 \mathrm{E}-12$ \\
$\mathrm{E}$ & $1.8 \mathrm{E}-12$ & $1.8 \mathrm{E}-12$ & $1.8 \mathrm{E}-12$ & $1.7 \mathrm{E}-12$ & $1.7 \mathrm{E}-12$ & $1.7 \mathrm{E}-12$ & $1.5 \mathrm{E}-12$ \\
$\mathrm{ENE}$ & $1.5 \mathrm{E}-12$ & $1.5 \mathrm{E}-12$ & $1.5 \mathrm{E}-12$ & $1.4 \mathrm{E}-12$ & $1.4 \mathrm{E}-12$ & $1.4 \mathrm{E}-12$ & $1.3 \mathrm{E}-12$ \\
$\mathrm{NE}$ & $2.2 \mathrm{E}-12$ & $2.2 \mathrm{E}-12$ & $2.1 \mathrm{E}-12$ & $2.0 \mathrm{E}-12$ & $2.0 \mathrm{E}-12$ & $2.0 \mathrm{E}-12$ & $1.8 \mathrm{E}-12$ \\
$\mathrm{NNE}$ & $1.5 \mathrm{E}-12$ & $1.5 \mathrm{E}-12$ & $1.4 \mathrm{E}-12$ & $1.4 \mathrm{E}-12$ & $1.4 \mathrm{E}-12$ & $1.3 \mathrm{E}-12$ & $1.2 \mathrm{E}-12$
\end{tabular}

Feb 20, $2008 \quad 02: 31 \mathrm{pm}$

SUMMARY

Page 8

INDIVIDUAL LIFETIME RISK (deaths)

(A11 Radionuclides and Pathways)

\begin{tabular}{|c|c|c|c|c|c|c|}
\hline \multirow[b]{2}{*}{ Direction } & \multicolumn{6}{|c|}{ Distance $(\mathrm{m})$} \\
\hline & 45196 & 45275 & 45654 & 45677 & 46668 & 47969 \\
\hline $\mathrm{N}$ & $9.5 \mathrm{E}-13$ & $9.5 \mathrm{E}-13$ & $9.4 \mathrm{E}-13$ & $9.4 \mathrm{E}-13$ & $9.2 \mathrm{E}-13$ & $8.9 \mathrm{E}-13$ \\
\hline NNW & $3.6 \mathrm{E}-13$ & $3.6 \mathrm{E}-13$ & $3.5 \mathrm{E}-13$ & $3.5 \mathrm{E}-13$ & 3. $4 \mathrm{E}-13$ & 3. $3 \mathrm{E}-13$ \\
\hline NW & $5.0 \mathrm{E}-13$ & 5. $0 \mathrm{E}-13$ & $5.0 \mathrm{E}-13$ & $5.0 \mathrm{E}-13$ & $4.9 \mathrm{E}-13$ & $4.7 \mathrm{E}-13$ \\
\hline WNW & $6.8 \mathrm{E}-13$ & $6.7 \mathrm{E}-13$ & $6.7 \mathrm{E}-13$ & $6.7 \mathrm{E}-13$ & $6.5 \mathrm{E}-13$ & $6.3 \mathrm{E}-13$ \\
\hline W & $7.6 \mathrm{E}-13$ & $7.6 \mathrm{E}-13$ & $7.5 \mathrm{E}-13$ & $7.5 \mathrm{E}-13$ & $7.4 \mathrm{E}-13$ & 7. $1 \mathrm{E}-13$ \\
\hline WSW & 1. $0 \mathrm{E}-12$ & $9.9 \mathrm{E}-13$ & $9.8 \mathrm{E}-13$ & $9.8 \mathrm{E}-13$ & $9.6 \mathrm{E}-13$ & $9.3 \mathrm{E}-13$ \\
\hline SW & $1.5 \mathrm{E}-12$ & $1.5 \mathrm{E}-12$ & 1. $5 \mathrm{E}-12$ & $1.5 \mathrm{E}-12$ & $1.5 \mathrm{E}-12$ & 1. $4 \mathrm{E}-12$ \\
\hline SSW & $2.5 \mathrm{E}-12$ & $2.5 \mathrm{E}-12$ & $2.5 \mathrm{E}-12$ & $2.5 \mathrm{E}-12$ & $2.5 \mathrm{E}-12$ & $2.4 \mathrm{E}-12$ \\
\hline $\mathrm{S}$ & $3.5 \mathrm{E}-12$ & $3.5 \mathrm{E}-12$ & $3.4 \mathrm{E}-12$ & $3.4 \mathrm{E}-12$ & $3.4 \mathrm{E}-12$ & $3.3 \mathrm{E}-12$ \\
\hline SSE & 3.0E-12 & 3. $0 \mathrm{E}-12$ & $2.9 \mathrm{E}-12$ & $2.9 \mathrm{E}-12$ & $2.8 \mathrm{E}-12$ & $2.8 \mathrm{E}-12$ \\
\hline SE & $1.7 \mathrm{E}-12$ & $1.7 \mathrm{E}-12$ & $1.6 \mathrm{E}-12$ & $1.6 \mathrm{E}-12$ & $1.6 \mathrm{E}-12$ & 1. $5 \mathrm{E}-12$ \\
\hline ESE & $1.1 \mathrm{E}-12$ & 1.1E-12 & 1. $1 \mathrm{E}-12$ & $1.1 \mathrm{E}-12$ & $1.1 \mathrm{E}-12$ & 1. $0 \mathrm{E}-12$ \\
\hline & $1.4 \mathrm{E}-12$ & $1.4 \mathrm{E}-12$ & 1. $4 \mathrm{E}-12$ & $1.4 \mathrm{E}-12$ & 1. $4 \mathrm{E}-12$ & 1. $3 \mathrm{E}-12$ \\
\hline ENE & 1. $2 \mathrm{E}-12$ & $1.2 \mathrm{E}-12$ & 1. $2 \mathrm{E}-12$ & $1.2 \mathrm{E}-12$ & 1. $2 \mathrm{E}-12$ & 1. $1 \mathrm{E}-12$ \\
\hline $\mathrm{NE}$ & $1.7 \mathrm{E}-12$ & $1.7 \mathrm{E}-12$ & $1.7 \mathrm{E}-12$ & $1.7 \mathrm{E}-12$ & $1.7 \mathrm{E}-12$ & 1. $6 \mathrm{E}-12$ \\
\hline NNE & 1. $2 \mathrm{E}-12$ & 1. $2 \mathrm{E}-12$ & $1.1 \mathrm{E}-12$ & $1.1 \mathrm{E}-12$ & $1.1 \mathrm{E}-12$ & 1. $1 \mathrm{E}-12$ \\
\hline
\end{tabular}




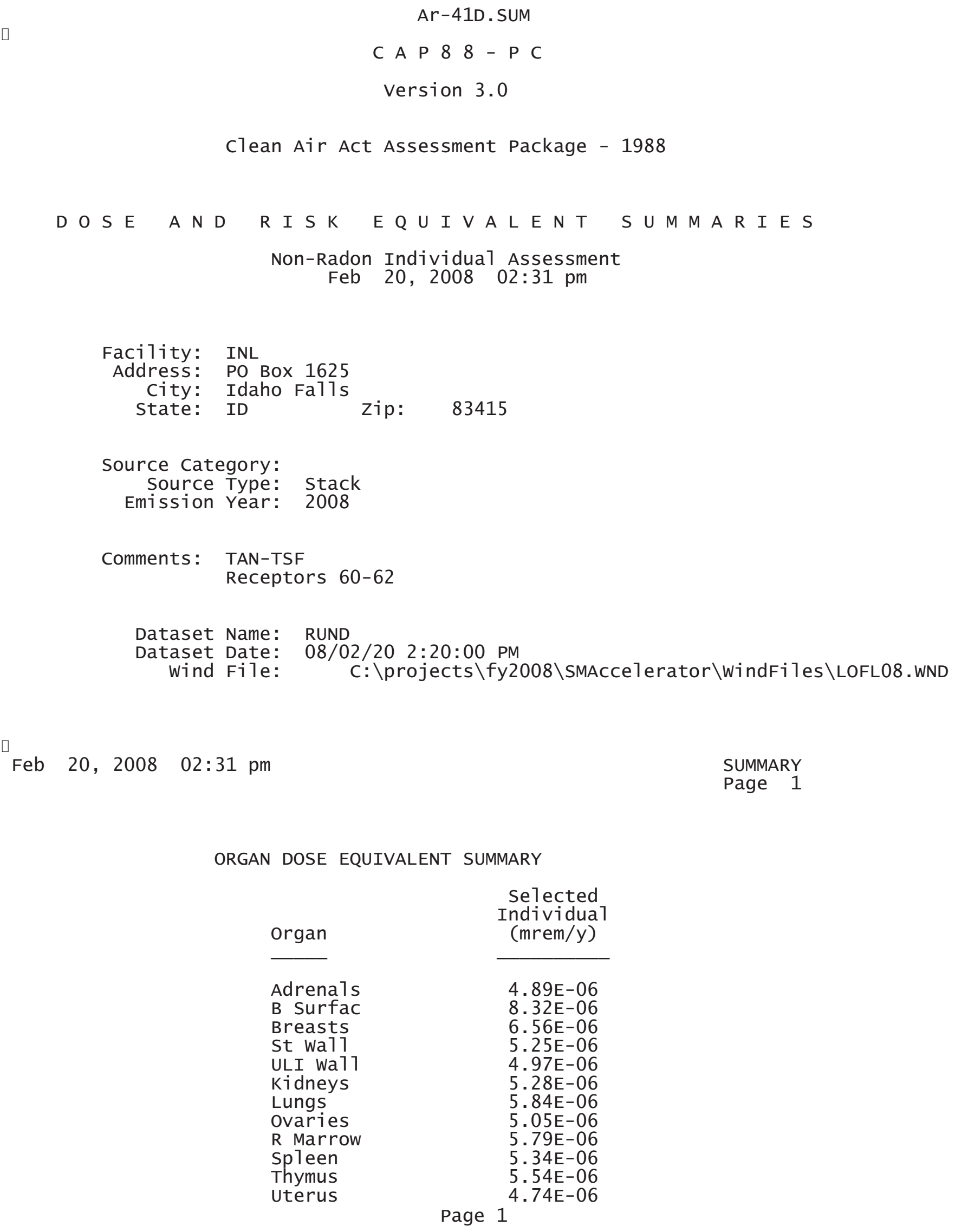




$\begin{array}{lr} & \text { Ar-41D. SUM } \\ \text { B1d wa11 } & 4.90 \mathrm{E}-06 \\ \text { Brain } & 6.29 \mathrm{E}-06 \\ \text { Esophagu } & 5.06 \mathrm{E}-06 \\ \text { SI wa11 } & 4.86 \mathrm{E}-06 \\ \text { LLI wa11 } & 4.93 \mathrm{E}-06 \\ \text { Liver } & 5.33 \mathrm{E}-06 \\ \text { Muscle } & 5.70 \mathrm{E}-06 \\ \text { Pancreas } & 4.78 \mathrm{E}-06 \\ \text { Skin } & 9.25 \mathrm{E}-06 \\ \text { Testes } & 5.80 \mathrm{E}-06 \\ \text { Thyroid } & 5.97 \mathrm{E}-06 \\ & \\ \text { EFFEC } & 5.63 \mathrm{E}-06\end{array}$

PATHWAY EFFECTIVE DOSE EQUIVALENT SUMMARY

\begin{tabular}{lc} 
Pathway & $\begin{array}{c}\text { Selected } \\
\text { Individua1 } \\
\text { (mrem/y) }\end{array}$ \\
\cline { 2 - 2 } INGESTION & $0.00 \mathrm{E}+00$ \\
INHALATION & $0.00 \mathrm{E}+00$ \\
AIR IMMERSION & $5.63 \mathrm{E}-06$ \\
GROUND SURFACE & $0.00 \mathrm{E}+00$ \\
INTERNAL & $0.00 \mathrm{E}+00$ \\
EXTERNAL & $5.63 \mathrm{E}-06$ \\
TOTAL & $5.63 \mathrm{E}-06$
\end{tabular}

Feb 20, $2008 \quad 02: 31 \mathrm{pm}$

SUMMARY

Page 2

NUCLIDE EFFECTIVE DOSE EQUIVALENT SUMMARY

Nuclide

Ar -41

TOTAL

Feb 20, 2008 02:31 pm

$$
\begin{gathered}
\begin{array}{c}
\text { Selected } \\
\text { Individua1 } \\
(\mathrm{mrem} / \mathrm{y})
\end{array} \\
\hline 5.63 \mathrm{E}-06 \\
5.63 \mathrm{E}-06
\end{gathered}
$$

SUMMARY

Page 3

CANCER RISK SUMMARY

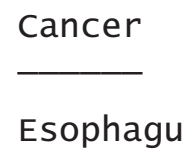

Esophagu

$$
\begin{aligned}
& \begin{array}{c}
\text { Selected Individual } \\
\text { Total Lifetime } \\
\text { Fatal Cancer Risk }
\end{array} \\
& 5.82 \mathrm{E}-14
\end{aligned}
$$




$\begin{array}{lr} & \text { Ar-41D. SUM } \\ \text { Stomach } & 2.12 \mathrm{E}-13 \\ \text { Colon } & 5.11 \mathrm{E}-13 \\ \text { Liver } & 8.08 \mathrm{E}-14 \\ \text { LUNG } & 5.72 \mathrm{E}-13 \\ \text { Bone } & 7.90 \mathrm{E}-15 \\ \text { Skin } & 9.25 \mathrm{E}-15 \\ \text { Breast } & 3.17 \mathrm{E}-13 \\ \text { Ovary } & 7.18 \mathrm{E}-14 \\ \text { B Tadder } & 1.18 \mathrm{E}-13 \\ \text { Kidneys } & 2.75 \mathrm{E}-14 \\ \text { Thyroid } & 1.91 \mathrm{E}-14 \\ \text { Leukemia } & 3.25 \mathrm{E}-13 \\ \text { Residua1 } & 7.63 \mathrm{E}-13 \\ \text { Tota1 } & 3.10 \mathrm{E}-12 \\ & \\ \text { TOTAL } & 6.19 \mathrm{E}-12\end{array}$

PATHWAY RISK SUMMARY

\begin{tabular}{lc} 
Pathway & $\begin{array}{c}\text { Selected Individua1 } \\
\text { Total Lifetime } \\
\text { Fatal Cancer Risk }\end{array}$ \\
\cline { 2 - 2 } INGESTION & $0.00 \mathrm{E}+00$ \\
INHALATION & $0.00 \mathrm{E}+00$ \\
AIR IMMERSION & $3.10 \mathrm{E}-12$ \\
GROUND SURFACE & $0.00 \mathrm{E}+00$ \\
INTERNAL & $0.00 \mathrm{E}+00$ \\
EXTERNAL & $3.10 \mathrm{E}-12$ \\
TOTAL & $3.10 \mathrm{E}-12$
\end{tabular}

Feb 20, $2008 \quad 02: 31$ pm

SUMMARY Page 4

NUCLIDE RISK SUMMARY

Nuclide

Ar -41

TOTAL

口

Feb 20, 2008 02:31 pm
Selected Individual

Total Lifetime Fatal Cancer Risk

3. $10 \mathrm{E}-12$

3. $10 \mathrm{E}-12$

SUMMARY

Page 5

INDIVIDUAL EFFECTIVE DOSE EQUIVALENT RATE (mrem/y) (A11 Radionuclides and Pathways)

Distance $(m)$ 


\begin{tabular}{|c|c|c|c|c|}
\hline Direction & 50024 & 54611 & Ar-41D. SUM & \\
\hline $\begin{array}{r}N \\
N N W \\
N W \\
W N W \\
W \\
W S W \\
\text { SW } \\
\text { SSW } \\
S \\
\text { SSE } \\
\text { SE } \\
\text { ESE } \\
\text { E } \\
\text { ENE } \\
\text { NE } \\
\text { NNE }\end{array}$ & $\begin{array}{l}1.5 \mathrm{E}-06 \\
5.8 \mathrm{E}-07 \\
8.2 \mathrm{E}-07 \\
1.1 \mathrm{E}-06 \\
1.2 \mathrm{E}-06 \\
1.6 \mathrm{E}-06 \\
2.5 \mathrm{E}-06 \\
4.1 \mathrm{E}-06 \\
5.6 \mathrm{E}-06 \\
4.8 \mathrm{E}-06 \\
2.7 \mathrm{E}-06 \\
1.8 \mathrm{E}-06 \\
2.3 \mathrm{E}-06 \\
2.0 \mathrm{E}-06 \\
2.8 \mathrm{E}-06 \\
1.9 \mathrm{E}-06\end{array}$ & $\begin{array}{l}1.4 \mathrm{E}-06 \\
5.3 \mathrm{E}-07 \\
7.4 \mathrm{E}-07 \\
1.0 \mathrm{E}-06 \\
1.1 \mathrm{E}-06 \\
1.5 \mathrm{E}-06 \\
2.3 \mathrm{E}-06 \\
3.7 \mathrm{E}-06 \\
5.1 \mathrm{E}-06 \\
4.3 \mathrm{E}-06 \\
2.4 \mathrm{E}-06 \\
1.6 \mathrm{E}-06 \\
2.1 \mathrm{E}-06 \\
1.8 \mathrm{E}-06 \\
2.5 \mathrm{E}-06 \\
1.7 \mathrm{E}-06\end{array}$ & $\begin{array}{l}1.3 \mathrm{E}-06 \\
4.9 \mathrm{E}-07 \\
6.9 \mathrm{E}-07 \\
9.2 \mathrm{E}-07 \\
1.0 \mathrm{E}-06 \\
1.4 \mathrm{E}-06 \\
2.1 \mathrm{E}-06 \\
3.4 \mathrm{E}-06 \\
4.7 \mathrm{E}-06 \\
4.0 \mathrm{E}-06 \\
2.2 \mathrm{E}-06 \\
1.5 \mathrm{E}-06 \\
2.0 \mathrm{E}-06 \\
1.6 \mathrm{E}-06 \\
2.3 \mathrm{E}-06 \\
1.6 \mathrm{E}-06\end{array}$ & \\
\hline Feb 20, & 2008 & $2: 31$ pm & & $\begin{array}{l}\text { SUMMARY } \\
\text { Page } 6\end{array}$ \\
\hline \multicolumn{5}{|c|}{$\begin{array}{l}\text { INDIVIDUAL LIFETIME RISK (deaths) } \\
\text { (A11 Radionuclides and Pathways) }\end{array}$} \\
\hline \multicolumn{5}{|c|}{ Distance (m) } \\
\hline Direction & 50024 & 54611 & 58610 & \\
\hline $\begin{array}{r}N \\
N N W \\
N W \\
W N W \\
W \\
W S W \\
S W \\
S S W \\
S \\
\text { SSE } \\
\text { SE } \\
\text { ESE } \\
\text { E } \\
\text { ENE } \\
N E \\
\text { NNE }\end{array}$ & $\begin{array}{l}8.5 \mathrm{E}-13 \\
3.2 \mathrm{E}-13 \\
4.5 \mathrm{E}-13 \\
6.0 \mathrm{E}-13 \\
6.8 \mathrm{E}-13 \\
8.9 \mathrm{E}-13 \\
1.4 \mathrm{E}-12 \\
2.3 \mathrm{E}-12 \\
3.1 \mathrm{E}-12 \\
2.6 \mathrm{E}-12 \\
1.5 \mathrm{E}-12 \\
1.0 \mathrm{E}-12 \\
1.3 \mathrm{E}-12 \\
1.1 \mathrm{E}-12 \\
1.5 \mathrm{E}-12 \\
1.0 \mathrm{E}-12\end{array}$ & $\begin{array}{l}7.7 \mathrm{E}-13 \\
2.9 \mathrm{E}-13 \\
4.1 \mathrm{E}-13 \\
5.5 \mathrm{E}-13 \\
6.2 \mathrm{E}-13 \\
8.1 \mathrm{E}-13 \\
1.2 \mathrm{E}-12 \\
2.0 \mathrm{E}-12 \\
2.8 \mathrm{E}-12 \\
2.4 \mathrm{E}-12 \\
1.3 \mathrm{E}-12 \\
9.0 \mathrm{E}-13 \\
1.2 \mathrm{E}-12 \\
9.7 \mathrm{E}-13 \\
1.4 \mathrm{E}-12 \\
9.3 \mathrm{E}-13\end{array}$ & $\begin{array}{l}7.1 \mathrm{E}-13 \\
2.7 \mathrm{E}-13 \\
3.8 \mathrm{E}-13 \\
5.1 \mathrm{E}-13 \\
5.7 \mathrm{E}-13 \\
7.4 \mathrm{E}-13 \\
1.1 \mathrm{E}-12 \\
1.9 \mathrm{E}-12 \\
2.6 \mathrm{E}-12 \\
2.2 \mathrm{E}-12 \\
1.2 \mathrm{E}-12 \\
8.4 \mathrm{E}-13 \\
1.1 \mathrm{E}-12 \\
9.0 \mathrm{E}-13 \\
1.3 \mathrm{E}-12 \\
8.6 \mathrm{E}-13\end{array}$ & \\
\hline
\end{tabular}




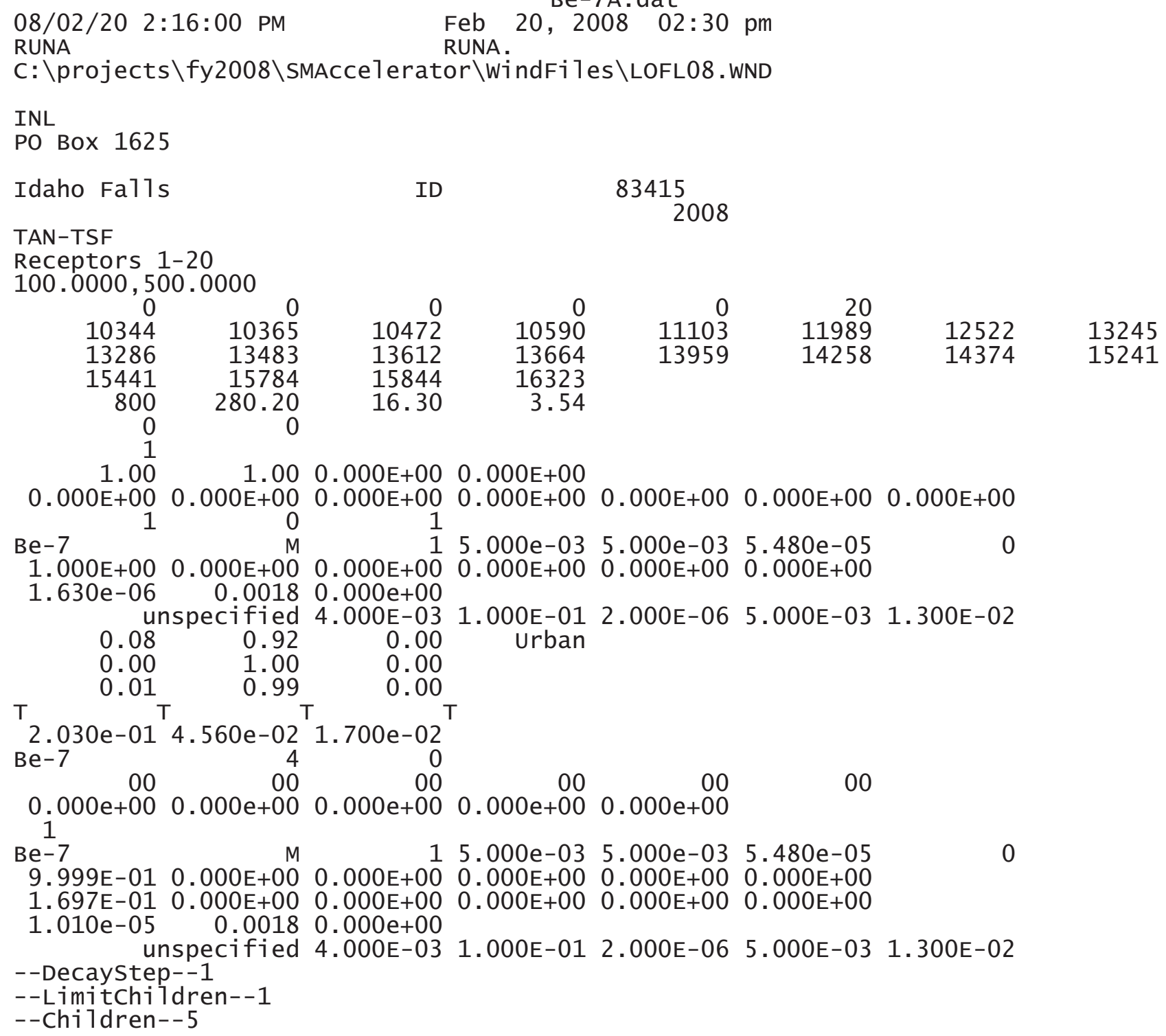


08/02/20 2:17:00 PM

RUNB

C: \projects \fy2008\SMAccelerator \WindFi 1es\LOFL08.WND

INL

PO Box 1625

Idaho Falls

TAN-TSF

Receptors 21-39

$100.0000,500.0000$

$\begin{array}{rr}0 & \\ 17035 & 17329 \\ 20457 & 21314 \\ 26794 & 27275 \\ 800 & 280.20 \\ 0 & 0 \\ 1 & \end{array}$

$\begin{array}{llll}1.00 & 1.00 & 0.000 \mathrm{E}+00 & 0.000 \mathrm{E}+00\end{array}$

$\begin{array}{r}0.000 \mathrm{E}+00 \\ 1\end{array} \quad 0.000 \mathrm{E}+$

$\mathrm{Be}-7$ $\begin{array}{lll}1 & 0 \\ M\end{array}$

$\begin{array}{rr}1.000 \mathrm{E}+00 & 0.000 \mathrm{E}+00 \\ 1.630 \mathrm{e}-06 & 0.0018\end{array}$

$0.000 \mathrm{E}+00$

1

$15.000 \mathrm{e}-03$

$0.000 \mathrm{E}+00$

$0.000 \mathrm{E}+00$

83415

2008

0.08

0.00

0.01

$\mathrm{T}$

$2.030 \mathrm{e}-01$

$\mathrm{Be}-7$

0000

0.92

1.00

0.99

0.00

0.00

1.000E-01

Urban

$0.000 \mathrm{E}+00 \quad 0.000 \mathrm{E}+00 \quad 0.000 \mathrm{E}+00$

$$
1
$$

Be-7

9. $999 \mathrm{E}-01 \quad 0.000 \mathrm{E}+00$

$\mathrm{M}$

1
-00

$5.000 \mathrm{e}-03 \quad 5.000 \mathrm{e}-03 \quad 5.480 \mathrm{e}-05$

0

00 $T$

$\begin{array}{llllllllllll}1.697 \mathrm{E}-01 & 0.000 \mathrm{E}+00 & 0.000 \mathrm{E}+00 & 0.000 \mathrm{E}+00 & 0.000 \mathrm{E}+00 & 0.000 \mathrm{E}+00\end{array}$

$1.010 \mathrm{e}-05 \quad 0.0018 \quad 0.000 \mathrm{e}+00$

$\begin{array}{lllll}\text { unspecified } 4.000 \mathrm{E}-03 & 1.000 \mathrm{E}-01 & 2.000 \mathrm{E}-06 & 5.000 \mathrm{E}-03 & 1.300 \mathrm{E}-02\end{array}$

--Decaystep--1

--LimitChildren--1

--Children--5 


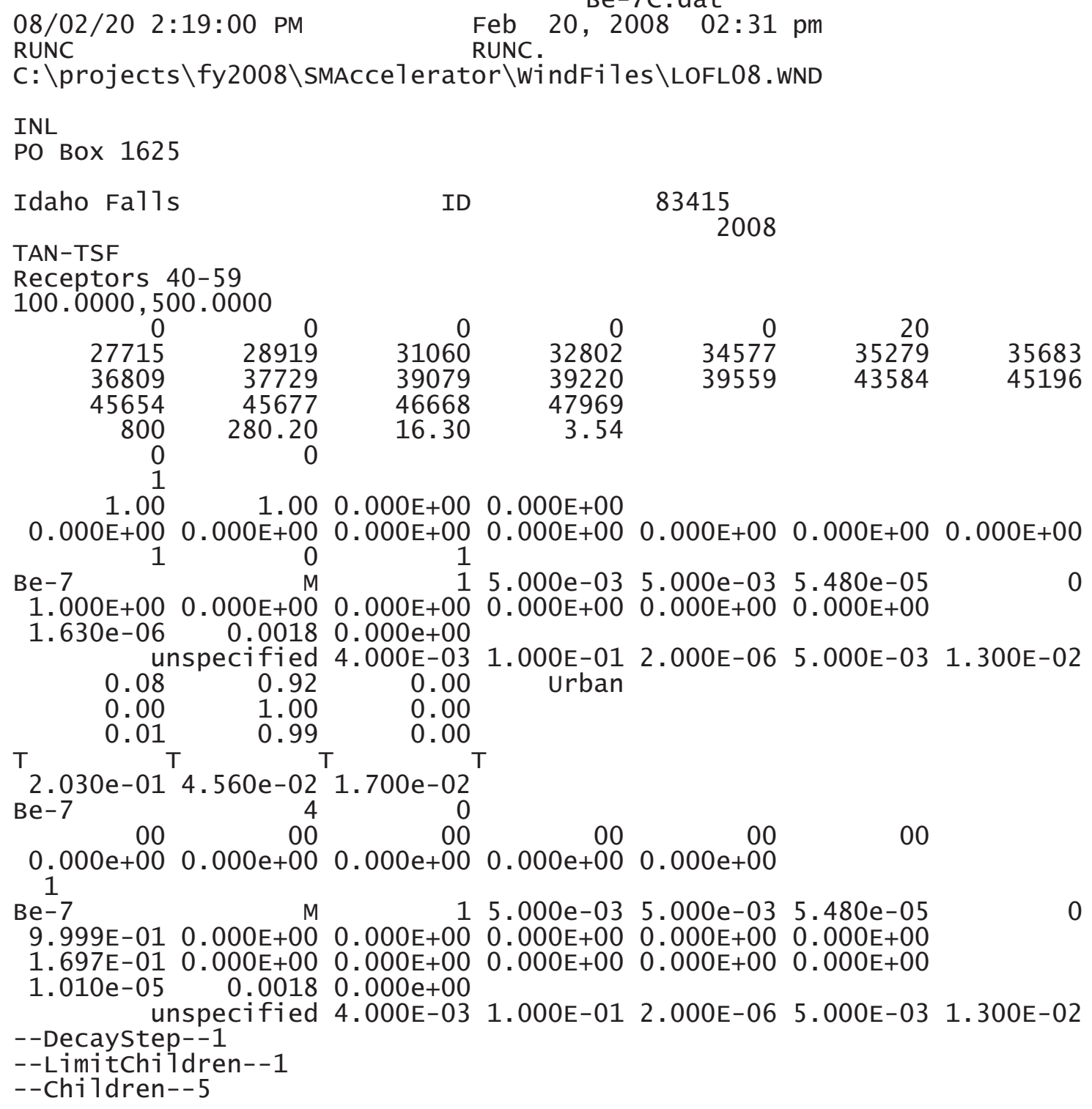

ID

83415

TAN-TSF

Receptors 40-59

$100.0000,500.0000$

$\begin{array}{rrrr}0 & 0 & 0 & \\ 27715 & 28919 & 31060 & 3280 \\ 36809 & 37729 & 39079 & 3922 \\ 45654 & 45677 & 46668 & 47969 \\ 800 & 280.20 & 16.30 & 3.54 \\ 0 & 0 & & \\ 1 & & & \end{array}$

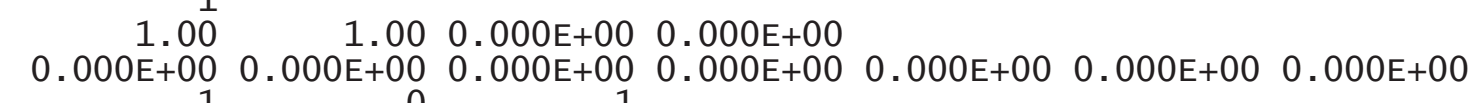

$\mathrm{Be}-7 \quad 1$

$\begin{array}{llllll}1.000 \mathrm{E}+00 & 0.000 \mathrm{E}+00 & 0.000 \mathrm{E}+00 & 0.000 \mathrm{E}+00 & 0.000 \mathrm{E}+00 & 0.000 \mathrm{E}+00\end{array}$

$\begin{array}{lr}1.630 \mathrm{e}-06 & 0.0018 \quad 0.000 \mathrm{e}+00\end{array}$

unspecified 4.000E-03 1.000E-01 2.000E-06 5.000E-03 $1.300 \mathrm{E}-02$

$\begin{array}{llll}0.08 & 0.92 & 0.00 & \text { Urban }\end{array}$

$0.00 \quad 1.00 \quad 0.00$

$\begin{array}{llll}\mathrm{T} & 0.01 & 0.99 & 0.00 \\ \mathrm{~T} & \mathrm{~T} & \mathrm{~T}\end{array}$

$\begin{array}{rrr}2.030 \mathrm{e}-01 & 4.560 \mathrm{e}-02 & 1.700 \mathrm{e}-02 \\ \mathrm{Be}-7 & 4 & 0\end{array}$

$0.000 \mathrm{e}+00 \quad 0.000 \mathrm{e}+00 \quad 0.000 \mathrm{e}+00 \quad 0.000 \mathrm{e}+00 \quad 0.000 \mathrm{e}+00$

2008 1

Be-7

M $\quad 15.000 \mathrm{e}-03 \quad 5.000 \mathrm{e}-03 \quad 5.480 \mathrm{e}-05$

00

$9.999 \mathrm{E}-010.000 \mathrm{E}+00 \quad 0.000 \mathrm{E}+00 \quad 0.000 \mathrm{E}+00 \quad 0.000 \mathrm{E}+00 \quad 0.000 \mathrm{E}+00$

$\begin{array}{lllllllll}1.697 \mathrm{E}-01 & 0.000 \mathrm{E}+00 & 0.000 \mathrm{E}+00 & 0.000 \mathrm{E}+00 & 0.000 \mathrm{E}+00 & 0.000 \mathrm{E}+00\end{array}$

$1.010 \mathrm{e}-05 \quad 0.0018 \quad 0.000 \mathrm{e}+00$

unspecified 4.000E-03 1.000E-01 2.000E-06 5.000E-03 $1.300 \mathrm{E}-02$

--Decaystep--1

--LimitChildren--1

--Children--5 
08/02/20 2:20:00 PM

RUND

C: \projects \fy2008\SMAccelerator \WindFi les \LOFL08.WND

INL

PO Box 1625

Idaho Falls

TAN-TSF

Receptors 60-62

$100.0000,500.0000$

$$
5002
$$

0024

0

800

0
1

1.00

$0.000 \mathrm{E}+000.000 \mathrm{E}$ 1

Be-7

$1.000 \mathrm{E}+00 \quad 0.000 \mathrm{E}+00$

1. $630 \mathrm{e}-06$

0.08

0.00

unspecified 4.000E-03

0.92

1.00

0.99
$\mathrm{T}$
$\mathrm{T}$

0.01

2.030e-01

0.00

0.00

16.30

0.00

ID

Feb 20, 2008 02:31 pm

RUND. 2008
Be-7D.dat $\mathrm{Be}-7$ 4 r. 0

$\begin{array}{rrrrr}00 & 00 & 00 & 00 & 00 \\ 0.000 \mathrm{e}+00 & 0.000 \mathrm{e}+00 & 0.000 \mathrm{e}+00 & 0.000 \mathrm{e}+00 & 0.000 \mathrm{e}+00\end{array}$ $0.000 \mathrm{E}+00 \quad 0.000 \mathrm{E}+00 \quad 0.000 \mathrm{E}+00$ 1

$\mathrm{Be}-7$

$15.000 \mathrm{e}-03 \quad 5.000 \mathrm{e}-03 \quad 5.480 \mathrm{e}-05$

00

9.999E-01 $0.000 \mathrm{E}+00 \quad 0.000 \mathrm{E}+00 \quad 0.000 \mathrm{E}+00 \quad 0.000 \mathrm{E}+00 \quad 0.000 \mathrm{E}+00$

$\begin{array}{lllllll}1.697 \mathrm{E}-01 & 0.000 \mathrm{E}+00 & 0.000 \mathrm{E}+00 & 0.000 \mathrm{E}+00 & 0.000 \mathrm{E}+00 & 0.000 \mathrm{E}+00\end{array}$

$1.010 \mathrm{e}-05 \quad 0.0018 \quad 0.000 \mathrm{e}+00$

unspecified 4.000E-03 1.000E-01 2.000E-06 5.000E-03 $1.300 \mathrm{E}-02$

--Decaystep--1

--LimitChildren--1

--Children--5 


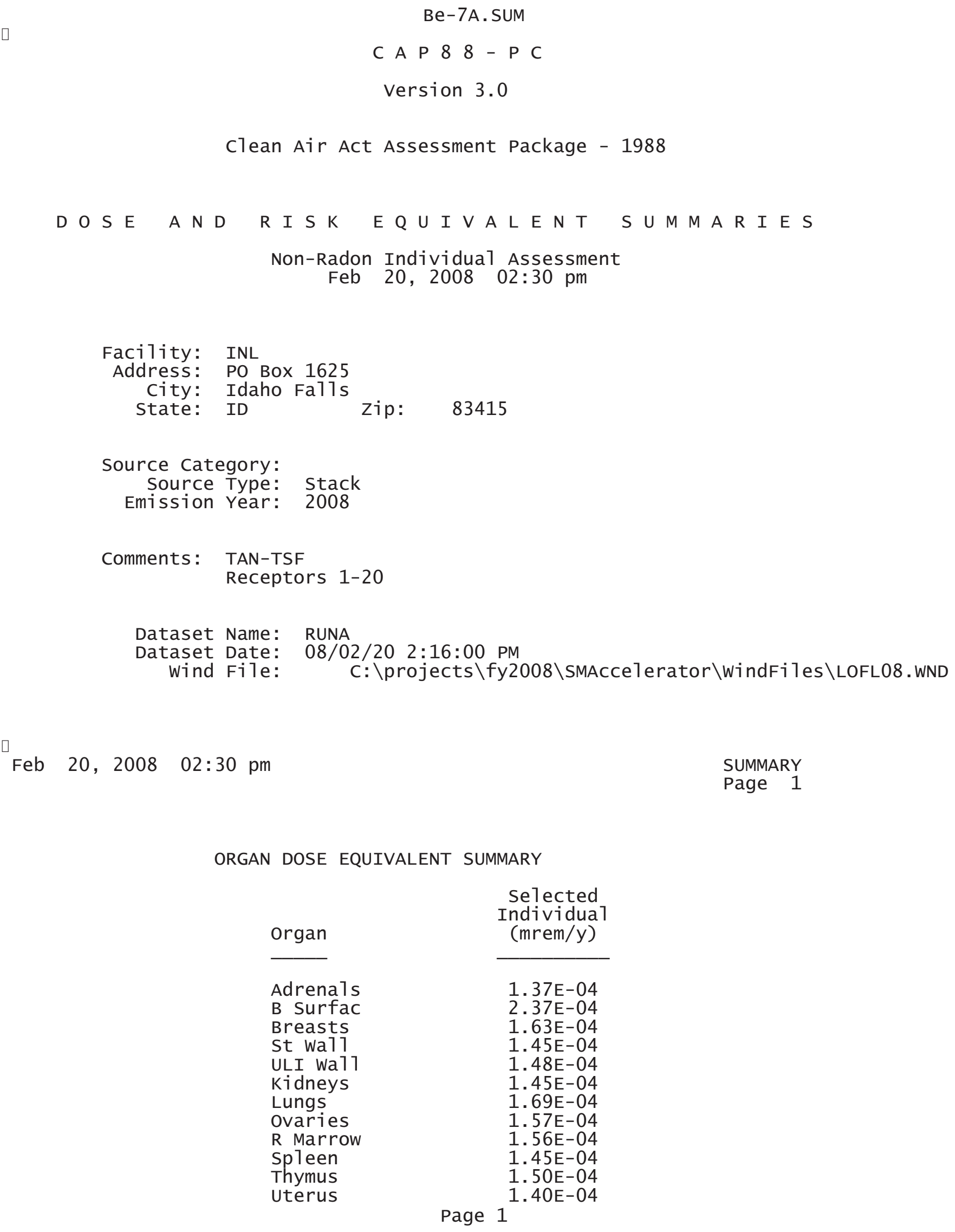

\begin{tabular}{lr} 
ORGAN DOSE EQUIVALENT SUMMARY \\
Selected \\
Organ & $\begin{array}{r}\text { Individual } \\
\text { (mrem/y) }\end{array}$ \\
\cline { 2 - 2 } Adrena1s & $1.37 \mathrm{E}-04$ \\
B Surfac & $2.37 \mathrm{E}-04$ \\
Breasts & $1.63 \mathrm{E}-04$ \\
St wa71 & $1.45 \mathrm{E}-04$ \\
ULI wa11 & $1.48 \mathrm{E}-04$ \\
Kidneys & $1.45 \mathrm{E}-04$ \\
Lungs & $1.69 \mathrm{E}-04$ \\
Ovaries & $1.57 \mathrm{E}-04$ \\
R Marrow & $1.56 \mathrm{E}-04$ \\
Spleen & $1.45 \mathrm{E}-04$ \\
Thymus & $1.50 \mathrm{E}-04$ \\
Uterus & $1.40 \mathrm{E}-04$ \\
& 1
\end{tabular}




$\begin{array}{lr} & \text { Be-7A.SUM } \\ \text { B1d wa11 } & 1.47 \mathrm{E}-04 \\ \text { Brain } & 1.42 \mathrm{E}-04 \\ \text { Esophagu } & 1.48 \mathrm{E}-04 \\ \text { SI wa11 } & 1.43 \mathrm{E}-04 \\ \text { LLI wa11 } & 1.56 \mathrm{E}-04 \\ \text { Liver } & 1.46 \mathrm{E}-04 \\ \text { Muscle } & 1.65 \mathrm{E}-04 \\ \text { Pancreas } & 1.34 \mathrm{E}-04 \\ \text { Skin } & 1.89 \mathrm{E}-04 \\ \text { Testes } & 1.66 \mathrm{E}-04 \\ \text { Thyroid } & 1.59 \mathrm{E}-04 \\ \text { EFFEC } & 1.59 \mathrm{E}-04\end{array}$

PATHWAY EFFECTIVE DOSE EQUIVALENT SUMMARY

\begin{tabular}{c}
$\begin{array}{c}\text { Selected } \\
\text { Individua1 } \\
\text { (mrem/y) }\end{array}$ \\
\hline $2.04 \mathrm{E}-06$ \\
$5.05 \mathrm{E}-06$ \\
$8.60 \mathrm{E}-07$ \\
$1.51 \mathrm{E}-04$ \\
$7.09 \mathrm{E}-06$ \\
$1.52 \mathrm{E}-04$ \\
$1.59 \mathrm{E}-04$
\end{tabular}
Feb 20, 2008 02:30 pm

NUCLIDE EFFECTIVE DOSE EQUIVALENT SUMMARY

Nuclide

Be-7

TOTAL

Feb 20, $2008 \quad 02: 30 \mathrm{pm}$

$$
\begin{gathered}
\begin{array}{c}
\text { Selected } \\
\text { Individua1 } \\
\text { (mrem/y) }
\end{array} \\
\hline 1.59 \mathrm{E}-04 \\
1.59 \mathrm{E}-04
\end{gathered}
$$$$
\text { 1. } 59 \mathrm{E}-04
$$$$
1.59 \mathrm{E}-04
$$
1. 59E-04
$1.59 \mathrm{E}-04$

1




$\begin{array}{lr} & \text { Be-7A.SUM } \\ \text { Stomach } & 5.89 \mathrm{E}-12 \\ \text { Colon } & 1.60 \mathrm{E}-11 \\ \text { Liver } & 2.22 \mathrm{E}-12 \\ \text { LUNG } & 1.67 \mathrm{E}-11 \\ \text { Bone } & 2.25 \mathrm{E}-13 \\ \text { Skin } & 1.89 \mathrm{E}-13 \\ \text { Breast } & 7.90 \mathrm{E}-12 \\ \text { Ovary } & 2.24 \mathrm{E}-12 \\ \text { Bladder } & 3.58 \mathrm{E}-12 \\ \text { Kidneys } & 7.53 \mathrm{E}-13 \\ \text { Thyroid } & 5.07 \mathrm{E}-13 \\ \text { Leukemia } & 8.78 \mathrm{E}-12 \\ \text { Residua1 } & 2.18 \mathrm{E}-11 \\ \text { Tota1 } & 8.82 \mathrm{E}-11 \\ & \\ \text { TOTAL } & 1.76 \mathrm{E}-10\end{array}$

PATHWAY RISK SUMMARY

\begin{tabular}{|c|c|}
\hline Pathway & $\begin{array}{c}\text { Total Lifetime } \\
\text { Fatal Cancer Risk }\end{array}$ \\
\hline $\begin{array}{l}\text { INGESTION } \\
\text { INHALATION } \\
\text { AIR IMMERSION } \\
\text { GROUND SURFACE } \\
\text { INTERNAL } \\
\text { EXTERNAL }\end{array}$ & $\begin{array}{l}1.41 \mathrm{E}-12 \\
3.69 \mathrm{E}-12 \\
4.68 \mathrm{E}-13 \\
8.27 \mathrm{E}-11 \\
5.10 \mathrm{E}-12 \\
8.31 \mathrm{E}-11\end{array}$ \\
\hline TOTAL & $8.82 \mathrm{E}-11$ \\
\hline
\end{tabular}

Feb 20, $200802: 30 \mathrm{pm} \quad \begin{aligned} & \text { SUMMARY } \\ & \text { Page } 4\end{aligned}$

NUCLIDE RISK SUMMARY

Nuclide

Be-7

TOTAL

$\square$

Feb 20, 2008 02:30 pm
Selected Individual

Total Lifetime Fatal Cancer Risk

$8.82 \mathrm{E}-11$

$8.82 \mathrm{E}-11$

SUMMARY

Page 5

INDIVIDUAL EFFECTIVE DOSE EQUIVALENT RATE (mrem/y) (A11 Radionuclides and Pathways)

Distance $(m)$ 


\begin{tabular}{|c|c|c|c|c|c|c|c|}
\hline Direction & 10344 & 10365 & 10472 & $\begin{array}{c}\text { Be-7A. } \\
10590\end{array}$ & JM 11103 & 11989 & 12522 \\
\hline $\mathrm{N}$ & $3.6 \mathrm{E}-05$ & $3.6 \mathrm{E}-05$ & $3.5 \mathrm{E}-05$ & $3.5 \mathrm{E}-05$ & $3.3 E-05$ & $3.0 \mathrm{E}-05$ & $2.8 \mathrm{E}-05$ \\
\hline NNW & $2.2 \mathrm{E}-05$ & $2.2 \mathrm{E}-05$ & $2.1 \mathrm{E}-05$ & $2.1 \mathrm{E}-05$ & $2.0 \mathrm{E}-05$ & $1.8 \mathrm{E}-05$ & $1.8 \mathrm{E}-05$ \\
\hline NW & $2.5 \mathrm{E}-05$ & $2.4 \mathrm{E}-05$ & $2.4 \mathrm{E}-05$ & $2.4 \mathrm{E}-05$ & $2.3 \mathrm{E}-05$ & $2.1 \mathrm{E}-05$ & 2. $0 \mathrm{E}-05$ \\
\hline WNW & $3.1 \mathrm{E}-05$ & $3.1 \mathrm{E}-05$ & $3.1 \mathrm{E}-05$ & $3.1 \mathrm{E}-05$ & $2.9 \mathrm{E}-05$ & $2.7 \mathrm{E}-05$ & 2. $5 \mathrm{E}-05$ \\
\hline W & $3.4 \mathrm{E}-05$ & $3.3 \mathrm{E}-05$ & $3.3 \mathrm{E}-05$ & $3.3 \mathrm{E}-05$ & $3.1 \mathrm{E}-05$ & $2.8 \mathrm{E}-05$ & $2.7 \mathrm{E}-05$ \\
\hline WSW & $5.3 \mathrm{E}-05$ & $5.3 \mathrm{E}-05$ & $5.2 \mathrm{E}-05$ & $5.1 \mathrm{E}-05$ & $4.9 \mathrm{E}-05$ & $4.4 \mathrm{E}-05$ & $4.2 \mathrm{E}-05$ \\
\hline SW & $8.4 \mathrm{E}-05$ & $8.4 \mathrm{E}-05$ & $8.3 \mathrm{E}-05$ & $8.1 \mathrm{E}-05$ & $7.7 \mathrm{E}-05$ & 7.0E-05 & $6.6 \mathrm{E}-05$ \\
\hline SSW & $1.1 \mathrm{E}-04$ & $1.1 \mathrm{E}-04$ & $1.1 \mathrm{E}-04$ & $1.1 \mathrm{E}-04$ & 1.0E-04 & $9.5 \mathrm{E}-05$ & $9.0 \mathrm{E}-05$ \\
\hline $\mathrm{S}$ & $1.6 \mathrm{E}-04$ & $1.6 \mathrm{E}-04$ & $1.6 \mathrm{E}-04$ & 1. $5 \mathrm{E}-04$ & 1. $5 \mathrm{E}-04$ & 1. 3E-04 & $1.2 \mathrm{E}-04$ \\
\hline SSE & 1. $4 \mathrm{E}-04$ & $1.4 \mathrm{E}-04$ & 1. 3E-04 & 1. 3E-04 & 1. 3E-04 & $1.1 \mathrm{E}-04$ & 1.1E-04 \\
\hline SE & $5.4 \mathrm{E}-05$ & $5.4 \mathrm{E}-05$ & $5.3 \mathrm{E}-05$ & $5.2 \mathrm{E}-05$ & $4.9 \mathrm{E}-05$ & $4.5 \mathrm{E}-05$ & $4.2 \mathrm{E}-05$ \\
\hline ESE & $3.4 \mathrm{E}-05$ & $3.4 \mathrm{E}-05$ & $3.3 \mathrm{E}-05$ & $3.3 \mathrm{E}-05$ & $3.1 \mathrm{E}-05$ & $2.8 \mathrm{E}-05$ & $2.7 \mathrm{E}-05$ \\
\hline E & 4.0E-05 & 4.0E-05 & 4.0E-05 & $3.9 \mathrm{E}-05$ & $3.7 \mathrm{E}-05$ & $3.3 \mathrm{E}-05$ & $3.2 \mathrm{E}-05$ \\
\hline ENE & $4.8 \mathrm{E}-05$ & $4.7 \mathrm{E}-05$ & $4.7 \mathrm{E}-05$ & $4.6 \mathrm{E}-05$ & $4.4 \mathrm{E}-05$ & 4.0E-05 & $3.8 \mathrm{E}-05$ \\
\hline NE & $8.5 E-05$ & $8.5 E-05$ & $8.4 \mathrm{E}-05$ & $8.3 \mathrm{E}-05$ & $7.8 \mathrm{E}-05$ & $7.1 \mathrm{E}-05$ & $6.7 \mathrm{E}-05$ \\
\hline NNE & $5.2 \mathrm{E}-05$ & $5.2 \mathrm{E}-05$ & $5.1 \mathrm{E}-05$ & $5.0 \mathrm{E}-05$ & $4.8 \mathrm{E}-05$ & $4.3 \mathrm{E}-05$ & $4.1 \mathrm{E}-05$ \\
\hline
\end{tabular}

Distance (m)

\begin{tabular}{|c|c|c|c|c|c|c|c|}
\hline Direction & n 13245 & 13286 & 13483 & 13612 & 13664 & 13959 & 14258 \\
\hline $\begin{array}{r}\text { N } \\
\text { NNW } \\
N W \\
\text { WNW } \\
W \\
\text { WSW } \\
\text { SW } \\
\text { SSW } \\
\text { S } \\
\text { SSE } \\
\text { SE } \\
\text { ESE } \\
\text { E } \\
\text { ENE } \\
\text { NE } \\
\text { NNE }\end{array}$ & $\begin{array}{l}2.7 \mathrm{E}-05 \\
1.7 \mathrm{E}-05 \\
1.9 \mathrm{E}-05 \\
2.4 \mathrm{E}-05 \\
2.5 \mathrm{E}-05 \\
3.9 \mathrm{E}-05 \\
6.2 \mathrm{E}-05 \\
8.4 \mathrm{E}-05 \\
1.2 \mathrm{E}-04 \\
1.0 \mathrm{E}-04 \\
3.9 \mathrm{E}-05 \\
2.5 \mathrm{E}-05 \\
2.9 \mathrm{E}-05 \\
3.5 \mathrm{E}-05 \\
6.3 \mathrm{E}-05 \\
3.8 \mathrm{E}-05\end{array}$ & $\begin{array}{l}2.6 \mathrm{E}-05 \\
1.6 \mathrm{E}-05 \\
1.9 \mathrm{E}-05 \\
2.4 \mathrm{E}-05 \\
2.5 \mathrm{E}-05 \\
3.9 \mathrm{E}-05 \\
6.2 \mathrm{E}-05 \\
8.4 \mathrm{E}-05 \\
1.2 \mathrm{E}-04 \\
1.0 \mathrm{E}-04 \\
3.9 \mathrm{E}-05 \\
2.5 \mathrm{E}-05 \\
2.9 \mathrm{E}-05 \\
3.5 \mathrm{E}-05 \\
6.2 \mathrm{E}-05 \\
3.8 \mathrm{E}-05\end{array}$ & $\begin{array}{l}2.6 \mathrm{E}-05 \\
1.6 \mathrm{E}-05 \\
1.8 \mathrm{E}-05 \\
2.3 \mathrm{E}-05 \\
2.5 \mathrm{E}-05 \\
3.8 \mathrm{E}-05 \\
6.1 \mathrm{E}-05 \\
8.2 \mathrm{E}-05 \\
1.1 \mathrm{E}-04 \\
9.9 \mathrm{E}-05 \\
3.8 \mathrm{E}-05 \\
2.4 \mathrm{E}-05 \\
2.9 \mathrm{E}-05 \\
3.4 \mathrm{E}-05 \\
6.1 \mathrm{E}-05 \\
3.8 \mathrm{E}-05\end{array}$ & $\begin{array}{l}2.6 \mathrm{E}-05 \\
1.6 \mathrm{E}-05 \\
1.8 \mathrm{E}-05 \\
2.3 \mathrm{E}-05 \\
2.4 \mathrm{E}-05 \\
3.8 \mathrm{E}-05 \\
6.0 \mathrm{E}-05 \\
8.1 \mathrm{E}-05 \\
1.1 \mathrm{E}-04 \\
9.7 \mathrm{E}-05 \\
3.8 \mathrm{E}-05 \\
2.4 \mathrm{E}-05 \\
2.8 \mathrm{E}-05 \\
3.4 \mathrm{E}-05 \\
6.0 \mathrm{E}-05 \\
3.7 \mathrm{E}-05\end{array}$ & $\begin{array}{l}2.6 \mathrm{E}-05 \\
1.6 \mathrm{E}-05 \\
1.8 \mathrm{E}-05 \\
2.3 \mathrm{E}-05 \\
2.4 \mathrm{E}-05 \\
3.8 \mathrm{E}-05 \\
6.0 \mathrm{E}-05 \\
8.1 \mathrm{E}-05 \\
1.1 \mathrm{E}-04 \\
9.7 \mathrm{E}-05 \\
3.8 \mathrm{E}-05 \\
2.4 \mathrm{E}-05 \\
2.8 \mathrm{E}-05 \\
3.4 \mathrm{E}-05 \\
6.0 \mathrm{E}-05 \\
3.7 \mathrm{E}-05\end{array}$ & $\begin{array}{l}2.5 \mathrm{E}-05 \\
1.6 \mathrm{E}-05 \\
1.8 \mathrm{E}-05 \\
2.2 \mathrm{E}-05 \\
2.4 \mathrm{E}-05 \\
3.7 \mathrm{E}-05 \\
5.8 \mathrm{E}-05 \\
7.9 \mathrm{E}-05 \\
1.1 \mathrm{E}-04 \\
9.4 \mathrm{E}-05 \\
3.7 \mathrm{E}-05 \\
2.3 \mathrm{E}-05 \\
2.7 \mathrm{E}-05 \\
3.3 \mathrm{E}-05 \\
5.9 \mathrm{E}-05 \\
3.6 \mathrm{E}-05\end{array}$ & $\begin{array}{l}2.4 \mathrm{E}-05 \\
1.5 \mathrm{E}-05 \\
1.7 \mathrm{E}-05 \\
2.2 \mathrm{E}-05 \\
2.3 \mathrm{E}-05 \\
3.6 \mathrm{E}-05 \\
5.7 \mathrm{E}-05 \\
7.6 \mathrm{E}-05 \\
1.1 \mathrm{E}-04 \\
9.2 \mathrm{E}-05 \\
3.6 \mathrm{E}-05 \\
2.3 \mathrm{E}-05 \\
2.7 \mathrm{E}-05 \\
3.2 \mathrm{E}-05 \\
5.7 \mathrm{E}-05 \\
3.5 \mathrm{E}-05\end{array}$ \\
\hline
\end{tabular}

$\square$

Feb 20, 2008 02:30 pm

SUMMARY

Page 6

INDIVIDUAL EFFECTIVE DOSE EQUIVALENT RATE (mrem/y) (A11 Radionuclides and Pathways)

\begin{tabular}{|c|c|c|c|c|c|c|}
\hline \multirow[b]{2}{*}{ Direction } & \multicolumn{6}{|c|}{ Distance (m) } \\
\hline & 14374 & 15241 & 15441 & 15784 & 15844 & 16323 \\
\hline $\begin{array}{r}N \\
N N W\end{array}$ & $\begin{array}{l}2.4 \mathrm{E}-05 \\
1.5 \mathrm{E}-05\end{array}$ & $\begin{array}{l}\text { 2. } 2 \mathrm{E}-05 \\
1.4 \mathrm{E}-05\end{array}$ & $\begin{array}{l}2.2 \mathrm{E}-05 \\
1.4 \mathrm{E}-05\end{array}$ & $\begin{array}{r}2.1 \mathrm{E}-05 \\
1.4 \mathrm{E}-05 \\
\text { Page }\end{array}$ & $\begin{array}{l}\text { 2. } 1 \mathrm{E}-05 \\
1.4 \mathrm{E}-05\end{array}$ & $\begin{array}{l}2.1 \mathrm{E}-05 \\
1.3 \mathrm{E}-05\end{array}$ \\
\hline
\end{tabular}




\begin{tabular}{rllllll} 
& \multicolumn{5}{c}{ Be-7A.SUM } \\
NW & $1.7 \mathrm{E}-05$ & $1.6 \mathrm{E}-05$ & $1.6 \mathrm{E}-05$ & $1.5 \mathrm{E}-05$ & $1.5 \mathrm{E}-05$ & $1.5 \mathrm{E}-05$ \\
WNW & $2.2 \mathrm{E}-05$ & $2.0 \mathrm{E}-05$ & $2.0 \mathrm{E}-05$ & $1.9 \mathrm{E}-05$ & $1.9 \mathrm{E}-05$ & $1.9 \mathrm{E}-05$ \\
$\mathrm{~W}$ & $2.3 \mathrm{E}-05$ & $2.1 \mathrm{E}-05$ & $2.1 \mathrm{E}-05$ & $2.0 \mathrm{E}-05$ & $2.0 \mathrm{E}-05$ & $2.0 \mathrm{E}-05$ \\
WSW & $3.6 \mathrm{E}-05$ & $3.3 \mathrm{E}-05$ & $3.3 \mathrm{E}-05$ & $3.2 \mathrm{E}-05$ & $3.2 \mathrm{E}-05$ & $3.1 \mathrm{E}-05$ \\
$\mathrm{SW}$ & $5.6 \mathrm{E}-05$ & $5.2 \mathrm{E}-05$ & $5.1 \mathrm{E}-05$ & $5.0 \mathrm{E}-05$ & $5.0 \mathrm{E}-05$ & $4.8 \mathrm{E}-05$ \\
$\mathrm{SSW}$ & $7.6 \mathrm{E}-05$ & $7.0 \mathrm{E}-05$ & $6.9 \mathrm{E}-05$ & $6.7 \mathrm{E}-05$ & $6.7 \mathrm{E}-05$ & $6.4 \mathrm{E}-05$ \\
$\mathrm{~S}$ & $1.0 \mathrm{E}-04$ & $9.6 \mathrm{E}-05$ & $9.5 \mathrm{E}-05$ & $9.2 \mathrm{E}-05$ & $9.2 \mathrm{E}-05$ & $8.8 \mathrm{E}-05$ \\
$\mathrm{SSE}$ & $9.1 \mathrm{E}-05$ & $8.4 \mathrm{E}-05$ & $8.3 \mathrm{E}-05$ & $8.1 \mathrm{E}-05$ & $8.0 \mathrm{E}-05$ & $7.7 \mathrm{E}-05$ \\
$\mathrm{SE}$ & $3.5 \mathrm{E}-05$ & $3.3 \mathrm{E}-05$ & $3.2 \mathrm{E}-05$ & $3.1 \mathrm{E}-05$ & $3.1 \mathrm{E}-05$ & $3.0 \mathrm{E}-05$ \\
$\mathrm{ESE}$ & $2.2 \mathrm{E}-05$ & $2.1 \mathrm{E}-05$ & $2.0 \mathrm{E}-05$ & $2.0 \mathrm{E}-05$ & $2.0 \mathrm{E}-05$ & $1.9 \mathrm{E}-05$ \\
$\mathrm{E}$ & $2.6 \mathrm{E}-05$ & $2.4 \mathrm{E}-05$ & $2.4 \mathrm{E}-05$ & $2.3 \mathrm{E}-05$ & $2.3 \mathrm{E}-05$ & $2.2 \mathrm{E}-05$ \\
$\mathrm{ENE}$ & $3.2 \mathrm{E}-05$ & $2.9 \mathrm{E}-05$ & $2.9 \mathrm{E}-05$ & $2.8 \mathrm{E}-05$ & $2.8 \mathrm{E}-05$ & $2.7 \mathrm{E}-05$ \\
$\mathrm{NE}$ & $5.6 \mathrm{E}-05$ & $5.2 \mathrm{E}-05$ & $5.2 \mathrm{E}-05$ & $5.0 \mathrm{E}-05$ & $5.0 \mathrm{E}-05$ & $4.8 \mathrm{E}-05$ \\
$\mathrm{NNE}$ & $3.5 \mathrm{E}-05$ & $3.2 \mathrm{E}-05$ & $3.2 \mathrm{E}-05$ & $3.1 \mathrm{E}-05$ & $3.1 \mathrm{E}-05$ & $3.0 \mathrm{E}-05$
\end{tabular}

Feb 20, $2008 \quad 02: 30 \mathrm{pm}$

SUMMARY

Page 7

INDIVIDUAL LIFETIME RISK (deaths)

(A11 Radionuclides and Pathways)

\begin{tabular}{|c|c|c|c|c|c|c|c|}
\hline \multirow[b]{2}{*}{ Direction } & \multicolumn{6}{|c|}{ Distance $(\mathrm{m})$} & \multirow[b]{2}{*}{12522} \\
\hline & 10344 & 10365 & 10472 & 10590 & 11103 & 11989 & \\
\hline $\begin{array}{r}N \\
N N W \\
N W \\
W N W \\
W \\
W S W \\
S W \\
S S W \\
S \\
S S E \\
S E \\
\text { ESE } \\
E \\
\text { ENE } \\
N E \\
N N E\end{array}$ & $\begin{array}{l}2.0 \mathrm{E}-11 \\
1.2 \mathrm{E}-11 \\
1.4 \mathrm{E}-11 \\
1.8 \mathrm{E}-11 \\
1.9 \mathrm{E}-11 \\
2.9 \mathrm{E}-11 \\
4.6 \mathrm{E}-11 \\
6.4 \mathrm{E}-11 \\
8.8 \mathrm{E}-11 \\
7.6 \mathrm{E}-11 \\
3.0 \mathrm{E}-11 \\
1.9 \mathrm{E}-11 \\
2.2 \mathrm{E}-11 \\
2.6 \mathrm{E}-11 \\
4.7 \mathrm{E}-11 \\
2.9 \mathrm{E}-11\end{array}$ & $\begin{array}{l}2.0 \mathrm{E}-11 \\
1.2 \mathrm{E}-11 \\
1.4 \mathrm{E}-11 \\
1.8 \mathrm{E}-11 \\
1.9 \mathrm{E}-11 \\
2.9 \mathrm{E}-11 \\
4.6 \mathrm{E}-11 \\
6.3 \mathrm{E}-11 \\
8.8 \mathrm{E}-11 \\
7.6 \mathrm{E}-11 \\
3.0 \mathrm{E}-11 \\
1.9 \mathrm{E}-11 \\
2.2 \mathrm{E}-11 \\
2.6 \mathrm{E}-11 \\
4.7 \mathrm{E}-11 \\
2.9 \mathrm{E}-11\end{array}$ & $\begin{array}{l}2.0 \mathrm{E}-11 \\
1.2 \mathrm{E}-11 \\
1.4 \mathrm{E}-11 \\
1.7 \mathrm{E}-11 \\
1.8 \mathrm{E}-11 \\
2.9 \mathrm{E}-11 \\
4.6 \mathrm{E}-11 \\
6.3 \mathrm{E}-11 \\
8.7 \mathrm{E}-11 \\
7.5 \mathrm{E}-11 \\
3.0 \mathrm{E}-11 \\
1.9 \mathrm{E}-11 \\
2.2 \mathrm{E}-11 \\
2.6 \mathrm{E}-11 \\
4.6 \mathrm{E}-11 \\
2.8 \mathrm{E}-11\end{array}$ & $\begin{array}{l}1.9 \mathrm{E}-11 \\
1.2 \mathrm{E}-11 \\
1.3 \mathrm{E}-11 \\
1.7 \mathrm{E}-11 \\
1.8 \mathrm{E}-11 \\
2.9 \mathrm{E}-11 \\
4.5 \mathrm{E}-11 \\
6.2 \mathrm{E}-11 \\
8.6 \mathrm{E}-11 \\
7.4 \mathrm{E}-11 \\
2.9 \mathrm{E}-11 \\
1.8 \mathrm{E}-11 \\
2.2 \mathrm{E}-11 \\
2.6 \mathrm{E}-11 \\
4.6 \mathrm{E}-11 \\
2.8 \mathrm{E}-11\end{array}$ & $\begin{array}{l}1.8 \mathrm{E}-11 \\
1.1 \mathrm{E}-11 \\
1.3 \mathrm{E}-11 \\
1.6 \mathrm{E}-11 \\
1.7 \mathrm{E}-11 \\
2.7 \mathrm{E}-11 \\
4.3 \mathrm{E}-11 \\
5.8 \mathrm{E}-11 \\
8.1 \mathrm{E}-11 \\
7.0 \mathrm{E}-11 \\
2.7 \mathrm{E}-11 \\
1.7 \mathrm{E}-11 \\
2.1 \mathrm{E}-11 \\
2.4 \mathrm{E}-11 \\
4.3 \mathrm{E}-11 \\
2.6 \mathrm{E}-11\end{array}$ & $\begin{array}{l}1.7 \mathrm{E}-11 \\
1.0 \mathrm{E}-11 \\
1.2 \mathrm{E}-11 \\
1.5 \mathrm{E}-11 \\
1.6 \mathrm{E}-11 \\
2.5 \mathrm{E}-11 \\
3.9 \mathrm{E}-11 \\
5.3 \mathrm{E}-11 \\
7.3 \mathrm{E}-11 \\
6.3 \mathrm{E}-11 \\
2.5 \mathrm{E}-11 \\
1.6 \mathrm{E}-11 \\
1.9 \mathrm{E}-11 \\
2.2 \mathrm{E}-11 \\
3.9 \mathrm{E}-11 \\
2.4 \mathrm{E}-11\end{array}$ & $\begin{array}{l}1.6 \mathrm{E}-11 \\
9.9 \mathrm{E}-12 \\
1.1 \mathrm{E}-11 \\
1.4 \mathrm{E}-11 \\
1.5 \mathrm{E}-11 \\
2.3 \mathrm{E}-11 \\
3.7 \mathrm{E}-11 \\
5.0 \mathrm{E}-11 \\
6.9 \mathrm{E}-11 \\
6.0 \mathrm{E}-11 \\
2.4 \mathrm{E}-11 \\
1.5 \mathrm{E}-11 \\
1.8 \mathrm{E}-11 \\
2.1 \mathrm{E}-11 \\
3.7 \mathrm{E}-11 \\
2.3 \mathrm{E}-11\end{array}$ \\
\hline \multicolumn{8}{|c|}{ Distance $(\mathrm{m})$} \\
\hline Direction & 13245 & 13286 & 13483 & 13612 & 13664 & 13959 & 14258 \\
\hline $\begin{array}{r}\mathrm{N} \\
\mathrm{NNW} \\
\mathrm{NW} \\
\text { WNW } \\
W \\
\text { WSW } \\
\text { SW } \\
\text { SSW }\end{array}$ & $\begin{array}{l}1.5 \mathrm{E}-11 \\
9.3 \mathrm{E}-12 \\
1.0 \mathrm{E}-11 \\
1.3 \mathrm{E}-11 \\
1.4 \mathrm{E}-11 \\
2.2 \mathrm{E}-11 \\
3.4 \mathrm{E}-11 \\
4.7 \mathrm{E}-11\end{array}$ & $\begin{array}{l}1.5 \mathrm{E}-11 \\
9.3 \mathrm{E}-12 \\
1.0 \mathrm{E}-11 \\
1.3 \mathrm{E}-11 \\
1.4 \mathrm{E}-11 \\
2.2 \mathrm{E}-11 \\
3.4 \mathrm{E}-11 \\
4.6 \mathrm{E}-11\end{array}$ & $\begin{array}{l}1.5 \mathrm{E}-11 \\
9.2 \mathrm{E}-12 \\
1.0 \mathrm{E}-11 \\
1.3 \mathrm{E}-11 \\
1.4 \mathrm{E}-11 \\
2.1 \mathrm{E}-11 \\
3.4 \mathrm{E}-11 \\
4.6 \mathrm{E}-11\end{array}$ & $\begin{array}{r}1.4 \mathrm{E}-11 \\
9.1 \mathrm{E}-12 \\
1.0 \mathrm{E}-11 \\
1.3 \mathrm{E}-11 \\
1.4 \mathrm{E}-11 \\
2.1 \mathrm{E}-11 \\
3.3 \mathrm{E}-11 \\
4.5 \mathrm{E}-11 \\
\text { Page }\end{array}$ & $\begin{array}{l}1.4 \mathrm{E}-11 \\
9.0 \mathrm{E}-12 \\
1.0 \mathrm{E}-11 \\
1.3 \mathrm{E}-11 \\
1.4 \mathrm{E}-11 \\
2.1 \mathrm{E}-11 \\
3.3 \mathrm{E}-11 \\
4.5 \mathrm{E}-11\end{array}$ & $\begin{array}{l}1.4 \mathrm{E}-11 \\
8.8 \mathrm{E}-12 \\
9.9 \mathrm{E}-12 \\
1.3 \mathrm{E}-11 \\
1.3 \mathrm{E}-11 \\
2.1 \mathrm{E}-11 \\
3.2 \mathrm{E}-11 \\
4.4 \mathrm{E}-11\end{array}$ & $\begin{array}{l}1.4 \mathrm{E}-11 \\
8.6 \mathrm{E}-12 \\
9.7 \mathrm{E}-12 \\
1.2 \mathrm{E}-11 \\
1.3 \mathrm{E}-11 \\
2.0 \mathrm{E}-11 \\
3.1 \mathrm{E}-11 \\
4.2 \mathrm{E}-11\end{array}$ \\
\hline
\end{tabular}




\begin{tabular}{rlllllll}
\multicolumn{7}{c}{ Be-7A.SUM } \\
S & $6.4 \mathrm{E}-11$ & $6.4 \mathrm{E}-11$ & $6.3 \mathrm{E}-11$ & $6.2 \mathrm{E}-11$ & $6.2 \mathrm{E}-11$ & $6.0 \mathrm{E}-11$ & $5.8 \mathrm{E}-11$ \\
SSE & $5.6 \mathrm{E}-11$ & $5.6 \mathrm{E}-11$ & $5.5 \mathrm{E}-11$ & $5.4 \mathrm{E}-11$ & $5.4 \mathrm{E}-11$ & $5.2 \mathrm{E}-11$ & $5.1 \mathrm{E}-11$ \\
$\mathrm{SE}$ & $2.2 \mathrm{E}-11$ & $2.2 \mathrm{E}-11$ & $2.2 \mathrm{E}-11$ & $2.1 \mathrm{E}-11$ & $2.1 \mathrm{E}-11$ & $2.1 \mathrm{E}-11$ & $2.0 \mathrm{E}-11$ \\
$\mathrm{ESE}$ & $1.4 \mathrm{E}-11$ & $1.4 \mathrm{E}-11$ & $1.4 \mathrm{E}-11$ & $1.4 \mathrm{E}-11$ & $1.3 \mathrm{E}-11$ & $1.3 \mathrm{E}-11$ & $1.3 \mathrm{E}-11$ \\
$\mathrm{E}$ & $1.6 \mathrm{E}-11$ & $1.6 \mathrm{E}-11$ & $1.6 \mathrm{E}-11$ & $1.6 \mathrm{E}-11$ & $1.6 \mathrm{E}-11$ & $1.5 \mathrm{E}-11$ & $1.5 \mathrm{E}-11$ \\
$\mathrm{ENE}$ & $2.0 \mathrm{E}-11$ & $2.0 \mathrm{E}-11$ & $1.9 \mathrm{E}-11$ & $1.9 \mathrm{E}-11$ & $1.9 \mathrm{E}-11$ & $1.8 \mathrm{E}-11$ & $1.8 \mathrm{E}-11$ \\
$\mathrm{NE}$ & $3.5 \mathrm{E}-11$ & $3.5 \mathrm{E}-11$ & $3.4 \mathrm{E}-11$ & $3.4 \mathrm{E}-11$ & $3.3 \mathrm{E}-11$ & $3.3 \mathrm{E}-11$ & $3.2 \mathrm{E}-11$ \\
$\mathrm{NNE}$ & $2.1 \mathrm{E}-11$ & $2.1 \mathrm{E}-11$ & $2.1 \mathrm{E}-11$ & $2.1 \mathrm{E}-11$ & $2.1 \mathrm{E}-11$ & $2.0 \mathrm{E}-11$ & $2.0 \mathrm{E}-11$
\end{tabular}

Feb 20, $2008 \quad 02: 30 \mathrm{pm}$

SUMMARY

Page 8

INDIVIDUAL LIFETIME RISK (deaths)

(A11 Radionuclides and Pathways)

\begin{tabular}{|c|c|c|c|c|c|c|}
\hline \multirow[b]{2}{*}{ Direction } & \multicolumn{6}{|c|}{ Distance $(\mathrm{m})$} \\
\hline & 14374 & 15241 & 15441 & 15784 & 15844 & 16323 \\
\hline $\mathrm{N}$ & 1. $4 \mathrm{E}-11$ & 1. $3 \mathrm{E}-11$ & 1. $2 \mathrm{E}-11$ & 1. $2 \mathrm{E}-11$ & $1.2 \mathrm{E}-11$ & $1.2 \mathrm{E}-11$ \\
\hline NNW & $\begin{array}{l}1.4 \mathrm{E}-1 \mathrm{I} \\
8.6 \mathrm{E}-12\end{array}$ & 8.0E-12 & $\begin{array}{l}1.2 \mathrm{E}-11 \\
7.9 \mathrm{E}-12\end{array}$ & $\frac{1}{7 .} \cdot 7 \mathrm{E}-12$ & $\frac{1}{7.25-1 \perp}$ & $\begin{array}{l}1.2 \mathrm{E}-1 \mathrm{I} \\
7.5 \mathrm{E}-12\end{array}$ \\
\hline NW & $9.6 \mathrm{E}-12$ & $9.0 \mathrm{E}-12$ & $8.8 \mathrm{E}-12$ & $8.6 \mathrm{E}-12$ & $8.6 \mathrm{E}-12$ & 8. $3 \mathrm{E}-12$ \\
\hline WNW & $1.2 \mathrm{E}-11$ & $1.1 \mathrm{E}-11$ & $1.1 \mathrm{E}-11$ & $1.1 \mathrm{E}-11$ & $1.1 \mathrm{E}-11$ & 1. $0 \mathrm{E}-11$ \\
\hline W & $1.3 \mathrm{E}-11$ & $1.2 \mathrm{E}-11$ & $1.2 \mathrm{E}-11$ & $1.1 \mathrm{E}-11$ & $1.1 \mathrm{E}-11$ & $1.1 \mathrm{E}-11$ \\
\hline WSW & $2.0 \mathrm{E}-11$ & $1.9 \mathrm{E}-11$ & $1.8 \mathrm{E}-11$ & $1.8 \mathrm{E}-11$ & $1.8 \mathrm{E}-11$ & $1.7 \mathrm{E}-11$ \\
\hline SW & $3.1 \mathrm{E}-11$ & $2.9 \mathrm{E}-11$ & $2.9 \mathrm{E}-11$ & $2.8 \mathrm{E}-11$ & $2.8 \mathrm{E}-11$ & $2.7 \mathrm{E}-11$ \\
\hline SSW & $4.2 \mathrm{E}-11$ & $3.9 \mathrm{E}-11$ & $3.8 \mathrm{E}-11$ & $3.7 \mathrm{E}-11$ & $3.7 \mathrm{E}-11$ & $3.6 \mathrm{E}-11$ \\
\hline $\mathrm{S}$ & $5.8 \mathrm{E}-11$ & $5.4 \mathrm{E}-11$ & $5.3 \mathrm{E}-11$ & $5.1 \mathrm{E}-11$ & $5.1 \mathrm{E}-11$ & $4.9 \mathrm{E}-11$ \\
\hline SSE & $5.0 \mathrm{E}-11$ & $4.7 \mathrm{E}-11$ & $4.6 \mathrm{E}-11$ & $4.5 \mathrm{E}-11$ & $4.5 \mathrm{E}-11$ & 4. $3 \mathrm{E}-11$ \\
\hline SE & $2.0 \mathrm{E}-11$ & $1.8 \mathrm{E}-11$ & $1.8 \mathrm{E}-11$ & $1.8 \mathrm{E}-11$ & $1.7 \mathrm{E}-11$ & $1.7 \mathrm{E}-11$ \\
\hline ESE & $1.3 \mathrm{E}-11$ & $1.2 \mathrm{E}-11$ & $1.2 \mathrm{E}-11$ & $1.1 \mathrm{E}-11$ & $1.1 \mathrm{E}-11$ & $1.1 \mathrm{E}-11$ \\
\hline & $1.5 \mathrm{E}-11$ & $1.4 \mathrm{E}-11$ & $1.4 \mathrm{E}-11$ & $1.3 \mathrm{E}-11$ & $1.3 \mathrm{E}-11$ & $1.3 \mathrm{E}-11$ \\
\hline ENE & $1.8 \mathrm{E}-11$ & $1.6 \mathrm{E}-11$ & $1.6 \mathrm{E}-11$ & $1.6 \mathrm{E}-11$ & $1.6 \mathrm{E}-11$ & $1.5 \mathrm{E}-11$ \\
\hline NE & $3.1 \mathrm{E}-11$ & $2.9 \mathrm{E}-11$ & $2.9 \mathrm{E}-11$ & $2.8 \mathrm{E}-11$ & $2.8 \mathrm{E}-11$ & $2.7 \mathrm{E}-11$ \\
\hline NNE & $1.9 \mathrm{E}-11$ & $1.8 \mathrm{E}-11$ & $1.8 \mathrm{E}-11$ & $1.7 \mathrm{E}-11$ & $1.7 \mathrm{E}-11$ & $1.7 \mathrm{E}-11$ \\
\hline
\end{tabular}




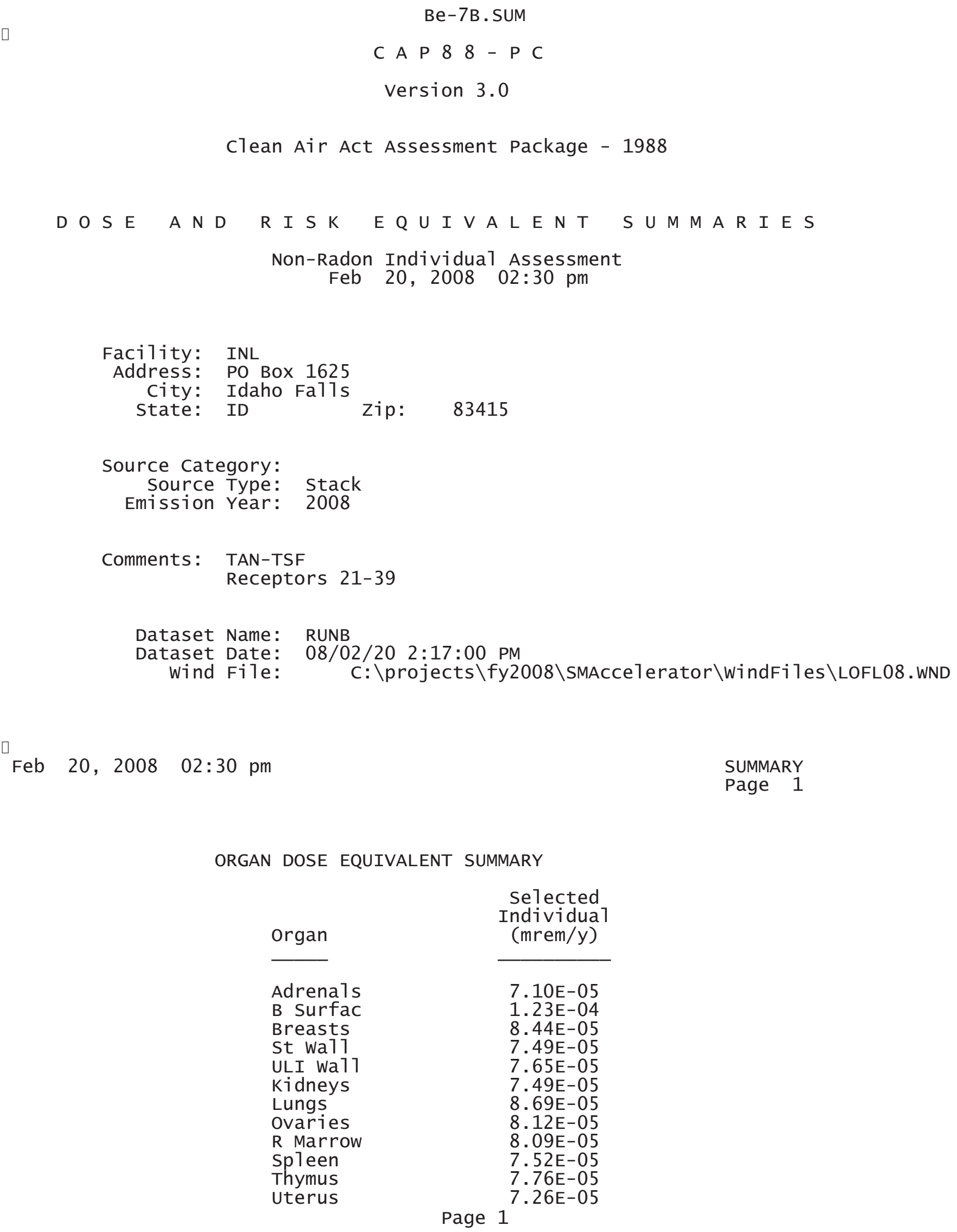

ORGAN DOSE EQUIVALENT SUMMARY

Organ

Adrenals

B Surfac Breasts

St wa11

ULI Wa11

Kidneys

Lungs

ovaries

R Marrow

spleen

Thymus

uterus

$$
\begin{gathered}
\text { Selected } \\
\text { Individual } \\
\text { (mrem/y) } \\
\hline \\
7.10 \mathrm{E}-05 \\
1.23 \mathrm{E}-04 \\
8.44 \mathrm{E}-05 \\
7.49 \mathrm{E}-05 \\
7.65 \mathrm{E}-05 \\
7.49 \mathrm{E}-05 \\
8.69 \mathrm{E}-05 \\
8.12 \mathrm{E}-05 \\
8.09 \mathrm{E}-05 \\
7.52 \mathrm{E}-05 \\
7.76 \mathrm{E}-05 \\
7.26 \mathrm{E}-05
\end{gathered}
$$$$
7.76 \mathrm{E}-05
$$

Page 1 


$\begin{array}{lr} & \text { Be-7B.SUM } \\ \text { Bld wa11 } & 7.61 \mathrm{E}-05 \\ \text { Brain } & 7.37 \mathrm{E}-05 \\ \text { Esophagu } & 7.58 \mathrm{E}-05 \\ \text { SI Wa11 } & 7.39 \mathrm{E}-05 \\ \text { LLI wa11 } & 8.04 \mathrm{E}-05 \\ \text { Liver } & 7.54 \mathrm{E}-05 \\ \text { Muscle } & 8.57 \mathrm{E}-05 \\ \text { Pancreas } & 6.92 \mathrm{E}-05 \\ \text { Skin } & 9.81 \mathrm{E}-05 \\ \text { Testes } & 8.61 \mathrm{E}-05 \\ \text { Thyroid } & 8.25 \mathrm{E}-05 \\ & \\ \text { EFFEC } & 8.23 \mathrm{E}-05\end{array}$

PATHWAY EFFECTIVE DOSE EQUIVALENT SUMMARY

\begin{tabular}{lcc} 
Pathway & $\begin{array}{c}\text { Selected } \\
\text { Individua } \\
\text { (mrem/y) }\end{array}$ \\
\cline { 2 - 3 } & $1.04 \mathrm{E}-06$ \\
INGESTION & $2.44 \mathrm{E}-06$ \\
INHALATION & $4.16 \mathrm{E}-07$ \\
AIR IMMERSION & $7.84 \mathrm{E}-05$ & \\
GROUND SURFACE & $3.48 \mathrm{E}-06$ & \\
INTERNAL & $7.88 \mathrm{E}-05$ & \\
EXTERNAL & $8.23 \mathrm{E}-05$ & SUMMARY \\
TOTAL & & Page 2
\end{tabular}

NUCLIDE EFFECTIVE DOSE EQUIVALENT SUMMARY

Nuclide

Be-7

TOTAL

Feb 20, $2008 \quad 02: 30$ pm

$$
\begin{gathered}
\begin{array}{c}
\text { Selected } \\
\text { Individual } \\
\text { (mrem/y) }
\end{array} \\
\hline 8.23 \mathrm{E}-05 \\
8.23 \mathrm{E}-05
\end{gathered}
$$

SUMMARY

Page 3

CANCER RISK SUMMARY

Cancer

Esophagu
Selected Individual

Total Lifetime Fatal Cancer Risk

$$
\text { Page } 2^{7.92 \mathrm{E}-13}
$$




$\begin{array}{lr} & \text { Be-7B.SUM } \\ \text { Stomach } & 3.05 \mathrm{E}-12 \\ \text { Colon } & 8.28 \mathrm{E}-12 \\ \text { Liver } & 1.15 \mathrm{E}-12 \\ \text { LUNG } & 8.58 \mathrm{E}-12 \\ \text { Bone } & 1.17 \mathrm{E}-13 \\ \text { Skin } & 9.80 \mathrm{E}-14 \\ \text { Breast } & 4.09 \mathrm{E}-12 \\ \text { Ovary } & 1.16 \mathrm{E}-12 \\ \text { Bladder } & 1.85 \mathrm{E}-12 \\ \text { Kidneys } & 3.90 \mathrm{E}-13 \\ \text { Thyroid } & 2.63 \mathrm{E}-13 \\ \text { Leukemia } & 4.54 \mathrm{E}-12 \\ \text { Residua1 } & 1.13 \mathrm{E}-11 \\ \text { Total } & 4.56 \mathrm{E}-11 \\ & \\ \text { TOTAL } & 9.12 \mathrm{E}-11\end{array}$

PATHWAY RISK SUMMARY

\begin{tabular}{|c|c|}
\hline Pathway & $\begin{array}{c}\text { Total Lifetime } \\
\text { Fatal Cancer Risk }\end{array}$ \\
\hline $\begin{array}{l}\text { INGESTION } \\
\text { INHALATION } \\
\text { AIR IMMERSION } \\
\text { GROUND SURFACE } \\
\text { INTERNAL } \\
\text { EXTERNAL }\end{array}$ & $\begin{array}{l}7.17 \mathrm{E}-13 \\
1.78 \mathrm{E}-12 \\
2.26 \mathrm{E}-13 \\
4.29 \mathrm{E}-11 \\
2.50 \mathrm{E}-12 \\
4.31 \mathrm{E}-11\end{array}$ \\
\hline TOTAL & $4.56 \mathrm{E}-11$ \\
\hline
\end{tabular}

Feb 20, $200802: 30 \mathrm{pm} \quad \begin{aligned} & \text { SUMMARY } \\ & \text { Page } 4\end{aligned}$

NUCLIDE RISK SUMMARY

Nuclide

Be-7

TOTAL

$\square$

Feb 20, 2008 02:30 pm
Selected Individual

Total Lifetime Fatal Cancer Risk

4. $56 \mathrm{E}-11$

$4.56 \mathrm{E}-11$

INDIVIDUAL EFFECTIVE DOSE EQUIVALENT RATE (mrem/y) (A11 Radionuclides and Pathways)

Distance $(m)$ 


\begin{tabular}{|c|c|c|c|c|c|c|c|}
\hline Direction & 17035 & 17329 & 18607 & $\begin{array}{c}\mathrm{Be}-7 \mathrm{~B} \\
18834\end{array}$ & M 18860 & 18890 & 19860 \\
\hline$N$ & 1.9E-05 & $1.8 \mathrm{E}-05$ & 1.7E-05 & $1.6 \mathrm{E}-05$ & $1.6 \mathrm{E}-05$ & $1.6 \mathrm{E}-05$ & 1. $5 \mathrm{E}-05$ \\
\hline NNW & $1.2 \mathrm{E}-05$ & $1.2 \mathrm{E}-05$ & $1.1 \mathrm{E}-05$ & $1.0 \mathrm{E}-05$ & $1.0 \mathrm{E}-05$ & $1.0 \mathrm{E}-05$ & $9.8 \mathrm{E}-06$ \\
\hline NW & $1.3 \mathrm{E}-05$ & 1. $3 \mathrm{E}-05$ & $1.2 \mathrm{E}-05$ & $1.2 \mathrm{E}-05$ & $1.2 \mathrm{E}-05$ & $1.2 \mathrm{E}-05$ & $1.1 \mathrm{E}-05$ \\
\hline WNW & $1.7 \mathrm{E}-05$ & $1.6 \mathrm{E}-05$ & 1. $5 \mathrm{E}-05$ & 1. $5 \mathrm{E}-05$ & $1.5 \mathrm{E}-05$ & 1. $5 \mathrm{E}-05$ & $1.4 \mathrm{E}-05$ \\
\hline W & $1.8 \mathrm{E}-05$ & $1.7 \mathrm{E}-05$ & $1.6 \mathrm{E}-05$ & $1.6 \mathrm{E}-05$ & $1.6 \mathrm{E}-05$ & $1.6 \mathrm{E}-05$ & 1. $5 \mathrm{E}-05$ \\
\hline WSW & $2.8 \mathrm{E}-05$ & $2.8 \mathrm{E}-05$ & $2.5 \mathrm{E}-05$ & $2.5 \mathrm{E}-05$ & $2.5 \mathrm{E}-05$ & $2.5 \mathrm{E}-05$ & 2. $3 \mathrm{E}-05$ \\
\hline SW & $4.5 \mathrm{E}-05$ & $4.4 \mathrm{E}-05$ & $4.0 \mathrm{E}-05$ & $3.9 \mathrm{E}-05$ & $3.9 \mathrm{E}-05$ & $3.9 \mathrm{E}-05$ & $3.6 \mathrm{E}-05$ \\
\hline SSW & $6.0 \mathrm{E}-05$ & $5.8 \mathrm{E}-05$ & $5.3 \mathrm{E}-05$ & $5.2 \mathrm{E}-05$ & $5.2 \mathrm{E}-05$ & $5.2 \mathrm{E}-05$ & $4.8 \mathrm{E}-05$ \\
\hline $\mathrm{S}$ & $8.2 \mathrm{E}-05$ & $8.0 \mathrm{E}-05$ & $7.3 \mathrm{E}-05$ & $7.2 \mathrm{E}-05$ & $7.1 \mathrm{E}-05$ & $7.1 \mathrm{E}-05$ & $6.6 \mathrm{E}-05$ \\
\hline SSE & $7.2 \mathrm{E}-05$ & $7.1 \mathrm{E}-05$ & $6.4 \mathrm{E}-05$ & $6.3 \mathrm{E}-05$ & $6.3 \mathrm{E}-05$ & $6.3 \mathrm{E}-05$ & $5.9 \mathrm{E}-05$ \\
\hline SE & $2.7 \mathrm{E}-05$ & $2.7 \mathrm{E}-05$ & $2.4 \mathrm{E}-05$ & $2.4 \mathrm{E}-05$ & $2.4 \mathrm{E}-05$ & $2.4 \mathrm{E}-05$ & 2. $2 \mathrm{E}-05$ \\
\hline ESE & 1.7E-05 & $1.7 \mathrm{E}-05$ & 1. $5 \mathrm{E}-05$ & $1.5 \mathrm{E}-05$ & $1.5 \mathrm{E}-05$ & $1.5 \mathrm{E}-05$ & 1. $4 \mathrm{E}-05$ \\
\hline$E$ & 2.0E-05 & 2.0E-05 & $1.8 \mathrm{E}-05$ & $1.7 \mathrm{E}-05$ & $1.7 \mathrm{E}-05$ & 1.7E-05 & 1. $6 \mathrm{E}-05$ \\
\hline ENE & $2.5 \mathrm{E}-05$ & $2.4 \mathrm{E}-05$ & $2.2 \mathrm{E}-05$ & $2.2 \mathrm{E}-05$ & $2.1 \mathrm{E}-05$ & $2.1 \mathrm{E}-05$ & 2.0E-05 \\
\hline NE & $4.5 \mathrm{E}-05$ & 4. $4 \mathrm{E}-05$ & $4.0 \mathrm{E}-05$ & $3.9 \mathrm{E}-05$ & $3.9 \mathrm{E}-05$ & $3.9 \mathrm{E}-05$ & $3.6 \mathrm{E}-05$ \\
\hline NNE & $2.7 \mathrm{E}-05$ & $2.7 \mathrm{E}-05$ & $2.4 \mathrm{E}-05$ & $2.4 \mathrm{E}-05$ & $2.4 \mathrm{E}-05$ & $2.4 \mathrm{E}-05$ & $2.2 \mathrm{E}-05$ \\
\hline
\end{tabular}

Distance (m)

\begin{tabular}{|c|c|c|c|c|c|c|c|}
\hline Direction & 19891 & 20457 & 21314 & 21349 & 22159 & 23521 & 24430 \\
\hline $\begin{array}{r}N \\
N N W \\
N W \\
W N W \\
W \\
W S W \\
\text { SW } \\
\text { SSW } \\
S \\
\text { SSE } \\
\text { SE } \\
\text { ESE } \\
\text { E } \\
\text { ENE } \\
N E \\
\text { NNE }\end{array}$ & $\begin{array}{l}1.5 \mathrm{E}-05 \\
9.8 \mathrm{E}-06 \\
1.1 \mathrm{E}-05 \\
1.4 \mathrm{E}-05 \\
1.5 \mathrm{E}-05 \\
2.3 \mathrm{E}-05 \\
3.6 \mathrm{E}-05 \\
4.8 \mathrm{E}-05 \\
6.6 \mathrm{E}-05 \\
5.9 \mathrm{E}-05 \\
2.2 \mathrm{E}-05 \\
1.4 \mathrm{E}-05 \\
1.6 \mathrm{E}-05 \\
2.0 \mathrm{E}-05 \\
3.6 \mathrm{E}-05 \\
2.2 \mathrm{E}-05\end{array}$ & $\begin{array}{l}1.5 \mathrm{E}-05 \\
9.4 \mathrm{E}-06 \\
1.0 \mathrm{E}-05 \\
1.3 \mathrm{E}-05 \\
1.4 \mathrm{E}-05 \\
2.2 \mathrm{E}-05 \\
3.5 \mathrm{E}-05 \\
4.6 \mathrm{E}-05 \\
6.3 \mathrm{E}-05 \\
5.6 \mathrm{E}-05 \\
2.1 \mathrm{E}-05 \\
1.3 \mathrm{E}-05 \\
1.5 \mathrm{E}-05 \\
1.9 \mathrm{E}-05 \\
3.5 \mathrm{E}-05 \\
2.1 \mathrm{E}-05\end{array}$ & $\begin{array}{l}1.4 \mathrm{E}-05 \\
9.0 \mathrm{E}-06 \\
9.9 \mathrm{E}-06 \\
1.3 \mathrm{E}-05 \\
1.3 \mathrm{E}-05 \\
2.1 \mathrm{E}-05 \\
3.3 \mathrm{E}-05 \\
4.4 \mathrm{E}-05 \\
6.0 \mathrm{E}-05 \\
5.3 \mathrm{E}-05 \\
2.0 \mathrm{E}-05 \\
1.2 \mathrm{E}-05 \\
1.4 \mathrm{E}-05 \\
1.8 \mathrm{E}-05 \\
3.3 \mathrm{E}-05 \\
2.0 \mathrm{E}-05\end{array}$ & $\begin{array}{l}1.4 \mathrm{E}-05 \\
8.9 \mathrm{E}-06 \\
9.8 \mathrm{E}-06 \\
1.3 \mathrm{E}-05 \\
1.3 \mathrm{E}-05 \\
2.1 \mathrm{E}-05 \\
3.3 \mathrm{E}-05 \\
4.4 \mathrm{E}-05 \\
6.0 \mathrm{E}-05 \\
5.3 \mathrm{E}-05 \\
2.0 \mathrm{E}-05 \\
1.2 \mathrm{E}-05 \\
1.4 \mathrm{E}-05 \\
1.8 \mathrm{E}-05 \\
3.3 \mathrm{E}-05 \\
2.0 \mathrm{E}-05\end{array}$ & $\begin{array}{l}1.3 \mathrm{E}-05 \\
8.5 \mathrm{E}-06 \\
9.3 \mathrm{E}-06 \\
1.2 \mathrm{E}-05 \\
1.2 \mathrm{E}-05 \\
2.0 \mathrm{E}-05 \\
3.1 \mathrm{E}-05 \\
4.1 \mathrm{E}-05 \\
5.6 \mathrm{E}-05 \\
5.0 \mathrm{E}-05 \\
1.8 \mathrm{E}-05 \\
1.1 \mathrm{E}-05 \\
1.3 \mathrm{E}-05 \\
1.7 \mathrm{E}-05 \\
3.1 \mathrm{E}-05 \\
1.9 \mathrm{E}-05\end{array}$ & $\begin{array}{l}1.2 \mathrm{E}-05 \\
7.9 \mathrm{E}-06 \\
8.5 \mathrm{E}-06 \\
1.1 \mathrm{E}-05 \\
1.1 \mathrm{E}-05 \\
1.8 \mathrm{E}-05 \\
2.9 \mathrm{E}-05 \\
3.7 \mathrm{E}-05 \\
5.1 \mathrm{E}-05 \\
4.5 \mathrm{E}-05 \\
1.6 \mathrm{E}-05 \\
1.0 \mathrm{E}-05 \\
1.2 \mathrm{E}-05 \\
1.5 \mathrm{E}-05 \\
2.8 \mathrm{E}-05 \\
1.7 \mathrm{E}-05\end{array}$ & $\begin{array}{l}1.1 \mathrm{E}-05 \\
7.4 \mathrm{E}-06 \\
8.0 \mathrm{E}-06 \\
1.0 \mathrm{E}-05 \\
1.1 \mathrm{E}-05 \\
1.7 \mathrm{E}-05 \\
2.7 \mathrm{E}-05 \\
3.5 \mathrm{E}-05 \\
4.7 \mathrm{E}-05 \\
4.3 \mathrm{E}-05 \\
1.5 \mathrm{E}-05 \\
9.3 \mathrm{E}-06 \\
1.1 \mathrm{E}-05 \\
1.4 \mathrm{E}-05 \\
2.6 \mathrm{E}-05 \\
1.6 \mathrm{E}-05\end{array}$ \\
\hline
\end{tabular}

$\square$

Feb 20, 2008 02:30 pm

SUMMARY

Page 6

INDIVIDUAL EFFECTIVE DOSE EQUIVALENT RATE (mrem/y) (A11 Radionuc1ides and Pathways)

\begin{tabular}{llllll}
\hline & \multicolumn{4}{c}{ Distance $(\mathrm{m})$} \\
\cline { 2 - 5 } Direction & 24545 & 25171 & 26794 & 27275 & 27389 \\
\hline
\end{tabular}

\begin{tabular}{rrrrrr}
\hline $\mathrm{N}$ & $1.1 \mathrm{E}-05$ & $1.0 \mathrm{E}-05$ & $9.4 \mathrm{E}-06$ & $9.2 \mathrm{E}-06$ & $9.2 \mathrm{E}-06$ \\
$\mathrm{NNW}$ & $7.4 \mathrm{E}-06$ & $7.1 \mathrm{E}-06$ & $6.6 \mathrm{E}-06$ & $\begin{array}{r}6.5 \mathrm{E}-06 \\
\text { Page }\end{array}$ & $6.5 \mathrm{E}-06$
\end{tabular}




\begin{tabular}{rccccc} 
& & & \multicolumn{3}{c}{ Be-7B.SUM } \\
NW & $7.9 \mathrm{E}-06$ & $7.6 \mathrm{E}-06$ & $7.1 \mathrm{E}-06$ & $6.9 \mathrm{E}-06$ & $6.9 \mathrm{E}-06$ \\
WNW & $1.0 \mathrm{E}-05$ & $9.6 \mathrm{E}-06$ & $8.9 \mathrm{E}-06$ & $8.7 \mathrm{E}-06$ & $8.7 \mathrm{E}-06$ \\
W & $1.1 \mathrm{E}-05$ & $1.0 \mathrm{E}-05$ & $9.3 \mathrm{E}-06$ & $9.1 \mathrm{E}-06$ & $9.1 \mathrm{E}-06$ \\
WSW & $1.7 \mathrm{E}-05$ & $1.6 \mathrm{E}-05$ & $1.5 \mathrm{E}-05$ & $1.5 \mathrm{E}-05$ & $1.5 \mathrm{E}-05$ \\
SW & $2.7 \mathrm{E}-05$ & $2.5 \mathrm{E}-05$ & $2.4 \mathrm{E}-05$ & $2.3 \mathrm{E}-05$ & $2.3 \mathrm{E}-05$ \\
SSW & $3.4 \mathrm{E}-05$ & $3.3 \mathrm{E}-05$ & $3.0 \mathrm{E}-05$ & $2.9 \mathrm{E}-05$ & $2.9 \mathrm{E}-05$ \\
$\mathrm{~S}$ & $4.7 \mathrm{E}-05$ & $4.5 \mathrm{E}-05$ & $4.1 \mathrm{E}-05$ & $4.0 \mathrm{E}-05$ & $4.0 \mathrm{E}-05$ \\
SSE & $4.2 \mathrm{E}-05$ & $4.0 \mathrm{E}-05$ & $3.7 \mathrm{E}-05$ & $3.6 \mathrm{E}-05$ & $3.6 \mathrm{E}-05$ \\
SE & $1.5 \mathrm{E}-05$ & $1.4 \mathrm{E}-05$ & $1.3 \mathrm{E}-05$ & $1.3 \mathrm{E}-05$ & $1.3 \mathrm{E}-05$ \\
ESE & $9.2 \mathrm{E}-06$ & $8.6 \mathrm{E}-06$ & $7.9 \mathrm{E}-06$ & $7.8 \mathrm{E}-06$ & $7.7 \mathrm{E}-06$ \\
$\mathrm{E}$ & $1.0 \mathrm{E}-05$ & $9.7 \mathrm{E}-06$ & $9.0 \mathrm{E}-06$ & $8.8 \mathrm{E}-06$ & $8.7 \mathrm{E}-06$ \\
ENE & $1.4 \mathrm{E}-05$ & $1.3 \mathrm{E}-05$ & $1.2 \mathrm{E}-05$ & $1.2 \mathrm{E}-05$ & $1.2 \mathrm{E}-05$ \\
NE & $2.6 \mathrm{E}-05$ & $2.5 \mathrm{E}-05$ & $2.3 \mathrm{E}-05$ & $2.3 \mathrm{E}-05$ & $2.3 \mathrm{E}-05$ \\
NNE & $1.6 \mathrm{E}-05$ & $1.5 \mathrm{E}-05$ & $1.4 \mathrm{E}-05$ & $1.4 \mathrm{E}-05$ & $1.4 \mathrm{E}-05$ \\
& & & & & \\
\hline
\end{tabular}

Feb 20, $2008 \quad 02: 30 \mathrm{pm}$

SUMMARY

Page 7

INDIVIDUAL LIFETIME RISK (deaths)

(A11 Radionuclides and Pathways)

\begin{tabular}{|c|c|c|c|c|c|c|c|}
\hline \multicolumn{8}{|c|}{ Distance $(\mathrm{m})$} \\
\hline Direction & 17035 & 17329 & 18607 & 18834 & 18860 & 18890 & 19860 \\
\hline $\begin{array}{r}N \\
N N W \\
N W \\
W N W \\
W \\
W S W \\
S W \\
S S W \\
S \\
S S E \\
S E \\
\text { ESE } \\
E \\
\text { ENE } \\
N E \\
N N E\end{array}$ & $\begin{array}{l}1.0 \mathrm{E}-11 \\
6.6 \mathrm{E}-12 \\
7.4 \mathrm{E}-12 \\
9.4 \mathrm{E}-12 \\
9.9 \mathrm{E}-12 \\
1.6 \mathrm{E}-11 \\
2.5 \mathrm{E}-11 \\
3.3 \mathrm{E}-11 \\
4.6 \mathrm{E}-11 \\
4.0 \mathrm{E}-11 \\
1.5 \mathrm{E}-11 \\
9.6 \mathrm{E}-12 \\
1.1 \mathrm{E}-11 \\
1.4 \mathrm{E}-11 \\
2.5 \mathrm{E}-11 \\
1.5 \mathrm{E}-11\end{array}$ & $\begin{array}{l}1.0 \mathrm{E}-11 \\
6.5 \mathrm{E}-12 \\
7.2 \mathrm{E}-12 \\
9.2 \mathrm{E}-12 \\
9.7 \mathrm{E}-12 \\
1.5 \mathrm{E}-11 \\
2.4 \mathrm{E}-11 \\
3.2 \mathrm{E}-11 \\
4.5 \mathrm{E}-11 \\
3.9 \mathrm{E}-11 \\
1.5 \mathrm{E}-11 \\
9.4 \mathrm{E}-12 \\
1.1 \mathrm{E}-11 \\
1.3 \mathrm{E}-11 \\
2.4 \mathrm{E}-11 \\
1.5 \mathrm{E}-11\end{array}$ & $\begin{array}{l}9.3 \mathrm{E}-12 \\
5.9 \mathrm{E}-12 \\
6.6 \mathrm{E}-12 \\
8.4 \mathrm{E}-12 \\
8.9 \mathrm{E}-12 \\
1.4 \mathrm{E}-11 \\
2.2 \mathrm{E}-11 \\
2.9 \mathrm{E}-11 \\
4.0 \mathrm{E}-11 \\
3.6 \mathrm{E}-11 \\
1.3 \mathrm{E}-11 \\
8.5 \mathrm{E}-12 \\
9.9 \mathrm{E}-12 \\
1.2 \mathrm{E}-11 \\
2.2 \mathrm{E}-11 \\
1.4 \mathrm{E}-11\end{array}$ & $\begin{array}{l}9.1 \mathrm{E}-12 \\
5.9 \mathrm{E}-12 \\
6.5 \mathrm{E}-12 \\
8.3 \mathrm{E}-12 \\
8.7 \mathrm{E}-12 \\
1.4 \mathrm{E}-11 \\
2.2 \mathrm{E}-11 \\
2.9 \mathrm{E}-11 \\
4.0 \mathrm{E}-11 \\
3.5 \mathrm{E}-11 \\
1.3 \mathrm{E}-11 \\
8.3 \mathrm{E}-12 \\
9.7 \mathrm{E}-12 \\
1.2 \mathrm{E}-11 \\
2.2 \mathrm{E}-11 \\
1.3 \mathrm{E}-11\end{array}$ & $\begin{array}{l}9.1 \mathrm{E}-12 \\
5.8 \mathrm{E}-12 \\
6.5 \mathrm{E}-12 \\
8.3 \mathrm{E}-12 \\
8.7 \mathrm{E}-12 \\
1.4 \mathrm{E}-11 \\
2.2 \mathrm{E}-11 \\
2.9 \mathrm{E}-11 \\
4.0 \mathrm{E}-11 \\
3.5 \mathrm{E}-11 \\
1.3 \mathrm{E}-11 \\
8.3 \mathrm{E}-12 \\
9.7 \mathrm{E}-12 \\
1.2 \mathrm{E}-11 \\
2.2 \mathrm{E}-11 \\
1.3 \mathrm{E}-11\end{array}$ & $\begin{array}{l}9.1 \mathrm{E}-12 \\
5.8 \mathrm{E}-12 \\
6.5 \mathrm{E}-12 \\
8.3 \mathrm{E}-12 \\
8.7 \mathrm{E}-12 \\
1.4 \mathrm{E}-11 \\
2.2 \mathrm{E}-11 \\
2.9 \mathrm{E}-11 \\
3.9 \mathrm{E}-11 \\
3.5 \mathrm{E}-11 \\
1.3 \mathrm{E}-11 \\
8.3 \mathrm{E}-12 \\
9.7 \mathrm{E}-12 \\
1.2 \mathrm{E}-11 \\
2.2 \mathrm{E}-11 \\
1.3 \mathrm{E}-11\end{array}$ & $\begin{array}{l}\text { 8.5E-12 } \\
5.5 \mathrm{E}-12 \\
6.1 \mathrm{E}-12 \\
7.7 \mathrm{E}-12 \\
8.1 \mathrm{E}-12 \\
1.3 \mathrm{E}-11 \\
2.0 \mathrm{E}-11 \\
2.7 \mathrm{E}-11 \\
3.7 \mathrm{E}-11 \\
3.3 \mathrm{E}-11 \\
1.2 \mathrm{E}-11 \\
7.7 \mathrm{E}-12 \\
8.9 \mathrm{E}-12 \\
1.1 \mathrm{E}-11 \\
2.0 \mathrm{E}-11 \\
1.2 \mathrm{E}-11\end{array}$ \\
\hline \multicolumn{8}{|c|}{ Distance $(\mathrm{m})$} \\
\hline Direction & 19891 & 20457 & 21314 & 21349 & 22159 & 23521 & 24430 \\
\hline $\begin{array}{r}N \\
N N W \\
N W \\
W N W \\
W \\
W S W \\
\text { SW } \\
\text { SSW }\end{array}$ & $\begin{array}{l}8 \cdot 5 \mathrm{E}-12 \\
5 \cdot 5 \mathrm{E}-12 \\
6.1 \mathrm{E}-12 \\
7.7 \mathrm{E}-12 \\
8.1 \mathrm{E}-12 \\
1.3 \mathrm{E}-11 \\
2.0 \mathrm{E}-11 \\
2.7 \mathrm{E}-11\end{array}$ & $\begin{array}{l}8.1 \mathrm{E}-12 \\
5.3 \mathrm{E}-12 \\
5.9 \mathrm{E}-12 \\
7.4 \mathrm{E}-12 \\
7.8 \mathrm{E}-12 \\
1.2 \mathrm{E}-11 \\
1.9 \mathrm{E}-11 \\
2.6 \mathrm{E}-11\end{array}$ & $\begin{array}{l}7.7 \mathrm{E}-12 \\
5.0 \mathrm{E}-12 \\
5.5 \mathrm{E}-12 \\
7.0 \mathrm{E}-12 \\
7.4 \mathrm{E}-12 \\
1.2 \mathrm{E}-11 \\
1.8 \mathrm{E}-11 \\
2.4 \mathrm{E}-11\end{array}$ & $\begin{array}{r}7.6 \mathrm{E}-12 \\
5.0 \mathrm{E}-12 \\
5.5 \mathrm{E}-12 \\
7.0 \mathrm{E}-12 \\
7.4 \mathrm{E}-12 \\
1.2 \mathrm{E}-11 \\
1.8 \mathrm{E}-11 \\
2.4 \mathrm{E}-11 \\
\text { Page }\end{array}$ & $\begin{array}{r}7.2 \mathrm{E}-12 \\
4.8 \mathrm{E}-12 \\
5.3 \mathrm{E}-12 \\
6.7 \mathrm{E}-12 \\
7.0 \mathrm{E}-12 \\
1.1 \mathrm{E}-11 \\
1.7 \mathrm{E}-11 \\
2.3 \mathrm{E}-11 \\
5\end{array}$ & $\begin{array}{l}6.5 \mathrm{E}-12 \\
4.4 \mathrm{E}-12 \\
4.8 \mathrm{E}-12 \\
6.1 \mathrm{E}-12 \\
6.4 \mathrm{E}-12 \\
1.0 \mathrm{E}-11 \\
1.6 \mathrm{E}-11 \\
2.1 \mathrm{E}-11\end{array}$ & $\begin{array}{l}6.1 \mathrm{E}-12 \\
4.2 \mathrm{E}-12 \\
4.5 \mathrm{E}-12 \\
5.7 \mathrm{E}-12 \\
5.9 \mathrm{E}-12 \\
9.5 \mathrm{E}-12 \\
1.5 \mathrm{E}-11 \\
1.9 \mathrm{E}-11\end{array}$ \\
\hline
\end{tabular}




\begin{tabular}{rlllllll} 
& \multicolumn{7}{c}{ Be-7B.SUM } \\
S & $3.7 \mathrm{E}-11$ & $3.5 \mathrm{E}-11$ & $3.3 \mathrm{E}-11$ & $3.3 \mathrm{E}-11$ & $3.1 \mathrm{E}-11$ & $2.8 \mathrm{E}-11$ & $2.6 \mathrm{E}-11$ \\
SSE & $3.2 \mathrm{E}-11$ & $3.1 \mathrm{E}-11$ & $2.9 \mathrm{E}-11$ & $2.9 \mathrm{E}-11$ & $2.8 \mathrm{E}-11$ & $2.5 \mathrm{E}-11$ & $2.4 \mathrm{E}-11$ \\
SE & $1.2 \mathrm{E}-11$ & $1.2 \mathrm{E}-11$ & $1.1 \mathrm{E}-11$ & $1.1 \mathrm{E}-11$ & $1.0 \mathrm{E}-11$ & $9.2 \mathrm{E}-12$ & $8.4 \mathrm{E}-12$ \\
ESE & $7.7 \mathrm{E}-12$ & $7.3 \mathrm{E}-12$ & $6.9 \mathrm{E}-12$ & $6.8 \mathrm{E}-12$ & $6.4 \mathrm{E}-12$ & $5.7 \mathrm{E}-12$ & $5.2 \mathrm{E}-12$ \\
$\mathrm{E}$ & $8.9 \mathrm{E}-12$ & $8.5 \mathrm{E}-12$ & $8.0 \mathrm{E}-12$ & $7.9 \mathrm{E}-12$ & $7.4 \mathrm{E}-12$ & $6.6 \mathrm{E}-12$ & $6.0 \mathrm{E}-12$ \\
ENE & $1.1 \mathrm{E}-11$ & $1.1 \mathrm{E}-11$ & $1.0 \mathrm{E}-11$ & $1.0 \mathrm{E}-11$ & $9.4 \mathrm{E}-12$ & $8.5 \mathrm{E}-12$ & $7.9 \mathrm{E}-12$ \\
NE & $2.0 \mathrm{E}-11$ & $1.9 \mathrm{E}-11$ & $1.8 \mathrm{E}-11$ & $1.8 \mathrm{E}-11$ & $1.7 \mathrm{E}-11$ & $1.6 \mathrm{E}-11$ & $1.5 \mathrm{E}-11$ \\
NNE & $1.2 \mathrm{E}-11$ & $1.2 \mathrm{E}-11$ & $1.1 \mathrm{E}-11$ & $1.1 \mathrm{E}-11$ & $1.1 \mathrm{E}-11$ & $9.7 \mathrm{E}-12$ & $9.1 \mathrm{E}-12$
\end{tabular}

Feb 20, $2008 \quad 02: 30 \mathrm{pm}$

SUMMARY

Page 8

INDIVIDUAL LIFETIME RISK (deaths)

(A11 Radionuclides and Pathways)

\begin{tabular}{|c|c|c|c|c|c|}
\hline \multirow[b]{2}{*}{ Direction } & \multicolumn{5}{|c|}{ Distance $(\mathrm{m})$} \\
\hline & 24545 & 25171 & 26794 & 27275 & 27389 \\
\hline $\begin{array}{r}N \\
N N W \\
N W \\
W N W \\
W \\
W S W \\
S W \\
S S W \\
S \\
\text { SSE } \\
\text { SE } \\
\text { ESE } \\
\text { E } \\
\text { ENE } \\
N E \\
\text { NNE }\end{array}$ & $\begin{array}{l}6.0 \mathrm{E}-12 \\
4.2 \mathrm{E}-12 \\
4.5 \mathrm{E}-12 \\
5.6 \mathrm{E}-12 \\
5.9 \mathrm{E}-12 \\
9.4 \mathrm{E}-12 \\
1.5 \mathrm{E}-11 \\
1.9 \mathrm{E}-11 \\
2.6 \mathrm{E}-11 \\
2.3 \mathrm{E}-11 \\
8.3 \mathrm{E}-12 \\
5.2 \mathrm{E}-12 \\
5.9 \mathrm{E}-12 \\
7.8 \mathrm{E}-12 \\
1.5 \mathrm{E}-11 \\
9.0 \mathrm{E}-12\end{array}$ & $\begin{array}{l}5.7 \mathrm{E}-12 \\
4.0 \mathrm{E}-12 \\
4.3 \mathrm{E}-12 \\
5.4 \mathrm{E}-12 \\
5.6 \mathrm{E}-12 \\
9.0 \mathrm{E}-12 \\
1.4 \mathrm{E}-11 \\
1.8 \mathrm{E}-11 \\
2.5 \mathrm{E}-11 \\
2.2 \mathrm{E}-11 \\
7.8 \mathrm{E}-12 \\
4.9 \mathrm{E}-12 \\
5.5 \mathrm{E}-12 \\
7.4 \mathrm{E}-12 \\
1.4 \mathrm{E}-11 \\
8.6 \mathrm{E}-12\end{array}$ & $\begin{array}{l}5.3 \mathrm{E}-12 \\
3 \cdot 8 \mathrm{E}-12 \\
4 \cdot 0 \mathrm{E}-12 \\
5.0 \mathrm{E}-12 \\
5.2 \mathrm{E}-12 \\
8.3 \mathrm{E}-12 \\
1.3 \mathrm{E}-11 \\
1.7 \mathrm{E}-11 \\
2.3 \mathrm{E}-11 \\
2.1 \mathrm{E}-11 \\
7.2 \mathrm{E}-12 \\
4.5 \mathrm{E}-12 \\
5 \cdot 1 \mathrm{E}-12 \\
6.9 \mathrm{E}-12 \\
1.3 \mathrm{E}-11 \\
8.0 \mathrm{E}-12\end{array}$ & $\begin{array}{l}5.2 \mathrm{E}-12 \\
3.7 \mathrm{E}-12 \\
3.9 \mathrm{E}-12 \\
4.9 \mathrm{E}-12 \\
5.1 \mathrm{E}-12 \\
8.2 \mathrm{E}-12 \\
1.3 \mathrm{E}-11 \\
1.6 \mathrm{E}-11 \\
2.2 \mathrm{E}-11 \\
2.0 \mathrm{E}-11 \\
7.1 \mathrm{E}-12 \\
4.4 \mathrm{E}-12 \\
5.0 \mathrm{E}-12 \\
6.7 \mathrm{E}-12 \\
1.3 \mathrm{E}-11 \\
7.8 \mathrm{E}-12\end{array}$ & $\begin{array}{l}5.2 \mathrm{E}-12 \\
3.7 \mathrm{E}-12 \\
3.9 \mathrm{E}-12 \\
4.9 \mathrm{E}-12 \\
5.1 \mathrm{E}-12 \\
8.1 \mathrm{E}-12 \\
1.3 \mathrm{E}-11 \\
1.6 \mathrm{E}-11 \\
2.2 \mathrm{E}-11 \\
2.0 \mathrm{E}-11 \\
7.0 \mathrm{E}-12 \\
4.4 \mathrm{E}-12 \\
4.9 \mathrm{E}-12 \\
6.7 \mathrm{E}-12 \\
1.3 \mathrm{E}-11 \\
7.8 \mathrm{E}-12\end{array}$ \\
\hline
\end{tabular}




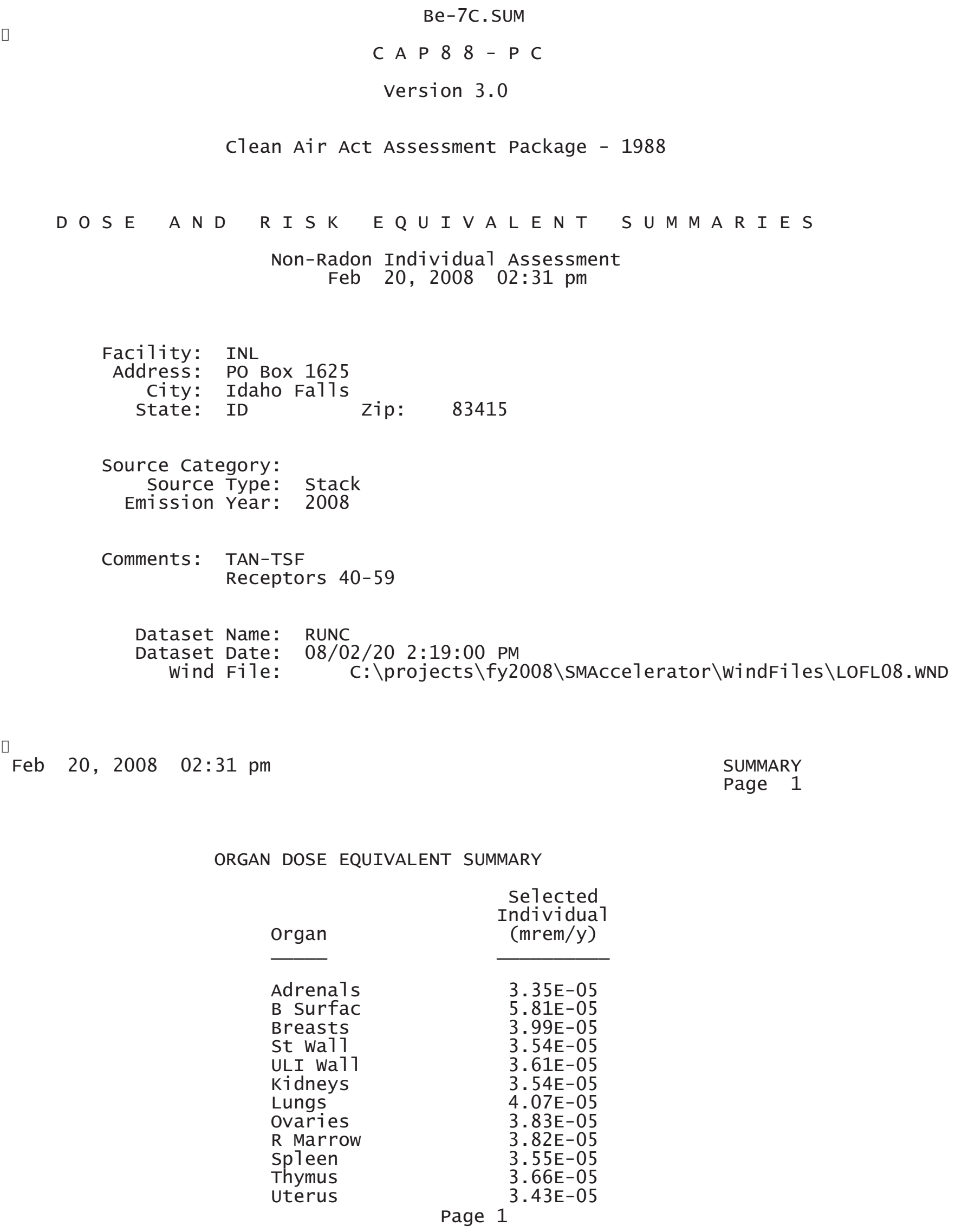




$\begin{array}{lr} & \text { Be-7C.SUM } \\ \text { Bld wa11 } & 3.60 \mathrm{E}-05 \\ \text { Brain } & 3.49 \mathrm{E}-05 \\ \text { Esophagu } & 3.54 \mathrm{E}-05 \\ \text { SI Wa11 } & 3.49 \mathrm{E}-05 \\ \text { LLI Wa11 } & 3.78 \mathrm{E}-05 \\ \text { Liver } & 3.56 \mathrm{E}-05 \\ \text { Muscle } & 4.05 \mathrm{E}-05 \\ \text { Pancreas } & 3.27 \mathrm{E}-05 \\ \text { Skin } & 4.64 \mathrm{E}-05 \\ \text { Testes } & 4.08 \mathrm{E}-05 \\ \text { Thyroid } & 3.90 \mathrm{E}-05 \\ & \\ \text { EFFEC } & 3.88 \mathrm{E}-05\end{array}$

PATHWAY EFFECTIVE DOSE EQUIVALENT SUMMARY

$$
\begin{gathered}
\text { Selected } \\
\text { Individual } \\
\text { (mrem/y) }
\end{gathered}
$$

Pathway

INGESTION

4. $57 \mathrm{E}-07$

INHALATION

1. $03 \mathrm{E}-06$

AIR IMMERSION

GROUND SURFACE

$1.76 \mathrm{E}-07$

INTERNAL

$3.72 \mathrm{E}-05$

$1.49 \mathrm{E}-06$

EXTERNAL

$3.73 \mathrm{E}-05$

TOTAL

$3.88 \mathrm{E}-05$

$\square$

Feb 20, $2008 \quad 02: 31 \mathrm{pm}$

SUMMARY

Page 2

NUCLIDE EFFECTIVE DOSE EQUIVALENT SUMMARY

Nuclide

Be-7

TOTAL

Feb 20, $2008 \quad 02: 31 \mathrm{pm}$

$$
\begin{gathered}
\begin{array}{c}
\text { Selected } \\
\text { Individua1 } \\
(\mathrm{mrem} / \mathrm{y})
\end{array} \\
\hline 3.88 \mathrm{E}-05 \\
3.88 \mathrm{E}-05
\end{gathered}
$$

SUMMARY

Page 3

CANCER RISK SUMMARY

Cancer

Esophagu
Selected Individual

Total Lifetime Fatal Cancer Risk

$$
\text { Page } 2^{3.73 E-13}
$$




$\begin{array}{lr} & \text { Be-7C. SUM } \\ \text { Stomach } & 1.44 \mathrm{E}-12 \\ \text { Colon } & 3.89 \mathrm{E}-12 \\ \text { Liver } & 5.42 \mathrm{E}-13 \\ \text { LUNG } & 4.02 \mathrm{E}-12 \\ \text { Bone } & 5.52 \mathrm{E}-14 \\ \text { Skin } & 4.64 \mathrm{E}-14 \\ \text { Breast } & 1.93 \mathrm{E}-12 \\ \text { Ovary } & 5.48 \mathrm{E}-13 \\ \text { Bladder } & 8.76 \mathrm{E}-13 \\ \text { Kidneys } & 1.84 \mathrm{E}-13 \\ \text { Thyroid } & 1.24 \mathrm{E}-13 \\ \text { Leukemia } & 2.15 \mathrm{E}-12 \\ \text { Residua1 } & 5.31 \mathrm{E}-12 \\ \text { Total } & 2.15 \mathrm{E}-11 \\ & \\ \text { TOTAL } & 4.30 \mathrm{E}-11\end{array}$

PATHWAY RISK SUMMARY

\begin{tabular}{lc}
\multirow{2}{*}{ Pathway } & \multicolumn{2}{c}{$\begin{array}{c}\text { Total Lifetime } \\
\text { Fatal Cancer Risk }\end{array}$} \\
\cline { 2 - 2 } & $3.15 \mathrm{E}-13$ \\
INGESTION & $7.54 \mathrm{E}-13$ \\
INHALATION & $9.56 \mathrm{E}-14$ \\
AIR IMMERSION & $2.03 \mathrm{E}-11$ \\
GROUND SURFACE & $1.07 \mathrm{E}-12$ \\
INTERNAL & $2.04 \mathrm{E}-11$ \\
EXTERNAL & $2.15 \mathrm{E}-11$ \\
TOTAL &
\end{tabular}

$\begin{array}{lll} & \text { Feb 20, } 2008 \quad 02: 31 \mathrm{pm} & \text { SUMMARY } \\ \text { Page } 4\end{array}$

NUCLIDE RISK SUMMARY

Nuclide

Be-7

TOTAL

$\square$

Feb 20, 2008 02:31 pm
Selected Individual

Total Lifetime Fatal Cancer Risk

$2.15 \mathrm{E}-11$

$2.15 \mathrm{E}-11$

SUMMARY

Page 5

INDIVIDUAL EFFECTIVE DOSE EQUIVALENT RATE (mrem/y) (A11 Radionuclides and Pathways)

Distance $(m)$ 


\begin{tabular}{|c|c|c|c|c|c|c|c|}
\hline Direction & 27715 & 28919 & 31060 & $\begin{array}{c}\text { Be-7C. } \\
32802\end{array}$ & M 34577 & 35279 & 35683 \\
\hline$N$ & $8.5 E-06$ & $8.1 \mathrm{E}-06$ & $7.4 \mathrm{E}-06$ & $6.9 \mathrm{E}-06$ & $6.4 \mathrm{E}-06$ & $6.2 \mathrm{E}-06$ & $6.1 \mathrm{E}-06$ \\
\hline NNW & $5.9 \mathrm{E}-06$ & $5.6 \mathrm{E}-06$ & $5.1 \mathrm{E}-06$ & $4.8 \mathrm{E}-06$ & $4.5 \mathrm{E}-06$ & $4.4 \mathrm{E}-06$ & 4. $3 \mathrm{E}-06$ \\
\hline NW & $6.3 \mathrm{E}-06$ & $6.0 \mathrm{E}-06$ & $5.5 \mathrm{E}-06$ & $5.1 \mathrm{E}-06$ & $4.8 \mathrm{E}-06$ & $4.7 \mathrm{E}-06$ & 4. $6 \mathrm{E}-06$ \\
\hline WNW & $8.1 \mathrm{E}-06$ & $7.7 \mathrm{E}-06$ & $7.0 \mathrm{E}-06$ & $6.5 \mathrm{E}-06$ & $6.1 \mathrm{E}-06$ & $5.9 \mathrm{E}-06$ & $5.8 \mathrm{E}-06$ \\
\hline W & $8.5 \mathrm{E}-06$ & $8.0 \mathrm{E}-06$ & $7.3 \mathrm{E}-06$ & $6.8 \mathrm{E}-06$ & $6.3 \mathrm{E}-06$ & $6.2 \mathrm{E}-06$ & $6.1 \mathrm{E}-06$ \\
\hline WSW & 1. $4 \mathrm{E}-05$ & 1. $3 \mathrm{E}-05$ & $1.2 \mathrm{E}-05$ & $1.1 \mathrm{E}-05$ & $1.0 \mathrm{E}-05$ & $1.0 \mathrm{E}-05$ & $9.9 \mathrm{E}-06$ \\
\hline SW & $2.2 \mathrm{E}-05$ & $2.1 \mathrm{E}-05$ & $1.9 \mathrm{E}-05$ & $1.8 \mathrm{E}-05$ & $1.7 \mathrm{E}-05$ & $1.6 \mathrm{E}-05$ & 1. $6 \mathrm{E}-05$ \\
\hline SSW & $2.8 \mathrm{E}-05$ & $2.7 \mathrm{E}-05$ & $2.4 \mathrm{E}-05$ & $2.3 \mathrm{E}-05$ & $2.1 \mathrm{E}-05$ & $2.1 \mathrm{E}-05$ & 2.0E-05 \\
\hline $\mathrm{S}$ & $3.9 \mathrm{E}-05$ & $3.7 \mathrm{E}-05$ & $3.3 \mathrm{E}-05$ & $3.1 \mathrm{E}-05$ & $2.9 \mathrm{E}-05$ & $2.8 \mathrm{E}-05$ & $2.7 \mathrm{E}-05$ \\
\hline SSE & $3.5 \mathrm{E}-05$ & $3.3 \mathrm{E}-05$ & $3.0 \mathrm{E}-05$ & $2.8 \mathrm{E}-05$ & $2.6 \mathrm{E}-05$ & $2.6 \mathrm{E}-05$ & 2. $5 \mathrm{E}-05$ \\
\hline SE & $1.2 \mathrm{E}-05$ & $1.1 \mathrm{E}-05$ & 1.0E-05 & $9.4 \mathrm{E}-06$ & $8.7 \mathrm{E}-06$ & $8.5 \mathrm{E}-06$ & $8.4 \mathrm{E}-06$ \\
\hline ESE & $7.1 \mathrm{E}-06$ & $6.7 \mathrm{E}-06$ & $6.1 \mathrm{E}-06$ & $5.7 E-06$ & $5.2 \mathrm{E}-06$ & $5.1 \mathrm{E}-06$ & 5. $0 \mathrm{E}-06$ \\
\hline E & $8.1 \mathrm{E}-06$ & $7.6 \mathrm{E}-06$ & $6.9 \mathrm{E}-06$ & $6.4 \mathrm{E}-06$ & $5.9 \mathrm{E}-06$ & $5.8 \mathrm{E}-06$ & $5.7 \mathrm{E}-06$ \\
\hline ENE & $1.1 \mathrm{E}-05$ & $1.1 \mathrm{E}-05$ & $9.7 \mathrm{E}-06$ & $9.0 \mathrm{E}-06$ & $8.4 \mathrm{E}-06$ & $8.2 \mathrm{E}-06$ & 8.0E-06 \\
\hline NE & $2.2 \mathrm{E}-05$ & $2.1 \mathrm{E}-05$ & $1.9 \mathrm{E}-05$ & $1.8 \mathrm{E}-05$ & $1.6 \mathrm{E}-05$ & $1.6 \mathrm{E}-05$ & 1. $6 \mathrm{E}-05$ \\
\hline NNE & 1. $3 \mathrm{E}-05$ & $1.2 \mathrm{E}-05$ & $1.1 \mathrm{E}-05$ & $1.1 \mathrm{E}-05$ & $9.9 \mathrm{E}-06$ & $9.7 \mathrm{E}-06$ & $9.5 \mathrm{E}-06$ \\
\hline
\end{tabular}

Distance (m)

\begin{tabular}{|c|c|c|c|c|c|c|c|}
\hline Direction & 36721 & 36809 & 37729 & 39079 & 39220 & 39559 & 43584 \\
\hline $\mathrm{N}$ & $5.9 \mathrm{E}-06$ & $5.9 \mathrm{E}-06$ & $5.7 \mathrm{E}-06$ & $5.4 \mathrm{E}-06$ & $5.4 \mathrm{E}-06$ & $5.3 E-06$ & $4.6 \mathrm{E}-06$ \\
\hline NNW & $4.2 \mathrm{E}-06$ & $4.2 \mathrm{E}-06$ & $4.1 \mathrm{E}-06$ & $3.9 \mathrm{E}-06$ & $3.9 \mathrm{E}-06$ & $3.8 \mathrm{E}-06$ & $3.4 \mathrm{E}-06$ \\
\hline NW & 4. $4 \mathrm{E}-06$ & 4. $4 \mathrm{E}-06$ & 4. $3 \mathrm{E}-06$ & $4.1 \mathrm{E}-06$ & $4.1 \mathrm{E}-06$ & $4.0 \mathrm{E}-06$ & $3.5 \mathrm{E}-06$ \\
\hline WNW & $5.6 \mathrm{E}-06$ & $5.6 \mathrm{E}-06$ & $5.4 \mathrm{E}-06$ & $5.2 \mathrm{E}-06$ & $5.1 \mathrm{E}-06$ & $5.1 \mathrm{E}-06$ & $4.4 \mathrm{E}-06$ \\
\hline W & $5.9 \mathrm{E}-06$ & $5.8 \mathrm{E}-06$ & $5.6 \mathrm{E}-06$ & $5.4 \mathrm{E}-06$ & $5.4 \mathrm{E}-06$ & $5.3 \mathrm{E}-06$ & $4.6 \mathrm{E}-06$ \\
\hline WSW & $9.6 \mathrm{E}-06$ & $9.5 \mathrm{E}-06$ & $9.2 \mathrm{E}-06$ & $8.8 \mathrm{E}-06$ & $8.7 \mathrm{E}-06$ & $8.6 \mathrm{E}-06$ & $7.5 \mathrm{E}-06$ \\
\hline SW & $1.5 \mathrm{E}-05$ & $1.5 \mathrm{E}-05$ & $1.5 \mathrm{E}-05$ & 1. $4 \mathrm{E}-05$ & $1.4 \mathrm{E}-05$ & 1. $4 \mathrm{E}-05$ & $1.2 \mathrm{E}-05$ \\
\hline SSW & $1.9 \mathrm{E}-05$ & $1.9 \mathrm{E}-05$ & $1.9 \mathrm{E}-05$ & $1.8 \mathrm{E}-05$ & $1.8 \mathrm{E}-05$ & $1.7 \mathrm{E}-05$ & $1.5 \mathrm{E}-05$ \\
\hline S & $2.6 \mathrm{E}-05$ & $2.6 \mathrm{E}-05$ & $2.5 \mathrm{E}-05$ & $2.4 \mathrm{E}-05$ & $2.4 \mathrm{E}-05$ & $2.4 \mathrm{E}-05$ & $2.1 \mathrm{E}-05$ \\
\hline SSE & $2.4 \mathrm{E}-05$ & $2.4 \mathrm{E}-05$ & $2.3 \mathrm{E}-05$ & $2.2 \mathrm{E}-05$ & $2.2 \mathrm{E}-05$ & $2.2 \mathrm{E}-05$ & $1.9 \mathrm{E}-05$ \\
\hline $\mathrm{SE}$ & $8.0 \mathrm{E}-06$ & $8.0 \mathrm{E}-06$ & $7.7 \mathrm{E}-06$ & $7.3 \mathrm{E}-06$ & $7.3 \mathrm{E}-06$ & $7.2 \mathrm{E}-06$ & $6.2 \mathrm{E}-06$ \\
\hline ESE & $4.8 \mathrm{E}-06$ & $4.8 \mathrm{E}-06$ & $4.6 \mathrm{E}-06$ & $4.4 \mathrm{E}-06$ & 4. $4 \mathrm{E}-06$ & $4.3 \mathrm{E}-06$ & $3.7 \mathrm{E}-06$ \\
\hline & $5.4 \mathrm{E}-06$ & $5.4 \mathrm{E}-06$ & $5.2 \mathrm{E}-06$ & $4.9 \mathrm{E}-06$ & $4.9 \mathrm{E}-06$ & $4.8 \mathrm{E}-06$ & $4.2 \mathrm{E}-06$ \\
\hline ENE & $7.7 \mathrm{E}-06$ & $7.7 \mathrm{E}-06$ & $7.4 \mathrm{E}-06$ & $7.1 \mathrm{E}-06$ & 7.0E-06 & 7.0E-06 & $6.0 \mathrm{E}-06$ \\
\hline $\mathrm{NE}$ & $1.5 \mathrm{E}-05$ & $1.5 \mathrm{E}-05$ & $1.5 \mathrm{E}-05$ & $1.4 \mathrm{E}-05$ & $1.4 \mathrm{E}-05$ & $1.4 \mathrm{E}-05$ & $1.2 \mathrm{E}-05$ \\
\hline NNE & $9.2 \mathrm{E}-06$ & $9.2 \mathrm{E}-06$ & $8.9 \mathrm{E}-06$ & $8.5 \mathrm{E}-06$ & $8.4 \mathrm{E}-06$ & $8.3 \mathrm{E}-06$ & 7. $3 \mathrm{E}-06$ \\
\hline
\end{tabular}

口

Feb 20, 2008 02:31 pm

SUMMARY

Page 6

INDIVIDUAL EFFECTIVE DOSE EQUIVALENT RATE (mrem/y) (A11 Radionuc1ides and Pathways)

\begin{tabular}{|c|c|c|c|c|c|c|}
\hline \multirow[b]{2}{*}{ Direction } & \multicolumn{6}{|c|}{ Distance (m) } \\
\hline & 45196 & 45275 & 45654 & 45677 & 46668 & 47969 \\
\hline
\end{tabular}

$\begin{array}{rrrrrrr}\mathrm{N} & 4.4 \mathrm{E}-06 & 4.4 \mathrm{E}-06 & 4.3 \mathrm{E}-06 & \begin{array}{c}4.3 \mathrm{E}-06 \\ \text { N }\end{array} & 4.2 \mathrm{E}-06 & 4.0 \mathrm{E}-06 \\ \mathrm{NNW} & 3.2 \mathrm{E}-06 & 3.2 \mathrm{E}-06 & 3.2 \mathrm{E}-06 & \begin{array}{c}3.2 \mathrm{E}-06 \\ \text { Page }\end{array} & 4.1 \mathrm{E}-06 & 3.0 \mathrm{E}-06\end{array}$




\begin{tabular}{|c|c|c|c|c|c|c|}
\hline & & & & Be-7C. & & \\
\hline NW & $3.3 \mathrm{E}-06$ & $3.3 E-06$ & $3.3 \mathrm{E}-06$ & $3.3 E-06$ & $3.2 \mathrm{E}-06$ & $3.1 \mathrm{E}-06$ \\
\hline WNW & $4.2 \mathrm{E}-06$ & $4.2 \mathrm{E}-06$ & $4.2 \mathrm{E}-06$ & $4.2 \mathrm{E}-06$ & $4.0 \mathrm{E}-06$ & $3.9 \mathrm{E}-06$ \\
\hline & $4.4 \mathrm{E}-06$ & $4.4 \mathrm{E}-06$ & $4.3 \mathrm{E}-06$ & $4.3 \mathrm{E}-06$ & $4.2 \mathrm{E}-06$ & $4.0 \mathrm{E}-06$ \\
\hline WSW & $7.2 \mathrm{E}-06$ & $7.1 \mathrm{E}-06$ & 7. $0 \mathrm{E}-06$ & 7.0E-06 & $6.8 \mathrm{E}-06$ & $6.6 \mathrm{E}-06$ \\
\hline SW & 1.1 $\mathrm{E}-05$ & 1.1E-05 & 1. $1 \mathrm{E}-05$ & 1.1E-05 & 05 & -05 \\
\hline SSW & $1.4 \mathrm{E}-05$ & $1.4 \mathrm{E}-05$ & $1.4 \mathrm{E}-05$ & $1.4 \mathrm{E}-05$ & $1.4 \mathrm{E}-05$ & $1.3 \mathrm{E}-05$ \\
\hline S & $1.9 \mathrm{E}-05$ & $1.9 \mathrm{E}-05$ & $1.9 \mathrm{E}-05$ & 1.9E-05 & 1.9E-05 & 5 \\
\hline SSE & $1.8 \mathrm{E}-05$ & $1.8 \mathrm{E}-05$ & $1.8 \mathrm{E}-05$ & $1.8 \mathrm{E}-05$ & $1.7 \mathrm{E}-05$ & 1 \\
\hline SE & $5.9 \mathrm{E}-06$ & $5.9 \mathrm{E}-06$ & $5.8 \mathrm{E}-06$ & $5.8 \mathrm{E}-06$ & $5.6 \mathrm{E}-06$ & $5.3 E-06$ \\
\hline ESE & $3.5 E-06$ & $3.5 \mathrm{E}-06$ & $3.5 \mathrm{E}-06$ & 3.5 & $3.3 \mathrm{E}-$ & \\
\hline $\mathrm{F}$ & $3.9 \mathrm{E}-0$ & $3.9 \mathrm{E}-06$ & $3.8 \mathrm{E}-06$ & 3.8 & $3.7 \mathrm{E}-06$ & $3.5 \mathrm{E}-06$ \\
\hline ENE & $5.7 \mathrm{E}-0$ & $5.7 E-06$ & $5.6 \mathrm{E}-06$ & $5.6 \mathrm{E}$ & $5.5 E-06$ & $52 \mathrm{~F}$ \\
\hline $\mathrm{NE}$ & $\perp$ & $1 E-05$ & $1.1 \mathrm{E}$ & & 1 . & 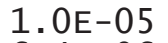 \\
\hline NNE & $7.0 \mathrm{E}-06$ & $6.9 \mathrm{E}-06$ & $6.9 \mathrm{E}-06$ & $6.9 \mathrm{E}-06$ & $6.6 \mathrm{E}-06$ & $6.4 \mathrm{E}-06$ \\
\hline
\end{tabular}

Feb 20, $2008 \quad 02: 31 \mathrm{pm}$

SUMMARY

Page 7

INDIVIDUAL LIFETIME RISK (deaths)

(A11 Radionuclides and Pathways)

\begin{tabular}{|c|c|c|c|c|c|c|c|}
\hline \multicolumn{8}{|c|}{ Distance $(\mathrm{m})$} \\
\hline Direction & 27715 & 28919 & 31060 & 32802 & 34577 & 35279 & 35683 \\
\hline $\begin{array}{r}\text { N } \\
\text { NNW } \\
N W \\
\text { WNW } \\
W \\
W S W \\
\text { SW } \\
\text { SSW } \\
S \\
\text { SSE } \\
\text { SE } \\
\text { ESE } \\
\text { E } \\
\text { ENE } \\
N E \\
\text { NNE }\end{array}$ & $\begin{array}{l}4.8 \mathrm{E}-12 \\
3.3 \mathrm{E}-12 \\
3.5 \mathrm{E}-12 \\
4.5 \mathrm{E}-12 \\
4.7 \mathrm{E}-12 \\
7.7 \mathrm{E}-12 \\
1.2 \mathrm{E}-11 \\
1.6 \mathrm{E}-11 \\
2.1 \mathrm{E}-11 \\
1.9 \mathrm{E}-11 \\
6.6 \mathrm{E}-12 \\
4.0 \mathrm{E}-12 \\
4.5 \mathrm{E}-12 \\
6.3 \mathrm{E}-12 \\
1.2 \mathrm{E}-11 \\
7.3 \mathrm{E}-12\end{array}$ & $\begin{array}{l}4.5 \mathrm{E}-12 \\
3.1 \mathrm{E}-12 \\
3.3 \mathrm{E}-12 \\
4.3 \mathrm{E}-12 \\
4.5 \mathrm{E}-12 \\
7.3 \mathrm{E}-12 \\
1.2 \mathrm{E}-11 \\
1.5 \mathrm{E}-11 \\
2.0 \mathrm{E}-11 \\
1.8 \mathrm{E}-11 \\
6.2 \mathrm{E}-12 \\
3.8 \mathrm{E}-12 \\
4.3 \mathrm{E}-12 \\
5.9 \mathrm{E}-12 \\
1.1 \mathrm{E}-11 \\
6.9 \mathrm{E}-12\end{array}$ & $\begin{array}{l}4.1 \mathrm{E}-12 \\
2.9 \mathrm{E}-12 \\
3.1 \mathrm{E}-12 \\
3.9 \mathrm{E}-12 \\
4.1 \mathrm{E}-12 \\
6.6 \mathrm{E}-12 \\
1.1 \mathrm{E}-11 \\
1.4 \mathrm{E}-11 \\
1.8 \mathrm{E}-11 \\
1.7 \mathrm{E}-11 \\
5.7 \mathrm{E}-12 \\
3.4 \mathrm{E}-12 \\
3.9 \mathrm{E}-12 \\
5.4 \mathrm{E}-12 \\
1.0 \mathrm{E}-11 \\
6.3 \mathrm{E}-12\end{array}$ & $\begin{array}{l}3.8 \mathrm{E}-12 \\
2.7 \mathrm{E}-12 \\
2.9 \mathrm{E}-12 \\
3.6 \mathrm{E}-12 \\
3.8 \mathrm{E}-12 \\
6.2 \mathrm{E}-12 \\
9.8 \mathrm{E}-12 \\
1.3 \mathrm{E}-11 \\
1.7 \mathrm{E}-11 \\
1.6 \mathrm{E}-11 \\
5.3 \mathrm{E}-12 \\
3.2 \mathrm{E}-12 \\
3.6 \mathrm{E}-12 \\
5.0 \mathrm{E}-12 \\
9.7 \mathrm{E}-12 \\
5.9 \mathrm{E}-12\end{array}$ & $\begin{array}{l}3.6 \mathrm{E}-12 \\
2.5 \mathrm{E}-12 \\
2.7 \mathrm{E}-12 \\
3.4 \mathrm{E}-12 \\
3.5 \mathrm{E}-12 \\
5.8 \mathrm{E}-12 \\
9.2 \mathrm{E}-12 \\
1.2 \mathrm{E}-11 \\
1.6 \mathrm{E}-11 \\
1.5 \mathrm{E}-11 \\
4.9 \mathrm{E}-12 \\
2.9 \mathrm{E}-12 \\
3.3 \mathrm{E}-12 \\
4.7 \mathrm{E}-12 \\
9.1 \mathrm{E}-12 \\
5.5 \mathrm{E}-12\end{array}$ & $\begin{array}{l}3.5 \mathrm{E}-12 \\
2.5 \mathrm{E}-12 \\
2.6 \mathrm{E}-12 \\
3.3 \mathrm{E}-12 \\
3.4 \mathrm{E}-12 \\
5.6 \mathrm{E}-12 \\
8.9 \mathrm{E}-12 \\
1.1 \mathrm{E}-11 \\
1.5 \mathrm{E}-11 \\
1.4 \mathrm{E}-11 \\
4.7 \mathrm{E}-12 \\
2.9 \mathrm{E}-12 \\
3.2 \mathrm{E}-12 \\
4.6 \mathrm{E}-12 \\
8.8 \mathrm{E}-12 \\
5.4 \mathrm{E}-12\end{array}$ & $\begin{array}{l}3.4 \mathrm{E}-12 \\
2.4 \mathrm{E}-12 \\
2.6 \mathrm{E}-12 \\
3.3 \mathrm{E}-12 \\
3.4 \mathrm{E}-12 \\
5.5 \mathrm{E}-12 \\
8.8 \mathrm{E}-12 \\
1.1 \mathrm{E}-11 \\
1.5 \mathrm{E}-11 \\
1.4 \mathrm{E}-11 \\
4.7 \mathrm{E}-12 \\
2.8 \mathrm{E}-12 \\
3.2 \mathrm{E}-12 \\
4.5 \mathrm{E}-12 \\
8.7 \mathrm{E}-12 \\
5.3 \mathrm{E}-12\end{array}$ \\
\hline \multicolumn{8}{|c|}{ Distance (m) } \\
\hline Direction & 36721 & 36809 & 37729 & 39079 & 39220 & 39559 & 43584 \\
\hline $\begin{array}{r}\text { N } \\
\text { NNW } \\
\text { NW } \\
\text { WNW } \\
W \\
\text { WSW } \\
\text { SW } \\
\text { SSW }\end{array}$ & $\begin{array}{l}3.3 \mathrm{E}-12 \\
2.4 \mathrm{E}-12 \\
2.5 \mathrm{E}-12 \\
3.1 \mathrm{E}-12 \\
3.3 \mathrm{E}-12 \\
5.3 \mathrm{E}-12 \\
8.5 \mathrm{E}-12 \\
1.1 \mathrm{E}-11\end{array}$ & $\begin{array}{l}3 \cdot 3 \mathrm{E}-12 \\
2 \cdot 3 \mathrm{E}-12 \\
2 \cdot 5 \mathrm{E}-12 \\
3 \cdot 1 \mathrm{E}-12 \\
3 \cdot 3 \mathrm{E}-12 \\
5 \cdot 3 \mathrm{E}-12 \\
8 \cdot 4 \mathrm{E}-12 \\
1.1 \mathrm{E}-11\end{array}$ & $\begin{array}{l}3.2 \mathrm{E}-12 \\
2.3 \mathrm{E}-12 \\
2.4 \mathrm{E}-12 \\
3.0 \mathrm{E}-12 \\
3.2 \mathrm{E}-12 \\
5.1 \mathrm{E}-12 \\
8.2 \mathrm{E}-12 \\
1.0 \mathrm{E}-11\end{array}$ & $\begin{array}{r}3.0 \mathrm{E}-12 \\
2.2 \mathrm{E}-12 \\
2.3 \mathrm{E}-12 \\
2.9 \mathrm{E}-12 \\
3.0 \mathrm{E}-12 \\
4.9 \mathrm{E}-12 \\
7.8 \mathrm{E}-12 \\
9.9 \mathrm{E}-12 \\
\text { Page }\end{array}$ & $\begin{array}{l}3.0 \mathrm{E}-12 \\
2.2 \mathrm{E}-12 \\
2.3 \mathrm{E}-12 \\
2.9 \mathrm{E}-12 \\
3.0 \mathrm{E}-12 \\
4.9 \mathrm{E}-12 \\
7.7 \mathrm{E}-12 \\
9.8 \mathrm{E}-12\end{array}$ & $\begin{array}{l}3.0 \mathrm{E}-12 \\
2.2 \mathrm{E}-12 \\
2.3 \mathrm{E}-12 \\
2.8 \mathrm{E}-12 \\
3.0 \mathrm{E}-12 \\
4.8 \mathrm{E}-12 \\
7.7 \mathrm{E}-12 \\
9.7 \mathrm{E}-12\end{array}$ & $\begin{array}{l}\text { 2. } 6 \mathrm{E}-12 \\
1.9 \mathrm{E}-12 \\
2.0 \mathrm{E}-12 \\
2.5 \mathrm{E}-12 \\
2.6 \mathrm{E}-12 \\
4.2 \mathrm{E}-12 \\
6.7 \mathrm{E}-12 \\
\text { 8.4E-12 }\end{array}$ \\
\hline
\end{tabular}




\begin{tabular}{rlllllll}
\multicolumn{7}{c}{ Be-7C.SUM } \\
S & $1.5 \mathrm{E}-11$ & $1.5 \mathrm{E}-11$ & $1.4 \mathrm{E}-11$ & $1.3 \mathrm{E}-11$ & $1.3 \mathrm{E}-11$ & $1.3 \mathrm{E}-11$ & $1.1 \mathrm{E}-11$ \\
SSE & $1.3 \mathrm{E}-11$ & $1.3 \mathrm{E}-11$ & $1.3 \mathrm{E}-11$ & $1.2 \mathrm{E}-11$ & $1.2 \mathrm{E}-11$ & $1.2 \mathrm{E}-11$ & $1.1 \mathrm{E}-11$ \\
SE & $4.5 \mathrm{E}-12$ & $4.5 \mathrm{E}-12$ & $4.3 \mathrm{E}-12$ & $4.1 \mathrm{E}-12$ & $4.1 \mathrm{E}-12$ & $4.0 \mathrm{E}-12$ & $3.5 \mathrm{E}-12$ \\
ESE & $2.7 \mathrm{E}-12$ & $2.7 \mathrm{E}-12$ & $2.6 \mathrm{E}-12$ & $2.5 \mathrm{E}-12$ & $2.5 \mathrm{E}-12$ & $2.4 \mathrm{E}-12$ & $2.1 \mathrm{E}-12$ \\
$\mathrm{E}$ & $3.0 \mathrm{E}-12$ & $3.0 \mathrm{E}-12$ & $2.9 \mathrm{E}-12$ & $2.8 \mathrm{E}-12$ & $2.8 \mathrm{E}-12$ & $2.7 \mathrm{E}-12$ & $2.3 \mathrm{E}-12$ \\
ENE & $4.3 \mathrm{E}-12$ & $4.3 \mathrm{E}-12$ & $4.2 \mathrm{E}-12$ & $4.0 \mathrm{E}-12$ & $3.9 \mathrm{E}-12$ & $3.9 \mathrm{E}-12$ & $3.4 \mathrm{E}-12$ \\
NE & $8.4 \mathrm{E}-12$ & $8.3 \mathrm{E}-12$ & $8.1 \mathrm{E}-12$ & $7.7 \mathrm{E}-12$ & $7.7 \mathrm{E}-12$ & $7.6 \mathrm{E}-12$ & $6.7 \mathrm{E}-12$ \\
NNE & $5.1 \mathrm{E}-12$ & $5.1 \mathrm{E}-12$ & $4.9 \mathrm{E}-12$ & $4.7 \mathrm{E}-12$ & $4.7 \mathrm{E}-12$ & $4.6 \mathrm{E}-12$ & $4.1 \mathrm{E}-12$
\end{tabular}

Feb 20, $2008 \quad 02: 31 \mathrm{pm}$

SUMMARY

Page 8

INDIVIDUAL LIFETIME RISK (deaths)

(A11 Radionuclides and Pathways)

\begin{tabular}{|c|c|c|c|c|c|c|}
\hline \multirow[b]{2}{*}{ Direction } & \multicolumn{6}{|c|}{ Distance $(\mathrm{m})$} \\
\hline & 45196 & 45275 & 45654 & 45677 & 46668 & 47969 \\
\hline $\begin{array}{r}N \\
N N W \\
N W \\
W N W \\
W \\
W S W \\
S W \\
\text { SSW } \\
S \\
\text { SSE } \\
\text { SE } \\
\text { ESE } \\
\text { E } \\
\text { ENE } \\
N E \\
\text { NNE }\end{array}$ & $\begin{array}{l}2.5 \mathrm{E}-12 \\
1.8 \mathrm{E}-12 \\
1.9 \mathrm{E}-12 \\
2.4 \mathrm{E}-12 \\
2.5 \mathrm{E}-12 \\
4.0 \mathrm{E}-12 \\
6.4 \mathrm{E}-12 \\
8.0 \mathrm{E}-12 \\
1.1 \mathrm{E}-11 \\
1.0 \mathrm{E}-11 \\
3.3 \mathrm{E}-12 \\
2.0 \mathrm{E}-12 \\
2.2 \mathrm{E}-12 \\
3.2 \mathrm{E}-12 \\
6.3 \mathrm{E}-12 \\
3.9 \mathrm{E}-12\end{array}$ & $\begin{array}{l}2.5 \mathrm{E}-12 \\
1.8 \mathrm{E}-12 \\
1.9 \mathrm{E}-12 \\
2.4 \mathrm{E}-12 \\
2.5 \mathrm{E}-12 \\
4.0 \mathrm{E}-12 \\
6.3 \mathrm{E}-12 \\
8.0 \mathrm{E}-12 \\
1.1 \mathrm{E}-11 \\
1.0 \mathrm{E}-11 \\
3.3 \mathrm{E}-12 \\
2.0 \mathrm{E}-12 \\
2.2 \mathrm{E}-12 \\
3.2 \mathrm{E}-12 \\
6.3 \mathrm{E}-12 \\
3.9 \mathrm{E}-12\end{array}$ & $\begin{array}{l}2.4 \mathrm{E}-12 \\
1.8 \mathrm{E}-12 \\
1.9 \mathrm{E}-12 \\
2.3 \mathrm{E}-12 \\
2.4 \mathrm{E}-12 \\
3.9 \mathrm{E}-12 \\
6.3 \mathrm{E}-12 \\
7.9 \mathrm{E}-12 \\
1.1 \mathrm{E}-11 \\
1.0 \mathrm{E}-11 \\
3.2 \mathrm{E}-12 \\
2.0 \mathrm{E}-12 \\
2.2 \mathrm{E}-12 \\
3.2 \mathrm{E}-12 \\
6.2 \mathrm{E}-12 \\
3.8 \mathrm{E}-12\end{array}$ & $\begin{array}{l}2.4 \mathrm{E}-12 \\
1.8 \mathrm{E}-12 \\
1.9 \mathrm{E}-12 \\
2.3 \mathrm{E}-12 \\
2.4 \mathrm{E}-12 \\
3.9 \mathrm{E}-12 \\
6.3 \mathrm{E}-12 \\
7.8 \mathrm{E}-12 \\
1.1 \mathrm{E}-11 \\
9.9 \mathrm{E}-12 \\
3.2 \mathrm{E}-12 \\
2.0 \mathrm{E}-12 \\
2.2 \mathrm{E}-12 \\
3.2 \mathrm{E}-12 \\
6.2 \mathrm{E}-12 \\
3.8 \mathrm{E}-12\end{array}$ & $\begin{array}{l}2.4 \mathrm{E}-12 \\
1.8 \mathrm{E}-12 \\
1.8 \mathrm{E}-12 \\
2.3 \mathrm{E}-12 \\
2.4 \mathrm{E}-12 \\
3.8 \mathrm{E}-12 \\
6.1 \mathrm{E}-12 \\
7.6 \mathrm{E}-12 \\
1.0 \mathrm{E}-11 \\
9.6 \mathrm{E}-12 \\
3.1 \mathrm{E}-12 \\
1.9 \mathrm{E}-12 \\
2.1 \mathrm{E}-12 \\
3.1 \mathrm{E}-12 \\
6.0 \mathrm{E}-12 \\
3.7 \mathrm{E}-12\end{array}$ & $\begin{array}{l}2.3 \mathrm{E}-12 \\
1.7 \mathrm{E}-12 \\
1.7 \mathrm{E}-12 \\
2.2 \mathrm{E}-12 \\
2.3 \mathrm{E}-12 \\
3.7 \mathrm{E}-12 \\
5.8 \mathrm{E}-12 \\
7.3 \mathrm{E}-12 \\
9.8 \mathrm{E}-12 \\
9.3 \mathrm{E}-12 \\
3.0 \mathrm{E}-12 \\
1.8 \mathrm{E}-12 \\
2.0 \mathrm{E}-12 \\
2.9 \mathrm{E}-12 \\
5.8 \mathrm{E}-12 \\
3.6 \mathrm{E}-12\end{array}$ \\
\hline
\end{tabular}




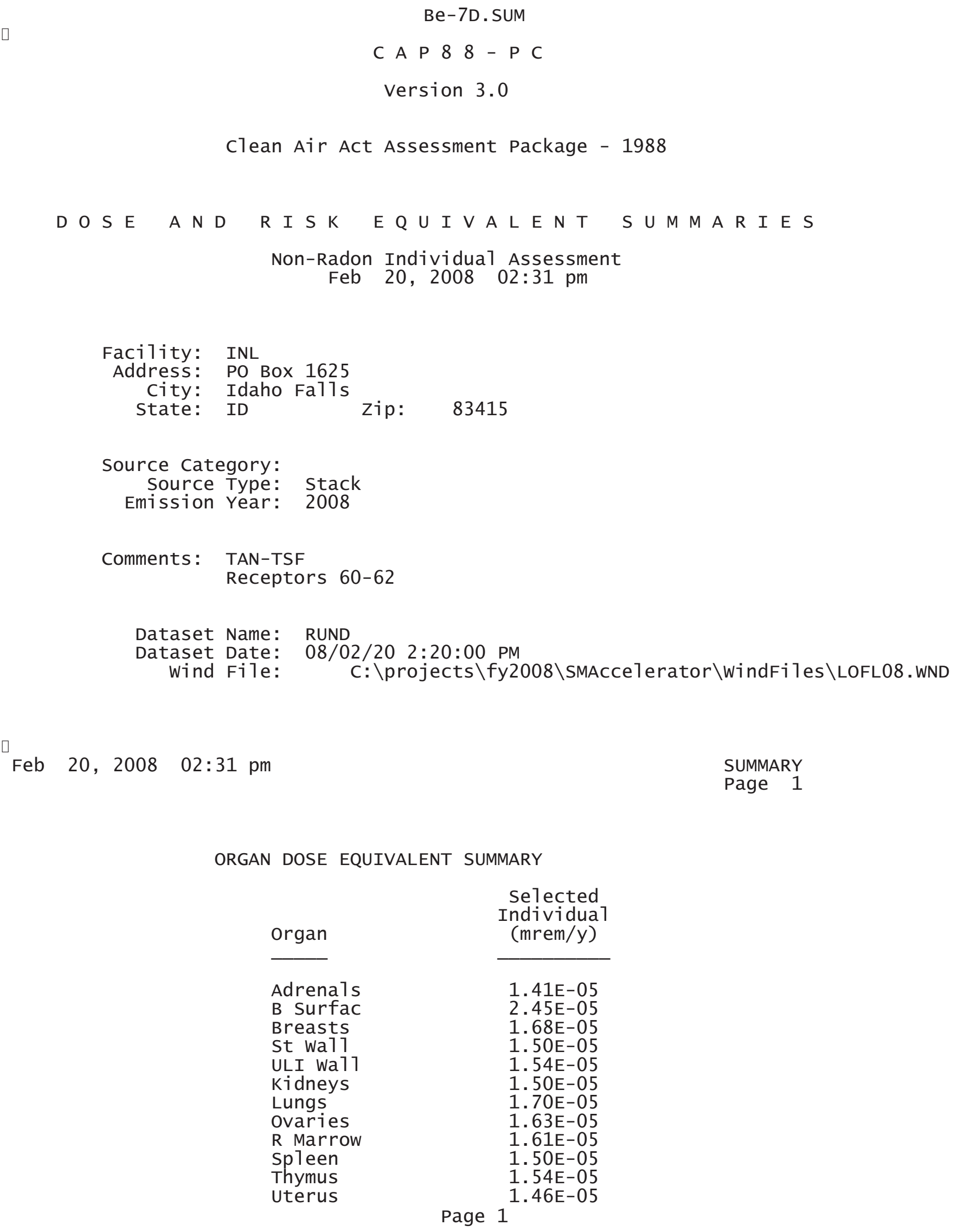




$\begin{array}{lr} & \text { Be-7D.SUM } \\ \text { Bld wa11 } & 1.52 \mathrm{E}-05 \\ \text { Brain } & 1.47 \mathrm{E}-05 \\ \text { Esophagu } & 1.48 \mathrm{E}-05 \\ \text { SI wa11 } & 1.48 \mathrm{E}-05 \\ \text { LLI wa11 } & 1.62 \mathrm{E}-05 \\ \text { Liver } & 1.50 \mathrm{E}-05 \\ \text { Muscle } & 1.71 \mathrm{E}-05 \\ \text { Pancreas } & 1.38 \mathrm{E}-05 \\ \text { Skin } & 1.96 \mathrm{E}-05 \\ \text { Testes } & 1.73 \mathrm{E}-05 \\ \text { Thyroid } & 1.65 \mathrm{E}-05 \\ & \\ \text { EFFEC } & 1.64 \mathrm{E}-05\end{array}$

PATHWAY EFFECTIVE DOSE EQUIVALENT SUMMARY

\begin{tabular}{c}
$\begin{array}{c}\text { Selected } \\
\text { Individua1 } \\
\text { (mrem/y) }\end{array}$ \\
\hline \\
$2.44 \mathrm{E}-07$ \\
$3.87 \mathrm{E}-07$ \\
$6.60 \mathrm{E}-08$ \\
$1.57 \mathrm{E}-05$ \\
$6.31 \mathrm{E}-07$ \\
$1.58 \mathrm{E}-05$ \\
$1.64 \mathrm{E}-05$
\end{tabular}
Feb 20, $2008 \quad 02: 31 \mathrm{pm}$

NUCLIDE EFFECTIVE DOSE EQUIVALENT SUMMARY

Nuclide

$\mathrm{Be}-7$

TOTAL

Feb 20, $2008 \quad 02: 31$ pm

$$
\begin{gathered}
\begin{array}{c}
\text { Selected } \\
\text { Individual } \\
\text { (mrem/y) }
\end{array} \\
\hline 1.64 \mathrm{E}-05 \\
1.64 \mathrm{E}-05
\end{gathered}
$$

SUMMARY

Page 3

CANCER RISK SUMMARY

Cancer

Esophagu
Selected Individual

Total Lifetime Fatal Cancer Risk

$$
\text { Page } 2^{1.57 \mathrm{E}-13}
$$




$\begin{array}{lr} & \text { Be-7D. SUM } \\ \text { Stomach } & 6.09 \mathrm{E}-13 \\ \text { Colon } & 1.67 \mathrm{E}-12 \\ \text { Liver } & 2.29 \mathrm{E}-13 \\ \text { LUNG } & 1.68 \mathrm{E}-12 \\ \text { Bone } & 2.33 \mathrm{E}-14 \\ \text { Skin } & 1.96 \mathrm{E}-14 \\ \text { Breast } & 8.15 \mathrm{E}-13 \\ \text { Ovary } & 2.33 \mathrm{E}-13 \\ \text { Bladder } & 3.72 \mathrm{E}-13 \\ \text { Kidneys } & 7.80 \mathrm{E}-14 \\ \text { Thyroid } & 5.25 \mathrm{E}-14 \\ \text { Leukemia } & 9.07 \mathrm{E}-13 \\ \text { Residua1 } & 2.24 \mathrm{E}-12 \\ \text { Tota1 } & 9.08 \mathrm{E}-12 \\ & \\ \text { TOTAL } & 1.82 \mathrm{E}-11\end{array}$

PATHWAY RISK SUMMARY

\begin{tabular}{|c|c|}
\hline Pathway & $\begin{array}{c}\text { Total Lifetime } \\
\text { Fatal Cancer Risk }\end{array}$ \\
\hline $\begin{array}{l}\text { INGESTION } \\
\text { INHALATION } \\
\text { AIR IMMERSION } \\
\text { GROUND SURFACE } \\
\text { INTERNAL } \\
\text { EXTERNAL }\end{array}$ & $\begin{array}{l}1.68 \mathrm{E}-13 \\
2.83 \mathrm{E}-13 \\
3.59 \mathrm{E}-14 \\
8.60 \mathrm{E}-12 \\
4.51 \mathrm{E}-13 \\
8.63 \mathrm{E}-12\end{array}$ \\
\hline TOTAL & $9.08 \mathrm{E}-12$ \\
\hline
\end{tabular}

$\begin{array}{lll} & \text { Feb 20, } 2008 \quad 02: 31 \mathrm{pm} & \text { SUMMARY } \\ \text { Page } 4\end{array}$

NUCLIDE RISK SUMMARY

Nuclide

Be-7

TOTAL

口

Feb 20, 2008 02:31 pm
Selected Individual

Total Lifetime Fatal Cancer Risk

$9.08 \mathrm{E}-12$

$9.08 \mathrm{E}-12$

INDIVIDUAL EFFECTIVE DOSE EQUIVALENT RATE (mrem/y) (A11 Radionuclides and Pathways)

Distance $(m)$ 


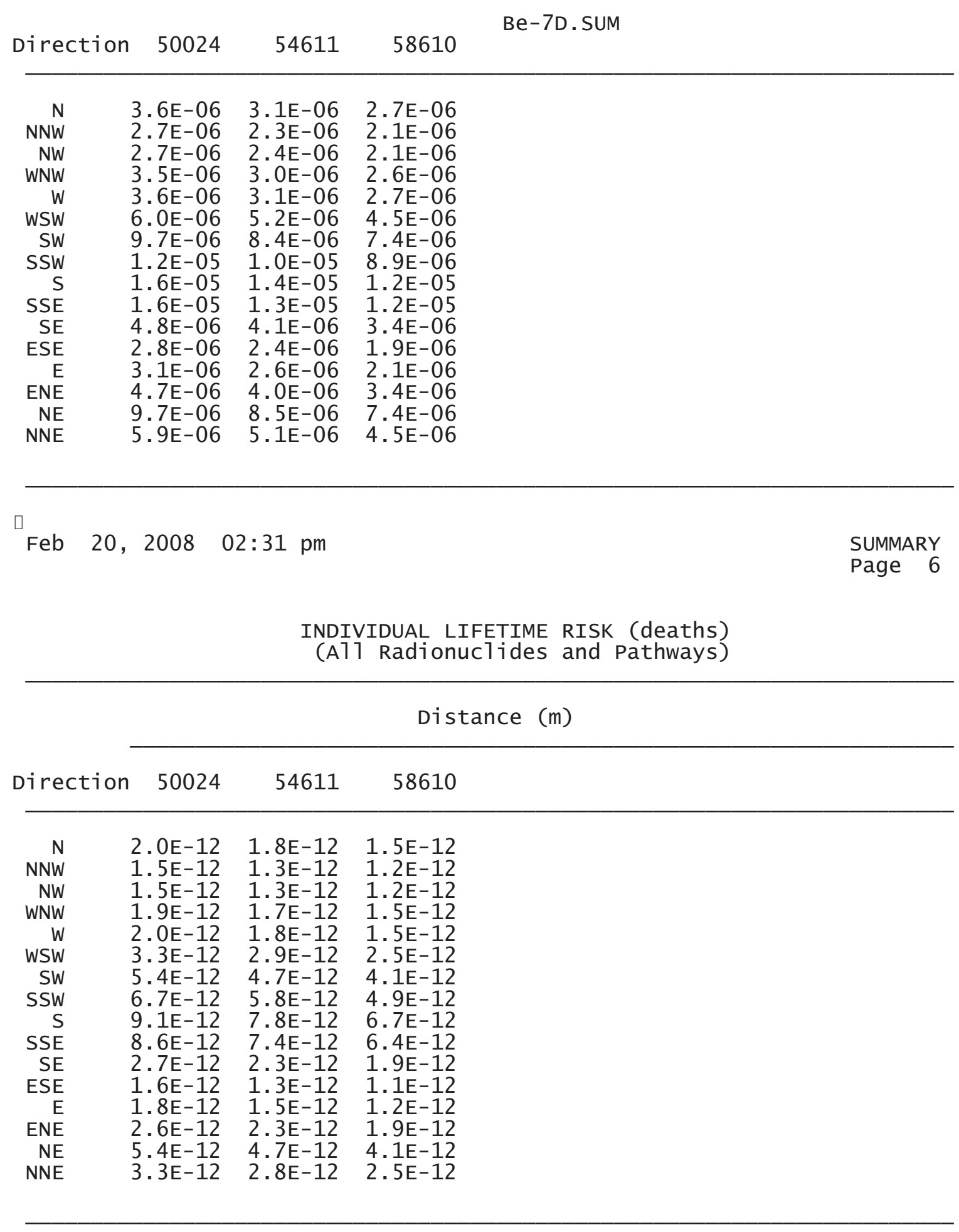




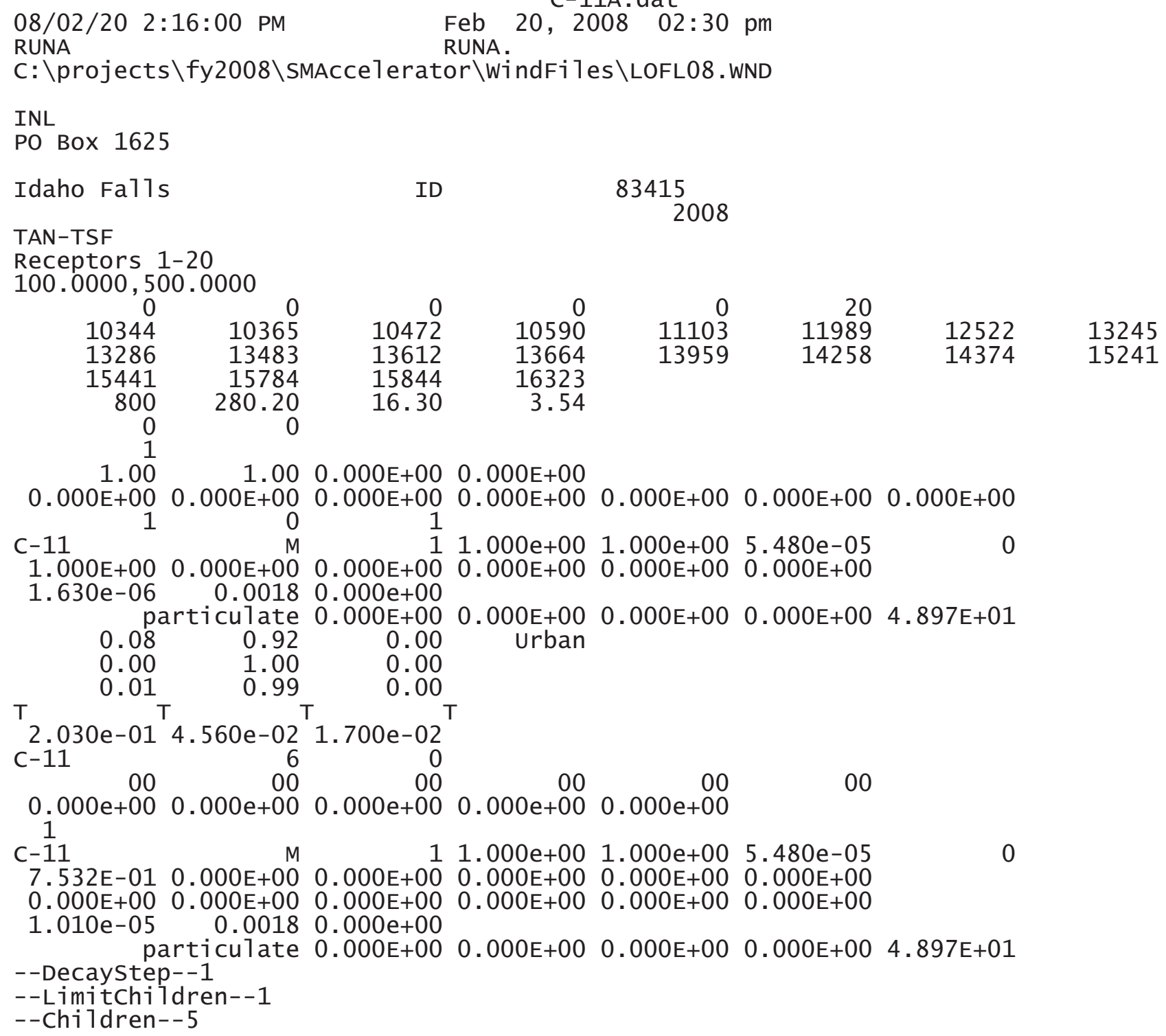




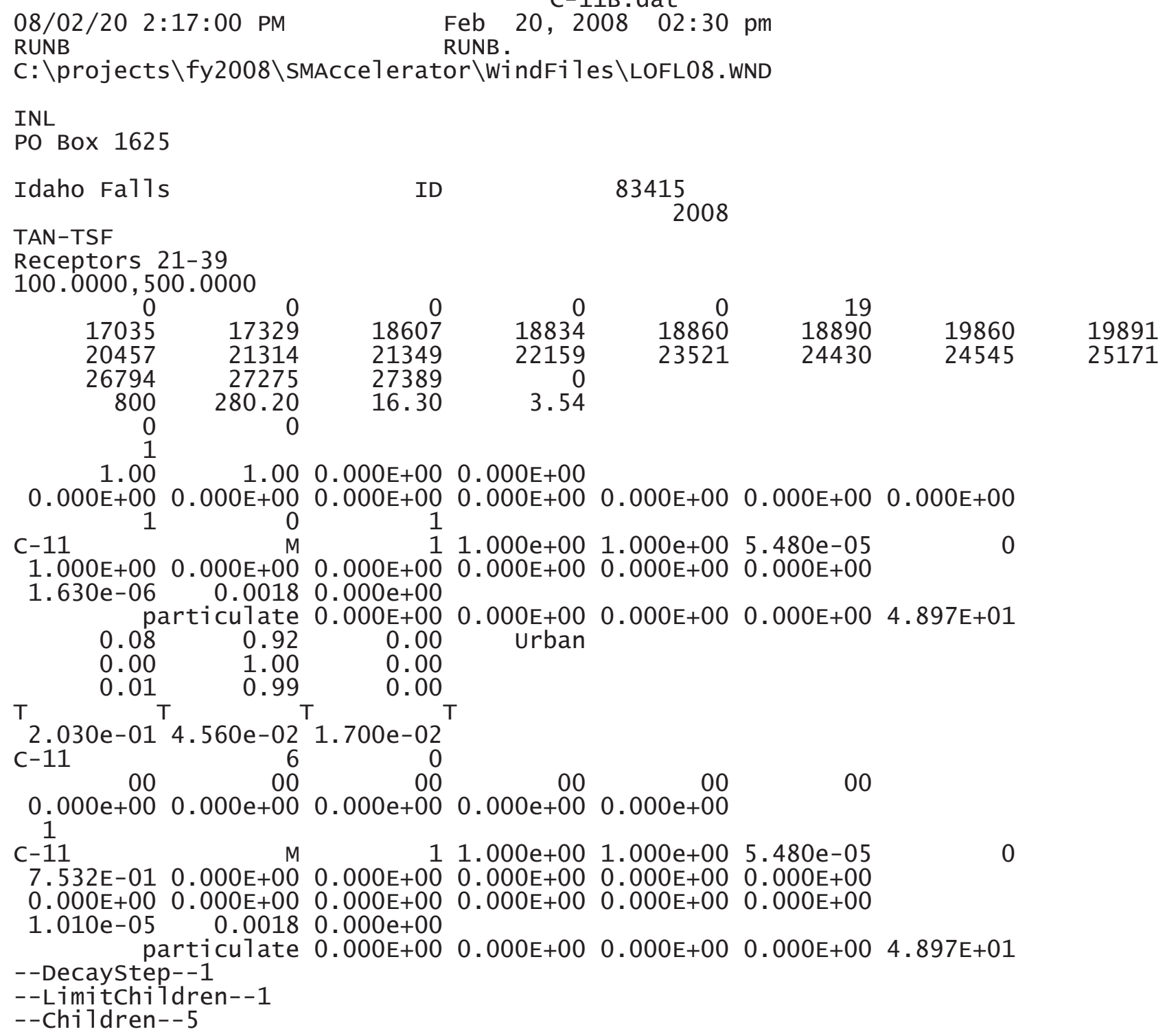




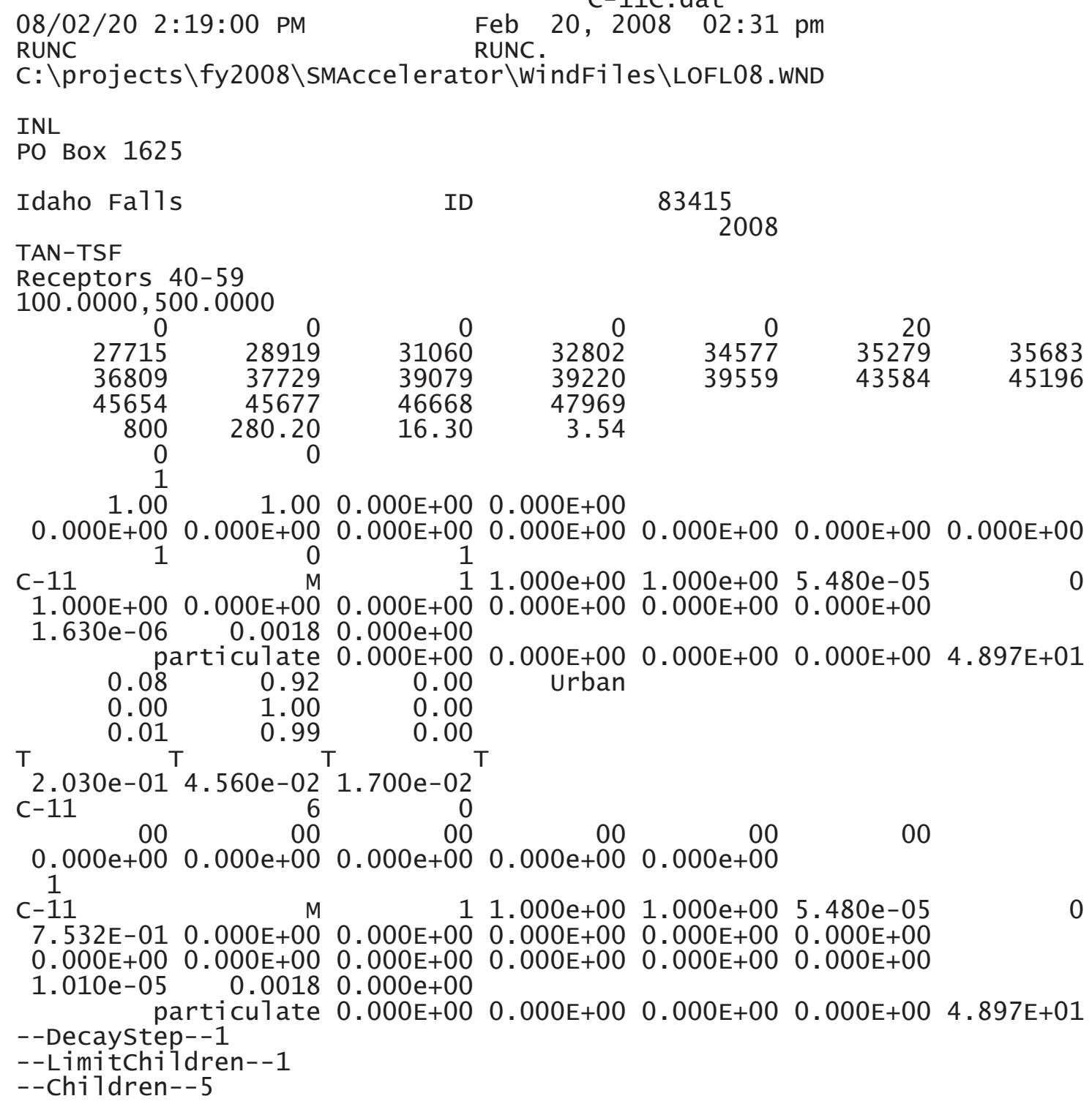

ID

83415

TAN-TSF

Receptors 40-59

$100.0000,500.0000$

$\begin{array}{rrrr}0 & 0 & 0 & \\ 27715 & 28919 & 31060 & 3280 \\ 36809 & 37729 & 39079 & 3922 \\ 45654 & 45677 & 46668 & 4796 \\ 800 & 280.20 & 16.30 & 3.5 \\ 0 & 0 & & \end{array}$




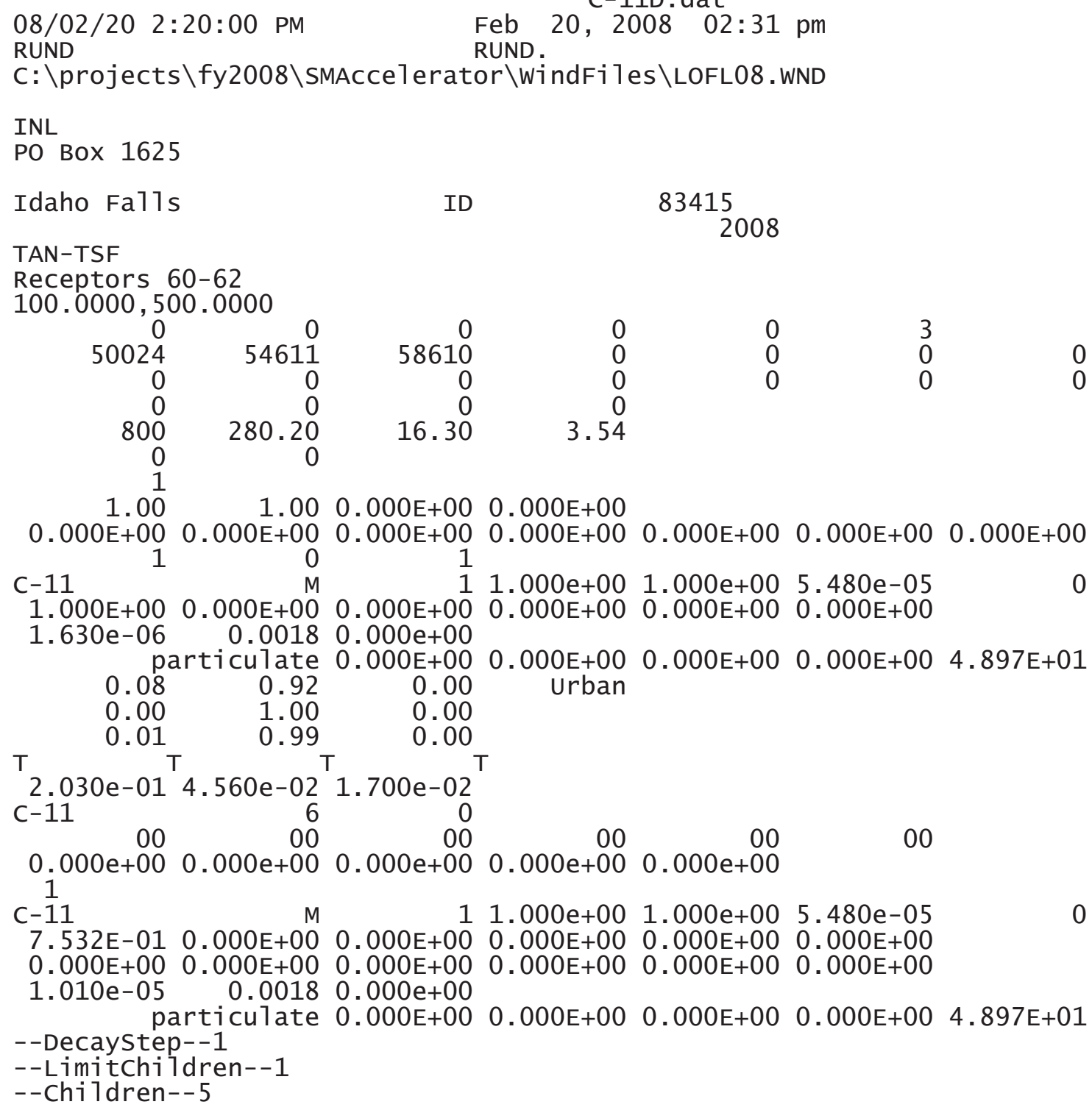




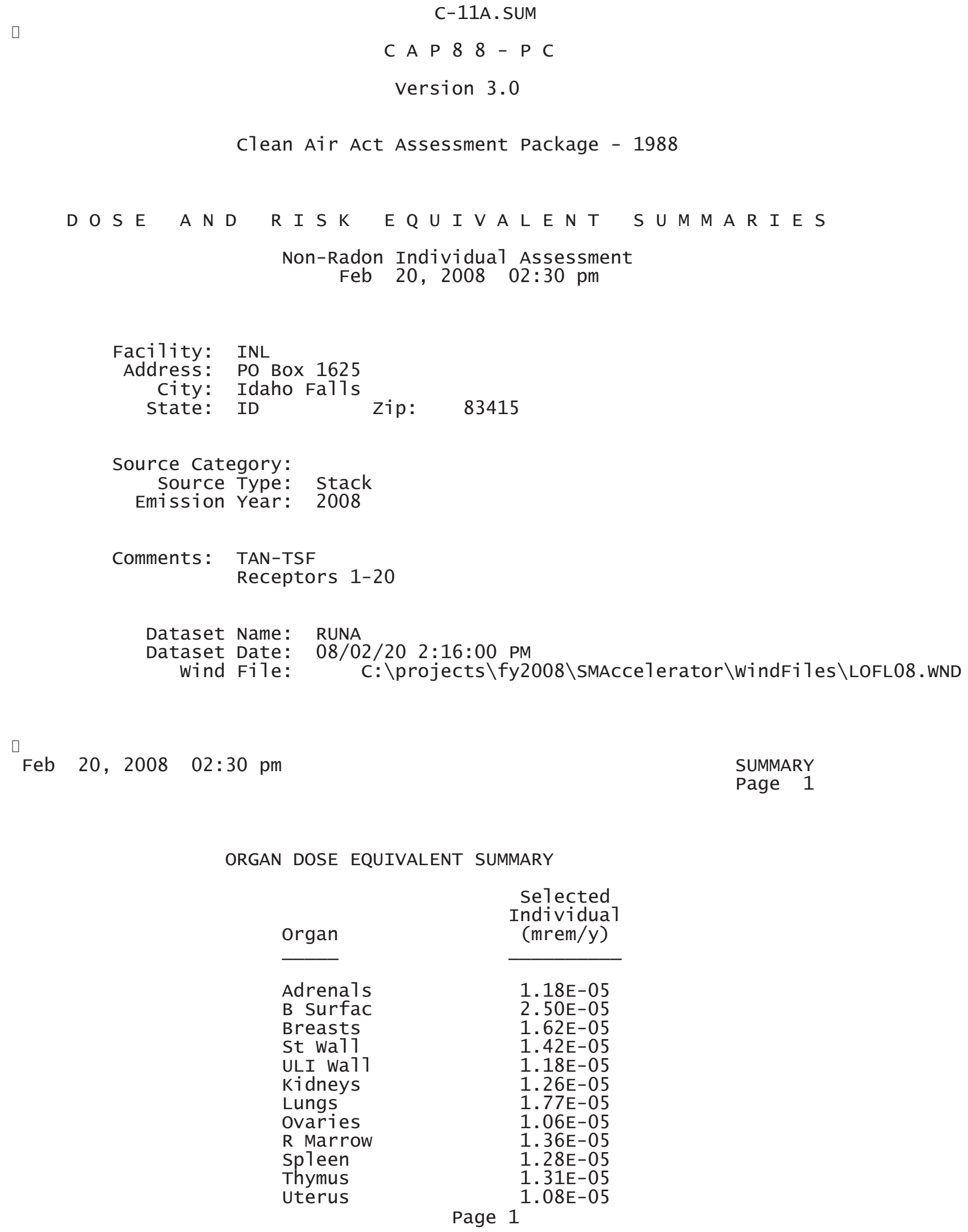




$\begin{array}{lr} & \text { C-11A.SUM } \\ \text { B1d wa11 } & 1.17 \mathrm{E}-05 \\ \text { Brain } & 1.50 \mathrm{E}-05 \\ \text { Esophagu } & 3.78 \mathrm{E}-05 \\ \text { SI wa11 } & 1.15 \mathrm{E}-05 \\ \text { LLI wa11 } & 1.13 \mathrm{E}-05 \\ \text { Liver } & 1.27 \mathrm{E}-05 \\ \text { Muscle } & 1.38 \mathrm{E}-05 \\ \text { Pancreas } & 1.11 \mathrm{E}-05 \\ \text { Skin } & 2.34 \mathrm{E}-05 \\ \text { Testes } & 1.42 \mathrm{E}-05 \\ \text { Thyroid } & 1.45 \mathrm{E}-05 \\ & \\ \text { EFFEC } & 1.48 \mathrm{E}-05\end{array}$

PATHWAY EFFECTIVE DOSE EQUIVALENT SUMMARY

$\begin{array}{lc}\text { Pathway } & \begin{array}{c}\text { selected } \\ \text { Individual } \\ \text { (mrem/y) }\end{array} \\ & \\ \text { INGESTION } & 0.00 \mathrm{E}+00 \\ \text { INHALATION } & 1.34 \mathrm{E}-06 \\ \text { AIR IMMERSION } & 1.35 \mathrm{E}-05 \\ \text { GROUND SURFACE } & 0.00 \mathrm{E}+00 \\ \text { INTERNAL } & 1.34 \mathrm{E}-06 \\ \text { EXTERNAL } & 1.35 \mathrm{E}-05 \\ \text { TOTAL } & 1.48 \mathrm{E}-05\end{array}$

Feb 20, $2008 \quad 02: 30$ pm

SUMMARY

Page 2

NUCLIDE EFFECTIVE DOSE EQUIVALENT SUMMARY

Nuclide

C-11

TOTAL

Feb 20, $2008 \quad 02: 30$ pm

$$
\begin{gathered}
\begin{array}{c}
\text { Selected } \\
\text { Individua } \\
(\mathrm{mrem} / \mathrm{y})
\end{array} \\
\hline 1.48 \mathrm{E}-05 \\
1.48 \mathrm{E}-05
\end{gathered}
$$$$
1.48 \mathrm{E}-05
$$ \\ $1.48 \mathrm{E}-05$}

SUMMARY

Page 3

Cancer

Esophagu
Selected Individual

Total Lifetime

Fatal Cancer Risk

$$
\text { Page } 2^{1.34 \mathrm{E}-13}
$$




$\begin{array}{lr} & \text { C-11A. SUM } \\ \text { Stomach } & 5.97 \mathrm{E}-13 \\ \text { Colon } & 1.20 \mathrm{E}-12 \\ \text { Liver } & 1.92 \mathrm{E}-13 \\ \text { LUNG } & 1.75 \mathrm{E}-12 \\ \text { Bone } & 2.37 \mathrm{E}-14 \\ \text { Skin } & 2.34 \mathrm{E}-14 \\ \text { Breast } & 7.85 \mathrm{E}-13 \\ \text { Ovary } & 1.50 \mathrm{E}-13 \\ \text { B Tadder } & 2.84 \mathrm{E}-13 \\ \text { Kidneys } & 6.57 \mathrm{E}-14 \\ \text { Thyroid } & 4.60 \mathrm{E}-14 \\ \text { Leukemia } & 7.64 \mathrm{E}-13 \\ \text { Residua1 } & 1.83 \mathrm{E}-12 \\ \text { Tota1 } & 7.85 \mathrm{E}-12 \\ & \\ \text { TOTAL } & 1.57 \mathrm{E}-11\end{array}$

PATHWAY RISK SUMMARY

\begin{tabular}{lc} 
Pathway & $\begin{array}{c}\text { Selected Individua1 } \\
\text { Tota1 Lifetime } \\
\text { Fatal Cancer Risk }\end{array}$ \\
\cline { 2 - 2 } INGESTION & $0.00 \mathrm{E}+00$ \\
INHALATION & $5.10 \mathrm{E}-13$ \\
AIR IMMERSION & $7.34 \mathrm{E}-12$ \\
GROUND SURFACE & $0.00 \mathrm{E}+00$ \\
INTERNAL & $5.10 \mathrm{E}-13$ \\
EXTERNAL & $7.34 \mathrm{E}-12$ \\
TOTAL & $7.85 \mathrm{E}-12$
\end{tabular}

Feb 20, $2008 \quad 02: 30$ pm

SUMMARY Page 4

NUCLIDE RISK SUMMARY

Nuclide

C-11

TOTAL

$\square$

Feb 20, 2008 02:30 pm
Selected Individual

Total Lifetime Fatal Cancer Risk

$7.85 \mathrm{E}-12$

7. $85 \mathrm{E}-12$

INDIVIDUAL EFFECTIVE DOSE EQUIVALENT RATE (mrem/y) (A11 Radionuclides and Pathways)

Distance $(m)$ 


\begin{tabular}{|c|c|c|c|c|c|c|c|}
\hline Direction & 10344 & 10365 & 10472 & $\begin{array}{l}C-11 \mathrm{~A} . \\
10590\end{array}$ & M 11103 & 11989 & 12522 \\
\hline$N$ & $2.9 \mathrm{E}-06$ & $2.8 \mathrm{E}-06$ & $2.8 \mathrm{E}-06$ & $2.8 \mathrm{E}-06$ & $2.6 \mathrm{E}-06$ & $2.3 E-06$ & $2.2 E-06$ \\
\hline NNW & 1.3E-06 & 1. $3 \mathrm{E}-06$ & 1. $3 \mathrm{E}-06$ & 1. $3 \mathrm{E}-06$ & $1.2 \mathrm{E}-06$ & $1.1 \mathrm{E}-06$ & 1. $0 \mathrm{E}-06$ \\
\hline NW & $1.6 \mathrm{E}-06$ & $1.6 \mathrm{E}-06$ & $1.6 \mathrm{E}-06$ & $1.6 \mathrm{E}-06$ & $1.5 \mathrm{E}-06$ & 1.3E-06 & 1. $3 \mathrm{E}-06$ \\
\hline WNW & $2.2 \mathrm{E}-06$ & $2.2 \mathrm{E}-06$ & $2.1 \mathrm{E}-06$ & $2.1 \mathrm{E}-06$ & $2.0 \mathrm{E}-06$ & $1.8 \mathrm{E}-06$ & $1.7 \mathrm{E}-06$ \\
\hline W & $2.3 \mathrm{E}-06$ & $2.3 \mathrm{E}-06$ & $2.2 \mathrm{E}-06$ & $2.2 \mathrm{E}-06$ & $2.1 \mathrm{E}-06$ & $1.9 \mathrm{E}-06$ & 1. $7 \mathrm{E}-06$ \\
\hline WSW & $3.6 \mathrm{E}-06$ & $3.6 \mathrm{E}-06$ & $3.6 \mathrm{E}-06$ & $3.5 \mathrm{E}-06$ & $3.3 \mathrm{E}-06$ & $3.0 \mathrm{E}-06$ & $2.8 \mathrm{E}-06$ \\
\hline SW & $6.5 \mathrm{E}-06$ & $6.5 \mathrm{E}-06$ & $6.4 \mathrm{E}-06$ & $6.3 \mathrm{E}-06$ & $5.9 \mathrm{E}-06$ & $5.3 \mathrm{E}-06$ & $5.0 \mathrm{E}-06$ \\
\hline SSW & 1.1E-05 & $1.0 \mathrm{E}-05$ & 1.0E-05 & 1.0E-05 & $9.5 \mathrm{E}-06$ & $8.6 \mathrm{E}-06$ & $8.1 \mathrm{E}-06$ \\
\hline $\mathrm{S}$ & 1. $5 \mathrm{E}-05$ & 1. $5 \mathrm{E}-05$ & 1. $5 \mathrm{E}-05$ & 1. $4 \mathrm{E}-05$ & 1. $3 \mathrm{E}-05$ & $1.2 \mathrm{E}-05$ & 1.1E-05 \\
\hline SSE & 1. $3 \mathrm{E}-05$ & 1. $3 \mathrm{E}-05$ & 1. $3 \mathrm{E}-05$ & 1. $3 \mathrm{E}-05$ & $1.2 \mathrm{E}-05$ & $1.1 \mathrm{E}-05$ & 1. $0 \mathrm{E}-05$ \\
\hline SE & $5.3 \mathrm{E}-06$ & $5.3 E-06$ & $5.2 \mathrm{E}-06$ & $5.1 \mathrm{E}-06$ & $4.8 \mathrm{E}-06$ & $4.3 \mathrm{E}-06$ & 4.0E-06 \\
\hline ESE & $3.3 \mathrm{E}-06$ & $3.2 \mathrm{E}-06$ & $3.2 \mathrm{E}-06$ & $3.2 \mathrm{E}-06$ & $3.0 \mathrm{E}-06$ & $2.7 \mathrm{E}-06$ & 2. $5 \mathrm{E}-06$ \\
\hline E & $4.1 \mathrm{E}-06$ & $4.1 \mathrm{E}-06$ & 4.0E-06 & $4.0 \mathrm{E}-06$ & $3.7 \mathrm{E}-06$ & $3.3 \mathrm{E}-06$ & $3.1 \mathrm{E}-06$ \\
\hline ENE & 4. $4 \mathrm{E}-06$ & 4. $4 \mathrm{E}-06$ & 4. $4 \mathrm{E}-06$ & $4.3 \mathrm{E}-06$ & $4.0 \mathrm{E}-06$ & $3.6 \mathrm{E}-06$ & $3.4 \mathrm{E}-06$ \\
\hline NE & $7.4 \mathrm{E}-06$ & 7.4E-06 & $7.3 E-06$ & $7.1 \mathrm{E}-06$ & $6.7 \mathrm{E}-06$ & $6.0 \mathrm{E}-06$ & $5.6 \mathrm{E}-06$ \\
\hline NNE & $4.2 \mathrm{E}-06$ & $4.2 \mathrm{E}-06$ & $4.1 \mathrm{E}-06$ & $4.0 \mathrm{E}-06$ & $3.8 \mathrm{E}-06$ & $3.4 \mathrm{E}-06$ & $3.2 \mathrm{E}-06$ \\
\hline
\end{tabular}

Distance (m)

\begin{tabular}{|c|c|c|c|c|c|c|c|}
\hline Direction & n 13245 & 13286 & 13483 & 13612 & 13664 & 13959 & 14258 \\
\hline $\mathrm{N}$ & $2.0 \mathrm{E}-06$ & $2.0 \mathrm{E}-06$ & $2.0 \mathrm{E}-06$ & $2.0 \mathrm{E}-06$ & $1.9 \mathrm{E}-06$ & $1.9 \mathrm{E}-06$ & $1.8 \mathrm{E}-06$ \\
\hline NNW & $9.4 \mathrm{E}-07$ & $9.4 \mathrm{E}-07$ & $9.2 \mathrm{E}-07$ & $9.1 \mathrm{E}-07$ & $9.0 \mathrm{E}-07$ & $8.8 \mathrm{E}-07$ & $8.5 \mathrm{E}-07$ \\
\hline NW & $1.2 \mathrm{E}-06$ & 1. $2 \mathrm{E}-06$ & 1.1 $\mathrm{E}-06$ & $1.1 \mathrm{E}-06$ & $1.1 \mathrm{E}-06$ & $1.1 \mathrm{E}-06$ & $1.1 \mathrm{E}-06$ \\
\hline WNW & $1.6 \mathrm{E}-06$ & $1.5 \mathrm{E}-06$ & $1.5 \mathrm{E}-06$ & $1.5 \mathrm{E}-06$ & $1.5 \mathrm{E}-06$ & $1.4 \mathrm{E}-06$ & $1.4 \mathrm{E}-06$ \\
\hline W & $1.6 \mathrm{E}-06$ & $1.6 \mathrm{E}-06$ & $1.6 \mathrm{E}-06$ & $1.6 \mathrm{E}-06$ & $1.5 \mathrm{E}-06$ & $1.5 \mathrm{E}-06$ & $1.5 \mathrm{E}-06$ \\
\hline WSW & $2.6 \mathrm{E}-06$ & $2.6 \mathrm{E}-06$ & $2.5 \mathrm{E}-06$ & $2.5 \mathrm{E}-06$ & $2.5 \mathrm{E}-06$ & $2.4 \mathrm{E}-06$ & $2.3 \mathrm{E}-06$ \\
\hline SW & $4.6 \mathrm{E}-06$ & $4.6 \mathrm{E}-06$ & $4.5 \mathrm{E}-06$ & $4.4 \mathrm{E}-06$ & $4.4 \mathrm{E}-06$ & $4.3 \mathrm{E}-06$ & $4.2 \mathrm{E}-06$ \\
\hline SSW & $7.4 \mathrm{E}-06$ & 7. $4 \mathrm{E}-06$ & 7. $3 \mathrm{E}-06$ & $7.2 \mathrm{E}-06$ & $7.1 \mathrm{E}-06$ & $6.9 \mathrm{E}-06$ & $6.7 \mathrm{E}-06$ \\
\hline $\mathrm{s}$ & $1.0 \mathrm{E}-05$ & $1.0 \mathrm{E}-05$ & 1. $0 \mathrm{E}-05$ & $1.0 \mathrm{E}-05$ & 1.0E-05 & $9.7 \mathrm{E}-06$ & $9.4 \mathrm{E}-06$ \\
\hline SSE & $9.4 \mathrm{E}-06$ & $9.4 \mathrm{E}-06$ & $9.2 \mathrm{E}-06$ & $9.1 \mathrm{E}-06$ & $9.0 \mathrm{E}-06$ & $8.7 \mathrm{E}-06$ & $8.5 \mathrm{E}-06$ \\
\hline SE & $3.7 \mathrm{E}-06$ & $3.7 \mathrm{E}-06$ & $3.6 \mathrm{E}-06$ & $3.6 \mathrm{E}-06$ & $3.6 \mathrm{E}-06$ & $3.5 \mathrm{E}-06$ & $3.4 \mathrm{E}-06$ \\
\hline ESE & $2.3 \mathrm{E}-06$ & $2.3 \mathrm{E}-06$ & $2.2 \mathrm{E}-06$ & $2.2 \mathrm{E}-06$ & $2.2 \mathrm{E}-06$ & $2.1 \mathrm{E}-06$ & $2.1 \mathrm{E}-06$ \\
\hline & $2.9 \mathrm{E}-06$ & $2.9 \mathrm{E}-06$ & $2.8 \mathrm{E}-06$ & $2.8 \mathrm{E}-06$ & $2.8 \mathrm{E}-06$ & $2.7 \mathrm{E}-06$ & $2.6 \mathrm{E}-06$ \\
\hline ENE & $3.2 \mathrm{E}-06$ & $3.1 \mathrm{E}-06$ & $3.1 \mathrm{E}-06$ & $3.0 \mathrm{E}-06$ & $3.0 \mathrm{E}-06$ & $2.9 \mathrm{E}-06$ & $2.8 \mathrm{E}-06$ \\
\hline $\mathrm{NE}$ & $5.2 \mathrm{E}-06$ & $5.2 \mathrm{E}-06$ & $5.1 \mathrm{E}-06$ & $5.0 \mathrm{E}-06$ & $5.0 \mathrm{E}-06$ & $4.8 \mathrm{E}-06$ & $4.7 \mathrm{E}-06$ \\
\hline NNE & $3.0 \mathrm{E}-06$ & $2.9 \mathrm{E}-06$ & $2.9 \mathrm{E}-06$ & $2.8 \mathrm{E}-06$ & $2.8 \mathrm{E}-06$ & $2.7 \mathrm{E}-06$ & $2.7 \mathrm{E}-06$ \\
\hline
\end{tabular}

$\square$

Feb 20, 2008 02:30 pm

SUMMARY

Page 6

INDIVIDUAL EFFECTIVE DOSE EQUIVALENT RATE (mrem/y) (A11 Radionuclides and Pathways)

\begin{tabular}{lllllll}
\hline & \multicolumn{5}{c}{ Distance $(\mathrm{m})$} \\
\cline { 2 - 6 } Direction & 14374 & 15241 & 15441 & 15784 & 15844 & 16323 \\
\hline
\end{tabular}

\begin{tabular}{|c|c|c|c|c|c|c|}
\hline $\begin{array}{r}\mathrm{N} \\
\text { NNW }\end{array}$ & $\begin{array}{l}\text { 1. } 8 \mathrm{E}-06 \\
\text { 8. } 4 \mathrm{E}-07\end{array}$ & $\begin{array}{l}1.7 \mathrm{E}-06 \\
7.8 \mathrm{E}-07\end{array}$ & $\begin{array}{l}1.6 \mathrm{E}-06 \\
7.6 \mathrm{E}-07\end{array}$ & $\begin{array}{r}\text { 1. } 6 \mathrm{E}-06 \\
\text { 7. } 4 \mathrm{E}-07 \\
\text { Page }\end{array}$ & $\begin{array}{r}1.6 \mathrm{E}-06 \\
7.4 \mathrm{E}-07\end{array}$ & $\begin{array}{l}1.5 \mathrm{E}-06 \\
7.1 \mathrm{E}-07\end{array}$ \\
\hline
\end{tabular}




\begin{tabular}{rllllll} 
& \multicolumn{5}{c}{$\mathrm{C}-11 \mathrm{~A} . \mathrm{SUM}$} \\
$\mathrm{NW}$ & $1.1 \mathrm{E}-06$ & $9.7 \mathrm{E}-07$ & $9.5 \mathrm{E}-07$ & $9.2 \mathrm{E}-07$ & $9.2 \mathrm{E}-07$ & $8.8 \mathrm{E}-07$ \\
WNW & $1.4 \mathrm{E}-06$ & $1.3 \mathrm{E}-06$ & $1.3 \mathrm{E}-06$ & $1.2 \mathrm{E}-06$ & $1.2 \mathrm{E}-06$ & $1.2 \mathrm{E}-06$ \\
$\mathrm{~W}$ & $1.4 \mathrm{E}-06$ & $1.3 \mathrm{E}-06$ & $1.3 \mathrm{E}-06$ & $1.3 \mathrm{E}-06$ & $1.2 \mathrm{E}-06$ & $1.2 \mathrm{E}-06$ \\
WSW & $2.3 \mathrm{E}-06$ & $2.1 \mathrm{E}-06$ & $2.1 \mathrm{E}-06$ & $2.0 \mathrm{E}-06$ & $2.0 \mathrm{E}-06$ & $1.9 \mathrm{E}-06$ \\
$\mathrm{SW}$ & $4.1 \mathrm{E}-06$ & $3.8 \mathrm{E}-06$ & $3.7 \mathrm{E}-06$ & $3.6 \mathrm{E}-06$ & $3.6 \mathrm{E}-06$ & $3.4 \mathrm{E}-06$ \\
$\mathrm{SSW}$ & $6.6 \mathrm{E}-06$ & $6.1 \mathrm{E}-06$ & $6.0 \mathrm{E}-06$ & $5.8 \mathrm{E}-06$ & $5.8 \mathrm{E}-06$ & $5.5 \mathrm{E}-06$ \\
$\mathrm{~S}$ & $9.3 \mathrm{E}-06$ & $8.5 \mathrm{E}-06$ & $8.3 \mathrm{E}-06$ & $8.1 \mathrm{E}-06$ & $8.0 \mathrm{E}-06$ & $7.7 \mathrm{E}-06$ \\
$\mathrm{SSE}$ & $8.4 \mathrm{E}-06$ & $7.7 \mathrm{E}-06$ & $7.6 \mathrm{E}-06$ & $7.3 \mathrm{E}-06$ & $7.3 \mathrm{E}-06$ & $7.0 \mathrm{E}-06$ \\
$\mathrm{SE}$ & $3.3 \mathrm{E}-06$ & $3.0 \mathrm{E}-06$ & $3.0 \mathrm{E}-06$ & $2.9 \mathrm{E}-06$ & $2.9 \mathrm{E}-06$ & $2.7 \mathrm{E}-06$ \\
$\mathrm{ESE}$ & $2.0 \mathrm{E}-06$ & $1.9 \mathrm{E}-06$ & $1.8 \mathrm{E}-06$ & $1.8 \mathrm{E}-06$ & $1.8 \mathrm{E}-06$ & $1.7 \mathrm{E}-06$ \\
$\mathrm{E}$ & $2.6 \mathrm{E}-06$ & $2.3 \mathrm{E}-06$ & $2.3 \mathrm{E}-06$ & $2.2 \mathrm{E}-06$ & $2.2 \mathrm{E}-06$ & $2.1 \mathrm{E}-06$ \\
$\mathrm{ENE}$ & $2.8 \mathrm{E}-06$ & $2.6 \mathrm{E}-06$ & $2.5 \mathrm{E}-06$ & $2.5 \mathrm{E}-06$ & $2.4 \mathrm{E}-06$ & $2.3 \mathrm{E}-06$ \\
$\mathrm{NE}$ & $4.6 \mathrm{E}-06$ & $4.2 \mathrm{E}-06$ & $4.2 \mathrm{E}-06$ & $4.0 \mathrm{E}-06$ & $4.0 \mathrm{E}-06$ & $3.8 \mathrm{E}-06$ \\
$\mathrm{NNE}$ & $2.6 \mathrm{E}-06$ & $2.4 \mathrm{E}-06$ & $2.4 \mathrm{E}-06$ & $2.3 \mathrm{E}-06$ & $2.3 \mathrm{E}-06$ & $2.2 \mathrm{E}-06$ \\
& & & & & &
\end{tabular}

Feb 20, $2008 \quad 02: 30 \mathrm{pm}$

SUMMARY

Page 7

INDIVIDUAL LIFETIME RISK (deaths)

(A11 Radionuclides and Pathways)

\begin{tabular}{|c|c|c|c|c|c|c|c|}
\hline \multicolumn{8}{|c|}{ Distance $(\mathrm{m})$} \\
\hline Direction & 10344 & 10365 & 10472 & 10590 & 11103 & 11989 & 12522 \\
\hline $\begin{array}{r}\text { N } \\
N N W \\
N W \\
W N W \\
W \\
W S W \\
\text { SW } \\
\text { SSW } \\
S \\
\text { SSE } \\
\text { SE } \\
\text { ESE } \\
\text { E } \\
\text { ENE } \\
N E \\
\text { NNE }\end{array}$ & $\begin{array}{l}1.5 \mathrm{E}-12 \\
6.9 \mathrm{E}-13 \\
8.6 \mathrm{E}-13 \\
1.1 \mathrm{E}-12 \\
1.2 \mathrm{E}-12 \\
1.9 \mathrm{E}-12 \\
3.4 \mathrm{E}-12 \\
5.6 \mathrm{E}-12 \\
7.9 \mathrm{E}-12 \\
7.0 \mathrm{E}-12 \\
2.8 \mathrm{E}-12 \\
1.7 \mathrm{E}-12 \\
2.2 \mathrm{E}-12 \\
2.3 \mathrm{E}-12 \\
3.9 \mathrm{E}-12 \\
2.2 \mathrm{E}-12\end{array}$ & $\begin{array}{l}1.5 \mathrm{E}-12 \\
6.9 \mathrm{E}-13 \\
8.6 \mathrm{E}-13 \\
1.1 \mathrm{E}-12 \\
1.2 \mathrm{E}-12 \\
1.9 \mathrm{E}-12 \\
3.4 \mathrm{E}-12 \\
5.6 \mathrm{E}-12 \\
7.8 \mathrm{E}-12 \\
7.0 \mathrm{E}-12 \\
2.8 \mathrm{E}-12 \\
1.7 \mathrm{E}-12 \\
2.2 \mathrm{E}-12 \\
2.3 \mathrm{E}-12 \\
3.9 \mathrm{E}-12 \\
2.2 \mathrm{E}-12\end{array}$ & $\begin{array}{l}1.5 \mathrm{E}-12 \\
6.8 \mathrm{E}-13 \\
8.5 \mathrm{E}-13 \\
1.1 \mathrm{E}-12 \\
1.2 \mathrm{E}-12 \\
1.9 \mathrm{E}-12 \\
3.4 \mathrm{E}-12 \\
5.5 \mathrm{E}-12 \\
7.7 \mathrm{E}-12 \\
6.9 \mathrm{E}-12 \\
2.7 \mathrm{E}-12 \\
1.7 \mathrm{E}-12 \\
2.1 \mathrm{E}-12 \\
2.3 \mathrm{E}-12 \\
3.8 \mathrm{E}-12 \\
2.2 \mathrm{E}-12\end{array}$ & $\begin{array}{l}1.5 \mathrm{E}-12 \\
6.7 \mathrm{E}-13 \\
8.4 \mathrm{E}-13 \\
1.1 \mathrm{E}-12 \\
1.2 \mathrm{E}-12 \\
1.9 \mathrm{E}-12 \\
3.3 \mathrm{E}-12 \\
5.4 \mathrm{E}-12 \\
7.6 \mathrm{E}-12 \\
6.8 \mathrm{E}-12 \\
2.7 \mathrm{E}-12 \\
1.7 \mathrm{E}-12 \\
2.1 \mathrm{E}-12 \\
2.3 \mathrm{E}-12 \\
3.8 \mathrm{E}-12 \\
2.1 \mathrm{E}-12\end{array}$ & $\begin{array}{l}1.4 \mathrm{E}-12 \\
6.3 \mathrm{E}-13 \\
7.9 \mathrm{E}-13 \\
1.0 \mathrm{E}-12 \\
1.1 \mathrm{E}-12 \\
1.7 \mathrm{E}-12 \\
3.1 \mathrm{E}-12 \\
5.0 \mathrm{E}-12 \\
7.1 \mathrm{E}-12 \\
6.3 \mathrm{E}-12 \\
2.5 \mathrm{E}-12 \\
1.6 \mathrm{E}-12 \\
2.0 \mathrm{E}-12 \\
2.1 \mathrm{E}-12 \\
3.5 \mathrm{E}-12 \\
2.0 \mathrm{E}-12\end{array}$ & $\begin{array}{l}1.2 \mathrm{E}-12 \\
5.7 \mathrm{E}-13 \\
7.1 \mathrm{E}-13 \\
9.4 \mathrm{E}-13 \\
9.8 \mathrm{E}-13 \\
1.6 \mathrm{E}-12 \\
2.8 \mathrm{E}-12 \\
4.5 \mathrm{E}-12 \\
6.4 \mathrm{E}-12 \\
5.7 \mathrm{E}-12 \\
2.3 \mathrm{E}-12 \\
1.4 \mathrm{E}-12 \\
1.8 \mathrm{E}-12 \\
1.9 \mathrm{E}-12 \\
3.2 \mathrm{E}-12 \\
1.8 \mathrm{E}-12\end{array}$ & $\begin{array}{l}1.2 \mathrm{E}-12 \\
5.4 \mathrm{E}-13 \\
6.7 \mathrm{E}-13 \\
8.9 \mathrm{E}-13 \\
9.3 \mathrm{E}-13 \\
1.5 \mathrm{E}-12 \\
2.6 \mathrm{E}-12 \\
4.3 \mathrm{E}-12 \\
6.0 \mathrm{E}-12 \\
5.4 \mathrm{E}-12 \\
2.1 \mathrm{E}-12 \\
1.3 \mathrm{E}-12 \\
1.7 \mathrm{E}-12 \\
1.8 \mathrm{E}-12 \\
3.0 \mathrm{E}-12 \\
1.7 \mathrm{E}-12\end{array}$ \\
\hline \multicolumn{8}{|c|}{ Distance (m) } \\
\hline Direction & 13245 & 13286 & 13483 & 13612 & 13664 & 13959 & 14258 \\
\hline $\begin{array}{r}\text { N } \\
\text { NNW } \\
\text { NW } \\
\text { WNW } \\
W \\
\text { WSW } \\
\text { SW } \\
\text { SSW }\end{array}$ & $\begin{array}{l}1.1 \mathrm{E}-12 \\
5.0 \mathrm{E}-13 \\
6.2 \mathrm{E}-13 \\
8.2 \mathrm{E}-13 \\
8.5 \mathrm{E}-13 \\
1.4 \mathrm{E}-12 \\
2.4 \mathrm{E}-12 \\
3.9 \mathrm{E}-12\end{array}$ & $\begin{array}{l}1.1 \mathrm{E}-12 \\
5.0 \mathrm{E}-13 \\
6.2 \mathrm{E}-13 \\
8.2 \mathrm{E}-13 \\
8.5 \mathrm{E}-13 \\
1.4 \mathrm{E}-12 \\
2.4 \mathrm{E}-12 \\
3.9 \mathrm{E}-12\end{array}$ & $\begin{array}{l}1.1 \mathrm{E}-12 \\
4.9 \mathrm{E}-13 \\
6.1 \mathrm{E}-13 \\
8.0 \mathrm{E}-13 \\
8.3 \mathrm{E}-13 \\
1.3 \mathrm{E}-12 \\
2.4 \mathrm{E}-12 \\
3.8 \mathrm{E}-12\end{array}$ & $\begin{array}{r}1.0 \mathrm{E}-12 \\
4.8 \mathrm{E}-13 \\
6.0 \mathrm{E}-13 \\
7.9 \mathrm{E}-13 \\
8.2 \mathrm{E}-13 \\
1.3 \mathrm{E}-12 \\
2.3 \mathrm{E}-12 \\
3.8 \mathrm{E}-12 \\
\text { Page }\end{array}$ & $\begin{array}{l}1.0 \mathrm{E}-12 \\
4.8 \mathrm{E}-13 \\
6.0 \mathrm{E}-13 \\
7.9 \mathrm{E}-13 \\
8.2 \mathrm{E}-13 \\
1.3 \mathrm{E}-12 \\
2.3 \mathrm{E}-12 \\
3.8 \mathrm{E}-12\end{array}$ & $\begin{array}{l}1.0 \mathrm{E}-12 \\
4.6 \mathrm{E}-13 \\
5.8 \mathrm{E}-13 \\
7.7 \mathrm{E}-13 \\
7.9 \mathrm{E}-13 \\
1.3 \mathrm{E}-12 \\
2.3 \mathrm{E}-12 \\
3.7 \mathrm{E}-12\end{array}$ & $\begin{array}{l}9.7 \mathrm{E}-13 \\
4.5 \mathrm{E}-13 \\
5.6 \mathrm{E}-13 \\
7.4 \mathrm{E}-13 \\
7.7 \mathrm{E}-13 \\
1.2 \mathrm{E}-12 \\
2.2 \mathrm{E}-12 \\
3.5 \mathrm{E}-12\end{array}$ \\
\hline
\end{tabular}




\begin{tabular}{rlllllll} 
& \multicolumn{7}{c}{$\mathrm{C}-11 \mathrm{~A} . \mathrm{SUM}$} \\
$\mathrm{S}$ & $5.5 \mathrm{E}-12$ & $5.5 \mathrm{E}-12$ & $5.4 \mathrm{E}-12$ & $5.3 \mathrm{E}-12$ & $5.3 \mathrm{E}-12$ & $5.1 \mathrm{E}-12$ & $5.0 \mathrm{E}-12$ \\
SSE & $5.0 \mathrm{E}-12$ & $5.0 \mathrm{E}-12$ & $4.9 \mathrm{E}-12$ & $4.8 \mathrm{E}-12$ & $4.8 \mathrm{E}-12$ & $4.6 \mathrm{E}-12$ & $4.5 \mathrm{E}-12$ \\
SE & $2.0 \mathrm{E}-12$ & $2.0 \mathrm{E}-12$ & $1.9 \mathrm{E}-12$ & $1.9 \mathrm{E}-12$ & $1.9 \mathrm{E}-12$ & $1.8 \mathrm{E}-12$ & $1.8 \mathrm{E}-12$ \\
ESE & $1.2 \mathrm{E}-12$ & $1.2 \mathrm{E}-12$ & $1.2 \mathrm{E}-12$ & $1.2 \mathrm{E}-12$ & $1.2 \mathrm{E}-12$ & $1.1 \mathrm{E}-12$ & $1.1 \mathrm{E}-12$ \\
$\mathrm{E}$ & $1.5 \mathrm{E}-12$ & $1.5 \mathrm{E}-12$ & $1.5 \mathrm{E}-12$ & $1.5 \mathrm{E}-12$ & $1.5 \mathrm{E}-12$ & $1.4 \mathrm{E}-12$ & $1.4 \mathrm{E}-12$ \\
$\mathrm{ENE}$ & $1.7 \mathrm{E}-12$ & $1.7 \mathrm{E}-12$ & $1.6 \mathrm{E}-12$ & $1.6 \mathrm{E}-12$ & $1.6 \mathrm{E}-12$ & $1.6 \mathrm{E}-12$ & $1.5 \mathrm{E}-12$ \\
$\mathrm{NE}$ & $2.7 \mathrm{E}-12$ & $2.7 \mathrm{E}-12$ & $2.7 \mathrm{E}-12$ & $2.6 \mathrm{E}-12$ & $2.6 \mathrm{E}-12$ & $2.5 \mathrm{E}-12$ & $2.5 \mathrm{E}-12$ \\
NNE & $1.6 \mathrm{E}-12$ & $1.6 \mathrm{E}-12$ & $1.5 \mathrm{E}-12$ & $1.5 \mathrm{E}-12$ & $1.5 \mathrm{E}-12$ & $1.5 \mathrm{E}-12$ & $1.4 \mathrm{E}-12$
\end{tabular}

Feb 20, $2008 \quad 02: 30 \mathrm{pm}$

SUMMARY

Page 8

INDIVIDUAL LIFETIME RISK (deaths)

(A11 Radionuclides and Pathways)

\begin{tabular}{|c|c|c|c|c|c|c|}
\hline \multirow[b]{2}{*}{ Direction } & \multicolumn{6}{|c|}{ Distance $(\mathrm{m})$} \\
\hline & 14374 & 15241 & 15441 & 15784 & 15844 & 16323 \\
\hline $\mathrm{N}$ & $9.6 \mathrm{E}-13$ & $8.8 \mathrm{E}-13$ & $8.7 \mathrm{E}-13$ & $8.4 \mathrm{E}-13$ & $8.3 \mathrm{E}-13$ & 8. $0 \mathrm{E}-13$ \\
\hline NNW & $4.5 \mathrm{E}-13$ & $4.1 \mathrm{E}-13$ & 4. $0 \mathrm{E}-13$ & $3.9 \mathrm{E}-13$ & $3.9 \mathrm{E}-13$ & $3.7 \mathrm{E}-13$ \\
\hline NW & $5.6 \mathrm{E}-13$ & $5.1 \mathrm{E}-13$ & $5.0 \mathrm{E}-13$ & $4.9 \mathrm{E}-13$ & $4.9 \mathrm{E}-13$ & $4.7 \mathrm{E}-13$ \\
\hline WNW & $7.4 \mathrm{E}-13$ & $6.8 \mathrm{E}-13$ & $6.7 \mathrm{E}-13$ & $6.5 \mathrm{E}-13$ & $6.4 \mathrm{E}-13$ & $6.2 \mathrm{E}-13$ \\
\hline W & $7.6 \mathrm{E}-13$ & $7.0 \mathrm{E}-13$ & $6.9 \mathrm{E}-13$ & $6.6 \mathrm{E}-13$ & $6.6 \mathrm{E}-13$ & $6.3 \mathrm{E}-13$ \\
\hline WSW & 1. $2 \mathrm{E}-12$ & 1. $1 \mathrm{E}-12$ & 1. $1 \mathrm{E}-12$ & $1.1 \mathrm{E}-12$ & $1.1 \mathrm{E}-12$ & 1. $0 \mathrm{E}-12$ \\
\hline SW & $2.2 \mathrm{E}-12$ & $2.0 \mathrm{E}-12$ & $2.0 \mathrm{E}-12$ & $1.9 \mathrm{E}-12$ & $1.9 \mathrm{E}-12$ & $1.8 \mathrm{E}-12$ \\
\hline SSW & $3.5 \mathrm{E}-12$ & $3.2 \mathrm{E}-12$ & $3.2 \mathrm{E}-12$ & $3.1 \mathrm{E}-12$ & $3.0 \mathrm{E}-12$ & $2.9 \mathrm{E}-12$ \\
\hline & $4.9 \mathrm{E}-12$ & $4.5 \mathrm{E}-12$ & $4.4 \mathrm{E}-12$ & $4.3 \mathrm{E}-12$ & $4.2 \mathrm{E}-12$ & $4.1 \mathrm{E}-12$ \\
\hline SSE & 4. $4 \mathrm{E}-12$ & $4.1 \mathrm{E}-12$ & 4.0E-12 & $3.9 \mathrm{E}-12$ & $3.9 \mathrm{E}-12$ & 3. $7 \mathrm{E}-12$ \\
\hline SE & $1.8 \mathrm{E}-12$ & $1.6 \mathrm{E}-12$ & $1.6 \mathrm{E}-12$ & $1.5 \mathrm{E}-12$ & 1. $5 \mathrm{E}-12$ & 1. $4 \mathrm{E}-12$ \\
\hline ESE & 1. $1 \mathrm{E}-12$ & $9.9 \mathrm{E}-13$ & $9.7 \mathrm{E}-13$ & $9.4 \mathrm{E}-13$ & $9.4 \mathrm{E}-13$ & $8.9 \mathrm{E}-13$ \\
\hline & $1.4 \mathrm{E}-12$ & 1. $2 \mathrm{E}-12$ & 1. $2 \mathrm{E}-12$ & $1.2 \mathrm{E}-12$ & 1. $2 \mathrm{E}-12$ & 1. $1 \mathrm{E}-12$ \\
\hline ENE & 1. $5 \mathrm{E}-12$ & $1.4 \mathrm{E}-12$ & 1. $3 \mathrm{E}-12$ & 1. $3 \mathrm{E}-12$ & 1. $3 \mathrm{E}-12$ & 1. $2 \mathrm{E}-12$ \\
\hline $\mathrm{NE}$ & $2.4 \mathrm{E}-12$ & $2.2 \mathrm{E}-12$ & $2.2 \mathrm{E}-12$ & $2.1 \mathrm{E}-12$ & $2.1 \mathrm{E}-12$ & 2. $0 \mathrm{E}-12$ \\
\hline NNE & 1. $4 \mathrm{E}-12$ & 1. $3 \mathrm{E}-12$ & 1. $3 \mathrm{E}-12$ & 1. $2 \mathrm{E}-12$ & 1. $2 \mathrm{E}-12$ & 1. $2 \mathrm{E}-12$ \\
\hline
\end{tabular}




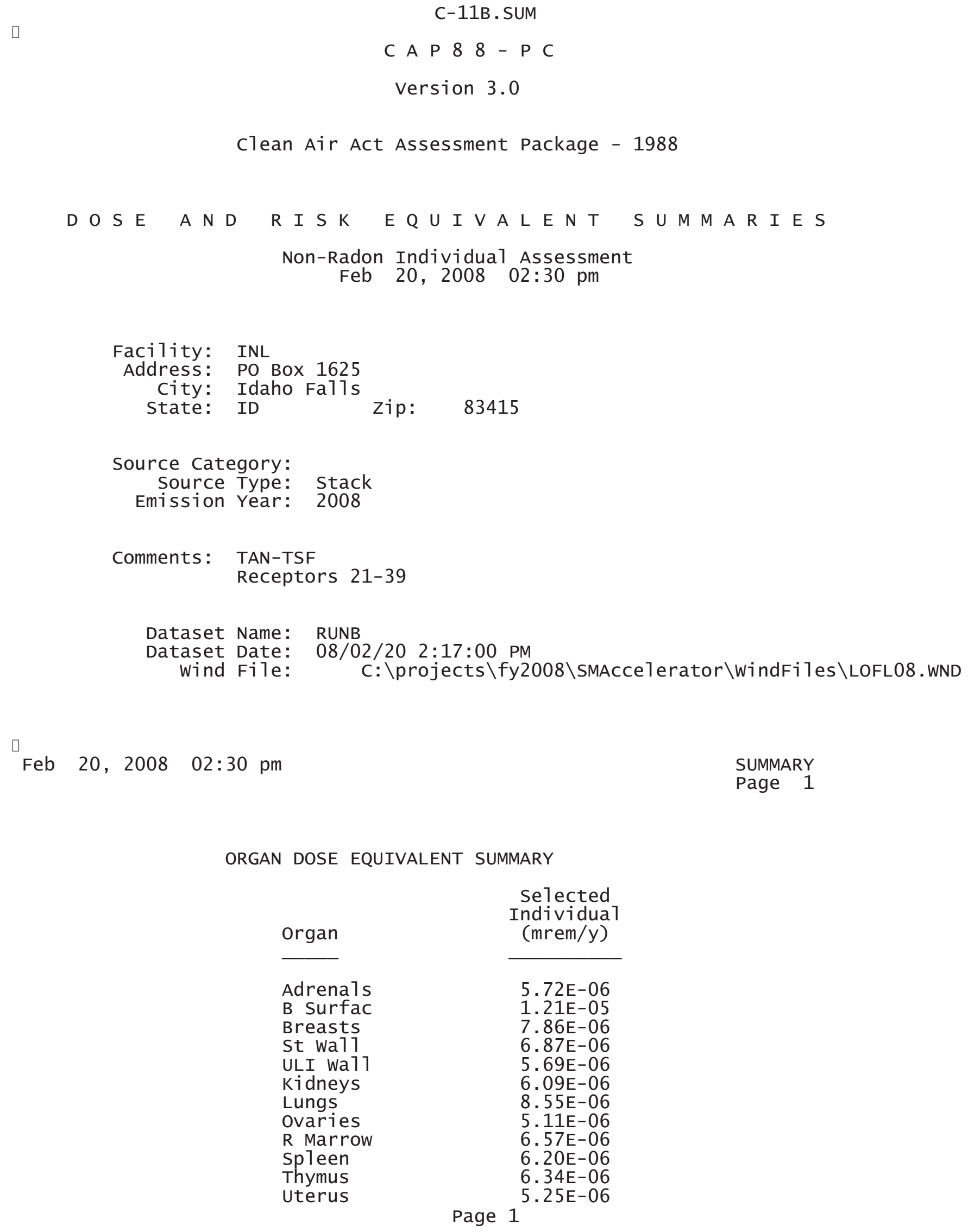




$\begin{array}{lr} & \text { C-11B.SUM } \\ \text { B1d wa11 } & 5.68 \mathrm{E}-06 \\ \text { Brain } & 7.26 \mathrm{E}-06 \\ \text { Esophagu } & 1.83 \mathrm{E}-05 \\ \text { SI wa11 } & 5.58 \mathrm{E}-06 \\ \text { LLI wa11 } & 5.49 \mathrm{E}-06 \\ \text { Liver } & 6.14 \mathrm{E}-06 \\ \text { Muscle } & 6.67 \mathrm{E}-06 \\ \text { Pancreas } & 5.39 \mathrm{E}-06 \\ \text { Skin } & 1.13 \mathrm{E}-05 \\ \text { Testes } & 6.85 \mathrm{E}-06 \\ \text { Thyroid } & 7.00 \mathrm{E}-06 \\ & \\ \text { EFFEC } & 7.18 \mathrm{E}-06\end{array}$

PATHWAY EFFECTIVE DOSE EQUIVALENT SUMMARY

\begin{tabular}{|c|c|}
\hline Pathway & $\begin{array}{c}\text { selected } \\
\text { Individual } \\
(\mathrm{mrem} / \mathrm{y})\end{array}$ \\
\hline $\begin{array}{l}\text { INGESTION } \\
\text { INHALATION } \\
\text { AIR IMMERSION } \\
\text { GROUND SURFACE } \\
\text { INTERNAL } \\
\text { EXTERNAL }\end{array}$ & $\begin{array}{l}0.00 \mathrm{E}+00 \\
6.47 \mathrm{E}-07 \\
6.53 \mathrm{E}-06 \\
0.00 \mathrm{E}+00 \\
6.47 \mathrm{E}-07 \\
6.53 \mathrm{E}-06\end{array}$ \\
\hline TOTAL & $7.18 \mathrm{E}-06$ \\
\hline
\end{tabular}

Feb 20, $2008 \quad 02: 30$ pm

SUMMARY

Page 2

NUCLIDE EFFECTIVE DOSE EQUIVALENT SUMMARY

Nuclide

C-11

TOTAL

Feb 20, $2008 \quad 02: 30$ pm

$$
\begin{gathered}
\begin{array}{c}
\text { Selected } \\
\text { Individual } \\
(\mathrm{mrem} / \mathrm{y})
\end{array} \\
\hline 7.18 \mathrm{E}-06 \\
7.18 \mathrm{E}-06
\end{gathered}
$$

SUMMARY

Page 3

CANCER RISK SUMMARY

Cancer

Esophagu
Selected Individual

Total Lifetime

Fatal Cancer Risk

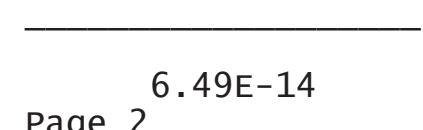




$\begin{array}{lr} & \text { C-11B. SUM } \\ \text { Stomach } & 2.89 \mathrm{E}-13 \\ \text { Colon } & 5.82 \mathrm{E}-13 \\ \text { Liver } & 9.31 \mathrm{E}-14 \\ \text { LUNG } & 8.44 \mathrm{E}-13 \\ \text { Bone } & 1.15 \mathrm{E}-14 \\ \text { Skin } & 1.13 \mathrm{E}-14 \\ \text { Breast } & 3.80 \mathrm{E}-13 \\ \text { Ovary } & 7.27 \mathrm{E}-14 \\ \text { B Tadder } & 1.37 \mathrm{E}-13 \\ \text { Kidneys } & 3.18 \mathrm{E}-14 \\ \text { Thyroid } & 2.23 \mathrm{E}-14 \\ \text { Leukemia } & 3.70 \mathrm{E}-13 \\ \text { Residua1 } & 8.84 \mathrm{E}-13 \\ \text { Tota1 } & 3.80 \mathrm{E}-12 \\ & \\ \text { TOTAL } & 7.59 \mathrm{E}-12\end{array}$

PATHWAY RISK SUMMARY

\begin{tabular}{lc} 
Pathway & $\begin{array}{c}\text { Selected Individual } \\
\text { Total Lifetime } \\
\text { Fatal Cancer Risk }\end{array}$ \\
\cline { 2 - 2 } & $0.00 \mathrm{E}+00$ \\
INGESTION & $2.47 \mathrm{E}-13$ \\
INHALATION & $3.55 \mathrm{E}-12$ \\
AIR IMMERSION & $0.00 \mathrm{E}+00$ \\
GROUND SURFACE & $2.47 \mathrm{E}-13$ \\
INTERNAL & $3.55 \mathrm{E}-12$ \\
EXTERNAL & $3.80 \mathrm{E}-12$ \\
TOTAL &
\end{tabular}

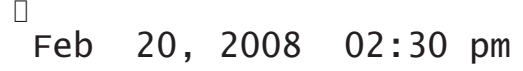

NUCLIDE RISK SUMMARY

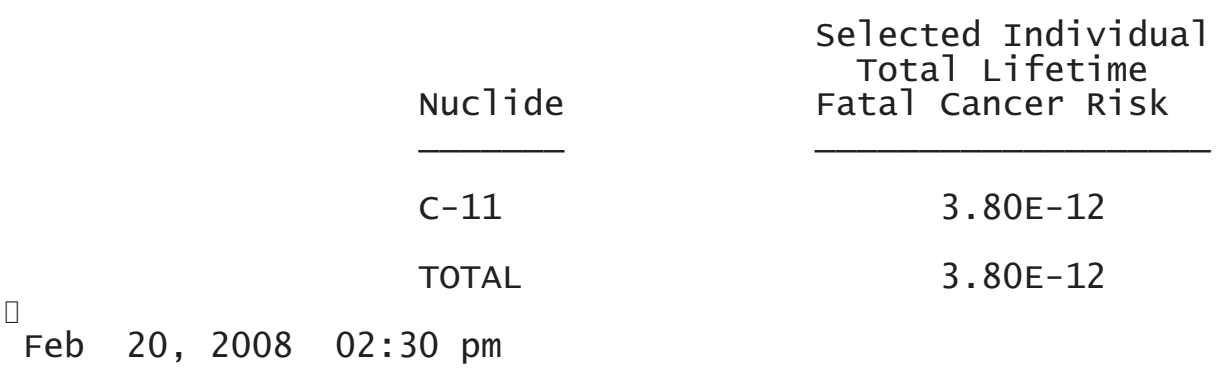

INDIVIDUAL EFFECTIVE DOSE EQUIVALENT RATE (mrem/y) (A11 Radionuclides and Pathways)

Distance (m) 


\begin{tabular}{|c|c|c|c|c|c|c|c|}
\hline Direction & 17035 & 17329 & 18607 & $\begin{array}{c}C-11 B \\
18834\end{array}$ & M 18860 & 18890 & 19860 \\
\hline$N$ & 1. $4 \mathrm{E}-06$ & $1.4 \mathrm{E}-06$ & $1.2 \mathrm{E}-06$ & $1.2 \mathrm{E}-06$ & $1.2 \mathrm{E}-06$ & 1.2E-06 & $1.1 \mathrm{E}-06$ \\
\hline NNW & $6.7 \mathrm{E}-07$ & $6.5 \mathrm{E}-07$ & $5.9 \mathrm{E}-07$ & $5.8 \mathrm{E}-07$ & $5.7 \mathrm{E}-07$ & $5.7 \mathrm{E}-07$ & $5.3 \mathrm{E}-07$ \\
\hline NW & $8.3 \mathrm{E}-07$ & $8.1 \mathrm{E}-07$ & $7.2 \mathrm{E}-07$ & $7.1 \mathrm{E}-07$ & $7.1 \mathrm{E}-07$ & $7.1 \mathrm{E}-07$ & $6.5 \mathrm{E}-07$ \\
\hline WNW & $1.1 \mathrm{E}-06$ & $1.1 \mathrm{E}-06$ & $9.6 \mathrm{E}-07$ & $9.4 \mathrm{E}-07$ & $9.4 \mathrm{E}-07$ & $9.3 \mathrm{E}-07$ & $8.6 \mathrm{E}-07$ \\
\hline W & $1.1 \mathrm{E}-06$ & $1.1 \mathrm{E}-06$ & $9.7 \mathrm{E}-07$ & $9.6 \mathrm{E}-07$ & $9.5 \mathrm{E}-07$ & $9.5 \mathrm{E}-07$ & $8.7 \mathrm{E}-07$ \\
\hline WSW & $1.8 \mathrm{E}-06$ & $1.8 \mathrm{E}-06$ & $1.6 \mathrm{E}-06$ & $1.6 \mathrm{E}-06$ & 1. $5 \mathrm{E}-06$ & 1. $5 \mathrm{E}-06$ & 1. $4 \mathrm{E}-06$ \\
\hline SW & $3.2 \mathrm{E}-06$ & $3.1 \mathrm{E}-06$ & $2.8 \mathrm{E}-06$ & $2.8 \mathrm{E}-06$ & $2.8 \mathrm{E}-06$ & $2.8 \mathrm{E}-06$ & $2.6 \mathrm{E}-06$ \\
\hline SSW & $5.2 \mathrm{E}-06$ & $5.0 \mathrm{E}-06$ & $4.5 \mathrm{E}-06$ & $4.4 \mathrm{E}-06$ & $4.4 \mathrm{E}-06$ & $4.4 \mathrm{E}-06$ & $4.1 \mathrm{E}-06$ \\
\hline $\mathrm{S}$ & $7.2 \mathrm{E}-06$ & $7.0 \mathrm{E}-06$ & $6.2 \mathrm{E}-06$ & $6.1 \mathrm{E}-06$ & $6.1 \mathrm{E}-06$ & $6.1 \mathrm{E}-06$ & $5.6 \mathrm{E}-06$ \\
\hline SSE & $6.6 \mathrm{E}-06$ & $6.4 \mathrm{E}-06$ & $5.8 \mathrm{E}-06$ & $5.7 \mathrm{E}-06$ & $5.6 \mathrm{E}-06$ & $5.6 \mathrm{E}-06$ & $5.2 \mathrm{E}-06$ \\
\hline SE & $2.6 \mathrm{E}-06$ & $2.5 \mathrm{E}-06$ & $2.2 \mathrm{E}-06$ & $2.2 \mathrm{E}-06$ & $2.2 \mathrm{E}-06$ & $2.2 \mathrm{E}-06$ & $2.0 \mathrm{E}-06$ \\
\hline ESE & $1.6 \mathrm{E}-06$ & 1.5E-06 & 1.4E-06 & 1. 3E-06 & 1. $3 \mathrm{E}-06$ & 1.3E-06 & 1. $2 \mathrm{E}-06$ \\
\hline $\mathrm{E}$ & $2.0 \mathrm{E}-06$ & $1.9 \mathrm{E}-06$ & $1.7 \mathrm{E}-06$ & $1.7 \mathrm{E}-06$ & $1.7 \mathrm{E}-06$ & $1.7 \mathrm{E}-06$ & $1.5 \mathrm{E}-06$ \\
\hline ENE & $2.2 \mathrm{E}-06$ & $2.1 \mathrm{E}-06$ & $1.9 \mathrm{E}-06$ & $1.9 \mathrm{E}-06$ & $1.9 \mathrm{E}-06$ & $1.9 \mathrm{E}-06$ & $1.7 \mathrm{E}-06$ \\
\hline $\mathrm{NE}$ & $3.6 \mathrm{E}-06$ & $3.5 \mathrm{E}-06$ & $3.1 \mathrm{E}-06$ & $3.1 \mathrm{E}-06$ & $3.1 \mathrm{E}-06$ & $3.1 \mathrm{E}-06$ & $2.8 \mathrm{E}-06$ \\
\hline NNE & $2.1 \mathrm{E}-06$ & $2.0 \mathrm{E}-06$ & $1.8 \mathrm{E}-06$ & $1.8 \mathrm{E}-06$ & $1.8 \mathrm{E}-06$ & $1.7 \mathrm{E}-06$ & $1.6 \mathrm{E}-06$ \\
\hline
\end{tabular}

Distance (m)

\begin{tabular}{|c|c|c|c|c|c|c|c|}
\hline Direction & 19891 & 20457 & 21314 & 21349 & 22159 & 23521 & 24430 \\
\hline $\begin{array}{r}N \\
N N W \\
N W \\
W N W \\
W \\
W S W \\
\text { SW } \\
\text { SSW } \\
S \\
\text { SSE } \\
\text { SE } \\
\text { ESE } \\
\text { E } \\
\text { ENE } \\
N E \\
\text { NNE }\end{array}$ & $\begin{array}{l}1.1 \mathrm{E}-06 \\
5.3 \mathrm{E}-07 \\
6.5 \mathrm{E}-07 \\
8.6 \mathrm{E}-07 \\
8.7 \mathrm{E}-07 \\
1.4 \mathrm{E}-06 \\
2.5 \mathrm{E}-06 \\
4.1 \mathrm{E}-06 \\
5.6 \mathrm{E}-06 \\
5.2 \mathrm{E}-06 \\
2.0 \mathrm{E}-06 \\
1.2 \mathrm{E}-06 \\
1.5 \mathrm{E}-06 \\
1.7 \mathrm{E}-06 \\
2.8 \mathrm{E}-06 \\
1.6 \mathrm{E}-06\end{array}$ & $\begin{array}{l}1.0 \mathrm{E}-06 \\
5.1 \mathrm{E}-07 \\
6.2 \mathrm{E}-07 \\
8.2 \mathrm{E}-07 \\
8.3 \mathrm{E}-07 \\
1.4 \mathrm{E}-06 \\
2.4 \mathrm{E}-06 \\
3.9 \mathrm{E}-06 \\
5.3 \mathrm{E}-06 \\
5.0 \mathrm{E}-06 \\
1.9 \mathrm{E}-06 \\
1.2 \mathrm{E}-06 \\
1.4 \mathrm{E}-06 \\
1.6 \mathrm{E}-06 \\
2.7 \mathrm{E}-06 \\
1.5 \mathrm{E}-06\end{array}$ & $\begin{array}{l}9.7 \mathrm{E}-07 \\
4.7 \mathrm{E}-07 \\
5.8 \mathrm{E}-07 \\
7.6 \mathrm{E}-07 \\
7.7 \mathrm{E}-07 \\
1.3 \mathrm{E}-06 \\
2.3 \mathrm{E}-06 \\
3.6 \mathrm{E}-06 \\
5.0 \mathrm{E}-06 \\
4.6 \mathrm{E}-06 \\
1.7 \mathrm{E}-06 \\
1.1 \mathrm{E}-06 \\
1.3 \mathrm{E}-06 \\
1.5 \mathrm{E}-06 \\
2.5 \mathrm{E}-06 \\
1.4 \mathrm{E}-06\end{array}$ & $\begin{array}{l}9.6 \mathrm{E}-07 \\
4.7 \mathrm{E}-07 \\
5.8 \mathrm{E}-07 \\
7.6 \mathrm{E}-07 \\
7.7 \mathrm{E}-07 \\
1.3 \mathrm{E}-06 \\
2.3 \mathrm{E}-06 \\
3.6 \mathrm{E}-06 \\
5.0 \mathrm{E}-06 \\
4.6 \mathrm{E}-06 \\
1.7 \mathrm{E}-06 \\
1.1 \mathrm{E}-06 \\
1.3 \mathrm{E}-06 \\
1.5 \mathrm{E}-06 \\
2.5 \mathrm{E}-06 \\
1.4 \mathrm{E}-06\end{array}$ & $\begin{array}{l}8.9 \mathrm{E}-07 \\
4.4 \mathrm{E}-07 \\
5.4 \mathrm{E}-07 \\
7.1 \mathrm{E}-07 \\
7.1 \mathrm{E}-07 \\
1.2 \mathrm{E}-06 \\
2.1 \mathrm{E}-06 \\
3.4 \mathrm{E}-06 \\
4.6 \mathrm{E}-06 \\
4.3 \mathrm{E}-06 \\
1.6 \mathrm{E}-06 \\
9.8 \mathrm{E}-07 \\
1.2 \mathrm{E}-06 \\
1.4 \mathrm{E}-06 \\
2.3 \mathrm{E}-06 \\
1.3 \mathrm{E}-06\end{array}$ & $\begin{array}{l}7.8 \mathrm{E}-07 \\
3.9 \mathrm{E}-07 \\
4.7 \mathrm{E}-07 \\
6.3 \mathrm{E}-07 \\
6.3 \mathrm{E}-07 \\
1.1 \mathrm{E}-06 \\
1.9 \mathrm{E}-06 \\
3.0 \mathrm{E}-06 \\
4.1 \mathrm{E}-06 \\
3.9 \mathrm{E}-06 \\
1.4 \mathrm{E}-06 \\
8.5 \mathrm{E}-07 \\
1.0 \mathrm{E}-06 \\
1.2 \mathrm{E}-06 \\
2.1 \mathrm{E}-06 \\
1.2 \mathrm{E}-06\end{array}$ & $\begin{array}{l}7.0 \mathrm{E}-07 \\
3.6 \mathrm{E}-07 \\
4.3 \mathrm{E}-07 \\
5.7 \mathrm{E}-07 \\
5.7 \mathrm{E}-07 \\
9.6 \mathrm{E}-07 \\
1.7 \mathrm{E}-06 \\
2.8 \mathrm{E}-06 \\
3.8 \mathrm{E}-06 \\
3.6 \mathrm{E}-06 \\
1.3 \mathrm{E}-06 \\
7.6 \mathrm{E}-07 \\
9.3 \mathrm{E}-07 \\
1.1 \mathrm{E}-06 \\
1.9 \mathrm{E}-06 \\
1.1 \mathrm{E}-06\end{array}$ \\
\hline
\end{tabular}

$\square$

Feb 20, 2008 02:30 pm

SUMMARY

Page 6

INDIVIDUAL EFFECTIVE DOSE EQUIVALENT RATE (mrem/y) (A11 Radionuc1ides and Pathways)

\begin{tabular}{llllll}
\hline & \multicolumn{4}{c}{ Distance $(\mathrm{m})$} \\
\cline { 2 - 5 } Direction & 24545 & 25171 & 26794 & 27275 & 27389 \\
\hline
\end{tabular}

\begin{tabular}{rrrrrr}
\hline$N$ & $6.9 \mathrm{E}-07$ & $6.4 \mathrm{E}-07$ & $5.8 \mathrm{E}-07$ & $5.7 \mathrm{E}-07$ & $5.7 \mathrm{E}-07$ \\
$\mathrm{NNW}$ & $3.6 \mathrm{E}-07$ & $3.4 \mathrm{E}-07$ & $3.1 \mathrm{E}-07$ & $\begin{array}{c}3.0 \mathrm{E}-07 \\
\text { Page }\end{array}$ & $3.0 \mathrm{E}-07$
\end{tabular}




\begin{tabular}{rlllll} 
& & & & \multicolumn{2}{c}{ C-11B.SUM } \\
NW & $4.2 \mathrm{E}-07$ & $4.0 \mathrm{E}-07$ & $3.6 \mathrm{E}-07$ & $3.5 \mathrm{E}-07$ & $3.5 \mathrm{E}-07$ \\
WNW & $5.6 \mathrm{E}-07$ & $5.2 \mathrm{E}-07$ & $4.8 \mathrm{E}-07$ & $4.6 \mathrm{E}-07$ & $4.6 \mathrm{E}-07$ \\
$\mathrm{~W}$ & $5.6 \mathrm{E}-07$ & $5.2 \mathrm{E}-07$ & $4.7 \mathrm{E}-07$ & $4.6 \mathrm{E}-07$ & $4.6 \mathrm{E}-07$ \\
WSW & $9.5 \mathrm{E}-07$ & $8.9 \mathrm{E}-07$ & $8.1 \mathrm{E}-07$ & $7.9 \mathrm{E}-07$ & $7.9 \mathrm{E}-07$ \\
SW & $1.7 \mathrm{E}-06$ & $1.6 \mathrm{E}-06$ & $1.5 \mathrm{E}-06$ & $1.4 \mathrm{E}-06$ & $1.4 \mathrm{E}-06$ \\
SSW & $2.7 \mathrm{E}-06$ & $2.6 \mathrm{E}-06$ & $2.3 \mathrm{E}-06$ & $2.3 \mathrm{E}-06$ & $2.3 \mathrm{E}-06$ \\
$\mathrm{~S}$ & $3.7 \mathrm{E}-06$ & $3.5 \mathrm{E}-06$ & $3.2 \mathrm{E}-06$ & $3.1 \mathrm{E}-06$ & $3.1 \mathrm{E}-06$ \\
SSE & $3.6 \mathrm{E}-06$ & $3.4 \mathrm{E}-06$ & $3.1 \mathrm{E}-06$ & $3.0 \mathrm{E}-06$ & $3.0 \mathrm{E}-06$ \\
SE & $1.2 \mathrm{E}-06$ & $1.1 \mathrm{E}-06$ & $1.0 \mathrm{E}-06$ & $1.0 \mathrm{E}-06$ & $1.0 \mathrm{E}-06$ \\
ESE & $7.4 \mathrm{E}-07$ & $6.8 \mathrm{E}-07$ & $6.2 \mathrm{E}-07$ & $6.0 \mathrm{E}-07$ & $6.0 \mathrm{E}-07$ \\
$\mathrm{E}$ & $9.1 \mathrm{E}-07$ & $8.3 \mathrm{E}-07$ & $7.6 \mathrm{E}-07$ & $7.4 \mathrm{E}-07$ & $7.3 \mathrm{E}-07$ \\
ENE & $1.1 \mathrm{E}-06$ & $1.0 \mathrm{E}-06$ & $9.5 \mathrm{E}-07$ & $9.2 \mathrm{E}-07$ & $9.2 \mathrm{E}-07$ \\
$\mathrm{NE}$ & $1.9 \mathrm{E}-06$ & $1.8 \mathrm{E}-06$ & $1.6 \mathrm{E}-06$ & $1.6 \mathrm{E}-06$ & $1.6 \mathrm{E}-06$ \\
NNE & $1.1 \mathrm{E}-06$ & $9.8 \mathrm{E}-07$ & $9.0 \mathrm{E}-07$ & $8.8 \mathrm{E}-07$ & $8.7 \mathrm{E}-07$ \\
& & & & & \\
\hline
\end{tabular}

Feb 20, $2008 \quad 02: 30$ pm

SUMMARY

Page 7

INDIVIDUAL LIFETIME RISK (deaths)

(A11 Radionuclides and Pathways)

\begin{tabular}{|c|c|c|c|c|c|c|c|}
\hline \multicolumn{8}{|c|}{ Distance $(\mathrm{m})$} \\
\hline Direction & 17035 & 17329 & 18607 & 18834 & 18860 & 18890 & 19860 \\
\hline $\begin{array}{r}\text { N } \\
N N W \\
N W \\
W N W \\
W \\
W S W \\
\text { SW } \\
\text { SSW } \\
S \\
\text { SSE } \\
\text { SE } \\
\text { ESE } \\
\text { E } \\
\text { ENE } \\
N E \\
\text { NNE }\end{array}$ & $\begin{array}{l}7.5 \mathrm{E}-13 \\
3.5 \mathrm{E}-13 \\
4.4 \mathrm{E}-13 \\
5.8 \mathrm{E}-13 \\
5.9 \mathrm{E}-13 \\
9.5 \mathrm{E}-13 \\
1.7 \mathrm{E}-12 \\
2.7 \mathrm{E}-12 \\
3.8 \mathrm{E}-12 \\
3.5 \mathrm{E}-12 \\
1.4 \mathrm{E}-12 \\
8.4 \mathrm{E}-13 \\
1.0 \mathrm{E}-12 \\
1.2 \mathrm{E}-12 \\
1.9 \mathrm{E}-12 \\
1.1 \mathrm{E}-12\end{array}$ & $\begin{array}{l}7.3 \mathrm{E}-13 \\
3.4 \mathrm{E}-13 \\
4.3 \mathrm{E}-13 \\
5.6 \mathrm{E}-13 \\
5.8 \mathrm{E}-13 \\
9.3 \mathrm{E}-13 \\
1.7 \mathrm{E}-12 \\
2.7 \mathrm{E}-12 \\
3.7 \mathrm{E}-12 \\
3.4 \mathrm{E}-12 \\
1.3 \mathrm{E}-12 \\
8.1 \mathrm{E}-13 \\
1.0 \mathrm{E}-12 \\
1.1 \mathrm{E}-12 \\
1.9 \mathrm{E}-12 \\
1.1 \mathrm{E}-12\end{array}$ & $\begin{array}{l}6.5 \mathrm{E}-13 \\
3.1 \mathrm{E}-13 \\
3.8 \mathrm{E}-13 \\
5.1 \mathrm{E}-13 \\
5.2 \mathrm{E}-13 \\
8.4 \mathrm{E}-13 \\
1.5 \mathrm{E}-12 \\
2.4 \mathrm{E}-12 \\
3.3 \mathrm{E}-12 \\
3.0 \mathrm{E}-12 \\
1.2 \mathrm{E}-12 \\
7.2 \mathrm{E}-13 \\
9.0 \mathrm{E}-13 \\
1.0 \mathrm{E}-12 \\
1.7 \mathrm{E}-12 \\
9.5 \mathrm{E}-13\end{array}$ & $\begin{array}{l}6.4 \mathrm{E}-13 \\
3.0 \mathrm{E}-13 \\
3.8 \mathrm{E}-13 \\
5.0 \mathrm{E}-13 \\
5.1 \mathrm{E}-13 \\
8.2 \mathrm{E}-13 \\
1.5 \mathrm{E}-12 \\
2.3 \mathrm{E}-12 \\
3.2 \mathrm{E}-12 \\
3.0 \mathrm{E}-12 \\
1.2 \mathrm{E}-12 \\
7.1 \mathrm{E}-13 \\
8.8 \mathrm{E}-13 \\
9.9 \mathrm{E}-13 \\
1.6 \mathrm{E}-12 \\
9.3 \mathrm{E}-13\end{array}$ & $\begin{array}{l}6.3 \mathrm{E}-13 \\
3.0 \mathrm{E}-13 \\
3.7 \mathrm{E}-13 \\
4.9 \mathrm{E}-13 \\
5.0 \mathrm{E}-13 \\
8.2 \mathrm{E}-13 \\
1.5 \mathrm{E}-12 \\
2.3 \mathrm{E}-12 \\
3.2 \mathrm{E}-12 \\
3.0 \mathrm{E}-12 \\
1.1 \mathrm{E}-12 \\
7.1 \mathrm{E}-13 \\
8.8 \mathrm{E}-13 \\
9.9 \mathrm{E}-13 \\
1.6 \mathrm{E}-12 \\
9.3 \mathrm{E}-13\end{array}$ & $\begin{array}{l}6.3 \mathrm{E}-13 \\
3.0 \mathrm{E}-13 \\
3.7 \mathrm{E}-13 \\
4.9 \mathrm{E}-13 \\
5.0 \mathrm{E}-13 \\
8.2 \mathrm{E}-13 \\
1.5 \mathrm{E}-12 \\
2.3 \mathrm{E}-12 \\
3.2 \mathrm{E}-12 \\
3.0 \mathrm{E}-12 \\
1.1 \mathrm{E}-12 \\
7.0 \mathrm{E}-13 \\
8.8 \mathrm{E}-13 \\
9.9 \mathrm{E}-13 \\
1.6 \mathrm{E}-12 \\
9.3 \mathrm{E}-13\end{array}$ & $\begin{array}{l}5.8 \mathrm{E}-13 \\
2.8 \mathrm{E}-13 \\
3.4 \mathrm{E}-13 \\
4.6 \mathrm{E}-13 \\
4.6 \mathrm{E}-13 \\
7.5 \mathrm{E}-13 \\
1.3 \mathrm{E}-12 \\
2.2 \mathrm{E}-12 \\
3.0 \mathrm{E}-12 \\
2.8 \mathrm{E}-12 \\
1.1 \mathrm{E}-12 \\
6.4 \mathrm{E}-13 \\
8.0 \mathrm{E}-13 \\
9.1 \mathrm{E}-13 \\
1.5 \mathrm{E}-12 \\
8.5 \mathrm{E}-13\end{array}$ \\
\hline \multicolumn{8}{|c|}{ Distance $(\mathrm{m})$} \\
\hline Direction & 19891 & 20457 & 21314 & 21349 & 22159 & 23521 & 24430 \\
\hline $\begin{array}{r}N \\
N N W \\
N W \\
\text { WNW } \\
W \\
\text { WSW } \\
\text { SW } \\
\text { SSW }\end{array}$ & $\begin{array}{l}5.8 \mathrm{E}-13 \\
2.8 \mathrm{E}-13 \\
3.4 \mathrm{E}-13 \\
4.5 \mathrm{E}-13 \\
4.6 \mathrm{E}-13 \\
7.5 \mathrm{E}-13 \\
1.3 \mathrm{E}-12 \\
2.1 \mathrm{E}-12\end{array}$ & $\begin{array}{l}5 \cdot 5 \mathrm{E}-13 \\
2 \cdot 7 \mathrm{E}-13 \\
3 \cdot 3 \mathrm{E}-13 \\
4.3 \mathrm{E}-13 \\
4 \cdot 4 \mathrm{E}-13 \\
7 \cdot 2 \mathrm{E}-13 \\
1.3 \mathrm{E}-12 \\
2.1 \mathrm{E}-12\end{array}$ & $\begin{array}{l}5 \cdot 1 \mathrm{E}-13 \\
2.5 \mathrm{E}-13 \\
3 \cdot 1 \mathrm{E}-13 \\
4.0 \mathrm{E}-13 \\
4 \cdot 1 \mathrm{E}-13 \\
6.7 \mathrm{E}-13 \\
1.2 \mathrm{E}-12 \\
1.9 \mathrm{E}-12\end{array}$ & $\begin{array}{r}5.1 \mathrm{E}-13 \\
2.5 \mathrm{E}-13 \\
3.0 \mathrm{E}-13 \\
4.0 \mathrm{E}-13 \\
4.1 \mathrm{E}-13 \\
6.7 \mathrm{E}-13 \\
1.2 \mathrm{E}-12 \\
1.9 \mathrm{E}-12 \\
\text { Page }\end{array}$ & $\begin{array}{l}4.7 \mathrm{E}-13 \\
2.3 \mathrm{E}-13 \\
2.8 \mathrm{E}-13 \\
3.8 \mathrm{E}-13 \\
3.8 \mathrm{E}-13 \\
6.3 \mathrm{E}-13 \\
1.1 \mathrm{E}-12 \\
1.8 \mathrm{E}-12\end{array}$ & $\begin{array}{l}4 \cdot 1 \mathrm{E}-13 \\
2 \cdot 1 \mathrm{E}-13 \\
2 \cdot 5 \mathrm{E}-13 \\
3 \cdot 3 \mathrm{E}-13 \\
3 \cdot 3 \mathrm{E}-13 \\
5 \cdot 6 \mathrm{E}-13 \\
1 \cdot 0 \mathrm{E}-12 \\
1.6 \mathrm{E}-12\end{array}$ & $\begin{array}{l}3.7 \mathrm{E}-13 \\
1.9 \mathrm{E}-13 \\
2.3 \mathrm{E}-13 \\
3.0 \mathrm{E}-13 \\
3.0 \mathrm{E}-13 \\
5.1 \mathrm{E}-13 \\
9.2 \mathrm{E}-13 \\
1.5 \mathrm{E}-12\end{array}$ \\
\hline
\end{tabular}




\begin{tabular}{rlllllll}
\multicolumn{7}{c}{$\mathrm{C}-11 \mathrm{~B} . \mathrm{SUM}$} \\
$\mathrm{S}$ & $3.0 \mathrm{E}-12$ & $2.8 \mathrm{E}-12$ & $2.6 \mathrm{E}-12$ & $2.6 \mathrm{E}-12$ & $2.5 \mathrm{E}-12$ & $2.2 \mathrm{E}-12$ & $2.0 \mathrm{E}-12$ \\
SSE & $2.7 \mathrm{E}-12$ & $2.6 \mathrm{E}-12$ & $2.5 \mathrm{E}-12$ & $2.5 \mathrm{E}-12$ & $2.3 \mathrm{E}-12$ & $2.1 \mathrm{E}-12$ & $1.9 \mathrm{E}-12$ \\
SE & $1.0 \mathrm{E}-12$ & $1.0 \mathrm{E}-12$ & $9.2 \mathrm{E}-13$ & $9.2 \mathrm{E}-13$ & $8.5 \mathrm{E}-13$ & $7.4 \mathrm{E}-13$ & $6.7 \mathrm{E}-13$ \\
ESE & $6.4 \mathrm{E}-13$ & $6.1 \mathrm{E}-13$ & $5.6 \mathrm{E}-13$ & $5.6 \mathrm{E}-13$ & $5.2 \mathrm{E}-13$ & $4.5 \mathrm{E}-13$ & $4.0 \mathrm{E}-13$ \\
$\mathrm{E}$ & $8.0 \mathrm{E}-13$ & $7.6 \mathrm{E}-13$ & $7.0 \mathrm{E}-13$ & $7.0 \mathrm{E}-13$ & $6.4 \mathrm{E}-13$ & $5.5 \mathrm{E}-13$ & $4.9 \mathrm{E}-13$ \\
ENE & $9.1 \mathrm{E}-13$ & $8.6 \mathrm{E}-13$ & $8.0 \mathrm{E}-13$ & $8.0 \mathrm{E}-13$ & $7.5 \mathrm{E}-13$ & $6.6 \mathrm{E}-13$ & $6.0 \mathrm{E}-13$ \\
NE & $1.5 \mathrm{E}-12$ & $1.4 \mathrm{E}-12$ & $1.3 \mathrm{E}-12$ & $1.3 \mathrm{E}-12$ & $1.2 \mathrm{E}-12$ & $1.1 \mathrm{E}-12$ & $1.0 \mathrm{E}-12$ \\
NNE & $8.5 \mathrm{E}-13$ & $8.1 \mathrm{E}-13$ & $7.6 \mathrm{E}-13$ & $7.5 \mathrm{E}-13$ & $7.0 \mathrm{E}-13$ & $6.2 \mathrm{E}-13$ & $5.7 \mathrm{E}-13$
\end{tabular}

Feb 20, $2008 \quad 02: 30 \mathrm{pm}$

SUMMARY

Page 8

INDIVIDUAL LIFETIME RISK (deaths)

(A11 Radionuclides and Pathways)

\begin{tabular}{|c|c|c|c|c|c|}
\hline \multirow[b]{2}{*}{ Direction } & \multicolumn{5}{|c|}{ Distance $(\mathrm{m})$} \\
\hline & 24545 & 25171 & 26794 & 27275 & 27389 \\
\hline $\begin{array}{r}N \\
N N W \\
N W \\
W N W \\
W \\
W S W \\
S W \\
\text { SSW } \\
S \\
\text { SSE } \\
\text { SE } \\
\text { ESE } \\
\text { E } \\
\text { ENE } \\
N E \\
\text { NNE }\end{array}$ & $\begin{array}{l}3.7 \mathrm{E}-13 \\
1.9 \mathrm{E}-13 \\
2.2 \mathrm{E}-13 \\
3.0 \mathrm{E}-13 \\
3.0 \mathrm{E}-13 \\
5.0 \mathrm{E}-13 \\
9.1 \mathrm{E}-13 \\
1.4 \mathrm{E}-12 \\
2.0 \mathrm{E}-12 \\
1.9 \mathrm{E}-12 \\
6.6 \mathrm{E}-13 \\
3.9 \mathrm{E}-13 \\
4.8 \mathrm{E}-13 \\
5.9 \mathrm{E}-13 \\
1.0 \mathrm{E}-12 \\
5.6 \mathrm{E}-13\end{array}$ & $\begin{array}{l}3.4 \mathrm{E}-13 \\
1.8 \mathrm{E}-13 \\
2.1 \mathrm{E}-13 \\
2.8 \mathrm{E}-13 \\
2.7 \mathrm{E}-13 \\
4.7 \mathrm{E}-13 \\
8.6 \mathrm{E}-13 \\
1.4 \mathrm{E}-12 \\
1.9 \mathrm{E}-12 \\
1.8 \mathrm{E}-12 \\
6.1 \mathrm{E}-13 \\
3.6 \mathrm{E}-13 \\
4.4 \mathrm{E}-13 \\
5.5 \mathrm{E}-13 \\
9.4 \mathrm{E}-13 \\
5.2 \mathrm{E}-13\end{array}$ & $\begin{array}{l}3.1 \mathrm{E}-13 \\
1.6 \mathrm{E}-13 \\
1.9 \mathrm{E}-13 \\
2.5 \mathrm{E}-13 \\
2.5 \mathrm{E}-13 \\
4.3 \mathrm{E}-13 \\
7.9 \mathrm{E}-13 \\
1.2 \mathrm{E}-12 \\
1.7 \mathrm{E}-12 \\
1.6 \mathrm{E}-12 \\
5.5 \mathrm{E}-13 \\
3.3 \mathrm{E}-13 \\
4.0 \mathrm{E}-13 \\
5.0 \mathrm{E}-13 \\
8.5 \mathrm{E}-13 \\
4.8 \mathrm{E}-13\end{array}$ & $\begin{array}{l}3.0 \mathrm{E}-13 \\
1.6 \mathrm{E}-13 \\
1.9 \mathrm{E}-13 \\
2.5 \mathrm{E}-13 \\
2.4 \mathrm{E}-13 \\
4.2 \mathrm{E}-13 \\
7.7 \mathrm{E}-13 \\
1.2 \mathrm{E}-12 \\
1.6 \mathrm{E}-12 \\
1.6 \mathrm{E}-12 \\
5.4 \mathrm{E}-13 \\
3.2 \mathrm{E}-13 \\
3.9 \mathrm{E}-13 \\
4.9 \mathrm{E}-13 \\
8.3 \mathrm{E}-13 \\
4.6 \mathrm{E}-13\end{array}$ & $\begin{array}{l}3.0 \mathrm{E}-13 \\
1.6 \mathrm{E}-13 \\
1.9 \mathrm{E}-13 \\
2.4 \mathrm{E}-13 \\
2.4 \mathrm{E}-13 \\
4.2 \mathrm{E}-13 \\
7.6 \mathrm{E}-13 \\
1.2 \mathrm{E}-12 \\
1.6 \mathrm{E}-12 \\
1.6 \mathrm{E}-12 \\
5.4 \mathrm{E}-13 \\
3.2 \mathrm{E}-13 \\
3.9 \mathrm{E}-13 \\
4.9 \mathrm{E}-13 \\
8.3 \mathrm{E}-13 \\
4.6 \mathrm{E}-13\end{array}$ \\
\hline
\end{tabular}




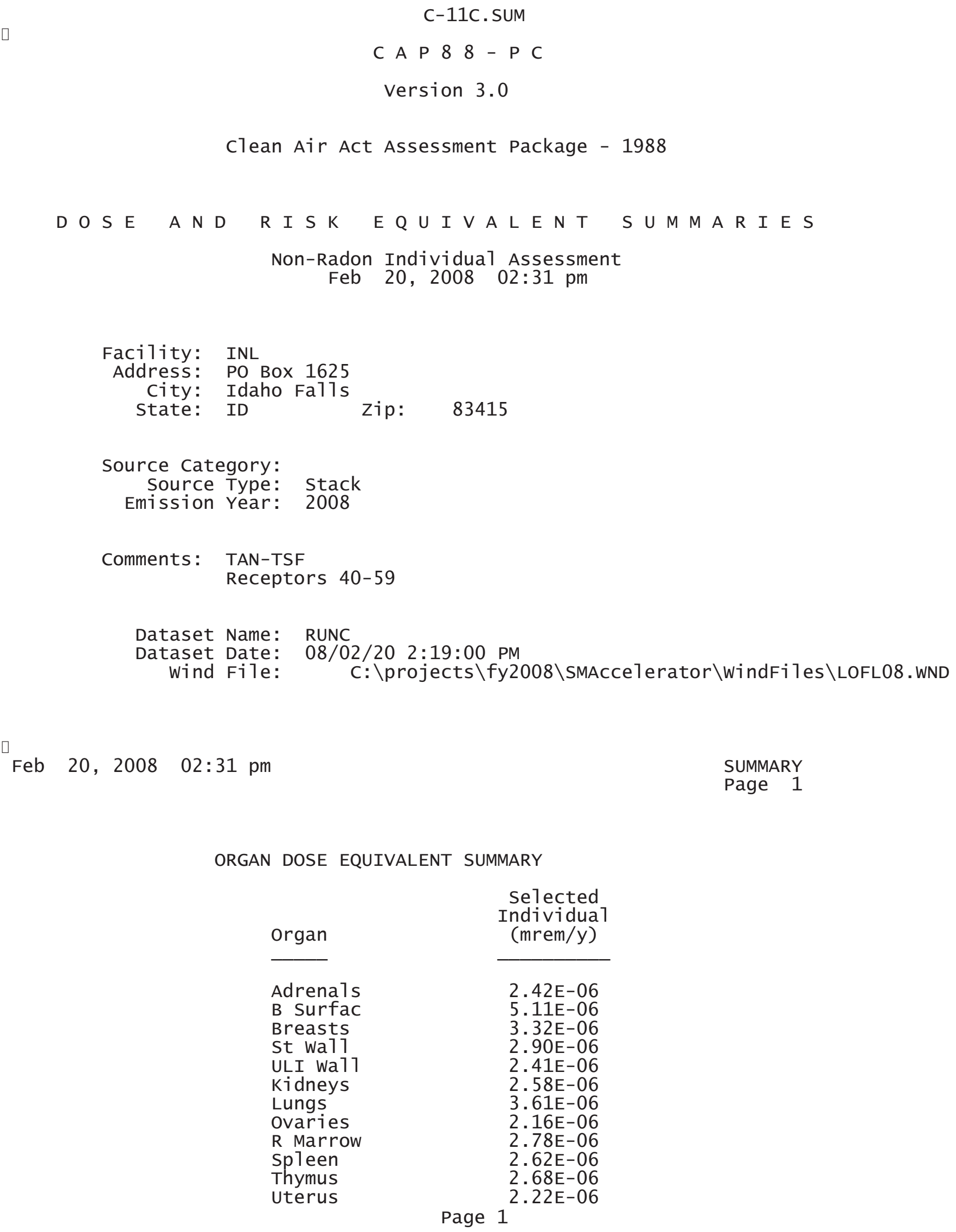

\begin{tabular}{lr} 
ORGAN DOSE EQUIVALENT SUMMARY \\
Organ & $\begin{array}{r}\text { Selected } \\
\text { Individual } \\
\text { (mrem/y) }\end{array}$ \\
\cline { 2 - 2 } Adrena1s & $2.42 \mathrm{E}-06$ \\
B Surfac & $5.11 \mathrm{E}-06$ \\
Breasts & $3.32 \mathrm{E}-06$ \\
St wa11 & $2.90 \mathrm{E}-06$ \\
ULI wa11 & $2.41 \mathrm{E}-06$ \\
Kidneys & $2.58 \mathrm{E}-06$ \\
Lungs & $3.61 \mathrm{E}-06$ \\
Ovaries & $2.16 \mathrm{E}-06$ \\
R Marrow & $2.78 \mathrm{E}-06$ \\
Spleen & $2.62 \mathrm{E}-06$ \\
Thymus & $2.68 \mathrm{E}-06$ \\
Uterus & $2.22 \mathrm{E}-06$
\end{tabular}




$\begin{array}{lr} & \text { C-11C.SUM } \\ \text { B1d wa11 } & 2.40 \mathrm{E}-06 \\ \text { Brain } & 3.07 \mathrm{E}-06 \\ \text { Esophagu } & 7.72 \mathrm{E}-06 \\ \text { SI wa11 } & 2.36 \mathrm{E}-06 \\ \text { LLI wa11 } & 2.32 \mathrm{E}-06 \\ \text { Liver } & 2.59 \mathrm{E}-06 \\ \text { Muscle } & 2.82 \mathrm{E}-06 \\ \text { Pancreas } & 2.28 \mathrm{E}-06 \\ \text { Skin } & 4.79 \mathrm{E}-06 \\ \text { Testes } & 2.89 \mathrm{E}-06 \\ \text { Thyroid } & 2.96 \mathrm{E}-06 \\ \text { EFFEC } & 3.03 \mathrm{E}-06\end{array}$

PATHWAY EFFECTIVE DOSE EQUIVALENT SUMMARY

\begin{tabular}{lc} 
Pathway & $\begin{array}{c}\text { Selected } \\
\text { Individual } \\
\text { (mrem/y) }\end{array}$ \\
\cline { 2 - 2 } & \\
INGESTION & $0.00 \mathrm{E}+00$ \\
INHALATION & $2.74 \mathrm{E}-07$ \\
AIR IMMERSION & $2.76 \mathrm{E}-06$ \\
GROUND SURFACE & $0.00 \mathrm{E}+00$ \\
INTERNAL & $2.74 \mathrm{E}-07$ \\
EXTERNAL & $2.76 \mathrm{E}-06$ \\
TOTAL & $3.03 \mathrm{E}-06$
\end{tabular}

Feb 20, 2008 02:31 pm

SUMMARY

Page 2

NUCLIDE EFFECTIVE DOSE EQUIVALENT SUMMARY

Nuclide

C-11

TOTAL

$$
\begin{gathered}
\begin{array}{c}
\text { Selected } \\
\text { Individua1 } \\
(\mathrm{mrem} / \mathrm{y})
\end{array} \\
\hline 3.03 \mathrm{E}-06 \\
3.03 \mathrm{E}-06
\end{gathered}
$$

Feb 20, $2008 \quad 02: 31 \mathrm{pm}$

SUMMARY

Page 3

CANCER RISK SUMMARY

Cancer

Esophagu
Selected Individual

Total Lifetime

Fatal Cancer Risk

Page $2^{2.74 E-14}$ 


$\begin{array}{lr} & \text { C-11C. SUM } \\ \text { Stomach } & 1.22 \mathrm{E}-13 \\ \text { Colon } & 2.46 \mathrm{E}-13 \\ \text { Liver } & 3.94 \mathrm{E}-14 \\ \text { LUNG } & 3.57 \mathrm{E}-13 \\ \text { Bone } & 4.85 \mathrm{E}-15 \\ \text { Skin } & 4.78 \mathrm{E}-15 \\ \text { Breast } & 1.60 \mathrm{E}-13 \\ \text { Ovary } & 3.07 \mathrm{E}-14 \\ \text { B Tadder } & 5.81 \mathrm{E}-14 \\ \text { Kidneys } & 1.34 \mathrm{E}-14 \\ \text { Thyroid } & 9.42 \mathrm{E}-15 \\ \text { Leukemia } & 1.56 \mathrm{E}-13 \\ \text { Residua1 } & 3.74 \mathrm{E}-13 \\ \text { Tota1 } & 1.61 \mathrm{E}-12 \\ & \\ \text { TOTAL } & 3.21 \mathrm{E}-12\end{array}$

PATHWAY RISK SUMMARY

\begin{tabular}{lc} 
Pathway & $\begin{array}{c}\text { Selected Individua1 } \\
\text { Tota1 Lifetime } \\
\text { Fatal Cancer Risk }\end{array}$ \\
\cline { 2 - 2 } INGESTION & $0.00 \mathrm{E}+00$ \\
INHALATION & $1.04 \mathrm{E}-13$ \\
AIR IMMERSION & $1.50 \mathrm{E}-12$ \\
GROUND SURFACE & $0.00 \mathrm{E}+00$ \\
INTERNAL & $1.04 \mathrm{E}-13$ \\
EXTERNAL & $1.50 \mathrm{E}-12$ \\
TOTAL & $1.61 \mathrm{E}-12$
\end{tabular}

Feb 20, $2008 \quad 02: 31$ pm

SUMMARY Page 4

NUCLIDE RISK SUMMARY

Nuclide

C-11

TOTAL

$\square$

Feb 20, 2008 02:31 pm
Selected Individual

Total Lifetime Fatal Cancer Risk

1. $61 \mathrm{E}-12$

1. $61 \mathrm{E}-12$

INDIVIDUAL EFFECTIVE DOSE EQUIVALENT RATE (mrem/y) (A11 Radionuclides and Pathways)

Distance $(m)$ 


\begin{tabular}{rccccccc}
\multicolumn{1}{c}{ Direction } & 27715 & 28919 & 31060 & 32802 & 34577 & 35279 & 35683 \\
\hline N & $5.6 \mathrm{E}-07$ & $5.2 \mathrm{E}-07$ & $4.7 \mathrm{E}-07$ & $4.3 \mathrm{E}-07$ & $4.0 \mathrm{E}-07$ & $3.8 \mathrm{E}-07$ & $3.8 \mathrm{E}-07$ \\
NNW & $2.9 \mathrm{E}-07$ & $2.8 \mathrm{E}-07$ & $2.5 \mathrm{E}-07$ & $2.3 \mathrm{E}-07$ & $2.1 \mathrm{E}-07$ & $2.1 \mathrm{E}-07$ & $2.1 \mathrm{E}-07$ \\
NW & $3.5 \mathrm{E}-07$ & $3.2 \mathrm{E}-07$ & $2.9 \mathrm{E}-07$ & $2.7 \mathrm{E}-07$ & $2.5 \mathrm{E}-07$ & $2.4 \mathrm{E}-07$ & $2.4 \mathrm{E}-07$ \\
WNW & $4.5 \mathrm{E}-07$ & $4.3 \mathrm{E}-07$ & $3.9 \mathrm{E}-07$ & $3.6 \mathrm{E}-07$ & $3.3 \mathrm{E}-07$ & $3.2 \mathrm{E}-07$ & $3.1 \mathrm{E}-07$ \\
W & $4.5 \mathrm{E}-07$ & $4.2 \mathrm{E}-07$ & $3.8 \mathrm{E}-07$ & $3.5 \mathrm{E}-07$ & $3.2 \mathrm{E}-07$ & $3.1 \mathrm{E}-07$ & $3.1 \mathrm{E}-07$ \\
WSW & $7.8 \mathrm{E}-07$ & $7.3 \mathrm{E}-07$ & $6.6 \mathrm{E}-07$ & $6.1 \mathrm{E}-07$ & $5.6 \mathrm{E}-07$ & $5.4 \mathrm{E}-07$ & $5.3 \mathrm{E}-07$ \\
SW & $1.4 \mathrm{E}-06$ & $1.3 \mathrm{E}-06$ & $1.2 \mathrm{E}-06$ & $1.1 \mathrm{E}-06$ & $1.0 \mathrm{E}-06$ & $9.9 \mathrm{E}-07$ & $9.7 \mathrm{E}-07$ \\
SSW & $2.2 \mathrm{E}-06$ & $2.1 \mathrm{E}-06$ & $1.9 \mathrm{E}-06$ & $1.7 \mathrm{E}-06$ & $1.6 \mathrm{E}-06$ & $1.5 \mathrm{E}-06$ & $1.5 \mathrm{E}-06$ \\
$\mathrm{~S}$ & $3.0 \mathrm{E}-06$ & $2.8 \mathrm{E}-06$ & $2.5 \mathrm{E}-06$ & $2.3 \mathrm{E}-06$ & $2.2 \mathrm{E}-06$ & $2.1 \mathrm{E}-06$ & $2.0 \mathrm{E}-06$ \\
SSE & $2.9 \mathrm{E}-06$ & $2.8 \mathrm{E}-06$ & $2.5 \mathrm{E}-06$ & $2.3 \mathrm{E}-06$ & $2.1 \mathrm{E}-06$ & $2.1 \mathrm{E}-06$ & $2.0 \mathrm{E}-06$ \\
$\mathrm{SE}$ & $1.0 \mathrm{E}-06$ & $9.3 \mathrm{E}-07$ & $8.4 \mathrm{E}-07$ & $7.7 \mathrm{E}-07$ & $7.0 \mathrm{E}-07$ & $6.8 \mathrm{E}-07$ & $6.7 \mathrm{E}-07$ \\
ESE & $5.9 \mathrm{E}-07$ & $5.5 \mathrm{E}-07$ & $4.9 \mathrm{E}-07$ & $4.5 \mathrm{E}-07$ & $4.1 \mathrm{E}-07$ & $4.0 \mathrm{E}-07$ & $3.9 \mathrm{E}-07$ \\
$\mathrm{E}$ & $7.2 \mathrm{E}-07$ & $6.7 \mathrm{E}-07$ & $6.0 \mathrm{E}-07$ & $5.5 \mathrm{E}-07$ & $5.0 \mathrm{E}-07$ & $4.9 \mathrm{E}-07$ & $4.8 \mathrm{E}-07$ \\
$\mathrm{ENE}$ & $9.0 \mathrm{E}-07$ & $8.5 \mathrm{E}-07$ & $7.6 \mathrm{E}-07$ & $7.0 \mathrm{E}-07$ & $6.4 \mathrm{E}-07$ & $6.2 \mathrm{E}-07$ & $6.1 \mathrm{E}-07$ \\
$\mathrm{NE}$ & $1.5 \mathrm{E}-06$ & $1.4 \mathrm{E}-06$ & $1.3 \mathrm{E}-06$ & $1.2 \mathrm{E}-06$ & $1.1 \mathrm{E}-06$ & $1.1 \mathrm{E}-06$ & $1.0 \mathrm{E}-06$ \\
$\mathrm{NNE}$ & $8.6 \mathrm{E}-07$ & $8.1 \mathrm{E}-07$ & $7.2 \mathrm{E}-07$ & $6.7 \mathrm{E}-07$ & $6.2 \mathrm{E}-07$ & $6.0 \mathrm{E}-07$ & $5.9 \mathrm{E}-07$ \\
\hline
\end{tabular}

Distance $(\mathrm{m})$

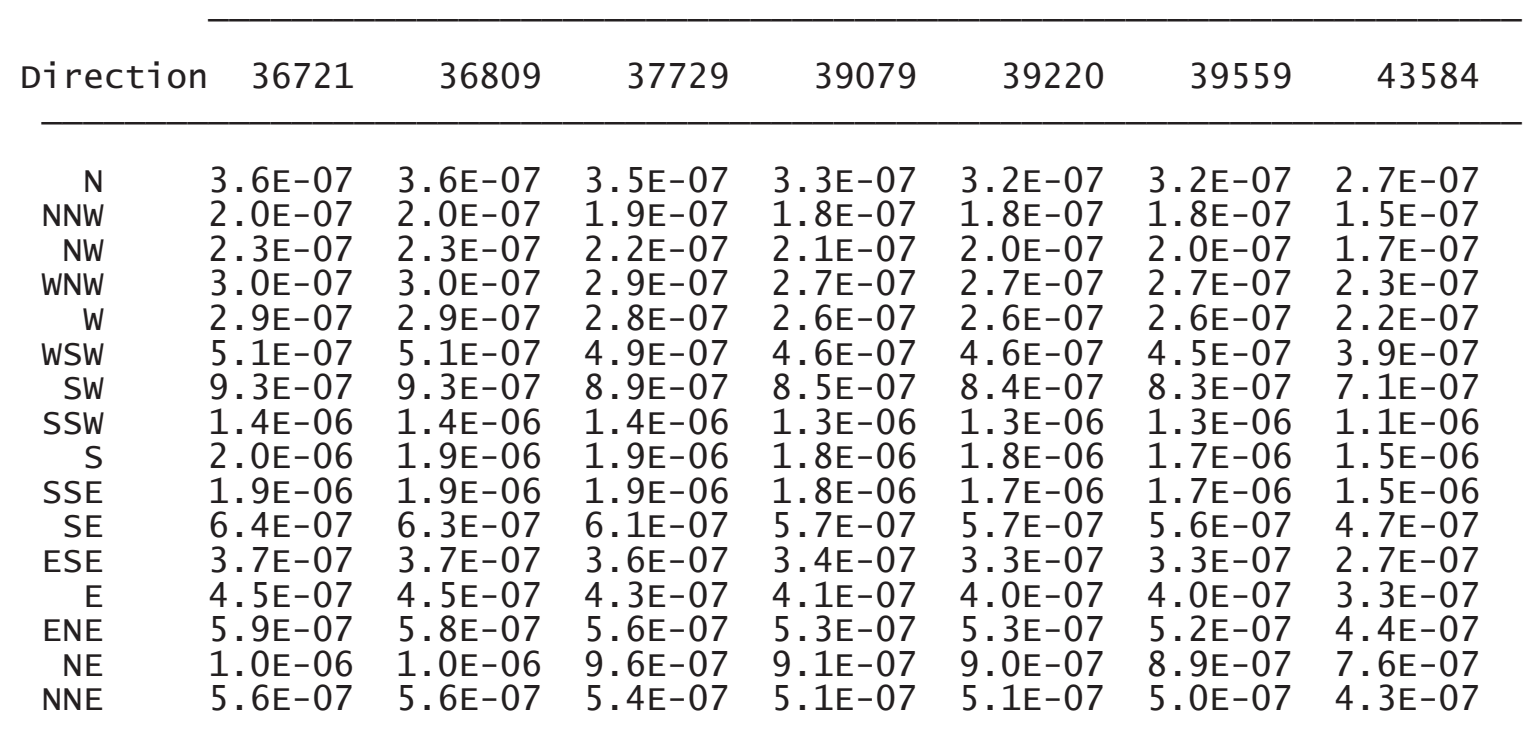

$\square$

Feb 20, $2008 \quad 02: 31 \mathrm{pm}$

SUMMARY

Page 6

INDIVIDUAL EFFECTIVE DOSE EQUIVALENT RATE (mrem/y) (A11 Radionuclides and Pathways)

\begin{tabular}{|c|c|c|c|c|c|c|}
\hline \multicolumn{7}{|c|}{ Distance (m) } \\
\hline Direction & 45196 & 45275 & 45654 & 45677 & 46668 & 47969 \\
\hline
\end{tabular}

\begin{tabular}{rrrrrrr}
\hline$N$ & $2.5 \mathrm{E}-07$ & $2.5 \mathrm{E}-07$ & $2.5 \mathrm{E}-07$ & $2.5 \mathrm{E}-07$ & $2.4 \mathrm{E}-07$ & $2.3 \mathrm{E}-07$ \\
$\mathrm{NNW}$ & $1.4 \mathrm{E}-07$ & $1.4 \mathrm{E}-07$ & $1.4 \mathrm{E}-07$ & $\begin{array}{c}1.4 \mathrm{E}-07 \\
\text { Page }\end{array}$ & $1.3 \mathrm{E}-07$ & $1.3 \mathrm{E}-07$
\end{tabular}




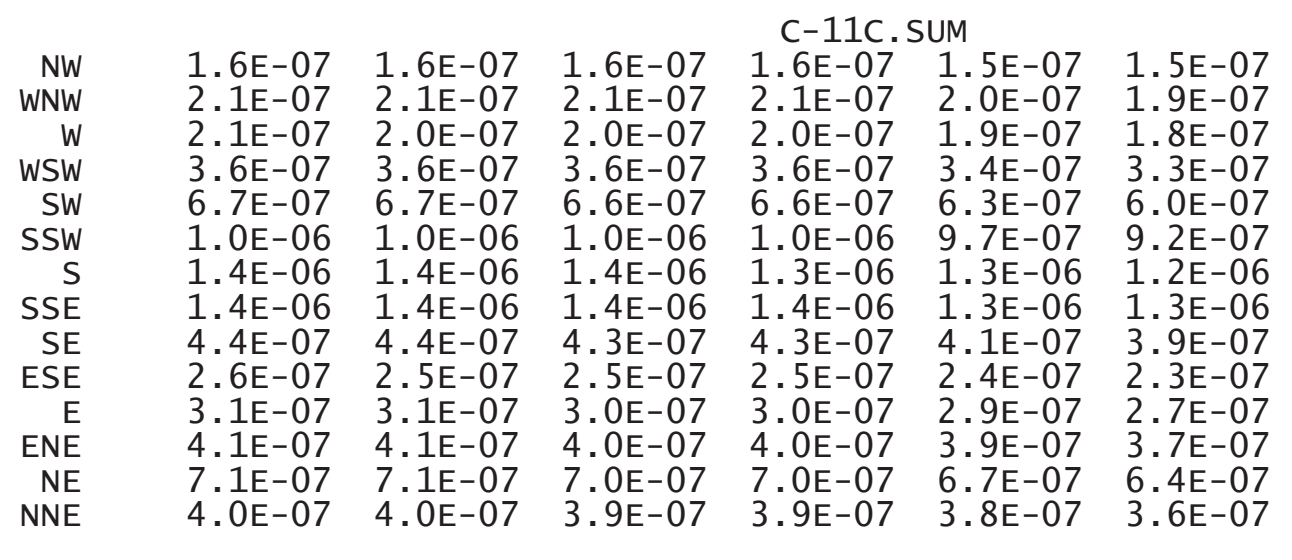

Feb 20, $2008 \quad 02: 31 \mathrm{pm}$

SUMMARY

Page 7

INDIVIDUAL LIFETIME RISK (deaths)

(A11 Radionuclides and Pathways)

\begin{tabular}{|c|c|c|c|c|c|c|c|}
\hline \multicolumn{8}{|c|}{ Distance $(\mathrm{m})$} \\
\hline Direction & 27715 & 28919 & 31060 & 32802 & 34577 & 35279 & 35683 \\
\hline $\begin{array}{r}N \\
N N W \\
N W \\
W N W \\
W \\
W S W \\
S W \\
S S W \\
S \\
S S E \\
\text { SE } \\
\text { ESE } \\
E \\
\text { ENE } \\
N E \\
\text { NNE }\end{array}$ & $\begin{array}{l}2.9 \mathrm{E}-13 \\
1.6 \mathrm{E}-13 \\
1.8 \mathrm{E}-13 \\
2.4 \mathrm{E}-13 \\
2.4 \mathrm{E}-13 \\
4.1 \mathrm{E}-13 \\
7.5 \mathrm{E}-13 \\
1.2 \mathrm{E}-12 \\
1.6 \mathrm{E}-12 \\
1.5 \mathrm{E}-12 \\
5.3 \mathrm{E}-13 \\
3.1 \mathrm{E}-13 \\
3.8 \mathrm{E}-13 \\
4.8 \mathrm{E}-13 \\
8.1 \mathrm{E}-13 \\
4.5 \mathrm{E}-13\end{array}$ & $\begin{array}{l}2.8 \mathrm{E}-13 \\
1.5 \mathrm{E}-13 \\
1.7 \mathrm{E}-13 \\
2.3 \mathrm{E}-13 \\
2.2 \mathrm{E}-13 \\
3.9 \mathrm{E}-13 \\
7.0 \mathrm{E}-13 \\
1.1 \mathrm{E}-12 \\
1.5 \mathrm{E}-12 \\
1.5 \mathrm{E}-12 \\
4.9 \mathrm{E}-13 \\
2.9 \mathrm{E}-13 \\
3.6 \mathrm{E}-13 \\
4.5 \mathrm{E}-13 \\
7.6 \mathrm{E}-13 \\
4.3 \mathrm{E}-13\end{array}$ & $\begin{array}{l}2.5 \mathrm{E}-13 \\
1.3 \mathrm{E}-13 \\
1.5 \mathrm{E}-13 \\
2.0 \mathrm{E}-13 \\
2.0 \mathrm{E}-13 \\
3.5 \mathrm{E}-13 \\
6.3 \mathrm{E}-13 \\
9.9 \mathrm{E}-13 \\
1.3 \mathrm{E}-12 \\
1.3 \mathrm{E}-12 \\
4.4 \mathrm{E}-13 \\
2.6 \mathrm{E}-13 \\
3.2 \mathrm{E}-13 \\
4.0 \mathrm{E}-13 \\
6.9 \mathrm{E}-13 \\
3.8 \mathrm{E}-13\end{array}$ & $\begin{array}{l}2.3 \mathrm{E}-13 \\
1.2 \mathrm{E}-13 \\
1.4 \mathrm{E}-13 \\
1.9 \mathrm{E}-13 \\
1.8 \mathrm{E}-13 \\
3.2 \mathrm{E}-13 \\
5.9 \mathrm{E}-13 \\
9.1 \mathrm{E}-13 \\
1.2 \mathrm{E}-12 \\
1.2 \mathrm{E}-12 \\
4.1 \mathrm{E}-13 \\
2.4 \mathrm{E}-13 \\
2.9 \mathrm{E}-13 \\
3.7 \mathrm{E}-13 \\
6.3 \mathrm{E}-13 \\
3.5 \mathrm{E}-13\end{array}$ & $\begin{array}{l}2.1 \mathrm{E}-13 \\
1.1 \mathrm{E}-13 \\
1.3 \mathrm{E}-13 \\
1.7 \mathrm{E}-13 \\
1.7 \mathrm{E}-13 \\
3.0 \mathrm{E}-13 \\
5.4 \mathrm{E}-13 \\
8.4 \mathrm{E}-13 \\
1.1 \mathrm{E}-12 \\
1.1 \mathrm{E}-12 \\
3.7 \mathrm{E}-13 \\
2.2 \mathrm{E}-13 \\
2.7 \mathrm{E}-13 \\
3.4 \mathrm{E}-13 \\
5.8 \mathrm{E}-13 \\
3.3 \mathrm{E}-13\end{array}$ & $\begin{array}{l}2.0 \mathrm{E}-13 \\
1.1 \mathrm{E}-13 \\
1.3 \mathrm{E}-13 \\
1.7 \mathrm{E}-13 \\
1.6 \mathrm{E}-13 \\
2.9 \mathrm{E}-13 \\
5.2 \mathrm{E}-13 \\
8.2 \mathrm{E}-13 \\
1.1 \mathrm{E}-12 \\
1.1 \mathrm{E}-12 \\
3.6 \mathrm{E}-13 \\
2.1 \mathrm{E}-13 \\
2.6 \mathrm{E}-13 \\
3.3 \mathrm{E}-13 \\
5.6 \mathrm{E}-13 \\
3.2 \mathrm{E}-13\end{array}$ & $\begin{array}{l}2.0 \mathrm{E}-13 \\
1.1 \mathrm{E}-13 \\
1.3 \mathrm{E}-13 \\
1.7 \mathrm{E}-13 \\
1.6 \mathrm{E}-13 \\
2.8 \mathrm{E}-13 \\
5.2 \mathrm{E}-13 \\
8.0 \mathrm{E}-13 \\
1.1 \mathrm{E}-12 \\
1.1 \mathrm{E}-12 \\
3.5 \mathrm{E}-13 \\
2.1 \mathrm{E}-13 \\
2.5 \mathrm{E}-13 \\
3.2 \mathrm{E}-13 \\
5.5 \mathrm{E}-13 \\
3.1 \mathrm{E}-13\end{array}$ \\
\hline \multicolumn{8}{|c|}{ Distance $(\mathrm{m})$} \\
\hline Direction & 36721 & 36809 & 37729 & 39079 & 39220 & 39559 & 43584 \\
\hline $\begin{array}{r}\mathrm{N} \\
N N W \\
N W \\
W N W \\
W \\
\text { WSW } \\
\text { SW } \\
\text { SSW }\end{array}$ & $\begin{array}{l}1.9 \mathrm{E}-13 \\
1.0 \mathrm{E}-13 \\
1.2 \mathrm{E}-13 \\
1.6 \mathrm{E}-13 \\
1.5 \mathrm{E}-13 \\
2.7 \mathrm{E}-13 \\
4.9 \mathrm{E}-13 \\
7.7 \mathrm{E}-13\end{array}$ & $\begin{array}{l}1.9 \mathrm{E}-13 \\
1.0 \mathrm{E}-13 \\
1.2 \mathrm{E}-13 \\
1.6 \mathrm{E}-13 \\
1.5 \mathrm{E}-13 \\
2.7 \mathrm{E}-13 \\
4.9 \mathrm{E}-13 \\
7.6 \mathrm{E}-13\end{array}$ & $\begin{array}{l}1.8 \mathrm{E}-13 \\
1.0 \mathrm{E}-13 \\
1.2 \mathrm{E}-13 \\
1.5 \mathrm{E}-13 \\
1.5 \mathrm{E}-13 \\
2.6 \mathrm{E}-13 \\
4.7 \mathrm{E}-13 \\
7.3 \mathrm{E}-13\end{array}$ & $\begin{array}{r}1.7 \mathrm{E}-13 \\
9.5 \mathrm{E}-14 \\
1.1 \mathrm{E}-13 \\
1.4 \mathrm{E}-13 \\
1.4 \mathrm{E}-13 \\
2.4 \mathrm{E}-13 \\
4.5 \mathrm{E}-13 \\
6.9 \mathrm{E}-13 \\
\text { Page }\end{array}$ & $\begin{array}{l}1.7 \mathrm{E}-13 \\
9.4 \mathrm{E}-14 \\
1.1 \mathrm{E}-13 \\
1.4 \mathrm{E}-13 \\
1.4 \mathrm{E}-13 \\
2.4 \mathrm{E}-13 \\
4.5 \mathrm{E}-13 \\
6.9 \mathrm{E}-13\end{array}$ & $\begin{array}{l}1.7 \mathrm{E}-13 \\
9.3 \mathrm{E}-14 \\
1.1 \mathrm{E}-13 \\
1.4 \mathrm{E}-13 \\
1.4 \mathrm{E}-13 \\
2.4 \mathrm{E}-13 \\
4.4 \mathrm{E}-13 \\
6.8 \mathrm{E}-13\end{array}$ & $\begin{array}{l}1.4 \mathrm{E}-13 \\
8.0 \mathrm{E}-14 \\
9.1 \mathrm{E}-14 \\
1.2 \mathrm{E}-13 \\
1.2 \mathrm{E}-13 \\
2.0 \mathrm{E}-13 \\
3.8 \mathrm{E}-13 \\
5.8 \mathrm{E}-13\end{array}$ \\
\hline
\end{tabular}




\begin{tabular}{rlllllll}
\multicolumn{7}{c}{$\mathrm{C}-11 \mathrm{C} . \mathrm{SUM}$} \\
$\mathrm{S}$ & $1.0 \mathrm{E}-12$ & $1.0 \mathrm{E}-12$ & $9.9 \mathrm{E}-13$ & $9.3 \mathrm{E}-13$ & $9.3 \mathrm{E}-13$ & $9.2 \mathrm{E}-13$ & $7.8 \mathrm{E}-13$ \\
SSE & $1.0 \mathrm{E}-12$ & $1.0 \mathrm{E}-12$ & $9.8 \mathrm{E}-13$ & $9.3 \mathrm{E}-13$ & $9.2 \mathrm{E}-13$ & $9.1 \mathrm{E}-13$ & $7.8 \mathrm{E}-13$ \\
SE & $3.4 \mathrm{E}-13$ & $3.4 \mathrm{E}-13$ & $3.2 \mathrm{E}-13$ & $3.0 \mathrm{E}-13$ & $3.0 \mathrm{E}-13$ & $3.0 \mathrm{E}-13$ & $2.5 \mathrm{E}-13$ \\
ESE & $2.0 \mathrm{E}-13$ & $2.0 \mathrm{E}-13$ & $1.9 \mathrm{E}-13$ & $1.8 \mathrm{E}-13$ & $1.8 \mathrm{E}-13$ & $1.7 \mathrm{E}-13$ & $1.5 \mathrm{E}-13$ \\
$\mathrm{E}$ & $2.4 \mathrm{E}-13$ & $2.4 \mathrm{E}-13$ & $2.3 \mathrm{E}-13$ & $2.2 \mathrm{E}-13$ & $2.1 \mathrm{E}-13$ & $2.1 \mathrm{E}-13$ & $1.8 \mathrm{E}-13$ \\
ENE & $3.1 \mathrm{E}-13$ & $3.1 \mathrm{E}-13$ & $3.0 \mathrm{E}-13$ & $2.8 \mathrm{E}-13$ & $2.8 \mathrm{E}-13$ & $2.7 \mathrm{E}-13$ & $2.3 \mathrm{E}-13$ \\
NE & $5.3 \mathrm{E}-13$ & $5.3 \mathrm{E}-13$ & $5.1 \mathrm{E}-13$ & $4.8 \mathrm{E}-13$ & $4.8 \mathrm{E}-13$ & $4.7 \mathrm{E}-13$ & $4.0 \mathrm{E}-13$ \\
NNE & $3.0 \mathrm{E}-13$ & $3.0 \mathrm{E}-13$ & $2.8 \mathrm{E}-13$ & $2.7 \mathrm{E}-13$ & $2.7 \mathrm{E}-13$ & $2.6 \mathrm{E}-13$ & $2.3 \mathrm{E}-13$
\end{tabular}

Feb 20, $2008 \quad 02: 31 \mathrm{pm}$

SUMMARY

Page 8

INDIVIDUAL LIFETIME RISK (deaths)

(A11 Radionuclides and Pathways)

\begin{tabular}{|c|c|c|c|c|c|c|}
\hline \multirow[b]{2}{*}{ Direction } & \multicolumn{6}{|c|}{ Distance $(\mathrm{m})$} \\
\hline & 45196 & 45275 & 45654 & 45677 & 46668 & 47969 \\
\hline $\begin{array}{r}\text { N } \\
N N W \\
N W \\
W N W \\
W \\
W S W \\
\text { SW } \\
\text { SSW } \\
\text { S } \\
\text { SSE } \\
\text { SE } \\
\text { ESE } \\
\text { E } \\
\text { ENE } \\
\text { NE } \\
\text { NNE }\end{array}$ & $\begin{array}{l}1.3 \mathrm{E}-13 \\
7.5 \mathrm{E}-14 \\
8.5 \mathrm{E}-14 \\
1.1 \mathrm{E}-13 \\
1.1 \mathrm{E}-13 \\
1.9 \mathrm{E}-13 \\
3.5 \mathrm{E}-13 \\
5.4 \mathrm{E}-13 \\
7.3 \mathrm{E}-13 \\
7.4 \mathrm{E}-13 \\
2.3 \mathrm{E}-13 \\
1.4 \mathrm{E}-13 \\
1.6 \mathrm{E}-13 \\
2.2 \mathrm{E}-13 \\
3.8 \mathrm{E}-13 \\
2.1 \mathrm{E}-13\end{array}$ & $\begin{array}{l}1.3 \mathrm{E}-13 \\
7.5 \mathrm{E}-14 \\
8.5 \mathrm{E}-14 \\
1.1 \mathrm{E}-13 \\
1.1 \mathrm{E}-13 \\
1.9 \mathrm{E}-13 \\
3.5 \mathrm{E}-13 \\
5.4 \mathrm{E}-13 \\
7.3 \mathrm{E}-13 \\
7.3 \mathrm{E}-13 \\
2.3 \mathrm{E}-13 \\
1.3 \mathrm{E}-13 \\
1.6 \mathrm{E}-13 \\
2.2 \mathrm{E}-13 \\
3.8 \mathrm{E}-13 \\
2.1 \mathrm{E}-13\end{array}$ & $\begin{array}{l}1.3 \mathrm{E}-13 \\
7.4 \mathrm{E}-14 \\
8.4 \mathrm{E}-14 \\
1.1 \mathrm{E}-13 \\
1.1 \mathrm{E}-13 \\
1.9 \mathrm{E}-13 \\
3.5 \mathrm{E}-13 \\
5.3 \mathrm{E}-13 \\
7.1 \mathrm{E}-13 \\
7.2 \mathrm{E}-13 \\
2.3 \mathrm{E}-13 \\
1.3 \mathrm{E}-13 \\
1.6 \mathrm{E}-13 \\
2.1 \mathrm{E}-13 \\
3.7 \mathrm{E}-13 \\
2.1 \mathrm{E}-13\end{array}$ & $\begin{array}{l}1.3 \mathrm{E}-13 \\
7.4 \mathrm{E}-14 \\
8.4 \mathrm{E}-14 \\
1.1 \mathrm{E}-13 \\
1.1 \mathrm{E}-13 \\
1.9 \mathrm{E}-13 \\
3.5 \mathrm{E}-13 \\
5.3 \mathrm{E}-13 \\
7.1 \mathrm{E}-13 \\
7.2 \mathrm{E}-13 \\
2.3 \mathrm{E}-13 \\
1.3 \mathrm{E}-13 \\
1.6 \mathrm{E}-13 \\
2.1 \mathrm{E}-13 \\
3.7 \mathrm{E}-13 \\
2.1 \mathrm{E}-13\end{array}$ & $\begin{array}{l}1.3 \mathrm{E}-13 \\
7.1 \mathrm{E}-14 \\
8.1 \mathrm{E}-14 \\
1.1 \mathrm{E}-13 \\
1.0 \mathrm{E}-13 \\
1.8 \mathrm{E}-13 \\
3.3 \mathrm{E}-13 \\
5.1 \mathrm{E}-13 \\
6.9 \mathrm{E}-13 \\
7.0 \mathrm{E}-13 \\
2.2 \mathrm{E}-13 \\
1.3 \mathrm{E}-13 \\
1.5 \mathrm{E}-13 \\
2.1 \mathrm{E}-13 \\
3.6 \mathrm{E}-13 \\
2.0 \mathrm{E}-13\end{array}$ & $\begin{array}{l}1.2 \mathrm{E}-13 \\
6.8 \mathrm{E}-14 \\
7.7 \mathrm{E}-14 \\
1.0 \mathrm{E}-13 \\
9.7 \mathrm{E}-14 \\
1.7 \mathrm{E}-13 \\
3.2 \mathrm{E}-13 \\
4.9 \mathrm{E}-13 \\
6.5 \mathrm{E}-13 \\
6.6 \mathrm{E}-13 \\
2.1 \mathrm{E}-13 \\
1.2 \mathrm{E}-13 \\
1.4 \mathrm{E}-13 \\
1.9 \mathrm{E}-13 \\
3.4 \mathrm{E}-13 \\
1.9 \mathrm{E}-13\end{array}$ \\
\hline
\end{tabular}




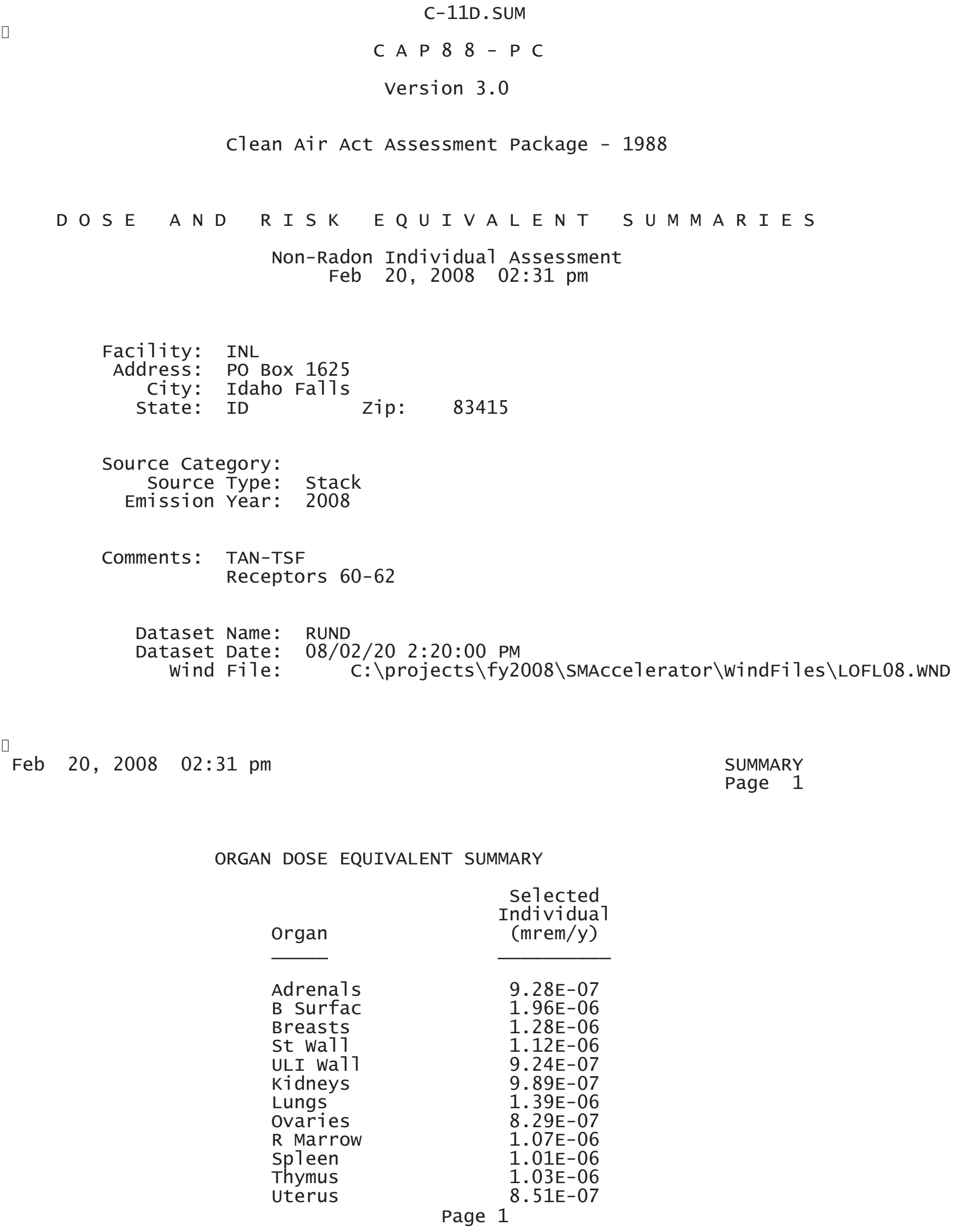
9. $28 \mathrm{E}-07$
$1.96 \mathrm{E}-06$
1. $28 \mathrm{E}-06$
1. $12 \mathrm{E}-06$
$9.24 \mathrm{E}-07$
9. 89E-07
1. $39 \mathrm{E}-06$
8.29E-07
$1.07 \mathrm{E}-06$
1. $01 \mathrm{E}-06$
1. 03E-06
8. 51E-07

Page 1 


$\begin{array}{lr} & \text { C-11D.SUM } \\ \text { B1d wa11 } & 9.22 \mathrm{E}-07 \\ \text { Brain } & 1.18 \mathrm{E}-06 \\ \text { Esophagu } & 2.97 \mathrm{E}-06 \\ \text { SI wa11 } & 9.06 \mathrm{E}-07 \\ \text { LLI wa11 } & 8.91 \mathrm{E}-07 \\ \text { Liver } & 9.96 \mathrm{E}-07 \\ \text { Muscle } & 1.08 \mathrm{E}-06 \\ \text { Pancreas } & 8.75 \mathrm{E}-07 \\ \text { Skin } & 1.84 \mathrm{E}-06 \\ \text { Testes } & 1.11 \mathrm{E}-06 \\ \text { Thyroid } & 1.14 \mathrm{E}-06 \\ & \\ \text { EFFEC } & 1.16 \mathrm{E}-06\end{array}$

PATHWAY EFFECTIVE DOSE EQUIVALENT SUMMARY

$\begin{array}{lc}\text { Pathway } & \begin{array}{c}\text { selected } \\ \text { Individual } \\ \text { (mrem/y) }\end{array} \\ & \\ \text { INGESTION } & 0.00 \mathrm{E}+00 \\ \text { INHALATION } & 1.05 \mathrm{E}-07 \\ \text { AIR IMMERSION } & 1.06 \mathrm{E}-06 \\ \text { GROUND SURFACE } & 0.00 \mathrm{E}+00 \\ \text { INTERNAL } & 1.05 \mathrm{E}-07 \\ \text { EXTERNAL } & 1.06 \mathrm{E}-06 \\ \text { TOTAL } & 1.16 \mathrm{E}-06\end{array}$

Feb 20, 2008 02:31 pm

SUMMARY

Page 2

NUCLIDE EFFECTIVE DOSE EQUIVALENT SUMMARY

Nuclide

C-11

TOTAL

Feb 20, $2008 \quad 02: 31 \mathrm{pm}$

$$
\begin{gathered}
\begin{array}{c}
\text { Selected } \\
\text { Individua1 } \\
\text { (mrem/y) }
\end{array} \\
\hline 1.16 \mathrm{E}-06 \\
1.16 \mathrm{E}-06
\end{gathered}
$$

CANCER RISK SUMMARY

Cancer

Esophagu
Selected Individual

Total Lifetime

Fatal Cancer Risk

$$
\text { Page } 2^{1.05 E-14}
$$




$\begin{array}{lr} & \text { C-11D. SUM } \\ \text { Stomach } & 4.69 \mathrm{E}-14 \\ \text { Colon } & 9.44 \mathrm{E}-14 \\ \text { Liver } & 1.51 \mathrm{E}-14 \\ \text { LUNG } & 1.37 \mathrm{E}-13 \\ \text { Bone } & 1.86 \mathrm{E}-15 \\ \text { Skin } & 1.84 \mathrm{E}-15 \\ \text { Breast } & 6.16 \mathrm{E}-14 \\ \text { Ovary } & 1.18 \mathrm{E}-14 \\ \text { B Tadder } & 2.23 \mathrm{E}-14 \\ \text { Kidneys } & 5.16 \mathrm{E}-15 \\ \text { Thyroid } & 3.62 \mathrm{E}-15 \\ \text { Leukemia } & 6.00 \mathrm{E}-14 \\ \text { Residua1 } & 1.43 \mathrm{E}-13 \\ \text { Tota1 } & 6.17 \mathrm{E}-13 \\ \text { TOTAL } & 1.23 \mathrm{E}-12\end{array}$

PATHWAY RISK SUMMARY

\begin{tabular}{lc} 
Pathway & \multicolumn{2}{c}{$\begin{array}{c}\text { Total Lifetime } \\
\text { Fatal Cancer Risk }\end{array}$} \\
\cline { 2 - 2 } & $0.00 \mathrm{E}+00$ \\
INGESTION & $4.01 \mathrm{E}-14$ \\
INHALATION & $5.76 \mathrm{E}-13$ \\
AIR IMMERSION & $0.00 \mathrm{E}+00$ \\
GROUND SURFACE & $4.01 \mathrm{E}-14$ \\
INTERNAL & $5.76 \mathrm{E}-13$ \\
EXTERNAL & $6.17 \mathrm{E}-13$ \\
TOTAL &
\end{tabular}

Feb 20, $2008 \quad 02: 31$ pm

SUMMARY Page 4

NUCLIDE RISK SUMMARY

Nuclide

C-11

TOTAL

口

Feb 20, 2008 02:31 pm
Selected Individual

Total Lifetime Fatal Cancer Risk

$6.17 \mathrm{E}-13$

$6.17 \mathrm{E}-13$

INDIVIDUAL EFFECTIVE DOSE EQUIVALENT RATE (mrem/y) (A11 Radionuclides and Pathways)

Distance $(m)$ 


$\begin{array}{lllll}\text { Direction } & 50024 & 54611 & 58610 & \text { C-11D.SUM }\end{array}$

\begin{tabular}{rlll}
\hline$N$ & $2.1 \mathrm{E}-07$ & $1.7 \mathrm{E}-07$ & $1.4 \mathrm{E}-07$ \\
$\mathrm{~N} W$ & $1.2 \mathrm{E}-07$ & $1.0 \mathrm{E}-07$ & $8.4 \mathrm{E}-08$ \\
$\mathrm{NW}$ & $1.3 \mathrm{E}-07$ & $1.1 \mathrm{E}-07$ & $9.0 \mathrm{E}-08$ \\
WNW & $1.8 \mathrm{E}-07$ & $1.5 \mathrm{E}-07$ & $1.2 \mathrm{E}-07$ \\
$\mathrm{~W}$ & $1.7 \mathrm{E}-07$ & $1.4 \mathrm{E}-07$ & $1.1 \mathrm{E}-07$ \\
WSW & $3.0 \mathrm{E}-07$ & $2.5 \mathrm{E}-07$ & $2.1 \mathrm{E}-07$ \\
$\mathrm{SW}$ & $5.6 \mathrm{E}-07$ & $4.7 \mathrm{E}-07$ & $3.9 \mathrm{E}-07$ \\
SSW & $8.5 \mathrm{E}-07$ & $7.1 \mathrm{E}-07$ & $5.8 \mathrm{E}-07$ \\
$\mathrm{~S}$ & $1.1 \mathrm{E}-06$ & $9.4 \mathrm{E}-07$ & $7.7 \mathrm{E}-07$ \\
$\mathrm{SSE}$ & $1.2 \mathrm{E}-06$ & $9.8 \mathrm{E}-07$ & $8.2 \mathrm{E}-07$ \\
$\mathrm{SE}$ & $3.6 \mathrm{E}-07$ & $2.9 \mathrm{E}-07$ & $2.3 \mathrm{E}-07$ \\
$\mathrm{ESE}$ & $2.1 \mathrm{E}-07$ & $1.6 \mathrm{E}-07$ & $1.2 \mathrm{E}-07$ \\
$\mathrm{E}$ & $2.5 \mathrm{E}-07$ & $2.0 \mathrm{E}-07$ & $1.5 \mathrm{E}-07$ \\
$\mathrm{ENE}$ & $3.4 \mathrm{E}-07$ & $2.8 \mathrm{E}-07$ & $2.2 \mathrm{E}-07$ \\
$\mathrm{NE}$ & $5.9 \mathrm{E}-07$ & $5.0 \mathrm{E}-07$ & $4.1 \mathrm{E}-07$ \\
$\mathrm{NNE}$ & $3.3 \mathrm{E}-07$ & $2.8 \mathrm{E}-07$ & $2.3 \mathrm{E}-07$ \\
& & &
\end{tabular}

$\square$

Feb 20, $2008 \quad 02: 31 \mathrm{pm}$

SUMMARY

Page 6

INDIVIDUAL LIFETIME RISK (deaths)

(A11 Radionuclides and Pathways)

\begin{tabular}{|c|c|c|c|}
\hline \multirow[b]{2}{*}{ Direction } & \multicolumn{3}{|r|}{ Distance $(\mathrm{m})$} \\
\hline & 50024 & 54611 & 58610 \\
\hline $\begin{array}{r}\text { N } \\
N N W \\
N W \\
W N W \\
W \\
W S W \\
\text { SW } \\
\text { SSW } \\
S \\
\text { SSE } \\
\text { SE } \\
\text { ESE } \\
\text { E } \\
\text { ENE } \\
N E \\
\text { NNE }\end{array}$ & $\begin{array}{l}1.1 \mathrm{E}-13 \\
6.3 \mathrm{E}-14 \\
7.1 \mathrm{E}-14 \\
9.3 \mathrm{E}-14 \\
8.9 \mathrm{E}-14 \\
1.6 \mathrm{E}-13 \\
3.0 \mathrm{E}-13 \\
4.5 \mathrm{E}-13 \\
6.0 \mathrm{E}-13 \\
6.2 \mathrm{E}-13 \\
1.9 \mathrm{E}-13 \\
1.1 \mathrm{E}-13 \\
1.3 \mathrm{E}-13 \\
1.8 \mathrm{E}-13 \\
3.1 \mathrm{E}-13 \\
1.8 \mathrm{E}-13\end{array}$ & $\begin{array}{l}9.0 \mathrm{E}-14 \\
5.3 \mathrm{E}-14 \\
5.9 \mathrm{E}-14 \\
7.7 \mathrm{E}-14 \\
7.4 \mathrm{E}-14 \\
1.3 \mathrm{E}-13 \\
2.5 \mathrm{E}-13 \\
3.8 \mathrm{E}-13 \\
5.0 \mathrm{E}-13 \\
5.2 \mathrm{E}-13 \\
1.5 \mathrm{E}-13 \\
8.7 \mathrm{E}-14 \\
1.0 \mathrm{E}-13 \\
1.5 \mathrm{E}-13 \\
2.6 \mathrm{E}-13 \\
1.5 \mathrm{E}-13\end{array}$ & $\begin{array}{l}7.2 \mathrm{E}-14 \\
4.4 \mathrm{E}-14 \\
4.8 \mathrm{E}-14 \\
6.2 \mathrm{E}-14 \\
6.0 \mathrm{E}-14 \\
1.1 \mathrm{E}-13 \\
2.1 \mathrm{E}-13 \\
3.1 \mathrm{E}-13 \\
4.1 \mathrm{E}-13 \\
4.3 \mathrm{E}-13 \\
1.2 \mathrm{E}-13 \\
6.6 \mathrm{E}-14 \\
7.8 \mathrm{E}-14 \\
1.2 \mathrm{E}-13 \\
2.2 \mathrm{E}-13 \\
1.2 \mathrm{E}-13\end{array}$ \\
\hline
\end{tabular}




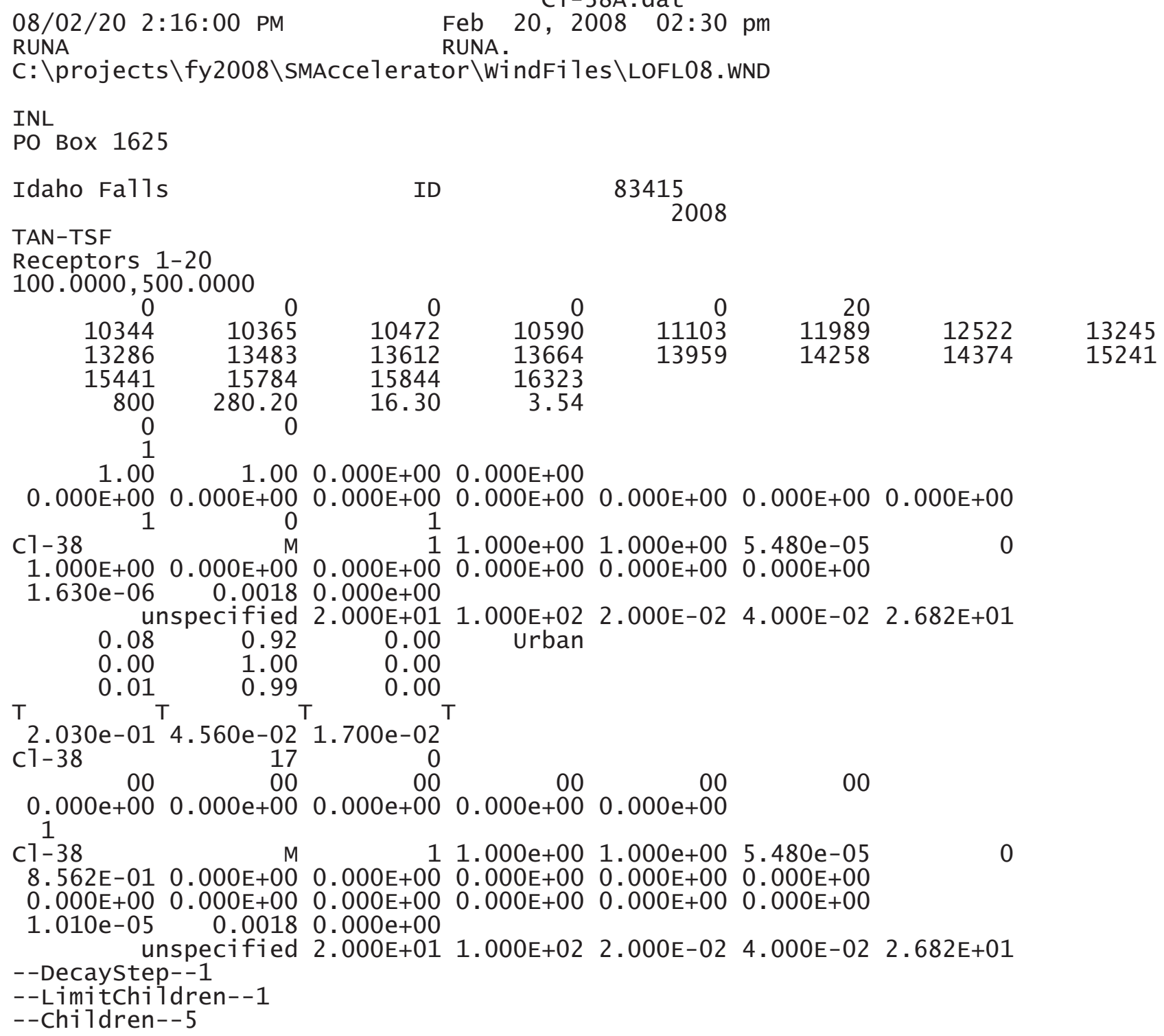




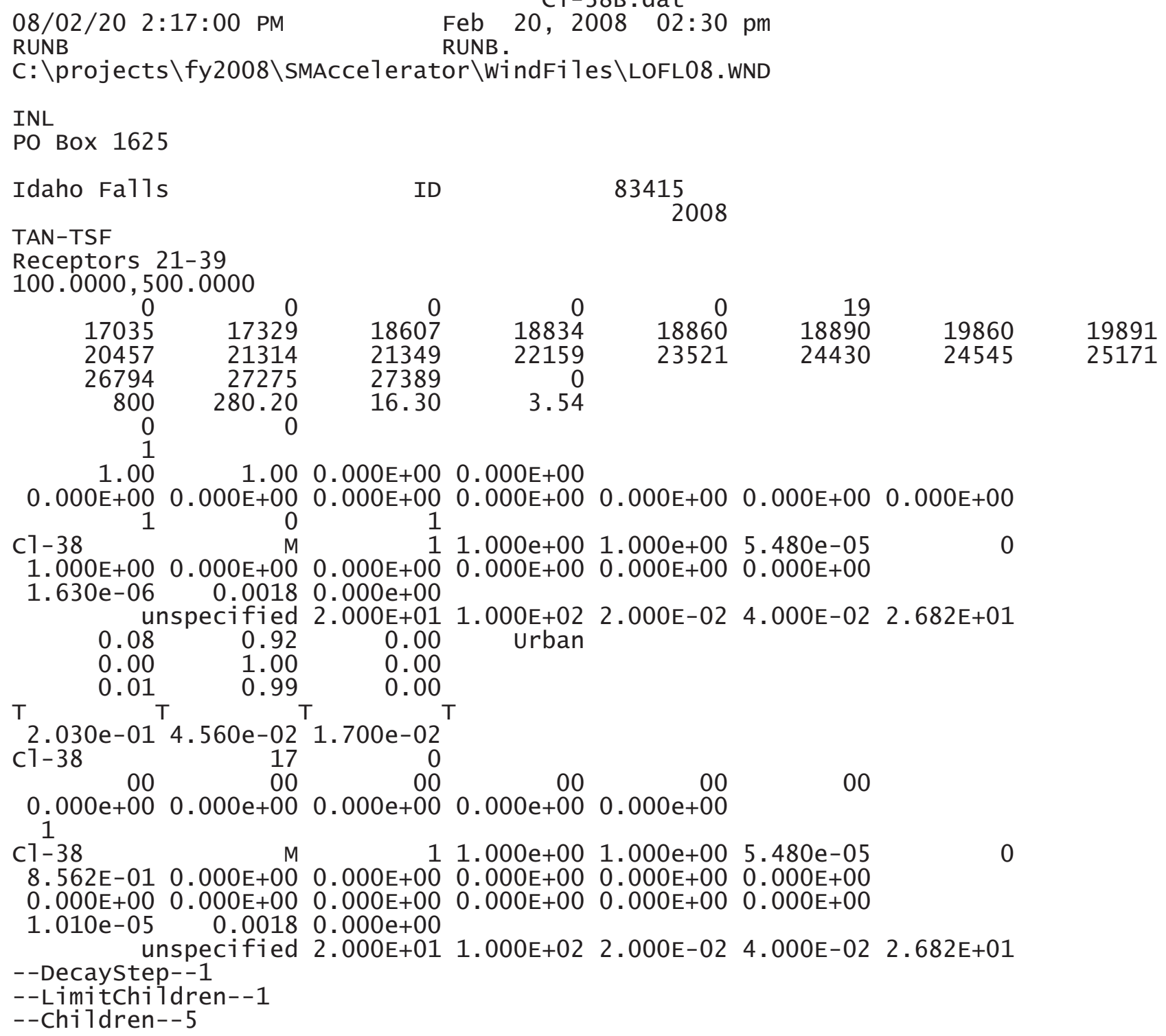




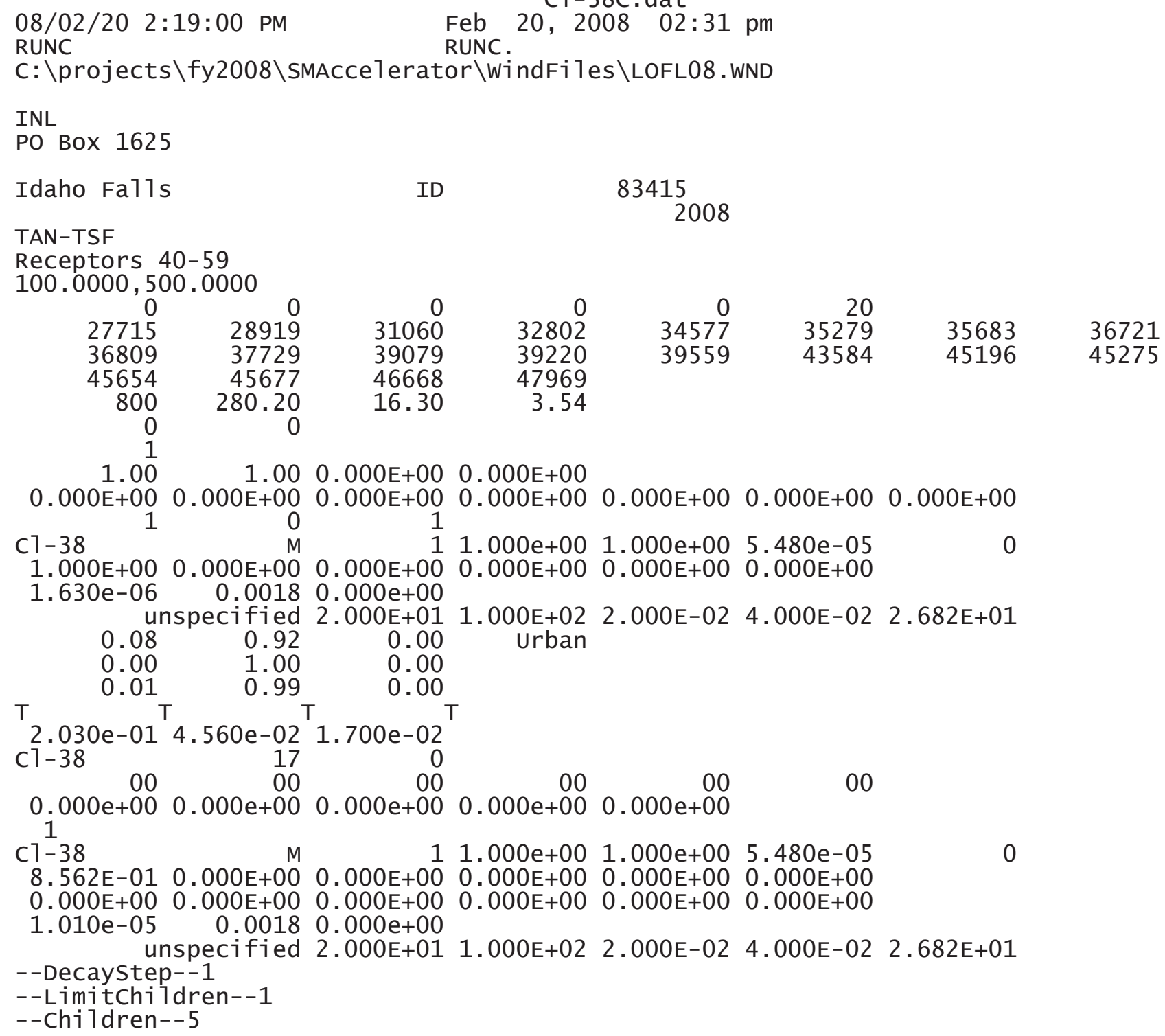

ID

83415

TAN-TSF

Receptors 40-59

$100.0000,500.0000$

$\begin{array}{rrrr}0 & 0 & 0 & \\ 27715 & 28919 & 31060 & 3280 \\ 36809 & 37729 & 39079 & 3922 \\ 45654 & 45677 & 46668 & 4796 \\ 800 & 280.20 & 16.30 & 3.5 \\ 0 & 0 & & \end{array}$




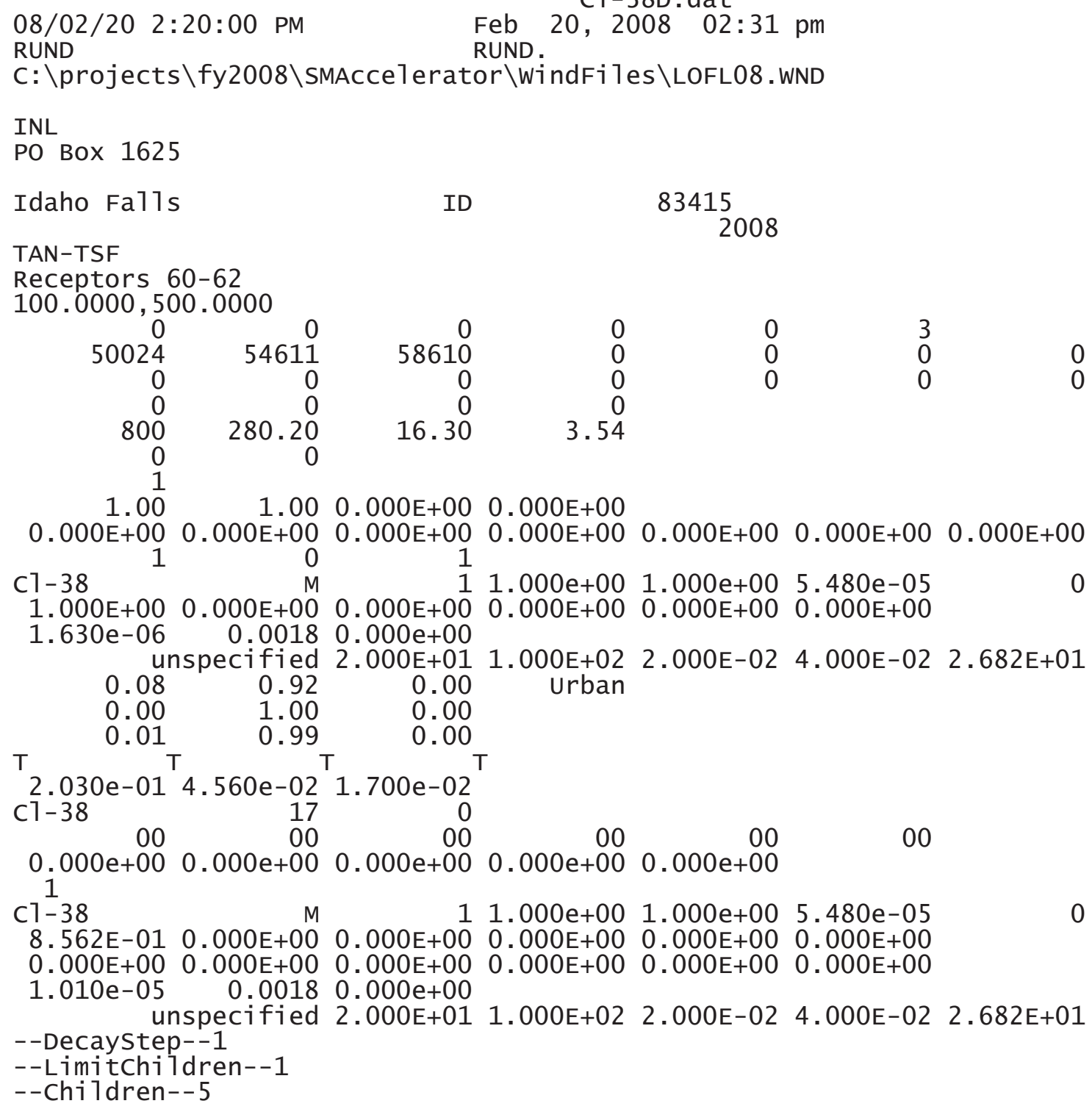




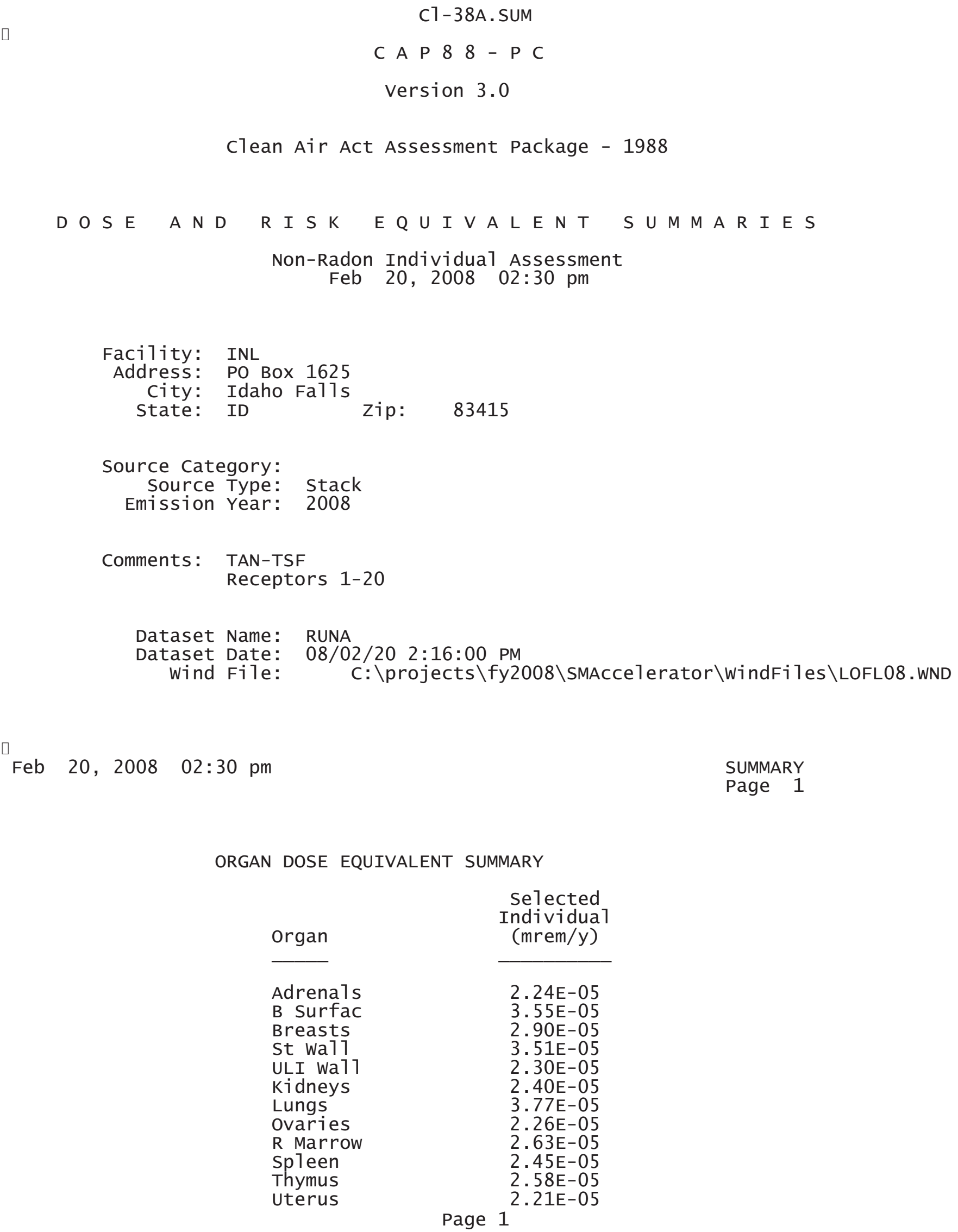

ORGAN DOSE EQUIVALENT SUMMARY

Organ

Adrenals

B Surfac

Breasts

St wa11

ULI Wa11

Kidneys

Lungs

ovaries

$\mathrm{R}$ Marrow

spleen

Thymus

uterus

$$
\begin{gathered}
\begin{array}{c}
\text { Selected } \\
\text { Individual } \\
\text { (mrem/y) }
\end{array} \\
\hline \\
2.24 \mathrm{E}-05 \\
3.55 \mathrm{E}-05 \\
2.90 \mathrm{E}-05 \\
3.51 \mathrm{E}-05 \\
2.30 \mathrm{E}-05 \\
2.40 \mathrm{E}-05 \\
3.77 \mathrm{E}-05 \\
2.26 \mathrm{E}-05 \\
2.63 \mathrm{E}-05 \\
2.45 \mathrm{E}-05 \\
2.58 \mathrm{E}-05 \\
2.21 \mathrm{E}-05
\end{gathered}
$$

Page 1 


$\begin{array}{lr} & \text { C1-38A. SUM } \\ \text { Bld wa11 } & 2.31 \mathrm{E}-05 \\ \text { Brain } & 2.81 \mathrm{E}-05 \\ \text { Esophagu } & 6.30 \mathrm{E}-05 \\ \text { SI wa11 } & 2.26 \mathrm{E}-05 \\ \text { LLI wa11 } & 2.25 \mathrm{E}-05 \\ \text { Liver } & 2.44 \mathrm{E}-05 \\ \text { Muscle } & 2.57 \mathrm{E}-05 \\ \text { Pancreas } & 2.25 \mathrm{E}-05 \\ \text { Skin } & 6.54 \mathrm{E}-05 \\ \text { Testes } & 2.57 \mathrm{E}-05 \\ \text { Thyroid } & 2.68 \mathrm{E}-05 \\ & \\ \text { EFFEC } & 2.94 \mathrm{E}-05\end{array}$

PATHWAY EFFECTIVE DOSE EQUIVALENT SUMMARY

\begin{tabular}{lcl} 
Pathway & $\begin{array}{c}\text { selected } \\
\text { Individual } \\
\text { (mrem/y) }\end{array}$ \\
\cline { 2 - 2 } INGESTION & $0.00 \mathrm{E}+00$ & \\
INHALATION & $3.88 \mathrm{E}-06$ \\
AIR IMMERSION & $2.55 \mathrm{E}-05$ & \\
GROUND SURFACE & $0.00 \mathrm{E}+00$ & \\
INTERNAL & $3.88 \mathrm{E}-06$ & \\
EXTERNAL & $2.55 \mathrm{E}-05$ & SUMMARY \\
TOTAL & $2.94 \mathrm{E}-05$ & Page 2
\end{tabular}

NUCLIDE EFFECTIVE DOSE EQUIVALENT SUMMARY

Nuclide

C1-38

TOTAL

Feb 20, $2008 \quad 02: 30$ pm

$$
\begin{gathered}
\begin{array}{c}
\text { Selected } \\
\text { Individual } \\
\text { (mrem/y) }
\end{array} \\
\hline 2.94 \mathrm{E}-05 \\
2.94 \mathrm{E}-05
\end{gathered}
$$

SUMMARY

Page 3

CANCER RISK SUMMARY

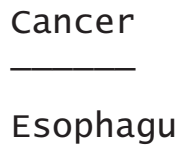

Esophagu

$$
\begin{aligned}
& \text { Selected Individual } \\
& \text { Total Lifetime } \\
& \text { Fatal Cancer Risk } \\
& \text { Page } 2^{2.68 \mathrm{E}-13}
\end{aligned}
$$




$\begin{array}{lr}\text { C1-38A. SUM } \\ \text { Stomach } & 1.59 \mathrm{E}-12 \\ \text { Colon } & 2.36 \mathrm{E}-12 \\ \text { Liver } & 3.71 \mathrm{E}-13 \\ \text { LUNG } & 3.77 \mathrm{E}-12 \\ \text { Bone } & 3.38 \mathrm{E}-14 \\ \text { Skin } & 6.55 \mathrm{E}-14 \\ \text { Breast } & 1.41 \mathrm{E}-12 \\ \text { Ovary } & 3.22 \mathrm{E}-13 \\ \text { Bladder } & 5.59 \mathrm{E}-13 \\ \text { Kidneys } & 1.25 \mathrm{E}-13 \\ \text { Thyroid } & 8.53 \mathrm{E}-14 \\ \text { Leukemia } & 1.48 \mathrm{E}-12 \\ \text { Residua1 } & 3.52 \mathrm{E}-12 \\ \text { Tota1 } & 1.59 \mathrm{E}-11 \\ & 3.19 \mathrm{E}-11 \\ \text { TOTAL } & \end{array}$

PATHWAY RISK SUMMARY

\begin{tabular}{lc} 
Pathway & \multicolumn{2}{c}{$\begin{array}{c}\text { Total Lifetime } \\
\text { Fatal Cancer Risk }\end{array}$} \\
\cline { 2 - 2 } & \\
\cline { 2 - 2 } INGESTION & $0.00 \mathrm{E}+00$ \\
INHALATION & $1.96 \mathrm{E}-12$ \\
AIR IMMERSION & $1.40 \mathrm{E}-11$ \\
GROUND SURFACE & $0.00 \mathrm{E}+00$ \\
INTERNAL & $1.96 \mathrm{E}-12$ \\
EXTERNAL & $1.40 \mathrm{E}-11$ \\
TOTAL & $1.59 \mathrm{E}-11$
\end{tabular}

$\begin{array}{lll} & \text { Feb 20, } 2008 \quad 02: 30 \mathrm{pm} & \text { SUMMARY } \\ \text { Page } 4\end{array}$

NUCLIDE RISK SUMMARY

Nuclide

C1-38

TOTAL

$\square$

Feb 20, 2008 02:30 pm
Selected Individual

Total Lifetime Fatal Cancer Risk

1. 59E-11

1. $59 \mathrm{E}-11$

INDIVIDUAL EFFECTIVE DOSE EQUIVALENT RATE (mrem/y) (A11 Radionuclides and Pathways)

Distance $(m)$ 


\begin{tabular}{|c|c|c|c|c|c|c|c|}
\hline \multirow[b]{2}{*}{ Direction } & \multirow[b]{2}{*}{10344} & \multicolumn{6}{|c|}{ C1-38A.SUM } \\
\hline & & 10365 & 10472 & 10590 & 11103 & 11989 & 12522 \\
\hline $\mathrm{N}$ & $5.7 E-06$ & $5.6 \mathrm{E}-06$ & $5.6 \mathrm{E}-06$ & $5.5 E-06$ & $5.1 \mathrm{E}-06$ & $4.6 \mathrm{E}-06$ & $4.4 E-06$ \\
\hline NNW & $2.6 \mathrm{E}-06$ & $2.6 \mathrm{E}-06$ & $2.5 \mathrm{E}-06$ & $2.5 \mathrm{E}-06$ & $2.4 \mathrm{E}-06$ & $2.1 \mathrm{E}-06$ & 2.0E-06 \\
\hline NW & $3.2 \mathrm{E}-06$ & $3.2 \mathrm{E}-06$ & $3.2 \mathrm{E}-06$ & $3.1 \mathrm{E}-06$ & $2.9 \mathrm{E}-06$ & $2.7 \mathrm{E}-06$ & $2.5 \mathrm{E}-06$ \\
\hline WNW & $4.3 E-06$ & $4.3 \mathrm{E}-06$ & $4.2 \mathrm{E}-06$ & $4.2 \mathrm{E}-06$ & $3.9 \mathrm{E}-06$ & $3.5 \mathrm{E}-06$ & $3.3 \mathrm{E}-06$ \\
\hline W & $4.5 \mathrm{E}-06$ & $4.5 \mathrm{E}-06$ & $4.4 \mathrm{E}-06$ & $4.3 \mathrm{E}-06$ & $4.1 \mathrm{E}-06$ & $3.7 \mathrm{E}-06$ & $3.5 \mathrm{E}-06$ \\
\hline WSW & $7.2 \mathrm{E}-06$ & $7.2 \mathrm{E}-06$ & $7.1 \mathrm{E}-06$ & $7.0 \mathrm{E}-06$ & $6.5 \mathrm{E}-06$ & $5.9 \mathrm{E}-06$ & $5.5 \mathrm{E}-06$ \\
\hline SW & 1.3E-05 & 1. $3 \mathrm{E}-05$ & 1.3E-05 & $1.2 \mathrm{E}-05$ & $1.2 \mathrm{E}-05$ & $1.1 \mathrm{E}-05$ & $9.9 \mathrm{E}-06$ \\
\hline SSW & $2.1 \mathrm{E}-05$ & $2.1 \mathrm{E}-05$ & $2.0 \mathrm{E}-05$ & $2.0 \mathrm{E}-05$ & $1.9 \mathrm{E}-05$ & 1.7E-05 & $1.6 \mathrm{E}-05$ \\
\hline $\mathrm{S}$ & $2.9 \mathrm{E}-05$ & $2.9 \mathrm{E}-05$ & $2.9 \mathrm{E}-05$ & $2.8 \mathrm{E}-05$ & $2.7 \mathrm{E}-05$ & $2.4 \mathrm{E}-05$ & $2.2 \mathrm{E}-05$ \\
\hline SSE & $2.6 \mathrm{E}-05$ & $2.6 \mathrm{E}-05$ & $2.6 \mathrm{E}-05$ & $2.5 \mathrm{E}-05$ & $2.4 \mathrm{E}-05$ & $2.1 \mathrm{E}-05$ & 2.0E-05 \\
\hline SE & $1.0 \mathrm{E}-05$ & $1.0 \mathrm{E}-05$ & 1.0E-05 & $1.0 \mathrm{E}-05$ & $9.5 \mathrm{E}-06$ & $8.5 \mathrm{E}-06$ & $8.0 \mathrm{E}-06$ \\
\hline ESE & $6.5 \mathrm{E}-06$ & $6.4 \mathrm{E}-06$ & $6.3 \mathrm{E}-06$ & $6.2 \mathrm{E}-06$ & $5.9 \mathrm{E}-06$ & $5.3 \mathrm{E}-06$ & $5.0 \mathrm{E}-06$ \\
\hline E & $8.1 \mathrm{E}-06$ & $8.1 \mathrm{E}-06$ & 8.0E-06 & $7.8 \mathrm{E}-06$ & $7.3 \mathrm{E}-06$ & $6.6 \mathrm{E}-06$ & $6.2 \mathrm{E}-06$ \\
\hline ENE & $8.8 \mathrm{E}-06$ & 8.7E-06 & $8.6 \mathrm{E}-06$ & $8.5 \mathrm{E}-06$ & 8.0E-06 & $7.2 \mathrm{E}-06$ & $6.8 \mathrm{E}-06$ \\
\hline NE & $1.5 \mathrm{E}-05$ & $1.5 \mathrm{E}-05$ & 1.4E-05 & 1. $4 \mathrm{E}-05$ & 1. $3 \mathrm{E}-05$ & $1.2 \mathrm{E}-05$ & 1.1E-05 \\
\hline NNE & $8.2 \mathrm{E}-06$ & $8.2 \mathrm{E}-06$ & $8.1 \mathrm{E}-06$ & $8.0 \mathrm{E}-06$ & $7.5 \mathrm{E}-06$ & $6.7 \mathrm{E}-06$ & $6.3 \mathrm{E}-06$ \\
\hline
\end{tabular}

Distance (m)

\begin{tabular}{|c|c|c|c|c|c|c|c|}
\hline Direction & 13245 & 13286 & 13483 & 13612 & 13664 & 13959 & 14258 \\
\hline $\mathrm{N}$ & $4.0 \mathrm{E}-06$ & 4. $0 \mathrm{E}-06$ & $3.9 \mathrm{E}-06$ & $3.9 \mathrm{E}-06$ & $3.9 \mathrm{E}-06$ & $3.7 E-06$ & $3.6 \mathrm{E}-06$ \\
\hline NNW & $1.9 \mathrm{E}-06$ & $1.9 \mathrm{E}-06$ & $1.8 \mathrm{E}-06$ & $1.8 \mathrm{E}-06$ & $1.8 \mathrm{E}-06$ & $1.7 \mathrm{E}-06$ & $1.7 \mathrm{E}-06$ \\
\hline NW & $2.3 \mathrm{E}-06$ & 2. $3 \mathrm{E}-06$ & $2.3 \mathrm{E}-06$ & $2.2 \mathrm{E}-06$ & $2.2 \mathrm{E}-06$ & $2.2 \mathrm{E}-06$ & $2.1 \mathrm{E}-06$ \\
\hline WNW & $3.1 \mathrm{E}-06$ & $3.1 \mathrm{E}-06$ & $3.0 \mathrm{E}-06$ & $3.0 \mathrm{E}-06$ & $3.0 \mathrm{E}-06$ & $2.9 \mathrm{E}-06$ & $2.8 \mathrm{E}-06$ \\
\hline & $3.2 \mathrm{E}-06$ & $3.2 \mathrm{E}-06$ & $3.1 \mathrm{E}-06$ & $3.1 \mathrm{E}-06$ & $3.1 \mathrm{E}-06$ & $3.0 \mathrm{E}-06$ & $2.9 \mathrm{E}-06$ \\
\hline WSW & $5.1 \mathrm{E}-06$ & $5.1 \mathrm{E}-06$ & $5.0 \mathrm{E}-06$ & $4.9 \mathrm{E}-06$ & $4.9 \mathrm{E}-06$ & $4.7 \mathrm{E}-06$ & $4.6 \mathrm{E}-06$ \\
\hline SW & $9.1 \mathrm{E}-06$ & $9.1 \mathrm{E}-06$ & $8.9 \mathrm{E}-06$ & $8.8 \mathrm{E}-06$ & $8.7 \mathrm{E}-06$ & $8.5 \mathrm{E}-06$ & $8.2 \mathrm{E}-06$ \\
\hline SSW & $1.5 \mathrm{E}-05$ & $1.5 \mathrm{E}-05$ & $1.4 \mathrm{E}-05$ & $1.4 \mathrm{E}-05$ & $1.4 \mathrm{E}-05$ & $1.4 \mathrm{E}-05$ & 1. $3 \mathrm{E}-05$ \\
\hline $\mathrm{S}$ & $2.1 \mathrm{E}-05$ & $2.1 \mathrm{E}-05$ & $2.0 \mathrm{E}-05$ & $2.0 \mathrm{E}-05$ & $2.0 \mathrm{E}-05$ & $1.9 \mathrm{E}-05$ & $1.9 \mathrm{E}-05$ \\
\hline SSE & $1.9 \mathrm{E}-05$ & $1.9 \mathrm{E}-05$ & $1.8 \mathrm{E}-05$ & $1.8 \mathrm{E}-05$ & $1.8 \mathrm{E}-05$ & $1.7 \mathrm{E}-05$ & $1.7 \mathrm{E}-05$ \\
\hline $\mathrm{SE}$ & $7.4 \mathrm{E}-06$ & $7.4 \mathrm{E}-06$ & $7.2 \mathrm{E}-06$ & $7.1 \mathrm{E}-06$ & $7.1 \mathrm{E}-06$ & $6.9 \mathrm{E}-06$ & $6.6 \mathrm{E}-06$ \\
\hline ESE & $4.6 \mathrm{E}-06$ & $4.5 \mathrm{E}-06$ & $4.5 \mathrm{E}-06$ & $4.4 \mathrm{E}-06$ & $4.4 \mathrm{E}-06$ & $4.2 \mathrm{E}-06$ & $4.1 \mathrm{E}-06$ \\
\hline & $5.7 \mathrm{E}-06$ & $5.7 \mathrm{E}-06$ & $5.6 \mathrm{E}-06$ & $5.5 \mathrm{E}-06$ & $5.5 \mathrm{E}-06$ & $5.3 \mathrm{E}-06$ & $5.1 \mathrm{E}-06$ \\
\hline ENE & $6.2 \mathrm{E}-06$ & $6.2 \mathrm{E}-06$ & $6.1 \mathrm{E}-06$ & $6.0 \mathrm{E}-06$ & $6.0 \mathrm{E}-06$ & $5.8 \mathrm{E}-06$ & $5.6 \mathrm{E}-06$ \\
\hline $\mathrm{NE}$ & $1.0 \mathrm{E}-05$ & $1.0 \mathrm{E}-05$ & $1.0 \mathrm{E}-05$ & $9.9 \mathrm{E}-06$ & $9.8 \mathrm{E}-06$ & $9.5 \mathrm{E}-06$ & $9.2 \mathrm{E}-06$ \\
\hline NNE & $5.8 \mathrm{E}-06$ & $5.8 \mathrm{E}-06$ & $5.7 \mathrm{E}-06$ & $5.6 \mathrm{E}-06$ & $5.6 \mathrm{E}-06$ & $5.4 \mathrm{E}-06$ & $5.3 \mathrm{E}-06$ \\
\hline
\end{tabular}

$\square$

Feb 20, 2008 02:30 pm

SUMMARY

Page 6

INDIVIDUAL EFFECTIVE DOSE EQUIVALENT RATE (mrem/y) (A11 Radionuclides and Pathways)

\begin{tabular}{|c|c|c|c|c|c|c|}
\hline \multirow[b]{2}{*}{ Direction } & \multicolumn{6}{|c|}{ Distance $(\mathrm{m})$} \\
\hline & 14374 & 15241 & 15441 & 15784 & 15844 & 16323 \\
\hline $\begin{array}{r}N \\
\text { NNW }\end{array}$ & $\begin{array}{l}3.6 \mathrm{E}-06 \\
1.7 \mathrm{E}-06\end{array}$ & $\begin{array}{l}3.3 \mathrm{E}-06 \\
1.5 \mathrm{E}-06\end{array}$ & $\begin{array}{l}3.2 \mathrm{E}-06 \\
1.5 \mathrm{E}-06\end{array}$ & $\begin{array}{r}3.1 \mathrm{E}-06 \\
1.5 \mathrm{E}-06 \\
\text { Page }\end{array}$ & $\begin{array}{r}3.1 \mathrm{E}-06 \\
1.5 \mathrm{E}-06\end{array}$ & $\begin{array}{l}3.0 \mathrm{E}-06 \\
1.4 \mathrm{E}-06\end{array}$ \\
\hline
\end{tabular}




\begin{tabular}{|c|c|c|c|c|c|c|}
\hline & & & & $38 \mathrm{~A}$ & & \\
\hline NW & $2.1 \mathrm{E}-06$ & $1.9 \mathrm{E}-06$ & 1.9E-06 & $1.8 \mathrm{E}-06$ & $1.8 \mathrm{E}-06$ & $1.7 \mathrm{E}-06$ \\
\hline WNW & $2.8 \mathrm{E}-06$ & $2.5 \mathrm{E}-06$ & $2.5 \mathrm{E}-06$ & $2.4 \mathrm{E}-06$ & $2.4 \mathrm{E}-06$ & 2. $3 \mathrm{E}-06$ \\
\hline & $2.8 \mathrm{E}-06$ & $2.6 \mathrm{E}-06$ & $2.6 \mathrm{E}-06$ & $2.5 \mathrm{E}-06$ & $2.5 \mathrm{E}-06$ & $2.4 \mathrm{E}-06$ \\
\hline WSW & $4.6 \mathrm{E}-06$ & $4.2 \mathrm{E}-06$ & $4.1 \mathrm{E}-06$ & $4.0 \mathrm{E}-06$ & $4.0 \mathrm{E}-06$ & $3.8 \mathrm{E}-06$ \\
\hline SW & $8.1 \mathrm{E}-06$ & $7.5 \mathrm{E}-06$ & 7. $4 \mathrm{E}-06$ & $7.1 \mathrm{E}-06$ & $7.1 \mathrm{E}-06$ & $6.8 \mathrm{E}-06$ \\
\hline SSW & 1. $3 \mathrm{E}-05$ & $1.2 \mathrm{E}-05$ & $1.2 \mathrm{E}-05$ & 1. $1 \mathrm{E}-05$ & $1.1 \mathrm{E}-05$ & $1.1 \mathrm{E}-05$ \\
\hline & $1.8 \mathrm{E}-05$ & $1.7 \mathrm{E}-05$ & $1.6 \mathrm{E}-05$ & $1.6 \mathrm{E}-05$ & $1.6 \mathrm{E}-05$ & $1.5 \mathrm{E}-05$ \\
\hline SSE & 1.7E-05 & 1. $5 \mathrm{E}-05$ & 1. $5 \mathrm{E}-05$ & 1. $5 \mathrm{E}-05$ & $1.4 \mathrm{E}-05$ & $1.4 \mathrm{E}-05$ \\
\hline SE & $6.6 \mathrm{E}-06$ & $6.0 \mathrm{E}-06$ & $5.9 \mathrm{E}-06$ & $5.7 \mathrm{E}-06$ & $5.7 \mathrm{E}-06$ & $5.4 \mathrm{E}-06$ \\
\hline ESE & 4.1E-06 & $3.7 E-06$ & $3.6 \mathrm{E}-06$ & $3.5 \mathrm{E}-06$ & $3.5 \mathrm{E}-06$ & $3.3 E-06$ \\
\hline & $1 E-06$ & $4.7 \mathrm{E}-06$ & $4.6 \mathrm{E}-06$ & $4.4 \mathrm{E}-06$ & $4.4 \mathrm{E}-06$ & $4.2 \mathrm{E}-06$ \\
\hline ENE & $5.6 \mathrm{E}-06$ & $5.1 \mathrm{E}-06$ & $5.0 \mathrm{E}-06$ & $4.9 \mathrm{E}-06$ & $4.8 \mathrm{E}-06$ & $4.6 \mathrm{E}-06$ \\
\hline $\mathrm{NE}$ & $1 \mathrm{E}-06$ & $8.4 \mathrm{E}-06$ & $8.2 \mathrm{E}-06$ & $8.0 \mathrm{E}-06$ & $7.9 \mathrm{E}-06$ & $6 \mathrm{E}-06$ \\
\hline NNE & $5.2 \mathrm{E}-06$ & $4.8 \mathrm{E}-06$ & $4.7 \mathrm{E}-06$ & $4.6 \mathrm{E}-06$ & $4.5 E-06$ & $4.3 \mathrm{E}-06$ \\
\hline
\end{tabular}

Feb 20, $2008 \quad 02: 30 \mathrm{pm}$

SUMMARY

Page 7

INDIVIDUAL LIFETIME RISK (deaths)

(A11 Radionuc1ides and Pathways)

\begin{tabular}{|c|c|c|c|c|c|c|c|}
\hline \multicolumn{8}{|c|}{ Distance $(\mathrm{m})$} \\
\hline Direction & 10344 & 10365 & 10472 & 10590 & 11103 & 11989 & 12522 \\
\hline $\begin{array}{r}N \\
N N W \\
N W \\
W N W \\
W \\
W S W \\
S W \\
S S W \\
S \\
S S E \\
S E \\
\text { ESE } \\
E \\
\text { ENE } \\
N E \\
N N E\end{array}$ & $\begin{array}{l}3.1 \mathrm{E}-12 \\
1.4 \mathrm{E}-12 \\
1.8 \mathrm{E}-12 \\
2.3 \mathrm{E}-12 \\
2.4 \mathrm{E}-12 \\
3.9 \mathrm{E}-12 \\
7.0 \mathrm{E}-12 \\
1.1 \mathrm{E}-11 \\
1.6 \mathrm{E}-11 \\
1.4 \mathrm{E}-11 \\
5.7 \mathrm{E}-12 \\
3.5 \mathrm{E}-12 \\
4.4 \mathrm{E}-12 \\
4.8 \mathrm{E}-12 \\
7.9 \mathrm{E}-12 \\
4.5 \mathrm{E}-12\end{array}$ & $\begin{array}{l}3.1 \mathrm{E}-12 \\
1.4 \mathrm{E}-12 \\
1.7 \mathrm{E}-12 \\
2.3 \mathrm{E}-12 \\
2.4 \mathrm{E}-12 \\
3.9 \mathrm{E}-12 \\
7.0 \mathrm{E}-12 \\
1.1 \mathrm{E}-11 \\
1.6 \mathrm{E}-11 \\
1.4 \mathrm{E}-11 \\
5.6 \mathrm{E}-12 \\
3.5 \mathrm{E}-12 \\
4.4 \mathrm{E}-12 \\
4.7 \mathrm{E}-12 \\
7.9 \mathrm{E}-12 \\
4.5 \mathrm{E}-12\end{array}$ & $\begin{array}{l}3.0 \mathrm{E}-12 \\
1.4 \mathrm{E}-12 \\
1.7 \mathrm{E}-12 \\
2.3 \mathrm{E}-12 \\
2.4 \mathrm{E}-12 \\
3.8 \mathrm{E}-12 \\
6.9 \mathrm{E}-12 \\
1.1 \mathrm{E}-11 \\
1.6 \mathrm{E}-11 \\
1.4 \mathrm{E}-11 \\
5.6 \mathrm{E}-12 \\
3.4 \mathrm{E}-12 \\
4.3 \mathrm{E}-12 \\
4.7 \mathrm{E}-12 \\
7.8 \mathrm{E}-12 \\
4.4 \mathrm{E}-12\end{array}$ & $\begin{array}{l}3.0 \mathrm{E}-12 \\
1.4 \mathrm{E}-12 \\
1.7 \mathrm{E}-12 \\
2.3 \mathrm{E}-12 \\
2.4 \mathrm{E}-12 \\
3.8 \mathrm{E}-12 \\
6.8 \mathrm{E}-12 \\
1.1 \mathrm{E}-11 \\
1.5 \mathrm{E}-11 \\
1.4 \mathrm{E}-11 \\
5.5 \mathrm{E}-12 \\
3.4 \mathrm{E}-12 \\
4.3 \mathrm{E}-12 \\
4.6 \mathrm{E}-12 \\
7.7 \mathrm{E}-12 \\
4.3 \mathrm{E}-12\end{array}$ & $\begin{array}{l}2.8 \mathrm{E}-12 \\
1.3 \mathrm{E}-12 \\
1.6 \mathrm{E}-12 \\
2.1 \mathrm{E}-12 \\
2.2 \mathrm{E}-12 \\
3.5 \mathrm{E}-12 \\
6.3 \mathrm{E}-12 \\
1.0 \mathrm{E}-11 \\
1.4 \mathrm{E}-11 \\
1.3 \mathrm{E}-11 \\
5.1 \mathrm{E}-12 \\
3.2 \mathrm{E}-12 \\
4.0 \mathrm{E}-12 \\
4.3 \mathrm{E}-12 \\
7.2 \mathrm{E}-12 \\
4.1 \mathrm{E}-12\end{array}$ & $\begin{array}{l}2.5 \mathrm{E}-12 \\
1.2 \mathrm{E}-12 \\
1.4 \mathrm{E}-12 \\
1.9 \mathrm{E}-12 \\
2.0 \mathrm{E}-12 \\
3.2 \mathrm{E}-12 \\
5.7 \mathrm{E}-12 \\
9.2 \mathrm{E}-12 \\
1.3 \mathrm{E}-11 \\
1.2 \mathrm{E}-11 \\
4.6 \mathrm{E}-12 \\
2.9 \mathrm{E}-12 \\
3.6 \mathrm{E}-12 \\
3.9 \mathrm{E}-12 \\
6.4 \mathrm{E}-12 \\
3.7 \mathrm{E}-12\end{array}$ & $\begin{array}{l}2.4 \mathrm{E}-12 \\
1.1 \mathrm{E}-12 \\
1.4 \mathrm{E}-12 \\
1.8 \mathrm{E}-12 \\
1.9 \mathrm{E}-12 \\
3.0 \mathrm{E}-12 \\
5.4 \mathrm{E}-12 \\
8.7 \mathrm{E}-12 \\
1.2 \mathrm{E}-11 \\
1.1 \mathrm{E}-11 \\
4.3 \mathrm{E}-12 \\
2.7 \mathrm{E}-12 \\
3.4 \mathrm{E}-12 \\
3.7 \mathrm{E}-12 \\
6.1 \mathrm{E}-12 \\
3.4 \mathrm{E}-12\end{array}$ \\
\hline \multicolumn{8}{|c|}{ Distance $(\mathrm{m})$} \\
\hline Direction & 13245 & 13286 & 13483 & 13612 & 13664 & 13959 & 14258 \\
\hline $\begin{array}{r}\mathrm{N} \\
\mathrm{NNW} \\
\mathrm{NW} \\
\text { WNW } \\
W \\
\text { WSW } \\
\text { SW } \\
\text { SSW }\end{array}$ & $\begin{array}{l}2.2 \mathrm{E}-12 \\
1.0 \mathrm{E}-12 \\
1.3 \mathrm{E}-12 \\
1.7 \mathrm{E}-12 \\
1.7 \mathrm{E}-12 \\
2.8 \mathrm{E}-12 \\
4.9 \mathrm{E}-12 \\
8.0 \mathrm{E}-12\end{array}$ & $\begin{array}{l}2.2 \mathrm{E}-12 \\
1.0 \mathrm{E}-12 \\
1.3 \mathrm{E}-12 \\
1.7 \mathrm{E}-12 \\
1.7 \mathrm{E}-12 \\
2.8 \mathrm{E}-12 \\
4.9 \mathrm{E}-12 \\
8.0 \mathrm{E}-12\end{array}$ & $\begin{array}{l}2.1 \mathrm{E}-12 \\
9.9 \mathrm{E}-13 \\
1.2 \mathrm{E}-12 \\
1.6 \mathrm{E}-12 \\
1.7 \mathrm{E}-12 \\
2.7 \mathrm{E}-12 \\
4.8 \mathrm{E}-12 \\
7.8 \mathrm{E}-12\end{array}$ & $\begin{array}{r}2.1 \mathrm{E}-12 \\
9.7 \mathrm{E}-13 \\
1.2 \mathrm{E}-12 \\
1.6 \mathrm{E}-12 \\
1.7 \mathrm{E}-12 \\
2.7 \mathrm{E}-12 \\
4.8 \mathrm{E}-12 \\
7.7 \mathrm{E}-12 \\
\text { Page }\end{array}$ & $\begin{array}{l}2.1 \mathrm{E}-12 \\
9.7 \mathrm{E}-13 \\
1.2 \mathrm{E}-12 \\
1.6 \mathrm{E}-12 \\
1.7 \mathrm{E}-12 \\
2.6 \mathrm{E}-12 \\
4.7 \mathrm{E}-12 \\
7.7 \mathrm{E}-12\end{array}$ & $\begin{array}{l}2.0 \mathrm{E}-12 \\
9.4 \mathrm{E}-13 \\
1.2 \mathrm{E}-12 \\
1.6 \mathrm{E}-12 \\
1.6 \mathrm{E}-12 \\
2.6 \mathrm{E}-12 \\
4.6 \mathrm{E}-12 \\
7.4 \mathrm{E}-12\end{array}$ & $\begin{array}{l}2.0 \mathrm{E}-12 \\
9.2 \mathrm{E}-13 \\
1.1 \mathrm{E}-12 \\
1.5 \mathrm{E}-12 \\
1.6 \mathrm{E}-12 \\
2.5 \mathrm{E}-12 \\
4.5 \mathrm{E}-12 \\
7.2 \mathrm{E}-12\end{array}$ \\
\hline
\end{tabular}




\begin{tabular}{rlllllll}
\multicolumn{7}{c}{ C1-38A. SUM } \\
S & $1.1 \mathrm{E}-11$ & $1.1 \mathrm{E}-11$ & $1.1 \mathrm{E}-11$ & $1.1 \mathrm{E}-11$ & $1.1 \mathrm{E}-11$ & $1.0 \mathrm{E}-11$ & $1.0 \mathrm{E}-11$ \\
SSE & $1.0 \mathrm{E}-11$ & $1.0 \mathrm{E}-11$ & $9.8 \mathrm{E}-12$ & $9.7 \mathrm{E}-12$ & $9.7 \mathrm{E}-12$ & $9.4 \mathrm{E}-12$ & $9.1 \mathrm{E}-12$ \\
SE & $4.0 \mathrm{E}-12$ & $4.0 \mathrm{E}-12$ & $3.9 \mathrm{E}-12$ & $3.9 \mathrm{E}-12$ & $3.8 \mathrm{E}-12$ & $3.7 \mathrm{E}-12$ & $3.6 \mathrm{E}-12$ \\
ESE & $2.5 \mathrm{E}-12$ & $2.5 \mathrm{E}-12$ & $2.4 \mathrm{E}-12$ & $2.4 \mathrm{E}-12$ & $2.4 \mathrm{E}-12$ & $2.3 \mathrm{E}-12$ & $2.2 \mathrm{E}-12$ \\
$\mathrm{E}$ & $3.1 \mathrm{E}-12$ & $3.1 \mathrm{E}-12$ & $3.0 \mathrm{E}-12$ & $3.0 \mathrm{E}-12$ & $3.0 \mathrm{E}-12$ & $2.9 \mathrm{E}-12$ & $2.8 \mathrm{E}-12$ \\
$\mathrm{ENE}$ & $3.4 \mathrm{E}-12$ & $3.4 \mathrm{E}-12$ & $3.3 \mathrm{E}-12$ & $3.3 \mathrm{E}-12$ & $3.2 \mathrm{E}-12$ & $3.1 \mathrm{E}-12$ & $3.1 \mathrm{E}-12$ \\
$\mathrm{NE}$ & $5.6 \mathrm{E}-12$ & $5.5 \mathrm{E}-12$ & $5.4 \mathrm{E}-12$ & $5.4 \mathrm{E}-12$ & $5.3 \mathrm{E}-12$ & $5.2 \mathrm{E}-12$ & $5.0 \mathrm{E}-12$ \\
NNE & $3.2 \mathrm{E}-12$ & $3.2 \mathrm{E}-12$ & $3.1 \mathrm{E}-12$ & $3.0 \mathrm{E}-12$ & $3.0 \mathrm{E}-12$ & $2.9 \mathrm{E}-12$ & $2.9 \mathrm{E}-12$
\end{tabular}

Feb 20, $2008 \quad 02: 30 \mathrm{pm}$

SUMMARY

Page 8

INDIVIDUAL LIFETIME RISK (deaths)

(A11 Radionuclides and Pathways)

\begin{tabular}{|c|c|c|c|c|c|c|}
\hline \multirow[b]{2}{*}{ Direction } & \multicolumn{6}{|c|}{ Distance $(\mathrm{m})$} \\
\hline & 14374 & 15241 & 15441 & 15784 & 15844 & 16323 \\
\hline $\begin{array}{r}N \\
N N W \\
N W \\
W N W \\
W \\
W S W \\
S W \\
S S W \\
S \\
\text { SSE } \\
\text { SE } \\
\text { ESE } \\
\text { E } \\
\text { ENE } \\
N E \\
\text { NNE }\end{array}$ & $\begin{array}{l}1.9 \mathrm{E}-12 \\
9.1 \mathrm{E}-13 \\
1.1 \mathrm{E}-12 \\
1.5 \mathrm{E}-12 \\
1.5 \mathrm{E}-12 \\
2.5 \mathrm{E}-12 \\
4.4 \mathrm{E}-12 \\
7.1 \mathrm{E}-12 \\
9.9 \mathrm{E}-12 \\
9.0 \mathrm{E}-12 \\
3.6 \mathrm{E}-12 \\
2.2 \mathrm{E}-12 \\
2.8 \mathrm{E}-12 \\
3.0 \mathrm{E}-12 \\
5.0 \mathrm{E}-12 \\
2.8 \mathrm{E}-12\end{array}$ & $\begin{array}{l}1.8 \mathrm{E}-12 \\
8.4 \mathrm{E}-13 \\
1.0 \mathrm{E}-12 \\
1.4 \mathrm{E}-12 \\
1.4 \mathrm{E}-12 \\
2.3 \mathrm{E}-12 \\
4.1 \mathrm{E}-12 \\
6.5 \mathrm{E}-12 \\
9.1 \mathrm{E}-12 \\
8.3 \mathrm{E}-12 \\
3.3 \mathrm{E}-12 \\
2.0 \mathrm{E}-12 \\
2.5 \mathrm{E}-12 \\
2.8 \mathrm{E}-12 \\
4.5 \mathrm{E}-12 \\
2 \cdot 6 \mathrm{E}-12\end{array}$ & $\begin{array}{l}1.8 \mathrm{E}-12 \\
8.2 \mathrm{E}-13 \\
1.0 \mathrm{E}-12 \\
1.4 \mathrm{E}-12 \\
1.4 \mathrm{E}-12 \\
2.2 \mathrm{E}-12 \\
4.0 \mathrm{E}-12 \\
6.4 \mathrm{E}-12 \\
8.9 \mathrm{E}-12 \\
8.1 \mathrm{E}-12 \\
3.2 \mathrm{E}-12 \\
2.0 \mathrm{E}-12 \\
2.5 \mathrm{E}-12 \\
2.7 \mathrm{E}-12 \\
4.5 \mathrm{E}-12 \\
2.5 \mathrm{E}-12\end{array}$ & $\begin{array}{l}1.7 \mathrm{E}-12 \\
8.0 \mathrm{E}-13 \\
9.9 \mathrm{E}-13 \\
1.3 \mathrm{E}-12 \\
1.3 \mathrm{E}-12 \\
2.2 \mathrm{E}-12 \\
3.9 \mathrm{E}-12 \\
6.2 \mathrm{E}-12 \\
8.7 \mathrm{E}-12 \\
7.9 \mathrm{E}-12 \\
3.1 \mathrm{E}-12 \\
1.9 \mathrm{E}-12 \\
2.4 \mathrm{E}-12 \\
2.6 \mathrm{E}-12 \\
4.3 \mathrm{E}-12 \\
2.5 \mathrm{E}-12\end{array}$ & $\begin{array}{l}1.7 \mathrm{E}-12 \\
7.9 \mathrm{E}-13 \\
9.8 \mathrm{E}-13 \\
1.3 \mathrm{E}-12 \\
1.3 \mathrm{E}-12 \\
2.2 \mathrm{E}-12 \\
3.8 \mathrm{E}-12 \\
6.2 \mathrm{E}-12 \\
8.6 \mathrm{E}-12 \\
7.8 \mathrm{E}-12 \\
3.1 \mathrm{E}-12 \\
1.9 \mathrm{E}-12 \\
2.4 \mathrm{E}-12 \\
2.6 \mathrm{E}-12 \\
4.3 \mathrm{E}-12 \\
2.5 \mathrm{E}-12\end{array}$ & $\begin{array}{l}1.6 \mathrm{E}-12 \\
7.6 \mathrm{E}-13 \\
9.4 \mathrm{E}-13 \\
1.2 \mathrm{E}-12 \\
1.3 \mathrm{E}-12 \\
2.1 \mathrm{E}-12 \\
3.7 \mathrm{E}-12 \\
5.9 \mathrm{E}-12 \\
8.2 \mathrm{E}-12 \\
7.5 \mathrm{E}-12 \\
2.9 \mathrm{E}-12 \\
1.8 \mathrm{E}-12 \\
2.3 \mathrm{E}-12 \\
2.5 \mathrm{E}-12 \\
4.1 \mathrm{E}-12 \\
2.3 \mathrm{E}-12\end{array}$ \\
\hline
\end{tabular}




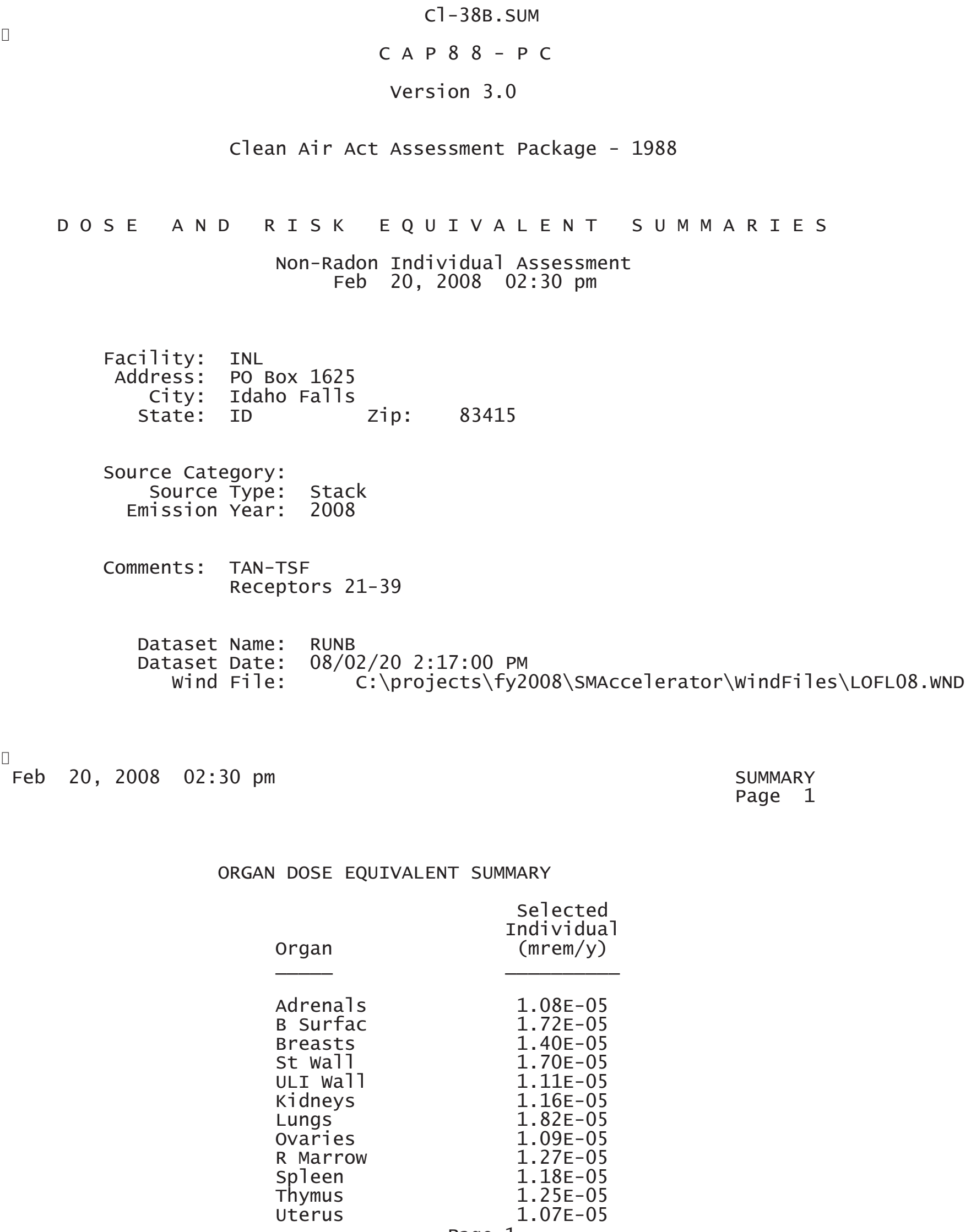

ORGAN DOSE EQUIVALENT SUMMARY

Organ

Adrenals

B Surfac Breasts

St wa11

ULI Wa11

Kidneys

Lungs

ovaries

R Marrow

spleen

Thymus

uterus

$$
\begin{gathered}
\begin{array}{c}
\text { Selected } \\
\text { Individual } \\
\text { (mrem/y) }
\end{array} \\
\hline \\
1.08 \mathrm{E}-05 \\
1.72 \mathrm{E}-05 \\
1.40 \mathrm{E}-05 \\
1.70 \mathrm{E}-05 \\
1.11 \mathrm{E}-05 \\
1.16 \mathrm{E}-05 \\
1.82 \mathrm{E}-05 \\
1.09 \mathrm{E}-05 \\
1.27 \mathrm{E}-05 \\
1.18 \mathrm{E}-05 \\
1.25 \mathrm{E}-05 \\
1.07 \mathrm{E}-05
\end{gathered}
$$




\begin{tabular}{lr} 
& \multicolumn{1}{c}{ C1-38B. SUM } \\
B1d wa11 & $1.12 \mathrm{E}-05$ \\
Brain & $1.36 \mathrm{E}-05$ \\
Esophagu & $3.05 \mathrm{E}-05$ \\
SI wa11 & $1.09 \mathrm{E}-05$ \\
LLI wa11 & $1.09 \mathrm{E}-05$ \\
Liver & $1.18 \mathrm{E}-05$ \\
Muscle & $1.24 \mathrm{E}-05$ \\
Pancreas & $1.09 \mathrm{E}-05$ \\
Skin & $3.17 \mathrm{E}-05$ \\
Testes & $1.24 \mathrm{E}-05$ \\
Thyroid & $1.29 \mathrm{E}-05$ \\
& \\
EFFEC & $1.42 \mathrm{E}-05$
\end{tabular}

PATHWAY EFFECTIVE DOSE EQUIVALENT SUMMARY

$\begin{array}{lc}\text { Pathway } & \begin{array}{c}\text { selected } \\ \text { Individual } \\ \text { (mrem/y) }\end{array} \\ & \\ \text { INGESTION } & 0.00 \mathrm{E}+00 \\ \text { INHALATION } & 1.87 \mathrm{E}-06 \\ \text { AIR IMMERSION } & 1.23 \mathrm{E}-05 \\ \text { GROUND SURFACE } & 0.00 \mathrm{E}+00 \\ \text { INTERNAL } & 1.87 \mathrm{E}-06 \\ \text { EXTERNAL } & 1.23 \mathrm{E}-05 \\ \text { TOTAL } & 1.42 \mathrm{E}-05\end{array}$

Feb 20, $2008 \quad 02: 30$ pm

SUMMARY

Page 2

NUCLIDE EFFECTIVE DOSE EQUIVALENT SUMMARY

Nuclide

C1-38

TOTAL

Feb 20, $2008 \quad 02: 30 \mathrm{pm}$

$$
\begin{gathered}
\begin{array}{c}
\text { Selected } \\
\text { Individua } \\
\text { (mrem/y) }
\end{array} \\
\hline 1.42 \mathrm{E}-05 \\
1.42 \mathrm{E}-05
\end{gathered}
$$$$
1.42 \mathrm{E}-05
$$$$
1.42 \mathrm{E}-05
$$
$1.42 \mathrm{E}-05$
$1.42 \mathrm{E}-05$

CANCER RISK SUMMARY

Cancer

Esophagu
Selected Individual

Total Lifetime Fatal Cancer Risk

$$
\text { Page } 2^{1.29 E-13}
$$




$\begin{array}{lr} & \text { C1-38B. SUM } \\ \text { Stomach } & 7.68 \mathrm{E}-13 \\ \text { Colon } & 1.14 \mathrm{E}-12 \\ \text { Liver } & 1.79 \mathrm{E}-13 \\ \text { LUNG } & 1.82 \mathrm{E}-12 \\ \text { Bone } & 1.63 \mathrm{E}-14 \\ \text { Skin } & 3.17 \mathrm{E}-14 \\ \text { Breast } & 6.81 \mathrm{E}-13 \\ \text { Ovary } & 1.56 \mathrm{E}-13 \\ \text { B Tadder } & 2.70 \mathrm{E}-13 \\ \text { Kidneys } & 6.07 \mathrm{E}-14 \\ \text { Thyroid } & 4.13 \mathrm{E}-14 \\ \text { Leukemia } & 7.16 \mathrm{E}-13 \\ \text { Residua1 } & 1.70 \mathrm{E}-12 \\ \text { Tota } 1 & 7.71 \mathrm{E}-12 \\ & \\ \text { TOTAL } & 1.54 \mathrm{E}-11\end{array}$

PATHWAY RISK SUMMARY

\begin{tabular}{lc} 
Pathway & $\begin{array}{c}\text { Selected Individua1 } \\
\text { Total Lifetime } \\
\text { Fatal Cancer Risk }\end{array}$ \\
\cline { 2 - 2 } INGESTION & $0.00 \mathrm{E}+00$ \\
INHALATION & $9.51 \mathrm{E}-13$ \\
AIR IMMERSION & $6.76 \mathrm{E}-12$ \\
GROUND SURFACE & $0.00 \mathrm{E}+00$ \\
INTERNAL & $9.51 \mathrm{E}-13$ \\
EXTERNAL & $6.76 \mathrm{E}-12$ \\
TOTAL & $7.71 \mathrm{E}-12$
\end{tabular}

Feb 20, $2008 \quad 02: 30$ pm

SUMMARY Page 4

NUCLIDE RISK SUMMARY

Nuclide

C1-38

TOTAL

口

Feb 20, 2008 02:30 pm
Selected Individual

Total Lifetime Fatal Cancer Risk

$7.71 \mathrm{E}-12$

7. $71 \mathrm{E}-12$

INDIVIDUAL EFFECTIVE DOSE EQUIVALENT RATE (mrem/y) (A11 Radionuclides and Pathways)

Distance $(m)$ 


\begin{tabular}{|c|c|c|c|c|c|c|c|}
\hline \multirow{2}{*}{ Direction } & \multirow{2}{*}{17035} & \multicolumn{6}{|c|}{ C1-38B. SUM } \\
\hline & & 17329 & 18607 & 10 & 18860 & 18890 & 19860 \\
\hline $\mathrm{N}$ & $2.8 \mathrm{E}-06$ & $2.7 \mathrm{E}-06$ & $2.4 \mathrm{E}-06$ & $2.4 \mathrm{E}-06$ & $2.4 \mathrm{E}-06$ & $2.4 \mathrm{E}-06$ & $2.2 \mathrm{E}-06$ \\
\hline NNW & 1. $3 \mathrm{E}-06$ & 1. $3 \mathrm{E}-06$ & $1.2 \mathrm{E}-06$ & $1.1 \mathrm{E}-06$ & $1.1 \mathrm{E}-06$ & $1.1 \mathrm{E}-06$ & 1. $1 \mathrm{E}-06$ \\
\hline NW & $1.6 \mathrm{E}-06$ & $1.6 \mathrm{E}-06$ & $1.4 \mathrm{E}-06$ & $1.4 \mathrm{E}-06$ & $1.4 \mathrm{E}-06$ & $1.4 \mathrm{E}-06$ & 1. $3 \mathrm{E}-06$ \\
\hline WNW & $2.2 \mathrm{E}-06$ & $2.1 \mathrm{E}-06$ & $1.9 \mathrm{E}-06$ & $1.9 \mathrm{E}-06$ & $1.9 \mathrm{E}-06$ & $1.8 \mathrm{E}-06$ & $1.7 \mathrm{E}-06$ \\
\hline W & 2. $2 \mathrm{E}-06$ & $2.2 \mathrm{E}-06$ & $1.9 \mathrm{E}-06$ & $1.9 \mathrm{E}-06$ & $1.9 \mathrm{E}-06$ & $1.9 \mathrm{E}-06$ & $1.7 \mathrm{E}-06$ \\
\hline WSW & $3.6 \mathrm{E}-06$ & $3.5 \mathrm{E}-06$ & $3.1 \mathrm{E}-06$ & $3.1 \mathrm{E}-06$ & $3.1 \mathrm{E}-06$ & $3.1 \mathrm{E}-06$ & $2.8 \mathrm{E}-06$ \\
\hline SW & $6.4 \mathrm{E}-06$ & $6.2 \mathrm{E}-06$ & $5.6 \mathrm{E}-06$ & $5.5 \mathrm{E}-06$ & $5.5 \mathrm{E}-06$ & $5.5 \mathrm{E}-06$ & $5.1 \mathrm{E}-06$ \\
\hline SSW & 1.0E-05 & $1.0 \mathrm{E}-05$ & $8.9 \mathrm{E}-06$ & $8.8 \mathrm{E}-06$ & $8.8 \mathrm{E}-06$ & $8.7 \mathrm{E}-06$ & $8.1 \mathrm{E}-06$ \\
\hline $\mathrm{S}$ & 1. $4 \mathrm{E}-05$ & 1. $4 \mathrm{E}-05$ & $1.2 \mathrm{E}-05$ & $1.2 \mathrm{E}-05$ & $1.2 \mathrm{E}-05$ & $1.2 \mathrm{E}-05$ & 1.1E-05 \\
\hline SSE & 1. $3 \mathrm{E}-05$ & 1. $3 \mathrm{E}-05$ & $1.1 \mathrm{E}-05$ & $1.1 \mathrm{E}-05$ & $1.1 \mathrm{E}-05$ & $1.1 \mathrm{E}-05$ & 1. $0 \mathrm{E}-05$ \\
\hline SE & $5.1 \mathrm{E}-06$ & $4.9 \mathrm{E}-06$ & $4.4 \mathrm{E}-06$ & $4.3 \mathrm{E}-06$ & $4.3 \mathrm{E}-06$ & $4.3 \mathrm{E}-06$ & $3.9 \mathrm{E}-06$ \\
\hline ESE & $3.1 \mathrm{E}-06$ & $3.0 \mathrm{E}-06$ & $2.7 \mathrm{E}-06$ & $2.6 \mathrm{E}-06$ & $2.6 \mathrm{E}-06$ & $2.6 \mathrm{E}-06$ & $2.4 \mathrm{E}-06$ \\
\hline $\mathrm{E}$ & $3.9 \mathrm{E}-06$ & $3.8 \mathrm{E}-06$ & $3.4 \mathrm{E}-06$ & $3.3 \mathrm{E}-06$ & $3.3 \mathrm{E}-06$ & $3.3 \mathrm{E}-06$ & $3.0 \mathrm{E}-06$ \\
\hline ENE & $4.3 E-06$ & $4.2 \mathrm{E}-06$ & $3.8 \mathrm{E}-06$ & $3.7 \mathrm{E}-06$ & $3.7 \mathrm{E}-06$ & $3.7 E-06$ & $3.4 \mathrm{E}-06$ \\
\hline NE & $7.1 \mathrm{E}-06$ & $6.9 \mathrm{E}-06$ & $6.2 \mathrm{E}-06$ & $6.1 \mathrm{E}-06$ & $6.1 \mathrm{E}-06$ & $6.1 \mathrm{E}-06$ & $5.6 \mathrm{E}-06$ \\
\hline NNE & $4.1 \mathrm{E}-06$ & $4.0 \mathrm{E}-06$ & $3.5 \mathrm{E}-06$ & $3.5 \mathrm{E}-06$ & $3.5 \mathrm{E}-06$ & $3.5 E-06$ & $3.2 \mathrm{E}-06$ \\
\hline
\end{tabular}

Distance (m)

\begin{tabular}{|c|c|c|c|c|c|c|c|}
\hline Direction & n 19891 & 20457 & 21314 & 21349 & 22159 & 23521 & 24430 \\
\hline $\mathrm{N}$ & $2.2 \mathrm{E}-06$ & $2.1 \mathrm{E}-06$ & $1.9 \mathrm{E}-06$ & $1.9 \mathrm{E}-06$ & $1.8 \mathrm{E}-06$ & 1. $5 \mathrm{E}-06$ & 1. $4 \mathrm{E}-06$ \\
\hline NNW & $1.0 \mathrm{E}-06$ & $1.0 \mathrm{E}-06$ & $9.4 \mathrm{E}-07$ & $9.3 \mathrm{E}-07$ & $8.8 \mathrm{E}-07$ & $7.8 \mathrm{E}-07$ & $7.2 \mathrm{E}-07$ \\
\hline NW & $1.3 \mathrm{E}-06$ & $1.2 \mathrm{E}-06$ & $1.1 \mathrm{E}-06$ & $1.1 \mathrm{E}-06$ & $1.1 \mathrm{E}-06$ & $9.4 \mathrm{E}-07$ & $8.5 \mathrm{E}-07$ \\
\hline WNW & $1.7 \mathrm{E}-06$ & $1.6 \mathrm{E}-06$ & $1.5 \mathrm{E}-06$ & $1.5 \mathrm{E}-06$ & $1.4 \mathrm{E}-06$ & 1. $2 \mathrm{E}-06$ & 1.1E-06 \\
\hline W & $1.7 \mathrm{E}-06$ & $1.6 \mathrm{E}-06$ & $1.5 \mathrm{E}-06$ & $1.5 \mathrm{E}-06$ & $1.4 \mathrm{E}-06$ & $1.2 \mathrm{E}-06$ & $1.1 \mathrm{E}-06$ \\
\hline WSW & $2.8 \mathrm{E}-06$ & $2.7 \mathrm{E}-06$ & $2.5 \mathrm{E}-06$ & $2.5 \mathrm{E}-06$ & $2.3 \mathrm{E}-06$ & $2.1 \mathrm{E}-06$ & $1.9 \mathrm{E}-06$ \\
\hline SW & $5.0 \mathrm{E}-06$ & $4.8 \mathrm{E}-06$ & $4.5 \mathrm{E}-06$ & $4.5 \mathrm{E}-06$ & $4.2 \mathrm{E}-06$ & $3.8 \mathrm{E}-06$ & $3.5 \mathrm{E}-06$ \\
\hline SSW & $8.0 \mathrm{E}-06$ & $7.7 \mathrm{E}-06$ & $7.2 \mathrm{E}-06$ & $7.1 \mathrm{E}-06$ & $6.7 \mathrm{E}-06$ & $5.9 \mathrm{E}-06$ & $5.4 \mathrm{E}-06$ \\
\hline $\mathrm{S}$ & $1.1 \mathrm{E}-05$ & 1.1E-05 & $9.9 \mathrm{E}-06$ & $9.8 \mathrm{E}-06$ & $9.2 \mathrm{E}-06$ & $8.2 \mathrm{E}-06$ & $7.5 \mathrm{E}-06$ \\
\hline SSE & $1.0 \mathrm{E}-05$ & $9.8 \mathrm{E}-06$ & $9.2 \mathrm{E}-06$ & $9.2 \mathrm{E}-06$ & $8.6 \mathrm{E}-06$ & $7.7 \mathrm{E}-06$ & $7.1 \mathrm{E}-06$ \\
\hline SE & $3.9 \mathrm{E}-06$ & $3.7 \mathrm{E}-06$ & $3.5 \mathrm{E}-06$ & $3.4 \mathrm{E}-06$ & $3.2 \mathrm{E}-06$ & $2.8 \mathrm{E}-06$ & $2.5 \mathrm{E}-06$ \\
\hline ESE & $2.4 \mathrm{E}-06$ & $2.3 \mathrm{E}-06$ & $2.1 \mathrm{E}-06$ & $2.1 \mathrm{E}-06$ & $1.9 \mathrm{E}-06$ & $1.7 \mathrm{E}-06$ & $1.5 \mathrm{E}-06$ \\
\hline & $3.0 \mathrm{E}-06$ & $2.8 \mathrm{E}-06$ & $2.6 \mathrm{E}-06$ & $2.6 \mathrm{E}-06$ & $2.4 \mathrm{E}-06$ & $2.1 \mathrm{E}-06$ & $1.8 \mathrm{E}-06$ \\
\hline ENE & $3.4 \mathrm{E}-06$ & $3.2 \mathrm{E}-06$ & $3.0 \mathrm{E}-06$ & $3.0 \mathrm{E}-06$ & $2.8 \mathrm{E}-06$ & $2.5 \mathrm{E}-06$ & $2.2 \mathrm{E}-06$ \\
\hline $\mathrm{NE}$ & $5.6 \mathrm{E}-06$ & $5.3 \mathrm{E}-06$ & $5.0 \mathrm{E}-06$ & $4.9 \mathrm{E}-06$ & $4.6 \mathrm{E}-06$ & $4.1 \mathrm{E}-06$ & $3.8 \mathrm{E}-06$ \\
\hline NNE & $3.2 \mathrm{E}-06$ & $3.0 \mathrm{E}-06$ & $2.8 \mathrm{E}-06$ & $2.8 \mathrm{E}-06$ & $2.6 \mathrm{E}-06$ & $2.3 \mathrm{E}-06$ & $2.1 \mathrm{E}-06$ \\
\hline
\end{tabular}

$\square$

Feb 20, 2008 02:30 pm

SUMMARY

Page 6

INDIVIDUAL EFFECTIVE DOSE EQUIVALENT RATE (mrem/y) (A11 Radionuclides and Pathways)

\begin{tabular}{llllll}
\hline & \multicolumn{4}{c}{ Distance $(\mathrm{m})$} \\
\cline { 2 - 5 } Direction & 24545 & 25171 & 26794 & 27275 & 27389 \\
\hline
\end{tabular}

\begin{tabular}{|c|c|c|c|c|}
\hline $\begin{array}{r}N \\
N N W\end{array}$ & $\begin{array}{l}1.4 \mathrm{E}-06 \\
7.1 \mathrm{E}-07\end{array}$ & $\begin{array}{l}1.3 \mathrm{E}-06 \\
6.7 \mathrm{E}-07\end{array}$ & $\begin{array}{l}1.2 \mathrm{E}-06 \\
6.1 \mathrm{E}-07\end{array}$ & $\begin{array}{cc}1.1 \mathrm{E}-06 & 1.1 \mathrm{E}-06 \\
6.0 \mathrm{E}-07 & 5.9 \mathrm{E}-07 \\
\text { Page } 4 & \end{array}$ \\
\hline
\end{tabular}




\begin{tabular}{rccccc} 
& & & \multicolumn{2}{c}{ C1-38B. SUM } \\
NW & $8.4 \mathrm{E}-07$ & $7.8 \mathrm{E}-07$ & $7.2 \mathrm{E}-07$ & $7.0 \mathrm{E}-07$ & $6.9 \mathrm{E}-07$ \\
WNW & $1.1 \mathrm{E}-06$ & $1.0 \mathrm{E}-06$ & $9.4 \mathrm{E}-07$ & $9.2 \mathrm{E}-07$ & $9.2 \mathrm{E}-07$ \\
$\mathrm{~W}$ & $1.1 \mathrm{E}-06$ & $1.0 \mathrm{E}-06$ & $9.4 \mathrm{E}-07$ & $9.1 \mathrm{E}-07$ & $9.1 \mathrm{E}-07$ \\
WSW & $1.9 \mathrm{E}-06$ & $1.8 \mathrm{E}-06$ & $1.6 \mathrm{E}-06$ & $1.6 \mathrm{E}-06$ & $1.6 \mathrm{E}-06$ \\
SW & $3.4 \mathrm{E}-06$ & $3.2 \mathrm{E}-06$ & $2.9 \mathrm{E}-06$ & $2.9 \mathrm{E}-06$ & $2.9 \mathrm{E}-06$ \\
SSW & $5.4 \mathrm{E}-06$ & $5.1 \mathrm{E}-06$ & $4.6 \mathrm{E}-06$ & $4.5 \mathrm{E}-06$ & $4.5 \mathrm{E}-06$ \\
$\mathrm{~S}$ & $7.4 \mathrm{E}-06$ & $6.9 \mathrm{E}-06$ & $6.3 \mathrm{E}-06$ & $6.2 \mathrm{E}-06$ & $6.1 \mathrm{E}-06$ \\
SSE & $7.0 \mathrm{E}-06$ & $6.6 \mathrm{E}-06$ & $6.1 \mathrm{E}-06$ & $5.9 \mathrm{E}-06$ & $5.9 \mathrm{E}-06$ \\
SE & $2.5 \mathrm{E}-06$ & $2.3 \mathrm{E}-06$ & $2.1 \mathrm{E}-06$ & $2.0 \mathrm{E}-06$ & $2.0 \mathrm{E}-06$ \\
ESE & $1.5 \mathrm{E}-06$ & $1.4 \mathrm{E}-06$ & $1.2 \mathrm{E}-06$ & $1.2 \mathrm{E}-06$ & $1.2 \mathrm{E}-06$ \\
$\mathrm{E}$ & $1.8 \mathrm{E}-06$ & $1.6 \mathrm{E}-06$ & $1.5 \mathrm{E}-06$ & $1.5 \mathrm{E}-06$ & $1.4 \mathrm{E}-06$ \\
ENE & $2.2 \mathrm{E}-06$ & $2.1 \mathrm{E}-06$ & $1.9 \mathrm{E}-06$ & $1.8 \mathrm{E}-06$ & $1.8 \mathrm{E}-06$ \\
NE & $3.7 \mathrm{E}-06$ & $3.5 \mathrm{E}-06$ & $3.2 \mathrm{E}-06$ & $3.1 \mathrm{E}-06$ & $3.1 \mathrm{E}-06$ \\
NNE & $2.1 \mathrm{E}-06$ & $1.9 \mathrm{E}-06$ & $1.8 \mathrm{E}-06$ & $1.7 \mathrm{E}-06$ & $1.7 \mathrm{E}-06$ \\
& & & & & \\
\hline
\end{tabular}

Feb 20, $2008 \quad 02: 30 \mathrm{pm}$

SUMMARY

Page 7

INDIVIDUAL LIFETIME RISK (deaths)

(A11 Radionuclides and Pathways)

\begin{tabular}{|c|c|c|c|c|c|c|c|}
\hline \multirow[b]{2}{*}{ Direction } & \multicolumn{6}{|c|}{ Distance $(\mathrm{m})$} & \multirow[b]{2}{*}{19860} \\
\hline & 17035 & 17329 & 18607 & 18834 & 18860 & 18890 & \\
\hline $\begin{array}{r}\text { N } \\
N N W \\
N W \\
W N W \\
W \\
W S W \\
\text { SW } \\
\text { SSW } \\
S \\
\text { SSE } \\
\text { SE } \\
\text { ESE } \\
\text { E } \\
\text { ENE } \\
N E \\
\text { NNE }\end{array}$ & $\begin{array}{l}1.5 \mathrm{E}-12 \\
7.2 \mathrm{E}-13 \\
8.9 \mathrm{E}-13 \\
1.2 \mathrm{E}-12 \\
1.2 \mathrm{E}-12 \\
1.9 \mathrm{E}-12 \\
3.5 \mathrm{E}-12 \\
5.6 \mathrm{E}-12 \\
7.7 \mathrm{E}-12 \\
7.1 \mathrm{E}-12 \\
2.8 \mathrm{E}-12 \\
1.7 \mathrm{E}-12 \\
2.1 \mathrm{E}-12 \\
2.4 \mathrm{E}-12 \\
3.9 \mathrm{E}-12 \\
2.2 \mathrm{E}-12\end{array}$ & $\begin{array}{l}1.5 \mathrm{E}-12 \\
7.0 \mathrm{E}-13 \\
8.6 \mathrm{E}-13 \\
1.1 \mathrm{E}-12 \\
1.2 \mathrm{E}-12 \\
1.9 \mathrm{E}-12 \\
3.4 \mathrm{E}-12 \\
5.4 \mathrm{E}-12 \\
7.5 \mathrm{E}-12 \\
6.9 \mathrm{E}-12 \\
2.7 \mathrm{E}-12 \\
1.7 \mathrm{E}-12 \\
2.1 \mathrm{E}-12 \\
2.3 \mathrm{E}-12 \\
3.8 \mathrm{E}-12 \\
2.1 \mathrm{E}-12\end{array}$ & $\begin{array}{l}1.3 \mathrm{E}-12 \\
6.3 \mathrm{E}-13 \\
7.8 \mathrm{E}-13 \\
1.0 \mathrm{E}-12 \\
1.0 \mathrm{E}-12 \\
1.7 \mathrm{E}-12 \\
3.0 \mathrm{E}-12 \\
4.8 \mathrm{E}-12 \\
6.7 \mathrm{E}-12 \\
6.2 \mathrm{E}-12 \\
2.4 \mathrm{E}-12 \\
1.5 \mathrm{E}-12 \\
1.8 \mathrm{E}-12 \\
2.0 \mathrm{E}-12 \\
3.4 \mathrm{E}-12 \\
1.9 \mathrm{E}-12\end{array}$ & $\begin{array}{l}1.3 \mathrm{E}-12 \\
6.2 \mathrm{E}-13 \\
7.6 \mathrm{E}-13 \\
1.0 \mathrm{E}-12 \\
1.0 \mathrm{E}-12 \\
1.7 \mathrm{E}-12 \\
3.0 \mathrm{E}-12 \\
4.8 \mathrm{E}-12 \\
6.6 \mathrm{E}-12 \\
6.1 \mathrm{E}-12 \\
2.3 \mathrm{E}-12 \\
1.4 \mathrm{E}-12 \\
1.8 \mathrm{E}-12 \\
2.0 \mathrm{E}-12 \\
3.3 \mathrm{E}-12 \\
1.9 \mathrm{E}-12\end{array}$ & $\begin{array}{l}1.3 \mathrm{E}-12 \\
6.2 \mathrm{E}-13 \\
7.6 \mathrm{E}-13 \\
1.0 \mathrm{E}-12 \\
1.0 \mathrm{E}-12 \\
1.7 \mathrm{E}-12 \\
3.0 \mathrm{E}-12 \\
4.7 \mathrm{E}-12 \\
6.6 \mathrm{E}-12 \\
6.1 \mathrm{E}-12 \\
2.3 \mathrm{E}-12 \\
1.4 \mathrm{E}-12 \\
1.8 \mathrm{E}-12 \\
2.0 \mathrm{E}-12 \\
3.3 \mathrm{E}-12 \\
1.9 \mathrm{E}-12\end{array}$ & $\begin{array}{l}1.3 \mathrm{E}-12 \\
6.1 \mathrm{E}-13 \\
7.6 \mathrm{E}-13 \\
1.0 \mathrm{E}-12 \\
1.0 \mathrm{E}-12 \\
1.7 \mathrm{E}-12 \\
3.0 \mathrm{E}-12 \\
4.7 \mathrm{E}-12 \\
6.5 \mathrm{E}-12 \\
6.0 \mathrm{E}-12 \\
2.3 \mathrm{E}-12 \\
1.4 \mathrm{E}-12 \\
1.8 \mathrm{E}-12 \\
2.0 \mathrm{E}-12 \\
3.3 \mathrm{E}-12 \\
1.9 \mathrm{E}-12\end{array}$ & $\begin{array}{l}1.2 \mathrm{E}-12 \\
5.7 \mathrm{E}-13 \\
7.0 \mathrm{E}-13 \\
9.2 \mathrm{E}-13 \\
9.4 \mathrm{E}-13 \\
1.5 \mathrm{E}-12 \\
2.7 \mathrm{E}-12 \\
4.4 \mathrm{E}-12 \\
6.0 \mathrm{E}-12 \\
5.6 \mathrm{E}-12 \\
2.1 \mathrm{E}-12 \\
1.3 \mathrm{E}-12 \\
1.6 \mathrm{E}-12 \\
1.8 \mathrm{E}-12 \\
3.0 \mathrm{E}-12 \\
1.7 \mathrm{E}-12\end{array}$ \\
\hline \multicolumn{8}{|c|}{ Distance (m) } \\
\hline Direction & 19891 & 20457 & 21314 & 21349 & 22159 & 23521 & 24430 \\
\hline $\begin{array}{r}\text { N } \\
\text { NNW } \\
\text { NW } \\
\text { WNW } \\
\text { W } \\
\text { WSW } \\
\text { SW } \\
\text { SSW }\end{array}$ & $\begin{array}{l}1.2 \mathrm{E}-12 \\
5.7 \mathrm{E}-13 \\
7.0 \mathrm{E}-13 \\
9.2 \mathrm{E}-13 \\
9.4 \mathrm{E}-13 \\
1.5 \mathrm{E}-12 \\
2.7 \mathrm{E}-12 \\
4.4 \mathrm{E}-12\end{array}$ & $\begin{array}{l}1.1 \mathrm{E}-12 \\
5.4 \mathrm{E}-13 \\
6.7 \mathrm{E}-13 \\
8.8 \mathrm{E}-13 \\
8.9 \mathrm{E}-13 \\
1.5 \mathrm{E}-12 \\
2.6 \mathrm{E}-12 \\
4.2 \mathrm{E}-12\end{array}$ & $\begin{array}{l}1.0 \mathrm{E}-12 \\
5.1 \mathrm{E}-13 \\
6.2 \mathrm{E}-13 \\
8.2 \mathrm{E}-13 \\
8.3 \mathrm{E}-13 \\
1.4 \mathrm{E}-12 \\
2.4 \mathrm{E}-12 \\
3.9 \mathrm{E}-12\end{array}$ & $\begin{array}{r}1.0 \mathrm{E}-12 \\
5.1 \mathrm{E}-13 \\
6.2 \mathrm{E}-13 \\
8.2 \mathrm{E}-13 \\
8.2 \mathrm{E}-13 \\
1.4 \mathrm{E}-12 \\
2.4 \mathrm{E}-12 \\
3.9 \mathrm{E}-12 \\
\text { Page }\end{array}$ & $\begin{array}{r}9.6 \mathrm{E}-13 \\
4.8 \mathrm{E}-13 \\
5.8 \mathrm{E}-13 \\
7.6 \mathrm{E}-13 \\
7.7 \mathrm{E}-13 \\
1.3 \mathrm{E}-12 \\
2.3 \mathrm{E}-12 \\
3.6 \mathrm{E}-12\end{array}$ & $\begin{array}{l}8.4 \mathrm{E}-13 \\
4.2 \mathrm{E}-13 \\
5.1 \mathrm{E}-13 \\
6.7 \mathrm{E}-13 \\
6.7 \mathrm{E}-13 \\
1.1 \mathrm{E}-12 \\
2.0 \mathrm{E}-12 \\
3.2 \mathrm{E}-12\end{array}$ & $\begin{array}{l}7.5 \mathrm{E}-13 \\
3.9 \mathrm{E}-13 \\
4.6 \mathrm{E}-13 \\
6.1 \mathrm{E}-13 \\
6.1 \mathrm{E}-13 \\
1.0 \mathrm{E}-12 \\
1.9 \mathrm{E}-12 \\
3.0 \mathrm{E}-12\end{array}$ \\
\hline
\end{tabular}




\begin{tabular}{rlllllll} 
& \multicolumn{7}{c}{$\mathrm{C} 1-38 \mathrm{~B} . \mathrm{SUM}$} \\
$\mathrm{S}$ & $6.0 \mathrm{E}-12$ & $5.7 \mathrm{E}-12$ & $5.3 \mathrm{E}-12$ & $5.3 \mathrm{E}-12$ & $5.0 \mathrm{E}-12$ & $4.4 \mathrm{E}-12$ & $4.1 \mathrm{E}-12$ \\
SSE & $5.6 \mathrm{E}-12$ & $5.3 \mathrm{E}-12$ & $5.0 \mathrm{E}-12$ & $5.0 \mathrm{E}-12$ & $4.7 \mathrm{E}-12$ & $4.2 \mathrm{E}-12$ & $3.9 \mathrm{E}-12$ \\
$\mathrm{SE}$ & $2.1 \mathrm{E}-12$ & $2.0 \mathrm{E}-12$ & $1.9 \mathrm{E}-12$ & $1.9 \mathrm{E}-12$ & $1.7 \mathrm{E}-12$ & $1.5 \mathrm{E}-12$ & $1.4 \mathrm{E}-12$ \\
$\mathrm{ESE}$ & $1.3 \mathrm{E}-12$ & $1.2 \mathrm{E}-12$ & $1.1 \mathrm{E}-12$ & $1.1 \mathrm{E}-12$ & $1.1 \mathrm{E}-12$ & $9.1 \mathrm{E}-13$ & $8.1 \mathrm{E}-13$ \\
$\mathrm{E}$ & $1.6 \mathrm{E}-12$ & $1.5 \mathrm{E}-12$ & $1.4 \mathrm{E}-12$ & $1.4 \mathrm{E}-12$ & $1.3 \mathrm{E}-12$ & $1.1 \mathrm{E}-12$ & $1.0 \mathrm{E}-12$ \\
$\mathrm{ENE}$ & $1.8 \mathrm{E}-12$ & $1.8 \mathrm{E}-12$ & $1.6 \mathrm{E}-12$ & $1.6 \mathrm{E}-12$ & $1.5 \mathrm{E}-12$ & $1.3 \mathrm{E}-12$ & $1.2 \mathrm{E}-12$ \\
$\mathrm{NE}$ & $3.0 \mathrm{E}-12$ & $2.9 \mathrm{E}-12$ & $2.7 \mathrm{E}-12$ & $2.7 \mathrm{E}-12$ & $2.5 \mathrm{E}-12$ & $2.2 \mathrm{E}-12$ & $2.0 \mathrm{E}-12$ \\
NNE & $1.7 \mathrm{E}-12$ & $1.6 \mathrm{E}-12$ & $1.5 \mathrm{E}-12$ & $1.5 \mathrm{E}-12$ & $1.4 \mathrm{E}-12$ & $1.3 \mathrm{E}-12$ & $1.1 \mathrm{E}-12$
\end{tabular}

Feb 20, $2008 \quad 02: 30 \mathrm{pm}$

SUMMARY

Page 8

INDIVIDUAL LIFETIME RISK (deaths)

(A11 Radionuclides and Pathways)

\begin{tabular}{|c|c|c|c|c|c|}
\hline \multirow[b]{2}{*}{ Direction } & \multicolumn{5}{|c|}{ Distance $(\mathrm{m})$} \\
\hline & 24545 & 25171 & 26794 & 27275 & 27389 \\
\hline $\begin{array}{r}N \\
N N W \\
N W \\
W N W \\
W \\
W S W \\
S W \\
S S W \\
S \\
S S E \\
S E \\
\text { ESE } \\
E \\
\text { ENE } \\
N E \\
N N E\end{array}$ & $\begin{array}{l}7.4 \mathrm{E}-13 \\
3.8 \mathrm{E}-13 \\
4.6 \mathrm{E}-13 \\
6.0 \mathrm{E}-13 \\
6.0 \mathrm{E}-13 \\
1.0 \mathrm{E}-12 \\
1.9 \mathrm{E}-12 \\
2.9 \mathrm{E}-12 \\
4.0 \mathrm{E}-12 \\
3.8 \mathrm{E}-12 \\
1.3 \mathrm{E}-12 \\
8.0 \mathrm{E}-13 \\
9.8 \mathrm{E}-13 \\
1.2 \mathrm{E}-12 \\
2.0 \mathrm{E}-12 \\
1.1 \mathrm{E}-12\end{array}$ & $\begin{array}{l}6 \cdot 9 \mathrm{E}-13 \\
3 \cdot 6 \mathrm{E}-13 \\
4 \cdot 2 \mathrm{E}-13 \\
5.6 \mathrm{E}-13 \\
5 \cdot 6 \mathrm{E}-13 \\
9 \cdot 6 \mathrm{E}-13 \\
1.7 \mathrm{E}-12 \\
2.7 \mathrm{E}-12 \\
3.8 \mathrm{E}-12 \\
3 \cdot 6 \mathrm{E}-12 \\
1 \cdot 2 \mathrm{E}-12 \\
7 \cdot 3 \mathrm{E}-13 \\
8.9 \mathrm{E}-13 \\
1.1 \mathrm{E}-12 \\
1 \cdot 9 \mathrm{E}-12 \\
1 \cdot 1 \mathrm{E}-12\end{array}$ & $\begin{array}{l}6 \cdot 3 \mathrm{E}-13 \\
3 \cdot 3 \mathrm{E}-13 \\
3 \cdot 9 \mathrm{E}-13 \\
5 \cdot 1 \mathrm{E}-13 \\
5 \cdot 1 \mathrm{E}-13 \\
8 \cdot 7 \mathrm{E}-13 \\
1 \cdot 6 \mathrm{E}-12 \\
2 \cdot 5 \mathrm{E}-12 \\
3.4 \mathrm{E}-12 \\
3 \cdot 3 \mathrm{E}-12 \\
1 \cdot 1 \mathrm{E}-12 \\
6 \cdot 7 \mathrm{E}-13 \\
8 \cdot 1 \mathrm{E}-13 \\
1 \cdot 0 \mathrm{E}-12 \\
1.7 \mathrm{E}-12 \\
9 \cdot 7 \mathrm{E}-13\end{array}$ & $\begin{array}{l}6.1 \mathrm{E}-13 \\
3.2 \mathrm{E}-13 \\
3.8 \mathrm{E}-13 \\
5.0 \mathrm{E}-13 \\
4.9 \mathrm{E}-13 \\
8.5 \mathrm{E}-13 \\
1.6 \mathrm{E}-12 \\
2.4 \mathrm{E}-12 \\
3.3 \mathrm{E}-12 \\
3.2 \mathrm{E}-12 \\
1.1 \mathrm{E}-12 \\
6 \cdot 5 \mathrm{E}-13 \\
7 \cdot 9 \mathrm{E}-13 \\
9.9 \mathrm{E}-13 \\
1.7 \mathrm{E}-12 \\
9 \cdot 4 \mathrm{E}-13\end{array}$ & $\begin{array}{l}6.1 \mathrm{E}-13 \\
3.2 \mathrm{E}-13 \\
3.8 \mathrm{E}-13 \\
5.0 \mathrm{E}-13 \\
4.9 \mathrm{E}-13 \\
8.5 \mathrm{E}-13 \\
1.5 \mathrm{E}-12 \\
2.4 \mathrm{E}-12 \\
3.3 \mathrm{E}-12 \\
3.2 \mathrm{E}-12 \\
1.1 \mathrm{E}-12 \\
6.4 \mathrm{E}-13 \\
7.9 \mathrm{E}-13 \\
9.9 \mathrm{E}-13 \\
1.7 \mathrm{E}-12 \\
9.4 \mathrm{E}-13\end{array}$ \\
\hline
\end{tabular}




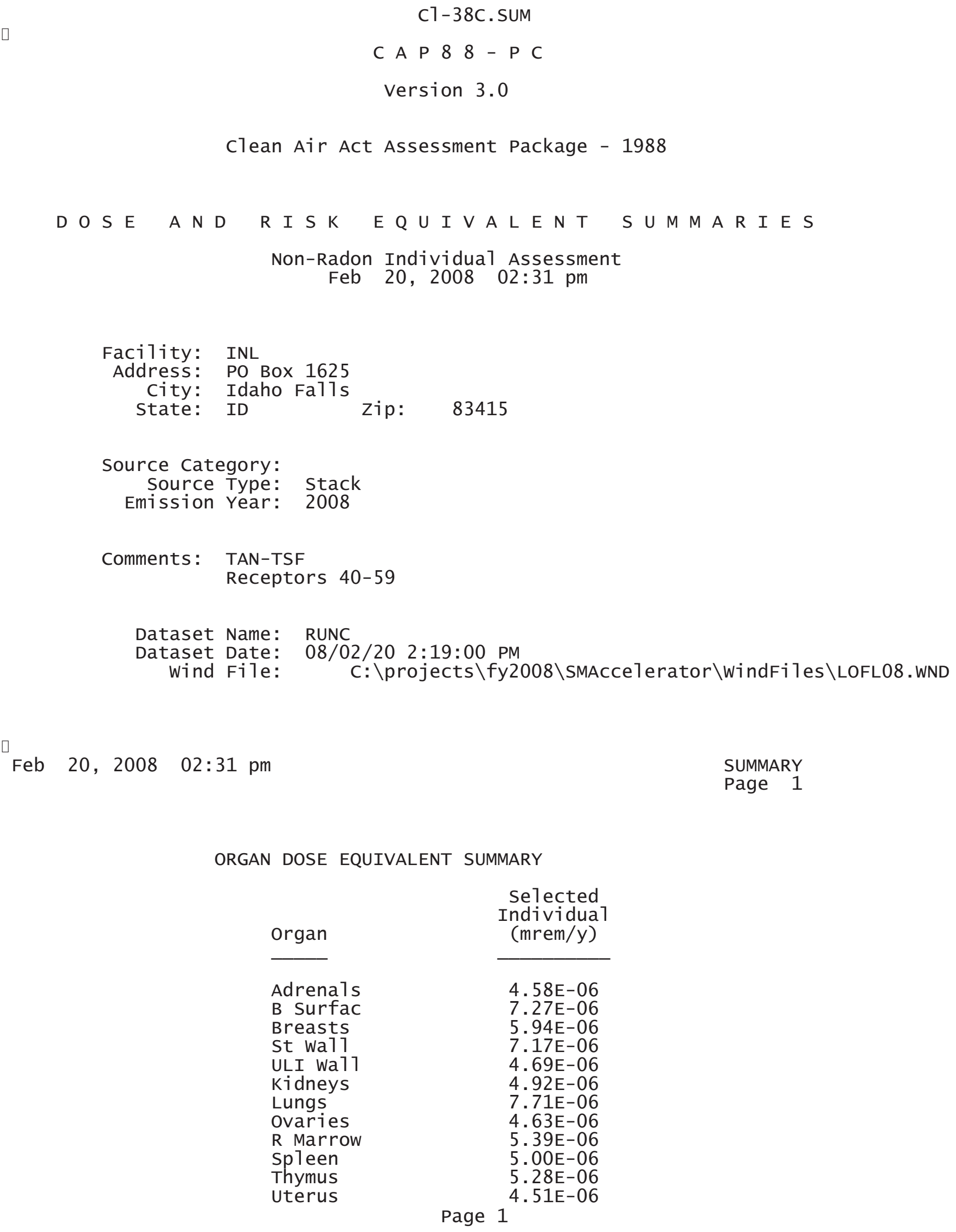




$\begin{array}{lr} & \text { C1-38C. SUM } \\ \text { B1d wa11 } & 4.73 \mathrm{E}-06 \\ \text { Brain } & 5.76 \mathrm{E}-06 \\ \text { Esophagu } & 1.29 \mathrm{E}-05 \\ \text { SI wa11 } & 4.63 \mathrm{E}-06 \\ \text { LLI wa11 } & 4.60 \mathrm{E}-06 \\ \text { Liver } & 4.98 \mathrm{E}-06 \\ \text { Muscle } & 5.25 \mathrm{E}-06 \\ \text { Pancreas } & 4.60 \mathrm{E}-06 \\ \text { Skin } & 1.34 \mathrm{E}-05 \\ \text { Testes } & 5.26 \mathrm{E}-06 \\ \text { Thyroid } & 5.47 \mathrm{E}-06 \\ & \\ \text { EFFEC } & 6.01 \mathrm{E}-06\end{array}$

PATHWAY EFFECTIVE DOSE EQUIVALENT SUMMARY

\begin{tabular}{lc} 
Pathway & $\begin{array}{c}\text { selected } \\
\text { Individual } \\
(\mathrm{mrem} / \mathrm{y})\end{array}$ \\
\cline { 2 - 2 } & $0.00 \mathrm{E}+00$ \\
INGESTION & $7.93 \mathrm{E}-07$ \\
INHALATION & $5.22 \mathrm{E}-06$ \\
AIR IMMERSION & $0.00 \mathrm{E}+00$ \\
GROUND SURFACE & $7.93 \mathrm{E}-07$ \\
INTERNAL & $5.22 \mathrm{E}-06$ \\
EXTERNAL & $6.01 \mathrm{E}-06$ \\
TOTAL &
\end{tabular}

Feb 20, $2008 \quad 02: 31 \mathrm{pm}$

SUMMARY

Page 2

NUCLIDE EFFECTIVE DOSE EQUIVALENT SUMMARY

Nuclide

C1-38

TOTAL

Feb 20, $2008 \quad 02: 31 \mathrm{pm}$

$$
\begin{gathered}
\begin{array}{c}
\text { Selected } \\
\text { Individua1 } \\
(\mathrm{mrem} / \mathrm{y})
\end{array} \\
\hline 6.01 \mathrm{E}-06 \\
6.01 \mathrm{E}-06
\end{gathered}
$$

CANCER RISK SUMMARY

Cancer

Esophagu
Selected Individual

Total Lifetime Fatal Cancer Risk

$$
\text { Page } 2^{5 \cdot 47 E-14}
$$




$\begin{array}{lr} & \mathrm{C} 1-38 \mathrm{C} . \mathrm{SUM} \\ \text { Stomach } & 3.25 \mathrm{E}-13 \\ \text { Colon } & 4.83 \mathrm{E}-13 \\ \text { Liver } & 7.58 \mathrm{E}-14 \\ \text { LUNG } & 7.70 \mathrm{E}-13 \\ \text { Bone } & 6.91 \mathrm{E}-15 \\ \text { Skin } & 1.34 \mathrm{E}-14 \\ \text { Breast } & 2.88 \mathrm{E}-13 \\ \text { Ovary } & 6.59 \mathrm{E}-14 \\ \text { Bladder } & 1.14 \mathrm{E}-13 \\ \text { Kidneys } & 2.56 \mathrm{E}-14 \\ \text { Thyroid } & 1.75 \mathrm{E}-14 \\ \text { Leukemia } & 3.03 \mathrm{E}-13 \\ \text { Residua1 } & 7.20 \mathrm{E}-13 \\ \text { Tota1 } & 3.26 \mathrm{E}-12 \\ & \\ \text { TOTAL } & 6.52 \mathrm{E}-12\end{array}$

PATHWAY RISK SUMMARY

\begin{tabular}{lc} 
Pathway & $\begin{array}{c}\text { Selected Individua1 } \\
\text { Total Lifetime } \\
\text { Fatal Cancer Risk }\end{array}$ \\
\cline { 2 - 2 } INGESTION & $0.00 \mathrm{E}+00$ \\
INHALATION & $4.02 \mathrm{E}-13$ \\
AIR IMMERSION & $2.86 \mathrm{E}-12$ \\
GROUND SURFACE & $0.00 \mathrm{E}+00$ \\
INTERNAL & $4.02 \mathrm{E}-13$ \\
EXTERNAL & $2.86 \mathrm{E}-12$ \\
TOTAL & $3.26 \mathrm{E}-12$
\end{tabular}

Feb 20, $2008 \quad 02: 31$ pm

SUMMARY Page 4

NUCLIDE RISK SUMMARY

Nuclide

C1 -38

TOTAL

$\square$

Feb 20, 2008 02:31 pm
Selected Individual

Total Lifetime Fatal Cancer Risk

$3.26 \mathrm{E}-12$

3. $26 \mathrm{E}-12$

SUMMARY

Page 5

INDIVIDUAL EFFECTIVE DOSE EQUIVALENT RATE (mrem/y) (A11 Radionuclides and Pathways)

Distance $(m)$ 


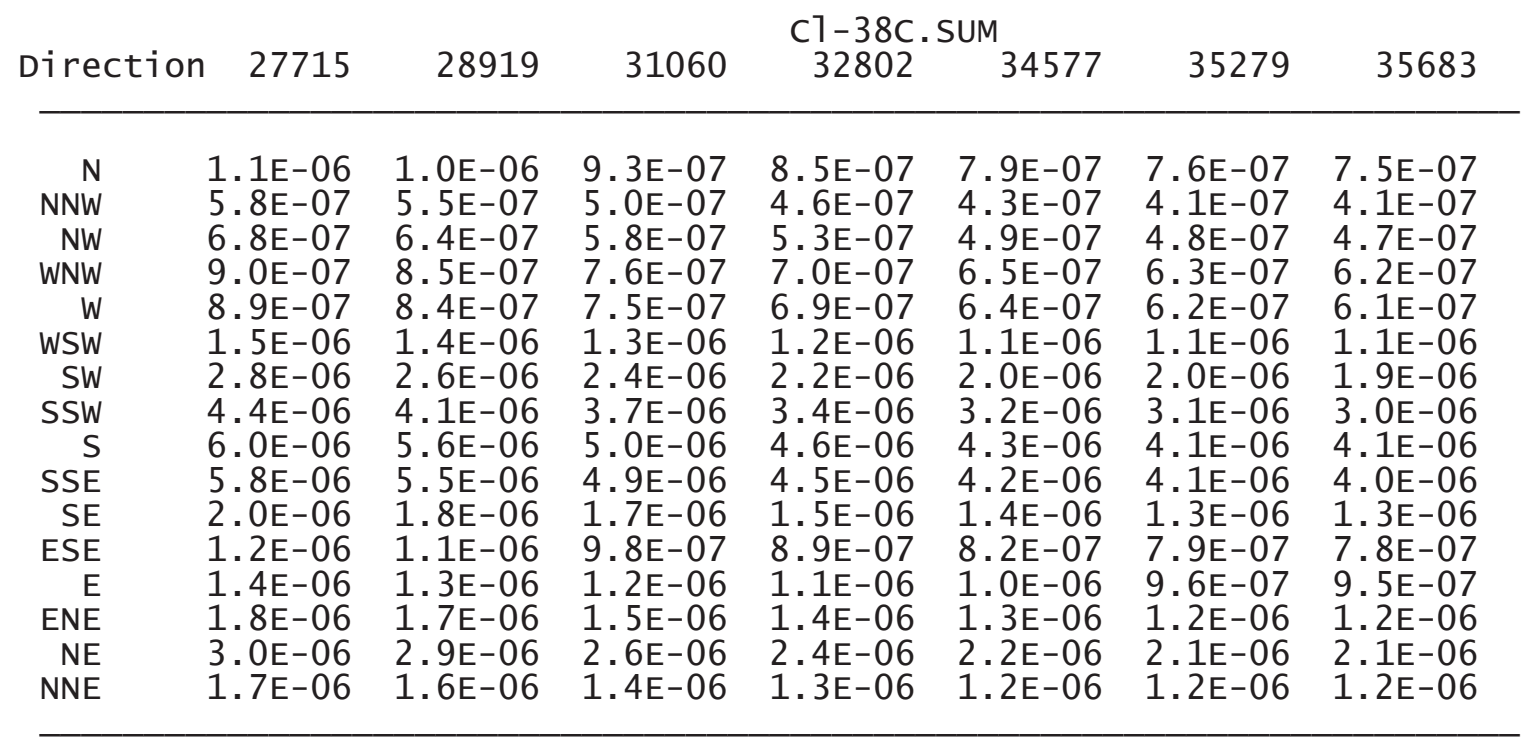

Distance (m)

\begin{tabular}{rrrrrrrr}
\cline { 3 - 7 } Direction & 36721 & 36809 & 37729 & 39079 & 39220 & 39559 & 43584 \\
\hline N & $7.1 \mathrm{E}-07$ & $7.1 \mathrm{E}-07$ & $6.8 \mathrm{E}-07$ & $6.5 \mathrm{E}-07$ & $6.4 \mathrm{E}-07$ & $6.3 \mathrm{E}-07$ & $5.3 \mathrm{E}-07$ \\
NNW & $3.9 \mathrm{E}-07$ & $3.9 \mathrm{E}-07$ & $3.7 \mathrm{E}-07$ & $3.5 \mathrm{E}-07$ & $3.5 \mathrm{E}-07$ & $3.5 \mathrm{E}-07$ & $3.0 \mathrm{E}-07$ \\
$\mathrm{NW}$ & $4.5 \mathrm{E}-07$ & $4.5 \mathrm{E}-07$ & $4.3 \mathrm{E}-07$ & $4.1 \mathrm{E}-07$ & $4.1 \mathrm{E}-07$ & $4.0 \mathrm{E}-07$ & $3.4 \mathrm{E}-07$ \\
WNW & $5.9 \mathrm{E}-07$ & $5.9 \mathrm{E}-07$ & $5.7 \mathrm{E}-07$ & $5.4 \mathrm{E}-07$ & $5.3 \mathrm{E}-07$ & $5.3 \mathrm{E}-07$ & $4.5 \mathrm{E}-07$ \\
$\mathrm{~W}$ & $5.8 \mathrm{E}-07$ & $5.8 \mathrm{E}-07$ & $5.5 \mathrm{E}-07$ & $5.2 \mathrm{E}-07$ & $5.2 \mathrm{E}-07$ & $5.1 \mathrm{E}-07$ & $4.3 \mathrm{E}-07$ \\
WSW & $1.0 \mathrm{E}-06$ & $1.0 \mathrm{E}-06$ & $9.7 \mathrm{E}-07$ & $9.2 \mathrm{E}-07$ & $9.1 \mathrm{E}-07$ & $9.0 \mathrm{E}-07$ & $7.7 \mathrm{E}-07$ \\
SW & $1.8 \mathrm{E}-06$ & $1.8 \mathrm{E}-06$ & $1.8 \mathrm{E}-06$ & $1.7 \mathrm{E}-06$ & $1.7 \mathrm{E}-06$ & $1.6 \mathrm{E}-06$ & $1.4 \mathrm{E}-06$ \\
SSW & $2.9 \mathrm{E}-06$ & $2.9 \mathrm{E}-06$ & $2.7 \mathrm{E}-06$ & $2.6 \mathrm{E}-06$ & $2.6 \mathrm{E}-06$ & $2.5 \mathrm{E}-06$ & $2.2 \mathrm{E}-06$ \\
$\mathrm{~S}$ & $3.9 \mathrm{E}-06$ & $3.9 \mathrm{E}-06$ & $3.7 \mathrm{E}-06$ & $3.5 \mathrm{E}-06$ & $3.5 \mathrm{E}-06$ & $3.4 \mathrm{E}-06$ & $2.9 \mathrm{E}-06$ \\
SSE & $3.8 \mathrm{E}-06$ & $3.8 \mathrm{E}-06$ & $3.7 \mathrm{E}-06$ & $3.5 \mathrm{E}-06$ & $3.5 \mathrm{E}-06$ & $3.4 \mathrm{E}-06$ & $2.9 \mathrm{E}-06$ \\
$\mathrm{SE}$ & $1.3 \mathrm{E}-06$ & $1.3 \mathrm{E}-06$ & $1.2 \mathrm{E}-06$ & $1.1 \mathrm{E}-06$ & $1.1 \mathrm{E}-06$ & $1.1 \mathrm{E}-06$ & $9.4 \mathrm{E}-07$ \\
ESE & $7.4 \mathrm{E}-07$ & $7.4 \mathrm{E}-07$ & $7.1 \mathrm{E}-07$ & $6.6 \mathrm{E}-07$ & $6.6 \mathrm{E}-07$ & $6.5 \mathrm{E}-07$ & $5.4 \mathrm{E}-07$ \\
$\mathrm{E}$ & $9.0 \mathrm{E}-07$ & $9.0 \mathrm{E}-07$ & $8.6 \mathrm{E}-07$ & $8.1 \mathrm{E}-07$ & $8.0 \mathrm{E}-07$ & $7.9 \mathrm{E}-07$ & $6.6 \mathrm{E}-07$ \\
ENE & $1.2 \mathrm{E}-06$ & $1.2 \mathrm{E}-06$ & $1.1 \mathrm{E}-06$ & $1.0 \mathrm{E}-06$ & $1.0 \mathrm{E}-06$ & $1.0 \mathrm{E}-06$ & $8.7 \mathrm{E}-07$ \\
NE & $2.0 \mathrm{E}-06$ & $2.0 \mathrm{E}-06$ & $1.9 \mathrm{E}-06$ & $1.8 \mathrm{E}-06$ & $1.8 \mathrm{E}-06$ & $1.8 \mathrm{E}-06$ & $1.5 \mathrm{E}-06$ \\
NNE & $1.1 \mathrm{E}-06$ & $1.1 \mathrm{E}-06$ & $1.1 \mathrm{E}-06$ & $1.0 \mathrm{E}-06$ & $1.0 \mathrm{E}-06$ & $9.9 \mathrm{E}-07$ & $8.4 \mathrm{E}-07$
\end{tabular}

口

Feb 20, 2008 02:31 pm

SUMMARY

Page 6

INDIVIDUAL EFFECTIVE DOSE EQUIVALENT RATE (mrem/y) (A11 Radionuclides and Pathways)

\begin{tabular}{|c|c|c|c|c|c|c|}
\hline \multirow[b]{2}{*}{ Direction } & \multicolumn{6}{|c|}{ Distance (m) } \\
\hline & 45196 & 45275 & 45654 & 45677 & 46668 & 47969 \\
\hline
\end{tabular}

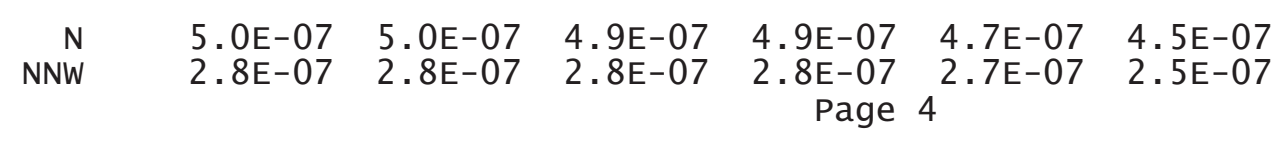




\begin{tabular}{|c|c|c|c|c|c|c|}
\hline & & & & $C 1-38 C$ & & \\
\hline NW & $3.2 \mathrm{E}-07$ & $3.2 \mathrm{E}-07$ & $3.1 \mathrm{E}-07$ & $3.1 \mathrm{E}-07$ & 3. $0 \mathrm{E}-07$ & $2.9 \mathrm{E}-07$ \\
\hline WNW & $4.2 \mathrm{E}-07$ & $4.2 \mathrm{E}-07$ & $4.1 \mathrm{E}-07$ & $4.1 \mathrm{E}-07$ & 4. $0 \mathrm{E}-07$ & $3.8 \mathrm{E}-07$ \\
\hline & 4.1E-07 & $4.0 \mathrm{E}-07$ & 4. $0 \mathrm{E}-07$ & 4. $0 \mathrm{E}-07$ & $3.8 \mathrm{E}-07$ & $3.6 \mathrm{E}-07$ \\
\hline WSW & 7. $2 \mathrm{E}-07$ & $7.2 \mathrm{E}-07$ & 7.1E-07 & $7.1 \mathrm{E}-07$ & $6.8 \mathrm{E}-07$ & $6.5 \mathrm{E}-07$ \\
\hline SW & 1. $3 \mathrm{E}-06$ & 1. 3E-06 & 1. $3 \mathrm{E}-06$ & 1. $3 \mathrm{E}-06$ & 1. $3 \mathrm{E}-06$ & -06 \\
\hline SSW & $2.0 \mathrm{E}-06$ & 2.0E-06 & $2.0 \mathrm{E}-06$ & $2.0 \mathrm{E}-06$ & $1.9 \mathrm{E}-06$ & $1.8 \mathrm{E}-06$ \\
\hline & $2.7 \mathrm{E}-06$ & $2.7 \mathrm{E}-06$ & $2.7 \mathrm{E}-06$ & $2.7 \mathrm{E}-06$ & $2.6 \mathrm{E}-06$ & 2. $4 \mathrm{E}-06$ \\
\hline SSE & $2.8 \mathrm{E}-06$ & $2.7 \mathrm{E}-06$ & $2.7 \mathrm{E}-06$ & $2.7 \mathrm{E}-06$ & $2.6 \mathrm{E}-06$ & -06 \\
\hline $\mathrm{SE}$ & $8.7 \mathrm{E}-07$ & $8.7 \mathrm{E}-07$ & $8.6 \mathrm{E}-07$ & $8.6 \mathrm{E}-07$ & $8.2 \mathrm{E}-07$ & $7.8 \mathrm{E}-07$ \\
\hline ESE & $5.1 \mathrm{E}-07$ & $5.0 \mathrm{E}-07$ & $5.0 \mathrm{E}-07$ & $4.9 \mathrm{E}-07$ & $4.7 \mathrm{E}-07$ & 4. $5 \mathrm{E}-07$ \\
\hline & $1 \mathrm{E}-07$ & $6.1 \mathrm{E}-07$ & $6.0 \mathrm{E}-07$ & $6.0 \mathrm{E}-07$ & $5.7 \mathrm{E}-07$ & $4 \mathrm{E}-07$ \\
\hline ENE & $8.1 \mathrm{E}-07$ & $8.1 \mathrm{E}-07$ & $8.0 \mathrm{E}-07$ & $8.0 \mathrm{E}-07$ & $7.7 \mathrm{E}-07$ & 7. $3 \mathrm{E}-07$ \\
\hline $\mathrm{NE}$ & $1.4 \mathrm{E}-\mathrm{C}$ & $1.4 \mathrm{E}-06$ & 1. $4 \mathrm{E}-06$ & $1.4 \mathrm{E}$ & $1.3 \mathrm{E}-06$ & 1. $3 \mathrm{E}-06$ \\
\hline NNE & $7.9 \mathrm{E}-07$ & $7.9 \mathrm{E}-07$ & $7.8 \mathrm{E}-07$ & $7.8 \mathrm{E}-07$ & $7.5 E-07$ & $7.1 \mathrm{E}-07$ \\
\hline
\end{tabular}

Feb 20, $2008 \quad 02: 31 \mathrm{pm}$

SUMMARY

Page 7

INDIVIDUAL LIFETIME RISK (deaths)

(A11 Radionuclides and Pathways)

\begin{tabular}{|c|c|c|c|c|c|c|c|}
\hline \multicolumn{8}{|c|}{ Distance (m) } \\
\hline Direction & 27715 & 28919 & 31060 & 32802 & 34577 & 35279 & 35683 \\
\hline $\begin{array}{r}\text { N } \\
N N W \\
N W \\
\text { WNW } \\
W \\
W S W \\
\text { SW } \\
\text { SSW } \\
S \\
\text { SSE } \\
\text { SE } \\
\text { ESE } \\
\text { E } \\
\text { ENE } \\
N E \\
\text { NNE }\end{array}$ & $\begin{array}{l}6.0 \mathrm{E}-13 \\
3.2 \mathrm{E}-13 \\
3.7 \mathrm{E}-13 \\
4.9 \mathrm{E}-13 \\
4.8 \mathrm{E}-13 \\
8.3 \mathrm{E}-13 \\
1.5 \mathrm{E}-12 \\
2.4 \mathrm{E}-12 \\
3.3 \mathrm{E}-12 \\
3.1 \mathrm{E}-12 \\
1.1 \mathrm{E}-12 \\
6.3 \mathrm{E}-13 \\
7.7 \mathrm{E}-13 \\
9.7 \mathrm{E}-13 \\
1.6 \mathrm{E}-12 \\
9.2 \mathrm{E}-13\end{array}$ & $\begin{array}{l}5.6 \mathrm{E}-13 \\
3.0 \mathrm{E}-13 \\
3.5 \mathrm{E}-13 \\
4.6 \mathrm{E}-13 \\
4.5 \mathrm{E}-13 \\
7.8 \mathrm{E}-13 \\
1.4 \mathrm{E}-12 \\
2.2 \mathrm{E}-12 \\
3.1 \mathrm{E}-12 \\
3.0 \mathrm{E}-12 \\
1.0 \mathrm{E}-12 \\
5.9 \mathrm{E}-13 \\
7.2 \mathrm{E}-13 \\
9.1 \mathrm{E}-13 \\
1.5 \mathrm{E}-12 \\
8.6 \mathrm{E}-13\end{array}$ & $\begin{array}{l}5.0 \mathrm{E}-13 \\
2.7 \mathrm{E}-13 \\
3.1 \mathrm{E}-13 \\
4.1 \mathrm{E}-13 \\
4.1 \mathrm{E}-13 \\
7.1 \mathrm{E}-13 \\
1.3 \mathrm{E}-12 \\
2.0 \mathrm{E}-12 \\
2.7 \mathrm{E}-12 \\
2.7 \mathrm{E}-12 \\
9.0 \mathrm{E}-13 \\
5.3 \mathrm{E}-13 \\
6.4 \mathrm{E}-13 \\
8.2 \mathrm{E}-13 \\
1.4 \mathrm{E}-12 \\
7.8 \mathrm{E}-13\end{array}$ & $\begin{array}{l}4.6 \mathrm{E}-13 \\
2.5 \mathrm{E}-13 \\
2.9 \mathrm{E}-13 \\
3.8 \mathrm{E}-13 \\
3.7 \mathrm{E}-13 \\
6.5 \mathrm{E}-13 \\
1.2 \mathrm{E}-12 \\
1.9 \mathrm{E}-12 \\
2.5 \mathrm{E}-12 \\
2.5 \mathrm{E}-12 \\
8.2 \mathrm{E}-13 \\
4.8 \mathrm{E}-13 \\
5.9 \mathrm{E}-13 \\
7.5 \mathrm{E}-13 \\
1.3 \mathrm{E}-12 \\
7.2 \mathrm{E}-13\end{array}$ & $\begin{array}{l}4.3 \mathrm{E}-13 \\
2.3 \mathrm{E}-13 \\
2.7 \mathrm{E}-13 \\
3.5 \mathrm{E}-13 \\
3.5 \mathrm{E}-13 \\
6.0 \mathrm{E}-13 \\
1.1 \mathrm{E}-12 \\
1.7 \mathrm{E}-12 \\
2.3 \mathrm{E}-12 \\
2.3 \mathrm{E}-12 \\
7.6 \mathrm{E}-13 \\
4.4 \mathrm{E}-13 \\
5.4 \mathrm{E}-13 \\
6.9 \mathrm{E}-13 \\
1.2 \mathrm{E}-12 \\
6.6 \mathrm{E}-13\end{array}$ & $\begin{array}{l}4.1 \mathrm{E}-13 \\
2.2 \mathrm{E}-13 \\
2.6 \mathrm{E}-13 \\
3.4 \mathrm{E}-13 \\
3.3 \mathrm{E}-13 \\
5.8 \mathrm{E}-13 \\
1.1 \mathrm{E}-12 \\
1.7 \mathrm{E}-12 \\
2.2 \mathrm{E}-12 \\
2.2 \mathrm{E}-12 \\
7.3 \mathrm{E}-13 \\
4.3 \mathrm{E}-13 \\
5.2 \mathrm{E}-13 \\
6.7 \mathrm{E}-13 \\
1.1 \mathrm{E}-12 \\
6.4 \mathrm{E}-13\end{array}$ & $\begin{array}{l}4.1 \mathrm{E}-13 \\
2.2 \mathrm{E}-13 \\
2.6 \mathrm{E}-13 \\
3.4 \mathrm{E}-13 \\
3.3 \mathrm{E}-13 \\
5.7 \mathrm{E}-13 \\
1.0 \mathrm{E}-12 \\
1.6 \mathrm{E}-12 \\
2.2 \mathrm{E}-12 \\
2.2 \mathrm{E}-12 \\
7.2 \mathrm{E}-13 \\
4.2 \mathrm{E}-13 \\
5.1 \mathrm{E}-13 \\
6.6 \mathrm{E}-13 \\
1.1 \mathrm{E}-12 \\
6.3 \mathrm{E}-13\end{array}$ \\
\hline \multicolumn{8}{|c|}{ Distance (m) } \\
\hline Direction & 36721 & 36809 & 37729 & 39079 & 39220 & 39559 & 43584 \\
\hline $\begin{array}{r}N \\
N N W \\
N W \\
\text { WNW } \\
W \\
\text { WSW } \\
\text { SW } \\
\text { SSW }\end{array}$ & $\begin{array}{l}3.9 \mathrm{E}-13 \\
2.1 \mathrm{E}-13 \\
2.4 \mathrm{E}-13 \\
3.2 \mathrm{E}-13 \\
3.1 \mathrm{E}-13 \\
5.5 \mathrm{E}-13 \\
1.0 \mathrm{E}-12 \\
1.6 \mathrm{E}-12\end{array}$ & $\begin{array}{l}3.9 \mathrm{E}-13 \\
2.1 \mathrm{E}-13 \\
2.4 \mathrm{E}-13 \\
3.2 \mathrm{E}-13 \\
3.1 \mathrm{E}-13 \\
5.5 \mathrm{E}-13 \\
1.0 \mathrm{E}-12 \\
1.6 \mathrm{E}-12\end{array}$ & $\begin{array}{l}3.7 \mathrm{E}-13 \\
2.0 \mathrm{E}-13 \\
2.3 \mathrm{E}-13 \\
3.1 \mathrm{E}-13 \\
3.0 \mathrm{E}-13 \\
5.3 \mathrm{E}-13 \\
9.6 \mathrm{E}-13 \\
1.5 \mathrm{E}-12\end{array}$ & $\begin{array}{r}3.5 \mathrm{E}-13 \\
1.9 \mathrm{E}-13 \\
2.2 \mathrm{E}-13 \\
2.9 \mathrm{E}-13 \\
2.8 \mathrm{E}-13 \\
5.0 \mathrm{E}-13 \\
9.1 \mathrm{E}-13 \\
1.4 \mathrm{E}-12 \\
\text { Page }\end{array}$ & $\begin{array}{l}3.5 \mathrm{E}-13 \\
1.9 \mathrm{E}-13 \\
2.2 \mathrm{E}-13 \\
2.9 \mathrm{E}-13 \\
2.8 \mathrm{E}-13 \\
4.9 \mathrm{E}-13 \\
9.0 \mathrm{E}-13 \\
1.4 \mathrm{E}-12\end{array}$ & $\begin{array}{l}3.4 \mathrm{E}-13 \\
1.9 \mathrm{E}-13 \\
2.2 \mathrm{E}-13 \\
2.9 \mathrm{E}-13 \\
2.8 \mathrm{E}-13 \\
4.9 \mathrm{E}-13 \\
8.9 \mathrm{E}-13 \\
1.4 \mathrm{E}-12\end{array}$ & $\begin{array}{l}2.9 \mathrm{E}-13 \\
1.6 \mathrm{E}-13 \\
1.8 \mathrm{E}-13 \\
2.4 \mathrm{E}-13 \\
2.4 \mathrm{E}-13 \\
4.2 \mathrm{E}-13 \\
7.6 \mathrm{E}-13 \\
1.2 \mathrm{E}-12\end{array}$ \\
\hline
\end{tabular}




\begin{tabular}{rlllllll} 
& \multicolumn{7}{c}{$\mathrm{C} 1-38 \mathrm{C} . \mathrm{SUM}$} \\
$\mathrm{S}$ & $2.1 \mathrm{E}-12$ & $2.1 \mathrm{E}-12$ & $2.0 \mathrm{E}-12$ & $1.9 \mathrm{E}-12$ & $1.9 \mathrm{E}-12$ & $1.9 \mathrm{E}-12$ & $1.6 \mathrm{E}-12$ \\
SSE & $2.1 \mathrm{E}-12$ & $2.1 \mathrm{E}-12$ & $2.0 \mathrm{E}-12$ & $1.9 \mathrm{E}-12$ & $1.9 \mathrm{E}-12$ & $1.8 \mathrm{E}-12$ & $1.6 \mathrm{E}-12$ \\
SE & $6.8 \mathrm{E}-13$ & $6.8 \mathrm{E}-13$ & $6.5 \mathrm{E}-13$ & $6.2 \mathrm{E}-13$ & $6.1 \mathrm{E}-13$ & $6.0 \mathrm{E}-13$ & $5.1 \mathrm{E}-13$ \\
ESE & $4.0 \mathrm{E}-13$ & $4.0 \mathrm{E}-13$ & $3.8 \mathrm{E}-13$ & $3.6 \mathrm{E}-13$ & $3.6 \mathrm{E}-13$ & $3.5 \mathrm{E}-13$ & $2.9 \mathrm{E}-13$ \\
$\mathrm{E}$ & $4.9 \mathrm{E}-13$ & $4.9 \mathrm{E}-13$ & $4.7 \mathrm{E}-13$ & $4.4 \mathrm{E}-13$ & $4.3 \mathrm{E}-13$ & $4.3 \mathrm{E}-13$ & $3.6 \mathrm{E}-13$ \\
ENE & $6.3 \mathrm{E}-13$ & $6.3 \mathrm{E}-13$ & $6.0 \mathrm{E}-13$ & $5.7 \mathrm{E}-13$ & $5.6 \mathrm{E}-13$ & $5.6 \mathrm{E}-13$ & $4.7 \mathrm{E}-13$ \\
NE & $1.1 \mathrm{E}-12$ & $1.1 \mathrm{E}-12$ & $1.0 \mathrm{E}-12$ & $9.7 \mathrm{E}-13$ & $9.7 \mathrm{E}-13$ & $9.5 \mathrm{E}-13$ & $8.1 \mathrm{E}-13$ \\
NNE & $6.0 \mathrm{E}-13$ & $6.0 \mathrm{E}-13$ & $5.8 \mathrm{E}-13$ & $5.5 \mathrm{E}-13$ & $5.4 \mathrm{E}-13$ & $5.4 \mathrm{E}-13$ & $4.6 \mathrm{E}-13$
\end{tabular}

Feb 20, $2008 \quad 02: 31 \mathrm{pm}$

SUMMARY

Page 8

INDIVIDUAL LIFETIME RISK (deaths)

(A11 Radionuclides and Pathways)

\begin{tabular}{|c|c|c|c|c|c|c|}
\hline \multirow[b]{2}{*}{ Direction } & \multicolumn{6}{|c|}{ Distance $(\mathrm{m})$} \\
\hline & 45196 & 45275 & 45654 & 45677 & 46668 & 47969 \\
\hline $\begin{array}{r}\text { N } \\
N N W \\
N W \\
W N W \\
W \\
W S W \\
\text { SW } \\
\text { SSW } \\
\text { S } \\
\text { SSE } \\
\text { SE } \\
\text { ESE } \\
\text { E } \\
\text { ENE } \\
\text { NE } \\
\text { NNE }\end{array}$ & $\begin{array}{l}2.7 \mathrm{E}-13 \\
1.5 \mathrm{E}-13 \\
1.7 \mathrm{E}-13 \\
2.3 \mathrm{E}-13 \\
2.2 \mathrm{E}-13 \\
3.9 \mathrm{E}-13 \\
7.2 \mathrm{E}-13 \\
1.1 \mathrm{E}-12 \\
1.5 \mathrm{E}-12 \\
1.5 \mathrm{E}-12 \\
4.7 \mathrm{E}-13 \\
2.7 \mathrm{E}-13 \\
3.3 \mathrm{E}-13 \\
4.4 \mathrm{E}-13 \\
7.6 \mathrm{E}-13 \\
4.3 \mathrm{E}-13\end{array}$ & $\begin{array}{l}2.7 \mathrm{E}-13 \\
1.5 \mathrm{E}-13 \\
1.7 \mathrm{E}-13 \\
2.3 \mathrm{E}-13 \\
2.2 \mathrm{E}-13 \\
3.9 \mathrm{E}-13 \\
7.1 \mathrm{E}-13 \\
1.1 \mathrm{E}-12 \\
1.5 \mathrm{E}-12 \\
1.5 \mathrm{E}-12 \\
4.7 \mathrm{E}-13 \\
2.7 \mathrm{E}-13 \\
3.3 \mathrm{E}-13 \\
4.4 \mathrm{E}-13 \\
7.6 \mathrm{E}-13 \\
4.3 \mathrm{E}-13\end{array}$ & $\begin{array}{l}2.7 \mathrm{E}-13 \\
1.5 \mathrm{E}-13 \\
1.7 \mathrm{E}-13 \\
2.2 \mathrm{E}-13 \\
2.2 \mathrm{E}-13 \\
3.8 \mathrm{E}-13 \\
7.0 \mathrm{E}-13 \\
1.1 \mathrm{E}-12 \\
1.4 \mathrm{E}-12 \\
1.5 \mathrm{E}-12 \\
4.6 \mathrm{E}-13 \\
2.7 \mathrm{E}-13 \\
3.2 \mathrm{E}-13 \\
4.3 \mathrm{E}-13 \\
7.5 \mathrm{E}-13 \\
4.2 \mathrm{E}-13\end{array}$ & $\begin{array}{l}2.7 \mathrm{E}-13 \\
1.5 \mathrm{E}-13 \\
1.7 \mathrm{E}-13 \\
2.2 \mathrm{E}-13 \\
2.2 \mathrm{E}-13 \\
3.8 \mathrm{E}-13 \\
7.0 \mathrm{E}-13 \\
1.1 \mathrm{E}-12 \\
1.4 \mathrm{E}-12 \\
1.5 \mathrm{E}-12 \\
4.6 \mathrm{E}-13 \\
2.7 \mathrm{E}-13 \\
3.2 \mathrm{E}-13 \\
4.3 \mathrm{E}-13 \\
7.5 \mathrm{E}-13 \\
4.2 \mathrm{E}-13\end{array}$ & $\begin{array}{l}2.6 \mathrm{E}-13 \\
1.4 \mathrm{E}-13 \\
1.6 \mathrm{E}-13 \\
2.2 \mathrm{E}-13 \\
2.1 \mathrm{E}-13 \\
3.7 \mathrm{E}-13 \\
6.8 \mathrm{E}-13 \\
1.0 \mathrm{E}-12 \\
1.4 \mathrm{E}-12 \\
1.4 \mathrm{E}-12 \\
4.5 \mathrm{E}-13 \\
2.6 \mathrm{E}-13 \\
3.1 \mathrm{E}-13 \\
4.2 \mathrm{E}-13 \\
7.2 \mathrm{E}-13 \\
4.1 \mathrm{E}-13\end{array}$ & $\begin{array}{l}2.4 \mathrm{E}-13 \\
1.4 \mathrm{E}-13 \\
1.6 \mathrm{E}-13 \\
2.0 \mathrm{E}-13 \\
2.0 \mathrm{E}-13 \\
3.5 \mathrm{E}-13 \\
6.5 \mathrm{E}-13 \\
9.9 \mathrm{E}-13 \\
1.3 \mathrm{E}-12 \\
1.3 \mathrm{E}-12 \\
4.2 \mathrm{E}-13 \\
2.4 \mathrm{E}-13 \\
2.9 \mathrm{E}-13 \\
3.9 \mathrm{E}-13 \\
6.9 \mathrm{E}-13 \\
3.9 \mathrm{E}-13\end{array}$ \\
\hline
\end{tabular}




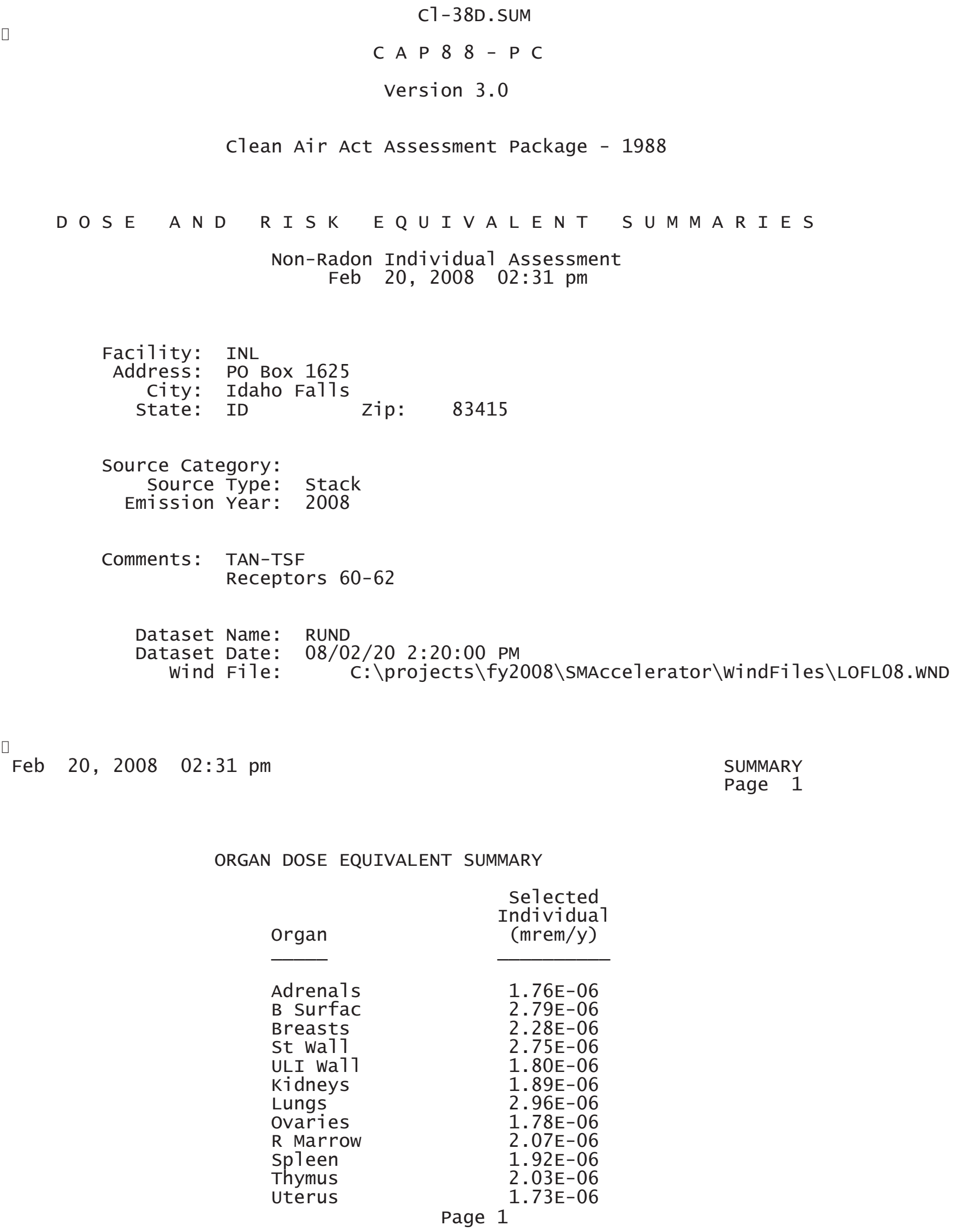




\begin{tabular}{lr} 
& \multicolumn{1}{c}{ C1-38D. SUM } \\
B1d wa11 & $1.82 \mathrm{E}-06$ \\
Brain & $2.21 \mathrm{E}-06$ \\
Esophagu & $4.95 \mathrm{E}-06$ \\
SI wa11 & $1.78 \mathrm{E}-06$ \\
LLI wa11 & $1.77 \mathrm{E}-06$ \\
Liver & $1.91 \mathrm{E}-06$ \\
Muscle & $2.02 \mathrm{E}-06$ \\
Pancreas & $1.77 \mathrm{E}-06$ \\
Skin & $5.14 \mathrm{E}-06$ \\
Testes & $2.02 \mathrm{E}-06$ \\
Thyroid & $2.10 \mathrm{E}-06$ \\
& $2.31 \mathrm{E}-06$
\end{tabular}

PATHWAY EFFECTIVE DOSE EQUIVALENT SUMMARY

\begin{tabular}{lcc} 
Pathway & $\begin{array}{c}\text { Selected } \\
\text { Individuat } \\
\text { (mrem/y) }\end{array}$ \\
\cline { 2 - 3 } & $0.00 \mathrm{E}+00$ \\
INGESTION & $3.04 \mathrm{E}-07$ \\
INHALATION & $2.00 \mathrm{E}-06$ & \\
AIR IMMERSION & $0.00 \mathrm{E}+00$ & \\
GROUND SURFACE & $3.04 \mathrm{E}-07$ & \\
INTERNAL & $2.00 \mathrm{E}-06$ & \\
EXTERNAL & $2.31 \mathrm{E}-06$ & SUMMARY \\
TOTAL & & Page 2 \\
pm & &
\end{tabular}

NUCLIDE EFFECTIVE DOSE EQUIVALENT SUMMARY

Nuclide

C1-38

TOTAL

Feb 20, $2008 \quad 02: 31$ pm

$$
\begin{gathered}
\begin{array}{c}
\text { Selected } \\
\text { Individual } \\
\text { (mrem/y) }
\end{array} \\
\hline 2.31 \mathrm{E}-06 \\
2.31 \mathrm{E}-06
\end{gathered}
$$

SUMMARY

Page 3

CANCER RISK SUMMARY

Cancer

Esophagu
Selected Individual

Total Lifetime Fatal Cancer Risk

Page $2^{2 \cdot 10 \mathrm{E}-14}$ 


$\begin{array}{lr} & \text { C1-38D. SUM } \\ \text { Stomach } & 1.25 \mathrm{E}-13 \\ \text { Colon } & 1.85 \mathrm{E}-13 \\ \text { Liver } & 2.91 \mathrm{E}-14 \\ \text { LUNG } & 2.96 \mathrm{E}-13 \\ \text { Bone } & 2.65 \mathrm{E}-15 \\ \text { Skin } & 5.14 \mathrm{E}-15 \\ \text { Breast } & 1.11 \mathrm{E}-13 \\ \text { Ovary } & 2.53 \mathrm{E}-14 \\ \text { B Tadder } & 4.39 \mathrm{E}-14 \\ \text { Kidneys } & 9.85 \mathrm{E}-15 \\ \text { Thyroid } & 6.70 \mathrm{E}-15 \\ \text { Leukemia } & 1.16 \mathrm{E}-13 \\ \text { Residual } & 2.76 \mathrm{E}-13 \\ \text { Total } & 1.25 \mathrm{E}-12 \\ & \\ \text { TOTAL } & 2.50 \mathrm{E}-12\end{array}$

PATHWAY RISK SUMMARY

\begin{tabular}{lc} 
Pathway & $\begin{array}{c}\text { Selected Individua1 } \\
\text { Tota1 Lifetime } \\
\text { Fatal Cancer Risk }\end{array}$ \\
\cline { 2 - 2 } INGESTION & $0.00 \mathrm{E}+00$ \\
INHALATION & $1.54 \mathrm{E}-13$ \\
AIR IMMERSION & $1.10 \mathrm{E}-12$ \\
GROUND SURFACE & $0.00 \mathrm{E}+00$ \\
INTERNAL & $1.54 \mathrm{E}-13$ \\
EXTERNAL & $1.10 \mathrm{E}-12$ \\
TOTAL & $1.25 \mathrm{E}-12$
\end{tabular}

Feb 20, $2008 \quad 02: 31$ pm

SUMMARY Page 4

NUCLIDE RISK SUMMARY

Nuclide

C1-38

TOTAL

$\square$

Feb 20, 2008 02:31 pm
Selected Individual

Total Lifetime Fatal Cancer Risk

1. $25 \mathrm{E}-12$

1. $25 \mathrm{E}-12$

SUMMARY

Page 5

INDIVIDUAL EFFECTIVE DOSE EQUIVALENT RATE (mrem/y) (A11 Radionuclides and Pathways)

Distance $(m)$ 


Direction $50024 \quad 54611 \quad 58610 \quad$ C1-38D.SUM

\begin{tabular}{|c|c|c|c|c|}
\hline $\begin{array}{r}N \\
N N W \\
N W \\
W N W \\
W \\
W S W \\
S W \\
S S W \\
S \\
S S E \\
S E \\
\text { ESE } \\
E \\
\text { ENE } \\
N E \\
N N E\end{array}$ & $\begin{array}{l}4.1 \mathrm{E}-07 \\
2.4 \mathrm{E}-07 \\
2.7 \mathrm{E}-07 \\
3.5 \mathrm{E}-07 \\
3.3 \mathrm{E}-07 \\
6.0 \mathrm{E}-07 \\
1.1 \mathrm{E}-06 \\
1.7 \mathrm{E}-06 \\
2.3 \mathrm{E}-06 \\
2.3 \mathrm{E}-06 \\
7.1 \mathrm{E}-07 \\
4.1 \mathrm{E}-07 \\
4.9 \mathrm{E}-07 \\
6.7 \mathrm{E}-07 \\
1.2 \mathrm{E}-06 \\
6.6 \mathrm{E}-07\end{array}$ & $\begin{array}{l}3.4 \mathrm{E}-07 \\
2.0 \mathrm{E}-07 \\
2.2 \mathrm{E}-07 \\
2.9 \mathrm{E}-07 \\
2.8 \mathrm{E}-07 \\
5.0 \mathrm{E}-07 \\
9.3 \mathrm{E}-07 \\
1.4 \mathrm{E}-06 \\
1.9 \mathrm{E}-06 \\
1.9 \mathrm{E}-06 \\
5.8 \mathrm{E}-07 \\
3.3 \mathrm{E}-07 \\
3.9 \mathrm{E}-07 \\
5.5 \mathrm{E}-07 \\
9.8 \mathrm{E}-07 \\
5.5 \mathrm{E}-07\end{array}$ & $\begin{array}{l}2.7 \mathrm{E}-07 \\
1.7 \mathrm{E}-07 \\
1.8 \mathrm{E}-07 \\
2.3 \mathrm{E}-07 \\
2.2 \mathrm{E}-07 \\
4.1 \mathrm{E}-07 \\
7.7 \mathrm{E}-07 \\
1.2 \mathrm{E}-06 \\
1.5 \mathrm{E}-06 \\
1.6 \mathrm{E}-06 \\
4.5 \mathrm{E}-07 \\
2.5 \mathrm{E}-07 \\
2.9 \mathrm{E}-07 \\
4.4 \mathrm{E}-07 \\
8.1 \mathrm{E}-07 \\
4.5 \mathrm{E}-07\end{array}$ & \\
\hline Feb 20, & , 2008 & $2: 31 \mathrm{pm}$ & & SUMMARY \\
\hline \multicolumn{5}{|c|}{$\begin{array}{l}\text { INDIVIDUAL LIFETIME RISK (deaths) } \\
\text { (A11 Radionuclides and Pathways) }\end{array}$} \\
\hline \multicolumn{5}{|c|}{ Distance (m) } \\
\hline Directior & ר 50024 & 54611 & 58610 & \\
\hline $\begin{array}{r}N \\
N N W \\
N W \\
W N W \\
W \\
W S W \\
\text { SW } \\
\text { SSW } \\
S \\
\text { SSE } \\
\text { SE } \\
\text { ESE } \\
\text { E } \\
\text { ENE } \\
N E \\
\text { NNE }\end{array}$ & $\begin{array}{l}2.2 \mathrm{E}-13 \\
1.3 \mathrm{E}-13 \\
1.4 \mathrm{E}-13 \\
1.9 \mathrm{E}-13 \\
1.8 \mathrm{E}-13 \\
3.3 \mathrm{E}-13 \\
6.0 \mathrm{E}-13 \\
9.2 \mathrm{E}-13 \\
1.2 \mathrm{E}-12 \\
1.3 \mathrm{E}-12 \\
3.9 \mathrm{E}-13 \\
2.2 \mathrm{E}-13 \\
2.7 \mathrm{E}-13 \\
3.6 \mathrm{E}-13 \\
6.4 \mathrm{E}-13 \\
3.6 \mathrm{E}-13\end{array}$ & $\begin{array}{l}1.8 \mathrm{E}-13 \\
1.1 \mathrm{E}-13 \\
1.2 \mathrm{E}-13 \\
1.6 \mathrm{E}-13 \\
1.5 \mathrm{E}-13 \\
2.7 \mathrm{E}-13 \\
5.0 \mathrm{E}-13 \\
7.7 \mathrm{E}-13 \\
1.0 \mathrm{E}-12 \\
1.1 \mathrm{E}-12 \\
3.1 \mathrm{E}-13 \\
1.8 \mathrm{E}-13 \\
2.1 \mathrm{E}-13 \\
3.0 \mathrm{E}-13 \\
5.3 \mathrm{E}-13 \\
3.0 \mathrm{E}-13\end{array}$ & $\begin{array}{l}1.5 \mathrm{E}-13 \\
9.0 \mathrm{E}-14 \\
9.6 \mathrm{E}-14 \\
1.3 \mathrm{E}-13 \\
1.2 \mathrm{E}-13 \\
2.2 \mathrm{E}-13 \\
4.2 \mathrm{E}-13 \\
6.3 \mathrm{E}-13 \\
8.3 \mathrm{E}-13 \\
8.8 \mathrm{E}-13 \\
2.4 \mathrm{E}-13 \\
1.3 \mathrm{E}-13 \\
1.6 \mathrm{E}-13 \\
2.4 \mathrm{E}-13 \\
4.4 \mathrm{E}-13 \\
2.4 \mathrm{E}-13\end{array}$ & \\
\hline
\end{tabular}


08/02/20 2:16:00 PM

RUNA

C: \projects \fy2008\SMAccelerator \WindFi 1es\LOFL08.WND

INL

PO Box 1625

Idaho Fal1s

TAN-TSF

Receptors 1-20

$100.0000,500.0000$

$\begin{array}{rrrr}0 & 0 & 0 & \\ 10344 & 10365 & 10472 & 1059 \\ 13286 & 13483 & 13612 & 1366 \\ 15441 & 15784 & 15844 & 1632 \\ 800 & 280.20 & 16.30 & 3.5 \\ 0 & 0 & & \\ 1 & & & \end{array}$

$\begin{array}{rrrr}1.00 & 1.00 & 0.000 \mathrm{E}+00 & 0.000 \mathrm{E}+00 \\ 0.000 \mathrm{E}+00 & 0.000 \mathrm{E}+00 & 0.000 \mathrm{E}+00 & 0.000 \mathrm{E}+00\end{array}$

C1-39 $2 \quad 0$

$1.000 \mathrm{E}+00 \quad 0.000 \mathrm{E}+00$

1. $630 \mathrm{e}-06$ 0.0018 $0 \quad 1$

$11.000 \mathrm{e}+00$

$0.000 E+00 \quad 0.000 E+00$

83415

2008

Ar-39

unspecified $2.000 \mathrm{E}+01$

$\mathrm{E}+02$

$0.000 \mathrm{e}+00$

$\mathrm{G}$

$0.000 \mathrm{E}+00$

$0.000 \mathrm{E}+00 \quad 0.000 \mathrm{E}+00$

$0.000 \mathrm{e}+00$

$0 \quad 0.000 \mathrm{e}+00$

$0.08 \quad 0.92$ unspecified $0.000 \mathrm{E}+00$

0.92

0.00
$0.000 E+00 \quad 0.000 E+00$ Urban

0.01

0.99

0.00

0.00

${ }^{\mathrm{T}} 2.030 \mathrm{e}-01^{\mathrm{T}} 4.560 \mathrm{e}-02^{\mathrm{T}} 1.700 \mathrm{e}-02$

C1-39 $00 \quad 00$

$0.000 \mathrm{e}+00$

$0.000 e+00$

0
00

Ar-39

18

$0.000 e+00$
0

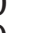

00

00

$0.000 e+00 \quad 0.000 e+00$

$\begin{array}{rr}0 & 20 \\ 11103 & 11989\end{array}$

$13959 \quad 14258$

12522

14374

13245

15241

00

$\begin{array}{rrrrr}00 & 00 & 00 & 00 & 00 \\ 0.000 \mathrm{e}+00 & 0.000 \mathrm{e}+00 & 0.000 \mathrm{e}+00 & 0.000 \mathrm{e}+00 & 0.000 \mathrm{e}+00\end{array}$

2

C1-39

$11.000 \mathrm{e}+00 \quad 1.000 \mathrm{e}+00 \quad 5.480 \mathrm{e}-05$

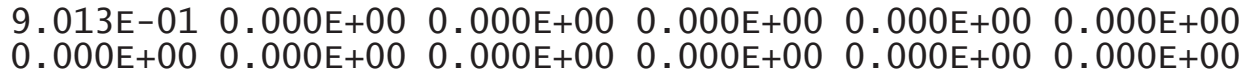

$1.010 \mathrm{e}-05 \quad 0.0018 \quad 0.000 \mathrm{e}+00$

unspecified 2.000E+01 $1.000 \mathrm{E}+02 \quad 2.000 \mathrm{E}-02 \quad 4.000 \mathrm{E}-02 \quad 1.795 \mathrm{E}+01$

$\begin{array}{crrrrr}\mathrm{Ar}-39 & \mathrm{G} & 0 & 0.000 \mathrm{e}+00 & 0.000 \mathrm{e}+00 & 5.480 \mathrm{e}-05 \\ 3.877 \mathrm{E}-08 & 0.000 \mathrm{E}+00 & 0.000 \mathrm{E}+00 & 0.000 \mathrm{E}+00 & 0.000 \mathrm{E}+00 & 0.000 \mathrm{E}+00\end{array}$

$\begin{array}{lllllllll}1.810 \mathrm{E}-07 & 0.000 \mathrm{E}+00 & 0.000 \mathrm{E}+00 & 0.000 \mathrm{E}+00 & 0.000 \mathrm{E}+00 & 0.000 \mathrm{E}+00\end{array}$

$0.000 \mathrm{e}+00$

$0 \quad 0.000 \mathrm{e}+00$

unspecified $0.000 \mathrm{E}+00 \quad 0.000 \mathrm{E}+00 \quad 0.000 \mathrm{E}+00 \quad 0.000 \mathrm{E}+00 \quad 7.053 \mathrm{E}-06$

--Decaystep--1

--Limitchildren--1

--Chi 1dren--5 


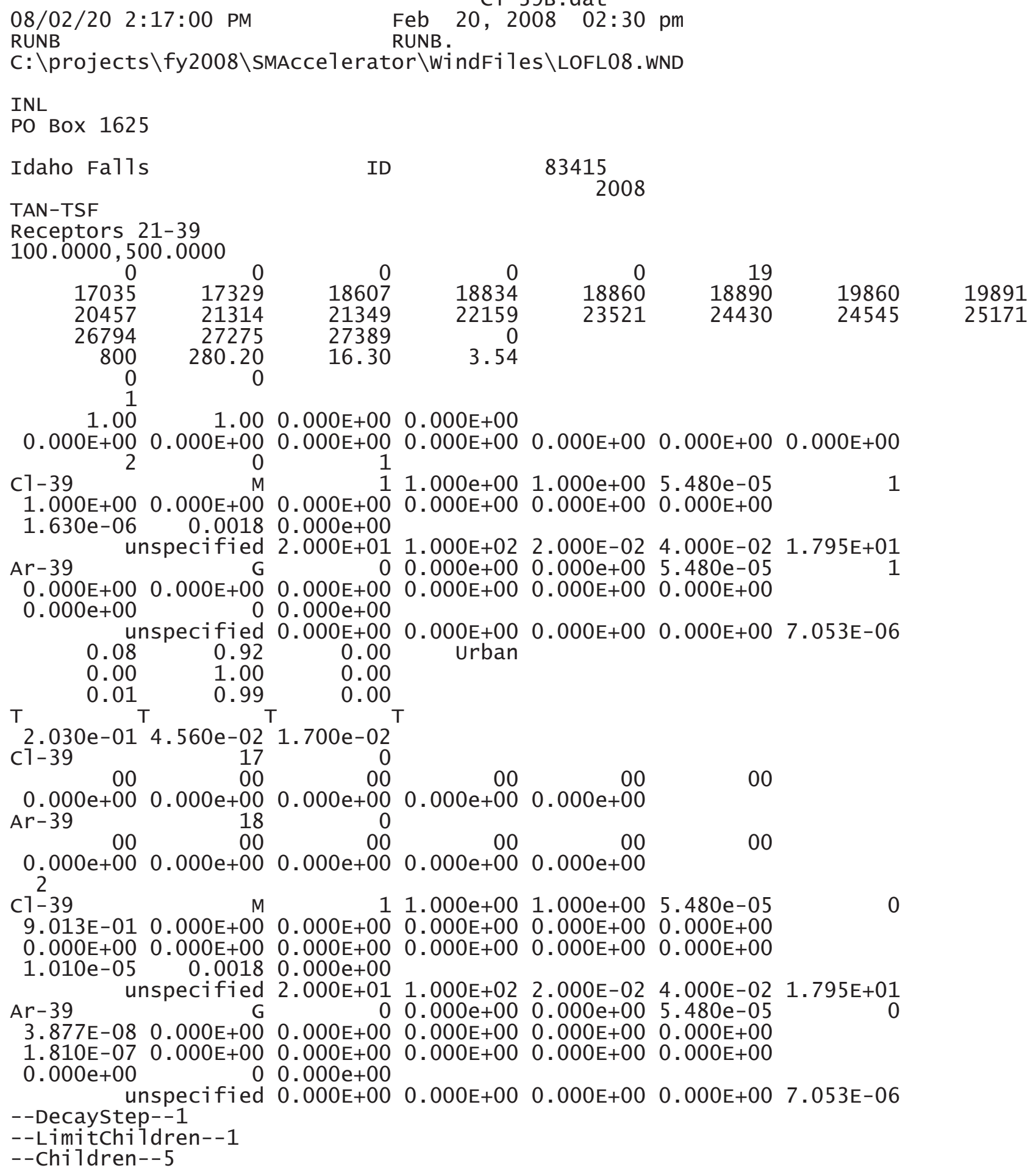




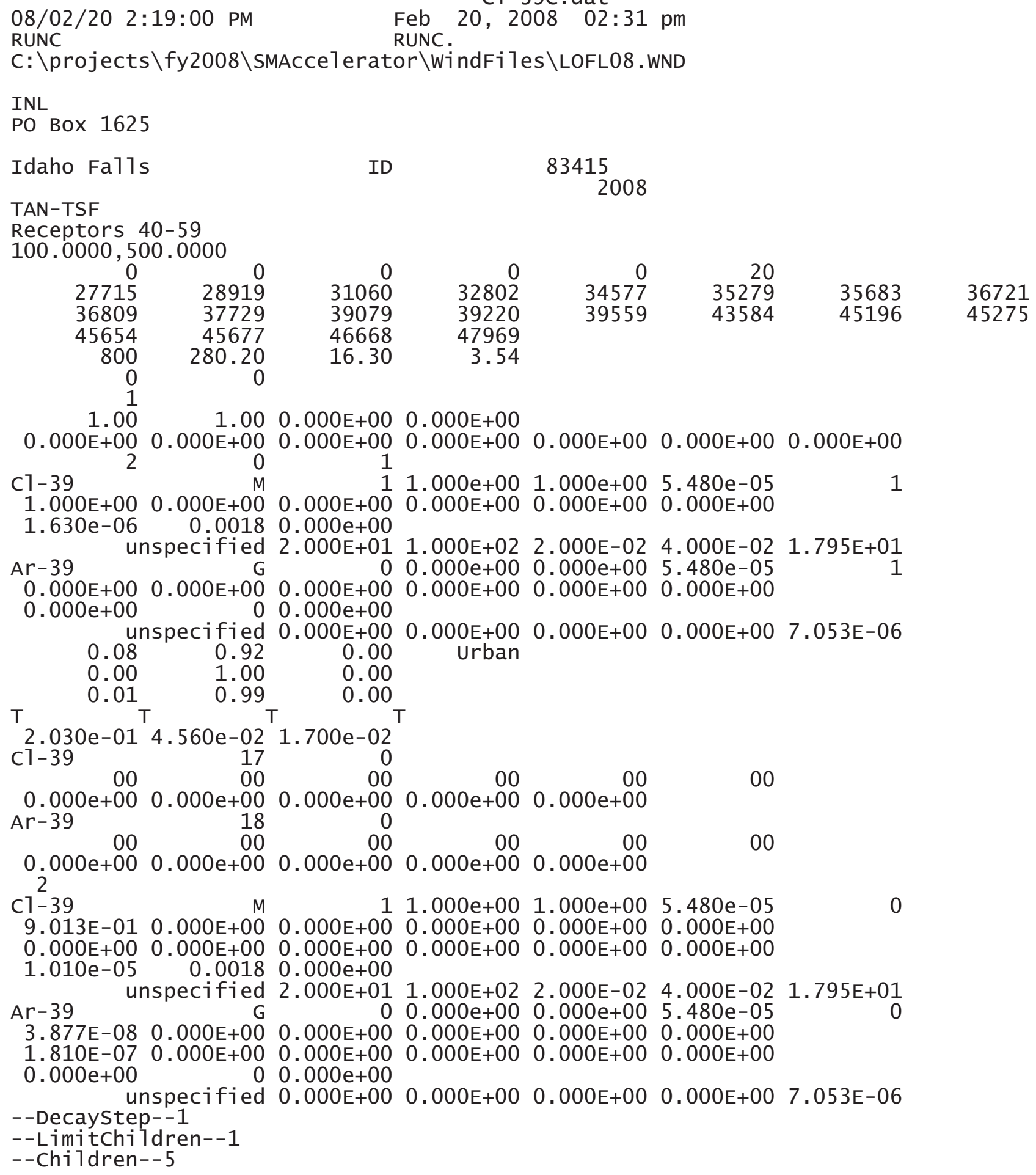




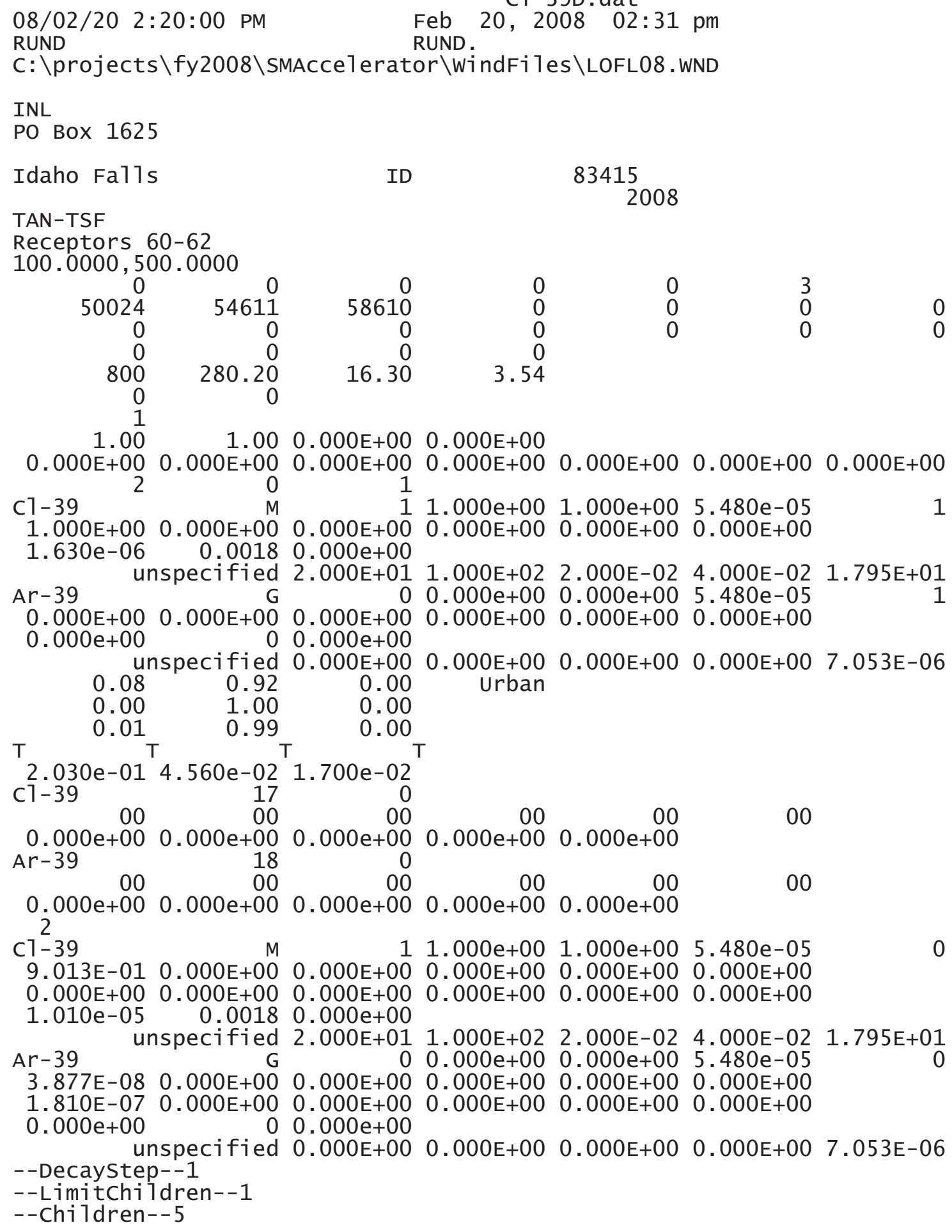




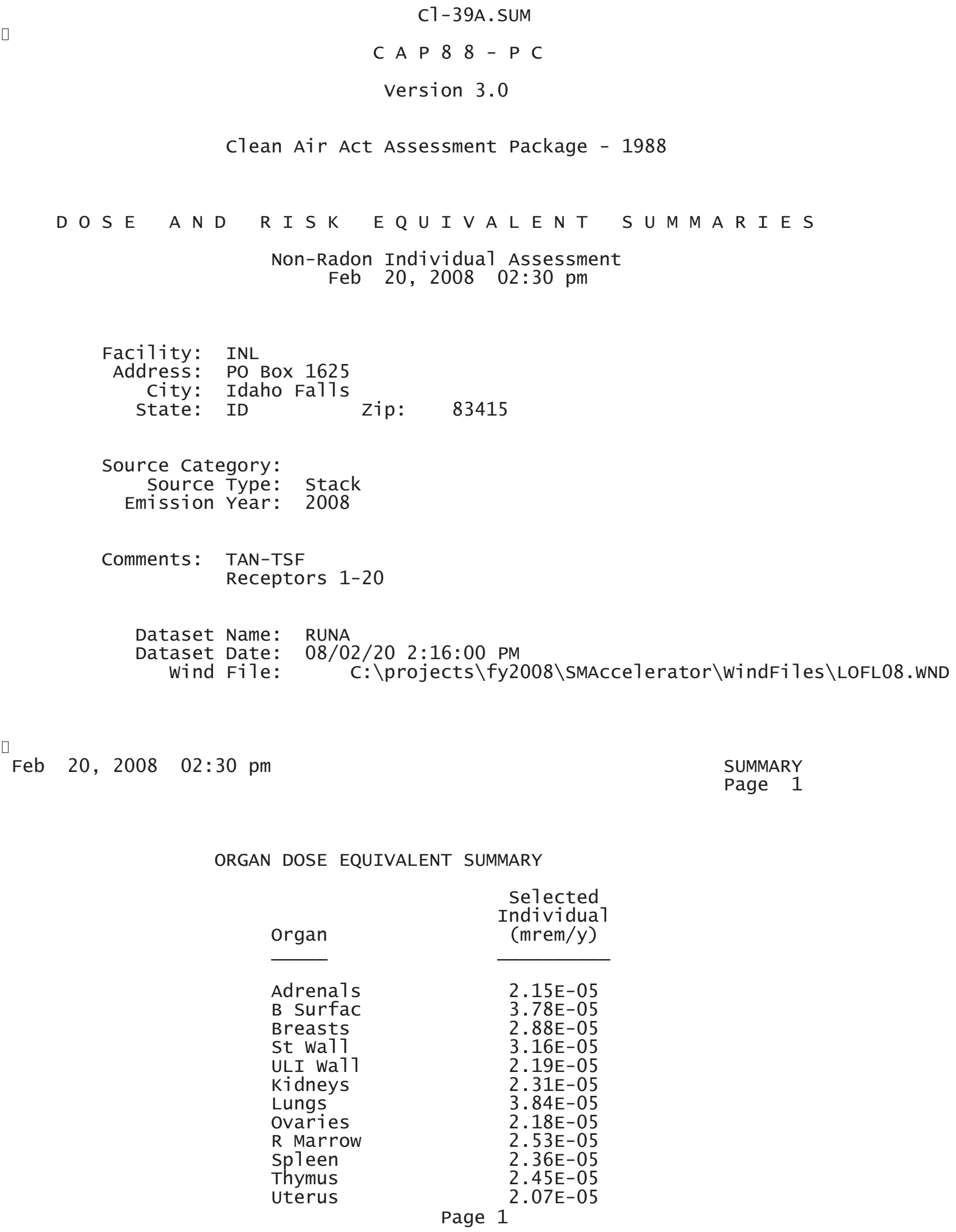

\begin{tabular}{lr} 
ORGAN DOSE EQUIVALENT SUMMARY \\
Organ & $\begin{array}{c}\text { Selected } \\
\text { Individual } \\
\text { (mrem/y) }\end{array}$ \\
\cline { 2 - 2 } Adrena1s & $2.15 \mathrm{E}-05$ \\
B Surfac & $3.78 \mathrm{E}-05$ \\
Breasts & $2.88 \mathrm{E}-05$ \\
St wa11 & $3.16 \mathrm{E}-05$ \\
ULI wa11 & $2.19 \mathrm{E}-05$ \\
Kidneys & $2.31 \mathrm{E}-05$ \\
Lungs & $3.84 \mathrm{E}-05$ \\
Ovaries & $2.18 \mathrm{E}-05$ \\
R Marrow & $2.53 \mathrm{E}-05$ \\
Spleen & $2.36 \mathrm{E}-05$ \\
Thymus & $2.45 \mathrm{E}-05$ \\
Uterus & $2.07 \mathrm{E}-05$ \\
& 1
\end{tabular}




\begin{tabular}{lr} 
& \multicolumn{1}{c}{ C1-39A. SUM } \\
Bld wa11 & $2.15 \mathrm{E}-05$ \\
Brain & $2.74 \mathrm{E}-05$ \\
Esophagu & $7.55 \mathrm{E}-05$ \\
SI wa11 & $2.14 \mathrm{E}-05$ \\
LLI wa11 & $2.15 \mathrm{E}-05$ \\
Liver & $2.34 \mathrm{E}-05$ \\
Muscle & $2.50 \mathrm{E}-05$ \\
Pancreas & $2.13 \mathrm{E}-05$ \\
Skin & $4.84 \mathrm{E}-05$ \\
Testes & $2.53 \mathrm{E}-05$ \\
Thyroid & $2.62 \mathrm{E}-05$ \\
& \\
EFFEC & $2.87 \mathrm{E}-05$
\end{tabular}

PATHWAY EFFECTIVE DOSE EQUIVALENT SUMMARY

\begin{tabular}{lc} 
Pathway & $\begin{array}{c}\text { selected } \\
\text { Individua1 } \\
\text { (mrem/y) }\end{array}$ \\
\cline { 2 - 2 } INGESTION & $0.00 \mathrm{E}+00$ \\
INHALATION & $4.18 \mathrm{E}-06$ \\
AIR IMMERSION & $2.45 \mathrm{E}-05$ \\
GROUND SURFACE & $0.00 \mathrm{E}+00$ \\
INTERNAL & $4.18 \mathrm{E}-06$ \\
EXTERNAL & $2.45 \mathrm{E}-05$ \\
TOTAL & $2.87 \mathrm{E}-05$
\end{tabular}

Feb 20, 2008 02:30 pm

SUMMARY

Page 2

NUCLIDE EFFECTIVE DOSE EQUIVALENT SUMMARY

Nuclide

C1 $1-39$

Ar-39

TOTAL

$$
\begin{gathered}
\begin{array}{c}
\text { selected } \\
\text { Individual } \\
\text { (mrem/y) }
\end{array} \\
\hline 2.87 \mathrm{E}-05 \\
2.83 \mathrm{E}-15 \\
2.87 \mathrm{E}-05
\end{gathered}
$$

Feb 20, $2008 \quad 02: 30 \mathrm{pm}$

SUMMARY

Page 3

CANCER RISK SUMMARY

Cancer

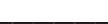

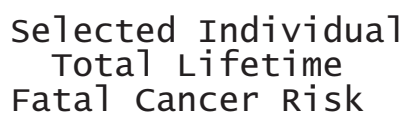

Page 2 


$\begin{array}{lr} & \text { C1-39A. SUM } \\ \text { Esophagu } & 2.57 \mathrm{E}-13 \\ \text { Stomach } & 1.41 \mathrm{E}-12 \\ \text { Colon } & 2.26 \mathrm{E}-12 \\ \text { Liver } & 3.56 \mathrm{E}-13 \\ \text { LUNG } & 3.84 \mathrm{E}-12 \\ \text { Bone } & 3.61 \mathrm{E}-14 \\ \text { Skin } & 4.84 \mathrm{E}-14 \\ \text { Breast } & 1.39 \mathrm{E}-12 \\ \text { Ovary } & 3.10 \mathrm{E}-13 \\ \text { B Tadder } & 5.22 \mathrm{E}-13 \\ \text { Kidneys } & 1.21 \mathrm{E}-13 \\ \text { Thyroid } & 8.34 \mathrm{E}-14 \\ \text { Leukemia } & 1.42 \mathrm{E}-12 \\ \text { Residual } & 3.38 \mathrm{E}-12 \\ \text { Total } & 1.54 \mathrm{E}-11 \\ & \\ \text { TOTAL } & 3.09 \mathrm{E}-11\end{array}$

PATHWAY RISK SUMMARY

Pathway

INGESTION

INHALATION

AIR IMMERSION

GROUND SURFACE

INTERNAL

EXTERNAL

TOTAL

Feb 20, 2008 02:30 pm
Selected Individual

Total Lifetime

Fatal Cancer Risk

$0.00 \mathrm{E}+00$

2.04E-12

1. $34 \mathrm{E}-11$

$0.00 \mathrm{E}+00$

2. $04 \mathrm{E}-12$

1. 34E-11

1. $54 \mathrm{E}-11$

SUMMARY

Page 4

NUCLIDE RISK SUMMARY

Nuclide

C1-39

Ar-39

TOTAL
Selected Individual Total Lifetime Fatal Cancer Risk

1. 54E-11

$3.59 \mathrm{E}-22$

1. 54E-11

Feb 20, 2008 02:30 pm

INDIVIDUAL EFFECTIVE DOSE EQUIVALENT RATE (mrem/y) (A11 Radionuclides and Pathways) 
C1-39A. SUM

\begin{tabular}{rrrrrrrr}
\cline { 2 - 7 } Direction & 10344 & 10365 & 10472 & 10590 & 11103 & 11989 & 12522 \\
\hline N & $5.5 \mathrm{E}-06$ & $5.5 \mathrm{E}-06$ & $5.4 \mathrm{E}-06$ & $5.3 \mathrm{E}-06$ & $5.0 \mathrm{E}-06$ & $4.5 \mathrm{E}-06$ & $4.3 \mathrm{E}-06$ \\
$\mathrm{NNW}$ & $2.5 \mathrm{E}-06$ & $2.5 \mathrm{E}-06$ & $2.5 \mathrm{E}-06$ & $2.4 \mathrm{E}-06$ & $2.3 \mathrm{E}-06$ & $2.1 \mathrm{E}-06$ & $2.0 \mathrm{E}-06$ \\
$\mathrm{NW}$ & $3.2 \mathrm{E}-06$ & $3.1 \mathrm{E}-06$ & $3.1 \mathrm{E}-06$ & $3.1 \mathrm{E}-06$ & $2.9 \mathrm{E}-06$ & $2.6 \mathrm{E}-06$ & $2.5 \mathrm{E}-06$ \\
WNW & $4.2 \mathrm{E}-06$ & $4.2 \mathrm{E}-06$ & $4.1 \mathrm{E}-06$ & $4.0 \mathrm{E}-06$ & $3.8 \mathrm{E}-06$ & $3.4 \mathrm{E}-06$ & $3.2 \mathrm{E}-06$ \\
$\mathrm{~W}$ & $4.4 \mathrm{E}-06$ & $4.4 \mathrm{E}-06$ & $4.3 \mathrm{E}-06$ & $4.2 \mathrm{E}-06$ & $4.0 \mathrm{E}-06$ & $3.6 \mathrm{E}-06$ & $3.4 \mathrm{E}-06$ \\
WSW & $7.0 \mathrm{E}-06$ & $7.0 \mathrm{E}-06$ & $6.9 \mathrm{E}-06$ & $6.8 \mathrm{E}-06$ & $6.4 \mathrm{E}-06$ & $5.7 \mathrm{E}-06$ & $5.4 \mathrm{E}-06$ \\
$\mathrm{SW}$ & $1.3 \mathrm{E}-05$ & $1.3 \mathrm{E}-05$ & $1.2 \mathrm{E}-05$ & $1.2 \mathrm{E}-05$ & $1.1 \mathrm{E}-05$ & $1.0 \mathrm{E}-05$ & $9.7 \mathrm{E}-06$ \\
$\mathrm{SSW}$ & $2.0 \mathrm{E}-05$ & $2.0 \mathrm{E}-05$ & $2.0 \mathrm{E}-05$ & $2.0 \mathrm{E}-05$ & $1.8 \mathrm{E}-05$ & $1.7 \mathrm{E}-05$ & $1.6 \mathrm{E}-05$ \\
$\mathrm{~S}$ & $2.9 \mathrm{E}-05$ & $2.9 \mathrm{E}-05$ & $2.8 \mathrm{E}-05$ & $2.8 \mathrm{E}-05$ & $2.6 \mathrm{E}-05$ & $2.3 \mathrm{E}-05$ & $2.2 \mathrm{E}-05$ \\
$\mathrm{SSE}$ & $2.5 \mathrm{E}-05$ & $2.5 \mathrm{E}-05$ & $2.5 \mathrm{E}-05$ & $2.5 \mathrm{E}-05$ & $2.3 \mathrm{E}-05$ & $2.1 \mathrm{E}-05$ & $2.0 \mathrm{E}-05$ \\
$\mathrm{SE}$ & $1.0 \mathrm{E}-05$ & $1.0 \mathrm{E}-05$ & $1.0 \mathrm{E}-05$ & $9.9 \mathrm{E}-06$ & $9.2 \mathrm{E}-06$ & $8.3 \mathrm{E}-06$ & $7.8 \mathrm{E}-06$ \\
$\mathrm{ESE}$ & $6.3 \mathrm{E}-06$ & $6.3 \mathrm{E}-06$ & $6.2 \mathrm{E}-06$ & $6.1 \mathrm{E}-06$ & $5.7 \mathrm{E}-06$ & $5.1 \mathrm{E}-06$ & $4.8 \mathrm{E}-06$ \\
$\mathrm{E}$ & $7.9 \mathrm{E}-06$ & $7.9 \mathrm{E}-06$ & $7.8 \mathrm{E}-06$ & $7.6 \mathrm{E}-06$ & $7.2 \mathrm{E}-06$ & $6.4 \mathrm{E}-06$ & $6.1 \mathrm{E}-06$ \\
$\mathrm{ENE}$ & $8.6 \mathrm{E}-06$ & $8.5 \mathrm{E}-06$ & $8.4 \mathrm{E}-06$ & $8.3 \mathrm{E}-06$ & $7.8 \mathrm{E}-06$ & $7.0 \mathrm{E}-06$ & $6.6 \mathrm{E}-06$ \\
$\mathrm{NE}$ & $1.4 \mathrm{E}-05$ & $1.4 \mathrm{E}-05$ & $1.4 \mathrm{E}-05$ & $1.4 \mathrm{E}-05$ & $1.3 \mathrm{E}-05$ & $1.2 \mathrm{E}-05$ & $1.1 \mathrm{E}-05$ \\
$\mathrm{NNE}$ & $8.0 \mathrm{E}-06$ & $8.0 \mathrm{E}-06$ & $7.9 \mathrm{E}-06$ & $7.8 \mathrm{E}-06$ & $7.3 \mathrm{E}-06$ & $6.6 \mathrm{E}-06$ & $6.2 \mathrm{E}-06$ \\
\hline
\end{tabular}

Distance (m)

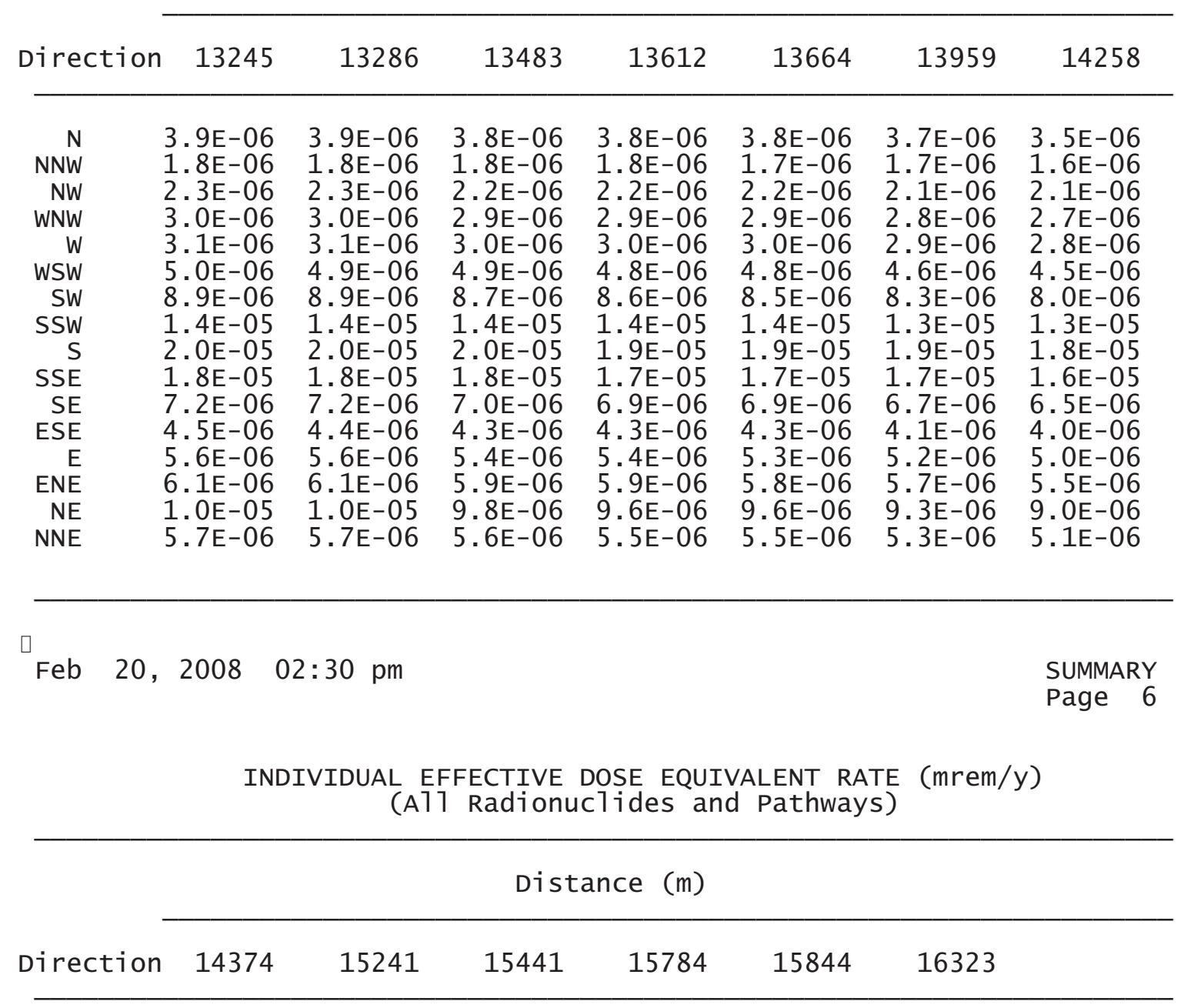

Page 4 


\begin{tabular}{|c|c|c|c|c|c|c|}
\hline & & & & $9 \mathrm{~A}$ & SUM & \\
\hline $\mathrm{N}$ & $3.5 \mathrm{E}-06$ & $3.2 \mathrm{E}-06$ & $3.2 \mathrm{E}-06$ & $3.1 \mathrm{E}-06$ & $3.0 \mathrm{E}-06$ & $2.9 \mathrm{E}-06$ \\
\hline NNW & $1.6 \mathrm{E}-06$ & $1.5 \mathrm{E}-06$ & 1. $5 \mathrm{E}-06$ & $1.4 \mathrm{E}-06$ & 1. $4 \mathrm{E}-06$ & 1. $4 \mathrm{E}-06$ \\
\hline NW & $2.0 \mathrm{E}-06$ & $1.9 \mathrm{E}-06$ & $1.8 \mathrm{E}-06$ & $1.8 \mathrm{E}-06$ & $1.8 \mathrm{E}-06$ & $1.7 \mathrm{E}-06$ \\
\hline WNW & $2.7 \mathrm{E}-06$ & $2.5 \mathrm{E}-06$ & $2.4 \mathrm{E}-06$ & $2.4 \mathrm{E}-06$ & $2.3 \mathrm{E}-06$ & $2.2 \mathrm{E}-06$ \\
\hline & $2.8 \mathrm{E}-06$ & $2.6 \mathrm{E}-06$ & $2.5 \mathrm{E}-06$ & $2.4 \mathrm{E}-06$ & $2.4 \mathrm{E}-06$ & 2. $3 \mathrm{E}-06$ \\
\hline WSW & $4.4 \mathrm{E}-06$ & $4.1 \mathrm{E}-06$ & 4. $0 \mathrm{E}-06$ & $3.9 E-06$ & $3.9 \mathrm{E}-06$ & $3.7 \mathrm{E}-06$ \\
\hline SW & $7.9 \mathrm{E}-06$ & 7. 3E-06 & 7. $2 \mathrm{E}-06$ & $6.9 \mathrm{E}-06$ & $6.9 \mathrm{E}-06$ & $6.6 \mathrm{E}-06$ \\
\hline SSW & 1. $3 \mathrm{E}-05$ & $1.2 \mathrm{E}-05$ & 1. $2 \mathrm{E}-05$ & 1. $1 \mathrm{E}-05$ & $1.1 \mathrm{E}-05$ & $1.1 \mathrm{E}-05$ \\
\hline & $1.8 \mathrm{E}-05$ & $1.6 \mathrm{E}-05$ & 1. $6 \mathrm{E}-05$ & $1.6 \mathrm{E}-05$ & 1. $5 \mathrm{E}-05$ & 1. $5 \mathrm{E}-05$ \\
\hline SSE & $1.6 \mathrm{E}-05$ & 1. $5 \mathrm{E}-05$ & 1. $5 \mathrm{E}-05$ & $1.4 \mathrm{E}$ & $1.4 \mathrm{E}-05$ & 1. $4 \mathrm{E}-05$ \\
\hline SE & $6.4 \mathrm{E}-06$ & $5.9 \mathrm{E}-06$ & $5.8 \mathrm{E}-06$ & $5.6 \mathrm{E}-06$ & $5.5 \mathrm{E}-06$ & $5.3 \mathrm{E}-06$ \\
\hline ESE & 4.0E-06 & $3.6 \mathrm{E}-06$ & $3.6 \mathrm{E}-06$ & $3.4 \mathrm{E}$ & $3.4 \mathrm{E}-06$ & $3 E-06$ \\
\hline & $5.0 \mathrm{E}-06$ & $4.5 \mathrm{E}-06$ & $4.4 \mathrm{E}-06$ & $4.3 \mathrm{E}-06$ & $4.3 \mathrm{E}-06$ & $4.1 \mathrm{E}-06$ \\
\hline ENE & $5.4 \mathrm{E}-06$ & $5.0 \mathrm{E}-06$ & $4.9 \mathrm{E}-06$ & 4. & $4.7 \mathrm{E}-06$ & $4.5 \mathrm{E}-06$ \\
\hline $\mathrm{NE}$ & $8.9 \mathrm{E}-06$ & $8.2 \mathrm{E}-06$ & $8.0 \mathrm{E}-06$ & $7.8 \mathrm{E}-06$ & $7.7 \mathrm{E}-06$ & $7.4 \mathrm{E}-06$ \\
\hline NNE & $5.1 \mathrm{E}-06$ & $4.7 E-06$ & $4.6 \mathrm{E}-06$ & $4.4 \mathrm{E}-06$ & $4.4 \mathrm{E}-06$ & $4.2 \mathrm{E}-06$ \\
\hline
\end{tabular}

Feb 20, 2008 02:30 pm

SUMMARY

Page 7

INDIVIDUAL LIFETIME RISK (deaths)

(A11 Radionuclides and Pathways)

\begin{tabular}{|c|c|c|c|c|c|c|c|}
\hline \multicolumn{8}{|c|}{ Distance $(\mathrm{m})$} \\
\hline Direction & 10344 & 10365 & 10472 & 10590 & 11103 & 11989 & 12522 \\
\hline $\begin{array}{r}\text { N } \\
N N W \\
N W \\
W N W \\
W \\
W S W \\
S W \\
S S W \\
S \\
S S E \\
S E \\
\text { ESE } \\
E \\
\text { ENE } \\
N E \\
\text { NNE }\end{array}$ & $\begin{array}{l}3.0 \mathrm{E}-12 \\
1.4 \mathrm{E}-12 \\
1.7 \mathrm{E}-12 \\
2.2 \mathrm{E}-12 \\
2.4 \mathrm{E}-12 \\
3.8 \mathrm{E}-12 \\
6.8 \mathrm{E}-12 \\
1.1 \mathrm{E}-11 \\
1.5 \mathrm{E}-11 \\
1.4 \mathrm{E}-11 \\
5.5 \mathrm{E}-12 \\
3.4 \mathrm{E}-12 \\
4.3 \mathrm{E}-12 \\
4.6 \mathrm{E}-12 \\
7.7 \mathrm{E}-12 \\
4.3 \mathrm{E}-12\end{array}$ & $\begin{array}{l}3.0 \mathrm{E}-12 \\
1.4 \mathrm{E}-12 \\
1.7 \mathrm{E}-12 \\
2.2 \mathrm{E}-12 \\
2.4 \mathrm{E}-12 \\
3.8 \mathrm{E}-12 \\
6.8 \mathrm{E}-12 \\
1.1 \mathrm{E}-11 \\
1.5 \mathrm{E}-11 \\
1.4 \mathrm{E}-11 \\
5.5 \mathrm{E}-12 \\
3.4 \mathrm{E}-12 \\
4.2 \mathrm{E}-12 \\
4.6 \mathrm{E}-12 \\
7.7 \mathrm{E}-12 \\
4.3 \mathrm{E}-12\end{array}$ & $\begin{array}{l}2.9 \mathrm{E}-12 \\
1.3 \mathrm{E}-12 \\
1.7 \mathrm{E}-12 \\
2.2 \mathrm{E}-12 \\
2.3 \mathrm{E}-12 \\
3.7 \mathrm{E}-12 \\
6.7 \mathrm{E}-12 \\
1.1 \mathrm{E}-11 \\
1.5 \mathrm{E}-11 \\
1.3 \mathrm{E}-11 \\
5.4 \mathrm{E}-12 \\
3.3 \mathrm{E}-12 \\
4.2 \mathrm{E}-12 \\
4.5 \mathrm{E}-12 \\
7.5 \mathrm{E}-12 \\
4.3 \mathrm{E}-12\end{array}$ & $\begin{array}{l}2.9 \mathrm{E}-12 \\
1.3 \mathrm{E}-12 \\
1.6 \mathrm{E}-12 \\
2.2 \mathrm{E}-12 \\
2.3 \mathrm{E}-12 \\
3.7 \mathrm{E}-12 \\
6.6 \mathrm{E}-12 \\
1.1 \mathrm{E}-11 \\
1.5 \mathrm{E}-11 \\
1.3 \mathrm{E}-11 \\
5.3 \mathrm{E}-12 \\
3.3 \mathrm{E}-12 \\
4.1 \mathrm{E}-12 \\
4.5 \mathrm{E}-12 \\
7.4 \mathrm{E}-12 \\
4.2 \mathrm{E}-12\end{array}$ & $\begin{array}{l}2.7 \mathrm{E}-12 \\
1.2 \mathrm{E}-12 \\
1.5 \mathrm{E}-12 \\
2.0 \mathrm{E}-12 \\
2.1 \mathrm{E}-12 \\
3.4 \mathrm{E}-12 \\
6.1 \mathrm{E}-12 \\
9.9 \mathrm{E}-12 \\
1.4 \mathrm{E}-11 \\
1.2 \mathrm{E}-11 \\
5.0 \mathrm{E}-12 \\
3.1 \mathrm{E}-12 \\
3.9 \mathrm{E}-12 \\
4.2 \mathrm{E}-12 \\
6.9 \mathrm{E}-12 \\
3.9 \mathrm{E}-12\end{array}$ & $\begin{array}{l}2.4 \mathrm{E}-12 \\
1.1 \mathrm{E}-12 \\
1.4 \mathrm{E}-12 \\
1.9 \mathrm{E}-12 \\
1.9 \mathrm{E}-12 \\
3.1 \mathrm{E}-12 \\
5.5 \mathrm{E}-12 \\
8.9 \mathrm{E}-12 \\
1.3 \mathrm{E}-11 \\
1.1 \mathrm{E}-11 \\
4.5 \mathrm{E}-12 \\
2.8 \mathrm{E}-12 \\
3.5 \mathrm{E}-12 \\
3.8 \mathrm{E}-12 \\
6.2 \mathrm{E}-12 \\
3.5 \mathrm{E}-12\end{array}$ & $\begin{array}{l}2.3 \mathrm{E}-12 \\
1.1 \mathrm{E}-12 \\
1.3 \mathrm{E}-12 \\
1.7 \mathrm{E}-12 \\
1.8 \mathrm{E}-12 \\
2.9 \mathrm{E}-12 \\
5.2 \mathrm{E}-12 \\
8.4 \mathrm{E}-12 \\
1.2 \mathrm{E}-11 \\
1.1 \mathrm{E}-11 \\
4.2 \mathrm{E}-12 \\
2.6 \mathrm{E}-12 \\
3.3 \mathrm{E}-12 \\
3.6 \mathrm{E}-12 \\
5.9 \mathrm{E}-12 \\
3.3 \mathrm{E}-12\end{array}$ \\
\hline \multicolumn{8}{|c|}{ Distance (m) } \\
\hline Direction & 13245 & 13286 & 13483 & 13612 & 13664 & 13959 & 14258 \\
\hline $\begin{array}{r}N \\
N N W \\
N W \\
W N W \\
W \\
W S W\end{array}$ & $\begin{array}{l}2.1 \mathrm{E}-12 \\
9.8 \mathrm{E}-13 \\
1.2 \mathrm{E}-12 \\
1.6 \mathrm{E}-12 \\
1.7 \mathrm{E}-12 \\
2.7 \mathrm{E}-12\end{array}$ & $\begin{array}{l}2.1 \mathrm{E}-12 \\
9.8 \mathrm{E}-13 \\
1.2 \mathrm{E}-12 \\
1.6 \mathrm{E}-12 \\
1.7 \mathrm{E}-12 \\
2.7 \mathrm{E}-12\end{array}$ & $\begin{array}{l}2.1 \mathrm{E}-12 \\
9.6 \mathrm{E}-13 \\
1.2 \mathrm{E}-12 \\
1.6 \mathrm{E}-12 \\
1.6 \mathrm{E}-12 \\
2.6 \mathrm{E}-12\end{array}$ & $\begin{array}{r}2.0 \mathrm{E}-12 \\
9.4 \mathrm{E}-13 \\
1.2 \mathrm{E}-12 \\
1.6 \mathrm{E}-12 \\
1.6 \mathrm{E}-12 \\
2.6 \mathrm{E}-12 \\
\text { Page }\end{array}$ & $\begin{array}{l}2.0 \mathrm{E}-12 \\
9.4 \mathrm{E}-13 \\
1.2 \mathrm{E}-12 \\
1.6 \mathrm{E}-12 \\
1.6 \mathrm{E}-12 \\
2.6 \mathrm{E}-12 \\
5\end{array}$ & $\begin{array}{l}2.0 \mathrm{E}-12 \\
9.1 \mathrm{E}-13 \\
1.1 \mathrm{E}-12 \\
1.5 \mathrm{E}-12 \\
1.6 \mathrm{E}-12 \\
2.5 \mathrm{E}-12\end{array}$ & $\begin{array}{l}1.9 \mathrm{E}-12 \\
8.9 \mathrm{E}-13 \\
1.1 \mathrm{E}-12 \\
1.5 \mathrm{E}-12 \\
1.5 \mathrm{E}-12 \\
2.4 \mathrm{E}-12\end{array}$ \\
\hline
\end{tabular}




\begin{tabular}{rlllllll}
\multicolumn{7}{c}{$\mathrm{C} 1-39 \mathrm{~A} . \mathrm{SUM}$} \\
$\mathrm{SW}$ & $4.8 \mathrm{E}-12$ & $4.8 \mathrm{E}-12$ & $4.7 \mathrm{E}-12$ & $4.6 \mathrm{E}-12$ & $4.6 \mathrm{E}-12$ & $4.5 \mathrm{E}-12$ & $4.3 \mathrm{E}-12$ \\
SSW & $7.7 \mathrm{E}-12$ & $7.7 \mathrm{E}-12$ & $7.6 \mathrm{E}-12$ & $7.5 \mathrm{E}-12$ & $7.4 \mathrm{E}-12$ & $7.2 \mathrm{E}-12$ & $7.0 \mathrm{E}-12$ \\
$\mathrm{~S}$ & $1.1 \mathrm{E}-11$ & $1.1 \mathrm{E}-11$ & $1.1 \mathrm{E}-11$ & $1.0 \mathrm{E}-11$ & $1.0 \mathrm{E}-11$ & $1.0 \mathrm{E}-11$ & $9.7 \mathrm{E}-12$ \\
SSE & $9.8 \mathrm{E}-12$ & $9.7 \mathrm{E}-12$ & $9.5 \mathrm{E}-12$ & $9.4 \mathrm{E}-12$ & $9.4 \mathrm{E}-12$ & $9.1 \mathrm{E}-12$ & $8.8 \mathrm{E}-12$ \\
SE & $3.9 \mathrm{E}-12$ & $3.9 \mathrm{E}-12$ & $3.8 \mathrm{E}-12$ & $3.7 \mathrm{E}-12$ & $3.7 \mathrm{E}-12$ & $3.6 \mathrm{E}-12$ & $3.5 \mathrm{E}-12$ \\
$\mathrm{ESE}$ & $2.4 \mathrm{E}-12$ & $2.4 \mathrm{E}-12$ & $2.3 \mathrm{E}-12$ & $2.3 \mathrm{E}-12$ & $2.3 \mathrm{E}-12$ & $2.2 \mathrm{E}-12$ & $2.2 \mathrm{E}-12$ \\
$\mathrm{E}$ & $3.0 \mathrm{E}-12$ & $3.0 \mathrm{E}-12$ & $2.9 \mathrm{E}-12$ & $2.9 \mathrm{E}-12$ & $2.9 \mathrm{E}-12$ & $2.8 \mathrm{E}-12$ & $2.7 \mathrm{E}-12$ \\
$\mathrm{ENE}$ & $3.3 \mathrm{E}-12$ & $3.3 \mathrm{E}-12$ & $3.2 \mathrm{E}-12$ & $3.2 \mathrm{E}-12$ & $3.1 \mathrm{E}-12$ & $3.0 \mathrm{E}-12$ & $3.0 \mathrm{E}-12$ \\
NE & $5.4 \mathrm{E}-12$ & $5.4 \mathrm{E}-12$ & $5.3 \mathrm{E}-12$ & $5.2 \mathrm{E}-12$ & $5.2 \mathrm{E}-12$ & $5.0 \mathrm{E}-12$ & $4.9 \mathrm{E}-12$ \\
NNE & $3.1 \mathrm{E}-12$ & $3.1 \mathrm{E}-12$ & $3.0 \mathrm{E}-12$ & $3.0 \mathrm{E}-12$ & $2.9 \mathrm{E}-12$ & $2.9 \mathrm{E}-12$ & $2.8 \mathrm{E}-12$
\end{tabular}

Feb 20, $2008 \quad 02: 30$ pm

SUMMARY

Page 8

INDIVIDUAL LIFETIME RISK (deaths)

(A11 Radionuclides and Pathways)

\begin{tabular}{|c|c|c|c|c|c|c|}
\hline \multirow[b]{2}{*}{ Direction } & \multicolumn{6}{|c|}{ Distance $(\mathrm{m})$} \\
\hline & n 14374 & 15241 & 15441 & 15784 & 15844 & 16323 \\
\hline $\begin{array}{r}N \\
N N W \\
N W \\
W N W \\
W \\
W S W \\
\text { SW } \\
\text { SSW } \\
S \\
\text { SSE } \\
\text { SE } \\
\text { ESE } \\
\text { E } \\
\text { ENE } \\
N E \\
\text { NNE }\end{array}$ & $\begin{array}{l}1.9 \mathrm{E}-12 \\
8.8 \mathrm{E}-13 \\
1.1 \mathrm{E}-12 \\
1.4 \mathrm{E}-12 \\
1.5 \mathrm{E}-12 \\
2.4 \mathrm{E}-12 \\
4.3 \mathrm{E}-12 \\
6.9 \mathrm{E}-12 \\
9.6 \mathrm{E}-12 \\
8.7 \mathrm{E}-12 \\
3.4 \mathrm{E}-12 \\
2.1 \mathrm{E}-12 \\
2.7 \mathrm{E}-12 \\
2.9 \mathrm{E}-12 \\
4.8 \mathrm{E}-12 \\
2.7 \mathrm{E}-12\end{array}$ & $\begin{array}{l}1.7 \mathrm{E}-12 \\
8.1 \mathrm{E}-13 \\
1.0 \mathrm{E}-12 \\
1.3 \mathrm{E}-12 \\
1.4 \mathrm{E}-12 \\
2.2 \mathrm{E}-12 \\
3.9 \mathrm{E}-12 \\
6.3 \mathrm{E}-12 \\
8.8 \mathrm{E}-12 \\
8.0 \mathrm{E}-12 \\
3.2 \mathrm{E}-12 \\
2.0 \mathrm{E}-12 \\
2.4 \mathrm{E}-12 \\
2.7 \mathrm{E}-12 \\
4.4 \mathrm{E}-12 \\
2.5 \mathrm{E}-12\end{array}$ & $\begin{array}{l}1.7 \mathrm{E}-12 \\
8.0 \mathrm{E}-13 \\
9.9 \mathrm{E}-13 \\
1.3 \mathrm{E}-12 \\
1.3 \mathrm{E}-12 \\
2.2 \mathrm{E}-12 \\
3.9 \mathrm{E}-12 \\
6.2 \mathrm{E}-12 \\
8.7 \mathrm{E}-12 \\
7.9 \mathrm{E}-12 \\
3.1 \mathrm{E}-12 \\
1.9 \mathrm{E}-12 \\
2.4 \mathrm{E}-12 \\
2.6 \mathrm{E}-12 \\
4.3 \mathrm{E}-12 \\
2.5 \mathrm{E}-12\end{array}$ & $\begin{array}{l}1.6 \mathrm{E}-12 \\
7.7 \mathrm{E}-13 \\
9.6 \mathrm{E}-13 \\
1.3 \mathrm{E}-12 \\
1.3 \mathrm{E}-12 \\
2.1 \mathrm{E}-12 \\
3.7 \mathrm{E}-12 \\
6.0 \mathrm{E}-12 \\
8.4 \mathrm{E}-12 \\
7.6 \mathrm{E}-12 \\
3.0 \mathrm{E}-12 \\
1.9 \mathrm{E}-12 \\
2.3 \mathrm{E}-12 \\
2.6 \mathrm{E}-12 \\
4.2 \mathrm{E}-12 \\
2.4 \mathrm{E}-12\end{array}$ & $\begin{array}{l}1.6 \mathrm{E}-12 \\
7.7 \mathrm{E}-13 \\
9.5 \mathrm{E}-13 \\
1.3 \mathrm{E}-12 \\
1.3 \mathrm{E}-12 \\
2.1 \mathrm{E}-12 \\
3.7 \mathrm{E}-12 \\
6.0 \mathrm{E}-12 \\
8.3 \mathrm{E}-12 \\
7.6 \mathrm{E}-12 \\
3.0 \mathrm{E}-12 \\
1.8 \mathrm{E}-12 \\
2.3 \mathrm{E}-12 \\
2.5 \mathrm{E}-12 \\
4.2 \mathrm{E}-12 \\
2.4 \mathrm{E}-12\end{array}$ & $\begin{array}{l}1.6 \mathrm{E}-12 \\
7.4 \mathrm{E}-13 \\
9.1 \mathrm{E}-13 \\
1.2 \mathrm{E}-12 \\
1.2 \mathrm{E}-12 \\
2.0 \mathrm{E}-12 \\
3.6 \mathrm{E}-12 \\
5.7 \mathrm{E}-12 \\
8.0 \mathrm{E}-12 \\
7.3 \mathrm{E}-12 \\
2.8 \mathrm{E}-12 \\
1.8 \mathrm{E}-12 \\
2.2 \mathrm{E}-12 \\
2.4 \mathrm{E}-12 \\
4.0 \mathrm{E}-12 \\
2.3 \mathrm{E}-12\end{array}$ \\
\hline
\end{tabular}




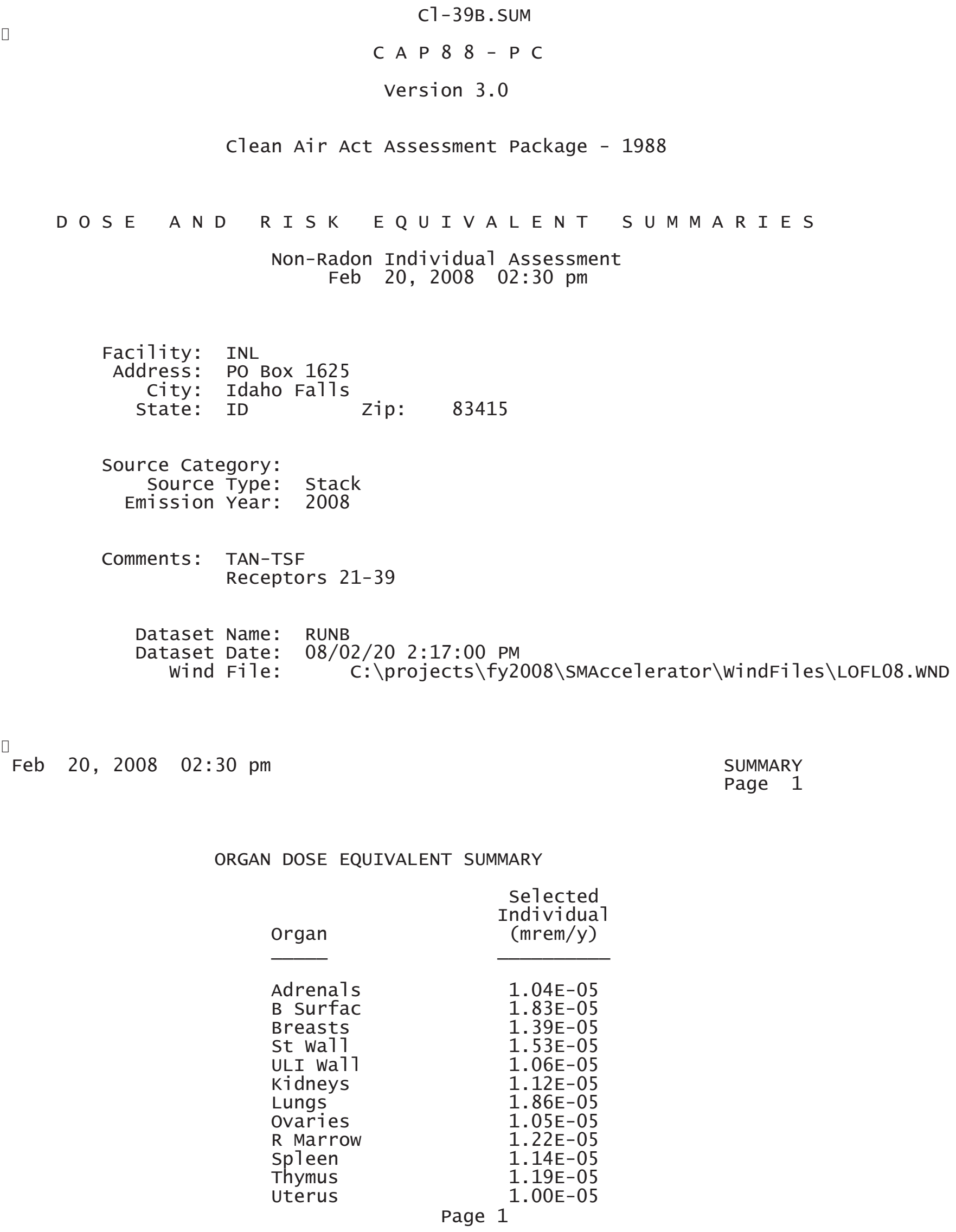

\begin{tabular}{lr} 
ORGAN DOSE EQUIVALENT SUMMARY \\
Selected \\
Organ & $\begin{array}{r}\text { Indididua } \\
\text { (mrem/y) }\end{array}$ \\
\cline { 2 - 2 } Adrena1s & $1.04 \mathrm{E}-05$ \\
B Surfac & $1.83 \mathrm{E}-05$ \\
Breasts & $1.39 \mathrm{E}-05$ \\
St wa11 & $1.53 \mathrm{E}-05$ \\
ULI wa11 & $1.06 \mathrm{E}-05$ \\
Kidneys & $1.12 \mathrm{E}-05$ \\
Lungs & $1.86 \mathrm{E}-05$ \\
Ovaries & $1.05 \mathrm{E}-05$ \\
R Marrow & $1.22 \mathrm{E}-05$ \\
Spleen & $1.14 \mathrm{E}-05$ \\
Thymus & $1.19 \mathrm{E}-05$ \\
Uterus & $1.00 \mathrm{E}-05$ \\
& 1
\end{tabular}




\begin{tabular}{lr} 
& \multicolumn{1}{c}{ C1-39B. SUM } \\
B1d wa11 & $1.04 \mathrm{E}-05$ \\
Brain & $1.32 \mathrm{E}-05$ \\
Esophagu & $3.65 \mathrm{E}-05$ \\
SI wa11 & $1.03 \mathrm{E}-05$ \\
LLI wa11 & $1.04 \mathrm{E}-05$ \\
Liver & $1.13 \mathrm{E}-05$ \\
Muscle & $1.21 \mathrm{E}-05$ \\
Pancreas & $1.03 \mathrm{E}-05$ \\
Skin & $2.34 \mathrm{E}-05$ \\
Testes & $1.22 \mathrm{E}-05$ \\
Thyroid & $1.27 \mathrm{E}-05$ \\
& \\
EFFEC & $1.39 \mathrm{E}-05$
\end{tabular}

PATHWAY EFFECTIVE DOSE EQUIVALENT SUMMARY

\begin{tabular}{lc} 
Pathway & $\begin{array}{c}\text { Selected } \\
\text { Individua1 } \\
\text { (mrem/y) }\end{array}$ \\
\cline { 2 - 2 } & \\
INGESTION & $0.00 \mathrm{E}+00$ \\
INHALATION & $2.02 \mathrm{E}-06$ \\
AIR IMMERSION & $1.18 \mathrm{E}-05$ \\
GROUND SURFACE & $0.00 \mathrm{E}+00$ \\
INTERNAL & $2.02 \mathrm{E}-06$ \\
EXTERNAL & $1.18 \mathrm{E}-05$ \\
TOTAL & $1.39 \mathrm{E}-05$
\end{tabular}

Feb 20, $2008 \quad 02: 30$ pm

SUMMARY

Page 2

NUCLIDE EFFECTIVE DOSE EQUIVALENT SUMMARY

Nuclide

C1-39

Ar-39

TOTAL

口

Feb 20, $2008 \quad 02: 30 \mathrm{pm}$

$$
\begin{gathered}
\begin{array}{c}
\text { Selected } \\
\text { Individua1 } \\
\text { (mrem/y) }
\end{array} \\
\hline \begin{array}{l}
1.39 \mathrm{E}-05 \\
1.55 \mathrm{E}-15 \\
1.39 \mathrm{E}-05
\end{array}
\end{gathered}
$$

SUMMARY

Page 3

CANCER RISK SUMMARY

Cancer

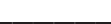

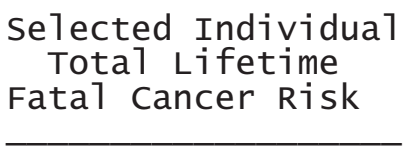

Page 2 


$\begin{array}{lr} & \text { C1-39B. SUM } \\ \text { Esophagu } & 1.24 \mathrm{E}-13 \\ \text { Stomach } & 6.81 \mathrm{E}-13 \\ \text { Colon } & 1.09 \mathrm{E}-12 \\ \text { Liver } & 1.72 \mathrm{E}-13 \\ \text { LUNG } & 1.86 \mathrm{E}-12 \\ \text { Bone } & 1.75 \mathrm{E}-14 \\ \text { Skin } & 2.34 \mathrm{E}-14 \\ \text { Breast } & 6.75 \mathrm{E}-13 \\ \text { Ovary } & 1.50 \mathrm{E}-13 \\ \text { B ladder } & 2.53 \mathrm{E}-13 \\ \text { Kidneys } & 5.84 \mathrm{E}-14 \\ \text { Thyroid } & 4.04 \mathrm{E}-14 \\ \text { Leukemia } & 6.88 \mathrm{E}-13 \\ \text { Residual } & 1.64 \mathrm{E}-12 \\ \text { Total } & 7.46 \mathrm{E}-12 \\ & \\ \text { TOTAL } & 1.49 \mathrm{E}-11\end{array}$

PATHWAY RISK SUMMARY

Pathway

INGESTION

INHALATION

AIR IMMERSION

GROUND SURFACE

INTERNAL

EXTERNAL

TOTAL

口

Feb 20, 2008 02:30 pm
Selected Individual

Total Lifetime

Fatal Cancer Risk

$0.00 \mathrm{E}+00$

$9.88 \mathrm{E}-13$

$6.48 \mathrm{E}-12$

$0.00 \mathrm{E}+00$

$9.88 \mathrm{E}-13$

$6.48 \mathrm{E}-12$

$7.46 \mathrm{E}-12$

NUCLIDE RISK SUMMARY

Nuclide

C1-39

Ar-39

TOTAL

Feb 20, 2008 02:30 pm
Selected Individual Total Lifetime Fatal Cancer Risk

$7.46 \mathrm{E}-12$

$1.96 \mathrm{E}-22$

$7.46 \mathrm{E}-12$

INDIVIDUAL EFFECTIVE DOSE EQUIVALENT RATE (mrem/y) (A11 Radionuclides and Pathways) 
C1-39B. SUM

\begin{tabular}{rrrrrrrr}
\cline { 2 - 7 } Direction & 17035 & 17329 & 18607 & 18834 & 18860 & 18890 & 19860 \\
\hline N & $2.7 \mathrm{E}-06$ & $2.7 \mathrm{E}-06$ & $2.4 \mathrm{E}-06$ & $2.3 \mathrm{E}-06$ & $2.3 \mathrm{E}-06$ & $2.3 \mathrm{E}-06$ & $2.1 \mathrm{E}-06$ \\
NNW & $1.3 \mathrm{E}-06$ & $1.3 \mathrm{E}-06$ & $1.1 \mathrm{E}-06$ & $1.1 \mathrm{E}-06$ & $1.1 \mathrm{E}-06$ & $1.1 \mathrm{E}-06$ & $1.0 \mathrm{E}-06$ \\
$\mathrm{NW}$ & $1.6 \mathrm{E}-06$ & $1.6 \mathrm{E}-06$ & $1.4 \mathrm{E}-06$ & $1.4 \mathrm{E}-06$ & $1.4 \mathrm{E}-06$ & $1.4 \mathrm{E}-06$ & $1.3 \mathrm{E}-06$ \\
WNW & $2.1 \mathrm{E}-06$ & $2.1 \mathrm{E}-06$ & $1.8 \mathrm{E}-06$ & $1.8 \mathrm{E}-06$ & $1.8 \mathrm{E}-06$ & $1.8 \mathrm{E}-06$ & $1.7 \mathrm{E}-06$ \\
$\mathrm{~W}$ & $2.2 \mathrm{E}-06$ & $2.1 \mathrm{E}-06$ & $1.9 \mathrm{E}-06$ & $1.8 \mathrm{E}-06$ & $1.8 \mathrm{E}-06$ & $1.8 \mathrm{E}-06$ & $1.7 \mathrm{E}-06$ \\
WSW & $3.5 \mathrm{E}-06$ & $3.4 \mathrm{E}-06$ & $3.1 \mathrm{E}-06$ & $3.0 \mathrm{E}-06$ & $3.0 \mathrm{E}-06$ & $3.0 \mathrm{E}-06$ & $2.8 \mathrm{E}-06$ \\
$\mathrm{SW}$ & $6.2 \mathrm{E}-06$ & $6.1 \mathrm{E}-06$ & $5.5 \mathrm{E}-06$ & $5.4 \mathrm{E}-06$ & $5.3 \mathrm{E}-06$ & $5.3 \mathrm{E}-06$ & $4.9 \mathrm{E}-06$ \\
SSW & $1.0 \mathrm{E}-05$ & $9.7 \mathrm{E}-06$ & $8.7 \mathrm{E}-06$ & $8.6 \mathrm{E}-06$ & $8.5 \mathrm{E}-06$ & $8.5 \mathrm{E}-06$ & $7.9 \mathrm{E}-06$ \\
$\mathrm{~S}$ & $1.4 \mathrm{E}-05$ & $1.4 \mathrm{E}-05$ & $1.2 \mathrm{E}-05$ & $1.2 \mathrm{E}-05$ & $1.2 \mathrm{E}-05$ & $1.2 \mathrm{E}-05$ & $1.1 \mathrm{E}-05$ \\
SSE & $1.3 \mathrm{E}-05$ & $1.2 \mathrm{E}-05$ & $1.1 \mathrm{E}-05$ & $1.1 \mathrm{E}-05$ & $1.1 \mathrm{E}-05$ & $1.1 \mathrm{E}-05$ & $1.0 \mathrm{E}-05$ \\
$\mathrm{SE}$ & $5.0 \mathrm{E}-06$ & $4.8 \mathrm{E}-06$ & $4.3 \mathrm{E}-06$ & $4.2 \mathrm{E}-06$ & $4.2 \mathrm{E}-06$ & $4.2 \mathrm{E}-06$ & $3.8 \mathrm{E}-06$ \\
ESE & $3.1 \mathrm{E}-06$ & $3.0 \mathrm{E}-06$ & $2.6 \mathrm{E}-06$ & $2.6 \mathrm{E}-06$ & $2.6 \mathrm{E}-06$ & $2.6 \mathrm{E}-06$ & $2.4 \mathrm{E}-06$ \\
$\mathrm{E}$ & $3.8 \mathrm{E}-06$ & $3.7 \mathrm{E}-06$ & $3.3 \mathrm{E}-06$ & $3.2 \mathrm{E}-06$ & $3.2 \mathrm{E}-06$ & $3.2 \mathrm{E}-06$ & $2.9 \mathrm{E}-06$ \\
$\mathrm{ENE}$ & $4.2 \mathrm{E}-06$ & $4.1 \mathrm{E}-06$ & $3.7 \mathrm{E}-06$ & $3.6 \mathrm{E}-06$ & $3.6 \mathrm{E}-06$ & $3.6 \mathrm{E}-06$ & $3.3 \mathrm{E}-06$ \\
$\mathrm{NE}$ & $6.9 \mathrm{E}-06$ & $6.8 \mathrm{E}-06$ & $6.1 \mathrm{E}-06$ & $5.9 \mathrm{E}-06$ & $5.9 \mathrm{E}-06$ & $5.9 \mathrm{E}-06$ & $5.5 \mathrm{E}-06$ \\
$\mathrm{NNE}$ & $4.0 \mathrm{E}-06$ & $3.9 \mathrm{E}-06$ & $3.5 \mathrm{E}-06$ & $3.4 \mathrm{E}-06$ & $3.4 \mathrm{E}-06$ & $3.4 \mathrm{E}-06$ & $3.1 \mathrm{E}-06$ \\
\hline
\end{tabular}

Distance (m)

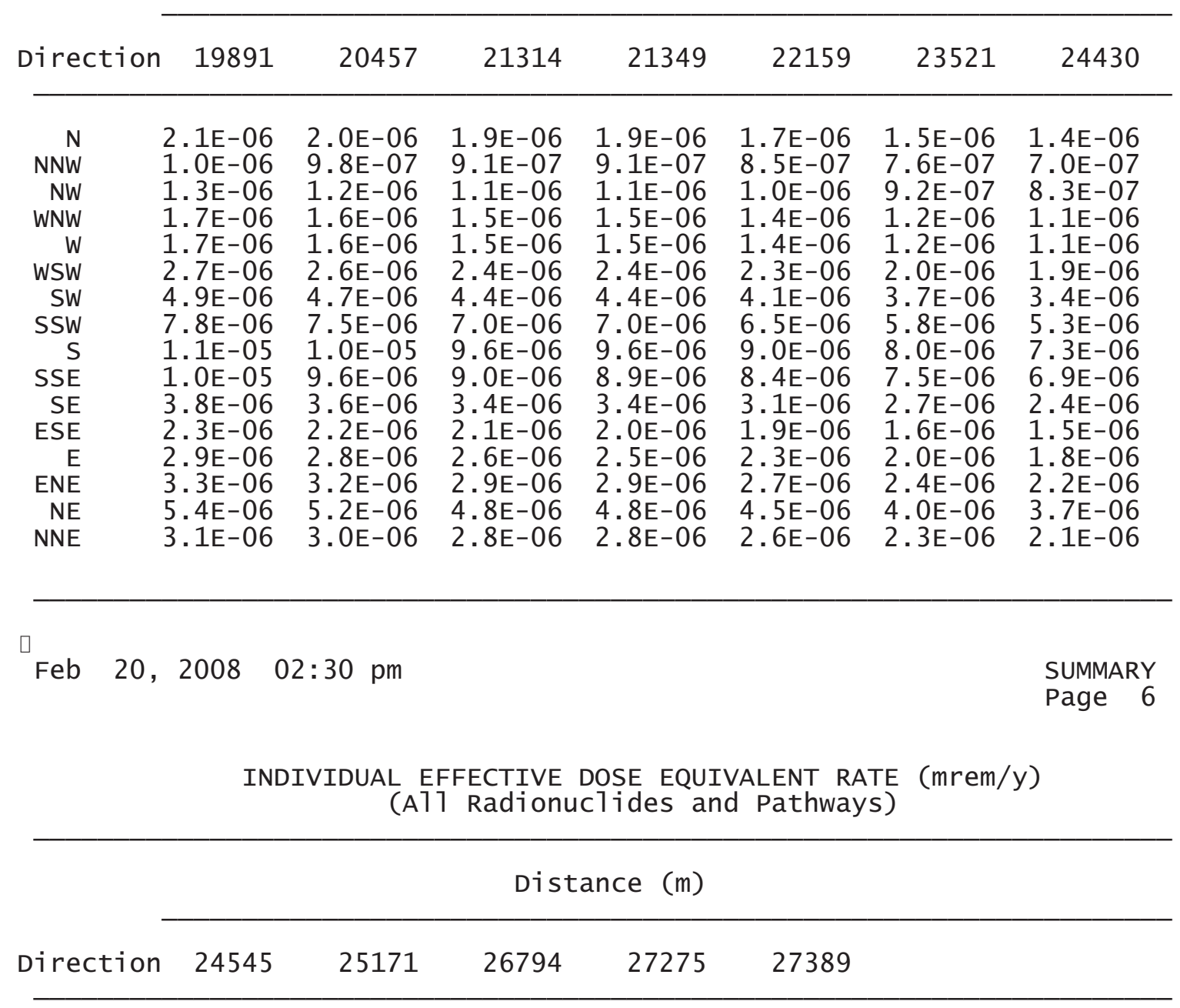

Page 4 


\begin{tabular}{rlllll} 
& & & \multicolumn{3}{c}{$\mathrm{C} 1-39 \mathrm{~B} . \mathrm{SUM}$} \\
$\mathrm{N}$ & $1.3 \mathrm{E}-06$ & $1.2 \mathrm{E}-06$ & $1.1 \mathrm{E}-06$ & $1.1 \mathrm{E}-06$ & $1.1 \mathrm{E}-06$ \\
$\mathrm{NNW}$ & $6.9 \mathrm{E}-07$ & $6.5 \mathrm{E}-07$ & $6.0 \mathrm{E}-07$ & $5.8 \mathrm{E}-07$ & $5.8 \mathrm{E}-07$ \\
$\mathrm{NW}$ & $8.2 \mathrm{E}-07$ & $7.6 \mathrm{E}-07$ & $7.0 \mathrm{E}-07$ & $6.8 \mathrm{E}-07$ & $6.8 \mathrm{E}-07$ \\
$\mathrm{WNW}$ & $1.1 \mathrm{E}-06$ & $1.0 \mathrm{E}-06$ & $9.2 \mathrm{E}-07$ & $9.0 \mathrm{E}-07$ & $8.9 \mathrm{E}-07$ \\
$\mathrm{~W}$ & $1.1 \mathrm{E}-06$ & $1.0 \mathrm{E}-06$ & $9.1 \mathrm{E}-07$ & $8.9 \mathrm{E}-07$ & $8.8 \mathrm{E}-07$ \\
$\mathrm{WSW}$ & $1.8 \mathrm{E}-06$ & $1.7 \mathrm{E}-06$ & $1.6 \mathrm{E}-06$ & $1.5 \mathrm{E}-06$ & $1.5 \mathrm{E}-06$ \\
$\mathrm{SW}$ & $3.3 \mathrm{E}-06$ & $3.1 \mathrm{E}-06$ & $2.9 \mathrm{E}-06$ & $2.8 \mathrm{E}-06$ & $2.8 \mathrm{E}-06$ \\
$\mathrm{SSW}$ & $5.3 \mathrm{E}-06$ & $4.9 \mathrm{E}-06$ & $4.5 \mathrm{E}-06$ & $4.4 \mathrm{E}-06$ & $4.4 \mathrm{E}-06$ \\
$\mathrm{~S}$ & $7.2 \mathrm{E}-06$ & $6.8 \mathrm{E}-06$ & $6.2 \mathrm{E}-06$ & $6.0 \mathrm{E}-06$ & $6.0 \mathrm{E}-06$ \\
$\mathrm{SSE}$ & $6.9 \mathrm{E}-06$ & $6.5 \mathrm{E}-06$ & $5.9 \mathrm{E}-06$ & $5.8 \mathrm{E}-06$ & $5.8 \mathrm{E}-06$ \\
$\mathrm{SE}$ & $2.4 \mathrm{E}-06$ & $2.2 \mathrm{E}-06$ & $2.0 \mathrm{E}-06$ & $2.0 \mathrm{E}-06$ & $2.0 \mathrm{E}-06$ \\
$\mathrm{ESE}$ & $1.4 \mathrm{E}-06$ & $1.3 \mathrm{E}-06$ & $1.2 \mathrm{E}-06$ & $1.2 \mathrm{E}-06$ & $1.2 \mathrm{E}-06$ \\
$\mathrm{E}$ & $1.8 \mathrm{E}-06$ & $1.6 \mathrm{E}-06$ & $1.5 \mathrm{E}-06$ & $1.4 \mathrm{E}-06$ & $1.4 \mathrm{E}-06$ \\
$\mathrm{ENE}$ & $2.2 \mathrm{E}-06$ & $2.0 \mathrm{E}-06$ & $1.8 \mathrm{E}-06$ & $1.8 \mathrm{E}-06$ & $1.8 \mathrm{E}-06$ \\
$\mathrm{NE}$ & $3.6 \mathrm{E}-06$ & $3.4 \mathrm{E}-06$ & $3.1 \mathrm{E}-06$ & $3.0 \mathrm{E}-06$ & $3.0 \mathrm{E}-06$ \\
$\mathrm{NNE}$ & $2.0 \mathrm{E}-06$ & $1.9 \mathrm{E}-06$ & $1.7 \mathrm{E}-06$ & $1.7 \mathrm{E}-06$ & $1.7 \mathrm{E}-06$
\end{tabular}

Feb 20, 2008 02:30 pm

SUMMARY

Page 7

INDIVIDUAL LIFETIME RISK (deaths)

(A11 Radionuclides and Pathways)

\begin{tabular}{|c|c|c|c|c|c|c|c|}
\hline \multirow[b]{2}{*}{ Direction } & \multicolumn{6}{|c|}{ Distance $(\mathrm{m})$} & \multirow[b]{2}{*}{19860} \\
\hline & 17035 & 17329 & 18607 & 18834 & 18860 & 18890 & \\
\hline $\begin{array}{r}N \\
N N W \\
N W \\
W N W \\
W \\
W S W \\
\text { SW } \\
\text { SSW } \\
S \\
\text { SSE } \\
\text { SE } \\
\text { ESE } \\
\text { E } \\
\text { ENE } \\
N E \\
\text { NNE }\end{array}$ & $\begin{array}{l}1.5 \mathrm{E}-12 \\
6.9 \mathrm{E}-13 \\
8.6 \mathrm{E}-13 \\
1.1 \mathrm{E}-12 \\
1.2 \mathrm{E}-12 \\
1.9 \mathrm{E}-12 \\
3.3 \mathrm{E}-12 \\
5.4 \mathrm{E}-12 \\
7.5 \mathrm{E}-12 \\
6.8 \mathrm{E}-12 \\
2.7 \mathrm{E}-12 \\
1.6 \mathrm{E}-12 \\
2.1 \mathrm{E}-12 \\
2.3 \mathrm{E}-12 \\
3.7 \mathrm{E}-12 \\
2.1 \mathrm{E}-12\end{array}$ & $\begin{array}{l}1.4 \mathrm{E}-12 \\
6.8 \mathrm{E}-13 \\
8.4 \mathrm{E}-13 \\
1.1 \mathrm{E}-12 \\
1.1 \mathrm{E}-12 \\
1.8 \mathrm{E}-12 \\
3.3 \mathrm{E}-12 \\
5.2 \mathrm{E}-12 \\
7.3 \mathrm{E}-12 \\
6.7 \mathrm{E}-12 \\
2.6 \mathrm{E}-12 \\
1.6 \mathrm{E}-12 \\
2.0 \mathrm{E}-12 \\
2.2 \mathrm{E}-12 \\
3.6 \mathrm{E}-12 \\
2.1 \mathrm{E}-12\end{array}$ & $\begin{array}{l}1.3 \mathrm{E}-12 \\
6.1 \mathrm{E}-13 \\
7.5 \mathrm{E}-13 \\
9.9 \mathrm{E}-13 \\
1.0 \mathrm{E}-12 \\
1.6 \mathrm{E}-12 \\
2.9 \mathrm{E}-12 \\
4.7 \mathrm{E}-12 \\
6.5 \mathrm{E}-12 \\
6.0 \mathrm{E}-12 \\
2.3 \mathrm{E}-12 \\
1.4 \mathrm{E}-12 \\
1.8 \mathrm{E}-12 \\
2.0 \mathrm{E}-12 \\
3.3 \mathrm{E}-12 \\
1.9 \mathrm{E}-12\end{array}$ & $\begin{array}{l}1.3 \mathrm{E}-12 \\
6.0 \mathrm{E}-13 \\
7.4 \mathrm{E}-13 \\
9.7 \mathrm{E}-13 \\
9.9 \mathrm{E}-13 \\
1.6 \mathrm{E}-12 \\
2.9 \mathrm{E}-12 \\
4.6 \mathrm{E}-12 \\
6.4 \mathrm{E}-12 \\
5.9 \mathrm{E}-12 \\
2.3 \mathrm{E}-12 \\
1.4 \mathrm{E}-12 \\
1.7 \mathrm{E}-12 \\
1.9 \mathrm{E}-12 \\
3.2 \mathrm{E}-12 \\
1.8 \mathrm{E}-12\end{array}$ & $\begin{array}{l}1.2 \mathrm{E}-12 \\
6.0 \mathrm{E}-13 \\
7.4 \mathrm{E}-13 \\
9.7 \mathrm{E}-13 \\
9.9 \mathrm{E}-13 \\
1.6 \mathrm{E}-12 \\
2.9 \mathrm{E}-12 \\
4.6 \mathrm{E}-12 \\
6.4 \mathrm{E}-12 \\
5.9 \mathrm{E}-12 \\
2.3 \mathrm{E}-12 \\
1.4 \mathrm{E}-12 \\
1.7 \mathrm{E}-12 \\
1.9 \mathrm{E}-12 \\
3.2 \mathrm{E}-12 \\
1.8 \mathrm{E}-12\end{array}$ & $\begin{array}{l}1.2 \mathrm{E}-12 \\
6.0 \mathrm{E}-13 \\
7.3 \mathrm{E}-13 \\
9.7 \mathrm{E}-13 \\
9.9 \mathrm{E}-13 \\
1.6 \mathrm{E}-12 \\
2.9 \mathrm{E}-12 \\
4.6 \mathrm{E}-12 \\
6.3 \mathrm{E}-12 \\
5.9 \mathrm{E}-12 \\
2.3 \mathrm{E}-12 \\
1.4 \mathrm{E}-12 \\
1.7 \mathrm{E}-12 \\
1.9 \mathrm{E}-12 \\
3.2 \mathrm{E}-12 \\
1.8 \mathrm{E}-12\end{array}$ & $\begin{array}{l}1.1 \mathrm{E}-12 \\
5.5 \mathrm{E}-13 \\
6.8 \mathrm{E}-13 \\
9.0 \mathrm{E}-13 \\
9.1 \mathrm{E}-13 \\
1.5 \mathrm{E}-12 \\
2.7 \mathrm{E}-12 \\
4.2 \mathrm{E}-12 \\
5.8 \mathrm{E}-12 \\
5.4 \mathrm{E}-12 \\
2.1 \mathrm{E}-12 \\
1.3 \mathrm{E}-12 \\
1.6 \mathrm{E}-12 \\
1.8 \mathrm{E}-12 \\
2.9 \mathrm{E}-12 \\
1.7 \mathrm{E}-12\end{array}$ \\
\hline
\end{tabular}

Distance (m)

\begin{tabular}{llllllll}
\cline { 2 - 5 } Direction & 19891 & 20457 & 21314 & 21349 & 22159 & 23521 & 24430 \\
\hline
\end{tabular}

$\begin{array}{rrrrrrrr}N & 1.1 \mathrm{E}-12 & 1.1 \mathrm{E}-12 & 1.0 \mathrm{E}-12 & 1.0 \mathrm{E}-12 & 9.3 \mathrm{E}-13 & 8.1 \mathrm{E}-13 & 7.3 \mathrm{E}-13 \\ \mathrm{~N} & 5.5 \mathrm{E}-13 & 5.3 \mathrm{E}-13 & 4.9 \mathrm{E}-13 & 4.9 \mathrm{E}-13 & 4.6 \mathrm{E}-13 & 4.1 \mathrm{E}-13 & 3.8 \mathrm{E}-13 \\ \mathrm{NW} & 6.8 \mathrm{E}-13 & 6.4 \mathrm{E}-13 & 6.0 \mathrm{E}-13 & 6.0 \mathrm{E}-13 & 5.6 \mathrm{E}-13 & 4.9 \mathrm{E}-13 & 4.5 \mathrm{E}-13 \\ \text { WNW } & 8.9 \mathrm{E}-13 & 8.5 \mathrm{E}-13 & 7.9 \mathrm{E}-13 & 7.9 \mathrm{E}-13 & 7.4 \mathrm{E}-13 & 6.5 \mathrm{E}-13 & 5.9 \mathrm{E}-13 \\ \text { W } & 9.1 \mathrm{E}-13 & 8.6 \mathrm{E}-13 & 8.0 \mathrm{E}-13 & 8.0 \mathrm{E}-13 & 7.4 \mathrm{E}-13 & 6.5 \mathrm{E}-13 & 5.9 \mathrm{E}-13 \\ \text { WSW } & 1.5 \mathrm{E}-12 & 1.4 \mathrm{E}-12 & 1.3 \mathrm{E}-12 & \begin{array}{c}1.3 \mathrm{E}-12 \\ \text { Page }\end{array} & 1.2 \mathrm{E}-12 & 1.1 \mathrm{E}-12 & 1.0 \mathrm{E}-12\end{array}$




\begin{tabular}{rlllllll}
\multicolumn{7}{c}{$\mathrm{C} 1-39 \mathrm{~B} \cdot \mathrm{SUM}$} \\
$\mathrm{SW}$ & $2.6 \mathrm{E}-12$ & $2.5 \mathrm{E}-12$ & $2.4 \mathrm{E}-12$ & $2.4 \mathrm{E}-12$ & $2.2 \mathrm{E}-12$ & $2.0 \mathrm{E}-12$ & $1.8 \mathrm{E}-12$ \\
SSW & $4.2 \mathrm{E}-12$ & $4.0 \mathrm{E}-12$ & $3.8 \mathrm{E}-12$ & $3.7 \mathrm{E}-12$ & $3.5 \mathrm{E}-12$ & $3.1 \mathrm{E}-12$ & $2.9 \mathrm{E}-12$ \\
$\mathrm{~S}$ & $5.8 \mathrm{E}-12$ & $5.6 \mathrm{E}-12$ & $5.2 \mathrm{E}-12$ & $5.2 \mathrm{E}-12$ & $4.8 \mathrm{E}-12$ & $4.3 \mathrm{E}-12$ & $3.9 \mathrm{E}-12$ \\
SSE & $5.4 \mathrm{E}-12$ & $5.2 \mathrm{E}-12$ & $4.8 \mathrm{E}-12$ & $4.8 \mathrm{E}-12$ & $4.5 \mathrm{E}-12$ & $4.0 \mathrm{E}-12$ & $3.7 \mathrm{E}-12$ \\
SE & $2.1 \mathrm{E}-12$ & $2.0 \mathrm{E}-12$ & $1.8 \mathrm{E}-12$ & $1.8 \mathrm{E}-12$ & $1.7 \mathrm{E}-12$ & $1.5 \mathrm{E}-12$ & $1.3 \mathrm{E}-12$ \\
$\mathrm{ESE}$ & $1.3 \mathrm{E}-12$ & $1.2 \mathrm{E}-12$ & $1.1 \mathrm{E}-12$ & $1.1 \mathrm{E}-12$ & $1.0 \mathrm{E}-12$ & $8.8 \mathrm{E}-13$ & $7.9 \mathrm{E}-13$ \\
$\mathrm{E}$ & $1.6 \mathrm{E}-12$ & $1.5 \mathrm{E}-12$ & $1.4 \mathrm{E}-12$ & $1.4 \mathrm{E}-12$ & $1.3 \mathrm{E}-12$ & $1.1 \mathrm{E}-12$ & $9.7 \mathrm{E}-13$ \\
$\mathrm{ENE}$ & $1.8 \mathrm{E}-12$ & $1.7 \mathrm{E}-12$ & $1.6 \mathrm{E}-12$ & $1.6 \mathrm{E}-12$ & $1.5 \mathrm{E}-12$ & $1.3 \mathrm{E}-12$ & $1.2 \mathrm{E}-12$ \\
$\mathrm{NE}$ & $2.9 \mathrm{E}-12$ & $2.8 \mathrm{E}-12$ & $2.6 \mathrm{E}-12$ & $2.6 \mathrm{E}-12$ & $2.4 \mathrm{E}-12$ & $2.2 \mathrm{E}-12$ & $2.0 \mathrm{E}-12$ \\
NNE & $1.7 \mathrm{E}-12$ & $1.6 \mathrm{E}-12$ & $1.5 \mathrm{E}-12$ & $1.5 \mathrm{E}-12$ & $1.4 \mathrm{E}-12$ & $1.2 \mathrm{E}-12$ & $1.1 \mathrm{E}-12$
\end{tabular}

$\square$

Feb 20, $200802: 30$ pm SUMMARY

Page 8

INDIVIDUAL LIFETIME RISK (deaths)

(A11 Radionuclides and Pathways)

\begin{tabular}{|c|c|c|c|c|c|}
\hline \multirow[b]{2}{*}{ Direction } & \multicolumn{5}{|c|}{ Distance $(\mathrm{m})$} \\
\hline & 24545 & 25171 & 26794 & 27275 & 27389 \\
\hline $\begin{array}{r}N \\
N N W \\
N W \\
W N W \\
W \\
W S W \\
\text { SW } \\
\text { SSW } \\
S \\
\text { SSE } \\
\text { SE } \\
\text { ESE } \\
\text { E } \\
\text { ENE } \\
\text { NE } \\
\text { NNE }\end{array}$ & $\begin{array}{l}7.2 \mathrm{E}-13 \\
3.7 \mathrm{E}-13 \\
4.4 \mathrm{E}-13 \\
5.8 \mathrm{E}-13 \\
5.8 \mathrm{E}-13 \\
9.9 \mathrm{E}-13 \\
1.8 \mathrm{E}-12 \\
2.8 \mathrm{E}-12 \\
3.9 \mathrm{E}-12 \\
3.7 \mathrm{E}-12 \\
1.3 \mathrm{E}-12 \\
7.7 \mathrm{E}-13 \\
9.5 \mathrm{E}-13 \\
1.2 \mathrm{E}-12 \\
2.0 \mathrm{E}-12 \\
1.1 \mathrm{E}-12\end{array}$ & $\begin{array}{l}6.7 \mathrm{E}-13 \\
3.5 \mathrm{E}-13 \\
4.1 \mathrm{E}-13 \\
5.4 \mathrm{E}-13 \\
5.4 \mathrm{E}-13 \\
9.3 \mathrm{E}-13 \\
1.7 \mathrm{E}-12 \\
2.7 \mathrm{E}-12 \\
3.6 \mathrm{E}-12 \\
3.5 \mathrm{E}-12 \\
1.2 \mathrm{E}-12 \\
7 \cdot 1 \mathrm{E}-13 \\
8.7 \mathrm{E}-13 \\
1.1 \mathrm{E}-12 \\
1.8 \mathrm{E}-12 \\
1.0 \mathrm{E}-12\end{array}$ & $\begin{array}{l}6.1 \mathrm{E}-13 \\
3.2 \mathrm{E}-13 \\
3.8 \mathrm{E}-13 \\
5.0 \mathrm{E}-13 \\
4.9 \mathrm{E}-13 \\
8.5 \mathrm{E}-13 \\
1.5 \mathrm{E}-12 \\
2.4 \mathrm{E}-12 \\
3.3 \mathrm{E}-12 \\
3.2 \mathrm{E}-12 \\
1.1 \mathrm{E}-12 \\
6.5 \mathrm{E}-13 \\
7.9 \mathrm{E}-13 \\
9.9 \mathrm{E}-13 \\
1.7 \mathrm{E}-12 \\
9.4 \mathrm{E}-13\end{array}$ & $\begin{array}{l}5.9 \mathrm{E}-13 \\
3.1 \mathrm{E}-13 \\
3.7 \mathrm{E}-13 \\
4.8 \mathrm{E}-13 \\
4.8 \mathrm{E}-13 \\
8.3 \mathrm{E}-13 \\
1.5 \mathrm{E}-12 \\
2.4 \mathrm{E}-12 \\
3.2 \mathrm{E}-12 \\
3.1 \mathrm{E}-12 \\
1.1 \mathrm{E}-12 \\
6.3 \mathrm{E}-13 \\
7.7 \mathrm{E}-13 \\
9.6 \mathrm{E}-13 \\
1.6 \mathrm{E}-12 \\
9.1 \mathrm{E}-13\end{array}$ & $\begin{array}{l}5.9 \mathrm{E}-13 \\
3.1 \mathrm{E}-13 \\
3.6 \mathrm{E}-13 \\
4.8 \mathrm{E}-13 \\
4.8 \mathrm{E}-13 \\
8.2 \mathrm{E}-13 \\
1.5 \mathrm{E}-12 \\
2.4 \mathrm{E}-12 \\
3.2 \mathrm{E}-12 \\
3.1 \mathrm{E}-12 \\
1.1 \mathrm{E}-12 \\
6.2 \mathrm{E}-13 \\
7.6 \mathrm{E}-13 \\
9.5 \mathrm{E}-13 \\
1.6 \mathrm{E}-12 \\
9.1 \mathrm{E}-13\end{array}$ \\
\hline
\end{tabular}




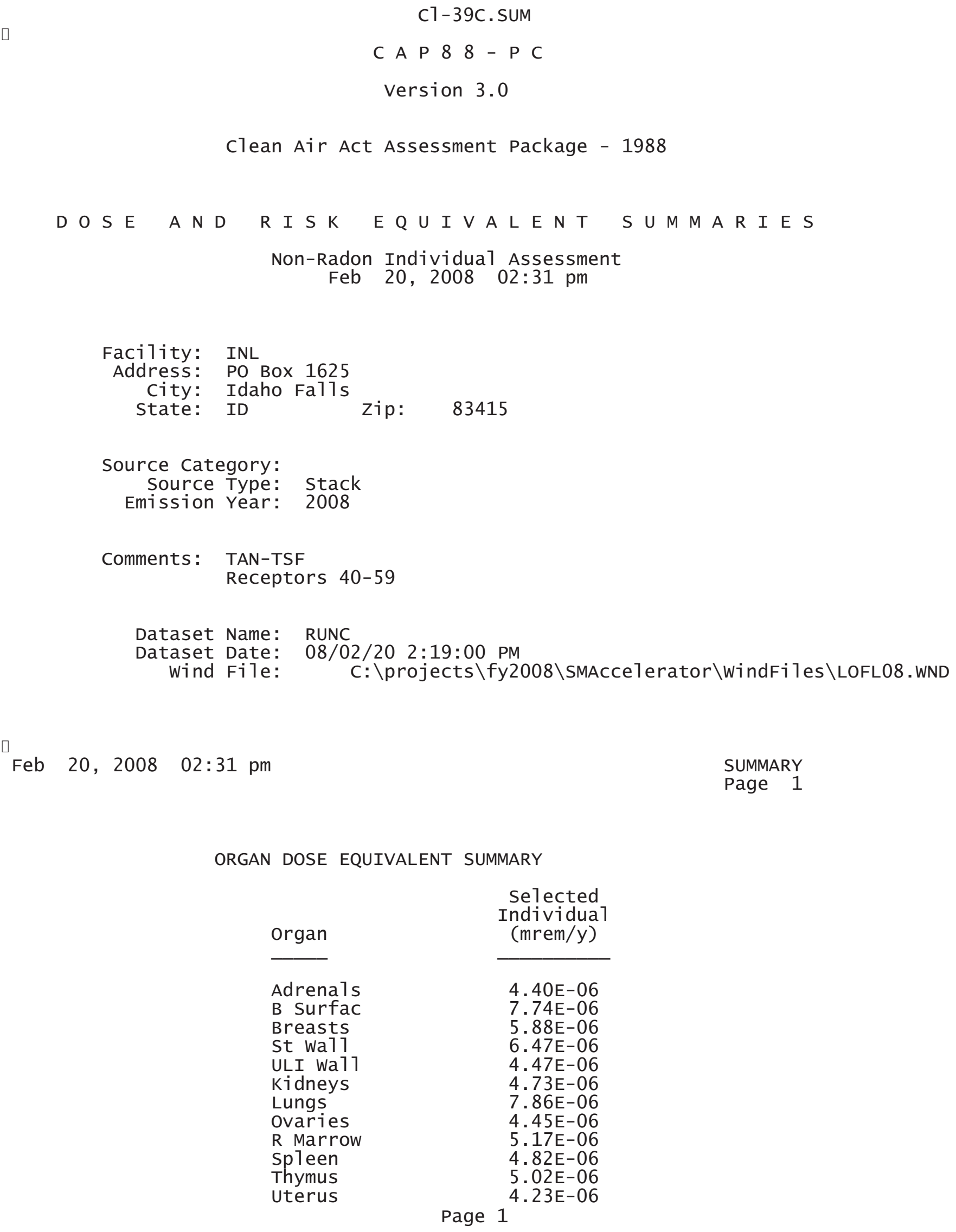




\begin{tabular}{lr} 
& \multicolumn{1}{c}{ C1-39C.SUM } \\
B1d wa11 & $4.40 \mathrm{E}-06$ \\
Brain & $5.60 \mathrm{E}-06$ \\
Esophagu & $1.54 \mathrm{E}-05$ \\
SI wa11 & $4.37 \mathrm{E}-06$ \\
LLI wa11 & $4.39 \mathrm{E}-06$ \\
Liver & $4.78 \mathrm{E}-06$ \\
Muscle & $5.11 \mathrm{E}-06$ \\
Pancreas & $4.35 \mathrm{E}-06$ \\
Skin & $9.89 \mathrm{E}-06$ \\
Testes & $5.16 \mathrm{E}-06$ \\
Thyroid & $5.35 \mathrm{E}-06$ \\
& \\
EFFEC & $5.86 \mathrm{E}-06$
\end{tabular}

PATHWAY EFFECTIVE DOSE EQUIVALENT SUMMARY

Selected Individual

Pathway (mrem/y)

INGESTION

$0.00 \mathrm{E}+00$

INHALATION

$8.55 \mathrm{E}-07$

AIR IMMERSION

GROUND SURFACE

5. $01 \mathrm{E}-06$

INTERNAL

$0.00 \mathrm{E}+00$

EXTERNAL

$8.55 \mathrm{E}-07$

5. $01 \mathrm{E}-06$

TOTAL

$5.86 \mathrm{E}-06$

$\square$

Feb 20, 2008 02:31 pm

SUMMARY

Page 2

NUCLIDE EFFECTIVE DOSE EQUIVALENT SUMMARY

Nuclide

C1-39

Ar-39

TOTAL

$$
\begin{gathered}
\begin{array}{c}
\text { Selected } \\
\text { Individual } \\
(\mathrm{mrem} / \mathrm{y})
\end{array} \\
\hline 5.86 \mathrm{E}-06 \\
8.64 \mathrm{E}-16 \\
5.86 \mathrm{E}-06
\end{gathered}
$$

口

Feb 20, $2008 \quad 02: 31 \mathrm{pm}$

SUMMARY

Page 3

CANCER RISK SUMMARY

Cancer

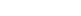

Selected Individual

Total Lifetime Fatal Cancer Risk

Page 2 


$\begin{array}{lr} & \mathrm{C} 1-39 \mathrm{C} . \mathrm{SUM} \\ \text { Esophagu } & 5.26 \mathrm{E}-14 \\ \text { Stomach } & 2.88 \mathrm{E}-13 \\ \text { Colon } & 4.61 \mathrm{E}-13 \\ \text { Liver } & 7.27 \mathrm{E}-14 \\ \text { LUNG } & 7.85 \mathrm{E}-13 \\ \text { Bone } & 7.38 \mathrm{E}-15 \\ \text { Skin } & 9.91 \mathrm{E}-15 \\ \text { Breast } & 2.85 \mathrm{E}-13 \\ \text { Ovary } & 6.34 \mathrm{E}-14 \\ \text { B Tadder } & 1.07 \mathrm{E}-13 \\ \text { Kidneys } & 2.47 \mathrm{E}-14 \\ \text { Thyroid } & 1.71 \mathrm{E}-14 \\ \text { Leukemia } & 2.91 \mathrm{E}-13 \\ \text { Residual } & 6.91 \mathrm{E}-13 \\ \text { Total } & 3.16 \mathrm{E}-12 \\ & \\ \text { TOTAL } & 6.31 \mathrm{E}-12\end{array}$

PATHWAY RISK SUMMARY

\begin{tabular}{lc} 
& \multicolumn{2}{c}{$\begin{array}{c}\text { Total Lifetime } \\
\text { Fathway }\end{array}$} \\
\cline { 2 - 2 } & $0.00 \mathrm{E}+00$ \\
INGESTION & $4.17 \mathrm{E}-13$ \\
INHALATION & $2.74 \mathrm{E}-12$ \\
AIR IMMERSION & $0.00 \mathrm{E}+00$ \\
GROUND SURFACE & $4.17 \mathrm{E}-13$ \\
INTERNAL & $2.74 \mathrm{E}-12$ \\
EXTERNAL & $3.16 \mathrm{E}-12$ \\
TOTAL & \\
$31 \mathrm{pm}$ &
\end{tabular}

NUCLIDE RISK SUMMARY

\begin{tabular}{l} 
Nuclide \\
\hline C1-39 \\
Ar-39 \\
TOTAL \\
31 pm
\end{tabular}

Selected Individual Total Lifetime Fatal Cancer Risk

$3.16 \mathrm{E}-12$

$1.10 \mathrm{E}-22$

$3.16 \mathrm{E}-12$

Feb 20, $2008 \quad 02: 31$ pm

INDIVIDUAL EFFECTIVE DOSE EQUIVALENT RATE (mrem/y) (A11 Radionuclides and Pathways) 
C1-39C. SUM

\begin{tabular}{|c|c|c|c|c|c|c|c|}
\hline Direction & 27715 & 28919 & 31060 & 32802 & 34577 & 35279 & 35683 \\
\hline$N$ & $1.1 \mathrm{E}-06$ & $1.0 \mathrm{E}-06$ & $9.1 \mathrm{E}-07$ & $8.3 E-07$ & $7.7 \mathrm{E}-07$ & $7.4 \mathrm{E}-07$ & 7. $3 \mathrm{E}-07$ \\
\hline NNW & $5.7 \mathrm{E}-07$ & $5.4 \mathrm{E}-07$ & $4.9 \mathrm{E}-07$ & $4.5 \mathrm{E}-07$ & $4.2 \mathrm{E}-07$ & $4.0 \mathrm{E}-07$ & $4.0 \mathrm{E}-07$ \\
\hline NW & $6.7 \mathrm{E}-07$ & $6.3 \mathrm{E}-07$ & $5.7 \mathrm{E}-07$ & $5.2 \mathrm{E}-07$ & $4.8 \mathrm{E}-07$ & $4.7 \mathrm{E}-07$ & $4.6 \mathrm{E}-07$ \\
\hline WNW & $8.8 \mathrm{E}-07$ & $8.3 \mathrm{E}-07$ & $7.4 \mathrm{E}-07$ & $6.9 \mathrm{E}-07$ & $6.3 \mathrm{E}-07$ & $6.1 \mathrm{E}-07$ & $6.0 \mathrm{E}-07$ \\
\hline W & $8.7 \mathrm{E}-07$ & $8.2 \mathrm{E}-07$ & $7.3 \mathrm{E}-07$ & $6.7 \mathrm{E}-07$ & $6.2 \mathrm{E}-07$ & $6.0 \mathrm{E}-07$ & $5.9 \mathrm{E}-07$ \\
\hline WSW & $1.5 \mathrm{E}-06$ & $1.4 \mathrm{E}-06$ & 1. $3 \mathrm{E}-06$ & $1.2 \mathrm{E}-06$ & $1.1 \mathrm{E}-06$ & $1.0 \mathrm{E}-06$ & 1. $0 \mathrm{E}-06$ \\
\hline SW & $2.7 \mathrm{E}-06$ & $2.6 \mathrm{E}-06$ & $2.3 \mathrm{E}-06$ & $2.1 \mathrm{E}-06$ & $2.0 \mathrm{E}-06$ & $1.9 \mathrm{E}-06$ & $1.9 \mathrm{E}-06$ \\
\hline SSW & $4.3 \mathrm{E}-06$ & $4.0 \mathrm{E}-06$ & $3.6 \mathrm{E}-06$ & $3.3 \mathrm{E}-06$ & $3.1 \mathrm{E}-06$ & $3.0 \mathrm{E}-06$ & $2.9 \mathrm{E}-06$ \\
\hline $\mathrm{S}$ & $5.9 \mathrm{E}-06$ & $5.5 \mathrm{E}-06$ & $4.9 \mathrm{E}-06$ & $4.5 \mathrm{E}-06$ & $4.2 \mathrm{E}-06$ & $4.0 \mathrm{E}-06$ & 4. $0 \mathrm{E}-06$ \\
\hline SSE & $5.7 \mathrm{E}-06$ & $5.3 \mathrm{E}-06$ & $4.8 \mathrm{E}-06$ & $4.4 \mathrm{E}-06$ & $4.1 \mathrm{E}-06$ & $4.0 \mathrm{E}-06$ & $3.9 \mathrm{E}-06$ \\
\hline SE & $1.9 \mathrm{E}-06$ & $1.8 \mathrm{E}-06$ & $1.6 \mathrm{E}-06$ & $1.5 \mathrm{E}-06$ & $1.4 \mathrm{E}-06$ & $1.3 \mathrm{E}-06$ & 1. $3 \mathrm{E}-06$ \\
\hline ESE & $1.1 \mathrm{E}-06$ & $1.1 \mathrm{E}-06$ & $9.5 \mathrm{E}-07$ & $8.7 \mathrm{E}-07$ & $8.0 \mathrm{E}-07$ & $7.7 \mathrm{E}-07$ & $7.6 \mathrm{E}-07$ \\
\hline & $1.4 \mathrm{E}-06$ & $1.3 \mathrm{E}-06$ & $1.2 \mathrm{E}-06$ & $1.1 \mathrm{E}-06$ & $9.7 \mathrm{E}-07$ & $9.4 \mathrm{E}-07$ & $9.2 \mathrm{E}-07$ \\
\hline ENE & $1.7 \mathrm{E}-06$ & $1.6 \mathrm{E}-06$ & $1.5 \mathrm{E}-06$ & $1.4 \mathrm{E}-06$ & $1.2 \mathrm{E}-06$ & $1.2 \mathrm{E}-06$ & $1.2 \mathrm{E}-06$ \\
\hline $\mathrm{NE}$ & $3.0 \mathrm{E}-06$ & $2.8 \mathrm{E}-06$ & $2.5 \mathrm{E}-06$ & $2.3 \mathrm{E}-06$ & $2.1 \mathrm{E}-06$ & $2.1 \mathrm{E}-06$ & $2.0 \mathrm{E}-06$ \\
\hline NNE & $1.7 \mathrm{E}-06$ & $1.6 \mathrm{E}-06$ & $1.4 \mathrm{E}-06$ & $1.3 \mathrm{E}-06$ & $1.2 \mathrm{E}-06$ & $1.2 \mathrm{E}-06$ & 1. $1 \mathrm{E}-06$ \\
\hline
\end{tabular}

Distance (m)

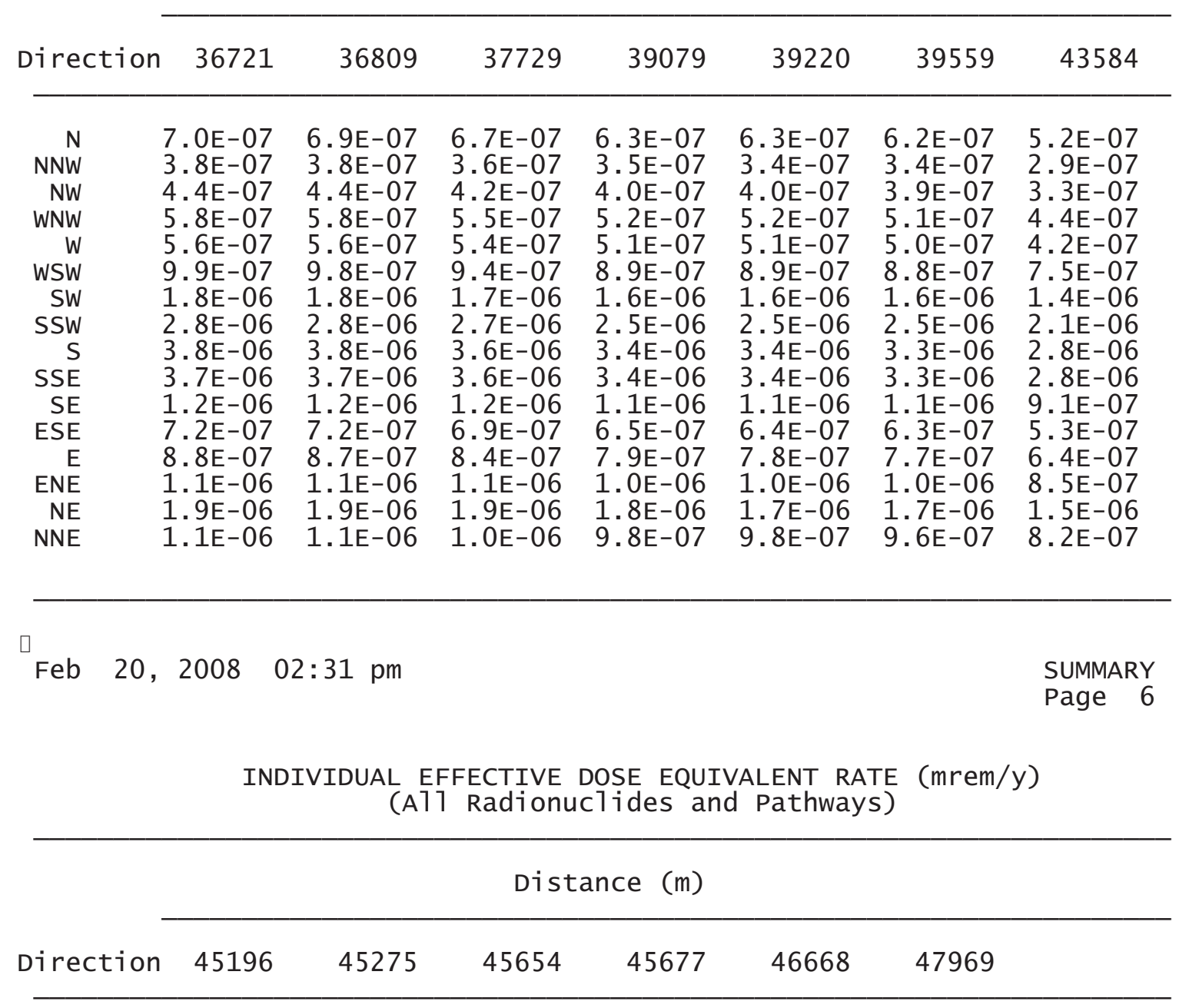

Page 4 


\begin{tabular}{rllllll} 
& \multicolumn{5}{c}{$\mathrm{Cl}-39 \mathrm{C} . \mathrm{SUM}$} \\
$\mathrm{N}$ & $4.9 \mathrm{E}-07$ & $4.9 \mathrm{E}-07$ & $4.8 \mathrm{E}-07$ & $4.8 \mathrm{E}-07$ & $4.6 \mathrm{E}-07$ & $4.4 \mathrm{E}-07$ \\
$\mathrm{NNW}$ & $2.7 \mathrm{E}-07$ & $2.7 \mathrm{E}-07$ & $2.7 \mathrm{E}-07$ & $2.7 \mathrm{E}-07$ & $2.6 \mathrm{E}-07$ & $2.5 \mathrm{E}-07$ \\
$\mathrm{NW}$ & $3.1 \mathrm{E}-07$ & $3.1 \mathrm{E}-07$ & $3.1 \mathrm{E}-07$ & $3.1 \mathrm{E}-07$ & $2.9 \mathrm{E}-07$ & $2.8 \mathrm{E}-07$ \\
WNW & $4.1 \mathrm{E}-07$ & $4.1 \mathrm{E}-07$ & $4.0 \mathrm{E}-07$ & $4.0 \mathrm{E}-07$ & $3.9 \mathrm{E}-07$ & $3.7 \mathrm{E}-07$ \\
$\mathrm{~W}$ & $4.0 \mathrm{E}-07$ & $3.9 \mathrm{E}-07$ & $3.9 \mathrm{E}-07$ & $3.9 \mathrm{E}-07$ & $3.7 \mathrm{E}-07$ & $3.5 \mathrm{E}-07$ \\
WSW & $7.0 \mathrm{E}-07$ & $7.0 \mathrm{E}-07$ & $6.9 \mathrm{E}-07$ & $6.9 \mathrm{E}-07$ & $6.6 \mathrm{E}-07$ & $6.3 \mathrm{E}-07$ \\
$\mathrm{SW}$ & $1.3 \mathrm{E}-06$ & $1.3 \mathrm{E}-06$ & $1.3 \mathrm{E}-06$ & $1.3 \mathrm{E}-06$ & $1.2 \mathrm{E}-06$ & $1.2 \mathrm{E}-06$ \\
SSW & $2.0 \mathrm{E}-06$ & $2.0 \mathrm{E}-06$ & $2.0 \mathrm{E}-06$ & $2.0 \mathrm{E}-06$ & $1.9 \mathrm{E}-06$ & $1.8 \mathrm{E}-06$ \\
$\mathrm{~S}$ & $2.7 \mathrm{E}-06$ & $2.6 \mathrm{E}-06$ & $2.6 \mathrm{E}-06$ & $2.6 \mathrm{E}-06$ & $2.5 \mathrm{E}-06$ & $2.4 \mathrm{E}-06$ \\
SSE & $2.7 \mathrm{E}-06$ & $2.7 \mathrm{E}-06$ & $2.6 \mathrm{E}-06$ & $2.6 \mathrm{E}-06$ & $2.5 \mathrm{E}-06$ & $2.4 \mathrm{E}-06$ \\
$\mathrm{SE}$ & $8.5 \mathrm{E}-07$ & $8.5 \mathrm{E}-07$ & $8.4 \mathrm{E}-07$ & $8.4 \mathrm{E}-07$ & $8.0 \mathrm{E}-07$ & $7.6 \mathrm{E}-07$ \\
ESE & $4.9 \mathrm{E}-07$ & $4.9 \mathrm{E}-07$ & $4.8 \mathrm{E}-07$ & $4.8 \mathrm{E}-07$ & $4.6 \mathrm{E}-07$ & $4.4 \mathrm{E}-07$ \\
$\mathrm{E}$ & $6.0 \mathrm{E}-07$ & $5.9 \mathrm{E}-07$ & $5.8 \mathrm{E}-07$ & $5.8 \mathrm{E}-07$ & $5.6 \mathrm{E}-07$ & $5.3 \mathrm{E}-07$ \\
$\mathrm{ENE}$ & $7.9 \mathrm{E}-07$ & $7.9 \mathrm{E}-07$ & $7.8 \mathrm{E}-07$ & $7.8 \mathrm{E}-07$ & $7.5 \mathrm{E}-07$ & $7.1 \mathrm{E}-07$ \\
$\mathrm{NE}$ & $1.4 \mathrm{E}-06$ & $1.4 \mathrm{E}-06$ & $1.4 \mathrm{E}-06$ & $1.4 \mathrm{E}-06$ & $1.3 \mathrm{E}-06$ & $1.2 \mathrm{E}-06$ \\
$\mathrm{NNE}$ & $7.7 \mathrm{E}-07$ & $7.7 \mathrm{E}-07$ & $7.6 \mathrm{E}-07$ & $7.6 \mathrm{E}-07$ & $7.3 \mathrm{E}-07$ & $7.0 \mathrm{E}-07$ \\
& & & & & &
\end{tabular}

Feb 20, $2008 \quad 02: 31$ pm

SUMMARY

Page 7

INDIVIDUAL LIFETIME RISK (deaths)

(A11 Radionuclides and Pathways)

\begin{tabular}{|c|c|c|c|c|c|c|c|}
\hline \multirow[b]{2}{*}{ Direction } & \multicolumn{6}{|c|}{ Distance $(\mathrm{m})$} & \multirow[b]{2}{*}{35683} \\
\hline & 27715 & 28919 & 31060 & 32802 & 34577 & 35279 & \\
\hline $\begin{array}{r}\text { N } \\
N N W \\
N W \\
W N W \\
W \\
W S W \\
S W \\
S S W \\
S \\
S S E \\
\text { SE } \\
\text { ESE } \\
\text { E } \\
\text { ENE } \\
N E \\
\text { NNE }\end{array}$ & $\begin{array}{l}5.8 \mathrm{E}-13 \\
3.1 \mathrm{E}-13 \\
3.6 \mathrm{E}-13 \\
4.7 \mathrm{E}-13 \\
4.7 \mathrm{E}-13 \\
8.1 \mathrm{E}-13 \\
1.5 \mathrm{E}-12 \\
2.3 \mathrm{E}-12 \\
3.2 \mathrm{E}-12 \\
3.0 \mathrm{E}-12 \\
1.0 \mathrm{E}-12 \\
6.1 \mathrm{E}-13 \\
7.5 \mathrm{E}-13 \\
9.4 \mathrm{E}-13 \\
1.6 \mathrm{E}-12 \\
8.9 \mathrm{E}-13\end{array}$ & $\begin{array}{l}5.4 \mathrm{E}-13 \\
2.9 \mathrm{E}-13 \\
3.4 \mathrm{E}-13 \\
4.4 \mathrm{E}-13 \\
4.4 \mathrm{E}-13 \\
7.6 \mathrm{E}-13 \\
1.4 \mathrm{E}-12 \\
2.2 \mathrm{E}-12 \\
3.0 \mathrm{E}-12 \\
2.9 \mathrm{E}-12 \\
9.7 \mathrm{E}-13 \\
5.7 \mathrm{E}-13 \\
7.0 \mathrm{E}-13 \\
8.8 \mathrm{E}-13 \\
1.5 \mathrm{E}-12 \\
8.4 \mathrm{E}-13\end{array}$ & $\begin{array}{l}4.9 \mathrm{E}-13 \\
2.6 \mathrm{E}-13 \\
3.0 \mathrm{E}-13 \\
4.0 \mathrm{E}-13 \\
3.9 \mathrm{E}-13 \\
6.8 \mathrm{E}-13 \\
1.2 \mathrm{E}-12 \\
2.0 \mathrm{E}-12 \\
2.7 \mathrm{E}-12 \\
2.6 \mathrm{E}-12 \\
8.7 \mathrm{E}-13 \\
5.1 \mathrm{E}-13 \\
6.2 \mathrm{E}-13 \\
7.9 \mathrm{E}-13 \\
1.3 \mathrm{E}-12 \\
7.5 \mathrm{E}-13\end{array}$ & $\begin{array}{l}4.5 \mathrm{E}-13 \\
2.4 \mathrm{E}-13 \\
2.8 \mathrm{E}-13 \\
3.7 \mathrm{E}-13 \\
3.6 \mathrm{E}-13 \\
6.3 \mathrm{E}-13 \\
1.2 \mathrm{E}-12 \\
1.8 \mathrm{E}-12 \\
2.4 \mathrm{E}-12 \\
2.4 \mathrm{E}-12 \\
8.0 \mathrm{E}-13 \\
4.7 \mathrm{E}-13 \\
5.7 \mathrm{E}-13 \\
7.3 \mathrm{E}-13 \\
1.2 \mathrm{E}-12 \\
6.9 \mathrm{E}-13\end{array}$ & $\begin{array}{l}4.1 \mathrm{E}-13 \\
2.2 \mathrm{E}-13 \\
2.6 \mathrm{E}-13 \\
3.4 \mathrm{E}-13 \\
3.3 \mathrm{E}-13 \\
5.8 \mathrm{E}-13 \\
1.1 \mathrm{E}-12 \\
1.7 \mathrm{E}-12 \\
2.2 \mathrm{E}-12 \\
2.2 \mathrm{E}-12 \\
7.3 \mathrm{E}-13 \\
4.3 \mathrm{E}-13 \\
5.2 \mathrm{E}-13 \\
6.7 \mathrm{E}-13 \\
1.1 \mathrm{E}-12 \\
6.4 \mathrm{E}-13\end{array}$ & $\begin{array}{l}4 \cdot 0 \mathrm{E}-13 \\
2 \cdot 2 \mathrm{E}-13 \\
2.5 \mathrm{E}-13 \\
3.3 \mathrm{E}-13 \\
3.2 \mathrm{E}-13 \\
5 \cdot 6 \mathrm{E}-13 \\
1.0 \mathrm{E}-12 \\
1.6 \mathrm{E}-12 \\
2.2 \mathrm{E}-12 \\
2.1 \mathrm{E}-12 \\
7 \cdot 1 \mathrm{E}-13 \\
4 \cdot 2 \mathrm{E}-13 \\
5 \cdot 1 \mathrm{E}-13 \\
6 \cdot 5 \mathrm{E}-13 \\
1.1 \mathrm{E}-12 \\
6 \cdot 2 \mathrm{E}-13\end{array}$ & $\begin{array}{l}3.9 \mathrm{E}-13 \\
2.1 \mathrm{E}-13 \\
2.5 \mathrm{E}-13 \\
3.3 \mathrm{E}-13 \\
3.2 \mathrm{E}-13 \\
5.5 \mathrm{E}-13 \\
1.0 \mathrm{E}-12 \\
1.6 \mathrm{E}-12 \\
2.1 \mathrm{E}-12 \\
2.1 \mathrm{E}-12 \\
7.0 \mathrm{E}-13 \\
4.1 \mathrm{E}-13 \\
5.0 \mathrm{E}-13 \\
6.4 \mathrm{E}-13 \\
1.1 \mathrm{E}-12 \\
6.1 \mathrm{E}-13\end{array}$ \\
\hline
\end{tabular}

Distance (m)

\begin{tabular}{|c|c|c|c|c|c|c|c|}
\hline Direction & 36721 & 36809 & 37729 & 39079 & 39220 & 39559 & 43584 \\
\hline $\begin{array}{r}N \\
N N W \\
N W \\
\text { WNW } \\
W \\
W S W\end{array}$ & $\begin{array}{l}3.8 \mathrm{E}-13 \\
2.0 \mathrm{E}-13 \\
2.4 \mathrm{E}-13 \\
3.1 \mathrm{E}-13 \\
3.0 \mathrm{E}-13 \\
5.3 \mathrm{E}-13\end{array}$ & $\begin{array}{l}3.7 \mathrm{E}-13 \\
2.0 \mathrm{E}-13 \\
2.4 \mathrm{E}-13 \\
3.1 \mathrm{E}-13 \\
3.0 \mathrm{E}-13 \\
5.3 \mathrm{E}-13\end{array}$ & $\begin{array}{l}3.6 \mathrm{E}-13 \\
2.0 \mathrm{E}-13 \\
2.3 \mathrm{E}-13 \\
3.0 \mathrm{E}-13 \\
2.9 \mathrm{E}-13 \\
5.1 \mathrm{E}-13\end{array}$ & $\begin{array}{r}3.4 \mathrm{E}-13 \\
1.9 \mathrm{E}-13 \\
2.1 \mathrm{E}-13 \\
2.8 \mathrm{E}-13 \\
2.7 \mathrm{E}-13 \\
4.8 \mathrm{E}-13 \\
\text { Page }\end{array}$ & $\begin{array}{l}3.4 \mathrm{E}-13 \\
1.8 \mathrm{E}-13 \\
2.1 \mathrm{E}-13 \\
2.8 \mathrm{E}-13 \\
2.7 \mathrm{E}-13 \\
4.8 \mathrm{E}-13\end{array}$ & $\begin{array}{l}3.3 \mathrm{E}-13 \\
1.8 \mathrm{E}-13 \\
2.1 \mathrm{E}-13 \\
2.8 \mathrm{E}-13 \\
2.7 \mathrm{E}-13 \\
4.7 \mathrm{E}-13\end{array}$ & $\begin{array}{l}2.8 \mathrm{E}-13 \\
1.6 \mathrm{E}-13 \\
1.8 \mathrm{E}-13 \\
2.4 \mathrm{E}-13 \\
2.3 \mathrm{E}-13 \\
4.0 \mathrm{E}-13\end{array}$ \\
\hline
\end{tabular}




\begin{tabular}{rlllllll}
\multicolumn{7}{c}{$\mathrm{C} 1-39 \mathrm{C} \cdot \mathrm{SUM}$} \\
SW & $9.7 \mathrm{E}-13$ & $9.7 \mathrm{E}-13$ & $9.3 \mathrm{E}-13$ & $8.8 \mathrm{E}-13$ & $8.7 \mathrm{E}-13$ & $8.6 \mathrm{E}-13$ & $7.4 \mathrm{E}-13$ \\
SSW & $1.5 \mathrm{E}-12$ & $1.5 \mathrm{E}-12$ & $1.4 \mathrm{E}-12$ & $1.4 \mathrm{E}-12$ & $1.4 \mathrm{E}-12$ & $1.3 \mathrm{E}-12$ & $1.1 \mathrm{E}-12$ \\
S & $2.0 \mathrm{E}-12$ & $2.0 \mathrm{E}-12$ & $1.9 \mathrm{E}-12$ & $1.8 \mathrm{E}-12$ & $1.8 \mathrm{E}-12$ & $1.8 \mathrm{E}-12$ & $1.5 \mathrm{E}-12$ \\
SSE & $2.0 \mathrm{E}-12$ & $2.0 \mathrm{E}-12$ & $1.9 \mathrm{E}-12$ & $1.8 \mathrm{E}-12$ & $1.8 \mathrm{E}-12$ & $1.8 \mathrm{E}-12$ & $1.5 \mathrm{E}-12$ \\
SE & $6.6 \mathrm{E}-13$ & $6.6 \mathrm{E}-13$ & $6.3 \mathrm{E}-13$ & $6.0 \mathrm{E}-13$ & $5.9 \mathrm{E}-13$ & $5.8 \mathrm{E}-13$ & $4.9 \mathrm{E}-13$ \\
ESE & $3.9 \mathrm{E}-13$ & $3.9 \mathrm{E}-13$ & $3.7 \mathrm{E}-13$ & $3.5 \mathrm{E}-13$ & $3.5 \mathrm{E}-13$ & $3.4 \mathrm{E}-13$ & $2.9 \mathrm{E}-13$ \\
$\mathrm{E}$ & $4.7 \mathrm{E}-13$ & $4.7 \mathrm{E}-13$ & $4.5 \mathrm{E}-13$ & $4.2 \mathrm{E}-13$ & $4.2 \mathrm{E}-13$ & $4.1 \mathrm{E}-13$ & $3.5 \mathrm{E}-13$ \\
$\mathrm{ENE}$ & $6.1 \mathrm{E}-13$ & $6.1 \mathrm{E}-13$ & $5.8 \mathrm{E}-13$ & $5.5 \mathrm{E}-13$ & $5.5 \mathrm{E}-13$ & $5.4 \mathrm{E}-13$ & $4.6 \mathrm{E}-13$ \\
NE & $1.0 \mathrm{E}-12$ & $1.0 \mathrm{E}-12$ & $1.0 \mathrm{E}-12$ & $9.4 \mathrm{E}-13$ & $9.4 \mathrm{E}-13$ & $9.2 \mathrm{E}-13$ & $7.9 \mathrm{E}-13$ \\
NNE & $5.8 \mathrm{E}-13$ & $5.8 \mathrm{E}-13$ & $5.6 \mathrm{E}-13$ & $5.3 \mathrm{E}-13$ & $5.3 \mathrm{E}-13$ & $5.2 \mathrm{E}-13$ & $4.4 \mathrm{E}-13$
\end{tabular}

Feb 20, $2008 \quad 02: 31$ pm

SUMMARY

Page 8

INDIVIDUAL LIFETIME RISK (deaths)

(A11 Radionuclides and Pathways)

\begin{tabular}{|c|c|c|c|c|c|c|}
\hline \multirow[b]{2}{*}{ Direction } & \multicolumn{6}{|c|}{ Distance $(\mathrm{m})$} \\
\hline & 45196 & 45275 & 45654 & 45677 & 46668 & 47969 \\
\hline $\begin{array}{r}N \\
N N W \\
N W \\
W N W \\
W \\
W S W \\
\text { SW } \\
\text { SSW } \\
S \\
\text { SSE } \\
\text { SE } \\
\text { ESE } \\
\text { E } \\
\text { ENE } \\
N E \\
\text { NNE }\end{array}$ & $\begin{array}{l}2.6 \mathrm{E}-13 \\
1.5 \mathrm{E}-13 \\
1.7 \mathrm{E}-13 \\
2.2 \mathrm{E}-13 \\
2.1 \mathrm{E}-13 \\
3.8 \mathrm{E}-13 \\
6.9 \mathrm{E}-13 \\
1.1 \mathrm{E}-12 \\
1.4 \mathrm{E}-12 \\
1.4 \mathrm{E}-12 \\
4.6 \mathrm{E}-13 \\
2.7 \mathrm{E}-13 \\
3.2 \mathrm{E}-13 \\
4.3 \mathrm{E}-13 \\
7.4 \mathrm{E}-13 \\
4.2 \mathrm{E}-13\end{array}$ & $\begin{array}{l}2.6 \mathrm{E}-13 \\
1.5 \mathrm{E}-13 \\
1.7 \mathrm{E}-13 \\
2.2 \mathrm{E}-13 \\
2.1 \mathrm{E}-13 \\
3.8 \mathrm{E}-13 \\
6.9 \mathrm{E}-13 \\
1.1 \mathrm{E}-12 \\
1.4 \mathrm{E}-12 \\
1.4 \mathrm{E}-12 \\
4.6 \mathrm{E}-13 \\
2.6 \mathrm{E}-13 \\
3.2 \mathrm{E}-13 \\
4.3 \mathrm{E}-13 \\
7.4 \mathrm{E}-13 \\
4.1 \mathrm{E}-13\end{array}$ & $\begin{array}{l}2.6 \mathrm{E}-13 \\
1.5 \mathrm{E}-13 \\
1.7 \mathrm{E}-13 \\
2.2 \mathrm{E}-13 \\
2.1 \mathrm{E}-13 \\
3.7 \mathrm{E}-13 \\
6.8 \mathrm{E}-13 \\
1.1 \mathrm{E}-12 \\
1.4 \mathrm{E}-12 \\
1.4 \mathrm{E}-12 \\
4.5 \mathrm{E}-13 \\
2.6 \mathrm{E}-13 \\
3.1 \mathrm{E}-13 \\
4.2 \mathrm{E}-13 \\
7.3 \mathrm{E}-13 \\
4.1 \mathrm{E}-13\end{array}$ & $\begin{array}{l}2.6 \mathrm{E}-13 \\
1.4 \mathrm{E}-13 \\
1.6 \mathrm{E}-13 \\
2.2 \mathrm{E}-13 \\
2.1 \mathrm{E}-13 \\
3.7 \mathrm{E}-13 \\
6.8 \mathrm{E}-13 \\
1.1 \mathrm{E}-12 \\
1.4 \mathrm{E}-12 \\
1.4 \mathrm{E}-12 \\
4.5 \mathrm{E}-13 \\
2.6 \mathrm{E}-13 \\
3.1 \mathrm{E}-13 \\
4.2 \mathrm{E}-13 \\
7.3 \mathrm{E}-13 \\
4.1 \mathrm{E}-13\end{array}$ & $\begin{array}{l}2.5 \mathrm{E}-13 \\
1.4 \mathrm{E}-13 \\
1.6 \mathrm{E}-13 \\
2.1 \mathrm{E}-13 \\
2.0 \mathrm{E}-13 \\
3.6 \mathrm{E}-13 \\
6.6 \mathrm{E}-13 \\
1.0 \mathrm{E}-12 \\
1.3 \mathrm{E}-12 \\
1.4 \mathrm{E}-12 \\
4.3 \mathrm{E}-13 \\
2.5 \mathrm{E}-13 \\
3.0 \mathrm{E}-13 \\
4.0 \mathrm{E}-13 \\
7.0 \mathrm{E}-13 \\
3.9 \mathrm{E}-13\end{array}$ & $\begin{array}{l}2.4 \mathrm{E}-13 \\
1.3 \mathrm{E}-13 \\
1.5 \mathrm{E}-13 \\
2.0 \mathrm{E}-13 \\
1.9 \mathrm{E}-13 \\
3.4 \mathrm{E}-13 \\
6.3 \mathrm{E}-13 \\
9.6 \mathrm{E}-13 \\
1.3 \mathrm{E}-12 \\
1.3 \mathrm{E}-12 \\
4.1 \mathrm{E}-13 \\
2.3 \mathrm{E}-13 \\
2.8 \mathrm{E}-13 \\
3.8 \mathrm{E}-13 \\
6.7 \mathrm{E}-13 \\
3.7 \mathrm{E}-13\end{array}$ \\
\hline
\end{tabular}




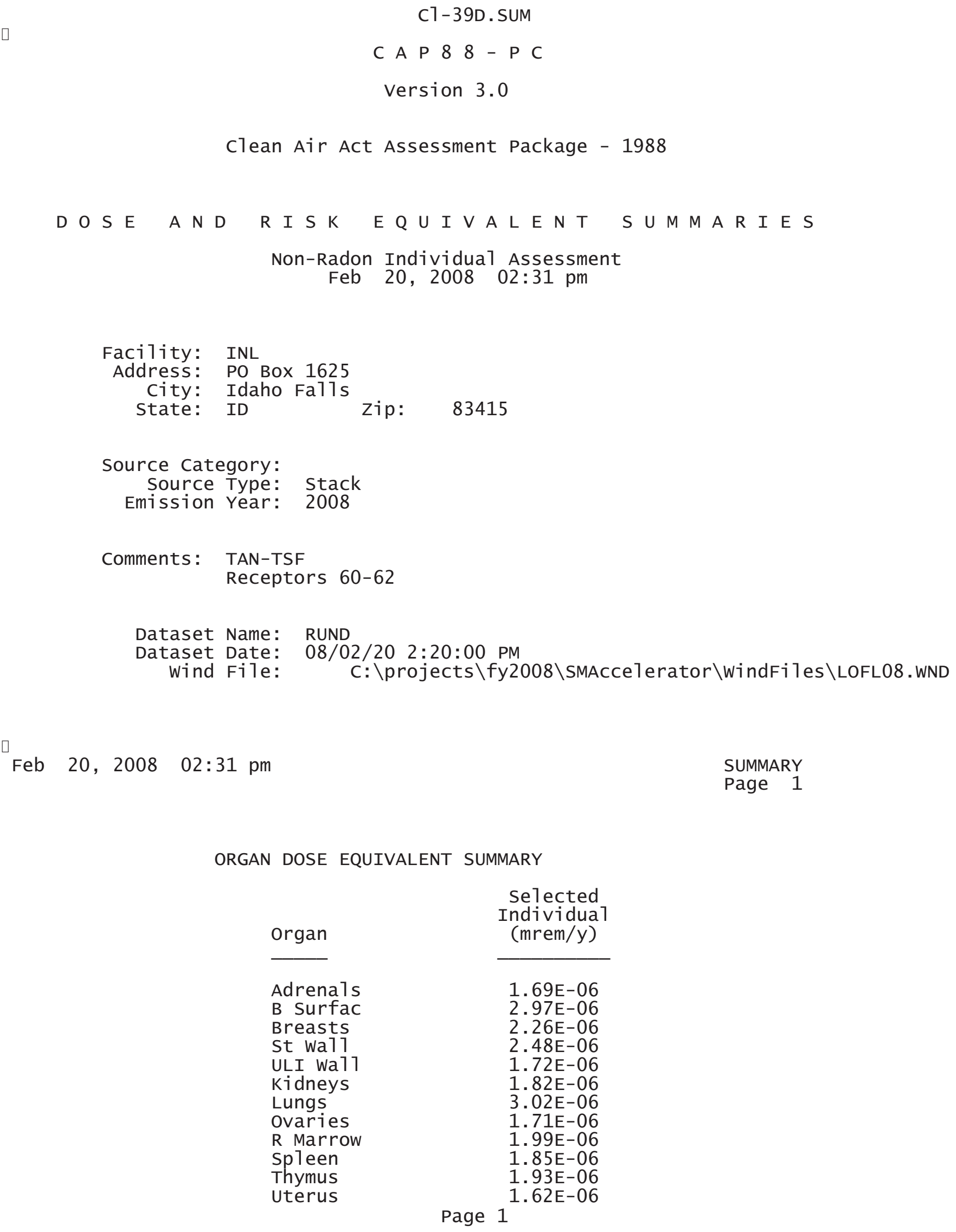

1. 69E-06

2. $97 \mathrm{E}-06$

2. 26E-06

2. $48 \mathrm{E}-06$

1. $72 \mathrm{E}-06$

1. 82E-06

3. $02 \mathrm{E}-06$

1. 71E-06

1. $99 \mathrm{E}-06$

1. 85E-06

1. $93 \mathrm{E}-06$

Page 1 


\begin{tabular}{lr} 
& \multicolumn{1}{c}{ C1-39D. SUM } \\
B1d wa11 & $1.69 \mathrm{E}-06$ \\
Brain & $2.15 \mathrm{E}-06$ \\
Esophagu & $5.93 \mathrm{E}-06$ \\
SI wa11 & $1.68 \mathrm{E}-06$ \\
LLI wa11 & $1.68 \mathrm{E}-06$ \\
Liver & $1.84 \mathrm{E}-06$ \\
Muscle & $1.96 \mathrm{E}-06$ \\
Pancreas & $1.67 \mathrm{E}-06$ \\
Skin & $3.80 \mathrm{E}-06$ \\
Testes & $1.98 \mathrm{E}-06$ \\
Thyroid & $2.05 \mathrm{E}-06$ \\
& \\
EFFEC & $2.25 \mathrm{E}-06$
\end{tabular}

PATHWAY EFFECTIVE DOSE EQUIVALENT SUMMARY

\begin{tabular}{lc} 
Pathway & $\begin{array}{c}\text { Selected } \\
\text { Individual } \\
\text { (mrem/y) }\end{array}$ \\
\cline { 2 - 2 } & \\
INGESTION & $0.00 \mathrm{E}+00$ \\
INHALATION & $3.28 \mathrm{E}-07$ \\
AIR IMMERSION & $1.92 \mathrm{E}-06$ \\
GROUND SURFACE & $0.00 \mathrm{E}+00$ \\
INTERNAL & $3.28 \mathrm{E}-07$ \\
EXTERNAL & $1.92 \mathrm{E}-06$ \\
TOTAL & $2.25 \mathrm{E}-06$
\end{tabular}

Feb 20, $2008 \quad 02: 31 \mathrm{pm}$

SUMMARY

Page 2

NUCLIDE EFFECTIVE DOSE EQUIVALENT SUMMARY

Nuclide

C1-39

Ar-39

TOTAL

$$
\begin{gathered}
\begin{array}{c}
\text { Selected } \\
\text { Individual } \\
\text { (mrem/y) }
\end{array} \\
\hline 2.25 \mathrm{E}-06 \\
3.65 \mathrm{E}-16 \\
2.25 \mathrm{E}-06
\end{gathered}
$$

Feb 20, $2008 \quad 02: 31 \mathrm{pm}$

SUMMARY

Page 3

CANCER RISK SUMMARY

Cancer

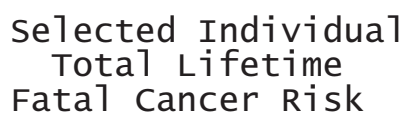

Page 2 


$\begin{array}{lr} & \text { C1-39D. SUM } \\ \text { Esophagu } & 2.02 \mathrm{E}-14 \\ \text { Stomach } & 1.10 \mathrm{E}-13 \\ \text { Colon } & 1.77 \mathrm{E}-13 \\ \text { Liver } & 2.79 \mathrm{E}-14 \\ \text { LUNG } & 3.01 \mathrm{E}-13 \\ \text { Bone } & 2.84 \mathrm{E}-15 \\ \text { Skin } & 3.80 \mathrm{E}-15 \\ \text { Breast } & 1.10 \mathrm{E}-13 \\ \text { Ovary } & 2.43 \mathrm{E}-14 \\ \text { B Tadder } & 4.10 \mathrm{E}-14 \\ \text { Kidneys } & 9.48 \mathrm{E}-15 \\ \text { Thyroid } & 6.55 \mathrm{E}-15 \\ \text { Leukemia } & 1.12 \mathrm{E}-13 \\ \text { Residual } & 2.65 \mathrm{E}-13 \\ \text { Total } & 1.21 \mathrm{E}-12 \\ & \\ \text { TOTAL } & 2.42 \mathrm{E}-12\end{array}$

PATHWAY RISK SUMMARY

Pathway

INGESTION
INHALATION
AIR IMMERSION
GROUND SURFACE
INTERNAL
EXTERNAL

TOTAL

$\square$

Feb 20, 2008 02:31 pm
Selected Individual

Tota1 Lifetime Fatal Cancer Risk

$0.00 \mathrm{E}+00$

1. $60 \mathrm{E}-13$

1. $05 \mathrm{E}-12$

$0.00 \mathrm{E}+00$

$1.60 \mathrm{E}-13$

1. $05 \mathrm{E}-12$

1. $21 \mathrm{E}-12$

NUCLIDE RISK SUMMARY

Nuclide

C1-39

Ar-39

TOTAL
Selected Individual Total Lifetime Fatal Cancer Risk

1. $21 \mathrm{E}-12$

$4.64 \mathrm{E}-23$

1. $21 \mathrm{E}-12$

Feb 20, $2008 \quad 02: 31 \mathrm{pm}$

INDIVIDUAL EFFECTIVE DOSE EQUIVALENT RATE (mrem/y) (A11 Radionuclides and Pathways) 
C1-39D. SUM

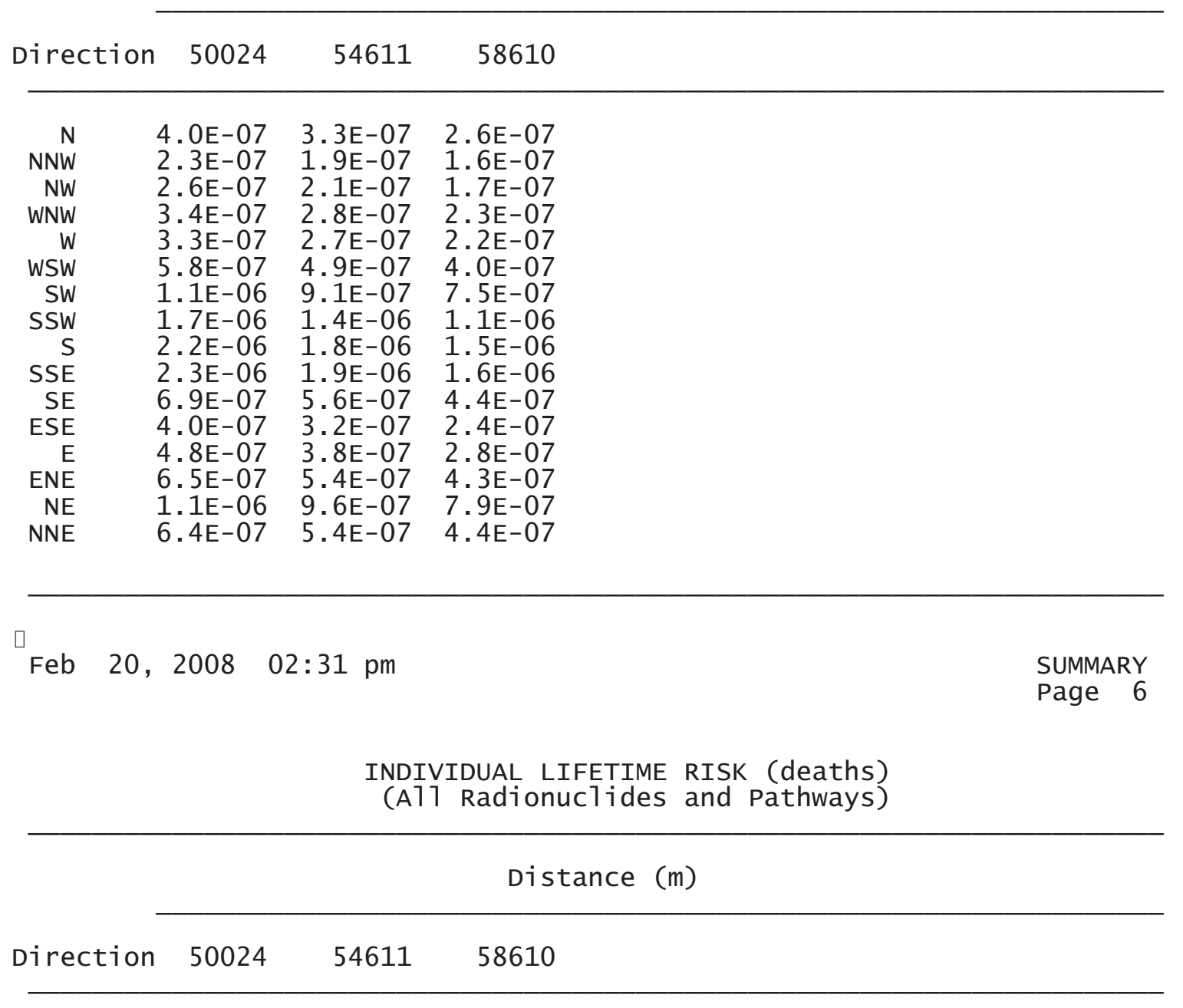

\begin{tabular}{rlll}
\hline$N$ & $2.2 \mathrm{E}-13$ & $1.8 \mathrm{E}-13$ & $1.4 \mathrm{E}-13$ \\
$\mathrm{~N} W$ & $1.2 \mathrm{E}-13$ & $1.0 \mathrm{E}-13$ & $8.7 \mathrm{E}-14$ \\
$\mathrm{NW}$ & $1.4 \mathrm{E}-13$ & $1.2 \mathrm{E}-13$ & $9.3 \mathrm{E}-14$ \\
WNW & $1.8 \mathrm{E}-13$ & $1.5 \mathrm{E}-13$ & $1.2 \mathrm{E}-13$ \\
$\mathrm{~W}$ & $1.8 \mathrm{E}-13$ & $1.5 \mathrm{E}-13$ & $1.2 \mathrm{E}-13$ \\
$\mathrm{WSW}$ & $3.1 \mathrm{E}-13$ & $2.6 \mathrm{E}-13$ & $2.2 \mathrm{E}-13$ \\
$\mathrm{SW}$ & $5.8 \mathrm{E}-13$ & $4.9 \mathrm{E}-13$ & $4.0 \mathrm{E}-13$ \\
$\mathrm{SSW}$ & $8.9 \mathrm{E}-13$ & $7.4 \mathrm{E}-13$ & $6.1 \mathrm{E}-13$ \\
$\mathrm{~S}$ & $1.2 \mathrm{E}-12$ & $9.8 \mathrm{E}-13$ & $8.1 \mathrm{E}-13$ \\
$\mathrm{SSE}$ & $1.2 \mathrm{E}-12$ & $1.0 \mathrm{E}-12$ & $8.5 \mathrm{E}-13$ \\
$\mathrm{SE}$ & $3.7 \mathrm{E}-13$ & $3.0 \mathrm{E}-13$ & $2.4 \mathrm{E}-13$ \\
$\mathrm{ESE}$ & $2.1 \mathrm{E}-13$ & $1.7 \mathrm{E}-13$ & $1.3 \mathrm{E}-13$ \\
$\mathrm{E}$ & $2.6 \mathrm{E}-13$ & $2.0 \mathrm{E}-13$ & $1.5 \mathrm{E}-13$ \\
$\mathrm{ENE}$ & $3.5 \mathrm{E}-13$ & $2.9 \mathrm{E}-13$ & $2.3 \mathrm{E}-13$ \\
$\mathrm{NE}$ & $6.2 \mathrm{E}-13$ & $5.2 \mathrm{E}-13$ & $4.3 \mathrm{E}-13$ \\
$\mathrm{NNE}$ & $3.5 \mathrm{E}-13$ & $2.9 \mathrm{E}-13$ & $2.4 \mathrm{E}-13$ \\
& & &
\end{tabular}




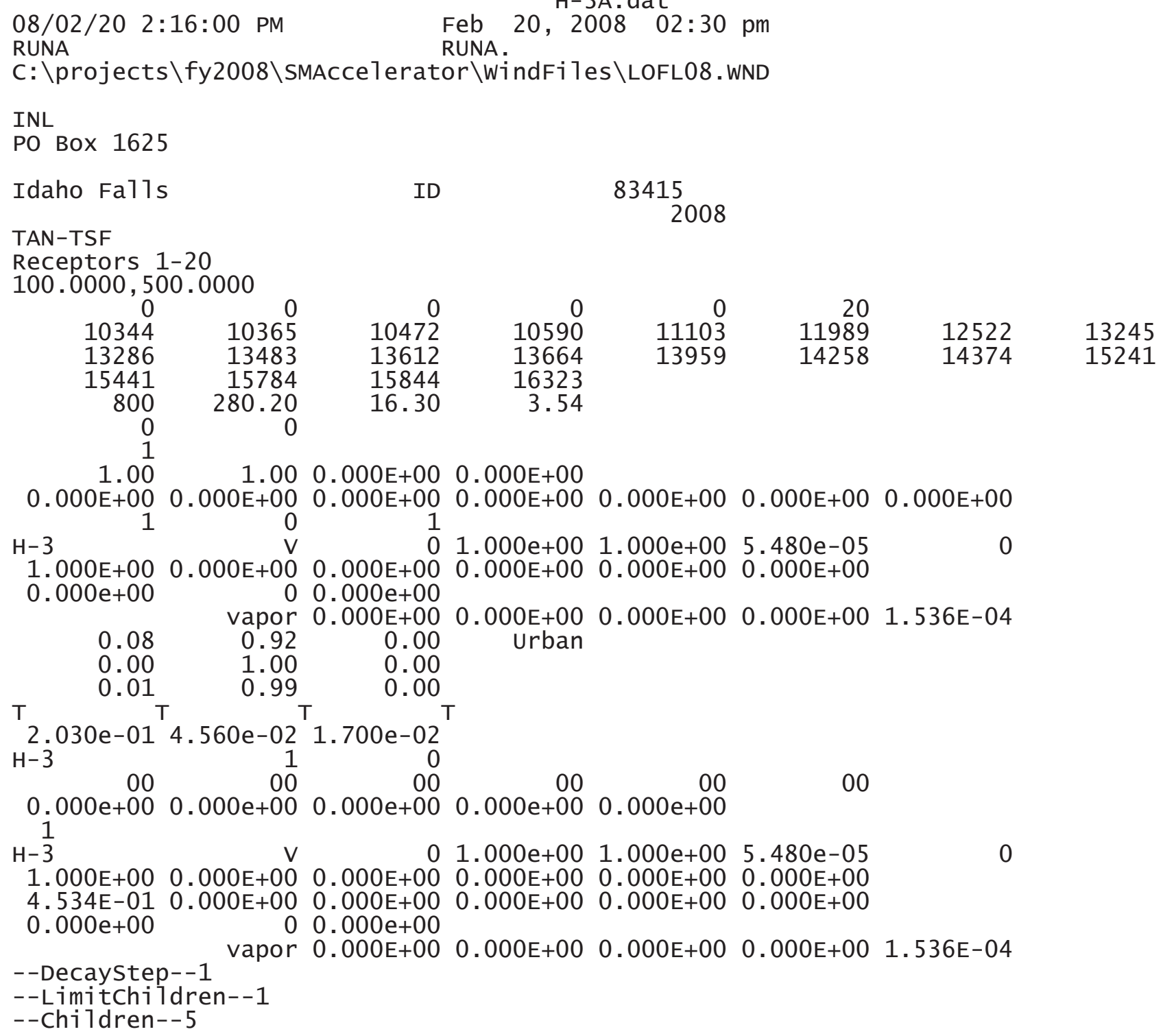




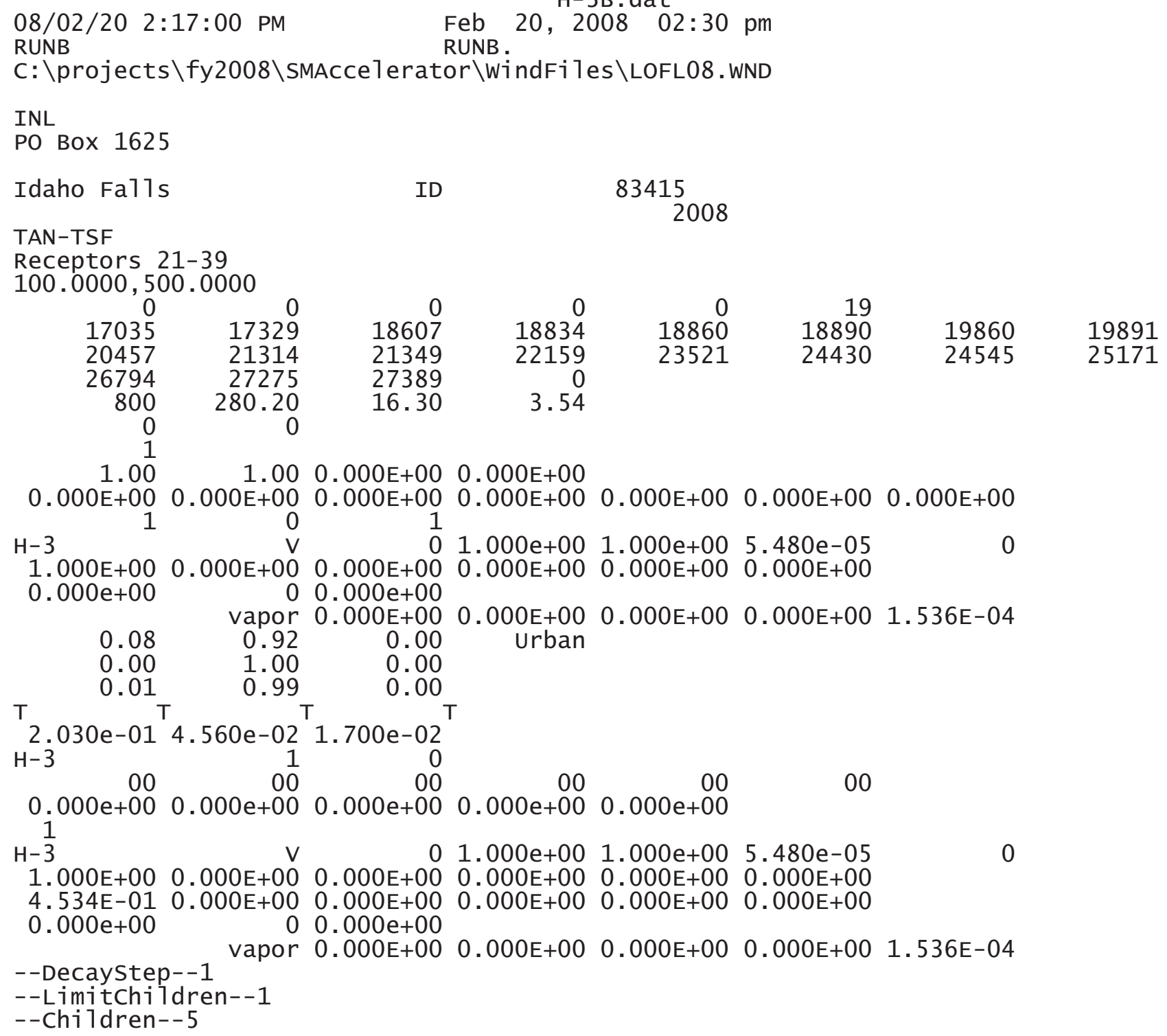




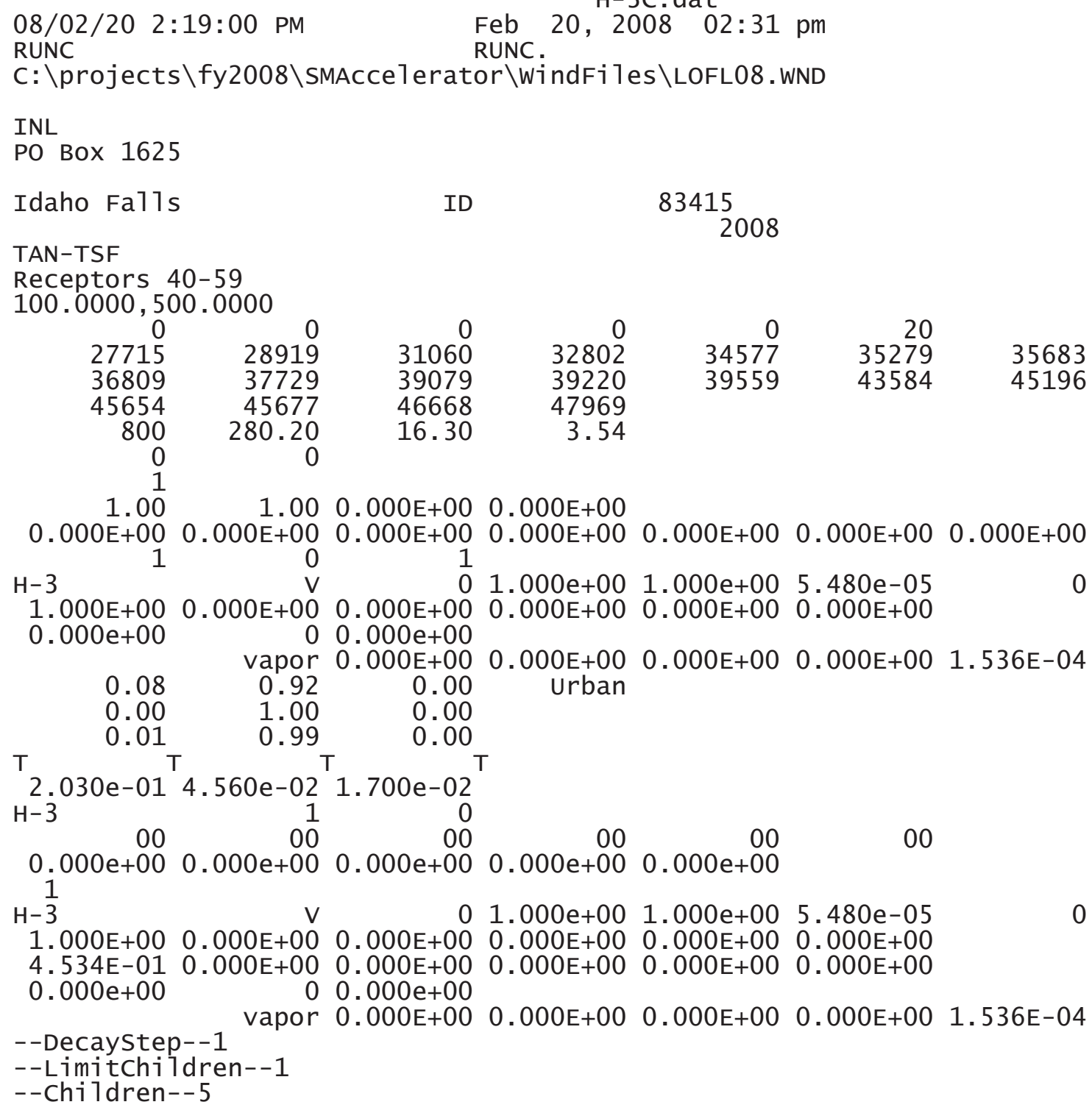

ID

83415

TAN-TSF

Receptors 40-59

$100.0000,500.0000$

$\begin{array}{rrrr}0 & 0 & 0 & \\ 27715 & 28919 & 31060 & 3280 \\ 36809 & 37729 & 39079 & 3922 \\ 45654 & 45677 & 46668 & 4796 \\ 800 & 280.20 & 16.30 & 3.5 \\ 0 & 0 & & \end{array}$




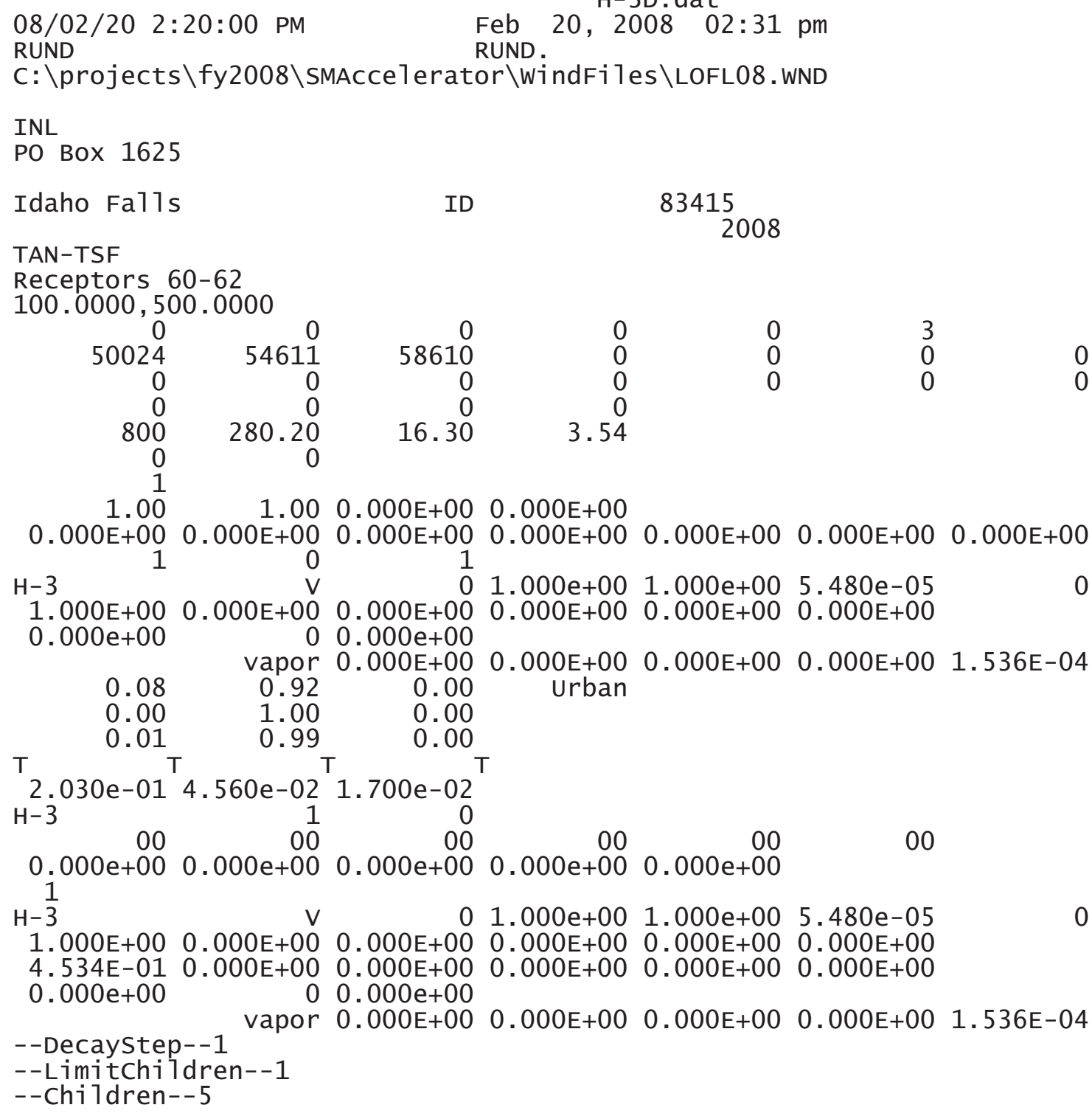

ID

83415

TAN-TSF

Receptors 60-62

$100.0000,500.0000$

$\begin{array}{rrrr}50024 & 54611 & 0 & \\ 0 & 0 & 0 & 0 \\ 0 & 0 & 0 & 0 \\ 800 & 280.20 & 16.30 & 3.54 \\ 0 & 0 & & \\ 1 & & & \end{array}$

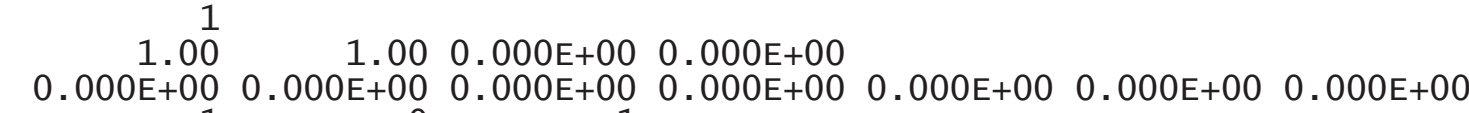

$\begin{array}{lllllll}\mathrm{H}-3 & 1 & 0 & 1 & & \\ 1 & \mathrm{~V} & 0 & 1.000 \mathrm{e}+00 & 1.000 \mathrm{e}+00 & 5.480 \mathrm{e}-05 & 0\end{array}$

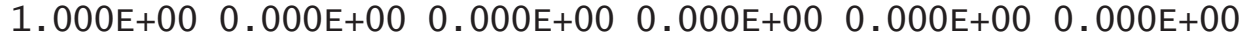

$0.000 \mathrm{e}+00 \quad 0.000 \mathrm{e}+00$

$\begin{array}{rrrr}0.08 & \text { vapor } & 0.000 \mathrm{E}+00 & 0.000 \mathrm{E}+00 \\ 0.92 & 0.92 & 0.00 & \text { Urban }\end{array}$

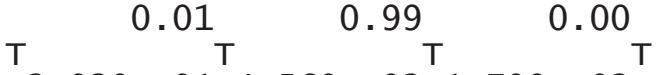

$\begin{array}{rrr}2.030 \mathrm{e}-01 & 4.560 \mathrm{e}-02 & 1.700 \mathrm{e}-02 \\ \mathrm{H}-3 & 0\end{array}$

$\begin{array}{rrrrr}00 & 00 & 00 & 00 & 00 \\ 0.000 e+00 & 0.000 e+00 & 0.000 e+00 & 0.000 e+00 & 0.000 e+00\end{array}$

H-3

$\begin{array}{lllllll}1.000 \mathrm{E}+00 & 0.000 \mathrm{E}+00 & 0.000 \mathrm{E}+00 & 0.000 \mathrm{E}+00 & 0.000 \mathrm{E}+00 & 0.000 \mathrm{E}+00\end{array}$

$\begin{array}{llllll}4.534 \mathrm{E}-01 & 0.000 \mathrm{E}+00 & 0.000 \mathrm{E}+00 & 0.000 \mathrm{E}+00 & 0.000 \mathrm{E}+00 & 0.000 \mathrm{E}+00\end{array}$

$0.000 \mathrm{e}+00$

$0 \quad 0.000 \mathrm{e}+00$

vapor $0.000 \mathrm{E}+00 \quad 0.000 \mathrm{E}+00 \quad 0.000 \mathrm{E}+00 \quad 0.000 \mathrm{E}+00 \quad 1.536 \mathrm{E}-04$

--Decaystep--1

--LimitChildren--1

--Children--5 


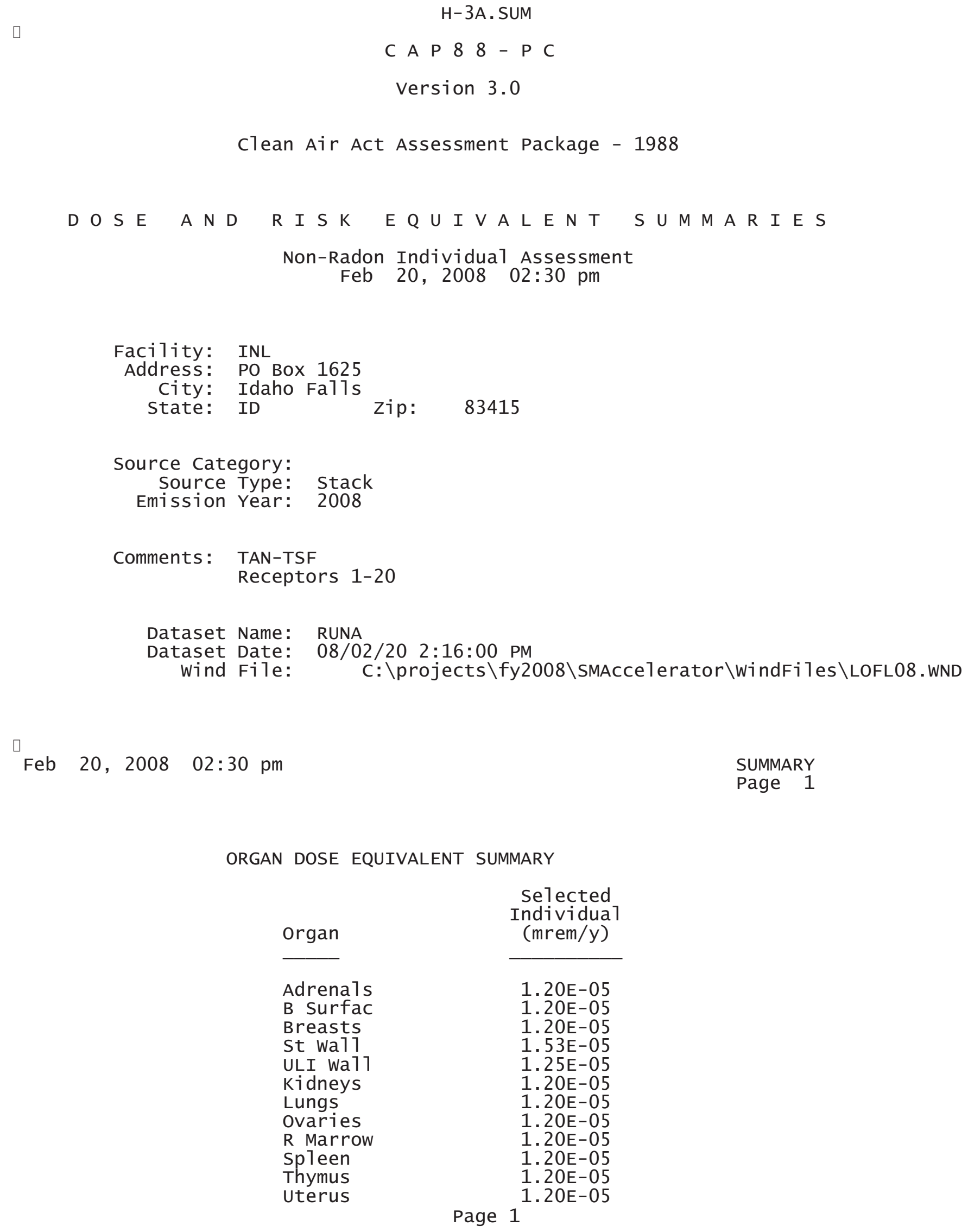




$\begin{array}{lr} & \mathrm{H}-3 \mathrm{~A} . \mathrm{SUM} \\ \text { B1d wa11 } & 1.20 \mathrm{E}-05 \\ \text { Brain } & 1.20 \mathrm{E}-05 \\ \text { Esophagu } & 1.20 \mathrm{E}-05 \\ \text { SI wa11 } & 1.21 \mathrm{E}-05 \\ \text { LLI wa11 } & 1.35 \mathrm{E}-05 \\ \text { Liver } & 1.20 \mathrm{E}-05 \\ \text { Muscle } & 1.20 \mathrm{E}-05 \\ \text { Pancreas } & 1.20 \mathrm{E}-05 \\ \text { Skin } & 1.20 \mathrm{E}-05 \\ \text { Testes } & 1.20 \mathrm{E}-05 \\ \text { Thyroid } & 1.20 \mathrm{E}-05 \\ & \\ \text { EFFEC } & 2.36 \mathrm{E}-05\end{array}$

PATHWAY EFFECTIVE DOSE EQUIVALENT SUMMARY

Selected Individual

Pathway (mrem/y)

INGESTION

1. $91 \mathrm{E}-05$

INHALATION

4. $46 \mathrm{E}-06$

AIR IMMERSION

GROUND SURFACE

INTERNAL

$0.00 \mathrm{E}+00$

EXTERNAL

$0.00 \mathrm{E}+00$

2. $36 \mathrm{E}-05$

$0.00 \mathrm{E}+00$

TOTAL

$2.36 \mathrm{E}-05$

$\square$

Feb 20, 2008 02:30 pm

SUMMARY

Page 2

NUCLIDE EFFECTIVE DOSE EQUIVALENT SUMMARY

Nuclide

$\mathrm{H}-3$

TOTAL

Feb 20, 2008 02:30 pm

$$
\begin{gathered}
\begin{array}{c}
\text { Selected } \\
\text { Individua1 } \\
\text { (mrem/y) }
\end{array} \\
\hline 2.36 \mathrm{E}-05 \\
2.36 \mathrm{E}-05
\end{gathered}
$$

SUMMARY

Page 3

CANCER RISK SUMMARY

Cancer

Esophagu
Selected Individual

Total Lifetime Fatal Cancer Risk

$$
\text { Page } 2^{2.72 \mathrm{E}-13}
$$




$\begin{array}{lr} & \text { H-3A.SUM } \\ \text { Stomach } & 1.37 \mathrm{E}-12 \\ \text { Colon } & 2.80 \mathrm{E}-12 \\ \text { Liver } & 3.81 \mathrm{E}-13 \\ \text { LUNG } & 2.31 \mathrm{E}-12 \\ \text { Bone } & 2.31 \mathrm{E}-14 \\ \text { Skin } & 2.45 \mathrm{E}-14 \\ \text { Breast } & 1.09 \mathrm{E}-12 \\ \text { Ovary } & 3.12 \mathrm{E}-13 \\ \text { B Tadder } & 6.12 \mathrm{E}-13 \\ \text { Kidneys } & 1.31 \mathrm{E}-13 \\ \text { Thyroid } & 7.55 \mathrm{E}-14 \\ \text { Leukemia } & 1.40 \mathrm{E}-12 \\ \text { Residua1 } & 3.69 \mathrm{E}-12 \\ \text { Tota1 } & 1.45 \mathrm{E}-11 \\ & \\ \text { TOTAL } & 2.90 \mathrm{E}-11\end{array}$

PATHWAY RISK SUMMARY

\begin{tabular}{lc} 
Pathway & $\begin{array}{c}\text { Selected Individual } \\
\text { Total Lifetime } \\
\text { Fatal Cancer Risk }\end{array}$ \\
\cline { 2 - 2 } INGESTION & $1.20 \mathrm{E}-11$ \\
INHALATION & $2.53 \mathrm{E}-12$ \\
AIR IMMERSION & $0.00 \mathrm{E}+00$ \\
GROUND SURFACE & $0.00 \mathrm{E}+00$ \\
INTERNAL & $1.45 \mathrm{E}-11$ \\
EXTERNAL & $0.00 \mathrm{E}+00$ \\
TOTAL & $1.45 \mathrm{E}-11$
\end{tabular}

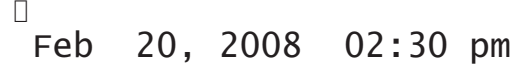

NUCLIDE RISK SUMMARY

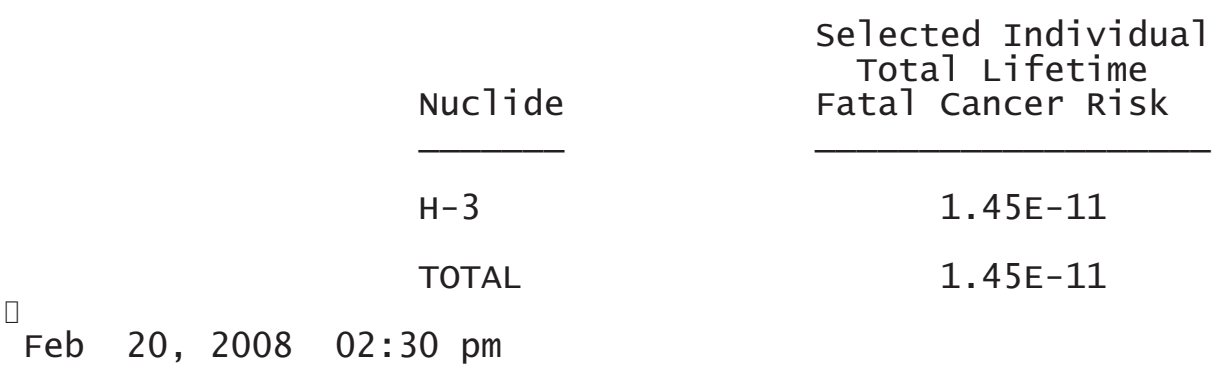

INDIVIDUAL EFFECTIVE DOSE EQUIVALENT RATE (mrem/y) (A11 Radionuclides and Pathways)

Distance (m) 


\begin{tabular}{|c|c|c|c|c|c|c|c|}
\hline Direction & 10344 & 10365 & 10472 & $\begin{array}{l}\text { H-3A.SUI } \\
10590\end{array}$ & JM 11103 & 11989 & 12522 \\
\hline $\mathrm{N}$ & $1.8 \mathrm{E}-05$ & $1.8 \mathrm{E}-05$ & $1.8 \mathrm{E}-05$ & $1.8 \mathrm{E}-05$ & $1.8 \mathrm{E}-05$ & $1.8 \mathrm{E}-05$ & $1.8 \mathrm{E}-05$ \\
\hline NNW & 1.7E-05 & $1.7 \mathrm{E}-05$ & $1.7 \mathrm{E}-05$ & 1.7E-05 & $1.7 \mathrm{E}-05$ & $1.7 \mathrm{E}-05$ & $1.7 \mathrm{E}-05$ \\
\hline NW & $1.7 \mathrm{E}-05$ & $1.7 \mathrm{E}-05$ & $1.7 \mathrm{E}-05$ & $1.7 \mathrm{E}-05$ & $1.7 \mathrm{E}-05$ & $1.7 \mathrm{E}-05$ & $1.7 \mathrm{E}-05$ \\
\hline WNW & $1.8 \mathrm{E}-05$ & $1.8 \mathrm{E}-05$ & $1.8 \mathrm{E}-05$ & $1.8 \mathrm{E}-05$ & $1.8 \mathrm{E}-05$ & $1.8 \mathrm{E}-05$ & $1.8 \mathrm{E}-05$ \\
\hline W & $1.8 \mathrm{E}-05$ & $1.8 \mathrm{E}-05$ & $1.8 \mathrm{E}-05$ & $1.8 \mathrm{E}-05$ & $1.8 \mathrm{E}-05$ & $1.8 \mathrm{E}-05$ & $1.8 \mathrm{E}-05$ \\
\hline WSW & $1.8 \mathrm{E}-05$ & $1.8 \mathrm{E}-05$ & $1.8 \mathrm{E}-05$ & $1.8 \mathrm{E}-05$ & $1.8 \mathrm{E}-05$ & $1.8 \mathrm{E}-05$ & $1.8 \mathrm{E}-05$ \\
\hline SW & $2.0 \mathrm{E}-05$ & $2.0 \mathrm{E}-05$ & $2.0 \mathrm{E}-05$ & $1.9 \mathrm{E}-05$ & $1.9 \mathrm{E}-05$ & $1.9 \mathrm{E}-05$ & $1.9 \mathrm{E}-05$ \\
\hline SSW & $2.2 \mathrm{E}-05$ & $2.2 \mathrm{E}-05$ & $2.1 \mathrm{E}-05$ & $2.1 \mathrm{E}-05$ & $2.1 \mathrm{E}-05$ & $2.1 \mathrm{E}-05$ & $2.1 \mathrm{E}-05$ \\
\hline $\mathrm{S}$ & $2.4 \mathrm{E}-05$ & $2.4 \mathrm{E}-05$ & 2. 3E-05 & $2.3 \mathrm{E}-05$ & $2.3 \mathrm{E}-05$ & $2.2 \mathrm{E}-05$ & $2.2 \mathrm{E}-05$ \\
\hline SSE & $2.2 \mathrm{E}-05$ & $2.2 \mathrm{E}-05$ & $2.2 \mathrm{E}-05$ & $2.2 \mathrm{E}-05$ & $2.2 \mathrm{E}-05$ & $2.1 \mathrm{E}-05$ & 2.1E-05 \\
\hline SE & $2.0 \mathrm{E}-05$ & $2.0 \mathrm{E}-05$ & $2.0 \mathrm{E}-05$ & $2.0 \mathrm{E}-05$ & 1.9E-05 & $1.9 \mathrm{E}-05$ & $1.9 \mathrm{E}-05$ \\
\hline ESE & 1.9E-05 & 1.9E-05 & $1.9 \mathrm{E}-05$ & 1.9E-05 & $1.8 \mathrm{E}-05$ & $1.8 \mathrm{E}-05$ & $1.8 \mathrm{E}-05$ \\
\hline E & 1.9E-05 & 1.9E-05 & $1.9 \mathrm{E}-05$ & $1.9 \mathrm{E}-05$ & 1.9E-05 & $1.9 \mathrm{E}-05$ & 1.9E-05 \\
\hline ENE & 1.9E-05 & $1.9 \mathrm{E}-05$ & $1.9 \mathrm{E}-05$ & $1.9 \mathrm{E}-05$ & 1.9E-05 & $1.8 \mathrm{E}-05$ & $1.8 \mathrm{E}-05$ \\
\hline $\mathrm{NE}$ & $2.0 \mathrm{E}-05$ & $2.0 \mathrm{E}-05$ & $2.0 \mathrm{E}-05$ & $2.0 \mathrm{E}-05$ & $2.0 \mathrm{E}-05$ & 1.9E-05 & 1.9E-05 \\
\hline NNE & $1.9 \mathrm{E}-05$ & $1.9 \mathrm{E}-05$ & $1.9 \mathrm{E}-05$ & $1.9 \mathrm{E}-05$ & 1.9E-05 & $1.8 \mathrm{E}-05$ & $1.8 \mathrm{E}-05$ \\
\hline
\end{tabular}

Distance $(\mathrm{m})$

\begin{tabular}{|c|c|c|c|c|c|c|c|}
\hline Direction & 13245 & 13286 & 13483 & 13612 & 13664 & 13959 & 14258 \\
\hline $\mathrm{N}$ & $1.8 \mathrm{E}-05$ & $1.8 \mathrm{E}-05$ & $1.8 \mathrm{E}-05$ & $1.8 \mathrm{E}-05$ & $1.8 \mathrm{E}-05$ & $1.8 \mathrm{E}-05$ & $1.8 \mathrm{E}-05$ \\
\hline NNW & $1.7 \mathrm{E}-05$ & $1.7 \mathrm{E}-05$ & $1.7 \mathrm{E}-05$ & $1.7 \mathrm{E}-05$ & $1.7 \mathrm{E}-05$ & $1.7 \mathrm{E}-05$ & $1.7 \mathrm{E}-05$ \\
\hline NW & $1.7 \mathrm{E}-05$ & $1.7 \mathrm{E}-05$ & $1.7 \mathrm{E}-05$ & $1.7 \mathrm{E}-05$ & $1.7 \mathrm{E}-05$ & $1.7 \mathrm{E}-05$ & $1.7 \mathrm{E}-05$ \\
\hline WNW & $1.7 \mathrm{E}-05$ & $1.7 \mathrm{E}-05$ & $1.7 \mathrm{E}-05$ & $1.7 \mathrm{E}-05$ & $1.7 \mathrm{E}-05$ & $1.7 \mathrm{E}-05$ & $1.7 \mathrm{E}-05$ \\
\hline W & $1.8 \mathrm{E}-05$ & $1.8 \mathrm{E}-05$ & $1.8 \mathrm{E}-05$ & $1.8 \mathrm{E}-05$ & $1.8 \mathrm{E}-05$ & $1.8 \mathrm{E}-05$ & $1.8 \mathrm{E}-05$ \\
\hline WSW & $1.8 \mathrm{E}-05$ & $1.8 \mathrm{E}-05$ & $1.8 \mathrm{E}-05$ & $1.8 \mathrm{E}-05$ & $1.8 \mathrm{E}-05$ & $1.8 \mathrm{E}-05$ & $1.8 \mathrm{E}-05$ \\
\hline SW & $1.9 \mathrm{E}-05$ & $1.9 \mathrm{E}-05$ & $1.9 \mathrm{E}-05$ & $1.9 \mathrm{E}-05$ & $1.9 \mathrm{E}-05$ & $1.9 \mathrm{E}-05$ & $1.9 \mathrm{E}-05$ \\
\hline SSW & $2.0 \mathrm{E}-05$ & 2.0E-05 & 2.0E-05 & 2.0E-05 & 2.0E-05 & 2.0E-05 & $2.0 \mathrm{E}-05$ \\
\hline & $2.2 \mathrm{E}-05$ & $2.2 \mathrm{E}-05$ & $2.2 \mathrm{E}-05$ & $2.2 \mathrm{E}-05$ & $2.2 \mathrm{E}-05$ & $2.1 \mathrm{E}-05$ & $2.1 \mathrm{E}-05$ \\
\hline SSE & $2.1 \mathrm{E}-05$ & $2.1 \mathrm{E}-05$ & 2.1E-05 & 2.1E-05 & 2. $1 \mathrm{E}-05$ & $2.1 \mathrm{E}-05$ & $2.1 \mathrm{E}-05$ \\
\hline SE & 1. $9 \mathrm{E}-05$ & 1. $9 \mathrm{E}-05$ & 1. $9 \mathrm{E}-05$ & 1. $9 \mathrm{E}-05$ & $1.9 \mathrm{E}-05$ & $1.9 \mathrm{E}-05$ & $1.9 \mathrm{E}-05$ \\
\hline ESE & $1.8 \mathrm{E}-05$ & $1.8 \mathrm{E}-05$ & $1.8 \mathrm{E}-05$ & $1.8 \mathrm{E}-05$ & $1.8 \mathrm{E}-05$ & $1.8 \mathrm{E}-05$ & $1.8 \mathrm{E}-05$ \\
\hline $\mathrm{E}$ & 1.9E-05 & $1.9 \mathrm{E}-05$ & $1.8 \mathrm{E}-05$ & $1.8 \mathrm{E}-05$ & $1.8 \mathrm{E}-05$ & $1.8 \mathrm{E}-05$ & $1.8 \mathrm{E}-05$ \\
\hline ENE & $1.8 \mathrm{E}-05$ & $1.8 \mathrm{E}-05$ & $1.8 \mathrm{E}-05$ & $1.8 \mathrm{E}-05$ & $1.8 \mathrm{E}-05$ & $1.8 \mathrm{E}-05$ & $1.8 \mathrm{E}-05$ \\
\hline $\mathrm{NE}$ & 1. $9 \mathrm{E}-05$ & 1. $9 \mathrm{E}-05$ & 1. $9 \mathrm{E}-05$ & 1. $9 \mathrm{E}-05$ & 1. $9 \mathrm{E}-05$ & 1. $9 \mathrm{E}-05$ & $1.9 \mathrm{E}-05$ \\
\hline NNE & $1.8 \mathrm{E}-05$ & $1.8 \mathrm{E}-05$ & $1.8 \mathrm{E}-05$ & $1.8 \mathrm{E}-05$ & $1.8 \mathrm{E}-05$ & $1.8 \mathrm{E}-05$ & $1.8 \mathrm{E}-05$ \\
\hline
\end{tabular}

$\square$

Feb 20, 2008 02:30 pm

SUMMARY

Page 6

INDIVIDUAL EFFECTIVE DOSE EQUIVALENT RATE (mrem/y) (A11 Radionuclides and Pathways)

\begin{tabular}{|c|c|c|c|c|c|c|}
\hline \multirow[b]{2}{*}{ Direction } & \multicolumn{6}{|c|}{ Distance (m) } \\
\hline & 14374 & 15241 & 15441 & 15784 & 15844 & 16323 \\
\hline $\begin{array}{r}N \\
N N W\end{array}$ & $\begin{array}{l}1.8 \mathrm{E}-05 \\
1.7 \mathrm{E}-05\end{array}$ & $\begin{array}{l}1.8 \mathrm{E}-05 \\
1.7 \mathrm{E}-05\end{array}$ & $\begin{array}{l}1.8 \mathrm{E}-05 \\
1.7 \mathrm{E}-05\end{array}$ & $\begin{array}{r}1.8 \mathrm{E}-05 \\
1.7 \mathrm{E}-05 \\
\text { Page }\end{array}$ & $\begin{array}{l}1.8 \mathrm{E}-05 \\
1.7 \mathrm{E}-05\end{array}$ & $\begin{array}{l}1.8 \mathrm{E}-05 \\
1.7 \mathrm{E}-05\end{array}$ \\
\hline
\end{tabular}




\begin{tabular}{|c|c|c|c|c|c|c|}
\hline & & & & $H-3 A$. & & \\
\hline NW & 1. $7 \mathrm{E}-05$ & 1.7E-05 & 1. $7 \mathrm{E}-05$ & 1. $7 \mathrm{E}-05$ & 1.7E-05 & $1.7 \mathrm{E}-05$ \\
\hline WNW & $1.7 \mathrm{E}-05$ & 1.7E-05 & $1.7 \mathrm{E}-05$ & 1.7E-05 & 1.7E-05 & 1. $7 \mathrm{E}-05$ \\
\hline W & $1.8 \mathrm{E}-05$ & $1.7 \mathrm{E}-05$ & $1.7 \mathrm{E}-05$ & $1.7 \mathrm{E}-05$ & $1.7 \mathrm{E}-05$ & $1.7 \mathrm{E}-05$ \\
\hline WSW & 1. $8 \mathrm{E}-05$ & $1.8 \mathrm{E}-05$ & $1.8 \mathrm{E}-05$ & $1.8 \mathrm{E}-05$ & $1.8 \mathrm{E}-05$ & $1.8 \mathrm{E}-05$ \\
\hline SW & 1. $9 \mathrm{E}-05$ & $1.8 \mathrm{E}-05$ & $1.8 \mathrm{E}-05$ & $1.8 \mathrm{E}-05$ & $1.8 \mathrm{E}-05$ & $1.8 \mathrm{E}-05$ \\
\hline SSW & $2.0 \mathrm{E}-05$ & $2.0 \mathrm{E}-05$ & $2.0 \mathrm{E}-05$ & $2.0 \mathrm{E}-05$ & $2.0 \mathrm{E}-05$ & $1.9 \mathrm{E}-05$ \\
\hline & $2.1 \mathrm{E}-05$ & $2.1 \mathrm{E}-05$ & $2.1 \mathrm{E}-05$ & $2.1 \mathrm{E}-05$ & $2.1 \mathrm{E}-05$ & $2.1 \mathrm{E}-05$ \\
\hline SSE & $2.0 \mathrm{E}-$ & $2.0 \mathrm{E}$ & $2.0 \mathrm{E}-05$ & $2.0 \mathrm{E}$ & $2.0 \mathrm{E}-05$ & $2.0 \mathrm{E}-05$ \\
\hline SE & $1.9 \mathrm{E}-05$ & $1.9 \mathrm{E}-05$ & $1.8 \mathrm{E}-05$ & $1.8 \mathrm{E}-05$ & $1.8 \mathrm{E}-05$ & $1.8 \mathrm{E}-05$ \\
\hline ESE & 1 & $1.8 \mathrm{E}$ & 1.8 & $1.8 \mathrm{E}-05$ & $1.8 \mathrm{E}$ & $1.8 \mathrm{E}-05$ \\
\hline & $1.8 \mathrm{E}$ & $1.8 \mathrm{E}-$ & $1.8 \mathrm{E}-05$ & $1.8 \mathrm{E}-$ & $1.8 \mathrm{E}-$ & $1.8 \mathrm{E}-05$ \\
\hline ENE & 1.8 & $1.8 \mathrm{E}$ & 1.8 & $1.8 \mathrm{E}$ & 1.8 & $1.8 \mathrm{E}-05$ \\
\hline NE & 1. & $1.9 \mathrm{E}$ & $1.9 \mathrm{E}-05$ & $1.9 \mathrm{E}-$ & 1. $9 \mathrm{E}-05$ & 1.9E-05 \\
\hline NNE & $1.8 \mathrm{E}-05$ & $1.8 \mathrm{E}-05$ & $1.8 \mathrm{E}-05$ & $1.8 \mathrm{E}-05$ & $1.8 \mathrm{E}-05$ & $1.8 \mathrm{E}-05$ \\
\hline
\end{tabular}

Feb 20, $2008 \quad 02: 30 \mathrm{pm}$

SUMMARY

Page 7

INDIVIDUAL LIFETIME RISK (deaths)

(A11 Radionuclides and Pathways)

\begin{tabular}{|c|c|c|c|c|c|c|c|}
\hline \multirow[b]{2}{*}{ Direction } & \multicolumn{6}{|c|}{ Distance (m) } & \multirow[b]{2}{*}{12522} \\
\hline & 10344 & 10365 & 10472 & 10590 & 11103 & 11989 & \\
\hline $\begin{array}{r}N \\
N N W \\
N W \\
W N W \\
W \\
W S W \\
S W \\
S S W \\
S \\
S S E \\
\text { SE } \\
\text { ESE } \\
E \\
\text { ENE } \\
N E \\
\text { NNE }\end{array}$ & $\begin{array}{l}1.1 \mathrm{E}-11 \\
1.1 \mathrm{E}-11 \\
1.1 \mathrm{E}-11 \\
1.1 \mathrm{E}-11 \\
1.1 \mathrm{E}-11 \\
1.1 \mathrm{E}-11 \\
1.2 \mathrm{E}-11 \\
1.3 \mathrm{E}-11 \\
1.4 \mathrm{E}-11 \\
1.4 \mathrm{E}-11 \\
1.2 \mathrm{E}-11 \\
1.2 \mathrm{E}-11 \\
1.2 \mathrm{E}-11 \\
1.2 \mathrm{E}-11 \\
1.2 \mathrm{E}-11 \\
1.2 \mathrm{E}-11\end{array}$ & $\begin{array}{l}1.1 \mathrm{E}-11 \\
1.1 \mathrm{E}-11 \\
1.1 \mathrm{E}-11 \\
1.1 \mathrm{E}-11 \\
1.1 \mathrm{E}-11 \\
1.1 \mathrm{E}-11 \\
1.2 \mathrm{E}-11 \\
1.3 \mathrm{E}-11 \\
1.4 \mathrm{E}-11 \\
1.4 \mathrm{E}-11 \\
1.2 \mathrm{E}-11 \\
1.2 \mathrm{E}-11 \\
1.2 \mathrm{E}-11 \\
1.2 \mathrm{E}-11 \\
1.2 \mathrm{E}-11 \\
1.2 \mathrm{E}-11\end{array}$ & $\begin{array}{l}1.1 \mathrm{E}-11 \\
1.1 \mathrm{E}-11 \\
1.1 \mathrm{E}-11 \\
1.1 \mathrm{E}-11 \\
1.1 \mathrm{E}-11 \\
1.1 \mathrm{E}-11 \\
1.2 \mathrm{E}-11 \\
1.3 \mathrm{E}-11 \\
1.4 \mathrm{E}-11 \\
1.4 \mathrm{E}-11 \\
1.2 \mathrm{E}-11 \\
1.2 \mathrm{E}-11 \\
1.2 \mathrm{E}-11 \\
1.2 \mathrm{E}-11 \\
1.2 \mathrm{E}-11 \\
1.2 \mathrm{E}-11\end{array}$ & $\begin{array}{l}1.1 \mathrm{E}-11 \\
1.1 \mathrm{E}-11 \\
1.1 \mathrm{E}-11 \\
1.1 \mathrm{E}-11 \\
1.1 \mathrm{E}-11 \\
1.1 \mathrm{E}-11 \\
1.2 \mathrm{E}-11 \\
1.3 \mathrm{E}-11 \\
1.4 \mathrm{E}-11 \\
1.4 \mathrm{E}-11 \\
1.2 \mathrm{E}-11 \\
1.2 \mathrm{E}-11 \\
1.2 \mathrm{E}-11 \\
1.2 \mathrm{E}-11 \\
1.2 \mathrm{E}-11 \\
1.2 \mathrm{E}-11\end{array}$ & $\begin{array}{l}1.1 \mathrm{E}-11 \\
1.1 \mathrm{E}-11 \\
1.1 \mathrm{E}-11 \\
1.1 \mathrm{E}-11 \\
1.1 \mathrm{E}-11 \\
1.1 \mathrm{E}-11 \\
1.2 \mathrm{E}-11 \\
1.3 \mathrm{E}-11 \\
1.4 \mathrm{E}-11 \\
1.4 \mathrm{E}-11 \\
1.2 \mathrm{E}-11 \\
1.1 \mathrm{E}-11 \\
1.2 \mathrm{E}-11 \\
1.2 \mathrm{E}-11 \\
1.2 \mathrm{E}-11 \\
1.2 \mathrm{E}-11\end{array}$ & $\begin{array}{l}1.1 \mathrm{E}-11 \\
1.1 \mathrm{E}-11 \\
1.1 \mathrm{E}-11 \\
1.1 \mathrm{E}-11 \\
1.1 \mathrm{E}-11 \\
1.1 \mathrm{E}-11 \\
1.2 \mathrm{E}-11 \\
1.3 \mathrm{E}-11 \\
1.4 \mathrm{E}-11 \\
1.3 \mathrm{E}-11 \\
1.2 \mathrm{E}-11 \\
1.1 \mathrm{E}-11 \\
1.2 \mathrm{E}-11 \\
1.1 \mathrm{E}-11 \\
1.2 \mathrm{E}-11 \\
1.1 \mathrm{E}-11\end{array}$ & $\begin{array}{l}1.1 \mathrm{E}-11 \\
1.1 \mathrm{E}-11 \\
1.1 \mathrm{E}-11 \\
1.1 \mathrm{E}-11 \\
1.1 \mathrm{E}-11 \\
1.1 \mathrm{E}-11 \\
1.2 \mathrm{E}-11 \\
1.3 \mathrm{E}-11 \\
1.4 \mathrm{E}-11 \\
1.3 \mathrm{E}-11 \\
1.2 \mathrm{E}-11 \\
1.1 \mathrm{E}-11 \\
1.2 \mathrm{E}-11 \\
1.1 \mathrm{E}-11 \\
1.2 \mathrm{E}-11 \\
1.1 \mathrm{E}-11\end{array}$ \\
\hline \multicolumn{8}{|c|}{ Distance $(\mathrm{m})$} \\
\hline Direction & 13245 & 13286 & 13483 & 13612 & 13664 & 13959 & 14258 \\
\hline $\begin{array}{r}N \\
N N W \\
N W \\
W N W \\
W \\
W S W \\
\text { SW } \\
\text { SSW }\end{array}$ & $\begin{array}{l}1.1 \mathrm{E}-11 \\
1.1 \mathrm{E}-11 \\
1.1 \mathrm{E}-11 \\
1.1 \mathrm{E}-11 \\
1.1 \mathrm{E}-11 \\
1.1 \mathrm{E}-11 \\
1.2 \mathrm{E}-11 \\
1.3 \mathrm{E}-11\end{array}$ & $\begin{array}{l}1 \cdot 1 \mathrm{E}-11 \\
1.1 \mathrm{E}-11 \\
1.1 \mathrm{E}-11 \\
1.1 \mathrm{E}-11 \\
1.1 \mathrm{E}-11 \\
1.1 \mathrm{E}-11 \\
1.2 \mathrm{E}-11 \\
1.3 \mathrm{E}-11\end{array}$ & $\begin{array}{l}1.1 \mathrm{E}-11 \\
1.1 \mathrm{E}-11 \\
1.1 \mathrm{E}-11 \\
1.1 \mathrm{E}-11 \\
1.1 \mathrm{E}-11 \\
1.1 \mathrm{E}-11 \\
1.2 \mathrm{E}-11 \\
1.2 \mathrm{E}-11\end{array}$ & $\begin{array}{r}1.1 \mathrm{E}-11 \\
1.1 \mathrm{E}-11 \\
1.1 \mathrm{E}-11 \\
1.1 \mathrm{E}-11 \\
1.1 \mathrm{E}-11 \\
1.1 \mathrm{E}-11 \\
1.2 \mathrm{E}-11 \\
1.2 \mathrm{E}-11 \\
\text { Page }\end{array}$ & $\begin{array}{l}1.1 \mathrm{E}-11 \\
1.1 \mathrm{E}-11 \\
1.1 \mathrm{E}-11 \\
1.1 \mathrm{E}-11 \\
1.1 \mathrm{E}-11 \\
1.1 \mathrm{E}-11 \\
1.2 \mathrm{E}-11 \\
1.2 \mathrm{E}-11 \\
5\end{array}$ & $\begin{array}{l}1 \cdot 1 \mathrm{E}-11 \\
1.1 \mathrm{E}-11 \\
1.1 \mathrm{E}-11 \\
1 \cdot 1 \mathrm{E}-11 \\
1.1 \mathrm{E}-11 \\
1.1 \mathrm{E}-11 \\
1.2 \mathrm{E}-11 \\
1.2 \mathrm{E}-11\end{array}$ & $\begin{array}{l}1.1 \mathrm{E}-11 \\
1.1 \mathrm{E}-11 \\
1.1 \mathrm{E}-11 \\
1.1 \mathrm{E}-11 \\
1.1 \mathrm{E}-11 \\
1.1 \mathrm{E}-11 \\
1.2 \mathrm{E}-11 \\
1.2 \mathrm{E}-11\end{array}$ \\
\hline
\end{tabular}




\begin{tabular}{rlllllll} 
& \multicolumn{7}{c}{$\mathrm{H}-3 \mathrm{~A} . \mathrm{SUM}$} \\
$\mathrm{S}$ & $1.3 \mathrm{E}-11$ & $1.3 \mathrm{E}-11$ & $1.3 \mathrm{E}-11$ & $1.3 \mathrm{E}-11$ & $1.3 \mathrm{E}-11$ & $1.3 \mathrm{E}-11$ & $1.3 \mathrm{E}-11$ \\
SSE & $1.3 \mathrm{E}-11$ & $1.3 \mathrm{E}-11$ & $1.3 \mathrm{E}-11$ & $1.3 \mathrm{E}-11$ & $1.3 \mathrm{E}-11$ & $1.3 \mathrm{E}-11$ & $1.3 \mathrm{E}-11$ \\
$\mathrm{SE}$ & $1.2 \mathrm{E}-11$ & $1.2 \mathrm{E}-11$ & $1.2 \mathrm{E}-11$ & $1.2 \mathrm{E}-11$ & $1.2 \mathrm{E}-11$ & $1.2 \mathrm{E}-11$ & $1.2 \mathrm{E}-11$ \\
$\mathrm{ESE}$ & $1.1 \mathrm{E}-11$ & $1.1 \mathrm{E}-11$ & $1.1 \mathrm{E}-11$ & $1.1 \mathrm{E}-11$ & $1.1 \mathrm{E}-11$ & $1.1 \mathrm{E}-11$ & $1.1 \mathrm{E}-11$ \\
$\mathrm{E}$ & $1.2 \mathrm{E}-11$ & $1.2 \mathrm{E}-11$ & $1.2 \mathrm{E}-11$ & $1.1 \mathrm{E}-11$ & $1.1 \mathrm{E}-11$ & $1.1 \mathrm{E}-11$ & $1.1 \mathrm{E}-11$ \\
$\mathrm{ENE}$ & $1.1 \mathrm{E}-11$ & $1.1 \mathrm{E}-11$ & $1.1 \mathrm{E}-11$ & $1.1 \mathrm{E}-11$ & $1.1 \mathrm{E}-11$ & $1.1 \mathrm{E}-11$ & $1.1 \mathrm{E}-11$ \\
$\mathrm{NE}$ & $1.2 \mathrm{E}-11$ & $1.2 \mathrm{E}-11$ & $1.2 \mathrm{E}-11$ & $1.2 \mathrm{E}-11$ & $1.2 \mathrm{E}-11$ & $1.2 \mathrm{E}-11$ & $1.2 \mathrm{E}-11$ \\
$\mathrm{NNE}$ & $1.1 \mathrm{E}-11$ & $1.1 \mathrm{E}-11$ & $1.1 \mathrm{E}-11$ & $1.1 \mathrm{E}-11$ & $1.1 \mathrm{E}-11$ & $1.1 \mathrm{E}-11$ & $1.1 \mathrm{E}-11$
\end{tabular}

Feb 20, $2008 \quad 02: 30 \mathrm{pm}$

SUMMARY

Page 8

INDIVIDUAL LIFETIME RISK (deaths)

(A11 Radionuclides and Pathways)

\begin{tabular}{|c|c|c|c|c|c|c|}
\hline \multirow[b]{2}{*}{ Direction } & \multicolumn{6}{|c|}{ Distance $(\mathrm{m})$} \\
\hline & 14374 & 15241 & 15441 & 15784 & 15844 & 16323 \\
\hline $\mathrm{N}$ & $1.1 \mathrm{E}-11$ & $1.1 \mathrm{E}-11$ & $1.1 \mathrm{E}-11$ & $1.1 \mathrm{E}-11$ & $1.1 \mathrm{E}-11$ & $1.1 \mathrm{E}-11$ \\
\hline NNW & $1.1 \mathrm{E}-11$ & $1.1 \mathrm{E}-11$ & $1.1 \mathrm{E}-11$ & $1.1 \mathrm{E}-11$ & $1.1 \mathrm{E}-11$ & $1.1 \mathrm{E}-11$ \\
\hline NW & $1.1 \mathrm{E}-11$ & $1.1 \mathrm{E}-11$ & $1.1 \mathrm{E}-11$ & $1.1 \mathrm{E}-11$ & $1.1 \mathrm{E}-11$ & $1.1 \mathrm{E}-11$ \\
\hline WNW & 1. $1 \mathrm{E}-11$ & $1.1 \mathrm{E}-11$ & 1. $1 \mathrm{E}-11$ & $1.1 \mathrm{E}-11$ & $1.1 \mathrm{E}-11$ & $1.1 \mathrm{E}-11$ \\
\hline W & $1.1 \mathrm{E}-11$ & $1.1 \mathrm{E}-11$ & $1.1 \mathrm{E}-11$ & $1.1 \mathrm{E}-11$ & $1.1 \mathrm{E}-11$ & $1.1 \mathrm{E}-11$ \\
\hline WSW & $1.1 \mathrm{E}-11$ & $1.1 \mathrm{E}-11$ & $1.1 \mathrm{E}-11$ & $1.1 \mathrm{E}-11$ & $1.1 \mathrm{E}-11$ & $1.1 \mathrm{E}-11$ \\
\hline SW & $1.2 \mathrm{E}-11$ & $1.1 \mathrm{E}-11$ & $1.1 \mathrm{E}-11$ & $1.1 \mathrm{E}-11$ & $1.1 \mathrm{E}-11$ & $1.1 \mathrm{E}-11$ \\
\hline SSW & $1.2 \mathrm{E}-11$ & $1.2 \mathrm{E}-11$ & $1.2 \mathrm{E}-11$ & $1.2 \mathrm{E}-11$ & $1.2 \mathrm{E}-11$ & $1.2 \mathrm{E}-11$ \\
\hline $\mathrm{S}$ & $1.3 \mathrm{E}-11$ & $1.3 \mathrm{E}-11$ & $1.3 \mathrm{E}-11$ & $1.3 \mathrm{E}-11$ & $1.3 \mathrm{E}-11$ & $1.3 \mathrm{E}-11$ \\
\hline SSE & $1.3 \mathrm{E}-11$ & $1.3 \mathrm{E}-11$ & $1.2 \mathrm{E}-11$ & $1.2 \mathrm{E}-11$ & $1.2 \mathrm{E}-11$ & $1.2 \mathrm{E}-11$ \\
\hline SE & $1.2 \mathrm{E}-11$ & $1.2 \mathrm{E}-11$ & $1.2 \mathrm{E}-11$ & $1.1 \mathrm{E}-11$ & $1.1 \mathrm{E}-11$ & $1.1 \mathrm{E}-11$ \\
\hline ESE & $1.1 \mathrm{E}-11$ & $1.1 \mathrm{E}-11$ & $1.1 \mathrm{E}-11$ & $1.1 \mathrm{E}-11$ & $1.1 \mathrm{E}-11$ & $1.1 \mathrm{E}-11$ \\
\hline $\mathrm{E}$ & $1.1 \mathrm{E}-11$ & $1.1 \mathrm{E}-11$ & $1.1 \mathrm{E}-11$ & $1.1 \mathrm{E}-11$ & $1.1 \mathrm{E}-11$ & $1.1 \mathrm{E}-11$ \\
\hline ENE & $1.1 \mathrm{E}-11$ & $1.1 \mathrm{E}-11$ & $1.1 \mathrm{E}-11$ & $1.1 \mathrm{E}-11$ & $1.1 \mathrm{E}-11$ & $1.1 \mathrm{E}-11$ \\
\hline $\mathrm{NE}$ & $1.2 \mathrm{E}-11$ & $1.2 \mathrm{E}-11$ & $1.2 \mathrm{E}-11$ & $1.2 \mathrm{E}-11$ & $1.2 \mathrm{E}-11$ & 1. $2 \mathrm{E}-11$ \\
\hline NNE & $1.1 \mathrm{E}-11$ & $1.1 \mathrm{E}-11$ & $1.1 \mathrm{E}-11$ & $1.1 \mathrm{E}-11$ & $1.1 \mathrm{E}-11$ & $1.1 \mathrm{E}-11$ \\
\hline
\end{tabular}




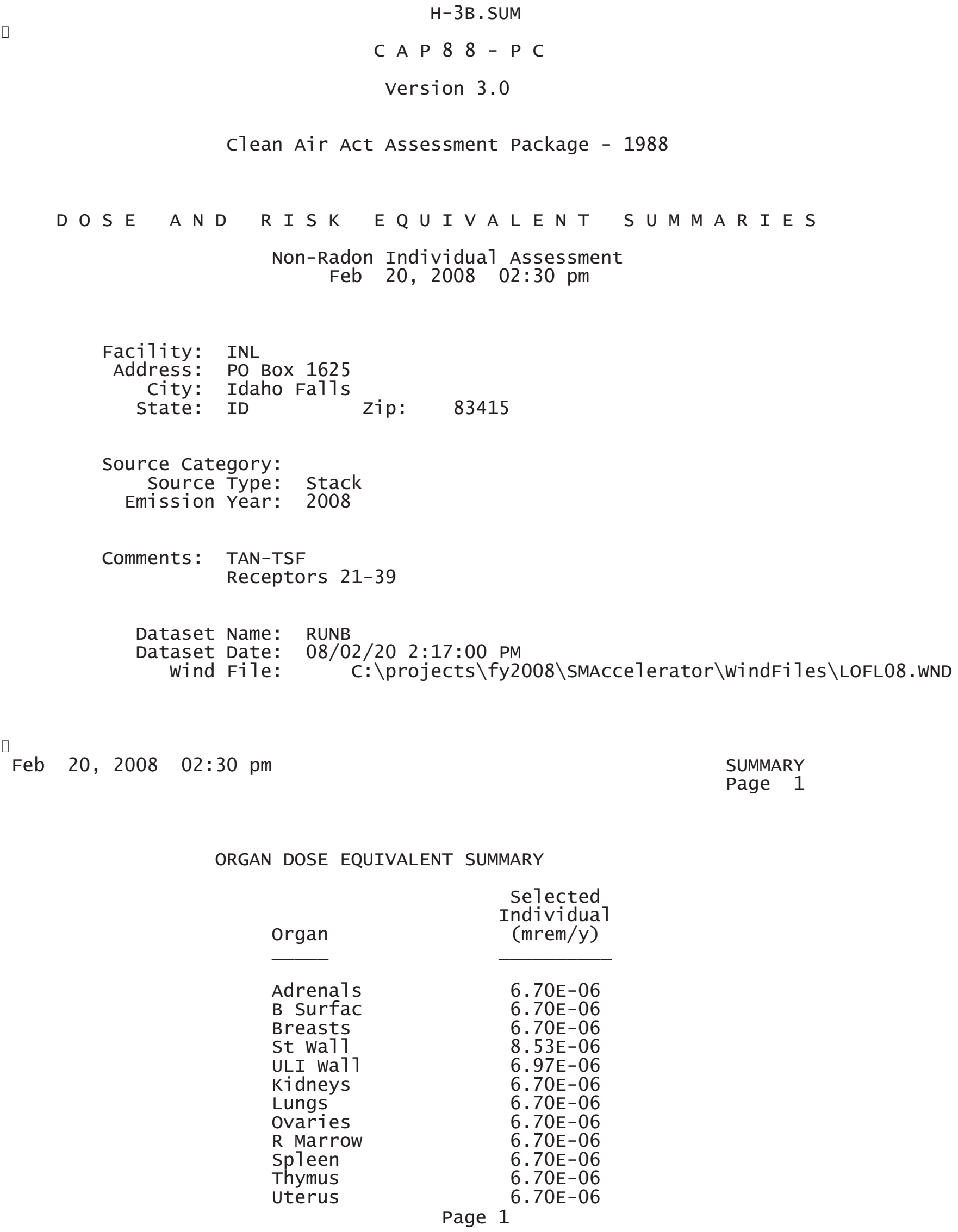

ORGAN DOSE EQUIVALENT SUMMARY

Organ

Adrenals

B Surfac Breasts

St wa11

ULI Wa71

Kidneys

Lungs

ovaries

R Marrow

spleen

Thymus

uterus

$$
\begin{gathered}
\begin{array}{c}
\text { Selected } \\
\text { Individual } \\
\text { (mrem/y) }
\end{array} \\
\hline 6.70 \mathrm{E}-06 \\
6.70 \mathrm{E}-06 \\
6.70 \mathrm{E}-06 \\
8.53 \mathrm{E}-06 \\
6.97 \mathrm{E}-06 \\
6.70 \mathrm{E}-06 \\
6.70 \mathrm{E}-06 \\
6.70 \mathrm{E}-06 \\
6.70 \mathrm{E}-06 \\
6.70 \mathrm{E}-06 \\
6.70 \mathrm{E}-06 \\
6.70 \mathrm{E}-06
\end{gathered}
$$

Page 1 


$\begin{array}{lr} & \text { H-3B.SUM } \\ \text { Bld wa11 } & 6.70 \mathrm{E}-06 \\ \text { Brain } & 6.70 \mathrm{E}-06 \\ \text { Esophagu } & 6.70 \mathrm{E}-06 \\ \text { SI wa11 } & 6.74 \mathrm{E}-06 \\ \text { LLI wa11 } & 7.51 \mathrm{E}-06 \\ \text { Liver } & 6.70 \mathrm{E}-06 \\ \text { Muscle } & 6.70 \mathrm{E}-06 \\ \text { Pancreas } & 6.70 \mathrm{E}-06 \\ \text { Skin } & 6.70 \mathrm{E}-06 \\ \text { Testes } & 6.70 \mathrm{E}-06 \\ \text { Thyroid } & 6.70 \mathrm{E}-06 \\ & \\ \text { EFFEC } & 1.31 \mathrm{E}-05\end{array}$

PATHWAY EFFECTIVE DOSE EQUIVALENT SUMMARY

\begin{tabular}{lcc} 
Pathway & $\begin{array}{c}\text { Selected } \\
\text { Individua1 } \\
\text { (mrem/y) }\end{array}$ \\
\cline { 2 - 2 } INGESTION & $1.07 \mathrm{E}-05$ \\
INHALATION & $2.43 \mathrm{E}-06$ \\
AIR IMMERSION & $0.00 \mathrm{E}+00$ & \\
GROUND SURFACE & $0.00 \mathrm{E}+00$ & \\
INTERNAL & $1.31 \mathrm{E}-05$ & \\
EXTERNAL & $0.00 \mathrm{E}+00$ & \\
TOTAL & $1.31 \mathrm{E}-05$ & SUMMARY \\
pm & & Page 2
\end{tabular}

NUCLIDE EFFECTIVE DOSE EQUIVALENT SUMMARY

Nuclide

$\mathrm{H}-3$

TOTAL

Feb 20, $2008 \quad 02: 30$ pm

$$
\begin{gathered}
\begin{array}{c}
\text { Selected } \\
\text { Individua1 } \\
\text { (mrem/y) }
\end{array} \\
\hline 1.31 \mathrm{E}-05 \\
1.31 \mathrm{E}-05
\end{gathered}
$$

SUMMARY

Page 3

CANCER RISK SUMMARY

Cancer

Esophagu
Selected Individual

Total Lifetime Fatal Cancer Risk

$$
\text { Page } 2^{1.52 \mathrm{E}-13}
$$




$\begin{array}{lr} & \text { H-3B.SUM } \\ \text { Stomach } & 7.64 \mathrm{E}-13 \\ \text { Colon } & 1.56 \mathrm{E}-12 \\ \text { Liver } & 2.12 \mathrm{E}-13 \\ \text { LUNG } & 1.29 \mathrm{E}-12 \\ \text { Bone } & 1.29 \mathrm{E}-14 \\ \text { Skin } & 1.37 \mathrm{E}-14 \\ \text { Breast } & 6.09 \mathrm{E}-13 \\ \text { Ovary } & 1.74 \mathrm{E}-13 \\ \text { B1adder } & 3.41 \mathrm{E}-13 \\ \text { Kidneys } & 7.31 \mathrm{E}-14 \\ \text { Thyroid } & 4.21 \mathrm{E}-14 \\ \text { Leukemia } & 7.79 \mathrm{E}-13 \\ \text { Residua1 } & 2.06 \mathrm{E}-12 \\ \text { Tota1 } & 8.09 \mathrm{E}-12 \\ & \\ \text { TOTAL } & 1.62 \mathrm{E}-11\end{array}$

PATHWAY RISK SUMMARY

\begin{tabular}{lc} 
Pathway & $\begin{array}{c}\text { Selected Individua1 } \\
\text { Tota1 Lifetime } \\
\text { Fatal Cancer Risk }\end{array}$ \\
\cline { 2 - 2 } INGESTION & $6.71 \mathrm{E}-12$ \\
INHALATION & $1.38 \mathrm{E}-12$ \\
AIR IMMERSION & $0.00 \mathrm{E}+00$ \\
GROUND SURFACE & $0.00 \mathrm{E}+00$ \\
INTERNAL & $8.09 \mathrm{E}-12$ \\
EXTERNAL & $0.00 \mathrm{E}+00$ \\
TOTAL & $8.09 \mathrm{E}-12$
\end{tabular}

Feb 20, $2008 \quad 02: 30$ pm

SUMMARY Page 4

NUCLIDE RISK SUMMARY

Nuclide

H-3

TOTAL

$\square$

Feb 20, 2008 02:30 pm
Selected Individual

Total Lifetime Fatal Cancer Risk

8.09E-12

8. 09E-12

INDIVIDUAL EFFECTIVE DOSE EQUIVALENT RATE (mrem/y) (A11 Radionuclides and Pathways)

Distance $(m)$ 


\begin{tabular}{rccccccc}
\multicolumn{1}{c}{ Direction } & 17035 & 17329 & 18607 & 18834 & 18860 & 18890 & 19860 \\
\hline N & $1.0 \mathrm{E}-05$ & $1.0 \mathrm{E}-05$ & $1.0 \mathrm{E}-05$ & $1.0 \mathrm{E}-05$ & $1.0 \mathrm{E}-05$ & $1.0 \mathrm{E}-05$ & $1.0 \mathrm{E}-05$ \\
NNW & $9.7 \mathrm{E}-06$ & $9.7 \mathrm{E}-06$ & $9.7 \mathrm{E}-06$ & $9.7 \mathrm{E}-06$ & $9.7 \mathrm{E}-06$ & $9.7 \mathrm{E}-06$ & $9.6 \mathrm{E}-06$ \\
NW & $9.8 \mathrm{E}-06$ & $9.8 \mathrm{E}-06$ & $9.8 \mathrm{E}-06$ & $9.8 \mathrm{E}-06$ & $9.8 \mathrm{E}-06$ & $9.8 \mathrm{E}-06$ & $9.8 \mathrm{E}-06$ \\
WNW & $1.0 \mathrm{E}-05$ & $1.0 \mathrm{E}-05$ & $9.9 \mathrm{E}-06$ & $9.9 \mathrm{E}-06$ & $9.9 \mathrm{E}-06$ & $9.9 \mathrm{E}-06$ & $9.9 \mathrm{E}-06$ \\
W & $1.0 \mathrm{E}-05$ & $1.0 \mathrm{E}-05$ & $1.0 \mathrm{E}-05$ & $1.0 \mathrm{E}-05$ & $1.0 \mathrm{E}-05$ & $1.0 \mathrm{E}-05$ & $1.0 \mathrm{E}-05$ \\
WSW & $1.0 \mathrm{E}-05$ & $1.0 \mathrm{E}-05$ & $1.0 \mathrm{E}-05$ & $1.0 \mathrm{E}-05$ & $1.0 \mathrm{E}-05$ & $1.0 \mathrm{E}-05$ & $1.0 \mathrm{E}-05$ \\
SW & $1.1 \mathrm{E}-05$ & $1.1 \mathrm{E}-05$ & $1.1 \mathrm{E}-05$ & $1.1 \mathrm{E}-05$ & $1.1 \mathrm{E}-05$ & $1.1 \mathrm{E}-05$ & $1.1 \mathrm{E}-05$ \\
SSW & $1.2 \mathrm{E}-05$ & $1.2 \mathrm{E}-05$ & $1.2 \mathrm{E}-05$ & $1.2 \mathrm{E}-05$ & $1.2 \mathrm{E}-05$ & $1.2 \mathrm{E}-05$ & $1.2 \mathrm{E}-05$ \\
$\mathrm{~S}$ & $1.3 \mathrm{E}-05$ & $1.3 \mathrm{E}-05$ & $1.3 \mathrm{E}-05$ & $1.3 \mathrm{E}-05$ & $1.3 \mathrm{E}-05$ & $1.3 \mathrm{E}-05$ & $1.3 \mathrm{E}-05$ \\
SSE & $1.3 \mathrm{E}-05$ & $1.2 \mathrm{E}-05$ & $1.2 \mathrm{E}-05$ & $1.2 \mathrm{E}-05$ & $1.2 \mathrm{E}-05$ & $1.2 \mathrm{E}-05$ & $1.2 \mathrm{E}-05$ \\
SE & $1.1 \mathrm{E}-05$ & $1.1 \mathrm{E}-05$ & $1.1 \mathrm{E}-05$ & $1.1 \mathrm{E}-05$ & $1.1 \mathrm{E}-05$ & $1.1 \mathrm{E}-05$ & $1.1 \mathrm{E}-05$ \\
ESE & $1.0 \mathrm{E}-05$ & $1.0 \mathrm{E}-05$ & $1.0 \mathrm{E}-05$ & $1.0 \mathrm{E}-05$ & $1.0 \mathrm{E}-05$ & $1.0 \mathrm{E}-05$ & $1.0 \mathrm{E}-05$ \\
$\mathrm{E}$ & $1.1 \mathrm{E}-05$ & $1.1 \mathrm{E}-05$ & $1.1 \mathrm{E}-05$ & $1.1 \mathrm{E}-05$ & $1.1 \mathrm{E}-05$ & $1.1 \mathrm{E}-05$ & $1.1 \mathrm{E}-05$ \\
ENE & $1.1 \mathrm{E}-05$ & $1.1 \mathrm{E}-05$ & $1.0 \mathrm{E}-05$ & $1.0 \mathrm{E}-05$ & $1.0 \mathrm{E}-05$ & $1.0 \mathrm{E}-05$ & $1.0 \mathrm{E}-05$ \\
$\mathrm{NE}$ & $1.1 \mathrm{E}-05$ & $1.1 \mathrm{E}-05$ & $1.1 \mathrm{E}-05$ & $1.1 \mathrm{E}-05$ & $1.1 \mathrm{E}-05$ & $1.1 \mathrm{E}-05$ & $1.1 \mathrm{E}-05$ \\
$\mathrm{NNE}$ & $1.1 \mathrm{E}-05$ & $1.1 \mathrm{E}-05$ & $1.0 \mathrm{E}-05$ & $1.0 \mathrm{E}-05$ & $1.0 \mathrm{E}-05$ & $1.0 \mathrm{E}-05$ & $1.0 \mathrm{E}-05$ \\
\hline
\end{tabular}

Distance $(\mathrm{m})$

\begin{tabular}{|c|c|c|c|c|c|c|c|}
\hline Direction & 19891 & 20457 & 21314 & 21349 & 22159 & 23521 & 24430 \\
\hline $\mathrm{N}$ & 1. $0 \mathrm{E}-05$ & 1. $0 \mathrm{E}-05$ & 1. $0 \mathrm{E}-05$ & 1.0E-05 & 1. $0 \mathrm{E}-05$ & $1.0 \mathrm{E}-05$ & 1. $0 \mathrm{E}-05$ \\
\hline NNW & $9.6 \mathrm{E}-06$ & $9.6 \mathrm{E}-06$ & $9.6 \mathrm{E}-06$ & $9.6 \mathrm{E}-06$ & $9.6 \mathrm{E}-06$ & $9.6 \mathrm{E}-06$ & $9.6 \mathrm{E}-06$ \\
\hline NW & $9.8 \mathrm{E}-06$ & $9.7 \mathrm{E}-06$ & $9.7 \mathrm{E}-06$ & $9.7 \mathrm{E}-06$ & $9.7 \mathrm{E}-06$ & $9.7 \mathrm{E}-06$ & $9.7 \mathrm{E}-06$ \\
\hline WNW & $9.9 \mathrm{E}-06$ & $9.9 \mathrm{E}-06$ & $9.9 \mathrm{E}-06$ & $9.9 \mathrm{E}-06$ & $9.8 \mathrm{E}-06$ & $9.8 \mathrm{E}-06$ & $9.8 \mathrm{E}-06$ \\
\hline W & $1.0 \mathrm{E}-05$ & $1.0 \mathrm{E}-05$ & $9.9 \mathrm{E}-06$ & $9.9 \mathrm{E}-06$ & $9.9 \mathrm{E}-06$ & $9.9 \mathrm{E}-06$ & $9.8 \mathrm{E}-06$ \\
\hline WSW & $1.0 \mathrm{E}-05$ & $1.0 \mathrm{E}-05$ & $1.0 \mathrm{E}-05$ & $1.0 \mathrm{E}-05$ & $1.0 \mathrm{E}-05$ & $1.0 \mathrm{E}-05$ & 1. $0 \mathrm{E}-05$ \\
\hline SW & $1.1 \mathrm{E}-05$ & $1.1 \mathrm{E}-05$ & $1.1 \mathrm{E}-05$ & $1.1 \mathrm{E}-05$ & $1.1 \mathrm{E}-05$ & 1. $0 \mathrm{E}-05$ & 1. $0 \mathrm{E}-05$ \\
\hline SSW & 1.2E-05 & $1.2 \mathrm{E}-05$ & $1.1 \mathrm{E}-05$ & $1.1 \mathrm{E}-05$ & $1.1 \mathrm{E}-05$ & $1.1 \mathrm{E}-05$ & $1.1 \mathrm{E}-05$ \\
\hline $\mathrm{S}$ & $1.2 \mathrm{E}-05$ & $1.2 \mathrm{E}-05$ & $1.2 \mathrm{E}-05$ & $1.2 \mathrm{E}-05$ & $1.2 \mathrm{E}-05$ & $1.2 \mathrm{E}-05$ & 1. $2 \mathrm{E}-05$ \\
\hline SSE & $1.2 \mathrm{E}-05$ & $1.2 \mathrm{E}-05$ & $1.2 \mathrm{E}-05$ & $1.2 \mathrm{E}-05$ & $1.2 \mathrm{E}-05$ & $1.2 \mathrm{E}-05$ & 1. $1 \mathrm{E}-05$ \\
\hline SE & $1.1 \mathrm{E}-05$ & $1.1 \mathrm{E}-05$ & $1.1 \mathrm{E}-05$ & $1.1 \mathrm{E}-05$ & $1.1 \mathrm{E}-05$ & $1.1 \mathrm{E}-05$ & 1. $0 \mathrm{E}-05$ \\
\hline ESE & $1.0 \mathrm{E}-05$ & 1. $0 \mathrm{E}-05$ & $1.0 \mathrm{E}-05$ & $1.0 \mathrm{E}-05$ & $1.0 \mathrm{E}-05$ & 1. $0 \mathrm{E}-05$ & 1. $0 \mathrm{E}-05$ \\
\hline E & $1.1 \mathrm{E}-05$ & $1.1 \mathrm{E}-05$ & $1.0 \mathrm{E}-05$ & $1.0 \mathrm{E}-05$ & 1.0E-05 & 1.0E-05 & 1. $0 \mathrm{E}-05$ \\
\hline ENE & $1.0 \mathrm{E}-05$ & $1.0 \mathrm{E}-05$ & 1.0E-05 & $1.0 \mathrm{E}-05$ & 1.0E-05 & 1.0E-05 & 1. $0 \mathrm{E}-05$ \\
\hline $\mathrm{NE}$ & $1.1 \mathrm{E}-05$ & 1.1E-05 & 1.1E-05 & $1.1 \mathrm{E}-05$ & $1.1 \mathrm{E}-05$ & $1.1 \mathrm{E}-05$ & 1. $1 \mathrm{E}-05$ \\
\hline NNE & $1.0 \mathrm{E}-05$ & 1.0E-05 & 1.0E-05 & 1.0E-05 & 1.0E-05 & 1.0E-05 & 1. $0 \mathrm{E}-05$ \\
\hline
\end{tabular}

$\square$

Feb 20, 2008 02:30 pm

SUMMARY

Page 6

INDIVIDUAL EFFECTIVE DOSE EQUIVALENT RATE (mrem/y) (A11 Radionuclides and Pathways)

\begin{tabular}{|c|c|c|c|c|c|}
\hline \multirow[b]{2}{*}{ Direction } & \multicolumn{5}{|c|}{ Distance (m) } \\
\hline & 24545 & 25171 & 26794 & 27275 & 27389 \\
\hline $\begin{array}{r}N \\
N N W\end{array}$ & $\begin{array}{l}1.0 \mathrm{E}-05 \\
9.6 \mathrm{E}-06\end{array}$ & $\begin{array}{l}1.0 \mathrm{E}-05 \\
9.6 \mathrm{E}-06\end{array}$ & $\begin{array}{l}9.9 \mathrm{E}-06 \\
9.5 \mathrm{E}-06\end{array}$ & $\begin{array}{r}9.9 \mathrm{E}-06 \\
9.5 \mathrm{E}-06 \\
\text { Page }\end{array}$ & $\begin{array}{l}9.9 \mathrm{E}-06 \\
9.5 \mathrm{E}-06 \\
4\end{array}$ \\
\hline
\end{tabular}




\begin{tabular}{rlllll} 
& & & \multicolumn{3}{c}{$\mathrm{H}-3 \mathrm{~B} . \mathrm{SUM}$} \\
$\mathrm{NW}$ & $9.7 \mathrm{E}-06$ & $9.7 \mathrm{E}-06$ & $9.6 \mathrm{E}-06$ & $9.6 \mathrm{E}-06$ & $9.6 \mathrm{E}-06$ \\
WNW & $9.8 \mathrm{E}-06$ & $9.8 \mathrm{E}-06$ & $9.7 \mathrm{E}-06$ & $9.7 \mathrm{E}-06$ & $9.7 \mathrm{E}-06$ \\
$\mathrm{~W}$ & $9.8 \mathrm{E}-06$ & $9.8 \mathrm{E}-06$ & $9.8 \mathrm{E}-06$ & $9.8 \mathrm{E}-06$ & $9.8 \mathrm{E}-06$ \\
WSW & $1.0 \mathrm{E}-05$ & $1.0 \mathrm{E}-05$ & $9.9 \mathrm{E}-06$ & $9.9 \mathrm{E}-06$ & $9.9 \mathrm{E}-06$ \\
$\mathrm{SW}$ & $1.0 \mathrm{E}-05$ & $1.0 \mathrm{E}-05$ & $1.0 \mathrm{E}-05$ & $1.0 \mathrm{E}-05$ & $1.0 \mathrm{E}-05$ \\
$\mathrm{SSW}$ & $1.1 \mathrm{E}-05$ & $1.1 \mathrm{E}-05$ & $1.1 \mathrm{E}-05$ & $1.1 \mathrm{E}-05$ & $1.1 \mathrm{E}-05$ \\
$\mathrm{~S}$ & $1.2 \mathrm{E}-05$ & $1.2 \mathrm{E}-05$ & $1.2 \mathrm{E}-05$ & $1.2 \mathrm{E}-05$ & $1.1 \mathrm{E}-05$ \\
$\mathrm{SSE}$ & $1.1 \mathrm{E}-05$ & $1.1 \mathrm{E}-05$ & $1.1 \mathrm{E}-05$ & $1.1 \mathrm{E}-05$ & $1.1 \mathrm{E}-05$ \\
$\mathrm{SE}$ & $1.0 \mathrm{E}-05$ & $1.0 \mathrm{E}-05$ & $1.0 \mathrm{E}-05$ & $1.0 \mathrm{E}-05$ & $1.0 \mathrm{E}-05$ \\
$\mathrm{ESE}$ & $1.0 \mathrm{E}-05$ & $1.0 \mathrm{E}-05$ & $1.0 \mathrm{E}-05$ & $1.0 \mathrm{E}-05$ & $1.0 \mathrm{E}-05$ \\
$\mathrm{E}$ & $1.0 \mathrm{E}-05$ & $1.0 \mathrm{E}-05$ & $1.0 \mathrm{E}-05$ & $1.0 \mathrm{E}-05$ & $1.0 \mathrm{E}-05$ \\
$\mathrm{ENE}$ & $1.0 \mathrm{E}-05$ & $1.0 \mathrm{E}-05$ & $1.0 \mathrm{E}-05$ & $1.0 \mathrm{E}-05$ & $1.0 \mathrm{E}-05$ \\
$\mathrm{NE}$ & $1.1 \mathrm{E}-05$ & $1.1 \mathrm{E}-05$ & $1.0 \mathrm{E}-05$ & $1.0 \mathrm{E}-05$ & $1.0 \mathrm{E}-05$ \\
$\mathrm{NNE}$ & $1.0 \mathrm{E}-05$ & $1.0 \mathrm{E}-05$ & $1.0 \mathrm{E}-05$ & $1.0 \mathrm{E}-05$ & $1.0 \mathrm{E}-05$ \\
& & & & &
\end{tabular}

Feb 20, $2008 \quad 02: 30$ pm

SUMMARY

Page 7

INDIVIDUAL LIFETIME RISK (deaths)

(A11 Radionuclides and Pathways)

\begin{tabular}{|c|c|c|c|c|c|c|c|}
\hline \multicolumn{8}{|c|}{ Distance $(\mathrm{m})$} \\
\hline Direction & 17035 & 17329 & 18607 & 18834 & 18860 & 18890 & 19860 \\
\hline $\begin{array}{r}\text { N } \\
\text { NNW } \\
N W \\
\text { WNW } \\
W \\
W S W \\
\text { SW } \\
\text { SSW } \\
\text { S } \\
\text { SSE } \\
\text { SE } \\
\text { ESE } \\
\text { E } \\
\text { ENE } \\
\text { NE } \\
\text { NNE }\end{array}$ & $\begin{array}{l}6.4 \mathrm{E}-12 \\
6.1 \mathrm{E}-12 \\
6.1 \mathrm{E}-12 \\
6.2 \mathrm{E}-12 \\
6.3 \mathrm{E}-12 \\
6.4 \mathrm{E}-12 \\
6.8 \mathrm{E}-12 \\
7.5 \mathrm{E}-12 \\
8.1 \mathrm{E}-12 \\
7.7 \mathrm{E}-12 \\
6.9 \mathrm{E}-12 \\
6.5 \mathrm{E}-12 \\
6.7 \mathrm{E}-12 \\
6.6 \mathrm{E}-12 \\
6.9 \mathrm{E}-12 \\
6.6 \mathrm{E}-12\end{array}$ & $\begin{array}{l}6.4 \mathrm{E}-12 \\
6.1 \mathrm{E}-12 \\
6.1 \mathrm{E}-12 \\
6.2 \mathrm{E}-12 \\
6.3 \mathrm{E}-12 \\
6.4 \mathrm{E}-12 \\
6.8 \mathrm{E}-12 \\
7.4 \mathrm{E}-12 \\
8.0 \mathrm{E}-12 \\
7.7 \mathrm{E}-12 \\
6.8 \mathrm{E}-12 \\
6.5 \mathrm{E}-12 \\
6.7 \mathrm{E}-12 \\
6.6 \mathrm{E}-12 \\
6.9 \mathrm{E}-12 \\
6.5 \mathrm{E}-12\end{array}$ & $\begin{array}{l}6.4 \mathrm{E}-12 \\
6.0 \mathrm{E}-12 \\
6.1 \mathrm{E}-12 \\
6.2 \mathrm{E}-12 \\
6.3 \mathrm{E}-12 \\
6.4 \mathrm{E}-12 \\
6.7 \mathrm{E}-12 \\
7.3 \mathrm{E}-12 \\
7.9 \mathrm{E}-12 \\
7.5 \mathrm{E}-12 \\
6.8 \mathrm{E}-12 \\
6.5 \mathrm{E}-12 \\
6.6 \mathrm{E}-12 \\
6.5 \mathrm{E}-12 \\
6.8 \mathrm{E}-12 \\
6.5 \mathrm{E}-12\end{array}$ & $\begin{array}{l}6.4 \mathrm{E}-12 \\
6.0 \mathrm{E}-12 \\
6.1 \mathrm{E}-12 \\
6.2 \mathrm{E}-12 \\
6.2 \mathrm{E}-12 \\
6.4 \mathrm{E}-12 \\
6.7 \mathrm{E}-12 \\
7.3 \mathrm{E}-12 \\
7.8 \mathrm{E}-12 \\
7.5 \mathrm{E}-12 \\
6.7 \mathrm{E}-12 \\
6.4 \mathrm{E}-12 \\
6.6 \mathrm{E}-12 \\
6.5 \mathrm{E}-12 \\
6.8 \mathrm{E}-12 \\
6.5 \mathrm{E}-12\end{array}$ & $\begin{array}{l}6.4 \mathrm{E}-12 \\
6.0 \mathrm{E}-12 \\
6.1 \mathrm{E}-12 \\
6.2 \mathrm{E}-12 \\
6.2 \mathrm{E}-12 \\
6.4 \mathrm{E}-12 \\
6.7 \mathrm{E}-12 \\
7.3 \mathrm{E}-12 \\
7.8 \mathrm{E}-12 \\
7.5 \mathrm{E}-12 \\
6.7 \mathrm{E}-12 \\
6.4 \mathrm{E}-12 \\
6.6 \mathrm{E}-12 \\
6.5 \mathrm{E}-12 \\
6.8 \mathrm{E}-12 \\
6.5 \mathrm{E}-12\end{array}$ & $\begin{array}{l}6.3 \mathrm{E}-12 \\
6.0 \mathrm{E}-12 \\
6.1 \mathrm{E}-12 \\
6.2 \mathrm{E}-12 \\
6.2 \mathrm{E}-12 \\
6.4 \mathrm{E}-12 \\
6.7 \mathrm{E}-12 \\
7.3 \mathrm{E}-12 \\
7.8 \mathrm{E}-12 \\
7.5 \mathrm{E}-12 \\
6.7 \mathrm{E}-12 \\
6.4 \mathrm{E}-12 \\
6.6 \mathrm{E}-12 \\
6 \cdot 5 \mathrm{E}-12 \\
6.8 \mathrm{E}-12 \\
6.5 \mathrm{E}-12\end{array}$ & $\begin{array}{l}6.3 \mathrm{E}-12 \\
6.0 \mathrm{E}-12 \\
6.1 \mathrm{E}-12 \\
6.2 \mathrm{E}-12 \\
6.2 \mathrm{E}-12 \\
6.3 \mathrm{E}-12 \\
6.6 \mathrm{E}-12 \\
7.2 \mathrm{E}-12 \\
7.7 \mathrm{E}-12 \\
7.4 \mathrm{E}-12 \\
6.7 \mathrm{E}-12 \\
6.4 \mathrm{E}-12 \\
6.6 \mathrm{E}-12 \\
6.5 \mathrm{E}-12 \\
6.8 \mathrm{E}-12 \\
6.4 \mathrm{E}-12\end{array}$ \\
\hline \multicolumn{8}{|c|}{ Distance (m) } \\
\hline Direction & 19891 & 20457 & 21314 & 21349 & 22159 & 23521 & 24430 \\
\hline $\begin{array}{r}\text { N } \\
\text { NNW } \\
\text { NW } \\
\text { WNW } \\
\text { W } \\
\text { WSW } \\
\text { SW } \\
\text { SSW }\end{array}$ & $\begin{array}{l}6.3 \mathrm{E}-12 \\
6.0 \mathrm{E}-12 \\
6.1 \mathrm{E}-12 \\
6.2 \mathrm{E}-12 \\
6.2 \mathrm{E}-12 \\
6.3 \mathrm{E}-12 \\
6.6 \mathrm{E}-12 \\
7.2 \mathrm{E}-12\end{array}$ & $\begin{array}{l}6.3 \mathrm{E}-12 \\
6.0 \mathrm{E}-12 \\
6.1 \mathrm{E}-12 \\
6.2 \mathrm{E}-12 \\
6.2 \mathrm{E}-12 \\
6.3 \mathrm{E}-12 \\
6.6 \mathrm{E}-12 \\
7.1 \mathrm{E}-12\end{array}$ & $\begin{array}{l}6.3 \mathrm{E}-12 \\
6.0 \mathrm{E}-12 \\
6.1 \mathrm{E}-12 \\
6.2 \mathrm{E}-12 \\
6.2 \mathrm{E}-12 \\
6.3 \mathrm{E}-12 \\
6.6 \mathrm{E}-12 \\
7.1 \mathrm{E}-12\end{array}$ & $\begin{array}{r}6.3 \mathrm{E}-12 \\
6.0 \mathrm{E}-12 \\
6.1 \mathrm{E}-12 \\
6.2 \mathrm{E}-12 \\
6.2 \mathrm{E}-12 \\
6.3 \mathrm{E}-12 \\
6.6 \mathrm{E}-12 \\
7.1 \mathrm{E}-12 \\
\text { Page }\end{array}$ & $\begin{array}{l}6.3 \mathrm{E}-12 \\
6 \cdot 0 \mathrm{E}-12 \\
6.1 \mathrm{E}-12 \\
6.1 \mathrm{E}-12 \\
6.2 \mathrm{E}-12 \\
6.3 \mathrm{E}-12 \\
6.6 \mathrm{E}-12 \\
7.0 \mathrm{E}-12\end{array}$ & $\begin{array}{l}6.2 \mathrm{E}-12 \\
6.0 \mathrm{E}-12 \\
6.1 \mathrm{E}-12 \\
6.1 \mathrm{E}-12 \\
6.2 \mathrm{E}-12 \\
6.3 \mathrm{E}-12 \\
6.5 \mathrm{E}-12 \\
6.9 \mathrm{E}-12\end{array}$ & $\begin{array}{l}6.2 \mathrm{E}-12 \\
6.0 \mathrm{E}-12 \\
6.0 \mathrm{E}-12 \\
6.1 \mathrm{E}-12 \\
6.1 \mathrm{E}-12 \\
6.2 \mathrm{E}-12 \\
6.5 \mathrm{E}-12 \\
6.9 \mathrm{E}-12\end{array}$ \\
\hline
\end{tabular}




\begin{tabular}{rlllllll} 
& \multicolumn{7}{c}{$\mathrm{H}-3 \mathrm{~B} \cdot \mathrm{SUM}$} \\
$\mathrm{S}$ & $7.7 \mathrm{E}-12$ & $7.6 \mathrm{E}-12$ & $7.6 \mathrm{E}-12$ & $7.6 \mathrm{E}-12$ & $7.5 \mathrm{E}-12$ & $7.4 \mathrm{E}-12$ & $7.3 \mathrm{E}-12$ \\
SSE & $7.4 \mathrm{E}-12$ & $7.4 \mathrm{E}-12$ & $7.3 \mathrm{E}-12$ & $7.3 \mathrm{E}-12$ & $7.2 \mathrm{E}-12$ & $7.1 \mathrm{E}-12$ & $7.1 \mathrm{E}-12$ \\
SE & $6.7 \mathrm{E}-12$ & $6.7 \mathrm{E}-12$ & $6.6 \mathrm{E}-12$ & $6.6 \mathrm{E}-12$ & $6.6 \mathrm{E}-12$ & $6.5 \mathrm{E}-12$ & $6.5 \mathrm{E}-12$ \\
ESE & $6.4 \mathrm{E}-12$ & $6.4 \mathrm{E}-12$ & $6.4 \mathrm{E}-12$ & $6.4 \mathrm{E}-12$ & $6.3 \mathrm{E}-12$ & $6.3 \mathrm{E}-12$ & $6.3 \mathrm{E}-12$ \\
$\mathrm{E}$ & $6.6 \mathrm{E}-12$ & $6.6 \mathrm{E}-12$ & $6.5 \mathrm{E}-12$ & $6.5 \mathrm{E}-12$ & $6.5 \mathrm{E}-12$ & $6.4 \mathrm{E}-12$ & $6.4 \mathrm{E}-12$ \\
$\mathrm{ENE}$ & $6.5 \mathrm{E}-12$ & $6.4 \mathrm{E}-12$ & $6.4 \mathrm{E}-12$ & $6.4 \mathrm{E}-12$ & $6.4 \mathrm{E}-12$ & $6.4 \mathrm{E}-12$ & $6.3 \mathrm{E}-12$ \\
NE & $6.8 \mathrm{E}-12$ & $6.7 \mathrm{E}-12$ & $6.7 \mathrm{E}-12$ & $6.7 \mathrm{E}-12$ & $6.6 \mathrm{E}-12$ & $6.6 \mathrm{E}-12$ & $6.6 \mathrm{E}-12$ \\
NNE & $6.4 \mathrm{E}-12$ & $6.4 \mathrm{E}-12$ & $6.4 \mathrm{E}-12$ & $6.4 \mathrm{E}-12$ & $6.4 \mathrm{E}-12$ & $6.3 \mathrm{E}-12$ & $6.3 \mathrm{E}-12$
\end{tabular}

Feb 20, $2008 \quad 02: 30 \mathrm{pm}$

SUMMARY

Page 8

INDIVIDUAL LIFETIME RISK (deaths)

(A11 Radionuclides and Pathways)

\begin{tabular}{|c|c|c|c|c|c|}
\hline \multirow[b]{2}{*}{ Direction } & \multicolumn{5}{|c|}{ Distance (m) } \\
\hline & 24545 & 25171 & 26794 & 27275 & 27389 \\
\hline $\begin{array}{r}\text { N } \\
N N W \\
N W \\
W N W \\
W \\
W S W \\
\text { SW } \\
\text { SSW } \\
S \\
\text { SSE } \\
\text { SE } \\
\text { ESE } \\
\text { E } \\
\text { ENE } \\
N E \\
\text { NNE }\end{array}$ & $\begin{array}{l}6.2 \mathrm{E}-12 \\
6 \cdot 0 \mathrm{E}-12 \\
6 \cdot 0 \mathrm{E}-12 \\
6.1 \mathrm{E}-12 \\
6.1 \mathrm{E}-12 \\
6.2 \mathrm{E}-12 \\
6.5 \mathrm{E}-12 \\
6.9 \mathrm{E}-12 \\
7.3 \mathrm{E}-12 \\
7.1 \mathrm{E}-12 \\
6.5 \mathrm{E}-12 \\
6.3 \mathrm{E}-12 \\
6 \cdot 4 \mathrm{E}-12 \\
6.3 \mathrm{E}-12 \\
6.6 \mathrm{E}-12 \\
6.3 \mathrm{E}-12\end{array}$ & $\begin{array}{l}6 \cdot 2 \mathrm{E}-12 \\
6 \cdot 0 \mathrm{E}-12 \\
6 \cdot 0 \mathrm{E}-12 \\
6 \cdot 1 \mathrm{E}-12 \\
6 \cdot 1 \mathrm{E}-12 \\
6.2 \mathrm{E}-12 \\
6 \cdot 5 \mathrm{E}-12 \\
6 \cdot 9 \mathrm{E}-12 \\
7 \cdot 3 \mathrm{E}-12 \\
7 \cdot 0 \mathrm{E}-12 \\
6 \cdot 5 \mathrm{E}-12 \\
6.3 \mathrm{E}-12 \\
6 \cdot 4 \mathrm{E}-12 \\
6 \cdot 3 \mathrm{E}-12 \\
6 \cdot 5 \mathrm{E}-12 \\
6 \cdot 3 \mathrm{E}-12\end{array}$ & $\begin{array}{l}6.2 \mathrm{E}-12 \\
6 \cdot 0 \mathrm{E}-12 \\
6.0 \mathrm{E}-12 \\
6.1 \mathrm{E}-12 \\
6.1 \mathrm{E}-12 \\
6.2 \mathrm{E}-12 \\
6.4 \mathrm{E}-12 \\
6.8 \mathrm{E}-12 \\
7.1 \mathrm{E}-12 \\
6.9 \mathrm{E}-12 \\
6.4 \mathrm{E}-12 \\
6.2 \mathrm{E}-12 \\
6.4 \mathrm{E}-12 \\
6.3 \mathrm{E}-12 \\
6.5 \mathrm{E}-12 \\
6.3 \mathrm{E}-12\end{array}$ & $\begin{array}{l}6.2 \mathrm{E}-12 \\
6 \cdot 0 \mathrm{E}-12 \\
6.0 \mathrm{E}-12 \\
6.1 \mathrm{E}-12 \\
6.1 \mathrm{E}-12 \\
6.2 \mathrm{E}-12 \\
6.4 \mathrm{E}-12 \\
6.8 \mathrm{E}-12 \\
7.1 \mathrm{E}-12 \\
6.9 \mathrm{E}-12 \\
6.4 \mathrm{E}-12 \\
6.2 \mathrm{E}-12 \\
6.4 \mathrm{E}-12 \\
6.3 \mathrm{E}-12 \\
6.5 \mathrm{E}-12 \\
6.3 \mathrm{E}-12\end{array}$ & $\begin{array}{l}6.2 \mathrm{E}-12 \\
6 \cdot 0 \mathrm{E}-12 \\
6.0 \mathrm{E}-12 \\
6.1 \mathrm{E}-12 \\
6.1 \mathrm{E}-12 \\
6.2 \mathrm{E}-12 \\
6.4 \mathrm{E}-12 \\
6.8 \mathrm{E}-12 \\
7.1 \mathrm{E}-12 \\
6.9 \mathrm{E}-12 \\
6 \cdot 4 \mathrm{E}-12 \\
6.2 \mathrm{E}-12 \\
6 \cdot 4 \mathrm{E}-12 \\
6.3 \mathrm{E}-12 \\
6.5 \mathrm{E}-12 \\
6.3 \mathrm{E}-12\end{array}$ \\
\hline
\end{tabular}




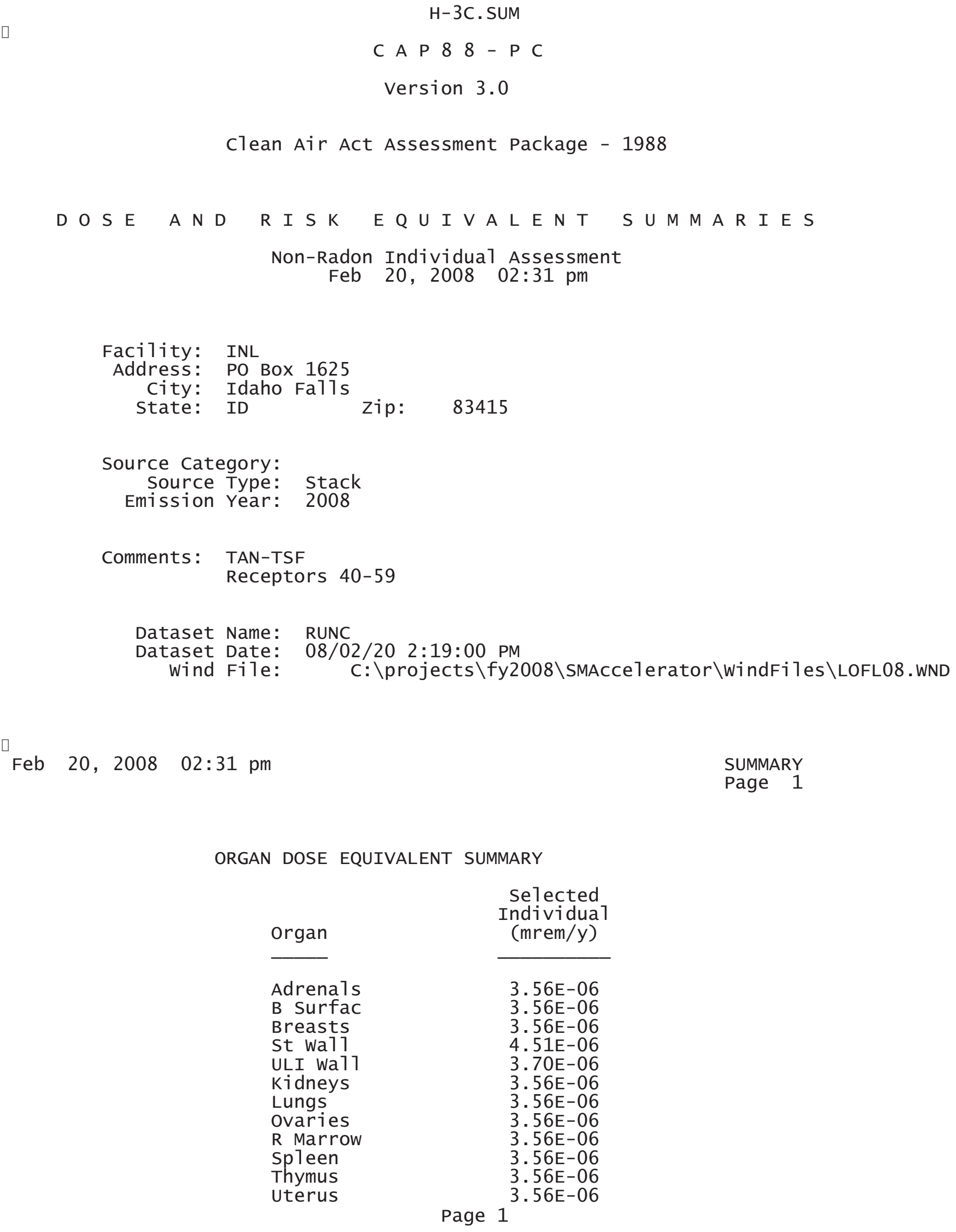

\begin{tabular}{lr} 
ORGAN DOSE EQUIVALENT SUMMARY \\
Organ & $\begin{array}{r}\text { Selected } \\
\text { Individual } \\
\text { (mrem/y) }\end{array}$ \\
\cline { 2 - 2 } Adrenals & $3.56 \mathrm{E}-06$ \\
B Surfac & $3.56 \mathrm{E}-06$ \\
Breasts & $3.56 \mathrm{E}-06$ \\
St wa11 & $4.51 \mathrm{E}-06$ \\
ULI wa11 & $3.70 \mathrm{E}-06$ \\
Kidneys & $3.56 \mathrm{E}-06$ \\
Lungs & $3.56 \mathrm{E}-06$ \\
Ovaries & $3.56 \mathrm{E}-06$ \\
R Marrow & $3.56 \mathrm{E}-06$ \\
Spleen & $3.56 \mathrm{E}-06$ \\
Thymus & $3.56 \mathrm{E}-06$ \\
Uterus & $3.56 \mathrm{E}-06$ \\
& 1
\end{tabular}




$\begin{array}{lr} & \mathrm{H}-3 \mathrm{C} . \mathrm{SUM} \\ \text { B1d wa11 } & 3.56 \mathrm{E}-06 \\ \text { Brain } & 3.56 \mathrm{E}-06 \\ \text { Esophagu } & 3.56 \mathrm{E}-06 \\ \text { SI wa11 } & 3.58 \mathrm{E}-06 \\ \text { LLI wa11 } & 3.98 \mathrm{E}-06 \\ \text { Liver } & 3.56 \mathrm{E}-06 \\ \text { Muscle } & 3.56 \mathrm{E}-06 \\ \text { Pancreas } & 3.56 \mathrm{E}-06 \\ \text { Skin } & 3.56 \mathrm{E}-06 \\ \text { Testes } & 3.56 \mathrm{E}-06 \\ \text { Thyroid } & 3.56 \mathrm{E}-06 \\ & \\ \text { EFFEC } & 6.95 \mathrm{E}-06\end{array}$

PATHWAY EFFECTIVE DOSE EQUIVALENT SUMMARY

\begin{tabular}{lc} 
Pathway & $\begin{array}{c}\text { selected } \\
\text { Individual } \\
\text { (mrem/y) }\end{array}$ \\
\cline { 2 - 2 } & \\
INGESTION & $5.59 \mathrm{E}-06$ \\
INHALATION & $1.36 \mathrm{E}-06$ \\
AIR IMMERSION & $0.00 \mathrm{E}+00$ \\
GROUND SURFACE & $0.00 \mathrm{E}+00$ \\
INTERNAL & $6.95 \mathrm{E}-06$ \\
EXTERNAL & $0.00 \mathrm{E}+00$ \\
TOTAL & $6.95 \mathrm{E}-06$
\end{tabular}

Feb 20, $2008 \quad 02: 31 \mathrm{pm}$

SUMMARY

Page 2

NUCLIDE EFFECTIVE DOSE EQUIVALENT SUMMARY

Nuclide

$\mathrm{H}-3$

TOTAL

Feb 20, $2008 \quad 02: 31$ pm

$$
\begin{gathered}
\begin{array}{c}
\text { Selected } \\
\text { Individual } \\
\text { (mrem/y) }
\end{array} \\
\hline 6.95 \mathrm{E}-06 \\
6.95 \mathrm{E}-06
\end{gathered}
$$

SUMMARY

Page 3

CANCER RISK SUMMARY

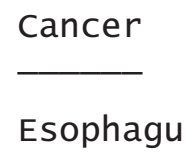

Esophagu

$$
\begin{aligned}
& \text { Selected Individual } \\
& \text { Total Lifetime } \\
& \text { Fatal Cancer Risk } \\
& \text { Page } 2^{8.02 \mathrm{E}-14}
\end{aligned}
$$




$\begin{array}{lr} & \mathrm{H}-3 \mathrm{C} . \mathrm{SUM} \\ \text { Stomach } & 4.02 \mathrm{E}-13 \\ \text { Colon } & 8.25 \mathrm{E}-13 \\ \text { Liver } & 1.12 \mathrm{E}-13 \\ \text { LUNG } & 6.82 \mathrm{E}-13 \\ \text { Bone } & 6.82 \mathrm{E}-15 \\ \text { Skin } & 7.22 \mathrm{E}-15 \\ \text { Breast } & 3.22 \mathrm{E}-13 \\ \text { Ovary } & 9.20 \mathrm{E}-14 \\ \text { B1adder } & 1.81 \mathrm{E}-13 \\ \text { Kidneys } & 3.86 \mathrm{E}-14 \\ \text { Thyroid } & 2.23 \mathrm{E}-14 \\ \text { Leukemia } & 4.12 \mathrm{E}-13 \\ \text { Residua1 } & 1.09 \mathrm{E}-12 \\ \text { Tota1 } & 4.27 \mathrm{E}-12 \\ & \\ \text { TOTAL } & 8.55 \mathrm{E}-12\end{array}$

PATHWAY RISK SUMMARY

\begin{tabular}{lc} 
Pathway & $\begin{array}{c}\text { Selected Individua1 } \\
\text { Total Lifetime } \\
\text { Fatal Cancer Risk }\end{array}$ \\
\cline { 2 - 2 } INGESTION & $3.50 \mathrm{E}-12$ \\
INHALATION & $7.71 \mathrm{E}-13$ \\
AIR IMMERSION & $0.00 \mathrm{E}+00$ \\
GROUND SURFACE & $0.00 \mathrm{E}+00$ \\
INTERNAL & $4.27 \mathrm{E}-12$ \\
EXTERNAL & $0.00 \mathrm{E}+00$ \\
TOTAL & $4.27 \mathrm{E}-12$
\end{tabular}

Feb 20, $2008 \quad 02: 31$ pm

SUMMARY Page 4

NUCLIDE RISK SUMMARY

Nuclide

$\mathrm{H}-3$

TOTAL

$\square$

Feb 20, 2008 02:31 pm
Selected Individual

Total Lifetime Fatal Cancer Risk

4. 27E-12

4. $27 \mathrm{E}-12$

SUMMARY

Page 5

INDIVIDUAL EFFECTIVE DOSE EQUIVALENT RATE (mrem/y) (A11 Radionuclides and Pathways)

Distance $(m)$ 


\begin{tabular}{rccccccc}
\multicolumn{1}{c}{ Direction } & 27715 & 28919 & 31060 & 32802 & 34577 & 35279 & 35683 \\
\hline N & $5.4 \mathrm{E}-06$ & $5.4 \mathrm{E}-06$ & $5.3 \mathrm{E}-06$ & $5.3 \mathrm{E}-06$ & $5.3 \mathrm{E}-06$ & $5.2 \mathrm{E}-06$ & $5.2 \mathrm{E}-06$ \\
NNW & $5.0 \mathrm{E}-06$ & $5.0 \mathrm{E}-06$ & $5.0 \mathrm{E}-06$ & $5.0 \mathrm{E}-06$ & $5.0 \mathrm{E}-06$ & $5.0 \mathrm{E}-06$ & $5.0 \mathrm{E}-06$ \\
$\mathrm{NW}$ & $5.1 \mathrm{E}-06$ & $5.1 \mathrm{E}-06$ & $5.1 \mathrm{E}-06$ & $5.1 \mathrm{E}-06$ & $5.1 \mathrm{E}-06$ & $5.0 \mathrm{E}-06$ & $5.0 \mathrm{E}-06$ \\
WNW & $5.2 \mathrm{E}-06$ & $5.2 \mathrm{E}-06$ & $5.2 \mathrm{E}-06$ & $5.1 \mathrm{E}-06$ & $5.1 \mathrm{E}-06$ & $5.1 \mathrm{E}-06$ & $5.1 \mathrm{E}-06$ \\
$\mathrm{~W}$ & $5.3 \mathrm{E}-06$ & $5.2 \mathrm{E}-06$ & $5.2 \mathrm{E}-06$ & $5.2 \mathrm{E}-06$ & $5.2 \mathrm{E}-06$ & $5.2 \mathrm{E}-06$ & $5.2 \mathrm{E}-06$ \\
WSW & $5.4 \mathrm{E}-06$ & $5.4 \mathrm{E}-06$ & $5.3 \mathrm{E}-06$ & $5.3 \mathrm{E}-06$ & $5.3 \mathrm{E}-06$ & $5.3 \mathrm{E}-06$ & $5.3 \mathrm{E}-06$ \\
$\mathrm{SW}$ & $5.8 \mathrm{E}-06$ & $5.7 \mathrm{E}-06$ & $5.6 \mathrm{E}-06$ & $5.6 \mathrm{E}-06$ & $5.5 \mathrm{E}-06$ & $5.5 \mathrm{E}-06$ & $5.5 \mathrm{E}-06$ \\
SSW & $6.4 \mathrm{E}-06$ & $6.3 \mathrm{E}-06$ & $6.2 \mathrm{E}-06$ & $6.1 \mathrm{E}-06$ & $6.0 \mathrm{E}-06$ & $6.0 \mathrm{E}-06$ & $6.0 \mathrm{E}-06$ \\
$\mathrm{~S}$ & $7.0 \mathrm{E}-06$ & $6.8 \mathrm{E}-06$ & $6.7 \mathrm{E}-06$ & $6.6 \mathrm{E}-06$ & $6.5 \mathrm{E}-06$ & $6.4 \mathrm{E}-06$ & $6.4 \mathrm{E}-06$ \\
$\mathrm{SSE}$ & $6.6 \mathrm{E}-06$ & $6.5 \mathrm{E}-06$ & $6.4 \mathrm{E}-06$ & $6.3 \mathrm{E}-06$ & $6.2 \mathrm{E}-06$ & $6.2 \mathrm{E}-06$ & $6.2 \mathrm{E}-06$ \\
$\mathrm{SE}$ & $5.8 \mathrm{E}-06$ & $5.8 \mathrm{E}-06$ & $5.7 \mathrm{E}-06$ & $5.6 \mathrm{E}-06$ & $5.6 \mathrm{E}-06$ & $5.6 \mathrm{E}-06$ & $5.6 \mathrm{E}-06$ \\
$\mathrm{ESE}$ & $5.5 \mathrm{E}-06$ & $5.5 \mathrm{E}-06$ & $5.4 \mathrm{E}-06$ & $5.4 \mathrm{E}-06$ & $5.3 \mathrm{E}-06$ & $5.3 \mathrm{E}-06$ & $5.3 \mathrm{E}-06$ \\
$\mathrm{E}$ & $5.7 \mathrm{E}-06$ & $5.6 \mathrm{E}-06$ & $5.6 \mathrm{E}-06$ & $5.5 \mathrm{E}-06$ & $5.5 \mathrm{E}-06$ & $5.5 \mathrm{E}-06$ & $5.5 \mathrm{E}-06$ \\
$\mathrm{ENE}$ & $5.5 \mathrm{E}-06$ & $5.5 \mathrm{E}-06$ & $5.5 \mathrm{E}-06$ & $5.4 \mathrm{E}-06$ & $5.4 \mathrm{E}-06$ & $5.4 \mathrm{E}-06$ & $5.4 \mathrm{E}-06$ \\
$\mathrm{NE}$ & $5.9 \mathrm{E}-06$ & $5.8 \mathrm{E}-06$ & $5.7 \mathrm{E}-06$ & $5.7 \mathrm{E}-06$ & $5.6 \mathrm{E}-06$ & $5.6 \mathrm{E}-06$ & $5.6 \mathrm{E}-06$ \\
NNE & $5.5 \mathrm{E}-06$ & $5.5 \mathrm{E}-06$ & $5.4 \mathrm{E}-06$ & $5.4 \mathrm{E}-06$ & $5.4 \mathrm{E}-06$ & $5.3 \mathrm{E}-06$ & $5.3 \mathrm{E}-06$ \\
\hline
\end{tabular}

Distance (m)

\begin{tabular}{|c|c|c|c|c|c|c|c|}
\hline Direction & 36721 & 36809 & 37729 & 39079 & 39220 & 39559 & 43584 \\
\hline $\mathrm{N}$ & $5.2 \mathrm{E}-06$ & $5.2 \mathrm{E}-06$ & $5.2 \mathrm{E}-06$ & $5.2 \mathrm{E}-06$ & $5.2 \mathrm{E}-06$ & $5.2 \mathrm{E}-06$ & $5.2 E-06$ \\
\hline NNW & $5.0 \mathrm{E}-06$ & $5.0 \mathrm{E}-06$ & $5.0 \mathrm{E}-06$ & $5.0 \mathrm{E}-06$ & $5.0 \mathrm{E}-06$ & $5.0 \mathrm{E}-06$ & $4.9 \mathrm{E}-06$ \\
\hline NW & $5.0 \mathrm{E}-06$ & $5.0 \mathrm{E}-06$ & $5.0 \mathrm{E}-06$ & $5.0 \mathrm{E}-06$ & $5.0 \mathrm{E}-06$ & $5.0 \mathrm{E}-06$ & $5.0 \mathrm{E}-06$ \\
\hline WNW & $5.1 \mathrm{E}-06$ & $5.1 \mathrm{E}-06$ & $5.1 \mathrm{E}-06$ & $5.1 \mathrm{E}-06$ & $5.1 \mathrm{E}-06$ & $5.1 \mathrm{E}-06$ & $5.1 \mathrm{E}-06$ \\
\hline W & $5.1 \mathrm{E}-06$ & $5.1 \mathrm{E}-06$ & $5.1 \mathrm{E}-06$ & $5.1 \mathrm{E}-06$ & $5.1 \mathrm{E}-06$ & $5.1 \mathrm{E}-06$ & $5.1 \mathrm{E}-06$ \\
\hline WSW & $5.3 \mathrm{E}-06$ & $5.2 \mathrm{E}-06$ & $5.2 \mathrm{E}-06$ & $5.2 \mathrm{E}-06$ & $5.2 \mathrm{E}-06$ & $5.2 \mathrm{E}-06$ & $5.2 \mathrm{E}-06$ \\
\hline SW & $5.5 \mathrm{E}-06$ & $5.5 \mathrm{E}-06$ & $5.5 \mathrm{E}-06$ & $5.4 \mathrm{E}-06$ & $5.4 \mathrm{E}-06$ & $5.4 \mathrm{E}-06$ & $5.4 \mathrm{E}-06$ \\
\hline SSW & $5.9 \mathrm{E}-06$ & $5.9 \mathrm{E}-06$ & $5.9 \mathrm{E}-06$ & $5.9 \mathrm{E}-06$ & $5.8 \mathrm{E}-06$ & $5.8 \mathrm{E}-06$ & $5.7 \mathrm{E}-06$ \\
\hline $\mathrm{s}$ & $6.3 \mathrm{E}-06$ & $6.3 \mathrm{E}-06$ & $6.3 \mathrm{E}-06$ & $6.2 \mathrm{E}-06$ & $6.2 \mathrm{E}-06$ & $6.2 \mathrm{E}-06$ & $6.1 \mathrm{E}-06$ \\
\hline SSE & $6.1 \mathrm{E}-06$ & $6.1 \mathrm{E}-06$ & $6.1 \mathrm{E}-06$ & $6.0 \mathrm{E}-06$ & $6.0 \mathrm{E}-06$ & $6.0 \mathrm{E}-06$ & $5.9 \mathrm{E}-06$ \\
\hline SE & $5.5 E-06$ & $5.5 E-06$ & $5.5 \mathrm{E}-06$ & $5.5 \mathrm{E}-06$ & $5.5 \mathrm{E}-06$ & $5.5 \mathrm{E}-06$ & $5.4 \mathrm{E}-06$ \\
\hline ESE & $5.3 E-06$ & $5.3 \mathrm{E}-06$ & $5.3 \mathrm{E}-06$ & $5.3 \mathrm{E}-06$ & $5.3 \mathrm{E}-06$ & $5.3 \mathrm{E}-06$ & $5.2 \mathrm{E}-06$ \\
\hline & $5.4 \mathrm{E}-06$ & $5.4 \mathrm{E}-06$ & $5.4 \mathrm{E}-06$ & $5.4 \mathrm{E}-06$ & $5.4 \mathrm{E}-06$ & $5.4 \mathrm{E}-06$ & $5.3 \mathrm{E}-06$ \\
\hline ENE & $5.3 E-06$ & $5.3 E-06$ & $5.3 E-06$ & $5.3 \mathrm{E}-06$ & $5.3 \mathrm{E}-06$ & $5.3 \mathrm{E}-06$ & $5.3 \mathrm{E}-06$ \\
\hline $\mathrm{NE}$ & $5.6 \mathrm{E}-06$ & $5.6 \mathrm{E}-06$ & $5.5 \mathrm{E}-06$ & $5.5 \mathrm{E}-06$ & $5.5 \mathrm{E}-06$ & $5.5 \mathrm{E}-06$ & $5.4 \mathrm{E}-06$ \\
\hline NNE & $5.3 E-06$ & $5.3 \mathrm{E}-06$ & $5.3 \mathrm{E}-06$ & $5.3 \mathrm{E}-06$ & $5.3 \mathrm{E}-06$ & $5.3 \mathrm{E}-06$ & $5.2 \mathrm{E}-06$ \\
\hline
\end{tabular}

口

Feb 20, 2008 02:31 pm

SUMMARY

Page 6

INDIVIDUAL EFFECTIVE DOSE EQUIVALENT RATE (mrem/y) (A11 Radionuclides and Pathways)

\begin{tabular}{|c|c|c|c|c|c|c|}
\hline \multirow[b]{2}{*}{ Direction } & \multicolumn{6}{|c|}{ Distance (m) } \\
\hline & 45196 & 45275 & 45654 & 45677 & 46668 & 47969 \\
\hline
\end{tabular}

$\begin{array}{rrrrrrr}\mathrm{N} & 5.1 \mathrm{E}-06 & 5.1 \mathrm{E}-06 & 5.1 \mathrm{E}-06 & 5.1 \mathrm{E}-06 & 5.1 \mathrm{E}-06 & 5.1 \mathrm{E}-06 \\ \mathrm{NNW} & 4.9 \mathrm{E}-06 & 4.9 \mathrm{E}-06 & 4.9 \mathrm{E}-06 & \begin{array}{c}4.9 \mathrm{E}-06 \\ \text { Page }\end{array} & 4.9 \mathrm{E}-06 & 4.9 \mathrm{E}-06\end{array}$




\begin{tabular}{rllllll} 
& & & \multicolumn{1}{c}{$\mathrm{H}-3 \mathrm{C} . \mathrm{SUM}$} \\
$\mathrm{NW}$ & $5.0 \mathrm{E}-06$ & $5.0 \mathrm{E}-06$ & $5.0 \mathrm{E}-06$ & $5.0 \mathrm{E}-06$ & $5.0 \mathrm{E}-06$ & $5.0 \mathrm{E}-06$ \\
WNW & $5.1 \mathrm{E}-06$ & $5.1 \mathrm{E}-06$ & $5.0 \mathrm{E}-06$ & $5.0 \mathrm{E}-06$ & $5.0 \mathrm{E}-06$ & $5.0 \mathrm{E}-06$ \\
$\mathrm{~W}$ & $5.1 \mathrm{E}-06$ & $5.1 \mathrm{E}-06$ & $5.1 \mathrm{E}-06$ & $5.1 \mathrm{E}-06$ & $5.1 \mathrm{E}-06$ & $5.1 \mathrm{E}-06$ \\
$\mathrm{WSW}$ & $5.2 \mathrm{E}-06$ & $5.2 \mathrm{E}-06$ & $5.2 \mathrm{E}-06$ & $5.2 \mathrm{E}-06$ & $5.1 \mathrm{E}-06$ & $5.1 \mathrm{E}-06$ \\
$\mathrm{SW}$ & $5.4 \mathrm{E}-06$ & $5.3 \mathrm{E}-06$ & $5.3 \mathrm{E}-06$ & $5.3 \mathrm{E}-06$ & $5.3 \mathrm{E}-06$ & $5.3 \mathrm{E}-06$ \\
$\mathrm{SSW}$ & $5.7 \mathrm{E}-06$ & $5.7 \mathrm{E}-06$ & $5.7 \mathrm{E}-06$ & $5.7 \mathrm{E}-06$ & $5.7 \mathrm{E}-06$ & $5.6 \mathrm{E}-06$ \\
$\mathrm{~S}$ & $6.0 \mathrm{E}-06$ & $6.0 \mathrm{E}-06$ & $6.0 \mathrm{E}-06$ & $6.0 \mathrm{E}-06$ & $6.0 \mathrm{E}-06$ & $5.9 \mathrm{E}-06$ \\
$\mathrm{SSE}$ & $5.8 \mathrm{E}-06$ & $5.8 \mathrm{E}-06$ & $5.8 \mathrm{E}-06$ & $5.8 \mathrm{E}-06$ & $5.8 \mathrm{E}-06$ & $5.8 \mathrm{E}-06$ \\
$\mathrm{SE}$ & $5.4 \mathrm{E}-06$ & $5.4 \mathrm{E}-06$ & $5.4 \mathrm{E}-06$ & $5.4 \mathrm{E}-06$ & $5.4 \mathrm{E}-06$ & $5.4 \mathrm{E}-06$ \\
$\mathrm{ESE}$ & $5.2 \mathrm{E}-06$ & $5.2 \mathrm{E}-06$ & $5.2 \mathrm{E}-06$ & $5.2 \mathrm{E}-06$ & $5.2 \mathrm{E}-06$ & $5.2 \mathrm{E}-06$ \\
$\mathrm{E}$ & $5.3 \mathrm{E}-06$ & $5.3 \mathrm{E}-06$ & $5.3 \mathrm{E}-06$ & $5.3 \mathrm{E}-06$ & $5.3 \mathrm{E}-06$ & $5.3 \mathrm{E}-06$ \\
$\mathrm{ENE}$ & $5.2 \mathrm{E}-06$ & $5.2 \mathrm{E}-06$ & $5.2 \mathrm{E}-06$ & $5.2 \mathrm{E}-06$ & $5.2 \mathrm{E}-06$ & $5.2 \mathrm{E}-06$ \\
$\mathrm{NE}$ & $5.4 \mathrm{E}-06$ & $5.4 \mathrm{E}-06$ & $5.4 \mathrm{E}-06$ & $5.4 \mathrm{E}-06$ & $5.4 \mathrm{E}-06$ & $5.4 \mathrm{E}-06$ \\
$\mathrm{NNE}$ & $5.2 \mathrm{E}-06$ & $5.2 \mathrm{E}-06$ & $5.2 \mathrm{E}-06$ & $5.2 \mathrm{E}-06$ & $5.2 \mathrm{E}-06$ & $5.2 \mathrm{E}-06$
\end{tabular}

Feb 20, $2008 \quad 02: 31 \mathrm{pm}$

SUMMARY

Page 7

INDIVIDUAL LIFETIME RISK (deaths)

(A11 Radionuclides and Pathways)

\begin{tabular}{|c|c|c|c|c|c|c|c|}
\hline \multicolumn{8}{|c|}{ Distance $(\mathrm{m})$} \\
\hline Direction & 27715 & 28919 & 31060 & 32802 & 34577 & 35279 & 35683 \\
\hline $\begin{array}{r}\text { N } \\
N N W \\
N W \\
\text { WNW } \\
W \\
W S W \\
\text { SW } \\
\text { SSW } \\
S \\
\text { SSE } \\
\text { SE } \\
\text { ESE } \\
\text { E } \\
\text { ENE } \\
N E \\
\text { NNE }\end{array}$ & $\begin{array}{l}3.3 \mathrm{E}-12 \\
3.1 \mathrm{E}-12 \\
3.2 \mathrm{E}-12 \\
3.3 \mathrm{E}-12 \\
3.3 \mathrm{E}-12 \\
3.4 \mathrm{E}-12 \\
3.6 \mathrm{E}-12 \\
3.9 \mathrm{E}-12 \\
4.3 \mathrm{E}-12 \\
4.1 \mathrm{E}-12 \\
3.6 \mathrm{E}-12 \\
3.4 \mathrm{E}-12 \\
3.5 \mathrm{E}-12 \\
3.4 \mathrm{E}-12 \\
3.6 \mathrm{E}-12 \\
3.4 \mathrm{E}-12\end{array}$ & $\begin{array}{l}3.3 \mathrm{E}-12 \\
3.1 \mathrm{E}-12 \\
3.2 \mathrm{E}-12 \\
3.2 \mathrm{E}-12 \\
3.3 \mathrm{E}-12 \\
3.4 \mathrm{E}-12 \\
3.5 \mathrm{E}-12 \\
3.9 \mathrm{E}-12 \\
4.2 \mathrm{E}-12 \\
4.0 \mathrm{E}-12 \\
3.6 \mathrm{E}-12 \\
3.4 \mathrm{E}-12 \\
3.5 \mathrm{E}-12 \\
3.4 \mathrm{E}-12 \\
3.6 \mathrm{E}-12 \\
3.4 \mathrm{E}-12\end{array}$ & $\begin{array}{l}3.3 \mathrm{E}-12 \\
3.1 \mathrm{E}-12 \\
3.2 \mathrm{E}-12 \\
3.2 \mathrm{E}-12 \\
3.3 \mathrm{E}-12 \\
3.3 \mathrm{E}-12 \\
3.5 \mathrm{E}-12 \\
3.8 \mathrm{E}-12 \\
4.1 \mathrm{E}-12 \\
3.9 \mathrm{E}-12 \\
3.5 \mathrm{E}-12 \\
3.4 \mathrm{E}-12 \\
3.5 \mathrm{E}-12 \\
3.4 \mathrm{E}-12 \\
3.6 \mathrm{E}-12 \\
3.4 \mathrm{E}-12\end{array}$ & $\begin{array}{l}3.3 \mathrm{E}-12 \\
3.1 \mathrm{E}-12 \\
3.2 \mathrm{E}-12 \\
3.2 \mathrm{E}-12 \\
3.2 \mathrm{E}-12 \\
3.3 \mathrm{E}-12 \\
3.5 \mathrm{E}-12 \\
3.8 \mathrm{E}-12 \\
4.0 \mathrm{E}-12 \\
3.9 \mathrm{E}-12 \\
3.5 \mathrm{E}-12 \\
3.3 \mathrm{E}-12 \\
3.4 \mathrm{E}-12 \\
3.4 \mathrm{E}-12 \\
3.5 \mathrm{E}-12 \\
3.4 \mathrm{E}-12\end{array}$ & $\begin{array}{l}3.3 \mathrm{E}-12 \\
3 \cdot 1 \mathrm{E}-12 \\
3.2 \mathrm{E}-12 \\
3.2 \mathrm{E}-12 \\
3.2 \mathrm{E}-12 \\
3.3 \mathrm{E}-12 \\
3.4 \mathrm{E}-12 \\
3.7 \mathrm{E}-12 \\
4 \cdot 0 \mathrm{E}-12 \\
3.8 \mathrm{E}-12 \\
3.5 \mathrm{E}-12 \\
3.3 \mathrm{E}-12 \\
3.4 \mathrm{E}-12 \\
3.3 \mathrm{E}-12 \\
3 \cdot 5 \mathrm{E}-12 \\
3 \cdot 3 \mathrm{E}-12\end{array}$ & $\begin{array}{l}3.3 \mathrm{E}-12 \\
3 \cdot 1 \mathrm{E}-12 \\
3 \cdot 2 \mathrm{E}-12 \\
3.2 \mathrm{E}-12 \\
3.2 \mathrm{E}-12 \\
3.3 \mathrm{E}-12 \\
3 \cdot 4 \mathrm{E}-12 \\
3 \cdot 7 \mathrm{E}-12 \\
4 \cdot 0 \mathrm{E}-12 \\
3 \cdot 8 \mathrm{E}-12 \\
3 \cdot 5 \mathrm{E}-12 \\
3.3 \mathrm{E}-12 \\
3 \cdot 4 \mathrm{E}-12 \\
3 \cdot 3 \mathrm{E}-12 \\
3 \cdot 5 \mathrm{E}-12 \\
3 \cdot 3 \mathrm{E}-12\end{array}$ & $\begin{array}{l}3.3 \mathrm{E}-12 \\
3.1 \mathrm{E}-12 \\
3.2 \mathrm{E}-12 \\
3.2 \mathrm{E}-12 \\
3.2 \mathrm{E}-12 \\
3.3 \mathrm{E}-12 \\
3.4 \mathrm{E}-12 \\
3.7 \mathrm{E}-12 \\
3.9 \mathrm{E}-12 \\
3.8 \mathrm{E}-12 \\
3.5 \mathrm{E}-12 \\
3.3 \mathrm{E}-12 \\
3.4 \mathrm{E}-12 \\
3.3 \mathrm{E}-12 \\
3.5 \mathrm{E}-12 \\
3.3 \mathrm{E}-12\end{array}$ \\
\hline \multicolumn{8}{|c|}{ Distance (m) } \\
\hline Direction & 36721 & 36809 & 37729 & 39079 & 39220 & 39559 & 43584 \\
\hline $\begin{array}{r}\text { N } \\
\text { NNW } \\
\text { NW } \\
\text { WNW } \\
W \\
\text { WSW } \\
\text { SW } \\
\text { SSW }\end{array}$ & $\begin{array}{l}3.3 \mathrm{E}-12 \\
3.1 \mathrm{E}-12 \\
3.1 \mathrm{E}-12 \\
3.2 \mathrm{E}-12 \\
3.2 \mathrm{E}-12 \\
3.3 \mathrm{E}-12 \\
3.4 \mathrm{E}-12 \\
3.7 \mathrm{E}-12\end{array}$ & $\begin{array}{l}3 \cdot 3 \mathrm{E}-12 \\
3 \cdot 1 \mathrm{E}-12 \\
3 \cdot 1 \mathrm{E}-12 \\
3 \cdot 2 \mathrm{E}-12 \\
3 \cdot 2 \mathrm{E}-12 \\
3 \cdot 3 \mathrm{E}-12 \\
3 \cdot 4 \mathrm{E}-12 \\
3 \cdot 7 \mathrm{E}-12\end{array}$ & $\begin{array}{l}3.3 \mathrm{E}-12 \\
3 \cdot 1 \mathrm{E}-12 \\
3.1 \mathrm{E}-12 \\
3.2 \mathrm{E}-12 \\
3.2 \mathrm{E}-12 \\
3.3 \mathrm{E}-12 \\
3.4 \mathrm{E}-12 \\
3.7 \mathrm{E}-12\end{array}$ & $\begin{array}{r}3.2 \mathrm{E}-12 \\
3.1 \mathrm{E}-12 \\
3.1 \mathrm{E}-12 \\
3.2 \mathrm{E}-12 \\
3.2 \mathrm{E}-12 \\
3.3 \mathrm{E}-12 \\
3.4 \mathrm{E}-12 \\
3.6 \mathrm{E}-12 \\
\text { Page }\end{array}$ & $\begin{array}{l}3.2 \mathrm{E}-12 \\
3.1 \mathrm{E}-12 \\
3.1 \mathrm{E}-12 \\
3.2 \mathrm{E}-12 \\
3.2 \mathrm{E}-12 \\
3.3 \mathrm{E}-12 \\
3.4 \mathrm{E}-12 \\
3.6 \mathrm{E}-12\end{array}$ & $\begin{array}{l}3.2 \mathrm{E}-12 \\
3 \cdot 1 \mathrm{E}-12 \\
3.1 \mathrm{E}-12 \\
3.2 \mathrm{E}-12 \\
3.2 \mathrm{E}-12 \\
3.3 \mathrm{E}-12 \\
3.4 \mathrm{E}-12 \\
3.6 \mathrm{E}-12\end{array}$ & $\begin{array}{l}3.2 \mathrm{E}-12 \\
3.1 \mathrm{E}-12 \\
3.1 \mathrm{E}-12 \\
3.2 \mathrm{E}-12 \\
3.2 \mathrm{E}-12 \\
3.2 \mathrm{E}-12 \\
3.3 \mathrm{E}-12 \\
3.6 \mathrm{E}-12\end{array}$ \\
\hline
\end{tabular}




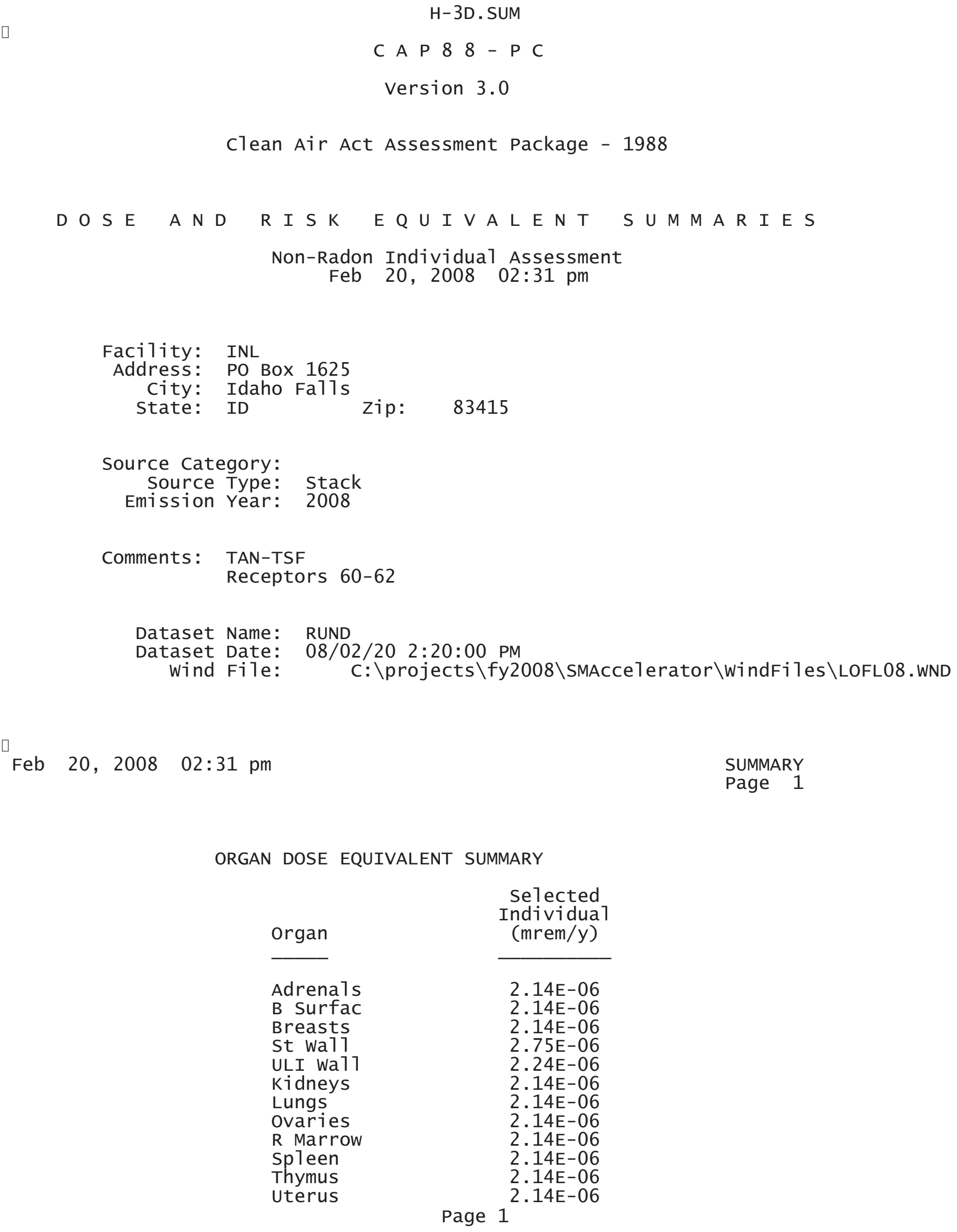

\begin{tabular}{lr} 
ORGAN DOSE EQUIVALENT SUMMARY \\
Organ & $\begin{array}{c}\text { Selected } \\
\text { Individual } \\
\text { (mrem/y) }\end{array}$ \\
\cline { 2 - 2 } Adrena1s & $2.14 \mathrm{E}-06$ \\
B Surfac & $2.14 \mathrm{E}-06$ \\
Breasts & $2.14 \mathrm{E}-06$ \\
St wa11 & $2.75 \mathrm{E}-06$ \\
ULI wa11 & $2.24 \mathrm{E}-06$ \\
Kidneys & $2.14 \mathrm{E}-06$ \\
Lungs & $2.14 \mathrm{E}-06$ \\
Ovaries & $2.14 \mathrm{E}-06$ \\
R Marrow & $2.14 \mathrm{E}-06$ \\
Spleen & $2.14 \mathrm{E}-06$ \\
Thymus & $2.14 \mathrm{E}-06$ \\
Uterus & $2.14 \mathrm{E}-06$
\end{tabular}




$\begin{array}{lr} & \text { H-3D.SUM } \\ \text { Bld wa11 } & 2.14 \mathrm{E}-06 \\ \text { Brain } & 2.14 \mathrm{E}-06 \\ \text { Esophagu } & 2.14 \mathrm{E}-06 \\ \text { SI wa11 } & 2.16 \mathrm{E}-06 \\ \text { LLI wa11 } & 2.41 \mathrm{E}-06 \\ \text { Liver } & 2.14 \mathrm{E}-06 \\ \text { Muscle } & 2.14 \mathrm{E}-06 \\ \text { Pancreas } & 2.14 \mathrm{E}-06 \\ \text { Skin } & 2.14 \mathrm{E}-06 \\ \text { Testes } & 2.14 \mathrm{E}-06 \\ \text { Thyroid } & 2.14 \mathrm{E}-06 \\ & \\ \text { EFFEC } & 4.25 \mathrm{E}-06\end{array}$

PATHWAY EFFECTIVE DOSE EQUIVALENT SUMMARY

\begin{tabular}{lcl} 
Pathway & $\begin{array}{c}\text { Selected } \\
\text { Individua1 } \\
\text { (mrem/y) }\end{array}$ \\
\cline { 2 - 2 } INGESTION & $3.57 \mathrm{E}-06$ \\
INHALATION & $6.78 \mathrm{E}-07$ \\
AIR IMMERSION & $0.00 \mathrm{E}+00$ & \\
GROUND SURFACE & $0.00 \mathrm{E}+00$ & \\
INTERNAL & $4.25 \mathrm{E}-06$ & \\
EXTERNAL & $0.00 \mathrm{E}+00$ & \\
TOTAL & $4.25 \mathrm{E}-06$ & SUMMARY \\
pm & & Page 2
\end{tabular}

NUCLIDE EFFECTIVE DOSE EQUIVALENT SUMMARY

Nuclide

$\mathrm{H}-3$

TOTAL

Feb 20, $2008 \quad 02: 31 \mathrm{pm}$

$$
\begin{gathered}
\begin{array}{c}
\text { Selected } \\
\text { Individual } \\
\text { (mrem/y) }
\end{array} \\
\hline 4.25 \mathrm{E}-06 \\
4.25 \mathrm{E}-06
\end{gathered}
$$

SUMMARY

Page 3

CANCER RISK SUMMARY

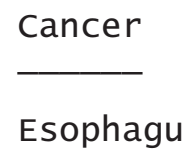

Esophagu

$$
\begin{aligned}
& \text { Selected Individual } \\
& \text { Total Lifetime } \\
& \text { Fatal Cancer Risk } \\
& \text { Page } 2^{4.89 E-14}
\end{aligned}
$$




$\begin{array}{lr} & \text { H-3D.SUM } \\ \text { Stomach } & 2.49 \mathrm{E}-13 \\ \text { Colon } & 5.08 \mathrm{E}-13 \\ \text { Liver } & 6.87 \mathrm{E}-14 \\ \text { LUNG } & 4.17 \mathrm{E}-13 \\ \text { Bone } & 4.17 \mathrm{E}-15 \\ \text { Skin } & 4.42 \mathrm{E}-15 \\ \text { Breast } & 1.97 \mathrm{E}-13 \\ \text { Ovary } & 5.61 \mathrm{E}-14 \\ \text { B1adder } & 1.10 \mathrm{E}-13 \\ \text { Kidneys } & 2.36 \mathrm{E}-14 \\ \text { Thyroid } & 1.36 \mathrm{E}-14 \\ \text { Leukemia } & 2.52 \mathrm{E}-13 \\ \text { Residua1 } & 6.67 \mathrm{E}-13 \\ \text { Tota1 } & 2.62 \mathrm{E}-12 \\ & \\ \text { TOTAL } & 5.24 \mathrm{E}-12\end{array}$

PATHWAY RISK SUMMARY

\begin{tabular}{lc} 
Pathway & $\begin{array}{c}\text { Selected Individual } \\
\text { Total Lifetime } \\
\text { Fatal Cancer Risk }\end{array}$ \\
\cline { 2 - 2 } & $2.24 \mathrm{E}-12$ \\
INGESTION & $3.84 \mathrm{E}-13$ \\
INHALATION & $0.00 \mathrm{E}+00$ \\
AIR IMMERSION & $0.00 \mathrm{E}+00$ \\
GROUND SURFACE & $2.62 \mathrm{E}-12$ \\
INTERNAL & $0.00 \mathrm{E}+00$ \\
EXTERNAL & $2.62 \mathrm{E}-12$ \\
TOTAL &
\end{tabular}

Feb 20, $2008 \quad 02: 31$ pm

SUMMARY Page 4

NUCLIDE RISK SUMMARY

Nuclide

$\mathrm{H}-3$

TOTAL

$\square$

Feb 20, 2008 02:31 pm
Selected Individual

Total Lifetime Fatal Cancer Risk

$2.62 \mathrm{E}-12$

$2.62 \mathrm{E}-12$

SUMMARY

Page 5

INDIVIDUAL EFFECTIVE DOSE EQUIVALENT RATE (mrem/y) (A11 Radionuclides and Pathways)

Distance $(m)$ 


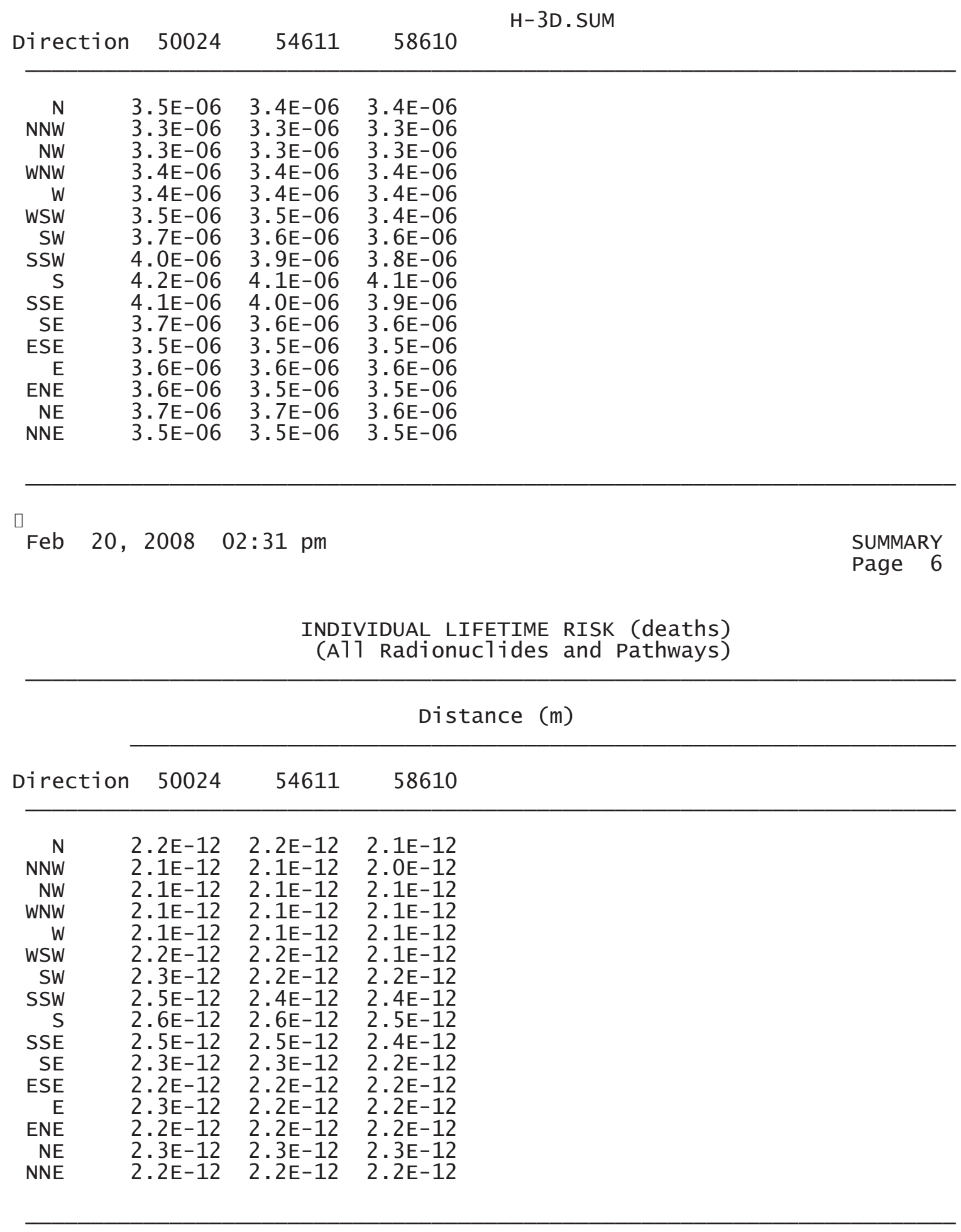




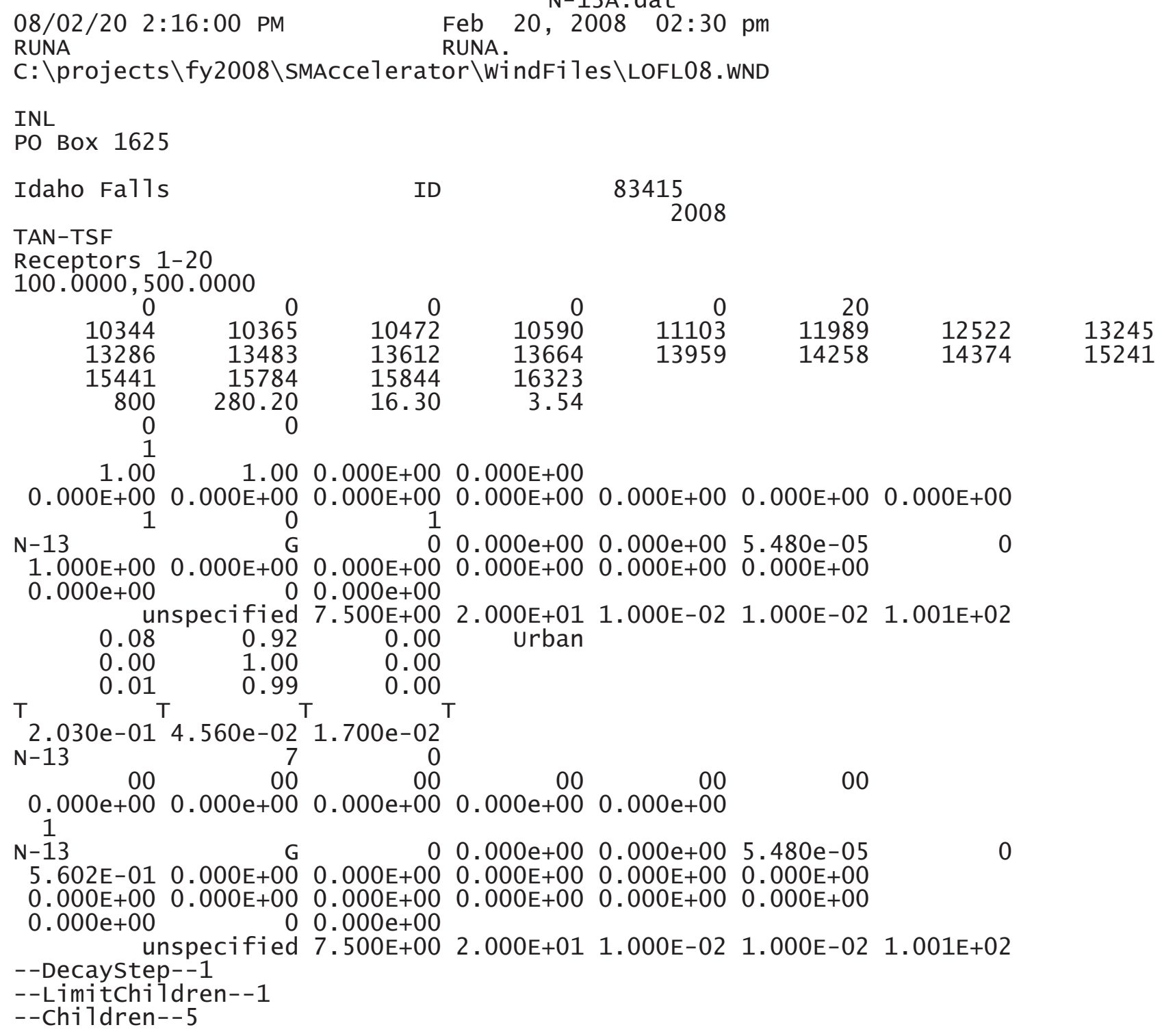




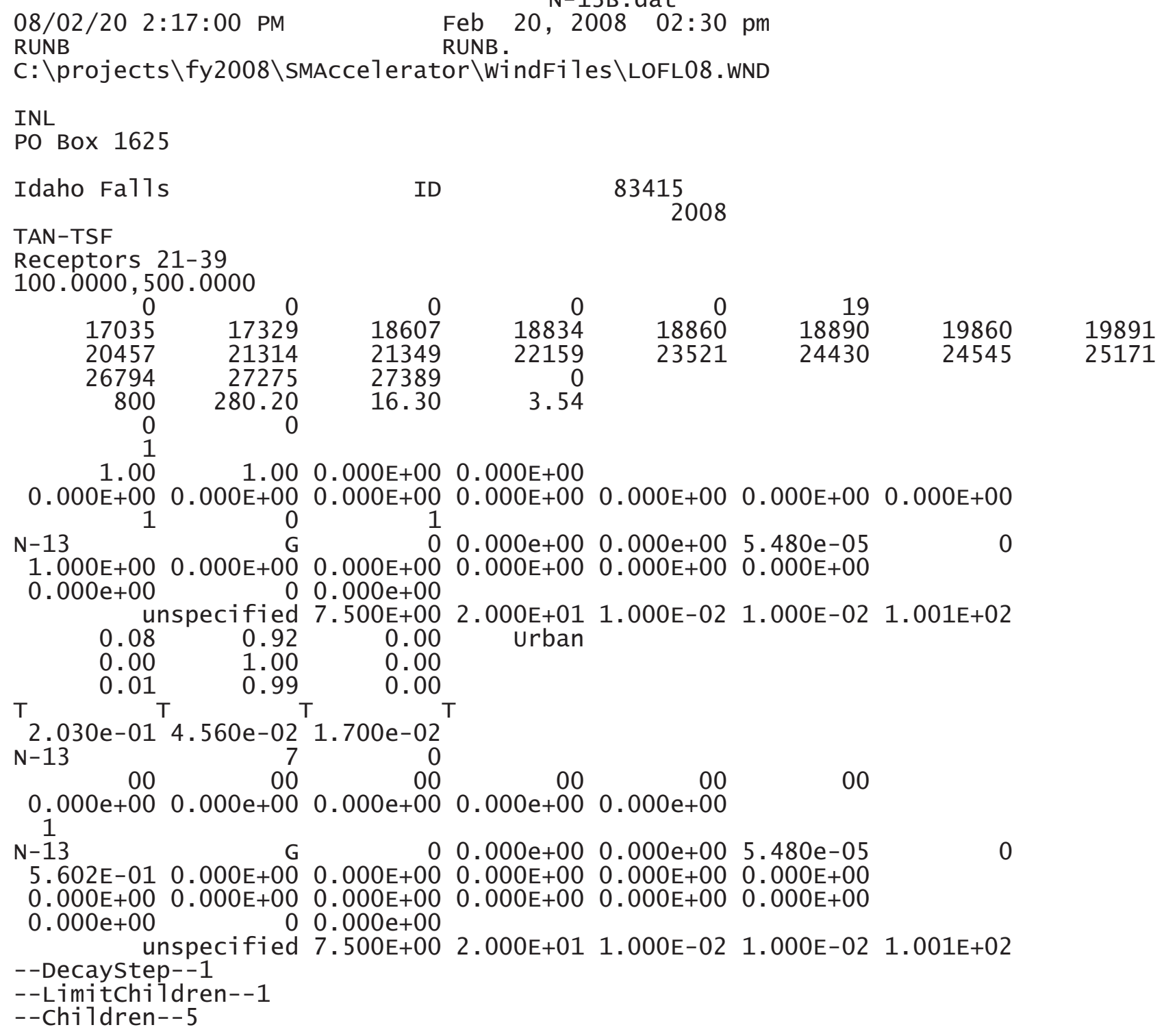




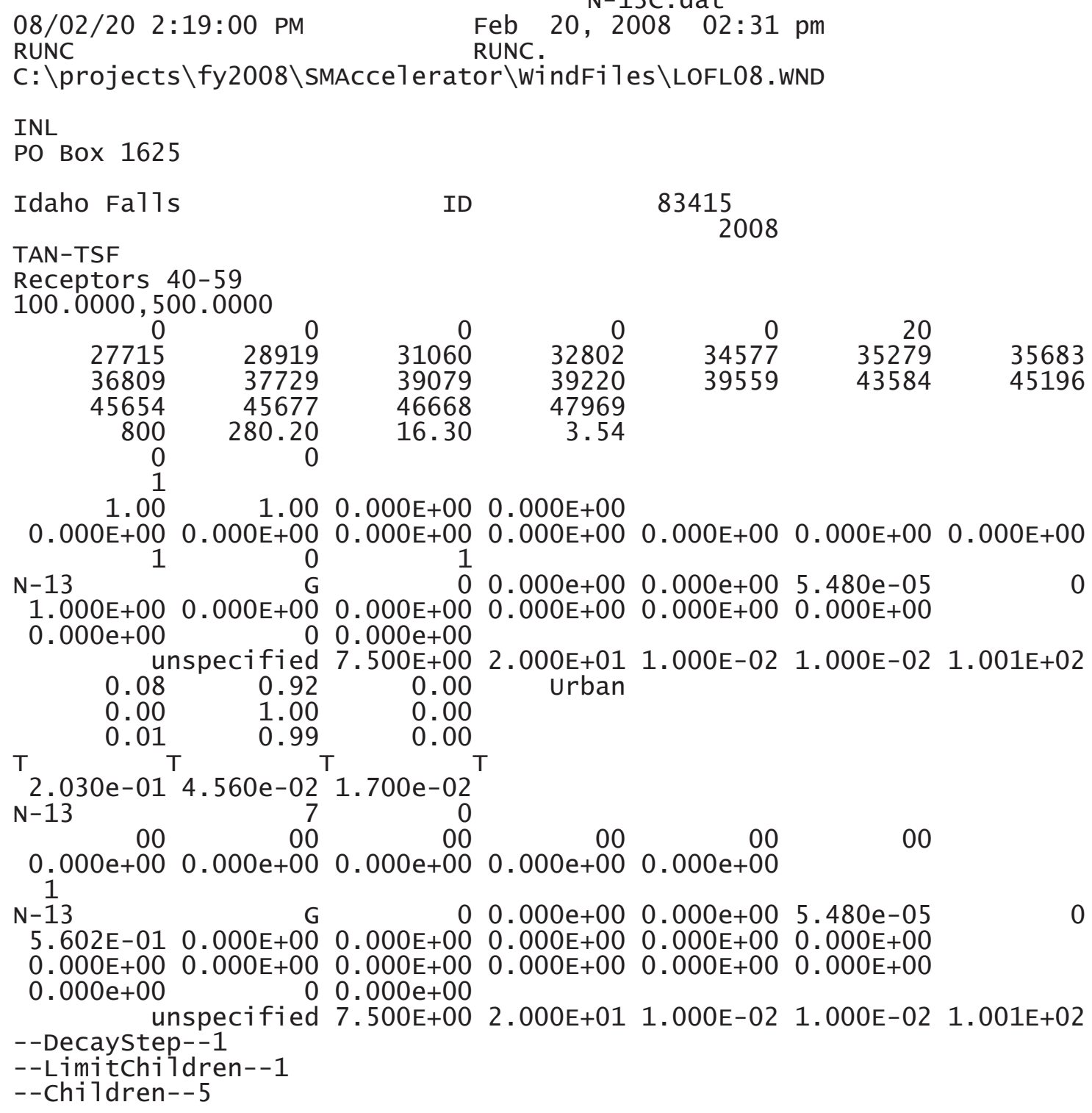

ID

83415

TAN-TSF

Receptors 40-59

$100.0000,500.0000$

$\begin{array}{rrrr}0 & 0 & 0 & \\ 27715 & 28919 & 31060 & 3280 \\ 36809 & 37729 & 39079 & 3922 \\ 45654 & 45677 & 46668 & 4796 \\ 800 & 280.20 & 16.30 & 3.5 \\ 0 & 0 & & \\ 1 & & & \end{array}$

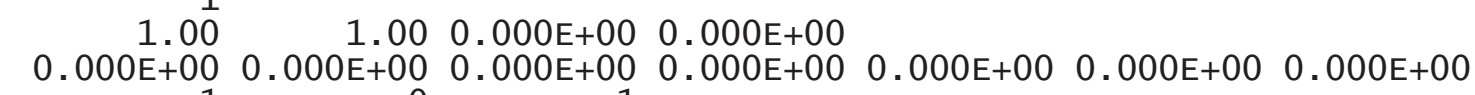

$\begin{array}{lllllll}\mathrm{N}-13 & \mathrm{G} & 0 & 0.000 \mathrm{e}+00 & 0.000 \mathrm{e}+00 & 5.480 \mathrm{e}-05 & 0\end{array}$

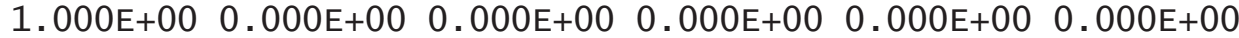

$0.000 \mathrm{e}+00 \quad 00.000 \mathrm{e}+00$

unspecified 7.500E+00 2.000E+01 1.000E-02 1.000E-02 $1.001 \mathrm{E}+02$

$\begin{array}{llll}0.08 & 0.92 & 0.00 & \text { Urban }\end{array}$

$0.00 \quad 1.00 \quad 0.00$

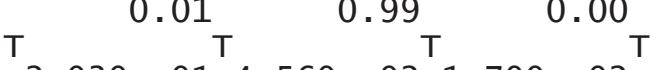

$\begin{array}{rrr}2.030 e-01 & 4.560 e-02 & 1.700 e-02 \\ N-13 & 0\end{array}$

$\begin{array}{rrrrr}00 & 00 & 00 & 00 & 00 \\ 0.000 \mathrm{e}+00 & 0.000 \mathrm{e}+00 & 0.000 \mathrm{e}+00 & 0.000 \mathrm{e}+00 & 0.000 \mathrm{e}+00\end{array}$

2008$$
1
$$

$\mathrm{N}-13$

G $\quad 0 \quad 0.000 \mathrm{e}+00 \quad 0.000 \mathrm{e}+00 \quad 5.480 \mathrm{e}-05$

00

$\begin{array}{lllllll}5.602 \mathrm{E}-01 & 0.000 \mathrm{E}+00 & 0.000 \mathrm{E}+00 & 0.000 \mathrm{E}+00 & 0.000 \mathrm{E}+00 & 0.000 \mathrm{E}+00\end{array}$

$\begin{array}{lllllllll}0.000 \mathrm{E}+00 & 0.000 \mathrm{E}+00 & 0.000 \mathrm{E}+00 & 0.000 \mathrm{E}+00 & 0.000 \mathrm{E}+00 & 0.000 \mathrm{E}+00\end{array}$

$0.000 \mathrm{e}+00$

$0 \quad 0.000 \mathrm{e}+00$

unspecified 7.500E+00 2.000E+01 1.000E-02 1.000E-02 1.001E+02

--Decaystep--1

--LimitChildren--1

--Children--5 


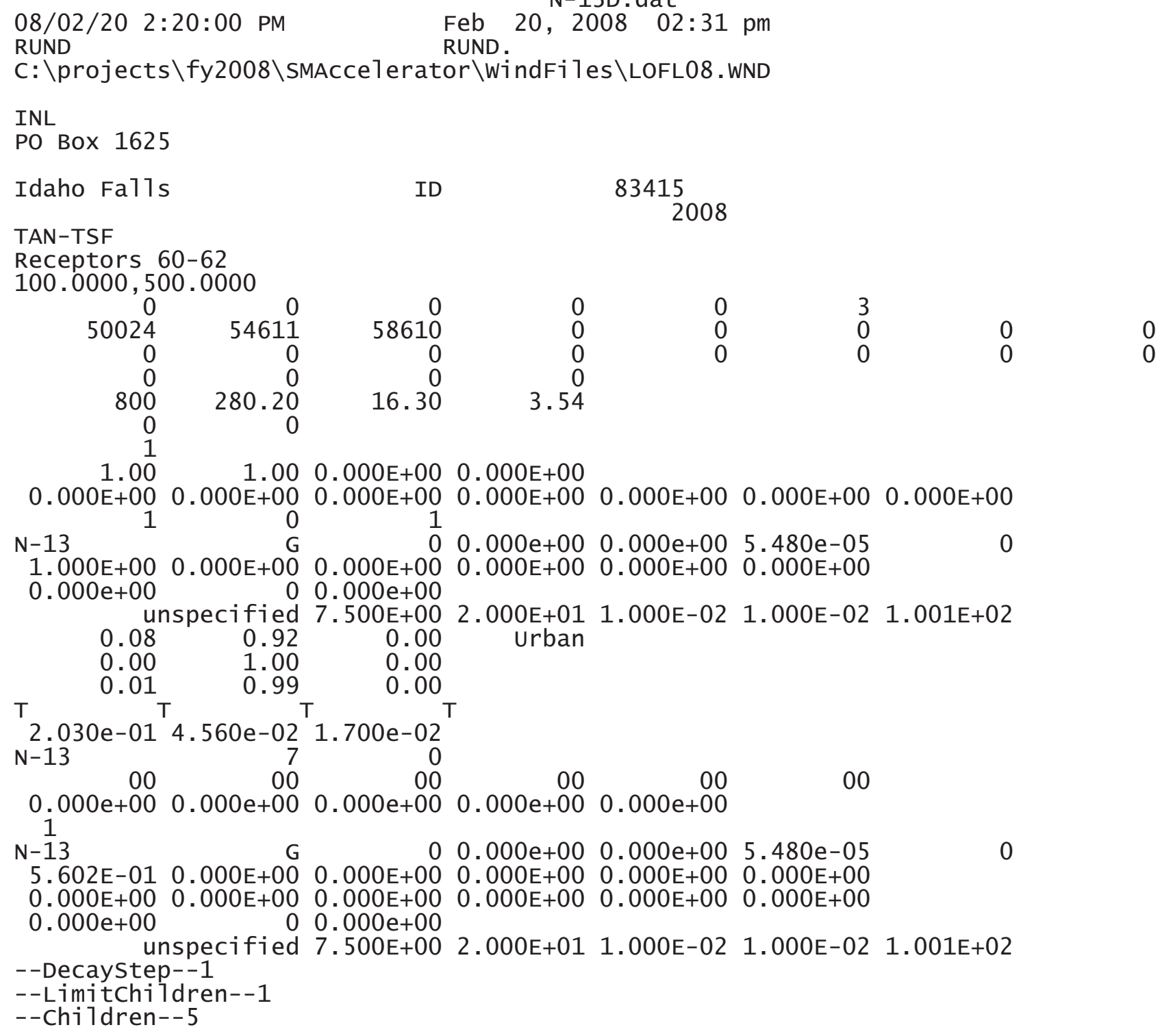




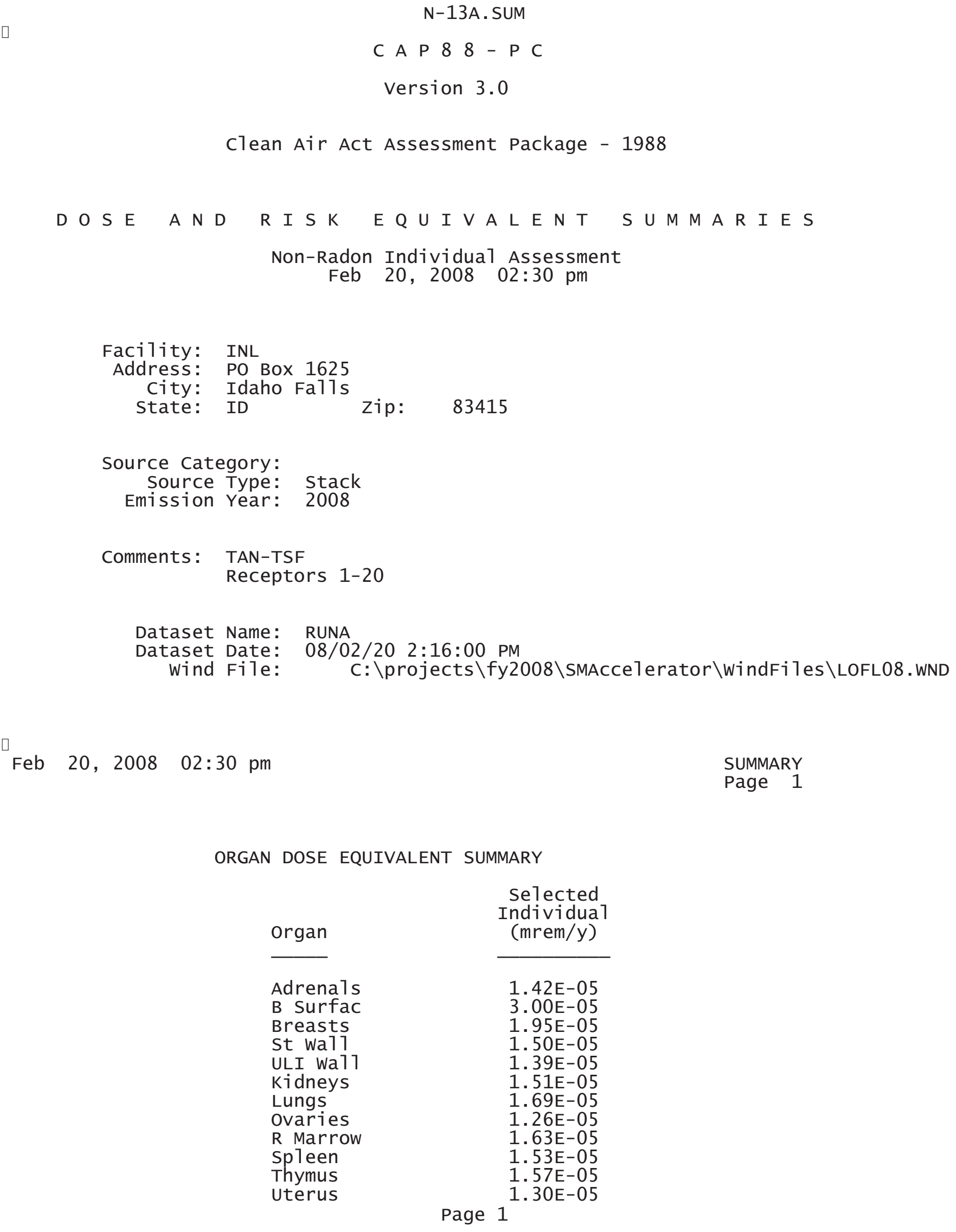

\begin{tabular}{lr} 
ORGAN DOSE EQUIVALENT SUMMARY \\
Organ & $\begin{array}{r}\text { Selected } \\
\text { Individual } \\
\text { (mrem/y) }\end{array}$ \\
\cline { 2 - 2 } Adrenals & $1.42 \mathrm{E}-05$ \\
B Surfac & $3.00 \mathrm{E}-05$ \\
Breasts & $1.95 \mathrm{E}-05$ \\
St wa11 & $1.50 \mathrm{E}-05$ \\
ULI wa11 & $1.39 \mathrm{E}-05$ \\
Kidneys & $1.51 \mathrm{E}-05$ \\
Lungs & $1.69 \mathrm{E}-05$ \\
Ovaries & $1.26 \mathrm{E}-05$ \\
R Marrow & $1.63 \mathrm{E}-05$ \\
Spleen & $1.53 \mathrm{E}-05$ \\
Thymus & $1.57 \mathrm{E}-05$ \\
Uterus & $1.30 \mathrm{E}-05$ \\
& Page 1
\end{tabular}




$\begin{array}{lr} & \mathrm{N}-13 \mathrm{~A} . \text { SUM } \\ \text { B1d wa11 } & 1.41 \mathrm{E}-05 \\ \text { Brain } & 1.80 \mathrm{E}-05 \\ \text { Esophagu } & 1.39 \mathrm{E}-05 \\ \text { SI wa11 } & 1.33 \mathrm{E}-05 \\ \text { LLI wa11 } & 1.36 \mathrm{E}-05 \\ \text { Liver } & 1.52 \mathrm{E}-05 \\ \text { Muscle } & 1.65 \mathrm{E}-05 \\ \text { Pancreas } & 1.33 \mathrm{E}-05 \\ \text { Skin } & 3.09 \mathrm{E}-05 \\ \text { Testes } & 1.70 \mathrm{E}-05 \\ \text { Thyroid } & 1.73 \mathrm{E}-05 \\ & \\ \text { EFFEC } & 1.63 \mathrm{E}-05\end{array}$

PATHWAY EFFECTIVE DOSE EQUIVALENT SUMMARY

$\begin{array}{lc}\text { Pathway } & \begin{array}{c}\text { selected } \\ \text { Individual } \\ \text { (mrem/y) }\end{array} \\ & \\ \text { INGESTION } & 0.00 \mathrm{E}+00 \\ \text { INHALATION } & 0.00 \mathrm{E}+00 \\ \text { AIR IMMERSION } & 1.63 \mathrm{E}-05 \\ \text { GROUND SURFACE } & 0.00 \mathrm{E}+00 \\ \text { INTERNAL } & 0.00 \mathrm{E}+00 \\ \text { EXTERNAL } & 1.63 \mathrm{E}-05 \\ \text { TOTAL } & 1.63 \mathrm{E}-05\end{array}$

Feb 20, 2008 02:30 pm

SUMMARY

Page 2

NUCLIDE EFFECTIVE DOSE EQUIVALENT SUMMARY

Nuclide

$\mathrm{N}-13$

TOTAL

Feb 20, $2008 \quad 02: 30 \mathrm{pm}$

$$
\begin{gathered}
\begin{array}{c}
\text { Selected } \\
\text { Individual } \\
\text { (mrem/y) }
\end{array} \\
\hline 1.63 \mathrm{E}-05 \\
1.63 \mathrm{E}-05
\end{gathered}
$$

SUMMARY

Page 3

CANCER RISK SUMMARY

Cancer

Esophagu
Selected Individual

Total Lifetime Fatal Cancer Risk

$$
\text { Page } 2^{1.60 \mathrm{E}-13}
$$




$\begin{array}{lr} & \mathrm{N}-13 \mathrm{~A} . \mathrm{SUM} \\ \text { Stomach } & 6.08 \mathrm{E}-13 \\ \text { Colon } & 1.42 \mathrm{E}-12 \\ \text { Liver } & 2.30 \mathrm{E}-13 \\ \text { LUNG } & 1.65 \mathrm{E}-12 \\ \text { Bone } & 2.85 \mathrm{E}-14 \\ \text { Skin } & 3.08 \mathrm{E}-14 \\ \text { Breast } & 9.39 \mathrm{E}-13 \\ \text { Ovary } & 1.80 \mathrm{E}-13 \\ \text { B Tadder } & 3.41 \mathrm{E}-13 \\ \text { Kidneys } & 7.86 \mathrm{E}-14 \\ \text { Thyroid } & 5.51 \mathrm{E}-14 \\ \text { Leukemia } & 9.14 \mathrm{E}-13 \\ \text { Residua1 } & 2.18 \mathrm{E}-12 \\ \text { Tota1 } & 8.82 \mathrm{E}-12 \\ & \\ \text { TOTAL } & 1.76 \mathrm{E}-11\end{array}$

PATHWAY RISK SUMMARY

\begin{tabular}{lc} 
Pathway & $\begin{array}{c}\text { Selected Individua1 } \\
\text { Total Lifetime } \\
\text { Fatal Cancer Risk }\end{array}$ \\
\cline { 2 - 2 } INGESTION & $0.00 \mathrm{E}+00$ \\
INHALATION & $0.00 \mathrm{E}+00$ \\
AIR IMMERSION & $8.82 \mathrm{E}-12$ \\
GROUND SURFACE & $0.00 \mathrm{E}+00$ \\
INTERNAL & $0.00 \mathrm{E}+00$ \\
EXTERNAL & $8.82 \mathrm{E}-12$ \\
TOTAL & $8.82 \mathrm{E}-12$
\end{tabular}

Feb 20, $2008 \quad 02: 30$ pm

SUMMARY Page 4

NUCLIDE RISK SUMMARY

Nuc7ide

$\mathrm{N}-13$

TOTAL

$\square$

Feb 20, 2008 02:30 pm
Selected Individual

Total Lifetime Fatal Cancer Risk

8. $82 \mathrm{E}-12$

$8.82 \mathrm{E}-12$

INDIVIDUAL EFFECTIVE DOSE EQUIVALENT RATE (mrem/y) (A11 Radionuclides and Pathways)

Distance $(m)$ 


\begin{tabular}{|c|c|c|c|c|c|c|c|}
\hline Direction & 10344 & 10365 & 10472 & $\begin{array}{c}N-13 A \\
10590\end{array}$ & 11103 & 11989 & 12522 \\
\hline $\mathrm{N}$ & $3.9 \mathrm{E}-06$ & $3.9 \mathrm{E}-06$ & $3.9 \mathrm{E}-06$ & $3.8 \mathrm{E}-06$ & $3.6 \mathrm{E}-06$ & $3.3 E-06$ & $3.2 \mathrm{E}-06$ \\
\hline NNW & $1.5 \mathrm{E}-06$ & $1.5 \mathrm{E}-06$ & 1. $5 \mathrm{E}-06$ & $1.4 \mathrm{E}-06$ & $1.4 \mathrm{E}-06$ & 1. $3 \mathrm{E}-06$ & 1. $2 \mathrm{E}-06$ \\
\hline NW & $2.1 \mathrm{E}-06$ & $2.1 \mathrm{E}-06$ & $2.0 \mathrm{E}-06$ & $2.0 \mathrm{E}-06$ & $1.9 \mathrm{E}-06$ & $1.7 \mathrm{E}-06$ & $1.7 \mathrm{E}-06$ \\
\hline WNW & $2.8 \mathrm{E}-06$ & $2.7 \mathrm{E}-06$ & $2.7 \mathrm{E}-06$ & $2.7 \mathrm{E}-06$ & $2.5 \mathrm{E}-06$ & 2. $3 \mathrm{E}-06$ & $2.2 \mathrm{E}-06$ \\
\hline W & $3.1 \mathrm{E}-06$ & $3.1 \mathrm{E}-06$ & $3.1 \mathrm{E}-06$ & $3.1 \mathrm{E}-06$ & $2.9 \mathrm{E}-06$ & $2.7 \mathrm{E}-06$ & $2.5 \mathrm{E}-06$ \\
\hline WSW & $4.2 \mathrm{E}-06$ & $4.2 \mathrm{E}-06$ & $4.2 \mathrm{E}-06$ & $4.1 \mathrm{E}-06$ & $3.9 \mathrm{E}-06$ & $3.6 \mathrm{E}-06$ & $3.4 \mathrm{E}-06$ \\
\hline SW & $6.9 \mathrm{E}-06$ & $6.9 \mathrm{E}-06$ & $6.8 \mathrm{E}-06$ & $6.7 \mathrm{E}-06$ & $6.4 \mathrm{E}-06$ & $5.8 \mathrm{E}-06$ & $5.5 \mathrm{E}-06$ \\
\hline SSW & $1.2 \mathrm{E}-05$ & $1.1 \mathrm{E}-05$ & $1.1 \mathrm{E}-05$ & $1.1 \mathrm{E}-05$ & $1.1 \mathrm{E}-05$ & $9.7 \mathrm{E}-06$ & $9.2 \mathrm{E}-06$ \\
\hline $\mathrm{S}$ & $1.6 \mathrm{E}-05$ & $1.6 \mathrm{E}-05$ & $1.6 \mathrm{E}-05$ & $1.6 \mathrm{E}-05$ & 1. $5 \mathrm{E}-05$ & $1.4 \mathrm{E}-05$ & 1. $3 \mathrm{E}-05$ \\
\hline SSE & $1.4 \mathrm{E}-05$ & 1. $3 \mathrm{E}-05$ & 1. $3 \mathrm{E}-05$ & 1. 3E-05 & $1.2 \mathrm{E}-05$ & $1.1 \mathrm{E}-05$ & $1.1 \mathrm{E}-05$ \\
\hline SE & $7.1 \mathrm{E}-06$ & $7.1 \mathrm{E}-06$ & $7.0 \mathrm{E}-06$ & $6.9 \mathrm{E}-06$ & $6.5 \mathrm{E}-06$ & $6.0 \mathrm{E}-06$ & $5.7 \mathrm{E}-06$ \\
\hline ESE & $4.7 \mathrm{E}-06$ & $4.7 \mathrm{E}-06$ & $4.6 \mathrm{E}-06$ & $4.6 \mathrm{E}-06$ & $4.3 \mathrm{E}-06$ & $4.0 \mathrm{E}-06$ & $3.8 \mathrm{E}-06$ \\
\hline $\mathrm{E}$ & $6.0 \mathrm{E}-06$ & $6.0 \mathrm{E}-06$ & $6.0 \mathrm{E}-06$ & $5.9 \mathrm{E}-06$ & $5.6 \mathrm{E}-06$ & $5.1 \mathrm{E}-06$ & $4.9 \mathrm{E}-06$ \\
\hline ENE & $5.2 \mathrm{E}-06$ & $5.2 \mathrm{E}-06$ & $5.2 \mathrm{E}-06$ & $5 \cdot 1 \mathrm{E}-06$ & $4.8 \mathrm{E}-06$ & 4. $4 \mathrm{E}-06$ & $4 \cdot 2 \mathrm{E}-06$ \\
\hline $\begin{array}{l}\text { NE } \\
\text { NNE }\end{array}$ & $5.0 \mathrm{E}-06$ & $5.0 \mathrm{E}-06$ & $4.9 \mathrm{E}-06$ & $\begin{array}{l}4.9 \mathrm{E}-06 \\
4.96\end{array}$ & $4.6 \mathrm{E}-06$ & $\begin{array}{l}0.6 \mathrm{E}-06 \\
4.2 \mathrm{E}-06\end{array}$ & 4. $0 \mathrm{E}-06$ \\
\hline
\end{tabular}

Distance (m)

\begin{tabular}{|c|c|c|c|c|c|c|c|}
\hline Direction & 13245 & 13286 & 13483 & 13612 & 13664 & 13959 & 14258 \\
\hline $\mathrm{N}$ & $3.0 \mathrm{E}-06$ & $3.0 \mathrm{E}-06$ & $2.9 \mathrm{E}-06$ & $2.9 \mathrm{E}-06$ & $2.9 \mathrm{E}-06$ & $2.8 \mathrm{E}-06$ & $2.8 \mathrm{E}-06$ \\
\hline NNW & $1.1 \mathrm{E}-06$ & $1.1 \mathrm{E}-06$ & $1.1 \mathrm{E}-06$ & $1.1 \mathrm{E}-06$ & $1.1 \mathrm{E}-06$ & $1.1 \mathrm{E}-06$ & 1. $0 \mathrm{E}-06$ \\
\hline NW & $1.6 \mathrm{E}-06$ & $1.6 \mathrm{E}-06$ & $1.5 \mathrm{E}-06$ & $1.5 \mathrm{E}-06$ & $1.5 \mathrm{E}-06$ & $1.5 \mathrm{E}-06$ & $1.4 \mathrm{E}-06$ \\
\hline WNW & $2.1 \mathrm{E}-06$ & $2.1 \mathrm{E}-06$ & 2. $0 \mathrm{E}-06$ & 2.0E-06 & 2.0E-06 & $2.0 \mathrm{E}-06$ & $1.9 \mathrm{E}-06$ \\
\hline & $2.4 \mathrm{E}-06$ & $2.4 \mathrm{E}-06$ & $2.3 \mathrm{E}-06$ & $2.3 \mathrm{E}-06$ & $2.3 \mathrm{E}-06$ & $2.2 \mathrm{E}-06$ & $2.2 \mathrm{E}-06$ \\
\hline WSW & $3.2 \mathrm{E}-06$ & $3.2 \mathrm{E}-06$ & $3.1 \mathrm{E}-06$ & $3.1 \mathrm{E}-06$ & $3.1 \mathrm{E}-06$ & $3.0 \mathrm{E}-06$ & $2.9 \mathrm{E}-06$ \\
\hline SW & $5.1 \mathrm{E}-06$ & $5.1 \mathrm{E}-06$ & $5.0 \mathrm{E}-06$ & $5.0 \mathrm{E}-06$ & $4.9 \mathrm{E}-06$ & $4.8 \mathrm{E}-06$ & $4.7 \mathrm{E}-06$ \\
\hline SSW & $8.6 \mathrm{E}-06$ & $8.5 \mathrm{E}-06$ & $8.4 \mathrm{E}-06$ & $8.3 \mathrm{E}-06$ & $8.3 \mathrm{E}-06$ & $8.0 \mathrm{E}-06$ & $7.8 \mathrm{E}-06$ \\
\hline $\mathrm{s}$ & 1. $2 \mathrm{E}-05$ & 1. $2 \mathrm{E}-05$ & 1. $2 \mathrm{E}-05$ & $1.2 \mathrm{E}-05$ & $1.2 \mathrm{E}-05$ & $1.1 \mathrm{E}-05$ & $1.1 \mathrm{E}-05$ \\
\hline SSE & $1.0 \mathrm{E}-05$ & $1.0 \mathrm{E}-05$ & $9.8 \mathrm{E}-06$ & $9.7 \mathrm{E}-06$ & $9.7 \mathrm{E}-06$ & $9.4 \mathrm{E}-06$ & $9.2 \mathrm{E}-06$ \\
\hline $\mathrm{SE}$ & $5.3 \mathrm{E}-06$ & $5.3 \mathrm{E}-06$ & $5.2 \mathrm{E}-06$ & $5.2 \mathrm{E}-06$ & $5.2 \mathrm{E}-06$ & $5.0 \mathrm{E}-06$ & $4.9 \mathrm{E}-06$ \\
\hline ESE & $3.5 \mathrm{E}-06$ & $3.5 \mathrm{E}-06$ & $3.5 \mathrm{E}-06$ & $3.4 \mathrm{E}-06$ & $3.4 \mathrm{E}-06$ & $3.3 \mathrm{E}-06$ & $3.3 \mathrm{E}-06$ \\
\hline & $4.6 \mathrm{E}-06$ & $4.6 \mathrm{E}-06$ & $4.5 \mathrm{E}-06$ & $4.4 \mathrm{E}-06$ & $4.4 \mathrm{E}-06$ & $4.3 \mathrm{E}-06$ & $4.2 \mathrm{E}-06$ \\
\hline ENE & $3.9 \mathrm{E}-06$ & $3.9 \mathrm{E}-06$ & $3.8 \mathrm{E}-06$ & $3.8 \mathrm{E}-06$ & $3.8 \mathrm{E}-06$ & $3.7 \mathrm{E}-06$ & $3.6 \mathrm{E}-06$ \\
\hline $\mathrm{NE}$ & $5.9 \mathrm{E}-06$ & $5.8 \mathrm{E}-06$ & $5.7 \mathrm{E}-06$ & $5.7 \mathrm{E}-06$ & $5.7 \mathrm{E}-06$ & $5.5 \mathrm{E}-06$ & $5.4 \mathrm{E}-06$ \\
\hline NNE & $3.7 \mathrm{E}-06$ & $3.7 \mathrm{E}-06$ & $3.7 \mathrm{E}-06$ & $3.6 \mathrm{E}-06$ & $3.6 \mathrm{E}-06$ & $3.5 \mathrm{E}-06$ & $3.4 \mathrm{E}-06$ \\
\hline
\end{tabular}

$\square$

Feb 20, 2008 02:30 pm

SUMMARY

Page 6

INDIVIDUAL EFFECTIVE DOSE EQUIVALENT RATE (mrem/y) (A11 Radionuc1ides and Pathways)

\begin{tabular}{|c|c|c|c|c|c|c|}
\hline \multirow[b]{2}{*}{ Direction } & \multicolumn{6}{|c|}{ Distance $(\mathrm{m})$} \\
\hline & 14374 & 15241 & 15441 & 15784 & 15844 & 16323 \\
\hline $\begin{array}{r}N \\
N N W\end{array}$ & $\begin{array}{l}2.7 \mathrm{E}-06 \\
1.0 \mathrm{E}-06\end{array}$ & $\begin{array}{l}2.6 \mathrm{E}-06 \\
9.6 \mathrm{E}-07\end{array}$ & $\begin{array}{l}2.5 \mathrm{E}-06 \\
9.4 \mathrm{E}-07\end{array}$ & $\begin{array}{r}2.5 \mathrm{E}-06 \\
9.2 \mathrm{E}-07 \\
\text { Page }\end{array}$ & $\begin{array}{l}2.4 \mathrm{E}-06 \\
9.2 \mathrm{E}-07 \\
4\end{array}$ & $\begin{array}{l}2.4 \mathrm{E}-06 \\
8.9 \mathrm{E}-07\end{array}$ \\
\hline
\end{tabular}




\begin{tabular}{|c|c|c|c|c|c|c|}
\hline \multicolumn{7}{|c|}{$\mathrm{N}-13 \mathrm{~A} . \mathrm{sum}$} \\
\hline NW & 1. $4 \mathrm{E}-06$ & 1. $3 \mathrm{E}-06$ & 1. $3 \mathrm{E}-06$ & 1. $3 \mathrm{E}-06$ & 1. $3 \mathrm{E}-06$ & 1. $2 \mathrm{E}-06$ \\
\hline WNW & $1.9 \mathrm{E}-06$ & $1.8 \mathrm{E}-06$ & $1.8 \mathrm{E}-06$ & $1.7 \mathrm{E}-06$ & $1.7 \mathrm{E}-06$ & $7 E-06$ \\
\hline & $2.2 \mathrm{E}-06$ & $2.0 \mathrm{E}-06$ & $2.0 \mathrm{E}-06$ & $2.0 \mathrm{E}-06$ & 2. $0 \mathrm{E}-06$ & $1.9 \mathrm{E}-06$ \\
\hline WSW & $2.9 \mathrm{E}-06$ & $2.7 \mathrm{E}-06$ & $2.7 \mathrm{E}-06$ & $2.6 \mathrm{E}-06$ & $2.6 \mathrm{E}-06$ & $2.5 \mathrm{E}-06$ \\
\hline SW & $4.7 \mathrm{E}-06$ & $4.3 \mathrm{E}-06$ & 4. $3 \mathrm{E}-06$ & $4.2 \mathrm{E}-06$ & $4.2 \mathrm{E}-06$ & 4. $0 \mathrm{E}-06$ \\
\hline SSW & $7.8 \mathrm{E}-06$ & 7. $3 \mathrm{E}-06$ & $7.1 \mathrm{E}-06$ & $7.0 \mathrm{E}-06$ & $6.9 \mathrm{E}-06$ & $6.7 \mathrm{E}-06$ \\
\hline & & 1. $0 \mathrm{E}-05$ & 1. $0 \mathrm{E}-05$ & $9.7 \mathrm{E}-06$ & $9.7 \mathrm{E}-06$ & 6 \\
\hline SSE & $9.1 \mathrm{E}-06$ & $8.5 E-06$ & $8.4 \mathrm{E}$ & $8.1 \mathrm{E}$ & $8.1 \mathrm{E}-$ & 7 \\
\hline $\mathrm{SE}$ & $4.9 \mathrm{E}-06$ & $4.5 \mathrm{E}-06$ & $4.5 \mathrm{E}-06$ & $4.4 \mathrm{E}-06$ & $4.3 \mathrm{E}-06$ & $4.2 \mathrm{E}-06$ \\
\hline ESE & & & & $2.9 \mathrm{~b}$ & $2.9 \mathrm{E}$ & \\
\hline & 4 & $3.9 \mathrm{E}-06$ & $3.8 \mathrm{E}-06$ & $3.7 \mathrm{E}$ & $3.7 \mathrm{E}-06$ & $E-06$ \\
\hline ENE & 2 & 3. & 3. & $3.2 \mathrm{l}$ & $3.2 \mathrm{E}-$ & \\
\hline $\mathrm{NE}$ & J. & -06 & $4.9 \mathrm{E}-06$ & $4.8 \mathrm{E}-06$ & $4.7 \mathrm{E}-06$ & $4.6 \mathrm{E}-06$ \\
\hline NNE & $E-06$ & $2 E-06$ & $1 E-06$ & $3.1 \mathrm{E}-06$ & $3.0 \mathrm{E}-06$ & $2.9 \mathrm{E}-06$ \\
\hline
\end{tabular}

Feb 20, $2008 \quad 02: 30 \mathrm{pm}$

SUMMARY

Page 7

INDIVIDUAL LIFETIME RISK (deaths)

(A11 Radionuclides and Pathways)

\begin{tabular}{|c|c|c|c|c|c|c|c|}
\hline \multicolumn{8}{|c|}{ Distance $(\mathrm{m})$} \\
\hline Direction & 10344 & 10365 & 10472 & 10590 & 11103 & 11989 & 12522 \\
\hline $\begin{array}{r}\text { N } \\
\text { NNW } \\
N W \\
\text { WNW } \\
W \\
W S W \\
\text { SW } \\
\text { SSW } \\
S \\
\text { SSE } \\
\text { SE } \\
\text { ESE } \\
\text { E } \\
\text { ENE } \\
N E \\
\text { NNE }\end{array}$ & $\begin{array}{l}2.1 \mathrm{E}-12 \\
8.1 \mathrm{E}-13 \\
1.1 \mathrm{E}-12 \\
1.5 \mathrm{E}-12 \\
1.7 \mathrm{E}-12 \\
2.3 \mathrm{E}-12 \\
3.8 \mathrm{E}-12 \\
6.3 \mathrm{E}-12 \\
8.8 \mathrm{E}-12 \\
7.3 \mathrm{E}-12 \\
3.8 \mathrm{E}-12 \\
2.5 \mathrm{E}-12 \\
3.3 \mathrm{E}-12 \\
2.8 \mathrm{E}-12 \\
4.3 \mathrm{E}-12 \\
2.7 \mathrm{E}-12\end{array}$ & $\begin{array}{l}2.1 \mathrm{E}-12 \\
8.0 \mathrm{E}-13 \\
1.1 \mathrm{E}-12 \\
1.5 \mathrm{E}-12 \\
1.7 \mathrm{E}-12 \\
2.3 \mathrm{E}-12 \\
3.7 \mathrm{E}-12 \\
6.2 \mathrm{E}-12 \\
8.8 \mathrm{E}-12 \\
7.3 \mathrm{E}-12 \\
3.8 \mathrm{E}-12 \\
2.5 \mathrm{E}-12 \\
3.3 \mathrm{E}-12 \\
2.8 \mathrm{E}-12 \\
4.3 \mathrm{E}-12 \\
2.7 \mathrm{E}-12\end{array}$ & $\begin{array}{l}2.1 \mathrm{E}-12 \\
8.0 \mathrm{E}-13 \\
1.1 \mathrm{E}-12 \\
1.5 \mathrm{E}-12 \\
1.7 \mathrm{E}-12 \\
2.3 \mathrm{E}-12 \\
3.7 \mathrm{E}-12 \\
6.2 \mathrm{E}-12 \\
8.7 \mathrm{E}-12 \\
7.2 \mathrm{E}-12 \\
3.8 \mathrm{E}-12 \\
2.5 \mathrm{E}-12 \\
3.2 \mathrm{E}-12 \\
2.8 \mathrm{E}-12 \\
4.2 \mathrm{E}-12 \\
2.7 \mathrm{E}-12\end{array}$ & $\begin{array}{l}2.1 \mathrm{E}-12 \\
7.9 \mathrm{E}-13 \\
1.1 \mathrm{E}-12 \\
1.5 \mathrm{E}-12 \\
1.7 \mathrm{E}-12 \\
2.2 \mathrm{E}-12 \\
3.7 \mathrm{E}-12 \\
6.1 \mathrm{E}-12 \\
8.6 \mathrm{E}-12 \\
7.1 \mathrm{E}-12 \\
3.7 \mathrm{E}-12 \\
2.5 \mathrm{E}-12 \\
3.2 \mathrm{E}-12 \\
2.8 \mathrm{E}-12 \\
4.2 \mathrm{E}-12 \\
2.6 \mathrm{E}-12\end{array}$ & $\begin{array}{l}2.0 \mathrm{E}-12 \\
7.4 \mathrm{E}-13 \\
1.0 \mathrm{E}-12 \\
1.4 \mathrm{E}-12 \\
1.6 \mathrm{E}-12 \\
2.1 \mathrm{E}-12 \\
3.4 \mathrm{E}-12 \\
5.7 \mathrm{E}-12 \\
8.1 \mathrm{E}-12 \\
6.7 \mathrm{E}-12 \\
3.5 \mathrm{E}-12 \\
2.3 \mathrm{E}-12 \\
3.0 \mathrm{E}-12 \\
2.6 \mathrm{E}-12 \\
4.0 \mathrm{E}-12 \\
2.5 \mathrm{E}-12\end{array}$ & $\begin{array}{l}1.8 \mathrm{E}-12 \\
6.8 \mathrm{E}-13 \\
9.5 \mathrm{E}-13 \\
1.3 \mathrm{E}-12 \\
1.4 \mathrm{E}-12 \\
1.9 \mathrm{E}-12 \\
3.1 \mathrm{E}-12 \\
5.2 \mathrm{E}-12 \\
7.4 \mathrm{E}-12 \\
6.1 \mathrm{E}-12 \\
3.2 \mathrm{E}-12 \\
2.2 \mathrm{E}-12 \\
2.8 \mathrm{E}-12 \\
2.4 \mathrm{E}-12 \\
3.6 \mathrm{E}-12 \\
2.3 \mathrm{E}-12\end{array}$ & $\begin{array}{l}1.7 \mathrm{E}-12 \\
6.5 \mathrm{E}-13 \\
9.0 \mathrm{E}-13 \\
1.2 \mathrm{E}-12 \\
1.4 \mathrm{E}-12 \\
1.8 \mathrm{E}-12 \\
3.0 \mathrm{E}-12 \\
5.0 \mathrm{E}-12 \\
7.0 \mathrm{E}-12 \\
5.8 \mathrm{E}-12 \\
3.1 \mathrm{E}-12 \\
2.1 \mathrm{E}-12 \\
2.6 \mathrm{E}-12 \\
2.3 \mathrm{E}-12 \\
3.4 \mathrm{E}-12 \\
2.2 \mathrm{E}-12\end{array}$ \\
\hline \multicolumn{8}{|c|}{ Distance (m) } \\
\hline Direction & 13245 & 13286 & 13483 & 13612 & 13664 & 13959 & 14258 \\
\hline $\begin{array}{r}\text { N } \\
\text { NNW } \\
\text { NW } \\
\text { WNW } \\
W \\
\text { WSW } \\
\text { SW } \\
\text { SSW }\end{array}$ & $\begin{array}{l}1.6 \mathrm{E}-12 \\
6.1 \mathrm{E}-13 \\
8.5 \mathrm{E}-13 \\
1.1 \mathrm{E}-12 \\
1.3 \mathrm{E}-12 \\
1.7 \mathrm{E}-12 \\
2.8 \mathrm{E}-12 \\
4.6 \mathrm{E}-12\end{array}$ & $\begin{array}{l}1 \cdot 6 \mathrm{E}-12 \\
6.1 \mathrm{E}-13 \\
8.4 \mathrm{E}-13 \\
1.1 \mathrm{E}-12 \\
1.3 \mathrm{E}-12 \\
1.7 \mathrm{E}-12 \\
2.8 \mathrm{E}-12 \\
4.6 \mathrm{E}-12\end{array}$ & $\begin{array}{l}1 \cdot 6 \mathrm{E}-12 \\
6.0 \mathrm{E}-13 \\
8.3 \mathrm{E}-13 \\
1.1 \mathrm{E}-12 \\
1.3 \mathrm{E}-12 \\
1.7 \mathrm{E}-12 \\
2.7 \mathrm{E}-12 \\
4.5 \mathrm{E}-12\end{array}$ & $\begin{array}{r}1.6 \mathrm{E}-12 \\
5.9 \mathrm{E}-13 \\
8.2 \mathrm{E}-13 \\
1.1 \mathrm{E}-12 \\
1.3 \mathrm{E}-12 \\
1.7 \mathrm{E}-12 \\
2.7 \mathrm{E}-12 \\
4.5 \mathrm{E}-12 \\
\text { Page }\end{array}$ & $\begin{array}{l}1.6 \mathrm{E}-12 \\
5.9 \mathrm{E}-13 \\
8.2 \mathrm{E}-13 \\
1.1 \mathrm{E}-12 \\
1.2 \mathrm{E}-12 \\
1.7 \mathrm{E}-12 \\
2.7 \mathrm{E}-12 \\
4.5 \mathrm{E}-12 \\
5\end{array}$ & $\begin{array}{l}1.5 \mathrm{E}-12 \\
5.7 \mathrm{E}-13 \\
8.0 \mathrm{E}-13 \\
1.1 \mathrm{E}-12 \\
1.2 \mathrm{E}-12 \\
1.6 \mathrm{E}-12 \\
2.6 \mathrm{E}-12 \\
4.4 \mathrm{E}-12\end{array}$ & $\begin{array}{l}1.5 \mathrm{E}-12 \\
5.6 \mathrm{E}-13 \\
7.8 \mathrm{E}-13 \\
1.0 \mathrm{E}-12 \\
1.2 \mathrm{E}-12 \\
1.6 \mathrm{E}-12 \\
2.6 \mathrm{E}-12 \\
4.3 \mathrm{E}-12\end{array}$ \\
\hline
\end{tabular}




\begin{tabular}{rlllllll}
\multicolumn{7}{c}{$\mathrm{N}-13 \mathrm{~A} . \mathrm{SUM}$} \\
$\mathrm{S}$ & $6.5 \mathrm{E}-12$ & $6.5 \mathrm{E}-12$ & $6.4 \mathrm{E}-12$ & $6.3 \mathrm{E}-12$ & $6.3 \mathrm{E}-12$ & $6.1 \mathrm{E}-12$ & $6.0 \mathrm{E}-12$ \\
$\mathrm{SSE}$ & $5.4 \mathrm{E}-12$ & $5.4 \mathrm{E}-12$ & $5.3 \mathrm{E}-12$ & $5.3 \mathrm{E}-12$ & $5.3 \mathrm{E}-12$ & $5.1 \mathrm{E}-12$ & $5.0 \mathrm{E}-12$ \\
$\mathrm{SE}$ & $2.9 \mathrm{E}-12$ & $2.9 \mathrm{E}-12$ & $2.8 \mathrm{E}-12$ & $2.8 \mathrm{E}-12$ & $2.8 \mathrm{E}-12$ & $2.7 \mathrm{E}-12$ & $2.7 \mathrm{E}-12$ \\
$\mathrm{ESE}$ & $1.9 \mathrm{E}-12$ & $1.9 \mathrm{E}-12$ & $1.9 \mathrm{E}-12$ & $1.9 \mathrm{E}-12$ & $1.9 \mathrm{E}-12$ & $1.8 \mathrm{E}-12$ & $1.8 \mathrm{E}-12$ \\
$\mathrm{E}$ & $2.5 \mathrm{E}-12$ & $2.5 \mathrm{E}-12$ & $2.4 \mathrm{E}-12$ & $2.4 \mathrm{E}-12$ & $2.4 \mathrm{E}-12$ & $2.3 \mathrm{E}-12$ & $2.3 \mathrm{E}-12$ \\
$\mathrm{ENE}$ & $2.1 \mathrm{E}-12$ & $2.1 \mathrm{E}-12$ & $2.1 \mathrm{E}-12$ & $2.1 \mathrm{E}-12$ & $2.1 \mathrm{E}-12$ & $2.0 \mathrm{E}-12$ & $2.0 \mathrm{E}-12$ \\
$\mathrm{NE}$ & $3.2 \mathrm{E}-12$ & $3.2 \mathrm{E}-12$ & $3.1 \mathrm{E}-12$ & $3.1 \mathrm{E}-12$ & $3.1 \mathrm{E}-12$ & $3.0 \mathrm{E}-12$ & $2.9 \mathrm{E}-12$ \\
$\mathrm{NNE}$ & $2.0 \mathrm{E}-12$ & $2.0 \mathrm{E}-12$ & $2.0 \mathrm{E}-12$ & $2.0 \mathrm{E}-12$ & $2.0 \mathrm{E}-12$ & $1.9 \mathrm{E}-12$ & $1.9 \mathrm{E}-12$
\end{tabular}

Feb 20, $2008 \quad 02: 30 \mathrm{pm}$

SUMMARY

Page 8

INDIVIDUAL LIFETIME RISK (deaths)

(A11 Radionuclides and Pathways)

\begin{tabular}{rcccccc}
\hline & \multicolumn{7}{c}{ Distance (m) } \\
\cline { 2 - 7 } Direction & 14374 & 15241 & 15441 & 15784 & 15844 & 16323 \\
\hline & & & & \\
N & $1.5 \mathrm{E}-12$ & $1.4 \mathrm{E}-12$ & $1.4 \mathrm{E}-12$ & $1.3 \mathrm{E}-12$ & $1.3 \mathrm{E}-12$ & $1.3 \mathrm{E}-12$ \\
NNW & $5.6 \mathrm{E}-13$ & $5.2 \mathrm{E}-13$ & $5.1 \mathrm{E}-13$ & $5.0 \mathrm{E}-13$ & $5.0 \mathrm{E}-13$ & $4.8 \mathrm{E}-13$ \\
$\mathrm{NW}$ & $7.7 \mathrm{E}-13$ & $7.3 \mathrm{E}-13$ & $7.1 \mathrm{E}-13$ & $7.0 \mathrm{E}-13$ & $6.9 \mathrm{E}-13$ & $6.7 \mathrm{E}-13$ \\
WNW & $1.0 \mathrm{E}-12$ & $9.7 \mathrm{E}-13$ & $9.6 \mathrm{E}-13$ & $9.3 \mathrm{E}-13$ & $9.3 \mathrm{E}-13$ & $9.0 \mathrm{E}-13$ \\
$\mathrm{~W}$ & $1.2 \mathrm{E}-12$ & $1.1 \mathrm{E}-12$ & $1.1 \mathrm{E}-12$ & $1.1 \mathrm{E}-12$ & $1.1 \mathrm{E}-12$ & $1.0 \mathrm{E}-12$ \\
WSW & $1.6 \mathrm{E}-12$ & $1.5 \mathrm{E}-12$ & $1.4 \mathrm{E}-12$ & $1.4 \mathrm{E}-12$ & $1.4 \mathrm{E}-12$ & $1.4 \mathrm{E}-12$ \\
$\mathrm{SW}$ & $2.5 \mathrm{E}-12$ & $2.4 \mathrm{E}-12$ & $2.3 \mathrm{E}-12$ & $2.3 \mathrm{E}-12$ & $2.3 \mathrm{E}-12$ & $2.2 \mathrm{E}-12$ \\
$\mathrm{SSW}$ & $4.2 \mathrm{E}-12$ & $3.9 \mathrm{E}-12$ & $3.9 \mathrm{E}-12$ & $3.8 \mathrm{E}-12$ & $3.8 \mathrm{E}-12$ & $3.6 \mathrm{E}-12$ \\
$\mathrm{~S}$ & $5.9 \mathrm{E}-12$ & $5.5 \mathrm{E}-12$ & $5.4 \mathrm{E}-12$ & $5.3 \mathrm{E}-12$ & $5.3 \mathrm{E}-12$ & $5.1 \mathrm{E}-12$ \\
$\mathrm{SSE}$ & $4.9 \mathrm{E}-12$ & $4.6 \mathrm{E}-12$ & $4.5 \mathrm{E}-12$ & $4.4 \mathrm{E}-12$ & $4.4 \mathrm{E}-12$ & $4.2 \mathrm{E}-12$ \\
$\mathrm{SE}$ & $2.6 \mathrm{E}-12$ & $2.5 \mathrm{E}-12$ & $2.4 \mathrm{E}-12$ & $2.4 \mathrm{E}-12$ & $2.4 \mathrm{E}-12$ & $2.3 \mathrm{E}-12$ \\
$\mathrm{ESE}$ & $1.8 \mathrm{E}-12$ & $1.6 \mathrm{E}-12$ & $1.6 \mathrm{E}-12$ & $1.6 \mathrm{E}-12$ & $1.6 \mathrm{E}-12$ & $1.5 \mathrm{E}-12$ \\
$\mathrm{E}$ & $2.3 \mathrm{E}-12$ & $2.1 \mathrm{E}-12$ & $2.1 \mathrm{E}-12$ & $2.0 \mathrm{E}-12$ & $2.0 \mathrm{E}-12$ & $2.0 \mathrm{E}-12$ \\
$\mathrm{ENE}$ & $1.9 \mathrm{E}-12$ & $1.8 \mathrm{E}-12$ & $1.8 \mathrm{E}-12$ & $1.7 \mathrm{E}-12$ & $1.7 \mathrm{E}-12$ & $1.7 \mathrm{E}-12$ \\
$\mathrm{NE}$ & $2.9 \mathrm{E}-12$ & $2.7 \mathrm{E}-12$ & $2.7 \mathrm{E}-12$ & $2.6 \mathrm{E}-12$ & $2.6 \mathrm{E}-12$ & $2.5 \mathrm{E}-12$ \\
$\mathrm{NNE}$ & $1.9 \mathrm{E}-12$ & $1.7 \mathrm{E}-12$ & $1.7 \mathrm{E}-12$ & $1.7 \mathrm{E}-12$ & $1.7 \mathrm{E}-12$ & $1.6 \mathrm{E}-12$ \\
& & & & & & \\
\hline
\end{tabular}




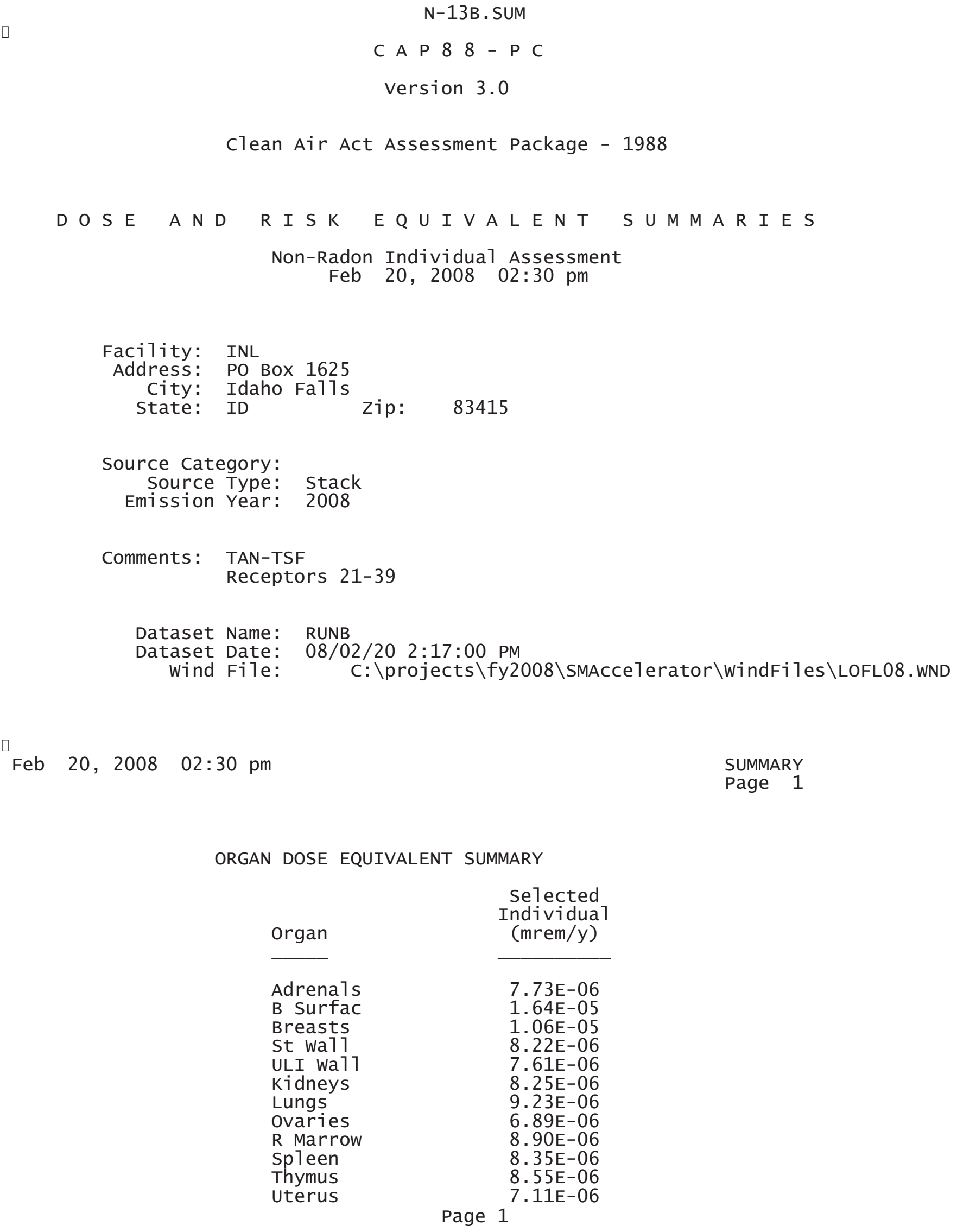




$\begin{array}{lr} & \mathrm{N}-13 \mathrm{~B} . \text { SUM } \\ \text { B1d wa11 } & 7.69 \mathrm{E}-06 \\ \text { Brain } & 9.83 \mathrm{E}-06 \\ \text { Esophagu } & 7.57 \mathrm{E}-06 \\ \text { SI wa11 } & 7.28 \mathrm{E}-06 \\ \text { LLI wa11 } & 7.42 \mathrm{E}-06 \\ \text { Liver } & 8.29 \mathrm{E}-06 \\ \text { Muscle } & 9.01 \mathrm{E}-06 \\ \text { Pancreas } & 7.24 \mathrm{E}-06 \\ \text { Skin } & 1.69 \mathrm{E}-05 \\ \text { Testes } & 9.28 \mathrm{E}-06 \\ \text { Thyroid } & 9.46 \mathrm{E}-06 \\ \text { EFFEC } & 8.88 \mathrm{E}-06\end{array}$

PATHWAY EFFECTIVE DOSE EQUIVALENT SUMMARY

Pathway $\begin{array}{r}\text { Individual } \\ (\mathrm{mrem} / \mathrm{y})\end{array}$

INGESTION $\quad 0.00 \mathrm{E}+00$

INHALATION $\quad 0.00 \mathrm{E}+00$

AIR IMMERSION $\quad 8.88 \mathrm{E}-06$

GROUND SURFACE $\quad 0.00 \mathrm{E}+00$

INTERNAL $\quad 0.00 \mathrm{E}+00$

EXTERNAL $\quad 8.88 \mathrm{E}-06$

TOTAL $\quad 8.88 \mathrm{E}-06$

$\square$

Feb 20, $2008 \quad 02: 30$ pm

SUMMARY

Page 2

NUCLIDE EFFECTIVE DOSE EQUIVALENT SUMMARY

Nuclide

$\mathrm{N}-13$

TOTAL

Feb 20, $2008 \quad 02: 30 \mathrm{pm}$
Selected Individua

(mrem/y)

8. $88 \mathrm{E}-06$

$8.88 \mathrm{E}-06$

SUMMARY

Page 3

CANCER RISK SUMMARY

Cancer

Esophagu
Selected Individual

Tota1 Lifetime

Fatal Cancer Risk

Page $2^{8.72 \mathrm{E}-14}$ 


$\begin{array}{lr} & \mathrm{N}-13 \mathrm{~B} . \mathrm{SUM} \\ \text { Stomach } & 3.32 \mathrm{E}-13 \\ \text { Colon } & 7.77 \mathrm{E}-13 \\ \text { Liver } & 1.26 \mathrm{E}-13 \\ \text { LUNG } & 9.01 \mathrm{E}-13 \\ \text { Bone } & 1.56 \mathrm{E}-14 \\ \text { Skin } & 1.68 \mathrm{E}-14 \\ \text { Breast } & 5.13 \mathrm{E}-13 \\ \text { Ovary } & 9.81 \mathrm{E}-14 \\ \text { B Tadder } & 1.86 \mathrm{E}-13 \\ \text { Kidneys } & 4.29 \mathrm{E}-14 \\ \text { Thyroid } & 3.01 \mathrm{E}-14 \\ \text { Leukemia } & 4.99 \mathrm{E}-13 \\ \text { Residua1 } & 1.19 \mathrm{E}-12 \\ \text { Tota1 } & 4.82 \mathrm{E}-12 \\ & \\ \text { TOTAL } & 9.63 \mathrm{E}-12\end{array}$

PATHWAY RISK SUMMARY

\begin{tabular}{lc} 
Pathway & $\begin{array}{c}\text { Selected Individual } \\
\text { Total Lifetime } \\
\text { Fatal Cancer Risk }\end{array}$ \\
\cline { 2 - 2 } & $0.00 \mathrm{E}+00$ \\
INGESTION & $0.00 \mathrm{E}+00$ \\
INHALATION & $4.82 \mathrm{E}-12$ \\
AIR IMMERSION & $0.00 \mathrm{E}+00$ \\
GROUND SURFACE & $0.00 \mathrm{E}+00$ \\
INTERNAL & $4.82 \mathrm{E}-12$ \\
EXTERNAL & $4.82 \mathrm{E}-12$ \\
TOTAL &
\end{tabular}

Feb 20, $2008 \quad 02: 30$ pm

SUMMARY Page 4

NUCLIDE RISK SUMMARY

Nuc7ide

$\mathrm{N}-13$

TOTAL

$\square$

Feb 20, 2008 02:30 pm
Selected Individual

Total Lifetime Fatal Cancer Risk

4. $82 \mathrm{E}-12$

$4.82 \mathrm{E}-12$

INDIVIDUAL EFFECTIVE DOSE EQUIVALENT RATE (mrem/y) (A11 Radionuclides and Pathways)

Distance $(m)$ 


\begin{tabular}{|c|c|c|c|c|c|c|c|}
\hline Direction & 17035 & 17329 & 18607 & $\begin{array}{c}N-13 B \\
18834\end{array}$ & M 18860 & 18890 & 19860 \\
\hline$N$ & $2.3 E-06$ & $2.2 \mathrm{E}-06$ & 2. $.0 \mathrm{E}-06$ & $2.0 \mathrm{E}-06$ & $2.0 \mathrm{E}-06$ & $2.0 \mathrm{E}-06$ & $1.9 \mathrm{E}-06$ \\
\hline NNW & $8.5 \mathrm{E}-07$ & $8.3 \mathrm{E}-07$ & $7.7 \mathrm{E}-07$ & $7.6 \mathrm{E}-07$ & $7.5 \mathrm{E}-07$ & $7.5 \mathrm{E}-07$ & 7. $1 \mathrm{E}-07$ \\
\hline NW & $1.2 \mathrm{E}-06$ & $1.2 \mathrm{E}-06$ & $1.1 \mathrm{E}-06$ & $1.1 \mathrm{E}-06$ & $1.1 \mathrm{E}-06$ & $1.1 \mathrm{E}-06$ & 1. $0 \mathrm{E}-06$ \\
\hline WNW & $1.6 \mathrm{E}-06$ & $1.6 \mathrm{E}-06$ & 1. $4 \mathrm{E}-06$ & $1.4 \mathrm{E}-06$ & $1.4 \mathrm{E}-06$ & $1.4 \mathrm{E}-06$ & 1. $3 \mathrm{E}-06$ \\
\hline W & $1.8 \mathrm{E}-06$ & $1.8 \mathrm{E}-06$ & $1.6 \mathrm{E}-06$ & $1.6 \mathrm{E}-06$ & $1.6 \mathrm{E}-06$ & $1.6 \mathrm{E}-06$ & $1.5 \mathrm{E}-06$ \\
\hline WSW & $2.4 \mathrm{E}-06$ & $2.3 \mathrm{E}-06$ & $2.2 \mathrm{E}-06$ & $2.1 \mathrm{E}-06$ & $2.1 \mathrm{E}-06$ & $2.1 \mathrm{E}-06$ & 2. $0 \mathrm{E}-06$ \\
\hline SW & $3.8 \mathrm{E}-06$ & $3.7 \mathrm{E}-06$ & $3.4 \mathrm{E}-06$ & $3.4 \mathrm{E}-06$ & $3.4 \mathrm{E}-06$ & $3.4 \mathrm{E}-06$ & $3.2 \mathrm{E}-06$ \\
\hline SSW & $6.4 \mathrm{E}-06$ & $6.2 \mathrm{E}-06$ & $5.7 \mathrm{E}-06$ & $5.6 \mathrm{E}-06$ & $5.6 \mathrm{E}-06$ & $5.6 \mathrm{E}-06$ & $5.3 \mathrm{E}-06$ \\
\hline $\mathrm{S}$ & $8.9 \mathrm{E}-06$ & $8.7 \mathrm{E}-06$ & $8.0 \mathrm{E}-06$ & $7.9 \mathrm{E}-06$ & $7.9 \mathrm{E}-06$ & $7.8 \mathrm{E}-06$ & $7.4 \mathrm{E}-06$ \\
\hline SSE & $7.4 \mathrm{E}-06$ & $7.3 \mathrm{E}-06$ & $6.7 \mathrm{E}-06$ & $6.6 \mathrm{E}-06$ & $6.6 \mathrm{E}-06$ & $6.6 \mathrm{E}-06$ & $6.2 \mathrm{E}-06$ \\
\hline SE & $4.0 \mathrm{E}-06$ & $3.9 \mathrm{E}-06$ & $3.6 \mathrm{E}-06$ & $3.6 \mathrm{E}-06$ & $3.6 \mathrm{E}-06$ & $3.6 \mathrm{E}-06$ & $3.4 \mathrm{E}-06$ \\
\hline ESE & $2.7 \mathrm{E}-06$ & $2.6 \mathrm{E}-06$ & $2.4 \mathrm{E}-06$ & $2.4 \mathrm{E}-06$ & $2.4 \mathrm{E}-06$ & $2.4 \mathrm{E}-06$ & 2. $2 \mathrm{E}-06$ \\
\hline $\mathrm{E}$ & $3.4 \mathrm{E}-06$ & $3.4 \mathrm{E}-06$ & $3.1 \mathrm{E}-06$ & $3.1 \mathrm{E}-06$ & $3.1 \mathrm{E}-06$ & $3.1 \mathrm{E}-06$ & $2.9 \mathrm{E}-06$ \\
\hline ENE & $2.9 \mathrm{E}-06$ & $2.9 \mathrm{E}-06$ & $2.7 \mathrm{E}-06$ & $2.6 \mathrm{E}-06$ & $2.6 \mathrm{E}-06$ & $2.6 \mathrm{E}-06$ & 2. $5 \mathrm{E}-06$ \\
\hline $\mathrm{NE}$ & 4. $3 \mathrm{E}-06$ & 4. $3 \mathrm{E}-06$ & $3.9 \mathrm{E}-06$ & $3.9 \mathrm{E}-06$ & $3.8 \mathrm{E}-06$ & $3.8 \mathrm{E}-06$ & $3.6 \mathrm{E}-06$ \\
\hline NNE & $2.8 \mathrm{E}-06$ & $2.8 \mathrm{E}-06$ & $2.5 \mathrm{E}-06$ & $2.5 \mathrm{E}-06$ & $2.5 \mathrm{E}-06$ & $2.5 \mathrm{E}-06$ & $2.4 \mathrm{E}-06$ \\
\hline
\end{tabular}

Distance (m)

\begin{tabular}{|c|c|c|c|c|c|c|c|}
\hline Direction & ค 19891 & 20457 & 21314 & 21349 & 22159 & 23521 & 24430 \\
\hline $\begin{array}{r}N \\
N N W \\
N W \\
W N W \\
W \\
W S W \\
\text { SW } \\
\text { SSW } \\
\text { S } \\
\text { SSE } \\
\text { SE } \\
\text { ESE } \\
\text { E } \\
\text { ENE } \\
\text { NE } \\
\text { NNE }\end{array}$ & $\begin{array}{l}1.9 \mathrm{E}-06 \\
7.1 \mathrm{E}-07 \\
1.0 \mathrm{E}-06 \\
1.3 \mathrm{E}-06 \\
1.5 \mathrm{E}-06 \\
2.0 \mathrm{E}-06 \\
3.2 \mathrm{E}-06 \\
5.3 \mathrm{E}-06 \\
7.4 \mathrm{E}-06 \\
6.2 \mathrm{E}-06 \\
3.4 \mathrm{E}-06 \\
2.2 \mathrm{E}-06 \\
2.9 \mathrm{E}-06 \\
2.5 \mathrm{E}-06 \\
3.6 \mathrm{E}-06 \\
2.3 \mathrm{E}-06\end{array}$ & $\begin{array}{l}1.8 \mathrm{E}-06 \\
6.9 \mathrm{E}-07 \\
9.6 \mathrm{E}-07 \\
1.3 \mathrm{E}-06 \\
1.5 \mathrm{E}-06 \\
1.9 \mathrm{E}-06 \\
3.1 \mathrm{E}-06 \\
5.1 \mathrm{E}-06 \\
7.1 \mathrm{E}-06 \\
6.0 \mathrm{E}-06 \\
3.2 \mathrm{E}-06 \\
2.2 \mathrm{E}-06 \\
2.8 \mathrm{E}-06 \\
2.4 \mathrm{E}-06 \\
3.5 \mathrm{E}-06 \\
2.3 \mathrm{E}-06\end{array}$ & $\begin{array}{l}1.8 \mathrm{E}-06 \\
6.6 \mathrm{E}-07 \\
9.2 \mathrm{E}-07 \\
1.2 \mathrm{E}-06 \\
1.4 \mathrm{E}-06 \\
1.8 \mathrm{E}-06 \\
2.9 \mathrm{E}-06 \\
4.9 \mathrm{E}-06 \\
6.8 \mathrm{E}-06 \\
5.7 \mathrm{E}-06 \\
3.1 \mathrm{E}-06 \\
2.1 \mathrm{E}-06 \\
2.7 \mathrm{E}-06 \\
2.3 \mathrm{E}-06 \\
3.3 \mathrm{E}-06 \\
2.2 \mathrm{E}-06\end{array}$ & $\begin{array}{l}1.8 \mathrm{E}-06 \\
6.6 \mathrm{E}-07 \\
9.2 \mathrm{E}-07 \\
1.2 \mathrm{E}-06 \\
1.4 \mathrm{E}-06 \\
1.8 \mathrm{E}-06 \\
2.9 \mathrm{E}-06 \\
4.9 \mathrm{E}-06 \\
6.8 \mathrm{E}-06 \\
5.7 \mathrm{E}-06 \\
3.1 \mathrm{E}-06 \\
2.1 \mathrm{E}-06 \\
2.7 \mathrm{E}-06 \\
2.3 \mathrm{E}-06 \\
3.3 \mathrm{E}-06 \\
2.2 \mathrm{E}-06\end{array}$ & $\begin{array}{l}1.7 \mathrm{E}-06 \\
6.3 \mathrm{E}-07 \\
8.8 \mathrm{E}-07 \\
1.2 \mathrm{E}-06 \\
1.3 \mathrm{E}-06 \\
1.8 \mathrm{E}-06 \\
2.8 \mathrm{E}-06 \\
4.7 \mathrm{E}-06 \\
6.5 \mathrm{E}-06 \\
5.4 \mathrm{E}-06 \\
3.0 \mathrm{E}-06 \\
2.0 \mathrm{E}-06 \\
2.6 \mathrm{E}-06 \\
2.2 \mathrm{E}-06 \\
3.2 \mathrm{E}-06 \\
2.1 \mathrm{E}-06\end{array}$ & $\begin{array}{l}1.6 \mathrm{E}-06 \\
5.9 \mathrm{E}-07 \\
8.3 \mathrm{E}-07 \\
1.1 \mathrm{E}-06 \\
1.3 \mathrm{E}-06 \\
1.7 \mathrm{E}-06 \\
2.6 \mathrm{E}-06 \\
4.4 \mathrm{E}-06 \\
6.0 \mathrm{E}-06 \\
5.1 \mathrm{E}-06 \\
2.8 \mathrm{E}-06 \\
1.9 \mathrm{E}-06 \\
2.4 \mathrm{E}-06 \\
2.0 \mathrm{E}-06 \\
3.0 \mathrm{E}-06 \\
1.9 \mathrm{E}-06\end{array}$ & $\begin{array}{l}1.5 \mathrm{E}-06 \\
5.7 \mathrm{E}-07 \\
7.9 \mathrm{E}-07 \\
1.1 \mathrm{E}-06 \\
1.2 \mathrm{E}-06 \\
1.6 \mathrm{E}-06 \\
2.5 \mathrm{E}-06 \\
4.2 \mathrm{E}-06 \\
5.8 \mathrm{E}-06 \\
4.9 \mathrm{E}-06 \\
2.7 \mathrm{E}-06 \\
1.8 \mathrm{E}-06 \\
2.3 \mathrm{E}-06 \\
1.9 \mathrm{E}-06 \\
2.8 \mathrm{E}-06 \\
1.9 \mathrm{E}-06\end{array}$ \\
\hline
\end{tabular}

$\square$

Feb 20, 2008 02:30 pm

SUMMARY

Page 6

INDIVIDUAL EFFECTIVE DOSE EQUIVALENT RATE (mrem/y) (A11 Radionuc1ides and Pathways)

\begin{tabular}{llllll}
\hline & \multicolumn{4}{c}{ Distance $(\mathrm{m})$} \\
\cline { 2 - 5 } Direction & 24545 & 25171 & 26794 & 27275 & 27389 \\
\hline
\end{tabular}

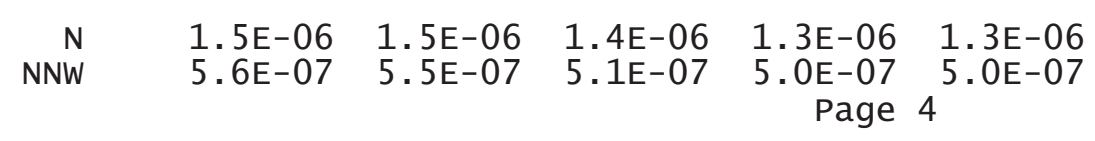




\begin{tabular}{rlllll} 
& & & & $\mathrm{N}-13 \mathrm{~B} . \mathrm{SUM}$ \\
$\mathrm{NW}$ & $7.9 \mathrm{E}-07$ & $7.7 \mathrm{E}-07$ & $7.2 \mathrm{E}-07$ & $7.0 \mathrm{E}-07$ & $7.0 \mathrm{E}-07$ \\
WNW & $1.1 \mathrm{E}-06$ & $1.0 \mathrm{E}-06$ & $9.6 \mathrm{E}-07$ & $9.4 \mathrm{E}-07$ & $9.4 \mathrm{E}-07$ \\
$\mathrm{~W}$ & $1.2 \mathrm{E}-06$ & $1.2 \mathrm{E}-06$ & $1.1 \mathrm{E}-06$ & $1.1 \mathrm{E}-06$ & $1.1 \mathrm{E}-06$ \\
WSW & $1.6 \mathrm{E}-06$ & $1.5 \mathrm{E}-06$ & $1.4 \mathrm{E}-06$ & $1.4 \mathrm{E}-06$ & $1.4 \mathrm{E}-06$ \\
$\mathrm{SW}$ & $2.5 \mathrm{E}-06$ & $2.4 \mathrm{E}-06$ & $2.3 \mathrm{E}-06$ & $2.2 \mathrm{E}-06$ & $2.2 \mathrm{E}-06$ \\
SSW & $4.1 \mathrm{E}-06$ & $4.0 \mathrm{E}-06$ & $3.7 \mathrm{E}-06$ & $3.7 \mathrm{E}-06$ & $3.6 \mathrm{E}-06$ \\
$\mathrm{~S}$ & $5.7 \mathrm{E}-06$ & $5.6 \mathrm{E}-06$ & $5.2 \mathrm{E}-06$ & $5.1 \mathrm{E}-06$ & $5.0 \mathrm{E}-06$ \\
SSE & $4.8 \mathrm{E}-06$ & $4.7 \mathrm{E}-06$ & $4.4 \mathrm{E}-06$ & $4.3 \mathrm{E}-06$ & $4.2 \mathrm{E}-06$ \\
SE & $2.6 \mathrm{E}-06$ & $2.6 \mathrm{E}-06$ & $2.4 \mathrm{E}-06$ & $2.3 \mathrm{E}-06$ & $2.3 \mathrm{E}-06$ \\
ESE & $1.8 \mathrm{E}-06$ & $1.7 \mathrm{E}-06$ & $1.6 \mathrm{E}-06$ & $1.6 \mathrm{E}-06$ & $1.6 \mathrm{E}-06$ \\
$\mathrm{E}$ & $2.3 \mathrm{E}-06$ & $2.2 \mathrm{E}-06$ & $2.1 \mathrm{E}-06$ & $2.0 \mathrm{E}-06$ & $2.0 \mathrm{E}-06$ \\
ENE & $1.9 \mathrm{E}-06$ & $1.9 \mathrm{E}-06$ & $1.7 \mathrm{E}-06$ & $1.7 \mathrm{E}-06$ & $1.7 \mathrm{E}-06$ \\
$\mathrm{NE}$ & $2.8 \mathrm{E}-06$ & $2.7 \mathrm{E}-06$ & $2.5 \mathrm{E}-06$ & $2.5 \mathrm{E}-06$ & $2.5 \mathrm{E}-06$ \\
NNE & $1.8 \mathrm{E}-06$ & $1.8 \mathrm{E}-06$ & $1.7 \mathrm{E}-06$ & $1.6 \mathrm{E}-06$ & $1.6 \mathrm{E}-06$ \\
& & & & & \\
\hline
\end{tabular}

Feb 20, $2008 \quad 02: 30 \mathrm{pm}$

SUMMARY

Page 7

INDIVIDUAL LIFETIME RISK (deaths)

(A11 Radionuclides and Pathways)

\begin{tabular}{|c|c|c|c|c|c|c|c|}
\hline \multicolumn{8}{|c|}{ Distance $(\mathrm{m})$} \\
\hline Direction & 17035 & 17329 & 18607 & 18834 & 18860 & 18890 & 19860 \\
\hline $\begin{array}{r}N \\
N N W \\
N W \\
W N W \\
W \\
W S W \\
\text { SW } \\
\text { SSW } \\
S \\
\text { SSE } \\
\text { SE } \\
\text { ESE } \\
\text { E } \\
\text { ENE } \\
\text { NE } \\
\text { NNE }\end{array}$ & $\begin{array}{l}1.2 \mathrm{E}-12 \\
4.6 \mathrm{E}-13 \\
6.4 \mathrm{E}-13 \\
8.6 \mathrm{E}-13 \\
9.8 \mathrm{E}-13 \\
1.3 \mathrm{E}-12 \\
2.1 \mathrm{E}-12 \\
3.4 \mathrm{E}-12 \\
4.8 \mathrm{E}-12 \\
4.0 \mathrm{E}-12 \\
2.2 \mathrm{E}-12 \\
1.4 \mathrm{E}-12 \\
1.9 \mathrm{E}-12 \\
1.6 \mathrm{E}-12 \\
2.4 \mathrm{E}-12 \\
1.5 \mathrm{E}-12\end{array}$ & $\begin{array}{l}1.2 \mathrm{E}-12 \\
4.5 \mathrm{E}-13 \\
6.3 \mathrm{E}-13 \\
8.4 \mathrm{E}-13 \\
9.6 \mathrm{E}-13 \\
1.3 \mathrm{E}-12 \\
2.0 \mathrm{E}-12 \\
3.4 \mathrm{E}-12 \\
4.7 \mathrm{E}-12 \\
4.0 \mathrm{E}-12 \\
2.1 \mathrm{E}-12 \\
1.4 \mathrm{E}-12 \\
1.8 \mathrm{E}-12 \\
1.6 \mathrm{E}-12 \\
2.3 \mathrm{E}-12 \\
1.5 \mathrm{E}-12\end{array}$ & $\begin{array}{l}1.1 \mathrm{E}-12 \\
4.2 \mathrm{E}-13 \\
5.8 \mathrm{E}-13 \\
7.8 \mathrm{E}-13 \\
8.8 \mathrm{E}-13 \\
1.2 \mathrm{E}-12 \\
1.9 \mathrm{E}-12 \\
3.1 \mathrm{E}-12 \\
4.3 \mathrm{E}-12 \\
3.6 \mathrm{E}-12 \\
2.0 \mathrm{E}-12 \\
1.3 \mathrm{E}-12 \\
1.7 \mathrm{E}-12 \\
1.4 \mathrm{E}-12 \\
2.1 \mathrm{E}-12 \\
1.4 \mathrm{E}-12\end{array}$ & $\begin{array}{l}1.1 \mathrm{E}-12 \\
4.1 \mathrm{E}-13 \\
5.7 \mathrm{E}-13 \\
7.7 \mathrm{E}-13 \\
8.7 \mathrm{E}-13 \\
1.2 \mathrm{E}-12 \\
1.8 \mathrm{E}-12 \\
3.1 \mathrm{E}-12 \\
4.3 \mathrm{E}-12 \\
3.6 \mathrm{E}-12 \\
1.9 \mathrm{E}-12 \\
1.3 \mathrm{E}-12 \\
1.7 \mathrm{E}-12 \\
1.4 \mathrm{E}-12 \\
2.1 \mathrm{E}-12 \\
1.4 \mathrm{E}-12\end{array}$ & $\begin{array}{l}1.1 \mathrm{E}-12 \\
4.1 \mathrm{E}-13 \\
5.7 \mathrm{E}-13 \\
7.7 \mathrm{E}-13 \\
8.7 \mathrm{E}-13 \\
1.2 \mathrm{E}-12 \\
1.8 \mathrm{E}-12 \\
3.1 \mathrm{E}-12 \\
4.3 \mathrm{E}-12 \\
3.6 \mathrm{E}-12 \\
1.9 \mathrm{E}-12 \\
1.3 \mathrm{E}-12 \\
1.7 \mathrm{E}-12 \\
1.4 \mathrm{E}-12 \\
2.1 \mathrm{E}-12 \\
1.4 \mathrm{E}-12\end{array}$ & $\begin{array}{l}1.1 \mathrm{E}-12 \\
4.1 \mathrm{E}-13 \\
5.7 \mathrm{E}-13 \\
7.7 \mathrm{E}-13 \\
8.7 \mathrm{E}-13 \\
1.2 \mathrm{E}-12 \\
1.8 \mathrm{E}-12 \\
3.1 \mathrm{E}-12 \\
4.3 \mathrm{E}-12 \\
3.6 \mathrm{E}-12 \\
1.9 \mathrm{E}-12 \\
1.3 \mathrm{E}-12 \\
1.7 \mathrm{E}-12 \\
1.4 \mathrm{E}-12 \\
2.1 \mathrm{E}-12 \\
1.4 \mathrm{E}-12\end{array}$ & $\begin{array}{l}1.0 \mathrm{E}-12 \\
3.9 \mathrm{E}-13 \\
5.4 \mathrm{E}-13 \\
7.2 \mathrm{E}-13 \\
8.2 \mathrm{E}-13 \\
1.1 \mathrm{E}-12 \\
1.7 \mathrm{E}-12 \\
2.9 \mathrm{E}-12 \\
4.0 \mathrm{E}-12 \\
3.4 \mathrm{E}-12 \\
1.8 \mathrm{E}-12 \\
1.2 \mathrm{E}-12 \\
1.6 \mathrm{E}-12 \\
1.3 \mathrm{E}-12 \\
2.0 \mathrm{E}-12 \\
1.3 \mathrm{E}-12\end{array}$ \\
\hline \multicolumn{8}{|c|}{ Distance $(\mathrm{m})$} \\
\hline Direction & 19891 & 20457 & 21314 & 21349 & 22159 & 23521 & 24430 \\
\hline $\begin{array}{r}N \\
N N W \\
N W \\
W N W \\
W \\
W S W \\
\text { SW } \\
\text { SSW }\end{array}$ & $\begin{array}{l}1.0 \mathrm{E}-12 \\
3.9 \mathrm{E}-13 \\
5.4 \mathrm{E}-13 \\
7.2 \mathrm{E}-13 \\
8.2 \mathrm{E}-13 \\
1.1 \mathrm{E}-12 \\
1.7 \mathrm{E}-12 \\
2.9 \mathrm{E}-12\end{array}$ & $\begin{array}{l}1.0 \mathrm{E}-12 \\
3.7 \mathrm{E}-13 \\
5.2 \mathrm{E}-13 \\
7.0 \mathrm{E}-13 \\
8.0 \mathrm{E}-13 \\
1.1 \mathrm{E}-12 \\
1.7 \mathrm{E}-12 \\
2.8 \mathrm{E}-12\end{array}$ & $\begin{array}{l}9.5 \mathrm{E}-13 \\
3.6 \mathrm{E}-13 \\
5.0 \mathrm{E}-13 \\
6.7 \mathrm{E}-13 \\
7.6 \mathrm{E}-13 \\
1.0 \mathrm{E}-12 \\
1.6 \mathrm{E}-12 \\
2.6 \mathrm{E}-12\end{array}$ & $\begin{array}{r}9.5 \mathrm{E}-13 \\
3.6 \mathrm{E}-13 \\
5.0 \mathrm{E}-13 \\
6.7 \mathrm{E}-13 \\
7.6 \mathrm{E}-13 \\
1.0 \mathrm{E}-12 \\
1.6 \mathrm{E}-12 \\
2.6 \mathrm{E}-12 \\
\text { Page }\end{array}$ & $\begin{array}{l}9.1 \mathrm{E}-13 \\
3.4 \mathrm{E}-13 \\
4.8 \mathrm{E}-13 \\
6.4 \mathrm{E}-13 \\
7.3 \mathrm{E}-13 \\
9.6 \mathrm{E}-13 \\
1.5 \mathrm{E}-12 \\
2.5 \mathrm{E}-12 \\
5\end{array}$ & $\begin{array}{l}8.5 \mathrm{E}-13 \\
3.2 \mathrm{E}-13 \\
4.5 \mathrm{E}-13 \\
6.0 \mathrm{E}-13 \\
6.8 \mathrm{E}-13 \\
9.0 \mathrm{E}-13 \\
1.4 \mathrm{E}-12 \\
2.4 \mathrm{E}-12\end{array}$ & $\begin{array}{l}8.2 \mathrm{E}-13 \\
3.1 \mathrm{E}-13 \\
4.3 \mathrm{E}-13 \\
5.8 \mathrm{E}-13 \\
6.5 \mathrm{E}-13 \\
8.6 \mathrm{E}-13 \\
1.4 \mathrm{E}-12 \\
2.3 \mathrm{E}-12\end{array}$ \\
\hline
\end{tabular}




\begin{tabular}{rlllllll} 
& \multicolumn{7}{c}{$\mathrm{N}-13 \mathrm{~B} \cdot \mathrm{SUM}$} \\
$\mathrm{S}$ & $4.0 \mathrm{E}-12$ & $3.9 \mathrm{E}-12$ & $3.7 \mathrm{E}-12$ & $3.7 \mathrm{E}-12$ & $3.5 \mathrm{E}-12$ & $3.3 \mathrm{E}-12$ & $3.1 \mathrm{E}-12$ \\
$\mathrm{SSE}$ & $3.4 \mathrm{E}-12$ & $3.2 \mathrm{E}-12$ & $3.1 \mathrm{E}-12$ & $3.1 \mathrm{E}-12$ & $3.0 \mathrm{E}-12$ & $2.8 \mathrm{E}-12$ & $2.6 \mathrm{E}-12$ \\
$\mathrm{SE}$ & $1.8 \mathrm{E}-12$ & $1.8 \mathrm{E}-12$ & $1.7 \mathrm{E}-12$ & $1.7 \mathrm{E}-12$ & $1.6 \mathrm{E}-12$ & $1.5 \mathrm{E}-12$ & $1.4 \mathrm{E}-12$ \\
$\mathrm{ESE}$ & $1.2 \mathrm{E}-12$ & $1.2 \mathrm{E}-12$ & $1.1 \mathrm{E}-12$ & $1.1 \mathrm{E}-12$ & $1.1 \mathrm{E}-12$ & $1.0 \mathrm{E}-12$ & $9.7 \mathrm{E}-13$ \\
$\mathrm{E}$ & $1.6 \mathrm{E}-12$ & $1.5 \mathrm{E}-12$ & $1.5 \mathrm{E}-12$ & $1.4 \mathrm{E}-12$ & $1.4 \mathrm{E}-12$ & $1.3 \mathrm{E}-12$ & $1.2 \mathrm{E}-12$ \\
$\mathrm{ENE}$ & $1.3 \mathrm{E}-12$ & $1.3 \mathrm{E}-12$ & $1.2 \mathrm{E}-12$ & $1.2 \mathrm{E}-12$ & $1.2 \mathrm{E}-12$ & $1.1 \mathrm{E}-12$ & $1.1 \mathrm{E}-12$ \\
$\mathrm{NE}$ & $2.0 \mathrm{E}-12$ & $1.9 \mathrm{E}-12$ & $1.8 \mathrm{E}-12$ & $1.8 \mathrm{E}-12$ & $1.7 \mathrm{E}-12$ & $1.6 \mathrm{E}-12$ & $1.5 \mathrm{E}-12$ \\
$\mathrm{NNE}$ & $1.3 \mathrm{E}-12$ & $1.2 \mathrm{E}-12$ & $1.2 \mathrm{E}-12$ & $1.2 \mathrm{E}-12$ & $1.1 \mathrm{E}-12$ & $1.1 \mathrm{E}-12$ & $1.0 \mathrm{E}-12$
\end{tabular}

Feb 20, $2008 \quad 02: 30 \mathrm{pm}$

SUMMARY

Page 8

INDIVIDUAL LIFETIME RISK (deaths)

(A11 Radionuclides and Pathways)

\begin{tabular}{|c|c|c|c|c|c|}
\hline \multirow[b]{2}{*}{ Direction } & \multicolumn{5}{|c|}{ Distance $(\mathrm{m})$} \\
\hline & 24545 & 25171 & 26794 & 27275 & 27389 \\
\hline $\begin{array}{r}N \\
N N W \\
N W \\
W N W \\
W \\
W S W \\
S W \\
S S W \\
S \\
S S E \\
S E \\
\text { ESE } \\
E \\
\text { ENE } \\
N E \\
N N E\end{array}$ & $\begin{array}{l}8.1 \mathrm{E}-13 \\
3.1 \mathrm{E}-13 \\
4.3 \mathrm{E}-13 \\
5.7 \mathrm{E}-13 \\
6.5 \mathrm{E}-13 \\
8.6 \mathrm{E}-13 \\
1.4 \mathrm{E}-12 \\
2.2 \mathrm{E}-12 \\
3.1 \mathrm{E}-12 \\
2.6 \mathrm{E}-12 \\
1.4 \mathrm{E}-12 \\
9.6 \mathrm{E}-13 \\
1.2 \mathrm{E}-12 \\
1.0 \mathrm{E}-12 \\
1.5 \mathrm{E}-12 \\
1.0 \mathrm{E}-12\end{array}$ & $\begin{array}{l}7 \cdot 9 \mathrm{E}-13 \\
3 \cdot 0 \mathrm{E}-13 \\
4 \cdot 2 \mathrm{E}-13 \\
5.6 \mathrm{E}-13 \\
6.3 \mathrm{E}-13 \\
8.3 \mathrm{E}-13 \\
1.3 \mathrm{E}-12 \\
2.2 \mathrm{E}-12 \\
3.0 \mathrm{E}-12 \\
2.5 \mathrm{E}-12 \\
1 \cdot 4 \mathrm{E}-12 \\
9 \cdot 3 \mathrm{E}-13 \\
1.2 \mathrm{E}-12 \\
1.0 \mathrm{E}-12 \\
1.5 \mathrm{E}-12 \\
9 \cdot 7 \mathrm{E}-13\end{array}$ & $\begin{array}{l}7.4 \mathrm{E}-13 \\
2.8 \mathrm{E}-13 \\
3.9 \mathrm{E}-13 \\
5.2 \mathrm{E}-13 \\
5.9 \mathrm{E}-13 \\
7.8 \mathrm{E}-13 \\
1.2 \mathrm{E}-12 \\
2.0 \mathrm{E}-12 \\
2.8 \mathrm{E}-12 \\
2.4 \mathrm{E}-12 \\
1.3 \mathrm{E}-12 \\
8.7 \mathrm{E}-13 \\
1.1 \mathrm{E}-12 \\
9.5 \mathrm{E}-13 \\
1.4 \mathrm{E}-12 \\
9.1 \mathrm{E}-13\end{array}$ & $\begin{array}{l}7.2 \mathrm{E}-13 \\
2.7 \mathrm{E}-13 \\
3.8 \mathrm{E}-13 \\
5.1 \mathrm{E}-13 \\
5.8 \mathrm{E}-13 \\
7.6 \mathrm{E}-13 \\
1.2 \mathrm{E}-12 \\
2.0 \mathrm{E}-12 \\
2.7 \mathrm{E}-12 \\
2.3 \mathrm{E}-12 \\
1.3 \mathrm{E}-12 \\
8.5 \mathrm{E}-13 \\
1.1 \mathrm{E}-12 \\
9.3 \mathrm{E}-13 \\
1.3 \mathrm{E}-12 \\
8.9 \mathrm{E}-13\end{array}$ & $\begin{array}{l}7.2 \mathrm{E}-13 \\
2.7 \mathrm{E}-13 \\
3.8 \mathrm{E}-13 \\
5.1 \mathrm{E}-13 \\
5.8 \mathrm{E}-13 \\
7.6 \mathrm{E}-13 \\
1.2 \mathrm{E}-12 \\
2.0 \mathrm{E}-12 \\
2.7 \mathrm{E}-12 \\
2.3 \mathrm{E}-12 \\
1.3 \mathrm{E}-12 \\
8.5 \mathrm{E}-13 \\
1.1 \mathrm{E}-12 \\
9.2 \mathrm{E}-13 \\
1.3 \mathrm{E}-12 \\
8.8 \mathrm{E}-13\end{array}$ \\
\hline
\end{tabular}




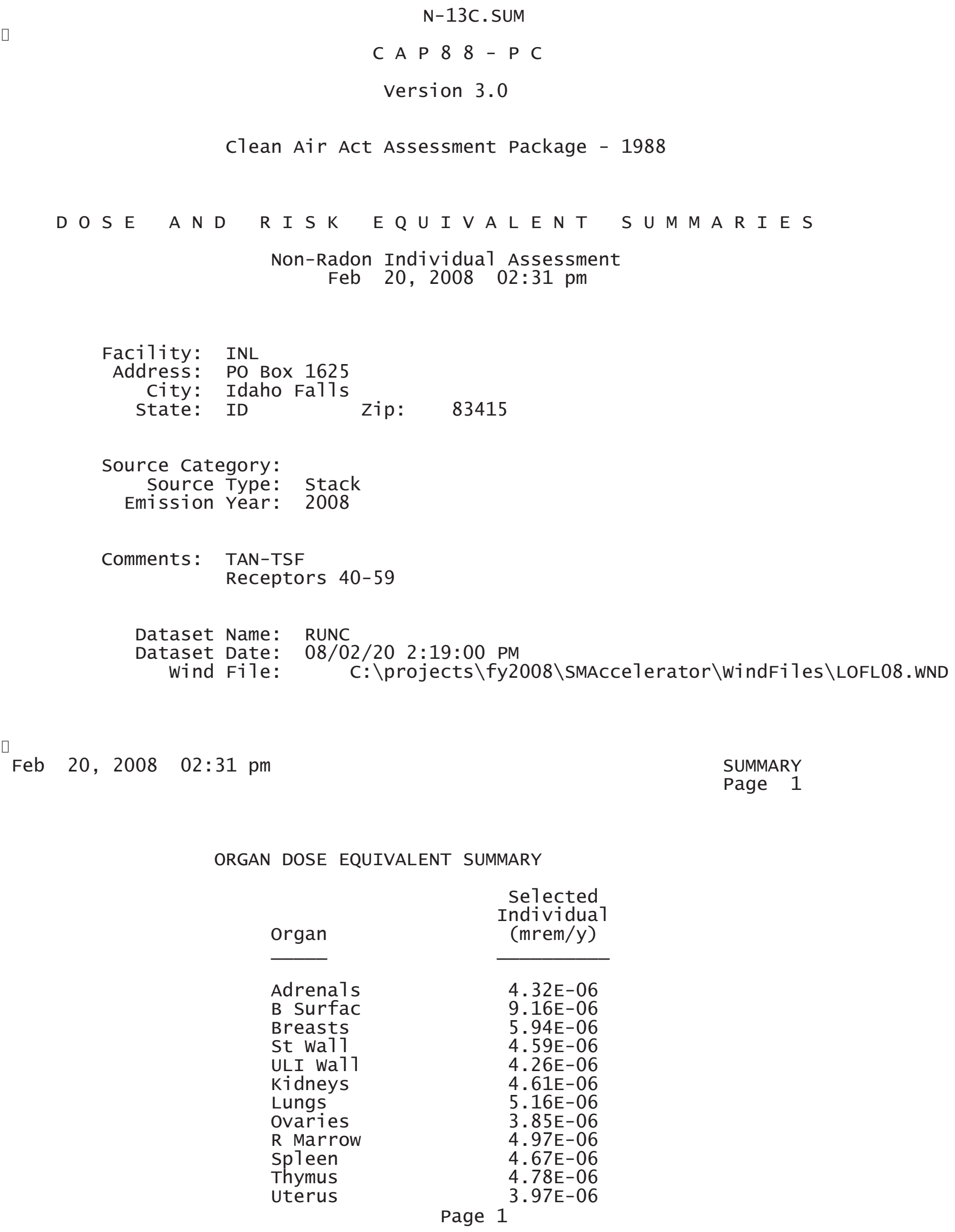




$\begin{array}{lr} & \mathrm{N}-13 \mathrm{C} . \text { SUM } \\ \text { B1d wa11 } & 4.30 \mathrm{E}-06 \\ \text { Brain } & 5.49 \mathrm{E}-06 \\ \text { Esophagu } & 4.23 \mathrm{E}-06 \\ \text { SI wa11 } & 4.07 \mathrm{E}-06 \\ \text { LLI wa11 } & 4.15 \mathrm{E}-06 \\ \text { Liver } & 4.64 \mathrm{E}-06 \\ \text { Muscle } & 5.04 \mathrm{E}-06 \\ \text { Pancreas } & 4.05 \mathrm{E}-06 \\ \text { Skin } & 9.43 \mathrm{E}-06 \\ \text { Testes } & 5.19 \mathrm{E}-06 \\ \text { Thyroid } & 5.29 \mathrm{E}-06 \\ & \\ \text { EFFEC } & 4.96 \mathrm{E}-06\end{array}$

PATHWAY EFFECTIVE DOSE EQUIVALENT SUMMARY

\begin{tabular}{lcc} 
Pathway & $\begin{array}{c}\text { selected } \\
\text { Individual } \\
\text { (mrem/y) }\end{array}$ \\
\cline { 2 - 3 } INGESTION & $0.00 \mathrm{E}+00$ & \\
INHALATION & $0.00 \mathrm{E}+00$ \\
AIR IMMERSION & $4.96 \mathrm{E}-06$ & \\
GROUND SURFACE & $0.00 \mathrm{E}+00$ & \\
INTERNAL & $0.00 \mathrm{E}+00$ & \\
EXTERNAL & $4.96 \mathrm{E}-06$ & \\
TOTAL & $4.96 \mathrm{E}-06$ & SUMMARY \\
Page 2
\end{tabular}

NUCLIDE EFFECTIVE DOSE EQUIVALENT SUMMARY

Nuclide

$\mathrm{N}-13$

TOTAL

Feb 20, $2008 \quad 02: 31$ pm

$$
\begin{gathered}
\begin{array}{c}
\text { Selected } \\
\text { Individual } \\
\text { (mrem/y) }
\end{array} \\
\hline 4.96 \mathrm{E}-06 \\
4.96 \mathrm{E}-06
\end{gathered}
$$

\section{CANCER RISK SUMMARY}

\section{Cancer}

Esophagu
Selected Individual

Total Lifetime Fatal Cancer Risk 


$\begin{array}{lr} & \mathrm{N}-13 \mathrm{C} . \mathrm{SUM} \\ \text { Stomach } & 1.86 \mathrm{E}-13 \\ \text { Colon } & 4.34 \mathrm{E}-13 \\ \text { Liver } & 7.03 \mathrm{E}-14 \\ \text { LUNG } & 5.04 \mathrm{E}-13 \\ \text { Bone } & 8.70 \mathrm{E}-15 \\ \text { Skin } & 9.40 \mathrm{E}-15 \\ \text { Breast } & 2.87 \mathrm{E}-13 \\ \text { Ovary } & 5.48 \mathrm{E}-14 \\ \text { B1adder } & 1.04 \mathrm{E}-13 \\ \text { Kidneys } & 2.40 \mathrm{E}-14 \\ \text { Thyroid } & 1.68 \mathrm{E}-14 \\ \text { Leukemia } & 2.79 \mathrm{E}-13 \\ \text { Residua1 } & 6.66 \mathrm{E}-13 \\ \text { Tota1 } & 2.69 \mathrm{E}-12 \\ & \\ \text { TOTAL } & 5.39 \mathrm{E}-12\end{array}$

PATHWAY RISK SUMMARY

\begin{tabular}{lc} 
& $\begin{array}{c}\text { Selected Individual } \\
\text { Total Lifetime } \\
\text { Fatal cancer Risk }\end{array}$ \\
\cline { 2 - 2 } Pathway & $0.00 \mathrm{E}+00$ \\
INGESTION & $0.00 \mathrm{E}+00$ \\
INHALATION & $2.69 \mathrm{E}-12$ \\
AIR IMMERSION & $0.00 \mathrm{E}+00$ \\
GROUND SURFACE & $0.00 \mathrm{E}+00$ \\
INTERNAL & $2.69 \mathrm{E}-12$ \\
EXTERNAL & $2.69 \mathrm{E}-12$ \\
TOTAL &
\end{tabular}

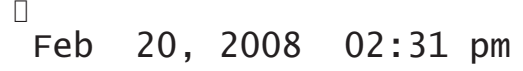

NUCLIDE RISK SUMMARY

Nuclide

$\mathrm{N}-13$

TOTAL

口

Feb 20, 2008 02:31 pm
Selected Individual

Total Lifetime Fatal Cancer Risk

2. $69 \mathrm{E}-12$

2. $69 \mathrm{E}-12$

INDIVIDUAL EFFECTIVE DOSE EQUIVALENT RATE (mrem/y) (A11 Radionuclides and Pathways)

Distance (m) 


\begin{tabular}{|c|c|c|c|c|c|c|c|}
\hline Direction & 27715 & 28919 & 31060 & $\begin{array}{c}N-13 C \\
32802\end{array}$ & M 34577 & 35279 & 35683 \\
\hline $\mathrm{N}$ & 1. $3 E-06$ & $1.2 \mathrm{E}-06$ & $1.2 \mathrm{E}-06$ & $1.1 \mathrm{E}-06$ & $1.0 \mathrm{E}-06$ & 1.0E-06 & $9.9 \mathrm{E}-07$ \\
\hline NNW & $4.9 \mathrm{E}-07$ & $4.7 \mathrm{E}-07$ & $4.3 \mathrm{E}-07$ & $4.1 \mathrm{E}-07$ & $3.8 \mathrm{E}-07$ & $3.8 \mathrm{E}-07$ & $3.7 \mathrm{E}-07$ \\
\hline NW & $6.9 \mathrm{E}-07$ & $6.6 \mathrm{E}-07$ & $6.1 \mathrm{E}-07$ & $5.7 \mathrm{E}-07$ & $5.4 \mathrm{E}-07$ & $5.3 \mathrm{E}-07$ & $5.2 \mathrm{E}-07$ \\
\hline WNW & $9.2 \mathrm{E}-07$ & $8.8 \mathrm{E}-07$ & $8.1 \mathrm{E}-07$ & $7.7 \mathrm{E}-07$ & $7.2 \mathrm{E}-07$ & $7.1 \mathrm{E}-07$ & 7.0E-07 \\
\hline W & $1.0 \mathrm{E}-06$ & $1.0 \mathrm{E}-06$ & $9.2 \mathrm{E}-07$ & $8.7 \mathrm{E}-07$ & $8.2 \mathrm{E}-07$ & $8.0 \mathrm{E}-07$ & 7. $9 \mathrm{E}-07$ \\
\hline WSW & $1.4 \mathrm{E}-06$ & 1. $3 \mathrm{E}-06$ & $1.2 \mathrm{E}-06$ & $1.1 \mathrm{E}-06$ & $1.1 \mathrm{E}-06$ & $1.0 \mathrm{E}-06$ & 1. $0 \mathrm{E}-06$ \\
\hline SW & $2.2 \mathrm{E}-06$ & $2.1 \mathrm{E}-06$ & $1.9 \mathrm{E}-06$ & $1.8 \mathrm{E}-06$ & $1.7 \mathrm{E}-06$ & $1.6 \mathrm{E}-06$ & 1. $6 \mathrm{E}-06$ \\
\hline SSW & $3.6 \mathrm{E}-06$ & $3.4 \mathrm{E}-06$ & $3.1 \mathrm{E}-06$ & $2.9 \mathrm{E}-06$ & $2.8 \mathrm{E}-06$ & $2.7 \mathrm{E}-06$ & $2.7 \mathrm{E}-06$ \\
\hline $\mathrm{S}$ & $5.0 \mathrm{E}-06$ & $4.7 \mathrm{E}-06$ & $4.3 \mathrm{E}-06$ & $4.1 \mathrm{E}-06$ & $3.8 \mathrm{E}-06$ & $3.7 \mathrm{E}-06$ & $3.7 \mathrm{E}-06$ \\
\hline SSE & $4.2 \mathrm{E}-06$ & $4.0 \mathrm{E}-06$ & $3.7 \mathrm{E}-06$ & $3.4 \mathrm{E}-06$ & $3.2 \mathrm{E}-06$ & $3.2 \mathrm{E}-06$ & $3.1 \mathrm{E}-06$ \\
\hline SE & $2.3 \mathrm{E}-06$ & $2.2 \mathrm{E}-06$ & 2.0E-06 & $1.9 \mathrm{E}-06$ & $1.8 \mathrm{E}-06$ & $1.7 \mathrm{E}-06$ & $1.7 \mathrm{E}-06$ \\
\hline ESE & $1.5 \mathrm{E}-06$ & 1. $5 \mathrm{E}-06$ & $1.4 \mathrm{E}-06$ & $1.3 \mathrm{E}-06$ & $1.2 \mathrm{E}-06$ & $1.2 \mathrm{E}-06$ & $1.2 \mathrm{E}-06$ \\
\hline E & $2.0 \mathrm{E}-06$ & $1.9 \mathrm{E}-06$ & $1.8 \mathrm{E}-06$ & $1.6 \mathrm{E}-06$ & $1.6 \mathrm{E}-06$ & 1. $5 \mathrm{E}-06$ & 1. $5 \mathrm{E}-06$ \\
\hline ENE & $1.7 \mathrm{E}-06$ & $1.6 \mathrm{E}-06$ & $1.5 \mathrm{E}-06$ & $1.4 \mathrm{E}-06$ & 1.3E-06 & 1. $3 \mathrm{E}-06$ & 1. $3 \mathrm{E}-06$ \\
\hline $\mathrm{NE}$ & $2.4 \mathrm{E}-06$ & $2.3 \mathrm{E}-06$ & $2.1 \mathrm{E}-06$ & $2.0 \mathrm{E}-06$ & $1.9 \mathrm{E}-06$ & $1.8 \mathrm{E}-06$ & $1.8 \mathrm{E}-06$ \\
\hline NNE & $1.6 \mathrm{E}-06$ & $1.5 \mathrm{E}-06$ & 1. $4 \mathrm{E}-06$ & $1.3 \mathrm{E}-06$ & $1.3 \mathrm{E}-06$ & $1.2 \mathrm{E}-06$ & $1.2 \mathrm{E}-06$ \\
\hline
\end{tabular}

Distance (m)

\begin{tabular}{|c|c|c|c|c|c|c|c|}
\hline Direction & 36721 & 36809 & 37729 & 39079 & 39220 & 39559 & 43584 \\
\hline $\mathrm{N}$ & $9.6 \mathrm{E}-07$ & $9.5 \mathrm{E}-07$ & $9.3 \mathrm{E}-07$ & $8.9 \mathrm{E}-07$ & $8.9 \mathrm{E}-07$ & $8.8 \mathrm{E}-07$ & $7.9 \mathrm{E}-07$ \\
\hline NNW & $3.6 \mathrm{E}-07$ & $3.6 \mathrm{E}-07$ & $3.5 \mathrm{E}-07$ & $3.4 \mathrm{E}-07$ & $3.3 \mathrm{E}-07$ & $3.3 \mathrm{E}-07$ & $3.0 \mathrm{E}-07$ \\
\hline NW & $5.1 \mathrm{E}-07$ & $5.0 \mathrm{E}-07$ & $4.9 \mathrm{E}-07$ & $4.7 \mathrm{E}-07$ & $4.7 \mathrm{E}-07$ & $4.7 \mathrm{E}-07$ & $4.2 \mathrm{E}-07$ \\
\hline WNW & $6.8 \mathrm{E}-07$ & $6.8 \mathrm{E}-07$ & $6.6 \mathrm{E}-07$ & $6.3 \mathrm{E}-07$ & $6.3 \mathrm{E}-07$ & $6.2 \mathrm{E}-07$ & $5.6 \mathrm{E}-07$ \\
\hline W & $7.7 \mathrm{E}-07$ & 7. $6 \mathrm{E}-07$ & $7.4 \mathrm{E}-07$ & 7. $2 \mathrm{E}-07$ & $7.1 \mathrm{E}-07$ & $7.1 \mathrm{E}-07$ & $6.3 \mathrm{E}-07$ \\
\hline WSW & $1.0 \mathrm{E}-06$ & 1. $0 \mathrm{E}-06$ & $9.7 \mathrm{E}-07$ & $9.4 \mathrm{E}-07$ & $9.3 \mathrm{E}-07$ & $9.2 \mathrm{E}-07$ & 8. $3 \mathrm{E}-07$ \\
\hline SW & $1.6 \mathrm{E}-06$ & $1.6 \mathrm{E}-06$ & $1.5 \mathrm{E}-06$ & $1.5 \mathrm{E}-06$ & $1.5 \mathrm{E}-06$ & $1.4 \mathrm{E}-06$ & 1. $3 \mathrm{E}-06$ \\
\hline SSW & $2.6 \mathrm{E}-06$ & $2.6 \mathrm{E}-06$ & $2.5 \mathrm{E}-06$ & $2.4 \mathrm{E}-06$ & $2.4 \mathrm{E}-06$ & $2.4 \mathrm{E}-06$ & $2.1 \mathrm{E}-06$ \\
\hline & $3.6 \mathrm{E}-06$ & $3.5 \mathrm{E}-06$ & $3.4 \mathrm{E}-06$ & $3.3 \mathrm{E}-06$ & $3.3 \mathrm{E}-06$ & $3.3 \mathrm{E}-06$ & $2.9 \mathrm{E}-06$ \\
\hline SSE & $3.0 \mathrm{E}-06$ & $3.0 \mathrm{E}-06$ & $2.9 \mathrm{E}-06$ & $2.8 \mathrm{E}-06$ & $2.8 \mathrm{E}-06$ & $2.8 \mathrm{E}-06$ & 2. $5 \mathrm{E}-06$ \\
\hline $\mathrm{SE}$ & $1.7 \mathrm{E}-06$ & $1.7 \mathrm{E}-06$ & $1.6 \mathrm{E}-06$ & $1.6 \mathrm{E}-06$ & $1.6 \mathrm{E}-06$ & $1.5 \mathrm{E}-06$ & $1.4 \mathrm{E}-06$ \\
\hline ESE & 1.1E-06 & 1.1E-06 & 1.1E-06 & $1.1 \mathrm{E}-06$ & 1.0E-06 & $1.0 \mathrm{E}-06$ & $9.3 \mathrm{E}-07$ \\
\hline F & $1.5 \mathrm{E}-06$ & $1.4 \mathrm{E}-06$ & $1.4 \mathrm{E}-06$ & $1.4 \mathrm{E}-06$ & $1.3 \mathrm{E}-06$ & $1.3 \mathrm{E}-06$ & $1.2 \mathrm{E}-06$ \\
\hline ENE & $1.2 \mathrm{E}-06$ & $1.2 \mathrm{E}-06$ & $1.2 \mathrm{E}-06$ & $1.1 \mathrm{E}-06$ & $1.1 \mathrm{E}-06$ & $1.1 \mathrm{E}-06$ & 1. $0 \mathrm{E}-06$ \\
\hline $\mathrm{NE}$ & $1.8 \mathrm{E}-06$ & $1.7 \mathrm{E}-06$ & $1.7 \mathrm{E}-06$ & $1.6 \mathrm{E}-06$ & $1.6 \mathrm{E}-06$ & $1.6 \mathrm{E}-06$ & $1.4 \mathrm{E}-06$ \\
\hline NNE & $1.2 \mathrm{E}-06$ & $1.2 \mathrm{E}-06$ & $1.1 \mathrm{E}-06$ & $1.1 \mathrm{E}-06$ & $1.1 \mathrm{E}-06$ & $1.1 \mathrm{E}-06$ & $9.6 \mathrm{E}-07$ \\
\hline
\end{tabular}

$\square$

Feb 20, 2008 02:31 pm

SUMMARY

Page 6

INDIVIDUAL EFFECTIVE DOSE EQUIVALENT RATE (mrem/y) (A11 Radionuc1ides and Pathways)

\begin{tabular}{|c|c|c|c|c|c|c|}
\hline \multirow[b]{2}{*}{ Direction } & \multicolumn{6}{|c|}{ Distance (m) } \\
\hline & 45196 & 45275 & 45654 & 45677 & 46668 & 47969 \\
\hline
\end{tabular}

\begin{tabular}{|c|c|c|c|c|c|c|}
\hline $\begin{array}{r}N \\
N N W\end{array}$ & $\begin{array}{l}7.6 \mathrm{E}-07 \\
2.9 \mathrm{E}-07\end{array}$ & $\begin{array}{l}7.6 \mathrm{E}-07 \\
2.8 \mathrm{E}-07\end{array}$ & $\begin{array}{l}7.5 \mathrm{E}-07 \\
2.8 \mathrm{E}-07\end{array}$ & $\begin{array}{r}7.5 \mathrm{E}-07 \\
2.8 \mathrm{E}-07 \\
\text { Page }\end{array}$ & $\begin{array}{r}7.3 \mathrm{E}-07 \\
2.8 \mathrm{E}-07\end{array}$ & $\begin{array}{l}7.1 \mathrm{E}-07 \\
2.7 \mathrm{E}-07\end{array}$ \\
\hline
\end{tabular}




$\begin{array}{rllllll} & & & \mathrm{N}-13 \mathrm{C} \cdot \mathrm{SUM} \\ \mathrm{NW} & 4.0 \mathrm{E}-07 & 4.0 \mathrm{E}-07 & 4.0 \mathrm{E}-07 & 4.0 \mathrm{E}-07 & 3.9 \mathrm{E}-07 & 3.8 \mathrm{E}-07 \\ \text { WNW } & 5.4 \mathrm{E}-07 & 5.4 \mathrm{E}-07 & 5.3 \mathrm{E}-07 & 5.3 \mathrm{E}-07 & 5.2 \mathrm{E}-07 & 5.0 \mathrm{E}-07 \\ \mathrm{~W} & 6.1 \mathrm{E}-07 & 6.1 \mathrm{E}-07 & 6.0 \mathrm{E}-07 & 6.0 \mathrm{E}-07 & 5.9 \mathrm{E}-07 & 5.7 \mathrm{E}-07 \\ \text { WSW } & 7.9 \mathrm{E}-07 & 7.9 \mathrm{E}-07 & 7.9 \mathrm{E}-07 & 7.9 \mathrm{E}-07 & 7.7 \mathrm{E}-07 & 7.4 \mathrm{E}-07 \\ \mathrm{SW} & 1.2 \mathrm{E}-06 & 1.2 \mathrm{E}-06 & 1.2 \mathrm{E}-06 & 1.2 \mathrm{E}-06 & 1.2 \mathrm{E}-06 & 1.2 \mathrm{E}-06 \\ \mathrm{SSW} & 2.0 \mathrm{E}-06 & 2.0 \mathrm{E}-06 & 2.0 \mathrm{E}-06 & 2.0 \mathrm{E}-06 & 2.0 \mathrm{E}-06 & 1.9 \mathrm{E}-06 \\ \mathrm{~S} & 2.8 \mathrm{E}-06 & 2.8 \mathrm{E}-06 & 2.8 \mathrm{E}-06 & 2.8 \mathrm{E}-06 & 2.7 \mathrm{E}-06 & 2.6 \mathrm{E}-06 \\ \mathrm{SSE} & 2.4 \mathrm{E}-06 & 2.4 \mathrm{E}-06 & 2.3 \mathrm{E}-06 & 2.3 \mathrm{E}-06 & 2.3 \mathrm{E}-06 & 2.2 \mathrm{E}-06 \\ \mathrm{SE} & 1.3 \mathrm{E}-06 & 1.3 \mathrm{E}-06 & 1.3 \mathrm{E}-06 & 1.3 \mathrm{E}-06 & 1.3 \mathrm{E}-06 & 1.2 \mathrm{E}-06 \\ \mathrm{ESE} & 8.9 \mathrm{E}-07 & 8.9 \mathrm{E}-07 & 8.8 \mathrm{E}-07 & 8.8 \mathrm{E}-07 & 8.6 \mathrm{E}-07 & 8.4 \mathrm{E}-07 \\ \mathrm{E} & 1.1 \mathrm{E}-06 & 1.1 \mathrm{E}-06 & 1.1 \mathrm{E}-06 & 1.1 \mathrm{E}-06 & 1.1 \mathrm{E}-06 & 1.1 \mathrm{E}-06 \\ \mathrm{ENE} & 9.6 \mathrm{E}-07 & 9.6 \mathrm{E}-07 & 9.5 \mathrm{E}-07 & 9.5 \mathrm{E}-07 & 9.3 \mathrm{E}-07 & 9.0 \mathrm{E}-07 \\ \mathrm{NE} & 1.4 \mathrm{E}-06 & 1.4 \mathrm{E}-06 & 1.4 \mathrm{E}-06 & 1.4 \mathrm{E}-06 & 1.3 \mathrm{E}-06 & 1.3 \mathrm{E}-06 \\ \mathrm{NNE} & 9.2 \mathrm{E}-07 & 9.2 \mathrm{E}-07 & 9.1 \mathrm{E}-07 & 9.1 \mathrm{E}-07 & 8.9 \mathrm{E}-07 & 8.6 \mathrm{E}-07\end{array}$

Feb 20, $2008 \quad 02: 31 \mathrm{pm}$

SUMMARY

Page 7

INDIVIDUAL LIFETIME RISK (deaths)

(A11 Radionuclides and Pathways)

\begin{tabular}{|c|c|c|c|c|c|c|c|}
\hline \multicolumn{8}{|c|}{ Distance $(\mathrm{m})$} \\
\hline Direction & 27715 & 28919 & 31060 & 32802 & 34577 & 35279 & 35683 \\
\hline $\begin{array}{r}\text { N } \\
\text { NNW } \\
N W \\
\text { WNW } \\
W \\
\text { WSW } \\
\text { SW } \\
\text { SSW } \\
S \\
\text { SSE } \\
\text { SE } \\
\text { ESE } \\
\text { E } \\
\text { ENE } \\
N E \\
\text { NNE }\end{array}$ & $\begin{array}{l}7.1 \mathrm{E}-13 \\
2.7 \mathrm{E}-13 \\
3.7 \mathrm{E}-13 \\
5.0 \mathrm{E}-13 \\
5.7 \mathrm{E}-13 \\
7.5 \mathrm{E}-13 \\
1.2 \mathrm{E}-12 \\
1.9 \mathrm{E}-12 \\
2.7 \mathrm{E}-12 \\
2.3 \mathrm{E}-12 \\
1.2 \mathrm{E}-12 \\
8.4 \mathrm{E}-13 \\
1.1 \mathrm{E}-12 \\
9.1 \mathrm{E}-13 \\
1.3 \mathrm{E}-12 \\
8.7 \mathrm{E}-13\end{array}$ & $\begin{array}{l}6.8 \mathrm{E}-13 \\
2.5 \mathrm{E}-13 \\
3.6 \mathrm{E}-13 \\
4.8 \mathrm{E}-13 \\
5.4 \mathrm{E}-13 \\
7.1 \mathrm{E}-13 \\
1.1 \mathrm{E}-12 \\
1.9 \mathrm{E}-12 \\
2.6 \mathrm{E}-12 \\
2.2 \mathrm{E}-12 \\
1.2 \mathrm{E}-12 \\
8.0 \mathrm{E}-13 \\
1.0 \mathrm{E}-12 \\
8.7 \mathrm{E}-13 \\
1.3 \mathrm{E}-12 \\
8.3 \mathrm{E}-13\end{array}$ & $\begin{array}{l}6.2 \mathrm{E}-13 \\
2.3 \mathrm{E}-13 \\
3.3 \mathrm{E}-13 \\
4.4 \mathrm{E}-13 \\
5.0 \mathrm{E}-13 \\
6.6 \mathrm{E}-13 \\
1.0 \mathrm{E}-12 \\
1.7 \mathrm{E}-12 \\
2.4 \mathrm{E}-12 \\
2.0 \mathrm{E}-12 \\
1.1 \mathrm{E}-12 \\
7.4 \mathrm{E}-13 \\
9.5 \mathrm{E}-13 \\
8.0 \mathrm{E}-13 \\
1.2 \mathrm{E}-12 \\
7.7 \mathrm{E}-13\end{array}$ & $\begin{array}{l}5.9 \mathrm{E}-13 \\
2.2 \mathrm{E}-13 \\
3.1 \mathrm{E}-13 \\
4.2 \mathrm{E}-13 \\
4.7 \mathrm{E}-13 \\
6.2 \mathrm{E}-13 \\
9.7 \mathrm{E}-13 \\
1.6 \mathrm{E}-12 \\
2.2 \mathrm{E}-12 \\
1.9 \mathrm{E}-12 \\
1.0 \mathrm{E}-12 \\
6.9 \mathrm{E}-13 \\
8.9 \mathrm{E}-13 \\
7.5 \mathrm{E}-13 \\
1.1 \mathrm{E}-12 \\
7.2 \mathrm{E}-13\end{array}$ & $\begin{array}{l}5.5 \mathrm{E}-13 \\
2.1 \mathrm{E}-13 \\
2.9 \mathrm{E}-13 \\
3.9 \mathrm{E}-13 \\
4.4 \mathrm{E}-13 \\
5.8 \mathrm{E}-13 \\
9.1 \mathrm{E}-13 \\
1.5 \mathrm{E}-12 \\
2.1 \mathrm{E}-12 \\
1.8 \mathrm{E}-12 \\
9.7 \mathrm{E}-13 \\
6.5 \mathrm{E}-13 \\
8.4 \mathrm{E}-13 \\
7.1 \mathrm{E}-13 \\
1.0 \mathrm{E}-12 \\
6.8 \mathrm{E}-13\end{array}$ & $\begin{array}{l}5.4 \mathrm{E}-13 \\
2.0 \mathrm{E}-13 \\
2.9 \mathrm{E}-13 \\
3.8 \mathrm{E}-13 \\
4.3 \mathrm{E}-13 \\
5.7 \mathrm{E}-13 \\
8.9 \mathrm{E}-13 \\
1.5 \mathrm{E}-12 \\
2.0 \mathrm{E}-12 \\
1.7 \mathrm{E}-12 \\
9.5 \mathrm{E}-13 \\
6.4 \mathrm{E}-13 \\
8.2 \mathrm{E}-13 \\
6.9 \mathrm{E}-13 \\
1.0 \mathrm{E}-12 \\
6.6 \mathrm{E}-13\end{array}$ & $\begin{array}{l}5.4 \mathrm{E}-13 \\
2.0 \mathrm{E}-13 \\
2.8 \mathrm{E}-13 \\
3.8 \mathrm{E}-13 \\
4.3 \mathrm{E}-13 \\
5.6 \mathrm{E}-13 \\
8.8 \mathrm{E}-13 \\
1.5 \mathrm{E}-12 \\
2.0 \mathrm{E}-12 \\
1.7 \mathrm{E}-12 \\
9.4 \mathrm{E}-13 \\
6.3 \mathrm{E}-13 \\
8.1 \mathrm{E}-13 \\
6.8 \mathrm{E}-13 \\
9.8 \mathrm{E}-13 \\
6.5 \mathrm{E}-13\end{array}$ \\
\hline \multicolumn{8}{|c|}{ Distance (m) } \\
\hline Direction & 36721 & 36809 & 37729 & 39079 & 39220 & 39559 & 43584 \\
\hline $\begin{array}{r}N \\
N N W \\
N W \\
\text { WNW } \\
W \\
\text { WSW } \\
\text { SW } \\
\text { SSW }\end{array}$ & $\begin{array}{l}5.2 \mathrm{E}-13 \\
1.9 \mathrm{E}-13 \\
2.7 \mathrm{E}-13 \\
3.7 \mathrm{E}-13 \\
4.2 \mathrm{E}-13 \\
5.4 \mathrm{E}-13 \\
8.5 \mathrm{E}-13 \\
1.4 \mathrm{E}-12\end{array}$ & $\begin{array}{l}5.2 \mathrm{E}-13 \\
1.9 \mathrm{E}-13 \\
2.7 \mathrm{E}-13 \\
3.7 \mathrm{E}-13 \\
4.1 \mathrm{E}-13 \\
5.4 \mathrm{E}-13 \\
8.5 \mathrm{E}-13 \\
1.4 \mathrm{E}-12\end{array}$ & $\begin{array}{l}5.0 \mathrm{E}-13 \\
1.9 \mathrm{E}-13 \\
2.7 \mathrm{E}-13 \\
3.6 \mathrm{E}-13 \\
4.0 \mathrm{E}-13 \\
5.3 \mathrm{E}-13 \\
8.2 \mathrm{E}-13 \\
1.4 \mathrm{E}-12\end{array}$ & $\begin{array}{r}4.8 \mathrm{E}-13 \\
1.8 \mathrm{E}-13 \\
2.6 \mathrm{E}-13 \\
3.4 \mathrm{E}-13 \\
3.9 \mathrm{E}-13 \\
5.1 \mathrm{E}-13 \\
7.9 \mathrm{E}-13 \\
1.3 \mathrm{E}-12 \\
\text { Page }\end{array}$ & $\begin{array}{l}4.8 \mathrm{E}-13 \\
1.8 \mathrm{E}-13 \\
2.6 \mathrm{E}-13 \\
3.4 \mathrm{E}-13 \\
3.9 \mathrm{E}-13 \\
5.1 \mathrm{E}-13 \\
7.9 \mathrm{E}-13 \\
1.3 \mathrm{E}-12\end{array}$ & $\begin{array}{l}4.8 \mathrm{E}-13 \\
1.8 \mathrm{E}-13 \\
2.5 \mathrm{E}-13 \\
3.4 \mathrm{E}-13 \\
3.8 \mathrm{E}-13 \\
5.0 \mathrm{E}-13 \\
7.8 \mathrm{E}-13 \\
1.3 \mathrm{E}-12\end{array}$ & $\begin{array}{l}\text { 4. 3E-13 } \\
1.6 \mathrm{E}-13 \\
2.3 \mathrm{E}-13 \\
3.0 \mathrm{E}-13 \\
3.4 \mathrm{E}-13 \\
4.5 \mathrm{E}-13 \\
7.0 \mathrm{E}-13 \\
1.2 \mathrm{E}-12\end{array}$ \\
\hline
\end{tabular}




\begin{tabular}{rlllllll}
\multicolumn{7}{c}{$\mathrm{N}-13 \mathrm{C} . \mathrm{SUM}$} \\
$\mathrm{S}$ & $1.9 \mathrm{E}-12$ & $1.9 \mathrm{E}-12$ & $1.9 \mathrm{E}-12$ & $1.8 \mathrm{E}-12$ & $1.8 \mathrm{E}-12$ & $1.8 \mathrm{E}-12$ & $1.6 \mathrm{E}-12$ \\
SSE & $1.6 \mathrm{E}-12$ & $1.6 \mathrm{E}-12$ & $1.6 \mathrm{E}-12$ & $1.5 \mathrm{E}-12$ & $1.5 \mathrm{E}-12$ & $1.5 \mathrm{E}-12$ & $1.3 \mathrm{E}-12$ \\
SE & $9.1 \mathrm{E}-13$ & $9.0 \mathrm{E}-13$ & $8.8 \mathrm{E}-13$ & $8.5 \mathrm{E}-13$ & $8.4 \mathrm{E}-13$ & $8.3 \mathrm{E}-13$ & $7.5 \mathrm{E}-13$ \\
ESE & $6.1 \mathrm{E}-13$ & $6.1 \mathrm{E}-13$ & $5.9 \mathrm{E}-13$ & $5.7 \mathrm{E}-13$ & $5.7 \mathrm{E}-13$ & $5.6 \mathrm{E}-13$ & $5.0 \mathrm{E}-13$ \\
$\mathrm{E}$ & $7.9 \mathrm{E}-13$ & $7.9 \mathrm{E}-13$ & $7.6 \mathrm{E}-13$ & $7.3 \mathrm{E}-13$ & $7.3 \mathrm{E}-13$ & $7.2 \mathrm{E}-13$ & $6.5 \mathrm{E}-13$ \\
ENE & $6.6 \mathrm{E}-13$ & $6.6 \mathrm{E}-13$ & $6.4 \mathrm{E}-13$ & $6.2 \mathrm{E}-13$ & $6.1 \mathrm{E}-13$ & $6.1 \mathrm{E}-13$ & $5.4 \mathrm{E}-13$ \\
NE & $9.5 \mathrm{E}-13$ & $9.5 \mathrm{E}-13$ & $9.2 \mathrm{E}-13$ & $8.8 \mathrm{E}-13$ & $8.8 \mathrm{E}-13$ & $8.7 \mathrm{E}-13$ & $7.8 \mathrm{E}-13$ \\
NNE & $6.3 \mathrm{E}-13$ & $6.3 \mathrm{E}-13$ & $6.1 \mathrm{E}-13$ & $5.9 \mathrm{E}-13$ & $5.9 \mathrm{E}-13$ & $5.8 \mathrm{E}-13$ & $5.2 \mathrm{E}-13$
\end{tabular}

Feb 20, $2008 \quad 02: 31 \mathrm{pm}$

SUMMARY

Page 8

INDIVIDUAL LIFETIME RISK (deaths)

(A11 Radionuclides and Pathways)

\begin{tabular}{|c|c|c|c|c|c|c|}
\hline \multirow[b]{2}{*}{ Direction } & \multicolumn{6}{|c|}{ Distance $(\mathrm{m})$} \\
\hline & 45196 & 45275 & 45654 & 45677 & 46668 & 47969 \\
\hline $\begin{array}{r}\text { N } \\
N N W \\
N W \\
W N W \\
W \\
W S W \\
\text { SW } \\
\text { SSW } \\
S \\
\text { SSE } \\
\text { SE } \\
\text { ESE } \\
\text { E } \\
\text { ENE } \\
N E \\
\text { NNE }\end{array}$ & $\begin{array}{l}4.1 \mathrm{E}-13 \\
1.5 \mathrm{E}-13 \\
2.2 \mathrm{E}-13 \\
2.9 \mathrm{E}-13 \\
3.3 \mathrm{E}-13 \\
4.3 \mathrm{E}-13 \\
6.7 \mathrm{E}-13 \\
1.1 \mathrm{E}-12 \\
1.5 \mathrm{E}-12 \\
1.3 \mathrm{E}-12 \\
7.2 \mathrm{E}-13 \\
4.8 \mathrm{E}-13 \\
6.2 \mathrm{E}-13 \\
5.2 \mathrm{E}-13 \\
7.5 \mathrm{E}-13 \\
5.0 \mathrm{E}-13\end{array}$ & $\begin{array}{l}4.1 \mathrm{E}-13 \\
1.5 \mathrm{E}-13 \\
2.2 \mathrm{E}-13 \\
2.9 \mathrm{E}-13 \\
3.3 \mathrm{E}-13 \\
4.3 \mathrm{E}-13 \\
6.7 \mathrm{E}-13 \\
1.1 \mathrm{E}-12 \\
1.5 \mathrm{E}-12 \\
1.3 \mathrm{E}-12 \\
7.2 \mathrm{E}-13 \\
4.8 \mathrm{E}-13 \\
6.2 \mathrm{E}-13 \\
5.2 \mathrm{E}-13 \\
7.4 \mathrm{E}-13 \\
5.0 \mathrm{E}-13\end{array}$ & $\begin{array}{l}4.1 \mathrm{E}-13 \\
1.5 \mathrm{E}-13 \\
2.2 \mathrm{E}-13 \\
2.9 \mathrm{E}-13 \\
3.3 \mathrm{E}-13 \\
4.3 \mathrm{E}-13 \\
6.6 \mathrm{E}-13 \\
1.1 \mathrm{E}-12 \\
1.5 \mathrm{E}-12 \\
1.3 \mathrm{E}-12 \\
7.1 \mathrm{E}-13 \\
4.8 \mathrm{E}-13 \\
6.2 \mathrm{E}-13 \\
5.2 \mathrm{E}-13 \\
7.4 \mathrm{E}-13 \\
5.0 \mathrm{E}-13\end{array}$ & $\begin{array}{l}4.1 \mathrm{E}-13 \\
1.5 \mathrm{E}-13 \\
2.2 \mathrm{E}-13 \\
2.9 \mathrm{E}-13 \\
3.3 \mathrm{E}-13 \\
4.3 \mathrm{E}-13 \\
6.6 \mathrm{E}-13 \\
1.1 \mathrm{E}-12 \\
1.5 \mathrm{E}-12 \\
1.3 \mathrm{E}-12 \\
7.1 \mathrm{E}-13 \\
4.8 \mathrm{E}-13 \\
6.2 \mathrm{E}-13 \\
5.2 \mathrm{E}-13 \\
7.4 \mathrm{E}-13 \\
4.9 \mathrm{E}-13\end{array}$ & $\begin{array}{l}4.0 \mathrm{E}-13 \\
1.5 \mathrm{E}-13 \\
2.1 \mathrm{E}-13 \\
2.8 \mathrm{E}-13 \\
3.2 \mathrm{E}-13 \\
4.2 \mathrm{E}-13 \\
6.5 \mathrm{E}-13 \\
1.1 \mathrm{E}-12 \\
1.5 \mathrm{E}-12 \\
1.2 \mathrm{E}-12 \\
6.9 \mathrm{E}-13 \\
4.7 \mathrm{E}-13 \\
6.0 \mathrm{E}-13 \\
5.0 \mathrm{E}-13 \\
7.2 \mathrm{E}-13 \\
4.8 \mathrm{E}-13\end{array}$ & $\begin{array}{l}3.9 \mathrm{E}-13 \\
1.4 \mathrm{E}-13 \\
2.0 \mathrm{E}-13 \\
2.7 \mathrm{E}-13 \\
3.1 \mathrm{E}-13 \\
4.0 \mathrm{E}-13 \\
6.3 \mathrm{E}-13 \\
1.0 \mathrm{E}-12 \\
1.4 \mathrm{E}-12 \\
1.2 \mathrm{E}-12 \\
6.7 \mathrm{E}-13 \\
4.5 \mathrm{E}-13 \\
5.8 \mathrm{E}-13 \\
4.9 \mathrm{E}-13 \\
7.0 \mathrm{E}-13 \\
4.7 \mathrm{E}-13\end{array}$ \\
\hline
\end{tabular}




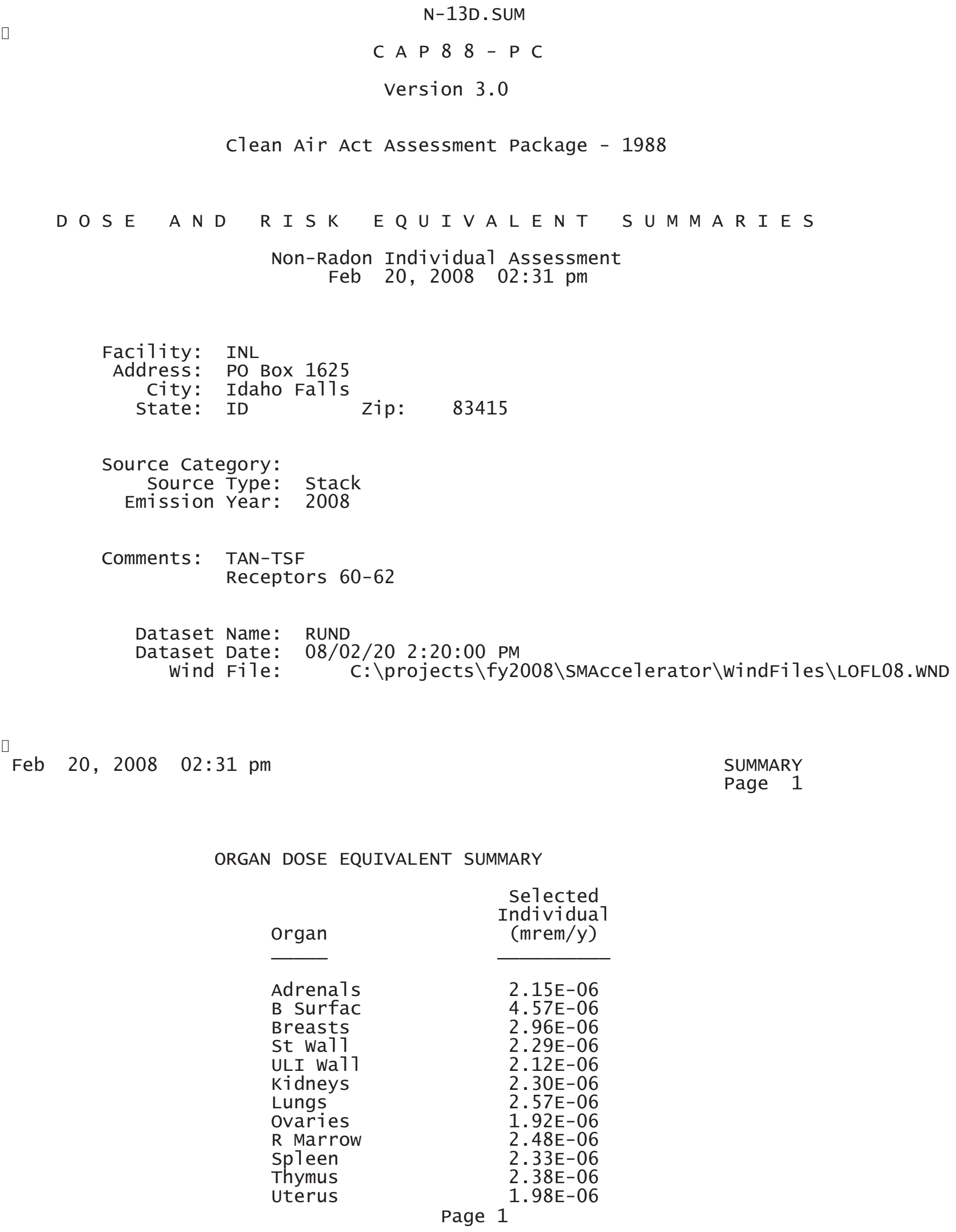




$\begin{array}{lr} & \mathrm{N}-13 \mathrm{D} . \text { SUM } \\ \text { B1d wa11 } & 2.14 \mathrm{E}-06 \\ \text { Brain } & 2.74 \mathrm{E}-06 \\ \text { Esophagu } & 2.11 \mathrm{E}-06 \\ \text { SI wa11 } & 2.03 \mathrm{E}-06 \\ \text { LLI wa11 } & 2.07 \mathrm{E}-06 \\ \text { Liver } & 2.31 \mathrm{E}-06 \\ \text { Muscle } & 2.51 \mathrm{E}-06 \\ \text { Pancreas } & 2.02 \mathrm{E}-06 \\ \text { Skin } & 4.70 \mathrm{E}-06 \\ \text { Testes } & 2.59 \mathrm{E}-06 \\ \text { Thyroid } & 2.63 \mathrm{E}-06 \\ \text { EFFEC } & 2.47 \mathrm{E}-06\end{array}$

PATHWAY EFFECTIVE DOSE EQUIVALENT SUMMARY

Pathway

INGESTION

INHALATION

AIR IMMERSION

GROUND SURFACE

INTERNAL

EXTERNAL

TOTAL selected

Individual

(mrem/y)

$0.00 \mathrm{E}+00$

$0.00 \mathrm{E}+00$

2. $47 \mathrm{E}-06$

$0.00 \mathrm{E}+00$

$0.00 \mathrm{E}+00$

$2.47 \mathrm{E}-06$

$2.47 \mathrm{E}-06$

$\square$

Feb 20, $2008 \quad 02: 31 \mathrm{pm}$

SUMMARY

Page 2

NUCLIDE EFFECTIVE DOSE EQUIVALENT SUMMARY

Nuclide

$\mathrm{N}-13$

TOTAL

Feb 20, $2008 \quad 02: 31$ pm

$$
\begin{gathered}
\begin{array}{c}
\text { Selected } \\
\text { Individual } \\
\text { (mrem/y) }
\end{array} \\
\hline 2.47 \mathrm{E}-06 \\
2.47 \mathrm{E}-06
\end{gathered}
$$

SUMMARY

Page 3

CANCER RISK SUMMARY

Cancer

Esophagu
Selected Individual

Total Lifetime

Fatal Cancer Risk

Page $2^{2.43 E-14}$ 


$\begin{array}{lr} & \mathrm{N}-13 \mathrm{D} . \mathrm{SUM} \\ \text { Stomach } & 9.25 \mathrm{E}-14 \\ \text { Colon } & 2.16 \mathrm{E}-13 \\ \text { Liver } & 3.50 \mathrm{E}-14 \\ \text { LUNG } & 2.51 \mathrm{E}-13 \\ \text { Bone } & 4.33 \mathrm{E}-15 \\ \text { Skin } & 4.68 \mathrm{E}-15 \\ \text { Breast } & 1.43 \mathrm{E}-13 \\ \text { Ovary } & 2.73 \mathrm{E}-14 \\ \text { B Tadder } & 5.18 \mathrm{E}-14 \\ \text { Kidneys } & 1.20 \mathrm{E}-14 \\ \text { Thyroid } & 8.38 \mathrm{E}-15 \\ \text { Leukemia } & 1.39 \mathrm{E}-13 \\ \text { Residua1 } & 3.32 \mathrm{E}-13 \\ \text { Tota1 } & 1.34 \mathrm{E}-12 \\ & \\ \text { TOTAL } & 2.68 \mathrm{E}-12\end{array}$

PATHWAY RISK SUMMARY

\begin{tabular}{lc} 
Pathway & $\begin{array}{c}\text { Selected Individua1 } \\
\text { Total Lifetime } \\
\text { Fatal Cancer Risk }\end{array}$ \\
\cline { 2 - 2 } INGESTION & $0.00 \mathrm{E}+00$ \\
INHALATION & $0.00 \mathrm{E}+00$ \\
AIR IMMERSION & $1.34 \mathrm{E}-12$ \\
GROUND SURFACE & $0.00 \mathrm{E}+00$ \\
INTERNAL & $0.00 \mathrm{E}+00$ \\
EXTERNAL & $1.34 \mathrm{E}-12$ \\
TOTAL & $1.34 \mathrm{E}-12$
\end{tabular}

Feb 20, $2008 \quad 02: 31$ pm

SUMMARY Page 4

NUCLIDE RISK SUMMARY

Nuclide

$\mathrm{N}-13$

TOTAL

$\square$

Feb 20, 2008 02:31 pm
Selected Individual

Total Lifetime Fatal Cancer Risk

1. $34 \mathrm{E}-12$

1. $34 \mathrm{E}-12$

SUMMARY

Page 5

INDIVIDUAL EFFECTIVE DOSE EQUIVALENT RATE (mrem/y) (A11 Radionuclides and Pathways)

Distance $(m)$ 


Direction $50024 \quad 54611 \quad 58610 \quad$ N-13D.SUM

\begin{tabular}{|c|c|c|c|c|}
\hline $\begin{array}{r}N \\
N N W \\
N W \\
W N W \\
W \\
W S W \\
S W \\
\text { SSW } \\
S \\
\text { SSE } \\
\text { SE } \\
\text { ESE } \\
\text { E } \\
\text { ENE } \\
\text { NE } \\
\text { NNE }\end{array}$ & $\begin{array}{l}6.8 \mathrm{E}-07 \\
2.6 \mathrm{E}-07 \\
3.6 \mathrm{E}-07 \\
4.8 \mathrm{E}-07 \\
5.4 \mathrm{E}-07 \\
7.1 \mathrm{E}-07 \\
1.1 \mathrm{E}-06 \\
1.8 \mathrm{E}-06 \\
2.5 \mathrm{E}-06 \\
2.1 \mathrm{E}-06 \\
1.2 \mathrm{E}-06 \\
8.0 \mathrm{E}-07 \\
1.0 \mathrm{E}-06 \\
8.6 \mathrm{E}-07 \\
1.2 \mathrm{E}-06 \\
8.2 \mathrm{E}-07\end{array}$ & $\begin{array}{l}6.1 \mathrm{E}-07 \\
2.3 \mathrm{E}-07 \\
3.3 \mathrm{E}-07 \\
4.4 \mathrm{E}-07 \\
4.9 \mathrm{E}-07 \\
6.4 \mathrm{E}-07 \\
1.0 \mathrm{E}-06 \\
1.6 \mathrm{E}-06 \\
2.2 \mathrm{E}-06 \\
1.9 \mathrm{E}-06 \\
1.1 \mathrm{E}-06 \\
7.2 \mathrm{E}-07 \\
9.3 \mathrm{E}-07 \\
7.8 \mathrm{E}-07 \\
1.1 \mathrm{E}-06 \\
7.5 \mathrm{E}-07\end{array}$ & $\begin{array}{l}5.7 \mathrm{E}-07 \\
2.1 \mathrm{E}-07 \\
3.0 \mathrm{E}-07 \\
4.0 \mathrm{E}-07 \\
4.6 \mathrm{E}-07 \\
5.9 \mathrm{E}-07 \\
9.2 \mathrm{E}-07 \\
1.5 \mathrm{E}-06 \\
2.1 \mathrm{E}-06 \\
1.7 \mathrm{E}-06 \\
9.9 \mathrm{E}-07 \\
6.7 \mathrm{E}-07 \\
8.6 \mathrm{E}-07 \\
7.2 \mathrm{E}-07 \\
1.0 \mathrm{E}-06 \\
6.9 \mathrm{E}-07\end{array}$ & \\
\hline Feb 20, & 2008 & $2: 31 \mathrm{pm}$ & & SUMMARY \\
\hline \multicolumn{5}{|c|}{$\begin{array}{l}\text { INDIVIDUAL LIFETIME RISK (deaths) } \\
\text { (A11 Radionuclides and Pathways) }\end{array}$} \\
\hline \multicolumn{5}{|c|}{ Distance (m) } \\
\hline Direction & 50024 & 54611 & 58610 & \\
\hline $\begin{array}{r}N \\
N N W \\
N W \\
W N W \\
W \\
W S W \\
\text { SW } \\
\text { SSW } \\
S \\
\text { SSE } \\
\text { SE } \\
\text { ESE } \\
\text { E } \\
\text { ENE } \\
\text { NE } \\
\text { NNE }\end{array}$ & $\begin{array}{l}3.7 \mathrm{E}-13 \\
1.4 \mathrm{E}-13 \\
2.0 \mathrm{E}-13 \\
2.6 \mathrm{E}-13 \\
3.0 \mathrm{E}-13 \\
3.9 \mathrm{E}-13 \\
6.0 \mathrm{E}-13 \\
9.8 \mathrm{E}-13 \\
1.3 \mathrm{E}-12 \\
1.1 \mathrm{E}-12 \\
6.4 \mathrm{E}-13 \\
4.3 \mathrm{E}-13 \\
5.6 \mathrm{E}-13 \\
4.7 \mathrm{E}-13 \\
6.6 \mathrm{E}-13 \\
4.5 \mathrm{E}-13\end{array}$ & $\begin{array}{l}3.3 \mathrm{E}-13 \\
1.3 \mathrm{E}-13 \\
1.8 \mathrm{E}-13 \\
2.4 \mathrm{E}-13 \\
2.7 \mathrm{E}-13 \\
3.5 \mathrm{E}-13 \\
5.4 \mathrm{E}-13 \\
8.9 \mathrm{E}-13 \\
1.2 \mathrm{E}-12 \\
1.0 \mathrm{E}-12 \\
5.8 \mathrm{E}-13 \\
3.9 \mathrm{E}-13 \\
5.0 \mathrm{E}-13 \\
4.2 \mathrm{E}-13 \\
6.0 \mathrm{E}-13 \\
4.0 \mathrm{E}-13\end{array}$ & $\begin{array}{l}3.1 \mathrm{E}-13 \\
1.2 \mathrm{E}-13 \\
1.6 \mathrm{E}-13 \\
2.2 \mathrm{E}-13 \\
2.5 \mathrm{E}-13 \\
3.2 \mathrm{E}-13 \\
5.0 \mathrm{E}-13 \\
8.2 \mathrm{E}-13 \\
1.1 \mathrm{E}-12 \\
9.5 \mathrm{E}-13 \\
5.3 \mathrm{E}-13 \\
3.6 \mathrm{E}-13 \\
4.7 \mathrm{E}-13 \\
3.9 \mathrm{E}-13 \\
5.5 \mathrm{E}-13 \\
3.7 \mathrm{E}-13\end{array}$ & \\
\hline
\end{tabular}




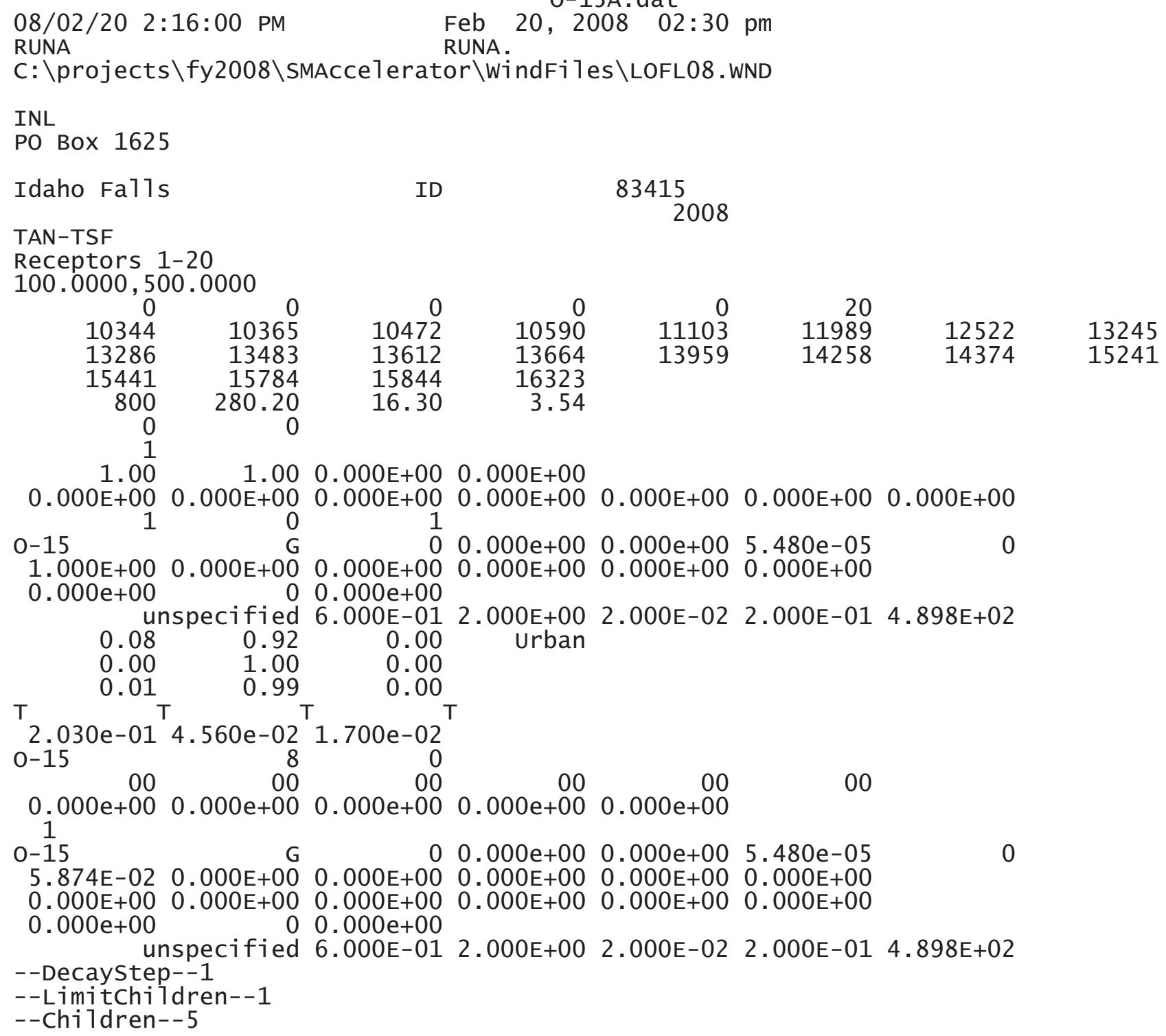




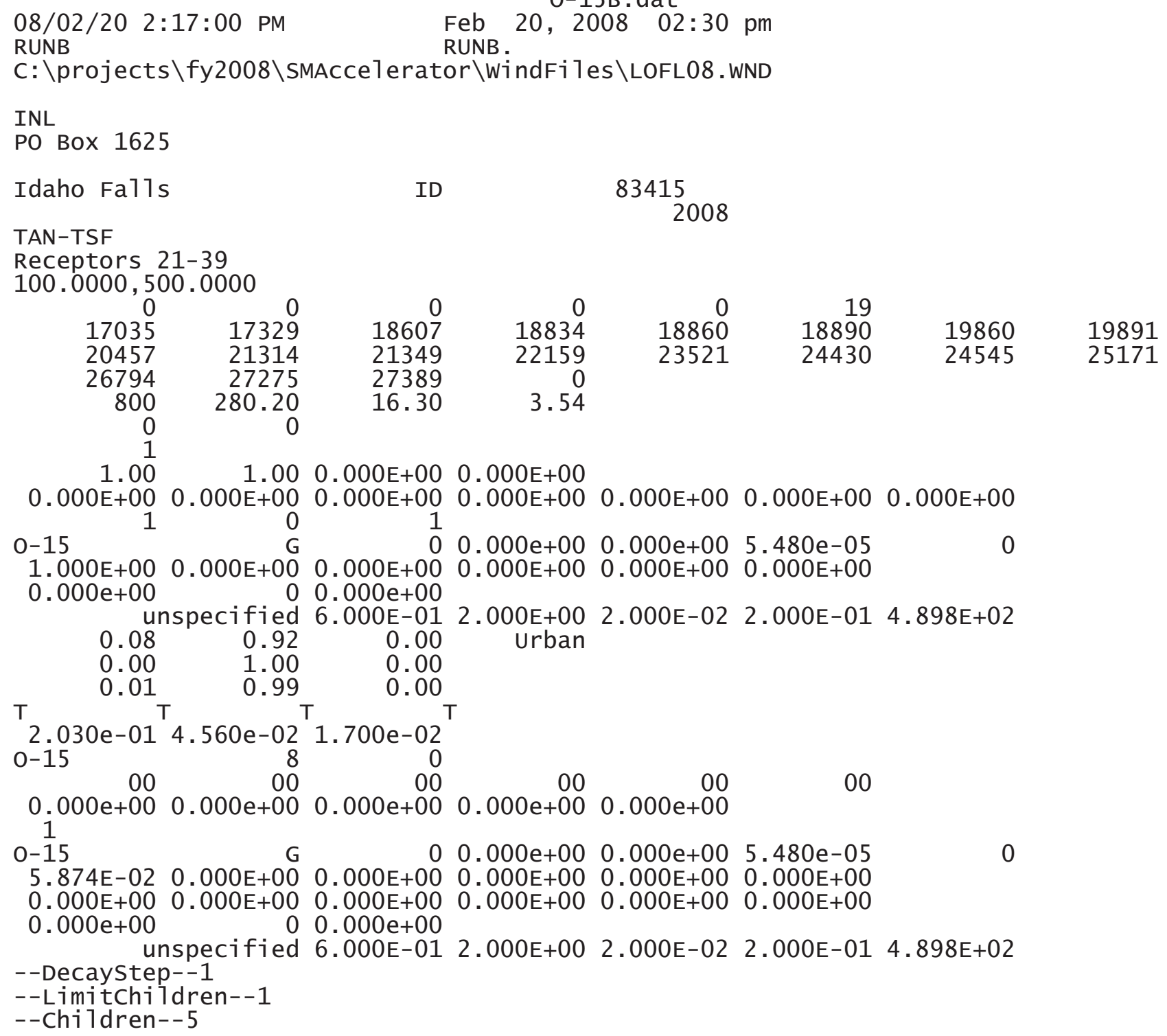




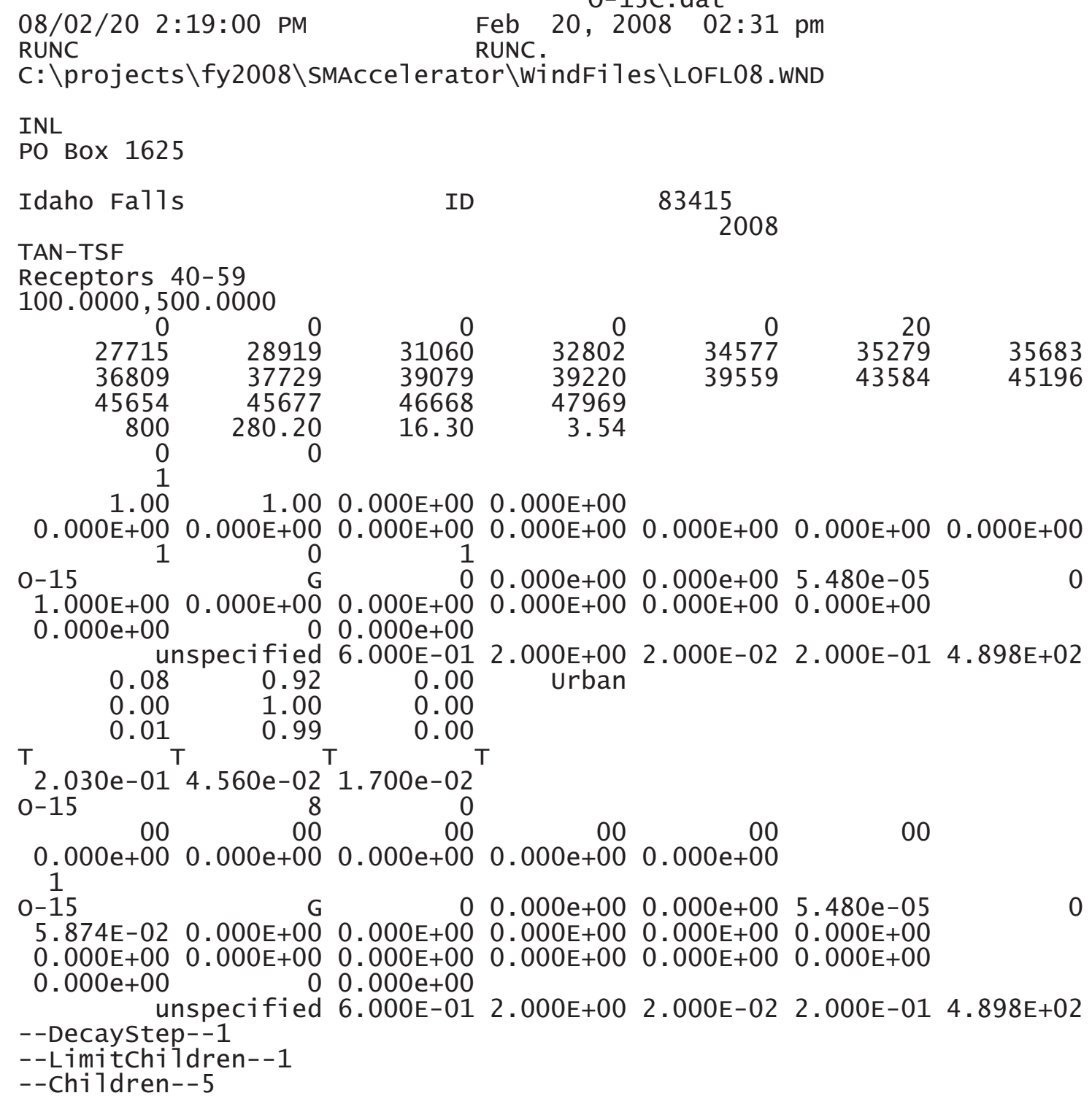

ID

83415

TAN-TSF

Receptors 40-59

$100.0000,500.0000$

$\begin{array}{rrrr}0 & 0 & 0 & \\ 27715 & 28919 & 31060 & 3280 \\ 36809 & 37729 & 39079 & 3922 \\ 45654 & 45677 & 46668 & 4796 \\ 800 & 280.20 & 16.30 & 3.5 \\ 0 & 0 & & \end{array}$




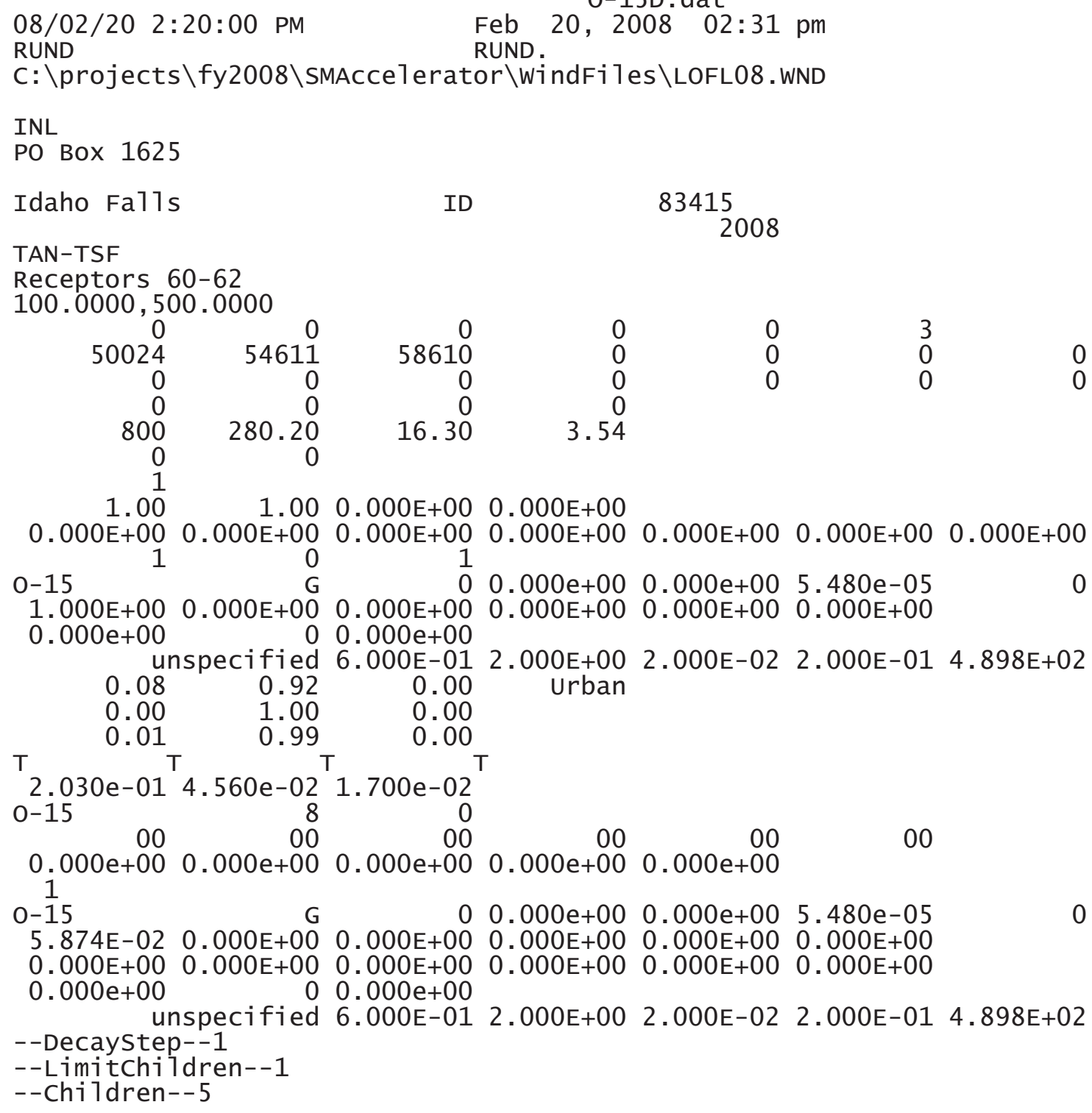




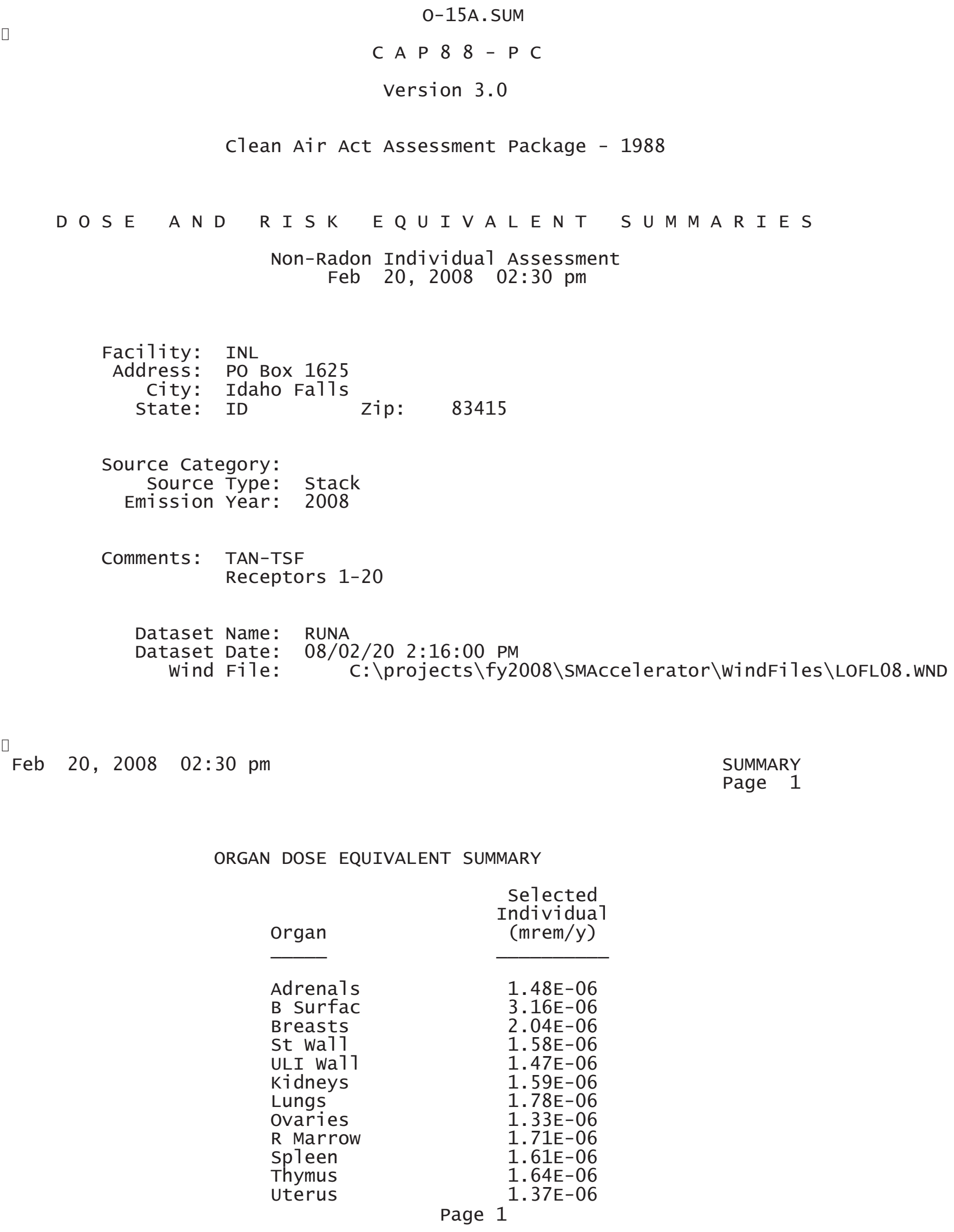

\begin{tabular}{lr} 
ORGAN DOSE EQUIVALENT SUMMARY \\
Selected \\
Organ & $\begin{array}{r}\text { Individua } \\
\text { (mrem/y) }\end{array}$ \\
\cline { 2 - 2 } Adrenals & $1.48 \mathrm{E}-06$ \\
B Surfac & $3.16 \mathrm{E}-06$ \\
Breasts & $2.04 \mathrm{E}-06$ \\
St wa11 & $1.58 \mathrm{E}-06$ \\
ULI wa11 & $1.47 \mathrm{E}-06$ \\
Kidneys & $1.59 \mathrm{E}-06$ \\
Lungs & $1.78 \mathrm{E}-06$ \\
Ovaries & $1.33 \mathrm{E}-06$ \\
R Marrow & $1.71 \mathrm{E}-06$ \\
Spleen & $1.61 \mathrm{E}-06$ \\
Thymus & $1.64 \mathrm{E}-06$ \\
Uterus & $1.37 \mathrm{E}-06$ \\
& 1
\end{tabular}




$\begin{array}{lr} & \text { 0-15A.SUM } \\ \text { B1d wa11 } & 1.48 \mathrm{E}-06 \\ \text { Brain } & 1.89 \mathrm{E}-06 \\ \text { Esophagu } & 1.46 \mathrm{E}-06 \\ \text { SI wa11 } & 1.40 \mathrm{E}-06 \\ \text { LLI wa11 } & 1.43 \mathrm{E}-06 \\ \text { Liver } & 1.60 \mathrm{E}-06 \\ \text { Muscle } & 1.73 \mathrm{E}-06 \\ \text { Pancreas } & 1.39 \mathrm{E}-06 \\ \text { Skin } & 3.88 \mathrm{E}-06 \\ \text { Testes } & 1.79 \mathrm{E}-06 \\ \text { Thyroid } & 1.82 \mathrm{E}-06 \\ \text { EFFEC } & 1.72 \mathrm{E}-06\end{array}$

PATHWAY EFFECTIVE DOSE EQUIVALENT SUMMARY

Pathway $\begin{array}{r}\text { Individual } \\ (\mathrm{mrem} / \mathrm{y})\end{array}$

INGESTION $\quad 0.00 \mathrm{E}+00$

INHALATION $\quad 0.00 \mathrm{E}+00$

AIR IMMERSION $\quad 1.72 \mathrm{E}-06$

GROUND SURFACE $\quad 0.00 \mathrm{E}+00$

INTERNAL $\quad 0.00 \mathrm{E}+00$

EXTERNAL $\quad 1.72 \mathrm{E}-06$

TOTAL $\quad 1.72 \mathrm{E}-06$

Feb 20, $2008 \quad 02: 30$ pm

SUMMARY

Page 2

NUCLIDE EFFECTIVE DOSE EQUIVALENT SUMMARY

Nuclide

$0-15$

TOTAL

Feb 20, $2008 \quad 02: 30 \mathrm{pm}$
Selected Individual

(mrem/y)

$1.72 \mathrm{E}-06$

$1.72 \mathrm{E}-06$

SUMMARY

Page 3

CANCER RISK SUMMARY

Cancer

Esophagu
Selected Individual

Total Lifetime

Fatal Cancer Risk

Page $2^{1.68 \mathrm{E}-14}$ 


\begin{tabular}{lr} 
& \multicolumn{1}{c}{ 0-15A.SUM } \\
Stomach & $6.38 \mathrm{E}-14$ \\
Colon & $1.50 \mathrm{E}-13$ \\
Liver & $2.42 \mathrm{E}-14$ \\
LUNG & $1.73 \mathrm{E}-13$ \\
Bone & $3.00 \mathrm{E}-15$ \\
Skin & $3.88 \mathrm{E}-15$ \\
Breast & $9.88 \mathrm{E}-14$ \\
Ovary & $1.89 \mathrm{E}-14$ \\
B Tadder & $3.58 \mathrm{E}-14$ \\
Kidneys & $8.24 \mathrm{E}-15$ \\
Thyroid & $5.78 \mathrm{E}-15$ \\
Leukemia & $9.62 \mathrm{E}-14$ \\
Residua1 & $2.29 \mathrm{E}-13$ \\
Tota1 & $9.29 \mathrm{E}-13$ \\
& \\
TOTAL & $1.86 \mathrm{E}-12$
\end{tabular}

PATHWAY RISK SUMMARY

\begin{tabular}{lc} 
Pathway & $\begin{array}{c}\text { Selected Individua1 } \\
\text { Total Lifetime } \\
\text { Fatal Cancer Risk }\end{array}$ \\
\cline { 2 - 2 } INGESTION & $0.00 \mathrm{E}+00$ \\
INHALATION & $0.00 \mathrm{E}+00$ \\
AIR IMMERSION & $9.29 \mathrm{E}-13$ \\
GROUND SURFACE & $0.00 \mathrm{E}+00$ \\
INTERNAL & $0.00 \mathrm{E}+00$ \\
EXTERNAL & $9.29 \mathrm{E}-13$ \\
TOTAL & $9.29 \mathrm{E}-13$
\end{tabular}

Feb 20, $2008 \quad 02: 30$ pm

SUMMARY Page 4

NUCLIDE RISK SUMMARY

Nuclide

$0-15$

TOTAL

$\square$

Feb 20, 2008 02:30 pm
Selected Individual

Total Lifetime Fatal Cancer Risk

$9.29 \mathrm{E}-13$

9. $29 \mathrm{E}-13$

INDIVIDUAL EFFECTIVE DOSE EQUIVALENT RATE (mrem/y) (A11 Radionuclides and Pathways)

Distance $(m)$ 


\begin{tabular}{rccccccc} 
Direction & 10344 & 10365 & 10472 & 10590 & 11103 & 11989 & 12522 \\
\hline N & $4.2 \mathrm{E}-07$ & $4.2 \mathrm{E}-07$ & $4.1 \mathrm{E}-07$ & $4.1 \mathrm{E}-07$ & $3.8 \mathrm{E}-07$ & $3.5 \mathrm{E}-07$ & $3.4 \mathrm{E}-07$ \\
NNW & $1.6 \mathrm{E}-07$ & $1.6 \mathrm{E}-07$ & $1.5 \mathrm{E}-07$ & $1.5 \mathrm{E}-07$ & $1.4 \mathrm{E}-07$ & $1.3 \mathrm{E}-07$ & $1.3 \mathrm{E}-07$ \\
NW & $2.2 \mathrm{E}-07$ & $2.2 \mathrm{E}-07$ & $2.1 \mathrm{E}-07$ & $2.1 \mathrm{E}-07$ & $2.0 \mathrm{E}-07$ & $1.8 \mathrm{E}-07$ & $1.8 \mathrm{E}-07$ \\
WNW & $2.9 \mathrm{E}-07$ & $2.9 \mathrm{E}-07$ & $2.9 \mathrm{E}-07$ & $2.8 \mathrm{E}-07$ & $2.7 \mathrm{E}-07$ & $2.5 \mathrm{E}-07$ & $2.3 \mathrm{E}-07$ \\
W & $3.3 \mathrm{E}-07$ & $3.3 \mathrm{E}-07$ & $3.3 \mathrm{E}-07$ & $3.2 \mathrm{E}-07$ & $3.1 \mathrm{E}-07$ & $2.8 \mathrm{E}-07$ & $2.7 \mathrm{E}-07$ \\
WSW & $4.5 \mathrm{E}-07$ & $4.5 \mathrm{E}-07$ & $4.4 \mathrm{E}-07$ & $4.4 \mathrm{E}-07$ & $4.1 \mathrm{E}-07$ & $3.8 \mathrm{E}-07$ & $3.6 \mathrm{E}-07$ \\
SW & $7.3 \mathrm{E}-07$ & $7.3 \mathrm{E}-07$ & $7.2 \mathrm{E}-07$ & $7.1 \mathrm{E}-07$ & $6.7 \mathrm{E}-07$ & $6.1 \mathrm{E}-07$ & $5.8 \mathrm{E}-07$ \\
SSW & $1.2 \mathrm{E}-06$ & $1.2 \mathrm{E}-06$ & $1.2 \mathrm{E}-06$ & $1.2 \mathrm{E}-06$ & $1.1 \mathrm{E}-06$ & $1.0 \mathrm{E}-06$ & $9.7 \mathrm{E}-07$ \\
$\mathrm{~S}$ & $1.7 \mathrm{E}-06$ & $1.7 \mathrm{E}-06$ & $1.7 \mathrm{E}-06$ & $1.7 \mathrm{E}-06$ & $1.6 \mathrm{E}-06$ & $1.4 \mathrm{E}-06$ & $1.4 \mathrm{E}-06$ \\
SSE & $1.4 \mathrm{E}-06$ & $1.4 \mathrm{E}-06$ & $1.4 \mathrm{E}-06$ & $1.4 \mathrm{E}-06$ & $1.3 \mathrm{E}-06$ & $1.2 \mathrm{E}-06$ & $1.1 \mathrm{E}-06$ \\
$\mathrm{SE}$ & $7.5 \mathrm{E}-07$ & $7.5 \mathrm{E}-07$ & $7.4 \mathrm{E}-07$ & $7.3 \mathrm{E}-07$ & $6.9 \mathrm{E}-07$ & $6.3 \mathrm{E}-07$ & $6.0 \mathrm{E}-07$ \\
ESE & $5.0 \mathrm{E}-07$ & $4.9 \mathrm{E}-07$ & $4.9 \mathrm{E}-07$ & $4.8 \mathrm{E}-07$ & $4.6 \mathrm{E}-07$ & $4.2 \mathrm{E}-07$ & $4.0 \mathrm{E}-07$ \\
$\mathrm{E}$ & $6.4 \mathrm{E}-07$ & $6.4 \mathrm{E}-07$ & $6.3 \mathrm{E}-07$ & $6.2 \mathrm{E}-07$ & $5.9 \mathrm{E}-07$ & $5.4 \mathrm{E}-07$ & $5.1 \mathrm{E}-07$ \\
$\mathrm{ENE}$ & $5.5 \mathrm{E}-07$ & $5.5 \mathrm{E}-07$ & $5.4 \mathrm{E}-07$ & $5.4 \mathrm{E}-07$ & $5.1 \mathrm{E}-07$ & $4.7 \mathrm{E}-07$ & $4.4 \mathrm{E}-07$ \\
NE & $8.4 \mathrm{E}-07$ & $8.4 \mathrm{E}-07$ & $8.3 \mathrm{E}-07$ & $8.1 \mathrm{E}-07$ & $7.7 \mathrm{E}-07$ & $7.0 \mathrm{E}-07$ & $6.6 \mathrm{E}-07$ \\
NNE & $5.3 \mathrm{E}-07$ & $5.3 \mathrm{E}-07$ & $5.2 \mathrm{E}-07$ & $5.1 \mathrm{E}-07$ & $4.9 \mathrm{E}-07$ & $4.5 \mathrm{E}-07$ & $4.2 \mathrm{E}-07$ \\
\hline
\end{tabular}

Distance $(\mathrm{m})$

\begin{tabular}{|c|c|c|c|c|c|c|c|}
\hline Direction & n 13245 & 13286 & 13483 & 13612 & 13664 & 13959 & 14258 \\
\hline $\begin{array}{r}\text { N } \\
\text { NNW } \\
N W \\
\text { WNW } \\
W \\
\text { WSW } \\
\text { SW } \\
\text { SSW } \\
\text { S } \\
\text { SSE } \\
\text { SE } \\
\text { ESE } \\
\text { E } \\
\text { ENE } \\
\text { NE } \\
\text { NNE }\end{array}$ & $\begin{array}{l}3.2 \mathrm{E}-07 \\
1.2 \mathrm{E}-07 \\
1.6 \mathrm{E}-07 \\
2.2 \mathrm{E}-07 \\
2.5 \mathrm{E}-07 \\
3.3 \mathrm{E}-07 \\
5.4 \mathrm{E}-07 \\
9.0 \mathrm{E}-07 \\
1.3 \mathrm{E}-06 \\
1.1 \mathrm{E}-06 \\
5.6 \mathrm{E}-07 \\
3.7 \mathrm{E}-07 \\
4.8 \mathrm{E}-07 \\
4.1 \mathrm{E}-07 \\
6.2 \mathrm{E}-07 \\
4.0 \mathrm{E}-07\end{array}$ & $\begin{array}{l}3.1 \mathrm{E}-07 \\
1.2 \mathrm{E}-07 \\
1.6 \mathrm{E}-07 \\
2.2 \mathrm{E}-07 \\
2.5 \mathrm{E}-07 \\
3.3 \mathrm{E}-07 \\
5.4 \mathrm{E}-07 \\
9.0 \mathrm{E}-07 \\
1.3 \mathrm{E}-06 \\
1.1 \mathrm{E}-06 \\
5.6 \mathrm{E}-07 \\
3.7 \mathrm{E}-07 \\
4.8 \mathrm{E}-07 \\
4.1 \mathrm{E}-07 \\
6.2 \mathrm{E}-07 \\
3.9 \mathrm{E}-07\end{array}$ & $\begin{array}{l}3.1 \mathrm{E}-07 \\
1.2 \mathrm{E}-07 \\
1.6 \mathrm{E}-07 \\
2.2 \mathrm{E}-07 \\
2.5 \mathrm{E}-07 \\
3.3 \mathrm{E}-07 \\
5.3 \mathrm{E}-07 \\
8.8 \mathrm{E}-07 \\
1.2 \mathrm{E}-06 \\
1.0 \mathrm{E}-06 \\
5.5 \mathrm{E}-07 \\
3.7 \mathrm{E}-07 \\
4.7 \mathrm{E}-07 \\
4.1 \mathrm{E}-07 \\
6.1 \mathrm{E}-07 \\
3.9 \mathrm{E}-07\end{array}$ & $\begin{array}{l}3.1 \mathrm{E}-07 \\
1.1 \mathrm{E}-07 \\
1.6 \mathrm{E}-07 \\
2.1 \mathrm{E}-07 \\
2.4 \mathrm{E}-07 \\
3.2 \mathrm{E}-07 \\
5.2 \mathrm{E}-07 \\
8.7 \mathrm{E}-07 \\
1.2 \mathrm{E}-06 \\
1.0 \mathrm{E}-06 \\
5.5 \mathrm{E}-07 \\
3.6 \mathrm{E}-07 \\
4.7 \mathrm{E}-07 \\
4.0 \mathrm{E}-07 \\
6.0 \mathrm{E}-07 \\
3.8 \mathrm{E}-07\end{array}$ & $\begin{array}{l}3.0 \mathrm{E}-07 \\
1.1 \mathrm{E}-07 \\
1.6 \mathrm{E}-07 \\
2.1 \mathrm{E}-07 \\
2.4 \mathrm{E}-07 \\
3.2 \mathrm{E}-07 \\
5.2 \mathrm{E}-07 \\
8.7 \mathrm{E}-07 \\
1.2 \mathrm{E}-06 \\
1.0 \mathrm{E}-06 \\
5.4 \mathrm{E}-07 \\
3.6 \mathrm{E}-07 \\
4.7 \mathrm{E}-07 \\
4.0 \mathrm{E}-07 \\
6.0 \mathrm{E}-07 \\
3.8 \mathrm{E}-07\end{array}$ & $\begin{array}{l}3.0 \mathrm{E}-07 \\
1.1 \mathrm{E}-07 \\
1.6 \mathrm{E}-07 \\
2.1 \mathrm{E}-07 \\
2.4 \mathrm{E}-07 \\
3.2 \mathrm{E}-07 \\
5.1 \mathrm{E}-07 \\
8.5 \mathrm{E}-07 \\
1.2 \mathrm{E}-06 \\
1.0 \mathrm{E}-06 \\
5.3 \mathrm{E}-07 \\
3.5 \mathrm{E}-07 \\
4.5 \mathrm{E}-07 \\
3.9 \mathrm{E}-07 \\
5.8 \mathrm{E}-07 \\
3.7 \mathrm{E}-07\end{array}$ & $\begin{array}{l}2.9 \mathrm{E}-07 \\
1.1 \mathrm{E}-07 \\
1.5 \mathrm{E}-07 \\
2.0 \mathrm{E}-07 \\
2.3 \mathrm{E}-07 \\
3.1 \mathrm{E}-07 \\
5.0 \mathrm{E}-07 \\
8.3 \mathrm{E}-07 \\
1.2 \mathrm{E}-06 \\
9.7 \mathrm{E}-07 \\
5.2 \mathrm{E}-07 \\
3.4 \mathrm{E}-07 \\
4.4 \mathrm{E}-07 \\
3.8 \mathrm{E}-07 \\
5.7 \mathrm{E}-07 \\
3.6 \mathrm{E}-07\end{array}$ \\
\hline
\end{tabular}

$\square$

Feb 20, 2008 02:30 pm

SUMMARY

Page 6

INDIVIDUAL EFFECTIVE DOSE EQUIVALENT RATE (mrem/y) (A11 Radionuclides and Pathways)

\begin{tabular}{|c|c|c|c|c|c|c|}
\hline \multirow[b]{2}{*}{ Direction } & \multicolumn{6}{|c|}{ Distance (m) } \\
\hline & 14374 & 15241 & 15441 & 15784 & 15844 & 16323 \\
\hline $\begin{array}{r}N \\
N N W\end{array}$ & $\begin{array}{l}2.9 \mathrm{E}-07 \\
1.1 \mathrm{E}-07\end{array}$ & $\begin{array}{l}2.7 \mathrm{E}-07 \\
1.0 \mathrm{E}-07\end{array}$ & $\begin{array}{l}2.7 \mathrm{E}-07 \\
1.0 \mathrm{E}-07\end{array}$ & $\begin{array}{r}2.6 \mathrm{E}-07 \\
9.7 \mathrm{E}-08 \\
\text { Page }\end{array}$ & $\begin{array}{r}2.6 \mathrm{E}-07 \\
9.7 \mathrm{E}-08 \\
4\end{array}$ & $\begin{array}{l}\text { 2. } 5 \mathrm{E}-07 \\
9.4 \mathrm{E}-08\end{array}$ \\
\hline
\end{tabular}




\begin{tabular}{|c|c|c|c|c|c|c|}
\hline \multicolumn{7}{|c|}{ O-15A. SUM } \\
\hline NW & 1. $5 \mathrm{E}-07$ & $1.4 \mathrm{E}-07$ & $1.4 \mathrm{E}-07$ & $1.4 \mathrm{E}-07$ & 1. $4 \mathrm{E}-07$ & 1. $3 E-07$ \\
\hline WNW & $2.0 \mathrm{E}-07$ & 1. $9 \mathrm{E}-07$ & 1. $9 \mathrm{E}-07$ & $1.8 \mathrm{E}-07$ & $1.8 \mathrm{E}-07$ & $1.7 \mathrm{E}-07$ \\
\hline W & 2. $3 \mathrm{E}-07$ & $2.1 \mathrm{E}-07$ & $2.1 \mathrm{E}-07$ & $2.1 \mathrm{E}-07$ & $2.1 \mathrm{E}-07$ & 2. $0 \mathrm{E}-07$ \\
\hline WSW & $3.0 \mathrm{E}-07$ & $2.9 \mathrm{E}-07$ & $2.8 \mathrm{E}-07$ & $2.7 \mathrm{E}-07$ & $2.7 \mathrm{E}-07$ & $6 \mathrm{E}-07$ \\
\hline SW & $4.9 \mathrm{E}-07$ & 4. $6 \mathrm{E}-07$ & 4. $5 \mathrm{E}-07$ & $4.4 \mathrm{E}-07$ & $4.4 \mathrm{E}-07$ & $4.2 \mathrm{E}-07$ \\
\hline SSW & $8.2 \mathrm{E}-07$ & $7.7 \mathrm{E}-07$ & 7. $5 \mathrm{E}-07$ & $7.3 \mathrm{E}-07$ & 7. 3E-07 & $7.1 \mathrm{E}-07$ \\
\hline $\mathrm{S}$ & $1.1 \mathrm{E}-06$ & $1.1 \mathrm{E}-06$ & $1.1 \mathrm{E}-06$ & 1.0E-06 & 1.0E-06 & $9.9 \mathrm{E}-07$ \\
\hline SSE & $9.6 \mathrm{E}-07$ & $9.0 \mathrm{E}-07$ & $8.8 \mathrm{E}-07$ & 8. $6 \mathrm{E}-07$ & $8.6 \mathrm{E}-07$ & $8.3 \mathrm{E}-07$ \\
\hline SE & $5.1 \mathrm{E}-07$ & $4.8 \mathrm{E}-07$ & $4.7 \mathrm{E}-07$ & 4. $6 \mathrm{E}-07$ & $4.6 \mathrm{E}-07$ & $4.4 \mathrm{E}-07$ \\
\hline ESE & $3.4 \mathrm{E}-07$ & $3.2 \mathrm{E}-07$ & $3.1 \mathrm{E}-07$ & $3.1 \mathrm{E}-07$ & $3.1 \mathrm{E}-07$ & $3.0 \mathrm{E}-07$ \\
\hline $\mathrm{E}$ & $4.4 \mathrm{E}-07$ & $4.1 \mathrm{E}-07$ & $4.1 \mathrm{E}-07$ & $4.0 \mathrm{E}-07$ & $3.9 \mathrm{E}-07$ & $3.8 \mathrm{E}-07$ \\
\hline ENE & $3.8 \mathrm{E}-07$ & 3. $5 \mathrm{E}-07$ & $3.5 \mathrm{E}-07$ & $3.4 \mathrm{E}-07$ & $3.4 \mathrm{E}-07$ & $3.3 \mathrm{E}-07$ \\
\hline NE & $5.6 E-07$ & $5.2 \mathrm{E}-07$ & $5.2 \mathrm{E}-07$ & $5.0 \mathrm{E}-07$ & $5.0 \mathrm{E}-07$ & $4.8 E-07$ \\
\hline NNE & $3.6 \mathrm{E}-07$ & $3.4 \mathrm{E}-07$ & $3.3 \mathrm{E}-07$ & $3.2 \mathrm{E}-07$ & $3.2 \mathrm{E}-07$ & $3.1 \mathrm{E}-07$ \\
\hline
\end{tabular}

Feb 20, $2008 \quad 02: 30 \mathrm{pm}$

SUMMARY

Page 7

INDIVIDUAL LIFETIME RISK (deaths)

(A11 Radionuclides and Pathways)

\begin{tabular}{|c|c|c|c|c|c|c|c|}
\hline \multicolumn{8}{|c|}{ Distance $(\mathrm{m})$} \\
\hline Direction & 10344 & 10365 & 10472 & 10590 & 11103 & 11989 & 12522 \\
\hline $\begin{array}{r}N \\
N N W \\
N W \\
W N W \\
W \\
W S W \\
S W \\
S S W \\
S \\
S S E \\
S E \\
\text { ESE } \\
E \\
\text { ENE } \\
N E \\
N N E\end{array}$ & $\begin{array}{l}2.3 \mathrm{E}-13 \\
8.5 \mathrm{E}-14 \\
1.2 \mathrm{E}-13 \\
1.6 \mathrm{E}-13 \\
1.8 \mathrm{E}-13 \\
2.4 \mathrm{E}-13 \\
4.0 \mathrm{E}-13 \\
6.6 \mathrm{E}-13 \\
9.3 \mathrm{E}-13 \\
7.7 \mathrm{E}-13 \\
4.1 \mathrm{E}-13 \\
2.7 \mathrm{E}-13 \\
3.5 \mathrm{E}-13 \\
3.0 \mathrm{E}-13 \\
4.5 \mathrm{E}-13 \\
2.9 \mathrm{E}-13\end{array}$ & $\begin{array}{l}2.3 \mathrm{E}-13 \\
8.5 \mathrm{E}-14 \\
1.2 \mathrm{E}-13 \\
1.6 \mathrm{E}-13 \\
1.8 \mathrm{E}-13 \\
2.4 \mathrm{E}-13 \\
3.9 \mathrm{E}-13 \\
6.6 \mathrm{E}-13 \\
9.3 \mathrm{E}-13 \\
7.7 \mathrm{E}-13 \\
4.0 \mathrm{E}-13 \\
2.7 \mathrm{E}-13 \\
3.4 \mathrm{E}-13 \\
3.0 \mathrm{E}-13 \\
4.5 \mathrm{E}-13 \\
2.9 \mathrm{E}-13\end{array}$ & $\begin{array}{l}2.2 \mathrm{E}-13 \\
8.4 \mathrm{E}-14 \\
1.2 \mathrm{E}-13 \\
1.6 \mathrm{E}-13 \\
1.8 \mathrm{E}-13 \\
2.4 \mathrm{E}-13 \\
3.9 \mathrm{E}-13 \\
6.5 \mathrm{E}-13 \\
9.1 \mathrm{E}-13 \\
7.6 \mathrm{E}-13 \\
4.0 \mathrm{E}-13 \\
2.6 \mathrm{E}-13 \\
3.4 \mathrm{E}-13 \\
2.9 \mathrm{E}-13 \\
4.5 \mathrm{E}-13 \\
2.8 \mathrm{E}-13\end{array}$ & $\begin{array}{l}2.2 \mathrm{E}-13 \\
8.3 \mathrm{E}-14 \\
1.1 \mathrm{E}-13 \\
1.5 \mathrm{E}-13 \\
1.8 \mathrm{E}-13 \\
2.4 \mathrm{E}-13 \\
3.8 \mathrm{E}-13 \\
6.4 \mathrm{E}-13 \\
9.0 \mathrm{E}-13 \\
7.5 \mathrm{E}-13 \\
3.9 \mathrm{E}-13 \\
2.6 \mathrm{E}-13 \\
3.4 \mathrm{E}-13 \\
2.9 \mathrm{E}-13 \\
4.4 \mathrm{E}-13 \\
2.8 \mathrm{E}-13\end{array}$ & $\begin{array}{l}2.1 \mathrm{E}-13 \\
7.8 \mathrm{E}-14 \\
1.1 \mathrm{E}-13 \\
1.5 \mathrm{E}-13 \\
1.7 \mathrm{E}-13 \\
2.2 \mathrm{E}-13 \\
3.6 \mathrm{E}-13 \\
6.0 \mathrm{E}-13 \\
8.5 \mathrm{E}-13 \\
7.1 \mathrm{E}-13 \\
3.7 \mathrm{E}-13 \\
2.5 \mathrm{E}-13 \\
3.2 \mathrm{E}-13 \\
2.8 \mathrm{E}-13 \\
4.2 \mathrm{E}-13 \\
2.6 \mathrm{E}-13\end{array}$ & $\begin{array}{l}1.9 \mathrm{E}-13 \\
7.2 \mathrm{E}-14 \\
1.0 \mathrm{E}-13 \\
1.3 \mathrm{E}-13 \\
1.5 \mathrm{E}-13 \\
2.0 \mathrm{E}-13 \\
3.3 \mathrm{E}-13 \\
5.5 \mathrm{E}-13 \\
7.8 \mathrm{E}-13 \\
6.5 \mathrm{E}-13 \\
3.4 \mathrm{E}-13 \\
2.3 \mathrm{E}-13 \\
2.9 \mathrm{E}-13 \\
2.5 \mathrm{E}-13 \\
3.8 \mathrm{E}-13 \\
2.4 \mathrm{E}-13\end{array}$ & $\begin{array}{l}1.8 \mathrm{E}-13 \\
6.8 \mathrm{E}-14 \\
9.5 \mathrm{E}-14 \\
1.3 \mathrm{E}-13 \\
1.5 \mathrm{E}-13 \\
1.9 \mathrm{E}-13 \\
3.1 \mathrm{E}-13 \\
5.2 \mathrm{E}-13 \\
7.4 \mathrm{E}-13 \\
6.1 \mathrm{E}-13 \\
3.3 \mathrm{E}-13 \\
2.2 \mathrm{E}-13 \\
2.8 \mathrm{E}-13 \\
2.4 \mathrm{E}-13 \\
3.6 \mathrm{E}-13 \\
2.3 \mathrm{E}-13\end{array}$ \\
\hline \multicolumn{8}{|c|}{ Distance $(\mathrm{m})$} \\
\hline Direction & 13245 & 13286 & 13483 & 13612 & 13664 & 13959 & 14258 \\
\hline $\begin{array}{r}N \\
N N W \\
N W \\
W N W \\
W \\
W S W \\
\text { SW } \\
\text { SSW }\end{array}$ & $\begin{array}{l}1.7 \mathrm{E}-13 \\
6.4 \mathrm{E}-14 \\
8.9 \mathrm{E}-14 \\
1.2 \mathrm{E}-13 \\
1.4 \mathrm{E}-13 \\
1.8 \mathrm{E}-13 \\
2.9 \mathrm{E}-13 \\
4.9 \mathrm{E}-13\end{array}$ & $\begin{array}{l}1.7 \mathrm{E}-13 \\
6.4 \mathrm{E}-14 \\
8.9 \mathrm{E}-14 \\
1.2 \mathrm{E}-13 \\
1.4 \mathrm{E}-13 \\
1.8 \mathrm{E}-13 \\
2.9 \mathrm{E}-13 \\
4.9 \mathrm{E}-13\end{array}$ & $\begin{array}{l}1.7 \mathrm{E}-13 \\
6.3 \mathrm{E}-14 \\
8.7 \mathrm{E}-14 \\
1.2 \mathrm{E}-13 \\
1.3 \mathrm{E}-13 \\
1.8 \mathrm{E}-13 \\
2.9 \mathrm{E}-13 \\
4.8 \mathrm{E}-13\end{array}$ & $\begin{array}{r}1.7 \mathrm{E}-13 \\
6.2 \mathrm{E}-14 \\
8.7 \mathrm{E}-14 \\
1.2 \mathrm{E}-13 \\
1.3 \mathrm{E}-13 \\
1.8 \mathrm{E}-13 \\
2.8 \mathrm{E}-13 \\
4.7 \mathrm{E}-13 \\
\text { Page }\end{array}$ & $\begin{array}{l}1.6 \mathrm{E}-13 \\
6.2 \mathrm{E}-14 \\
8.6 \mathrm{E}-14 \\
1.2 \mathrm{E}-13 \\
1.3 \mathrm{E}-13 \\
1.7 \mathrm{E}-13 \\
2.8 \mathrm{E}-13 \\
4.7 \mathrm{E}-13\end{array}$ & $\begin{array}{l}1.6 \mathrm{E}-13 \\
6.0 \mathrm{E}-14 \\
8.4 \mathrm{E}-14 \\
1.1 \mathrm{E}-13 \\
1.3 \mathrm{E}-13 \\
1.7 \mathrm{E}-13 \\
2.8 \mathrm{E}-13 \\
4.6 \mathrm{E}-13\end{array}$ & $\begin{array}{l}1.6 \mathrm{E}-13 \\
5.9 \mathrm{E}-14 \\
8.2 \mathrm{E}-14 \\
1.1 \mathrm{E}-13 \\
1.3 \mathrm{E}-13 \\
1.7 \mathrm{E}-13 \\
2.7 \mathrm{E}-13 \\
4.5 \mathrm{E}-13\end{array}$ \\
\hline
\end{tabular}




\begin{tabular}{rlllllll}
\multicolumn{7}{c}{ O-15A.SUM } \\
S & $6.9 \mathrm{E}-13$ & $6.8 \mathrm{E}-13$ & $6.7 \mathrm{E}-13$ & $6.6 \mathrm{E}-13$ & $6.6 \mathrm{E}-13$ & $6.4 \mathrm{E}-13$ & $6.3 \mathrm{E}-13$ \\
SSE & $5.7 \mathrm{E}-13$ & $5.7 \mathrm{E}-13$ & $5.6 \mathrm{E}-13$ & $5.6 \mathrm{E}-13$ & $5.5 \mathrm{E}-13$ & $5.4 \mathrm{E}-13$ & $5.3 \mathrm{E}-13$ \\
SE & $3.0 \mathrm{E}-13$ & $3.0 \mathrm{E}-13$ & $3.0 \mathrm{E}-13$ & $3.0 \mathrm{E}-13$ & $2.9 \mathrm{E}-13$ & $2.9 \mathrm{E}-13$ & $2.8 \mathrm{E}-13$ \\
ESE & $2.0 \mathrm{E}-13$ & $2.0 \mathrm{E}-13$ & $2.0 \mathrm{E}-13$ & $2.0 \mathrm{E}-13$ & $2.0 \mathrm{E}-13$ & $1.9 \mathrm{E}-13$ & $1.9 \mathrm{E}-13$ \\
$\mathrm{E}$ & $2.6 \mathrm{E}-13$ & $2.6 \mathrm{E}-13$ & $2.6 \mathrm{E}-13$ & $2.5 \mathrm{E}-13$ & $2.5 \mathrm{E}-13$ & $2.5 \mathrm{E}-13$ & $2.4 \mathrm{E}-13$ \\
ENE & $2.2 \mathrm{E}-13$ & $2.2 \mathrm{E}-13$ & $2.2 \mathrm{E}-13$ & $2.2 \mathrm{E}-13$ & $2.2 \mathrm{E}-13$ & $2.1 \mathrm{E}-13$ & $2.1 \mathrm{E}-13$ \\
NE & $3.4 \mathrm{E}-13$ & $3.3 \mathrm{E}-13$ & $3.3 \mathrm{E}-13$ & $3.2 \mathrm{E}-13$ & $3.2 \mathrm{E}-13$ & $3.1 \mathrm{E}-13$ & $3.1 \mathrm{E}-13$ \\
NNE & $2.1 \mathrm{E}-13$ & $2.1 \mathrm{E}-13$ & $2.1 \mathrm{E}-13$ & $2.1 \mathrm{E}-13$ & $2.1 \mathrm{E}-13$ & $2.0 \mathrm{E}-13$ & $2.0 \mathrm{E}-13$
\end{tabular}

Feb 20, $2008 \quad 02: 30 \mathrm{pm}$

SUMMARY

Page 8

INDIVIDUAL LIFETIME RISK (deaths)

(A11 Radionuclides and Pathways)

\begin{tabular}{|c|c|c|c|c|c|c|}
\hline \multirow[b]{2}{*}{ Direction } & \multicolumn{6}{|c|}{ Distance $(\mathrm{m})$} \\
\hline & 14374 & 15241 & 15441 & 15784 & 15844 & 16323 \\
\hline $\begin{array}{r}N \\
N N W \\
N W \\
W N W \\
W \\
W S W \\
S W \\
S S W \\
S \\
\text { SSE } \\
\text { SE } \\
\text { ESE } \\
\text { E } \\
\text { ENE } \\
N E \\
\text { NNE }\end{array}$ & $\begin{array}{l}1.6 \mathrm{E}-13 \\
5.8 \mathrm{E}-14 \\
8.1 \mathrm{E}-14 \\
1.1 \mathrm{E}-13 \\
1.2 \mathrm{E}-13 \\
1.7 \mathrm{E}-13 \\
2.7 \mathrm{E}-13 \\
4.4 \mathrm{E}-13 \\
6.2 \mathrm{E}-13 \\
5.2 \mathrm{E}-13 \\
2.8 \mathrm{E}-13 \\
1.8 \mathrm{E}-13 \\
2.4 \mathrm{E}-13 \\
2.0 \mathrm{E}-13 \\
3.0 \mathrm{E}-13 \\
1.9 \mathrm{E}-13\end{array}$ & $\begin{array}{l}1.5 \mathrm{E}-13 \\
5.5 \mathrm{E}-14 \\
7.6 \mathrm{E}-14 \\
1.0 \mathrm{E}-13 \\
1.2 \mathrm{E}-13 \\
1.5 \mathrm{E}-13 \\
2.5 \mathrm{E}-13 \\
4.1 \mathrm{E}-13 \\
5.8 \mathrm{E}-13 \\
4.9 \mathrm{E}-13 \\
2.6 \mathrm{E}-13 \\
1.7 \mathrm{E}-13 \\
2.2 \mathrm{E}-13 \\
1.9 \mathrm{E}-13 \\
2.8 \mathrm{E}-13 \\
1.8 \mathrm{E}-13\end{array}$ & $\begin{array}{l}1.4 \mathrm{E}-13 \\
5.4 \mathrm{E}-14 \\
7.5 \mathrm{E}-14 \\
1.0 \mathrm{E}-13 \\
1.1 \mathrm{E}-13 \\
1.5 \mathrm{E}-13 \\
2.4 \mathrm{E}-13 \\
4.1 \mathrm{E}-13 \\
5.7 \mathrm{E}-13 \\
4.8 \mathrm{E}-13 \\
2.6 \mathrm{E}-13 \\
1.7 \mathrm{E}-13 \\
2.2 \mathrm{E}-13 \\
1.9 \mathrm{E}-13 \\
2.8 \mathrm{E}-13 \\
1.8 \mathrm{E}-13\end{array}$ & $\begin{array}{l}1.4 \mathrm{E}-13 \\
5.3 \mathrm{E}-14 \\
7.3 \mathrm{E}-14 \\
9.8 \mathrm{E}-14 \\
1.1 \mathrm{E}-13 \\
1.5 \mathrm{E}-13 \\
2.4 \mathrm{E}-13 \\
4.0 \mathrm{E}-13 \\
5.6 \mathrm{E}-13 \\
4.7 \mathrm{E}-13 \\
2.5 \mathrm{E}-13 \\
1.7 \mathrm{E}-13 \\
2.1 \mathrm{E}-13 \\
1.8 \mathrm{E}-13 \\
2.7 \mathrm{E}-13 \\
1.8 \mathrm{E}-13\end{array}$ & $\begin{array}{l}1.4 \mathrm{E}-13 \\
5.2 \mathrm{E}-14 \\
7.3 \mathrm{E}-14 \\
9.8 \mathrm{E}-14 \\
1.1 \mathrm{E}-13 \\
1.5 \mathrm{E}-13 \\
2.4 \mathrm{E}-13 \\
4.0 \mathrm{E}-13 \\
5.5 \mathrm{E}-13 \\
4.6 \mathrm{E}-13 \\
2.5 \mathrm{E}-13 \\
1.7 \mathrm{E}-13 \\
2.1 \mathrm{E}-13 \\
1.8 \mathrm{E}-13 \\
2.7 \mathrm{E}-13 \\
1.7 \mathrm{E}-13\end{array}$ & $\begin{array}{l}1.4 \mathrm{E}-13 \\
5.1 \mathrm{E}-14 \\
7.1 \mathrm{E}-14 \\
9.5 \mathrm{E}-14 \\
1.1 \mathrm{E}-13 \\
1.4 \mathrm{E}-13 \\
2.3 \mathrm{E}-13 \\
3.8 \mathrm{E}-13 \\
5.3 \mathrm{E}-13 \\
4.5 \mathrm{E}-13 \\
2.4 \mathrm{E}-13 \\
1.6 \mathrm{E}-13 \\
2.1 \mathrm{E}-13 \\
1.8 \mathrm{E}-13 \\
2.6 \mathrm{E}-13 \\
1.7 \mathrm{E}-13\end{array}$ \\
\hline
\end{tabular}




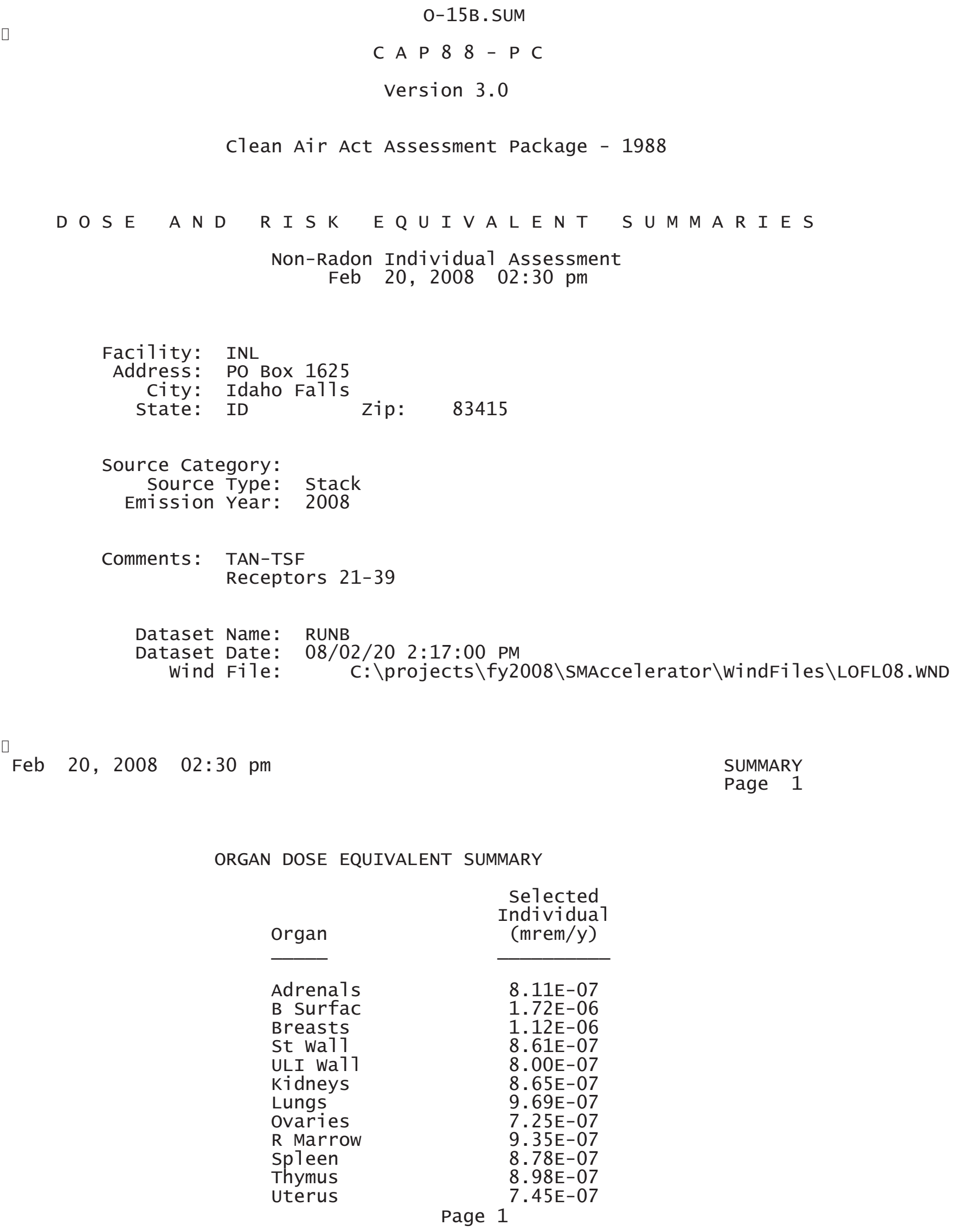

\begin{tabular}{lr} 
ORGAN DOSE EQUIVALENT SUMMARY \\
$\begin{array}{c}\text { Selected } \\
\text { Individual } \\
\text { (mrem/y) }\end{array}$ \\
\cline { 2 - 2 } Organ & $8.11 \mathrm{E}-07$ \\
\hline Adrena1s & $1.72 \mathrm{E}-06$ \\
B Surfac & $1.12 \mathrm{E}-06$ \\
Breasts & $8.61 \mathrm{E}-07$ \\
St wa17 & $8.00 \mathrm{E}-07$ \\
ULI wa71 & $8.65 \mathrm{E}-07$ \\
Kidneys & $9.69 \mathrm{E}-07$ \\
Lungs & $7.25 \mathrm{E}-07$ \\
Ovaries & $9.35 \mathrm{E}-07$ \\
R Marrow & $8.78 \mathrm{E}-07$ \\
Spleen & $8.98 \mathrm{E}-07$ \\
Thymus & $7.45 \mathrm{E}-07$ \\
Uterus &
\end{tabular}




$\begin{array}{lr} & \text { O-15B.SUM } \\ \text { B1d wa11 } & 8.08 \mathrm{E}-07 \\ \text { Brain } & 1.03 \mathrm{E}-06 \\ \text { Esophagu } & 7.96 \mathrm{E}-07 \\ \text { SI wa11 } & 7.66 \mathrm{E}-07 \\ \text { LLI Wa11 } & 7.80 \mathrm{E}-07 \\ \text { Liver } & 8.72 \mathrm{E}-07 \\ \text { Muscle } & 9.47 \mathrm{E}-07 \\ \text { Pancreas } & 7.60 \mathrm{E}-07 \\ \text { Skin } & 2.12 \mathrm{E}-06 \\ \text { Testes } & 9.75 \mathrm{E}-07 \\ \text { Thyroid } & 9.94 \mathrm{E}-07 \\ & \\ \text { EFFEC } & 9.37 \mathrm{E}-07\end{array}$

PATHWAY EFFECTIVE DOSE EQUIVALENT SUMMARY

\begin{tabular}{lc} 
Pathway & $\begin{array}{c}\text { selected } \\
\text { Individual } \\
(\mathrm{mrem} / \mathrm{y})\end{array}$ \\
\cline { 2 - 2 } INGESTION & $0.00 \mathrm{E}+00$ \\
INHALATION & $0.00 \mathrm{E}+00$ \\
AIR IMMERSION & $9.37 \mathrm{E}-07$ \\
GROUND SURFACE & $0.00 \mathrm{E}+00$ \\
INTERNAL & $0.00 \mathrm{E}+00$ \\
EXTERNAL & $9.37 \mathrm{E}-07$ \\
TOTAL & $9.37 \mathrm{E}-07$
\end{tabular}

Feb 20, $2008 \quad 02: 30$ pm

SUMMARY

Page 2

NUCLIDE EFFECTIVE DOSE EQUIVALENT SUMMARY

Nuclide

$0-15$

TOTAL

Feb 20, $2008 \quad 02: 30 \mathrm{pm}$

$$
\begin{gathered}
\begin{array}{c}
\text { Selected } \\
\text { Individual } \\
(\mathrm{mrem} / \mathrm{y})
\end{array} \\
\hline 9.37 \mathrm{E}-07 \\
9.37 \mathrm{E}-07
\end{gathered}
$$

SUMMARY

Page 3

CANCER RISK SUMMARY

Cancer

Esophagu
Selected Individual

Total Lifetime

Fatal Cancer Risk

Page $2^{9 \cdot 16 \mathrm{E}-15}$ 


\begin{tabular}{lr} 
& \multicolumn{1}{c}{ 0-15B. SUM } \\
Stomach & $3.48 \mathrm{E}-14$ \\
Colon & $8.17 \mathrm{E}-14$ \\
Liver & $1.32 \mathrm{E}-14$ \\
LUNG & $9.47 \mathrm{E}-14$ \\
Bone & $1.64 \mathrm{E}-15$ \\
Skin & $2.12 \mathrm{E}-15$ \\
Breast & $5.40 \mathrm{E}-14$ \\
Ovary & $1.03 \mathrm{E}-14$ \\
B Tadder & $1.95 \mathrm{E}-14$ \\
Kidneys & $4.50 \mathrm{E}-15$ \\
Thyroid & $3.16 \mathrm{E}-15$ \\
Leukemia & $5.25 \mathrm{E}-14$ \\
Residua1 & $1.25 \mathrm{E}-13$ \\
Tota1 & $5.07 \mathrm{E}-13$ \\
& \\
TOTAL & $1.01 \mathrm{E}-12$
\end{tabular}

PATHWAY RISK SUMMARY

\begin{tabular}{lc} 
Pathway & $\begin{array}{c}\text { Selected Individual } \\
\text { Total Lifetime } \\
\text { Fatal Cancer Risk }\end{array}$ \\
\cline { 2 - 2 } INGESTION & $0.00 \mathrm{E}+00$ \\
INHALATION & $0.00 \mathrm{E}+00$ \\
AIR IMMERSION & $5.07 \mathrm{E}-13$ \\
GROUND SURFACE & $0.00 \mathrm{E}+00$ \\
INTERNAL & $0.00 \mathrm{E}+00$ \\
EXTERNAL & $5.07 \mathrm{E}-13$ \\
TOTAL & $5.07 \mathrm{E}-13$
\end{tabular}

Feb 20, $2008 \quad 02: 30$ pm

SUMMARY Page 4

NUCLIDE RISK SUMMARY

Nuclide

$0-15$

TOTAL

$\square$

Feb 20, 2008 02:30 pm
Selected Individual

Total Lifetime Fatal Cancer Risk

$5.07 E-13$

$5.07 \mathrm{E}-13$

INDIVIDUAL EFFECTIVE DOSE EQUIVALENT RATE (mrem/y) (A11 Radionuclides and Pathways)

Distance $(m)$ 


\begin{tabular}{rccccccc} 
Direction & 17035 & 17329 & 18607 & 18834 & 18860 & 18890 & 19860 \\
\hline N & $2.4 \mathrm{E}-07$ & $2.3 \mathrm{E}-07$ & $2.2 \mathrm{E}-07$ & $2.1 \mathrm{E}-07$ & $2.1 \mathrm{E}-07$ & $2.1 \mathrm{E}-07$ & $2.0 \mathrm{E}-07$ \\
NNW & $8.9 \mathrm{E}-08$ & $8.8 \mathrm{E}-08$ & $8.1 \mathrm{E}-08$ & $8.0 \mathrm{E}-08$ & $8.0 \mathrm{E}-08$ & $8.0 \mathrm{E}-08$ & $7.5 \mathrm{E}-08$ \\
NW & $1.2 \mathrm{E}-07$ & $1.2 \mathrm{E}-07$ & $1.1 \mathrm{E}-07$ & $1.1 \mathrm{E}-07$ & $1.1 \mathrm{E}-07$ & $1.1 \mathrm{E}-07$ & $1.1 \mathrm{E}-07$ \\
WNW & $1.7 \mathrm{E}-07$ & $1.6 \mathrm{E}-07$ & $1.5 \mathrm{E}-07$ & $1.5 \mathrm{E}-07$ & $1.5 \mathrm{E}-07$ & $1.5 \mathrm{E}-07$ & $1.4 \mathrm{E}-07$ \\
W & $1.9 \mathrm{E}-07$ & $1.9 \mathrm{E}-07$ & $1.7 \mathrm{E}-07$ & $1.7 \mathrm{E}-07$ & $1.7 \mathrm{E}-07$ & $1.7 \mathrm{E}-07$ & $1.6 \mathrm{E}-07$ \\
WSW & $2.5 \mathrm{E}-07$ & $2.5 \mathrm{E}-07$ & $2.3 \mathrm{E}-07$ & $2.2 \mathrm{E}-07$ & $2.2 \mathrm{E}-07$ & $2.2 \mathrm{E}-07$ & $2.1 \mathrm{E}-07$ \\
SW & $4.0 \mathrm{E}-07$ & $4.0 \mathrm{E}-07$ & $3.6 \mathrm{E}-07$ & $3.6 \mathrm{E}-07$ & $3.6 \mathrm{E}-07$ & $3.6 \mathrm{E}-07$ & $3.4 \mathrm{E}-07$ \\
SSW & $6.7 \mathrm{E}-07$ & $6.6 \mathrm{E}-07$ & $6.0 \mathrm{E}-07$ & $6.0 \mathrm{E}-07$ & $6.0 \mathrm{E}-07$ & $5.9 \mathrm{E}-07$ & $5.6 \mathrm{E}-07$ \\
$\mathrm{~S}$ & $9.4 \mathrm{E}-07$ & $9.2 \mathrm{E}-07$ & $8.4 \mathrm{E}-07$ & $8.3 \mathrm{E}-07$ & $8.3 \mathrm{E}-07$ & $8.3 \mathrm{E}-07$ & $7.8 \mathrm{E}-07$ \\
SSE & $7.9 \mathrm{E}-07$ & $7.7 \mathrm{E}-07$ & $7.1 \mathrm{E}-07$ & $7.0 \mathrm{E}-07$ & $7.0 \mathrm{E}-07$ & $6.9 \mathrm{E}-07$ & $6.5 \mathrm{E}-07$ \\
$\mathrm{SE}$ & $4.2 \mathrm{E}-07$ & $4.1 \mathrm{E}-07$ & $3.8 \mathrm{E}-07$ & $3.8 \mathrm{E}-07$ & $3.8 \mathrm{E}-07$ & $3.8 \mathrm{E}-07$ & $3.5 \mathrm{E}-07$ \\
ESE & $2.8 \mathrm{E}-07$ & $2.8 \mathrm{E}-07$ & $2.6 \mathrm{E}-07$ & $2.5 \mathrm{E}-07$ & $2.5 \mathrm{E}-07$ & $2.5 \mathrm{E}-07$ & $2.4 \mathrm{E}-07$ \\
$\mathrm{E}$ & $3.6 \mathrm{E}-07$ & $3.6 \mathrm{E}-07$ & $3.3 \mathrm{E}-07$ & $3.2 \mathrm{E}-07$ & $3.2 \mathrm{E}-07$ & $3.2 \mathrm{E}-07$ & $3.1 \mathrm{E}-07$ \\
ENE & $3.1 \mathrm{E}-07$ & $3.0 \mathrm{E}-07$ & $2.8 \mathrm{E}-07$ & $2.8 \mathrm{E}-07$ & $2.8 \mathrm{E}-07$ & $2.8 \mathrm{E}-07$ & $2.6 \mathrm{E}-07$ \\
NE & $4.6 \mathrm{E}-07$ & $4.5 \mathrm{E}-07$ & $4.1 \mathrm{E}-07$ & $4.1 \mathrm{E}-07$ & $4.1 \mathrm{E}-07$ & $4.1 \mathrm{E}-07$ & $3.8 \mathrm{E}-07$ \\
NNE & $3.0 \mathrm{E}-07$ & $2.9 \mathrm{E}-07$ & $2.7 \mathrm{E}-07$ & $2.6 \mathrm{E}-07$ & $2.6 \mathrm{E}-07$ & $2.6 \mathrm{E}-07$ & $2.5 \mathrm{E}-07$ \\
\hline
\end{tabular}

Distance $(\mathrm{m})$

\begin{tabular}{|c|c|c|c|c|c|c|c|}
\hline Direction & 19891 & 20457 & 21314 & 21349 & 22159 & 23521 & 24430 \\
\hline $\begin{array}{r}N \\
N N W \\
N W \\
W N W \\
W \\
W S W \\
\text { SW } \\
\text { SSW } \\
S \\
\text { SSE } \\
\text { SE } \\
\text { ESE } \\
\text { E } \\
\text { ENE } \\
N E \\
\text { NNE }\end{array}$ & $\begin{array}{l}2.0 \mathrm{E}-07 \\
7.5 \mathrm{E}-08 \\
1.1 \mathrm{E}-07 \\
1.4 \mathrm{E}-07 \\
1.6 \mathrm{E}-07 \\
2.1 \mathrm{E}-07 \\
3.4 \mathrm{E}-07 \\
5.6 \mathrm{E}-07 \\
7.8 \mathrm{E}-07 \\
6.5 \mathrm{E}-07 \\
3.5 \mathrm{E}-07 \\
2.4 \mathrm{E}-07 \\
3.0 \mathrm{E}-07 \\
2.6 \mathrm{E}-07 \\
3.8 \mathrm{E}-07 \\
2.5 \mathrm{E}-07\end{array}$ & $\begin{array}{l}1.9 \mathrm{E}-07 \\
7.3 \mathrm{E}-08 \\
1.0 \mathrm{E}-07 \\
1.4 \mathrm{E}-07 \\
1.5 \mathrm{E}-07 \\
2.0 \mathrm{E}-07 \\
3.3 \mathrm{E}-07 \\
5.4 \mathrm{E}-07 \\
7.5 \mathrm{E}-07 \\
6.3 \mathrm{E}-07 \\
3.4 \mathrm{E}-07 \\
2.3 \mathrm{E}-07 \\
3.0 \mathrm{E}-07 \\
2.5 \mathrm{E}-07 \\
3.7 \mathrm{E}-07 \\
2.4 \mathrm{E}-07\end{array}$ & $\begin{array}{l}1.9 \mathrm{E}-07 \\
6.9 \mathrm{E}-08 \\
9.7 \mathrm{E}-08 \\
1.3 \mathrm{E}-07 \\
1.5 \mathrm{E}-07 \\
2.0 \mathrm{E}-07 \\
3.1 \mathrm{E}-07 \\
5.2 \mathrm{E}-07 \\
7.2 \mathrm{E}-07 \\
6.0 \mathrm{E}-07 \\
3.3 \mathrm{E}-07 \\
2.2 \mathrm{E}-07 \\
2.8 \mathrm{E}-07 \\
2.4 \mathrm{E}-07 \\
3.5 \mathrm{E}-07 \\
2.3 \mathrm{E}-07\end{array}$ & $\begin{array}{l}1.8 \mathrm{E}-07 \\
6.9 \mathrm{E}-08 \\
9.7 \mathrm{E}-08 \\
1.3 \mathrm{E}-07 \\
1.5 \mathrm{E}-07 \\
1.9 \mathrm{E}-07 \\
3.1 \mathrm{E}-07 \\
5.1 \mathrm{E}-07 \\
7.1 \mathrm{E}-07 \\
6.0 \mathrm{E}-07 \\
3.3 \mathrm{E}-07 \\
2.2 \mathrm{E}-07 \\
2.8 \mathrm{E}-07 \\
2.4 \mathrm{E}-07 \\
3.5 \mathrm{E}-07 \\
2.3 \mathrm{E}-07\end{array}$ & $\begin{array}{l}1.8 \mathrm{E}-07 \\
6.7 \mathrm{E}-08 \\
9.3 \mathrm{E}-08 \\
1.2 \mathrm{E}-07 \\
1.4 \mathrm{E}-07 \\
1.9 \mathrm{E}-07 \\
3.0 \mathrm{E}-07 \\
4.9 \mathrm{E}-07 \\
6.8 \mathrm{E}-07 \\
5.8 \mathrm{E}-07 \\
3.1 \mathrm{E}-07 \\
2.1 \mathrm{E}-07 \\
2.7 \mathrm{E}-07 \\
2.3 \mathrm{E}-07 \\
3.4 \mathrm{E}-07 \\
2.2 \mathrm{E}-07\end{array}$ & $\begin{array}{l}1.7 \mathrm{E}-07 \\
6.2 \mathrm{E}-08 \\
8.7 \mathrm{E}-08 \\
1.2 \mathrm{E}-07 \\
1.3 \mathrm{E}-07 \\
1.7 \mathrm{E}-07 \\
2.8 \mathrm{E}-07 \\
4.6 \mathrm{E}-07 \\
6.4 \mathrm{E}-07 \\
5.4 \mathrm{E}-07 \\
2.9 \mathrm{E}-07 \\
2.0 \mathrm{E}-07 \\
2.5 \mathrm{E}-07 \\
2.1 \mathrm{E}-07 \\
3.1 \mathrm{E}-07 \\
2.0 \mathrm{E}-07\end{array}$ & $\begin{array}{l}1.6 \mathrm{E}-07 \\
6.0 \mathrm{E}-08 \\
8.4 \mathrm{E}-08 \\
1.1 \mathrm{E}-07 \\
1.3 \mathrm{E}-07 \\
1.7 \mathrm{E}-07 \\
2.6 \mathrm{E}-07 \\
4.4 \mathrm{E}-07 \\
6.1 \mathrm{E}-07 \\
5.1 \mathrm{E}-07 \\
2.8 \mathrm{E}-07 \\
1.9 \mathrm{E}-07 \\
2.4 \mathrm{E}-07 \\
2.1 \mathrm{E}-07 \\
3.0 \mathrm{E}-07 \\
2.0 \mathrm{E}-07\end{array}$ \\
\hline
\end{tabular}

$\square$

Feb 20, $2008 \quad 02: 30 \mathrm{pm}$

SUMMARY

Page 6

INDIVIDUAL EFFECTIVE DOSE EQUIVALENT RATE (mrem/y) (A11 Radionuclides and Pathways)

\begin{tabular}{llllll}
\hline & \multicolumn{4}{c}{ Distance $(\mathrm{m})$} \\
\cline { 2 - 5 } Direction & 24545 & 25171 & 26794 & 27275 & 27389 \\
\hline
\end{tabular}

\begin{tabular}{rrrrrr}
\hline $\mathrm{N}$ & $1.6 \mathrm{E}-07$ & $1.5 \mathrm{E}-07$ & $1.4 \mathrm{E}-07$ & $1.4 \mathrm{E}-07$ & $1.4 \mathrm{E}-07$ \\
$\mathrm{NNW}$ & $5.9 \mathrm{E}-08$ & $5.8 \mathrm{E}-08$ & $5.4 \mathrm{E}-08$ & $5.3 \mathrm{E}-08$ & $5.3 \mathrm{E}-08$ \\
& & & \multicolumn{4}{c}{$\begin{array}{c}\text { Page } \\
\end{array}$} & &
\end{tabular}




\begin{tabular}{rlllll} 
& & & & \multicolumn{2}{c}{ O-15B.SUM } \\
NW & $8.3 \mathrm{E}-08$ & $8.1 \mathrm{E}-08$ & $7.6 \mathrm{E}-08$ & $7.4 \mathrm{E}-08$ & $7.4 \mathrm{E}-08$ \\
WNW & $1.1 \mathrm{E}-07$ & $1.1 \mathrm{E}-07$ & $1.0 \mathrm{E}-07$ & $9.9 \mathrm{E}-08$ & $9.9 \mathrm{E}-08$ \\
$\mathrm{~W}$ & $1.3 \mathrm{E}-07$ & $1.2 \mathrm{E}-07$ & $1.1 \mathrm{E}-07$ & $1.1 \mathrm{E}-07$ & $1.1 \mathrm{E}-07$ \\
WSW & $1.7 \mathrm{E}-07$ & $1.6 \mathrm{E}-07$ & $1.5 \mathrm{E}-07$ & $1.5 \mathrm{E}-07$ & $1.5 \mathrm{E}-07$ \\
SW & $2.6 \mathrm{E}-07$ & $2.6 \mathrm{E}-07$ & $2.4 \mathrm{E}-07$ & $2.3 \mathrm{E}-07$ & $2.3 \mathrm{E}-07$ \\
SSW & $4.4 \mathrm{E}-07$ & $4.2 \mathrm{E}-07$ & $3.9 \mathrm{E}-07$ & $3.9 \mathrm{E}-07$ & $3.8 \mathrm{E}-07$ \\
$\mathrm{~S}$ & $6.1 \mathrm{E}-07$ & $5.9 \mathrm{E}-07$ & $5.5 \mathrm{E}-07$ & $5.3 \mathrm{E}-07$ & $5.3 \mathrm{E}-07$ \\
SSE & $5.1 \mathrm{E}-07$ & $4.9 \mathrm{E}-07$ & $4.6 \mathrm{E}-07$ & $4.5 \mathrm{E}-07$ & $4.5 \mathrm{E}-07$ \\
SE & $2.8 \mathrm{E}-07$ & $2.7 \mathrm{E}-07$ & $2.5 \mathrm{E}-07$ & $2.5 \mathrm{E}-07$ & $2.5 \mathrm{E}-07$ \\
ESE & $1.9 \mathrm{E}-07$ & $1.8 \mathrm{E}-07$ & $1.7 \mathrm{E}-07$ & $1.7 \mathrm{E}-07$ & $1.7 \mathrm{E}-07$ \\
$\mathrm{E}$ & $2.4 \mathrm{E}-07$ & $2.3 \mathrm{E}-07$ & $2.2 \mathrm{E}-07$ & $2.1 \mathrm{E}-07$ & $2.1 \mathrm{E}-07$ \\
ENE & $2.0 \mathrm{E}-07$ & $2.0 \mathrm{E}-07$ & $1.8 \mathrm{E}-07$ & $1.8 \mathrm{E}-07$ & $1.8 \mathrm{E}-07$ \\
$\mathrm{NE}$ & $3.0 \mathrm{E}-07$ & $2.9 \mathrm{E}-07$ & $2.7 \mathrm{E}-07$ & $2.6 \mathrm{E}-07$ & $2.6 \mathrm{E}-07$ \\
NNE & $1.9 \mathrm{E}-07$ & $1.9 \mathrm{E}-07$ & $1.8 \mathrm{E}-07$ & $1.7 \mathrm{E}-07$ & $1.7 \mathrm{E}-07$ \\
& & & & & \\
\hline
\end{tabular}

Feb 20, $2008 \quad 02: 30 \mathrm{pm}$

SUMMARY

Page 7

INDIVIDUAL LIFETIME RISK (deaths)

(A11 Radionuclides and Pathways)

\begin{tabular}{|c|c|c|c|c|c|c|c|}
\hline \multicolumn{8}{|c|}{ Distance $(\mathrm{m})$} \\
\hline Direction & 17035 & 17329 & 18607 & 18834 & 18860 & 18890 & 19860 \\
\hline $\begin{array}{r}N \\
N N W \\
N W \\
W N W \\
W \\
W S W \\
S W \\
S S W \\
S \\
S S E \\
S E \\
\text { ESE } \\
E \\
\text { ENE } \\
N E \\
N N E\end{array}$ & $\begin{array}{l}1.3 \mathrm{E}-13 \\
4.8 \mathrm{E}-14 \\
6.7 \mathrm{E}-14 \\
9.0 \mathrm{E}-14 \\
1.0 \mathrm{E}-13 \\
1.4 \mathrm{E}-13 \\
2.2 \mathrm{E}-13 \\
3.6 \mathrm{E}-13 \\
5.1 \mathrm{E}-13 \\
4.3 \mathrm{E}-13 \\
2.3 \mathrm{E}-13 \\
1.5 \mathrm{E}-13 \\
2.0 \mathrm{E}-13 \\
1.7 \mathrm{E}-13 \\
2.5 \mathrm{E}-13 \\
1.6 \mathrm{E}-13\end{array}$ & $\begin{array}{l}1.3 \mathrm{E}-13 \\
4.7 \mathrm{E}-14 \\
6.6 \mathrm{E}-14 \\
8.9 \mathrm{E}-14 \\
1.0 \mathrm{E}-13 \\
1.3 \mathrm{E}-13 \\
2.1 \mathrm{E}-13 \\
3.6 \mathrm{E}-13 \\
5.0 \mathrm{E}-13 \\
4.2 \mathrm{E}-13 \\
2.2 \mathrm{E}-13 \\
1.5 \mathrm{E}-13 \\
1.9 \mathrm{E}-13 \\
1.6 \mathrm{E}-13 \\
2.4 \mathrm{E}-13 \\
1.6 \mathrm{E}-13\end{array}$ & $\begin{array}{l}1.2 \mathrm{E}-13 \\
4.4 \mathrm{E}-14 \\
6.1 \mathrm{E}-14 \\
8.2 \mathrm{E}-14 \\
9.3 \mathrm{E}-14 \\
1.2 \mathrm{E}-13 \\
2.0 \mathrm{E}-13 \\
3.3 \mathrm{E}-13 \\
4.6 \mathrm{E}-13 \\
3.8 \mathrm{E}-13 \\
2.1 \mathrm{E}-13 \\
1.4 \mathrm{E}-13 \\
1.8 \mathrm{E}-13 \\
1.5 \mathrm{E}-13 \\
2.2 \mathrm{E}-13 \\
1.4 \mathrm{E}-13\end{array}$ & $\begin{array}{l}1.2 \mathrm{E}-13 \\
4.3 \mathrm{E}-14 \\
6.0 \mathrm{E}-14 \\
8.1 \mathrm{E}-14 \\
9.2 \mathrm{E}-14 \\
1.2 \mathrm{E}-13 \\
1.9 \mathrm{E}-13 \\
3.2 \mathrm{E}-13 \\
4.5 \mathrm{E}-13 \\
3.8 \mathrm{E}-13 \\
2.0 \mathrm{E}-13 \\
1.4 \mathrm{E}-13 \\
1.8 \mathrm{E}-13 \\
1.5 \mathrm{E}-13 \\
2.2 \mathrm{E}-13 \\
1.4 \mathrm{E}-13\end{array}$ & $\begin{array}{l}1.1 \mathrm{E}-13 \\
4.3 \mathrm{E}-14 \\
6.0 \mathrm{E}-14 \\
8.1 \mathrm{E}-14 \\
9.2 \mathrm{E}-14 \\
1.2 \mathrm{E}-13 \\
1.9 \mathrm{E}-13 \\
3.2 \mathrm{E}-13 \\
4.5 \mathrm{E}-13 \\
3.8 \mathrm{E}-13 \\
2.0 \mathrm{E}-13 \\
1.4 \mathrm{E}-13 \\
1.8 \mathrm{E}-13 \\
1.5 \mathrm{E}-13 \\
2.2 \mathrm{E}-13 \\
1.4 \mathrm{E}-13\end{array}$ & $\begin{array}{l}1.1 \mathrm{E}-13 \\
4.3 \mathrm{E}-14 \\
6.0 \mathrm{E}-14 \\
8.1 \mathrm{E}-14 \\
9.2 \mathrm{E}-14 \\
1.2 \mathrm{E}-13 \\
1.9 \mathrm{E}-13 \\
3.2 \mathrm{E}-13 \\
4.5 \mathrm{E}-13 \\
3.8 \mathrm{E}-13 \\
2.0 \mathrm{E}-13 \\
1.4 \mathrm{E}-13 \\
1.7 \mathrm{E}-13 \\
1.5 \mathrm{E}-13 \\
2.2 \mathrm{E}-13 \\
1.4 \mathrm{E}-13\end{array}$ & $\begin{array}{l}1.1 \mathrm{E}-13 \\
4.1 \mathrm{E}-14 \\
5.7 \mathrm{E}-14 \\
7.6 \mathrm{E}-14 \\
8.7 \mathrm{E}-14 \\
1.1 \mathrm{E}-13 \\
1.8 \mathrm{E}-13 \\
3.0 \mathrm{E}-13 \\
4.2 \mathrm{E}-13 \\
3.5 \mathrm{E}-13 \\
1.9 \mathrm{E}-13 \\
1.3 \mathrm{E}-13 \\
1.7 \mathrm{E}-13 \\
1.4 \mathrm{E}-13 \\
2.1 \mathrm{E}-13 \\
1.3 \mathrm{E}-13\end{array}$ \\
\hline \multicolumn{8}{|c|}{ Distance $(\mathrm{m})$} \\
\hline Direction & 19891 & 20457 & 21314 & 21349 & 22159 & 23521 & 24430 \\
\hline $\begin{array}{r}N \\
N N W \\
N W \\
W N W \\
W \\
W S W \\
\text { SW } \\
\text { SSW }\end{array}$ & $\begin{array}{l}1.1 \mathrm{E}-13 \\
4.1 \mathrm{E}-14 \\
5.7 \mathrm{E}-14 \\
7.6 \mathrm{E}-14 \\
8.6 \mathrm{E}-14 \\
1.1 \mathrm{E}-13 \\
1.8 \mathrm{E}-13 \\
3.0 \mathrm{E}-13\end{array}$ & $\begin{array}{l}1.0 \mathrm{E}-13 \\
3.9 \mathrm{E}-14 \\
5.5 \mathrm{E}-14 \\
7.4 \mathrm{E}-14 \\
8.4 \mathrm{E}-14 \\
1.1 \mathrm{E}-13 \\
1.8 \mathrm{E}-13 \\
2.9 \mathrm{E}-13\end{array}$ & $\begin{array}{l}1.0 \mathrm{E}-13 \\
3.8 \mathrm{E}-14 \\
5.3 \mathrm{E}-14 \\
7.1 \mathrm{E}-14 \\
8.0 \mathrm{E}-14 \\
1.1 \mathrm{E}-13 \\
1.7 \mathrm{E}-13 \\
2.8 \mathrm{E}-13\end{array}$ & $\begin{array}{r}1.0 \mathrm{E}-13 \\
3.8 \mathrm{E}-14 \\
5.3 \mathrm{E}-14 \\
7.0 \mathrm{E}-14 \\
8.0 \mathrm{E}-14 \\
1.1 \mathrm{E}-13 \\
1.7 \mathrm{E}-13 \\
2.8 \mathrm{E}-13 \\
\text { Page }\end{array}$ & $\begin{array}{l}9.6 \mathrm{E}-14 \\
3.6 \mathrm{E}-14 \\
5.0 \mathrm{E}-14 \\
6.8 \mathrm{E}-14 \\
7.7 \mathrm{E}-14 \\
1.0 \mathrm{E}-13 \\
1.6 \mathrm{E}-13 \\
2.7 \mathrm{E}-13\end{array}$ & $\begin{array}{l}9.0 \mathrm{E}-14 \\
3.4 \mathrm{E}-14 \\
4.7 \mathrm{E}-14 \\
6.3 \mathrm{E}-14 \\
7.2 \mathrm{E}-14 \\
9.5 \mathrm{E}-14 \\
1.5 \mathrm{E}-13 \\
2.5 \mathrm{E}-13\end{array}$ & $\begin{array}{l}8.6 \mathrm{E}-14 \\
3.2 \mathrm{E}-14 \\
4.5 \mathrm{E}-14 \\
6.1 \mathrm{E}-14 \\
6.9 \mathrm{E}-14 \\
9.1 \mathrm{E}-14 \\
1.4 \mathrm{E}-13 \\
2.4 \mathrm{E}-13\end{array}$ \\
\hline
\end{tabular}




\begin{tabular}{rlllllll} 
& \multicolumn{7}{c}{ O-15B.SUM } \\
S & $4.2 \mathrm{E}-13$ & $4.1 \mathrm{E}-13$ & $3.9 \mathrm{E}-13$ & $3.9 \mathrm{E}-13$ & $3.7 \mathrm{E}-13$ & $3.4 \mathrm{E}-13$ & $3.3 \mathrm{E}-13$ \\
SSE & $3.5 \mathrm{E}-13$ & $3.4 \mathrm{E}-13$ & $3.3 \mathrm{E}-13$ & $3.3 \mathrm{E}-13$ & $3.1 \mathrm{E}-13$ & $2.9 \mathrm{E}-13$ & $2.8 \mathrm{E}-13$ \\
SE & $1.9 \mathrm{E}-13$ & $1.9 \mathrm{E}-13$ & $1.8 \mathrm{E}-13$ & $1.8 \mathrm{E}-13$ & $1.7 \mathrm{E}-13$ & $1.6 \mathrm{E}-13$ & $1.5 \mathrm{E}-13$ \\
ESE & $1.3 \mathrm{E}-13$ & $1.2 \mathrm{E}-13$ & $1.2 \mathrm{E}-13$ & $1.2 \mathrm{E}-13$ & $1.1 \mathrm{E}-13$ & $1.1 \mathrm{E}-13$ & $1.0 \mathrm{E}-13$ \\
$\mathrm{E}$ & $1.7 \mathrm{E}-13$ & $1.6 \mathrm{E}-13$ & $1.5 \mathrm{E}-13$ & $1.5 \mathrm{E}-13$ & $1.5 \mathrm{E}-13$ & $1.4 \mathrm{E}-13$ & $1.3 \mathrm{E}-13$ \\
ENE & $1.4 \mathrm{E}-13$ & $1.4 \mathrm{E}-13$ & $1.3 \mathrm{E}-13$ & $1.3 \mathrm{E}-13$ & $1.2 \mathrm{E}-13$ & $1.2 \mathrm{E}-13$ & $1.1 \mathrm{E}-13$ \\
NE & $2.1 \mathrm{E}-13$ & $2.0 \mathrm{E}-13$ & $1.9 \mathrm{E}-13$ & $1.9 \mathrm{E}-13$ & $1.8 \mathrm{E}-13$ & $1.7 \mathrm{E}-13$ & $1.6 \mathrm{E}-13$ \\
NNE & $1.3 \mathrm{E}-13$ & $1.3 \mathrm{E}-13$ & $1.2 \mathrm{E}-13$ & $1.2 \mathrm{E}-13$ & $1.2 \mathrm{E}-13$ & $1.1 \mathrm{E}-13$ & $1.1 \mathrm{E}-13$
\end{tabular}

Feb 20, $2008 \quad 02: 30 \mathrm{pm}$

SUMMARY

Page 8

INDIVIDUAL LIFETIME RISK (deaths)

(A11 Radionuclides and Pathways)

\begin{tabular}{|c|c|c|c|c|c|}
\hline \multirow[b]{2}{*}{ Direction } & \multicolumn{5}{|c|}{ Distance $(\mathrm{m})$} \\
\hline & 24545 & 25171 & 26794 & 27275 & 27389 \\
\hline $\begin{array}{r}N \\
N N W \\
N W \\
W N W \\
W \\
W S W \\
S W \\
\text { SSW } \\
S \\
\text { SSE } \\
\text { SE } \\
\text { ESE } \\
\text { E } \\
\text { ENE } \\
\text { NE } \\
\text { NNE }\end{array}$ & $\begin{array}{l}8.6 \mathrm{E}-14 \\
3.2 \mathrm{E}-14 \\
4.5 \mathrm{E}-14 \\
6.0 \mathrm{E}-14 \\
6.8 \mathrm{E}-14 \\
9.0 \mathrm{E}-14 \\
1.4 \mathrm{E}-13 \\
2.4 \mathrm{E}-13 \\
3.3 \mathrm{E}-13 \\
2.8 \mathrm{E}-13 \\
1.5 \mathrm{E}-13 \\
1.0 \mathrm{E}-13 \\
1.3 \mathrm{E}-13 \\
1.1 \mathrm{E}-13 \\
1.6 \mathrm{E}-13 \\
1.1 \mathrm{E}-13\end{array}$ & $\begin{array}{l}8.3 \mathrm{E}-14 \\
3.1 \mathrm{E}-14 \\
4.4 \mathrm{E}-14 \\
5.9 \mathrm{E}-14 \\
6.7 \mathrm{E}-14 \\
8.8 \mathrm{E}-14 \\
1.4 \mathrm{E}-13 \\
2.3 \mathrm{E}-13 \\
3.2 \mathrm{E}-13 \\
2.7 \mathrm{E}-13 \\
1.5 \mathrm{E}-13 \\
9.8 \mathrm{E}-14 \\
1.3 \mathrm{E}-13 \\
1.1 \mathrm{E}-13 \\
1.6 \mathrm{E}-13 \\
1.0 \mathrm{E}-13\end{array}$ & $\begin{array}{l}7.8 \mathrm{E}-14 \\
2.9 \mathrm{E}-14 \\
4.1 \mathrm{E}-14 \\
5.5 \mathrm{E}-14 \\
6.2 \mathrm{E}-14 \\
8.2 \mathrm{E}-14 \\
1.3 \mathrm{E}-13 \\
2.1 \mathrm{E}-13 \\
3.0 \mathrm{E}-13 \\
2.5 \mathrm{E}-13 \\
1.4 \mathrm{E}-13 \\
9.2 \mathrm{E}-14 \\
1.2 \mathrm{E}-13 \\
1.0 \mathrm{E}-13 \\
1.5 \mathrm{E}-13 \\
9.6 \mathrm{E}-14\end{array}$ & $\begin{array}{l}7.6 \mathrm{E}-14 \\
2.9 \mathrm{E}-14 \\
4.0 \mathrm{E}-14 \\
5.4 \mathrm{E}-14 \\
6.1 \mathrm{E}-14 \\
8.0 \mathrm{E}-14 \\
1.3 \mathrm{E}-13 \\
2.1 \mathrm{E}-13 \\
2.9 \mathrm{E}-13 \\
2.4 \mathrm{E}-13 \\
1.3 \mathrm{E}-13 \\
9.0 \mathrm{E}-14 \\
1.2 \mathrm{E}-13 \\
9.8 \mathrm{E}-14 \\
1.4 \mathrm{E}-13 \\
9.4 \mathrm{E}-14\end{array}$ & $\begin{array}{l}7.6 \mathrm{E}-14 \\
2.8 \mathrm{E}-14 \\
4.0 \mathrm{E}-14 \\
5.3 \mathrm{E}-14 \\
6.1 \mathrm{E}-14 \\
8.0 \mathrm{E}-14 \\
1.3 \mathrm{E}-13 \\
2.1 \mathrm{E}-13 \\
2.9 \mathrm{E}-13 \\
2.4 \mathrm{E}-13 \\
1.3 \mathrm{E}-13 \\
8.9 \mathrm{E}-14 \\
1.2 \mathrm{E}-13 \\
9.7 \mathrm{E}-14 \\
1.4 \mathrm{E}-13 \\
9.3 \mathrm{E}-14\end{array}$ \\
\hline
\end{tabular}




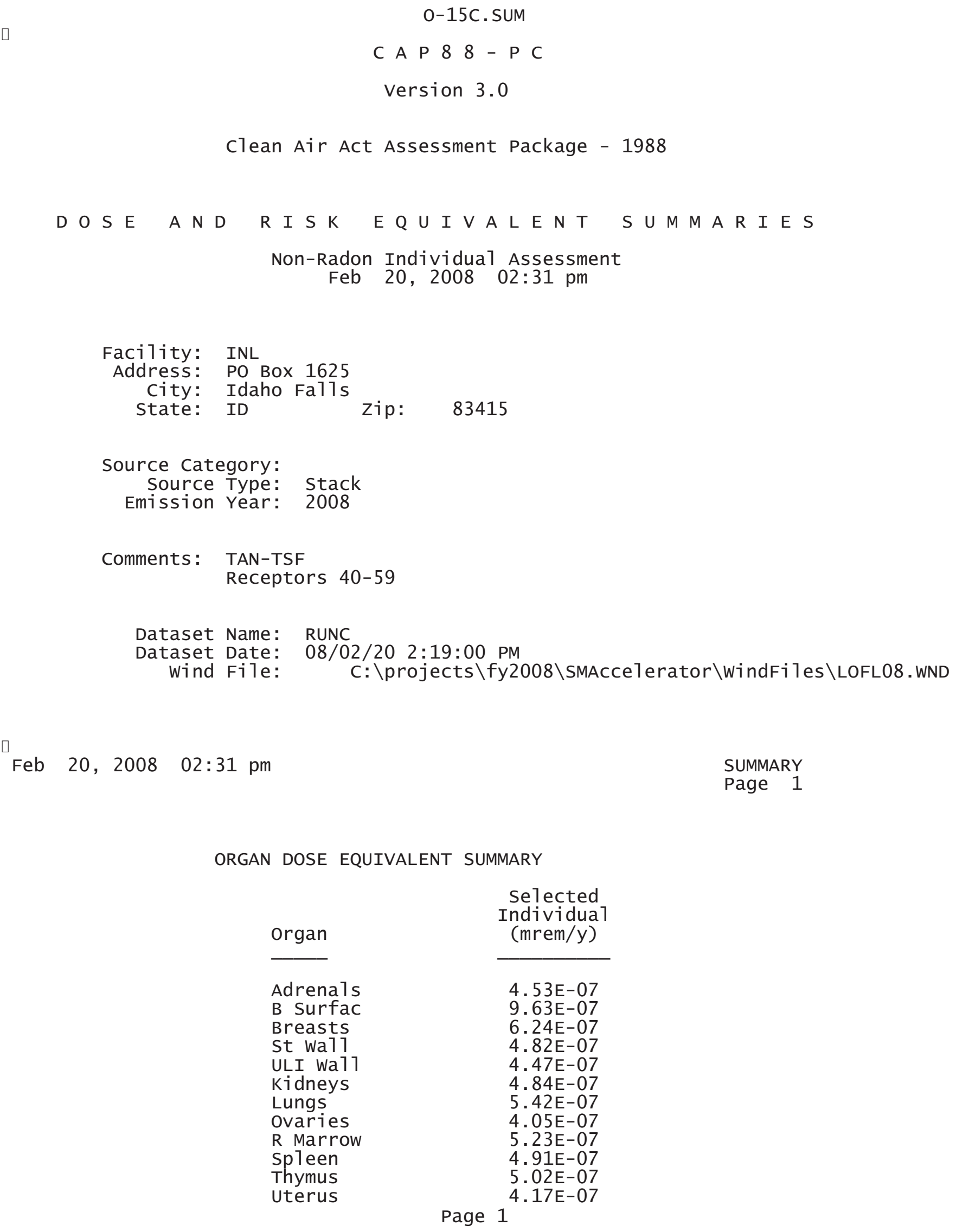

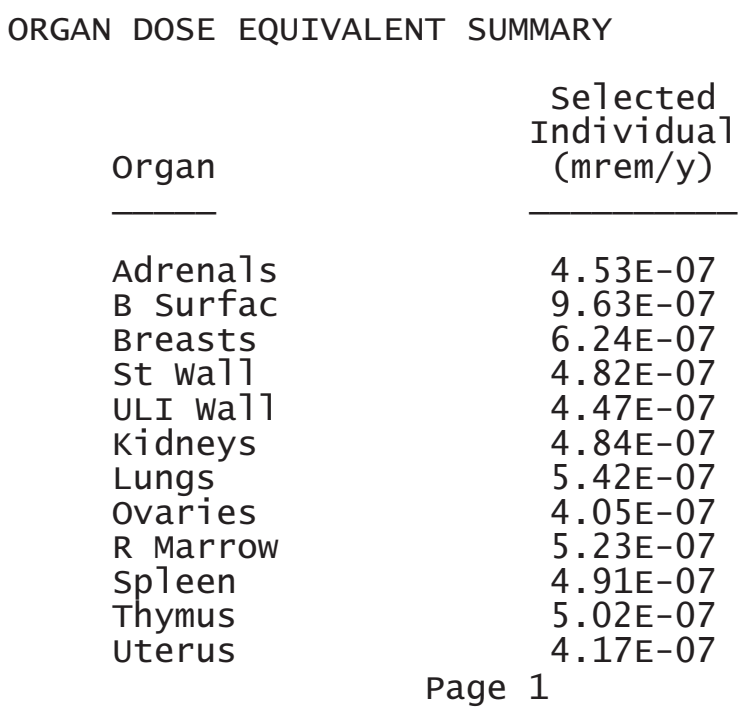




$\begin{array}{lr} & \text { 0-15C.SUM } \\ \text { B1d wa11 } & 4.52 \mathrm{E}-07 \\ \text { Brain } & 5.77 \mathrm{E}-07 \\ \text { Esophagu } & 4.45 \mathrm{E}-07 \\ \text { SI wa11 } & 4.28 \mathrm{E}-07 \\ \text { LLI wa11 } & 4.36 \mathrm{E}-07 \\ \text { Liver } & 4.87 \mathrm{E}-07 \\ \text { Muscle } & 5.29 \mathrm{E}-07 \\ \text { Pancreas } & 4.25 \mathrm{E}-07 \\ \text { Skin } & 1.18 \mathrm{E}-06 \\ \text { Testes } & 5.45 \mathrm{E}-07 \\ \text { Thyroid } & 5.56 \mathrm{E}-07 \\ & \\ \text { EFFEC } & 5.24 \mathrm{E}-07\end{array}$

PATHWAY EFFECTIVE DOSE EQUIVALENT SUMMARY

\begin{tabular}{lc} 
Pathway & $\begin{array}{c}\text { selected } \\
\text { Individual } \\
\text { (mrem/y) }\end{array}$ \\
\cline { 2 - 2 } INGESTION & $0.00 \mathrm{E}+00$ \\
INHALATION & $0.00 \mathrm{E}+00$ \\
AIR IMMERSION & $5.24 \mathrm{E}-07$ \\
GROUND SURFACE & $0.00 \mathrm{E}+00$ \\
INTERNAL & $0.00 \mathrm{E}+00$ \\
EXTERNAL & $5.24 \mathrm{E}-07$ \\
TOTAL & $5.24 \mathrm{E}-07$
\end{tabular}

Feb 20, $2008 \quad 02: 31 \mathrm{pm}$

SUMMARY

Page 2

NUCLIDE EFFECTIVE DOSE EQUIVALENT SUMMARY

Nuclide

$0-15$

TOTAL

Feb 20, 2008 02:31 pm

$$
\begin{gathered}
\begin{array}{c}
\text { Selected } \\
\text { Individual } \\
\text { (mrem/y) }
\end{array} \\
\hline 5.24 \mathrm{E}-07 \\
5.24 \mathrm{E}-07
\end{gathered}
$$

SUMMARY

Page 3

CANCER RISK SUMMARY

Cancer

Esophagu
Selected Individual

Total Lifetime

Fatal Cancer Risk

$$
\text { Page } 2^{5 \cdot 12 \mathrm{E}-15}
$$




\begin{tabular}{lr} 
& \multicolumn{1}{c}{ 0-15C. SUM } \\
Stomach & $1.95 \mathrm{E}-14$ \\
Colon & $4.57 \mathrm{E}-14$ \\
Liver & $7.39 \mathrm{E}-15$ \\
LUNG & $5.29 \mathrm{E}-14$ \\
Bone & $9.14 \mathrm{E}-16$ \\
Skin & $1.18 \mathrm{E}-15$ \\
Breast & $3.02 \mathrm{E}-14$ \\
Ovary & $5.76 \mathrm{E}-15$ \\
B Tadder & $1.09 \mathrm{E}-14$ \\
Kidneys & $2.52 \mathrm{E}-15$ \\
Thyroid & $1.76 \mathrm{E}-15$ \\
Leukemia & $2.94 \mathrm{E}-14$ \\
Residua1 & $6.98 \mathrm{E}-14$ \\
Tota1 & $2.84 \mathrm{E}-13$ \\
& \\
TOTAL & $5.67 \mathrm{E}-13$
\end{tabular}

PATHWAY RISK SUMMARY

\begin{tabular}{lc} 
Pathway & $\begin{array}{c}\text { Selected Individual } \\
\text { Total Lifetime } \\
\text { Fatal Cancer Risk }\end{array}$ \\
\cline { 2 - 2 } INGESTION & $0.00 \mathrm{E}+00$ \\
INHALATION & $0.00 \mathrm{E}+00$ \\
AIR IMMERSION & $2.84 \mathrm{E}-13$ \\
GROUND SURFACE & $0.00 \mathrm{E}+00$ \\
INTERNAL & $0.00 \mathrm{E}+00$ \\
EXTERNAL & $2.84 \mathrm{E}-13$ \\
TOTAL & $2.84 \mathrm{E}-13$
\end{tabular}

Feb 20, $2008 \quad 02: 31$ pm

SUMMARY Page 4

NUCLIDE RISK SUMMARY

Nuclide

0-15

TOTAL

$\square$

Feb 20, 2008 02:31 pm
Selected Individual

Total Lifetime Fatal Cancer Risk

$2.84 \mathrm{E}-13$

$2.84 \mathrm{E}-13$

INDIVIDUAL EFFECTIVE DOSE EQUIVALENT RATE (mrem/y) (A11 Radionuclides and Pathways)

Distance $(m)$ 


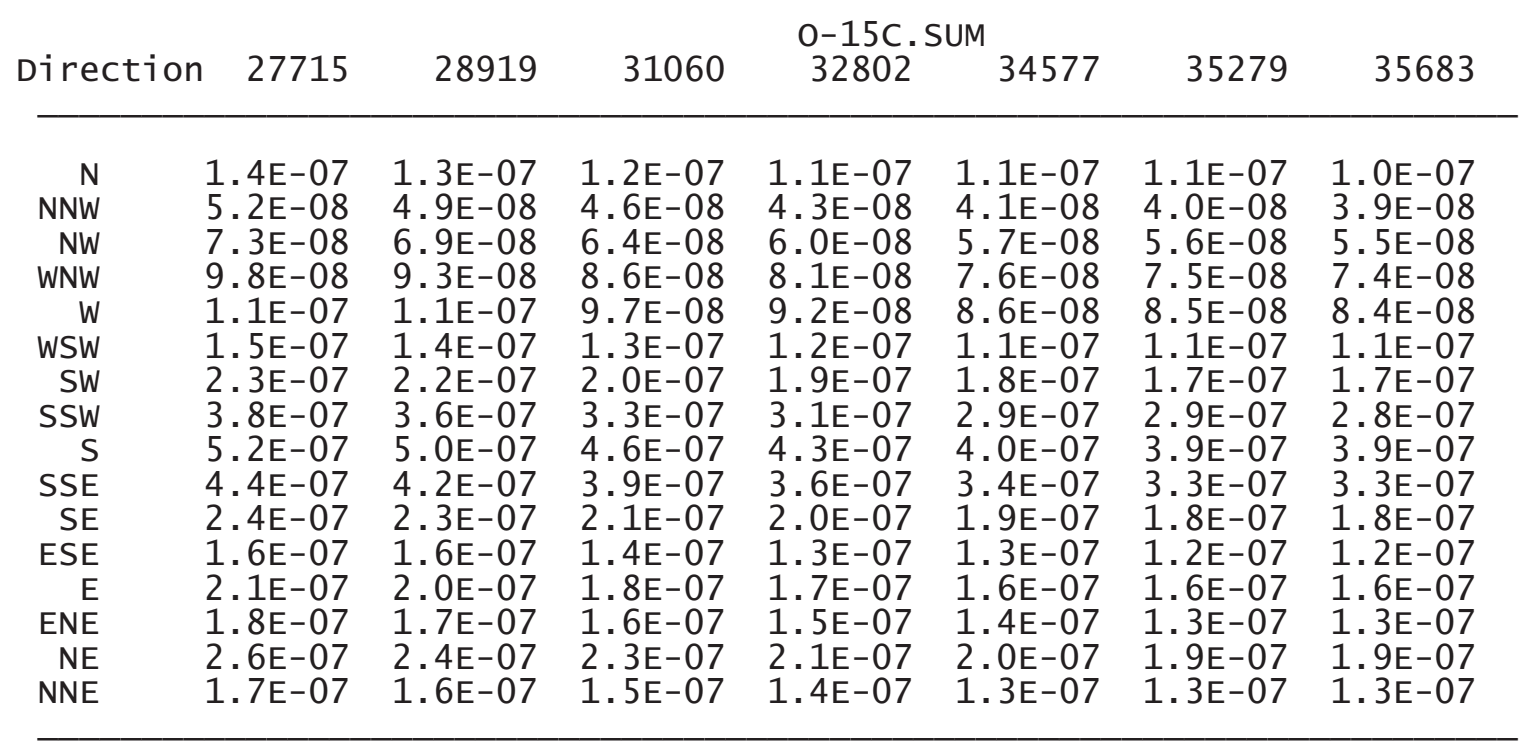

Distance $(m)$

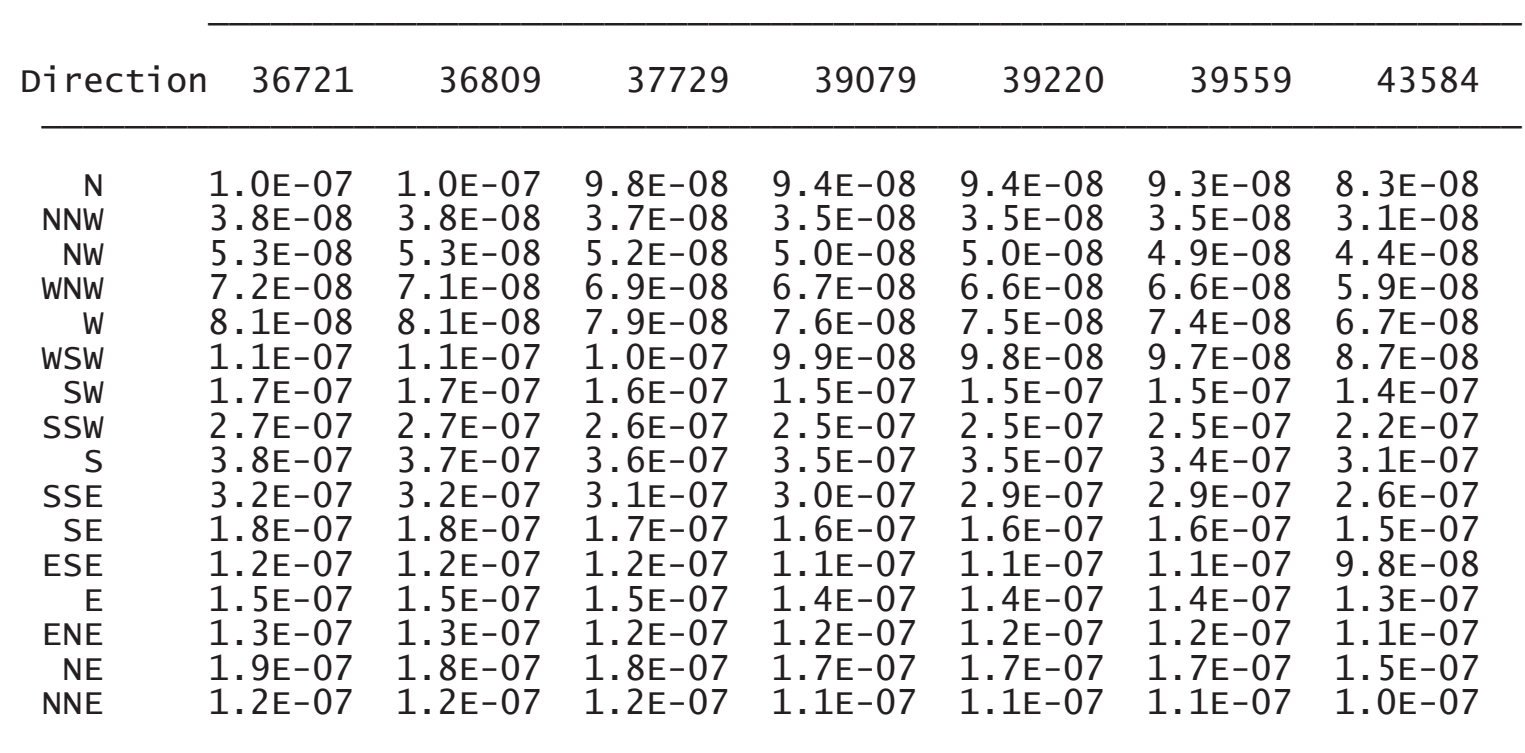

$\square$

Feb 20, $2008 \quad 02: 31 \mathrm{pm}$

SUMMARY

Page 6

INDIVIDUAL EFFECTIVE DOSE EQUIVALENT RATE (mrem/y) (A11 Radionuclides and Pathways)

\begin{tabular}{lllllll}
\hline & \multicolumn{5}{c}{ Distance $(\mathrm{m})$} \\
\cline { 2 - 6 } Direction & 45196 & 45275 & 45654 & 45677 & 46668 & 47969 \\
\hline
\end{tabular}

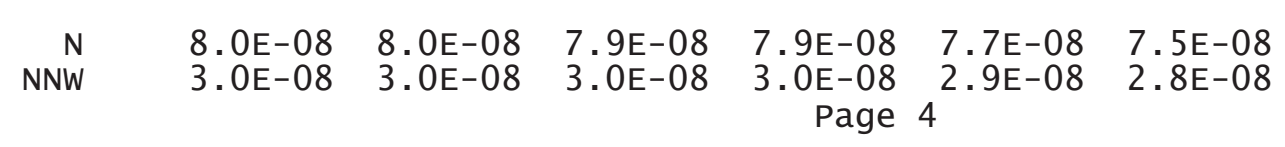




\begin{tabular}{|c|c|c|c|c|c|c|}
\hline & & & & $0-15 \mathrm{C}$ & UM & \\
\hline NW & $4.2 \mathrm{E}-08$ & 4. $2 \mathrm{E}-08$ & 4. $2 \mathrm{E}-08$ & $4.2 \mathrm{E}-08$ & $4.1 \mathrm{E}-08$ & $4.0 \mathrm{E}-08$ \\
\hline WNW & $5.7 \mathrm{E}-08$ & $5.7 \mathrm{E}-08$ & $5.6 \mathrm{E}-08$ & $5.6 \mathrm{E}-08$ & $5.5 \mathrm{E}-08$ & 5. $3 \mathrm{E}-08$ \\
\hline & $6.4 \mathrm{E}-08$ & $6.4 \mathrm{E}-08$ & $6.4 \mathrm{E}-08$ & $6.4 \mathrm{E}-08$ & $6.2 \mathrm{E}-08$ & $6.0 \mathrm{E}-08$ \\
\hline WSW & $8.4 \mathrm{E}-08$ & $8.4 \mathrm{E}-08$ & $8.3 E-08$ & $8.3 \mathrm{E}-08$ & $8.1 \mathrm{E}-08$ & $7.8 \mathrm{E}-08$ \\
\hline SW & & 1. $3 \mathrm{E}-07$ & 1. $3 \mathrm{E}-07$ & 1. $3 \mathrm{E}-07$ & 1. $3 \mathrm{E}-07$ & $1.2 \mathrm{E}-07$ \\
\hline SSW & $2.1 \mathrm{E}-07$ & $2.1 \mathrm{E}-07$ & $2.1 \mathrm{E}-07$ & $2.1 \mathrm{E}-07$ & $2.1 \mathrm{E}-07$ & $2.0 \mathrm{E}-07$ \\
\hline & $2.9 \mathrm{E}-07$ & $2.9 \mathrm{E}-07$ & $2.9 \mathrm{E}-07$ & 2.9 & $2.8 \mathrm{E}-07$ & $2.7 \mathrm{E}-07$ \\
\hline SSE & $2.5 \mathrm{E}-07$ & $2.5 \mathrm{E}$ & $2.5 \mathrm{E}$ & 2.5 & $2.4 \mathrm{E}$ & 2. $3 \mathrm{E}-07$ \\
\hline SE & $1.4 \mathrm{E}-07$ & $1.4 \mathrm{E}-07$ & $1.4 \mathrm{E}-07$ & $1.4 \mathrm{E}-07$ & 1. $3 \mathrm{E}-07$ & 1. $3 \mathrm{E}-07$ \\
\hline ESE & & $9.4 \mathrm{E}$ & & $9.3 \mathrm{k}$ & $9.1 \mathrm{E}$ & 8 \\
\hline & -07 & 1. $2 \mathrm{E}-07$ & 1. $2 \mathrm{E}-07$ & 1. $2 \mathrm{E}-07$ & $1.2 \mathrm{E}-07$ & $1 \mathrm{E}-07$ \\
\hline ENE & 07 & 1.0E-07 & $1.0 \mathrm{E}$ & 1.0E-07 & 9.8 & 0 \\
\hline $\mathrm{NE}$ & $E_{-}$ & $1.4 \mathrm{E}-07$ & $1.4 \mathrm{E}-07$ & $1.4 \mathrm{E}-07$ & $1.4 \mathrm{E}-07$ & 1. $4 \mathrm{E}-07$ \\
\hline NNE & $9.7 \mathrm{E}-08$ & $9.7 \mathrm{E}-08$ & $9.6 \mathrm{E}-08$ & $9.6 \mathrm{E}-08$ & $9.4 \mathrm{E}-08$ & $9.1 \mathrm{E}-08$ \\
\hline
\end{tabular}

Feb 20, 2008 02:31 pm

SUMMARY

Page 7

INDIVIDUAL LIFETIME RISK (deaths)

(A11 Radionuclides and Pathways)

\begin{tabular}{|c|c|c|c|c|c|c|c|}
\hline \multicolumn{8}{|c|}{ Distance $(\mathrm{m})$} \\
\hline Direction & 27715 & 28919 & 31060 & 32802 & 34577 & 35279 & 35683 \\
\hline $\begin{array}{r}N \\
N N W \\
N W \\
W N W \\
W \\
W S W \\
S W \\
S S W \\
S \\
S S E \\
\text { SE } \\
\text { ESE } \\
E \\
\text { ENE } \\
N E \\
\text { NNE }\end{array}$ & $\begin{array}{l}7.5 \mathrm{E}-14 \\
2.8 \mathrm{E}-14 \\
3.9 \mathrm{E}-14 \\
5.3 \mathrm{E}-14 \\
6.0 \mathrm{E}-14 \\
7.9 \mathrm{E}-14 \\
1.2 \mathrm{E}-13 \\
2.1 \mathrm{E}-13 \\
2.8 \mathrm{E}-13 \\
2.4 \mathrm{E}-13 \\
1.3 \mathrm{E}-13 \\
8.8 \mathrm{E}-14 \\
1.1 \mathrm{E}-13 \\
9.6 \mathrm{E}-14 \\
1.4 \mathrm{E}-13 \\
9.2 \mathrm{E}-14\end{array}$ & $\begin{array}{l}7.1 \mathrm{E}-14 \\
2.7 \mathrm{E}-14 \\
3.8 \mathrm{E}-14 \\
5.0 \mathrm{E}-14 \\
5.7 \mathrm{E}-14 \\
7.5 \mathrm{E}-14 \\
1.2 \mathrm{E}-13 \\
2.0 \mathrm{E}-13 \\
2.7 \mathrm{E}-13 \\
2.3 \mathrm{E}-13 \\
1.3 \mathrm{E}-13 \\
8.4 \mathrm{E}-14 \\
1.1 \mathrm{E}-13 \\
9.2 \mathrm{E}-14 \\
1.3 \mathrm{E}-13 \\
8.8 \mathrm{E}-14\end{array}$ & $\begin{array}{l}6.6 \mathrm{E}-14 \\
2.5 \mathrm{E}-14 \\
3.5 \mathrm{E}-14 \\
4.7 \mathrm{E}-14 \\
5.3 \mathrm{E}-14 \\
6.9 \mathrm{E}-14 \\
1.1 \mathrm{E}-13 \\
1.8 \mathrm{E}-13 \\
2.5 \mathrm{E}-13 \\
2.1 \mathrm{E}-13 \\
1.2 \mathrm{E}-13 \\
7.8 \mathrm{E}-14 \\
1.0 \mathrm{E}-13 \\
8.4 \mathrm{E}-14 \\
1.2 \mathrm{E}-13 \\
8.1 \mathrm{E}-14\end{array}$ & $\begin{array}{l}6.2 \mathrm{E}-14 \\
2.3 \mathrm{E}-14 \\
3.3 \mathrm{E}-14 \\
4.4 \mathrm{E}-14 \\
5.0 \mathrm{E}-14 \\
6.5 \mathrm{E}-14 \\
1.0 \mathrm{E}-13 \\
1.7 \mathrm{E}-13 \\
2.3 \mathrm{E}-13 \\
2.0 \mathrm{E}-13 \\
1.1 \mathrm{E}-13 \\
7.3 \mathrm{E}-14 \\
9.4 \mathrm{E}-14 \\
7.9 \mathrm{E}-14 \\
1.1 \mathrm{E}-13 \\
7.6 \mathrm{E}-14\end{array}$ & $\begin{array}{l}5.8 \mathrm{E}-14 \\
2.2 \mathrm{E}-14 \\
3.1 \mathrm{E}-14 \\
4.1 \mathrm{E}-14 \\
4.7 \mathrm{E}-14 \\
6.1 \mathrm{E}-14 \\
9.6 \mathrm{E}-14 \\
1.6 \mathrm{E}-13 \\
2.2 \mathrm{E}-13 \\
1.8 \mathrm{E}-13 \\
1.0 \mathrm{E}-13 \\
6.9 \mathrm{E}-14 \\
8.9 \mathrm{E}-14 \\
7.5 \mathrm{E}-14 \\
1.1 \mathrm{E}-13 \\
7.1 \mathrm{E}-14\end{array}$ & $\begin{array}{l}5.7 \mathrm{E}-14 \\
2.1 \mathrm{E}-14 \\
3.0 \mathrm{E}-14 \\
4.0 \mathrm{E}-14 \\
4.6 \mathrm{E}-14 \\
6.0 \mathrm{E}-14 \\
9.4 \mathrm{E}-14 \\
1.5 \mathrm{E}-13 \\
2.1 \mathrm{E}-13 \\
1.8 \mathrm{E}-13 \\
1.0 \mathrm{E}-13 \\
6.7 \mathrm{E}-14 \\
8.7 \mathrm{E}-14 \\
7.3 \mathrm{E}-14 \\
1.0 \mathrm{E}-13 \\
7.0 \mathrm{E}-14\end{array}$ & $\begin{array}{l}5.6 \mathrm{E}-14 \\
2.1 \mathrm{E}-14 \\
3.0 \mathrm{E}-14 \\
4.0 \mathrm{E}-14 \\
4.5 \mathrm{E}-14 \\
5.9 \mathrm{E}-14 \\
9.3 \mathrm{E}-14 \\
1.5 \mathrm{E}-13 \\
2.1 \mathrm{E}-13 \\
1.8 \mathrm{E}-13 \\
9.9 \mathrm{E}-14 \\
6.6 \mathrm{E}-14 \\
8.6 \mathrm{E}-14 \\
7.2 \mathrm{E}-14 \\
1.0 \mathrm{E}-13 \\
6.9 \mathrm{E}-14\end{array}$ \\
\hline \multicolumn{8}{|c|}{ Distance $(\mathrm{m})$} \\
\hline Direction & 36721 & 36809 & 37729 & 39079 & 39220 & 39559 & 43584 \\
\hline $\begin{array}{r}\mathrm{N} \\
N N W \\
N W \\
W N W \\
W \\
\text { WSW } \\
\text { SW } \\
\text { SSW }\end{array}$ & $\begin{array}{l}5.5 \mathrm{E}-14 \\
2.1 \mathrm{E}-14 \\
2.9 \mathrm{E}-14 \\
3.9 \mathrm{E}-14 \\
4.4 \mathrm{E}-14 \\
5.7 \mathrm{E}-14 \\
9.0 \mathrm{E}-14 \\
1.5 \mathrm{E}-13\end{array}$ & $\begin{array}{l}5.4 \mathrm{E}-14 \\
2.0 \mathrm{E}-14 \\
2.9 \mathrm{E}-14 \\
3.9 \mathrm{E}-14 \\
4.4 \mathrm{E}-14 \\
5.7 \mathrm{E}-14 \\
8.9 \mathrm{E}-14 \\
1.5 \mathrm{E}-13\end{array}$ & $\begin{array}{l}5.3 \mathrm{E}-14 \\
2.0 \mathrm{E}-14 \\
2.8 \mathrm{E}-14 \\
3.8 \mathrm{E}-14 \\
4.2 \mathrm{E}-14 \\
5.6 \mathrm{E}-14 \\
8.7 \mathrm{E}-14 \\
1.4 \mathrm{E}-13\end{array}$ & $\begin{array}{r}5.1 \mathrm{E}-14 \\
1.9 \mathrm{E}-14 \\
2.7 \mathrm{E}-14 \\
3.6 \mathrm{E}-14 \\
4.1 \mathrm{E}-14 \\
5.3 \mathrm{E}-14 \\
8.3 \mathrm{E}-14 \\
1.4 \mathrm{E}-13 \\
\text { Page }\end{array}$ & $\begin{array}{l}5.1 \mathrm{E}-14 \\
1.9 \mathrm{E}-14 \\
2.7 \mathrm{E}-14 \\
3.6 \mathrm{E}-14 \\
4.1 \mathrm{E}-14 \\
5.3 \mathrm{E}-14 \\
8.3 \mathrm{E}-14 \\
1.4 \mathrm{E}-13\end{array}$ & $\begin{array}{l}5.0 \mathrm{E}-14 \\
1.9 \mathrm{E}-14 \\
2.7 \mathrm{E}-14 \\
3.6 \mathrm{E}-14 \\
4.0 \mathrm{E}-14 \\
5.3 \mathrm{E}-14 \\
8.2 \mathrm{E}-14 \\
1.4 \mathrm{E}-13\end{array}$ & $\begin{array}{l}4.5 \mathrm{E}-14 \\
1.7 \mathrm{E}-14 \\
2.4 \mathrm{E}-14 \\
3.2 \mathrm{E}-14 \\
3.6 \mathrm{E}-14 \\
4.7 \mathrm{E}-14 \\
7.4 \mathrm{E}-14 \\
1.2 \mathrm{E}-13\end{array}$ \\
\hline
\end{tabular}




\begin{tabular}{rlllllll} 
& \multicolumn{7}{c}{ O-15C.SUM } \\
S & $2.0 \mathrm{E}-13$ & $2.0 \mathrm{E}-13$ & $2.0 \mathrm{E}-13$ & $1.9 \mathrm{E}-13$ & $1.9 \mathrm{E}-13$ & $1.9 \mathrm{E}-13$ & $1.7 \mathrm{E}-13$ \\
SSE & $1.7 \mathrm{E}-13$ & $1.7 \mathrm{E}-13$ & $1.7 \mathrm{E}-13$ & $1.6 \mathrm{E}-13$ & $1.6 \mathrm{E}-13$ & $1.6 \mathrm{E}-13$ & $1.4 \mathrm{E}-13$ \\
SE & $9.5 \mathrm{E}-14$ & $9.5 \mathrm{E}-14$ & $9.3 \mathrm{E}-14$ & $8.9 \mathrm{E}-14$ & $8.9 \mathrm{E}-14$ & $8.8 \mathrm{E}-14$ & $7.9 \mathrm{E}-14$ \\
ESE & $6.4 \mathrm{E}-14$ & $6.4 \mathrm{E}-14$ & $6.2 \mathrm{E}-14$ & $6.0 \mathrm{E}-14$ & $6.0 \mathrm{E}-14$ & $5.9 \mathrm{E}-14$ & $5.3 \mathrm{E}-14$ \\
$\mathrm{E}$ & $8.3 \mathrm{E}-14$ & $8.3 \mathrm{E}-14$ & $8.0 \mathrm{E}-14$ & $7.7 \mathrm{E}-14$ & $7.7 \mathrm{E}-14$ & $7.6 \mathrm{E}-14$ & $6.8 \mathrm{E}-14$ \\
ENE & $7.0 \mathrm{E}-14$ & $7.0 \mathrm{E}-14$ & $6.8 \mathrm{E}-14$ & $6.5 \mathrm{E}-14$ & $6.5 \mathrm{E}-14$ & $6.4 \mathrm{E}-14$ & $5.7 \mathrm{E}-14$ \\
NE & $1.0 \mathrm{E}-13$ & $1.0 \mathrm{E}-13$ & $9.7 \mathrm{E}-14$ & $9.3 \mathrm{E}-14$ & $9.3 \mathrm{E}-14$ & $9.2 \mathrm{E}-14$ & $8.2 \mathrm{E}-14$ \\
NNE & $6.7 \mathrm{E}-14$ & $6.7 \mathrm{E}-14$ & $6.5 \mathrm{E}-14$ & $6.2 \mathrm{E}-14$ & $6.2 \mathrm{E}-14$ & $6.1 \mathrm{E}-14$ & $5.5 \mathrm{E}-14$
\end{tabular}

Feb 20, $2008 \quad 02: 31 \mathrm{pm}$

SUMMARY

Page 8

INDIVIDUAL LIFETIME RISK (deaths)

(A11 Radionuclides and Pathways)

\begin{tabular}{|c|c|c|c|c|c|c|}
\hline \multirow[b]{2}{*}{ Direction } & \multicolumn{6}{|c|}{ Distance $(\mathrm{m})$} \\
\hline & 45196 & 45275 & 45654 & 45677 & 46668 & 47969 \\
\hline$N$ & $4.3 E-14$ & $4.3 E-14$ & $4.3 E-14$ & $4.3 E-14$ & $4.2 \mathrm{E}-14$ & $4.1 \mathrm{E}-14$ \\
\hline NNW & $1.6 \mathrm{E}-14$ & $1.6 \mathrm{E}-14$ & $1.6 \mathrm{E}-14$ & $1.6 \mathrm{E}-14$ & $1.6 \mathrm{E}-14$ & $1.5 \mathrm{E}-14$ \\
\hline NW & $2.3 \mathrm{E}-14$ & $2.3 \mathrm{E}-14$ & $2.3 \mathrm{E}-14$ & $2.3 \mathrm{E}-14$ & $2.2 \mathrm{E}-14$ & 2. $2 \mathrm{E}-14$ \\
\hline WNW & $3.1 \mathrm{E}-14$ & $3.1 \mathrm{E}-14$ & $3.0 \mathrm{E}-14$ & $3.0 \mathrm{E}-14$ & $3.0 \mathrm{E}-14$ & $2.9 \mathrm{E}-14$ \\
\hline W & $3.5 \mathrm{E}-14$ & $3.5 \mathrm{E}-14$ & $3.4 \mathrm{E}-14$ & $3.4 \mathrm{E}-14$ & $3.4 \mathrm{E}-14$ & $3.3 \mathrm{E}-14$ \\
\hline WSW & $4.5 \mathrm{E}-14$ & $4.5 \mathrm{E}-14$ & $4.5 \mathrm{E}-14$ & $4.5 \mathrm{E}-14$ & $4.4 \mathrm{E}-14$ & 4. $2 \mathrm{E}-14$ \\
\hline SW & $7.1 \mathrm{E}-14$ & 7.0E-14 & $7.0 \mathrm{E}-14$ & 7.0E-14 & $6.8 \mathrm{E}-14$ & $6.6 \mathrm{E}-14$ \\
\hline SSW & 1. $2 \mathrm{E}-13$ & $1.2 \mathrm{E}-13$ & 1. $1 \mathrm{E}-13$ & $1.1 \mathrm{E}-13$ & $1.1 \mathrm{E}-13$ & 1. $1 \mathrm{E}-13$ \\
\hline $\mathrm{S}$ & $1.6 \mathrm{E}-13$ & $1.6 \mathrm{E}-13$ & $1.6 \mathrm{E}-13$ & $1.6 \mathrm{E}-13$ & $1.5 \mathrm{E}-13$ & $1.5 \mathrm{E}-13$ \\
\hline SSE & 1. $3 \mathrm{E}-13$ & 1. $3 \mathrm{E}-13$ & $1.3 \mathrm{E}-13$ & $1.3 \mathrm{E}-13$ & $1.3 \mathrm{E}-13$ & 1. $3 \mathrm{E}-13$ \\
\hline $\mathrm{SE}$ & $7.5 \mathrm{E}-14$ & $7.5 \mathrm{E}-14$ & $7.5 \mathrm{E}-14$ & $7.5 \mathrm{E}-14$ & $7.3 \mathrm{E}-14$ & 7.1E-14 \\
\hline ESE & $5.1 \mathrm{E}-14$ & $5.1 \mathrm{E}-14$ & $5.0 \mathrm{E}-14$ & $5.0 \mathrm{E}-14$ & $4.9 \mathrm{E}-14$ & $4.8 \mathrm{E}-14$ \\
\hline $\mathrm{E}$ & $6.6 \mathrm{E}-14$ & $6.6 \mathrm{E}-14$ & $6.5 \mathrm{E}-14$ & $6.5 \mathrm{E}-14$ & $6.3 \mathrm{E}-14$ & $6.1 \mathrm{E}-14$ \\
\hline ENE & $5.5 \mathrm{E}-14$ & $5.5 \mathrm{E}-14$ & $5.4 \mathrm{E}-14$ & $5.4 \mathrm{E}-14$ & $5.3 \mathrm{E}-14$ & $5.1 \mathrm{E}-14$ \\
\hline $\mathrm{NE}$ & $7.9 \mathrm{E}-14$ & $7.8 \mathrm{E}-14$ & $7.8 \mathrm{E}-14$ & $7.8 \mathrm{E}-14$ & $7.6 \mathrm{E}-14$ & 7. $3 \mathrm{E}-14$ \\
\hline NNE & $5.3 \mathrm{E}-14$ & $5.3 \mathrm{E}-14$ & $5.2 \mathrm{E}-14$ & $5.2 \mathrm{E}-14$ & $5.1 \mathrm{E}-14$ & $4.9 \mathrm{E}-14$ \\
\hline
\end{tabular}




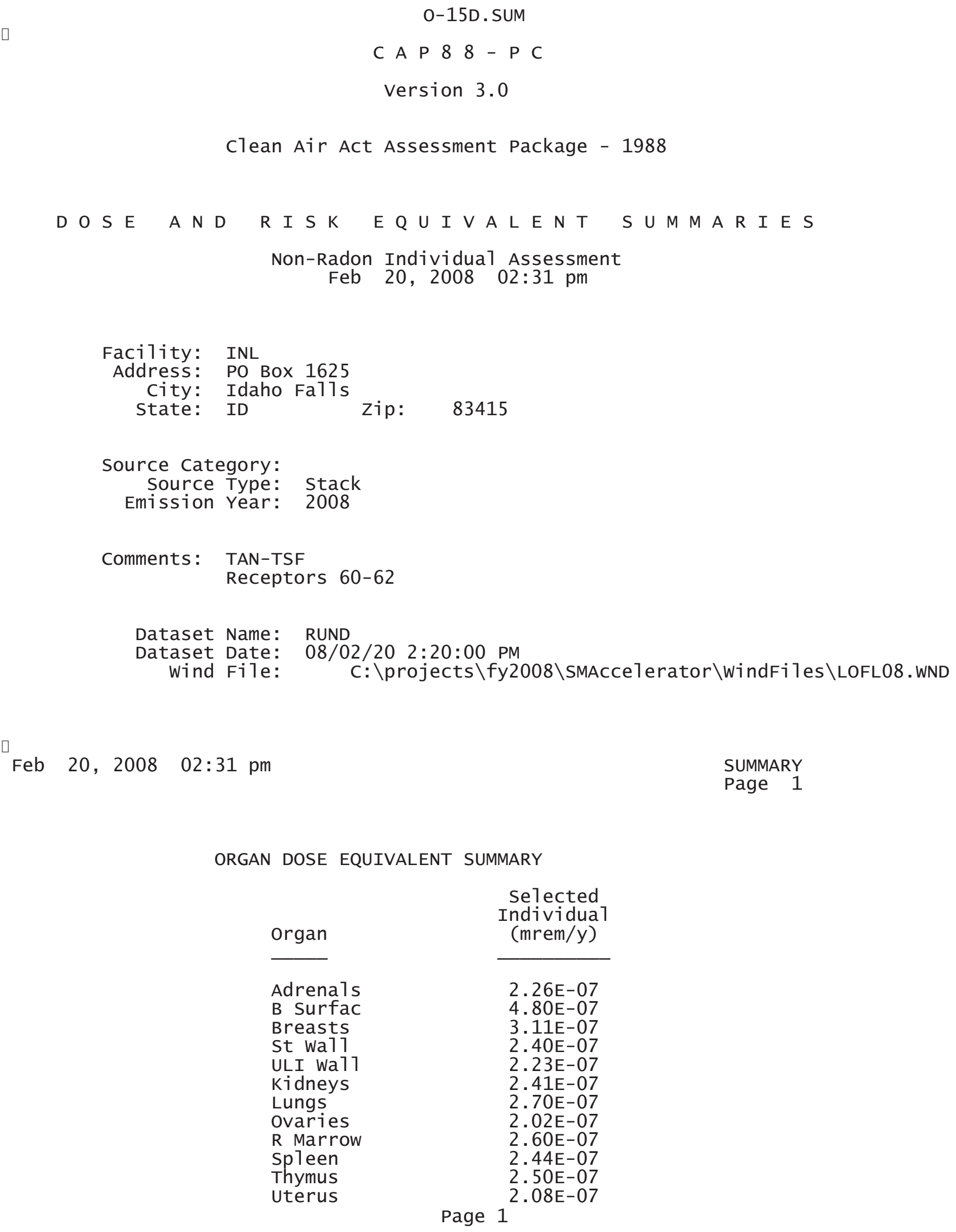




$\begin{array}{lr} & \text { 0-15D.SUM } \\ \text { B1d wa11 } & 2.25 \mathrm{E}-07 \\ \text { Brain } & 2.88 \mathrm{E}-07 \\ \text { Esophagu } & 2.22 \mathrm{E}-07 \\ \text { SI wa11 } & 2.13 \mathrm{E}-07 \\ \text { LLI wa11 } & 2.17 \mathrm{E}-07 \\ \text { Liver } & 2.43 \mathrm{E}-07 \\ \text { Muscle } & 2.64 \mathrm{E}-07 \\ \text { Pancreas } & 2.12 \mathrm{E}-07 \\ \text { Skin } & 5.90 \mathrm{E}-07 \\ \text { Testes } & 2.72 \mathrm{E}-07 \\ \text { Thyroid } & 2.77 \mathrm{E}-07 \\ \text { EFFEC } & 2.61 \mathrm{E}-07\end{array}$

PATHWAY EFFECTIVE DOSE EQUIVALENT SUMMARY

$\begin{array}{lc}\text { Pathway } & \begin{array}{c}\text { selected } \\ \text { Individual } \\ \text { (mrem/y) }\end{array} \\ & \\ \text { INGESTION } & 0.00 \mathrm{E}+00 \\ \text { INHALATION } & 0.00 \mathrm{E}+00 \\ \text { AIR IMMERSION } & 2.61 \mathrm{E}-07 \\ \text { GROUND SURFACE } & 0.00 \mathrm{E}+00 \\ \text { INTERNAL } & 0.00 \mathrm{E}+00 \\ \text { EXTERNAL } & 2.61 \mathrm{E}-07 \\ \text { TOTAL } & 2.61 \mathrm{E}-07\end{array}$

Feb 20, 2008 02:31 pm

SUMMARY

Page 2

NUCLIDE EFFECTIVE DOSE EQUIVALENT SUMMARY

Nuclide

$0-15$

TOTAL

Feb 20, $2008 \quad 02: 31 \mathrm{pm}$

$$
\begin{gathered}
\begin{array}{c}
\text { Selected } \\
\text { Individual } \\
\text { (mrem/y) }
\end{array} \\
\hline 2.61 \mathrm{E}-07 \\
2.61 \mathrm{E}-07
\end{gathered}
$$

SUMMARY

Page 3

CANCER RISK SUMMARY

Cancer

Esophagu
Selected Individual

Total Lifetime

Fatal Cancer Risk

Page $2^{2.55 E-15}$ 


\begin{tabular}{lr} 
& \multicolumn{1}{c}{ 0-15D. SUM } \\
Stomach & $9.70 \mathrm{E}-15$ \\
Colon & $2.27 \mathrm{E}-14$ \\
Liver & $3.68 \mathrm{E}-15$ \\
LUNG & $2.64 \mathrm{E}-14$ \\
Bone & $4.55 \mathrm{E}-16$ \\
Skin & $5.90 \mathrm{E}-16$ \\
Breast & $1.50 \mathrm{E}-14$ \\
Ovary & $2.87 \mathrm{E}-15$ \\
B Tadder & $5.45 \mathrm{E}-15$ \\
Kidneys & $1.25 \mathrm{E}-15$ \\
Thyroid & $8.79 \mathrm{E}-16$ \\
Leukemia & $1.46 \mathrm{E}-14$ \\
Residua1 & $3.48 \mathrm{E}-14$ \\
Tota1 & $1.41 \mathrm{E}-13$ \\
& \\
TOTAL & $2.82 \mathrm{E}-13$
\end{tabular}

PATHWAY RISK SUMMARY

\begin{tabular}{lc} 
Pathway & $\begin{array}{c}\text { Selected Individua1 } \\
\text { Total Lifetime } \\
\text { Fatal Cancer Risk }\end{array}$ \\
\cline { 2 - 2 } INGESTION & $0.00 \mathrm{E}+00$ \\
INHALATION & $0.00 \mathrm{E}+00$ \\
AIR IMMERSION & $1.41 \mathrm{E}-13$ \\
GROUND SURFACE & $0.00 \mathrm{E}+00$ \\
INTERNAL & $0.00 \mathrm{E}+00$ \\
EXTERNAL & $1.41 \mathrm{E}-13$ \\
TOTAL & $1.41 \mathrm{E}-13$
\end{tabular}

Feb 20, $2008 \quad 02: 31$ pm

SUMMARY Page 4

NUCLIDE RISK SUMMARY

Nuclide

0-15

TOTAL

$\square$

Feb 20, 2008 02:31 pm
Selected Individual

Total Lifetime Fatal Cancer Risk

1. $41 \mathrm{E}-13$

1. $41 \mathrm{E}-13$

INDIVIDUAL EFFECTIVE DOSE EQUIVALENT RATE (mrem/y) (A11 Radionuclides and Pathways)

Distance $(m)$ 


Direction $50024 \quad 54611 \quad 58610 \quad$ O-15D.SUM

\begin{tabular}{|c|c|c|c|c|}
\hline $\begin{array}{r}N \\
N N W \\
N W \\
W N W \\
W \\
W S W \\
S W \\
\text { SSW } \\
S \\
\text { SSE } \\
\text { SE } \\
\text { ESE } \\
\text { E } \\
\text { ENE } \\
\text { NE } \\
\text { NNE }\end{array}$ & $\begin{array}{l}7.1 \mathrm{E}-08 \\
2.7 \mathrm{E}-08 \\
3.8 \mathrm{E}-08 \\
5.1 \mathrm{E}-08 \\
5.7 \mathrm{E}-08 \\
7.5 \mathrm{E}-08 \\
1.2 \mathrm{E}-07 \\
1.9 \mathrm{E}-07 \\
2.6 \mathrm{E}-07 \\
2.2 \mathrm{E}-07 \\
1.2 \mathrm{E}-07 \\
8.4 \mathrm{E}-08 \\
1.1 \mathrm{E}-07 \\
9.1 \mathrm{E}-08 \\
1.3 \mathrm{E}-07 \\
8.7 \mathrm{E}-08\end{array}$ & $\begin{array}{l}6.5 \mathrm{E}-08 \\
2.4 \mathrm{E}-08 \\
3.4 \mathrm{E}-08 \\
4.6 \mathrm{E}-08 \\
5.2 \mathrm{E}-08 \\
6.8 \mathrm{E}-08 \\
1.1 \mathrm{E}-07 \\
1.7 \mathrm{E}-07 \\
2.4 \mathrm{E}-07 \\
2.0 \mathrm{E}-07 \\
1.1 \mathrm{E}-07 \\
7.6 \mathrm{E}-08 \\
9.8 \mathrm{E}-08 \\
8.2 \mathrm{E}-08 \\
1.2 \mathrm{E}-07 \\
7.9 \mathrm{E}-08\end{array}$ & $\begin{array}{l}6.0 \mathrm{E}-08 \\
2.3 \mathrm{E}-08 \\
3.2 \mathrm{E}-08 \\
4.3 \mathrm{E}-08 \\
4.8 \mathrm{E}-08 \\
6.3 \mathrm{E}-08 \\
9.7 \mathrm{E}-08 \\
1.6 \mathrm{E}-07 \\
2.2 \mathrm{E}-07 \\
1.8 \mathrm{E}-07 \\
1.0 \mathrm{E}-07 \\
7.0 \mathrm{E}-08 \\
9.1 \mathrm{E}-08 \\
7.6 \mathrm{E}-08 \\
1.1 \mathrm{E}-07 \\
7.3 \mathrm{E}-08\end{array}$ & \\
\hline Feb 20, & 2008 & $2: 31 \mathrm{pm}$ & & SUMMARY \\
\hline \multicolumn{5}{|c|}{$\begin{array}{l}\text { INDIVIDUAL LIFETIME RISK (deaths) } \\
\text { (A11 Radionuclides and Pathways) }\end{array}$} \\
\hline \multicolumn{5}{|c|}{ Distance $(\mathrm{m})$} \\
\hline Direction & 50024 & 54611 & 58610 & \\
\hline $\begin{array}{r}N \\
N N W \\
N W \\
W N W \\
W \\
W S W \\
\text { SW } \\
\text { SSW } \\
S \\
\text { SSE } \\
\text { SE } \\
\text { ESE } \\
\text { E } \\
\text { ENE } \\
\text { NE } \\
\text { NNE }\end{array}$ & $\begin{array}{l}3.9 \mathrm{E}-14 \\
1.5 \mathrm{E}-14 \\
2.1 \mathrm{E}-14 \\
2.8 \mathrm{E}-14 \\
3.1 \mathrm{E}-14 \\
4.1 \mathrm{E}-14 \\
6.3 \mathrm{E}-14 \\
1.0 \mathrm{E}-13 \\
1.4 \mathrm{E}-13 \\
1.2 \mathrm{E}-13 \\
6.7 \mathrm{E}-14 \\
4.6 \mathrm{E}-14 \\
5.9 \mathrm{E}-14 \\
4.9 \mathrm{E}-14 \\
7.0 \mathrm{E}-14 \\
4.7 \mathrm{E}-14\end{array}$ & $\begin{array}{l}3.5 \mathrm{E}-14 \\
1.3 \mathrm{E}-14 \\
1.9 \mathrm{E}-14 \\
2.5 \mathrm{E}-14 \\
2.8 \mathrm{E}-14 \\
3.7 \mathrm{E}-14 \\
5.7 \mathrm{E}-14 \\
9.3 \mathrm{E}-14 \\
1.3 \mathrm{E}-13 \\
1.1 \mathrm{E}-13 \\
6.1 \mathrm{E}-14 \\
4.1 \mathrm{E}-14 \\
5.3 \mathrm{E}-14 \\
4.4 \mathrm{E}-14 \\
6.3 \mathrm{E}-14 \\
4.3 \mathrm{E}-14\end{array}$ & $\begin{array}{l}3.2 \mathrm{E}-14 \\
1.2 \mathrm{E}-14 \\
1.7 \mathrm{E}-14 \\
2.3 \mathrm{E}-14 \\
2.6 \mathrm{E}-14 \\
3.4 \mathrm{E}-14 \\
5.2 \mathrm{E}-14 \\
8.6 \mathrm{E}-14 \\
1.2 \mathrm{E}-13 \\
1.0 \mathrm{E}-13 \\
5.6 \mathrm{E}-14 \\
3.8 \mathrm{E}-14 \\
4.9 \mathrm{E}-14 \\
4.1 \mathrm{E}-14 \\
5.8 \mathrm{E}-14 \\
3.9 \mathrm{E}-14\end{array}$ & \\
\hline
\end{tabular}


08/02/20 2:16:00 PM

RUNA

C: \projects \fy2008\SMAccelerator \WindFi 1es\LOFL08 . WND

INL

PO Box 1625

Idaho Fal1s

TAN-TSF

Receptors 1-20

$100.0000,500.0000$

$\begin{array}{rrrr}0 & 0 & 0 & \\ 10344 & 10365 & 10472 & 1059 \\ 13286 & 13483 & 13612 & 1366 \\ 15441 & 15784 & 15844 & 1632 \\ 800 & 280.20 & 16.30 & 3.5 \\ 0 & 0 & & \\ 1 & & & \end{array}$

$\begin{array}{llll}1.00 & 1.00 & 0.000 \mathrm{E}+00 & 0.000 \mathrm{E}+00\end{array}$

$0.000 \mathrm{E}+00 \quad 0.000 \mathrm{E}+00 \quad 0.000 \mathrm{E}+00 \quad 0.000 \mathrm{E}+00$

$0.000 \mathrm{E}+0060.000 \mathrm{E}+00$

$\mathrm{Rn}-220$ 0

1. $000 \mathrm{E}+00 \quad 0.000 \mathrm{E}+00$

$0.000 \mathrm{e}+00$ $\mathrm{G}$

0

$0 \quad 0.000 \mathrm{e}+00$

$0.000 e+00 \quad 0.000 e+00 \quad 5.480 e-05$

$0.000 \mathrm{E}+00$

$\begin{array}{rrr}\mathrm{PO}-216 & \mathrm{M} & 1 \\ 0.000 \mathrm{E}+00 & 0.000 \mathrm{E}+00 & 0.000 \mathrm{E}+00 \\ 1.630 \mathrm{e}-06 & 0.0018 & 0.000 \mathrm{e}+00 \\ & \text { inorganic } & 1.000 \mathrm{E}-03\end{array}$

unspecified $0.000 \mathrm{E}+00$

$\mathrm{Pb}-212$

inorganic

$0.000 \mathrm{E}+00 \quad 0.000 \mathrm{E}+00 \quad 0.000 \mathrm{E}+00$

$1.630 \mathrm{e}-06 \quad 0.0018 \quad 0.000 \mathrm{e}+00$

$\mathrm{Bi}-212$

unspecified

$\begin{array}{rrr}0.000 \mathrm{E}+00 & 0.000 \mathrm{E}+00 & 0.000 \mathrm{E}+00 \\ 1.630 \mathrm{e}-06 & 0.0018 & 0.000 \mathrm{e}+00\end{array}$

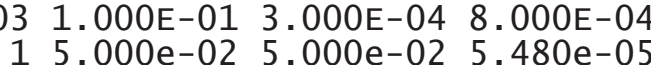
unspecified 1.000E-01

Po-212

$0.000 \mathrm{E}+00 \quad 0.000 \mathrm{E}+00 \quad 0.000 \mathrm{E}+00$

$\begin{array}{lll}0.000 \mathrm{E}+00 & 0.000 \mathrm{E}+00 & 0.000 \mathrm{E}+00 \\ 1.000 \mathrm{e}-01 & 1.000 \mathrm{e}-01 & 5.480 \mathrm{e}-05 \\ . .000 \mathrm{E}+00 & 0.000 \mathrm{E}+00 & 0.000 \mathrm{E}+00\end{array}$

$0.000 \mathrm{E}+00 \quad 0.000 \mathrm{E}+00 \quad 0.000 \mathrm{E}+00$

1.000E-01 4.000E-04 5.000E-03

$2.000 \mathrm{e}-012.000 \mathrm{e}-01 \quad 5.480 \mathrm{e}-05$

$0.000 \mathrm{E}+00 \quad 0.000 \mathrm{E}+00 \quad 0.000 \mathrm{E}+00$

$3.992 \mathrm{E}+05$

$\begin{array}{rrr}0.000 \mathrm{E}+00 & 0.000 \mathrm{E}+00 & 0.000 \mathrm{E}+00 \\ 1.630 \mathrm{e}-06 & 0.0018 & 0.000 \mathrm{e}+00 \\ & \text { inorganic } & 1.000 \mathrm{E}-03\end{array}$

T1-208

$\mathrm{M}$

$0.000 \mathrm{E}+00$

$\begin{array}{llll}51 & 5.000 \mathrm{E}-01 & 1.000 \mathrm{E}-03 & 2.000 \mathrm{E}-03 \\ 1 & 1.000 \mathrm{e}-01 & 1.000 \mathrm{e}-01 & 5.480 \mathrm{e}-05\end{array}$

$\begin{array}{rrr}0.000 \mathrm{E}+00 & 0.000 \mathrm{E}+00 & 0.000 \mathrm{E}+00 \\ 1.630 \mathrm{e}-06 & 0.0018 & 0.000 \mathrm{e}+00\end{array}$

unspecified 2.000E-0

$\begin{array}{lll}0.08 & 0.92 & 0.00\end{array}$

$\begin{array}{lll}0.00 & 1.00 & 0.00\end{array}$

$\begin{array}{lll}\mathrm{T} & \mathrm{T} & \mathrm{T}\end{array}$

$2.030 \mathrm{e}-014.560 \mathrm{e}-02 \quad 1.700 \mathrm{e}-02$

$\mathrm{Rn}-220$

$\begin{array}{rrr}00 & 00 & 00 \\ 0.000 \mathrm{e}+00 & 0.000 \mathrm{e}+00 & 0.000 \mathrm{e}+00\end{array}$

Po-216

$0.000 \mathrm{e}+00 \quad 0.000 \mathrm{e}+00$

$\mathrm{Pb}-212$

$00 \quad 82$

$0.000 \mathrm{e}+00 \quad 0.000 \mathrm{e}+00$

$\mathrm{Bi}-212$

$\begin{array}{rrr}00 & 00 & 00\end{array}$

$0.000 \mathrm{e}+00 \quad 0.000 \mathrm{e}+00$
$0.000 \mathrm{E}+00 \quad 0.000 \mathrm{E}+00 \quad 0.000 \mathrm{E}+00$

1.000E-01 4.000E-04 5.000E-03 1.963E+11

$1.000 \mathrm{e}+00 \quad 1.000 \mathrm{e}+00 \quad 5.480 \mathrm{e}-05$

6.000E-01 3.000E-03 2.000E-02 3.251E+02

Urban $T$ $\begin{array}{rrr}00 & 00 & 00\end{array}$

$\begin{array}{rrrr}00 & 00 & 00 & 00\end{array}$

$0.000 e+00 \quad 0.000 e+00 \quad 0.000 e+00$

$\begin{array}{llll}00 & 00 & 00 & 00\end{array}$

$0.000 e+00 \quad 0.000 e+00$

$\begin{array}{rrr}00 & 00 & 00\end{array}$ 


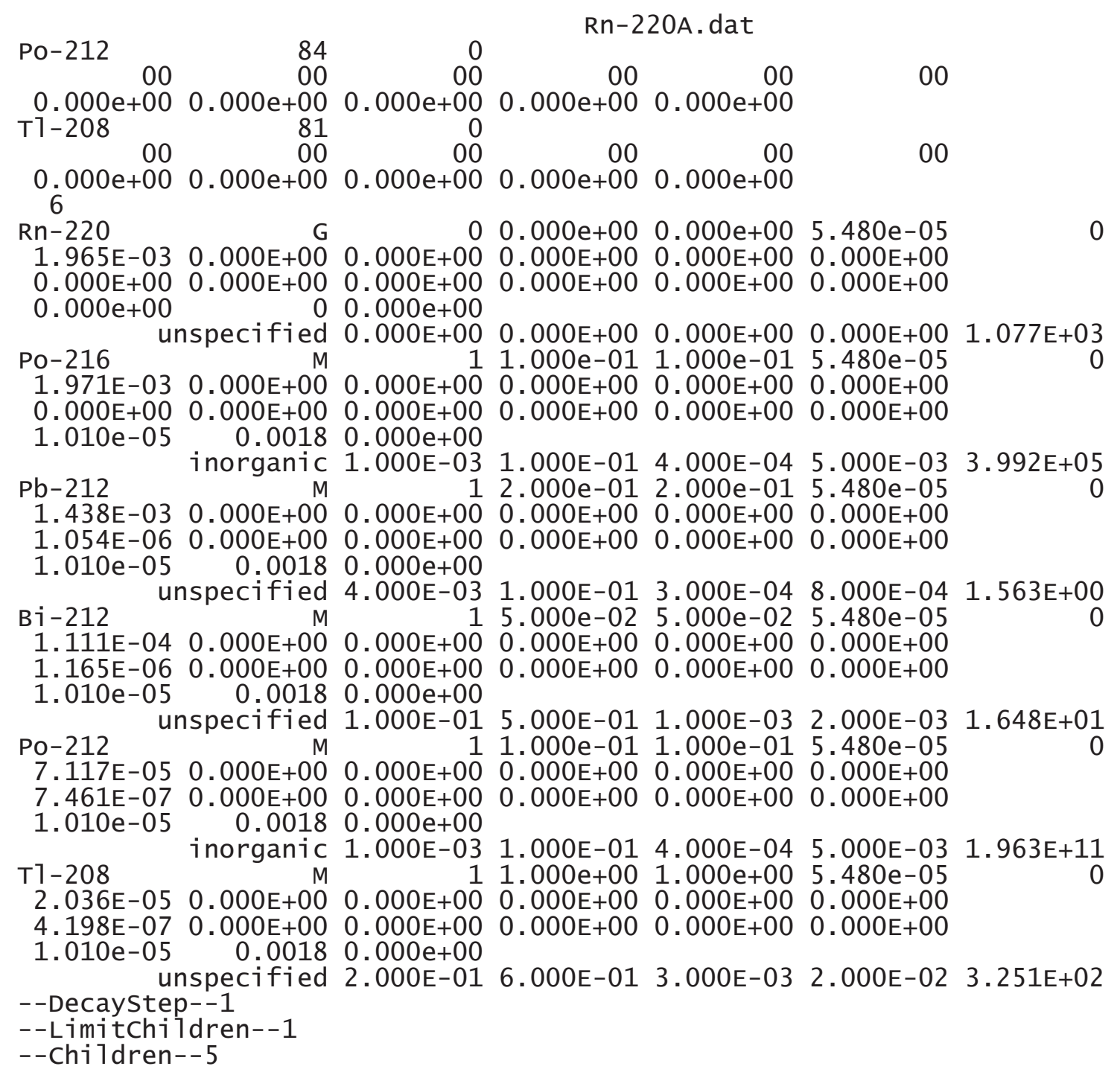




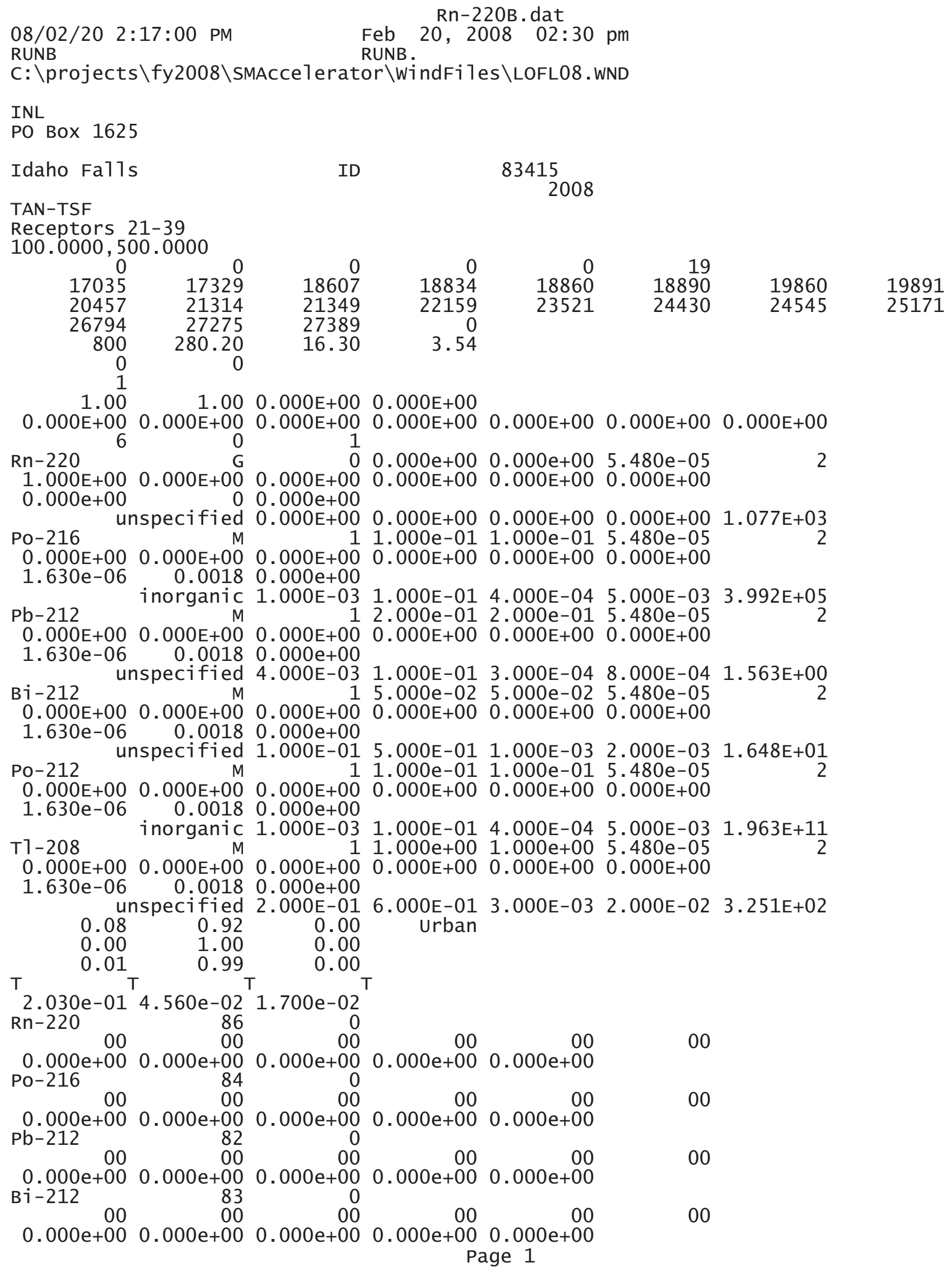




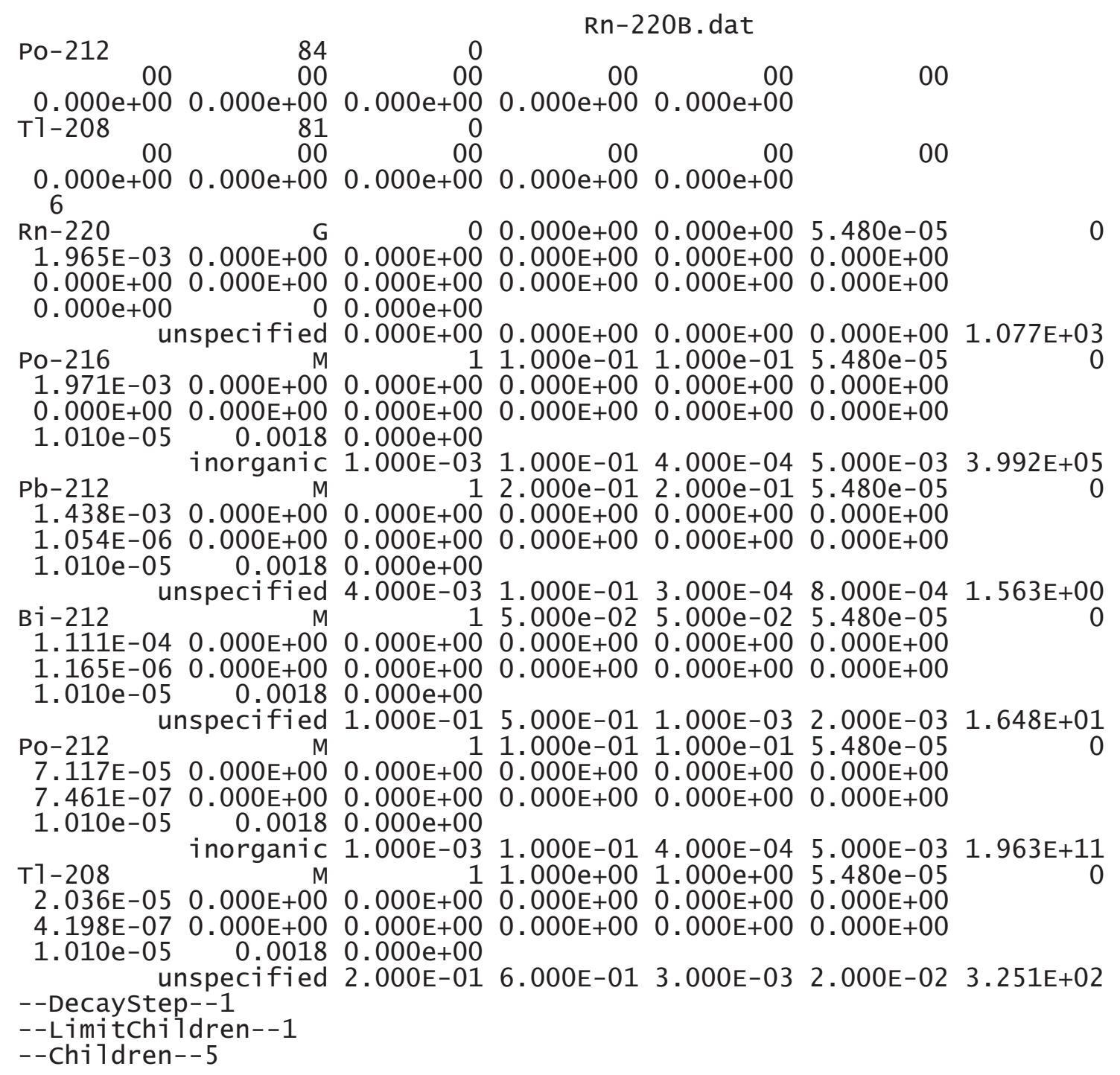




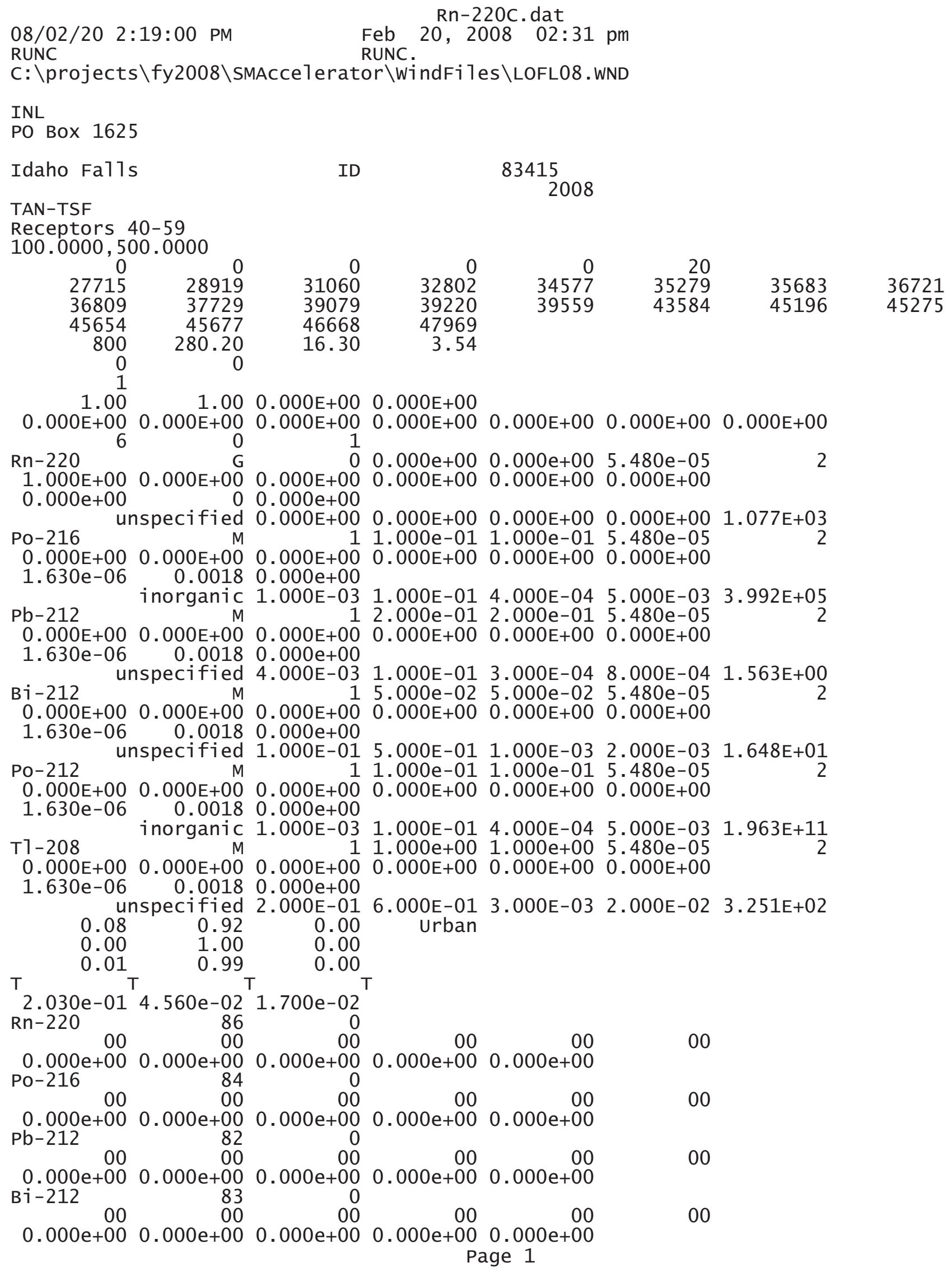




\begin{tabular}{|c|c|c|c|c|c|c|}
\hline \multirow{4}{*}{$\begin{array}{l}\text { Po-212 } 00 \\
0.000 \mathrm{e}+00 \\
\mathrm{~T} 1-208\end{array}$} & \multicolumn{6}{|c|}{ Rn-220C.dat } \\
\hline & 84 & & & & & \\
\hline & $\begin{array}{r}00 \\
000 e+00\end{array}$ & $\begin{array}{r}00 \\
0.000 e+00\end{array}$ & $\begin{array}{r}00 \\
0.000 e+00\end{array}$ & 00 & 00 & \\
\hline & & & & & & \\
\hline $\begin{array}{r}00 \\
0.000 e+00 \\
6\end{array}$ & $\begin{array}{r}00 \\
0.000 \mathrm{e}+00\end{array}$ & $\begin{array}{r}00 \\
0.000 e+00\end{array}$ & $\begin{array}{r}00 \\
0.000 e+00\end{array}$ & $\begin{array}{r}00 \\
0.000 \mathrm{e}+00\end{array}$ & 00 & \\
\hline $\begin{array}{l}\mathrm{Rn}-220 \\
1.965 \mathrm{E}-03 \\
0.000 \mathrm{E}+00 \\
0.000 \mathrm{e}+00\end{array}$ & $\begin{array}{r}\mathrm{G} \\
0.000 \mathrm{E}+00 \\
0.000 \mathrm{E}+00\end{array}$ & $\begin{array}{l}0 \\
0.000 \mathrm{E}+00 \\
0.000 \mathrm{E}+00 \\
0.000 \mathrm{e}+00\end{array}$ & $\begin{array}{l}0.000 \mathrm{e}+00 \\
0.000 \mathrm{E}+00 \\
0.000 \mathrm{E}+00\end{array}$ & $\begin{array}{l}0.000 \mathrm{e}+00 \\
0.000 \mathrm{E}+00 \\
0.000 \mathrm{E}+00\end{array}$ & $\begin{array}{l}5.480 \mathrm{e}-05 \\
0.000 \mathrm{E}+00 \\
0.000 \mathrm{E}+00\end{array}$ & 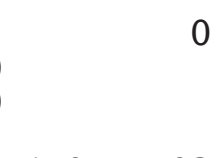 \\
\hline $\begin{array}{l}\text { Po-216 un } \\
1.971 \mathrm{E}-03 \\
0.000 \mathrm{E}+00 \\
1.010 \mathrm{e}-05\end{array}$ & $\begin{array}{r}\text { ispecified } \\
M \\
0.000 \mathrm{E}+00 \\
0.000 \mathrm{E}+00 \\
0.0018\end{array}$ & $\begin{array}{r}0.000 \mathrm{E}+00 \\
1 \\
0.000 \mathrm{E}+00 \\
0.000 \mathrm{E}+00 \\
0.000 \mathrm{e}+00\end{array}$ & $\begin{array}{l}0.000 \mathrm{E}+00 \\
1.000 \mathrm{e}-01 \\
0.000 \mathrm{E}+00 \\
0.000 \mathrm{E}+00\end{array}$ & $\begin{array}{l}0.000 \mathrm{E}+00 \\
1.000 \mathrm{e}-01 \\
0.000 \mathrm{E}+00 \\
0.000 \mathrm{E}+00\end{array}$ & $\begin{array}{l}0.000 \mathrm{E}+00 \\
5.480 \mathrm{e}-05 \\
0.000 \mathrm{E}+00 \\
0.000 \mathrm{E}+00\end{array}$ & $\begin{array}{r}1.077 \mathrm{E}+03 \\
0\end{array}$ \\
\hline $\begin{array}{l}\mathrm{Pb}-212 \\
1.438 \mathrm{E}-03 \\
1.054 \mathrm{E}-06 \\
1.010 \mathrm{e}-05\end{array}$ & $\begin{array}{r}\text { inorganic } \\
\mathrm{M} \\
0.000 \mathrm{E}+00 \\
0.000 \mathrm{E}+00 \\
0.0018\end{array}$ & $\begin{array}{r}1.000 \mathrm{E}-03 \\
1 \\
0.000 \mathrm{E}+00 \\
0.000 \mathrm{E}+00 \\
0.000 \mathrm{e}+00\end{array}$ & $\begin{array}{l}1.000 \mathrm{E}-01 \\
2.000 \mathrm{e}-01 \\
0.000 \mathrm{E}+00 \\
0.000 \mathrm{E}+00\end{array}$ & $\begin{array}{l}4.000 \mathrm{E}-04 \\
2.000 \mathrm{e}-01 \\
0.000 \mathrm{E}+00 \\
0.000 \mathrm{E}+00\end{array}$ & $\begin{array}{l}5.000 \mathrm{E}-03 \\
5.480 \mathrm{e}-05 \\
0.000 \mathrm{E}+00 \\
0.000 \mathrm{E}+00\end{array}$ & $\begin{array}{r}3.992 \mathrm{E}+05 \\
0\end{array}$ \\
\hline $\begin{array}{l}\mathrm{Bi}-212 \\
1.111 \mathrm{E}-04 \\
1.165 \mathrm{E}-06 \\
1.010 \mathrm{e}-05\end{array}$ & $\begin{array}{r}\text { sspecified } \\
M \\
0.000 \mathrm{E}+00 \\
0.000 \mathrm{E}+00 \\
0.0018\end{array}$ & $\begin{array}{r}4.000 \mathrm{E}-03 \\
1 \\
0.000 \mathrm{E}+00 \\
0.000 \mathrm{E}+00 \\
0.000 \mathrm{e}+00\end{array}$ & $\begin{array}{l}1.000 \mathrm{E}-01 \\
5.000 \mathrm{e}-02 \\
0.000 \mathrm{E}+00 \\
0.000 \mathrm{E}+00\end{array}$ & $\begin{array}{l}3.000 \mathrm{E}-04 \\
5.000 \mathrm{e}-02 \\
0.000 \mathrm{E}+00 \\
0.000 \mathrm{E}+00\end{array}$ & $\begin{array}{l}8.000 \mathrm{E}-04 \\
5.480 \mathrm{e}-05 \\
0.000 \mathrm{E}+00 \\
0.000 \mathrm{E}+00\end{array}$ & $\begin{array}{r}1.563 \mathrm{E}+00 \\
0\end{array}$ \\
\hline $\begin{array}{l}\text { Po-212 Un } \\
7.117 \mathrm{E}-05 \\
7.461 \mathrm{E}-07 \\
1.010 \mathrm{e}-05\end{array}$ & $\begin{array}{r}\text { ispecified } \\
M \\
0.000 \mathrm{E}+00 \\
0.000 \mathrm{E}+00 \\
0.0018\end{array}$ & $\begin{array}{r}1.000 \mathrm{E}-01 \\
1 \\
0.000 \mathrm{E}+00 \\
0.000 \mathrm{E}+00 \\
0.000 \mathrm{e}+00\end{array}$ & $\begin{array}{l}5.000 \mathrm{E}-01 \\
1.000 \mathrm{e}-01 \\
0.000 \mathrm{E}+00 \\
0.000 \mathrm{E}+00\end{array}$ & $\begin{array}{l}1.000 \mathrm{E}-03 \\
1.000 \mathrm{e}-01 \\
0.000 \mathrm{E}+00 \\
0.000 \mathrm{E}+00\end{array}$ & $\begin{array}{l}2.000 \mathrm{E}-03 \\
5.480 \mathrm{e}-05 \\
0.000 \mathrm{E}+00 \\
0.000 \mathrm{E}+00\end{array}$ & $1.648 \mathrm{E}+01$ \\
\hline $\begin{array}{l}\mathrm{T} 1-208 \\
2.036 \mathrm{E}-05 \\
4.198 \mathrm{E}-07 \\
1.010 \mathrm{e}-05\end{array}$ & $\begin{array}{r}\text { inorganic } \\
M \\
0.000 \mathrm{E}+00 \\
0.000 \mathrm{E}+00 \\
0.0018\end{array}$ & $\begin{array}{r}1.000 \mathrm{E}-03 \\
1 \\
0.000 \mathrm{E}+00 \\
0.000 \mathrm{E}+00 \\
0.000 \mathrm{e}+00\end{array}$ & $\begin{array}{l}1.000 \mathrm{E}-01 \\
1.000 \mathrm{e}+00 \\
0.000 \mathrm{E}+00 \\
0.000 \mathrm{E}+00\end{array}$ & $\begin{array}{l}4.000 \mathrm{E}-04 \\
1.000 \mathrm{e}+00 \\
0.000 \mathrm{E}+00 \\
0.000 \mathrm{E}+00\end{array}$ & $\begin{array}{l}5.000 \mathrm{E}-03 \\
5.480 \mathrm{e}-05 \\
0.000 \mathrm{E}+00 \\
0.000 \mathrm{E}+00\end{array}$ & $\begin{array}{r}1.963 \mathrm{E}+11 \\
0\end{array}$ \\
\hline $\begin{array}{l}\text { un } \\
\text {--Decaystep } \\
\text {--Limitchi } \\
\text {--Children- }\end{array}$ & $\begin{array}{l}\text { ispecified } \\
\text { - }\end{array}$ & $2.000 \mathrm{E}-01$ & $6.000 E-01$ & $3.000 \mathrm{E}-03$ & $2.000 \mathrm{E}-02$ & $3.251 E+02$ \\
\hline
\end{tabular}


08/02/20 2:20:00 PM

RUND

C: \projects \fy2008\SMAccelerator \WindFi 1es\LOFL08 . WND

INL

PO Box 1625

Idaho Fal1s

TAN-TSF

Receptors 60-62

$100.0000,500.0000$

$\begin{array}{rrrr}50024 & 0 & 0 & 0 \\ 0 & 54611 & 58610 & 0 \\ 0 & 0 & 0 & 0 \\ 800 & 0 & 0 & 0 \\ 0 & 280.20 & 16.30 & 3.54 \\ 1 & 0 & & \end{array}$

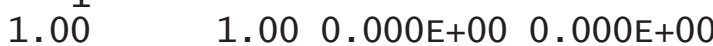

$\begin{array}{llll}0.000 \mathrm{E}+00 & 0.000 \mathrm{E}+00 \quad 0.000 \mathrm{E}+00 \quad 0.000 \mathrm{E}+00 & 0\end{array}$

$60.00 \mathrm{E}+00$

$\mathrm{Rn}-220$ $0.000 \mathrm{H}+1$

$\begin{array}{lll}1.000 \mathrm{E}+00 & 0.000 \mathrm{E}+00 \quad 0.000 \mathrm{E}+00 & 0\end{array}$

$0.000 \mathrm{e}+00$

$0.000 \mathrm{E}+00 \quad 0.000 \mathrm{E}+00 \quad 0.000 \mathrm{E}+00$

$0.000 \mathrm{e}+00$ $0.000 \mathrm{e}+00$

Po-216 unspecified $0.000 \mathrm{E}+00$

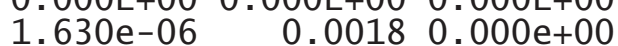

inorganic $1.000 \mathrm{E}-03 \quad 1.000 \mathrm{E}-01 \quad 4.000 \mathrm{E}-04 \quad 5.000 \mathrm{E}-03$

$\mathrm{Pb}-212$

$\mathrm{M}$

$\begin{array}{llllll}0.000 \mathrm{E}+00 & 0.000 \mathrm{E}+00 & 0.000 \mathrm{E}+00 & 0.000 \mathrm{E}+00 & 0.000 \mathrm{E}+00 & 0.000 \mathrm{E}+00\end{array}$

$\begin{array}{lll}1.630 \mathrm{e}-06 & 0.0018 \quad 0.000 \mathrm{e}+00\end{array}$

unspecified 4.000E-03 1.000E-01 3.000E-04 8.000E-04 $1.563 \mathrm{E}+00$

$\mathrm{Bi}-212$

$\mathrm{M} 0.000 \mathrm{~T}+00$

$\begin{array}{rrr}0.000 \mathrm{E}+00 & 0.000 \mathrm{E}+00 & 0.000 \mathrm{E}+00 \\ 1.630 \mathrm{e}-06 & 0.0018 & 0.000 \mathrm{e}+00\end{array}$

$\begin{array}{rrr}1.630 \mathrm{e}-06 & 0.0018 & 0.000 \mathrm{e}+00 \\ \text { unspecified } & 1.000 \mathrm{E}-01\end{array}$

Po-212

$0.000 \mathrm{E}+00 \quad 0.000 \mathrm{E}+00 \quad 0.000 \mathrm{E}+00 \quad 0.000 \mathrm{E}+00 \quad 0.000 \mathrm{E}+00 \quad 0.000 \mathrm{E}+00$

$1.630 \mathrm{e}-06 \quad 0.0018 \quad 0.000 \mathrm{e}+00$

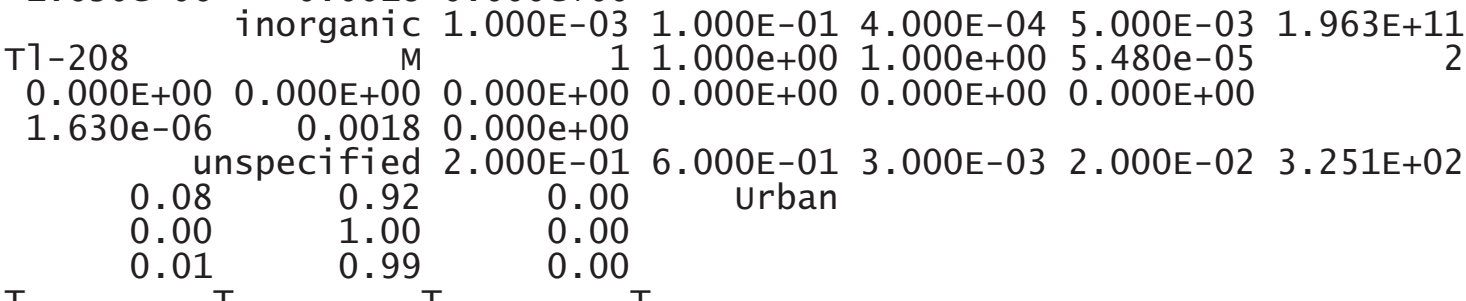

$\mathrm{T}^{\mathrm{T}} \mathrm{T}^{\mathrm{T}} \mathrm{T}^{\mathrm{T}} \mathrm{T}^{\mathrm{T}}$

2.030e-01 4.560e-02 $1.700 \mathrm{e}-02$

$\mathrm{Rn}-220$

$00 \quad 00 \quad 00$

$0.000 \mathrm{e}+00 \quad 0.000 \mathrm{e}+00 \quad 0.000 \mathrm{e}+00$

Po-216

$0.000 \mathrm{e}+00 \quad 0.000 \mathrm{e}+00$

$\mathrm{Pb}-212$

$00 \quad 82$

$0.000 \mathrm{e}+00 \quad 0.000 \mathrm{e}+00$

$\mathrm{Bi}-212$

$00 \quad 00$

$0.000 \mathrm{e}+00 \quad 0.000 \mathrm{e}+00$
$0.000 \mathrm{E}+00 \quad 0.000 \mathrm{E}+00 \quad 0.000 \mathrm{E}+00 \quad 1.077 \mathrm{E}+03$

$12.000 \mathrm{e}-01 \quad 2.000 \mathrm{e}-01 \quad 5.480 \mathrm{e}-05$

$15.000 \mathrm{e}-02 \quad 5.000 \mathrm{e}-02 \quad 5.480 \mathrm{e}-05$

$0.000 \mathrm{E}+00 \quad 0.000 \mathrm{E}+00 \quad 0.000 \mathrm{E}+00$

$3.992 \mathrm{E}+05$

$11.000 \mathrm{e}-01 \quad 1.000 \mathrm{e}-01 \quad 5.480 \mathrm{e}-05$

0

00

$0.000 e+00 \quad 0.000 e+00$

00

00

$0.000 e+00$

$0.000 e+00 \quad 0.000 e+00$

1. $648 \mathrm{E}+01$

2

\section{.}




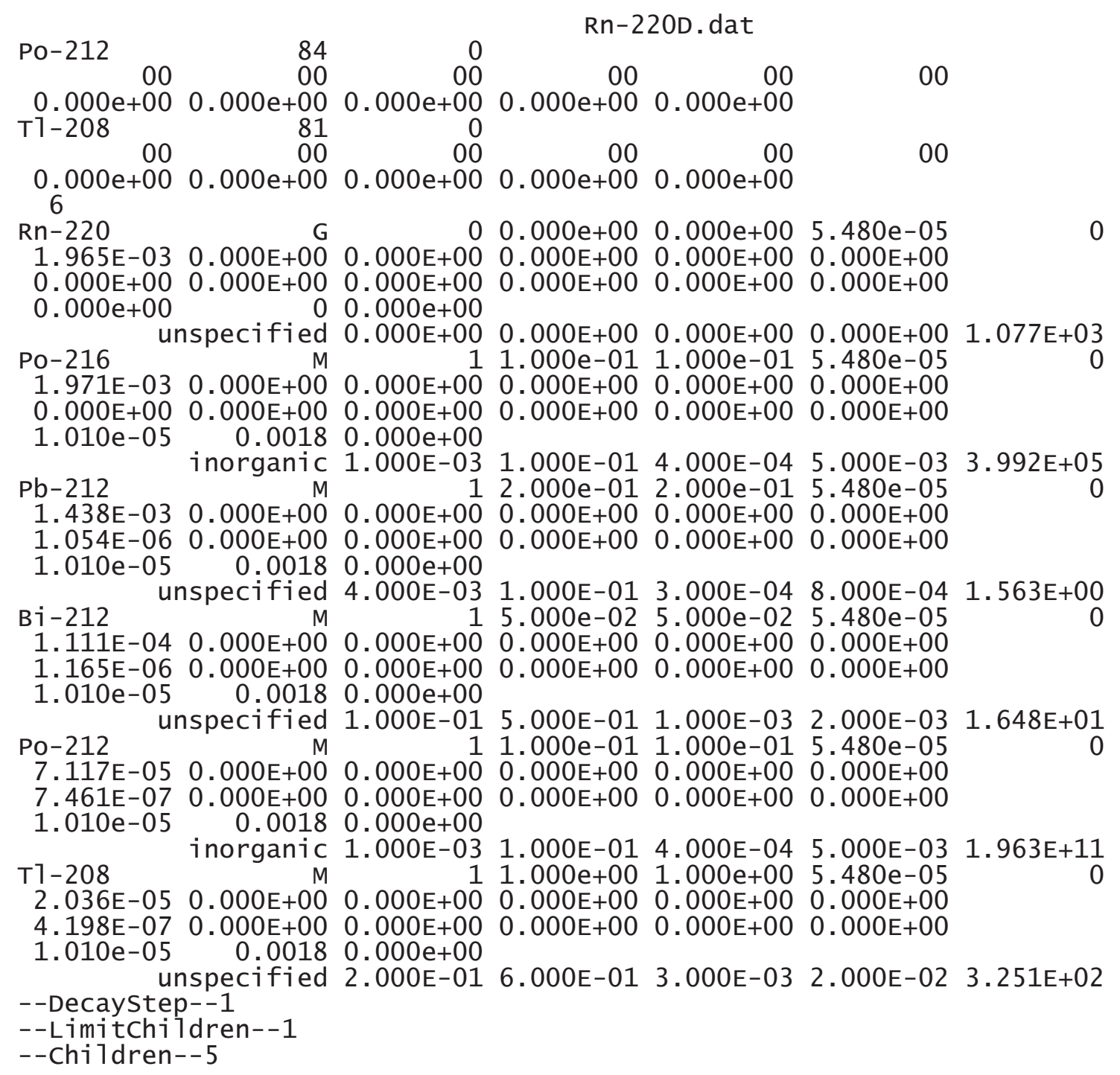




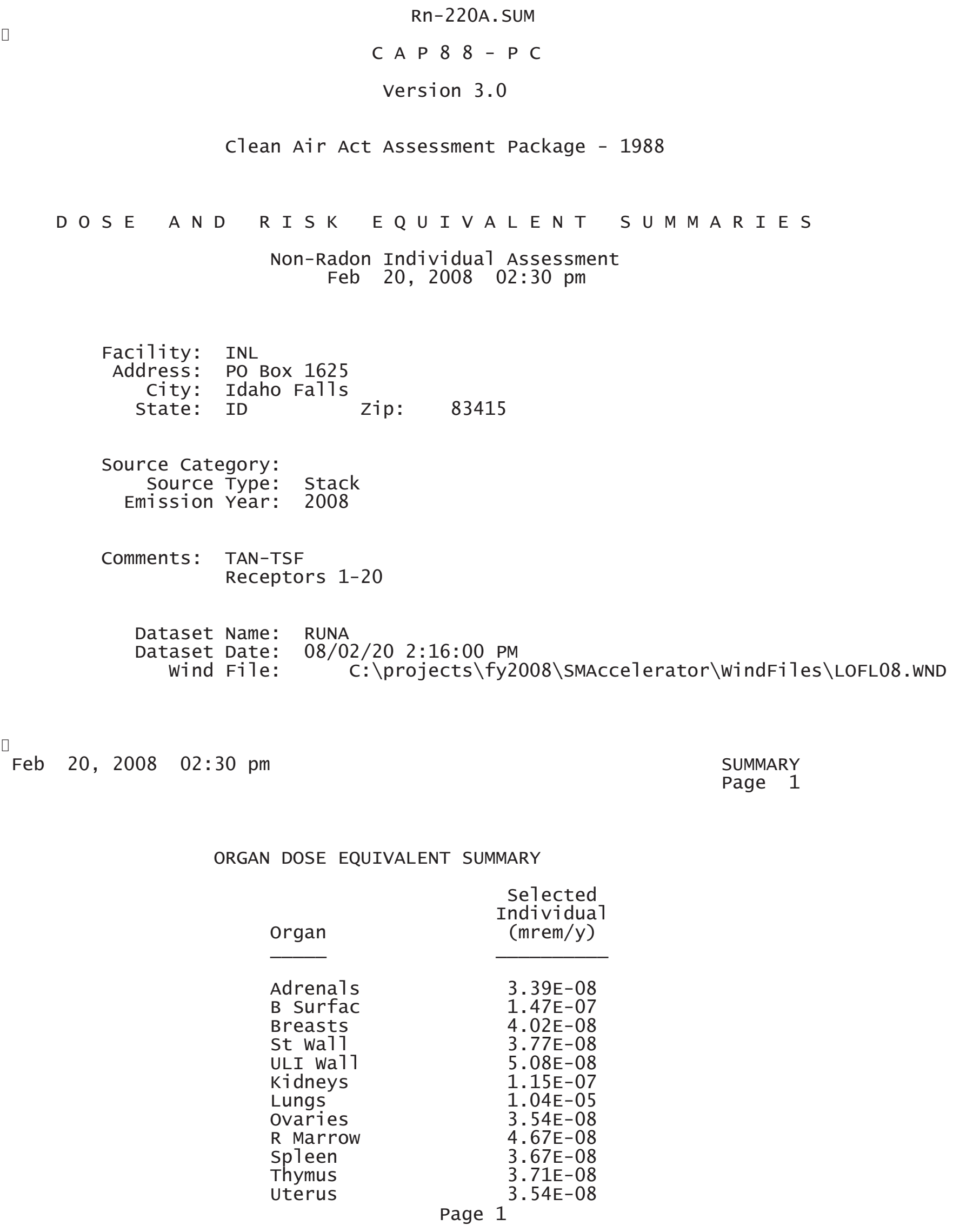

\begin{tabular}{lr} 
ORGAN DOSE EQUIVALENT SUMMARY \\
Selected \\
Organ & $\begin{array}{r}\text { Individual } \\
\text { (mrem/y) }\end{array}$ \\
\cline { 2 - 2 } Adrena1s & $3.39 \mathrm{E}-08$ \\
B Surfac & $1.47 \mathrm{E}-07$ \\
Breasts & $4.02 \mathrm{E}-08$ \\
St wa11 & $3.77 \mathrm{E}-08$ \\
ULI wa71 & $5.08 \mathrm{E}-08$ \\
Kidneys & $1.15 \mathrm{E}-07$ \\
Lungs & $1.04 \mathrm{E}-05$ \\
Ovaries & $3.54 \mathrm{E}-08$ \\
R Marrow & $4.67 \mathrm{E}-08$ \\
Spleen & $3.67 \mathrm{E}-08$ \\
Thymus & $3.71 \mathrm{E}-08$ \\
Uterus & $3.54 \mathrm{E}-08$ \\
& 1
\end{tabular}




$\begin{array}{lr} & \text { Rn-220A. SUM } \\ \text { B1d wa11 } & 3.85 \mathrm{E}-08 \\ \text { Brain } & 3.70 \mathrm{E}-08 \\ \text { Esophagu } & 3.83 \mathrm{E}-06 \\ \text { SI wa11 } & 3.96 \mathrm{E}-08 \\ \text { LLI wa11 } & 5.36 \mathrm{E}-08 \\ \text { Liver } & 5.73 \mathrm{E}-08 \\ \text { Muscle } & 4.03 \mathrm{E}-08 \\ \text { Pancreas } & 3.46 \mathrm{E}-08 \\ \text { Skin } & 2.14 \mathrm{E}-07 \\ \text { Testes } & 4.08 \mathrm{E}-08 \\ \text { Thyroid } & 3.77 \mathrm{E}-08 \\ & \\ \text { EFFEC } & 2.51 \mathrm{E}-05\end{array}$

PATHWAY EFFECTIVE DOSE EQUIVALENT SUMMARY

selected Individual

Pathway (mrem/y)

INGESTION

2. $08 \mathrm{E}-13$

INHALATION

2. $51 \mathrm{E}-05$

AIR IMMERSION

5. $30 \mathrm{E}-09$

GROUND SURFACE

$3.12 \mathrm{E}-08$

INTERNAL

2. $51 \mathrm{E}-05$

EXTERNAL

$3.65 \mathrm{E}-08$

TOTAL

$2.51 \mathrm{E}-05$

$\square$

Feb 20, 2008 02:30 pm

SUMMARY

Page 2

NUCLIDE EFFECTIVE DOSE EQUIVALENT SUMMARY

Nuclide

$\mathrm{Rn}-220$

Po-216

$\mathrm{Pb}-212$

$\mathrm{Bi}-212$

Po-212

$\mathrm{T} 1-208$

TOTAL

$$
\begin{gathered}
\begin{array}{c}
\text { selected } \\
\text { Individual } \\
\text { (mrem/y) }
\end{array} \\
\hline 2.15 \mathrm{E}-11 \\
6.00 \mathrm{E}-13 \\
2.48 \mathrm{E}-05 \\
3.49 \mathrm{E}-07 \\
0.00 \mathrm{E}+00 \\
2.49 \mathrm{E}-08 \\
2.51 \mathrm{E}-05
\end{gathered}
$$

Feb 20, $2008 \quad 02: 30 \mathrm{pm}$

SUMMARY

Page 3

CANCER RISK SUMMARY 


\section{Cancer \\ Esophagu \\ Stomach \\ colon \\ Liver \\ LUNG \\ Bone \\ Skin \\ Breast \\ ovary \\ Bladder \\ Kidneys \\ Thyroid \\ Leukemia \\ Residua 1 \\ Tota1}

TOTAL

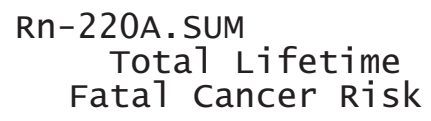

Rn-220A. SUM

Total Lifetime Fatal Cancer Risk

1. $41 \mathrm{E}-15$

7. $63 \mathrm{E}-15$

1. $01 \mathrm{E}-13$

9. $14 \mathrm{E}-15$

2. $14 \mathrm{E}-11$

2.95E-15

$3.05 \mathrm{E}-16$

4. $26 \mathrm{E}-15$

$1.83 \mathrm{E}-15$

3. $24 \mathrm{E}-15$

$9.88 \mathrm{E}-15$

$4.26 \mathrm{E}-16$

$3.18 \mathrm{E}-15$

$1.96 \mathrm{E}-14$

2. $16 \mathrm{E}-11$

4. 31E-11

PATHWAY RISK SUMMARY

Pathway

INGESTION

INHALATION

AIR IMMERSION

GROUND SURFACE

INTERNAL

EXTERNAL

TOTAL
Selected Individual Total Lifetime Fatal Cancer Risk

2.07E-19

2. $16 \mathrm{E}-11$

2. $84 \mathrm{E}-15$

1. $65 \mathrm{E}-14$

2. $16 \mathrm{E}-11$

1. $94 \mathrm{E}-14$

$2.16 \mathrm{E}-11$

$\square$

Feb 20, 2008 02:30 pm

SUMMARY Page 4

NUCLIDE RISK SUMMARY

Nuclide

$\mathrm{Rn}-220$

Po-216

$\mathrm{Pb}-212$

Bi -212

Po-212

T1-208

TOTAL
Selected Individual

Total Lifetime Fatal Cancer Risk

1.17E-17

3. $28 \mathrm{E}-19$

$2.13 \mathrm{E}-11$

2. $24 \mathrm{E}-13$

$0.00 \mathrm{E}+00$

1. $36 \mathrm{E}-14$

$2.16 \mathrm{E}-11$

$\square$

Feb 20, 2008 02:30 pm

Page 3 
Rn-220A. SUM

Page 5

INDIVIDUAL EFFECTIVE DOSE EQUIVALENT RATE (mrem/y)

(A11 Radionuclides and Pathways)

\begin{tabular}{|c|c|c|c|c|c|c|c|}
\hline \multirow[b]{2}{*}{ Direction } & \multicolumn{6}{|c|}{ Distance (m) } & \multirow[b]{2}{*}{12522} \\
\hline & 10344 & 10365 & 10472 & 10590 & 11103 & 11989 & \\
\hline $\mathrm{N}$ & $4.8 \mathrm{E}-06$ & $4.8 \mathrm{E}-06$ & $4.8 \mathrm{E}-06$ & $4.7 \mathrm{E}-06$ & $4.4 \mathrm{E}-06$ & $4.0 \mathrm{E}-06$ & $3.7 \mathrm{E}-06$ \\
\hline NNW & $2.2 \mathrm{E}-06$ & $2.2 \mathrm{E}-06$ & $2.2 \mathrm{E}-06$ & $2.1 \mathrm{E}-06$ & $2.0 \mathrm{E}-06$ & $1.8 \mathrm{E}-06$ & $1.7 \mathrm{E}-06$ \\
\hline NW & $2.8 \mathrm{E}-06$ & $2.8 \mathrm{E}-06$ & $2.7 \mathrm{E}-06$ & $2.7 \mathrm{E}-06$ & $2.5 \mathrm{E}-06$ & $2.3 \mathrm{E}-06$ & $2.2 \mathrm{E}-06$ \\
\hline WNW & $3.7 \mathrm{E}-06$ & $3.7 \mathrm{E}-06$ & $3.6 \mathrm{E}-06$ & $3.6 \mathrm{E}-06$ & $3.3 \mathrm{E}-06$ & $3.0 \mathrm{E}-06$ & $2.8 \mathrm{E}-06$ \\
\hline W & $3.8 \mathrm{E}-06$ & $3.8 \mathrm{E}-06$ & $3.8 \mathrm{E}-06$ & $3.7 \mathrm{E}-06$ & $3.5 \mathrm{E}-06$ & $3.1 \mathrm{E}-06$ & 3. $0 \mathrm{E}-06$ \\
\hline WSW & $6.1 \mathrm{E}-06$ & $6.1 \mathrm{E}-06$ & $6.0 \mathrm{E}-06$ & $6.0 \mathrm{E}-06$ & $5.6 \mathrm{E}-06$ & $5.0 \mathrm{E}-06$ & $4.7 \mathrm{E}-06$ \\
\hline SW & 1.1E-05 & 1.1E-05 & 1.1E-05 & 1.1E-05 & $1.0 \mathrm{E}-05$ & $9.0 \mathrm{E}-06$ & $8.5 \mathrm{E}-06$ \\
\hline SSW & $1.8 \mathrm{E}-05$ & $1.8 \mathrm{E}-05$ & $1.8 \mathrm{E}-05$ & $1.7 \mathrm{E}-05$ & $1.6 \mathrm{E}-05$ & 1.5E-05 & 1. $4 \mathrm{E}-05$ \\
\hline $\mathrm{S}$ & 2. $5 \mathrm{E}-05$ & 2. $5 \mathrm{E}-05$ & $2.5 \mathrm{E}-05$ & $2.4 \mathrm{E}-05$ & $2.3 \mathrm{E}-05$ & $2.0 \mathrm{E}-05$ & 1. $9 \mathrm{E}-05$ \\
\hline SSE & $2.2 \mathrm{E}-05$ & $2.2 \mathrm{E}-05$ & $2.2 \mathrm{E}-05$ & $2.2 \mathrm{E}-05$ & $2.0 \mathrm{E}-05$ & $1.8 \mathrm{E}-05$ & $1.7 \mathrm{E}-05$ \\
\hline SE & 8.9E-06 & 8.9E-06 & $8.8 \mathrm{E}-06$ & 8.6E-06 & $8.1 \mathrm{E}-06$ & $7.3 \mathrm{E}-06$ & $6.9 \mathrm{E}-06$ \\
\hline ESE & $5.5 \mathrm{E}-06$ & $5.5 \mathrm{E}-06$ & $5.4 \mathrm{E}-06$ & 5. $3 \mathrm{E}-06$ & $5.0 \mathrm{E}-06$ & 4.5E-06 & $4.2 \mathrm{E}-06$ \\
\hline E & $6.9 \mathrm{E}-06$ & $6.9 \mathrm{E}-06$ & $6.8 \mathrm{E}-06$ & $6.7 \mathrm{E}-06$ & $6.3 \mathrm{E}-06$ & $5.6 \mathrm{E}-06$ & $5.3 \mathrm{E}-06$ \\
\hline ENE & $7.5 \mathrm{E}-06$ & $7.5 \mathrm{E}-06$ & $7.4 \mathrm{E}-06$ & $7.3 \mathrm{E}-06$ & $6.8 \mathrm{E}-06$ & $6.1 \mathrm{E}-06$ & $5.8 \mathrm{E}-06$ \\
\hline NE & $1.2 \mathrm{E}-05$ & $1.2 \mathrm{E}-05$ & $1.2 \mathrm{E}-05$ & $1.2 \mathrm{E}-05$ & 1.1E-05 & $1.0 \mathrm{E}-05$ & $9.5 \mathrm{E}-06$ \\
\hline NNE & $7.1 \mathrm{E}-06$ & 7.0E-06 & $6.9 \mathrm{E}-06$ & $6.8 \mathrm{E}-06$ & $6.4 \mathrm{E}-06$ & $5.8 \mathrm{E}-06$ & $5.4 \mathrm{E}-06$ \\
\hline
\end{tabular}

Distance $(m)$

\begin{tabular}{|c|c|c|c|c|c|c|c|}
\hline Direction & n 13245 & 13286 & 13483 & 13612 & 13664 & 13959 & 14258 \\
\hline $\mathrm{N}$ & $3.5 \mathrm{E}-06$ & $3.4 \mathrm{E}-06$ & $3.4 \mathrm{E}-06$ & $3.3 E-06$ & $3.3 \mathrm{E}-06$ & $3.2 \mathrm{E}-06$ & $3.1 \mathrm{E}-06$ \\
\hline NNW & $1.6 \mathrm{E}-06$ & $1.6 \mathrm{E}-06$ & $1.6 \mathrm{E}-06$ & $1.5 \mathrm{E}-06$ & $1.5 \mathrm{E}-06$ & $1.5 \mathrm{E}-06$ & $1.4 \mathrm{E}-06$ \\
\hline NW & $2.0 \mathrm{E}-06$ & $2.0 \mathrm{E}-06$ & $1.9 \mathrm{E}-06$ & $1.9 \mathrm{E}-06$ & $1.9 \mathrm{E}-06$ & $1.9 \mathrm{E}-06$ & $1.8 \mathrm{E}-06$ \\
\hline WNW & $2.6 \mathrm{E}-06$ & $2.6 \mathrm{E}-06$ & $2.6 \mathrm{E}-06$ & 2. $5 \mathrm{E}-06$ & $2.5 \mathrm{E}-06$ & $2.5 \mathrm{E}-06$ & $2.4 \mathrm{E}-06$ \\
\hline W & $2.7 \mathrm{E}-06$ & $2.7 \mathrm{E}-06$ & $2.7 \mathrm{E}-06$ & $2.6 \mathrm{E}-06$ & $2.6 \mathrm{E}-06$ & $2.5 \mathrm{E}-06$ & $2.5 \mathrm{E}-06$ \\
\hline WSW & $4.4 \mathrm{E}-06$ & 4. $3 \mathrm{E}-06$ & 4. $3 \mathrm{E}-06$ & $4.2 \mathrm{E}-06$ & $4.2 \mathrm{E}-06$ & $4.1 \mathrm{E}-06$ & $3.9 \mathrm{E}-06$ \\
\hline SW & $7.8 \mathrm{E}-06$ & $7.8 \mathrm{E}-06$ & $7.6 \mathrm{E}-06$ & 7. $5 \mathrm{E}-06$ & $7.5 \mathrm{E}-06$ & $7.3 \mathrm{E}-06$ & 7.0E-06 \\
\hline SSW & 1. $3 \mathrm{E}-05$ & 1. $3 \mathrm{E}-05$ & $1.2 \mathrm{E}-05$ & $1.2 \mathrm{E}-05$ & $1.2 \mathrm{E}-05$ & $1.2 \mathrm{E}-05$ & $1.1 \mathrm{E}-05$ \\
\hline & $1.8 \mathrm{E}-05$ & $1.8 \mathrm{E}-05$ & $1.7 \mathrm{E}-05$ & 1.7E-05 & $1.7 \mathrm{E}-05$ & $1.6 \mathrm{E}-05$ & 1. $6 \mathrm{E}-05$ \\
\hline SSE & $1.6 \mathrm{E}-05$ & $1.6 \mathrm{E}-05$ & $1.6 \mathrm{E}-05$ & $1.5 \mathrm{E}-05$ & $1.5 \mathrm{E}-05$ & $1.5 \mathrm{E}-05$ & $1.4 \mathrm{E}-05$ \\
\hline SE & $6.3 \mathrm{E}-06$ & $6.3 \mathrm{E}-06$ & $6.2 \mathrm{E}-06$ & $6.1 \mathrm{E}-06$ & $6.0 \mathrm{E}-06$ & $5.9 \mathrm{E}-06$ & $5.7 \mathrm{E}-06$ \\
\hline ESE & $3.9 \mathrm{E}-06$ & $3.9 \mathrm{E}-06$ & $3.8 \mathrm{E}-06$ & $3.8 \mathrm{E}-06$ & $3.7 \mathrm{E}-06$ & $3.6 \mathrm{E}-06$ & $3.5 \mathrm{E}-06$ \\
\hline & $4.9 \mathrm{E}-06$ & $4.9 \mathrm{E}-06$ & $4.8 \mathrm{E}-06$ & 4.7E-06 & 4.7E-06 & $4.5 \mathrm{E}-06$ & 4. $4 \mathrm{E}-06$ \\
\hline ENE & $5.3 \mathrm{E}-06$ & $5.3 \mathrm{E}-06$ & $5.2 \mathrm{E}-06$ & $5.1 \mathrm{E}-06$ & $5.1 \mathrm{E}-06$ & $5.0 \mathrm{E}-06$ & $4.8 \mathrm{E}-06$ \\
\hline $\mathrm{NE}$ & $8.8 \mathrm{E}-06$ & $8.7 \mathrm{E}-06$ & $8.6 \mathrm{E}-06$ & $8.4 \mathrm{E}-06$ & $8.4 \mathrm{E}-06$ & $8.1 \mathrm{E}-06$ & $7.9 \mathrm{E}-06$ \\
\hline NNE & $5.0 \mathrm{E}-06$ & $5.0 \mathrm{E}-06$ & $4.9 \mathrm{E}-06$ & $4.8 \mathrm{E}-06$ & $4.8 \mathrm{E}-06$ & $4.6 \mathrm{E}-06$ & 4. $5 \mathrm{E}-06$ \\
\hline
\end{tabular}

口

Feb 20, $2008 \quad 02: 30 \mathrm{pm}$

SUMMARY

Page 6

INDIVIDUAL EFFECTIVE DOSE EQUIVALENT RATE (mrem/y) (A11 Radionuclides and Pathways)

Page 4 
Rn-220A. SUM

\begin{tabular}{|c|c|c|c|c|c|c|}
\hline \multirow[b]{2}{*}{ Direction } & \multicolumn{6}{|c|}{ Distance $(\mathrm{m})$} \\
\hline & 14374 & 15241 & 15441 & 15784 & 15844 & 16323 \\
\hline $\mathrm{N}$ & $3.1 \mathrm{E}-06$ & $2.8 \mathrm{E}-06$ & $2.8 \mathrm{E}-06$ & $2.7 \mathrm{E}-06$ & $2.7 \mathrm{E}-06$ & $2.5 \mathrm{E}-06$ \\
\hline NNW & 1. $4 \mathrm{E}-06$ & 1. $3 \mathrm{E}-06$ & 1. $3 \mathrm{E}-06$ & 1. $3 \mathrm{E}-06$ & 1. $3 \mathrm{E}-06$ & 1. $2 \mathrm{E}-06$ \\
\hline NW & 1. $8 \mathrm{E}-06$ & 1. $6 \mathrm{E}-06$ & 1. $6 \mathrm{E}-06$ & $1.6 \mathrm{E}-06$ & $1.6 \mathrm{E}-06$ & $1.5 \mathrm{E}-06$ \\
\hline WNW & $2.4 \mathrm{E}-06$ & 2. $2 \mathrm{E}-06$ & $2.1 \mathrm{E}-06$ & $2.1 \mathrm{E}-06$ & $2.1 \mathrm{E}-06$ & 2. $0 \mathrm{E}-06$ \\
\hline W & $2.4 \mathrm{E}-06$ & 2. $2 \mathrm{E}-06$ & $2.2 \mathrm{E}-06$ & $2.1 \mathrm{E}-06$ & $2.1 \mathrm{E}-06$ & 2. $0 \mathrm{E}-06$ \\
\hline WSW & $3.9 \mathrm{E}-06$ & $3.6 \mathrm{E}-06$ & $3.5 \mathrm{E}-06$ & $3.4 \mathrm{E}-06$ & $3.4 \mathrm{E}-06$ & 3. $3 \mathrm{E}-06$ \\
\hline SW & 7. $0 \mathrm{E}-06$ & $6.4 \mathrm{E}-06$ & $6.3 \mathrm{E}-06$ & $6.1 \mathrm{E}-06$ & $6.1 \mathrm{E}-06$ & $5.8 \mathrm{E}-06$ \\
\hline SSW & $1.1 \mathrm{E}-05$ & 1. $0 \mathrm{E}-05$ & 1. $0 \mathrm{E}-05$ & $9.8 \mathrm{E}-06$ & $9.8 \mathrm{E}-06$ & $9.3 \mathrm{E}-06$ \\
\hline $\mathrm{s}$ & $1.6 \mathrm{E}-05$ & $1.4 \mathrm{E}-05$ & $1.4 \mathrm{E}-05$ & $1.4 \mathrm{E}-05$ & 1. $4 \mathrm{E}-05$ & 1. $3 \mathrm{E}-05$ \\
\hline SSE & $1.4 \mathrm{E}-05$ & $1.3 \mathrm{E}-05$ & $1.3 \mathrm{E}-05$ & $1.2 \mathrm{E}-05$ & $1.2 \mathrm{E}-05$ & $1.2 \mathrm{E}-05$ \\
\hline $\mathrm{SE}$ & $5.6 \mathrm{E}-06$ & $5.1 \mathrm{E}-06$ & $5.0 \mathrm{E}-06$ & $4.9 \mathrm{E}-06$ & $4.9 \mathrm{E}-06$ & $4.6 \mathrm{E}-06$ \\
\hline ESE & $3.5 \mathrm{E}-06$ & $3.2 \mathrm{E}-06$ & $3.1 \mathrm{E}-06$ & $3.0 \mathrm{E}-06$ & $3.0 \mathrm{E}-06$ & $2.9 \mathrm{E}-06$ \\
\hline $\mathrm{E}$ & $4.3 \mathrm{E}-06$ & $4.0 \mathrm{E}-06$ & $3.9 \mathrm{E}-06$ & $3.8 \mathrm{E}-06$ & $3.7 \mathrm{E}-06$ & $3.6 \mathrm{E}-06$ \\
\hline ENE & $4.8 \mathrm{E}-06$ & $4.4 \mathrm{E}-06$ & $4.3 \mathrm{E}-06$ & $4.2 \mathrm{E}-06$ & $4.1 \mathrm{E}-06$ & $4.0 \mathrm{E}-06$ \\
\hline $\mathrm{NE}$ & $7.8 \mathrm{E}-06$ & $7.2 \mathrm{E}-06$ & $7.0 \mathrm{E}-06$ & $6.8 \mathrm{E}-06$ & $6.8 \mathrm{E}-06$ & $6.5 \mathrm{E}-06$ \\
\hline NNE & $4.5 \mathrm{E}-06$ & $4.1 \mathrm{E}-06$ & $4.0 \mathrm{E}-06$ & $3.9 \mathrm{E}-06$ & $3.9 \mathrm{E}-06$ & $3.7 \mathrm{E}-06$ \\
\hline
\end{tabular}

Feb 20, $2008 \quad 02: 30 \mathrm{pm}$

SUMMARY

Page 7

INDIVIDUAL LIFETIME RISK (deaths)

(A11 Radionuclides and Pathways)

\begin{tabular}{|c|c|c|c|c|c|c|c|}
\hline \multicolumn{8}{|c|}{ Distance $(\mathrm{m})$} \\
\hline Direction & 10344 & 10365 & 10472 & 10590 & 11103 & 11989 & 12522 \\
\hline $\begin{array}{r}\text { N } \\
N N W \\
N W \\
\text { WNW } \\
W \\
W S W \\
\text { SW } \\
\text { SSW } \\
\text { S } \\
\text { SSE } \\
\text { SE } \\
\text { ESE } \\
\text { E } \\
\text { ENE } \\
\text { NE } \\
\text { NNE }\end{array}$ & $\begin{array}{l}4.2 \mathrm{E}-12 \\
1.9 \mathrm{E}-12 \\
2.4 \mathrm{E}-12 \\
3.1 \mathrm{E}-12 \\
3.3 \mathrm{E}-12 \\
5.3 \mathrm{E}-12 \\
9.5 \mathrm{E}-12 \\
1.5 \mathrm{E}-11 \\
2.2 \mathrm{E}-11 \\
1.9 \mathrm{E}-11 \\
7.7 \mathrm{E}-12 \\
4.7 \mathrm{E}-12 \\
5.9 \mathrm{E}-12 \\
6.4 \mathrm{E}-12 \\
1.1 \mathrm{E}-11 \\
6.1 \mathrm{E}-12\end{array}$ & $\begin{array}{l}4.1 \mathrm{E}-12 \\
1.9 \mathrm{E}-12 \\
2.4 \mathrm{E}-12 \\
3.1 \mathrm{E}-12 \\
3.3 \mathrm{E}-12 \\
5.3 \mathrm{E}-12 \\
9.4 \mathrm{E}-12 \\
1.5 \mathrm{E}-11 \\
2.2 \mathrm{E}-11 \\
1.9 \mathrm{E}-11 \\
7 \cdot 6 \mathrm{E}-12 \\
4.7 \mathrm{E}-12 \\
5 \cdot 9 \mathrm{E}-12 \\
6.4 \mathrm{E}-12 \\
1.1 \mathrm{E}-11 \\
6.0 \mathrm{E}-12\end{array}$ & $\begin{array}{l}4.1 \mathrm{E}-12 \\
1.9 \mathrm{E}-12 \\
2.3 \mathrm{E}-12 \\
3.1 \mathrm{E}-12 \\
3.2 \mathrm{E}-12 \\
5.2 \mathrm{E}-12 \\
9.3 \mathrm{E}-12 \\
1.5 \mathrm{E}-11 \\
2.1 \mathrm{E}-11 \\
1.9 \mathrm{E}-11 \\
7 \cdot 5 \mathrm{E}-12 \\
4.7 \mathrm{E}-12 \\
5.8 \mathrm{E}-12 \\
6.3 \mathrm{E}-12 \\
1.1 \mathrm{E}-11 \\
6.0 \mathrm{E}-12\end{array}$ & $\begin{array}{l}4.0 \mathrm{E}-12 \\
1.8 \mathrm{E}-12 \\
2.3 \mathrm{E}-12 \\
3.0 \mathrm{E}-12 \\
3.2 \mathrm{E}-12 \\
5.1 \mathrm{E}-12 \\
9.2 \mathrm{E}-12 \\
1.5 \mathrm{E}-11 \\
2.1 \mathrm{E}-11 \\
1.9 \mathrm{E}-11 \\
7.4 \mathrm{E}-12 \\
4.6 \mathrm{E}-12 \\
5.8 \mathrm{E}-12 \\
6.2 \mathrm{E}-12 \\
1.0 \mathrm{E}-11 \\
5.9 \mathrm{E}-12\end{array}$ & $\begin{array}{l}3.8 \mathrm{E}-12 \\
1.7 \mathrm{E}-12 \\
2.2 \mathrm{E}-12 \\
2.9 \mathrm{E}-12 \\
3.0 \mathrm{E}-12 \\
4.8 \mathrm{E}-12 \\
8.6 \mathrm{E}-12 \\
1.4 \mathrm{E}-11 \\
2.0 \mathrm{E}-11 \\
1.7 \mathrm{E}-11 \\
7.0 \mathrm{E}-12 \\
4.3 \mathrm{E}-12 \\
5.4 \mathrm{E}-12 \\
5.9 \mathrm{E}-12 \\
9.7 \mathrm{E}-12 \\
5.5 \mathrm{E}-12\end{array}$ & $\begin{array}{l}3.4 \mathrm{E}-12 \\
1.6 \mathrm{E}-12 \\
2.0 \mathrm{E}-12 \\
2.6 \mathrm{E}-12 \\
2.7 \mathrm{E}-12 \\
4.3 \mathrm{E}-12 \\
7.7 \mathrm{E}-12 \\
1.2 \mathrm{E}-11 \\
1.8 \mathrm{E}-11 \\
1.6 \mathrm{E}-11 \\
6.3 \mathrm{E}-12 \\
3.9 \mathrm{E}-12 \\
4.8 \mathrm{E}-12 \\
5.3 \mathrm{E}-12 \\
8.7 \mathrm{E}-12 \\
4.9 \mathrm{E}-12\end{array}$ & $\begin{array}{l}3.2 \mathrm{E}-12 \\
1.5 \mathrm{E}-12 \\
1.8 \mathrm{E}-12 \\
2.4 \mathrm{E}-12 \\
2.5 \mathrm{E}-12 \\
4.1 \mathrm{E}-12 \\
7.3 \mathrm{E}-12 \\
1.2 \mathrm{E}-11 \\
1.6 \mathrm{E}-11 \\
1.5 \mathrm{E}-11 \\
5.9 \mathrm{E}-12 \\
3.6 \mathrm{E}-12 \\
4.6 \mathrm{E}-12 \\
5.0 \mathrm{E}-12 \\
8.2 \mathrm{E}-12 \\
4.7 \mathrm{E}-12\end{array}$ \\
\hline \multicolumn{8}{|c|}{ Distance (m) } \\
\hline Direction & 13245 & 13286 & 13483 & $\begin{array}{r}13612 \\
\text { Page }\end{array}$ & 13664 & 13959 & 14258 \\
\hline
\end{tabular}


Rn-220A. SUM

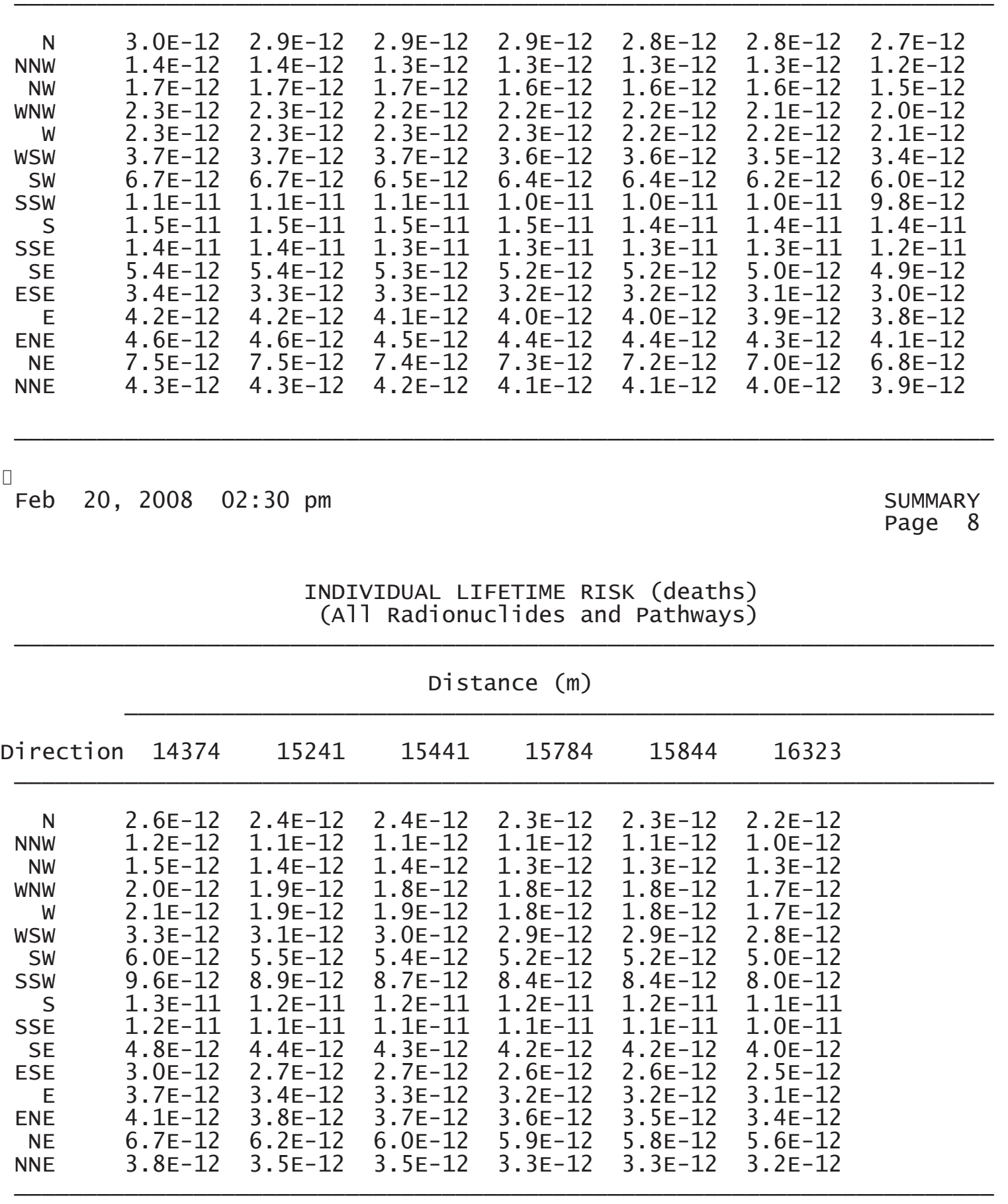




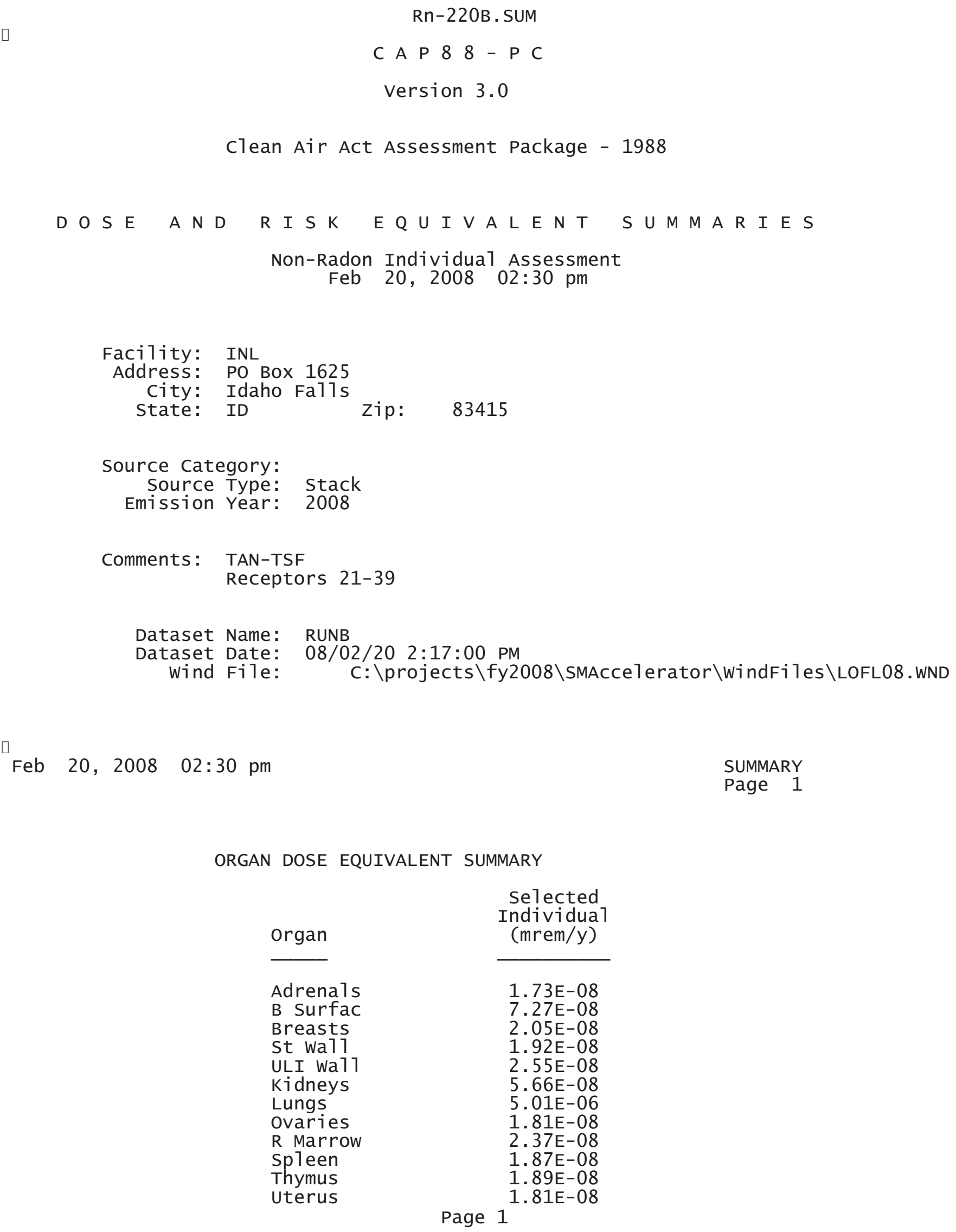




$\begin{array}{lr} & \text { Rn-220B. SUM } \\ \text { B1d wa11 } & 1.97 \mathrm{E}-08 \\ \text { Brain } & 1.88 \mathrm{E}-08 \\ \text { Esophagu } & 1.86 \mathrm{E}-06 \\ \text { SI wa11 } & 2.01 \mathrm{E}-08 \\ \text { LLI wa11 } & 2.69 \mathrm{E}-08 \\ \text { Liver } & 2.87 \mathrm{E}-08 \\ \text { Muscle } & 2.06 \mathrm{E}-08 \\ \text { Pancreas } & 1.77 \mathrm{E}-08 \\ \text { Skin } & 1.10 \mathrm{E}-07 \\ \text { Testes } & 2.09 \mathrm{E}-08 \\ \text { Thyroid } & 1.92 \mathrm{E}-08 \\ \text { EFFEC } & 1.22 \mathrm{E}-05\end{array}$

PATHWAY EFFECTIVE DOSE EQUIVALENT SUMMARY

selected Individual

Pathway (mrem/y)

INGESTION

1.05E-13

INHALATION

1.21E-05

AIR IMMERSION

GROUND SURFACE

2. $57 \mathrm{E}-09$

INTERNAL

1. $62 \mathrm{E}-08$

$1.21 \mathrm{E}-05$

EXTERNAL

$1.87 \mathrm{E}-08$

TOTAL

1. $22 \mathrm{E}-05$

$\square$

Feb 20, 2008 02:30 pm

SUMMARY

Page 2

NUCLIDE EFFECTIVE DOSE EQUIVALENT SUMMARY

Nuclide

$\mathrm{Rn}-220$

Po-216

$\mathrm{Pb}-212$

$\mathrm{Bi}-212$

Po-212

$\mathrm{T} 1-208$

TOTAL
Selected Individual

(mrem/y)

1. $17 \mathrm{E}-11$

2. $90 \mathrm{E}-13$

1. $20 \mathrm{E}-05$

$1.69 \mathrm{E}-07$

$0.00 \mathrm{E}+00$

1.29E-08

1. $22 \mathrm{E}-05$

Feb 20, 2008 02:30 pm

SUMMARY

Page 3

CANCER RISK SUMMARY

Selected Individual

Page 2 


\begin{tabular}{lc} 
Cancer & $\begin{array}{r}\text { Rn-220B.SUM } \\
\text { Total Lifetime } \\
\text { Fatal Cancer Risk }\end{array}$ \\
\cline { 2 - 2 } $\begin{array}{l}\text { Esophagu } \\
\text { Stomach }\end{array}$ & $6.92 \mathrm{E}-16$ \\
Colon & $3.73 \mathrm{E}-15$ \\
Liver & $4.90 \mathrm{E}-14$ \\
LUNG & $4.44 \mathrm{E}-15$ \\
Bone & $1.04 \mathrm{E}-11$ \\
Skin & $1.43 \mathrm{E}-15$ \\
Breast & $1.54 \mathrm{E}-16$ \\
Ovary & $2.11 \mathrm{E}-15$ \\
Bladder & $8.99 \mathrm{E}-16$ \\
Kidneys & $1.59 \mathrm{E}-15$ \\
Thyroid & $4.78 \mathrm{E}-15$ \\
Leukemia & $2.09 \mathrm{E}-16$ \\
Residua1 & $1.60 \mathrm{E}-15$ \\
Tota1 & $9.63 \mathrm{E}-15$ \\
TOTAL & $1.04 \mathrm{E}-11$ \\
& $2.09 \mathrm{E}-11$
\end{tabular}

PATHWAY RISK SUMMARY

Pathway

INGESTION

INHALATION

AIR IMMERSION

GROUND SURFACE

INTERNAL

EXTERNAL

TOTAL

$\square$

Feb 20, 2008 02:30 pm
Selected Individual

Total Lifetime Fatal Cancer Risk

1. $05 \mathrm{E}-19$

1. 04E-11

1. 37E-15

8.57E-15

1. 04E-11

9. $94 \mathrm{E}-15$

1. 04E-11
SUMMARY Page 4

NUCLIDE RISK SUMMARY

Nuclide

$$
\begin{aligned}
& \mathrm{Rn}-220 \\
& \mathrm{PO}-216 \\
& \mathrm{~Pb}-212 \\
& \mathrm{~B} \mathrm{i}-212 \\
& \mathrm{PO}-212 \\
& \mathrm{~T} \mathrm{~T}-208
\end{aligned}
$$

TOTAL
Selected Individual

Total Lifetime Fatal Cancer Risk

6. $40 \mathrm{E}-18$

1. $59 \mathrm{E}-19$

$1.03 \mathrm{E}-11$

1. $08 \mathrm{E}-13$

$0.00 \mathrm{E}+00$

$7.02 \mathrm{E}-15$

1. 04E-11

Feb 20, 2008 02:30 pm

Page 3 
Rn-220B.SUM

Page 5

INDIVIDUAL EFFECTIVE DOSE EQUIVALENT RATE (mrem/y)

(A11 Radionuclides and Pathways)

\begin{tabular}{|c|c|c|c|c|c|c|c|}
\hline \multirow[b]{2}{*}{ Direction } & \multicolumn{6}{|c|}{ Distance (m) } & \multirow[b]{2}{*}{19860} \\
\hline & 17035 & 17329 & 18607 & 18834 & 18860 & 18890 & \\
\hline $\mathrm{N}$ & $2.4 \mathrm{E}-06$ & 2. $3 \mathrm{E}-06$ & $2.1 \mathrm{E}-06$ & $2.0 \mathrm{E}-06$ & $2.0 \mathrm{E}-06$ & $2.0 \mathrm{E}-06$ & 1. $9 \mathrm{E}-06$ \\
\hline NNW & $1.1 \mathrm{E}-06$ & 1. $1 \mathrm{E}-06$ & $9.9 \mathrm{E}-07$ & $9.7 \mathrm{E}-07$ & $9.7 \mathrm{E}-07$ & $9.7 \mathrm{E}-07$ & $9.0 \mathrm{E}-07$ \\
\hline NW & $1.4 \mathrm{E}-06$ & $1.4 \mathrm{E}-06$ & $1.2 \mathrm{E}-06$ & $1.2 \mathrm{E}-06$ & $1.2 \mathrm{E}-06$ & $1.2 \mathrm{E}-06$ & 1. $1 \mathrm{E}-06$ \\
\hline WNW & 1.9E-06 & $1.8 \mathrm{E}-06$ & $1.6 \mathrm{E}-06$ & $1.6 \mathrm{E}-06$ & $1.6 \mathrm{E}-06$ & $1.6 \mathrm{E}-06$ & 1. $5 \mathrm{E}-06$ \\
\hline W & $1.9 \mathrm{E}-06$ & $1.8 \mathrm{E}-06$ & 1.7E-06 & 1. $6 \mathrm{E}-06$ & $1.6 \mathrm{E}-06$ & $1.6 \mathrm{E}-06$ & $1.5 \mathrm{E}-06$ \\
\hline WSW & $3.1 \mathrm{E}-06$ & $3.0 \mathrm{E}-06$ & $2.7 \mathrm{E}-06$ & $2.6 \mathrm{E}-06$ & $2.6 \mathrm{E}-06$ & $2.6 \mathrm{E}-06$ & $2.4 \mathrm{E}-06$ \\
\hline SW & $5.5 \mathrm{E}-06$ & $5.3 \mathrm{E}-06$ & $4.8 \mathrm{E}-06$ & $4.7 \mathrm{E}-06$ & $4.7 \mathrm{E}-06$ & $4.7 \mathrm{E}-06$ & $4.3 E-06$ \\
\hline SSW & $8.8 \mathrm{E}-06$ & $8.5 \mathrm{E}-06$ & 7.7E-06 & $7.5 \mathrm{E}-06$ & $7.5 \mathrm{E}-06$ & $7.5 \mathrm{E}-06$ & $6.9 \mathrm{E}-06$ \\
\hline $\mathrm{S}$ & $1.2 \mathrm{E}-05$ & $1.2 \mathrm{E}-05$ & 1.1. $\mathrm{E}-05$ & $1.0 \mathrm{E}-05$ & 1.0E-05 & 1.0E-05 & $9.5 \mathrm{E}-06$ \\
\hline SSE & $1.1 \mathrm{E}-05$ & $1.1 \mathrm{E}-05$ & $9.8 \mathrm{E}-06$ & $9.6 \mathrm{E}-06$ & $9.6 \mathrm{E}-06$ & $9.5 \mathrm{E}-06$ & $8.8 \mathrm{E}-06$ \\
\hline SE & 4. $3 \mathrm{E}-06$ & 4. $2 \mathrm{E}-06$ & $3.8 \mathrm{E}-06$ & $3.7 \mathrm{E}-06$ & $3.7 \mathrm{E}-06$ & $3.7 \mathrm{E}-06$ & $3.4 \mathrm{E}-06$ \\
\hline ESE & $2.7 \mathrm{E}-06$ & $2.6 \mathrm{E}-06$ & $2.3 \mathrm{E}-06$ & $2.3 \mathrm{E}-06$ & $2.3 \mathrm{E}-06$ & $2.3 \mathrm{E}-06$ & $2.1 \mathrm{E}-06$ \\
\hline E & $3.3 \mathrm{E}-06$ & 3. 3E-06 & $2.9 \mathrm{E}-06$ & $2.8 \mathrm{E}-06$ & $2.8 \mathrm{E}-06$ & $2.8 \mathrm{E}-06$ & $2.6 \mathrm{E}-06$ \\
\hline ENE & $3.7 \mathrm{E}-06$ & $3.6 \mathrm{E}-06$ & $3.2 \mathrm{E}-06$ & $3.2 \mathrm{E}-06$ & $3.2 \mathrm{E}-06$ & $3.2 \mathrm{E}-06$ & $2.9 \mathrm{E}-06$ \\
\hline NE & $6.1 \mathrm{E}-06$ & $5.9 \mathrm{E}-06$ & $5.3 \mathrm{E}-06$ & $5.2 \mathrm{E}-06$ & $5.2 \mathrm{E}-06$ & $5.2 \mathrm{E}-06$ & $4.8 \mathrm{E}-06$ \\
\hline NNE & $3.5 \mathrm{E}-06$ & $3.4 \mathrm{E}-06$ & $3.0 \mathrm{E}-06$ & $3.0 \mathrm{E}-06$ & $3.0 \mathrm{E}-06$ & $3.0 \mathrm{E}-06$ & $2.7 \mathrm{E}-06$ \\
\hline
\end{tabular}

Distance (m)

\begin{tabular}{|c|c|c|c|c|c|c|c|}
\hline Direction & 19891 & 20457 & 21314 & 21349 & 22159 & 23521 & 24430 \\
\hline $\mathrm{N}$ & $1.9 \mathrm{E}-06$ & $1.8 \mathrm{E}-06$ & $1.6 \mathrm{E}-06$ & $1.6 \mathrm{E}-06$ & 1. $5 \mathrm{E}-06$ & 1. $3 \mathrm{E}-06$ & $1.2 \mathrm{E}-06$ \\
\hline NNW & $9.0 \mathrm{E}-07$ & $8.6 \mathrm{E}-07$ & $8.0 \mathrm{E}-07$ & 8.0E-07 & $7.5 \mathrm{E}-07$ & $6.7 \mathrm{E}-07$ & $6.1 \mathrm{E}-07$ \\
\hline NW & $1.1 \mathrm{E}-06$ & $1.1 \mathrm{E}-06$ & $9.8 \mathrm{E}-07$ & $9.8 \mathrm{E}-07$ & $9.1 \mathrm{E}-07$ & 8. $0 \mathrm{E}-07$ & $7.3 \mathrm{E}-07$ \\
\hline WNW & $1.5 \mathrm{E}-06$ & $1.4 \mathrm{E}-06$ & $1.3 \mathrm{E}-06$ & $1.3 \mathrm{E}-06$ & $1.2 \mathrm{E}-06$ & 1.1E-06 & $9.6 \mathrm{E}-07$ \\
\hline W & $1.5 \mathrm{E}-06$ & $1.4 \mathrm{E}-06$ & $1.3 \mathrm{E}-06$ & 1. $3 \mathrm{E}-06$ & $1.2 \mathrm{E}-06$ & $1.1 \mathrm{E}-06$ & $9.6 \mathrm{E}-07$ \\
\hline WSW & $2.4 \mathrm{E}-06$ & $2.3 \mathrm{E}-06$ & $2.1 \mathrm{E}-06$ & $2.1 \mathrm{E}-06$ & $2.0 \mathrm{E}-06$ & $1.8 \mathrm{E}-06$ & $1.6 \mathrm{E}-06$ \\
\hline SW & $4.3 \mathrm{E}-06$ & $4.1 \mathrm{E}-06$ & $3.8 \mathrm{E}-06$ & $3.8 \mathrm{E}-06$ & $3.6 \mathrm{E}-06$ & $3.2 \mathrm{E}-06$ & $3.0 \mathrm{E}-06$ \\
\hline SSW & $6.9 E-06$ & $6.6 \mathrm{E}-06$ & $6.1 \mathrm{E}-06$ & $6.1 \mathrm{E}-06$ & $5.7 \mathrm{E}-06$ & $5.1 \mathrm{E}-06$ & $4.7 \mathrm{E}-06$ \\
\hline $\mathrm{S}$ & $9.5 \mathrm{E}-06$ & $9.1 \mathrm{E}-06$ & $8.4 \mathrm{E}-06$ & $8.4 \mathrm{E}-06$ & $7.9 \mathrm{E}-06$ & $7.0 \mathrm{E}-06$ & $6.4 \mathrm{E}-06$ \\
\hline SSE & $8.8 \mathrm{E}-06$ & $8.4 \mathrm{E}-06$ & $7.9 \mathrm{E}-06$ & $7.8 \mathrm{E}-06$ & $7.4 \mathrm{E}-06$ & $6.6 \mathrm{E}-06$ & $6.1 \mathrm{E}-06$ \\
\hline SE & $3.4 \mathrm{E}-06$ & $3.2 \mathrm{E}-06$ & $3.0 \mathrm{E}-06$ & $2.9 \mathrm{E}-06$ & $2.7 \mathrm{E}-06$ & $2.4 \mathrm{E}-06$ & $2.1 \mathrm{E}-06$ \\
\hline ESE & $2.1 \mathrm{E}-06$ & $2.0 \mathrm{E}-06$ & $1.8 \mathrm{E}-06$ & $1.8 \mathrm{E}-06$ & $1.7 \mathrm{E}-06$ & $1.4 \mathrm{E}-06$ & $1.3 \mathrm{E}-06$ \\
\hline $\mathrm{E}$ & $2.6 \mathrm{E}-06$ & $2.4 \mathrm{E}-06$ & $2.2 \mathrm{E}-06$ & $2.2 \mathrm{E}-06$ & $2.1 \mathrm{E}-06$ & $1.8 \mathrm{E}-06$ & $1.6 \mathrm{E}-06$ \\
\hline ENE & $2.9 \mathrm{E}-06$ & $2.8 \mathrm{E}-06$ & $2.6 \mathrm{E}-06$ & $2.6 \mathrm{E}-06$ & $2.4 \mathrm{E}-06$ & $2.1 \mathrm{E}-06$ & $1.9 \mathrm{E}-06$ \\
\hline $\mathrm{NE}$ & $4.8 \mathrm{E}-06$ & $4.6 \mathrm{E}-06$ & $4.2 \mathrm{E}-06$ & $4.2 \mathrm{E}-06$ & $4.0 \mathrm{E}-06$ & $3.5 \mathrm{E}-06$ & $3.2 \mathrm{E}-06$ \\
\hline NNE & $2.7 \mathrm{E}-06$ & $2.6 \mathrm{E}-06$ & $2.4 \mathrm{E}-06$ & $2.4 \mathrm{E}-06$ & $2.3 \mathrm{E}-06$ & $2.0 \mathrm{E}-06$ & $1.8 \mathrm{E}-06$ \\
\hline
\end{tabular}

$\square$

Feb 20, $2008 \quad 02: 30 \mathrm{pm}$

SUMMARY

Page 6

INDIVIDUAL EFFECTIVE DOSE EQUIVALENT RATE (mrem/y) (A11 Radionuclides and Pathways)

Page 4 
Rn-220B. SUM

\begin{tabular}{|c|c|c|c|c|c|}
\hline \multirow[b]{2}{*}{ Direction } & \multicolumn{5}{|c|}{ Distance (m) } \\
\hline & 24545 & 25171 & 26794 & 27275 & 27389 \\
\hline $\mathrm{N}$ & 1. $2 \mathrm{E}-06$ & $1.1 \mathrm{E}-06$ & $9.9 \mathrm{E}-07$ & $9.6 \mathrm{E}-07$ & $9.6 \mathrm{E}-07$ \\
\hline NNW & $6.1 \mathrm{E}-07$ & $5.7 \mathrm{E}-07$ & $5.2 \mathrm{E}-07$ & $5.1 \mathrm{E}-07$ & $5.1 \mathrm{E}-07$ \\
\hline NW & $7.2 \mathrm{E}-07$ & $6.7 \mathrm{E}-07$ & $6.1 \mathrm{E}-07$ & $6.0 \mathrm{E}-07$ & $5.9 \mathrm{E}-07$ \\
\hline WNW & $9.5 \mathrm{E}-07$ & $8.8 \mathrm{E}-07$ & $8.1 \mathrm{E}-07$ & $7.9 \mathrm{E}-07$ & $7.8 \mathrm{E}-07$ \\
\hline W & $9.5 \mathrm{E}-07$ & $8.8 \mathrm{E}-07$ & $8.0 \mathrm{E}-07$ & $7.8 \mathrm{E}-07$ & $7.8 \mathrm{E}-07$ \\
\hline WSW & $1.6 \mathrm{E}-06$ & $1.5 \mathrm{E}-06$ & 1. $4 \mathrm{E}-06$ & $1.3 \mathrm{E}-06$ & $1.3 \mathrm{E}-06$ \\
\hline SW & $2.9 \mathrm{E}-06$ & $2.8 \mathrm{E}-06$ & $2.5 \mathrm{E}-06$ & $2.5 \mathrm{E}-06$ & $2.4 \mathrm{E}-06$ \\
\hline SSW & $4.6 \mathrm{E}-06$ & $4.3 \mathrm{E}-06$ & $4.0 \mathrm{E}-06$ & $3.9 \mathrm{E}-06$ & $3.8 \mathrm{E}-06$ \\
\hline $\mathrm{S}$ & $6.3 \mathrm{E}-06$ & $5.9 \mathrm{E}-06$ & $5.4 \mathrm{E}-06$ & $5.3 \mathrm{E}-06$ & $5.2 \mathrm{E}-06$ \\
\hline SSE & $6.0 \mathrm{E}-06$ & $5.7 \mathrm{E}-06$ & $5.2 \mathrm{E}-06$ & $5.1 \mathrm{E}-06$ & $5.0 \mathrm{E}-06$ \\
\hline SE & $2.1 \mathrm{E}-06$ & 1.9E-06 & $1.8 \mathrm{E}-06$ & $1.7 \mathrm{E}-06$ & 1.7E-06 \\
\hline ESE & 1. $3 \mathrm{E}-06$ & $1.2 \mathrm{E}-06$ & $1.1 \mathrm{E}-06$ & $1.0 \mathrm{E}-06$ & $1.0 \mathrm{E}-06$ \\
\hline $\mathrm{E}$ & 1. $5 \mathrm{E}-06$ & $1.4 \mathrm{E}-06$ & 1. $3 \mathrm{E}-06$ & $1.2 \mathrm{E}-06$ & $1.2 \mathrm{E}-06$ \\
\hline ENE & 1. $9 \mathrm{E}-06$ & $1.8 \mathrm{E}-06$ & $1.6 \mathrm{E}-06$ & $1.6 \mathrm{E}-06$ & 1. $6 \mathrm{E}-06$ \\
\hline NE & $3.2 \mathrm{E}-06$ & $3.0 \mathrm{E}-06$ & 2.7E-06 & $2.7 \mathrm{E}-06$ & $2.6 \mathrm{E}-06$ \\
\hline NNE & $1.8 \mathrm{E}-06$ & $1.7 \mathrm{E}-06$ & 1. $5 \mathrm{E}-06$ & $1.5 \mathrm{E}-06$ & $1.5 \mathrm{E}-06$ \\
\hline
\end{tabular}

Feb 20, 2008 02:30 pm

SUMMARY

Page 7

INDIVIDUAL LIFETIME RISK (deaths)

(A11 Radionuclides and Pathways)

\begin{tabular}{|c|c|c|c|c|c|c|c|}
\hline \multirow[b]{2}{*}{ Direction } & \multicolumn{6}{|c|}{ Distance $(\mathrm{m})$} & \multirow[b]{2}{*}{19860} \\
\hline & 17035 & 17329 & 18607 & 18834 & 18860 & 18890 & \\
\hline $\begin{array}{r}\text { N } \\
N N W \\
N W \\
\text { WNW } \\
W \\
W S W \\
\text { SW } \\
\text { SSW } \\
S \\
\text { SSE } \\
\text { SE } \\
\text { ESE } \\
\text { E } \\
\text { ENE } \\
N E \\
\text { NNE }\end{array}$ & $\begin{array}{l}2.1 \mathrm{E}-12 \\
9.7 \mathrm{E}-13 \\
1.2 \mathrm{E}-12 \\
1.6 \mathrm{E}-12 \\
1.6 \mathrm{E}-12 \\
2.6 \mathrm{E}-12 \\
4.7 \mathrm{E}-12 \\
7.5 \mathrm{E}-12 \\
1.0 \mathrm{E}-11 \\
9.6 \mathrm{E}-12 \\
3.7 \mathrm{E}-12 \\
2.3 \mathrm{E}-12 \\
2.9 \mathrm{E}-12 \\
3.2 \mathrm{E}-12 \\
5.2 \mathrm{E}-12 \\
3.0 \mathrm{E}-12\end{array}$ & $\begin{array}{l}2.0 \mathrm{E}-12 \\
9.5 \mathrm{E}-13 \\
1.2 \mathrm{E}-12 \\
1.5 \mathrm{E}-12 \\
1.6 \mathrm{E}-12 \\
2.6 \mathrm{E}-12 \\
4.6 \mathrm{E}-12 \\
7.3 \mathrm{E}-12 \\
1.0 \mathrm{E}-11 \\
9.3 \mathrm{E}-12 \\
3.6 \mathrm{E}-12 \\
2.2 \mathrm{E}-12 \\
2.8 \mathrm{E}-12 \\
3.1 \mathrm{E}-12 \\
5.1 \mathrm{E}-12 \\
2.9 \mathrm{E}-12\end{array}$ & $\begin{array}{l}1.8 \mathrm{E}-12 \\
8.5 \mathrm{E}-13 \\
1.1 \mathrm{E}-12 \\
1.4 \mathrm{E}-12 \\
1.4 \mathrm{E}-12 \\
2.3 \mathrm{E}-12 \\
4.1 \mathrm{E}-12 \\
6.6 \mathrm{E}-12 \\
9.1 \mathrm{E}-12 \\
8.4 \mathrm{E}-12 \\
3.2 \mathrm{E}-12 \\
2.0 \mathrm{E}-12 \\
2.5 \mathrm{E}-12 \\
2.8 \mathrm{E}-12 \\
4.6 \mathrm{E}-12 \\
2.6 \mathrm{E}-12\end{array}$ & $\begin{array}{l}1.7 \mathrm{E}-12 \\
8.4 \mathrm{E}-13 \\
1.0 \mathrm{E}-12 \\
1.4 \mathrm{E}-12 \\
1.4 \mathrm{E}-12 \\
2.3 \mathrm{E}-12 \\
4.0 \mathrm{E}-12 \\
6.4 \mathrm{E}-12 \\
8.9 \mathrm{E}-12 \\
8.2 \mathrm{E}-12 \\
3.2 \mathrm{E}-12 \\
1.9 \mathrm{E}-12 \\
2.4 \mathrm{E}-12 \\
2.7 \mathrm{E}-12 \\
4.5 \mathrm{E}-12 \\
2.6 \mathrm{E}-12\end{array}$ & $\begin{array}{l}1.7 \mathrm{E}-12 \\
8.3 \mathrm{E}-13 \\
1.0 \mathrm{E}-12 \\
1.4 \mathrm{E}-12 \\
1.4 \mathrm{E}-12 \\
2.3 \mathrm{E}-12 \\
4.0 \mathrm{E}-12 \\
6.4 \mathrm{E}-12 \\
8.9 \mathrm{E}-12 \\
8.2 \mathrm{E}-12 \\
3.2 \mathrm{E}-12 \\
1.9 \mathrm{E}-12 \\
2.4 \mathrm{E}-12 \\
2.7 \mathrm{E}-12 \\
4.5 \mathrm{E}-12 \\
2.6 \mathrm{E}-12\end{array}$ & $\begin{array}{l}1.7 \mathrm{E}-12 \\
8.3 \mathrm{E}-13 \\
1.0 \mathrm{E}-12 \\
1.4 \mathrm{E}-12 \\
1.4 \mathrm{E}-12 \\
2.2 \mathrm{E}-12 \\
4.0 \mathrm{E}-12 \\
6.4 \mathrm{E}-12 \\
8.9 \mathrm{E}-12 \\
8.2 \mathrm{E}-12 \\
3.1 \mathrm{E}-12 \\
1.9 \mathrm{E}-12 \\
2.4 \mathrm{E}-12 \\
2.7 \mathrm{E}-12 \\
4.4 \mathrm{E}-12 \\
2.5 \mathrm{E}-12\end{array}$ & $\begin{array}{l}1.6 \mathrm{E}-12 \\
7.7 \mathrm{E}-13 \\
9.5 \mathrm{E}-13 \\
1.3 \mathrm{E}-12 \\
1.3 \mathrm{E}-12 \\
2.1 \mathrm{E}-12 \\
3.7 \mathrm{E}-12 \\
5.9 \mathrm{E}-12 \\
8.2 \mathrm{E}-12 \\
7.6 \mathrm{E}-12 \\
2.9 \mathrm{E}-12 \\
1.8 \mathrm{E}-12 \\
2.2 \mathrm{E}-12 \\
2.5 \mathrm{E}-12 \\
4.1 \mathrm{E}-12 \\
2.3 \mathrm{E}-12\end{array}$ \\
\hline \multicolumn{8}{|c|}{ Distance $(\mathrm{m})$} \\
\hline Direction & 19891 & 20457 & 21314 & $\begin{array}{r}21349 \\
\text { Page }\end{array}$ & 22159 & 23521 & 24430 \\
\hline
\end{tabular}


Rn-220B. SUM

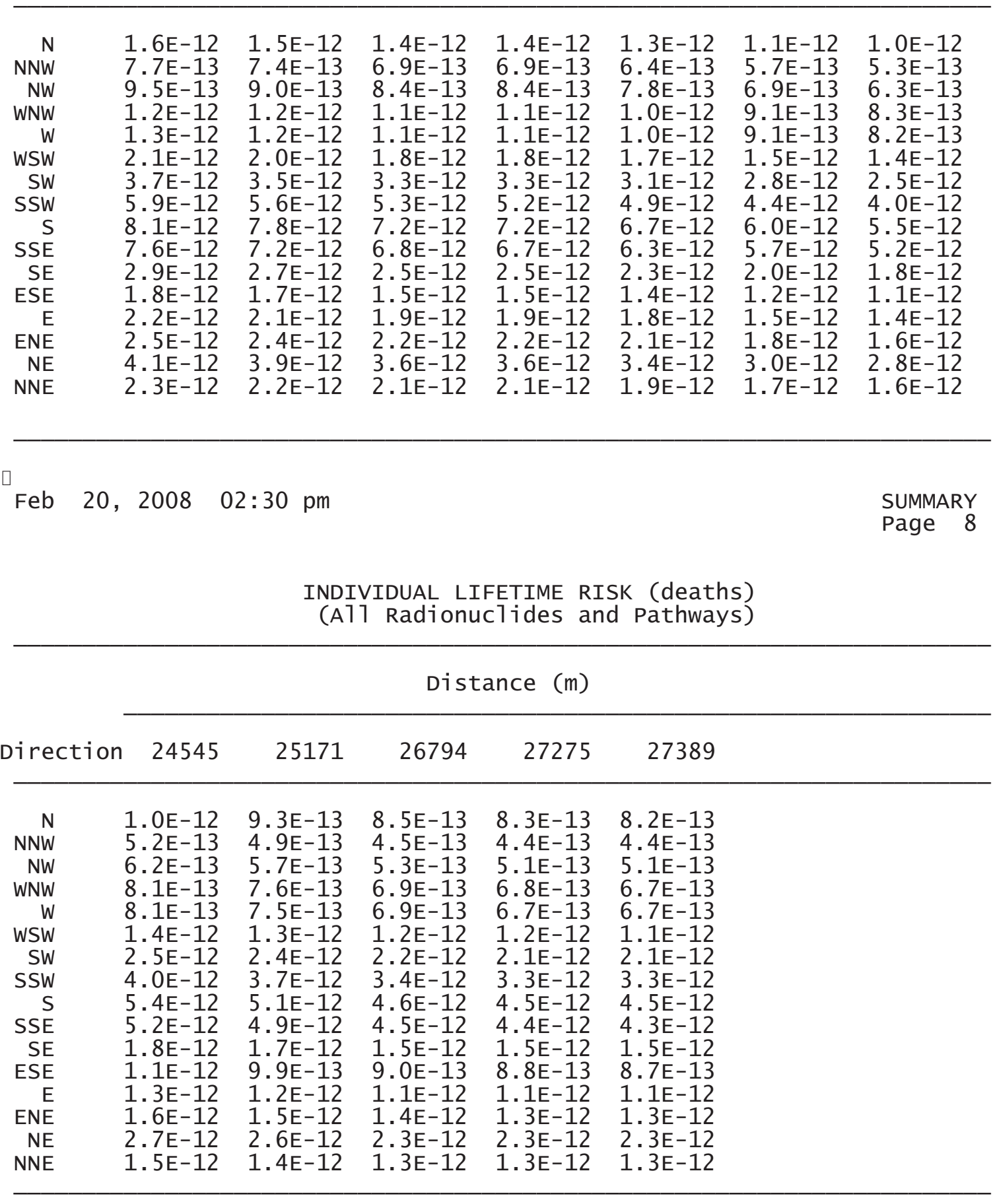




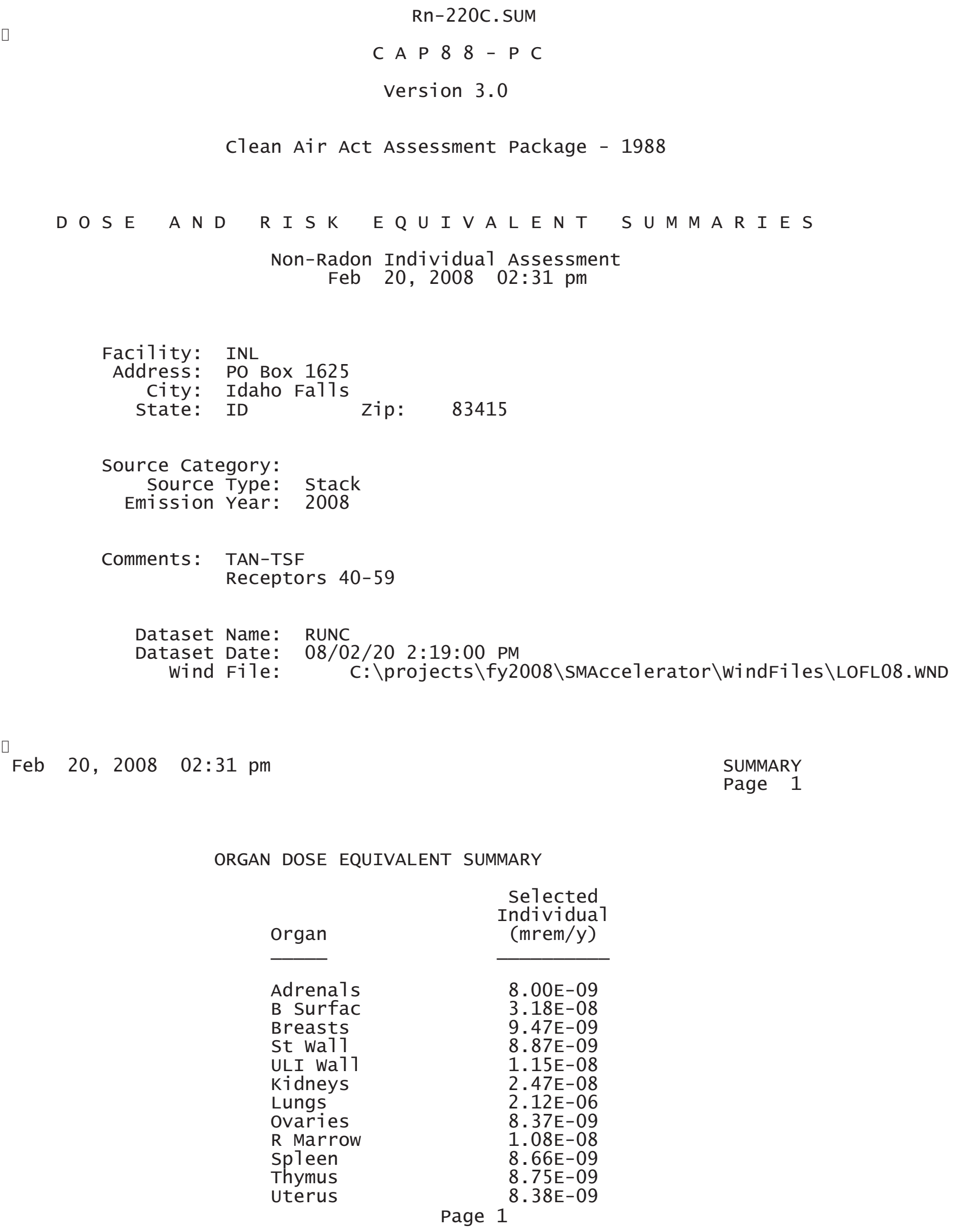

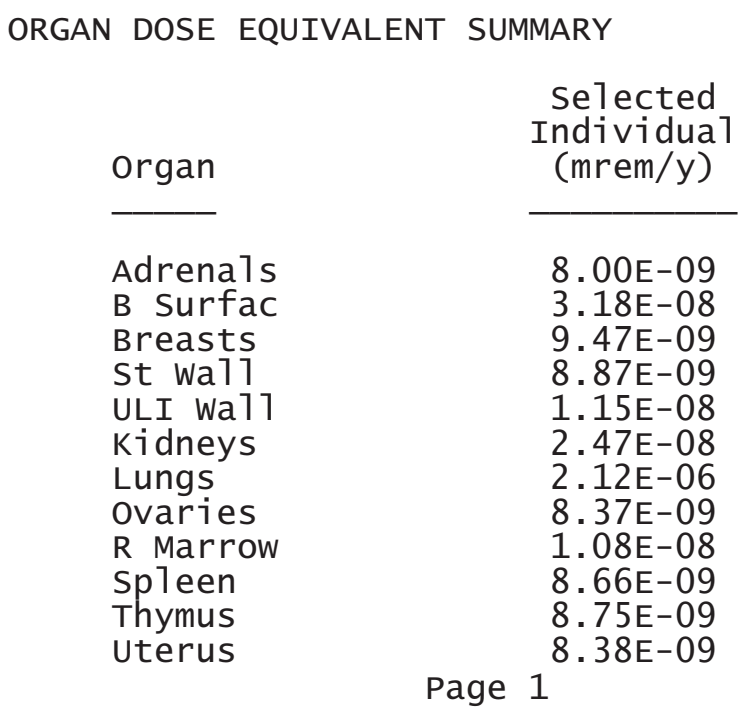




$\begin{array}{lr} & \text { Rn-220C. SUM } \\ \text { Bld wa11 } & 9.12 \mathrm{E}-09 \\ \text { Brain } & 8.70 \mathrm{E}-09 \\ \text { Esophagu } & 7.85 \mathrm{E}-07 \\ \text { SI Wa11 } & 9.25 \mathrm{E}-09 \\ \text { LLI wa11 } & 1.21 \mathrm{E}-08 \\ \text { Liver } & 1.29 \mathrm{E}-08 \\ \text { Muscle } & 9.52 \mathrm{E}-09 \\ \text { Pancreas } & 8.18 \mathrm{E}-09 \\ \text { Skin } & 5.19 \mathrm{E}-08 \\ \text { Testes } & 9.66 \mathrm{E}-09 \\ \text { Thyroid } & 8.88 \mathrm{E}-09 \\ & \\ \text { EFFEC } & 5.14 \mathrm{E}-06\end{array}$

PATHWAY EFFECTIVE DOSE EQUIVALENT SUMMARY

selected Individual

Pathway (mrem/y)

INGESTION $4.53 \mathrm{E}-14$

INHALATION $\quad 5.13 \mathrm{E}-06$

AIR IMMERSION $\quad 1.09 \mathrm{E}-09$

GROUND SURFACE $\quad 7.66 \mathrm{E}-09$

INTERNAL $\quad 5.13 \mathrm{E}-06$

EXTERNAL $\quad 8.75 \mathrm{E}-09$

TOTAL $5.14 \mathrm{E}-06$

Feb 20, $2008 \quad 02: 31$ pm

SUMMARY

Page 2

NUCLIDE EFFECTIVE DOSE EQUIVALENT SUMMARY

Nuclide

$\mathrm{Rn}-220$

Po-216

$\mathrm{Pb}-212$

Bi-212

Po-212

$\mathrm{T} 1-208$

TOTAL

$$
\begin{gathered}
\begin{array}{c}
\text { Selected } \\
\text { Individua1 } \\
\text { (mrem/y) }
\end{array} \\
\hline 6.55 \mathrm{E}-12 \\
1.23 \mathrm{E}-13 \\
5.06 \mathrm{E}-06 \\
7.15 \mathrm{E}-08 \\
0.00 \mathrm{E}+00 \\
6.06 \mathrm{E}-09 \\
5.14 \mathrm{E}-06
\end{gathered}
$$

Feb 20, $2008 \quad 02: 31$ pm

SUMMARY

Page 3

CANCER RISK SUMMARY 


\begin{tabular}{lc} 
Cancer & $\begin{array}{c}\text { Rn-220C.SUM } \\
\text { Total Lifetime } \\
\text { Fatal Cancer Risk }\end{array}$ \\
\cline { 2 - 2 } $\begin{array}{l}\text { Esophagu } \\
\text { Stomach }\end{array}$ & $3.00 \mathrm{E}-16$ \\
Colon & $1.61 \mathrm{E}-15$ \\
Liver & $2.08 \mathrm{E}-14$ \\
LUNG & $1.89 \mathrm{E}-15$ \\
Bone & $4.38 \mathrm{E}-12$ \\
Skin & $6.05 \mathrm{E}-16$ \\
Breast & $7.05 \mathrm{E}-17$ \\
Ovary & $9.32 \mathrm{E}-16$ \\
Bladder & $3.90 \mathrm{E}-16$ \\
Kidneys & $6.93 \mathrm{E}-16$ \\
Thyroid & $2.03 \mathrm{E}-15$ \\
Leukemia & $9.09 \mathrm{E}-17$ \\
Residua1 & $7.20 \mathrm{E}-16$ \\
Total & $4.18 \mathrm{E}-15$ \\
TotAL & $4.41 \mathrm{E}-12$ \\
& $8.82 \mathrm{E}-12$
\end{tabular}

PATHWAY RISK SUMMARY

Pathway

INGESTION

INHALATION

AIR IMMERSION

GROUND SURFACE

INTERNAL

EXTERNAL

TOTAL
Selected Individual Total Lifetime Fatal Cancer Risk

4. $50 \mathrm{E}-20$

$4.41 \mathrm{E}-12$

$5.82 \mathrm{E}-16$

$4.06 \mathrm{E}-15$

4. $41 \mathrm{E}-12$

$4.64 \mathrm{E}-15$

4. $41 \mathrm{E}-12$

$\square$

Feb 20, $2008 \quad 02: 31$ pm

SUMMARY Page 4

NUCLIDE RISK SUMMARY

Nuclide

$\mathrm{Rn}-220$
$\mathrm{PO}-216$
$\mathrm{~Pb}-212$
$\mathrm{Bi}-212$
$\mathrm{PO}-212$
$\mathrm{~T} \mathrm{~T}-208$

TOTAL
Selected Individual

Total Lifetime Fatal Cancer Risk

$3.58 \mathrm{E}-18$

$6.72 \mathrm{E}-20$

$4.36 \mathrm{E}-12$

4. $59 \mathrm{E}-14$

$0.00 \mathrm{E}+00$

$3.31 \mathrm{E}-15$

$4.41 \mathrm{E}-12$

Feb 20, 2008 02:31 pm

Page 3 
Rn-220C.SUM

Page 5

INDIVIDUAL EFFECTIVE DOSE EQUIVALENT RATE (mrem/y)

(A11 Radionuclides and Pathways)

\begin{tabular}{|c|c|c|c|c|c|c|c|}
\hline \multirow[b]{2}{*}{ Direction } & \multicolumn{6}{|c|}{ Distance $(\mathrm{m})$} & \multirow[b]{2}{*}{35683} \\
\hline & n 27715 & 28919 & 31060 & 32802 & 34577 & 35279 & \\
\hline $\mathrm{N}$ & $9.4 \mathrm{E}-07$ & $8.8 \mathrm{E}-07$ & $7.9 \mathrm{E}-07$ & 7. $3 \mathrm{E}-07$ & $6.7 \mathrm{E}-07$ & $6.5 \mathrm{E}-07$ & $6.4 \mathrm{E}-07$ \\
\hline NNW & $5.0 \mathrm{E}-07$ & $4.7 \mathrm{E}-07$ & 4. $3 \mathrm{E}-07$ & $3.9 \mathrm{E}-07$ & $3.6 \mathrm{E}-07$ & $3.5 \mathrm{E}-07$ & $3.5 \mathrm{E}-07$ \\
\hline NW & $5.8 \mathrm{E}-07$ & $5.5 \mathrm{E}-07$ & $5.0 \mathrm{E}-07$ & $4.6 \mathrm{E}-07$ & $4.2 \mathrm{E}-07$ & $4.1 \mathrm{E}-07$ & $4.0 \mathrm{E}-07$ \\
\hline WNW & $7.7 \mathrm{E}-07$ & $7.2 \mathrm{E}-07$ & $6.5 \mathrm{E}-07$ & $6.0 \mathrm{E}-07$ & $5.6 \mathrm{E}-07$ & $5.4 \mathrm{E}-07$ & $5.3 E-07$ \\
\hline W & $7.6 \mathrm{E}-07$ & $7.2 \mathrm{E}-07$ & $6.4 \mathrm{E}-07$ & $5.9 \mathrm{E}-07$ & $5.5 \mathrm{E}-07$ & $5.3 \mathrm{E}-07$ & $5.2 \mathrm{E}-07$ \\
\hline WSW & 1. $3 \mathrm{E}-06$ & $1.2 \mathrm{E}-06$ & $1.1 \mathrm{E}-06$ & $1.0 \mathrm{E}-06$ & $9.5 \mathrm{E}-07$ & $9.2 \mathrm{E}-07$ & $9.0 \mathrm{E}-07$ \\
\hline SW & $2.4 \mathrm{E}-06$ & 2. $3 \mathrm{E}-06$ & $2.0 \mathrm{E}-06$ & $1.9 \mathrm{E}-06$ & 1.7E-06 & $1.7 \mathrm{E}-06$ & $1.7 \mathrm{E}-06$ \\
\hline SSW & $3.8 \mathrm{E}-06$ & $3.5 \mathrm{E}-06$ & $3.2 \mathrm{E}-06$ & $2.9 \mathrm{E}-06$ & $2.7 \mathrm{E}-06$ & $2.6 \mathrm{E}-06$ & $2.6 \mathrm{E}-06$ \\
\hline $\mathrm{S}$ & $5.1 \mathrm{E}-06$ & $4.8 \mathrm{E}-06$ & 4. 3E-06 & 4.0E-06 & $3.6 \mathrm{E}-06$ & $3.5 \mathrm{E}-06$ & $3.5 \mathrm{E}-06$ \\
\hline SSE & $5.0 \mathrm{E}-06$ & $4.7 \mathrm{E}-06$ & $4.2 \mathrm{E}-06$ & $3.9 \mathrm{E}-06$ & $3.6 \mathrm{E}-06$ & $3.5 \mathrm{E}-06$ & $3.4 \mathrm{E}-06$ \\
\hline SE & 1.7E-06 & $1.6 \mathrm{E}-06$ & $1.4 \mathrm{E}-06$ & 1. $3 \mathrm{E}-06$ & $1.2 \mathrm{E}-06$ & $1.2 \mathrm{E}-06$ & $1.1 \mathrm{E}-06$ \\
\hline ESE & $1.0 \mathrm{E}-06$ & $9.3 \mathrm{E}-07$ & $8.4 \mathrm{E}-07$ & $7.7 \mathrm{E}-07$ & $7.0 \mathrm{E}-07$ & $6.8 \mathrm{E}-07$ & $6.7 \mathrm{E}-07$ \\
\hline E & $1.2 \mathrm{E}-06$ & $1.1 \mathrm{E}-06$ & 1. $0 \mathrm{E}-06$ & $9.3 \mathrm{E}-07$ & $8.5 \mathrm{E}-07$ & $8.2 \mathrm{E}-07$ & $8.1 \mathrm{E}-07$ \\
\hline ENE & $1.5 \mathrm{E}-06$ & $1.4 \mathrm{E}-06$ & $1.3 \mathrm{E}-06$ & 1. $2 \mathrm{E}-06$ & $1.1 \mathrm{E}-06$ & $1.1 \mathrm{E}-06$ & 1. $0 \mathrm{E}-06$ \\
\hline NE & $2.6 \mathrm{E}-06$ & $2.4 \mathrm{E}-06$ & $2.2 \mathrm{E}-06$ & 2. $0 \mathrm{E}-06$ & 1. $9 \mathrm{E}-06$ & $1.8 \mathrm{E}-06$ & $1.8 \mathrm{E}-06$ \\
\hline NNE & 1. $5 \mathrm{E}-06$ & $1.4 \mathrm{E}-06$ & 1.2E-06 & $1.1 \mathrm{E}-06$ & 1.0E-06 & 1.0E-06 & 1.0E-06 \\
\hline
\end{tabular}

Distance $(m)$

\begin{tabular}{|c|c|c|c|c|c|c|c|}
\hline Direction & 36721 & 36809 & 37729 & 39079 & 39220 & 39559 & 43584 \\
\hline $\mathrm{N}$ & $6.1 \mathrm{E}-07$ & $6.1 \mathrm{E}-07$ & $5.9 \mathrm{E}-07$ & $5.5 \mathrm{E}-07$ & $5.5 E-07$ & $5.4 \mathrm{E}-07$ & $4.6 \mathrm{E}-07$ \\
\hline NNW & $3.3 \mathrm{E}-07$ & $3.3 \mathrm{E}-07$ & $3.2 \mathrm{E}-07$ & $3.0 \mathrm{E}-07$ & $3.0 \mathrm{E}-07$ & $3.0 \mathrm{E}-07$ & $2.6 \mathrm{E}-07$ \\
\hline NW & $3.9 \mathrm{E}-07$ & $3.8 \mathrm{E}-07$ & $3.7 \mathrm{E}-07$ & $3.5 \mathrm{E}-07$ & $3.5 \mathrm{E}-07$ & $3.4 \mathrm{E}-07$ & $2.9 \mathrm{E}-07$ \\
\hline WNW & $5.1 \mathrm{E}-07$ & $5.1 \mathrm{E}-07$ & $4.9 \mathrm{E}-07$ & $4.6 \mathrm{E}-07$ & $4.6 \mathrm{E}-07$ & $4.5 \mathrm{E}-07$ & $3.8 \mathrm{E}-07$ \\
\hline w & $5.0 \mathrm{E}-07$ & $4.9 \mathrm{E}-07$ & $4.7 \mathrm{E}-07$ & $4.5 \mathrm{E}-07$ & $4.4 \mathrm{E}-07$ & $4.4 \mathrm{E}-07$ & $3.7 \mathrm{E}-07$ \\
\hline WSW & $8.6 \mathrm{E}-07$ & $8.6 \mathrm{E}-07$ & $8.3 \mathrm{E}-07$ & $7.8 \mathrm{E}-07$ & $7.8 \mathrm{E}-07$ & $7.7 \mathrm{E}-07$ & $6.6 \mathrm{E}-07$ \\
\hline SW & $1.6 \mathrm{E}-06$ & $1.6 \mathrm{E}-06$ & $1.5 \mathrm{E}-06$ & $1.4 \mathrm{E}-06$ & $1.4 \mathrm{E}-06$ & $1.4 \mathrm{E}-06$ & 1. $2 \mathrm{E}-06$ \\
\hline SSW & $2.5 \mathrm{E}-06$ & $2.4 \mathrm{E}-06$ & $2.4 \mathrm{E}-06$ & $2.2 \mathrm{E}-06$ & $2.2 \mathrm{E}-06$ & $2.2 \mathrm{E}-06$ & 1. $9 \mathrm{E}-06$ \\
\hline & $3.3 \mathrm{E}-06$ & $3.3 \mathrm{E}-06$ & $3.2 \mathrm{E}-06$ & $3.0 \mathrm{E}-06$ & $3.0 \mathrm{E}-06$ & $2.9 \mathrm{E}-06$ & 2. $5 \mathrm{E}-06$ \\
\hline SSE & $3.3 \mathrm{E}-06$ & $3.3 \mathrm{E}-06$ & $3.1 \mathrm{E}-06$ & $3.0 \mathrm{E}-06$ & $3.0 \mathrm{E}-06$ & $2.9 \mathrm{E}-06$ & $2.5 \mathrm{E}-06$ \\
\hline SE & 1.1E-06 & $1.1 \mathrm{E}-06$ & 1.0E-06 & $9.7 \mathrm{E}-07$ & $9.7 \mathrm{E}-07$ & $9.5 \mathrm{E}-07$ & $8.0 \mathrm{E}-07$ \\
\hline ESE & $6.3 \mathrm{E}-07$ & $6.3 \mathrm{E}-07$ & $6.0 \mathrm{E}-07$ & $5.7 \mathrm{E}-07$ & $5.6 \mathrm{E}-07$ & $5.6 \mathrm{E}-07$ & $4.6 \mathrm{E}-07$ \\
\hline E & $7.7 \mathrm{E}-07$ & $7.7 \mathrm{E}-07$ & $7.3 \mathrm{E}-07$ & $6.9 \mathrm{E}-07$ & $6.8 \mathrm{E}-07$ & $6.7 \mathrm{E}-07$ & $5.6 \mathrm{E}-07$ \\
\hline ENE & $9.9 \mathrm{E}-07$ & $9.9 \mathrm{E}-07$ & $9.5 \mathrm{E}-07$ & $9.0 \mathrm{E}-07$ & $8.9 E-07$ & $8.8 \mathrm{E}-07$ & $7.4 \mathrm{E}-07$ \\
\hline $\mathrm{NE}$ & $1.7 \mathrm{E}-06$ & $1.7 \mathrm{E}-06$ & $1.6 \mathrm{E}-06$ & $1.5 \mathrm{E}-06$ & $1.5 \mathrm{E}-06$ & $1.5 \mathrm{E}-06$ & 1. $3 \mathrm{E}-06$ \\
\hline NNE & $9.5 \mathrm{E}-07$ & $9.5 \mathrm{E}-07$ & $9.1 \mathrm{E}-07$ & $8.6 \mathrm{E}-07$ & $8.6 \mathrm{E}-07$ & $8.5 \mathrm{E}-07$ & 7. $2 \mathrm{E}-07$ \\
\hline
\end{tabular}

$\square$

Feb 20, $2008 \quad 02: 31 \mathrm{pm}$

SUMMARY

Page 6

INDIVIDUAL EFFECTIVE DOSE EQUIVALENT RATE (mrem/y) (A11 Radionuclides and Pathways)

Page 4 
Rn-220C. SUM

\begin{tabular}{|c|c|c|c|c|c|c|}
\hline \multirow[b]{2}{*}{ Direction } & \multicolumn{6}{|c|}{ Distance $(\mathrm{m})$} \\
\hline & 45196 & 45275 & 45654 & 45677 & 46668 & 47969 \\
\hline $\mathrm{N}$ & 4. $3 \mathrm{E}-07$ & 4. $3 \mathrm{E}-07$ & 4. $2 \mathrm{E}-07$ & $4.2 \mathrm{E}-07$ & $4.0 \mathrm{E}-07$ & $3.8 \mathrm{E}-07$ \\
\hline NNW & 2. $4 \mathrm{E}-07$ & 2. $4 \mathrm{E}-07$ & 2. $4 \mathrm{E}-07$ & 2. $4 \mathrm{E}-07$ & 2. $3 \mathrm{E}-07$ & $2.2 \mathrm{E}-07$ \\
\hline NW & $2.7 \mathrm{E}-07$ & $2.7 \mathrm{E}-07$ & $2.7 \mathrm{E}-07$ & $2.7 \mathrm{E}-07$ & $2.6 \mathrm{E}-07$ & $2.5 \mathrm{E}-07$ \\
\hline WNW & $3.6 \mathrm{E}-07$ & $3.6 \mathrm{E}-07$ & $3.5 \mathrm{E}-07$ & $3.5 \mathrm{E}-07$ & $3.4 \mathrm{E}-07$ & $3.2 \mathrm{E}-07$ \\
\hline W & $3.5 \mathrm{E}-07$ & $3.5 \mathrm{E}-07$ & $3.4 \mathrm{E}-07$ & $3.4 \mathrm{E}-07$ & $3.3 \mathrm{E}-07$ & $3.1 \mathrm{E}-07$ \\
\hline WSW & $6.2 \mathrm{E}-07$ & $6.1 \mathrm{E}-07$ & $6.1 \mathrm{E}-07$ & $6.1 \mathrm{E}-07$ & $5.8 \mathrm{E}-07$ & $5.5 \mathrm{E}-07$ \\
\hline SW & 1. $1 \mathrm{E}-06$ & 1. $1 \mathrm{E}-06$ & 1. $1 \mathrm{E}-06$ & $1.1 \mathrm{E}-06$ & $1.1 \mathrm{E}-06$ & 1. $0 \mathrm{E}-06$ \\
\hline SSW & $1.7 \mathrm{E}-06$ & $1.7 \mathrm{E}-06$ & $1.7 \mathrm{E}-06$ & $1.7 \mathrm{E}-06$ & $1.6 \mathrm{E}-06$ & $1.6 \mathrm{E}-06$ \\
\hline $\mathrm{S}$ & 2. $3 \mathrm{E}-06$ & 2. 3E-06 & $2.3 \mathrm{E}-06$ & $2.3 \mathrm{E}-06$ & $2.2 \mathrm{E}-06$ & $2.1 \mathrm{E}-06$ \\
\hline SSE & $2.4 \mathrm{E}-06$ & $2.3 \mathrm{E}-06$ & $2.3 \mathrm{E}-06$ & $2.3 \mathrm{E}-06$ & $2.2 \mathrm{E}-06$ & $2.1 \mathrm{E}-06$ \\
\hline SE & $7.5 \mathrm{E}-07$ & 7. $4 \mathrm{E}-07$ & 7. $3 \mathrm{E}-07$ & 7. $3 \mathrm{E}-07$ & $7.0 \mathrm{E}-07$ & $6.6 \mathrm{E}-07$ \\
\hline ESE & $4.3 \mathrm{E}-07$ & $4.3 \mathrm{E}-07$ & $4.2 \mathrm{E}-07$ & $4.2 \mathrm{E}-07$ & $4.1 \mathrm{E}-07$ & $3.8 \mathrm{E}-07$ \\
\hline $\mathrm{E}$ & $5.2 \mathrm{E}-07$ & $5.2 \mathrm{E}-07$ & $5.1 \mathrm{E}-07$ & $5.1 \mathrm{E}-07$ & $4.9 \mathrm{E}-07$ & $4.6 \mathrm{E}-07$ \\
\hline ENE & $7.0 \mathrm{E}-07$ & $6.9 \mathrm{E}-07$ & $6.8 \mathrm{E}-07$ & $6.8 \mathrm{E}-07$ & $6.6 \mathrm{E}-07$ & $6.2 \mathrm{E}-07$ \\
\hline $\begin{array}{r}\text { NE } \\
\text { NNE }\end{array}$ & 1.2E-06 & 1. $2 \mathrm{E}-06$ & 1.2E-06 & $1.2 \mathrm{E}-06$ & $1.1 \mathrm{E}-06$ & $1.1 \mathrm{E}-06$ \\
\hline NNE & $6.8 \mathrm{E}-07$ & $6.8 \mathrm{E}-07$ & $6.7 \mathrm{E}-07$ & $6.7 \mathrm{E}-07$ & $6.4 \mathrm{E}-07$ & $6.1 \mathrm{E}-07$ \\
\hline
\end{tabular}

Feb 20, 2008 02:31 pm

SUMMARY

Page 7

INDIVIDUAL LIFETIME RISK (deaths)

(A11 Radionuclides and Pathways)

\begin{tabular}{|c|c|c|c|c|c|c|c|}
\hline \multirow[b]{2}{*}{ Direction } & \multicolumn{6}{|c|}{ Distance $(\mathrm{m})$} & \multirow[b]{2}{*}{35683} \\
\hline & 27715 & 28919 & 31060 & 32802 & 34577 & 35279 & \\
\hline $\begin{array}{r}\text { N } \\
\text { NNW } \\
N W \\
W N W \\
W \\
W S W \\
\text { SW } \\
\text { SSW } \\
\text { S } \\
\text { SSE } \\
\text { SE } \\
\text { ESE } \\
\text { E } \\
\text { ENE } \\
\text { NE } \\
\text { NNE }\end{array}$ & $\begin{array}{l}8.1 \mathrm{E}-13 \\
4.3 \mathrm{E}-13 \\
5.0 \mathrm{E}-13 \\
6.6 \mathrm{E}-13 \\
6.5 \mathrm{E}-13 \\
1.1 \mathrm{E}-12 \\
2.1 \mathrm{E}-12 \\
3.2 \mathrm{E}-12 \\
4.4 \mathrm{E}-12 \\
4.3 \mathrm{E}-12 \\
1.4 \mathrm{E}-12 \\
8.6 \mathrm{E}-13 \\
1.0 \mathrm{E}-12 \\
1.3 \mathrm{E}-12 \\
2.2 \mathrm{E}-12 \\
1.2 \mathrm{E}-12\end{array}$ & $\begin{array}{l}7.6 \mathrm{E}-13 \\
4.0 \mathrm{E}-13 \\
4.7 \mathrm{E}-13 \\
6.2 \mathrm{E}-13 \\
6.1 \mathrm{E}-13 \\
1.1 \mathrm{E}-12 \\
1.9 \mathrm{E}-12 \\
3.0 \mathrm{E}-12 \\
4.1 \mathrm{E}-12 \\
4.0 \mathrm{E}-12 \\
1.4 \mathrm{E}-12 \\
8.0 \mathrm{E}-13 \\
9.8 \mathrm{E}-13 \\
1.2 \mathrm{E}-12 \\
2.1 \mathrm{E}-12 \\
1.2 \mathrm{E}-12\end{array}$ & $\begin{array}{l}6.8 \mathrm{E}-13 \\
3.7 \mathrm{E}-13 \\
4.3 \mathrm{E}-13 \\
5.6 \mathrm{E}-13 \\
5.5 \mathrm{E}-13 \\
9.6 \mathrm{E}-13 \\
1.7 \mathrm{E}-12 \\
2.7 \mathrm{E}-12 \\
3.7 \mathrm{E}-12 \\
3.6 \mathrm{E}-12 \\
1.2 \mathrm{E}-12 \\
7.2 \mathrm{E}-13 \\
8.7 \mathrm{E}-13 \\
1.1 \mathrm{E}-12 \\
1.9 \mathrm{E}-12 \\
1.1 \mathrm{E}-12\end{array}$ & $\begin{array}{l}6.3 \mathrm{E}-13 \\
3.4 \mathrm{E}-13 \\
3.9 \mathrm{E}-13 \\
5.2 \mathrm{E}-13 \\
5.1 \mathrm{E}-13 \\
8.8 \mathrm{E}-13 \\
1.6 \mathrm{E}-12 \\
2.5 \mathrm{E}-12 \\
3.4 \mathrm{E}-12 \\
3.3 \mathrm{E}-12 \\
1.1 \mathrm{E}-12 \\
6.6 \mathrm{E}-13 \\
8.0 \mathrm{E}-13 \\
1.0 \mathrm{E}-12 \\
1.7 \mathrm{E}-12 \\
9.7 \mathrm{E}-13\end{array}$ & $\begin{array}{l}5.8 \mathrm{E}-13 \\
3.1 \mathrm{E}-13 \\
3.6 \mathrm{E}-13 \\
4.8 \mathrm{E}-13 \\
4.7 \mathrm{E}-13 \\
8.1 \mathrm{E}-13 \\
1.5 \mathrm{E}-12 \\
2.3 \mathrm{E}-12 \\
3.1 \mathrm{E}-12 \\
3.1 \mathrm{E}-12 \\
1.0 \mathrm{E}-12 \\
6.0 \mathrm{E}-13 \\
7.3 \mathrm{E}-13 \\
9.4 \mathrm{E}-13 \\
1.6 \mathrm{E}-12 \\
9.0 \mathrm{E}-13\end{array}$ & $\begin{array}{l}5.6 \mathrm{E}-13 \\
3.0 \mathrm{E}-13 \\
3.5 \mathrm{E}-13 \\
4.6 \mathrm{E}-13 \\
4.5 \mathrm{E}-13 \\
7.9 \mathrm{E}-13 \\
1.4 \mathrm{E}-12 \\
2.2 \mathrm{E}-12 \\
3.0 \mathrm{E}-12 \\
3.0 \mathrm{E}-12 \\
9.9 \mathrm{E}-13 \\
5.8 \mathrm{E}-13 \\
7.1 \mathrm{E}-13 \\
9.1 \mathrm{E}-13 \\
1.6 \mathrm{E}-12 \\
8.7 \mathrm{E}-13\end{array}$ & $\begin{array}{l}5.5 \mathrm{E}-13 \\
3.0 \mathrm{E}-13 \\
3.5 \mathrm{E}-13 \\
4.5 \mathrm{E}-13 \\
4.5 \mathrm{E}-13 \\
7.8 \mathrm{E}-13 \\
1.4 \mathrm{E}-12 \\
2.2 \mathrm{E}-12 \\
3.0 \mathrm{E}-12 \\
2.9 \mathrm{E}-12 \\
9.7 \mathrm{E}-13 \\
5.7 \mathrm{E}-13 \\
6.9 \mathrm{E}-13 \\
8.9 \mathrm{E}-13 \\
1.5 \mathrm{E}-12 \\
8.5 \mathrm{E}-13\end{array}$ \\
\hline \multicolumn{8}{|c|}{ Distance $(\mathrm{m})$} \\
\hline Direction & 36721 & 36809 & 37729 & $\begin{array}{r}39079 \\
\text { Page }\end{array}$ & $5 \quad 39220$ & 39559 & 43584 \\
\hline
\end{tabular}




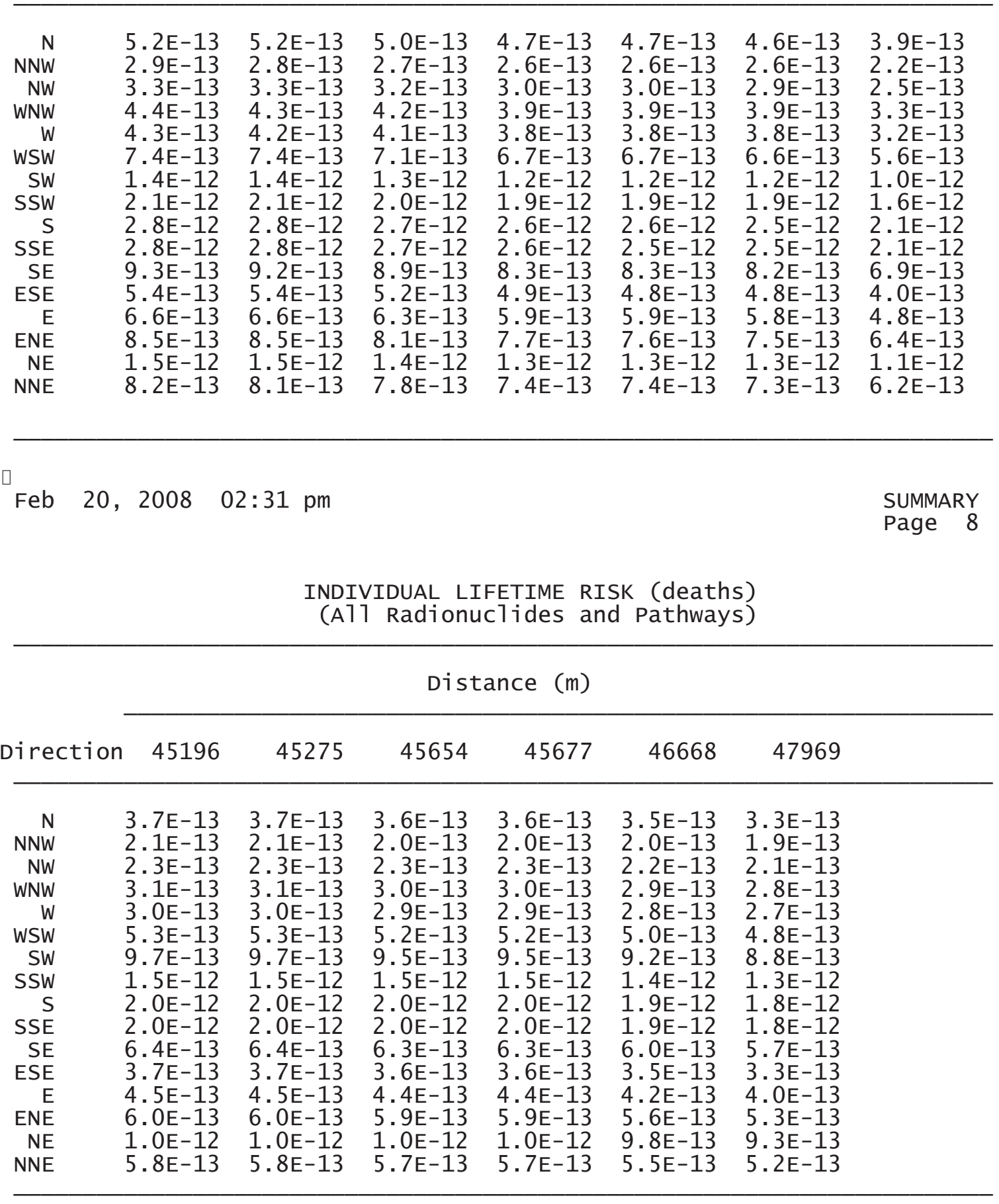




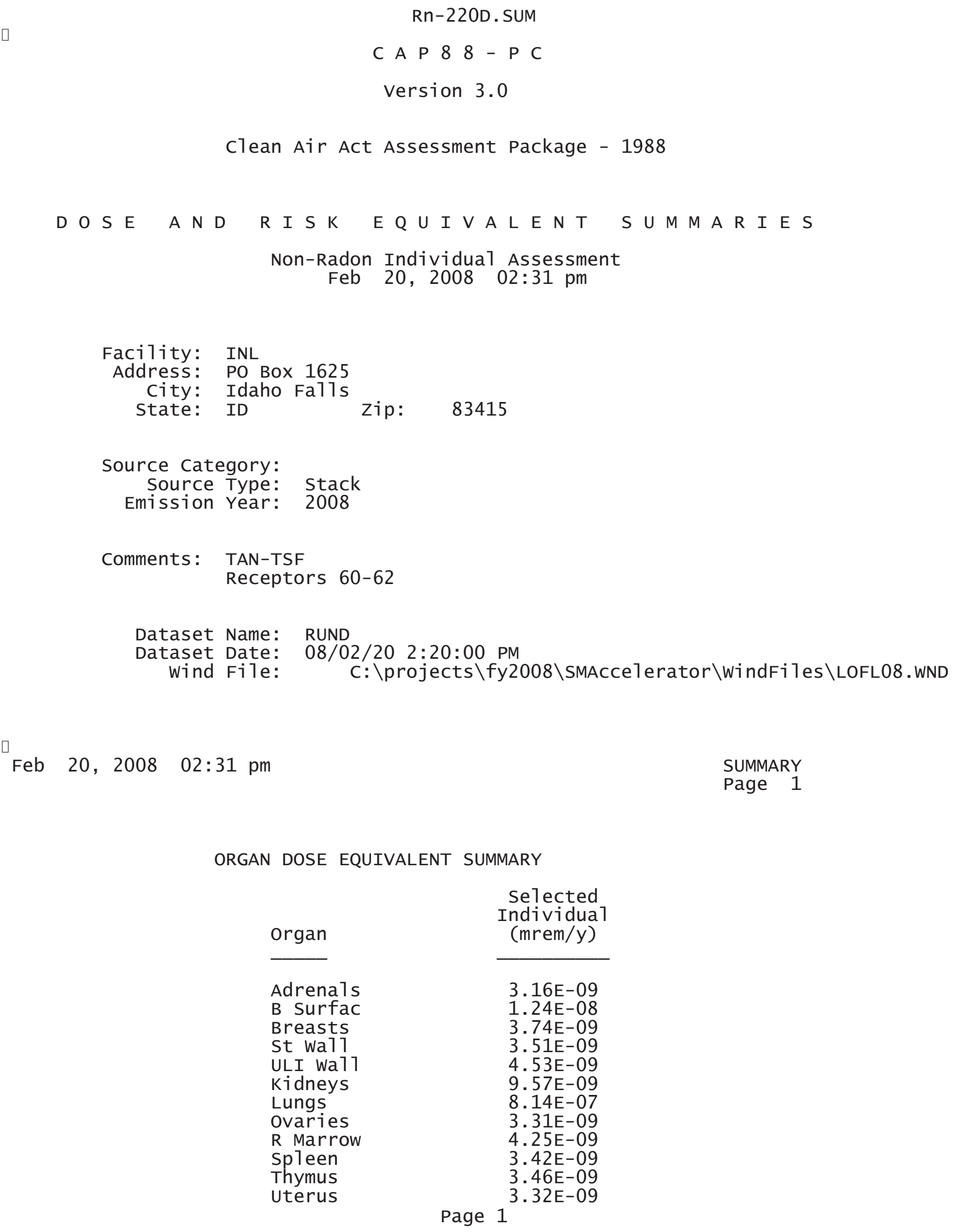




$\begin{array}{lr} & \text { Rn-220D. SUM } \\ \text { B1d wa11 } & 3.61 \mathrm{E}-09 \\ \text { Brain } & 3.44 \mathrm{E}-09 \\ \text { Esophagu } & 3.02 \mathrm{E}-07 \\ \text { SI wa11 } & 3.65 \mathrm{E}-09 \\ \text { LLI wa11 } & 4.76 \mathrm{E}-09 \\ \text { Liver } & 5.04 \mathrm{E}-09 \\ \text { Muscle } & 3.77 \mathrm{E}-09 \\ \text { Pancreas } & 3.23 \mathrm{E}-09 \\ \text { Skin } & 2.06 \mathrm{E}-08 \\ \text { Testes } & 3.82 \mathrm{E}-09 \\ \text { Thyroid } & 3.51 \mathrm{E}-09 \\ & \\ \text { EFFEC } & 1.97 \mathrm{E}-06\end{array}$

PATHWAY EFFECTIVE DOSE EQUIVALENT SUMMARY

\begin{tabular}{lcc} 
Pathway & $\begin{array}{c}\text { Selected } \\
\text { Individua1 } \\
\text { (mrem/y) }\end{array}$ \\
\cline { 2 - 3 } & $2.56 \mathrm{E}-14$ \\
INGESTION & $1.97 \mathrm{E}-06$ \\
INHALATION & $4.18 \mathrm{E}-10$ \\
AIR IMMERSION & $3.05 \mathrm{E}-09$ & \\
GROUND SURFACE & $3.97 \mathrm{E}-06$ & \\
INTERNAL & & \\
EXTERNAL & $1.97 \mathrm{E}-06$ & \\
TOTAL & & SUMMARY \\
pm & & Page 2
\end{tabular}

NUCLIDE EFFECTIVE DOSE EQUIVALENT SUMMARY

Nuclide

$\mathrm{Rn}-220$

Po-216

$\mathrm{Pb}-212$

$\mathrm{Bi}-212$

Po-212

$\mathrm{T} 1-208$

TOTAL

$$
\begin{gathered}
\begin{array}{c}
\text { selected } \\
\text { Individual } \\
\text { (mrem/y) }
\end{array} \\
\hline 2.77 \mathrm{E}-12 \\
4.71 \mathrm{E}-14 \\
1.94 \mathrm{E}-06 \\
2.75 \mathrm{E}-08 \\
0.00 \mathrm{E}+00 \\
2.41 \mathrm{E}-09 \\
1.97 \mathrm{E}-06
\end{gathered}
$$

Feb 20, $2008 \quad 02: 31 \mathrm{pm}$

SUMMARY

Page 3

CANCER RISK SUMMARY 


\begin{tabular}{lc} 
Cancer & $\begin{array}{c}\text { Rn-220D.SUM } \\
\text { Total Lifetime } \\
\text { Fatal Cancer Risk }\end{array}$ \\
\cline { 2 - 2 } $\begin{array}{l}\text { Esophagu } \\
\text { Stomach }\end{array}$ & $1.16 \mathrm{E}-16$ \\
Colon & $6.21 \mathrm{E}-16$ \\
Liver & $8.00 \mathrm{E}-15$ \\
LUNG & $7.26 \mathrm{E}-16$ \\
Bone & $1.68 \mathrm{E}-12$ \\
Skin & $2.32 \mathrm{E}-16$ \\
Breast & $2.78 \mathrm{E}-17$ \\
Ovary & $3.63 \mathrm{E}-16$ \\
Bladder & $1.51 \mathrm{E}-16$ \\
Kidneys & $2.69 \mathrm{E}-16$ \\
Thyroid & $7.79 \mathrm{E}-16$ \\
Leukemia & $3.52 \mathrm{E}-17$ \\
Residua1 & $2.82 \mathrm{E}-16$ \\
Total & $1.62 \mathrm{E}-15$ \\
TotAL & $1.69 \mathrm{E}-12$ \\
& $3.39 \mathrm{E}-12$
\end{tabular}

PATHWAY RISK SUMMARY

\begin{tabular}{|c|c|}
\hline Pathway & $\begin{array}{l}\text { Total Lifetime } \\
\text { Fatal Cancer Risk }\end{array}$ \\
\hline $\begin{array}{l}\text { INGESTION } \\
\text { INHALATION } \\
\text { AIR IMMERSION } \\
\text { GROUND SURFACE } \\
\text { INTERNAL } \\
\text { EXTERNAL }\end{array}$ & $\begin{array}{l}2.55 \mathrm{E}-20 \\
1.69 \mathrm{E}-12 \\
2.23 \mathrm{E}-16 \\
1.62 \mathrm{E}-15 \\
1.69 \mathrm{E}-12 \\
1.84 \mathrm{E}-15\end{array}$ \\
\hline TOTAL & $1.69 \mathrm{E}-12$ \\
\hline
\end{tabular}

Feb 20, 2008 02:31 pm

SUMMARY Page 4

NUCLIDE RISK SUMMARY

Nuclide

$\mathrm{Rn}-220$

Po-216

$\mathrm{Pb}-212$

Bi -212

Po-212

$\mathrm{T} 7-208$

TOTAL
Selected Individual

Total Lifetime Fatal Cancer Risk

1. $51 \mathrm{E}-18$

2. $58 \mathrm{E}-20$

$1.68 \mathrm{E}-12$

$1.76 \mathrm{E}-14$

$0.00 \mathrm{E}+00$

1. $32 \mathrm{E}-15$

1. $69 \mathrm{E}-12$

Feb 20, 2008 02:31 pm 
Rn-220D.SUM

Page 5

INDIVIDUAL EFFECTIVE DOSE EQUIVALENT RATE (mrem/y)

(A11 Radionuclides and Pathways)

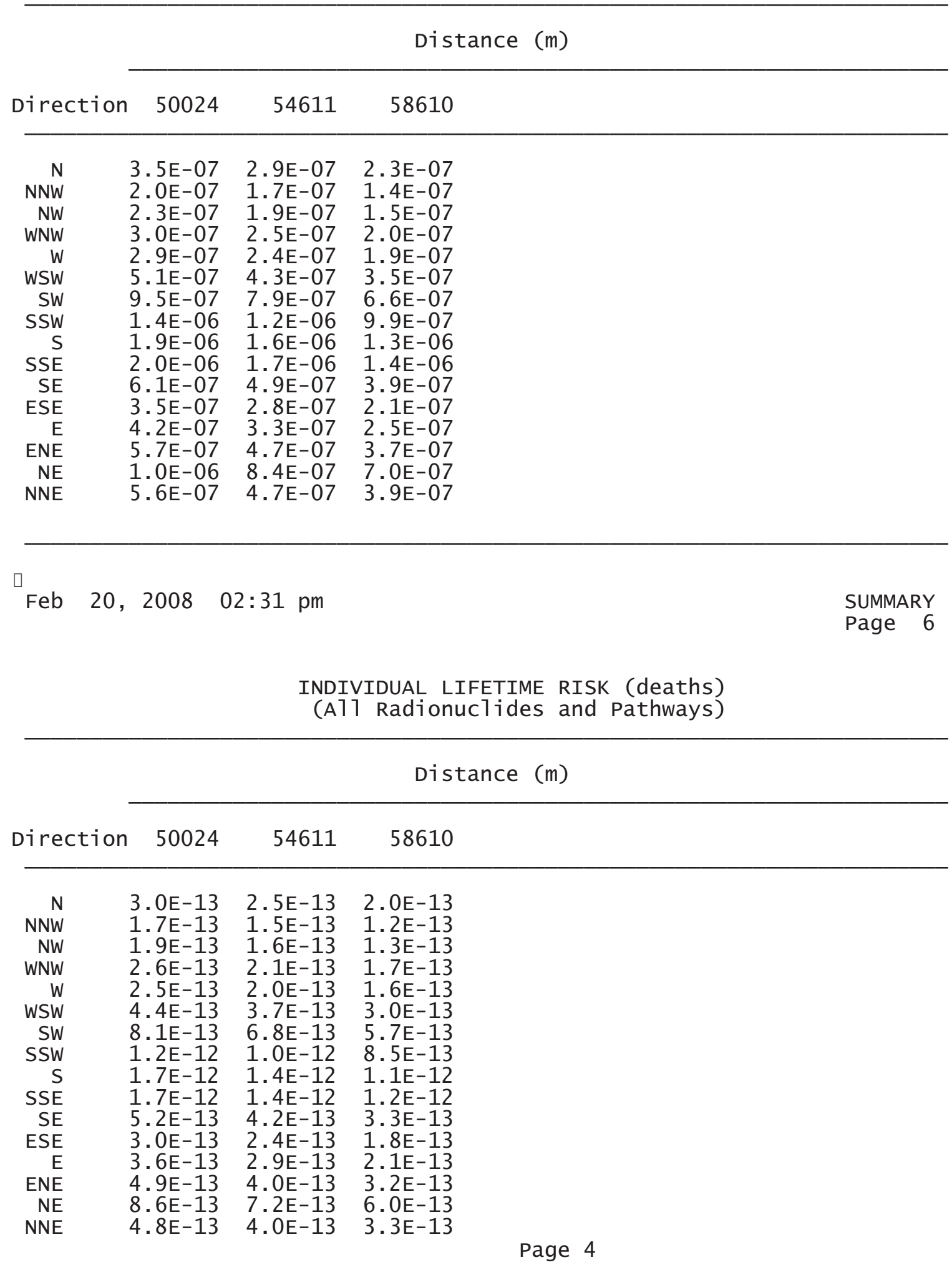


Rn-220D. SUM

Page 5 